\title{
SELECTED FLOW CHARACTERISTICS OF STREAMS IN THE \\ WILLAMETTE RIVER BASIN, OREGON
}

Flow Duration

Annual Low Flow

Annual High Flow

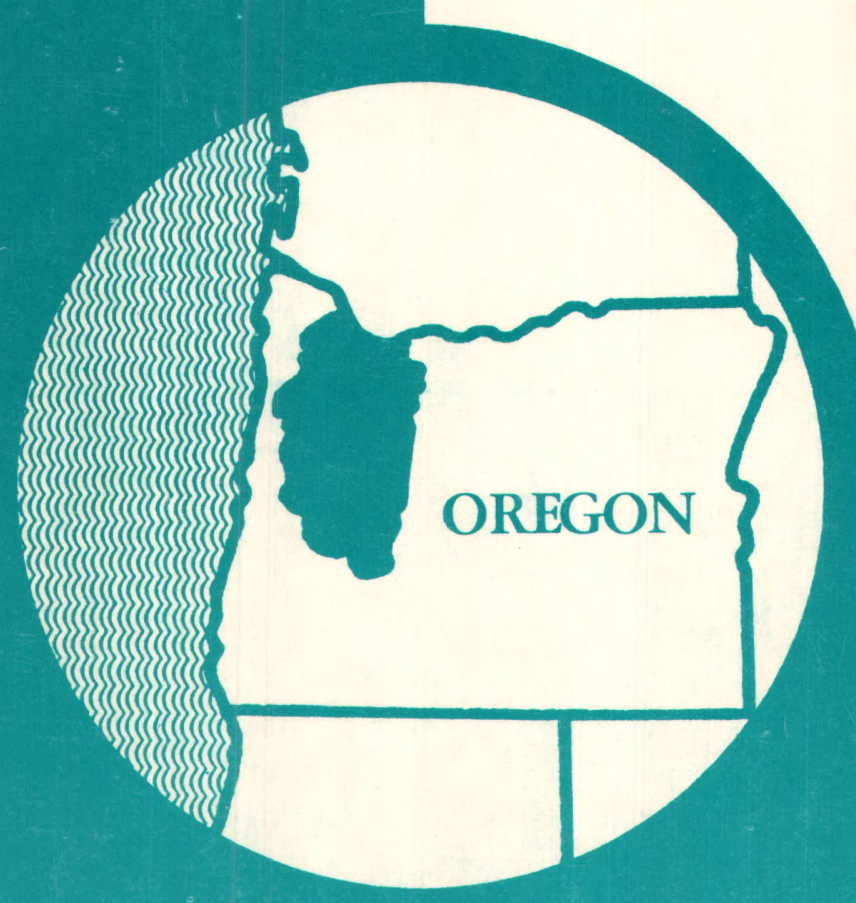

OPEN-FILE REPORT

U.S. Department of the Interior

Geological Survey

Water Resources Division

Portland, Oregon

1966 


\author{
UNITED STATES \\ DEPARTMENT OF THE INTERIOR \\ GEOLOGICAL SURVEY \\ WATER RESOURCES DIVISION
}

SELECTED FLOW CHARACTERISTICS OF STREAMS

IN THE WILLAMETTE RIVER BASIN, OREGON

By

C. H. Swift III

Data derived from streamflow records

crllected in cooperation with:

Oregon State Engineer

City of Portland, Bureau of Water Works City of Eugene, Water and Electric Board

U.S. Corps of Engineers

U.S. Bureau of Reclamation

Portland General Electric Company

other local and Federal agencies 
Abstract

Introduction

Purpose

Scope -

Data in the report

Selection of discharge records

Uses of the data

Explanation of summaries

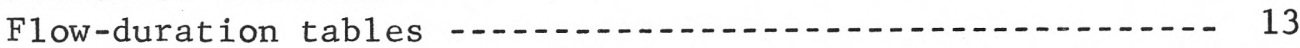

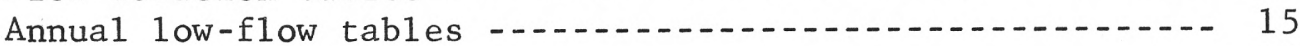

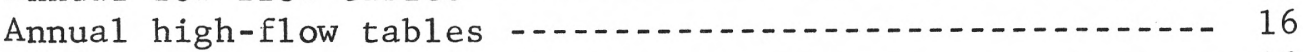

Techniques for using summary data

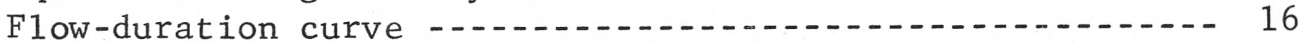

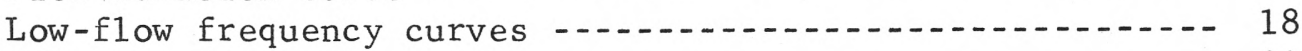

High-flow frequency curves

Selected bibliography -

Oregon streamflow records computer processed

Sandy River basin

Sandy River:

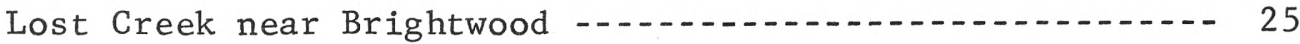

Little Zigzag River near Rhododendron -............ 26

Zigzag River at Rhododendron -....................... 27

Stil1 Creek at Rhododendron -.................. 27

Sandy River above Salmon River, at Brightwood -........... 28

Salmon River near Government Camp _.......... 29

Salmon River below Linney Creek _.................. 30

Salmon River at Welches _........................... 32

Salmon River above Boulder Creek, near Brightwood -.....- 33

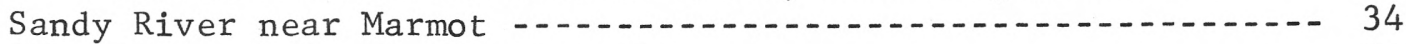

Bul1 Run River near Bul1 Run ......................... 36

Little Sandy River near Marmot ................... 39

Little Sandy River near Bul1 Run ................ 40

Sandy River below Bull Run River, near Bull Run .... 42

Willamette River basin

Middle Fork Willamette River near Oakridge -............ 44

Hills Creek above Hills Creek Reservoir, near Oakridge --- 44

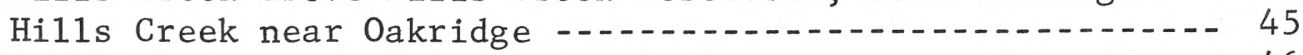

Middle Fork Willamette River above Salt Creek, near Oakridge -- 46

Salt Creek near Oakridge ........ 48

Salmon Creek near Oakridge

North Fork of Middle Fork Willamette River near Oakridge - 50

Middle Fork Willamette River below North Fork, near Oakridge -- 52

Middle Fork Willamette River near Dexter ....... 54

Fal1 Creek above Winberry Creek, near Lowe11 _...... 55 
Oregon streamflow records computer processed--Continued

Willamette River basin--Continued

Fa11 Creek below Winberry Creek, near Fall Creek -......- 56

Little Fal1 Creek near Fall Creek ............ 58

Middle Fork Willamette River at Jasper

Coast Fork Willamette River at London ...... 60

Coast Fork Willamette River below Cottage Grove Dam -...- 62

Row River above Pitcher Creek, near Dorena -...... 64

Row River near Cottage Grove

Mosby Creek near Cottage Grove -.............. 68

Mosby Creek at mouth, near Cottage Grove -.... 69

Coast Fork Willamette River at Saginaw -....... 70

Coast Fork Willamette River near Goshen ........ 72

Willamette River at Springfield _... 74

McKenzie River at outlet of Clear Lake -...... 76

McKenzie River near Belknap Springs -........ 77

McKenzie River at McKenzie Bridge . 78

South Fork McKenzie River above Cougar Reservoir, near Rainbow -.......... 81

South Fork McKenzie River near Rainbow -......... 82

B1ue River above Quentin Creek _..................... 83

Lookout Creek near Blue River -.............. 84

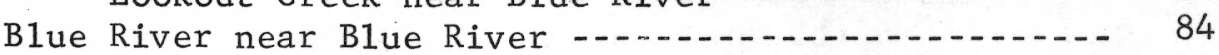

McKenzie River near Vida

Gate Creek at Vida

McKenzie River near Springfield _....... 89

Mohawk River near Springfield _.................. 90

McKenzie River near Coburg -

Willamette River at Harrisburg - 93

Long Tom River near Noti

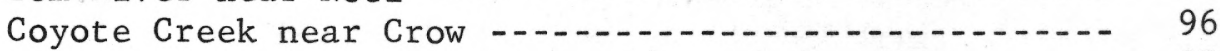

Amazon Creek near Eugene -

Long Tom River at Monroe - 98

Marys River:

Rock Creek near Philomath _... 100

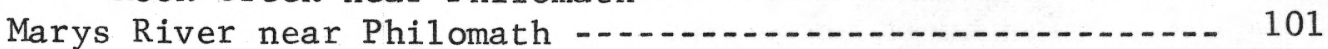

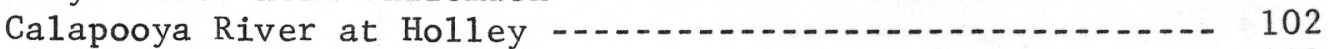

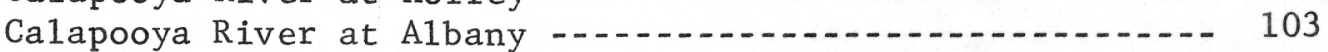

Willamette River at Albany - 105

Santiam River:

North Santiam River below Boulder Creek, near Detroit -.-- 109

Breitenbush River above Canyon Creek, near Detroit -- 110

North Santiam River at Niagara _.......... 112

Little North Santiam River near Mehama ........... 114 


\section{CONTENTS}

Oregon streamflow records computer processed--Continued

Page

Willamette River basin--Continued

North Santiam River at Mehama

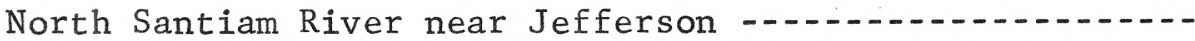

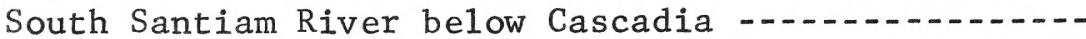

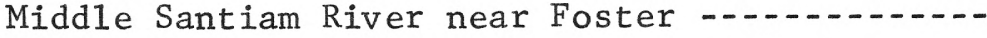

Middle Santiam River at mouth, near Foster ----

Wiley Creek near Foster

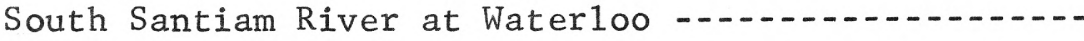

Santiam River at Jefferson

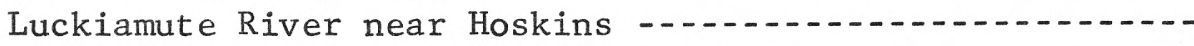

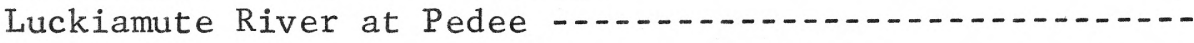

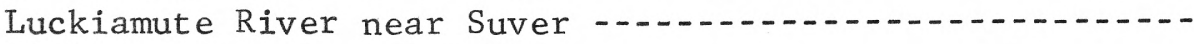

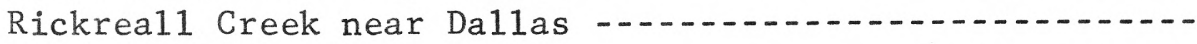

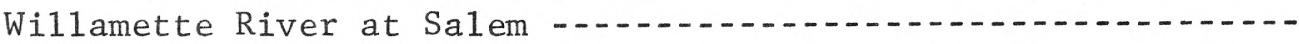

Mil1 Creek at penitentiary annex, near Salem -........

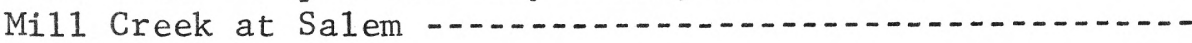

Yamhil1 River:

South Yamhil1 River near Willamina -

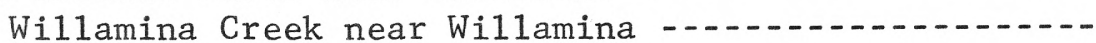

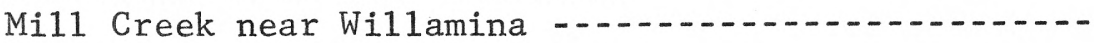

South Yamhil1 River near Whiteson

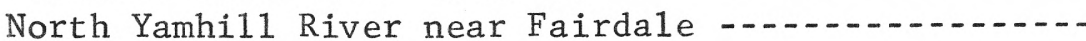

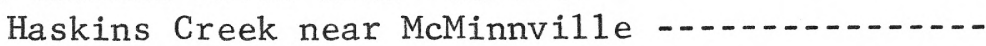

Haskins Creek below reservoir, near

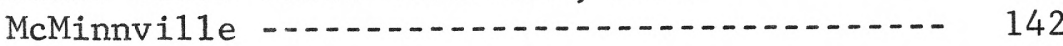

North Yamhill River near Pike .................. 143

North Yamhil1 River at Pike -................... 144

Willamette River at Wilsonville

Mola1la River above Pine Creek, near Wilhoit -......-. 146

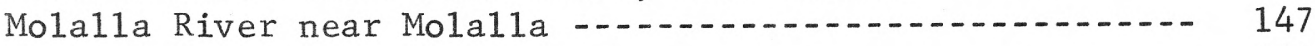

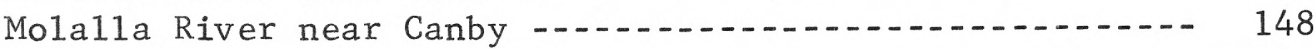

Pudding River near Mount Angel _................... 149

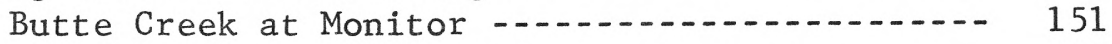

Pudding River at Aurora ............................ 152

Tualatin River at Gaston -... 154

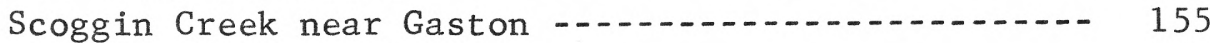

Tualatin River near Dilley _........................... 157

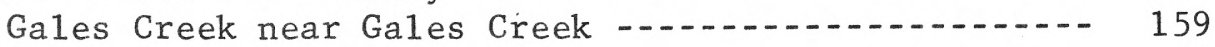

Gales Creek near Forest Grove .................. 160

Dairy Creek:

East Fork Dairy Creek at Mountaindale -...-.-. 161

McKay Creek near North Plains ............. 162

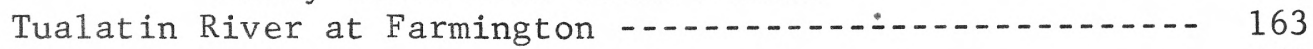

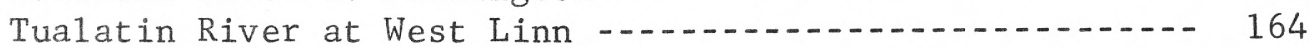


Oregon streamflow records computer processed--Continued

Willamette River basin--Continued

Clackamas River at Big Bottom -...... 166

Oak Grove Fork at Timothy Meadows _................ 168

Oak Grove Fork near Government Camp -............ 169

Oak Grove Fork above powerplant intake -...... 170

Clackamas River above Three Lynx Creek -.............- 172

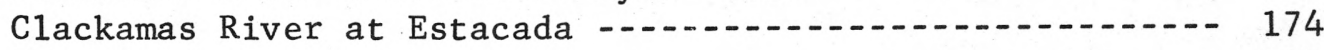

Johnson Creek at Sycamore

\section{ILLUSTRATIONS}

Figure 1. Map of Willamette and Sandy River basins showing

$$
\text { locations of stream-gaging stations -........... } 9
$$

2. Bar chart of complete years of computer-processed records - 10

3. Illustrated explanation of summaries - 14

4. Duration curve of daily flow

5. Low-flow and high-flow frequency curves

\section{TABLES}

Table 1A. Ranking of annual low flows for selected periods of

Page consecutive days for Fa11 Creek below Winberry Creek,

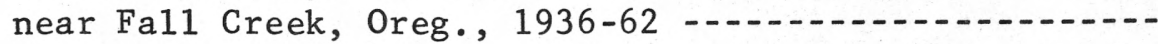

1B. Ranking of annual high flows for selected periods of consecutive days for Fal1 Creek below Winberry Creek,

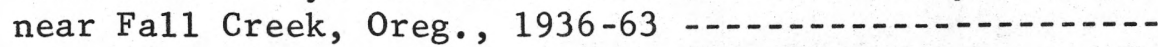

2. Arithmetic values of linear measurement (X) for selected recurrence intervals (R.I.) 


\title{
SELECTED FLOW CHARACTERISTICS OF STREAMS \\ IN THE WILLAMETTE RIVER BASIN, OREGON
}

\author{
By C. H. Swift III
}

\begin{abstract}
Flow-duration, annual low-flow, and annual high-flow tables through September 30, 1963, are given in this report for 110 stream-gaging stations in the Willamette and Sandy River basins. These tables summarize the basic data needed to define the streamflow characteristics at the gaging stations. The content of each of the three summary tables is described, and techniques for preparing flow-duration curves, low-flow frequency curves, and high-flow frequency curves are explained.
\end{abstract}

\section{INTRODUCTION}

\section{Purpose}

This report presents three summaries of streamflow records by years--flow duration, low flow, and high flow. These summaries provide a common foundation for the analyses of flow characteristics of streams within the Willamette and Sandy River basins. Technique for using each of the summaries to determine flow characteristics is explained and illustrated.

\section{$\underline{\text { Scope }}$}

This report covers an area of about 12,000 square miles in northwestern Oregon. The area is bounded by the Columbia River on the north, the Cascade Range on the east, the Calapooya Mountains on the south, and the Coast Range on the west.

The first part of this report explains concepts, record selection, the three summaries, and techniques for using the summaries. The second part of the report presents the summaries (flow duration, annual low flow, and annual high flow) for 110 gaging stations.

An electronic computer was used to process all summaries for this report except those for Still Creek at Rhododendron and North Santiam River near Jefferson. The discharge record at Rhododendron was partly estimated and the summaries were prepared manually. Separate preparation of the annual low-flow summary for the station near Jefferson was necessary because high flows had not been recorded. In addition, flow- 
duration summary tables for periods of regulated and unregulated flows at some stations were prepared manually.

The report was prepared under the general direction of $R$. B. Sanderson, district engineer, Surface Water Branch, Water Resources Division, U.S. Geological Survey; and A. R. Leonard, chairman, Hydrology Committee of the Willamette Basin Task Force.

DATA IN THE REPORT

\section{Selection of Discharge Records}

The summary data in this report were derived from records of daily discharge for gaging stations in the Willamette River basin. Those records have been collected and published by the U.S. Geological Survey in cooperation with the State of Oregon and other agencies since 1896. Certain qualifications were established to select discharge records for processing into summary data. The qualifications are as follows:

1. Records of streamflow only--excluding canal, ditch, lake, and reservoir records.

2. Complete years of daily discharge.

3. A minimum of 5 years of record.

4. Records useful in streamflow analysis--generally, but not always, excluding records entirely composed of regulated flow.

The published discharge record for some stations has been supplemented with unpublished daily discharges to increase the length of record. These unpublished data were used only if they had previously been used to determine published monthly mean discharges. These unpublished daily discharges and those of low flows for the station, North Santiam River near Jefferson, are available in the files of the U.S. Geological Survey, Water Resources Division, Portland.

The location of each gaging station for which the record was selected for processing into summaries is shown on figure 1 by a triangle accompanied by the station number. The station number is a combination identification and order number that refers to a national numbering system; the number and station name are shown on each summary.

Figure 2 includes a bar chart to show complete years of processed record at each gaging station, the number and name of each gaging station in downstream order (the same order in which the summaries are presented), and the size of the drainage area at each station. A total of 2,514 years of processed record is given in the summaries. 


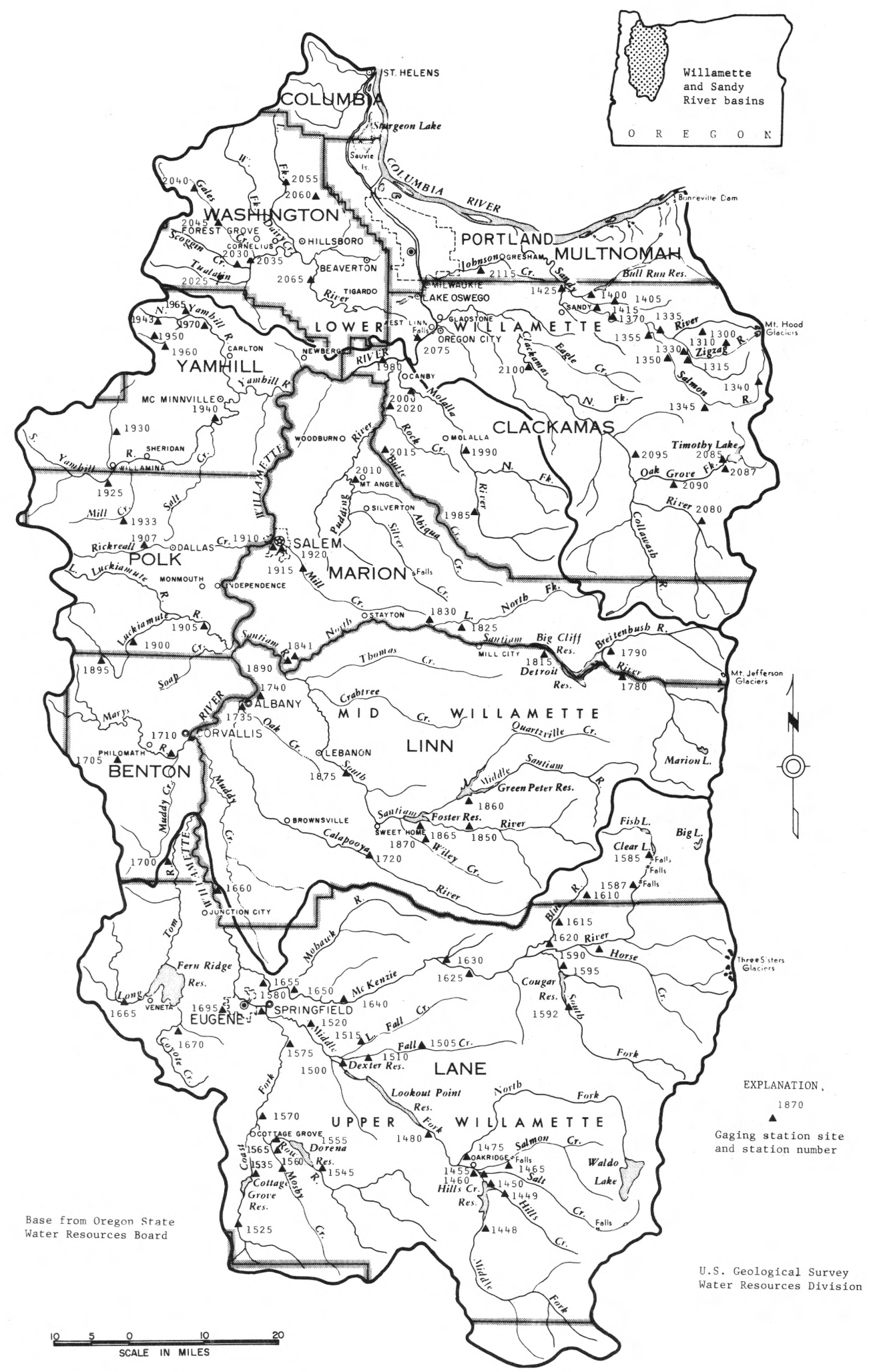

Figure 1.--Location of stream-gaging stations in the Willamette and Sandy River basins. 


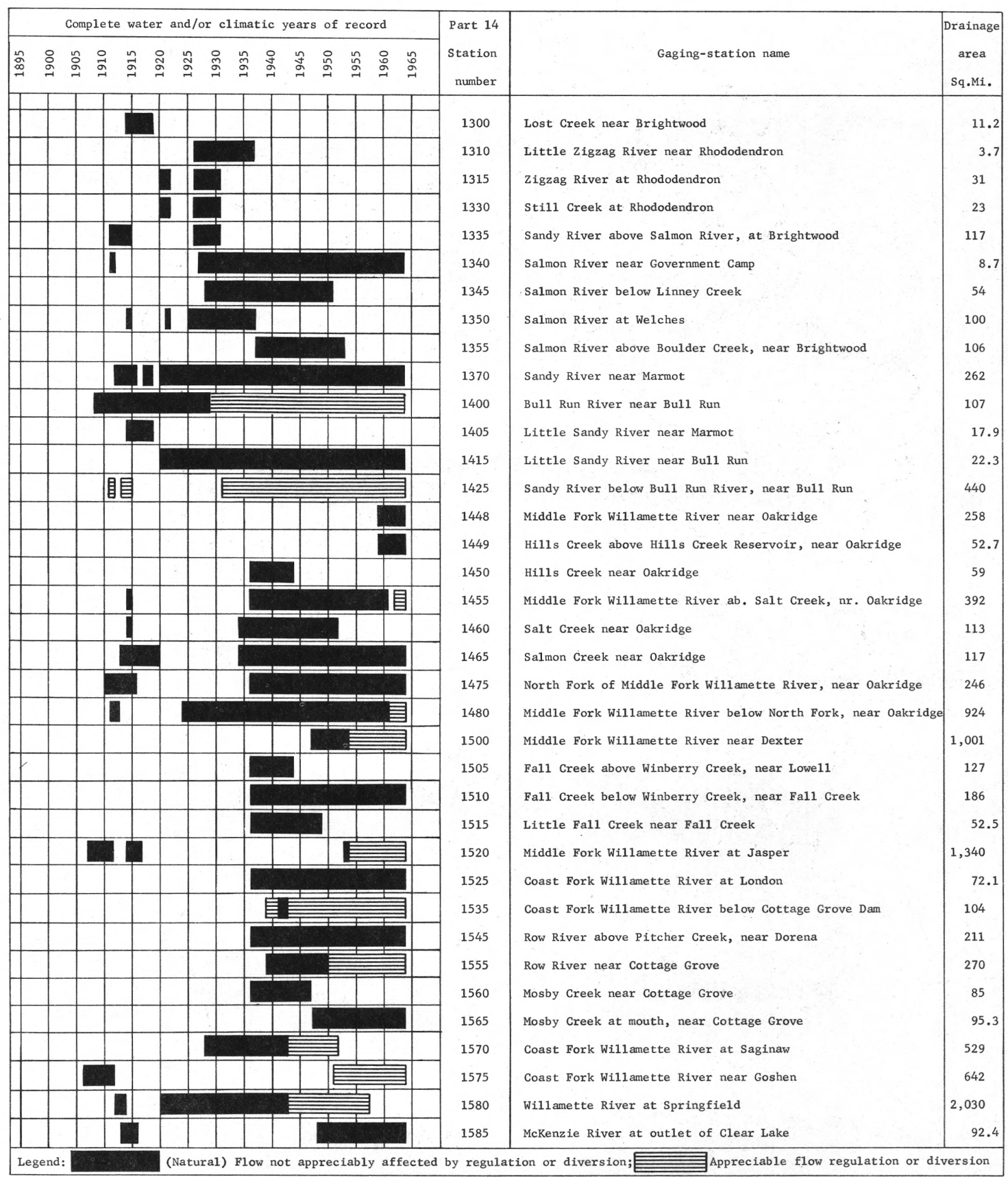

Figure 2.--Bar chart of complete years of processed record at gaging stations. 


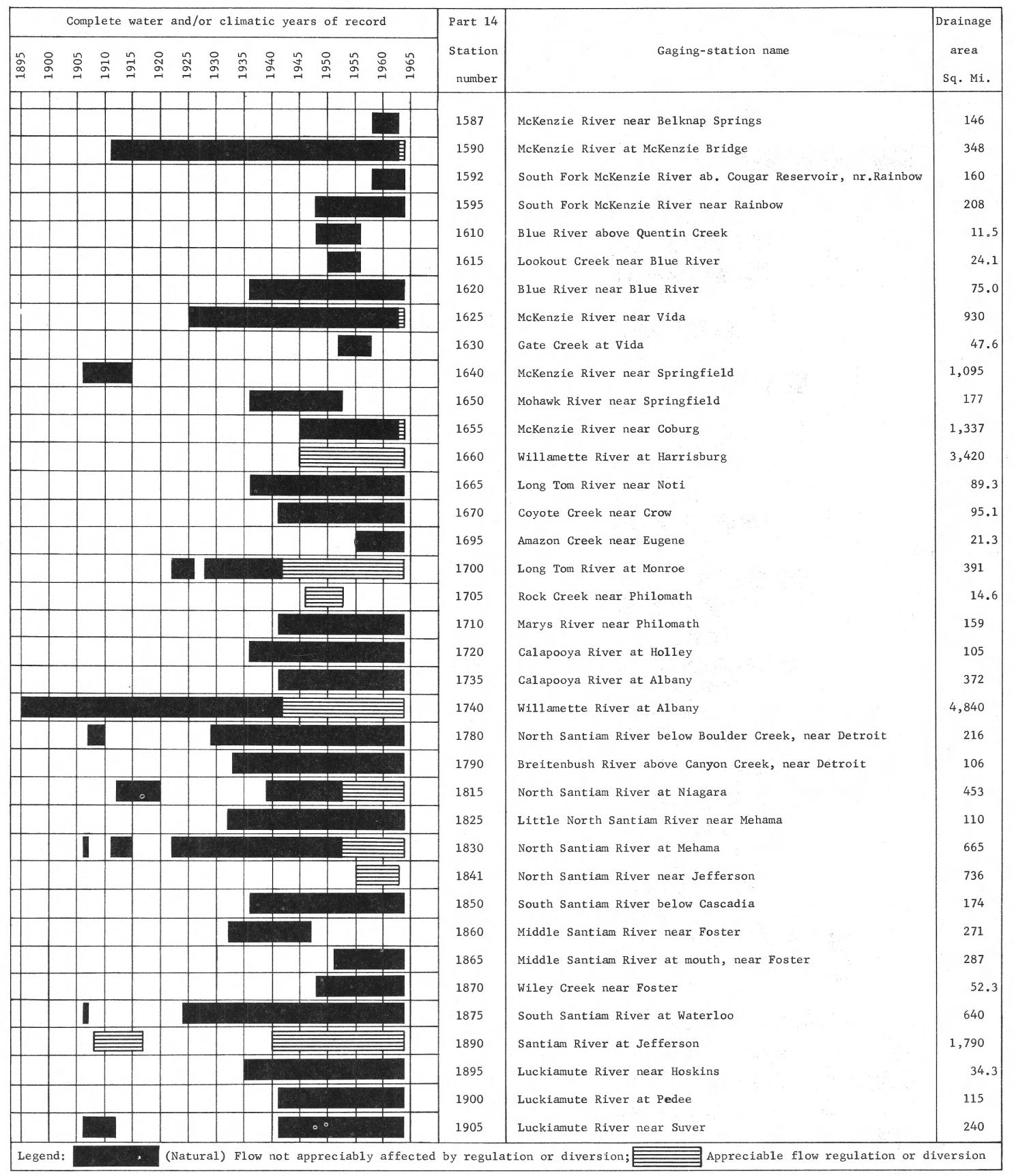

Figure 2.--(Continued) Bar chart of complete years of processed record at gaging stations. 


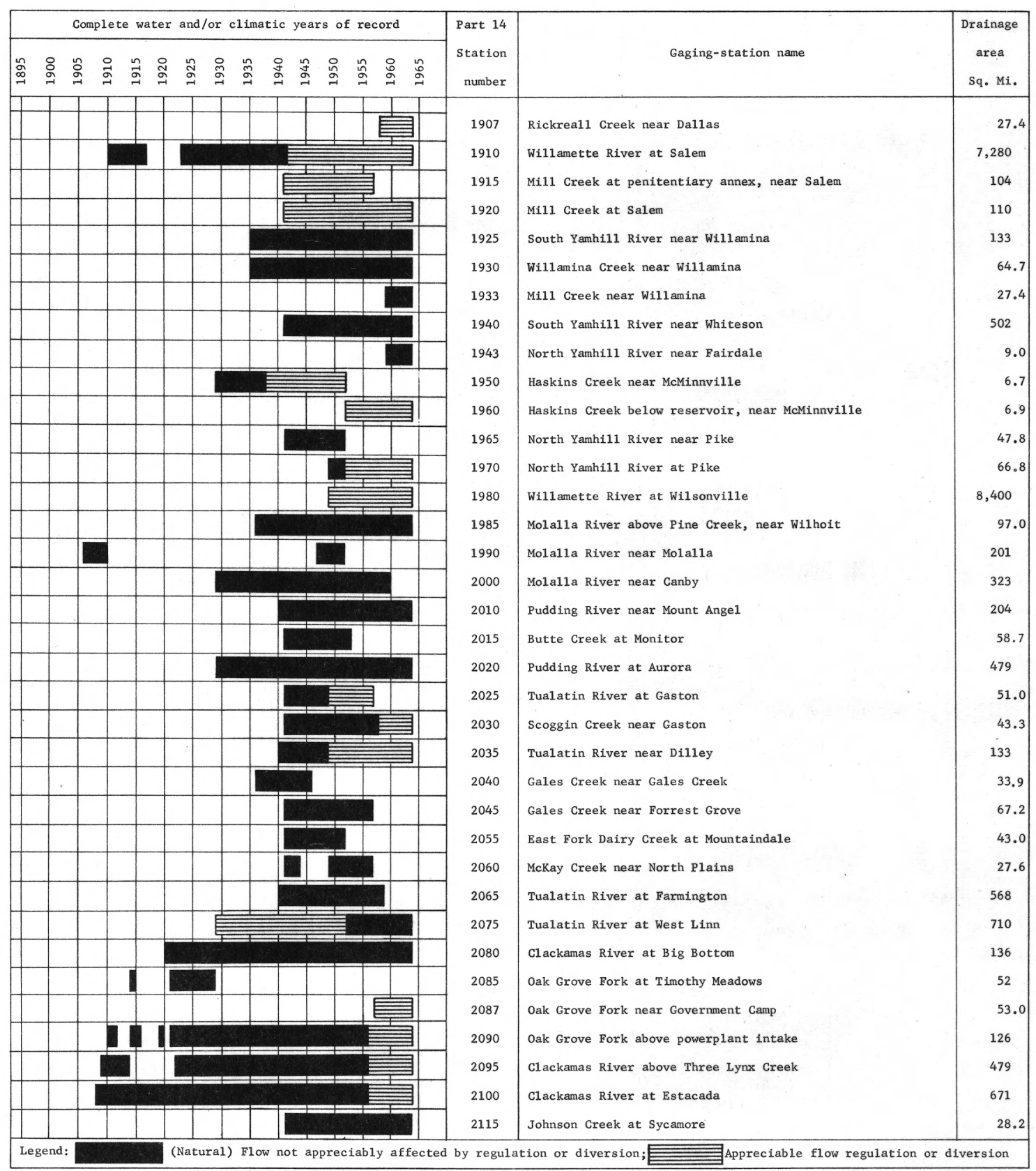

Figure 2.--(Continued) Bar chart of complete years of processed record at gaging stations. 
The descriptions of the gaging stations selected for this report are in the water-supply papers and open-file reports 1 isted under U.S. Geological Survey in the selected bibliography. They should be consulted by users of the summaries because the remarks paragraph of the descriptions contains information pertaining to the accuracy of the records and conditions affecting natural flow. That information is important because records that were processed into summaries for some of the stations are composed of separate periods of regulated and unregulated flows. Al'so included in each description are location, drainage area, records available, station names under which the record may have been previously pub1ished, type of equipment, datum, average discharge, and recorded minimum and maximum instantaneous discharges.

\section{Uses of the Data}

The comparison and study of the flow characteristics at different gaging stations provide the basis for analysis and appraisal of the streamflow resource. The three summaries for each gaging station contain the basic data which are most frequently used in analyses of streamflow. Means, medians, frequency and probability distributions, and variability are flow characteristics that can be determined from the summary data by statistical methods. The techniques for using the data contained in the summaries, which are described in this report, are those used by the U.S. Geological Survey. Other agencies often use different techniques in making similar types of analyses.

\section{Explanation of Summaries}

\section{Flow-Duration Tables}

The first summary that is presented for each gaging station consists of two related tables--the annual flow-duration table and the flow-duration summary table. The annual flow-duration table, illustrated at the top of figure 3, shows the number of daily discharges in as many as 34 different classes for each water year (year ending September 30 ). This table portrays the annual range, distribution, and variability of flows for the period of record. The first column lists water years, and other column headings across the table are numbers that designate classes of discharge. The class limit of each class is the lowest discharge that would be contained in that class and is listed, in cubic feet per second, in the flow-duration summary table. The body of the table shows the number of days that the daily discharge equaled or exceeded the class limit of each class but was less than the class limit of the next higher class. The last column in the annual table is the sum of all daily discharges for each water year and is used primarily as a check on the electronic computer, although it can be used to compute the mean discharge for the year. 
Wiley Creek near Foster, Oreg. Station name NTATION NUMRER 14-1870.00

DURATION TABLE OF DAILY DISCHARGE

ANNUAL FLOW-DURATION TABLE

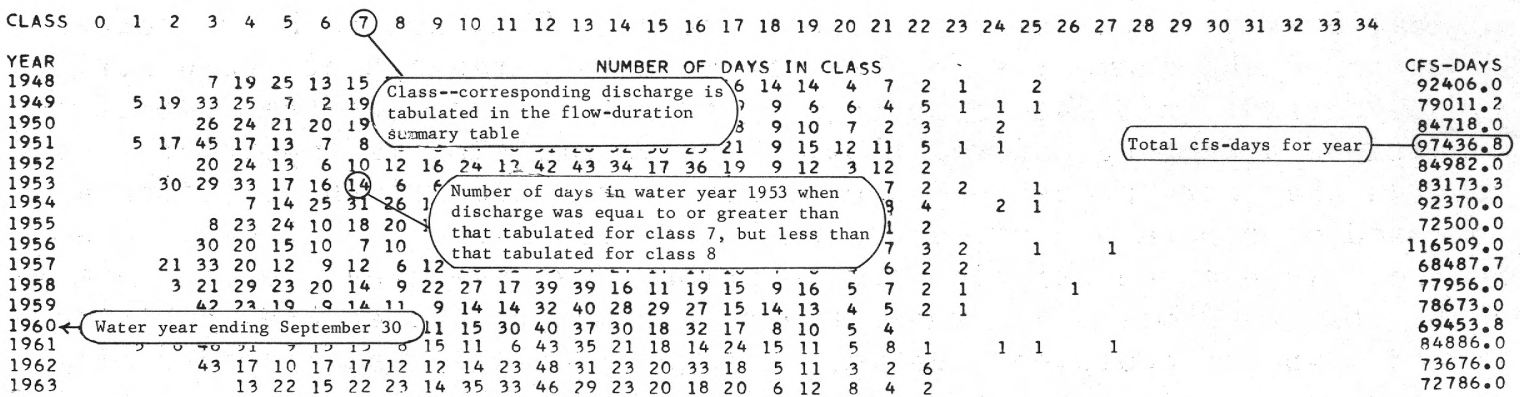

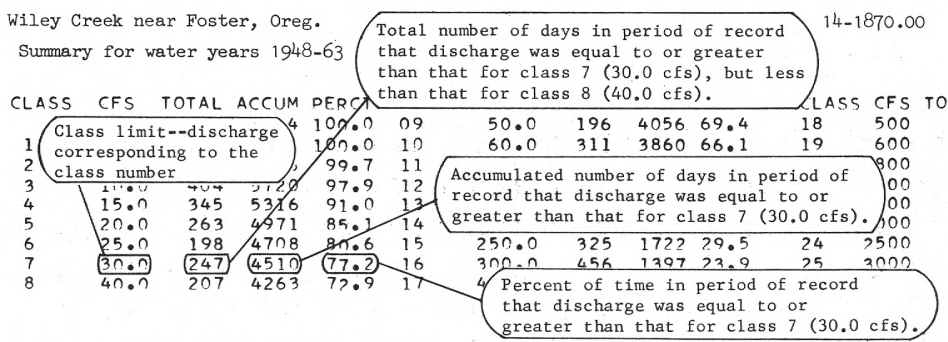

W'iley Creek near Foster, Oreg.

STATION NUMBER $14-1870.00$

FLOW-DURATION SUMMARY TABLE

LOWEST MEAN DISCHARGE, IN CFS, FOR THE FOLLOWING NUMBER OF CONSECUTIVE DAYS IN YEAR BEGINNING APRIL I

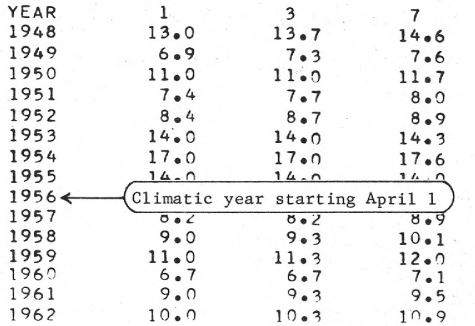

Wiley Creek near Foster, Oreg.

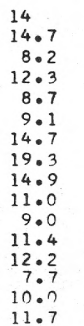

STATION NUMBER
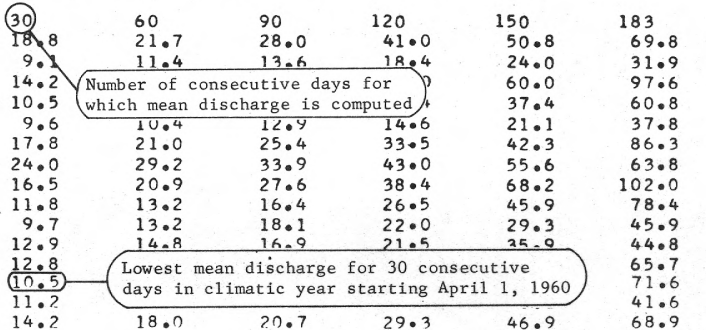

274

112.0

236.0

64.0

86.4

217.0

119.0

295.0

124.0

142.0

140.0

97.9
152.0

152.0

HIGHEST MEAN DISCHARGF, IN CFS, FOR THF FOLLOWING NUMBER OF CONSFCUTIVF DAYS IN YEAR ENDING SEPTEMBER 30

\begin{tabular}{|c|c|c|c|c|c|c|c|c|c|c|c|}
\hline YEAR & 1 & 3 & 7 & 15 & (30) & 60 & 90 & 120 & 150 & 183 & 274 \\
\hline 1948 & 3620.0 & 2730.0 & 1790.0 & 1090.0 & 684.0 & 543.0 & 481.0 & $428 \cdot 0$ & 422.0 & 398.0 & 327.0 \\
\hline 1949 & 3310.0 & $2300 \cdot 0$ & 1740.0 & 1130.0 & & 524.0 & 455.0 & $437 \cdot 0$ & 407.0 & 387.0 & 284.0 \\
\hline 1950 & $27 a n . n$ & 2.02n:n & 12000 & 806.0 & 660.0 & Number of & consecutive $d$ & ys for & 449.0 & 413.0 & 302.0 \\
\hline 19514 & Water yea & ending $S$ & mber 30$)$, & 942.0 & 821.0 & which mean & discharge is & computed & 524.0 & 476.0 & 351.0 \\
\hline 1952 & 1310.0 & $1420 \cdot n$ & 1220.0 & 826.0 & 588.0 & $4+400 \mathrm{~V}$ & 408.0 & 433.0 & 416.0 & 385.0 & 299.0 \\
\hline 1953 & 3390.0 & $2500 \cdot n$ & 1670.0 & 1250.0 & 1020.0 & 697.0 & 579.0 & $496 \cdot 0$ & 459.0 & $416 \cdot 0$ & 299.0 \\
\hline 1954 & 3100.0 & $233 n \cdot n$ & 1360.0 & 994.0 & 922.0 & 663.0 & 651.0 & $570 \cdot 0$ & 515.0 & 445.0 & 325.0 \\
\hline 1955 & 1540.0 & $141 n \cdot n$ & $9 \cap n \cdot n$ & 586.0 & 566.0 & 469.0 & 391.0 & $361 \cdot 0$ & $354 \cdot 0$ & $33 n \cdot n$ & 256.0 \\
\hline 1956 & 5500.0 & 3550.0 & $2710 . n$ & 1440.0 & 1060.0 & 897.0 & 741.0 & 639.0 & $6 n 1 \cdot 0$ & $54 \pi \cdot n$ & 418.0 \\
\hline 1957 & 2420.0 & $1900 \cdot n$ & 1240.0 & 987.0 & 735.0 & $53 ? \cdot 0$ & $4 n 3.0$ & $389 \cdot 0$ & $34 ? \cdot 0$ & 321.0 & 245.0 \\
\hline 1958 & 4080.0 & 2750.0 & 1660.0 & 1160.0 & 770.0 & 686.0 & 581.0 & $478 \cdot 0$ & 438.0 & 382.0 & 277.0 \\
\hline 1959 & 2280.0 & 1560.0 & 1080.0 & $702 \cdot 0$ & & \multirow{2}{*}{\multicolumn{4}{|c|}{$\begin{array}{l}\text { Highest mean discharge for } 30 \text { consecutive } \\
\text { days in water year ending September } 30,1960\end{array}$}} & $374 \cdot 0$ & 279.0 \\
\hline 1960 & 1380.0 & 1230.0 & 824.0 & 617.0 & 553 & & & & & $315 \cdot 0$ & 247.0 \\
\hline 1961 & $5770 \cdot 0$ & $324 n \cdot n$ & $3070 \cdot \hat{n}$ & $1350^{\circ}$ ? & 939.0 & & & & & $426 \cdot n$ & $305 \cdot 0$ \\
\hline 1962 & $\begin{array}{l}1970.0 \\
1840.0\end{array}$ & $1640 \cdot 0$ & $109 n \cdot n$ & $732 . n$ & 615.0 & 462.0 & 371.0 & 349.0 & 36 & $346 . n$ & 263.0 \\
\hline 1963 & 1840.0 & 1230.7 & $851 \cdot 0$ & 675.0 & 511.0 & 457.0 & $39 n \cdot 0$ & $364 \cdot 0$ & 310.0 & $326 \cdot 0$ & 252.0 \\
\hline
\end{tabular}

Figure 3.--Explanation of the data shown on the three summaries. 
shows:

The flow-duration summary table, also illustrated in figure 3 ,

1. Total number of days in period of record that discharge equaled or exceeded the class limit of a particular class but was less than the class limit of the next higher class.

2. Accumulated number of days in period of record that discharge equaled or exceeded the class limit of a particular class.

3. Percentage of time in period of record that the class limit was equaled or exceeded.

This table is in a form suitable for preparation of a flow-duration curve for the period of record, but similar tables can be prepared manually for any number of the water years 1 isted in the annual flowduration table.

Three flow-duration summary tables were prepared for some stations for which the record included both a period of regulated flow and a period of unregulated flow. The three summary tables for those stations are presented in the following order: entire period, unregulated period, and regulated period. The annual tables for those stations are divided by a horizontal line (or lines) to separate the periods of regulated from nonregulated flow.

\section{Annua1 Low-F1ow Tables}

The annual low-flow table, which is the second summary presented for each gaging station, shows the lowest mean discharges for certain preselected periods, ranging from 1 to 274 consecutive days. This table is based on a climatic year, beginning on April 1, in order to allow the low-water season to be complete in one year. The chronological sequence of low flows is preserved by using periods of consecutive days that are included entirely within each climatic year. The table provides the data needed to prepare frequency curves of annual low flows.

The low-flow summary is illustrated by the table near the middle of figure 3. The first column 1ists climatic years and the other column headings across the top of the table are periods of consecutive days. Lowest mean discharge, in cubic feet per second, is shown in the body of the table for the number of consecutive days indicated by the column heading and for the climatic year indicated in the first column. The discharges listed for a particular number of consecutive days will give some idea of the range and frequency of annual low flows that has been experienced for that number of consecutive days. The low-flow frequency curves, described later in this report under "Techniques for using summary data," provide a clearer basis for interpretation. 


\section{Annua1 High-F1ow Tables}

The annual high-flow table, which is the third summary presented for each gaging station, shows highest mean discharges for each water year of record for the same periods of consecutive days as the low-flow summary, except for the 15-day period. This table is based on a water year, ending on September 30, in order to allow the high-water season to be complete in one year. The chronological sequence of high flows is preserved by using periods of consecutive days that are included entirely within each water year. The table provides the data needed to prepare frequency curves of annual high flows.

The high-flow summary is illustrated by the bottom table in figure 3. The first column lists water years, and other column headings are periods of consecutive days. Highest mean discharge, in cubic feet per second, is shown in the body of the table for the number of consecutive days indicated by the column heading and for the water year indicated in the first column. Some idea of the range and frequency of annual high flows can be gained by scanning individual discharge columns in the table. However, a clearer interpretation is provided by high-flow frequency curves, which are described in this report under "Techniques for using summary data."

Techniques for Using Summary Data

\section{Flow-Duration Curve}

The flow-duration curve represents the integrated effects of climate, topography, and geology on the outflow of the contributing drainage basin and, thus, provides a useful medium for studying and comparing flow and drainage-basin characteristics. A flow-duration curve (Searcy, 1959) can be constructed from the flow-duration summary table by plotting the discharges as ordinates and the corresponding percentages of time as abscissas. Logarithmic-probability paper gives good definition to the extreme points and helps to straighten the curve, but rectangular coordinates are more suitable for computations that involve the area under the curve, such as estimates of potential waterpower.

A smooth curve is usually drawn to fit the plotted points, but straight lines can be used to connect successive points if it is desired to avoid differences in interpretation. Whatever method is used, the curve represents only an average of the data for the period on which the computation was based. Duration curves for different periods of record are usually somewhat dissimilar. Figure 4 illustrates a flow-duration curve on logarithmic-probability paper for Fall Creek below Winberry Creek. This curve shows that a daily discharge of $41 \mathrm{cfs}$ (cubic feet per second) was exceeded or at least equaled on 90 percent of the days in the period 1936-63 at the Fall Creek gaging station. 


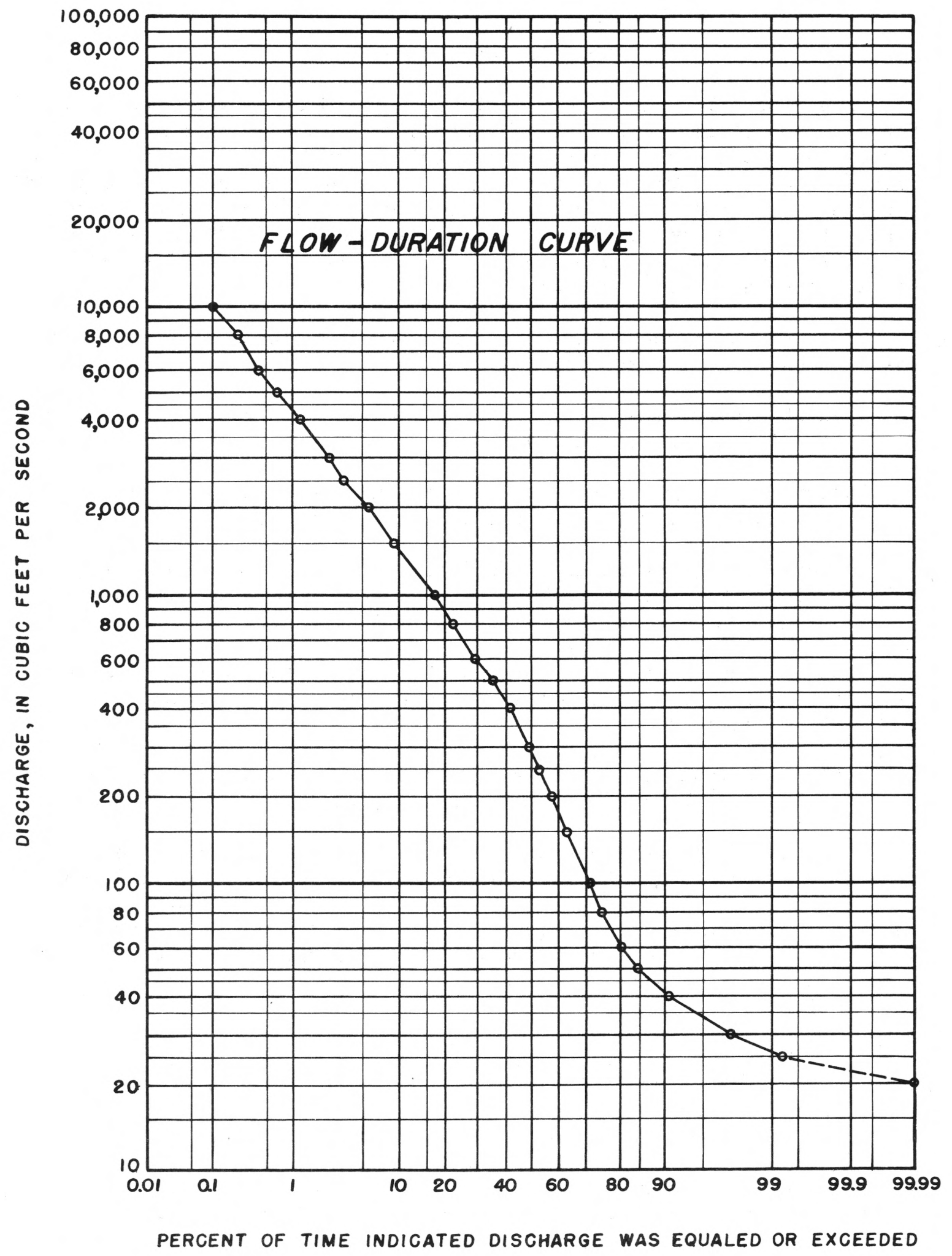

Figure 4.--Duration curve of daily flow, Fall Creek below Winberry Creek, near Fa11 Creek, Oreg., 1936-63. 


\section{Low-F1ow Frequency Curves}

The annual low flows for a selected period of consecutive days and for a series of climatic years can be used to estimate the average number of years between recurrences of similar low flows. The recurrence interva1 in years, thus obtained, is one way of expressing the expected frequency of annual low flows, but it should be remembered that the interval of time between low flows of the same magnitude may vary considerably and that the recorded low-flow extremes may be exceeded by those which have not been recorded.

A low-flow frequency curve is prepared from the data in the annual low-flow table in the following manner:

1. Rank the lowest mean discharges, for a selected number of consecutive days in the table, in order of magnitude.

2. Assign order numbers to ranked discharges in sequence, beginning with order number 1 to the lowest ranked discharge.

3. Compute recurrence interval for each ranked discharge by using the formula $(\mathrm{N}+1) / \mathrm{M}$, where $\mathrm{N}$ is the number of years for which discharges were ranked and $\mathrm{M}$ is the assigned order number.

4. Plot each discharge as an ordinate for the corresponding recurrence interval on the abscissa and draw a smooth curve through the plotted points.

Table 1A illustrates the results of steps 1,2 , and 3 for 7 and 30 consecutive days for Fall Creek below Winberry Creek.

Low-flow frequency curves for 7 and 30 consecutive days were constructed (using step 4 above) from the discharges and corresponding recurrence intervals in table $1 \mathrm{~A}$ and are included in the family of curves shown in the lower part of figure 5. The smoothed curve for 30 consecutive days shows that the lowest mean discharge is expected to be less than $25 \mathrm{cfs}$ at intervals that average 15 years in 1 ength for Fall Creek below Winberry Creek.

The graph paper used by the U.S. Geological Survey for frequency curves has an abscissa scale of recurrence interval in years that tends to linearize frequency curves (Powell, 1943) and has an ordinate scale which may be either logarithmic or arithmetic. This graph paper is not readily available but the abscissa can be duplicated on the arithmetic scale of semilogarithmic graph paper by using table 2, which gives values (X) of linear measurement on the arithmetic scale for selected recurrence intervals (R.I.). The initial point for measurement $(X=0)$ 
Table 1B.--Ranking of annual high flows for selected periods of consecutive days for Fall Creek below Winberry Creek, near Fall Creek, Oreg., 1936-63

\begin{tabular}{|c|c|c|c|c|c|c|c|c|c|c|c|}
\hline \multirow[b]{3}{*}{$\begin{array}{l}\text { ¿ } \\
\dot{0} \\
z \\
\dot{y} \\
\dot{y} \\
\dot{0}\end{array}$} & \multirow[b]{3}{*}{ 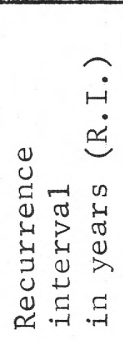 } & \multicolumn{4}{|c|}{ Number of consecutive days } & \multirow[b]{3}{*}{ 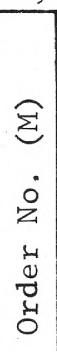 } & \multirow[b]{3}{*}{ 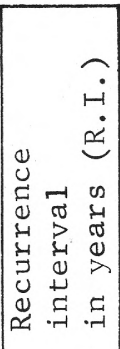 } & \multirow{2}{*}{\multicolumn{4}{|c|}{ 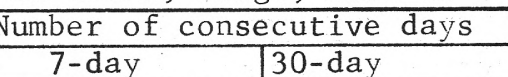 }} \\
\hline & & \multicolumn{2}{|c|}{ 7-day } & \multicolumn{2}{|c|}{ 30-day } & & & & & & \\
\hline & & 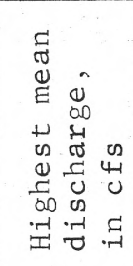 & $\begin{array}{l}\stackrel{y}{\pi} \\
\underset{\nu}{\circlearrowright}\end{array}$ & 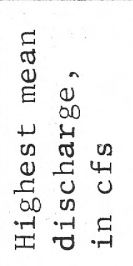 & 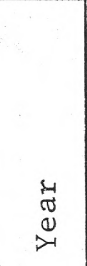 & & & 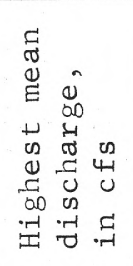 & 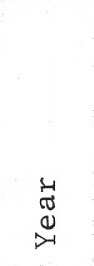 & 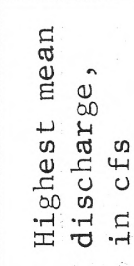 & 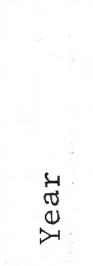 \\
\hline 1 & 29.0 & 7,050 & 1943 & 3,190 & 1943 & 15 & 1.93 & 3,340 & 1936 & 1,760 & 1938 \\
\hline 2 & 14.5 & 5,290 & 1957 & 2,910 & 1956 & 16 & 1.81 & 3,240 & 1950 & 1,760 & 1960 \\
\hline 3 & 9.67 & 5,090 & 1949 & 2,860 & 1954 & 17 & 1.71 & 3,060 & 1952 & 1,750 & 1937 \\
\hline 4 & 7.25 & 5,070 & 1947 & 2,750 & 1953 & 18 & 1.61 & 2,950 & 1945 & 1,750 & 1948 \\
\hline 5 & 5.80 & 4,870 & 1961 & 2,510 & 1961 & 19 & 1.53 & 2,940 & 1939 & 1,710 & 1952 \\
\hline 6 & 4.83 & 4,780 & 1956 & 2,450 & 1951 & 20 & 1.45 & 2,910 & 1938 & 1,620 & 1955 \\
\hline 7 & 4.14 & 4,740 & 1951 & 2,310 & 1946 & 21 & 1.38 & 2,900 & 1955 & 1,530 & 1939 \\
\hline 8 & 3.62 & 4,350 & 1946 & 2,290 & 1957 & 22 & 1.32 & 2,760 & 1960 & 1,530 & 1963 \\
\hline 9 & 3.22 & 4,280 & 1953 & 2,260 & 1947 & 23 & 1.26 & 2,680 & 1963 & 1,470 & 1940 \\
\hline 10 & 2.90 & 4,240 & 1948 & 2,190 & 1949 & 24 & 1.21 & 2,590 & 1959 & 1,390 & 1959 \\
\hline 11 & 2.64 & 4,180 & 1954 & 1,970 & 1958 & 25 & 1.16 & 2,560 & 1942 & 1,370 & 1945 \\
\hline 12 & 2.42 & 3,710 & 1958 & 1,950 & 1962 & 26 & 1.12 & 1,890 & 1940 & 1,360 & 1942 \\
\hline 13 & 2.23 & 3,540 & 1937 & 1,930 & 1936 & 27 & 1.07 & 1,480 & 1944 & 907 & 1941 \\
\hline 14 & 2.07 & 3,390 & 1962 & 1,840 & 1950 & 28 & 1.04 & 1,450 & 1941 & 851 & 1944 \\
\hline
\end{tabular}

Table 1A.--Ranking of annual low flows for selected periods of consecutive days for Fall Creek below Winberry Creek, near Fall Creek, Oreg., 1936-62

\begin{tabular}{|c|c|c|c|c|c|c|c|c|c|c|c|}
\hline \multirow[b]{3}{*}{$\begin{array}{l}\widehat{E} \\
\dot{0} \\
z \\
\dot{y} \\
\dot{y} \\
\dot{y}\end{array}$} & \multirow[b]{3}{*}{ 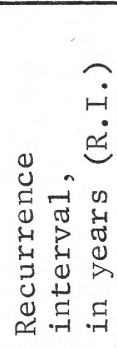 } & \multirow{2}{*}{\multicolumn{4}{|c|}{\begin{tabular}{c|c} 
Number of consecutive days \\
$7-$ day & $30-$ day
\end{tabular}}} & \multirow[b]{3}{*}{ 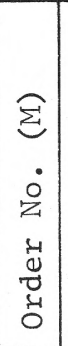 } & \multirow[b]{3}{*}{ 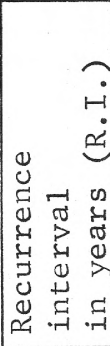 } & \multirow{2}{*}{\multicolumn{4}{|c|}{\begin{tabular}{l|l|l} 
Number of consecutive days \\
$7-$ day & $30-$ day
\end{tabular}}} \\
\hline & & & & & & & & & & & \\
\hline & & 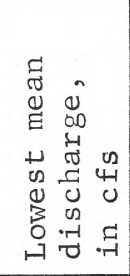 & $\overbrace{\nu=-1}^{\mathbb{D}}$ & 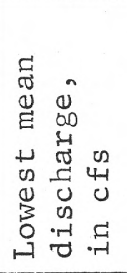 & 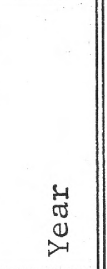 & & & 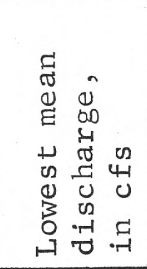 & $\underset{\tilde{D}}{\stackrel{D}{\nu}}$ & 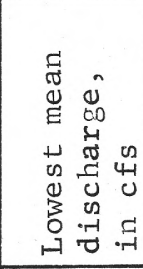 & $\begin{array}{l}\stackrel{\mathscr{J}}{0} \\
\stackrel{\nu}{\circlearrowright}\end{array}$ \\
\hline 1 & 28.0 & 20.0 & 1940 & 22.1 & 1940 & 15 & 1.87 & 32.7 & 1957 & 36.6 & 1959 \\
\hline 2 & 14.0 & 20.7 & 1939 & 25.2 & 1936 & 16 & & 32.9 & 196 & 36.8 & 1961 \\
\hline 3 & .33 & 22.9 & 1936 & 26.0 & 1939 & 17 & 1. & 33.0 & 1956 & 39.2 & 1960 \\
\hline 4 & 7.00 & 25.0 & 1952 & 27.5 & 1952 & 18 & 1. & 34.3 & 1960 & 40.0 & 1962 \\
\hline 5 & 5.60 & 25.7 & 1938 & 30.6 & 1938 & 19 & 1.47 & 36.7 & 1955 & 41.2 & 1943 \\
\hline 6 & 4.67 & 25.7 & 1951 & 32.0 & 1951 & 20 & 1.40 & 37.9 & 1937 & 41.6 & 1955 \\
\hline 7 & 4.00 & 26.4 & 1944 & 32.8 & 1944 & 21 & 1.33 & 39.4 & 1943 & 45.3 & 1950 \\
\hline 8 & 3.50 & 27.0 & 1945 & 33.7 & 1942 & 22 & 1. & 41.6 & 19 & 50.6 & 1953 \\
\hline 9 & & 29.4 & 19 & 34.1 & 1957 & 23 & & 41.7 & 19 & 50.7 & 1948 \\
\hline 10 & 2.80 & 30.4 & 1946 & 34.5 & 1949 & 24 & & 42.1 & 19 & 52.2 & 1937 \\
\hline 11 & 2.55 & 31.3 & 1942 & 34.9 & 1958 & 25 & 1.12 & 42.6 & 1941 & 58.2 & 1941 \\
\hline 12 & 2.33 & 31.3 & 1949 & 35.9 & 1945 & 26 & 1.08 & 49.6 & 1947 & 62.7 & 1947 \\
\hline 13 & 2.15 & 31.9 & 1961 & 36.2 & 1946 & 27 & 1.04 & 51.3 & 1954 & 66.6 & 1954 \\
\hline 14 & 2.00 & 32.0 & 1959 & 36.6 & 1956 & & & & & & \\
\hline
\end{tabular}




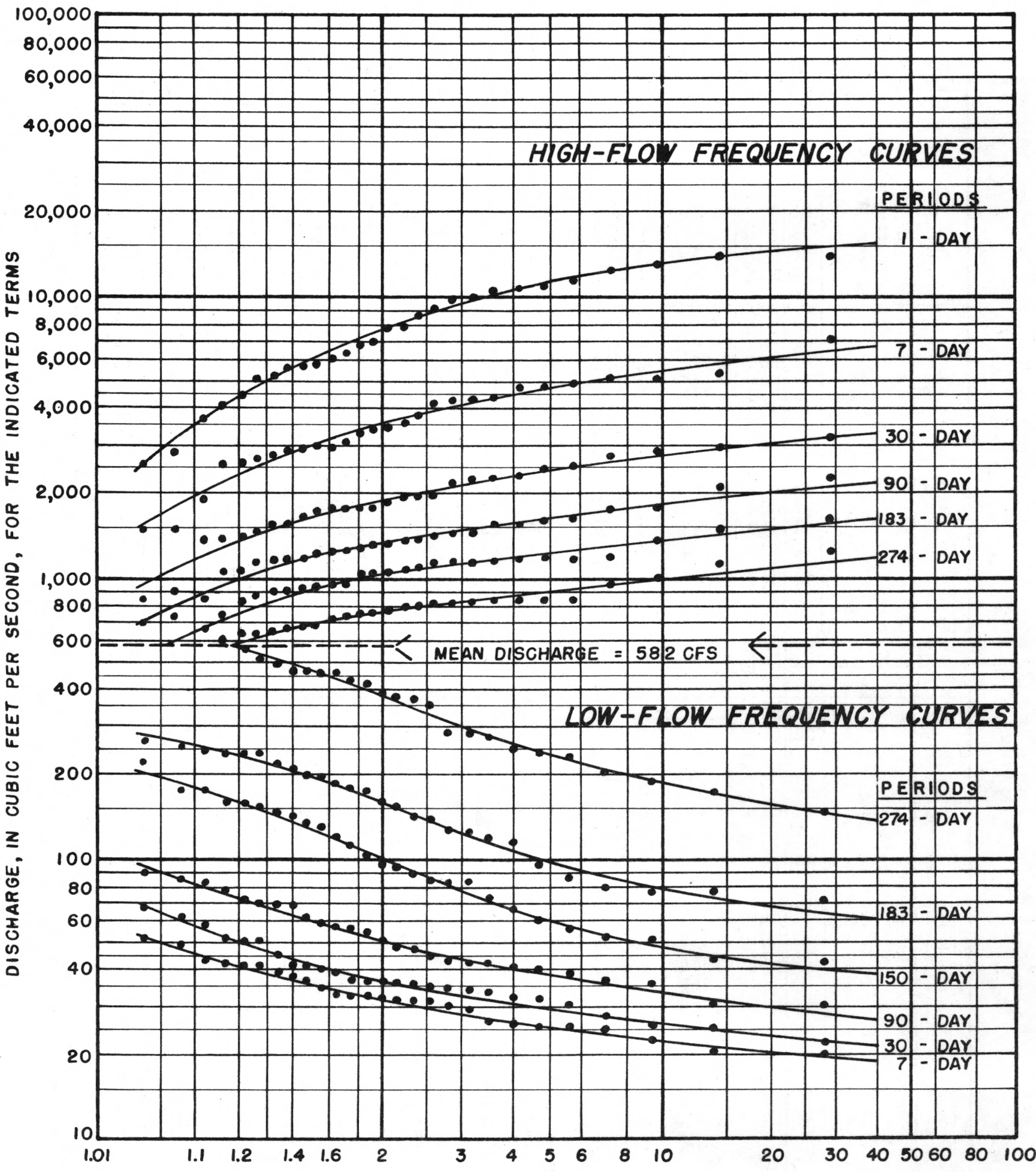

RECURRENCE INTERVAL, IN YEARS

Figure 5.--Recurrence intervals of annual low flows (1936-62) and annual high flows (1936-63) for selected periods of consecutive days for Fall Creek below Winberry Creek, near Fal1 Creek, Oreg. 
Table 2.--Arithmetic values of 1 inear measurement (X) for selected recurrence intervals (R.I.)

$\underline{\perp}$ Produced by solution of the equation $x=-\log _{\underline{e}}\left[-\log _{\underline{e}}\left(1-\frac{1}{\text { R.I.I. }}\right)\right]-T$

\begin{tabular}{|rr|rr|rr|rr|rc|}
\hline R.I. & X & R.I. & X & R.I. & X & R.I. & $X$ & R.I. & $X$ \\
\hline 1.01 & -1.53 & 1.4 & -0.22 & 3.0 & 0.90 & 10 & 2.25 & 40 & 3.68 \\
1.05 & -1.10 & 1.6 & +.02 & 4.0 & 1.25 & 15 & 2.67 & 50 & 3.90 \\
1.1 & -.88 & 1.8 & .21 & 5.0 & 1.50 & 20 & 2.97 & 60 & 4.07 \\
1.2 & -.58 & 2.0 & .37 & 6.0 & 1.70 & 25 & 3.20 & 80 & 4.34 \\
1.3 & -.39 & 2.5 & .67 & 8.0 & 2.01 & 30 & 3.40 & 100 & 4.60 \\
\hline
\end{tabular}


occurs at a recurrence interval of 1.582 years, and other measurements are made to the left (minus) or right (plus) of the initial point.

\section{High-F1ow Frequency Curves}

The technique used in the preparation of high-flow frequency curves is similar to that used for low-flow frequency curves, except that the arrayed highest mean discharges for a selected number of consecutive days are assigned order numbers from the highest to the lowest with the highest number 1 , as shown in table 1B. The family of curves in the upper part of figure 5 illustrates that the same graph paper may be used to construct both high-flow and low-flow frequency curves. The smoothed curve for 30 consecutive days shows that the highest mean discharge is expected to be greater than 2,950 cfs at intervals that average 15 years in length for Fall Creek below Winberry Creek.

A frequency curve of annual flow shows how much the mean flows for a selected period of consecutive days have differed throughout the years of record. The curve represents an estimate of how much future flows might be expected to differ over a similar period of years. The amount by which annual high flows differ throughout time is influenced largely by climate and topography, whereas the amount annual low flows differ is largely controlled by climate and geology.

\section{SELECTED BIBLIOGRAPHY}

Goddard, G. C., Jr., 1963, Water-supply characteristics of North Carolina streams: U.S. Geo1. Survey Water-Supp1y Paper 1761.

Hardison, C. H., and Martin, R. O. R., 1963, Low-flow frequency curves for selected long-term stream-gaging stations in Eastern United States: U.S. Geo1. Survey Water-Supp1y Paper 1669-G.

1963, Water-supp1y characteristics of streams in the Delaware River basin and in southern New Jersey: U.S. Geol. Survey WaterSupp1y Paper 1669-N.

Langbein, W. B., and Iseri, K. T., 1960, Genera1 introduction and hydrologic definitions: U.S. Geol. Survey Water-Supp1y Paper 1541-A.

Powe11, R. W., 1943, A simple method of estimating flood frequencies: Civil Eng., v. 13, no. 2 .

Rantz, S. E., 1964, Surface-water hydrology of coastal basins of northern California: U.S. Geol. Survey Water-Supply Paper 1758.

Riggs, H. C., 1961, Frequency of natural events: Jour. Hydraulics Div., Am. Soc. Civil Engineers, v. 87, no. HY1, January. 
Searcy, J. K., 1959, Flow-duration curves: U.S. Geol. Survey WaterSupp1y Paper 1542-A.

Sta11, J. B., 1964, Low flows of Illinois streams for impounding reservoir design: Illinois State Water Survey Bu11. 51.

U.S. Army, Corps of Engineers, 1955, Stream flow volume-durationfrequency studies: Civil Works Inv. Proj. CW-152, Tech. Rept. 1.

U.S. Geological Survey, 1958, Compilation of records of surface waters of the United States through September 1950, part 14, Pacific slope basins in Oregon and lower Columbia River basin: U.S. Geol. Survey Water-Supp1y Paper 1318.

1963, Compilation of records of surface waters of the United States, October 1950 to September 1960, part 14, Pacific slope basins in Oregon and lower Columbia River basin: U.S. Geol. Survey Water-Supp1y Paper 1738.

, Surface water records of Oregon, 1961: U.S. Geol. Survey open-file report.

, Surface water records of Oregon, 1962: U.S. Geol. Survey open-file report.

, Surface water records of Oregon, 1963: U.S. Geol. Survey open-file report.

1899-1960, Surface water supply of the United States, part 14, Pacific slope basins in Oregon and lower Columbia River basin: (Water-supply papers 1isted in table below.)

\begin{tabular}{|lc|cc|cc|cc|rr|}
\hline Year & WSP & Year & WSP & Year & WSP & Year & WSP & Year & WSP \\
\hline 1899 & 38 & 1912 & $332-C$ & 1925 & 614 & 1937 & 834 & 1949 & 1154 \\
1900 & 51 & 1913 & $362-C$ & 1926 & 634 & 1938 & 864 & 1950 & 1184 \\
1901 & 75 & 1914 & 394 & 1927 & 654 & 1939 & 884 & 1951 & 1218 \\
1902 & 85 & 1915 & 414 & 1928 & 674 & 1940 & 904 & 1952 & 1248 \\
1903 & 100 & 1916 & 444 & 1929 & 694 & 1941 & 934 & 1953 & 1288 \\
1904 & 135 & 1917 & 464 & 1930 & 709 & 1942 & 964 & 1954 & 1348 \\
1905 & 178 & 1918 & 484 & 1931 & 724 & 1943 & 984 & 1955 & 1398 \\
1906 & 214 & $1919-20$ & 514 & 1932 & 739 & 1944 & 1014 & 1956 & 1448 \\
$1907-8$ & 252 & 1921 & 534 & 1933 & 754 & 1945 & 1044 & 1957 & 1518 \\
1909 & 272 & 1922 & 554 & 1934 & 769 & 1946 & 1064 & 1958 & 1568 \\
1910 & 292 & 1923 & 574 & 1935 & 794 & 1947 & 1094 & 1959 & 1638 \\
1911 & 312 & 1924 & 594 & 1936 & 814 & 1948 & 1124 & 1960 & 1718 \\
\hline
\end{tabular}

Note: Earlier records of streamflow are published in reports listed in WSP 1718. 



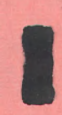

I

I

I

】

\

I

I

I

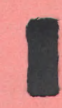

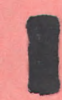

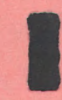

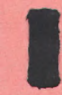

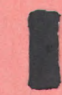

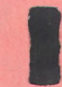

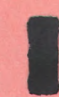

I

I 
I

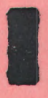

I

I

I

I

I

I

I

I

I 


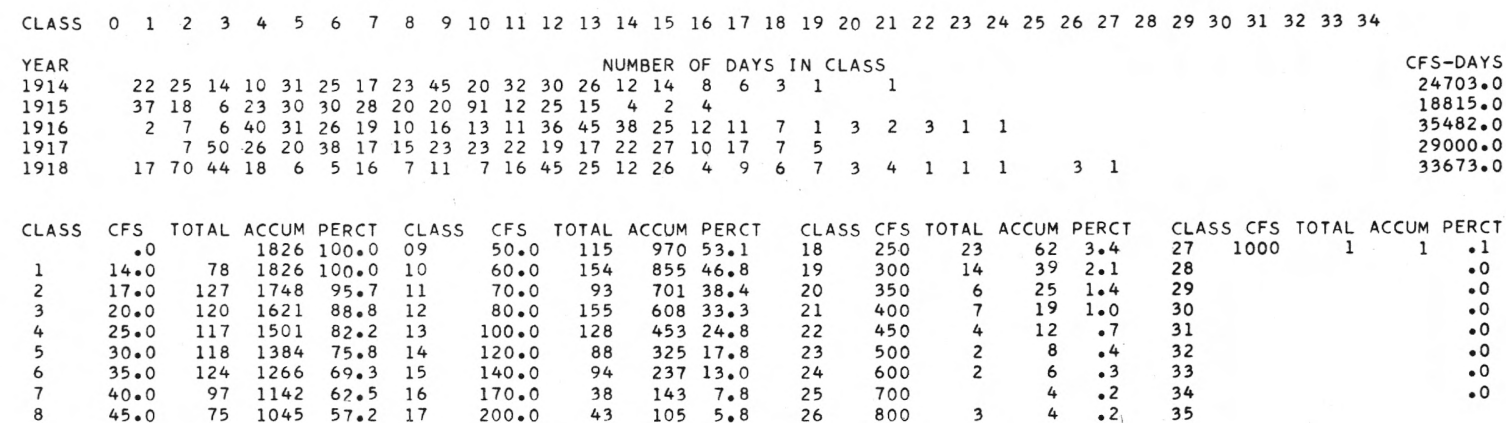

LOST CREAKK NEAR BRIGHWWOOD ORBG.

STATION NUMBER $\quad 14-1300.00$

LOWEST MEAN DISCHARGE, IN CFS, FOR THE FOLLOWING NUMBER OF CONSECUTIVE DAYS IN YEAR BEGINNING APRIL 1

\begin{tabular}{|c|c|c|c|c|c|c|c|c|c|c|c|}
\hline YEAR & 1 & 3 & 7 & 14 & 30 & 60 & 90 & 120 & 150 & 183 & 274 \\
\hline $\begin{array}{l}1914 \\
1915 \\
1916\end{array}$ & $\begin{array}{l}15.0 \\
15.0 \\
21.0\end{array}$ & $\begin{array}{l}15.0 \\
15.0 \\
21.0\end{array}$ & $\begin{array}{l}15.0 \\
15.0 \\
21.1\end{array}$ & $\begin{array}{l}15.1 \\
15.1 \\
21.9\end{array}$ & $\begin{array}{l}15.9 \\
15.8 \\
23.2\end{array}$ & $\begin{array}{l}18.4 \\
16.5 \\
26.1\end{array}$ & $\begin{array}{l}24.8 \\
18.7 \\
28.6\end{array}$ & $\begin{array}{l}31 \cdot 3 \\
22 \cdot 4 \\
34 \cdot 9\end{array}$ & $\begin{array}{l}37 \cdot 2 \\
27 \cdot 2 \\
43.6\end{array}$ & $\begin{array}{l}41 \cdot 3 \\
35 \cdot 1 \\
46 \cdot 2\end{array}$ & $\begin{array}{l}46.0 \\
63.7 \\
52.3\end{array}$ \\
\hline 1917 & 17.0 & 17.0 & 17.0 & 17.0 & 17.1 & 18.6 & 20.0 & 22.0 & 31.9 & 52.1 & $\begin{array}{r}52.3 \\
103.0\end{array}$ \\
\hline
\end{tabular}

LOST CREATK NEAR BRIGHITOOD ORBG

STATION NUMBER $14-1300.00$

HIGHEST MEAN DISCHARGE, IN CFS, FOR THE FOLLOWING NUMBER OF CONSECUTIVE DAYS IN YEAR ENDING SEPTEMBER 30

\begin{tabular}{|c|c|c|c|c|c|c|c|c|c|c|c|}
\hline YEAR & 1 & 3 & 7 & 15 & 30 & 60 & 90 & 120 & 150 & 183 & 274 \\
\hline 1914 & 438.0 & 305.0 & 218.0 & 183.0 & 148.0 & 129.0 & 111.0 & 109.0 & 100.0 & 93.0 & 82.2 \\
\hline 1915 & 182.0 & $179 \cdot 0$ & 144.0 & 115.0 & 90.9 & 77.0 & 74.0 & $70 \cdot 7$ & 67.8 & 63.6 & 61.5 \\
\hline 1916 & 630.0 & 458.0 & 372.0 & 303.0 & 226.0 & 164.0 & 140.0 & $132 \cdot 0$ & 136.0 & 133.0 & 120.0 \\
\hline 1917 & 340.0 & 313.0 & 284.0 & 220.0 & 196.0 & 192.0 & 170.0 & 148.0 & 127.0 & 115.0 & 97.9 \\
\hline 1918 & 1020.0 & 938.0 & 713.0 & 505.0 & 404.0 & 291.0 & 230.0 & 190.0 & 171.0 & 157.0 & 117.0 \\
\hline
\end{tabular}


CLASS $00 \begin{array}{llllllllllllllllllllllllllllllllllll} & 2 & 3 & 4 & 5 & 6 & 7 & 8 & 9 & 10 & 11 & 12 & 13 & 14 & 15 & 16 & 17 & 18 & 19 & 20 & 21 & 22 & 23 & 24 & 25 & 26 & 27 & 28 & 29 & 30 & 31 & 32 & 33 & 34\end{array}$

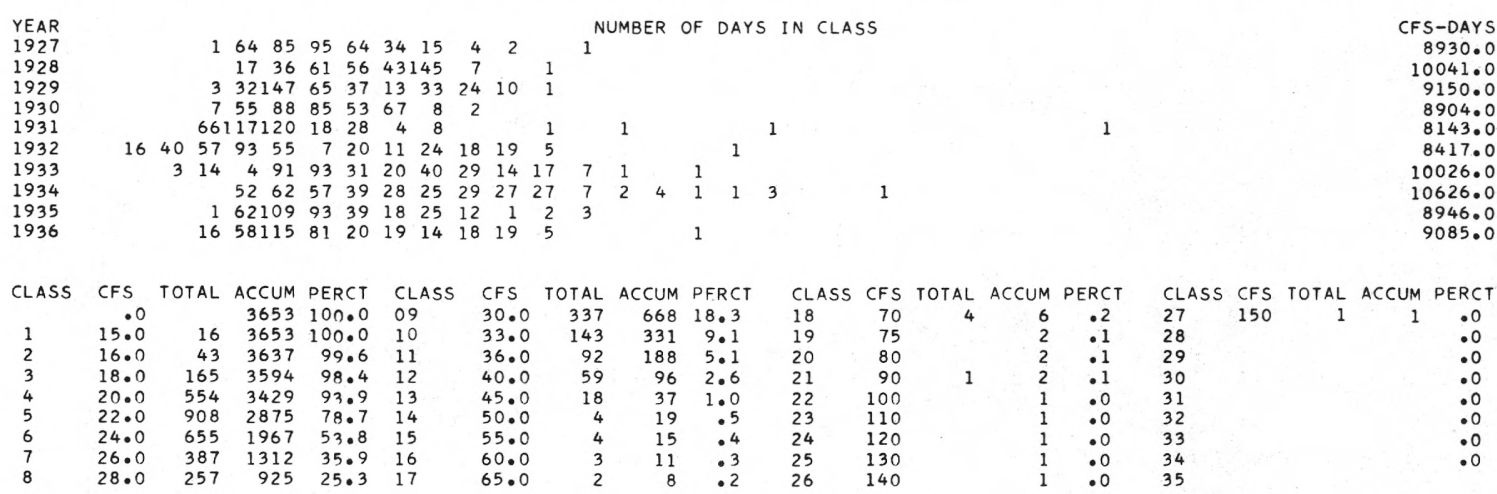

LITTLE ZIGZAG RIVER NR RHODODENDRON OREG.

STATION NUMBER $14-1310.00$

LOWEST MEAN DISCHARGE, IN CFS, FOR THE FOLLOWING NUMBER OF CONSECUTIVE DAYS IN YEAR BEGINNING APRIL 1

\begin{tabular}{|c|c|c|c|c|c|c|c|c|c|c|c|}
\hline YEAR & 1 & 3 & 7 & 14 & 30 & 60 & 90 & 120 & 150 & 183 & 274 \\
\hline 1926 & 18.0 & 18.0 & 18.6 & 18.6 & 19.7 & 20.4 & 20.5 & 21.0 & 21.3 & 21.6 & 22.7 \\
\hline 1927 & 20.0 & 20.0 & 20.0 & 20.0 & 20.9 & 21.3 & 21.7 & 21.9 & 22.3 & 23.2 & 24.9 \\
\hline 1928 & 19.0 & 19.3 & 19.7 & 19.8 & 21.2 & 21.7 & 21.8 & 21.8 & 21.8 & 22.1 & 23.1 \\
\hline 1929 & 21.0 & 21.0 & 21.1 & 21.6 & 22.2 & 22.9 & 22.9 & 23.0 & 23.2 & 23.4 & 24.6 \\
\hline 1930 & 18.0 & 18.0 & 18.6 & 18.8 & 19.2 & 19.5 & 19.9 & $20 \cdot 4$ & 20.5 & 21.3 & 21.3 \\
\hline 1931 & 15.0 & 15.0 & 15.0 & 15.1 & 15.5 & 17.9 & 18.3 & 18.7 & 18.9 & 19.1 & 15.7 \\
\hline 1932 & 16.0 & 16.0 & 16.0 & 16.0 & 16.7 & 18.1 & 19.2 & 20.0 & 21.0 & 22.4 & 23.4 \\
\hline 1933 & 20.0 & 20.0 & 20.1 & 20.8 & 21.9 & 22.4 & 23.2 & 23.8 & 24.6 & 26.7 & 36.1 \\
\hline 1934 & 20.0 & 20.0 & 20.0 & 20.0 & 20.5 & 20.6 & 20.9 & 21.8 & 22.2 & 22.8 & 23.4 \\
\hline 1935 & 18.0 & 18.7 & 18.7 & 19.1 & 19.7 & 20.4 & 20.6 & 20.6 & 20.8 & 21.3 & 22.6 \\
\hline
\end{tabular}

LITTIE ZIGZAG RIVER NR RHODODENDRON OREG

STATION NUMBER $\quad 14-1310.00$

HIGHEST MEAN DISCHARGE, IN CFS, FOR THE FOLLOWING NUMBER OF CONSECUTIVE DAYS IN YEAR ENDING SEPTEMBER 30

\begin{tabular}{|c|c|c|c|c|c|c|c|c|c|c|c|}
\hline YEAR & 1 & 3 & 7 & 15 & 30 & 60 & 90 & 120 & 150 & 183 & 274 \\
\hline 1927 & 47.0 & 35.7 & 32.1 & 30.5 & 28.5 & 28.0 & 27.2 & $26 \cdot 6$ & 26.0 & 25.5 & 25.4 \\
\hline 1928 & 42.0 & $34 . ?$ & 32.4 & 32.1 & 31.8 & 31.0 & 30.7 & 30.5 & 30.3 & 30.3 & 28.9 \\
\hline $19>9$ & 40.0 & 39.7 & 38.4 & 36.8 & 35.0 & 33.3 & 31.5 & 30.2 & 29.1 & 28.0 & 26.0 \\
\hline 1930 & 35.0 & 32.7 & $32 \cdot 1$ & 29.8 & 28.8 & 28.2 & 28.0 & 27.8 & 27.1 & 26.5 & 25.4 \\
\hline 1931 & 150.0 & 86.7 & 52.7 & 39.0 & 32.7 & 28.5 & 26.6 & $25 \cdot 4$ & $24 \cdot 2$ & 23.4 & 23.0 \\
\hline 1932 & 68.0 & $50 \cdot 0$ & 38.7 & 38.2 & 37.0 & $34 \cdot 1$ & 33.0 & $30 \cdot 6$ & 28.9 & 27.3 & 24.4 \\
\hline 1933 & 64.0 & 53.0 & 49.0 & 45.5 & 42.6 & 38.0 & 35.5 & $33 \cdot 1$ & 31.3 & 29.9 & 29.2 \\
\hline 1934 & 97.0 & 80.0 & 68.4 & 55.9 & 48.9 & 44.0 & 39.7 & 38.8 & 37.1 & 35.0 & 31.7 \\
\hline 1935 & 46.0 & 44.3 & 36.0 & 32.7 & 32.2 & 29.5 & 27.9 & 26.7 & 26.3 & 26.2 & 25.7 \\
\hline 1936 & 62.0 & 45.3 & 37.9 & 37.5 & 36.2 & 33.5 & 30.9 & $29 \cdot 1$ & 28.2 & 28.0 & 26.2 \\
\hline
\end{tabular}


cous
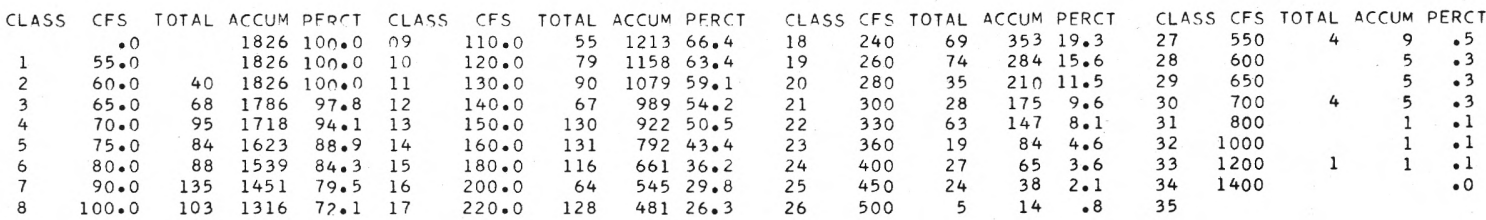

ZIGZAG RTVER AT RHODODENDRON ORBG.

STATION NUMBER $\quad 14-1315.00$ LOWEST MEAN DISCHARGE, IN CFS, FOR THE FOLLOWING NUMBER OF CONSECUTIVE DAYS IN YEAR BEGINNING APRIL 1

\begin{tabular}{|c|c|c|c|c|c|c|c|c|c|c|c|}
\hline YEAR & 1 & 3 & 7 & 14 & 30 & 60 & 90 & 120 & 150 & 183 & 274 \\
\hline 1920 & 72.0 & 74.0 & 76.1 & 80.1 & 81.3 & 88.9 & 103.0 & 118.0 & 125.0 & 135.0 & 165.0 \\
\hline 1926 & 59.0 & 60.0 & 61.1 & 62.4 & 64.1 & 70.5 & 75.5 & 79.5 & 86.1 & 92.4 & 118.0 \\
\hline 1927 & 72.0 & 72.7 & 73.4 & 75.6 & 83.9 & 99.0 & 118.0 & 142.0 & 149.0 & 171.0 & 192.0 \\
\hline 1928 & 70.0 & 70.0 & 70.0 & 70.0 & 72.2 & 73.8 & 79.6 & 85.4 & 88.0 & 90.1 & 98.0 \\
\hline 1929 & 62.0 & 62.0 & 63.1 & 63.6 & 64.7 & 67.0 & 67.8 & 69.9 & 74.1 & 91.1 & 126.0 \\
\hline
\end{tabular}

ZIGZAG RIVER AT RHODODENDRON OREG.

DIATION NUMBER $14-1315.00$

HIGHEST MEAN DISCHARGE, IN CFS, FOR THE FOLLOWING NUMBER OF CONSECUTIVE DAYS IN YEAR ENDING SEPTEMBER 30

\begin{tabular}{|c|c|c|c|c|c|c|c|c|c|c|c|}
\hline YEAR & 1 & 3 & 7 & 15 & 30 & 60 & 90 & 120 & 150 & 183 & 274 \\
\hline 1921 & 546.0 & 506.0 & 417.0 & 342.0 & $281 \cdot 0$ & 266.0 & 260.0 & $257 \cdot 0$ & 253.0 & 248.0 & 223.0 \\
\hline $19 ? 7$ & 770.0 & 538.0 & 364.0 & 313.0 & 296.0 & 272.0 & 236.0 & $223 \cdot 0$ & 211.0 & 203.0 & 190.0 \\
\hline 1928 & 1270.0 & 920.0 & 682.0 & 497.0 & 376.0 & 300.0 & 271.0 & $251 \cdot 0$ & $25,2 \cdot 0$ & 254.0 & 228.0 \\
\hline 1929 & 565.0 & 473.0 & 471.0 & 471.0 & 456.0 & 374.0 & 317.0 & $278 \cdot 0$ & 244.0 & 215.0 & 178.0 \\
\hline 1930 & 400.0 & 330.0 & 330.0 & 330.0 & 319.0 & 250.0 & 226.0 & $220 \cdot 0$ & 206.0 & 198.0 & 160.0 \\
\hline
\end{tabular}

STILL CREEK AT RHODODENDRON OREGON

\section{(computed by hand)}

YEAR
1921
1927
1928
1929
1930

STATION NUMBER

$14-1330.00$

DURATION TABLE OF DAIIY DISCHARGE

NUMBER OF DAYS IN CLASS

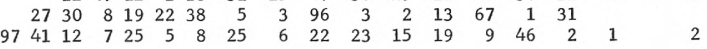

ERCT
.5
.3
.3
.3
.1
.1
.1
.0

STILL CREEK AT RHODODENDRON OREGON

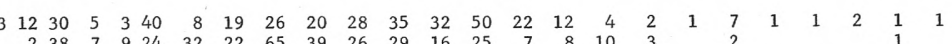
$\begin{array}{rrrrrrrrrrrrrrrrrrrrrrrrrrr}12 & 47 & 12 & 1 & 18 & 32 & 15 & 47 & 36 & 29 & 21 & 24 & 30 & 12 & 8 & 5 & 3 & 3 & 4 & 1 & 2 & 2 & 1 & 1\end{array}$

\begin{tabular}{|c|c|c|c|c|c|c|c|c|c|c|}
\hline CLASS & CFS & TOTAL & ACCUM & PERCT & CLASS & CFS & TOTAL & ACCUM & PERCT & CLASS \\
\hline & .0 & & 1826 & 100.0 & 09 & 50.0 & 128 & 1352 & 74.0 & 18 \\
\hline 1 & 14.0 & & 1826 & 100.0 & 10 & 60.0 & 102 & 1224 & 67.0 & 19 \\
\hline 2 & 17.0 & & 1826 & 100.0 & 11 & 70.0 & 65 & 1122 & 61.4 & 20 \\
\hline 3 & 20.0 & 97 & 1826 & 100.0 & 12 & 80.0 & 256 & 1057 & 57.9 & 21 \\
\hline 4 & 25.0 & 71 & 1729 & 94.7 & 13 & 100.0 & 121 & 801 & 43.9 & 22 \\
\hline 5 & 30.0 & $\begin{array}{l}11 \\
68\end{array}$ & 1658 & 90.8 & 14 & 120.0 & 100 & 680 & 37.2 & 23 \\
\hline 6 & 35.0 & 130 & 1590 & 87.1 & 15 & 140.0 & 117 & 580 & 31.8 & 24 \\
\hline 7 & 40.0 & 68 & 1460 & 80.0 & 16 & 170.0 & 148 & 463 & 25.4 & 25 \\
\hline 8 & 45.0 & 40 & 1392 & 76.2 & 17 & 200.0 & 152 & 315 & 17.2 & 26 \\
\hline
\end{tabular}

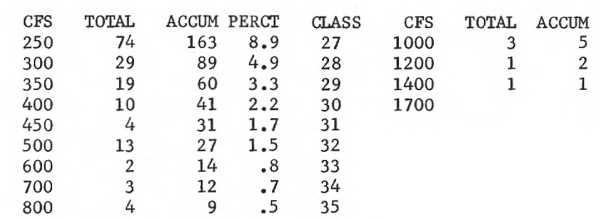

CFS-DAYS (Computed by hand)

LOWEST MEAN DISCHARGE, IN CFS, FOR THE FOLLOWING NUMBER OF CONSECUTIVE DAYS IN YEAR BEGINNING APRIL 1

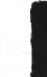

$\begin{array}{lccc}\text { YEAR } & 1 & 3 & 7 \\ 1920 & 30.0 & 31.3 & 31.7 \\ 1926 & 16.0 & 17.0 & 18.0 \\ 1927 & 34.0 & 34.7 & 35.3 \\ 1928 & 34.0 & 34.0 & 34.0 \\ 1929 & 22.0 & 22.0 & 22.0\end{array}$

$\begin{array}{ll}7 & 14 \\ 31.7 & 33.2 \\ 18.0 & 19.4 \\ 35.3 & 36.1 \\ 34.0 & 34.0 \\ 22.0 & 22.0\end{array}$

30
35.4
23.5
36.4
34.4
22.1

60
38.8
25.2
40.3
35.8
23.6

$$
\begin{aligned}
& 90 \\
& 46.0 \\
& 27.6 \\
& 51.1 \\
& 37.0 \\
& 25.1
\end{aligned}
$$

120
59.1
31.8
75.0
41.5
26.6
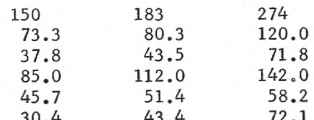

STATION NUMBER

$14-1330.00$

STILL CREEK AT RHODODENDRON OREGON

HIGHEST MEAN DISCHARGE, IN CFS, FOR THE FOLLOWING NUMBER OF CONSECUTIVE DAYS IN YEAR ENDING SEPTEMBER 30

$\begin{array}{lr}\text { YEAR } & 1 \\ 1921 & 1230.0 \\ 1927 & 1100.0 \\ 1928 & 1600.0 \\ 1929 & 765.0 \\ 1930 & 425.0\end{array}$

$\begin{array}{rr}3 & 7 \\ 860.0 & 595.0 \\ 643.0 & 385.0 \\ 1228.0 & 904.0 \\ 250.0 & 250.0 \\ 315.0 & 289.0\end{array}$

30
302.0
264.0
431.0
250.0

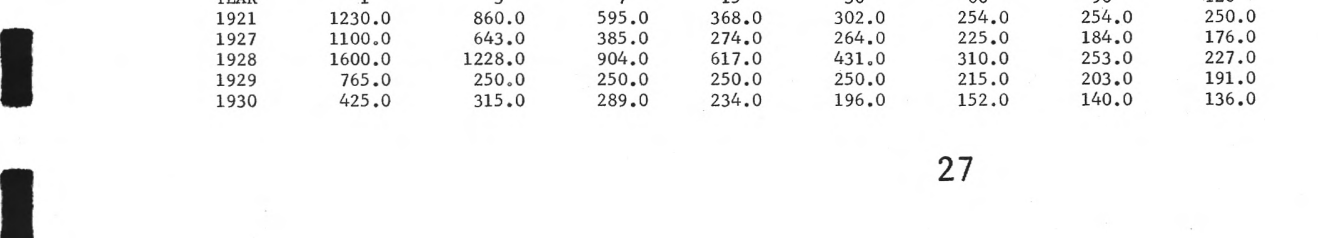

60
254.0
225.0
310.0
215.0
152.0

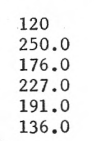


SANDY RIVER AB SALMON RIVER AT BRIGHTWOOD OREGON STATION NUMBER
DURATION TABLE OF DAILY DISCHARGE

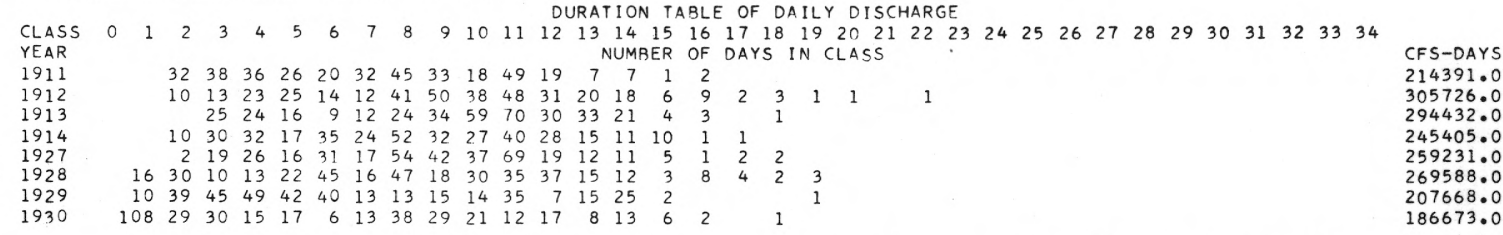

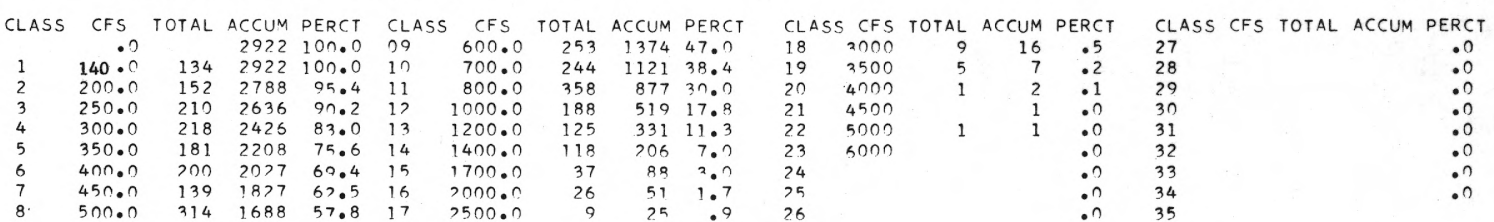

SANDY RIVER AB SALMON RIVER AT BRIGHTWOOD OREGON STATION NUMBER

$14-1335.00$

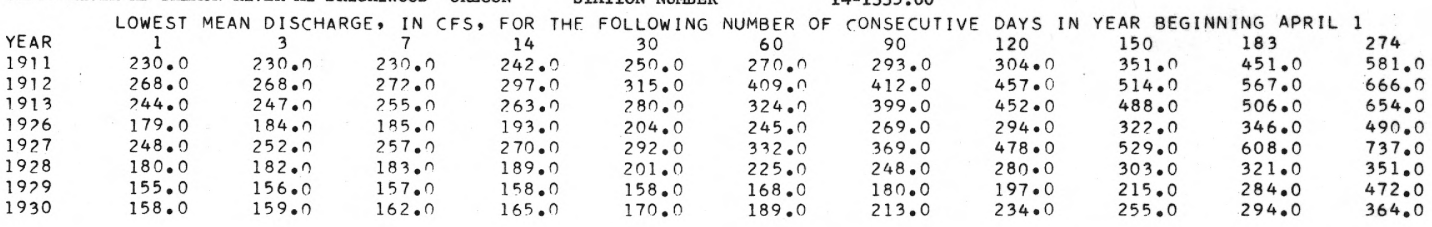

SANDY RIVER AB SALMON RIVER AT BRIGHTWOOD OREGON STATION NUMBER

$\begin{array}{cccccc} & \text { HIGHEST } & \text { MEAN DISCHARGE, IN } & \text { CFS, FOR } & \text { THE } & \text { FOLLOWING } \\ \text { YEAR } & 1 & 3 & 7 & 15 & 30 \\ 1911 & 2360.0 & 2190.0 & 1700.0 & 1390.0 & 1160.0 \\ 1912 & 5920.0 & 4640.0 & 3080.0 & 2150.0 & 1870.0 \\ 1913 & 3150.0 & 2450.0 & 1780.0 & 1530.0 & 1370.0 \\ 1914 & 2620.0 & 2050.0 & 1650.0 & 1500.0 & 1320.0 \\ 1927 & 3410.0 & 2590.0 & 1740.0 & 1270.0 & 1120.0 \\ 1928 & 3500.0 & 3500.0 & 3100.0 & 2310.0 & 1700.0 \\ 1929 & 3520.0 & 2280.0 & 1610.0 & 1560.0 & 1490.0 \\ 1930 & 3160.0 & 2410.0 & 1990.0 & 1810.0 & 1440.0\end{array}$

14-1335.00

$\begin{array}{cccccl}\text { NUMBER OF } & \text { CONSECUTIVE DAYS IN } & \text { YEAR ENDING SEPTEMBER } & 30 \\ 60 & 90 & 120 & 150 & 183 & 274 \\ 919.0 & 829.0 & 740.0 & 723.0 & 730.0 & 683.0 \\ 1570.0 & 1280.0 & 1240.0 & 1170.0 & 1090.0 & 982.0 \\ 1320.0 & 1230.0 & 1150.0 & 1070.0 & 1060.0 & 956.0 \\ 1190.0 & 1050.0 & 1030.0 & 950.0 & 888.0 & 786.0 \\ 1010.0 & 897.0 & 902.0 & 848.0 & 858.0 & 809.0 \\ 1340.0 & 1140.0 & 1050.0 & 1040.0 & 1020.0 & 888.0 \\ 1270.0 & 1150.0 & 1040.0 & 908.0 & 820.0 & 675.0 \\ 1080.0 & 929.0 & 875.0 & 812.0 & 802.0 & 625.0\end{array}$
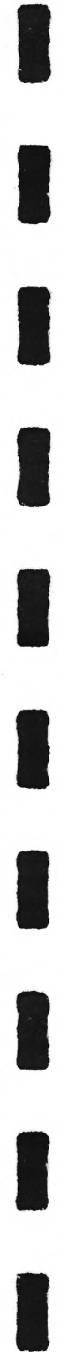
Salmon River near Government Camp, Oreg. STATION NUMBER 14-1340.00

DURATION TABLE OF DAILY DISCHARGE

$\begin{array}{lllllllllllllllllllllllllllllllllllll}\text { CLASS } & 0 & 1 & 2 & 3 & 4 & 5 & 6 & 7 & 8 & 9 & 10 & 11 & 12 & 13 & 14 & 15 & 16 & 17 & 18 & 19 & 20 & 21 & 22 & 23 & 24 & 25 & 26 & 27 & 28 & 29 & 30 & 31 & 32 & 33 & 34\end{array}$

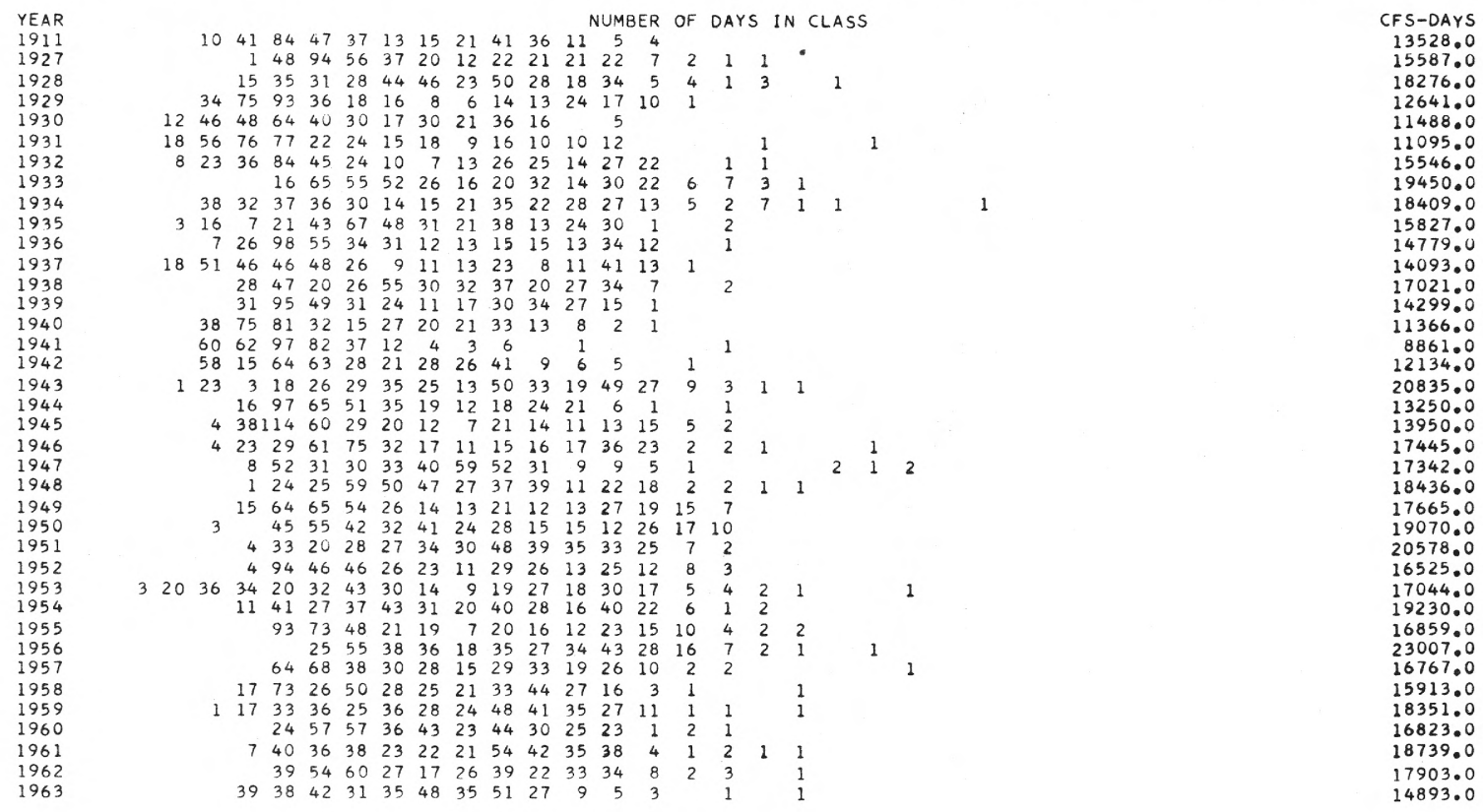

Salmon River near Government Camp, Oreg.

STATION NUMBER $\quad 14-1340.00$

Summary for water years 1911, 1927-63
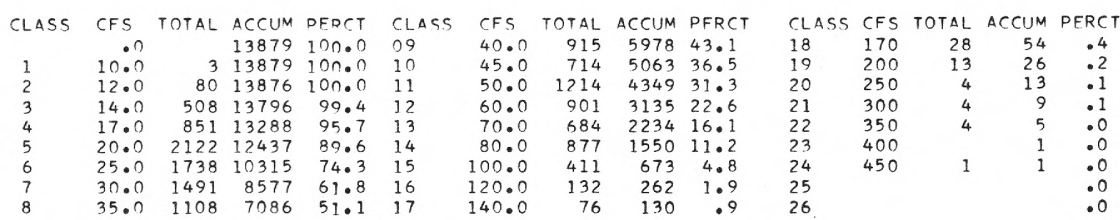

$\begin{array}{lc}\text { CLASS CFS TOTAL ACCUM PERCT } \\ 27 & : 0 \\ 28 & : 0 \\ 29 & .0 \\ 30 & .0 \\ 31 & .0 \\ 32 & .0 \\ 33 & .0 \\ 34 & .0\end{array}$

Salmon River near Government Camp, Oreg.

STATION NUMBER $14-1340.00$

LOWEST MEAN DISCHARGE, IN CFS, FOR THE FOLLOWING NUMBER OF CONSECUTIVE DAYS IN YEAR BEGINNING APRIL I

YEAR
1911
1927
1928
1929
1930
1931
1932
1933
1934
1935
1936
1937
1938
1939
1940
1941
1942
1943
1944
1945
1946
1947
1948
1949
1950
1951
1952
1953
1954
1955
1956
1957
1958
1959
1950
1961
1962
1962

1
12.0
20.0
15.0
12.0
12.0
13.0
20.0
21.0
13.0
16.0
12.0
17.0
18.0
15.0
14.0
14.0
13.0
21.0
16.0
16.0
21.0
18.0
22.0
14.0
14.0
18.0
11.0
18.0
20.0
23.0
22.0
17.0
16.0
21.0
19.0
20.0
22.0
19

3
$13 \cdot 3$
20.3
15.0
12.7
12.7
13.0
20.0
22.3
13.3
16.0
12.0
17.0
18.0
15.7
15.3
$14 \cdot 3$
13.7
21.0
16.0
16.0
21.7
18.0
22.0
$14 \cdot 3$
24.0
18.0
11.0
18.0
20.0
23.0
23.3
17.7
16.7
21.7
19.0
20.0
22.3
19

7
14.6
22.0
15.0
12.9
13.0
13.0
20.3
22.7
13.7
16.1
12.0
17.6
18.0
15.7
15.4
15.1
13.9
21.0
16.4
16.4
21.9
18.6
22.0
16.7
24.0
18.9
11.6
18.3
20.3
24.4
23.7
17.9
16.9
22.3
19.4
20.9
22.9

\begin{tabular}{|c|}
\hline $\begin{array}{l}4 \\
5.6 \\
3.2 \\
5.0 \\
3.1 \\
3.8 \\
3.6 \\
0.9 \\
4.5 \\
3.8 \\
8.7 \\
2.9 \\
8.5 \\
8.6 \\
5.8 \\
5.7 \\
5.9 \\
4.2 \\
1.1 \\
6.7 \\
6.9 \\
3.1 \\
9.5 \\
2.0 \\
8.3 \\
4.5 \\
9.5 \\
2.0 \\
8.8 \\
1.4 \\
5.4 \\
4.3 \\
8.6 \\
7.9 \\
2.9 \\
0.6 \\
1.4 \\
3.6\end{array}$ \\
\hline
\end{tabular}

30
16.4
24.9
15.1
13.7
16.0
14.3
22.0
28.5
15.1
19.9
13.3
20.5
20.0
16.5
17.2
16.0
14.5
23.6
18.0
17.9
23.8
21.3
23.1
21.0
26.8
20.8
13.6
20.4
22.9
27.0
25.6
20.1
20.4
23.7
21.5
22.9
24.6

60
20.5
29.5
16.4
15.3
16.5
14.9
23.6
30.6
15.4
20.3
15.1
21.3
20.2
17.5
17.4
16.7
15.1
26.1
19.8
20.3
26.0
22.2
25.8
24.0
30.8
24.5
14.5
21.4
25.4
36.0
28.5
21.1
21.7
26.2
23.0
25.7
26.8

90
20.7
33.7
17.4
17.2
17.7
16.0
25.6
37.0
16.1
20.7
17.1
23.8
21.7
18.5
18.0
17.2
16.4
27.5
20.6
21.0
29.6
24.6
27.8
24.7
34.7
25.0
15.4
23.2
27.1
40.3
30.8
21.4
23.0
29.7
25.7
27.4
28.4

120
$22 \cdot 7$
$38 \cdot 1$
$18 \cdot 5$
$19 \cdot 0$
$18 \cdot 4$
$17 \cdot 7$
$29 \cdot 9$
$39 \cdot 2$
$17 \cdot 5$
$21 \cdot 2$
$18 \cdot 3$
$27 \cdot 7$
$24 \cdot 0$
$19 \cdot 2$
$18 \cdot 5$
$18 \cdot 2$
$19 \cdot 7$
$30 \cdot 0$
$21 \cdot 1$
$22 \cdot 5$
$32 \cdot 2$
$28 \cdot 2$
$30 \cdot 0$
$27 \cdot 1$
$41 \cdot 7$
$27 \cdot 8$
$16 \cdot 7$
$26 \cdot 2$
$27 \cdot 5$
$48 \cdot 1$
$32 \cdot 6$
$22 \cdot 6$
$27 \cdot 0$
$34 \cdot 6$
$27 \cdot 9$
$28 \cdot 3$
$29 \cdot 8$

150
$27 \cdot 4$
$41 \cdot 7$
$19 \cdot 4$
$21 \cdot 7$
$18 \cdot 4$
$18 \cdot 2$
$37 \cdot 0$
$45 \cdot 2$
$20 \cdot 0$
$22 \cdot 6$
$18 \cdot 4$
$33 \cdot 9$
$26 \cdot 7$
$20 \cdot 7$
$19 \cdot 1$
$18 \cdot 8$
$23 \cdot 5$
$31 \cdot 7$
$21 \cdot 8$
$24 \cdot 6$
$36 \cdot 9$
$32 \cdot 0$
$30 \cdot 3$
$29 \cdot 1$
$47 \cdot 0$
$29 \cdot 4$
$18 \cdot 5$
$32 \cdot 3$
$27 \cdot 9$
$55 \cdot 8$
$37 \cdot 0$
$24 \cdot 2$
$33 \cdot 0$
$37 \cdot 2$
$32 \cdot 7$
30.2
34.4

\begin{tabular}{ll}
183 & 274 \\
27.5 & 35.9 \\
49.5 & 48.8 \\
19.7 & 22.5 \\
22.8 & 30.2 \\
19.0 & 21.6 \\
19.0 & 21.5 \\
39.9 & 38.4 \\
54.4 & 65.2 \\
23.3 & 30.4 \\
25.8 & 29.5 \\
19.1 & 23.6 \\
38.5 & 41.0 \\
28.7 & 30.5 \\
22.6 & 29.1 \\
20.9 & 23.4 \\
20.6 & 25.5 \\
29.5 & 37.0 \\
31.1 & 36.5 \\
22.9 & 27.9 \\
26.9 & 32.1 \\
44.6 & 49.2 \\
35.5 & 40.5 \\
29.6 & 32.4 \\
29.2 & 35.7 \\
50.0 & 53.6 \\
31.3 & 32.4 \\
21.9 & 36.7 \\
38.9 & 41.0 \\
27.9 & 31.8 \\
56.4 & 55.4 \\
39.7 & 44.7 \\
27.7 & 33.8 \\
39.2 & 44.4 \\
38.8 & 37.9 \\
35.0 & 44.6 \\
33.7 & 39.1 \\
38.0 & 42.0 \\
& \\
\hline
\end{tabular}


Salmon River near Government Camp, Oreg.

STATION NUMBER

$14-1340.00$

HIGHEST MEAN DISCHARGE, IN CFS, FOR THE FOLLOWING NUMBER OF CONSECUTIVE DAYS IN YEAR ENDING SEPTEMBER 30

\begin{tabular}{|c|c|c|c|c|c|c|c|c|c|c|c|}
\hline YEAR & 1 & 3 & 7 & 15 & 30 & 60 & 90 & 120 & 150 & 183 & 274 \\
\hline 1911 & 115.0 & 114.0 & 101.0 & 86.7 & 71.2 & 60.4 & 53.5 & $47 \cdot 8$ & 43.0 & 42.4 & 42.3 \\
\hline 1927 & 194.0 & 132.0 & $114 . \mathrm{C}$ & 100.0 & 89.5 & 82.1 & 71.1 & 61.0 & 55.9 & 51.4 & 46.9 \\
\hline 1928 & 250.0 & 184.0 & 162.0 & 134.0 & 99.5 & 77.7 & 66.8 & 63.0 & 57.4 & 60.8 & 58.3 \\
\hline 1929 & 124.0 & 117.0 & 112.0 & 105.0 & 90.6 & 81.9 & 71.1 & $61 \cdot 2$ & 54.9 & 49.1 & 38.8 \\
\hline 1930 & 96.0 & 80.7 & 76.1 & 63.1 & 55.3 & 51.6 & 48.7 & $47 \cdot 9$ & 45.7 & 42.7 & 36.7 \\
\hline 1931 & 330.0 & 198.0 & 122.0 & 83.9 & 72.2 & 68.8 & 59.6 & $52 \cdot 6$ & 46.9 & 42.8 & 35.1 \\
\hline 1932 & 194.0 & 148.0 & 113.0 & 121.0 & 103.0 & 94.7 & 84.9 & 79.0 & 70.8 & 62.8 & 49.9 \\
\hline 1933 & 209.0 & 181.0 & 165.0 & 145.0 & 127.0 & 108.0 & 93.0 & $82 \cdot 7$ & 73.2 & 65.7 & 60.5 \\
\hline 1934 & 493.0 & 321.0 & 233.0 & 168.0 & 129.0 & 112.0 & 95.5 & 88.9 & $81 \cdot 5$ & 75.8 & 61.3 \\
\hline 1935 & 163.0 & 135.0 & 97.4 & 82.9 & 81.3 & 75.6 & 68.2 & $60 \cdot 7$ & 55.7 & 52.6 & 50.0 \\
\hline 1936 & 157.0 & 111.0 & 102.0 & 95.8 & 94.1 & 88.1 & 77.4 & $67 \cdot 2$ & 60.1 & 55.3 & 46.9 \\
\hline 1937 & 125.0 & 112.0 & 103.0 & 97.4 & 95.2 & 91.4 & 81.6 & 72.1 & $64 \cdot 2$ & 57.8 & 45.6 \\
\hline 1938 & 146.0 & 121.0 & 99.6 & 93.0 & 86.2 & 82.3 & 72.7 & $64 \cdot 2$ & 59.1 & 59.0 & 55.0 \\
\hline 1939 & 109.0 & 88.0 & 82.7 & 79.6 & 77.5 & 71.2 & 67.4 & 62.5 & 55.7 & 49.9 & 45.2 \\
\hline 1940 & 110.0 & 83.0 & 77.7 & 66.5 & 58.4 & 54.8 & 52.4 & $49 \cdot 2$ & $44 \cdot 7$ & 42.6 & 35.4 \\
\hline 1941 & 146.0 & $92 \cdot 0$ & 66.1 & $51 \cdot 3$ & 38.8 & 31.8 & 29.5 & $28 \cdot 9$ & 28.6 & 28.9 & 26.7 \\
\hline 1942 & 124.0 & 98.7 & 79.6 & 66.5 & 58.2 & 53.6 & 51.3 & $46 \cdot 4$ & 42.7 & 40.6 & 38.4 \\
\hline 1943 & 221.0 & 149.0 & 134.0 & 118.0 & 114.0 & 98.3 & 94.8 & 86.7 & 78.9 & 71.6 & 67.4 \\
\hline 1944 & 140.0 & 88.0 & 77.9 & 73.1 & 72.1 & 65.3 & 56.7 & 50.7 & $46 \cdot 1$ & 42.9 & 40.6 \\
\hline 1945 & 153.0 & 134.0 & 122.0 & 116.0 & 103.0 & 84.7 & 70.3 & 59.8 & 55.7 & 53.1 & 44.0 \\
\hline 1946 & 315.0 & 203.0 & 134.0 & 116.0 & 109.0 & 98.7 & 87.9 & $76 \cdot 7$ & 67.8 & 61.9 & 55.7 \\
\hline 1947 & 384.0 & 364.0 & 265.0 & 162.0 & 109.0 & 78.3 & 70.3 & 63.5 & 63.5 & 62.2 & 55.4 \\
\hline 1948 & 220.0 & 140.0 & 112.0 & 109.0 & 101.0 & 84.7 & 74.4 & 66.0 & 60.8 & 57.9 & 56.8 \\
\hline 1949 & 169.0 & 164.0 & 153.0 & 137.0 & 128.0 & 113.0 & 99.5 & 86.8 & 76.0 & 68.0 & 55.8 \\
\hline 1950 & 168.0 & 151.0 & 145.0 & 141.0 & 133.0 & 118.0 & 99.3 & $86 \cdot 4$ & 79.3 & $73 \cdot 3$ & 60.4 \\
\hline 1951 & 151.0 & 132.0 & 117.0 & 114.0 & 106.0 & 92.3 & 83.7 & $74 \cdot 3$ & $70 \cdot 6$ & 69.3 & 65.8 \\
\hline 1952 & 152.0 & 144.0 & 132.0 & 124.0 & 109.0 & 93.4 & 83.7 & $75 \cdot 2$ & $66 \cdot 6$ & 59.0 & 50.6 \\
\hline 1953 & 352.0 & 256.0 & 177.0 & 125.0 & 115.0 & 86.8 & 78.0 & $68 \cdot 2$ & 69.1 & 69.6 & 57.0 \\
\hline 1954 & 182.0 & 134.0 & 115.0 & 109.0 & 104.0 & 98.7 & 88.4 & $78 \cdot 2$ & 70.5 & 65.7 & 62.2 \\
\hline 1955 & 203.0 & 197.0 & 175.0 & 149.0 & 133.0 & 112.0 & 95.3 & 81.5 & 72.5 & $64 \cdot 3$ & 52.2 \\
\hline 1956 & 300.0 & 203.0 & 143.0 & 137.0 & 125.0 & 110.0 & 99.0 & 87.8 & 78.2 & 70.4 & 71.2 \\
\hline 1957 & 362.0 & 220.0 & 154.0 & 113.0 & 84.5 & 78.5 & 70.4 & $66 \cdot 2$ & 60.5 & 60.8 & 53.3 \\
\hline 1958 & 234.0 & 157.0 & 113.0 & 86.5 & 79.7 & 77.2 & $70 \cdot 3$ & $62 \cdot 6$ & 60.0 & 57.6 & 50.7 \\
\hline 1959 & 228.0 & 158.0 & 115.0 & 92.7 & 82.4 & 73.9 & 69.2 & 62.5 & 60.1 & 62.2 & 58.0 \\
\hline 1960 & 147.0 & 119.0 & 92.9 & 84.5 & 80.8 & 73.7 & 69.5 & $64 \cdot 2$ & 58.0 & 54.1 & 51.2 \\
\hline 1961 & 249.0 & 154.0 & 113.0 & 102.0 & 84.8 & 77.3 & 71.4 & 71.6 & 70.8 & 67.4 & 59.6 \\
\hline 1962 & 234.0 & 170.0 & 117.0 & 92.4 & 88.5 & 83.8 & 78.5 & $70 \cdot 0$ & 62.7 & 63.6 & 56.4 \\
\hline 1963 & 239.0 & $136 . ?$ & 93.3 & 73.7 & 68.1 & 56.0 & 54.1 & 53.2 & 51.9 & 51.6 & 47.0 \\
\hline
\end{tabular}

Salmon River below Linney Creek, Oreg. STATION NUMBER 14-1345.00

DURATION TABLE OF DAILY DISCHARGE

CLASS $0 \begin{array}{llllllllllllllllllllllllllllllllllll} & 1 & 2 & 3 & 4 & 5 & 6 & 7 & 8 & 9 & 10 & 11 & 12 & 13 & 14 & 15 & 16 & 17 & 18 & 19 & 20 & 21 & 22 & 23 & 24 & 25 & 26 & 27 & 28 & 29 & 30 & 31 & 32 & 33 & 34\end{array}$

YEAR
1928
1929
1930
1931
1932
1933
1934
1935
1936
1937
1938
1939
1940
1941
1942
1943
1944
1945
1946
1947
1948
1949
1950
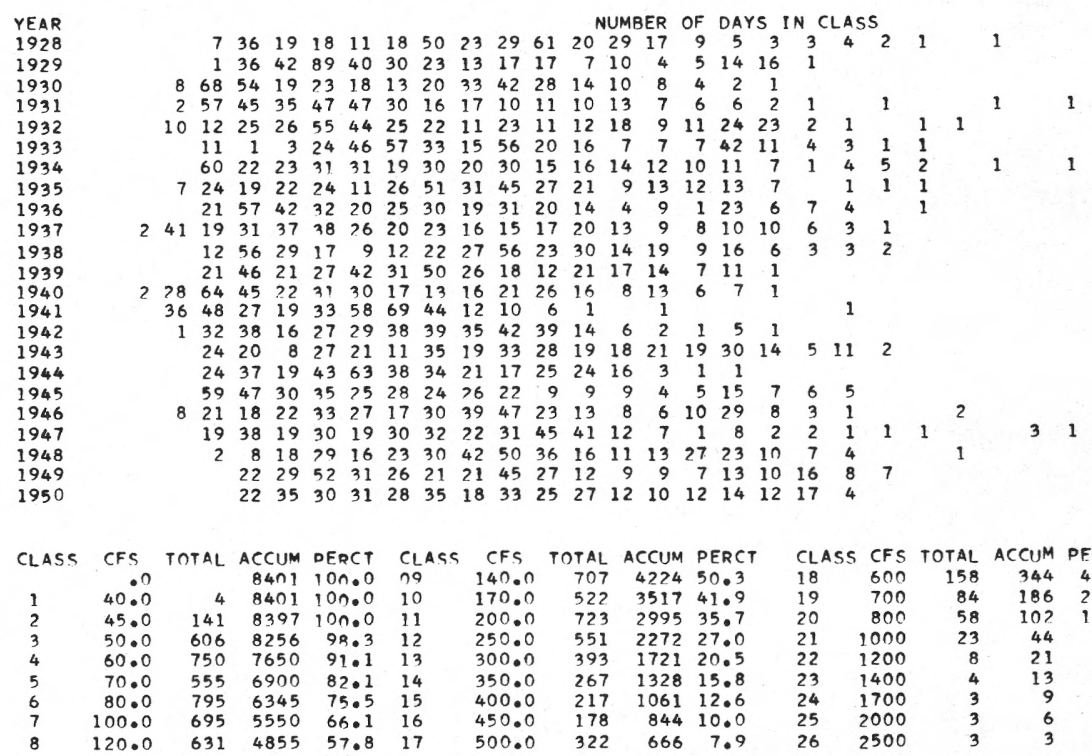

$\begin{array}{lrrrr}\text { CLASS CFS } & \text { TOTAL } & \text { ACCUM } & \text { PERCT } \\ 18 & 600 & 158 & 344 & 4.1 \\ 19 & 700 & 84 & 186 & 2.2 \\ 20 & 800 & 58 & 102 & 1.2 \\ 21 & 1000 & 23 & 44 & .5 \\ 22 & 1200 & 8 & 21 & .2 \\ 23 & 1400 & 4 & 13 & .2 \\ 24 & 1700 & 3 & 9 & 11 \\ 25 & 2000 & 3 & 6 & 11 \\ 26 & 2500 & 3 & 3 & .0\end{array}$


Salmon River below Linney Creek, Oreg.

STATION NUMBER $14-1345.00$

LOWEST MEAN DISCHARGE, IN CFS, FOR THE FOLLOWING NUMBER OF CONSECUTIVE DAYS IN YEAR BEGINNING APRIL I

\begin{tabular}{|c|c|c|c|c|c|c|c|c|c|c|c|}
\hline YEAR & 1 & 3 & 7 & 14 & 30 & 60 & 90 & 120 & 150 & 183 & 274 \\
\hline 1928 & 50.0 & 51.3 & 53.1 & 58.1 & 62.6 & 65.6 & 70.1 & 74.0 & 84.6 & 89.5 & $\begin{array}{r}94.9 \\
\end{array}$ \\
\hline 1929 & 48.0 & 48.0 & 48.3 & 50.1 & 52.6 & 57.8 & 59.8 & 63.6 & 72.0 & 93.5 & $140 \cdot 0$ \\
\hline 1930 & 49.0 & 49.7 & 50.4 & 55.0 & 55.7 & 56.9 & 58.7 & 61.4 & 66.1 & 69.6 & 87.7 \\
\hline 1931 & 48.0 & 48.0 & $48 \cdot 3$ & 48.9 & 53.6 & 55.2 & 57.8 & 65.4 & 72.9 & 79.2 & 103.0 \\
\hline 1932 & 54.0 & 55.3 & 56.0 & 56.9 & 61.6 & 70.3 & 78.2 & 89.7 & $117 \cdot 0$ & 154.0 & 163.0 \\
\hline 1933 & 79.0 & 79.3 & 79.9 & 82.0 & 94.8 & 97.4 & 107.0 & $129 \cdot 0$ & 139.0 & 183.0 & 273.0 \\
\hline 1934 & 48.0 & 48.7 & 49.4 & 49.7 & 51.8 & 52.6 & 53.8 & $58 \cdot 1$ & $65 \cdot 1$ & 78.5 & 135.0 \\
\hline 1935 & 51.0 & 51.7 & 52.7 & 53.1 & 56.8 & 60.2 & 63.7 & 66.3 & 70.0 & 79.0 & 134.0 \\
\hline 1936 & 44.0 & 44.7 & 45.3 & 46.0 & 46.8 & 48.9 & 54.5 & $59 \cdot 4$ & 66.7 & $72 \cdot 4$ & 92.3 \\
\hline 1937 & 61.0 & 61.7 & 61.9 & 64.9 & 67.1 & 74.6 & 80.0 & $91 \cdot 2$ & 119.0 & 159.0 & 202.0 \\
\hline 1938 & 36.0 & 56.7 & 57.9 & 58.4 & 59.6 & 62.3 & 64.6 & $70 \cdot 5$ & 83.2 & 99.8 & 121.0 \\
\hline 1939 & 55.0 & 55.3 & 55.4 & 56.8 & 60.3 & 63.8 & 63.7 & 64.9 & 69.6 & 83.1 & 118.0 \\
\hline 1940 & 44.0 & $44 \cdot 3$ & 45.6 & 47.0 & 48.7 & 49.8 & 51.7 & $55 \cdot 2$ & 60.4 & 67.8 & 96.7 \\
\hline 1941 & 45.0 & 45.3 & 45.9 & 47.9 & 50.0 & 53.9 & 56.8 & $60 \cdot 9$ & 66.8 & 81.9 & 122.0 \\
\hline 1942 & 49.0 & 49.7 & 49.9 & 50.9 & 52.0 & 54.5 & 60.0 & $70 \cdot 5$ & 87.8 & 113.0 & 191.0 \\
\hline 1943 & 58.0 & 59.0 & 59.7 & 61.5 & 64.8 & 73.3 & 85.0 & $103 \cdot 0$ & 113.0 & 11400 & 129.0 \\
\hline 1944 & 50.0 & 51.0 & 51.3 & 31.4 & 54.9 & 58.4 & 60.4 & 63.0 & 66.9 & 70.5 & 116.0 \\
\hline 1945 & 48.0 & 48.3 & 48.9 & 49.3 & 52.6 & 58.7 & 60.9 & 67.2 & 74.6 & 93.1 & 152.0 \\
\hline 1946 & 58.0 & 58.3 & 39.3 & 61.9 & 65.4 & 70.1 & 80.4 & 97.6 & 117.0 & 155.0 & 234.0 \\
\hline 1947 & 54.0 & 54.0 & 55.4 & 58.2 & 60.9 & 63.9 & 70.3 & 83.4 & 97.2 & 125.0 & 192.0 \\
\hline 1948 & 69.0 & 69.0 & 69.6 & 70.2 & 75.6 & 84.9 & 93.6 & $99 \cdot 3$ & 117.0 & 147.0 & 160.0 \\
\hline 1949 & 62.0 & 62.7 & 63.1 & 63.9 & 69.4 & $74 \cdot 8$ & 79.7 & $83 \cdot 4$ & 98.1 & 114.0 & 155 \\
\hline
\end{tabular}

Salmon River below Linney Creek, Oreg.

STATION NUMBER

$14-1345.00$

HIGHEST MEAN DISCHARGE, IN CFS, FOR THE FOLLOWING NUMBER OF CONSECUTIVF DAYS IN YEAR ENDING SEPTFMBFR 30

YEAR
1998
1929
1930
1931
1932
1933
1934
1935
1936
1937
1938
1939
1940
1941
1942
1943
1944
1945
1946
1947
1948
1949
1950

1
1920.0
714.0
610.0
2530.0
1480.0
1320.0
2650.0
1240.0
1300.0
1080.0
1110.0
600.0
680.0
834.0
621.0
1100.0
505.0
884.0
1600.0
2500.0
1610.0
1180.0
874.0

3
1310.0
694.0
563.0
1860.0
1230.0
1150.0
1860.0
1030.0
927.0
972.0
918.0
583.0
517.0
528.0
595.0
919.0
403.0
858.0
1360.0
2380.0
1080.0
1100.0
845.0

$170^{7} .0$

871.0

871.0
663.0
457.0

791.0

682.0
773.0

1000.0

574.0
715.0

715.0

$666 . ?$

465.0

440.0

280.0
365.0

365.0

739.0
375.0

375.0
699.0

646.0

$1070 . ?$

626.0
929.0
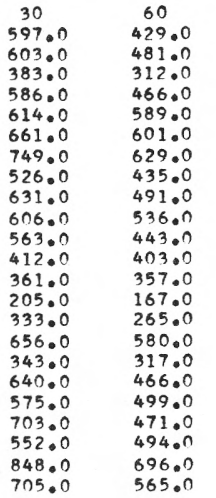

90
363.0
399.0
290.0
368.0
533.0
495.0
501.0
356.0
399.0
460.0
374.0
343.0
335.0
156.0
240.0
528.0
274.0
372.0
413.0
420.0
413.0
560.0
492.0

120
$331 \cdot 0$
$347 \cdot 0$
$270 \cdot 0$
$309 \cdot 0$
$471 \cdot 0$
$426 \cdot 0$
$449 \cdot 0$
$313 \cdot 0$
$355 \cdot 0$
$401 \cdot 0$
$338 \cdot 0$
$795 \cdot 0$
$291 \cdot 0$
$149 \cdot 0$
$226 \cdot 0$
$448 \cdot 0$
$243 \cdot 0$
343.0
$366 \cdot 0$
$379 \cdot 0$
$382 \cdot 0$
$479 \cdot 0$
462.0

150

337.0
302.0
248.0

406.0

371.0

399.0

344.0

351.0

340.0

267.0

262.0

222.0

409.

$225 \circ$

348.0

369.0
347.0
421.0

421.0

$\begin{array}{ll}183 & 274 \\ 344.0 & 296.0 \\ 263.0 & 212.0 \\ 237.0 & 186.0 \\ 244.0 & 190.0 \\ 365.0 & 282.0 \\ 336.0 & 308.0 \\ 363.0 & 277.0 \\ 298.0 & 261.0 \\ 320.0 & 244.0 \\ 308.0 & 237.0 \\ 347.0 & 286.0 \\ 253.0 & 215.0 \\ 238.0 & 182.0 \\ 145.0 & 128.0 \\ 228.0 & 198.0 \\ 398.0 & 356.0 \\ 203.0 & 183.0 \\ 287.0 & 218.0 \\ 345.0 & 280.0 \\ 350.0 & 279.0 \\ 341.0 & 329.0 \\ 366.0 & 311.0 \\ 378.0 & 304.0\end{array}$

296.0

86.0

82.0

08.0

1.0

37.0

86.0

198.0

56.0

18.0

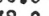

135.

(565.

(1)

(1) 
CLASS $0 \begin{array}{llllllllllllllllllllllllllllllllllll} & 0 & 2 & 3 & 4 & 5 & 6 & 7 & 8 & 9 & 10 & 11 & 12 & 13 & 14 & 15 & 16 & 17 & 18 & 19 & 20 & 21 & 22 & 23 & 24 & 25 & 26 & 27 & 28 & 29 & 30 & 31 & 32 & 33 & 34\end{array}$

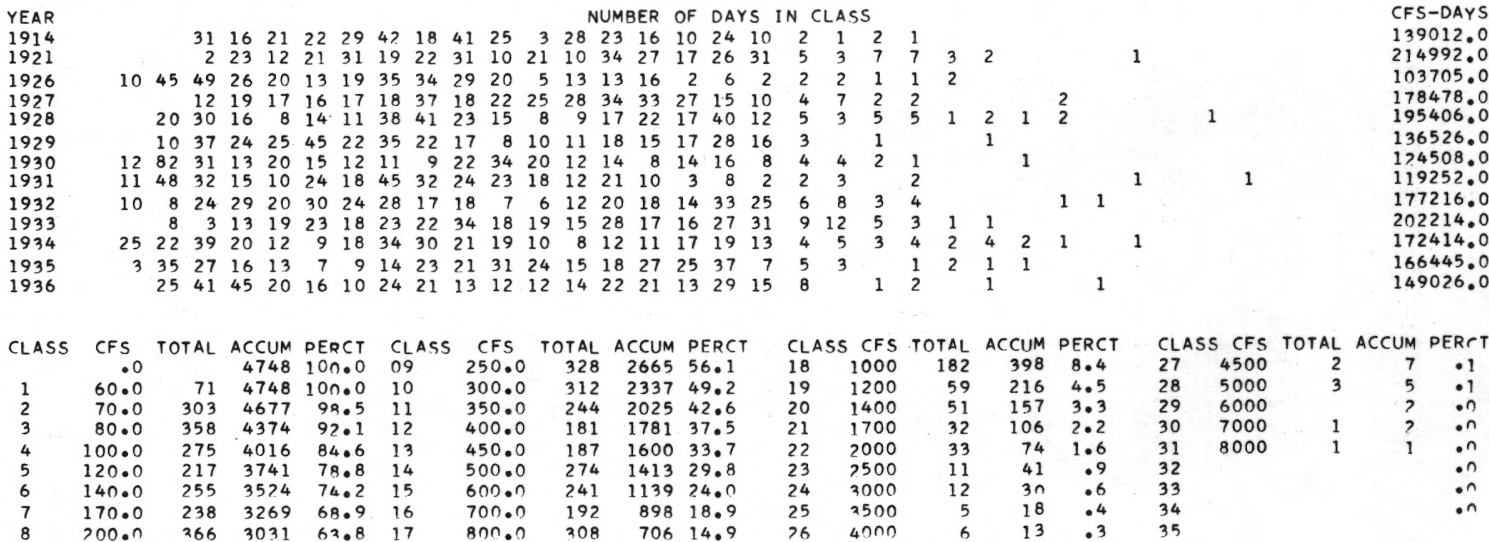

Salmon River at Welches, Oreg.

STATION NUMBER $14-1350.00$

LOWEST MEAN DISCHARGE, IN CFS, FOR THE FOLLOWING NUMBER OF CONSECUTIVE DAYS IN YEAR BEGINNING APRIL 1

\begin{tabular}{|c|c|c|c|c|c|c|c|c|c|c|c|}
\hline YEAR & 1 & 3 & 7 & 14 & 30 & 60 & 90 & 120 & 150 & 183 & 274 \\
\hline 1925 & 69.0 & 69.0 & 71.6 & 73.3 & 77.4 & 81.3 & 87.2 & $94 \cdot 1$ & $11 n \cdot 0$ & 137.0 & 254.0 \\
\hline 1926 & 68.0 & 68.0 & 68.4 & 69.9 & 73.6 & 88.1 & 93.4 & 109.0 & 132.0 & 157.0 & 273.0 \\
\hline 1977 & 92.0 & $94 . n$ & 94.9 & 97.4 & 105.0 & 130.0 & 166.0 & $252 \cdot 0$ & 291.0 & $367: 0$ & 505.0 \\
\hline 1928 & 75.0 & 75.0 & 75.0 & 76.7 & 83.1 & 88.0 & 100.0 & $108 \cdot 0$ & 129.0 & 165.0 & 198.0 \\
\hline 1929 & 65.0 & 65.0 & 67.0 & 69.2 & 71.5 & 78.9 & 82.4 & 86.1 & 98.3 & 149.0 & 310.0 \\
\hline 1930 & 69.0 & 71.0 & 72.0 & 72.9 & 75.6 & 78.1 & 88.3 & $97 \cdot 2$ & 113.0 & 148.0 & 208.0 \\
\hline 1931 & 65.0 & 65.0 & 66.7 & 68.7 & 73.0 & 77.5 & 80.1 & 97.5 & 121.0 & 153.0 & 234.0 \\
\hline 1932 & 73.0 & 73.0 & $74 \cdot 3$ & 77.7 & 84.0 & 96.7 & 112.0 & $136 \cdot 0$ & 188.0 & 302.0 & 385.0 \\
\hline 1933 & 93.0 & 93.0 & 95.6 & 102.0 & 130.0 & 147.0 & 154.0 & 197.0 & 272.0 & 366.0 & 567.0 \\
\hline 1934 & 68.0 & 68.0 & 68.0 & 68.4 & 69.4 & 74.2 & 76.8 & 85.1 & 103.0 & 136.0 & 319.0 \\
\hline 1935 & 72.0 & 72.0 & 72.9 & 75.5 & 80.1 & 82.8 & 87.1 & 96.2 & 106.0 & 125.0 & 279.0 \\
\hline
\end{tabular}

Salmon River at Welches, Oreg.

STATION NUMBER $14-1350.00$

HIGHEST MEAN DISCHARGE. IN CFS, FOR THE FOLLOWING NUMBER OF CONSECUTIVE DAYS IN YEAR FNDING SEPTEMBER 30

\begin{tabular}{|c|c|c|c|c|c|c|c|c|c|c|c|}
\hline YEAR & 1 & 3 & 7 & 15 & 30 & 60 & 90 & 120 & 150 & 183 & 274 \\
\hline 1914 & 2290.0 & 1400.0 & 1140.0 & 907.0 & 808.0 & 768.0 & 659.0 & 650.0 & 591.0 & 542.0 & 459.0 \\
\hline 1921 & 5230.0 & 3210.0 & 2700.0 & 1880.0 & 1220.0 & 1010.0 & 991.0 & $952 \cdot 0$ & 970.0 & 897.0 & 731.0 \\
\hline 1926 & 2920.0 & 2090.0 & 1410.0 & 965.0 & 908.0 & 678.0 & 627.0 & 563.0 & 505.0 & 455.0 & 345.0 \\
\hline 1977 & 4470.0 & 2710.0 & 1670.0 & 1100.0 & 954.0 & 799.0 & 733.0 & $718 \cdot 0$ & 665.0 & 671.0 & 588.0 \\
\hline 1978 & 7000.0 & $4370 . n$ & 3720.0 & 2460.0 & 1660.0 & 1170.0 & 935.0 & $8>6 \cdot 0$ & $83 n .0$ & 825.0 & 676.0 \\
\hline 1979 & 3300.0 & $1920 \cdot 0$ & 1250.0 & $10 \mathrm{an} \cdot 0$ & 1030.0 & 878.0 & 810.0 & 727.0 & 625.0 & 568.0 & $46 ? .0$ \\
\hline 1930 & 3540.0 & $2090 . n$ & 1480.0 & 1320.0 & 1080.0 & 788.0 & 667.0 & 630.0 & 585.0 & 575.0 & 478.0 \\
\hline 1931 & 8000.0 & 5110.0 & 2950.0 & 1880.0 & 1320.0 & 905.0 & 718.0 & 635.0 & $\begin{array}{l}566.0 \\
847.0\end{array}$ & 514.0 & 407.0 \\
\hline $\begin{array}{l}1932 \\
1933\end{array}$ & $\begin{array}{l}4690.0 \\
3280.0\end{array}$ & $\begin{array}{l}3670.0 \\
2620.0\end{array}$ & $\begin{array}{l}2480.0 \\
1940.0\end{array}$ & $\begin{array}{l}1900.0 \\
1480.0\end{array}$ & 1390.0 & 1180.0 & $\begin{array}{r}1090.0 \\
972.0\end{array}$ & $956 \cdot 0$ & 847.0 & $\begin{array}{l}779.0 \\
749.0\end{array}$ & $\begin{array}{l}612.0 \\
688.0\end{array}$ \\
\hline $\begin{array}{l}1933 \\
1934\end{array}$ & $\begin{array}{l}3280.0 \\
5270.0\end{array}$ & $\begin{array}{l}2620.0 \\
4380.0\end{array}$ & $\begin{array}{l}1940.0 \\
3460.0\end{array}$ & $\begin{array}{l}1480.0 \\
2370.0\end{array}$ & $\begin{array}{l}1310.0 \\
1770.0\end{array}$ & $\begin{array}{l}1140.0 \\
1400.0\end{array}$ & $\begin{array}{r}972.0 \\
1100.0\end{array}$ & $\begin{array}{l}849.0 \\
967.0\end{array}$ & $\begin{array}{l}749.0 \\
867.0\end{array}$ & $\begin{array}{l}749.0 \\
803.0\end{array}$ & 602.0 \\
\hline $\begin{array}{l}1934 \\
1935\end{array}$ & $\begin{array}{l}5270.0 \\
3790.0\end{array}$ & $\begin{array}{l}4380.0 \\
3230.0\end{array}$ & $\begin{array}{l}3460.0 \\
2420.0\end{array}$ & $\begin{array}{l}2370.0 \\
1450.0\end{array}$ & $\begin{array}{r}1770.0 \\
976.0\end{array}$ & $\begin{array}{r}1400.0 \\
825.0\end{array}$ & $\begin{array}{r}1100.0 \\
729.0\end{array}$ & 676.0 & 674.0 & 662.0 & 578.0 \\
\hline 36 & 4800.0 & 3030.0 & 020.0 & 1580.0 & 1090.0 & 835.0 & 754.0 & 733.0 & 744.0 & 685.0 & 512.0 \\
\hline
\end{tabular}



Salmon River above Boulder Creek,
near Brightwood, Oreg.
STATION NUMBER 14-1355.00

DURATION TABLF OF DAILY DISCHARGF

CLASS $0 \begin{array}{lllllllllllllllllllllllllllllllllllll} & 0 & 1 & 3 & 4 & 5 & 6 & 7 & 8 & 9 & 10 & 11 & 12 & 13 & 14 & 15 & 16 & 17 & 18 & 19 & 20 & 21 & 22 & 23 & 24 & 25 & 26 & 27 & 28 & 29 & 30 & 31 & 32 & 33 & 34\end{array}$

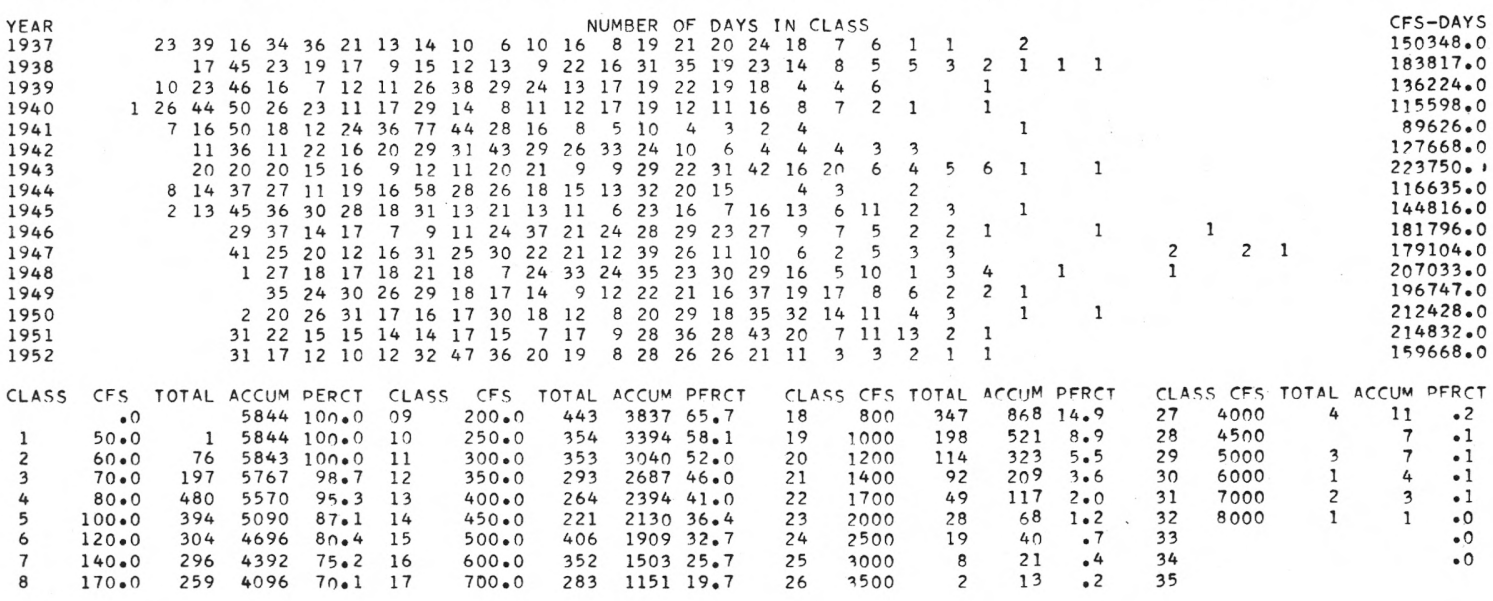

Salmon River above Boulder Creek, near Brightwood, Oreg.

STATION NUMBER 14-1355.00

LOWEST MEAN DISCHARGE, IN CFS, FOR THE FOLLOWING NUMBER OF CONSECUTIVE DAYS IN YEAR BEGINNING APRIL 1

\begin{tabular}{|c|c|c|c|c|c|c|c|c|c|c|c|}
\hline YEAR & 1 & 3 & 7 & 14 & 30 & 60 & 90 & 120 & 150 & 183 & 274 \\
\hline 1937 & 88.0 & 88.7 & $90 \cdot 3$ & 97.9 & 99.3 & 107.0 & $120 \cdot 0$ & $143 \cdot 0$ & 221.0 & 309.0 & 479.0 \\
\hline 1938 & 73.0 & 74.3 & 75.9 & 76.9 & 79.7 & 84.8 & 89.1 & 97.9 & $122 \cdot 0$ & $158 \cdot 0$ & 265.0 \\
\hline 1939 & 67.0 & 67.7 & 67.9 & 69.3 & 73.9 & 83.0 & 90.4 & 96.5 & 103.0 & 132.0 & 213.0 \\
\hline 1940 & 59.0 & $60 \cdot 3$ & 62.9 & 67.5 & 69.1 & 71.6 & 77.4 & 81.9 & 95.5 & $115 \cdot 0$ & 210.0 \\
\hline 1941 & 74.0 & 74.0 & 76.0 & 79.1 & 83.4 & 92.2 & 108.0 & $132 \cdot 0$ & 144.0 & 183.0 & 285.0 \\
\hline 1942 & 71.0 & 71.3 & 71.7 & 75.7 & 76.9 & 80.5 & 89.0 & $108 \cdot 0$ & 156.0 & 215.0 & 436.0 \\
\hline 1943 & 88.0 & 88.0 & 90.0 & 91.4 & 94.9 & $108 \cdot 0$ & 124.0 & 168.0 & 204.0 & 235.0 & 278.0 \\
\hline 1944 & 65.0 & 65.3 & 66.0 & $70 \cdot 8$ & 76.6 & 88.2 & 88.0 & $96 \cdot 3$ & $105 \cdot 0$ & $121 \cdot 0$ & 232.0 \\
\hline 1945 & 81.0 & $82 \cdot 3$ & 83.3 & 85.6 & 97.6 & 109.0 & $108 \cdot 0$ & $116 \cdot 0$ & $145 \cdot 0$ & 209.0 & 407.0 \\
\hline 1946 & 89.0 & 91.0 & 92.7 & 96.9 & 101.0 & 107.0 & 120.0 & 164.0 & 215.0 & 281.0 & 520.0 \\
\hline 1947 & 87.0 & 87.7 & 89.9 & 92.0 & 98.6 & 103.0 & 120.0 & $155 \cdot 0$ & 175.0 & 227.0 & 403. \\
\hline 1948 & 100.0 & $100 \cdot 0$ & 100.0 & 103.0 & 111.0 & 127.0 & 152.0 & 174.0 & 205.0 & 296.0 & $384^{\circ}$ \\
\hline 1949 & 97.0 & 99.7 & 104.0 & 107.0 & 114.0 & 126.0 & 148.0 & $170 \cdot 0$ & 185.0 & 254.0 & 375.0 \\
\hline 1950 & 101.0 & 102.0 & $104 \cdot ?$ & $108 \cdot 0$ & 118.0 & 140.0 & 180.0 & 231.0 & 353.0 & 466.0 & 595. \\
\hline 1951 & 80.0 & 80.0 & 81.1 & 83.2 & 90.6 & 101.0 & 121.0 & 155.0 & 217.0 & 266.0 & 358.0 \\
\hline
\end{tabular}

Salmon River above Boulder Creek, near Brightwood, Oreg.

STATION NUMBER 14-1355.00

HIGHEST MEAN DISCHARGE, IN CFS, FOR THE FOLLOWING NUMBER OF CONSECUTIVE DAYS IN YEAR ENDING SEPTEMBER 30

\begin{tabular}{|c|c|c|c|c|c|c|c|c|c|c|c|}
\hline YEAR & 1 & 3 & 7 & 15 & 30 & 60 & 90 & 120 & 150 & 183 & 274 \\
\hline 1937 & 3190.0 & 2930.0 & 1980.0 & $1380 \cdot 0$ & 1240.0 & 1060.0 & 950.0 & $871 \cdot 0$ & 777.0 & 667.0 & 522.0 \\
\hline 1938 & 4020.0 & 3130.0 & $2220 \cdot 0$ & $1410 \cdot 0$ & 1190.0 & 989.0 & 919.0 & $803 \cdot 0$ & $800 \cdot 0$ & 811.0 & 640.0 \\
\hline 1939 & 2710.0 & 1830.0 & $1380 \cdot 0$ & 1100.0 & 909.0 & 777.0 & $698 \cdot 0$ & $616 \cdot 0$ & 601.0 & 574.0 & 468.0 \\
\hline 1940 & 2640.0 & $1840 \cdot 0$ & $1520 \cdot 0$ & 1050.0 & 968.0 & 835.0 & $749 \cdot 0$ & $635 \cdot 0$ & $601 \cdot 0$ & 534.0 & $\begin{array}{l}395.0 \\
293.0\end{array}$ \\
\hline 1941 & 3030.0 & 1790.0 & 1130.0 & 796.0 & 543.0 & 453.0 & $404 \cdot 0$ & $361 \cdot 0$ & $335 \cdot 0$ & $\begin{array}{l}339.0 \\
488.0\end{array}$ & $\begin{array}{l}293.0 \\
428.0\end{array}$ \\
\hline 1942 & 1830.0 & 1570.0 & 1260.0 & 827.0 & 794.0 & 602.0 & 552.0 & $516 \cdot 0$ & 491.0 & $\begin{array}{l}488.0 \\
927.0\end{array}$ & $\begin{array}{l}428.0 \\
780.0\end{array}$ \\
\hline 1943 & 4140.0 & $266 n \cdot n$ & 2380.0 & $1840 . ?$ & 1420.0 & $1210 \cdot 0$ & $1090 \cdot 0$ & $962 \cdot 0$ & $949 \cdot 0$ & $\begin{array}{l}927.0 \\
447.0\end{array}$ & $\begin{array}{l}780.0 \\
394.0\end{array}$ \\
\hline 1944 & 1980.0 & $1340 \cdot n$ & $809 \cdot 0$ & 671.0 & 638.0 & $593 . ?$ & $561 \cdot 0$ & $515 \cdot 0$ & $471 \cdot 0$ & & $\begin{array}{l}394.0 \\
494.0\end{array}$ \\
\hline 1945 & 3390.0 & $2110 \cdot 0$ & 1720.0 & $135 n \cdot 0$ & $1270 \cdot 0$ & 953.0 & $798 \cdot 0$ & $782 \cdot 0$ & 749.0 & 749.0 & $\begin{array}{l}494.0 \\
627.0\end{array}$ \\
\hline 1946 & $6600 \cdot 0$ & $4280 \cdot$ ? & $2650 \cdot 0$ & $1850 \cdot 0$ & $1300 \cdot 0$ & 972.0 & $840 \cdot 0$ & $780 \cdot 0$ & $\begin{array}{l}769 \cdot 0 \\
851.0\end{array}$ & 786.0 & 618.0 \\
\hline $\begin{array}{l}1947 \\
1948\end{array}$ & $\begin{array}{l}8680.0 \\
5230.0\end{array}$ & $\begin{array}{l}7940.0 \\
3360.0\end{array}$ & $\begin{array}{l}5450.0 \\
2060.0\end{array}$ & $\begin{array}{l}2950.0 \\
1490.0\end{array}$ & $\begin{array}{l}1950.0 \\
1050.0\end{array}$ & $\begin{array}{r}1230.0 \\
856.0\end{array}$ & $\begin{array}{r}1060.0 \\
886.0\end{array}$ & $\begin{array}{l}923.0 \\
803.0\end{array}$ & 784.0 & 736.0 & 705.0 \\
\hline 1949 & 3180.0 & 2600.0 & 1820.0 & 1690.0 & 1460.0 & 1180.0 & 1020.0 & 952.0 & 832.0 & $800 \cdot 0$ & 668.0 \\
\hline 1950 & 4130.0 & 3060.0 & 2020.0 & $1590 \cdot 0$ & 1240.0 & 1030.0 & 989.0 & $1010 \cdot 0$ & 935.0 & $861 \cdot 0$ & 716.0 \\
\hline 1951 & 2580.0 & $1900 \cdot 0$ & 1670.0 & 1300.0 & 1210.0 & 1040.0 & 1030.0 & 975.0 & 886.0 & 871.0 & 744.0 \\
\hline 1952 & 2650.0 & $1700 \cdot 0$ & 1380.0 & 968.0 & 773.0 & 735.0 & $628 \cdot 0$ & $624 \cdot 0$ & $570 \cdot 0$ & 583.0 & 534.0 \\
\hline
\end{tabular}


CLASS $\begin{array}{lllllllllllllllllllllllllllllllllllll} & 0 & 2 & 3 & 4 & 5 & 6 & 7 & 8 & 9 & 10 & 11 & 12 & 13 & 14 & 15 & 16 & 17 & 18 & 19 & 20 & 21 & 22 & 23 & 24 & 25 & 26 & 27 & 28 & 29 & 30 & 31 & 32 & 33 & 34\end{array}$

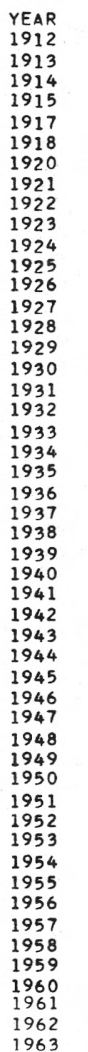

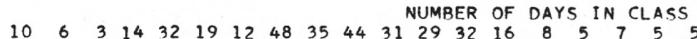

$\begin{array}{rrrrrrrrrrrrrrrrrrrrrr}5 & 12 & 21 & 27 & 17 & 5 & 52 & 38 & 39 & 27 & 28 & 37 & 27 & 18 & 4 & 2 & 2 & 1 & 1 & & 2 & 1 \\ 26 & 11 & 21 & 34 & 30 & 28 & 51 & 33 & 32 & 30 & 16 & 22 & 12 & 5 & 5 & 3 & 1 & & & & \end{array}$

$\begin{array}{rrrrrrrrrrrrrrrr}5 & 26 & 11 & 21 & 34 & 30 & 28 & 51 & 33 & 32 & 30 & 16 & 22 & 12 \\ 3 & 21 & 16 & 13 & 6 & 21 & 31 & 46 & 63 & 55 & 38 & 26 & 17 & 5 & 3\end{array}$

$\begin{array}{llllllllllllllllllll}10 & 18 & 2 & 29 & 23 & 16 & 28 & 35 & 28 & 12 & 38 & 26 & 26 & 27 & 15 & 13 & 11 & 5 & 3\end{array}$

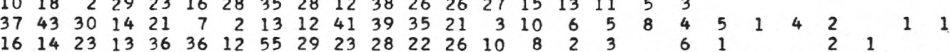

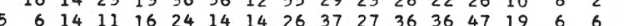

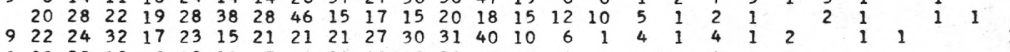

$\begin{array}{lllllllllllllllll}13 & 38 & 55 & 18 & 13 & 29 & 11 & 7 & 24 & 31 & 32 & 42 & 23 & 12 & 3 & 4 & 4\end{array}$

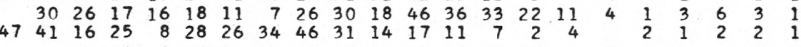

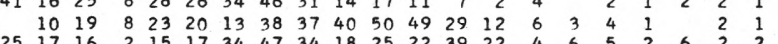

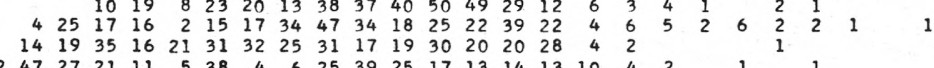

$\begin{array}{lllllllllllllllllll}42 & 47 & 27 & 21 & 11 & 5 & 38 & 4 & 6 & 25 & 39 & 25 & 17 & 13 & 14 & 13 & 10 & 4 & 2\end{array}$

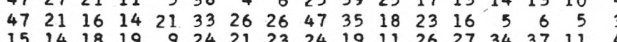

$\begin{array}{llllllllllllllllllllllll}18 & 15 & 14 & 18 & 19 & 9 & 24 & 21 & 23 & 24 & 19 & 11 & 26 & 27 & 34 & 37 & 11 & 4 & 4 & 3 & 3\end{array}$

$\begin{array}{rrrrrrrrrrrrrrrrrr}4 & 7 & 5 & 7 & 16 & 4 & 23 & 18 & 16 & 42 & 31 & 20 & 31 & 36 & 33 & 29 & 16 & 8 \\ 11 & 26 & 27 & 24 & 19 & 14 & 8 & 27 & 33 & 47 & 23 & 11 & 9 & 10 & 18 & 15 & 12 & 6\end{array}$

$\begin{array}{llllllllllllllllllllllll}1 & 23 & 30 & 22 & 13 & 20 & 35 & 12 & 21 & 27 & 23 & 30 & 18 & 23 & 30 & 13 & 12 & 4 & 1 & 4 & 1 & 2 & 1\end{array}$

$\begin{array}{rrrrrrrrrrrrrrrrrrrrrrrr}25 & 11 & 25 & 22 & 28 & 17 & 17 & 8 & 17 & 21 & 20 & 29 & 20 & 26 & 21 & 6 & 6 & 3 & 1 & 2 & 1 & \\ 25 & 31 & 19 & 7 & 12 & 21 & 12 & 18 & 23 & 16 & 28 & 36 & 31 & 37 & 16 & 6 & 6 & 3 & 2 & 8 & 3 & 2 & 1 & 2\end{array}$

$\begin{array}{llllllllllllllllllllll}34 & 33 & 15 & 11 & 2 & 14 & 14 & 16 & 61 & 40 & 26 & 29 & 27 & 20 & 7 & 6 & 6 & 3 & 1\end{array}$

$\begin{array}{rrrrrrrrrrrrrrrrrrrrrr}19 & 49 & 34 & 26 & 21 & 19 & 27 & 17 & 17 & 19 & 13 & 23 & 20 & 13 & 18 & 13 & 9 & 4 & 3 & 1 & 1 \\ 2 & 33 & 28 & 12 & 17 & 8 & 54 & 48 & 40 & 54 & 27 & 16 & 7 & 9 & 4 & 2 & & 3 & & & 1\end{array}$

$\begin{array}{rrrrrrrrrrrrrrrrrrrrr}2 & 33 & 28 & 12 & 17 & 8 & 54 & 48 & 40 & 54 & 27 & 16 & 7 & 9 & 4 & 2 & & 3 & & \\ 13 & 18 & 8 & 12 & 11 & 12 & 15 & 21 & 23 & 59 & 54 & 49 & 30 & 11 & 11 & 4 & 5 & 4 & 2 & 3\end{array}$

$\begin{array}{lllllllllllllllllllll}3 & 12 & 20 & 11 & 12 & 13 & 8 & 13 & 26 & 28 & 13 & 34 & 24 & 46 & 28 & 11 & 13\end{array}$

$\begin{array}{rrrrrrrrrrrrrrrrrrr}11 & 38 & 23 & 12 & 13 & 33 & 35 & 29 & 45 & 30 & 23 & 35 & 24 & 7 & 1 & 4 & 1 & \\ 13 & 38 & 36 & 19 & 22 & 33 & 16 & 15 & 31 & 21 & 14 & 28 & 17 & 15 & 21 & 8 & 6 & 6\end{array}$

$\begin{array}{rrrrrrrrrrrrrrrrrrr}8 & 28 & 19 & 17 & 13 & 14 & 16 & 15 & 31 & 21 & 14 & 28 & 17 & 15 & 21 & 8 & 6 & 6 \\ 15 & 48 & 35 & 41 & 47 & 34 & 17 & 5 & 5 & 1\end{array}$

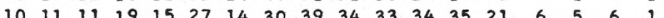

$\begin{array}{rllllllllllllllll}10 & 11 & 11 & 19 & 15 & 27 & 14 & 30 & 39 & 34 & 33 & 34 & 35 & 21 & 6 \\ 2 & 27 & 18 & 24 & 36 & 24 & 18 & 33 & 18 & 19 & 25 & 16 & 37 & 28 & 13\end{array}$

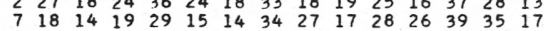

$\begin{array}{lllllllllllllllllllll}4 & 22 & 30 & 11 & 6 & 17 & 16 & 12 & 27 & 17 & 16 & 37 & 38 & 44 & 28 & 10 & 8 & 5 & 4 & 12\end{array}$

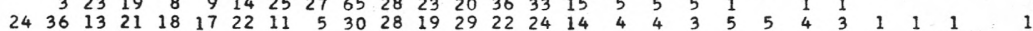

$\begin{array}{lllllllllllllllllllllll}14 & 17 & 12 & 14 & 38 & 16 & 6 & 22 & 23 & 23 & 41 & 40 & 44 & 21 & 13 & 7 & 5 & 1 & 1 & 4 & 1 & 2\end{array}$

$\begin{array}{rrrrrrrrrrrrrrrrrrrrrrrrrr}14 & 9 & 20 & 25 & 21 & 20 & 66 & 38 & 38 & 22 & 17 & 32 & 15 & 9 & 4 & 5 & 2 & 6 & & 1 & 1 & \\ 10 & 17 & 16 & 22 & 14 & 12 & 34 & 26 & 14 & 34 & 27 & 57 & 28 & 14 & 13 & 10 & 6 & 2 & 2 & 2 & 3 & 2\end{array}$

$\begin{array}{llllllllllllllllllllllllllll}1 & 28 & 37 & 14 & 12 & 18 & 24 & 27 & 42 & 28 & 28 & 23 & 21 & 30 & 9 & 4 & 4 & 4 & 1 & 4 & 2 & 1 & 2 & & & 1\end{array}$

$\begin{array}{rllllllllllllllll}23 & 45 & 24 & 11 & 18 & 16 & 11 & 58 & 39 & 15 & 28 & 12 & 20 & 11 & 8 & 10\end{array}$

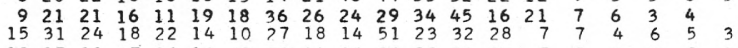

$\begin{array}{llllllllllllllllllll}17 & 26 & 16 & 8 & 36 & 44 & 33 & 36 & 23 & 24 & 20 & 7 & 8 & 6 & 2 & 2 & 4 & 2 & 4 & 1\end{array}$

$\begin{array}{lllllllllllllllllllllll}13 & 31 & 19 & 16 & 16 & 25 & 24 & 42 & 36 & 25 & 44 & 27 & 20 & 9 & 7 & 4 & 1 & 2 & 1 & 1 & 1 & & 1\end{array}$

CFS-DAYS

574645.0

556177.0

416428.0

346482.0
567468.0

590227.0

478799.0

617641.0

495702.0

506349.0

382461.0
536565.0

536565.0
$32149 \% .0$

516706.0

543420.0

398728.0

356019.0

355143.0
515672.0

600037.0

513250.0

484963.0

448313.0

417846.0
546872.0

408138.0

$35220 \% .0$

280559.0

.

648258.0

44340.0

42980.0

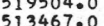

594603.0

553947.0

595803.0

4583.0

512550.0

571692.0

510562.0

693753.0

477234.0

487992.0

581653.0

553019.0

608135.0

570460.0

Sandy Rilver near Marmot, ureg.

Summary for water years 1912-15, 1917-18, 1920-63 STATION NUMBER 14-1370.00

CLASS CFS TOTAL ACCUM PERCT CLASS CFS TOTAL ACCUM PERCT

$\begin{array}{ccrlllllll}\text { CLASS } & \text { CFS } & \text { TOTAL ACCUM } & \text { PERCT } & \text { CLASS } & \text { CFS } & \text { TOTAL } & \text { ACCUM } & \text { PERCT } \\ & .0 & & 18262 & 100.0 & 09 & 700.0 & 899 & 11807 & 64.7 \\ 1 & 200.0 & 205 & 18262 & 100.0 & 10 & 800.0 & 1850 & 10908 & 59.7 \\ 2 & 250.0 & 545 & 18057 & 98.9 & 11 & 1000.0 & 1473 & 9058 & 49.6 \\ 3 & 300.0 & 952 & 17512 & 95.9 & 12 & 1200.0 & 1258 & 7585 & 41.5 \\ 4 & 350.0 & 1072 & 16560 & 90.7 & 13 & 1400.0 & 1518 & 6327 & 34.6 \\ 5 & 400.0 & 786 & 15488 & 84.8 & 14 & 1700.0 & 1258 & 4809 & 26.03 \\ 6 & 450.0 & 722 & 14702 & 80.5 & 15 & 2000.0 & 1376 & 3551 & 19.4 \\ 7 & 500.0 & 1183 & 13980 & 76.6 & 16 & 2500.0 & 813 & 2175 & 11.9 \\ 8 & 600.0 & 990 & 12797 & 70.1 & 17 & 3000.0 & 415 & 1362 & 7.5\end{array}$

\begin{tabular}{|c|c|c|c|c|c|c|c|c|c|}
\hline & $5 S$ CFS & TOTAL & ACCUM & PERCT & $\mathrm{CL}$ & SS CFS & TOTAL & ACCUM & PERC \\
\hline 18 & 3500 & 272 & 947 & 5.2 & 27 & 14000 & 8 & 17 & 01 \\
\hline 19 & 4000 & 180 & 675 & 3.7 & 28 & 17000 & 8 & 9 & \\
\hline 20 & 4500 & 114 & 495 & 2.7 & 29 & 20000 & 1 & 1 & .0 \\
\hline & 500 & 153 & 381 & 2.1 & 30 & & & & .0 \\
\hline 2 & 6000 & 81 & 228 & 1.2 & 31 & & & & .0 \\
\hline & 700 & & 147 & & 32 & & & & 0 \\
\hline 24 & 8000 & 47 & 97 & .5 & 33 & & & & 0 \\
\hline 5 & 10000 & 23 & 50 & . 3 & 34 & & & & \\
\hline
\end{tabular}


STATION NUMBER $\quad 14-1370.00$

LOWEST MEAN DISCHARGE, IN CFS, FOR THE FOLLOWING NUMBER OF CONSECUTIVE DAYS IN YEAR BEGINNING APRIL I

\begin{tabular}{|c|c|c|c|c|c|c|c|c|c|c|c|}
\hline YEAR & 1 & 3 & 7 & 14 & 30 & 60 & 90 & 120 & 150 & 183 & 274 \\
\hline $\begin{array}{l}1912 \\
1913 \\
1914\end{array}$ & $\begin{array}{l}460.0 \\
362.0 \\
329.0\end{array}$ & $\begin{array}{l}473.0 \\
365.0 \\
336.0\end{array}$ & $\begin{array}{l}488.0 \\
377.0 \\
347.0\end{array}$ & $\begin{array}{l}495 \bullet 0 \\
391 \bullet 0 \\
359.0\end{array}$ & $\begin{array}{l}509.0 \\
426.0 \\
372.0\end{array}$ & $\begin{array}{l}655.0 \\
489.0 \\
424.0\end{array}$ & $\begin{array}{l}651.0 \\
589.0 \\
502.0\end{array}$ & $\begin{array}{l}723 \cdot 0 \\
718 \cdot 0 \\
576 \cdot 0\end{array}$ & $\begin{array}{l}829.0 \\
799.0 \\
639.0\end{array}$ & $\begin{array}{l}958.0 \\
824.0 \\
708.0\end{array}$ & $\begin{array}{r}1200.0 \\
1090.0 \\
820.0\end{array}$ \\
\hline $\begin{array}{l}1917 \\
1920 \\
1921\end{array}$ & $\begin{array}{l}302.0 \\
313.0 \\
281.0\end{array}$ & $\begin{array}{l}309 \cdot 0 \\
331.0 \\
282.0\end{array}$ & $\begin{array}{l}324.0 \\
342.0 \\
292.0\end{array}$ & $\begin{array}{l}328.0 \\
383 \bullet 0 \\
315.0\end{array}$ & $\begin{array}{l}338.0 \\
399.0 \\
361.0\end{array}$ & $\begin{array}{l}374.0 \\
454.0 \\
412.0\end{array}$ & $\begin{array}{l}411.0 \\
548.0 \\
442.0\end{array}$ & $\begin{array}{l}465 \cdot 0 \\
669 \cdot 0 \\
515 \cdot 0\end{array}$ & $\begin{array}{l}645.0 \\
811.0 \\
587.0\end{array}$ & $\begin{array}{r}1010.0 \\
880.0 \\
782.0\end{array}$ & $\begin{array}{l}1800 \cdot 0 \\
1280 \bullet 0 \\
1100.0\end{array}$ \\
\hline $\begin{array}{l}1922 \\
1923 \\
1924\end{array}$ & $\begin{array}{l}290.0 \\
316.0 \\
267.0\end{array}$ & $\begin{array}{l}290 \cdot 0 \\
321 \cdot 0 \\
280 \cdot 0\end{array}$ & $\begin{array}{l}293.0 \\
337.0 \\
287.0\end{array}$ & $\begin{array}{l}299.0 \\
347.0 \\
292.0\end{array}$ & $\begin{array}{l}327.0 \\
361.0 \\
313.0\end{array}$ & $\begin{array}{l}345.0 \\
394.0 \\
336.0\end{array}$ & $\begin{array}{l}377.0 \\
410.0 \\
359.0\end{array}$ & $\begin{array}{l}410 \cdot 0 \\
441 \bullet 0 \\
386 \bullet 0\end{array}$ & $\begin{array}{l}455.0 \\
550.0 \\
426.0\end{array}$ & $\begin{array}{l}506 \cdot 0 \\
725.0 \\
520.0\end{array}$ & $\begin{array}{r}1150.0 \\
1110.0 \\
975.0\end{array}$ \\
\hline $\begin{array}{l}1925 \\
1926 \\
1927\end{array}$ & $\begin{array}{l}253.0 \\
274.0 \\
360.0\end{array}$ & $\begin{array}{l}259.0 \\
278.0 \\
363.0\end{array}$ & $\begin{array}{l}262.0 \\
294.0 \\
370.0\end{array}$ & $\begin{array}{l}265.0 \\
298.0 \\
386.0\end{array}$ & $\begin{array}{l}278.0 \\
307.0 \\
411.0\end{array}$ & $\begin{array}{l}297.0 \\
352.0 \\
486.0\end{array}$ & $\begin{array}{l}322.0 \\
374 \bullet 0 \\
607 \bullet 0\end{array}$ & $\begin{array}{l}360 \cdot 0 \\
431 \cdot 0 \\
833 \cdot 0\end{array}$ & $\begin{array}{l}414 \cdot 0 \\
484 \cdot 0 \\
921 \cdot 0\end{array}$ & $\begin{array}{r}504.0 \\
556.0 \\
1110.0\end{array}$ & $\begin{array}{r}803.0 \\
864.0 \\
1430.0\end{array}$ \\
\hline $\begin{array}{l}1928 \\
1929 \\
1930\end{array}$ & $\begin{array}{l}290.0 \\
220.0 \\
253.0\end{array}$ & $\begin{array}{l}293.0 \\
220.0 \\
253.0\end{array}$ & $\begin{array}{l}297.0 \\
221.0 \\
258.0\end{array}$ & $\begin{array}{l}307.0 \\
225.0 \\
259.0\end{array}$ & $\begin{array}{l}326.0 \\
232.0 \\
278.0\end{array}$ & $\begin{array}{l}354.0 \\
261.0 \\
300.0\end{array}$ & $\begin{array}{l}395 \bullet 0 \\
271 \bullet 0 \\
339 \bullet 0\end{array}$ & $\begin{array}{l}422.0 \\
293.0 \\
375.0\end{array}$ & $\begin{array}{l}489 \cdot 0 \\
338 \cdot 0 \\
417 \cdot 0\end{array}$ & $\begin{array}{l}576.0 \\
479.0 \\
528.0\end{array}$ & $\begin{array}{l}671.0 \\
884.0 \\
661.0\end{array}$ \\
\hline $\begin{array}{l}1931 \\
1932 \\
1933\end{array}$ & $\begin{array}{l}210.0 \\
246.0 \\
341.0\end{array}$ & $\begin{array}{l}211.0 \\
246.0 \\
344.0\end{array}$ & $\begin{array}{l}212.0 \\
252.0 \\
355.0\end{array}$ & $\begin{array}{l}216.0 \\
264.0 \\
382.0\end{array}$ & $\begin{array}{l}256.0 \\
283.0 \\
422.0\end{array}$ & $\begin{array}{l}276.0 \\
322.0 \\
501.0\end{array}$ & $\begin{array}{l}294.0 \\
374.0 \\
536.0\end{array}$ & $\begin{array}{l}357 \cdot 0 \\
453 \cdot 0 \\
666 \cdot 0\end{array}$ & $\begin{array}{l}433 \cdot 0 \\
596 \cdot 0 \\
862 \cdot 0\end{array}$ & $\begin{array}{r}517.0 \\
889.0 \\
1150.0\end{array}$ & $\begin{array}{r}798.0 \\
1190.0 \\
1670.0\end{array}$ \\
\hline $\begin{array}{l}1934 \\
1935 \\
1936\end{array}$ & $\begin{array}{l}228.0 \\
244.0 \\
218.0\end{array}$ & $\begin{array}{l}229.0 \\
251.0 \\
219.0\end{array}$ & $\begin{array}{l}232.0 \\
258.0 \\
221.0\end{array}$ & $\begin{array}{l}241.0 \\
274.0 \\
228.0\end{array}$ & $\begin{array}{l}257.0 \\
299.0 \\
235.0\end{array}$ & $\begin{array}{l}268.0 \\
310.0 \\
249.0\end{array}$ & $\begin{array}{l}288 \cdot 0 \\
324 \cdot 0 \\
278.0\end{array}$ & $\begin{array}{l}318 \cdot 0 \\
369 \cdot 0 \\
313 \cdot 0\end{array}$ & $\begin{array}{l}370.0 \\
402.0 \\
368.0\end{array}$ & $\begin{array}{l}453.0 \\
467.0 \\
463.0\end{array}$ & $\begin{array}{l}979.0 \\
826.0 \\
631.0\end{array}$ \\
\hline $\begin{array}{l}1937 \\
1938 \\
1939\end{array}$ & $\begin{array}{l}278.0 \\
253.0 \\
253.0\end{array}$ & $\begin{array}{l}279.0 \\
260.0 \\
254.0\end{array}$ & $\begin{array}{l}284.0 \\
268.0 \\
261.0\end{array}$ & $\begin{array}{l}309 \cdot 0 \\
287.0 \\
275.0\end{array}$ & $\begin{array}{l}320.0 \\
291.0 \\
288.0\end{array}$ & $\begin{array}{l}359 \cdot 0 \\
312 \cdot 0 \\
313.0\end{array}$ & $\begin{array}{l}420 \cdot 0 \\
326 \bullet 0 \\
339 \cdot 0\end{array}$ & $\begin{array}{l}490 \cdot 0 \\
364 \cdot 0 \\
355 \cdot 0\end{array}$ & $\begin{array}{l}710.0 \\
440.0 \\
383.0\end{array}$ & $\begin{array}{l}946.0 \\
569.0 \\
485.0\end{array}$ & $\begin{array}{r}1470.0 \\
833.0 \\
697.0\end{array}$ \\
\hline $\begin{array}{l}1940 \\
1941 \\
1942\end{array}$ & $\begin{array}{l}213.0 \\
238.0 \\
214.0\end{array}$ & $\begin{array}{l}216 \cdot 0 \\
247 \cdot 0 \\
217.0\end{array}$ & $\begin{array}{l}222.0 \\
264.0 \\
219.0\end{array}$ & $\begin{array}{l}241.0 \\
277.0 \\
233.0\end{array}$ & $\begin{array}{l}248.0 \\
288.0 \\
236.0\end{array}$ & $\begin{array}{l}262.0 \\
332.0 \\
255.0\end{array}$ & $\begin{array}{l}283.0 \\
395 \bullet 0 \\
298.0\end{array}$ & $\begin{array}{l}309 \cdot 0 \\
472 \cdot 0 \\
383.0\end{array}$ & $\begin{array}{l}351 \cdot 0 \\
511 \bullet 0 \\
541.0\end{array}$ & $\begin{array}{l}421.0 \\
620.0 \\
717.0\end{array}$ & $\begin{array}{r}681.0 \\
888.0 \\
1320.0\end{array}$ \\
\hline $\begin{array}{l}1943 \\
1944 \\
1945\end{array}$ & $\begin{array}{l}310.0 \\
270.0 \\
280.0\end{array}$ & $\begin{array}{l}316.0 \\
273.0 \\
289.0\end{array}$ & $\begin{array}{l}320.0 \\
276.0 \\
293.0\end{array}$ & $\begin{array}{l}324.0 \\
282.0 \\
298.0\end{array}$ & $\begin{array}{l}340 \cdot 0 \\
311.0 \\
324.0\end{array}$ & $\begin{array}{l}374.0 \\
343.0 \\
375.0\end{array}$ & $\begin{array}{l}428.0 \\
342.0 \\
373.0\end{array}$ & $\begin{array}{l}546 \cdot 0 \\
367 \cdot 0 \\
406 \cdot 0\end{array}$ & $\begin{array}{l}638.0 \\
401.0 \\
490.0\end{array}$ & $\begin{array}{l}705.0 \\
450.0 \\
683.0\end{array}$ & $\begin{array}{r}830.0 \\
742.0 \\
1190.0\end{array}$ \\
\hline $\begin{array}{l}1946 \\
1947\end{array}$ & $\begin{array}{l}299.0 \\
310.0\end{array}$ & $\begin{array}{l}302.0 \\
320.0\end{array}$ & $\begin{array}{l}307.0 \\
331.0\end{array}$ & $\begin{array}{l}326.0 \\
340.0\end{array}$ & $\begin{array}{l}331.0 \\
360.0\end{array}$ & $\begin{array}{l}364.0 \\
371.0\end{array}$ & $\begin{array}{l}415.0 \\
440.0\end{array}$ & $\begin{array}{l}564 \cdot 0 \\
566 \cdot 0\end{array}$ & $\begin{array}{l}726 \cdot 0 \\
614 \cdot 0\end{array}$ & $\begin{array}{l}891.0 \\
735.0\end{array}$ & $\begin{array}{l}1470.0 \\
1270.0\end{array}$ \\
\hline 1948 & 318.0 & 318.0 & 325.0 & 339.0 & 378.0 & 438.0 & 513.0 & $590 \cdot 0$ & 672.0 & 896.0 & 1140.0 \\
\hline $\begin{array}{l}1949 \\
1950\end{array}$ & $\begin{array}{l}329.0 \\
345.0\end{array}$ & $\begin{array}{l}337.0 \\
345.0\end{array}$ & $\begin{array}{l}362.0 \\
346.0\end{array}$ & $\begin{array}{l}372.0 \\
358.0\end{array}$ & $\begin{array}{l}393.0 \\
395.0\end{array}$ & $\begin{array}{l}429.0 \\
458.0\end{array}$ & $\begin{array}{l}496.0 \\
567.0\end{array}$ & $\begin{array}{l}564 \cdot 0 \\
707 \cdot 0\end{array}$ & $\begin{array}{r}594.0 \\
1020.0\end{array}$ & $\begin{array}{r}755.0 \\
1320.0\end{array}$ & $\begin{array}{l}1100.0 \\
1690.0\end{array}$ \\
\hline 1951 & 293.0 & 293.0 & 305.0 & 316.0 & 327.0 & 359.0 & 418.0 & $504 \cdot 0$ & 650.0 & 805.0 & 1070.0 \\
\hline $\begin{array}{l}1952 \\
1953 \\
1954\end{array}$ & $\begin{array}{l}207.0 \\
325.0\end{array}$ & $\begin{array}{l}211.0 \\
332.0\end{array}$ & $\begin{array}{l}219.0 \\
339.0\end{array}$ & $\begin{array}{l}230.0 \\
353.0\end{array}$ & $\begin{array}{l}248.0 \\
369.0\end{array}$ & $\begin{array}{l}254.0 \\
384.0\end{array}$ & $\begin{array}{l}273.0 \\
402.0\end{array}$ & $\begin{array}{l}300 \cdot 0 \\
438 \cdot 0\end{array}$ & $\begin{array}{l}352 \cdot 0 \\
521 \cdot 0\end{array}$ & $\begin{array}{l}424.0 \\
770.0\end{array}$ & $\begin{array}{r}813.0 \\
1350.0\end{array}$ \\
\hline 1954 & 365.0 & $372 . n$ & 377.0 & 399.0 & 476.0 & 507.0 & 579.0 & $645 \cdot 0$ & 750.0 & 813.0 & 1010.0 \\
\hline $\begin{array}{l}1955 \\
1956 \\
1957\end{array}$ & $\begin{array}{l}361.0 \\
362.0 \\
296.0\end{array}$ & $\begin{array}{l}367 \cdot n \\
366 \cdot n \\
307 \cdot 0\end{array}$ & $\begin{array}{l}393.0 \\
371.0 \\
313.0\end{array}$ & $\begin{array}{l}429.0 \\
385.0 \\
322.0\end{array}$ & $\begin{array}{l}466.0 \\
396.0 \\
332.0\end{array}$ & $\begin{array}{l}501.0 \\
446.0 \\
358.0\end{array}$ & $\begin{array}{l}646.0 \\
488.0 \\
364.0\end{array}$ & $\begin{array}{l}863 \cdot 0 \\
591 \cdot 0 \\
387.0\end{array}$ & $\begin{array}{r}1170.0 \\
746.0 \\
449.0\end{array}$ & $\begin{array}{r}1520.0 \\
838.0 \\
553.0\end{array}$ & $\begin{array}{l}1820.0 \\
1120.0 \\
1070.0\end{array}$ \\
\hline 1958 & 308.0 & $\begin{array}{l}307 \cdot 0 \\
309.0\end{array}$ & $\begin{array}{l}313.0 \\
320.0\end{array}$ & $\begin{array}{l}322.0 \\
352.0\end{array}$ & $\begin{array}{l}332.0 \\
372.0\end{array}$ & $\begin{array}{l}358.0 \\
384.0\end{array}$ & $\begin{array}{l}364.0 \\
416.0\end{array}$ & 454.0 & $\begin{array}{l}449.0 \\
532.0\end{array}$ & $\begin{array}{l}553.0 \\
636.0\end{array}$ & $\begin{array}{l}1070.0 \\
1270.0\end{array}$ \\
\hline 1959 & 370.0 & 383.0 & 395.0 & 404.0 & 416.0 & 498.0 & 569.0 & $730 \cdot 0$ & 968.0 & 1120.0 & 1230.0 \\
\hline 1960 & 312.0 & $312 . ?$ & 317.0 & 335.0 & 354.0 & 389.0 & 405.0 & 459.0 & 593.0 & 866.0 & 1310.0 \\
\hline 1961 & 318.0 & 323.0 & 337.0 & 347.0 & 361.0 & $424 \cdot 0$ & 450.0 & $509 \cdot 0$ & 616.0 & 765.0 & 1260.0 \\
\hline 1962 & 328.0 & 331.0 & 336.0 & 356.0 & 384.0 & 414.0 & 463.0 & 566.0 & 618.0 & & 1150 \\
\hline
\end{tabular}

STATION NUMBER $\quad 14-1370.00$

HIGHEST MEAN DISCHARGE, IN CFS, FOR THE FOLLOWING NUMBER OF CONSECUTIVE DAYS IN YEAR ENDING SEPTEMBER 30

YEA
191
191
191
191
1917
191
1920
1921
192
1923
1924
1925
1926
1927
192
1929
1930
1931
1932
1933
1934
1935
1936
193
1938
1939
1940
1941
1942
1943
1944
1945
1946
1947
1948
1949
1950
1951
1952
195
1954
1955
1956
1955
195
195
1960
196
1962
1963

\begin{tabular}{|c|c|c|}
\hline 1 & 3 & 7 \\
\hline $\begin{array}{r}11700.0 \\
10800.0 \\
4820.0\end{array}$ & $\begin{array}{r}10000.0 \\
8180.0 \\
3740.0\end{array}$ & $\begin{array}{l}6820 \cdot 0 \\
5100 \cdot 0 \\
3230.0\end{array}$ \\
\hline $\begin{array}{r}3500.0 \\
5250.0 \\
19800.0\end{array}$ & $\begin{array}{r}2650.0 \\
4830.0 \\
15100.0\end{array}$ & $\begin{array}{r}2090.0 \\
4330.0 \\
11000.0\end{array}$ \\
\hline $\begin{array}{l}12500 \cdot 0 \\
14300 \cdot 0 \\
17000.0\end{array}$ & $\begin{array}{r}9690.0 \\
9370.0 \\
13700.0\end{array}$ & $\begin{array}{l}6570 \cdot 0 \\
7630.0 \\
8250.0\end{array}$ \\
\hline $\begin{array}{r}23500 \cdot 0 \\
7410.0 \\
7200.0\end{array}$ & $\begin{array}{r}16000.0 \\
5170.0 \\
6220.0\end{array}$ & $\begin{array}{l}9980 \cdot 0 \\
4190 \cdot 0 \\
5580 \cdot 0\end{array}$ \\
\hline $\begin{array}{r}7700 \cdot 0 \\
7320 \cdot 0 \\
13000.0\end{array}$ & $\begin{array}{l}6020 \cdot 0 \\
5790 \cdot 0 \\
8920.0\end{array}$ & $\begin{array}{l}4180.0 \\
3950.0 \\
7400.0\end{array}$ \\
\hline $\begin{array}{r}6340.0 \\
7500.0 \\
19800.0\end{array}$ & $\begin{array}{r}4200.0 \\
5270.0 \\
12300.0\end{array}$ & $\begin{array}{l}2950.0 \\
4110 \cdot 0 \\
7250.0\end{array}$ \\
\hline $\begin{array}{r}10100.0 \\
8820.0 \\
14500.0\end{array}$ & $\begin{array}{r}8290.0 \\
7000.0 \\
10900.0\end{array}$ & $\begin{array}{l}5690.0 \\
5500.0 \\
8620.0\end{array}$ \\
\hline $\begin{array}{r}9800.0 \\
11100.0 \\
7690.0\end{array}$ & $\begin{array}{l}7650.0 \\
7720.0 \\
6880.0\end{array}$ & $\begin{array}{l}5810.0 \\
5750 \cdot 0 \\
4830.0\end{array}$ \\
\hline $\begin{array}{r}10600 \cdot 0 \\
7310 \cdot 0 \\
6350.0\end{array}$ & $\begin{array}{l}8660.0 \\
5010 \cdot 0 \\
4900.0\end{array}$ & $\begin{array}{l}6110 \cdot 0 \\
3790 \cdot 0 \\
4270.0\end{array}$ \\
\hline $\begin{array}{r}6960.0 \\
4700.0\end{array}$ & $\begin{array}{l}4670.0 \\
4210.0\end{array}$ & $\begin{array}{l}3110.0 \\
3430.0 \\
6970.0\end{array}$ \\
\hline $\begin{array}{r}12600.0 \\
4980.0\end{array}$ & $\begin{array}{l}8150.0 \\
3640.0\end{array}$ & $\begin{array}{l}6970 \cdot 0 \\
2290.0\end{array}$ \\
\hline 8210.0 & 5420.0 & $\begin{array}{l}4590.0 \\
6720.0\end{array}$ \\
\hline 17300.0 & 11200.0 & $6720 . n$ \\
\hline $\begin{array}{r}17200.0 \\
13800.0 \\
9150.0\end{array}$ & $\begin{array}{r}15900.0 \\
9030.0 \\
7040.0\end{array}$ & $\begin{array}{r}11700.0 \\
5500.0 \\
4890.0\end{array}$ \\
\hline 11400.0 & 8780.0 & 5670.0 \\
\hline 6470.0 & $5510 \cdot n$ & 5080.0 \\
\hline 7060.0 & 5270.0 & 4360.0 \\
\hline 17800.0 & 13500.0 & 9430.0 \\
\hline 8570.0 & 6710.0 & 5040.0 \\
\hline 9730.0 & 7540.0 & $4990 . n$ \\
\hline 13800.0 & 10400.0 & $665 n . n$ \\
\hline 14800.0 & $9810 \cdot n$ & 6820.0 \\
\hline 15700.0 & $10200 \cdot 0$ & 6350.0 \\
\hline 1600.0 & 7490.0 & 4850.0 \\
\hline 5720.0 & 4600.0 & 3920.0 \\
\hline 19100.0 & 11900.0 & 7960.0 \\
\hline 10000.0 & 8870.0 & 6450.0 \\
\hline
\end{tabular}

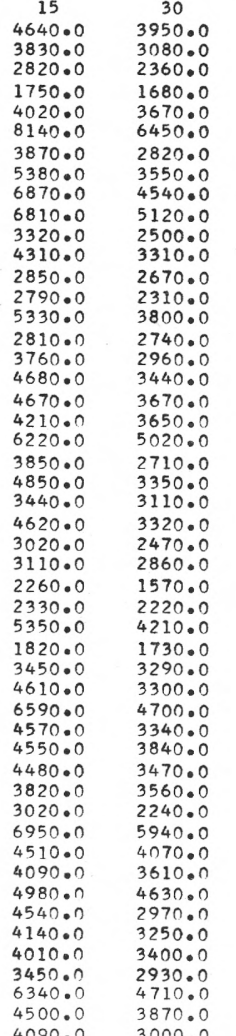

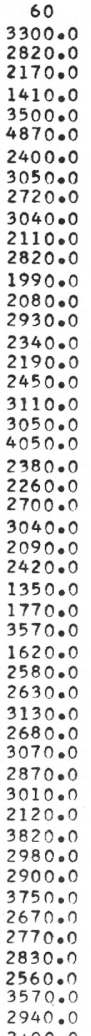

\begin{tabular}{|c|}
\hline $\begin{array}{l}2630.0 \\
2500.0 \\
1920.0\end{array}$ \\
\hline $\begin{array}{l}1300.0 \\
3130.0 \\
3840.0\end{array}$ \\
\hline $\begin{array}{l}2180 \cdot 0 \\
2880 \bullet 0 \\
2230 \bullet 0\end{array}$ \\
\hline $\begin{array}{l}2500.0 \\
2070 \cdot 0 \\
2620.0\end{array}$ \\
\hline $\begin{array}{l}1820 \cdot 0 \\
1920.0\end{array}$ \\
\hline $\begin{array}{l}2430.0 \\
2160.0 \\
1840.0\end{array}$ \\
\hline $\begin{array}{l}1950.0 \\
2910.0 \\
2670.0 \\
3170.0\end{array}$ \\
\hline $\begin{array}{l}2150 \cdot 0 \\
2110 \bullet 0 \\
2430.0\end{array}$ \\
\hline $\begin{array}{l}2780.0 \\
1930.0\end{array}$ \\
\hline $2160 \cdot 0$ \\
\hline $\begin{array}{l}1230.0 \\
1630.0\end{array}$ \\
\hline 3210.0 \\
\hline $\begin{array}{l}1550 \cdot 0 \\
2210\end{array}$ \\
\hline $2310 \cdot 0$ \\
\hline $\begin{array}{l}2870.0 \\
2690.0 \\
2750.0\end{array}$ \\
\hline $\begin{array}{l}2660 \cdot 0 \\
2930 \cdot 0\end{array}$ \\
\hline $\begin{array}{l}1800.0 \\
3060.0\end{array}$ \\
\hline $\begin{array}{l}2730.0 \\
2520.0\end{array}$ \\
\hline $\begin{array}{r}252000 \\
3440\end{array}$ \\
\hline $\begin{array}{l}3440.0 \\
2350.0\end{array}$ \\
\hline $\begin{array}{l}2350.0 \\
2610.0\end{array}$ \\
\hline 2780.0 \\
\hline $\begin{array}{l}2450.0 \\
3000.0\end{array}$ \\
\hline+40. \\
\hline
\end{tabular}

\begin{tabular}{|c|}
\hline $\begin{array}{l}2560 \cdot 0 \\
2310 \cdot 0 \\
1840 \cdot 0\end{array}$ \\
\hline $\begin{array}{l}1250 \cdot 0 \\
2780.0 \\
3240.0\end{array}$ \\
\hline $\begin{array}{l}1870 \cdot 0 \\
2670 \cdot 0 \\
1880 \cdot 0\end{array}$ \\
\hline $\begin{array}{l}2400 \cdot 0 \\
1860 \cdot 0 \\
2380 \cdot 0\end{array}$ \\
\hline $\begin{array}{l}1620.0 \\
1890.0 \\
2190.0\end{array}$ \\
\hline $\begin{array}{l}1970 \cdot 0 \\
1740.0 \\
1730 \cdot 0\end{array}$ \\
\hline $\begin{array}{l}2570 \cdot 0 \\
2400 \cdot 0 \\
2810 \cdot 0\end{array}$ \\
\hline $\begin{array}{l}2000 \cdot 0 \\
2150 \cdot 0 \\
2280 \cdot 0\end{array}$ \\
\hline $\begin{array}{l}2420.0 \\
1730 \cdot 0 \\
1840.0\end{array}$ \\
\hline $\begin{array}{l}1110 \cdot 0 \\
1530.0 \\
2810.0\end{array}$ \\
\hline $\begin{array}{l}1440 \cdot 0 \\
2180 \cdot 0 \\
2170 \cdot 0\end{array}$ \\
\hline $\begin{array}{l}2520 \cdot 0 \\
2280 \cdot 0 \\
2540 \cdot 0\end{array}$ \\
\hline $\begin{array}{l}2700 \cdot 0 \\
2730 \cdot 0 \\
1810 \cdot 0\end{array}$ \\
\hline $2700 \cdot 0$ \\
\hline $\begin{array}{r}2520 \cdot 0 \\
2220 \cdot 0\end{array}$ \\
\hline $2930 \cdot 0$ \\
\hline $\begin{array}{l}2100 \cdot 0 \\
2220 \cdot 0\end{array}$ \\
\hline $2440 \cdot 0$ \\
\hline $\begin{array}{r}2380 \cdot 0 \\
2860 \cdot 0\end{array}$ \\
\hline 2310.0 \\
\hline
\end{tabular}

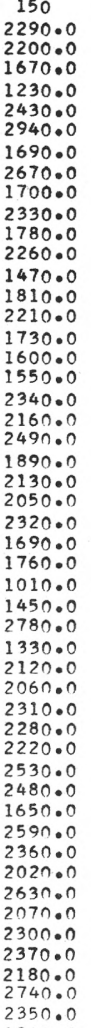

\begin{tabular}{|c|c|}
\hline 183 & 274 \\
\hline $\begin{array}{l}2150.0 \\
2120.0 \\
1560.0\end{array}$ & $\begin{array}{l}1890.0 \\
1850.0 \\
1360.0\end{array}$ \\
\hline $\begin{array}{l}1180 \cdot 0 \\
2240 \cdot 0 \\
2700 \cdot 0\end{array}$ & $\begin{array}{l}1110.0 \\
1910.0 \\
2030.0\end{array}$ \\
\hline $\begin{array}{l}1760 \cdot 0 \\
2480 \cdot 0 \\
1860.0\end{array}$ & $\begin{array}{l}1540.0 \\
2090.0 \\
1680.0\end{array}$ \\
\hline $\begin{array}{l}2190 \cdot 0 \\
1660 \cdot 0 \\
2200 \cdot 0\end{array}$ & $\begin{array}{l}1710.0 \\
1280.0 \\
1830.0\end{array}$ \\
\hline $\begin{array}{l}1330 \cdot 0 \\
1820 \cdot 0 \\
2190.0\end{array}$ & $\begin{array}{l}1040.0 \\
1660.0 \\
1830.0\end{array}$ \\
\hline $\begin{array}{l}1600 \cdot 0 \\
1580.0 \\
1450.0\end{array}$ & $\begin{array}{l}1330.0 \\
1210.0 \\
1190.0\end{array}$ \\
\hline $\begin{array}{l}2190 \cdot 0 \\
2180.0 \\
2330.0\end{array}$ & $\begin{array}{l}1770.0 \\
2020.0 \\
1770.0\end{array}$ \\
\hline $\begin{array}{l}1870 \cdot 0 \\
1970 \cdot 0 \\
1790 \cdot 0\end{array}$ & $\begin{array}{l}1650.0 \\
1520.0 \\
1430.0\end{array}$ \\
\hline $2360 \cdot 0$ & $\begin{array}{l}1880.0 \\
1380.0\end{array}$ \\
\hline $\begin{array}{l}1630 \cdot 0 \\
1570 \cdot 0\end{array}$ & $\begin{array}{l}1380.0 \\
1190.0\end{array}$ \\
\hline 1000.0 & 903.0 \\
\hline 1430.0 & 1300.0 \\
\hline 2670.0 & 2240.0 \\
\hline $1250 \cdot 0$ & 1140.0 \\
\hline $1880 \cdot 0$ & 1440.0 \\
\hline 2050.0 & 1770.0 \\
\hline $2170 \cdot 0$ & 1750.0 \\
\hline 2110.0 & 2000.0 \\
\hline $2200 \cdot 0$ & 1850.0 \\
\hline $2350 \cdot 0$ & 1980.0 \\
\hline $2400 \cdot 0$ & 2040.0 \\
\hline 1680.0 & 1560.0 \\
\hline 2360.0 & 1770.0 \\
\hline $2230 \cdot 0$ & 1930.0 \\
\hline 1960.0 & 1670.0 \\
\hline 2590.0 & 2350.0 \\
\hline 1930.0 & 1620.0 \\
\hline 2090.0 & 1650.0 \\
\hline 2290.0 & 1910.0 \\
\hline 1970.0 & $1860 \cdot 0$ \\
\hline $2610 \cdot 0$ & 2080.0 \\
\hline 2290.0 & 1930.0 \\
\hline 1820.0 & 1510.0 \\
\hline
\end{tabular}




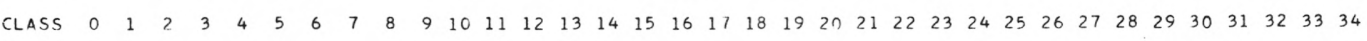

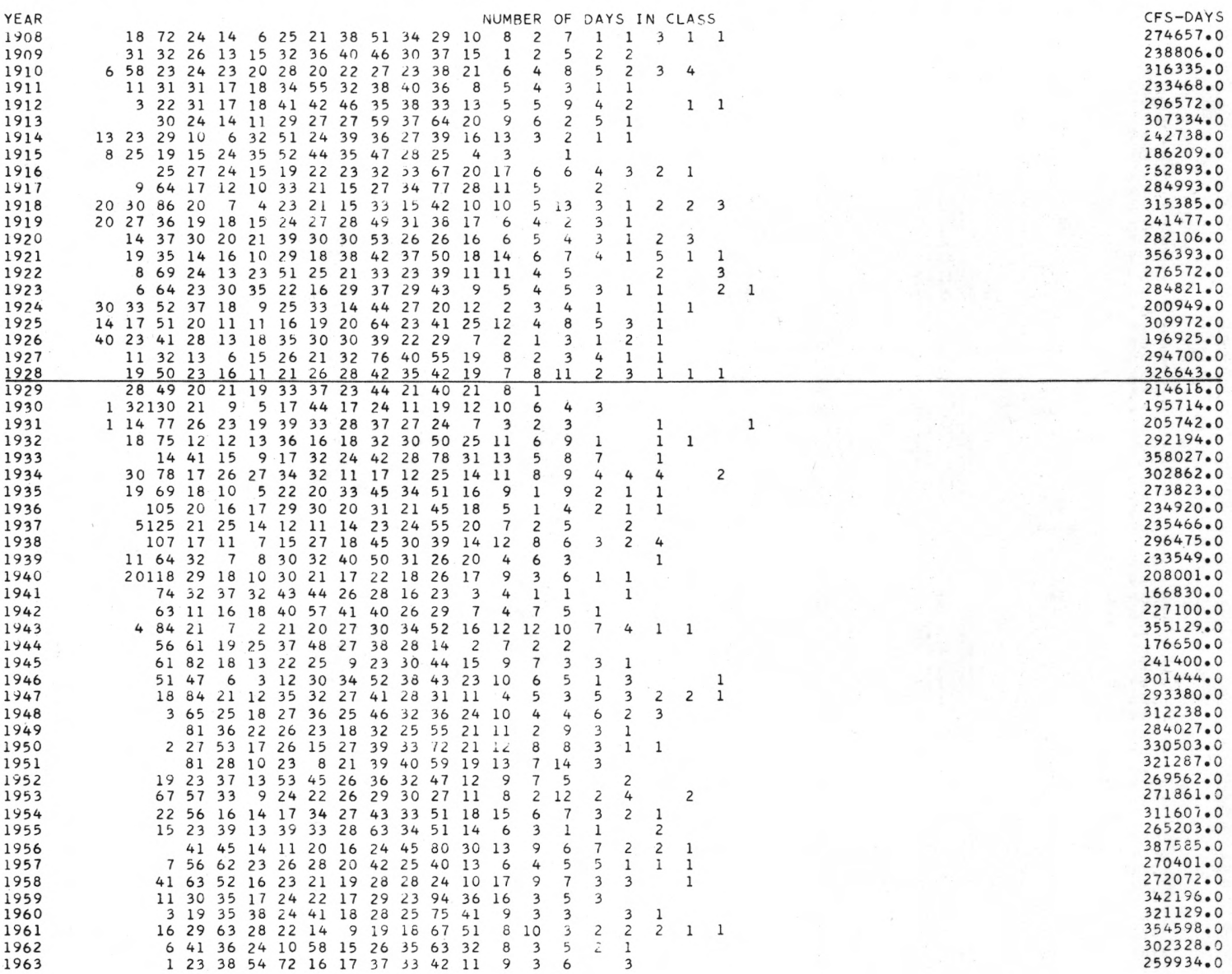

BULL RUN RIVER NR BULL RUN OREG

\begin{tabular}{lrrrrl} 
CLASS & \multicolumn{1}{c}{ CFS } & TOTAL ACCUM & PERCT & CLAS \\
& .0 & & 20454 & 100.0 & 09 \\
1 & 60.0 & 153 & 20454 & 100.0 & 10 \\
2 & 80.0 & 566 & 20301 & 99.3 & 11 \\
3 & 100.0 & 2531 & 19735 & 96.5 & 12 \\
4 & 150.0 & 1807 & 17204 & 84.1 & 13 \\
5 & 200.0 & 1282 & 15397 & 75.3 & 14 \\
6 & 250.0 & 940 & 14115 & 69.0 & 15 \\
7 & 300.0 & 1626 & 13175 & 64.4 & 16 \\
8 & 400.0 & 1605 & 11549 & 56.5 & 17
\end{tabular}

Bull Run River near

Bu11 Run, Oreg.

STATION NUMBER $\quad 14-1400.00$

Summary for water years $1908-28$

\begin{tabular}{lrr} 
CL.ASS & \multicolumn{1}{c}{ CFS } & TOTAL \\
& .0 & \\
1 & 60.0 & 151 \\
2 & 80.0 & 385 \\
3 & 100.0 & 900 \\
4 & 150.0 & 480 \\
5 & 200.0 & 332 \\
6 & 250.0 & 352 \\
7 & 300.0 & 655 \\
8 & 400.0 & 578
\end{tabular}

Bu11 Run River near
Bu11 Run, Oreg.

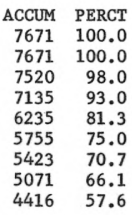

CFS TOTAL ACCUM PERCT $500.0 \quad 1385 \quad 9944 \quad 48.6$ $600.0 \quad 2134 \quad 8559 \quad 41.8$ $800.0 \quad 1630 \quad 6425 \quad 31.4$ $1000.0 \quad 2467 \quad 4795 \quad 23.4$ $\begin{array}{llll}1500.0 & 974 & 2328 & 11.4\end{array}$ $2000.0 \quad 483 \quad 1354 \quad 6.6$ $\begin{array}{llll}2500.0 & 255 & 871 & 4.3\end{array}$ $3000.0 \quad 303 \quad 616 \quad 3.0$ $4000 \cdot 0$

STATION NUMBER

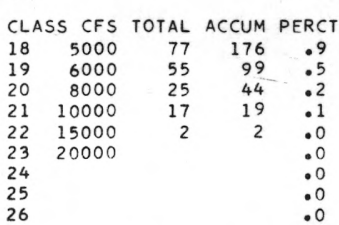

$14-1400.00$

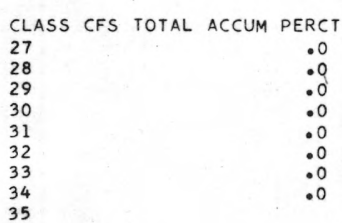

.0
.0
.0
.0
.0
.0
.0

Summary for water years 1929-63

\begin{tabular}{rrrrlrr}
\multicolumn{1}{l}{ CFS } & TOTAL & ACCUM & PERCT & CLASS & CFS & TOTAL \\
.0 & & 12783 & 100.0 & 09 & 500.0 & 783 \\
60.0 & 2 & 12783 & 100.0 & 10 & 600.0 & 1224 \\
80.0 & 181 & 12781 & 100.0 & 11 & 800.0 & 978 \\
100.0 & 1631 & 12600 & 98.6 & 12 & 1000.0 & 1597 \\
150.0 & 1327 & 10969 & 85.8 & 13 & 1500.0 & 656 \\
200.0 & 950 & 9642 & 75.4 & 14 & 2000.0 & 322 \\
250.0 & 588 & 8692 & 68.0 & 15 & 2500.0 & 172 \\
300.0 & 971 & 8104 & 63.4 & 16 & 3000.0 & 195 \\
400.0 & 1027 & 7133 & 55.8 & 17 & 4000.0 & 83
\end{tabular}

$\begin{array}{rrl}\text { ACCUM } & \text { PERCT } & \text { CLASS } \\ 6106 & 47.8 & 18 \\ 5323 & 41.6 & 19 \\ 4099 & 32.1 & 20 \\ 3121 & 24.4 & 21 \\ 1524 & 11.9 & 22 \\ 868 & 6.8 & 23 \\ 546 & 4.3 & 24 \\ 374 & 2.9 & 25 \\ 179 & 1.4 & 26\end{array}$

$\begin{array}{rrrrr}\text { CFS } & \text { TOTAL } & \text { ACCUM } & \text { PRRCT } & \\ 5000 & 27 & 80 & 1.0 & 27 \\ 6000 & 25 & 53 & .7 & 28 \\ 8000 & 15 & 28 & .4 & 29 \\ 10000 & 12 & 13 & .2 & 3 \\ 15000 & 1 & 1 & .0 & 3 \\ 20000 & & & .0 & 3 \\ & & & .0 & 3 \\ & & & .0 & 3 \\ & & & .0 & 3\end{array}$

$14-1400.00$

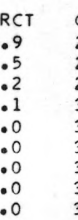

$\begin{array}{rlr}\text { CLASS } & \text { CFS } \\ .0 & 09 & 500.0 \\ .0 & 10 & 600.0 \\ .0 & 11 & 800.0 \\ .0 & 12 & 1000.0 \\ .3 & 13 & 1500.0 \\ .0 & 14 & 2000.0 \\ .7 & 15 & 2500.0 \\ .1 & 16 & 3000.0 \\ .6 & 17 & 4000.0\end{array}$

STATION NUMBER

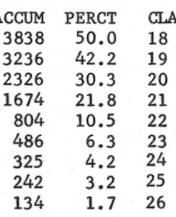

\begin{tabular}{|c|c|c|c|c|c|c|c|c|}
\hline CFS & TOTAL & ACCUM & PERCT & CLASS & CFS & TOTAL & ACCUM & PERCT \\
\hline 5000 & 50 & 96 & .8 & 27 & & & & .0 \\
\hline 6000 & 30 & 46 & .4 & 28 & & & & .0 \\
\hline 8000 & 10 & 16 & .1 & 29 & & & & .0 \\
\hline 10000 & 5 & 6 & .0 & 30 & & & & .0 \\
\hline 15000 & 1 & 1 & .0 & 31 & & & & .0 \\
\hline \multirow[t]{4}{*}{20000} & & & .0 & 32 & & & & .0 \\
\hline & & & .0 & 33 & & & & .0 \\
\hline & & & .0 & 34 & & & & .0 \\
\hline & & & 0 & 35 & & & & \\
\hline
\end{tabular}


BULL RUN RIVER NR BULL RUN OREG]

STATION NUMBER $\quad 14-1400 \cdot 00$

LOWEST MEAN DISCHARGE, IN CFS, FOR THE FOLLOWING NUMBER OF CONSECUTIVE DAYS IN YEAR BEGINNING APRIL 1

\begin{tabular}{|c|c|c|c|c|c|c|c|c|c|c|c|}
\hline YEAR & 1 & 3 & 7 & 14 & 30 & 60 & 90 & 120 & 150 & 183 & 274 \\
\hline 1908 & 85.0 & 86.3 & 90.4 & 93.4 & 96.5 & 118.0 & 122.0 & 201.0 & 286.0 & $347 \cdot 0$ & 525.0 \\
\hline 1909 & 82.0 & 83.3 & 85.7 & 91.6 & 95.7 & 118.0 & 145.0 & $186 \cdot 0$ & 233.0 & 356.0 & 736.0 \\
\hline 1910 & 72.0 & 72.7 & 74.9 & 78.7 & 82.3 & 85.8 & 99.8 & $136 \cdot 0$ & $186 \cdot 0$ & 239.0 & 515.0 \\
\hline 1911 & 80.0 & 81.0 & 84.4 & 91.9 & 110.0 & 139.0 & 189.0 & $220 \cdot 0$ & 253.0 & 391.0 & 610.0 \\
\hline 1912 & 92.0 & 94.7 & 104.0 & 112.0 & 138.0 & 217.0 & 294.0 & 315.0 & $378 \cdot 0$ & $446 \cdot 0$ & 650.0 \\
\hline 1913 & $107 \cdot 0$ & $108 \cdot 0$ & 111.0 & 121.0 & 136.0 & 190.0 & 212.0 & $307 \cdot 0$ & 395.0 & 452.0 & 627.0 \\
\hline 1914 & 72.0 & 72.0 & 72.0 & 74.2 & 80.7 & 97.6 & 151.0 & $218 \cdot 0$ & $266 \cdot 0$ & $327 \cdot 0$ & 445.0 \\
\hline 1915 & 74.0 & 74.0 & $74 \cdot 3$ & 79.2 & 86.8 & 107.0 & 169.0 & $212 \cdot 0$ & $264 \cdot 0$ & $306 \cdot 0$ & 638.0 \\
\hline 1916 & 87.0 & 88.0 & 91.0 & 95.9 & 108.0 & 137.0 & 158.0 & 231.0 & 370.0 & 461.0 & 595.0 \\
\hline 1917 & 88.0 & 88.7 & 90.9 & 94.3 & 99.5 & 114.0 & 121.0 & $142 \cdot 0$ & 208.0 & 423.0 & 1010.0 \\
\hline 1918 & 68.0 & 68.7 & 69.7 & 72.0 & 77.7 & 90.6 & 99.4 & 109.0 & $150 \cdot 0$ & 238.0 & 413.0 \\
\hline 1919 & 70.0 & 70.7 & 71.4 & 73.6 & 80.8 & 97.7 & $132 \cdot 0$ & $164 \cdot 0$ & 217.0 & 326.0 & 640.0 \\
\hline 1920 & 91.0 & 92.3 & 95.9 & 98.1 & 106.0 & 129.0 & 193.0 & 278.0 & 388.0 & 455.0 & 728.0 \\
\hline 1921 & 85.0 & 86.0 & 89.7 & 95.6 & 102.0 & 117.0 & 148.0 & $194 \cdot 0$ & 251.0 & 330.0 & 651.0 \\
\hline 1922 & 90.0 & 92.0 & 94.0 & 99.4 & 110.0 & $117 \cdot 0$ & 126.0 & $138 \cdot 0$ & 194.0 & 237.0 & 626.0 \\
\hline 1923 & 109.0 & $112 \cdot 0$ & 115.0 & 126.0 & 131.0 & 139.0 & 145.0 & $168 \cdot 0$ & 205.0 & 286.0 & 552.0 \\
\hline 1924 & 71.0 & 71.0 & $71 \cdot 6$ & 73.7 & 78.3 & 87.5 & 98.4 & $132 \cdot 0$ & $160 \cdot 0$ & 194.0 & 545.0 \\
\hline 1925 & 64.0 & 65.3 & 65.7 & 66.0 & 72.7 & 77.8 & 87.4 & $95 \cdot 1$ & 127.0 & 198.0 & 429.0 \\
\hline 1926 & 63.0 & 63.0 & 66.3 & 71.1 & 76.7 & 107.0 & 135.0 & $190 \cdot 0$ & $219 \cdot 0$ & 268.0 & $48 \varepsilon \cdot 0$ \\
\hline 1927 & 88.0 & 88.0 & 91.4 & 95.1 & 100.0 & 130.0 & 220.0 & 370.0 & 482.0 & 574.0 & 876.0 \\
\hline 1928 & 83.0 & 89.3 & 96.0 & 97.5 & 110.0 & 139.0 & 159.0 & 171.0 & 199.0 & 235.0 & 393.0 \\
\hline 1929 & 76.0 & 81.7 & 84.1 & 96.0 & 98.6 & 103.0 & 105.0 & 106.0 & 112.0 & 183.0 & 404.0 \\
\hline 1930 & 72.0 & 94.0 & 94.9 & 96.6 & 103.0 & 113.0 & 116.0 & 130.0 & 165.0 & 254.0 & 376.0 \\
\hline 1931 & 86.0 & 86.0 & 86.3 & 89.1 & 95.4 & 115.0 & 123.0 & $152 \cdot 0$ & 181.0 & 212.0 & 447.0 \\
\hline 1932 & 105.0 & 105.0 & 107.0 & 109.0 & 117.0 & 123.0 & 132.0 & $154 \cdot 0$ & 232.0 & 354.0 & 716.0 \\
\hline 1933 & 147.0 & 149.0 & 152.0 & 155.0 & 157.0 & 197.0 & 309.0 & $378 \cdot 0$ & 547.0 & 699.0 & 1050.0 \\
\hline 1934 & 80.0 & 82.0 & $82 \cdot 1$ & 84.8 & 88.9 & 94.1 & 97.1 & 102.0 & 117.0 & 164.0 & 570.0 \\
\hline 1935 & 110.0 & 110.0 & 111.0 & 112.0 & 116.0 & 117.0 & 123.0 & $129 \cdot 0$ & 165.0 & 198.0 & 393.0 \\
\hline 1936 & 95.0 & 95.0 & 98.3 & 112.0 & 118.0 & 119.0 & $121 \cdot 0$ & $122 \cdot 0$ & 132.0 & 179.0 & 333.0 \\
\hline 1937 & 103.0 & 103.0 & 105.0 & 106.0 & 110.0 & 117.0 & 125.0 & $176 \cdot 0$ & 335.0 & 469.0 & 830.0 \\
\hline 1938 & 89.0 & 89.7 & 96.4 & 102.0 & 107.0 & 114.0 & 119.0 & $124 \cdot 0$ & 138.0 & 215.0 & 482.0 \\
\hline 1939 & 83.0 & 83.7 & 86.4 & 94.6 & 104.0 & 109.0 & 122.0 & $154 \cdot 0$ & 182.0 & 244.0 & 408.0 \\
\hline 1940 & 87.0 & 88.3 & 89.0 & 94.6 & 101.0 & 109.0 & 112.0 & $115 \cdot 0$ & 121.0 & 165.0 & 377.0 \\
\hline 1941 & 113.0 & 116.0 & 121.0 & 127.0 & 128.0 & 139.0 & 177.0 & $251 \cdot 0$ & 303.0 & 324.0 & 535.0 \\
\hline 1942 & 96.0 & 96.7 & 99.1 & 106.0 & 109.0 & 114.0 & 121.0 & $151 \cdot 0$ & 244.0 & 341.0 & 792.0 \\
\hline 1943 & 132.0 & 132.0 & 133.0 & 136.0 & 136.0 & 140.0 & 143.0 & 177.0 & 273.0 & 351.0 & 421.0 \\
\hline 1944 & 112.0 & 112.0 & 114.0 & 115.0 & 131.0 & 137.0 & 140.0 & $146 \cdot 0$ & 174.0 & 229.0 & 370.0 \\
\hline 1945 & 117.0 & 119.0 & $120 \cdot 0$ & 121.0 & 127.0 & 136.0 & 142.0 & 149.0 & 170.0 & 336.0 & 724.0 \\
\hline 1946 & 127.0 & $128 \cdot 0$ & 134.0 & 136.0 & 140.0 & 146.0 & 159.0 & $238 \cdot 0$ & 303.0 & 392.0 & 778.0 \\
\hline 1947 & 146.0 & 147.0 & 148.0 & 150.0 & 160.0 & 165.0 & $181 \cdot 0$ & 273.0 & 289.0 & 345.0 & 695.0 \\
\hline 1948 & 147.0 & 148.0 & 151.0 & 153.0 & 156.0 & 169.0 & 185.0 & 236.0 & 294.0 & 379.0 & 588.0 \\
\hline 1949 & 144.0 & 147.0 & 156.0 & 174.0 & 182.0 & 196.0 & 203.0 & $244 \cdot 0$ & 304.0 & 354.0 & 599.0 \\
\hline 1950 & 180.0 & 180.0 & 181.0 & 182.0 & 190.0 & 203.0 & 225.0 & $350 \cdot 0$ & 518.0 & 625.0 & 921.0 \\
\hline 1951 & 150.0 & 151.0 & 152.0 & 158.0 & 160.0 & 173.0 & 180.0 & 201.0 & 283.0 & 411.0 & 602.0 \\
\hline 1952 & 116.0 & 117.0 & 119.0 & 122.0 & 123.0 & 128.0 & 137.0 & $145 \cdot 0$ & 160.0 & 212.0 & 394.0 \\
\hline 1953 & 126.0 & 128.0 & 135.0 & 135.0 & 146.0 & 156.0 & 161.0 & $172 \cdot 0$ & $200 \cdot 0$ & 328.0 & 716.0 \\
\hline 1954 & 117.0 & 118.0 & 119.0 & 134.0 & 190.0 & 211.0 & 233.0 & $306 \cdot 0$ & 378.0 & 434.0 & 565.0 \\
\hline 1955 & 158.0 & 160.0 & 165.0 & 183.0 & 200.0 & 217.0 & 278.0 & $445 \cdot 0$ & 633.0 & 718.0 & 1060.0 \\
\hline 1956 & 155.0 & 171.0 & 173.0 & 177.0 & 180.0 & 189.0 & 202.0 & $235 \cdot 0$ & 376.0 & 462.0 & 619.0 \\
\hline 1957 & 121.0 & 124.0 & 125.0 & 132.0 & 141.0 & 150.0 & 170.0 & $180 \cdot 0$ & 200.0 & 250.0 & 587.0 \\
\hline 1958 & 118.0 & $121 \cdot 0$ & $128 \cdot 0$ & 136.0 & 151.0 & $180 \cdot 0$ & 199.0 & 230.0 & $227 \cdot 0$ & 273.0 & 723.0 \\
\hline 1959 & 126.0 & 140.0 & 146.0 & 151.0 & 186.0 & 196.0 & 241.0 & $334 \cdot 0$ & 479.0 & 562.0 & 732.0 \\
\hline 1960 & 119.0 & 135.0 & 146.0 & 185.0 & 221.0 & 237.0 & 271.0 & 279.0 & 298.0 & 492.0 & 803.0 \\
\hline 1961 & 113.0 & 129.0 & 144.0 & 160.0 & 200.0 & 206.0 & 212.0 & $212 \cdot 0$ & 246.0 & 353.0 & 646.0 \\
\hline 1962 & 143.0 & 155.0 & $158 \cdot 0$ & 162.0 & 184.0 & 199.0 & 258.0 & 299.0 & 346.0 & 393.0 & 610.0 \\
\hline
\end{tabular}


BULL RUN RIVER NR BULL RIN OREG.

STATION NUMBER

$14-1400.00$

HIGHEST MEAN DISCHARGE, IN CFS, FOR THE FOLLOWING NUMBER OF CONSECUTIVE DAYS IN YEAR ENDING SEPIEMBER 30

\begin{tabular}{|c|c|c|c|c|c|c|c|c|c|c|c|}
\hline YEAR & 1 & 3 & 7 & 15 & 30 & 60 & 90 & 120 & 150 & 183 & 274 \\
\hline $\begin{array}{l}1908 \\
1909\end{array}$ & 10900.0 & $7510 \cdot 0$ & 4480.0 & 2820.0 & 2190.0 & 1850.0 & 1440.0 & $1420 \cdot 0$ & $1300 \cdot 0$ & 1230.0 & 958.0 \\
\hline $\begin{array}{l}1909 \\
1910\end{array}$ & 5860.0 & 5380.0 & 3970.0 & 2330.0 & 1770.0 & 1330.0 & 1160.0 & $1050 \cdot 0$ & 1020.0 & 972.0 & 826.0 \\
\hline $\begin{array}{l}1910 \\
1911\end{array}$ & 9490.0 & $8070 \cdot 0$ & 5550.0 & 3960.0 & 3080.0 & 1990.0 & 1630.0 & $1600 \cdot 0$ & 1620.0 & 1500.0 & 1120.0 \\
\hline $\begin{array}{l}1911 \\
1912\end{array}$ & 5080.0 & $3600 \cdot n$ & $2420 \cdot 0$ & 1900.0 & 1660.0 & 1200.0 & $\begin{array}{l}1130.0 \\
1570.0\end{array}$ & $\begin{array}{r}947.0 \\
1570.0\end{array}$ & $\begin{array}{r}916.0 \\
1340.0\end{array}$ & $\begin{array}{r}878.0 \\
1210.0\end{array}$ & $\begin{array}{l}79 C \cdot 0 \\
982.0\end{array}$ \\
\hline $\begin{array}{l}1912 \\
1913\end{array}$ & $\begin{array}{r}10400.0 \\
5980.0\end{array}$ & $\begin{array}{l}8140 \cdot 0 \\
4540.0\end{array}$ & $\begin{array}{l}4960.0 \\
3090.0\end{array}$ & $\begin{array}{l}2920.0 \\
2240.0\end{array}$ & $\begin{array}{l}2320.0 \\
1630.0\end{array}$ & $\begin{array}{l}1880.0 \\
1380.0\end{array}$ & 1280.0 & 1190.0 & $\begin{array}{l}1340.0 \\
1180.0\end{array}$ & 1170.0 & 1050.0 \\
\hline 1914 & 5230.0 & 3450.0 & 2470.0 & 1810.0 & 1550.0 & 1340.0 & 1220.0 & $1170 \cdot 0$ & 1070.0 & 992.0 & 828.0 \\
\hline 1915 & 3320.0 & $2150 \cdot 0$ & 1540.0 & 1320.0 & 994.0 & 760.0 & 747.0 & 705.0 & 684.0 & 688.0 & 618.0 \\
\hline 1916 & 8830.0 & 5770.0 & 3760.0 & 3240.0 & 2280.0 & 1770.0 & 1580.0 & $1470 \bullet 0$ & 1510.0 & 1460.0 & 1250.0 \\
\hline 1917 & 4720.0 & 3370.0 & 2410.0 & 1690.0 & 1510.0 & 1440.0 & 1420.0 & $1240 \cdot 0$ & 1130.0 & 1110.0 & 997.0 \\
\hline 1918 & $12900 \cdot 0$ & 10400.0 & 8480.0 & 5740.0 & 4420.0 & 3160.0 & 2460.0 & $2010 \cdot 0$ & 1780.0 & $1580 \cdot 0$ & 1120.0 \\
\hline $\begin{array}{l}1919 \\
1920\end{array}$ & 5060.0 & 3750.0 & 3410.0 & $2250 \cdot 0$ & $1500 \cdot 0$ & 1250.0 & 1190.0 & $1150 \cdot 0$ & 1070.0 & $1020 \cdot 0$ & $840 \cdot 0$ \\
\hline 1920 & 9890.0 & 7150.0 & 5070.0 & 2830.0 & 2010.0 & 1570.0 & 1490.0 & $1240 \cdot 0$ & $1120 \cdot 0$ & $1130 \cdot 0$ & 912.0 \\
\hline 1921 & 11400.0 & 6580.0 & 5830.0 & 3890.0 & 2490.0 & $2100 \cdot 0$ & 1940.0 & $1740 \cdot 0$ & 1660.0 & 1560.0 & 1240.0 \\
\hline 1922 & 14500.0 & $11400 \cdot 0$ & 6580.0 & 5430.0 & 3250.0 & 1920.0 & 1410.0 & 1190.0 & 1110.0 & 1130.0 & $968 \cdot 0$ \\
\hline 1923 & 17200.0 & 13300.0 & 7750.0 & 5480.0 & 3940.0 & 2190.0 & 1710.0 & $1560 \cdot 0$ & 1420.0 & 1290.0 & 993.0 \\
\hline 1924 & 8200.0 & 4520.0 & 3010.0 & 2200.0 & 1570.0 & 1340.0 & 1330.0 & $1160 \cdot 0$ & $1060 \cdot 0$ & 943.0 & $700 \cdot 0$ \\
\hline 1925 & $6020 \cdot 0$ & $5150 \cdot 0$ & 4630.0 & 3360.0 & 2460.0 & $2000 \cdot 0$ & 1820.0 & $1700 \cdot 0$ & 1530.0 & 1420.0 & $110 C \cdot 0$ \\
\hline 1926 & 7090.0 & 4580.0 & $2950 \cdot 0$ & 1890.0 & 1740.0 & 1280.0 & 1230.0 & $1110 \cdot 0$ & 991.0 & 867.0 & 655.0 \\
\hline 1927 & 7250.0 & $4660 \cdot 0$ & 3070.0 & 2010.0 & 1560.0 & 1350.0 & 1290.0 & $1210 \cdot 0$ & 1120.0 & 1080.0 & 952.0 \\
\hline 1928 & 10600.0 & 7870.0 & 5590.0 & 3970.0 & 2840.0 & 2070.0 & 1670.0 & $1500 \cdot 0$ & 1490.0 & 1430.0 & 1140.0 \\
\hline 1929 & 2510.0 & 2080.0 & 1990.0 & 1540.0 & 1160.0 & 1080.0 & 1000.0 & 945.0 & 858.0 & 845.0 & 743.0 \\
\hline 1930 & 4990.0 & 3780.0 & 3030.0 & 2860.0 & 2080.0 & 1430.0 & 1230.0 & $1120 \cdot 0$ & 1010.0 & 946.0 & 679.0 \\
\hline 1931 & 16400.0 & $9090 \cdot 0$ & 5110.0 & 3140.0 & 2230.0 & 1470.0 & 1200.0 & $1050 \cdot 0$ & 993.0 & 905.0 & 706.0 \\
\hline 1932 & 9070.0 & 6440.0 & 3920.0 & 3260.0 & 2350.0 & 1920.0 & 1610.0 & 1410.0 & $1330 \cdot 0$ & 1250.0 & 1030.0 \\
\hline 1933 & 6820.0 & 4980.0 & 3340.0 & $2800 \cdot 0$ & 2090.0 & 1650.0 & 1580.0 & 1340.0 & $1280 \cdot 0$ & 1290.0 & 1210.0 \\
\hline 1934 & $12300 \cdot 0$ & 8420.0 & 6460.0 & 4310.0 & 3460.0 & 2730.0 & 2160.0 & $1810 \cdot 0$ & $1620 \cdot 0$ & 1470.0 & 1070.0 \\
\hline 1935 & 7280.0 & 5270.0 & 3870.0 & 2410.0 & 1780.0 & 1680.0 & 1470.0 & $1330 \cdot 0$ & 1240.0 & 1170.0 & 961.0 \\
\hline 1936 & 7420.0 & 5520.0 & 3930.0 & 3380.0 & 2330.0 & 1490.0 & 1330.0 & $1230 \cdot 0$ & 1180.0 & 1070.0 & 815.0 \\
\hline 1937 & 5220.0 & 4690.0 & 3030.0 & $2120 \cdot 0$ & 1750.0 & 1500.0 & 1390.0 & $1290 \cdot 0$ & 1160.0 & $1030 \cdot 0$ & 821.0 \\
\hline 1938 & 7450.0 & 5810.0 & 4060.0 & $2570 \cdot 0$ & 2090.0 & 1940.0 & 1740.0 & 1460.0 & $1400 \cdot 0$ & $1390 \cdot 0$ & 1040.0 \\
\hline 1939 & 6290.0 & 3910.0 & 2400.0 & 1490.0 & 1300.0 & 1100.0 & 1060.0 & 1040.0 & 1040.0 & $1010 \cdot 0$ & 811.0 \\
\hline 1940 & 5500.0 & 4040.0 & 3210.0 & 2360.0 & 2070.0 & 1550.0 & 1320.0 & $1220 \cdot 0$ & 1130.0 & 986.0 & 723.0 \\
\hline 1941 & 5520.0 & 3480.0 & 2190.0 & 1510.0 & 1140.0 & 947.0 & 907.0 & $782 \cdot 0$ & 687.0 & 618.0 & 537.0 \\
\hline 1942 & 4770.0 & 3620.0 & 2650.0 & 1670.0 & 1580.0 & 1190.0 & 1120.0 & 995.0 & $901 \cdot 0$ & 862.0 & 774.0 \\
\hline 1943 & 9930.0 & $6060 \cdot 0$ & $4800 \cdot 0$ & 3500.0 & 2810.0 & 2350.0 & 1980.0 & $1810 \cdot 0$ & $1650 \cdot 0$ & $1610 \cdot 0$ & 1250.0 \\
\hline 1944 & 3900.0 & $2520 \cdot 0$ & 1610.0 & 1330.0 & 914.0 & 811.0 & 747.0 & 705.0 & 672.0 & 673.0 & 595.0 \\
\hline 1945 & 5440.0 & 3620.0 & 2880.0 & 2340.0 & 1690.0 & 1360.0 & 1200.0 & $1210 \cdot 0$ & 1230.0 & 1100.0 & 834.0 \\
\hline 1946 & 12600.0 & 7220.0 & 4320.0 & 3140.0 & 2220.0 & 1800.0 & 1620.0 & 1520.0 & 1420.0 & 1310.0 & 1050.0 \\
\hline 1947 & 11300.0 & 9780.0 & 6900.0 & 3790.0 & 3110.0 & 2120.0 & 1750.0 & 1590.0 & 1420.0 & 1320.0 & 1020.0 \\
\hline 1948 & 7610.0 & $5200 \cdot 0$ & 3660.0 & 2670.0 & 2230.0 & 1590.0 & 1630.0 & 1340.0 & $1350 \cdot 0$ & 1230.0 & 1080.0 \\
\hline 1949 & 5740.0 & 4150.0 & 3210.0 & 2430.0 & 2210.0 & 1460.0 & 1340.0 & 1190.0 & 1040.0 & 1170.0 & 968.0 \\
\hline 1950 & 7180.0 & 5160.0 & 3210.0 & 2620.0 & 2030.0 & 1690.0 & 1520.0 & 1450.0 & 1370.0 & 1310.0 & $113 C .0$ \\
\hline 1951 & 4480.0 & 3680.0 & 2890.0 & 2410.0 & 2090.0 & 1770.0 & 1770.0 & $1680 \cdot 0$ & 1470.0 & 1370.0 & 1110.0 \\
\hline 1952 & 5550.0 & 4420.0 & 3530.0 & $2200 \cdot 0$ & 1380.0 & 1200.0 & 1130.0 & 1130.0 & 1030.0 & $1000 \cdot 0$ & 913.0 \\
\hline 1953 & 9580.0 & 8100.0 & 5640.0 & $4400 \cdot 0$ & 3700.0 & 2350.0 & 1830.0 & $1560 \cdot 0$ & 1430.0 & $1300 \cdot 0$ & 946.0 \\
\hline 1954 & 7660.0 & 5020.0 & 3490.0 & 2930.0 & 2830.0 & 1980.0 & 1720.0 & $1600 \cdot 0$ & 1460.0 & 1320.0 & 1070.0 \\
\hline 1955 & 6610.0 & 5640.0 & 3250.0 & 1860.0 & 1520.0 & 1250.0 & $1180 \cdot 0$ & $1050 \cdot 0$ & 987.0 & $1000 \cdot 0$ & 888.0 \\
\hline 1956 & 8460.0 & 6750.0 & $4080 \cdot 0$ & 2930.0 & 2660.0 & $2220 \cdot 0$ & 2050.0 & $1790 \cdot 0$ & $1580 \cdot 0$ & 1520.0 & 1340.0 \\
\hline 1957 & 8400.0 & 5890.0 & 4450.0 & 2910.0 & 1850.0 & 1570.0 & 1310.0 & 1260.0 & 1190.0 & $1130 \cdot 0$ & 922.0 \\
\hline 1958 & 9530.0 & 5600.0 & 3690.0 & 2680.0 & 2080.0 & 1820.0 & 1660.0 & 1440.0 & 1400.0 & 1280.0 & 939.0 \\
\hline 1959 & 4700.0 & 3960.0 & 2920.0 & 2760.0 & 2170.0 & 1890.0 & 1760.0 & $1560 \cdot C$ & 1480.0 & 1390.0 & 1120.0 \\
\hline 1960 & 6890.0 & $4500 \cdot 0$ & 2860.0 & 2350.0 & 1890.0 & 1500.0 & 1300.0 & $1310 \cdot 0$ & 1150.0 & 1130.0 & 1080.0 \\
\hline 1961 & 10100.0 & 6200.0 & 4340.0 & 3530.0 & 2700.0 & 2170.0 & 1810.0 & $1780 \cdot 0$ & $1730 \cdot 0$ & 1620.0 & 1220.0 \\
\hline 1962 & 5030.0 & 4140.0 & 2920.0 & 2280.0 & 2080.0 & 1630.0 & 1380.0 & $1240 \cdot 0$ & 1230.0 & $1200 \cdot 0$ & 1020.0 \\
\hline 1963 & 5700.0 & 4460.0 & 2960.0 & $2620 \cdot 0$ & 1840.0 & 1270.0 & 1110.0 & $1060 \cdot 0$ & $1100 \cdot 0$ & 1110.0 & 862.0 \\
\hline
\end{tabular}




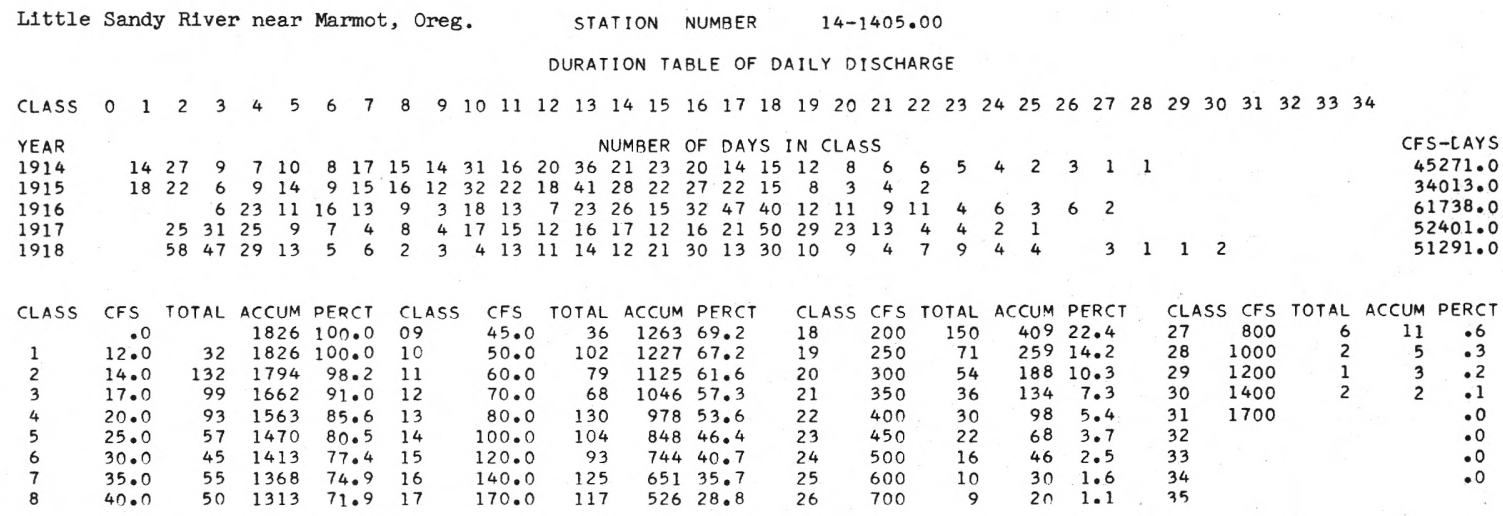

$\begin{array}{ll}18 & 2 \\ 19 & 2 \\ 20 & 3 \\ 21 & 3 \\ 22 & 4 \\ 23 & 4 \\ 24 & 5 \\ 25 & 6 \\ 26 & 7\end{array}$

$\begin{array}{lr}\text { CFS } & \text { TOTAL } \\ 200 & 15 \\ 250 & 71 \\ 300 & 54 \\ 350 & 36 \\ 400 & 30 \\ 450 & 22 \\ 500 & 16 \\ 600 & 10 \\ 700 & \end{array}$

$\begin{array}{rrr}150 & 409 & 22.4 \\ 71 & 259 & 14.2 \\ 54 & 188 & 10.3 \\ 36 & 134 & 7.3 \\ 30 & 98 & 5.4 \\ 22 & 68 & 3.7 \\ 16 & 46 & 2.5 \\ 10 & 30 & 1.6 \\ 9 & 20 & 1.1\end{array}$

Little Sandy River near Marmot, Oreg.

STATION NUMBER

$14-1405.00$

LOWEST MEAN DISCHARGE, IN CFS, FOR THE FOLLOWING NUMBER OF CONSECUTIVE DAYS IN YEAR BEGINNING APRIL 1

$\begin{array}{lcccccccrrrr}\text { YEAR } & 1 & 3 & 7 & 14 & 30 & 60 & 90 & 120 & 150 & 183 & 274 \\ 1914 & 12.0 & 12.0 & 12.0 & 12.2 & 13.4 & 16.1 & 23.0 & 36.7 & 41.2 & 52.4 & 72.7 \\ 1915 & 12.0 & 12.0 & 12.4 & 13.4 & 13.9 & 16.6 & 28.9 & 40.5 & 52.8 & 61.5 & 11.0 \\ 1916 & 14.0 & 14.3 & 14.7 & 15.6 & 17.8 & 23.1 & 26.2 & 38.5 & 64.3 & 83.7 & 106.0 \\ 1917 & 15.0 & 15.0 & 15.0 & 15.5 & 16.9 & 19.3 & 19.1 & 22.2 & 34.4 & 77.8 & 154.0 \\ 1918 & 14.0 & 14.0 & 14.0 & 14.0 & 14.8 & 16.7 & 17.5 & 19.1 & 27.6 & 43.1 & 73.1\end{array}$

Little Sandy River near Marmot, Oreg. STATION NUMBER $14-1405.00$ HIGHEST MEAN DISCHARGF, IN CFS, FOR THE FOLLOWING NUMBER OF CONSECUTIVF DAYS IN YEAR. ENDING SEPTFMBER 30

\begin{tabular}{|c|c|c|c|c|c|c|c|c|c|c|c|}
\hline YEAR & 1 & 3 & 7 & 15 & 30 & 60 & 90 & 120 & 150 & 183 & 274 \\
\hline 1914 & 1000.0 & 750.0 & 647.0 & 400.0 & 244.0 & 230.0 & 202.0 & 194.0 & 192.0 & 185.0 & 158.0 \\
\hline 915 & 418.0 & 309.0 & 241.0 & 208.0 & 160.0 & 137.0 & 140.0 & 131.0 & 127.0 & 122.0 & 113.0 \\
\hline 1916 & 815.0 & 749.0 & 609.0 & 501.0 & 379.0 & 291.0 & 264.0 & $248 \cdot 0$ & 245.0 & 240.0 & 212.0 \\
\hline 17 & 651.0 & 452.0 & 362.0 & 335.0 & 301.0 & 282.0 & 274.0 & $239 \cdot 0$ & 217.0 & 210.0 & 185.0 \\
\hline 918 & 1670.0 & 1370.0 & 1070.0 & 750.0 & 588.0 & 456.0 & 364.0 & 302.0 & 273.0 & 253.0 & 182.0 \\
\hline
\end{tabular}


CLASS $0 \begin{array}{llllllllllllllllllllllllllllllllllll} & 0 & 2 & 3 & 4 & 5 & 6 & 7 & 8 & 9 & 10 & 11 & 12 & 13 & 14 & 15 & 16 & 17 & 18 & 19 & 20 & 21 & 22 & 23 & 24 & 25 & 26 & 27 & 28 & 29 & 30 & 31 & 32 & 33 & 34\end{array}$

YEAR
1920
1921
1922
1923
1924
1925
1926
1927
1928
1929
1930
1931
1932
1933
1934
1935
1936
1937
1938
1939
1940
1941
1942
1943
1944
1945
1946
1947
1948
1949
1950
1951
1952
1953
1954
1955
1956
1957
1958
1959
1960
1961
1962
1963
1

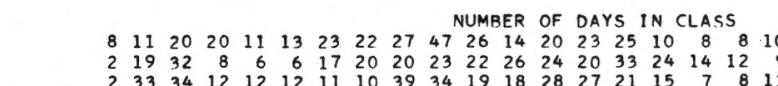
$\begin{array}{rrrrrrrrrrrrrrrrrrr}2 & 19 & 32 & 8 & 6 & 6 & 17 & 20 & 20 & 23 & 22 & 26 & 24 & 20 & 33 & 24 & 14 & 12 & 9 \\ 2 & 33 & 34 & 12 & 12 & 12 & 11 & 10 & 39 & 34 & 19 & 18 & 28 & 27 & 21 & 15 & 7 & 8 & 13\end{array}$ $19 \begin{array}{rrrrrrrrrrrrrrrrrrrrrr}14 & 17 & 7 & 35 & 6 & 3 & 5 & 16 & 26 & 40 & 18 & 17 & 31 & 34 & 37 & 18 & 11 & 5 & 3 & 7 & 3 \\ 34 & 22 & 12 & 34 & 15 & 12 & 13 & 13 & 7 & 20 & 33 & 25 & 21 & 29 & 19 & 14 & 7 & 3 & 4 & 3 & 2\end{array}$

$\begin{array}{rrrrrrrrrrrrrrrrrrrr}3 & 45 & 24 & 17 & 9 & 6 & 7 & 16 & 11 & 30 & 22 & 19 & 45 & 24 & 31 & 13 & 8 & 7 & 11 \\ 34 & 16 & 15 & 20 & 13 & 14 & 30 & 22 & 37 & 45 & 32 & 19 & 23 & 13 & 15 & 4 & 2 & 2 & 2\end{array}$

$\begin{array}{lllllllllllllllllllllll}1 & 16 & 18 & 9 & 5 & 5 & 13 & 11 & 22 & 23 & 30 & 45 & 51 & 44 & 31 & 14 & 9 & 4 & 6 & 1 & 4 & 3 & 2\end{array}$

$\begin{array}{lllllllllllllllllll}20 & 31 & 13 & 3 & 4 & 12 & 23 & 32 & 40 & 32 & 12 & 29 & 21 & 29 & 22 & 8 & 4 & 11\end{array}$

$\begin{array}{llllllllllllllllllllll}21 & 26 & 8 & 9 & 14 & 13 & 9 & 12 & 17 & 33 & 29 & 20 & 20 & 24 & 26 & 28 & 27 & 14 & 6 & 6 & 2\end{array}$ $\begin{array}{rrrrrrrrrrrrrrrrrrrrrrr}8 & 81 & 33 & 10 & 16 & 6 & 6 & 6 & 7 & 26 & 12 & 31 & 30 & 16 & 16 & 10 & 11 & 7 & 9 & 9 & 8 & 4 & 2 \\ 19 & 36 & 11 & 6 & 12 & 8 & 16 & 6 & 17 & 21 & 36 & 45 & 29 & 21 & 35 & 15 & 11 & 5 & 3 & 6 & 1 & 2 & 2\end{array}$ $\begin{array}{llllllllllll}38 & 20 & 10 & 14 & 15 & 23 & 47 & 20 & 17 & 10 & 10\end{array}$

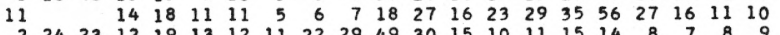

$\begin{array}{llllllllllllllllllllll}2 & 24 & 23 & 12 & 19 & 13 & 12 & 11 & 22 & 29 & 49 & 30 & 15 & 10 & 11 & 15 & 14 & 8 & 7 & 8 & 9 & 5\end{array}$

$\begin{array}{rrrrrrrrrrrrrrrrrrrrr}17 & 34 & 14 & 16 & 11 & 9 & 5 & 8 & 13 & 19 & 29 & 27 & 24 & 30 & 27 & 39 & 15 & 7 & 6 & 8 \\ 5 & 25 & 23 & 19 & 9 & 13 & 14 & 26 & 23 & 26 & 33 & 19 & 16 & 23 & 18 & 28 & 18 & 9 & 6 & 3 & 4\end{array}$

$\begin{array}{rrrrrrrrrrrrrrrrrrrrr} & 8 & 52 & 29 & 33 & 10 & 9 & 9 & 25 & 15 & 11 & 17 & 10 & 8 & 17 & 21 & 30 & 26 & 10 & 13 & 4 \\ 15 & 13 & 31 & 16 & 17 & 10 & 19 & 5 & 12 & 9 & 13 & 22 & 15 & 17 & 40 & 26 & 22 & 22 & 6 & 5 & 12\end{array}$

$\begin{array}{lllllllrlllllllllllllll}20 & 30 & 11 & 15 & 10 & 8 & 5 & 9 & 8 & 34 & 28 & 38 & 27 & 37 & 24 & 19 & 10 & 12 & 7 & 5 & 2 & 5\end{array}$

$\begin{array}{rrrrrrrrrrrrrrrrrrrrrrrr}24 & 23 & 26 & 29 & 12 & 10 & 12 & 12 & 11 & 16 & 20 & 30 & 20 & 16 & 16 & 17 & 10 & 14 & 17 & 8 & 9 & 6 & 4 \\ & 5 & 9 & 22 & 12 & 13 & 9 & 7 & 24 & 39 & 35 & 45 & 48 & 21 & 21 & 13 & 15 & 12 & 5 & 2 & 2 & 2 & 1\end{array}$

$\begin{array}{lllllllllllllllllllllllll}5 & 6 & 25 & 10 & 8 & 8 & 3 & 2 & 22 & 12 & 38 & 58 & 36 & 33 & 32 & 20 & 17 & 8 & 6 & 2 & 5 & 2 & 7\end{array}$

$\begin{array}{rrrrrrrrrrrrrrrrrrrrrrrr}3 & 17 & 26 & 21 & 22 & 8 & 5 & 5 & 8 & 3 & 25 & 19 & 23 & 18 & 29 & 25 & 32 & 18 & 9 & 13 & 11 & 8 & 9 \\ 5 & 28 & 23 & 4 & 13 & 11 & 15 & 10 & 34 & 16 & 49 & 51 & 28 & 15 & 26 & 14 & 7 & 8 & 2 & 2 & 1 & 2 & 2\end{array}$

$\begin{array}{lllllllllllllllllllllll}11 & 25 & 8 & 28 & 14 & 11 & 21 & 25 & 10 & 34 & 29 & 15 & 14 & 14 & 18 & 35 & 14 & 8 & 14 & 7 & 4 & 5\end{array}$

$\begin{array}{llllllllllllllllllllll}26 & 14 & 30 & 16 & 6 & 6 & 6 & 6 & 17 & 28 & 28 & 18 & 39 & 37 & 30 & 20 & 12 & 7 & 8 & 4 & 4 \\ 7 & 18 & 23 & 8 & 18 & 13 & 14 & 16 & 28 & 34 & 28 & 19 & 23 & 27 & 23 & 17 & 6 & 7 & 8 & 4 & 1\end{array}$ $\begin{array}{llllllllllllll}6 & 17 & 28 & 28 & 18 & 39 & 37 & 30 & 20 & 11 & 7 & 8 & 4 \\ 16 & 34 & 23 & 37 & 19 & 23 & 27 & 23 & 17 & 6 & 7 & 8 & 4\end{array}$ \begin{tabular}{rrrrrrrrrrrrrrrrrrrr}
12 & 7 & 17 & 16 & 9 & 20 & 13 & 34 & 23 & 37 & 29 & 30 & 17 & 28 & 18 & 17 & 8 & 9 \\
9 & 24 & 27 & 11 & 8 & 39 & 17 & 23 & 18 & 18 & 22 & 21 & 23 & 33 & 24 & 13 & 8 & 14 \\
\hline & 24 & 17 & 14 & 3 & 15 & 15 & 12 & 18 & 19 & 23 & 28 & 21 & 41 & 31 & 12 & 10 & 15
\end{tabular}

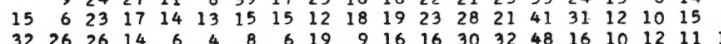
$\begin{array}{rrrrrrrrrrrrrrrrrrrrrrr}11 & 25 & 14 & 13 & 5 & 7 & 4 & 8 & 6 & 19 & 9 & 16 & 16 & 30 & 32 & 48 & 16 & 10 & 12 & 11 & 11 & 8 \\ 9 & 3 & 13 & 47 & 23 & 22 & 22 & 31 & 32 & 14 & 9 & 5 & 4 & 9 & 2\end{array}$

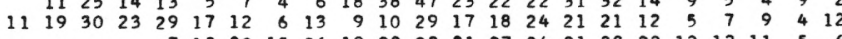
$\begin{array}{lllllllllllllllllllll}13 & 20 & 13 & 20 & 15 & 26 & 19 & 28 & 32 & 21 & 27 & 24 & 21 & 38 & 22 & 12 & 12 & 11 & 5 & 6\end{array}$ $\begin{array}{rrrrrrrrrrrrrrrrrrrr}13 & 20 & 17 & 6 & 6 & 20 & 12 & 31 & 42 & 23 & 25 & 39 & 28 & 36 & 15 & 8 & 10 & 8 & 2 & 2 \\ 19 & 28 & 14 & 9 & 13 & 6 & 11 & 23 & 19 & 19 & 30 & 39 & 44 & 21 & 20 & 5 & 19 & 7 & 4\end{array}$

$\begin{array}{llllllllllllllllllllllll}30 & 14 & 30 & 13 & 11 & 6 & 9 & 21 & 33 & 33 & 23 & 24 & 29 & 19 & 26 & 10 & 4 & 8 & 7 & 3 & 7 \\ 4 & 25 & 22 & 4 & 12 & 13 & 35 & 18 & 38 & 34 & 26 & 26 & 22 & 14 & 14 & 7 & 11 & 7 & 8 & 6 & 3\end{array}$

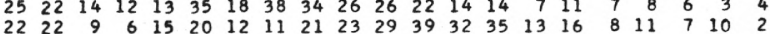
$\begin{array}{llllllllllllllllllllll}11 & 24 & 21 & 14 & 9 & 7 & 23 & 37 & 38 & 17 & 16 & 18 & 24 & 40 & 26 & 16 & 6 & 9 & 5 & 1 & 3\end{array}$

$\begin{array}{lllllllllllllllllllllllll}12 & 19 & 32 & 11 & 16 & 14 & 17 & 6 & 16 & 15 & 19 & 25 & 28 & 36 & 30 & 22 & 12 & 3 & 15 & 3 & 7 & 4\end{array}$ $\begin{array}{rrrrrrrrrrrrrrrrrrrrr}11 & 29 & 21 & 12 & 13 & 15 & 15 & 23 & 20 & 31 & 32 & 38 & 25 & 30 & 15 & 10 & 1 & 7 & 6 & 7 \\ 7 & 17 & 12 & 19 & 10 & 23 & 26 & 47 & 38 & 30 & 17 & 22 & 25 & 31 & 13 & 5 & 5 & 5 & 7 & 4\end{array}$

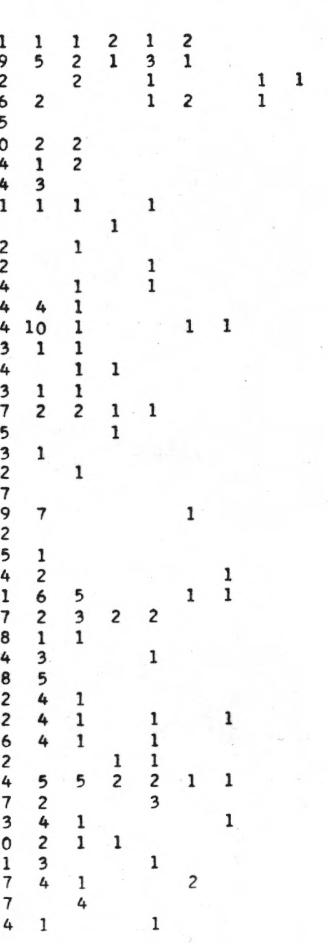

CFS-DAYS

58984.0

71506.0

55885.0

54771.0

35372.0

55650.0

36151.0

54483.0

58211.0

47229.0

36429.0

39342.0

53006.0

65469.0

52028.0

48713.0

45850.0

45257.0

55280.0

46356.0

37843.0

32181.0

$4418^{*}$. 0

63304.0

32054.0

47561.0

55857.0

59090.0

65912.0

56778.0

62710.0

62395.0

52629.0

53803.0

62746.0

52988.0

80559.0

51734.0

49478.0

63423.0

56251.0

60605.0

54781.0

49153.0

Little Sandy River near Bull Run, Oreg.

Summary for water years 1920-63

STATION NUMBER

$14-1415.00$

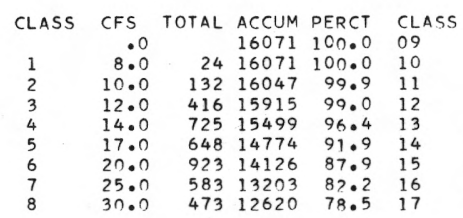

CFS TOTAL ACCUM PERCT

$35.0 \quad 404 \quad 12147 \quad 75.6$

40.07441174373 .

$50.0 \quad 688 \quad 1099968.4$

$\begin{array}{rrrr}60.0 & 1214 & 10311 & 64.2 \\ 80.0 & 1331 & 9097 & 56.6\end{array}$

$\begin{array}{rrrr}80.0 & 1331 & 9097 & 56.6 \\ 100.0 & 1011 & 7766 & 48.3\end{array}$

100.01011776648 .3

$120.0 \quad 925675542.0$

$140.0 \quad 1197 \quad 5830 \quad 36$.

\begin{tabular}{|c|c|c|c|c|c|c|c|c|c|}
\hline CLASS & CFS & TOTAL & ACCUM & PERCT & CLASS & CFS & TOTAL & ACCUM & PERCT \\
\hline 18 & 200 & 1219 & 3591 & 22.3 & 27 & 1200 & 13 & 57 & .4 \\
\hline 19 & 250 & 691 & 2372 & 14.8 & 28 & 1400 & 23 & 44 & .3 \\
\hline 20 & 300 & 407 & 1681 & 10.5 & 29 & 1700 & 11 & 21 & .1 \\
\hline 21 & 350 & 310 & 1274 & 7.9 & 30 & 2000 & 6 & 10 & - 1 \\
\hline 22 & 400 & 358 & 964 & 6.0 & 31 & 2500 & 2 & 4 & .0 \\
\hline 23 & 500 & 186 & 606 & 3.8 & 32 & 3000 & 1 & 2 & .0 \\
\hline 24 & 600 & 225 & 420 & 2.6 & 33 & 3500 & 1 & 1 & .0 \\
\hline 25 & 800 & 94 & 195 & 1.2 & 34 & & & & .0 \\
\hline 26 & 1000 & 44 & 101 & & 35 & & & & \\
\hline
\end{tabular}


LOWEST MEAN DISCHARGE, IN CFS, FOR THE FOLLOWING NUMBER OF CONSECUTIVE DAYS IN YEAR BEGINNING APRIL 1

\begin{tabular}{|c|c|c|c|c|c|c|c|c|c|c|c|}
\hline YEAR & 1 & 3 & 7 & 14 & 30 & 60 & 90 & 120 & 150 & 183 & 274 \\
\hline $\begin{array}{l}1920 \\
1921 \\
1922\end{array}$ & $\begin{array}{l}15.0 \\
16.0 \\
16.0\end{array}$ & $\begin{array}{l}15.3 \\
16.3 \\
16.3\end{array}$ & $\begin{array}{l}15.7 \\
17.3 \\
16.7\end{array}$ & $\begin{array}{l}16.8 \\
18.4 \\
17.2\end{array}$ & $\begin{array}{l}19.9 \\
18.6 \\
20.9\end{array}$ & $\begin{array}{l}26.3 \\
20.8 \\
21.4\end{array}$ & $\begin{array}{l}37.5 \\
27.11 \\
24.6\end{array}$ & $\begin{array}{l}55 \cdot 2 \\
34 \cdot 0 \\
27 \cdot 6\end{array}$ & $\begin{array}{l}82.6 \\
43.2 \\
41.6\end{array}$ & $\begin{array}{l}89 \cdot 2 \\
57 \cdot 1 \\
51.0\end{array}$ & $\begin{array}{l}158.0 \\
122.0 \\
130.0\end{array}$ \\
\hline $\begin{array}{l}1923 \\
1924 \\
1925\end{array}$ & $\begin{array}{l}12.0 \\
10.0 \\
14.0\end{array}$ & $\begin{array}{l}12.7 \\
10.0 \\
14.3\end{array}$ & $\begin{array}{l}12.9 \\
10.4 \\
14.7\end{array}$ & $\begin{array}{l}12.9 \\
10.9 \\
15.0\end{array}$ & $\begin{array}{l}14.1 \\
12.2 \\
16.6\end{array}$ & $\begin{array}{l}17.8 \\
13.0 \\
17.7\end{array}$ & $\begin{array}{l}23.4 \\
14.8 \\
18.2\end{array}$ & $\begin{array}{l}25 \cdot 1 \\
23 \cdot 3 \\
20 \cdot 7\end{array}$ & $\begin{array}{l}37.4 \\
29.5 \\
26.9\end{array}$ & $\begin{array}{l}55 \cdot 6 \\
36.5 \\
41.7\end{array}$ & $\begin{array}{l}98.4 \\
98.1 \\
80.3\end{array}$ \\
\hline $\begin{array}{l}1926 \\
1927\end{array}$ & 14.0 & $\begin{array}{l}14.0 \\
16.7\end{array}$ & 14.3 & 14.6 & 16.4 & 24.1 & $\begin{array}{l}31.4 \\
44.2\end{array}$ & $\begin{array}{l}41.0 \\
77.3\end{array}$ & $\begin{array}{l}45.0 \\
96.1\end{array}$ & $\begin{array}{r}53.2 \\
113.0\end{array}$ & $\begin{array}{r}88.8 \\
155.0\end{array}$ \\
\hline 1928 & 18.0 & 18.0 & 18.3 & 18.7 & 19.7 & 21.4 & 29.1 & 39.4 & 44.9 & 51.7 & 81.5 \\
\hline 1929 & 12.0 & 12.0 & 12.0 & 12.1 & 13.4 & 14.4 & 15.0 & 14.9 & 16.8 & 34.4 & 89.0 \\
\hline $\begin{array}{l}1930 \\
1931\end{array}$ & $\begin{array}{l}11.0 \\
10.0\end{array}$ & $\begin{array}{l}11.0 \\
10.3\end{array}$ & $\begin{array}{l}11.3 \\
10.7\end{array}$ & $\begin{array}{l}11.6 \\
10.9\end{array}$ & $\begin{array}{l}11.8 \\
11.4\end{array}$ & $\begin{array}{l}12.7 \\
13.1\end{array}$ & $\begin{array}{l}14.7 \\
15.4\end{array}$ & $\begin{array}{l}22.9 \\
26.5\end{array}$ & $\begin{array}{l}33.4 \\
33.4\end{array}$ & $\begin{array}{l}52.0 \\
41.2\end{array}$ & $\begin{array}{l}72.9 \\
84.5\end{array}$ \\
\hline 1932 & 10.0 & 10.0 & 10.0 & 10.2 & 11.7 & 13.6 & 15.8 & 21.7 & 38.3 & 68.0 & 128.0 \\
\hline 1933 & 18.0 & 18.0 & 18.4 & 19.9 & 20.7 & 26.2 & 51.8 & 65.7 & $100 \cdot 0$ & 125.0 & 176.0 \\
\hline 1934 & 11.0 & 11.3 & 11.7 & 11.9 & 13.0 & 14.0 & 15.5 & 18.4 & 23.6 & 33.9 & 99.6 \\
\hline $\begin{array}{l}1935 \\
1936\end{array}$ & $\begin{array}{l}12.0 \\
12.0\end{array}$ & $\begin{array}{l}12.0 \\
12.7\end{array}$ & $\begin{array}{l}12.0 \\
12.9\end{array}$ & $\begin{array}{l}12.7 \\
13.5\end{array}$ & $\begin{array}{l}16.0 \\
14.7\end{array}$ & $\begin{array}{l}16.3 \\
15.9\end{array}$ & $\begin{array}{l}19.2 \\
18.2\end{array}$ & $\begin{array}{l}23 \cdot 3 \\
18 \cdot 4\end{array}$ & $\begin{array}{l}32.4 \\
21.5\end{array}$ & $\begin{array}{l}39.6 \\
33.9\end{array}$ & $\begin{array}{l}77.3 \\
62.7\end{array}$ \\
\hline 1937 & 16.0 & 16.0 & 16.4 & 17.6 & 19.9 & 20.4 & 21.8 & 32.9 & 61.5 & 89.2 & 153.0 \\
\hline 1938 & 10.0 & 10.0 & 10.6 & 10.8 & 12.1 & 13.8 & 15.8 & $21 \cdot 1$ & 25.5 & 42.0 & 90.3 \\
\hline 1939 & 12.0 & 12.0 & 12.0 & 12.4 & 14.2 & 14.9 & 18.3 & 26.9 & 33.4 & 48.2 & 77.9 \\
\hline 1940 & 8.0 & 8.3 & 9.0 & 9.2 & 9.6 & 10.3 & 11.5 & 14.1 & 17.5 & 30.1 & 69.6 \\
\hline 1941 & 11.0 & 11.0 & 11.3 & 11.9 & 13.4 & 17.5 & 33.1 & $48 \cdot 8$ & 59.3 & 64.5 & 102.0 \\
\hline 1942 & 11.0 & 11.0 & 11.3 & 11.9 & 12.5 & 13.7 & 16.6 & $26 \cdot 3$ & 45.6 & 68.3 & 145.0 \\
\hline 1943 & 12.0 & 12.0 & 12.6 & 13.6 & 13.7 & 17.0 & 19.5 & 29.3 & 49.0 & 60.8 & 73.6 \\
\hline 1944 & 10.0 & 10.0 & 10.9 & 12.6 & 13.2 & 15.2 & 23.4 & 28.8 & -4.6 & 44.3 & 70.3 \\
\hline 1945 & 13.0 & 13.0 & 13.0 & 13.5 & 14.6 & 17.5 & 22.9 & $26 \cdot 6$ & 32.8 & 72.1 & 135.0 \\
\hline 1946 & 14.0 & 14.3 & 14.7 & 15.2 & 17.3 & 18.8 & 21.0 & $35 \cdot 7$ & 51.8 & 71.9 & 149.0 \\
\hline 1947 & 16.0 & 16.0 & 17.4 & 18.4 & 22.8 & 25.5 & 31.5 & $57 \cdot 4$ & 60.4 & 73.1 & 150.0 \\
\hline $\begin{array}{l}1948 \\
1949\end{array}$ & $\begin{array}{l}17.0 \\
19.0\end{array}$ & $\begin{array}{l}17.0 \\
19.0\end{array}$ & $\begin{array}{l}17.7 \\
19.1\end{array}$ & $\begin{array}{l}18.5 \\
19.4\end{array}$ & $\begin{array}{l}23.9 \\
20.5\end{array}$ & $\begin{array}{l}35.8 \\
27.1\end{array}$ & $\begin{array}{l}38.9 \\
32.0\end{array}$ & $\begin{array}{l}56 \cdot 0 \\
39.3\end{array}$ & $\begin{array}{l}66.7 \\
52.1\end{array}$ & $\begin{array}{l}76.9 \\
61.8\end{array}$ & $\begin{array}{l}118.0 \\
113.0\end{array}$ \\
\hline 1950 & 14.0 & 14.0 & 14.0 & 15.0 & 17.6 & 22.0 & 28.9 & 54.9 & 94.7 & 119.0 & 173.0 \\
\hline 1951 & 14.0 & 14.7 & 14.9 & 15.1 & 16.1 & 16.8 & 19.4 & $24 \cdot 3$ & 45.4 & 74.7 & 109.0 \\
\hline 1952 & 11.0 & 11.0 & 11.0 & 11.3 & 12.0 & 13.7 & 14.8 & $15 \cdot 7$ & 18.9 & 35.4 & 77.5 \\
\hline 1953 & 16.0 & 16.0 & 16.0 & 16.4 & 19.7 & 21.5 & 24.6 & 29.2 & 36.3 & 67.6 & 145.0 \\
\hline 1954 & 23.0 & $24 \cdot 3$ & 24.9 & 26.9 & 35.6 & 41.7 & 44.5 & $58 \cdot 6$ & 71.9 & 87.4 & 114.0 \\
\hline 1955 & 17.0 & 17.7 & 17.9 & 18.1 & 20.2 & 29.6 & 43.0 & 80.1 & $122 \cdot 0$ & 140.0 & 228.0 \\
\hline 1956 & 22.0 & 22.3 & $23 . n$ & 23.9 & 25.6 & 30.2 & 33.7 & $42 \cdot 4$ & 68.0 & 85.8 & 116.0 \\
\hline 1957 & 15.0 & 15.0 & 15.3 & 15.9 & 16.1 & 18.2 & 21.6 & 30.1 & 36.5 & 48.1 & 107.0 \\
\hline 1958 & 16.0 & 16.0 & 16.4 & 17.6 & 18.1 & 20.4 & 26.9 & 38.4 & 42.9 & 50.2 & 129.0 \\
\hline 1959 & 17.0 & 17.7 & 17.9 & 17.9 & 19.2 & 25.1 & 39.6 & 57.6 & 87.3 & 103.0 & 123.0 \\
\hline 1960 & 18.0 & 18.7 & 18.9 & 19.7 & 21.6 & 29.8 & $29 \cdot C$ & 35.4 & $5 ? \cdot 9$ & 88.2 & 140.0 \\
\hline 1961 & 15.0 & 15.3 & 15.7 & 16.1 & 17.0 & 20.8 & 27.0 & 31.0 & 46.9 & 64.3 & 117.0 \\
\hline 1962 & 18.0 & 18.0 & 18.6 & 20.0 & 22.3 & 26.7 & 28.6 & 35.8 & 49.2 & 68.3 & 110.0 \\
\hline
\end{tabular}

Little Sandy River near Bull Run, Oreg.

STATION NUMBER $14-1415.00$

HIGHEST MEAN DISCHARGE, IN CFS, FOR THE FOLLOWING NUMBER OF CONSECUTIVE DAYS IN YEAR ENDING SEPTEMBER 30

\begin{tabular}{|c|c|c|c|}
\hline 1840.0 & & 17 & 15 \\
\hline $\begin{array}{l}1840.0 \\
1980.0\end{array}$ & $\begin{array}{l}1540 . n \\
1440.0\end{array}$ & $102 n \cdot n$ & $\begin{array}{l}566.0 \\
785.0\end{array}$ \\
\hline $\begin{array}{l}3260.0 \\
2970.0\end{array}$ & $\begin{array}{l}2440.0 \\
2200.0\end{array}$ & $\begin{array}{l}1340.0 \\
1330.0\end{array}$ & $\begin{array}{l}994.0 \\
958.0\end{array}$ \\
\hline 750.0 & 591.0 & $537 \cdot 0$ & 384.0 \\
\hline $1130 \cdot 0$ & 897.0 & $667 \cdot 0$ & 514.0 \\
\hline 1100.0 & 773.0 & 489.0 & 324.0 \\
\hline 945.0 & 631.0 & 437.0 & 309.0 \\
\hline 1480.0 & 1000.0 & 770.0 & 579.0 \\
\hline 1280.0 & 732.0 & 449.0 & 328.0 \\
\hline 1010.0 & 695.0 & 554.0 & 482.0 \\
\hline 3500.0 & 1850.0 & 1050.0 & 628.0 \\
\hline 1640.0 & 1120.0 & 680.0 & 549.0 \\
\hline 1080.0 & 850.0 & $573 . n$ & 520.0 \\
\hline 2050.0 & $1200 \cdot 0$ & 956.0 & 651.0 \\
\hline 1120.0 & 853.0 & 619.0 & 401.0 \\
\hline 1330.0 & $1030 \cdot 0$ & 711.0 & 594.0 \\
\hline 1020.0 & 894.0 & 593.0 & 419.0 \\
\hline 1490.0 & $1100 \cdot 0$ & 758.0 & 476.0 \\
\hline 1210.0 & 813.0 & 498.0 & 306.0 \\
\hline $880 \cdot 0$ & 699.0 & 564.0 & 422.0 \\
\hline 1020.0 & 687.0 & 446.0 & 299.0 \\
\hline 792.0 & 623.0 & 465.0 & 300.0 \\
\hline 1930.0 & 1110.0 & 828.0 & 611.0 \\
\hline 641.0 & 472.0 & 280.0 & 235.0 \\
\hline 943.0 & 708.0 & 544.0 & 389.0 \\
\hline 2470.0 & 1280.0 & 776.0 & 531.0 \\
\hline 2350.0 & 1740.0 & 1170.0 & 674.0 \\
\hline 1690.0 & $1130 . n$ & 794.0 & 677.0 \\
\hline 1100.0 & 797.0 & 628.0 & 471.0 \\
\hline 1400.0 & 983.0 & 624.0 & 509.0 \\
\hline 896.0 & 756.0 & 591.0 & 524.0 \\
\hline 1020.0 & $848 \cdot 0$ & 674.0 & 423.0 \\
\hline 2130.0 & $1540 . n$ & 1060.0 & 826.0 \\
\hline 1450.0 & 920.0 & 676.0 & 591.0 \\
\hline 1560.0 & 1160.0 & 679.0 & 391.0 \\
\hline 2460.0 & 1710.0 & 983.0 & 731.0 \\
\hline 1680.0 & 1120.0 & $801 \cdot 0$ & 533.0 \\
\hline 2060.0 & 1120.0 & 683.0 & 481.0 \\
\hline 1370.0 & 860.0 & 570.0 & 530.0 \\
\hline 1610.0 & $1000 \cdot 0$ & 560.0 & 405.0 \\
\hline & 1110.0 & 732.0 & 607.0 \\
\hline 1130.0 & $\begin{array}{l}975 \cdot 0 \\
893.0\end{array}$ & $\begin{array}{l}678.0 \\
562.0\end{array}$ & $\begin{array}{l}463 \cdot 0 \\
471.0\end{array}$ \\
\hline
\end{tabular}

30
382.0
508.0
605.0
701.0
274.0
392.0
308.0
259.0
433.0
291.0
356.0
437.0
411.0
358.0
530.0
304.0
425.0
343.0
391.0
243.0
364.0
214.0
277.0
486.0
175.0
375.0
381.0
556.0
478.0
417.0
394.0
434.0
273.0
702.0
553.0
296.0
608.0
367.0
365.0
379.0
292.0
457.0
390.0
337.0

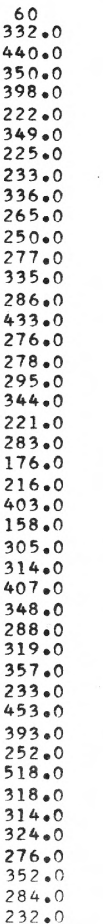

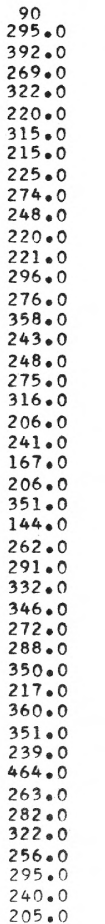

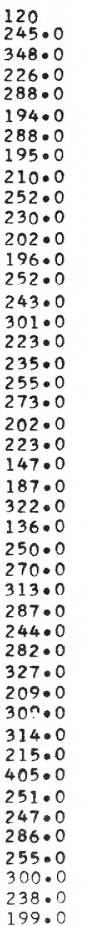

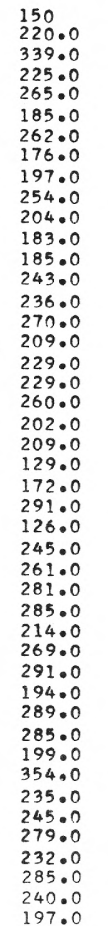

$\begin{array}{ll}183 & 274 \\ 236.0 & 194.0 \\ 316.0 & 251.0 \\ 227.0 & 196.0 \\ 243.0 & 193.0 \\ 167.0 & 124.0 \\ 250.0 & 196.0 \\ 155.0 & 119.0 \\ 195.0 & 173.0 \\ 249.0 & 201.0 \\ 199.0 & 166.0 \\ 179.0 & 129.0 \\ 170.0 & 138.0 \\ 227.0 & 188.0 \\ 237.0 & 221.0 \\ 247.0 & 184.0 \\ 202.0 & 172.0 \\ 207.0 & 160.0 \\ 202.0 & 159.0 \\ 260.0 & 196.0 \\ 198.0 & 163.0 \\ 181.0 & 134.0 \\ 115.0 & 104.0 \\ 166.0 & 152.0 \\ 284.0 & 225.0 \\ 121.0 & 109.0 \\ 216.0 & 165.0 \\ 243.0 & 197.0 \\ 264.0 & 207.0 \\ 263.0 & 226.0 \\ 236.0 & 196.0 \\ 258.0 & 219.0 \\ 273.0 & 221.0 \\ 195.0 & 182.0 \\ 264.0 & 191.0 \\ 258.0 & 215.0 \\ 204.0 & 181.0 \\ 331.0 & 282.0 \\ 223.0 & 182.0 \\ 226.0 & 171.0 \\ 259.0 & 209.0 \\ 206.0 & 195.0 \\ 269.0 & 212.0 \\ 227.0 & 191.0 \\ 202.0 & 165.0 \\ & \end{array}$




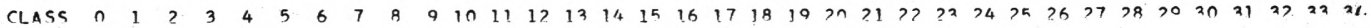

$\begin{array}{llllllllllllllll}26 & 23 & 8 & 19 & 18 & 62 & 83 & 44 & 32 & 21 & 8 & 6 & 9 & 3 & 3\end{array}$

$\begin{array}{lllllllllllllllllllll}1 & 2 & 1 & 2 & 18 & 28 & 24 & 23 & 13 & 24 & 27 & 36 & 26 & 48 & 28 & 16 & 17 & 12 & 11 & 7 & 1\end{array}$

$\begin{array}{rrrrrrrrrrrrrrrrrrr}5 & 5 & 2 & 21 & 28 & 19 & 36 & 32 & 70 & 52 & 37 & 26 & 22 & 6 & 1 & 3 & 7 & 1 \\ 2 & 4 & 1 & 1 & 19 & 38 & 28 & 42 & 19 & 61 & 25 & 17 & 24 & 31 & 18 & 9 & 16 & 4 & 5\end{array}$

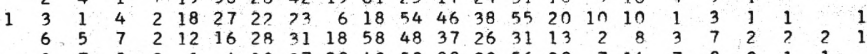

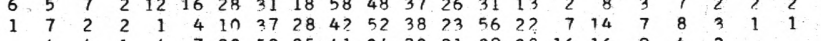

$\begin{array}{lllllllllllllllllllllll}1 & 4 & 4 & 1 & 4 & 7 & 20 & 52 & 35 & 41 & 34 & 23 & 21 & 39 & 33 & 16 & 16 & 9 & 4 & 2 & \\ 1 & & 2 & 4 & 3 & 8 & 16 & 41 & 23 & 35 & 34 & 26 & 34 & 44 & 41 & 2 ? & 18 & 5 & 6 & 1 & 1\end{array}$

$\begin{array}{rrrrrrrrrrrrrrrrrrrrrrr}3 & 7 & 2 & 6 & 1 & & 9 & 11 & 5 & 56 & 11 & 27 & 23 & 25 & 35 & 57 & 34 & 13 & 18 & 11 & 10 \\ 2 & 1 & 4 & 1 & 1 & 2 & 1 & 21 & 25 & 24 & 6 & 75 & 49 & 32 & 31 & 47 & 17 & 11 & 8 & 4 & 4\end{array}$

$\begin{array}{lllllllllllllllllllllllllll}12 & 2 & 5 & 4 & 7 & 26 & 39 & 29 & 30 & 12 & 24 & 36 & 35 & 20 & 3 & 15 & 7 & 10 & 8 & 8 & ? & 1 & 1\end{array}$

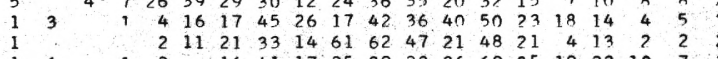

$\begin{array}{lllllllllllllllllll}1 & 6 & 1 & 2 & 16 & 41 & 17 & 25 & 29 & 23 & 36 & 69 & 35 & 19 & 23 & 10 & 7 & 2 & 4\end{array}$

$\begin{array}{rrrrrrrrrrrrrrrrrrrrrr}8 & 4 & 1 & 1 & 11 & -28 & 35 & 23 & 16 & 57 & 43 & 27 & 31 & 37 & 17 & 9 & 8 & 2 & 8 & ? & 1 & \\ 4 & 3 & 3 & 1 & 8 & 28 & 41 & 29 & 21 & 61 & 42 & 26 & 16 & 24 & 15 & 10 & 17 & 6 & 4 & 3 & & 1\end{array}$

$\begin{array}{llllllllllllllllll}1 & 1 & 2 & 23 & 24 & 37 & 17 & 20 & 31 & 30 & 40 & 60 & 33 & 17 & 18 & 6 & 5 \\ 1 & 1 & 13 & 22 & 79 & 26 & 49 & 34 & 20 & 25 & 58 & 20 & 16 & 17 & 6 & 3\end{array}$

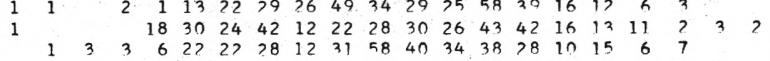

Sandy River below Bull Run River,

near Bull Run, Oreg.

$\begin{array}{rrrrrrrrrrrrrrrrr}6 & 22 & 27 & 78 & 12 & 31 & 58 & 40 & 34 & 38 & 78 & 10 & 15 & 6 & 7 \\ 1 & 20 & 47 & 76 & 43 & 52 & 45 & 32 & 79 & 37 & 14 & 4 & 11 & 5 & 3 & 1\end{array}$
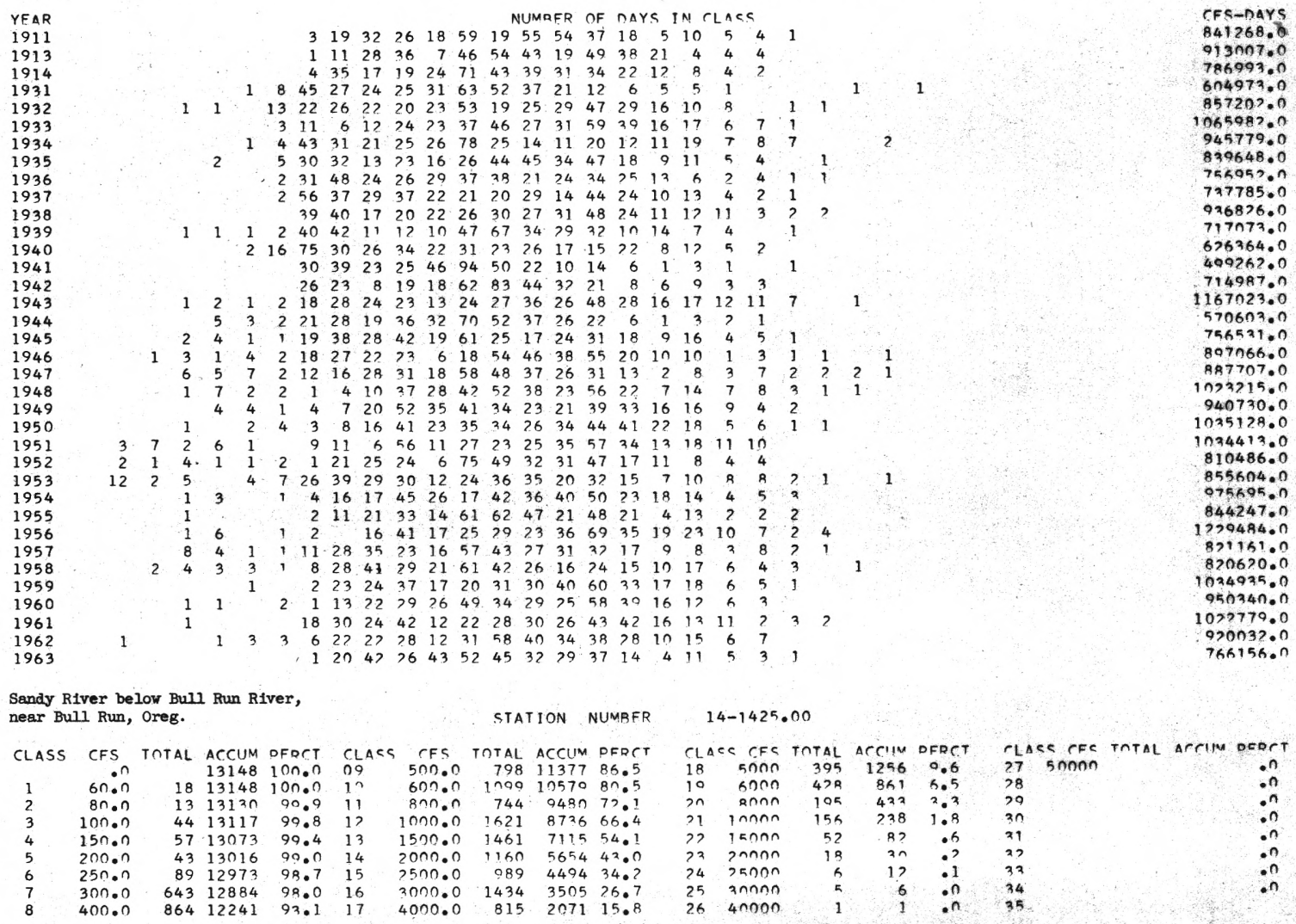

STATION NUMBFR $\quad 14-1425.00$

LOWEST MFAN DISCHARGF, IN CFS, FOR THF FOLLOWINT, NUMRFP OF RONCFTUTIVF NAYC IN YFAR AEGINNTNG APRIL,

\begin{tabular}{|c|c|c|c|c|c|}
\hline YEAR & 1 & 3 & 7 & 14 & 30 \\
\hline 1913 & 360.0 & 395.0 & $446 . n$ & 453.0 & 533.0 \\
\hline 1931 & 129.0 & 202.0 & 266.0 & 279.0 & $3 \cap 9.0$ \\
\hline 1932 & 262.0 & 287.0 & 308.0 & 324.0 & 363.0 \\
\hline 1933 & 318.0 & 431.0 & 519.0 & 533.0 & 557.0 \\
\hline 1934 & 242.0 & 291.0 & 318.0 & 325.0 & 351.0 \\
\hline 1935 & 170.0 & 317.0 & 348.0 & 358.0 & 389.0 \\
\hline 1936 & 291.0 & $3 \cap 2.0$ & $312 . n$ & 326.0 & 343.0 \\
\hline 1937 & 357.0 & $362 \cdot n$ & $387 . n$ & 419.0 & $431 . n$ \\
\hline 1938 & 128.0 & $2 n 2 \cdot n$ & $3 n 8 \cdot n$ & $336 . n$ & $364 \cdot ?$ \\
\hline 1939 & $280 . n$ & $305 . n$ & $316 \cdot 0$ & 373.0 & 350.0 \\
\hline 1940 & $208 . n$ & 282.0 & 305.0 & 314.0 & 316.0 \\
\hline 1941 & 320.0 & $331 . ?$ & 363.0 & 376.0 & $395 . ?$ \\
\hline 1942 & 141.0 & 205.0 & 228.0 & 328.0 & 345.0 \\
\hline 1943 & 322.0 & $401 . n$ & 408.0 & 421.0 & 426.0 \\
\hline 1944 & 152.0 & 260.0 & $328 . ?$ & $363 . n$ & 389.0 \\
\hline 1945 & 93.0 & $221 . n$ & 312.0 & 341.0 & 417.0 \\
\hline 1946 & 107.0 & 228.7 & 297.0 & 331.0 & 378.0 \\
\hline 1947 & 111.0 & 237.0 & 382.0 & $418 . ?$ & 440.0 \\
\hline 1948 & 118.0 & 242.0 & 411.7 & $443 . n$ & 497.0 \\
\hline 1949 & $135 . n$ & $28 n \cdot n$ & 425.0 & 472.0 & 490.0 \\
\hline 1950 & $205 . n$ & 383.0 & 431.0 & 481.0 & 533.0 \\
\hline 1951 & 75.0 & $108 . n$ & 352.0 & $355 . ?$ & 370.0 \\
\hline 1952 & 63.0 & 143.7 & $235 . ?$ & 255.0 & 294.0 \\
\hline 1953 & 87.0 & 330.0 & $380 . ?$ & $381 . ?$ & 416.0 \\
\hline 1954 & 181.0 & 441.0 & 469.0 & 522.0 & 688.0 \\
\hline 1955 & 119.0 & $373 . n$ & $503 . ?$ & 530.0 & 558.0 \\
\hline 1956 & 133.0 & $349 . ?$ & $464 . n$ & 501.0 & 509.0 \\
\hline 1957 & 99.0 & 242.0 & 353.0 & 364.0 & 378.0 \\
\hline 1958 & 140.0 & $315 . ?$ & 374.0 & 423.0 & 431.0 \\
\hline 1959 & 446.0 & $458 . ?$ & $466 . ?$ & $474 . ?$ & 486.0 \\
\hline 1960 & 135.0 & $318 . n$ & 384.0 & 448.0 & 517.0 \\
\hline 1961 & 222.0 & $335 . n$ & $376 \cdot n$ & $405 \cdot ?$ & $434 . ?$ \\
\hline 1962 & 79.0 & $274 . n$ & 337.0 & 396.0 & 455.0 \\
\hline
\end{tabular}

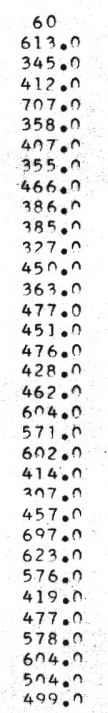

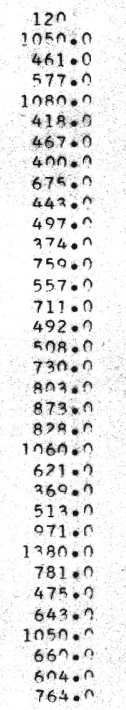

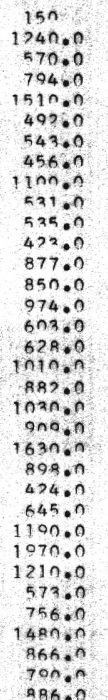

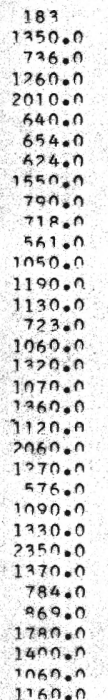

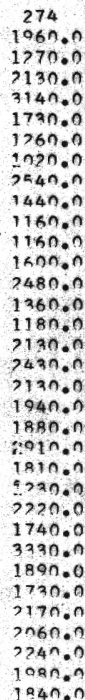


Sandy River below Bull Run River, near Bull Run, Oreg.

STATION NUMBER $14-1425.00$

HIGHEST MFAN DISTHARGF, IN CFC, FOR THF FOLLOWINF MUMAFP OF CONGFCIIIIVF OAY T TM YFAR FNOING SEPTFMRFR 30 $156 n n$. 0 $10900^{3} \cdot 0 \quad 8377^{7} \cdot n$ $34 n 0 . n$ 12700.0 45000.0 22400.0 $185 n \pi . n$ 33900.0 20700.0 22600.0 15800.0 21500.0 18100.0 1610000 1610n. 0 116nก. 104000 1600 16000 1600.0 34600.0 27100.0

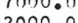
(a) (2) 269nn. $169 n 0 . n$ 13500.0 $24000 \cdot$ ? $15000 . n$ $16100 . n$ $14200 . n$ $174 n 0 . n$ $11600 . n$ $\ln 600 . n$ 9980.0 $8930 . n$ 71500 ? $7150 \cdot n$ 1 4 400 . 22900.ก 19600.ก 17400 ?

. ก 16500.? 12900.0 11100.?

$14400 . n$ litnn?

245nn.?

18100.0

19.

$153 n n \cdot n$

$188 \mathrm{n} \cdot \mathrm{n}$

17200.0

$17100.0 \quad 12600.0$

$13200.0 \quad 10500.0$

100.016200 .0

$14000.0 \quad 11800.0$ $8370 . n$
782.0 .0 $6890 . n$ linm? กดกก.? 968 กำ $18600 . n$ $11000 \cdot ?$ 11600.0
9580.0 12300.0 7450.0 89nก.? $6340 . n$ 7ต3ก. (4) (4) 8910.0 133nก.? I 1.  106 385 $947 n$. ingnn:? $914^{n}$. $12200 . n$ 12500.0 11200.0 8620.0 1160ก 11607.0 8220.0

\begin{tabular}{|c|c|}
\hline 15 & 30 \\
\hline & \\
\hline & $498 n \cdot n$ \\
\hline $588 \cap \cdot n$ & $4670 . n$ \\
\hline $93 n 0 . n$ & $66 n n \cdot n$ \\
\hline 8890.0 & 6570.0 \\
\hline 7570.0 & $5970 . ?$ \\
\hline $12800 \cdot 0$ & $1010 n \cdot 0$ \\
\hline 7140.0 & $5170 . n$ \\
\hline 9790.0 & 6780.0 \\
\hline $671 n \cdot 0$ & 5620.0 \\
\hline $7660 . n$ & $6300 . n$ \\
\hline 5010.0 & 4040.0 \\
\hline $657 n \cdot n$ & $503 n$. \\
\hline $445 n \cdot ?$ & $3 n 8 \cap . n$ \\
\hline $461 n \cdot n$ & $45 n n \cdot 0$ \\
\hline $1 n n n \cdot ?$ & $846 \cap .0$ \\
\hline 3350.0 & 3000.0 \\
\hline 6270.0 & 5700.0 \\
\hline 9170.0 & $642 \cap .0$ \\
\hline $2300 \cdot 0$ & $9260 \cdot 0$ \\
\hline $8470 . ?$ & 6390.0 \\
\hline $735 n \cdot n$ & $636 n .0$ \\
\hline $8 \cap 8 n \cdot ?$ & 6230.0 \\
\hline 7780.0 & $6810 . n$ \\
\hline $621 n^{\circ} n$ & $4040 \cdot 0$ \\
\hline $8903 n$.? & $\begin{array}{l}11 \mathrm{nnn} \cdot \mathrm{n} \\
796 n \cdot n\end{array}$ \\
\hline $615 n .0$ & 5360.0 \\
\hline 9120.0 & 8250.0 \\
\hline 8340.0 & 5640.0 \\
\hline 7590.0 & 5820.0 \\
\hline 7710.0 & 6230.0 \\
\hline $6 n \pi n \cdot n$ & $5160 \cdot ?$ \\
\hline 0100.0 & 7830.0 \\
\hline $6650 \cdot n$ & 6120.0 \\
\hline 780.0 & 5330.0 \\
\hline
\end{tabular}

60 4260.0 $4540 . n$ $4130 . n$ $43 n n$. 5460 .? $499 n$.? $8170 . ?$ $47 n \pi$. 4440 . 4820 . 5530.0 3670 . ? $472 n$. 2700. $344 n$. ? 7280 . ? 7750. 4530.0 516n. 6inc. 4850 . 4810 . ? $515 n$. ? $561 n$. ? उदnก: 7nsn. 568 . ? 4350 . ก 30 . 4850 . 5140 . ก 5370.0
$463 n .0$ 626n. $4700 . ?$
3630.0

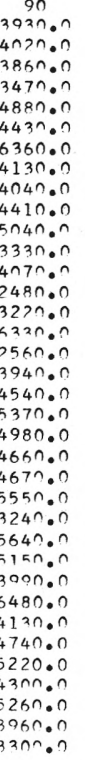

$24 \mathrm{nn} \cdot$ ?

27

4?

5460 .

392n.

41,

4

317n.

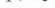

a

$43 n$.

391n.

$421 n$.

4720.0

4170. .

4220.0

$17 n$.

5) $17 n$.

$484 n$. ?

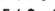

政

$83 n$.

$406 n \cdot 0$

$4640 . n$

$426 n$. s 277n.
150
2230

2680? s8n. asn.

(3)

38in.

$424 n$.

$4 n$.

$36 n$.

1940.0

7740.0

$2,4 n$. n

ann.?

$93 n$.

$4>3 n \cdot n$

$363 n$ ?

$44 n n$.n

$464 n \cdot n$

$54 n$. $4260 . n$ x>8n. 5nin.? 369n. 4120 . 4470 . $382 n \cdot ?$ 4860 . $288 \pi \cdot ?$ 3annon 284n.n

ann.n $2110 . n$

$214 n . n \quad 2 k 5 n . n$

Ran.n marn.

argnon posn.n

$26>0 . n$

$4480 . n \quad 321 n \cdot n$

44n.n papn.n

34kn.n $2 k 2 n .0$

3220.0 ग56n.ก

2>8n.?

295n.0 ग17n.n

$184 n . n \quad 162 n . n$

$266 n .0$ 241n.n

sinn.n

$341 n$. n sknn.n

3950.0 3n8n.? $385 n \cdot n \quad 35 n n$. 160 321n. 2an.n $3 k>n$. n 2000.0 275n.? $412 n \cdot n$ anin.n 209n.n 225n.n कर्थ 410.0 284n.0 3750.0 2830.n $4250.0 \quad 3440.0$ $348 n$.n $\quad 253 n$.n 4610.0 356n.n a $770 . n \quad a 15 n . n$ 


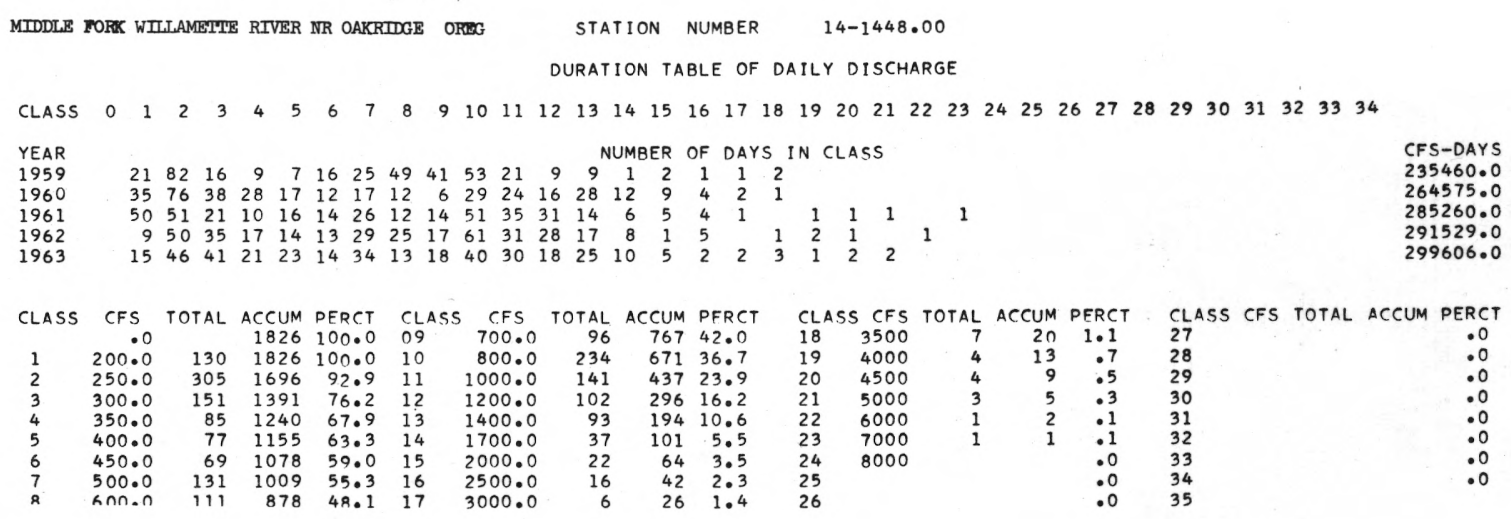

MIDDLE FORK WTLLAMETTTE RTVER NR OAKRTIGE ORBG

STATION NUMBER $14-1448.00$

LOWEST MEAN DISCHARGE, IN CFS, FOR THE FOLLOWING NUMBER OF CONSECUTIVE DAYS IN YEAR BEGINNING APRIL 1

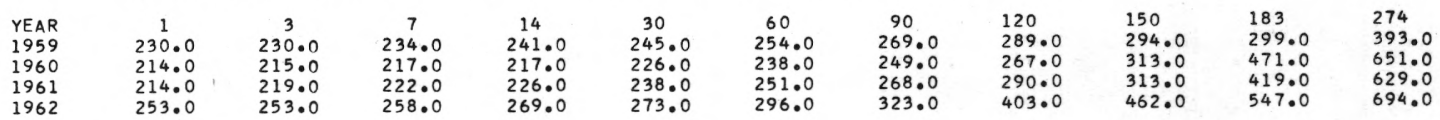

MIDDLE FOFK WIHLAMEIVE RTVER NR OAKRTDGE OREG STATION NUMBER $14-1448.00$

HIGHEST MEAN DISCHARGE, IN CFS, FOR THE FOLLOWING NUMBER OF CONSECUTIVE DAYS IN YEAR ENDING SEPTEMBER 30

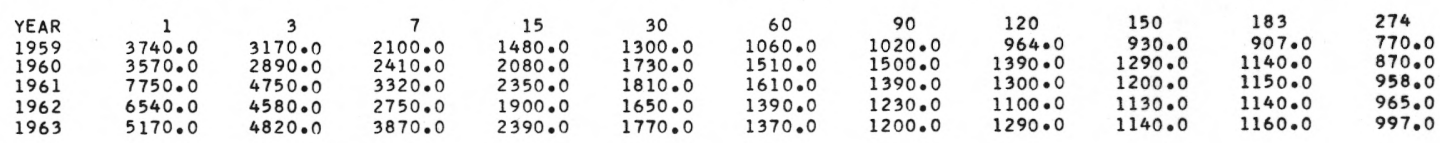

HIILS CRIEFK AB HILIS CREFEK RESERVOIR NR OAKRIDGE OREG STATION NUMBER 14-1449.00

DURATION TABLE OF DAILY DISCHARGE

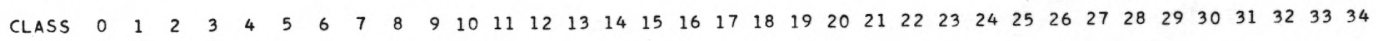

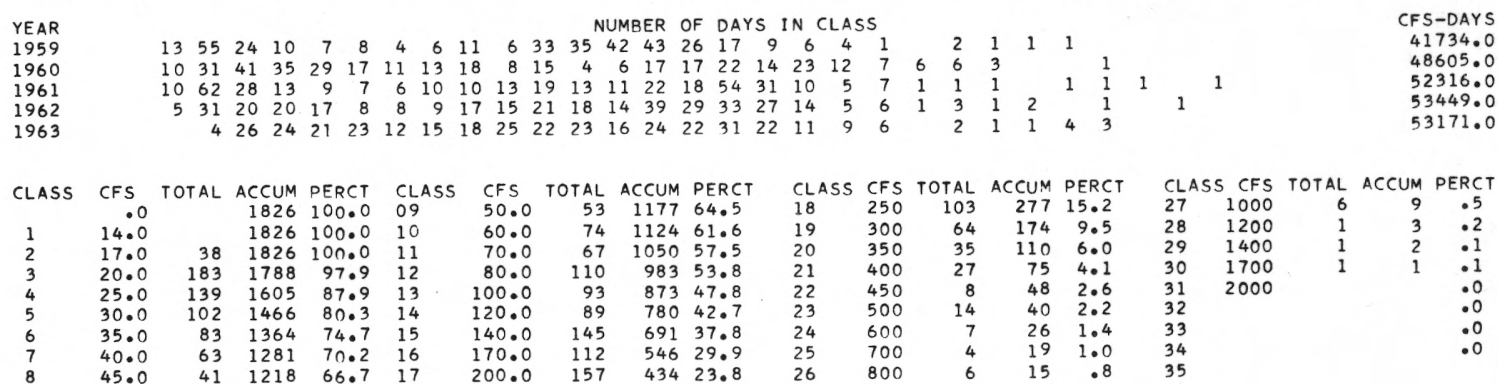

HIIIS CREETK AB HIIIS CRIERK RRSERVOIR NR OAKRIDGE OREG STATION NUMBER 14-1449.00

LOWEST MEAN DISCHARGE, IN CFS, FOR THE FOLLOWING NUMBER OF CONSECUTIVE DAYS IN YEAR REGINNING APRIL 1

\begin{tabular}{|c|c|c|c|c|c|c|c|c|c|c|c|}
\hline YEAR & 1 & 3 & 7 & 14 & 30 & 60 & 90 & 120 & 150 & 183 & 274 \\
\hline 1959 & 18.0 & 18.0 & 18.9 & 19.6 & 20.5 & 22.7 & 28.0 & 31.9 & 33.1 & 34.5 & 60.5 \\
\hline 1960 & 18.0 & 18.0 & 18.0 & 18.4 & 19.9 & 22.4 & 23.6 & $25 \cdot 4$ & 32.9 & 68.8 & 114.0 \\
\hline 1961 & 18.0 & 18.3 & 19.0 & 19.6 & 21.3 & 22.1 & 24.7 & $28 \cdot 9$ & 36.4 & 60.4 & 105.0 \\
\hline 1962 & 20.0 & 20.3 & 20.9 & 22.0 & 23.2 & 27.9 & 32.4 & $51 \cdot 1$ & 62.7 & 87.8 & 118.0 \\
\hline
\end{tabular}

HIIIS CREITK AB HIILS CRIATK RESERVOIR NR OAKRTDGE OREG STATION NUMBER 14-1449.00 HIGHEST MEAN DISCHARGF, IN CFS, FOR THE FOLLOWING NUMBER OF CONSECUTIVE DAYS IN YEAR ENDING SEPTEMBFR 30

\begin{tabular}{|c|c|c|c|c|c|c|c|c|c|c|c|}
\hline YEAR & 1 & 3 & 7 & 15 & 30 & 60 & 90 & 120 & 150 & 183 & 274 \\
\hline 1959 & 986.0 & 637.0 & 427.0 & 286.0 & 256.0 & 201.0 & 194.0 & 178.0 & 180.0 & 172.0 & 144.0 \\
\hline 1960 & 1100.0 & 757.0 & 493.0 & 422.0 & 375.0 & 313.0 & 322.0 & 296.0 & 269.0 & 232.0 & 168.0 \\
\hline 1961 & 1720.0 & 1170.0 & 770.0 & 526.0 & 391.0 & 328.0 & 279.0 & $266 \cdot 0$ & 241.0 & 230.0 & $\begin{array}{l}183.0 \\
185.0\end{array}$ \\
\hline 1962 & 1500.0 & 1030.0 & 603.0 & 414.0 & 333.0 & 283.0 & $\begin{array}{l}258.0 \\
226.0\end{array}$ & $\begin{array}{l}222.0 \\
243.0\end{array}$ & $\begin{array}{l}214.0 \\
216.0\end{array}$ & $\begin{array}{l}221.0 \\
213.0\end{array}$ & 182.0 \\
\hline 1963 & $1130 \cdot 0$ & 1010.0 & 730.0 & 438.0 & 340.0 & 282.0 & & & & & \\
\hline
\end{tabular}




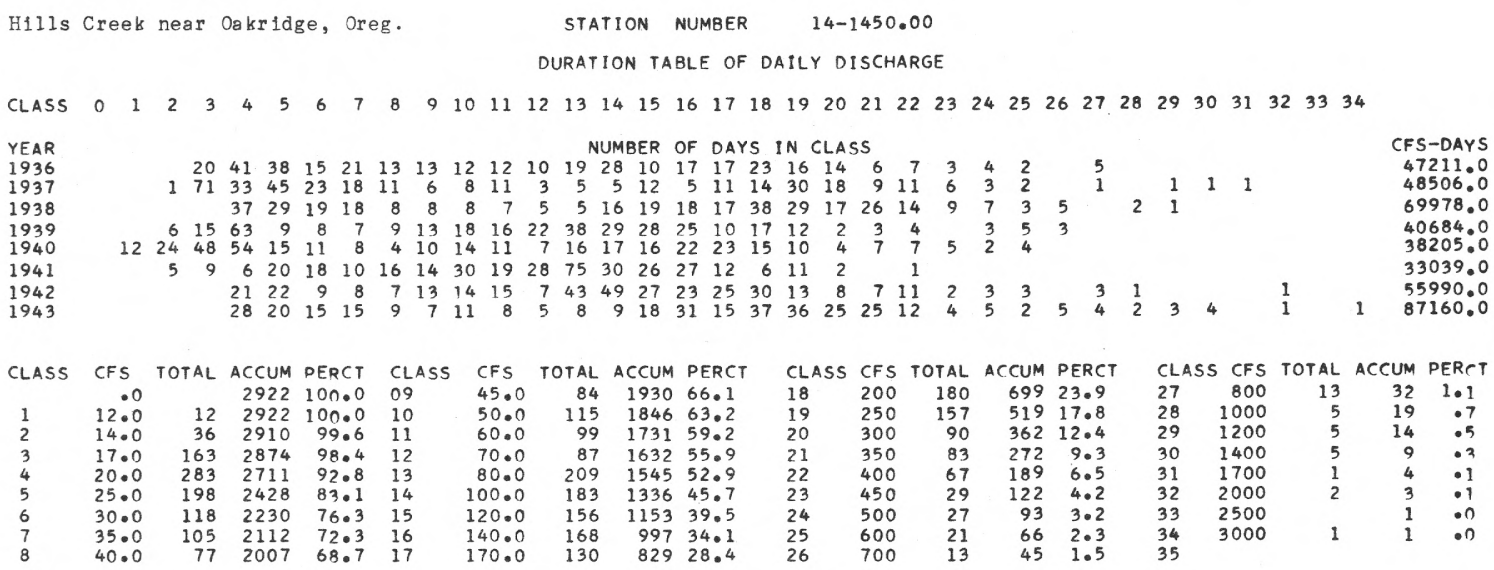

Hills Creek near Oakridge, Oreg. STATION NUMBER 14-1450.00

LOWEST MEAN DISCHARGE, IN CFS, FOR THE FOLLOWING NUMBER OF CONSECUTIVE DAYS IN YEAR BEGINNING APRIL 1

$\begin{array}{llllllllrrrr}\text { YEAR } & 1 & 3 & 7 & 14 & 30 & 60 & 90 & 120 & 150 & 183 & 274 \\ 1936 & 15.0 & 17.0 & 17.0 & 17.0 & 17.5 & 17.8 & 18.7 & 19.6 & 21.3 & 22.5 & 38.7 \\ 1937 & 22.0 & 22.0 & 23.0 & 25.0 & 26.0 & 28.1 & 30.2 & 33.2 & 59.6 & 103.0 & 166.0 \\ 1938 & 19.0 & 19.3 & 19.6 & 20.7 & 21.3 & 22.6 & 24.9 & 29.5 & 39.2 & 54.0 & 76.4 \\ 1939 & 16.0 & 16.0 & 16.1 & 16.8 & 18.0 & 20.5 & 22.0 & 22.3 & 23.7 & 33.3 & 63.6 \\ 1940 & 12.0 & 12.0 & 12.0 & 12.6 & 14.0 & 17.0 & 18.9 & 20.7 & 24.6 & 34.9 & 60.7 \\ 1941 & 26.0 & 26.3 & 28.0 & 28.5 & 29.9 & 39.0 & 41.3 & 46.3 & 61.5 & 74.0 & 131.0 \\ 1942 & 20.0 & 20.0 & 20.0 & 20.2 & 21.0 & 23.6 & 26.1 & 33.6 & 51.0 & 90.0 & 174.0\end{array}$

Hills Creek near Oakridge, Oreg. STATION NUMBER 14-1450.00

HIGHEST MEAN DISCHARGE, IN CFS, FOR THE FOLLOWING NUMBER OF CONSECUTIVE DAYS IN YEAR ENDING SEPTEMBER 30

\begin{tabular}{|c|c|c|c|c|c|c|c|c|c|c|c|}
\hline YEAR & $\stackrel{1}{960.0}$ & 3 & 7 & 15 & 30 & 60 & 90 & 120 & 150 & 183 & 274 \\
\hline 1936 & 960.0 & $827 \cdot 0$ & 691.0 & 600.0 & 405.0 & 288.0 & 241.0 & 250.0 & 246.0 & 223.0 & 164.0 \\
\hline 1937 & 1860.0 & 1620.0 & 1030.0 & 630.0 & 446.0 & 382.0 & 353.0 & $333 \cdot 0$ & 285.0 & 241.0 & 170.0 \\
\hline 1938 & 1210.0 & $808 \cdot 0$ & 577.0 & 440.0 & 366.0 & 347.0 & 321.0 & $313 \cdot 0$ & 314.0 & 309.0 & 246.0 \\
\hline 1939 & 755.0 & 748.0 & 693.0 & 550.0 & 395.0 & 283.0 & 245.0 & $213 \cdot 0$ & 190.0 & 178.0 & 142.0 \\
\hline 1940 & 691.0 & 632.0 & 527.0 & 413.0 & 346.0 & 317.0 & 265.0 & $227 \cdot 0$ & 210.0 & 186.0 & 133.0 \\
\hline 1941 & 418.0 & 323.0 & 264.0 & 215.0 & 167.0 & 152.0 & 131.0 & $117 \cdot 0$ & 120.0 & 121.0 & 109.0 \\
\hline 1942 & 2020.0 & 1260.0 & 750.0 & 421.0 & 358.0 & 335.0 & 294.0 & $251 \cdot 0$ & 225.0 & 210.0 & 193.0 \\
\hline 1943 & 3290.0 & 2310.0 & $1700 \cdot 0$ & 1030.0 & 684.0 & 605.0 & 521.0 & $444 \cdot 0$ & 409.0 & 379.0 & 308.0 \\
\hline
\end{tabular}


M1ddle Fork Willamette River above Salt

$14-1455.00$

DURATION TABLE OF DAILY DISCHARGE

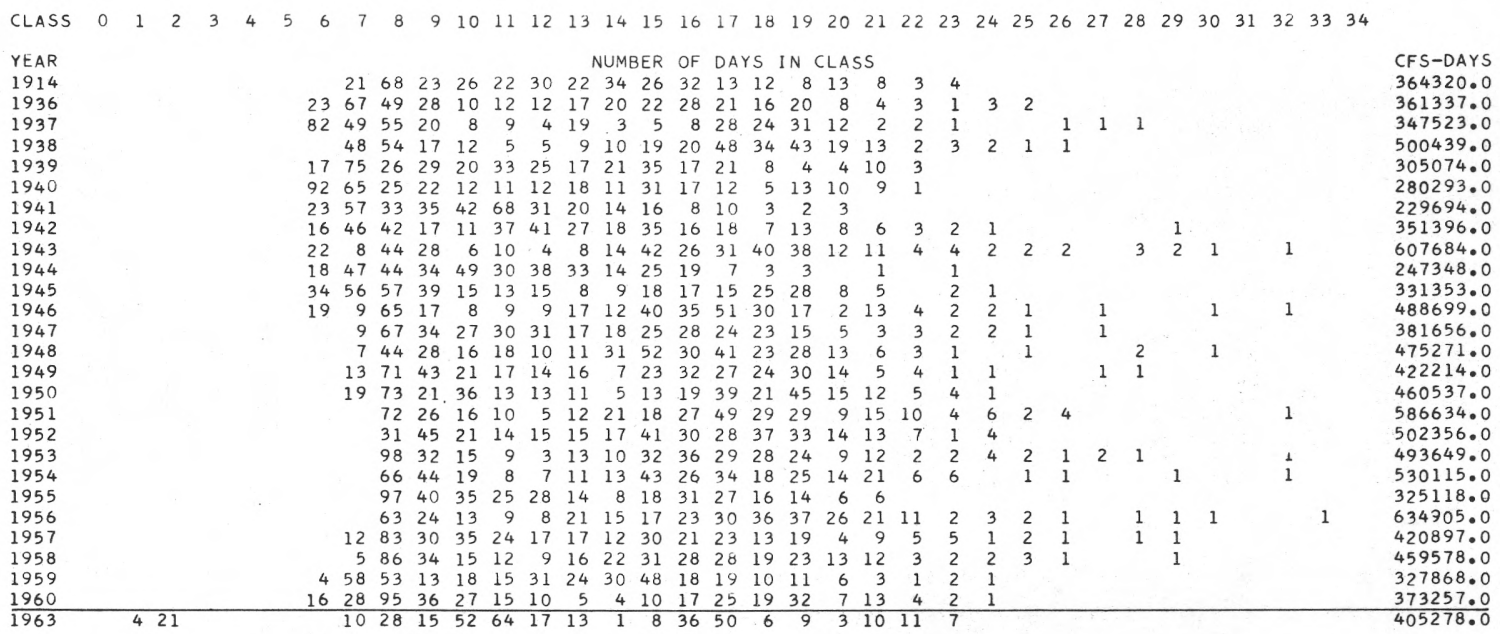

Middle Fork W1llamette R1ver above Salt

Middle Fork Willamette R1ver above Salt
Creek, near Oakridge, Oreg.

$\begin{array}{rrrrl}\text { CFS } & \text { TOTAL ACCUM } & \text { PERCT } & \text { CLASS } \\ .0 & & 9862 & 100.0 & 09 \\ 90.0 & 4 & 9862 & 100.0 & 10 \\ 100.0 & 21 & 9858 & 100.0 & 11 \\ 120.0 & & 9837 & 99.7 & 12 \\ 140.0 & & 9837 & 99.7 & 13 \\ 170.0 & & 9837 & 99.7 & 14 \\ 200.0 & 366 & 9837 & 99.7 & 15 \\ 250.0 & 709 & 9471 & 96.0 & 16 \\ 300.0 & 1589 & 8762 & 88.8 & 17\end{array}$

STATION NUMBEE CFS TOTAL ACCUM PERCT $\begin{array}{lllll}400.0 & 774 & 7173 & 72.7\end{array}$ $500.0 \quad 585 \quad 6399 \quad 64.9$ $600.0 \quad 542 \quad 581459.0$ $\begin{array}{lll}6000 & 444 & 5272 \quad 53.5\end{array}$ $000431 \quad 4828 \quad 49$. $900.0 \quad 394 \quad 4397 \quad 44.6$ $1000.0 \quad 723 \quad 4003 \quad 4 n$. $\begin{array}{llll}1200.0 & 645 & 3280 & 33.3 \\ 1400.0 & 748 & 2635 & 26.7\end{array}$
$14-1455.00$

$\begin{array}{lrrrr}\text { CLASS } & \text { CFS } & \text { TOTAL } & \text { ACCUM } & \text { PERCT } \\ 18 & 1700 & 529 & 1887 & 19.1 \\ 19 & 2000 & 594 & 1358 & 13.8 \\ 20 & 2500 & 257 & 764 & 7.7 \\ 21 & 3000 & 243 & 507 & 5.1 \\ 22 & 4000 & 100 & 264 & 2.7 \\ 23 & 5000 & 61 & 164 & 1.7 \\ 24 & 6000 & 37 & 103 & 1.0 \\ 25 & 7000 & 20 & 66 & .7 \\ 26 & 8000 & 13 & 46 & .5\end{array}$

$\begin{array}{lrrrr}\text { CLASS CFS } & \text { TOTAL } & \text { ACCUM } & \text { PERCT } \\ 27 & 9000 & 6 & 33 & \bullet 3 \\ 28 & 10000 & 10 & 27 & .3 \\ 29 & 12000 & 7 & 17 & .2 \\ 30 & 14000 & 4 & 10 & .1 \\ 31 & 17000 & & 6 & .1 \\ 32 & 20000 & 5 & 6 & .1 \\ 33 & 25000 & 1 & 1 & .0 \\ 34 & & & & .0\end{array}$

Middle Fork Willamette River above Salt Creek, near Oakridge, Oreg.

STATION NUMBER

$14-1455.00$

Summary for water years 1914, 1936-60,

\begin{tabular}{crrrrlr} 
CLASS & \multicolumn{1}{c}{ CFS } & TOTAL & ACCUM & PERCT & \multicolumn{2}{l}{ CLASS } \\
& .0 & & 9497 & 100.0 & 09 & 400.0 \\
1 & 90.0 & & 9497 & 100.0 & 10 & 500.0 \\
2 & 100.0 & & 9497 & 100.0 & 11 & 600.0 \\
3 & 120.0 & & 9497 & 100.0 & 12 & 700.0 \\
4 & 140.0 & & 9497 & 100.0 & 13 & 800.0 \\
5 & 170.0 & & 9497 & 100.0 & 14 & 900.0 \\
6 & 200.0 & 366 & 9497 & 100.0 & 15 & 1000.0 \\
7 & 250.0 & 699 & 9131 & 96.1 & 16 & 1200.0 \\
8 & 300.0 & 1561 & 8432 & 88.8 & 17 & 1400.0
\end{tabular}

Middle Fork Willamette River above Salt Creek, near Oakridge, Oreg.

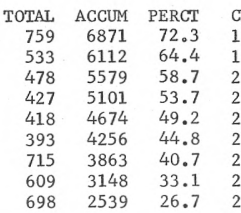

STATION NUMBER

$\begin{array}{lr}\text { CLASS } & \text { CFS } \\ 18 & 1700 \\ 19 & 2000 \\ 20 & 2500 \\ 21 & 3000 \\ 22 & 4000 \\ 23 & 5000 \\ 24 & 6000 \\ 25 & 7000 \\ 26 & 8000\end{array}$
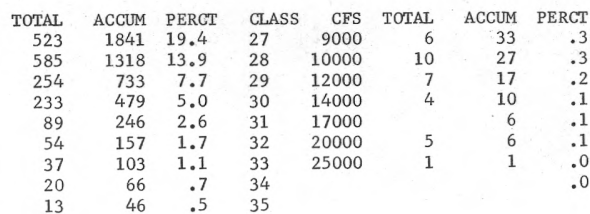

Summary for water year 1963

\begin{tabular}{lrrrrl} 
CLASS & \multicolumn{1}{c}{ CFS } & TOTAL & ACCUM & PERCT & CLASS \\
& .0 & & 365 & 100.0 & 09 \\
1 & 90.0 & 4 & 365 & 100.0 & 10 \\
2 & 100.0 & 21 & 361 & 98.9 & 11 \\
3 & 120.0 & & 340 & 93.2 & 12 \\
4 & 140.0 & & 340 & 93.2 & 13 \\
5 & 170.0 & & 340 & 93.2 & 14 \\
6 & 200.0 & & 340 & 93.2 & 15 \\
7 & 250.0 & 10 & 340 & 93.2 & 16 \\
8 & 300.0 & 28 & 330 & 90.4 & 17
\end{tabular}

CFS
400.0
500.0
600.0
700.0
800.0
900.0
1000.0
1200.0
1400.0

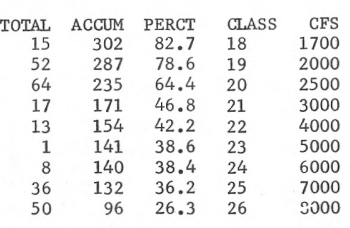

$\begin{array}{rrrlrlrr}\text { TOTAL } & \text { ACCUM } & \text { PERCT } & \text { CLASS } & \text { CFS } & \text { TOTAL } & \text { ACCUM } & \text { PERCT } \\ 6 & 46 & 12.6 & 27 & 9000 & & & .0 \\ 9 & 40 & 11.0 & 28 & 10000 & & .0 \\ 3 & 31 & 8.5 & 29 & 12000 & & .0 \\ 10 & 28 & 7.7 & 30 & 14000 & & .0 \\ 11 & 18 & 4.9 & 31 & 17000 & & .0 \\ 7 & 7 & 1.9 & 32 & 20000 & & .0 \\ & & .0 & 33 & 25000 & & .0 \\ & & .0 & 34 & & & & .0\end{array}$

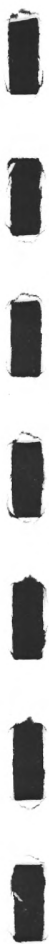

I
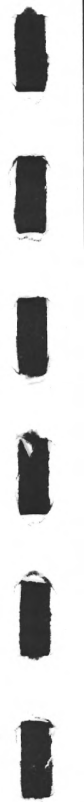

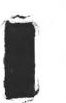


Middle Fork Willamette River above Salt

Creek, near Oakridge, Oreg.

STATION NUMBER $14-1455.00$

LOWEST MEAN DISCHARGE, IN CFS, FOR THF FOLLOWING NUMBER OF CONSECUTIVE DAYS IN YEAR BEGINNING APRIL 1

\begin{tabular}{|c|c|c|c|c|c|c|c|c|c|c|c|}
\hline YEAR & 1 & 3 & 7 & 14 & 30 & 60 & 90 & 120 & 150 & 183 & 274 \\
\hline 1936 & 201.0 & 201.0 & 201.0 & 204.0 & 210.0 & 215.0 & 227.0 & $239 \cdot 0$ & 253.0 & $259 \cdot 0$ & 390.0 \\
\hline 1937 & 260.0 & 262.0 & 267.0 & 281.0 & 288.0 & 304.0 & 327.0 & $356 \cdot 0$ & 546.0 & 810.0 & 1210.0 \\
\hline 1938 & 256.0 & 257.0 & 262.0 & 269.0 & 273.0 & 279.0 & 293.0 & $329 \cdot 0$ & 393.0 & 496.0 & 659.0 \\
\hline 1939 & 231.0 & 231.0 & 231.0 & 231.0 & 234.0 & 252.0 & 253.0 & $255 \cdot 0$ & 267.0 & 322.0 & 511.0 \\
\hline 1940 & 220.0 & 220.0 & 221.0 & 225.0 & 229.0 & 242.0 & 252.0 & $258 \cdot 0$ & 277.0 & 329.0 & 501.0 \\
\hline 1941 & 237.0 & 242.0 & 254.0 & 256.0 & 262.0 & 292.0 & 295.0 & $311 \cdot 0$ & 374.0 & 435.0 & 801.0 \\
\hline 1942 & 221.0 & 223.0 & $225 \cdot 0$ & 227.0 & 234.0 & 243.0 & 259.0 & $297 \cdot 0$ & $386 \cdot 0$ & $580 \cdot 0$ & 1240.0 \\
\hline 1943 & 326.0 & 329.0 & 330.0 & 333.0 & 339.0 & 362.0 & 396.0 & $512 \cdot 0$ & $570 \cdot 0$ & 586.0 & 690.0 \\
\hline $\begin{array}{l}1944 \\
1945\end{array}$ & 232.0 & 232.0 & 233.0 & 236.0 & 244.0 & 250.0 & 255.0 & $272 \cdot 0$ & $291 \cdot 0$ & 312.0 & 483.0 \\
\hline 1945 & 232.0 & 235.0 & 238.0 & 241.0 & 249.0 & 259.0 & 270.0 & 289.0 & 343.0 & 535.0 & 1150.0 \\
\hline $\begin{array}{l}1946 \\
1947\end{array}$ & 306.0 & 307.0 & 312.0 & 327.0 & 329.0 & 348.0 & 369.0 & $418 \cdot 0$ & 491.0 & 661.0 & $1000 \cdot 0$ \\
\hline 1947 & 283.0 & 283.0 & $284 . ?$ & 285.0 & 299.0 & 324.0 & 377.0 & 429.0 & 524.0 & 630.0 & 871.0 \\
\hline 1948 & 315.0 & 318.0 & 324.0 & 326.0 & 347.0 & 380.0 & 391.0 & $427 \cdot 0$ & 527.0 & 720.0 & 945.0 \\
\hline 1949 & 270.0 & 273.0 & $277 . n$ & $280 \cdot 0$ & 299.0 & 319.0 & 325.0 & $336 \cdot 0$ & $378 \cdot 0$ & 464.0 & 784.0 \\
\hline 1950 & 310.0 & 310.0 & 315.0 & 318.0 & 326.0 & 356.0 & $411 \cdot 0$ & $531 \cdot 0$ & 829.0 & 1020.0 & 1590.0 \\
\hline 1951 & $320 \cdot 0$ & 320.0 & 323.0 & 326.0 & 337.0 & $351 \cdot 0$ & $380 \cdot 0$ & $425 \cdot 0$ & 517.0 & $639 \cdot 0$ & 984.0 \\
\hline 1952 & 320.0 & 320.0 & 321.0 & 325.0 & 329.0 & 339.0 & 355.0 & 373.0 & 416.0 & $502 \cdot 0$ & 908.0 \\
\hline 1953 & 320.0 & 321.0 & 325.0 & 329.0 & 346.0 & 374.0 & $386 \cdot 0$ & $411 \cdot 0$ & 541.0 & $804 \cdot 0$ & 1380.0 \\
\hline 1954 & 325.0 & 325.0 & 326.0 & 329.0 & 353.0 & 365.0 & 375.0 & $390 \cdot 0$ & $404 \cdot 0$ & 436.0 & 595.0 \\
\hline 1955 & 311.0 & 314.0 & 318.0 & 322.0 & 337.0 & 355.0 & 397.0 & $458 \cdot 0$ & 554.0 & 802.0 & 1490.0 \\
\hline 1956 & 312.0 & 312.0 & 315.0 & 320.0 & 331.0 & 350.0 & 379.0 & $488 \cdot 0$ & 603.0 & 717.0 & 955.0 \\
\hline 1957 & 289.0 & 289.0 & 293.0 & 296.0 & 303.0 & 320.0 & 345.0 & $350 \cdot 0$ & 378.0 & 441.0 & 816.0 \\
\hline 1958 & 284.0 & 286.0 & 290.0 & 296.0 & 308.0 & $322 \cdot 0$ & 334.0 & 363.0 & 469.0 & 605.0 & 883.0 \\
\hline 1959 & 248.0 & 249.0 & 253.0 & 257.0 & 264.0 & 279.0 & 302.0 & $325 \cdot 0$ & 335.0 & 347.0 & 481.0 \\
\hline 1960 & 225.0 & 225.0 & 230.0 & 240.0 & 250.0 & 279.0 & 294.0 & $311 \cdot 0$ & 376.0 & 606.0 & 922.0 \\
\hline 1962 & & & 99.3 & 100.0 & 134.0 & 360.0 & 398.0 & $752 \cdot 0$ & 866.0 & 914.0 & 1070.0 \\
\hline
\end{tabular}

M1ddle Fork W1llamette R1ver above Salt

STATION NUMBER

$14-1455.00$

Crek, near Oakridge, Oreg.

\begin{tabular}{|c|c|c|c|c|c|c|c|c|c|c|c|}
\hline YEAR & 1 & 3 & 7 & 15 & 30 & 60 & 90 & 120 & 150 & 183 & 274 \\
\hline 1914 & 5170.0 & 5060.0 & 4650.0 & 3000.0 & 2520.0 & 2080.0 & 1910.0 & $1720 \cdot 0$ & 1610.0 & 1470.0 & $1210 \cdot 0$ \\
\hline 1936 & 7430.0 & 6190.0 & 5140.0 & 4440.0 & 2960.0 & 2150.0 & 1840.0 & 1810.0 & 1770.0 & 1620.0 & 1230.0 \\
\hline 1937 & $11900 \cdot 0$ & $10200 \cdot 0$ & 6350.0 & 3920.0 & 2950.0 & 2530.0 & $2340 \cdot 0$ & $2150 \cdot 0$ & $1890 \cdot 0$ & 1630.0 & 1190.0 \\
\hline 1938 & 8940.0 & 5700.0 & 3920.0 & 3030.0 & 2460.0 & $2310 \cdot 0$ & 2170.0 & $2180 \cdot 0$ & 2140.0 & 2100.0 & 1720.0 \\
\hline 1939 & 4300.0 & 4170.0 & 3850.0 & 3100.0 & 2390.0 & 1830.0 & 1630.0 & 1450.0 & 1330.0 & 1260.0 & 1030.0 \\
\hline 1940 & 4540.0 & 3630.0 & 3260.0 & 2600.0 & 2360.0 & 2100.0 & 1760.0 & 1530.0 & 1420.0 & 1270.0 & 936.0 \\
\hline 1941 & 2810.0 & 2290.0 & 1770.0 & 1640.0 & 1260.0 & 1120.0 & 981.0 & 891.0 & 867.0 & 842.0 & 746.0 \\
\hline 1942 & $12000 \cdot 0$ & 7130.0 & 4120.0 & 2550.0 & 2290.0 & 2080.0 & 1870.0 & $1610 \cdot 0$ & 1440.0 & 1340.0 & 1190.0 \\
\hline 1943 & 22500.0 & $17000 \cdot 0$ & 12400.0 & 7650.0 & 5100.0 & 4440.0 & 3690.0 & $3140 \cdot 0$ & $2830 \cdot 0$ & $2600 \cdot 0$ & 2110.0 \\
\hline 1944 & 5830.0 & 3870.0 & $2350 \cdot 0$ & 1620.0 & 1210.0 & 1090.0 & 998.0 & 973.0 & 926.0 & 871.0 & 808.0 \\
\hline 1945 & 6370.0 & 5020.0 & 4100.0 & 3060.0 & 2260.0 & 2000.0 & 1750.0 & $1780 \cdot 0$ & 1660.0 & 1490.0 & 1120.0 \\
\hline 1946 & 24600.0 & 16100.0 & 9010.0 & 6520.0 & $4180 \cdot 0$ & 3050.0 & 2520.0 & 2340.0 & 2120.0 & 2040.0 & 1680.0 \\
\hline 1947 & 9000.0 & 6600.0 & 4400.0 & 3390.0 & 2930.0 & 1910.0 & 1830.0 & $1670 \cdot 0$ & 1670.0 & 1570.0 & 1270.0 \\
\hline 1948 & 15900.0 & $11500 \cdot 0$ & 7070.0 & 4500.0 & 2800.0 & 2260.0 & 1900.0 & $1810 \cdot 0$ & 1810.0 & $1800 \cdot 0$ & 1600.0 \\
\hline 1949 & $10600 \cdot 0$ & 7300.0 & 4630.0 & 3260.0 & $2700 \cdot 0$ & 2210.0 & 2210.0 & $2010 \cdot 0$ & 1770.0 & 1730.0 & 1420.0 \\
\hline 1950 & 6620.0 & $5850 \cdot 0$ & 4580.0 & 3010.0 & $2780 \cdot 0$ & 2480.0 & 2330.0 & $2190 \cdot 0$ & $2190 \cdot 0$ & 2030.0 & 1570.0 \\
\hline 1951 & 20800.0 & 12700.0 & 7980.0 & 4750.0 & 3940.0 & 3280.0 & 3020.0 & 3040.0 & 2720.0 & 2530.0 & 2020.0 \\
\hline 1952 & 6470.0 & 5770.0 & 4620.0 & 3230.0 & 2580.0 & 2290.0 & 2060.0 & $2030 \cdot 0$ & 1920.0 & 1930.0 & 1680.0 \\
\hline 1953 & 20200.0 & 13400.0 & $8600 \cdot 0$ & 6140.0 & 5100.0 & 3440.0 & 2760.0 & $2420 \cdot 0$ & 2310.0 & $2200 \cdot 0$ & 1690.0 \\
\hline 1954 & 22100.0 & 14400.0 & 8190.0 & 5320.0 & 4540.0 & 3170.0 & 3120.0 & $2740 \cdot 0$ & 2520.0 & $2310 \cdot 0$ & $1800 \cdot 0$ \\
\hline 1955 & 3920.0 & 3460.0 & $2890 \cdot 0$ & $2220 \cdot 0$ & 2020.0 & 1840.0 & 1750.0 & $1550 \cdot 0$ & 1400.0 & 1340.0 & 1070.0 \\
\hline 1956 & 26100.0 & 17600.0 & 10400.0 & 6920.0 & 4970.0 & 4280.0 & 3430.0 & $2910 \cdot 0$ & 2740.0 & 2670.0 & 2180.0 \\
\hline 1957 & 12500.0 & 9410.0 & 6160.0 & 4890.0 & 3720.0 & 2750.0 & $2260 \cdot 0$ & $2040 \cdot 0$ & 1900.0 & $1760 \cdot 0$ & 1430.0 \\
\hline 1958 & 13100.0 & 9380.0 & 6290.0 & 4520.0 & 3570.0 & 2880.0 & 2580.0 & $2180 \cdot 0$ & 2090.0 & $2000 \cdot 0$ & 1560.0 \\
\hline 1959 & 6280.0 & 5300.0 & 3500.0 & 2350.0 & 2120.0 & 1700.0 & 1580.0 & 1460.0 & 1420.0 & 1340.0 & 1100.0 \\
\hline 1960 & 6070.0 & 5250.0 & 3710.0 & 3000.0 & 2720.0 & 2290.0 & 2260.0 & $2100 \cdot 0$ & 1920.0 & 1680.0 & 1250.0 \\
\hline 1963 & 5140.0 & $5010 . n$ & 5010.0 & 4480.0 & 3310.0 & 2600.0 & 12130.0 & $1710 \cdot 0$ & 1490.0 & 1250.0 & 1260.0 \\
\hline
\end{tabular}


LOWEST MEAN DISCHARGE, IN CFS, FOR THE FOLLOWING NUMBER, OF CONSECUTIVF DAYS IN YEAR REGINNING APRIL 1

\begin{tabular}{|c|c|c|c|c|c|c|c|c|c|c|c|}
\hline $\begin{array}{l}\text { YEAR } \\
1934 \\
1935\end{array}$ & $\begin{array}{c}1 \\
82.0 \\
88.0\end{array}$ & $\begin{array}{c}3 \\
82.3 \\
88.0\end{array}$ & $\begin{array}{c}7 \\
83.6 \\
89.4\end{array}$ & $\begin{array}{l}14 \\
84.7 \\
90.9\end{array}$ & $\begin{array}{l}30 \\
85.2 \\
92.1\end{array}$ & $\begin{array}{l}60 \\
86.2 \\
94.6\end{array}$ & $\begin{array}{l}90 \\
88.7 \\
99.4\end{array}$ & $\begin{array}{l}120 \\
94.6 \\
105.0\end{array}$ & $\begin{array}{l}150 \\
110.0 \\
109.0\end{array}$ & $\begin{array}{l}183 \\
122.0 \\
119.0\end{array}$ & $\begin{array}{l}274 \\
188.0 \\
195.0\end{array}$ \\
\hline $\begin{array}{l}1936 \\
1937\end{array}$ & $\begin{array}{r}66.0 \\
105.0\end{array}$ & $\begin{array}{r}76.7 \\
105.0\end{array}$ & $\begin{array}{r}89.0 \\
107.0\end{array}$ & $\begin{array}{r}90.2 \\
111.0\end{array}$ & $\begin{array}{r}92.6 \\
112.0\end{array}$ & $\begin{array}{r}94.2 \\
115.0\end{array}$ & $\begin{array}{r}97.4 \\
120.0\end{array}$ & $\begin{array}{r}99.6 \\
129.0\end{array}$ & $\begin{array}{l}104.0 \\
166.0\end{array}$ & $\begin{array}{l}105.0 \\
221.0\end{array}$ & $\begin{array}{l}143.0 \\
294.0\end{array}$ \\
\hline 1938 & 106.0 & 108.0 & 109.0 & 110.0 & 113.0 & 115.0 & 117.0 & 125.0 & 141.0 & 161.0 & 198.0 \\
\hline $\begin{array}{l}1939 \\
1940\end{array}$ & $\begin{array}{l}96.0 \\
78.0\end{array}$ & $\begin{array}{l}96.0 \\
78.0\end{array}$ & $\begin{array}{l}96.9 \\
78 \cdot 1\end{array}$ & $\begin{array}{l}98.1 \\
78.6\end{array}$ & $\begin{array}{r}101.0 \\
80.1\end{array}$ & $\begin{array}{r}105.0 \\
83.7\end{array}$ & $\begin{array}{r}107.0 \\
86.0\end{array}$ & $\begin{array}{r}108.0 \\
87.9\end{array}$ & $\begin{array}{r}112.0 \\
93.5\end{array}$ & $\begin{array}{l}132.0 \\
106.0\end{array}$ & $\begin{array}{l}179.0 \\
140.0\end{array}$ \\
\hline 1941 & 87.0 & 87.3 & 90.4 & 93.1 & 95.1 & 107.0 & 112.0 & 117.0 & 138.0 & 154.0 & 231.0 \\
\hline $\begin{array}{l}1942 \\
1943 \\
1944\end{array}$ & $\begin{array}{r}96.0 \\
129.0 \\
74.0\end{array}$ & $\begin{array}{r}96.3 \\
129.0 \\
74.0\end{array}$ & $\begin{array}{r}96.9 \\
130.0 \\
75.1\end{array}$ & $\begin{array}{r}97.3 \\
132.0 \\
76.7\end{array}$ & $\begin{array}{r}98.5 \\
135.0 \\
81.5\end{array}$ & $\begin{array}{r}100.0 \\
144.0 \\
86.1\end{array}$ & $\begin{array}{r}103.0 \\
153.0 \\
89.7\end{array}$ & $\begin{array}{r}115.0 \\
180.0 \\
95.2\end{array}$ & $\begin{array}{r}143.0 \\
187.0 \\
99.5\end{array}$ & $\begin{array}{l}201.0 \\
186.0 \\
104.0\end{array}$ & $\begin{array}{l}325.0 \\
217.0 \\
172.0\end{array}$ \\
\hline $\begin{array}{l}1945 \\
1946\end{array}$ & $\begin{array}{r}96.0 \\
130.0\end{array}$ & $\begin{array}{r}96.0 \\
131.0\end{array}$ & $\begin{array}{r}96.9 \\
133.0\end{array}$ & $\begin{array}{r}98.0 \\
135.0\end{array}$ & $\begin{array}{l}103.0 \\
139.0\end{array}$ & $\begin{array}{l}107.0 \\
141.0\end{array}$ & $\begin{array}{l}108.0 \\
145.0\end{array}$ & $\begin{array}{l}115 \cdot 0 \\
162.0\end{array}$ & $\begin{array}{l}135.0 \\
192.0\end{array}$ & $\begin{array}{l}183.0 \\
251.0\end{array}$ & $\begin{array}{l}283.0 \\
285.0\end{array}$ \\
\hline 1947 & 124.0 & 124.0 & 124.0 & 126.0 & 129.0 & 133.0 & 144.0 & 160.0 & 193.0 & 223.0 & 291.0 \\
\hline 1948 & 110.0 & 110.0 & 113.0 & 114.0 & 120.0 & 130.0 & 143.0 & 156.0 & 189.0 & 237.0 & 261.0 \\
\hline 1949 & 120.0 & 120.0 & 121.0 & 122.0 & 126.0 & 127.0 & 137.0 & 137.0 & 148.0 & 167.0 & 241.0 \\
\hline 1950 & 128.0 & 128.0 & $130 . \mathrm{C}$ & 130.0 & 132.0 & 139.0 & 155.0 & 201.0 & 308.0 & 352.0 & 424.0 \\
\hline
\end{tabular}

HIGHEST MEAN DISCHARGF, IN CFS, FOR THE FOLLOWING NUMBER OF CONSECUTIVE DAYS IN YEAR FNDING SEPTEMBER 30

\begin{tabular}{|c|c|c|c|c|c|c|c|c|c|c|c|}
\hline YEAR & 1 & 3 & 7 & 15 & 30 & 60 & 90 & 120 & 150 & 183 & 274 \\
\hline 1914 & 766.0 & $738 \cdot 0$ & 685.0 & 608.0 & 505.0 & 453.0 & 435.0 & $407 \cdot 0$ & 399.0 & 379.0 & 327.0 \\
\hline 1934 & 1100.0 & $898 \cdot 0$ & 678.0 & 501.0 & 411.0 & 350.0 & 322.0 & $321 \cdot 0$ & 298.0 & 275.0 & 230.0 \\
\hline 1935 & 1650.0 & 1320.0 & 964.0 & 661.0 & 517.0 & 491.0 & 443.0 & 393.0 & 378.0 & 389.0 & 339.0 \\
\hline 1936 & 970.0 & $865 . n$ & 746.0 & 672.0 & 638.0 & 578.0 & 491.0 & $454 \cdot 0$ & 431.0 & 415.0 & 322.0 \\
\hline 1937 & 1710.0 & 1490.0 & 1050.0 & 713.0 & 607.0 & 595.0 & 553.0 & 507.0 & 451.0 & 395.0 & 302.0 \\
\hline 1938 & 1240.0 & $99 n \cdot n$ & 804.0 & 646.0 & 592.0 & 566.0 & 541.0 & $496 \cdot 0$ & 494.0 & 493.0 & 418.0 \\
\hline 1939 & 864.0 & $851 \cdot 0$ & $800 \cdot 0$ & 665.0 & 568.0 & 524.0 & 485.0 & $443 \cdot 0$ & 406.0 & 373.0 & 317.0 \\
\hline 1940 & 670.0 & $613 . ?$ & 559.0 & 495.0 & 433.0 & 412.0 & 384.0 & 343.0 & 315.0 & 293.0 & 232.0 \\
\hline 1941 & 438.0 & $421 \cdot 0$ & 389.0 & 340.0 & 290.0 & 257.0 & 235.0 & $213 \cdot 0$ & 205.0 & 206.0 & 197.0 \\
\hline 1942 & 2210.0 & 1440.0 & 932.0 & 608.0 & 532.0 & 473.0 & 411.0 & $364 \cdot 0$ & 335.0 & 334.0 & 321.0 \\
\hline 1943 & 3500.0 & 2830.0 & 2130.0 & 1420.0 & 998.0 & 910.0 & 768.0 & $665 \cdot 0$ & 617.0 & 587.0 & 524.0 \\
\hline 1944 & 1100.0 & $843 . n$ & 556.0 & 480.0 & 435.0 & 398.0 & 376.0 & $346 \cdot 0$ & 325.0 & 298.0 & 270.0 \\
\hline 1945 & 1290.0 & 1130.0 & 915.0 & 859.0 & 772.0 & 642.0 & 537.0 & $514 \cdot 0$ & 479.0 & 443.0 & 335.0 \\
\hline 1946 & 2830.0 & 2240.0 & 1440.0 & 1080.0 & 754.0 & 564.0 & 504.0 & 476.0 & 480.0 & 484.0 & 422.0 \\
\hline 1947 & 1860.0 & 1470.0 & 1050.0 & 698.0 & 655.0 & 477.0 & 445.0 & $405 \cdot 0$ & 408.0 & 411.0 & 356.0 \\
\hline 1948 & 2560.0 & $1870 . n$ & 1250.0 & 871.0 & 710.0 & 597.0 & 542.0 & 519.0 & 495.0 & 496.0 & 456.0 \\
\hline 1949 & 1660.0 & $1290 . n$ & 918.0 & $862 \cdot 0$ & 736.0 & 634.0 & 546.0 & $520 \cdot 0$ & 467.0 & 437.0 & 386.0 \\
\hline 1950 & 1260.0 & $\ln 8 n .0$ & $93 n \cdot n$ & $8 \cap 2 \cdot n$ & 779.0 & 672.0 & 589.0 & 567.0 & 548.0 & 523.0 & 419.0 \\
\hline 1951 & 3220.0 & 2010.0 & 1490.0 & 1010.0 & 798.0 & 707.0 & 648.0 & 658.0 & 603.0 & $584 \cdot 0$ & 514.0 \\
\hline
\end{tabular}


$\begin{array}{lllllllllllllllllllllllllllllllllll}0 & 1 & 2 & 3 & 4 & 5 & 6 & 7 & 8 & 9 & 10 & 11 & 12 & 13 & 14 & 15 & 16 & 17 & 18 & 19 & 20 & 21 & 22 & 23 & 24 & 25 & 26 & 27 & 28 & 29 & 30 & 31 & 32 & 33 & 34\end{array}$

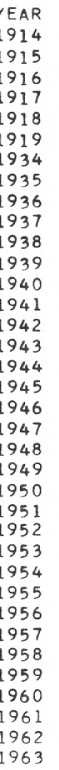

$\begin{array}{llllllllllllllll}13 & 25 & \text { NUMBER OF DAYS IN CLASS } & 25 & 19 & 45 & 26 & 22 & 49 & 28 & 34 & 11 & 14 & 9 & 10 & 1\end{array}$

$\begin{array}{lllllllllllllll}16 & 48 & 44 & 43 & 43 & 40 & 40 & 25 & 20 & 15 & 13 & 12 & 5 & 1\end{array}$

$\begin{array}{rrrrrrrrrrrrrrrrr}27 & 16 & 2 & 31 & 39 & 9 & 6 & 3 & 6 & 38107 & 17 & 12 & 22 & 18 & 8 & 4 \\ 1 & 27 & 43 & 36 & 25 & 27 & 21 & 21 & 14 & 23 & 19 & 15 & 45 & 25 & 8 & 15\end{array}$

$\begin{array}{lllllllllllllllllll}43 & 38 & 51 & 26 & 10 & 5 & 8 & 8 & 24 & 15 & 33 & 20 & 15 & 39 & 12 & 2 & 4 & 11\end{array}$

$\begin{array}{lllllllllllllllll}1 & 44 & 47 & 38 & 50 & 15 & 18 & 12 & 13 & 17 & 22 & 16 & 20 & 25 & 16\end{array}$

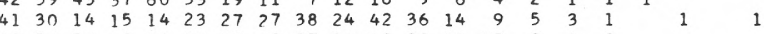

$\begin{array}{lllllllllllrlllllllllll}2 & 18 & 73 & 32 & 51 & 25 & 23 & 14 & 3 & 11 & 3 & 5 & 22 & 32 & 16 & 25 & 5 & 1 & 1 & 1 & 1 & 1 \\ & & 41 & 53 & 21 & 17 & 15 & 17 & 12 & 10 & 38 & 39 & 29 & 28 & 30 & 7 & 5 & 1 & 1 & & 1\end{array}$

$\begin{array}{rrrrrrrrrrrrrrrrr}33 & 51 & 21 & 20 & 31 & 44 & 31 & 27 & 21 & 17 & 23 & 11 & 16 & 8 & 3 & 3 & 5\end{array}$

$\begin{array}{llllllllllllllll}40100 & 26 & 20 & 29 & 23 & 25 & 20 & 17 & 19 & 8 & 14 & 13 & 7 & 4 & 1\end{array}$

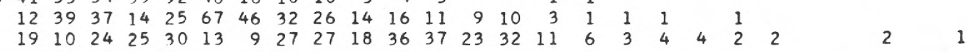

$\begin{array}{lllllllllllllllll}36 & 31 & 36 & 18103 & 30 & 34 & 23 & 11 & 9 & 22 & 8 & 3 & & 1\end{array}$

$\begin{array}{llllllllllllllllllllll}40 & 55 & 66 & 25 & 16 & 20 & 12 & 19 & 13 & 14 & 24 & 17 & 10 & 17 & 11 & 2 & 2 & 1 & 1 & 1 \\ 22 & 6 & 55 & 22 & 11 & 13 & 16 & 24 & 30 & 27 & 50 & 32 & 18 & 16 & 10 & 5 & 4 & 1 & 1 & & 1\end{array}$

$\begin{array}{rrrrrrrrrrrrrrrrrrrrrrr}55 & 22 & 11 & 13 & 16 & 24 & 30 & 27 & 50 & 32 & 18 & 16 & 10 & 5 & 4 & 1 & 1 & & & & 1 & & 1 \\ 57 & 31 & 35 & 43 & 40 & 19 & 8 & 24 & 31 & 22 & 29 & 13 & 3 & 1 & 3 & 2 & 1 & 1 & & 1 & & 1 & \\ 27 & 36 & 29 & 23 & 14 & 19 & 38 & 34 & 47 & 29 & 22 & 26 & 9 & 6 & 3 & & 1 & 1 & 1 & 1 & & & \\ 46 & 52 & 46 & 33 & 23 & 7 & 12 & 15 & 35 & 26 & 23 & 27 & 10 & 4 & 1 & 3 & & 1 & 1 & & & \\ 38 & 45 & 58 & 27 & 17 & 9 & 9 & 12 & 36 & 20 & 19 & 47 & 19 & 4 & 5 & & & & & & \end{array}$

$\begin{array}{rrrrrrrrrrrrrrrrrrrrr}38 & 45 & 58 & 27 & 17 & 9 & 9 & 12 & 36 & 20 & 19 & 47 & 19 & 4 & 5 & & & & \\ 56 & 38 & 31 & 15 & 10 & 14 & 20 & 19 & 38 & 33 & 32 & 23 & 7 & 8 & 8 & 4 & 7 & 1 & 1\end{array}$

$\begin{array}{lllllllllllllllll}18 & 42 & 42 & 20 & 22 & 20 & 39 & 21 & 31 & 22 & 34 & 30 & 13 & 5 & 7\end{array}$

$\begin{array}{lllllllllllllllllllll}60 & 52 & 42 & 10 & 10 & 28 & 33 & 16 & 26 & 25 & 19 & 16 & 9 & 3 & 4 & 3 & 3 & 1 & 1 & 1\end{array}$

$\begin{array}{rrrrrrrrrrrrrrrr}14 & 74 & 34 & 14 & 12 & 20 & 29 & 32 & 43 & 21 & 18 & 24 & 19 & 2 & 4 \\ 60 & 53 & 33 & 36 & 21 & 26 & 13 & 5 & 27 & 28 & 21 & 17 & 7 & 5 & 3\end{array}$

$\begin{array}{llllllllllllllllllll}3 & 45 & 41 & 13 & 11 & 23 & 21 & 17 & 40 & 35 & 27 & 32 & 26 & 10 & 11 & 1 & 5 & 1 & 3\end{array}$

$\begin{array}{rrrrrrrrrrrrrrrrrrrr}26 & 68 & 34 & 26 & 37 & 15 & 19 & 25 & 41 & 21 & 9 & 15 & 6 & 4 & 8 & 5 & 2 & 1 & 2 \\ 59 & 44 & 33 & 9 & 18 & 29 & 22 & 23 & 46 & 20 & 13 & 22 & 6 & 9 & 3 & 6 & 2 & & 1\end{array}$

$\begin{array}{rrrrrrrrrrrrrrrr}15 & 83 & 20 & 13 & 8 & 25 & 51 & 32 & 23 & 49 & 16 & 8 & 11 & 5 & 3 \\ 17 & 34 & 55 & 69 & 25 & 18 & 11 & 9 & 10 & 21 & 19 & 19 & 34 & 12 & 13\end{array}$

$\begin{array}{rrrrrrrrrrrrrrr}17 & 34 & 55 & 69 & 25 & 18 & 11 & 9 & 10 & 21 & 19 & 19 & 34 & 12 & 13 \\ 52 & 56 & 24 & 8 & 20 & 18 & 18 & 16 & 14 & 33 & 49 & 25 & 20 & 3 & 2\end{array}$

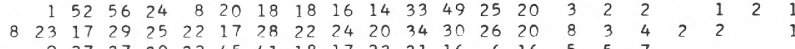

Salmon Creek near Oakridge, Oreg.

STATION NUMBER $\quad 14-1465.00$

Summary for water years 1914-19, 1934-63
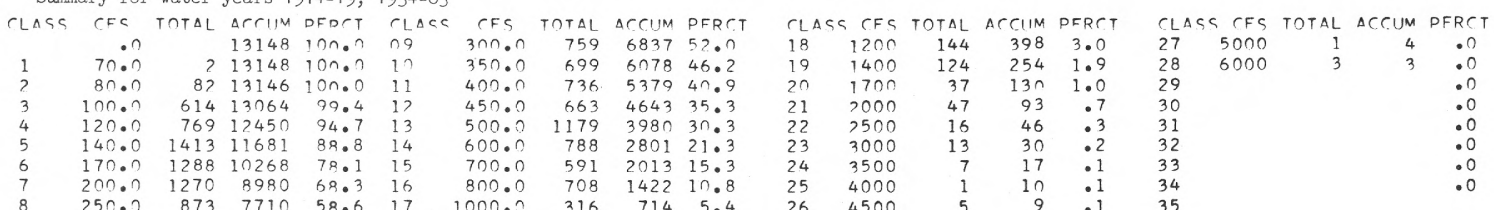

Salmon Creek near Oakridge, Oreg.

STATION NUMBER

$14-1465.00$

LOWEST MEAN DISCHARGF, IN CFS, FOR THF FOLLOWING NUMBER OF CONSFCUTIVF DAYS IN YEAR REGINNING APRIL I

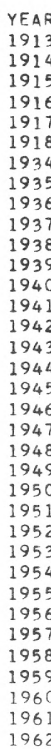

$\begin{array}{rrrrr}1 & 3 & 7 & 14 & 30 \\ 156.0 & 156.0 & 156.0 & 158.0 & 164.0 \\ 112.0 & 112.0 & 113.0 & 114.0 & 118.0 \\ 101.0 & 105.0 & 107.0 & 111.0 & 113.0 \\ 132.0 & 137.0 & 139.0 & 143.0 & 169.0 \\ 110.0 & 110.0 & 110.0 & 113.0 & 125.0 \\ 110.0 & 110.0 & 110.0 & 110.0 & 113.0 \\ 107.0 & 109.0 & 110.0 & 110.0 & 115.0 \\ 107.0 & 107.0 & 108.0 & 110.0 & 114.0 \\ 78.0 & 86.3 & 94.1 & 94.0 & 99.0 \\ 121.0 & 121.0 & 124.0 & 133.0 & 134.0 \\ 110.0 & 111.0 & 112.0 & 115.0 & 121.0 \\ 101.0 & 101.0 & 102.0 & 103.0 & 106.0 \\ 90.0 & 90.3 & 91.0 & 92.3 & 94.7 \\ 101.0 & 101.0 & 104.0 & 105.0 & 110.0 \\ 112.0 & 113.0 & 114.0 & 116.0 & 119.0 \\ 148.0 & 149.0 & 150.0 & 153.0 & 158.0 \\ 100.0 & 100.0 & 100.0 & 102.0 & 107.0 \\ 108.0 & 109.0 & 111.0 & 112.0 & 117.0 \\ 142.0 & 142.0 & 143.0 & 148.0 & 149.0 \\ 144.0 & 145.0 & 146.0 & 149.0 & 155.0 \\ 152.0 & 154.0 & 157.0 & 157.0 & 165.0 \\ 142.0 & 142.0 & 144.0 & 146.0 & 155.0 \\ 164.0 & 165.0 & 168.0 & 169.0 & 174.0 \\ 140.0 & 140.0 & 140.0 & 142.0 & 147.0 \\ 138.0 & 138.0 & 140.0 & 142.0 & 148.0 \\ 172.0 & 173.0 & 174.0 & 176.0 & 182.0 \\ 143.0 & 145.0 & 146.0 & 150.0 & 161.0 \\ 160.0 & 161.0 & 163.0 & 165.0 & 172.0 \\ 171.0 & 172.0 & 173.0 & 176.0 & 182.0 \\ 142.0 & 143.0 & 144.0 & 146.0 & 161.0 \\ 140.0 & 140.0 & 141.0 & 145.0 & 151.0 \\ 135.0 & 135.0 & 135.0 & 140.0 & 141.0 \\ 124.0 & 124.0 & 125.0 & 128.0 & 135.0 \\ 117.0 & 117.0 & 118.0 & 121.0 & 129.0 \\ 94.0 & 94.7 & 96.0 & 99.1 & 107.0\end{array}$

60
$179 \cdot 0$
$135 \bullet 0$
$117 \bullet 0$
$176 \bullet 0$
$134 \cdot 0$
$122 \bullet 0$
$115 \bullet 0$
$118 \cdot 0$
$104 \bullet 0$
$142 \bullet 0$
$127 \bullet 0$
$119 \bullet 0$
$99 \bullet 1$
$126 \bullet 0$
$122 \bullet 0$
$168 \bullet 0$
$112 \bullet 0$
$124 \bullet 0$
$155 \bullet 0$
$163 \bullet 0$
$178 \bullet 0$
$161 \bullet 0$
$186 \bullet 0$
$156 \bullet 0$
$151 \bullet 0$
$187 \bullet 0$
$167 \bullet 0$
$179 \bullet 0$
$190 \bullet 0$
$169 \bullet 0$
$159 \bullet 0$
$147 \bullet 0$
$144 \bullet 0$
$136 \bullet 0$
$130 \bullet 0$

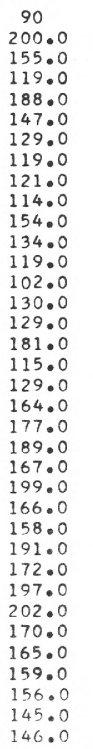

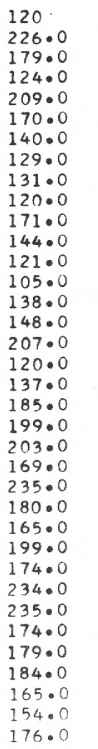

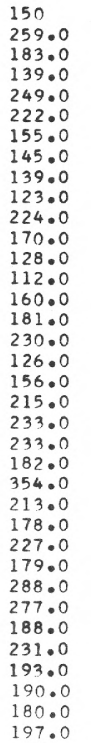

\begin{tabular}{|c|c|}
\hline 83 & 274 \\
\hline 82.0 & 391.0 \\
\hline 184.0 & 237.0 \\
\hline 195.0 & 317.0 \\
\hline 274.0 & 335.0 \\
\hline 343.0 & 540.0 \\
\hline 166.0 & 246.0 \\
\hline 159.0 & 290.0 \\
\hline 154.0 & 279.0 \\
\hline 127.0 & 172.0 \\
\hline 296.0 & 404.0 \\
\hline 197.0 & 256.0 \\
\hline 152.0 & 209.0 \\
\hline 127.0 & 190.0 \\
\hline 175.0 & 275.0 \\
\hline 240.0 & 431.0 \\
\hline 230.0 & 262.0 \\
\hline 132.0 & 208.0 \\
\hline 213.0 & 389.0 \\
\hline 275.0 & 414.0 \\
\hline 271.0 & 394.0 \\
\hline 303.0 & 361.0 \\
\hline 211.0 & 302.0 \\
\hline 427.0 & 565.0 \\
\hline $260 \cdot 0$ & 358.0 \\
\hline 195.0 & 346.0 \\
\hline 314.0 & 483.0 \\
\hline 195.0 & 259.0 \\
\hline 405.0 & 590.0 \\
\hline 317.0 & 439.0 \\
\hline 207.0 & 345.0 \\
\hline 297.0 & 382.0 \\
\hline 208.0 & 253.0 \\
\hline 283.0 & 388.0 \\
\hline 228.0 & $3<6$ \\
\hline 244.0 & 326. \\
\hline
\end{tabular}


Salmon Creek near Oakridge, Oreg.

STATION NUMBER $\quad 14-1465.00$

HIGHEST MEAN DISCHARGE, IN CFS, FOR THF FOLLOWING NUMBER OF CONSECUTIVE DAYS IN YEAR ENDING SEPTEMBER 30

\begin{tabular}{|c|c|c|c|c|c|c|c|c|c|c|c|}
\hline YEAR & 1 & 3 & 7 & 15 & 30 & 60 & 90 & 120 & 150 & 183 & 274 \\
\hline 1914 & 1430.0 & 1330.0 & 1140.0 & 1040.0 & 818.0 & 688.0 & 626.0 & $598 \cdot 0$ & $560 \cdot 0$ & 522.0 & 453.0 \\
\hline 1915 & 805.0 & 712.0 & 695.0 & 631.0 & 516.0 & 422.0 & 413.0 & $407 \cdot 0$ & 398.0 & 370.0 & 317.0 \\
\hline 1916 & 2210.0 & 1790.0 & 1420.0 & 1300.0 & 1010.0 & 883.0 & 774.0 & $723 \cdot 0$ & 714.0 & 687.0 & 611.0 \\
\hline 1917 & 1560.0 & 1480.0 & 1420.0 & 1250.0 & 1150.0 & 1110.0 & 1050.0 & $978 \cdot 0$ & 863.0 & 787.0 & 646.0 \\
\hline 1918 & 2800.0 & $2350 \cdot n$ & 2230.0 & 1690.0 & 1430.0 & 1190.0 & 976.0 & $915 \cdot 0$ & 858.0 & 780.0 & 591.0 \\
\hline 1919 & 1840.0 & 1750.0 & 1370.0 & 1190.0 & 1100.0 & 962.0 & 820.0 & $740 \cdot 0$ & 731.0 & 654.0 & 507.0 \\
\hline 1934 & 1720.0 & 1510.0 & 1160.0 & 844.0 & 649.0 & 535.0 & 483.0 & $467 \cdot 0$ & 425.0 & 387.0 & 314.0 \\
\hline 1935 & 3360.0 & 2380.0 & 1670.0 & 1120.0 & 835.0 & 670.0 & 634.0 & $578 \cdot 0$ & 576.0 & 579.0 & 506.0 \\
\hline 1936 & 1540.0 & 1340.0 & 1170.0 & 1060.0 & 826.0 & 700.0 & 638.0 & $614 \cdot 0$ & 624.0 & 578.0 & 448.0 \\
\hline 1937 & 2740.0 & 2310.0 & 1700.0 & 1160.0 & 947.0 & 859.0 & 784.0 & $731 \cdot 0$ & $640 \cdot 0$ & 559.0 & 420.0 \\
\hline 1938 & 2640.0 & 1890.0 & 1340.0 & 970.0 & 825.0 & 760.0 & 715.0 & $708 \cdot 0$ & 675.0 & 668.0 & 555.0 \\
\hline 1939 & $1600 \cdot 0$ & 1580.0 & 1470.0 & $1160 \cdot 0$ & 920.0 & 738.0 & 644.0 & $577 \cdot 0$ & $525 \cdot 0$ & $501 \cdot 0$ & 421.0 \\
\hline 1940 & 1040.0 & 862.0 & 797.0 & 702.0 & 638.0 & 568.0 & 499.0 & 433.0 & 407.0 & 369.0 & 288.0 \\
\hline 1941 & 1210.0 & 978.0 & 710.0 & $538 \cdot 0$ & 388.0 & 360.0 & 333.0 & $302 \cdot 0$ & 277.0 & 274.0 & 250.0 \\
\hline 1942 & 2930.0 & 2030.0 & 1270.0 & 808.0 & 697.0 & 647.0 & 575.0 & 512.0 & 470.0 & 444.0 & 410.0 \\
\hline 1943 & $6>90.0$ & 4640.0 & $3230 . n$ & 2130.0 & 1500.0 & $1380 . ?$ & 1180.0 & $1030 \cdot 0$ & 938.0 & 868.0 & 722.0 \\
\hline 1944 & 1520.0 & 1180.0 & 760.0 & 574.0 & 530.0 & 465.0 & 429.0 & 402.0 & 370.0 & 346.0 & 318.0 \\
\hline 1945 & 3260.0 & $2350 . n$ & $16 ? 0.0$ & 1160.0 & 906.0 & 737.0 & 647.0 & $656 \cdot 0$ & 626.0 & 563.0 & 427.0 \\
\hline 1946 & 5620.0 & 4100.0 & 2460.0 & $18+0.0$ & 1270.0 & 883.0 & 824.0 & $746 \cdot 0$ & 727.0 & 690.0 & 581.0 \\
\hline 1947 & 4700.0 & 3830.0 & 2470.0 & 1470.0 & 1170.0 & 788.0 & 744.0 & 669.0 & 675.0 & 640.0 & 524.0 \\
\hline 1948 & 3840.0 & $3050 \cdot 0$ & 1960.0 & $1340 \cdot 0$ & 912.0 & 771.0 & 714.0 & 693.0 & 654.0 & 650.0 & 613.0 \\
\hline 1949 & 3010.0 & 2250.0 & 1550.0 & 1250.0 & 1080.0 & 882.0 & 796.0 & $741 \cdot 0$ & 650.0 & 632.0 & 538.0 \\
\hline 1950 & 1560.0 & $1360 \cdot 0$ & 1150.0 & 1040.0 & 942.0 & 845.0 & 812.0 & $826 \cdot 0$ & 786.0 & 733.0 & 579.0 \\
\hline 1951 & 3990.0 & $2540 . ?$ & $2190 \cdot 0$ & 1390.0 & 1240.0 & 950.0 & 921.0 & 933.0 & 856.0 & 821.0 & 670.0 \\
\hline 1952 & 1570.0 & 1510.0 & 1270.0 & 1040.0 & 924.0 & 845.0 & 758.0 & 721.0 & 677.0 & 656.0 & 592.0 \\
\hline 1953 & 4680.0 & 3240.0 & 2550.0 & 1740.0 & 1600.0 & 1070.0 & 883.0 & 796.0 & 776.0 & 736.0 & 571.0 \\
\hline 1954 & 4500.0 & 2950.0 & 1890.0 & 1370.0 & 1310.0 & 939.0 & 899.0 & 809.0 & 764.0 & 719.0 & 594.0 \\
\hline 1955 & 1610.0 & 1540.0 & $1380 \cdot ?$ & 1080.0 & 984.0 & 835.0 & 772.0 & $691 \cdot 0$ & 625.0 & 585.0 & 472.0 \\
\hline 1956 & 6250.0 & 4260.0 & 2690.0 & 2040.0 & 1600.0 & 1360.0 & 1140.0 & $971 \cdot 0$ & 920.0 & 921.0 & 785.0 \\
\hline 1957 & 6920.0 & 4820.0 & 3160.7 & 1930.0 & 1200.0 & 965.0 & 823.0 & $825 \cdot 0$ & 773.0 & 723.0 & 595.0 \\
\hline 1958 & 3600.0 & 2660.0 & 1940.0 & 1400.0 & 1090.0 & 937.0 & 868.0 & $749 \cdot 0$ & $746 \cdot 0$ & 714.0 & 579.0 \\
\hline 1959 & 1710.0 & 1410.0 & 1030.0 & 834.0 & 703.0 & 621.0 & 631.0 & $584 \cdot 0$ & 571.0 & 552.0 & 469.0 \\
\hline 1960 & 1380.0 & 1330.0 & 1270.0 & 1110.0 & 973.0 & 851.0 & 855.0 & $787 \cdot 0$ & 721.0 & 637.0 & 510.0 \\
\hline 1961 & 3470.0 & 2400.0 & 1620.0 & 1230.0 & 966.0 & 841.0 & 733.0 & $700 \cdot 0$ & 664.0 & $642 \cdot 0$ & 533.0 \\
\hline 1962 & 3050.0 & 2120.0 & 1560.0 & 1130.0 & 922.0 & 815.0 & 700.0 & $648 \cdot 0$ & 670.0 & 672.0 & 555.0 \\
\hline 1963 & $161 \cap .0$ & 1440.0 & $1200 . n$ & $905 . ?$ & 784.0 & 648.0 & 582.0 & 604.0 & 542.0 & 542.0 & 464.0 \\
\hline
\end{tabular}

North Fork of Middle Fork

Willamette River near Oakridge, Oreg.

STATION NUMBER $14-1475 \cdot 00$

DURATION TABLF OF DAILY DISCHARGE

CLASS $0 \begin{array}{llllllllllllllllllllllllllllllllllll} & 1 & 2 & 3 & 4 & 5 & 6 & 7 & 8 & 9 & 10 & 11 & 12 & 13 & 14 & 15 & 16 & 17 & 18 & 19 & 20 & 21 & 22 & 23 & 24 & 25 & 26 & 27 & 28 & 29 & 30 & 31 & 32 & 33 & 34\end{array}$

\begin{tabular}{|c|c|c|c|c|c|c|c|c|c|c|c|c|c|c|c|c|c|c|c|c|c|c|c|c|c|c|c|c|c|c|c|c|}
\hline EAR & & & & & & & & & & & & & & NUMB & & of & DAY & YS 1 & $I N C$ & CLASS & & & & & & & & & & & & CFS-DAYS \\
\hline 10 & & & 64 & 34 & 18 & 75 & 8 & 9 & 16 & 14 & 10 & 13 & 23 & 23 & 29 & 16 & 9 & 20 & 9 & 6 & 4 & 5 & 3 & & 2 & 1 & & 2 & & & 2 & 293250.0 \\
\hline 911 & & & 22 & 52 & 27 & 17 & 13 & 5 & 13 & 17 & 13 & 46 & 45 & 24 & 31 & 16 & 7 & 4 & 2 & 3 & 3 & 2 & 1 & & 1 & 1 & & & & & & 212262.0 \\
\hline 912 & & & & 1 & & 49 & 35 & 8 & 4 & 1 & 32 & 8 & 11 & 26 & 48 & 40 & 14 & 52 & 9 & 8 & 4 & 4 & 3 & 2 & 2 & 2 & & 1 & 2 & & & 367967.0 \\
\hline 913 & & & & & & 30 & 31 & 31 & & & & & 31 & & 89 & & 61 & 62 & 30 & & & & & & & & & & & & & 343220.0 \\
\hline 914 & & & 7 & 20 & 23 & 30 & 16 & 17 & 10 & 18 & 25 & 27 & 47 & 21 & 38 & 25 & 10 & 13 & 11 & 6 & 1 & & & & & & & & & & & 236995.0 \\
\hline 1915 & & & 5 & 45 & 16 & 17 & 43 & 38 & 23 & 17 & 11 & 19 & 20 & 27 & 55 & 29 & & & & & & & & & & & & & & & & 179667.0 \\
\hline 1936 & & & 17 & 52 & 29 & 31 & 23 & 19 & 5 & 9 & 3 & 9 & 23 & 23 & 22 & 33 & 20 & 23 & 8 & 8 & 1 & 3 & 2 & 1 & 2 & & & & & & & 260790.0 \\
\hline 937 & & 29 & 43 & 36 & 31 & 45 & 15 & 7 & 4 & 8 & 3 & 5 & 16 & 6 & 12 & 25 & 21 & 25 & 16 & 12 & 1 & 1 & & & 1 & 2 & 1 & & & & & 246721.0 \\
\hline 1938 & 1 & 1 & 39 & 28 & 23 & 2.5 & 10 & 8 & 2 & 9 & 6 & 5 & 8 & 14 & 19 & 64 & 39 & 28 & 13 & 15 & 6 & & 1 & & & & 1 & & & & & 312777.0 \\
\hline 1939 & & 28 & 38 & 22 & 14 & 12 & 10 & 15 & 19 & 15 & 9 & 34 & 30 & 19 & 36 & 17 & 16 & 14 & 5 & 4 & 5 & 3 & & & & & & & & & & 227084.0 \\
\hline 1940 & 25 & 59 & 48 & 23 & 13 & 15 & 12 & 16 & 13 & 9 & 10 & 21 & 13 & 17 & 28 & 9 & 9 & 11 & 7 & 7 & 1 & & & & & & & & & & & 172725.0 \\
\hline 1941 & & 16 & 24 & 25 & 23 & 26 & 18 & 35 & 51 & 35 & 19 & 36 & 11 & 11 & 19 & 6 & 4 & 2 & 2 & 1 & 1 & & & & & & & & & & & 153116.0 \\
\hline 1942 & & 6 & 27 & 18 & 14 & 30 & 14 & 12 & 6 & 6 & 28 & 55 & 39 & 25 & 26 & 14 & 15 & 12 & 7 & 5 & 2 & 1 & & 2 & & & 1 & & & & & 239259.0 \\
\hline 1943 & & 16 & 7 & 21 & 16 & 23 & 16 & 10 & 6 & 6 & 3 & 7 & 26 & 20 & 30 & 27 & 25 & 42 & 21 & 17 & 8 & 2 & 3 & 1 & 4 & 2 & 2 & 1 & 1 & 1 & 1 & 417394.0 \\
\hline 1944 & & 17 & 36 & 28 & 12 & 17 & 18 & 21 & 27 & 35 & 22 & 32 & 31 & 16 & 26 & 17 & 5 & 2 & 2 & & & 1 & 1 & & & & & & & & & 174406.0 \\
\hline 1945 & & 30 & 35 & 28 & 21 & 41 & 20 & 16 & 9 & 4 & 4 & 13 & 11 & 9 & $3 ?$ & 22 & 21 & 18 & 13 & 10 & 3 & 3 & & & 1 & & 1 & & & & & 246294.0 \\
\hline 1946 & & 19 & 10 & 46 & 20 & 17 & 6 & 8 & 4 & 3 & 4 & 5 & 25 & 30 & 39 & 44 & 30 & 18 & 9 & 9 & 8 & 5 & 2 & 1 & & & 1 & & & 1 & 1 & 333422.0 \\
\hline 1947 & & & 4 & 40 & 18 & 17 & 19 & 15 & 23 & 15 & 26 & 21 & 19 & 22 & 28 & 20 & 16 & 36 & 10 & 4 & 1 & 3 & 1 & $i$ & 1 & 1 & 1 & 2 & & 1 & & 302620.0 \\
\hline 1948 & & & & 25 & 16 & 21 & 18 & 9 & 13 & 9 & 7 & 11 & 13 & 20 & 69 & 27 & 37 & 31 & 15 & 9 & 6 & 5 & & I & & $i$ & 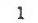 & 1 & 1 & & & 1.0 \\
\hline 1949 & & 1 & 21 & 25 & 19 & 31 & 11 & 28 & 16 & 15 & 9 & 16 & 15 & 6 & 20 & 25 & 27 & $\begin{array}{l}31 \\
19\end{array}$ & 27 & 22 & 6 & & 1 & 2 & & 1 & 1 & & 1 & & & 322132.0 \\
\hline 1950 & & 3 & 5 & 36 & 29 & 24 & 14 & 11 & 14 & 20 & 14 & 11 & 11 & 8 & 24 & 22 & 28 & 38 & 23 & 15 & 6 & 7 & 1 & 1 & & & & & & & & 326187.0 \\
\hline 1951 & & & 19 & 35 & 19 & 23 & 18 & 9 & 7 & 6 & 6 & 8 & 13 & 17 & 36 & 37 & $3 n$ & 28 & 15 & 9 & 8 & 6 & 4 & 2 & 5 & 3 & & 1 & 1 & & & 379391.0 \\
\hline 1952 & & & & 15 & 30 & 24 & 11 & 11 & 7 & 10 & 10 & 29 & 34 & 17 & 47 & 17 & 30 & 32 & 14 & 15 & 6 & 5 & 2 & & & & & & & & & 328281.0 \\
\hline 1953 & & & 6 & 67 & 19 & 29 & 22 & 14 & 7 & 4 & 6 & 11 & 18 & 21 & 37 & 22 & 26 & 20 & 8 & 7 & 5 & 5 & & 2 & & 5 & 1 & 2 & & & 1 & 320755.0 \\
\hline 1954 & & & & 18 & 43 & 48 & 18 & 7 & 6 & 4 & 9 & 21 & 28 & 23 & 34 & 22 & 19 & 17 & 16 & 19 & 4 & 2 & 2 & 1 & & 3 & & & & 1 & & 315521.0 \\
\hline 1955 & & & & 28 & 44 & 46 & 24 & 17 & 8 & 6 & 10 & 39 & 15 & 18 & 16 & 24 & 22 & 22 & 15 & 6 & 4 & 1 & & & & & & & & & & 247453.0 \\
\hline 1956 & & & & 18 & 18 & 32 & 18 & 9 & 7 & 7 & 4 & 9 & 15 & 25 & 34 & 47 & 17 & 30 & 27 & 18 & 12 & 7 & 1 & 2 & 1 & 4 & 1 & 2 & & 1 & & 429594.0 \\
\hline 1957 & & & 6 & 50 & 23 & 16 & 19 & 20 & 13 & 23 & 11 & 33 & 21 & 17 & 37 & 10 & 10 & 13 & 10 & 12 & 6 & 4 & 1 & 3 & 3 & 1 & 1 & 1 & & 1 & & 301573.0 \\
\hline 1958 & & & & 47 & 34 & 20 & 22 & 14 & 4 & 9 & 3 & 28 & 28 & 24 & 36 & 22 & 12 & 12 & 17 & 14 & 7 & 5 & 2 & 1 & 2 & 1 & & 1 & & & & 53.0 \\
\hline 1959 & & & 30 & 53 & 17 & 13 & 14 & 6 & 7 & 10 & 7 & 27 & 33 & 40 & 42 & 23 & 15 & 6 & 9 & 7 & 1 & 3 & 2 & & & & & & & & & 241856.0 \\
\hline 1960 & & & 21 & 28 & 22 & 26 & 31 & 27 & 30 & 11 & 11 & 12 & 12 & 9 & 21 & 16 & 24 & 28 & 15 & 12 & 8 & 2 & & & & & & & & & & 271058.0 \\
\hline 1961 & & 3 & 36 & 39 & 24 & 21 & 14 & 6 & 5 & 4 & 8 & 17 & 18 & 23 & 35 & 27 & 24 & 30 & 10 & 10 & 5 & 1 & 1 & & 1 & & 1 & 2 & & & & 295503.0 \\
\hline 1962 & & 2 & 9 & 34 & 18 & 29 & 15 & 16 & 5 & 8 & 11 & 21 & 23 & 27 & 44 & 15 & 22 & 30 & 14 & 8 & 6 & 3 & 1 & 2 & 1 & 1 & & & & & & 303496.0 \\
\hline 1963 & & 1 & 13 & 33 & 15 & 23 & 23 & 20 & 19 & 19 & 11 & 26 & 26 & 12 & 30 & 30 & 20 & 13 & 13 & 5 & 6 & 5 & 2 & & & & & & & & & 267417.0 \\
\hline
\end{tabular}

NORTH FORK OF MIDDLE FORK WTLAMETTE RIVER

NEAR OAKRIDGE OREG
SUIMMARY FOR WATER YEARS 1910-15, 1936-63

STATION NUMBER $\quad 14-1475.00$
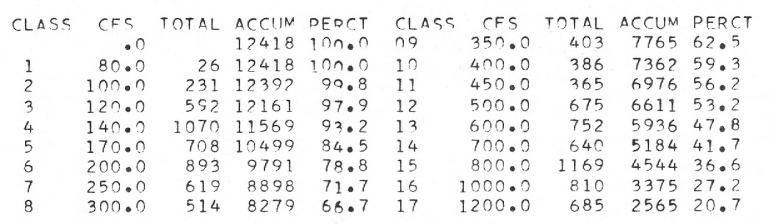

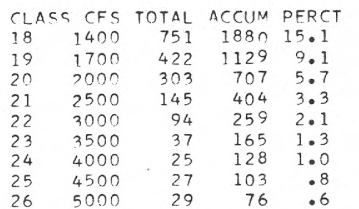

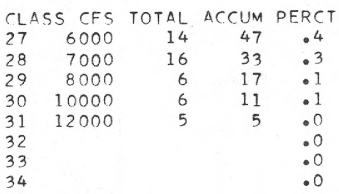


North Fork of Middle Fork

Willamette River near Oakridge, Oreg. STATION NUMBER 14-1475.00

LOWEST MEAN DISCHARGF, IN CFS, FOR THE FOLLOWING NUMBER OF CONSECUTIVE DAYS IN YEAR BEGINNING APRIL 1

\begin{tabular}{|c|c|c|c|c|c|c|c|c|c|c|c|}
\hline YEAR & 1 & 3 & 7 & 14 & 30 & 60 & 90 & 120 & 150 & 183 & 274 \\
\hline $\begin{array}{l}1910 \\
1911\end{array}$ & $\begin{array}{l}122.0 \\
120.0\end{array}$ & $\begin{array}{l}122 \cdot 0 \\
120 \cdot 0\end{array}$ & $\begin{array}{l}122.0 \\
121.0\end{array}$ & $\begin{array}{l}122.0 \\
127.0\end{array}$ & $\begin{array}{l}122.0 \\
139.0\end{array}$ & $\begin{array}{l}124.0 \\
161.0\end{array}$ & $\begin{array}{l}134.0 \\
179.0\end{array}$ & $\begin{array}{l}139.0 \\
203.0\end{array}$ & $\begin{array}{l}156.0 \\
244.0\end{array}$ & $\begin{array}{l}190.0 \\
359.0\end{array}$ & $\begin{array}{l}449.0 \\
568.0\end{array}$ \\
\hline 1912 & 220.0 & $220 . n$ & 220.0 & 220.0 & 220.0 & 250.0 & 266.0 & 310.0 & 419.0 & 506.0 & 704.0 \\
\hline 1913 & 180.0 & $181 . n$ & 197.0 & 204.0 & 207.0 & 233.0 & 284.0 & $360 \cdot 0$ & 427.0 & 465.0 & 697.0 \\
\hline 1914 & 130.0 & 133.0 & 136.0 & 140.0 & 151.0 & 178.0 & 207.0 & $238 \cdot 0$ & 264.0 & 279.0 & 396.0 \\
\hline 1915 & 133.0 & 135.0 & 137.0 & 140.0 & 142.0 & 144.0 & 149.0 & $162 \cdot 0$ & 185.0 & 288.0 & 558.0 \\
\hline 1936 & 114.0 & 115.0 & 116.0 & 116.0 & 118.0 & 122.0 & 133.0 & $142 \cdot 0$ & 157.0 & 171.0 & 263.0 \\
\hline 1937 & 84.0 & $108 . n$ & $129 . n$ & 143.0 & 147.0 & 157.0 & 183.0 & $214 \cdot 0$ & 332.0 & 510.0 & 768.0 \\
\hline 1938 & 120.0 & $122 \cdot 0$ & $123 . ?$ & 128.0 & 132.0 & 134.0 & 139.0 & 156.0 & 206.0 & 265.0 & 438.0 \\
\hline 1939 & 100.0 & $100 \cdot 0$ & 101.0 & 105.0 & 111.0 & 122.0 & 131.0 & $135 \cdot 0$ & 145.0 & 190.0 & 322.0 \\
\hline 1940 & 93.0 & 93.0 & 93.3 & 93.4 & 96.2 & 106.0 & 113.0 & $118 \cdot 0$ & 135.0 & 173.0 & 330.0 \\
\hline 1941 & 125.0 & $126 \cdot 0$ & 130.0 & 132.0 & 140.0 & 171.0 & 182.0 & 199.0 & 237.0 & 275.0 & 519.0 \\
\hline 1942 & 114.0 & 114.0 & 116.0 & 118.0 & 122.0 & 126.0 & 139.0 & $172 \cdot 0$ & 237.0 & 356.0 & 796.0 \\
\hline 1943 & 135.0 & 137.0 & 139.0 & 144.0 & 153.0 & 175.0 & 202.0 & 273.0 & 360.0 & 369.0 & 457.0 \\
\hline 1944 & 101.0 & 101.0 & 103.0 & 105.0 & 113.0 & 122.0 & 124.0 & $140 \cdot 0$ & 154.0 & 173.0 & 322.0 \\
\hline 1945 & 109.0 & 110.0 & 110.0 & 112.0 & 119.0 & 128.0 & $134 \cdot 0$ & $152 \cdot 0$ & 192.0 & 313.0 & 771.0 \\
\hline 1946 & 135.0 & 136.0 & 138.0 & 146.0 & 148.0 & 153.0 & 164.0 & $216 \cdot 0$ & 264.0 & 379.0 & 765.0 \\
\hline 1947 & 142.0 & $143 . n$ & 145.0 & 149.0 & 160.0 & 172.0 & 206.0 & 257.0 & 312.0 & 406.0 & 679.0 \\
\hline 1948 & 14200 & $151 \cdot 0$ & 155.0 & 157.0 & 169.0 & 201.0 & 230.0 & $257 \cdot 0$ & 315.0 & 464.0 & 654.0 \\
\hline 1949 & 116.0 & 117.0 & 119.0 & 120.0 & 133.0 & 144.0 & 172.0 & 177.0 & 199.0 & 270.0 & 476.0 \\
\hline 1950 & 134.0 & $134 \cdot 0$ & 139.0 & 143.0 & 155.0 & 183.0 & 227.0 & 296.0 & $520 \cdot 0$ & 687.0 & 1060.0 \\
\hline 1951 & 122.0 & $127 \cdot 0$ & $128 \cdot 0$ & $130 \cdot 0$ & 138.0 & 149.0 & 170.0 & $206 \cdot 0$ & $276 \cdot 0$ & 389.0 & 618.0 \\
\hline 1952 & 138.0 & 138.0 & 139.0 & 140.0 & 142.0 & 147.0 & 154.0 & 166.0 & 190.0 & 231.0 & 524.0 \\
\hline 1953 & 155.0 & 156.0 & 160.0 & 163.0 & $\begin{array}{l}170.0 \\
184.0\end{array}$ & 180.0 & 191.0 & $\begin{array}{l}204.0 \\
200.0\end{array}$ & 255.0 & 426.0 & 858.0 \\
\hline $\begin{array}{l}1954 \\
1955\end{array}$ & $\begin{array}{l}156.0 \\
160.0\end{array}$ & $\begin{array}{l}158.0 \\
161.0\end{array}$ & $\begin{array}{l}158.0 \\
163.0\end{array}$ & $\begin{array}{l}163.0 \\
169.0\end{array}$ & $\begin{array}{l}184.0 \\
184.0\end{array}$ & 196.0 & 238.0 & 293.0 & 392.0 & 608.0 & $\begin{array}{r}386.0 \\
1090.0\end{array}$ \\
\hline 1956 & 146.0 & 147.0 & 149.0 & 153.0 & 162.0 & 174.0 & 198.0 & $257 \cdot 0$ & 358.0 & 426.0 & 655.0 \\
\hline $\begin{array}{l}1957 \\
1958\end{array}$ & 136.0 & $137 \cdot 0$ & 139.0 & 140.0 & 142.0 & 157.0 & 173.0 & 183.0 & 212.0 & 250.0 & 543.0 \\
\hline $\begin{array}{l}1958 \\
1959\end{array}$ & $\begin{array}{l}123.0 \\
131.0\end{array}$ & $\begin{array}{l}125.0 \\
133.0\end{array}$ & 133.0 & 139.0 & 146.0 & 157.0 & $169 \cdot 0$ & 197.0 & 278.0 & 386.0 & 622.0 \\
\hline 1960 & 116.0 & $\begin{array}{l}133.0 \\
117.0\end{array}$ & 134.0 & $125 \cdot 0$ & 139.0 & 148.0 & 172.0 & 212.0 & 238.0 & 275.0 & 358.0 \\
\hline 1961 & 116.0 & 118.0 & $\begin{array}{l}120.0 \\
119.0\end{array}$ & $123 \cdot 0$ & 130.0 & 147.0 & $156 \cdot 0$ & $170 \cdot 0$ & $210 \cdot 0$ & 394.0 & 650.0 \\
\hline 1962 & 137.0 & $138 \cdot 0$ & 140.0 & $\begin{array}{l}122.0 \\
146.0\end{array}$ & $\begin{array}{l}129.0 \\
156.0\end{array}$ & $\begin{array}{l}140.0 \\
172.0\end{array}$ & $\begin{array}{l}154.0 \\
194.0\end{array}$ & $\begin{array}{l}175.0 \\
256.0\end{array}$ & $\begin{array}{l}229.0 \\
299.0\end{array}$ & $\begin{array}{l}299.0 \\
396.0\end{array}$ & $\begin{array}{l}633.0 \\
580.0\end{array}$ \\
\hline
\end{tabular}

North Fork of Middle Fork
Willamette River near Oakridge, Oreg.

STATION NUMBER $14-1475.00$

HIGHEST MEAN DISCHARGF, IN CFS, FOR THE FOLLOWING NUMBER OF CONSECUTIVE DAYS IN YEAR ENDING SEPTEMBER 30

\begin{tabular}{|c|c|c|c|c|c|c|c|c|c|c|c|}
\hline YEAR & 1 & 3 & 7 & 15 & 30 & 60 & 90 & 120 & 150 & 183 & 274 \\
\hline 1910 & 13700.0 & 11200.0 & 6910.0 & 4470.0 & 3120.0 & 1850.0 & 1550.0 & 1690.0 & 1540.0 & 1400.0 & 1030.0 \\
\hline 1911 & 5150.0 & 4330.0 & 3420.0 & 2590.0 & 1770.0 & 1160.0 & 974.0 & 862.0 & 871.0 & 876.0 & 721.0 \\
\hline 1912 & 8800.0 & 7720.0 & 5580.0 & 3920.0 & 2840.0 & 2380.0 & 1880.0 & 1740.0 & 1550.0 & 1460.0 & 1260.0 \\
\hline 1913 & 1700.0 & 1700.0 & 1700.0 & 1700.0 & 1700.0 & 1650.0 & 1520.0 & $1440 \cdot 0$ & 1370.0 & 1340.0 & 1170.0 \\
\hline 1914 & 2890.0 & 2440.0 & 1980.0 & 1820.0 & 1520.0 & 1320.0 & 1180.0 & $1100 \cdot 0$ & 1010.0 & 948.0 & 797.0 \\
\hline 1915 & 1170.0 & 1170.0 & 1130.0 & 1050.0 & 917.0 & $819 \cdot 0$ & 824.0 & $790 \cdot 0$ & 779.0 & 722.0 & 595.0 \\
\hline 1936 & 4860.0 & 4080.0 & 3200.0 & 3030.0 & 2080.0 & 1520.0 & 1370.0 & 1370.0 & 1330.0 & $1200 \cdot 0$ & 898.0 \\
\hline 1937 & 6080.0 & $5850 \cdot 0$ & 4350.0 & 2870.0 & 2170.0 & $1900 \cdot 0$ & 1700.0 & $1570 \cdot 0$ & 1380.0 & 1180.0 & 857.0 \\
\hline 1938 & 6200.0 & 4260.0 & 2850.0 & 1940.0 & 1600.0 & 1550.0 & 1450.0 & $1440 \cdot 0$ & 1400.0 & 1380.0 & 1090.0 \\
\hline 1939 & 3230.0 & $3200 \cdot 0$ & 2950.0 & 2300.0 & 1820.0 & 1440.0 & 1280.0 & $1120 \cdot 0$ & 1040.0 & $985 \cdot 0$ & 786.0 \\
\hline 1940 & 2770.0 & 2280.0 & 2070.0 & 1750.0 & 1620.0 & 1370.0 & 1150.0 & $994 \cdot 0$ & 915.0 & 808.0 & 591.0 \\
\hline 1941 & 2500.0 & 1880.0 & 1470.0 & 1220.0 & 927.0 & 829.0 & 771.0 & $686 \cdot 0$ & 612.0 & 588.0 & 506.0 \\
\hline 1942 & 6610.0 & 4420.0 & 2720.0 & 1810.0 & 1580.0 & 1420.0 & 1280.0 & $1120 \cdot 0$ & 1010.0 & 938.0 & 816.0 \\
\hline 1943 & 13200.0 & 10200.0 & 7180.0 & 4780.0 & 3260.0 & 2960.0 & 2530.0 & 2180.0 & 2010.0 & 1840.0 & 1460.0 \\
\hline 1944 & 3670.0 & 2840.0 & 1750.0 & 1110.0 & 996.0 & 888.0 & 804.0 & $760 \cdot 0$ & 700.0 & $662 \cdot 0$ & 589.0 \\
\hline 1945 & 6850.0 & 4940.0 & 3600.0 & 2640.0 & 1770.0 & 1470.0 & 1420.0 & 1390.0 & 1330.0 & 1170.0 & 855.0 \\
\hline 1946 & 12900.0 & $9860 \cdot n$ & 6010.0 & $4200 \cdot ?$ & 2870.0 & 2030.0 & 1780.0 & 1660.0 & 1540.0 & 1480.0 & 1170.0 \\
\hline 1947 & $10>00 \cdot 0$ & 8560.0 & 5520.0 & 3230.0 & 2800.0 & 1780.0 & 1640.0 & $1480 \cdot 0$ & 1450.0 & 1340.0 & 1040.0 \\
\hline 1948 & 8570.0 & 6650.0 & 4320.0 & 3000.0 & 1980.0 & 1630.0 & 1470.0 & 1430.0 & 1390.0 & 1340.0 & 1230.0 \\
\hline $\begin{array}{l}1949 \\
1950\end{array}$ & $\begin{array}{l}8190.0 \\
4260.0\end{array}$ & $\begin{array}{l}5970.0 \\
3570.0\end{array}$ & $\begin{array}{l}3960.0 \\
2850.0\end{array}$ & $\begin{array}{l}2630.0 \\
2510.0\end{array}$ & $\begin{array}{l}2210.0 \\
2260.0\end{array}$ & 1840.0 & $\begin{array}{l}1810.0 \\
1740.0\end{array}$ & $1610 \cdot 0$ & 1370.0 & 1400.0 & $\begin{array}{l}1120.0 \\
1130.0\end{array}$ \\
\hline 1951 & 8690.0 & 5550.0 & 4820.0 & 3040.0 & 2720.0 & 2070.0 & $\begin{array}{l}1740.0 \\
2010.0\end{array}$ & $\begin{array}{l}1720 \cdot 0 \\
2020 \cdot 0\end{array}$ & $\begin{array}{l}1630.0 \\
1800.0\end{array}$ & $\begin{array}{l}1470.0 \\
1700.0\end{array}$ & $\begin{array}{l}1130.0 \\
1330.0\end{array}$ \\
\hline 1952 & 3740.0 & 3570.0 & 2940.0 & 2150.0 & 1900.0 & 1630.0 & 1470.0 & 1420.0 & 1310.0 & 1300.0 & 1120.0 \\
\hline 1953 & 12700.0 & $8680 \cdot 0$ & 5870.0 & 4080.0 & 3770.0 & 2490.0 & 1970.0 & $1740 \cdot 0$ & 1650.0 & 1520.0 & 1120.0 \\
\hline 1954 & 10300.0 & 7110.0 & 4340.0 & 3110.0 & 2920.0 & 2010.0 & 1890.0 & $1690 \cdot 0$ & 1560.0 & 1410.0 & 1090.0 \\
\hline 1955 & 3190.0 & $2560 \cdot 0$ & $2300 \cdot 0$ & $1810 \cdot 0$ & 1700.0 & 1540.0 & 1460.0 & $1280 \cdot 0$ & 1170.0 & 1100.0 & 840.0 \\
\hline 1956 & 11600.0 & 8690.0 & $5550 \cdot 0$ & 4240.0 & 3340.0 & 2850.0 & 2330.0 & 1960.0 & 1860.0 & 1840.0 & 1490.0 \\
\hline 1957 & 10600.0 & 8210.0 & 5340.0 & 3560.0 & 2780.0 & 2080.0 & 1670.0 & $1600 \cdot 0$ & 1460.0 & 1320.0 & 1050.0 \\
\hline 1958 & 7320.0 & 5270.0 & 3790.0 & 2790.0 & 2260.0 & 2070.0 & 1850.0 & $1550 \cdot 0$ & 1500.0 & 1390.0 & 1070.0 \\
\hline 1959 & 3880.0 & 3460.0 & 2430.0 & 1700.0 & 1390.0 & 1190.0 & 1210.0 & $1120 \cdot 0$ & 1100.0 & 1040.0 & 828.0 \\
\hline 1960 & 3440.0 & 3220.0 & 2590.0 & $2200 \cdot 0$ & 1930.0 & 1650.0 & 1630.0 & $1530 \cdot 0$ & 1380.0 & $1200 \cdot 0$ & 931.0 \\
\hline 1961 & 7560.0 & $5830 \cdot n$ & 4090.0 & 3000.0 & 2270.0 & 1900.0 & 1580.0 & $1430 \cdot 0$ & 1410.0 & $1330 \cdot 0$ & 1030. \\
\hline $\begin{array}{l}1962 \\
1963\end{array}$ & $\begin{array}{l}5270.0 \\
3670.0\end{array}$ & $\begin{array}{l}4080.0 \\
3370.0\end{array}$ & 3170.0 & $2320 \cdot 0$ & 1930.0 & 1660.0 & 1350.0 & $1270 \cdot 0$ & $1320 \cdot 0$ & 1310.0 & $\begin{array}{r}1050.0 \\
910.0\end{array}$ \\
\hline 1903 & 3610.0 & $3310 \cdot 0$ & & & & 1320.0 & & & & & 91 \\
\hline
\end{tabular}


Middle Fork Willamette River below North Fork, near Oakridge, Oreg.
STATION NUMBER $\quad 14-1480.00$

DURATION TABLE OF DAILY DISCHARGE

CLASS $00 \begin{array}{lllllllllllllllllllllllllllllllllll} & 2 & 2 & 3 & 4 & 5 & 6 & 7 & 8 & 9 & 10 & 11 & 12 & 13 & 14 & 15 & 16 & 17 & 18 & 19 & 20 & 21 & 22 & 23 & 24 & 25 & 26 & 27 & 28 & 29 & 30 & 31 & 32 & 33 & 34\end{array}$

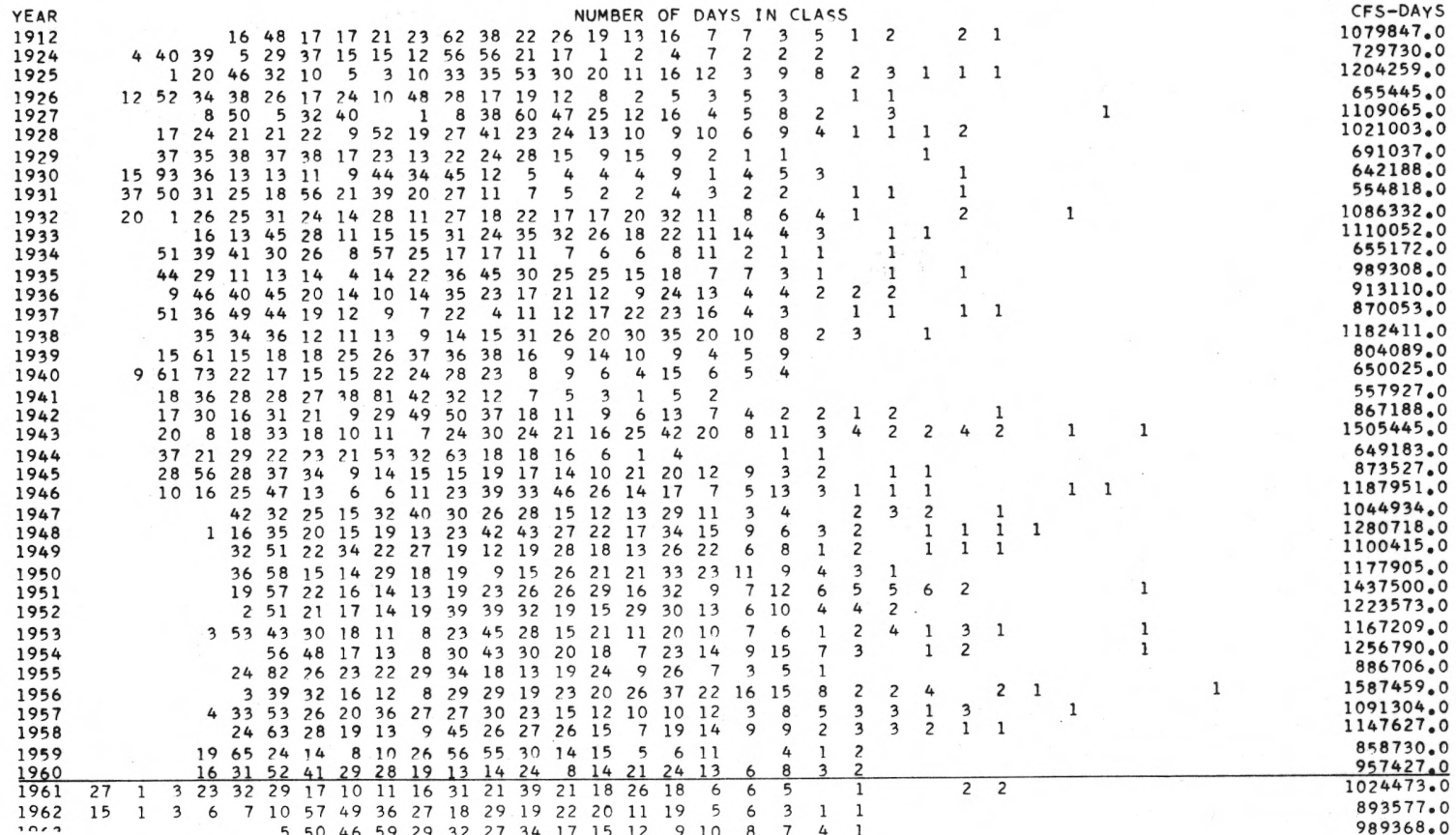

Middle Fork W1llamette R. below North Fork near Oakridge, Oreg.

Summary for water years 1912, 1924-63
STATION NUMBER 14-1480.00

\begin{tabular}{rrrrrrrrrrr}
\multicolumn{2}{c}{ CLASS CFS } & TOTAL & ACCUM & PERCT & \multicolumn{2}{l}{ CLASS CFS } & TOTAL & ACCUM \\
18 & 7000 & 235 & 771 & 5.1 & 27 & 35000 & 4 & 11 & .1 \\
19 & 8000 & 248 & 536 & 3.6 & 28 & 40000 & 2 & 7 & .0 \\
20 & 10000 & 99 & 288 & 1.9 & 29 & 45000 & 4 & 5 & .0 \\
21 & 12000 & 57 & 189 & 1.3 & 30 & 50000 & & 1 & .0 \\
22 & 14000 & 46 & 132 & .9 & 31 & 60000 & 1 & 1 & .0 \\
23 & 17000 & 28 & 86 & .6 & 32 & & & & .0 \\
24 & 20000 & 30 & 58 & .4 & 33 & & & & .0 \\
25 & 25000 & 15 & 28 & .2 & 34 & & & & .0
\end{tabular}


Middle Fork Willamette River below

STATION NUMBER $\quad 14-1480.00$

LOWEST MEAN DISCHARGE, IN CFS, FOR THE FOLLOWING NUMBER OF CONSECUTIVE DAYS IN YEAR BEGINNING APRIL 1

\begin{tabular}{|c|c|c|c|c|c|c|c|c|c|c|c|}
\hline $\begin{array}{l}\text { YEAR } \\
1911 \\
1924\end{array}$ & $\begin{array}{l}1 \\
660.0 \\
495.0\end{array}$ & $\begin{array}{l}3 \\
660.0 \\
495.0\end{array}$ & $\begin{array}{c}7 \\
660.0 \\
521.0\end{array}$ & $\begin{array}{l}14 \\
660.0 \\
523.0\end{array}$ & $\begin{array}{l}30 \\
690.0 \\
543.0\end{array}$ & $\begin{array}{c}60 \\
766.0 \\
576.0\end{array}$ & $\begin{array}{l}90 \\
849.0 \\
606.0\end{array}$ & $\begin{array}{l}120 \\
869 \cdot 0 \\
641 \cdot 0\end{array}$ & $\begin{array}{c}150 \\
1090.0 \\
731.0\end{array}$ & $\begin{array}{r}183 \\
1440.0 \\
983.0\end{array}$ & $\begin{array}{c}274 \\
1890.0 \\
2100.0\end{array}$ \\
\hline 1925 & 500.0 & $500 . n$ & 556.0 & 508.0 & 627.0 & 678.0 & 707.0 & $751 \cdot 0$ & 850.0 & 1040.0 & 1810.0 \\
\hline 1926 & 480.0 & $480 \cdot 0$ & $480 . n$ & $484 \cdot 0$ & 515.0 & 582.0 & 613.0 & $654 \cdot 0$ & $704 \cdot 0$ & 788.0 & 1680.0 \\
\hline 1927 & 710.0 & 710.0 & 710.0 & 710.0 & 710.0 & 884.0 & 1050.0 & 1180.0 & 1430.0 & 1770.0 & 2530.0 \\
\hline 1928 & 555.0 & 564.0 & $578 \cdot 0$ & 588.0 & 610.0 & 648.0 & $661 \cdot 0$ & $707 \cdot 0$ & 738.0 & 785.0 & 1060.0 \\
\hline $\begin{array}{l}1929 \\
1930\end{array}$ & $\begin{array}{l}450.0 \\
495.0\end{array}$ & $\begin{array}{r}458.0 \\
495.0\end{array}$ & 482.0 & $484 \cdot 0$ & $\begin{array}{l}496.0 \\
525.0\end{array}$ & $\begin{array}{l}527.0 \\
545.0\end{array}$ & $\begin{array}{l}533.0 \\
573.0\end{array}$ & $\begin{array}{l}552 \cdot 0 \\
587.0\end{array}$ & $\begin{array}{l}621.0 \\
646.0\end{array}$ & $\begin{array}{l}896.0 \\
778.0\end{array}$ & 1730.0 \\
\hline 1931 & 450.0 & $\begin{array}{l}495.0 \\
450.0\end{array}$ & $\begin{array}{l}495.0 \\
460.0\end{array}$ & $\begin{array}{l}515 \cdot 0 \\
464.0\end{array}$ & $\begin{array}{l}525.0 \\
476.0\end{array}$ & $\begin{array}{l}545.0 \\
483.0\end{array}$ & $\begin{array}{l}573.0 \\
492.0\end{array}$ & $\begin{array}{l}587 \cdot 0 \\
562 \cdot 0\end{array}$ & $\begin{array}{l}646.0 \\
634.0\end{array}$ & $\begin{array}{l}778.0 \\
726.0\end{array}$ & $\begin{array}{l}1010.0 \\
1190.0\end{array}$ \\
\hline 1932 & 604.0 & $604 \cdot 0$ & 609.0 & 616.0 & 636.0 & 681.0 & 731.0 & 833.0 & 1200.0 & 1380.0 & 2040.0 \\
\hline 1933 & 695.0 & 695.0 & 700.0 & 710.0 & 759.0 & 836.0 & 882.0 & $894 \cdot 0$ & 1040.0 & 1470.0 & 2080.0 \\
\hline 1934 & 517.0 & 520.0 & 527.0 & 534.0 & 542.0 & 549.0 & 572.0 & 619.0 & 725.0 & $842 \cdot 0$ & 1810.0 \\
\hline 1935 & 545.0 & $545 \cdot 0$ & 551.0 & 565.0 & $\begin{array}{l}581.0 \\
563.0\end{array}$ & $\begin{array}{l}600.0 \\
578.0\end{array}$ & 634.0 & 688.0 & 748.0 & $865 \cdot 0$ & 1750.0 \\
\hline 1936 & 545.0 & $545 \cdot 0$ & 546.0 & 549.0 & 563.0 & 578.0 & 608.0 & 638.0 & 687.0 & 721.0 & 1050.0 \\
\hline 1937 & 618.0 & 629.0 & 662.0 & 702.0 & 719.0 & 742.0 & 816.0 & 903.0 & 1320.0 & 1910.0 & 2880.0 \\
\hline 1938 & $\begin{array}{l}618.0 \\
560.0\end{array}$ & 618.0 & $\begin{array}{l}622.0 \\
563.0\end{array}$ & 638.0 & 655.0 & 669.0 & 700.0 & 776.0 & 959.0 & 1170.0 & 1650.0 \\
\hline 1940 & 488.0 & 488.0 & $\begin{array}{l}563.0 \\
493.0\end{array}$ & $\begin{array}{l}575.0 \\
499.0\end{array}$ & $601 \cdot 0$ & $632 \cdot 0$ & 654.0 & $654 \cdot 0$ & $69 ? .0$ & 843.0 & 1260.0 \\
\hline 1941 & 600.0 & 608.0 & 630.0 & $\begin{array}{l}499.0 \\
632.0\end{array}$ & $\begin{array}{l}513.0 \\
653.0\end{array}$ & 551.0 & 581.0 & $598 \cdot 0$ & 649.0 & 777.0 & 1230.0 \\
\hline 1942 & 538.0 & 538.0 & 542.0 & $\begin{array}{l}632.0 \\
553.0\end{array}$ & & 751.0 & 768.0 & $81 ? \cdot 0$ & $968 \cdot 0$ & 1100.0 & 1960.0 \\
\hline 1943 & 728.0 & 730.0 & 734.0 & 741.0 & $\begin{array}{l}568.0 \\
762.0\end{array}$ & $\begin{array}{l}586.0 \\
836.0\end{array}$ & $\begin{array}{l}632.0 \\
921.0\end{array}$ & $\begin{array}{r}745 \cdot 0 \\
1180.0\end{array}$ & $\begin{array}{r}969.0 \\
1400.0\end{array}$ & $\begin{array}{l}1430.0 \\
1430.0\end{array}$ & $\begin{array}{l}2910.0 \\
1740.0\end{array}$ \\
\hline 1944 & 503.0 & 505.0 & 510.0 & 516.0 & 536.0 & 555.0 & 574.0 & 630.0 & 683.0 & 739.0 & \\
\hline 1945 & 588.0 & 593.0 & 593.0 & 597.0 & 616.0 & 640.0 & 659.0 & 707.0 & 848.0 & 1330.0 & $\begin{array}{l}1260.0 \\
2770.0\end{array}$ \\
\hline 1946 & 707.0 & $714 \cdot 0$ & $724 \cdot 0$ & 747.0 & 760.0 & 794.0 & 845.0 & $1010 \cdot 0$ & 1190.0 & 1620.0 & 2660.0 \\
\hline 1947 & 696.0 & $702 \cdot 0$ & 710.0 & $721 \cdot 0$ & 750.0 & 798.0 & 926.0 & $1090 \cdot 0$ & 1320.0 & 1640.0 & 2450.0 \\
\hline 1948 & 769.0 & 785.0 & $791 \cdot 0$ & 799.0 & 842.0 & 948.0 & 1000.0 & $1100 \cdot 0$ & 1330.0 & 1870.0 & 2450.0 \\
\hline $\begin{array}{l}1949 \\
1950\end{array}$ & $\begin{array}{l}708.0 \\
750: 0\end{array}$ & $714 \cdot 0$ & 719.0 & 725.0 & 762.0 & 788.0 & 842.0 & $858 \cdot 0$ & 944.0 & 1180.0 & 1960.0 \\
\hline 1951 & 750.0 & 752.0 & $\begin{array}{l}769.0 \\
760.0\end{array}$ & $\begin{array}{l}784.0 \\
771.0\end{array}$ & $\begin{array}{l}814.0 \\
791.0\end{array}$ & $\begin{array}{l}880.0 \\
8>2.0\end{array}$ & $\begin{array}{r}1000 \cdot 0 \\
902.0\end{array}$ & $\begin{array}{l}1270.0 \\
1020.0\end{array}$ & $\begin{array}{l}2050.0 \\
1260.0\end{array}$ & $\begin{array}{l}2560.0 \\
1580.0\end{array}$ & $\begin{array}{l}3950.0 \\
2420.0\end{array}$ \\
\hline 1952 & 695.0 & 698.0 & 704.0 & 722.0 & 728.0 & 745.0 & 785.0 & 826.0 & 921.0 & $\begin{array}{l}1580.0 \\
1090.0\end{array}$ & $\begin{array}{l}2430.0 \\
2100.0\end{array}$ \\
\hline 1953 & 820.0 & 823.0 & 839.0 & 852.0 & 881.0 & 038.0 & 978.0 & 1030.0 & 1270.0 & 1900.0 & 3290.0 \\
\hline 1954 & 800.0 & 800.0 & $814 \cdot 0$ & 876.0 & 924.0 & 970.0 & 982.0 & 995.0 & 1020.0 & 1100.0 & 1570.0 \\
\hline 1955 & 710.0 & 717.0 & 719.0 & 736.0 & 789.0 & 841.0 & 990.0 & $1180 \cdot 0$ & 1490.0 & 2190.0 & 3950.0 \\
\hline 1956 & 790.0 & $797 \cdot 0$ & 810.0 & $824 \cdot 0$ & 854.0 & 909.0 & 991.0 & 1240.0 & 1500.0 & 1800.0 & 2470.0 \\
\hline 1957 & 680.0 & 680.0 & 691.0 & 709.0 & 734.0 & 789.0 & 867.0 & 885.0 & 978.0 & 1110.0 & 2130.0 \\
\hline 1958 & 683.0 & 692.0 & 705.0 & 718.0 & 744.0 & 787.0 & 831.0 & 919.0 & 1230.0 & 1670.0 & 2300.0 \\
\hline 1959 & 666.0 & $669 \cdot 0$ & 674.0 & 685.0 & 699.0 & 725.0 & 801.0 & 945.0 & 968.0 & 1050.0 & 1380.0 \\
\hline 1960 & 610.0 & $610 . n$ & 619.0 & 636.0 & 668.0 & 728.0 & 770.0 & $813 \cdot 0$ & 961.0 & 1540.0 & 2340.0 \\
\hline 1961 & 334.0 & 338.0 & $343 . n$ & 352.0 & 370.0 & 450.0 & 547.0 & $656 \cdot 0$ & 742.0 & 1090.0 & 1780.0 \\
\hline 1962 & 942.0 & 962.0 & 991.0 & 1070.0 & 1140.0 & 1180.0 & 1230.0 & 1470.0 & 1750.0 & 2000.0 & 2440.0 \\
\hline
\end{tabular}

Middle Fork Willamette River below

STATION NUMBER $\quad 14-1480.00$

North Fork, near Oakridge, Oreg.

HIGHEST MEAN DISCHARGE, IN CFS, FOR THE FOLLOWING NUMBER OF CONSECUTIVE DAYS IN YEAR ENDING SEPTEMBER 30

\begin{tabular}{|c|c|c|c|c|c|c|c|c|c|c|c|}
\hline YEAR & 1 & 3 & 7 & 15 & 30 & 60 & 90 & 120 & 150 & 183 & 274 \\
\hline $\begin{array}{l}1912 \\
1924 \\
1925\end{array}$ & $\begin{array}{l}28200 \cdot 0 \\
11400 \cdot 0 \\
27000.0\end{array}$ & $\begin{array}{r}22000 \cdot 0 \\
8040.0 \\
17900.0\end{array}$ & $\begin{array}{r}13900.0 \\
7350.0 \\
13800.0\end{array}$ & $\begin{array}{r}9810.0 \\
5990.0 \\
11600.0\end{array}$ & $\begin{array}{l}7380.0 \\
4350.0 \\
8170.0\end{array}$ & $\begin{array}{l}6450.0 \\
3920.0 \\
6990.0\end{array}$ & $\begin{array}{l}5140.0 \\
3760.0 \\
6180.0\end{array}$ & $\begin{array}{l}4610 \cdot 0 \\
3370 \cdot 0 \\
5760.0\end{array}$ & $\begin{array}{l}4370.0 \\
3280.0 \\
5140.0\end{array}$ & $\begin{array}{l}4260.0 \\
3100 \cdot 0 \\
4890 \cdot 0\end{array}$ & $\begin{array}{l}3610.0 \\
2460.0 \\
4140.0\end{array}$ \\
\hline $\begin{array}{l}1926 \\
1927 \\
1928\end{array}$ & $\begin{array}{l}14400.0 \\
40000.0 \\
22000.0\end{array}$ & $\begin{array}{l}12200.0 \\
23800.0 \\
16800.0\end{array}$ & $\begin{array}{r}9590.0 \\
15300.0 \\
13600.0\end{array}$ & $\begin{array}{l}6960.0 \\
9960.0 \\
9060.0\end{array}$ & $\begin{array}{l}5890.0 \\
7110.0 \\
6340.0\end{array}$ & $\begin{array}{l}4510.0 \\
5370.0 \\
5530.0\end{array}$ & $\begin{array}{l}3940.0 \\
5110.0 \\
4800.0\end{array}$ & $\begin{array}{l}3460 \cdot 0 \\
5080.0 \\
4460.0\end{array}$ & $\begin{array}{l}3160.0 \\
4750.0 \\
4470.0\end{array}$ & $\begin{array}{l}2860.0 \\
4500.0 \\
4360.0\end{array}$ & $\begin{array}{l}2190.0 \\
3750.0 \\
3470.0\end{array}$ \\
\hline $\begin{array}{l}1929 \\
1930\end{array}$ & $\begin{array}{r}18200.0 \\
73500.0\end{array}$ & $\begin{array}{l}11600.0 \\
14000.0\end{array}$ & $\begin{array}{l}7440.0 \\
0000.0\end{array}$ & $\begin{array}{l}5120.0 \\
7800.0\end{array}$ & $\begin{array}{l}4340.0 \\
5860.0\end{array}$ & $\begin{array}{l}4120.0 \\
4090.0\end{array}$ & $\begin{array}{l}4000.0 \\
3810.0\end{array}$ & $\begin{array}{l}3620.0 \\
3330.0\end{array}$ & $\begin{array}{l}3220.0 \\
3030.0\end{array}$ & $\begin{array}{l}2970.0 \\
2870.0\end{array}$ & $\begin{array}{l}2310.0 \\
2160.0\end{array}$ \\
\hline 1931 & 22500.0 & 17000.0 & 11500.0 & 7870.0 & 6080.0 & 4190.0 & 3370.0 & 2880.0 & 2560.0 & 2360.0 & 1850.0 \\
\hline $\begin{array}{l}1932 \\
1933 \\
1934\end{array}$ & $\begin{array}{l}36600.0 \\
17800.0 \\
16500.0\end{array}$ & $\begin{array}{l}27100.0 \\
14200.0 \\
12700.0\end{array}$ & $\begin{array}{r}17400.0 \\
11800.0 \\
9120.0\end{array}$ & $\begin{array}{r}13100.0 \\
9380.0 \\
6660.0\end{array}$ & $\begin{array}{l}9110.0 \\
7900.0 \\
5120.0\end{array}$ & $\begin{array}{l}7320.0 \\
6270.0 \\
4350.0\end{array}$ & $\begin{array}{l}65>0.0 \\
5580.0 \\
3670.0\end{array}$ & $\begin{array}{l}5920.0 \\
5130.0 \\
3500.0\end{array}$ & $\begin{array}{l}5160.0 \\
4710.0 \\
3160.0\end{array}$ & $\begin{array}{l}4800.0 \\
4470.0 \\
2810.0\end{array}$ & $\begin{array}{l}3730.0 \\
3770.0 \\
2190.0\end{array}$ \\
\hline $\begin{array}{l}1935 \\
1936 \\
1937\end{array}$ & $\begin{array}{l}20100.0 \\
14500.0 \\
25500.0\end{array}$ & $\begin{array}{l}15300.0 \\
12400.0 \\
20900.0\end{array}$ & $\begin{array}{l}11400.0 \\
10600.0 \\
14100.0\end{array}$ & $\begin{array}{l}7670.0 \\
9490.0 \\
9270.0\end{array}$ & $\begin{array}{l}5510.0 \\
6690.0 \\
7010.0\end{array}$ & $\begin{array}{l}4710.0 \\
5040.0 \\
6100.0\end{array}$ & $\begin{array}{l}4430.0 \\
4480.0 \\
5630.0\end{array}$ & $\begin{array}{l}4060.0 \\
4530.0 \\
5300.0\end{array}$ & $\begin{array}{l}4000.0 \\
4480.0 \\
4660.0\end{array}$ & $\begin{array}{l}3980.0 \\
4100.0 \\
4020.0\end{array}$ & $\begin{array}{l}3400.0 \\
3100.0 \\
2970.0\end{array}$ \\
\hline $\begin{array}{l}1938 \\
1939 \\
1940\end{array}$ & $\begin{array}{r}19200.0 \\
9850.0 \\
8560.0\end{array}$ & $\begin{array}{r}13300.0 \\
9780.0 \\
8020.0\end{array}$ & $\begin{array}{r}9300.0 \\
9230.0 \\
7250.0\end{array}$ & $\begin{array}{l}7040.0 \\
7550.0 \\
6090.0\end{array}$ & $\begin{array}{l}5690.0 \\
6210.0 \\
5530.0\end{array}$ & $\begin{array}{l}5570.0 \\
4850.0 \\
4860.0\end{array}$ & $\begin{array}{l}5180.0 \\
4370.0 \\
4080.0\end{array}$ & $\begin{array}{l}5220.0 \\
3860.0 \\
3510.0\end{array}$ & $\begin{array}{l}5130.0 \\
3530.0 \\
3240.0\end{array}$ & $\begin{array}{l}5020.0 \\
3350.0 \\
2900.0\end{array}$ & $\begin{array}{l}4060.0 \\
2720.0 \\
2170.0\end{array}$ \\
\hline $\begin{array}{l}1941 \\
1947 \\
1943\end{array}$ & $\begin{array}{r}6630.0 \\
26000.0 \\
49800.0\end{array}$ & $\begin{array}{r}5680 \cdot 0 \\
16800.0 \\
37800.0\end{array}$ & $\begin{array}{r}4460.0 \\
9950.0 \\
27000.0\end{array}$ & $\begin{array}{r}3860.0 \\
6410.0 \\
17100.0\end{array}$ & $\begin{array}{r}2940.0 \\
5590.0 \\
11500.0\end{array}$ & $\begin{array}{r}2640.0 \\
5050.0 \\
10400.0\end{array}$ & $\begin{array}{l}2460.0 \\
4500.0 \\
8870.0\end{array}$ & $\begin{array}{l}2790.0 \\
3910.0 \\
7630.0\end{array}$ & $\begin{array}{l}2020.0 \\
7510.0 \\
6980.0\end{array}$ & $\begin{array}{l}2040.0 \\
3280.0 \\
6440.0\end{array}$ & $\begin{array}{l}1800.0 \\
2920.0 \\
5230.0\end{array}$ \\
\hline $\begin{array}{l}1944 \\
1945 \\
1946\end{array}$ & $\begin{array}{l}11900.0 \\
18500.0 \\
40800.0\end{array}$ & $\begin{array}{r}9100.0 \\
14600.0 \\
31800.0\end{array}$ & $\begin{array}{r}5700.0 \\
11100.0 \\
18900.0\end{array}$ & $\begin{array}{r}3790.0 \\
8340.0 \\
13700.0\end{array}$ & $\begin{array}{l}3440.0 \\
6050.0 \\
9220.0\end{array}$ & $\begin{array}{l}3090.0 \\
5260.0 \\
6820.0\end{array}$ & $\begin{array}{l}2810.0 \\
4720.0 \\
5930.0\end{array}$ & $\begin{array}{l}2710.0 \\
4770.0 \\
5550.0\end{array}$ & $\begin{array}{l}2530.0 \\
4490.0 \\
5130.0\end{array}$ & $\begin{array}{l}2350.0 \\
4000.0 \\
4960.0\end{array}$ & $\begin{array}{l}2150.0 \\
2980.0 \\
4080.0\end{array}$ \\
\hline $\begin{array}{l}1947 \\
1948 \\
1949\end{array}$ & $\begin{array}{l}27800.0 \\
32900.0 \\
26000.0\end{array}$ & $\begin{array}{l}21200.0 \\
25100.0 \\
18800.0\end{array}$ & $\begin{array}{l}14300.0 \\
16300.0 \\
12500.0\end{array}$ & $\begin{array}{r}8730.0 \\
11000.0 \\
8570.0\end{array}$ & $\begin{array}{l}7920.0 \\
6980.0 \\
7250.0\end{array}$ & $\begin{array}{l}5280.0 \\
5830.0 \\
5840.0\end{array}$ & $\begin{array}{l}5100.0 \\
5020.0 \\
5780.0\end{array}$ & $\begin{array}{l}4660 \cdot 0 \\
4870 \cdot 0 \\
5240.0\end{array}$ & $\begin{array}{l}4680.0 \\
4830.0 \\
4550.0\end{array}$ & $\begin{array}{l}4400.0 \\
4750.0 \\
4540.0\end{array}$ & $\begin{array}{l}3520.0 \\
4330.0 \\
3720.0\end{array}$ \\
\hline $\begin{array}{l}1950 \\
1951 \\
1952\end{array}$ & $\begin{array}{l}14500.0 \\
47600.0 \\
15100.0\end{array}$ & $\begin{array}{l}12900.0 \\
28100.0 \\
12900.0\end{array}$ & $\begin{array}{l}10100.0 \\
19600.0 \\
10900.0\end{array}$ & $\begin{array}{r}7680.0 \\
11900.0 \\
7770.0\end{array}$ & $\begin{array}{l}7140.0 \\
9740.0 \\
6470.0\end{array}$ & $\begin{array}{l}6250.0 \\
7940.0 \\
5640.0\end{array}$ & $\begin{array}{l}5900.0 \\
7350.0 \\
5100.0\end{array}$ & $\begin{array}{l}5670.0 \\
7470.0 \\
4990.0\end{array}$ & $\begin{array}{l}5600.0 \\
6690.0 \\
4720.0\end{array}$ & $\begin{array}{l}5160.0 \\
6240.0 \\
4730.0\end{array}$ & $\begin{array}{l}4010.0 \\
4950.0 \\
4120.0\end{array}$ \\
\hline 1953 & 45000.0 & $30600 . n$ & 19800.0 & 14100.0 & $12>00.0$ & $8>00.0$ & 6580.0 & 5790.0 & 5570.0 & 5240.0 & 4000.0 \\
\hline $\begin{array}{l}1994 \\
1955\end{array}$ & $\begin{array}{l}47>00.0 \\
10500.0\end{array}$ & $\begin{array}{r}29600.0 \\
8790.0\end{array}$ & $\begin{array}{r}17400.0 \\
7880.0\end{array}$ & $\begin{array}{r}12000.0 \\
6160.0\end{array}$ & $\begin{array}{r}10700.0 \\
5690.0\end{array}$ & $\begin{array}{l}7480.0 \\
5210.0\end{array}$ & $\begin{array}{l}7200.0 \\
4940.0\end{array}$ & $\begin{array}{l}6360.0 \\
4360.0\end{array}$ & $\begin{array}{l}5880.0 \\
3960.0\end{array}$ & $\begin{array}{l}5400.0 \\
3750.0\end{array}$ & $\begin{array}{l}4250.0 \\
>940.0\end{array}$ \\
\hline 1956 & 60300.0 & 39700.0 & 23800.0 & 16500.0 & 12200.0 & 10300.0 & 8360.0 & 7120.0 & 6720.0 & 6580.0 & 5430.0 \\
\hline 1957 & 39000.0 & 27800.0 & 17500.0 & 12100.0 & 9410.0 & $\begin{array}{l}7110.0 \\
7360.0\end{array}$ & $\begin{array}{l}5840.0 \\
6510.0\end{array}$ & $5340 \cdot 0$ & 4970.0 & $4600 \cdot 0$ & 3710.0 \\
\hline 1958 & 29700.0 & 21200.0 & $14500 \cdot 0$ & 10400.0 & 8430.0 & 7360.0 & 6510.0 & $5470 \cdot 0$ & 5260.0 & 4970.0 & 3890.0 \\
\hline 1959 & 13600.0 & 11500.0 & 7870.0 & 5550.0 & 4830.0 & 4020.0 & 3910.0 & $3690 \cdot 0$ & 3640.0 & 3480.0 & 2889.0 \\
\hline 1960 & 12600.0 & 11300.0 & 8940.0 & 7360.0 & 6550.0 & 5570.0 & 5520.0 & 5180.0 & 4730.0 & 4160.0 & 3210.0 \\
\hline 1961 & 27100.0 & 20100.0 & 13800.0 & 9640.0 & 7310.0 & 6200.0 & 5230.0 & 4810.0 & 4640.0 & 4400.0 & 3530.0 \\
\hline 1962 & 13000.0 & $9970 \cdot n$ & 8520.0 & 6970.0 & 5500.0 & $\begin{array}{l}4650.0 \\
4610.0\end{array}$ & 3850.0 & $3330 \cdot 0$ & 3410.0 & 3460.0 & 2940.0 \\
\hline 1963 & 12200.0 & 11000.0 & 9340.0 & 8320.0 & 6380.0 & 4610.0 & 3870.0 & 3570.0 & 3360.0 & 3560.0 & 3190.0 \\
\hline
\end{tabular}


Middle Fork W1llamette River near Dexter, Oreg.
STATION NUMBER $\quad 14-1500.00$

DURATION TABLE OF DAILY DISCHARGE

\begin{tabular}{|c|c|c|c|c|c|c|c|c|c|c|c|c|c|c|c|c|c|c|c|c|c|c|c|c|c|c|c|c|c|c|c|c|c|}
\hline CLASS & 0 & 1 & 2 & 3 & 4 & 5 & 6 & 7 & 8 & 9 & 10 & 11 & 12 & 1314 & $4 \quad 15$ & 16 & 171 & 181 & 19 & 20 & 21 & 22 & 23 & 24 & 25 & 26 & 27 & 28 & 29 & 30 & 3132 & 3334 & \\
\hline YEAR & & & & & & & & & & & & & & NUM & MBER & OF & DAYS & $S$ II & $N C$ & CLAS & & & & & & & & & & & & & CFS-DAYS \\
\hline 1947 & & & & & & & & & & & & 33 & 34 & $25 \quad 15$ & 527 & 41 & 333 & 304 & $45^{\circ}$ & 23 & 28 & 11 & 6 & 4 & 2 & & 3 & 3 & 1 & 1 & & & 1109806.0 \\
\hline 1948 & & & & & & & & & & & & 16 & 31 & 2218 & $8 \quad 12$ & 13 & 184 & 427 & 75 & 37 & 33 & 21 & 11 & 5 & 5 & 1 & 2 & & 1 & 2 & 1 & & 1355101.0 \\
\hline 1949 & & & & & & & & & & & & 26 & 58 & 2129 & 926 & 15 & 26 & 94 & 40 & 39 & 29 & 19 & 11 & 6 & 5 & $i$ & 2 & & 2 & 1 & & & 1181978.0 \\
\hline 1950 & & & & & & & & & & & & 12 & 68 & $24 \quad 17$ & $\begin{array}{ll}7 & 21\end{array}$ & 21 & 23 & 83 & 31 & 45 & 33 & 30 & 10 & 12 & 3 & 4 & 2 & 1 & & & & & 1243307.0 \\
\hline 1951 & & & & & & & & & & & & 20 & 54 & 2314 & $\begin{array}{ll}4 & 12\end{array}$ & 8 & 171 & 185 & 54 & 50 & 31 & 15 & 7 & 8 & 12 & 5 & 6 & 5 & 5 & & & 1 & 1565795.0 \\
\hline 1952 & & & & & & & & & & & & 3 & 41 & $28 \quad 20$ & 011 & 10 & 284 & 415 & 57 & 32 & 41 & 15 & 11 & 11 & 7 & 3 & 5 & 2 & & & & & 1374619.0 \\
\hline 1953 & & & & & & & & & & & & 9 & 81 & $28 \quad 20$ & $\begin{array}{l}0 \\
0\end{array}$ & 12 & 183 & 305 & 56 & 34 & 17 & 17 & 8 & 4 & 5 & 2 & 4 & 1 & 3 & & 1 & & 1245413.0 \\
\hline 1954 & & & & & & & & & & & & 1 & 24 & 2140 & 048 & 70 & 132 & 223 & 31 & 16 & 10 & 17 & 15 & 13 & 12 & 7 & & 2 & 3 & & & & 1289986.0 \\
\hline 1955 & & & & & & & & 1 & 1 & & 3 & 5 & 12 & 1948 & 866 & 68 & 262 & 234 & 47 & 9 & 18 & 10 & 7 & 2 & & & & & & & & & $866100 \cdot 0$ \\
\hline 1956 & & & & & & & & 1 & & & & & & 3125 & $5 \quad 33$ & 20 & 391 & 164 & 43 & 42 & 15 & 33 & 12 & 12 & 14 & 1 & 29 & & & & & & 1702900.0 \\
\hline 1957 & & & & & & & & & & & & 4 & 10 & 7330 & 016 & 10 & 555 & 552 & 20 & 19 & 22 & 18 & 9 & 8 & 8 & 1 & 7 & & & & & & 1180251.0 \\
\hline 1958 & & & & & & 4 & & & & & & & & 2669 & 921 & 19 & 554 & 424 & 48 & 14 & 21 & 6 & 10 & 11 & 5 & 12 & 2 & & & & & & 1214090.0 \\
\hline 1959 & & & & & & & & & & & & & & 1479 & 989 & 26 & 244 & 44 & 48 & 15 & 8 & 6 & 2 & 5 & 4 & 1 & & & & & & & 911740.0 \\
\hline 1960 & & & & & & 1 & & & & & & & 1 & 2764 & 454 & 52 & 183 & 36 & 47 & 21 & 12 & 9 & 11 & 7 & 4 & 2 & & & & & & & 1021840.0 \\
\hline 1961 & & 1 & & & & & 1 & & & 2 & & & & 641 & 129 & 52 & 745 & 54 & 49 & 15 & 6 & 8 & 2 & 6 & 6 & 13 & & & & & & & 1129170.0 \\
\hline 1962 & & & & & & & & & & & & 2 & 7 & 12102 & 254 & 63 & 312 & 292 & 25 & 10 & 6 & 2 & 3 & 18 & 1 & & & & & & & & 874033.0 \\
\hline 1963 & & & & & & & & & & & & & & 3386 & 685 & 19 & 241 & 113 & 38 & 16 & 5 & 4 & 13 & 14 & 6 & 11 & & & & & & & 1079280.0 \\
\hline
\end{tabular}

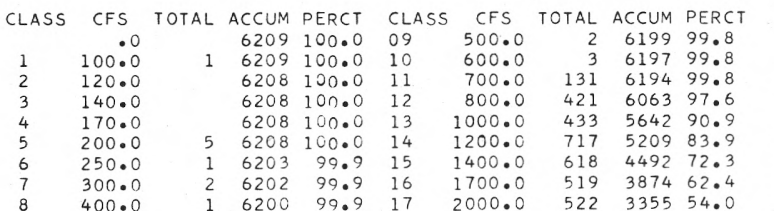

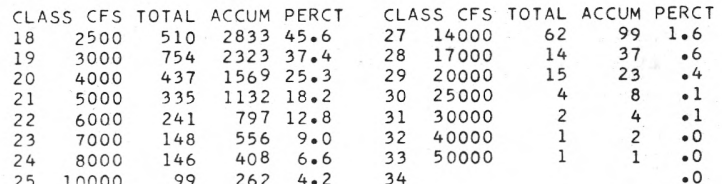
$\begin{array}{rrrrr}24 & 8000 & 146 & 408 & 6.6 \\ 25 & 10000 & 99 & 262 & 4.2\end{array}$ $\begin{array}{llllll}25 & 10000 & 99 & 262 & 4.2 & 34 \\ 26 & 12000 & 64 & 163 & 2.6 & 35\end{array}$
Madle fiork Willamette River near Dexter, Oreg.

Sumnary for water years 1947-53

$\begin{array}{ccrrr}\text { CLASS } & \text { CFS } & \text { TOTAL } & \text { ACCUM } & \text { PERCT } \\ & .0 & 2557 & 100.0 \\ 1 & 100.0 & & 2557 & 100.0 \\ 2 & 120.0 & & 2557 & 100.0 \\ 3 & 140.0 & & 2557 & 100.0 \\ 4 & 170.0 & & 2557 & 100.0 \\ 5 & 200.0 & & 2557 & 100.0 \\ 6 & 250.0 & & 2557 & 100.0 \\ 7 & 300.0 & & 2557 & 100.0 \\ 8 & 400.0 & & 2557 & 100.0\end{array}$

Middle Fork Willamette River near Dexter, Oreg.

Summary for water years 1954-63

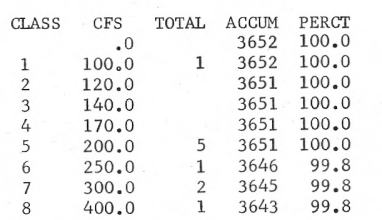

\begin{tabular}{rlr} 
& \multicolumn{1}{l}{ CLASS } & CFS \\
0 & 09 & 500.0 \\
0 & 10 & 600.0 \\
0 & 11 & 700.0 \\
0 & 12 & 800.0 \\
0 & 13 & 1000.0 \\
0 & 14 & 1200.0 \\
8 & 15 & 1400.0 \\
8 & 16 & 1700.0 \\
8 & 17 & 2000.0
\end{tabular}

$\begin{array}{lr}\text { CLASS } & \text { CFS } \\ 09 & 500.0 \\ 10 & 600.0 \\ 11 & 700.0 \\ 12 & 800.0 \\ 13 & 1000.0 \\ 14 & 1200.0 \\ 15 & 1400.0 \\ 16 & 1700.0 \\ 17 & 2000.0\end{array}$

STATION NUMBER

$14-1500.00$

$\begin{array}{rrrr}\text { TAL } & \text { ACCUM } & \text { PERCT } & \text { C } \\ & 2557 & 100.0 & 1 \\ & 2557 & 100.0 & 1 \\ 119 & 2557 & 100.0 & 2 \\ 367 & 2438 & 95.3 & 2 \\ 171 & 2071 & 81.0 & 2 \\ 133 & 1900 & 74.3 & 23 \\ 123 & 1767 & 69.1 & 24 \\ 120 & 1644 & 64.3 & 2 \\ 163 & 1524 & 59.6 & 26\end{array}$

STATION NUMBER

$\begin{array}{lr}\text { CLASS } & \text { C } \\ 18 & 25 \\ 19 & 3000 \\ 20 & 4000 \\ 21 & 5000 \\ 22 & 6000 \\ 23 & 7000 \\ 24 & 8000 \\ 25 & 10000 \\ 26 & 12000\end{array}$

CFS
2500
3000
4000
5000
6000
7000
8000
10000
12000

TOTAL A
178
358
260
212
128
64
50
39
16

\begin{tabular}{rrll} 
ACCUM & PERCT & \multicolumn{2}{l}{ CLASS CFS } \\
1361 & 53.2 & 27 & 14000 \\
1183 & 46.3 & 28 & 17000 \\
825 & 32.3 & 29 & 20000 \\
565 & 22.1 & 30 & 25000 \\
353 & 13.8 & 31 & 30000 \\
225 & 8.8 & 32 & 40000 \\
161 & 6.3 & 33 & 50000 \\
111 & 4.3 & 34 & \\
72 & 2.8 & 35 &
\end{tabular}

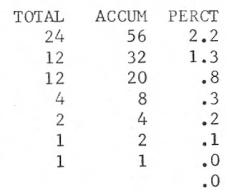

$14-1500.00$

\begin{tabular}{|c|c|c|c|c|c|c|c|c|c|c|c|}
\hline YEAR & 1 & 3 & 7 & 14 & 30 & 60 & 90 & 120 & 150 & 183 & 274 \\
\hline 1947 & 724.0 & 724.0 & 727.0 & 739.0 & 780.0 & 832.0 & 967.0 & $1130 \cdot 0$ & $1380 \cdot 0$ & 1710.0 & 2550.0 \\
\hline 1948 & 780.0 & 783.0 & 791.0 & 797.0 & 859.0 & $959 . ?$ & 1010.0 & $1120 \cdot 0$ & 1360.0 & 1960.0 & 2600.0 \\
\hline 1949 & 716.0 & 721.0 & 723.0 & 731.0 & 778.0 & 804.0 & 873.0 & $886 \cdot 0$ & 969.0 & 1210.0 & 2070.0 \\
\hline 1950 & 803.0 & $808 \cdot 0$ & 813.0 & 829.0 & $851 \cdot 0$ & 923.0 & 1060.0 & $1340 \cdot 0$ & 2180.0 & 2690.0 & 4230.0 \\
\hline 1951 & 752.0 & $755 \cdot 0$ & 759.0 & 767.0 & 789.0 & 825.0 & 904.0 & 1030.0 & 1290.0 & $1620 \cdot 0$ & 2630.0 \\
\hline 1952 & 775.0 & 790.0 & 794.0 & 798.0 & 799.0 & 818.0 & 857.0 & $898 \cdot 0$ & 1000.0 & 1200.0 & 2300.0 \\
\hline 953 & 765.0 & 795.0 & 810.0 & 825.0 & 872.0 & 966.0 & 1010.0 & 1080.0 & 1320.0 & 2020.0 & 3520.0 \\
\hline 1954 & 370.0 & 573.0 & 696.0 & 848.6 & 911.0 & 1280.0 & 1420.0 & 1470.0 & 1510.0 & 1580.0 & 1750.0 \\
\hline 1955 & 350.0 & 1100.0 & 1210.0 & 1290.0 & 1350.0 & 1540.0 & 1720.0 & $2180 \cdot 0$ & 2560.0 & 2800.0 & 4070.0 \\
\hline 1956 & 756.0 & 769.0 & 868.0 & 1070.0 & 1110.0 & 1290.0 & 1480.0 & 1630.0 & 2100.0 & 2570.0 & 3010.0 \\
\hline 1957 & 200.0 & 567.0 & 1020.0 & 1020.0 & 1030.0 & 1060.0 & 1270.0 & $1570 \cdot 0$ & 1690.0 & $1840 \cdot 0$ & 2370.0 \\
\hline 1958 & 1090.0 & 1100.0 & 1120.0 & 1140.0 & 1210.0 & 1360.0 & 1710.0 & $2010 \cdot 0$ & 2170.0 & 2320.0 & 2740.0 \\
\hline 1959 & 200.0 & 770.0 & 1050.0 & 1200.0 & $122 \cdot 0$ & 1320.0 & 1460.0 & $1580 \cdot 0$ & 1710.0 & 1780.0 & 1920.0 \\
\hline 1960 & 100.0 & 663.0 & 1050.0 & 1160.0 & 1410.0 & 1510.0 & 1600.0 & $1740 \cdot 0$ & 1970.0 & 2310.0 & 2820.0 \\
\hline 1961 & $700 \cdot 0$ & 822.0 & $921 \cdot ?$ & 1060.0 & 1170.0 & 1260.0 & 1700.0 & $1810 \cdot 0$ & 1790.0 & $1900 \cdot 0$ & 2460.0 \\
\hline 1962 & $1000 \cdot 0$ & $1010 \cdot 0$ & $1050 . ?$ & 1120.0 & 1200.0 & 1210.0 & 1270.0 & $1810 \cdot 0$ & 2070.0 & 2080.0 & 2880.0 \\
\hline
\end{tabular}


Middle Fork Willamette River near

STATION NUMBER $\quad 14-1500.00$

Dexter, Oreg.

\begin{tabular}{|c|c|c|c|c|c|c|c|c|c|c|c|}
\hline YEAR & 1 & 3 & 7 & 15 & 30 & 60 & 90 & 120 & 150 & 183 & 274 \\
\hline 1947 & 29800.0 & 23200.0 & 15500.0 & 9320.0 & 8740.0 & 5770.0 & $5500 \cdot 0$ & $5000 \cdot 0$ & $5000 \cdot 0$ & 4680.0 & \\
\hline 1948 & $36200 \cdot 0$ & 27400.0 & 17900.0 & 12000.0 & 7550.0 & 6440.0 & 5530.0 & $5250 \cdot 0$ & $5200 \cdot 0$ & 5090.0 & 4600.0 \\
\hline 1949 & $29800 \cdot 0$ & 22200.0 & 14300.0 & 9470.0 & 7600.0 & 6160.0 & 6240.0 & $5640 \cdot 0$ & $4890 \cdot 0$ & 4920.0 & 4010.0 \\
\hline 1950 & 17100.0 & $15300 \cdot 0$ & $11400 \cdot 0$ & 7780.0 & 7430.0 & 6520.0 & 6220.0 & $5940 \cdot 0$ & 5910.0 & 5460.0 & 4230.0 \\
\hline 1951 & $50100 \cdot 0$ & $30300 . ?$ & 21300.0 & 13000.0 & 10800.0 & 8640.0 & 8020.0 & $8180 \cdot 0$ & 7370.0 & $6860 \cdot 0$ & 5420.0 \\
\hline 1952 & 19000.0 & 16500.0 & 13500.0 & 9360.0 & 7550.0 & 6400.0 & 5960.0 & $5720 \cdot 0$ & 5490.0 & 5430.0 & 4650.0 \\
\hline 1953 & $43200 \cdot 0$ & 32200.0 & 20800.0 & 14700.0 & 12600.0 & 8570.0 & 6950.0 & $6130 \cdot 0$ & 5930.0 & 5570.0 & 4260.0 \\
\hline 1954 & 21900.0 & $21300 \cdot 0$ & $17100 \cdot 0$ & 12600.0 & 11100.0 & 8110.0 & 7940.0 & $6980 \cdot 0$ & $6340 \cdot 0$ & $5450 \cdot 0$ & 4260.0 \\
\hline 1955 & $8450 \cdot 0$ & 8020.0 & 7240.0 & $5930 \cdot 0$ & 5290.0 & 4050.0 & 4100.0 & $3660 \cdot 0$ & 3240.0 & 3160.0 & 2690.0 \\
\hline 1956 & 16100.0 & $15900 \cdot 0$ & 15200.0 & 14900.0 & 13400.0 & $11500 \cdot 0$ & 9590.0 & $8220 \cdot 0$ & 6960.0 & 6430.0 & 5670.0 \\
\hline 1957 & 14900.0 & 14800.0 & 14600.0 & 10500.0 & 8160.0 & 6130.0 & $5020 \cdot 0$ & $5030 \cdot 0$ & 4850.0 & $4760 \cdot 0$ & $3780 \cdot 0$ \\
\hline 1958 & $14600 \cdot 0$ & 14200.0 & 13400.0 & 10500.0 & 8220.0 & 7430.0 & 6490.0 & $5620 \cdot 0$ & 4930.0 & $4450 \cdot 0$ & 3830.0 \\
\hline 1959 & $12000 \cdot 0$ & 11700.0 & 9270.0 & 6930.0 & 5070.0 & 4280.0 & 4400.0 & $3990 \cdot 0$ & 3640.0 & $3220 \cdot 0$ & 2810.0 \\
\hline 1960 & $12000 \cdot 0$ & $11900 \cdot n$ & $9000 \cdot 0$ & $6820 \cdot n$ & $6000 \cdot 0$ & 4870.0 & $4800 \cdot 0$ & $4520 \cdot 0$ & 4020.0 & $3570 \cdot 0$ & 3170.0 \\
\hline 61 & $13200 \bullet 0$ & $13100 \cdot 0$ & $12600 \cdot 0$ & 11800.0 & 7710.0 & 5570.0 & $4550 \cdot 0$ & $4620 \cdot 0$ & 4320.0 & 4060.0 & 3470.0 \\
\hline 962 & $10100 \cdot 0$ & 9870.0 & $9520 \cdot 0$ & 9090.0 & $6480 \cdot 0$ & 5350.0 & 4030.0 & $3450 \cdot 0$ & $3020 \cdot 0$ & 2970.0 & $2650 \cdot 0$ \\
\hline 963 & $13200 \cdot 0$ & 13200.0 & $13000 \cdot 0$ & $11100 \cdot 0$ & 9520.0 & 7140.0 & 5760.0 & $4660 \cdot 0$ & 3980.0 & 3480.0 & 3370.0 \\
\hline
\end{tabular}

Fall Creek above Winberry Creek, near Loweli, Oreg.
STATION NUMBFR $14-1505.00$

DURATION TABLE OF DAILY DISCHARGE

CLASS $0 \begin{array}{lllllllllllllllllllllllllllllllllll} & 0 & 2 & 3 & 4 & 5 & 6 & 7 & 8 & 9 & 10 & 11 & 12 & 13 & 14 & 15 & 16 & 17 & 18 & 19 & 20 & 21 & 22 & 23 & 24 & 25 & 26 & 27 & 28 & 29 & 30 & 31 & 32 & 33 & 34\end{array}$

YEAR NUMBER OF DAYS IN CLASS

$\begin{array}{lrllllllllllllllllllllllll}1936 & 7 & 7 & 16 & 41 & 23 & 12 & 19 & 16 & 24 & 16 & 20 & 30 & 31 & 24 & 12 & 15 & 19 & 21 & 5 & 4 & 2 & 2\end{array}$

$\begin{array}{lllllllllllllllllllllllllllll}1937 & 1 & 11 & 46 & 7 & 15 & 27 & 14 & 20 & 17 & 32 & 19 & 11 & 13 & 18 & 23 & 15 & 16 & 30 & 12 & 11 & 3 & 1 & 2 & 1 \\ 1938 & & & 18 & 28 & 28 & 13 & 9 & 15 & 19 & 21 & 14 & 11 & 10 & 17 & 20 & 22 & 35 & 39 & 31 & 8 & 3 & 1 & 2 & 1 \\ 1939 & & 13 & 16 & 34 & 21 & 9 & 13 & 13 & 24 & 37 & 26 & 21 & 17 & 21 & 21 & 14 & 25 & 12 & 13 & 9 & 3 & 1 & 1 & 1\end{array}$

1940

1941

$\begin{array}{llllllllllllllllrrrrr}17 & 24 & 21 & 66 & 25 & 15 & 20 & 9 & 27 & 21 & 13 & 21 & 26 & 14 & 5 & 14 & 8 & 16 & 4 \\ 111 & 3 & 18 & 21 & 19 & 25 & 32 & 57 & 54 & 27 & 18 & 28 & 16 & 11 & 11 & 7 & 5 & 2\end{array}$

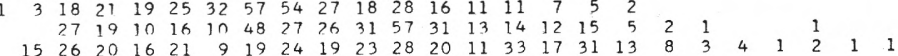

137394.0

176129.0

123943.0

84539.0

130453.0

1943

217565 .
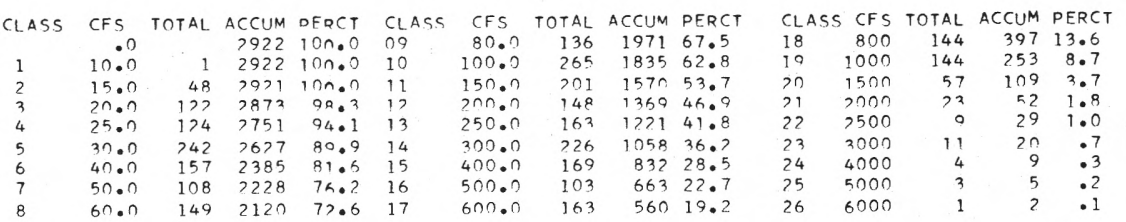

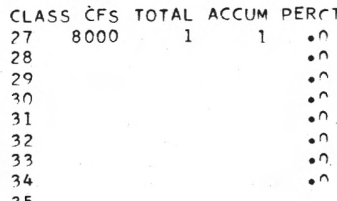

Fall Creek above Winberry Creek, near Lowell, Oreg.

STATION NUMBER $14-1505.00$ LOWEST MEAN DISCHARGF, IN CFS, FOR THE FOLLOWING NUMRFR OF IONSECUTIVE DAYS IN YEAR BFGINNING APRIL 1

\begin{tabular}{|c|c|c|c|c|c|c|c|c|c|c|c|}
\hline YEAR & 1 & 3 & 7 & 14 & 30 & 60 & 90 & 120 & 150 & 183 & 274 \\
\hline 1936 & 14.0 & 16.0 & 17.1 & 18.1 & 19.8 & 20.7 & 25.7 & $28 \cdot 2$ & 35.3 & 58.9 & 127.0 \\
\hline 1937 & 32.0 & 32.3 & $34 \cdot 1$ & 37.5 & 45.3 & 49.5 & 57.0 & $92 \cdot 5$ & 170.0 & $199 \cdot 0$ & 370.0 \\
\hline 1938 & 22.0 & 22.0 & $22 \cdot 6$ & 23.4 & 25.6 & 28.6 & 31.2 & 35.2 & 46.8 & $88 \cdot 8$ & $\begin{array}{l}213.0 \\
111.0\end{array}$ \\
\hline 1939 & 15.0 & 15.0 & 15.0 & 16.0 & 19.6 & 24.9 & 32.1 & $41 \cdot 3$ & 44.9 & $62 \cdot 3$ & $\begin{array}{l}111.0 \\
165.0\end{array}$ \\
\hline 1940 & 18.0 & 18.0 & $18 . ?$ & 18.6 & 20.3 & 24.0 & 27.2 & $29 \cdot 5$ & 35.8 & 55.8 & $\begin{array}{l}165.0 \\
277.0\end{array}$ \\
\hline 1941 & 34.0 & 35.0 & 36.9 & 39.1 & 42.7 & 56.2 & 69.0 & $81 \cdot 4$ & 109.0 & 133.0 & $\begin{array}{l}277.0 \\
457.0\end{array}$ \\
\hline 1942 & 28.0 & $28 \cdot 0$ & 28.1 & 29.1 & 31.2 & 33.7 & $41 \cdot 1$ & $61 \cdot 3$ & 114.0 & $177 \cdot 0$ & \\
\hline
\end{tabular}

Fall Creek above Winberry Creek,

STATION NUMBER $14-1505.00$ near Lowell, Oreg.

HIGHEST MEAN DISCHARGE, IN CFS, FOR THE FOLLOWING NUMBER OF CONSECUTIVE DAYS IN YEAR ENDING SEPTEMBER 30

\begin{tabular}{|c|c|c|c|c|c|c|c|c|c|c|c|}
\hline YEAR & 1 & 3 & 7 & 15 & 30 & 60 & 90 & 120 & 150 & 183 & 274 \\
\hline 1936 & 3900.0 & 3080.0 & 2510.0 & 2180.0 & 1480.0 & 1130.0 & 939.0 & 836.0 & 755.0 & 664.0 & 488.0 \\
\hline 1937 & 4800.0 & 3990.0 & 2600.0 & 1730.0 & 1290.0 & 1740.0 & 1040.0 & $874 \cdot 0$ & 798.0 & 679.0 & 495.0 \\
\hline 1928 & $4200 . ?$ & 2990.0 & $>110 . ?$ & $17 n n .0$ & $1>80.0$ & 1070.0 & 1030.0 & 947.0 & $929 \cdot n$ & 868.0 & 621.0 \\
\hline 1939 & 4500.0 & 3310.0 & 2150.0 & $1470 . n$ & 1120.0 & 1030.0 & 857.0 & 757.0 & 690.0 & 610.0 & 443.0 \\
\hline 1940 & $19 \cap 0.0$ & 1510.0 & $3390 . ?$ & 1200.0 & 1กBก.ก & $8 \cap 8.0$ & 62.8 .0 & 544.0 & $488 . n$ & 418.0 & 299.0 \\
\hline 1941 & 1840.0 & $1290 . ?$ & $905 . ?$ & 727.0 & $6 \cap 2.0$ & 511.0 & 479.0 & 408.0 & 351.0 & 320.0 & 276.0 \\
\hline 1942 & 5600.0 & 3140.0 & $1750 . ?$ & 1060.0 & $9>0.0$ & 835.0 & 738.0 & $651 \cdot 0$ & 583.0 & 529.0 & 455.0 \\
\hline 1943 & 9100.0 & 7540.0 & $4880 . ?$ & 3070.0 & 2210.0 & 1840.0 & 1600.0 & $1320 \cdot 0$ & 1180.0 & 1040.0 & 779.0 \\
\hline
\end{tabular}




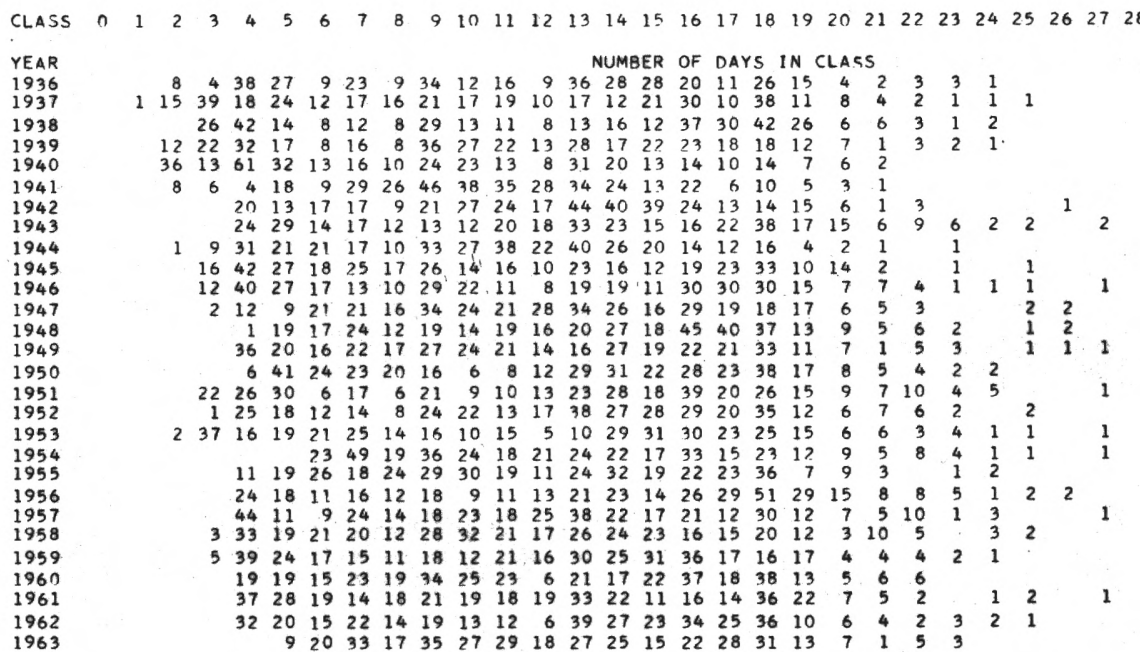

CFS-DAYS 179891 . 188381.0 235356.0 169073.0 113583.0 183339.0 309802.0 123067.0 178898.0 228201.0 215672.0 268921.0 217364.0 231630.0 275356.0 235766.0 226414.0 238701.0 191040.0 338302.0 233200.0 209793.0 192322.0

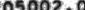
223747.0

222881.0

206120.0

Fall Creek below Winberry Creek near

STATION NUMBER $\quad 14-1510.00$

Creek below Winbe
Fall Creek, Oreg.

Summary for water years 1936-63
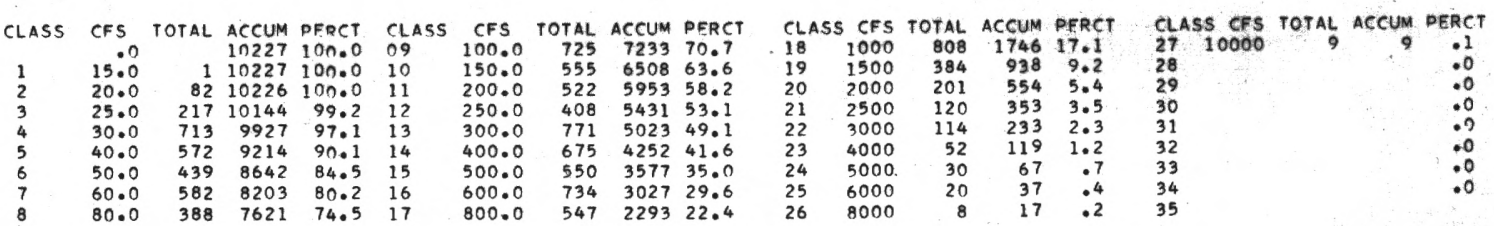

Fall Creek below Winberry Creek

STATION NUMBER $14-1510.00$

near $\mathrm{Fall} C \mathrm{Creek}$, Oreg.

LOWEST MEAN DISCHARGF, IN CFS, FOR THF. FOLLOWING NUMBER OF CONSECUTIVE DAYS IN YEAR BFGINNING APRIL 1

\begin{tabular}{|c|c|c|c|c|c|c|c|c|c|c|c|}
\hline YEAR & 1 & 3 & 7 & 14 & 30 & 60 & $9 n$ & 120 & 150 & 183 & 274 \\
\hline 1937 & 36.0 & $\begin{array}{l}36.7 \\
25.3\end{array}$ & $\begin{array}{l}37.9 \\
25.7\end{array}$ & 42.3 & 52.2 & 58.2 & $\begin{array}{l}68.3 \\
36.6\end{array}$ & $\begin{array}{r}115 \bullet 0 \\
41 \cdot 1\end{array}$ & $\begin{array}{r}220.0 \\
56.0\end{array}$ & $\begin{array}{l}265.0 \\
119.0\end{array}$ & $\begin{array}{l}490.0 \\
284.0\end{array}$ \\
\hline $\begin{array}{l}1938 \\
1939\end{array}$ & $\begin{array}{l}25.0 \\
20.0\end{array}$ & $\begin{array}{l}25.3 \\
20.7\end{array}$ & $\begin{array}{l}25.7 \\
20.7\end{array}$ & $\begin{array}{l}26 \cdot 7 \\
22 \cdot 0\end{array}$ & $\begin{array}{l}30.6 \\
26.0\end{array}$ & $\begin{array}{l}34.3 \\
32.4\end{array}$ & $\begin{array}{l}36.6 \\
38.9\end{array}$ & $\begin{array}{l}41 \cdot 1 \\
50 \cdot 2\end{array}$ & $\begin{array}{l}56.0 \\
51.4\end{array}$ & 79.5 & 14.7 .0 \\
\hline 1940 & 20.0 & 20.0 & 20.0 & 20.2 & 22.1 & 26.3 & 30.5 & $33 \cdot 8$ & 43.4 & 71.2 & 232.0 \\
\hline 1941 & 41.0 & 41.0 & $42 \cdot 6$ & 47.7 & 58.2 & 74.8 & 85.6 & $102 \cdot 0$ & 140.0 & 176.0 & 389.0 \\
\hline 1942 & 31.0 & 31.0 & 31.3 & 32.0 & 33.7 & 36.9 & 44.9 & 71.1 & 144.0 & 242.0 & 638.0 \\
\hline 1943 & 38.0 & 38.7 & 39.4 & 40.4 & 41.2 & $52 \cdot 6$ & 61.8 & $115 \cdot 0$ & 177.0 & $209 \cdot 0$ & 282.0 \\
\hline 1944 & 24.0 & 24.7 & 26.4 & 27.8 & 32.8 & 40.3 & 40.7 & $46 \cdot 3$ & 60.4 & 77.2 & 189.0 \\
\hline $\begin{array}{l}1946 \\
1947\end{array}$ & $\begin{array}{l}28.0 \\
49.0\end{array}$ & 49.0 & $\begin{array}{l}30.4 \\
49.6\end{array}$ & $\begin{array}{l}34.2 \\
51.5\end{array}$ & $\begin{array}{l}36.2 \\
62.7\end{array}$ & $\begin{array}{l}40.7 \\
66.1\end{array}$ & 90.3 & $127 \cdot 0$ & 158.0 & 194.0 & 512.0 \\
\hline $\begin{array}{l}1948 \\
1949\end{array}$ & $\begin{array}{l}38.0 \\
30.0\end{array}$ & $\begin{array}{l}40.3 \\
30.3\end{array}$ & $\begin{array}{l}42 \cdot 1 \\
31.3\end{array}$ & $\begin{array}{l}43.0 \\
31.7\end{array}$ & $\begin{array}{l}50.7 \\
34.5\end{array}$ & $\begin{array}{l}60.6 \\
42.6\end{array}$ & $\begin{array}{l}83.5 \\
47.6\end{array}$ & $\begin{array}{r}102.0 \\
61.9\end{array}$ & $\begin{array}{r}132.0 \\
71.9\end{array}$ & $\begin{array}{r}187.0 \\
86.4\end{array}$ & $\begin{array}{l}460.0 \\
274.0\end{array}$ \\
\hline 1950 & 38.0 & 39.0 & 41.6 & 43.2 & 45.3 & 53.7 & 68.8 & $120 \cdot 0$ & 175.0 & 256.0 & 639.0 \\
\hline $\begin{array}{l}1951 \\
1952\end{array}$ & $\begin{array}{l}25.0 \\
24.0\end{array}$ & $\begin{array}{l}25.3 \\
24.3\end{array}$ & $\begin{array}{l}25.7 \\
25.0\end{array}$ & $\begin{array}{l}26.7 \\
25.9\end{array}$ & $\begin{array}{l}32.0 \\
27.5\end{array}$ & $\begin{array}{l}34.8 \\
29.9\end{array}$ & 40.0 & $\begin{array}{l}54.5 \\
39.2\end{array}$ & $\begin{array}{l}93.9 \\
52.5\end{array}$ & $\begin{array}{r}159.0 \\
95.7\end{array}$ & $\begin{array}{l}463.0 \\
201.0\end{array}$ \\
\hline 1953 & 40.0 & 40.7 & 41.7 & 42.3 & 50.6 & 60.5 & $\begin{array}{l}36.0 \\
69.6\end{array}$ & 82.4 & 103.0 & 240.0 & 629.0 \\
\hline 1954 & 51.0 & 51.0 & $51 \cdot 3$ & 53.7 & 66.6 & 71.4 & 78.1 & 95.4 & 111.0 & 140.0 & 250.0 \\
\hline 1955 & 36.0 & 36.0 & 36.7 & 37.8 & 41.6 & 54.2 & 71.0 & $106 \cdot 0$ & 153.0 & 239.0 & 790.0 \\
\hline 1956 & 31.0 & 31.7 & 33.0 & 34.2 & 36.6 & 41.1 & 51.0 & 82.2 & 159.0 & 241.0 & 459.0 \\
\hline 1957 & 30.0 & 30.3 & 32.7 & 33.1 & 34.1 & 44.3 & 57.0 & 69.3 & 88.7 & 126.0 & 368.0 \\
\hline 1958 & 29.0 & 29.0 & 29.4 & 32.4 & 34.9 & 40.0 & 42.7 & 55.5 & 95.5 & 123.0 & 378.0 \\
\hline 1959 & 31.0 & 31.0 & 32.0 & 33.9 & 36.6 & 44.5 & 58.9 & 95.2 & 128.0 & 152.0 & 242.0 \\
\hline 1960 & 33.0 & 33.7 & 34.3 & 35.0 & 39.2 & 52.2 & 56.3 & $65 \cdot 2$ & 83.5 & 219.0 & 429.0 \\
\hline 1961 & 31.0 & 31.0 & 31.9 & 32.8 & 36.8 & 45.4 & 48.3 & 59.9 & 85.7 & 137.0 & 458.0 \\
\hline 1962 & 32.0 & 32.0 & 32.9 & 34.2 & 40.0 & 50.0 & 55.0 & 78.1 & 119.0 & 198.0 & 352.0 \\
\hline
\end{tabular}




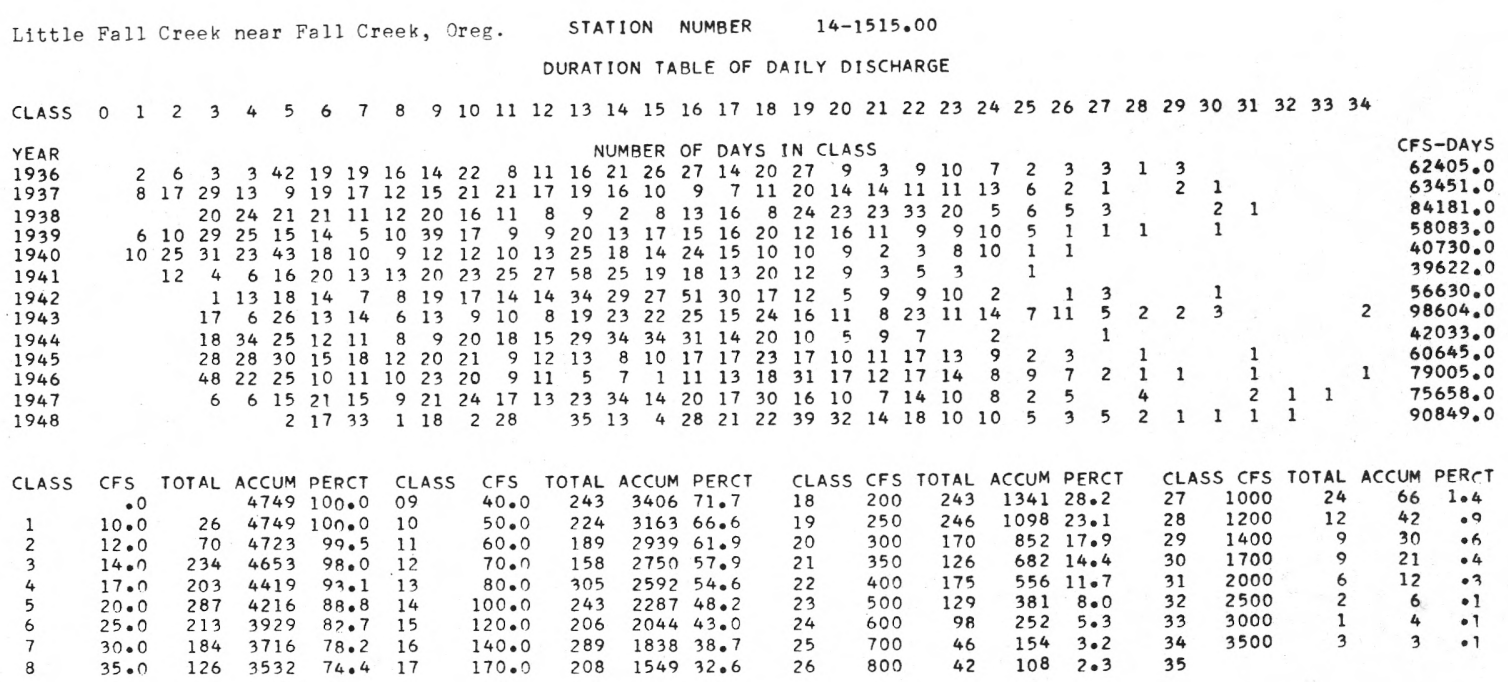

Little Fall Creek near Fall Creek, Oreg. STATION NUMBER 14-1515.00

LOWEST MEAN DISCHARGF, IN CFS, FOR THE FOLLOWING NUMBER OF CONSECUTIVE DAYS IN YEAR BEGINNING APRIL 1

\begin{tabular}{|c|c|c|c|c|c|c|c|c|c|c|c|}
\hline YEAR & 1 & 3 & 7 & 14 & 30 & 60 & 90 & 120 & 150 & 183 & 274 \\
\hline $\begin{array}{l}1936 \\
1937\end{array}$ & $\begin{array}{l}10.0 \\
24.0\end{array}$ & $\begin{array}{l}10.7 \\
24.0\end{array}$ & $\begin{array}{l}10.9 \\
24.4\end{array}$ & $\begin{array}{l}11.6 \\
26.2\end{array}$ & $\begin{array}{l}12.8 \\
29.0\end{array}$ & $\begin{array}{l}14.1 \\
31.6\end{array}$ & $\begin{array}{l}17.0 \\
35.2\end{array}$ & $\begin{array}{l}19.4 \\
48.2\end{array}$ & $\begin{array}{l}23.8 \\
78.2\end{array}$ & $\begin{array}{l}30.7 \\
89.0\end{array}$ & $\begin{array}{r}60.1 \\
174.0\end{array}$ \\
\hline 1938 & 15.0 & 15.0 & 15.1 & 15.5 & 17.8 & 18.2 & 19.1 & $21 \cdot 8$ & 26.8 & 41.0 & 100.0 \\
\hline 1939 & 11.0 & 11.0 & 11.1 & 11.8 & 13.6 & 15.8 & 18.4 & $22 \cdot 6$ & 23.3 & 29.9 & 53.4 \\
\hline 1940 & 10.0 & 10.0 & 10.3 & 11.0 & 12.2 & 14.5 & 15.9 & 17.5 & 22.1 & 31.7 & 82.2 \\
\hline 1941 & 21.0 & 21.3 & 22.4 & 23.5 & 25.1 & 33.9 & 36.2 & $41 \cdot 4$ & 50.7 & 60.1 & 123.0 \\
\hline 1942 & 15.0 & $15 \cdot 3$ & 15.7 & 16.3 & 17.5 & 19.2 & 24.3 & $34 \cdot 1$ & $51 \cdot 3$ & 73.3 & 197.0 \\
\hline 1943 & 18.0 & 18.0 & $18 \cdot 3$ & 19.1 & 20.2 & 25.7 & 30.1 & $46 \cdot 0$ & 67.1 & 80.9 & 99.0 \\
\hline 1944 & 14.0 & 14.0 & 14.0 & 14.2 & 16.1 & 17.6 & 18.2 & $20 \cdot 9$ & 25.4 & 30.9 & 65.7 \\
\hline 1945 & 14.0 & 14.7 & 15.0 & 15.4 & 17.6 & 20.9 & 21.5 & $24 \cdot 7$ & 31.8 & 62.0 & 186.0 \\
\hline 1746 & 14.0 & 14.0 & 14.4 & 15.2 & 16.5 & 17.3 & 18.5 & $22 \cdot 7$ & 28.2 & 37.7 & 149.0 \\
\hline 1947 & 23.0 & $23 . n$ & 23.0 & 23.6 & $28 \cdot 1$ & 29.9 & 37.0 & $47 \cdot 6$ & 56.0 & 72.1 & 173.0 \\
\hline
\end{tabular}

Little Fall Creek near Fall Creek, Oreg. STATION NUMBER 14-1515.00

HIGHEST MEAN DISCHARGE, IN CFS, FOR THE FOLLOWING NUMRER OF CONSECUTIVE DAYS IN YEAR ENDING SEPTEMBER 30

\begin{tabular}{|c|c|c|c|c|c|c|c|c|c|c|c|}
\hline YEAR & 1 & 3 & 7 & 15 & 30 & 60 & 90 & 120 & 150 & 183 & 274 \\
\hline 1936 & 1590.0 & $1320 \cdot n$ & 1130.0 & 1000.0 & 698.0 & 506.0 & 429.0 & $369 \cdot 0$ & 330.0 & 294.0 & 219.0 \\
\hline 1937 & 1990.0 & 1660.0 & 1170.0 & 827.0 & 621.0 & 574.0 & 482.0 & $402 \cdot 0$ & 361.0 & 309.0 & 226.0 \\
\hline 1938 & 2240.0 & 1710.0 & $1190 . n$ & 901.0 & 648.0 & 536.0 & 513.0 & $471 \cdot 0$ & 456.0 & 416.0 & 300.0 \\
\hline 1939 & 1760.0 & 1390.0 & 958.0 & 657.0 & 523.0 & 473.0 & 397.0 & 357.0 & 322.0 & 285.0 & 206.0 \\
\hline 1940 & 820.0 & 714.0 & 671.0 & 602.0 & 523.0 & 370.0 & 289.0 & $256 \cdot 0$ & 229.0 & 199.0 & 143.0 \\
\hline 1941 & 700.0 & 548.0 & 393.0 & 332.0 & 286.0 & 251.0 & 233.0 & $201 \cdot 0$ & 176.0 & 160.0 & 135.0 \\
\hline 1942 & 1720.0 & $1110 \cdot n$ & 761.0 & $548 \cdot 0$ & 428.0 & 372.0 & 327.0 & $292 \cdot 0$ & $259 \cdot 0$ & 236.0 & 195.0 \\
\hline 1943 & 3820.0 & $3190 \cdot 0$ & 2150.0 & 1430.0 & 1050.0 & 842.0 & 727.0 & $601 \cdot 0$ & 530.0 & 469.0 & 351.0 \\
\hline 1944 & 1180.0 & 733.0 & 434.0 & 351.0 & 258.0 & 219.0 & 223.0 & $198 \cdot 0$ & 181.0 & 178.0 & 146.0 \\
\hline 1945 & 2210.0 & 1460.0 & 950.0 & 722.0 & 471.0 & $412 \cdot 0$ & 402.0 & 379.0 & 346.0 & 299.0 & 214.0 \\
\hline 1946 & 3810.0 & 2390.0 & 1410.0 & 1100.0 & 757.0 & 575.0 & 533.0 & 497.0 & 461.0 & 401.0 & 282.0 \\
\hline 1947 & 3250.0 & $2790 . n$ & 1790.0 & 1010.0 & 798.0 & 493.0 & 473.0 & $403 \cdot 0$ & 395.0 & 354.0 & 265.0 \\
\hline 1948 & 2590.0 & $1850 \cdot n$ & 1230.0 & 816.0 & 577.0 & 480.0 & 444.0 & $424 \cdot 0$ & 412.0 & 397.0 & 319.0 \\
\hline
\end{tabular}


MIDDLE FORK WHILAMETTE RIVER AT JASPER OREG.

STATION NUMBER $\quad 14-1520.00$

DURATION TABLE OF DAILY DISCHARGE

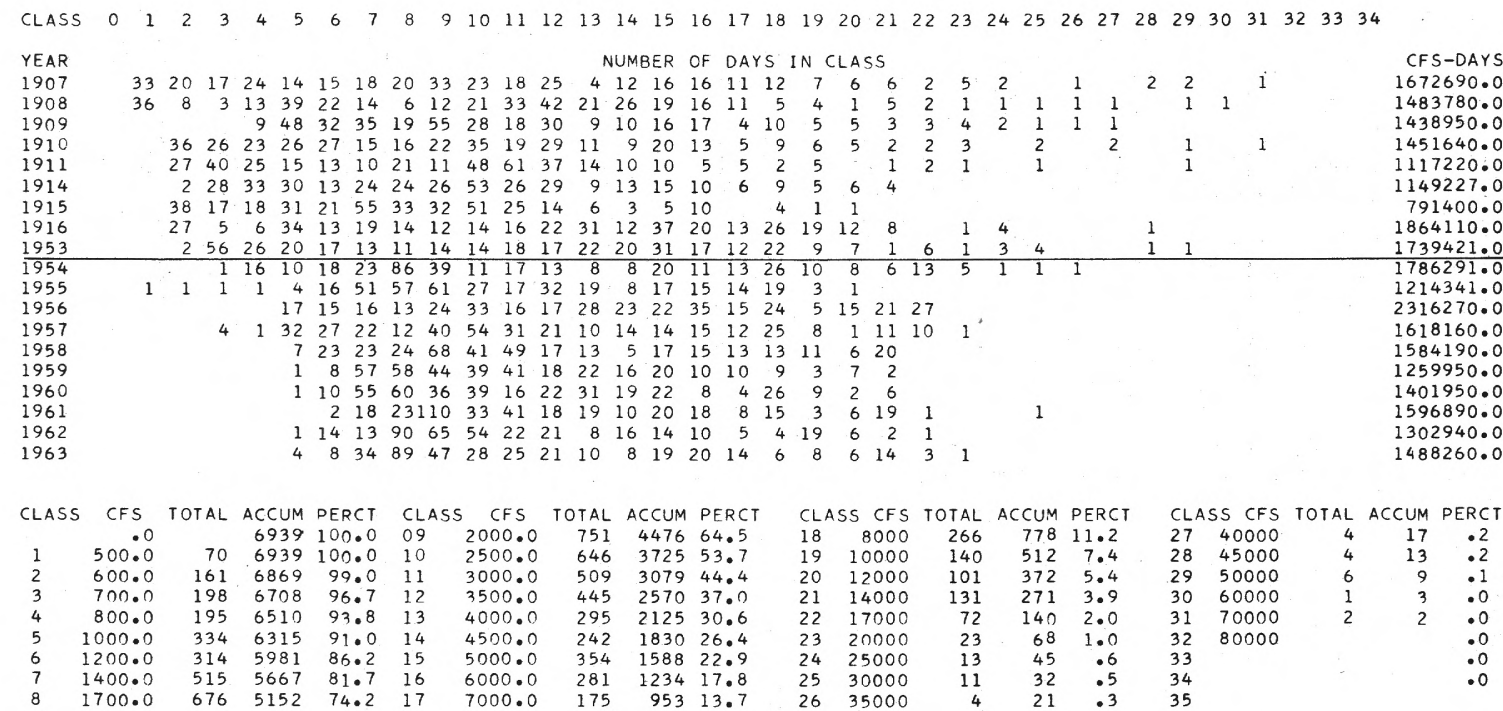

Middle Fork Willamette River

STATION NUMBER

$14-1520.00$

Summary for water years 1907-11, 1914-16, 1953

\begin{tabular}{|c|c|c|c|c|c|c|c|}
\hline LASS & GFS & TOTAL & ACCUM & PERCT & CLA: & CFS & or \\
\hline & .0 & & 3287 & 100.0 & 09 & 2000.0 & \\
\hline 1 & 500.0 & 69 & 3287 & 100.0 & 10 & 2500.0 & \\
\hline 2 & 600.0 & 160 & 3218 & 97.9 & 11 & 3000.0 & \\
\hline 3 & 700.0 & 192 & 3058 & 93.0 & 12 & 3500.0 & \\
\hline 4 & 800.0 & 177 & 2866 & 87.2 & 13 & 4000.0 & \\
\hline 5 & 1000.0 & 2 & 26 & & 14 & 4500.0 & \\
\hline 6 & 1200.0 & 173 & 2432 & 74.0 & 15 & 5000.0 & \\
\hline 7 & 1400.0 & 203 & 2259 & 68.7 & 16 & 6000.0 & \\
\hline 8 & 1700.0 & 164 & 2056 & 62.5 & 17 & 7000.0 & \\
\hline
\end{tabular}
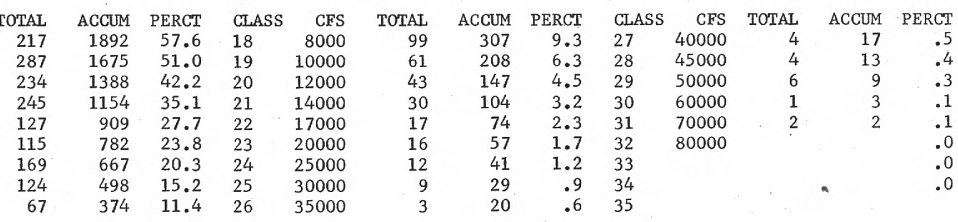

Middle Fork Willamette River

STATION NUMBER

$14-1520.00$

Summary for water years 1954-63

\begin{tabular}{lrrrrrr} 
CLASS & \multicolumn{1}{c}{ CFS } & TOTAL & ACCUM & PERCT & \multicolumn{2}{l}{ CLASS CFS } \\
& .0 & & 3652 & 100.0 & 09 & 2000.0 \\
1 & 500.0 & 1 & 3652 & 100.0 & 10 & 2500.0 \\
2 & 600.0 & 1 & 3651 & 100.0 & 11 & 3000.0 \\
3 & 700.0 & 6 & 3650 & 99.9 & 12 & 3500.0 \\
4 & 800.0 & 18 & 3644 & 99.8 & 13 & 4000.0 \\
5 & 1000.0 & 77 & 3626 & 99.3 & 14 & 4500.0 \\
6 & 1200.0 & 141 & 3549 & 97.2 & 15 & 5000.0 \\
7 & 1400.0 & 312 & 3408 & 93.3 & 16 & 6000.0 \\
8 & 1700.0 & 512 & 3096 & 84.8 & 17 & 7000.0
\end{tabular}

$\begin{array}{rrrll}\text { TOTAL } & \text { ACCUM } & \text { PERCT } & \text { CLASS } \\ 534 & 2584 & 70.8 & 18 & \\ 359 & 2050 & 56.1 & 19 & 100 \\ 275 & 1691 & 46.3 & 20 & 1 \\ 200 & 1416 & 38.8 & 21 & 140 \\ 168 & 1216 & 33.3 & 22 & 170 \\ 127 & 1048 & 28.7 & 23 & 200 \\ 185 & 921 & 25.2 & 24 & 250 \\ 157 & 736 & 20.2 & 25 & 300 \\ 108 & 579 & 15.9 & 26 & 350\end{array}$

CFS
8000
10000
12000
14000
17000
20000
25000
30000
35000

$\begin{array}{rrrllllr}\text { TOTAL } & \text { ACCUM } & \text { PERCT } & \text { CLASS } & \text { CFS } & \text { TOTAL } & \text { ACCUM } & \text { PERCT } \\ 167 & 471 & 12.9 & 27 & 40000 & & & .0 \\ 79 & 304 & 8.3 & 28 & 45000 & & .0 \\ 58 & 225 & 6.2 & 29 & 50000 & & .0 \\ 101 & 167 & 4.6 & 30 & 60000 & & .0 \\ 55 & 66 & 1.8 & 31 & 70000 & & .0 \\ 7 & 11 & .3 & 32 & 80000 & & .0 \\ 1 & 4 & .1 & 33 & & & .0 \\ 2 & 3 & .1 & 34 & & & .0 \\ 1 & 1 & .0 & 35 & & & & \end{array}$


MIDDLE FORK WHIAMETHE RTVER AT JASPBR ORBG.

STATION NUMBER 14-1520.00 LOWEST MEAN DISCHARGE, IN CFS, FOR THE FOLLOWING NUMBER OF CONSECUTIVE DAYS IN YEAR BEGINNING APRIL 1

\begin{tabular}{|c|c|c|c|c|c|c|c|c|c|c|c|}
\hline YEAR & 1 & 3 & 7 & 14 & 30 & 60 & 90 & 120 & 150 & 183 & 274 \\
\hline 1907 & 530.0 & 530.0 & $\begin{array}{r}530.0 \\
937.0\end{array}$ & $\begin{array}{l}530.0 \\
968.0\end{array}$ & 564.0 & 572.0 & 589.0 & 620.0 & 723.0 & 937.0 & 3140.0 \\
\hline $\begin{array}{l}1908 \\
1909\end{array}$ & $\begin{array}{r}880.0 \\
1050.0\end{array}$ & $\begin{array}{r}907.0 \\
1050.0\end{array}$ & $\begin{array}{r}937.0 \\
1080.0\end{array}$ & $\begin{array}{r}968.0 \\
1110.0\end{array}$ & $\begin{array}{r}988.0 \\
1140.0\end{array}$ & $\begin{array}{l}1060.0 \\
1160.0\end{array}$ & 1160.0 & $\begin{array}{l}1590.0 \\
1290.0\end{array}$ & $\begin{array}{l}1780.0 \\
1460.0\end{array}$ & $\begin{array}{l}1940.0 \\
1800.0\end{array}$ & $\begin{array}{l}2770.0 \\
3470.0\end{array}$ \\
\hline $\begin{array}{l}1909 \\
1910\end{array}$ & $\begin{array}{r}1050.0 \\
670.0\end{array}$ & $\begin{array}{r}1050.0 \\
670.0\end{array}$ & $\begin{array}{r}1080.0 \\
670.0\end{array}$ & $\begin{array}{r}1110.0 \\
670.0\end{array}$ & $\begin{array}{r}1140.0 \\
676.0\end{array}$ & $\begin{array}{r}1160.0 \\
715.0\end{array}$ & $\begin{array}{r}1190.0 \\
770.0\end{array}$ & $\begin{array}{r}1290.0 \\
790.0\end{array}$ & 883.0 & $\begin{array}{l}1800.0 \\
1090.0\end{array}$ & $\begin{array}{l}3470.0 \\
2510.0\end{array}$ \\
\hline $\begin{array}{l}1910 \\
1914\end{array}$ & 670.0 & 685.0 & 709.0 & 734.0 & 753.0 & 842.0 & 984.0 & 1120.0 & 1290.0 & 1330.0 & $\begin{array}{l}2510.0 \\
1790.0\end{array}$ \\
\hline 1915 & 610.0 & 610.0 & 610.0 & 623.0 & 635.0 & 643.0 & 677.0 & 747.0 & 873.0 & 1440.0 & 2950.0 \\
\hline 1916 & 850.0 & 850.0 & 850.0 & 868.0 & 905.0 & 1020.0 & 1130.0 & $1270 \cdot 0$ & 1810.0 & 2210.0 & 3020.0 \\
\hline 195 & 750.0 & 753.0 & 768.0 & 788.0 & 885.0 & 1020.0 & 1090.0 & 1190.0 & 1500.0 & 2440.0 & 4720.0 \\
\hline 1954 & 543.0 & 743.0 & 1070.0 & 1240.0 & 1430.0 & 1710.0 & 1800.0 & 1810.0 & 1820.0 & 1890.0 & 2260.0 \\
\hline 1955 & 1320.0 & 1330.0 & 1360.0 & 1410.0 & 1480.0 & 1670.0 & 1870.0 & 2440.0 & 3030.0 & 3280.0 & 5580.0 \\
\hline 1956 & 760.0 & 768.0 & 877.0 & 1110.0 & 1190.0 & 1420.0 & 1610.0 & 1810.0 & 2400.0 & 3020.0 & $395 \mathrm{C} .0$ \\
\hline 1957 & 1060.0 & 1110.0 & 1140.0 & 1150.0 & 1170.0 & 1240.0 & 1550.0 & 1810.0 & 1970.0 & 2170.0 & 3130.0 \\
\hline 1958 & 1170.0 & 1180.0 & 1190.0 & 1220.0 & 1300.0 & 1480.0 & 1900.0 & $2190 \cdot 0$ & 2420.0 & 2630.0 & 3370.0 \\
\hline 1959 & 1150.0 & 1180.0 & 1320.0 & 1400.0 & 1440.0 & 1490.0 & 1570.0 & 1770.0 & 2130.0 & 2200.0 & 2410.0 \\
\hline 1960 & 1300.0 & 1340.0 & 1380.0 & 148010 & 1570.0 & 1640.0 & 1730.0 & 1900.0 & 2160.0 & 2830.0 & 3780.0 \\
\hline 1961 & 1170.0 & 1220.0 & 1380.0 & 1460.0 & 1710.0 & 2030.0 & 2080.0 & $2090 \cdot 0$ & 2090.0 & 2270.0 & 3450.0 \\
\hline 1962 & 1110.0 & 1140.0 & 1190.0 & 1300.0 & 1650.0 & 1770.0 & 1820.0 & 2050.0 & 2470.0 & 2720.0 & 376 \\
\hline
\end{tabular}

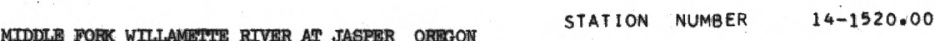
HIGHEST MEAN DISCHARGE, IN CFS, FOR THE FOLLOWING NUMBER OF CONSECUTIVE DAYS IN YEAR ENDING SEPTEMBER 30

\begin{tabular}{|c|c|c|c|c|c|c|c|c|c|c|c|}
\hline YEAR & 1 & 3 & 7 & 15 & 30 & 60 & 90 & 120 & 150 & 183 & 274 \\
\hline 1907 & 78800.0 & $63500 . n$ & $43400 \cdot 0$ & 28600.0 & $16800 \cdot 0$ & 12800.0 & 10400.0 & 9540.0 & 8690.0 & 7930.0 & 5870.0 \\
\hline 1908 & 65300.0 & 53400.0 & 41200.0 & 24300.0 & 15700.0 & 10200.0 & 8210.0 & 7470.0 & 6880.0 & 6420.0 & 5140.0 \\
\hline 1909 & 40400.0 & 31500.0 & 30000.0 & 19500.0 & 15000.0 & 11300.0 & 9160.0 & $7600 \cdot 0$ & 6630.0 & 6040.0 & 4850.0 \\
\hline 1910 & 77300.0 & $59700 . n$ & 37100.0 & 22300.0 & 14900.0 & 8950.0 & 7620.0 & $8410 \cdot 0$ & 7500.0 & 6750.0 & 5040.0 \\
\hline 1911 & 51100.0 & 34700.0 & 21600.0 & 14600.0 & 9750.0 & 6810.0 & 5820.0 & 5070.0 & 4760.0 & 4660.0 & 3780.0 \\
\hline 1914 & 16600.0 & 16000.0 & $136 n 0.0$ & 10200.0 & 7300.0 & 6880.0 & 6100.0 & 5680.0 & 5100.0 & 4760.0 & 3870.0 \\
\hline 1915 & 12600.0 & 9690.0 & 6890.0 & 6050.0 & 4520.0 & 3710.0 & 3400.0 & 3310.0 & 3380.0 & 3090.0 & $261 C, 0$ \\
\hline 1916 & 48400.0 & $33800 \cdot 0$ & $2350 n \cdot 0$ & 17600.0 & 12300.0 & 10600.0 & 8830.0 & 8510.0 & 8280.0 & 792.0 .0 & 6470.0 \\
\hline 1953 & 50800.0 & 43500.0 & 29500.0 & 21000.0 & 19200.0 & 13100.0 & 10700.0 & $9230 \cdot 0$ & 8760.0 & 8080,0 & 6080.0 \\
\hline 1954 & 37600.0 & 32000.0 & 24300.0 & 18200.0 & 16300.0 & 12000.0 & 11600.0 & $10100 \cdot 0$ & $919 \pi .0$ & 7880.0 & 6000.0 \\
\hline 1955 & 12600.0 & 10600.0 & 8960.0 & 8650.0 & 7670.0 & 5940.0 & 5840.0 & 5170.0 & 4690.0 & 476000 & 3830.0 \\
\hline 1956 & 19800.0 & 19600.0 & 19200.0 & 18100.0 & 17400.0 & 16100.0 & 13500.0 & $11400 \cdot 0$ & 10000.0 & 9270.0 & 7850.0 \\
\hline 1957 & 21400.0 & 18300.0 & 17800.0 & 15200.0 & 11700.0 & 8990.0 & 7040.0 & $7530 \cdot 0$ & 7000.0 & 6810.0 & 5330.0 \\
\hline 1958 & 16300.0 & 16300.0 & 15300.0 & 13500.0 & 10800.0 & 9830.0 & 9140.0 & 7740.0 & 6710.0 & 619 & 5130.0 \\
\hline 1959 & $14200 \cdot 0$ & 13700.0 & $12100 \cdot 0$ & 9550.0 & 7410.0 & 6020.0 & 6130.0 & $5590 \cdot 0$ & 5070.0 & 4720.0 & 4040.0 \\
\hline 1960 & 16600.0 & $15000 . n$ & 12300.0 & 9260.0 & 8400.0 & 7360.0 & 6930.0 & 6650.0 & 5940.0 & 5310.0 & 4520.0 \\
\hline 1961 & 30100.0 & 18700.0 & 16700.0 & 16100.0 & 12400.0 & 9220.0 & 7110.0 & $7280 \cdot 0$ & 6830.0 & 6170.0 & 5110 \\
\hline 1962 & 18100.0 & 14700.0 & 13300.0 & 11700.0 & 9250.0 & 8230.0 & 6280.0 & $5330 \cdot 0$ & 5060.0 & 4930.0 & 4140.0 \\
\hline 963 & 20200.0 & $18900 . ?$ & 15600.0 & 14500.0 & 12400.0 & 8750.0 & 7090.0 & $5810 \cdot 0$ & $5290 . Q$ & 5290.0 & 4780.0 \\
\hline
\end{tabular}

Coast Fork Willamette River at London, Oreg.

\section{STATION NUMBER $\quad 14-1525.00$}

DURATION TABLE OF DAILY DISCHARGE

CLASS $0 \begin{array}{llllllllllllllllllllllllllllllllllllllllllll} & 0 & 2 & 3 & 4 & 5 & 6 & 7 & 8 & 9 & 10 & 11 & 12 & 13 & 14 & 15 & 16 & 17 & 18 & 19 & 20 & 21 & 22 & 23 & 24 & 25 & 26 & 27 & 28 & 29 & 30 & 31 & 32 & 33 & 34\end{array}$

YEAR
1936
1937
1938
1939
1940
1941
1942
1943
1944
1945
1946
1947
1948
1949
1950
1951
1952
1953
1954
1955
1956
1957
1958
1959
1960
1961
1962
1963

$$
\text { NUMBER OF DAYS IN CLASS }
$$

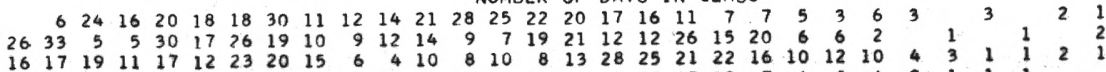

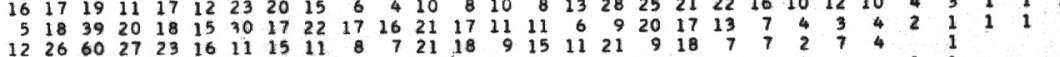

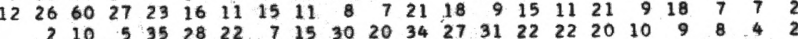

$\begin{array}{rrrrrrrrrrrrrrrrrrrrrrrrrrrrrrr}10 & 5 & 35 & 28 & 22 & 7 & 15 & 30 & 20 & 34 & 27 & 31 & 22 & 22 & 20 & 10 & 9 & 8 & 4 & 2 & & 10 & 1 & & & 1 \\ 11 & 15 & 23 & 19 & 28 & 15 & 9 & 10 & 11 & 29 & 19 & 31 & 37 & 23 & 21 & 12 & 16 & 13 & 3 & 4 & 4 & 5 & 2 & 3 & 1 & & \\ 16 & 18 & 28 & 18 & 19 & 8 & 8 & 13 & 8 & 23 & 18 & 16 & 21 & 18 & 23 & 10 & 18 & 17 & 15 & 10 & 9 & 10 & 8 & 2 & 2 & 3 & 2 & 3 & 1\end{array}$

$\begin{array}{llllllllllllllllllll}30 & 31 & 16 & 9 & 19 & 19 & 20 & 19 & 18 & 36 & 31 & 19 & 15 & 16 & 14 & 9 & 18 & 12 & 4\end{array}$

$\begin{array}{llllllllllllllllllllll}43 & 26 & 26 & 23 & 26 & 14 & 16 & 12 & 12 & 13 & 14 & 10 & 24 & 18 & 14 & 12 & 15 & 16 & 10 & 10 & 3\end{array}$

$\begin{array}{llllllllllllllllllllllllllllll}7 & 3 & 32 & 21 & 21 & 26 & 13 & 19 & 29 & 21 & 13 & 17 & 20 & 19 & 13 & 20 & 12 & 12 & 4 & 5 & 7 & 4 & 1 & 1 & 1 & 1\end{array}$

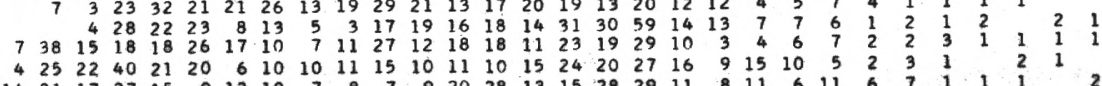

$\begin{array}{rrrrrrrrrrrrrrrrrrrrrrrrrrrrrrrrrr}14 & 31 & 17 & 27 & 15 & 9 & 12 & 10 & 7 & 8 & 7 & 9 & 20 & 28 & 13 & 15 & 28 & 29 & 11 & 8 & 11 & 6 & 11 & 6 & 7 & 1 \\ & 18 & 16 & 24 & 10 & 44 & 25 & 22 & 13 & 6 & 16 & 17 & 11 & 12 & 19 & 24 & 23 & 33 & 16 & 10 & 9 & 6 & 4 & 5 & 8 & 5\end{array}$ $\begin{array}{lllllllllllllllllllllllllllllll}28 & 35 & 33 & 12 & 20 & 14 & 5 & 6 & 6 & 8 & 10 & 11 & 26 & 20 & 21 & 16 & 27 & 16 & 14 & 7 & 4 & 14 & 4 & 2 & 2\end{array}$

\begin{tabular}{rrrrrrrrrrrrrrrrrrrrrrrrrr}
5 & 22 & 29 & 29 & 46 & 14 & 17 & 25 & 13 & 23 & 5 & 7 & 15 & 11 & 19 & 15 & 33 & 16 & 11 & 11 & 3 & 11 & 4 & 8 \\
\hline
\end{tabular}

$\begin{array}{lllllllllllllllllllllllllllllllllllll}4 & 18 & 20 & 11 & 12 & 23 & 13 & 6 & 8 & 9 & 25 & 14 & 11 & 14 & 15 & 20 & 15 & 20 & 28 & 19 & 13 & 8 & 11 & 11 & 3 & 6 & 2 & 3 & 1 & 2 & 1\end{array}$

$\begin{array}{rrrrrrrrrrrrrrrrrrrrrrrrrrrrrrrrrr}2 & 7 & 34 & 18 & 20 & 14 & 14 & 10 & 11 & 10 & 20 & 32 & 22 & 12 & 20 & 14 & 28 & 8 & 17 & 13 & 8 & 5 & 9 & 5 & 3 & 4 & 3 & 2 & 2 \\ 2 & 31 & 29 & 22 & 7 & 27 & 13 & 13 & 25 & 14 & 19 & 7 & 9 & 20 & 18 & 16 & 17 & 22 & 13 & 10 & 7 & 6 & 4 & 4 & 3 & 1 & 3 & & 2 & 1\end{array}$

$\begin{array}{lllllllllllllllllllllllllll}24 & 39 & 29 & 9 & 20 & 12 & 10 & 9 & 18 & 26 & 16 & 25 & 23 & 13 & 27 & 13 & 15 & 9 & 8 & 5 & 3 & 3 & 3 & 2 & 3 & 1\end{array}$

$\begin{array}{rllllllllllllllllllllllllll}1 & 36 & 16 & 39 & 20 & 31 & 19 & 13 & 7 & 9 & 11 & 15 & 10 & 22 & 14 & 24 & 9 & 21 & 16 & 13 & 2 & 5 & 5 & 4 & 2 \\ & 35 & \end{array}$

$\begin{array}{rrrrrrrrrrrrrrrrrrrrrrrrrr}20 & 35 & 21 & 22 & 9 & 20 & 14 & 12 & 19 & 17 & 13 & 16 & 23 & 19 & 9 & 14 & 14 & 11 & 14 & 12 & 6 & 10 & 7 & 2 & 2 & \\ 3 & 15 & 14 & 36 & 15 & 19 & 25 & 14 & 8 & 5 & 7 & 20 & 28 & 30 & 18 & 23 & 19 & 25 & 12 & 8 & 2 & 3 & 4 & 3 & 2 & 3 \\ & 6 & 17 & 25 & 16 & 24 & 14 & 25 & 17 & 10 & 30 & 18 & 23 & 21 & 17 & 17 & 10 & 20 & 18 & 12 & 7 & 7 & 4 & 3 & 1 & 1\end{array}$

\begin{abstract}
$\begin{array}{llllllllllllllllllllll}6 & 17 & 25 & 16 & 24 & 14 & 25 & 17 & 10 & 30 & 18 & 23 & 21 & 17 & 17 & 10 & 20 & 18 & 12\end{array}$
\end{abstract}
STATION NUMBER 14-1.525.00

\begin{tabular}{ccccccccccccccc} 
CLASS & CFS & TOTAL ACCUM PFRCT & CLASS & CFS & TOTAL & ACCUM PERCT & \multicolumn{2}{c}{ CLASS CFS TOTAL ACCUM PERCT } \\
& .0 & & 10227 & 100.0 & 09 & 50.0 & 390 & 6578 & 64.3 & 18 & 250 & 446 & 2541 & 24.8 \\
1 & 10.0 & 61 & 10227 & 100.0 & 10 & 60.0 & 347 & 6188 & 60.5 & 19 & 300 & 621 & 2095 & 20.5 \\
2 & 12.0 & 173 & 10166 & 99.4 & 11 & 70.0 & 314 & 5841 & 57.1 & 20 & 400 & 392 & 1474 & 14.4 \\
3 & 14.0 & 659 & 9993 & 97.7 & 12 & 80.0 & 552 & 5527 & 54.0 & 21 & 500 & 287 & 1082 & 10.6 \\
4 & 17.0 & 502 & 9334 & 91.3 & 13 & 100.0 & 444 & 4975 & 48.6 & 22 & 600 & 182 & 795 & 7.8 \\
5 & 20.0 & 723 & 8832 & 86.4 & 14 & 120.0 & 435 & 4531 & 44.3 & 23 & 700 & 161 & 613 & 6.0 \\
6 & 25.0 & 458 & 8109 & 79.3 & 15 & 140.0 & 535 & 4096 & 40.1 & 24 & 800 & 161 & 452 & 4.4 \\
7 & 30.0 & 629 & 7651 & 74.8 & 16 & 170.0 & 445 & 3561 & 34.8 & 25 & 1000 & 89 & 291 & 2.8 \\
8 & 40.0 & 444 & 7022 & 68.7 & 17 & 200.0 & 575 & 3116 & 30.5 & 26 & 1200 & 75 & 202 & 2.0
\end{tabular}
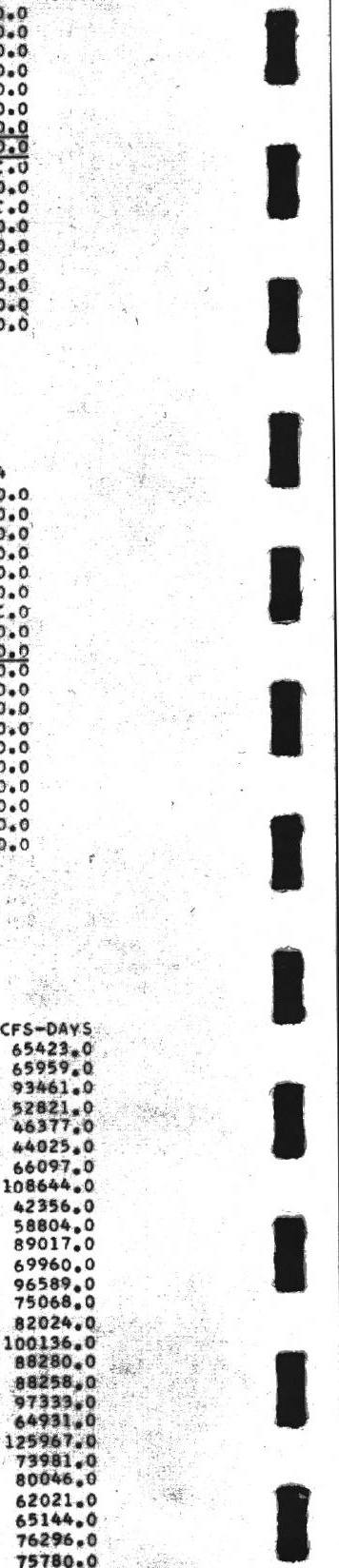
Coast Fork Willamette River at

STATION NUMBER $14-1525.00$

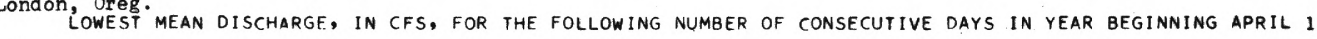

\begin{tabular}{|c|c|c|c|c|c|c|c|c|c|c|c|}
\hline YEAR & 1 & 3 & 7 & 14 & 30 & 60 & 90 & 120 & 150 & 183 & 274 \\
\hline 1936 & 10.0 & 10.0 & 10.4 & 11.1 & 11.7 & 11.7 & 13.2 & $\begin{array}{l}14.5 \\
40.5\end{array}$ & $\begin{array}{l}17.8 \\
78.3\end{array}$ & 23.2 & 53.2 \\
\hline $\begin{array}{l}1938 \\
1939\end{array}$ & $\begin{array}{l}10.0 \\
10.0\end{array}$ & $\begin{array}{l}10.0 \\
10.7\end{array}$ & 10.4 & 11.2 & 12.6 & $\begin{array}{l}13.9 \\
16.1\end{array}$ & $\begin{array}{l}16.1 \\
16.4\end{array}$ & $\begin{array}{l}18.2 \\
17.7\end{array}$ & $\begin{array}{r}23.9 \\
18.0\end{array}$ & $\begin{array}{l}40.8 \\
22.3\end{array}$ & $\begin{array}{r}91.3 \\
48.9\end{array}$ \\
\hline 1940 & 10.0 & 10.0 & 10.6 & 10.9 & 12.0 & $\begin{array}{l}10.1 \\
14.4\end{array}$ & 16.1 & 18.4 & 22.4 & 33.3 & 87.4 \\
\hline 1941 & 20.0 & 20.0 & 20.7 & 21.3 & 22.6 & 28.9 & 30.9 & 33.3 & 43.3 & 56.6 & 154.0 \\
\hline 1942 & 14.0 & 14.7 & 15.0 & 15.5 & 16.3 & 17.6 & 20.7 & $27 \cdot 2$ & 43.4 & 73.3 & 211.0 \\
\hline 1943 & 17.0 & 17.0 & 17.4 & 18.4 & 18.6 & 22.3 & 25.7 & 39.5 & 59.1 & 72.0 & 97.7 \\
\hline 1944 & 12.0 & 12.0 & 12.3 & 13.1 & 14.3 & 16.4 & 16.6 & $18 \cdot 7$ & 24.5 & 29.7 & 58.4 \\
\hline 1945 & 14.0 & 14.3 & 14.7 & 15.9 & 16.9 & 19.0 & 19.8 & $22 \cdot 5$ & 28.5 & 58.0 & 207.0 \\
\hline 1946 & 14.0 & 14.0 & $14 \cdot 1$ & 15.1 & 15.6 & 17.2 & 18.3 & 22.0 & 31.5 & 39.2 & 123.0 \\
\hline 1947 & 22.0 & 22.3 & 22.7 & 23.3 & 26.8 & 28.6 & 34.1 & $41 \cdot 8$ & 52.2 & 63.5 & 163.0 \\
\hline 1948 & 18.0 & 18.0 & 19.0 & 20.1 & 21.5 & 25.2 & 33.1 & 35.8 & 45.7 & 63.5 & $160 \cdot 0$ \\
\hline 1949 & 12.0 & 12.0 & 12.4 & 13.7 & 14.4 & 16.5 & 18.1 & $21 \cdot 3$ & 24.1 & 30.6 & 96.3 \\
\hline 1950 & 13.0 & 13.3 & 13.6 & 13.9 & 15.0 & 17.2 & 20.9 & $32 \cdot 2$ & 44.1 & 65.4 & 212.0 \\
\hline 1951 & 12.0 & 12.0 & $12 \cdot 3$ & $12 \cdot 6$ & 13.8 & 15.3 & 17.6 & $21 \cdot 9$ & 30.6 & 45.8 & 159.0 \\
\hline 1952 & 14.0 & $14 . n$ & 14.1 & 14.2 & 14.9 & 16.9 & 17.9 & 19.7 & 23.9 & 35.6 & 82.0 \\
\hline 1953 & 16.0 & 16.7 & 16.9 & 17.0 & 19.4 & 23.7 & 28.0 & 33.5 & 43.8 & 92.8 & 224.0 \\
\hline 1954 & 18.0 & 19.3 & 20.9 & 23.4 & 26.5 & 28.8 & 30.4 & $32 \cdot 9$ & 37.7 & 45.1 & 94.4 \\
\hline 1955 & 15.0 & 15.7 & 16.1 & 16.9 & 18.1 & 22.2 & 26.9 & 33.4 & 43.3 & 62.3 & 283.0 \\
\hline 1956 & 13.0 & 13.0 & 13.7 & 14.1 & 15.9 & 17.1 & 20.7 & $29 \cdot 8$ & 47.8 & 71.7 & 132.0 \\
\hline 1957 & 11.0 & 11.3 & $12 \cdot 1$ & 13.1 & 14.3 & 16.7 & 20.8 & $25 \cdot 8$ & 29.3 & 39.8 & 135.0 \\
\hline 1958 & 13.0 & 13.3 & 14.0 & 14.5 & 15.3 & 15.7 & 17.6 & $22 \cdot 3$ & 35.3 & 48.1 & 106.0 \\
\hline 1959 & 16.0 & 16.0 & 16.0 & 16.7 & 17.3 & 19.0 & 22.7 & 27.9 & 28.9 & 32.6 & 56.3 \\
\hline 1960 & 12.0 & 12.3 & 12.9 & 13.4 & 14.9 & 16.9 & 18.8 & $21 \cdot 0$ & 27.0 & 65.8 & 125.0 \\
\hline 1961 & 12.0 & 12.0 & 12.6 & 13.4 & 14.0 & 16.7 & 17.6 & 22.9 & 30.4 & 41.5 & 166.0 \\
\hline 1962 & 14.0 & 14.0 & 14.9 & 15.6 & 19.5 & 22.6 & 24.6 & $32 \cdot 2$ & 47.0 & 62.1 & 116.0 \\
\hline
\end{tabular}

Coast Fork Willamette River at

STATION NUMBER $\quad 14-1525.00$

London, Oreg.

HIGHEST MEAN DISCHARGE, IN CFS, FOR THE FOLLOWING NUMBER OF CONSECUTIVE DAYS IN YEAR ENDING SEPTEMBER 30

\begin{tabular}{|c|c|c|c|c|c|c|c|c|c|c|c|}
\hline $\begin{array}{l}\text { YEAR } \\
1936 \\
1937\end{array}$ & $\begin{array}{c}1 \\
2760.0 \\
2520.0\end{array}$ & $\begin{array}{c}3 \\
2150.0 \\
2260.0\end{array}$ & $\begin{array}{c}7 \\
1580.0 \\
1410.0\end{array}$ & $\begin{array}{c}15 \\
1250.0 \\
902.0\end{array}$ & $\begin{array}{c}30 \\
811.0 \\
622.0\end{array}$ & $\begin{array}{l}60 \\
577.0 \\
570.0\end{array}$ & $\begin{array}{l}90 \\
461.0 \\
498.0\end{array}$ & $\begin{array}{l}120 \\
399 \cdot 0 \\
417 \cdot 0\end{array}$ & $\begin{array}{l}150 \\
355.0 \\
381.0\end{array}$ & $\begin{array}{l}183 \\
313.0 \\
330.0\end{array}$ & $\begin{array}{l}274 \\
232.0 \\
236.0\end{array}$ \\
\hline $\begin{array}{l}1938 \\
1939 \\
1940\end{array}$ & $\begin{array}{l}2530.0 \\
1930.0 \\
1340.0\end{array}$ & $\begin{array}{l}1950.0 \\
1270.0 \\
1020.0\end{array}$ & $\begin{array}{r}1530.0 \\
875.0 \\
799.0\end{array}$ & $\begin{array}{r}1140.0 \\
654.0 \\
632.0\end{array}$ & $\begin{array}{l}799.0 \\
527.0 \\
584.0\end{array}$ & $\begin{array}{l}683.0 \\
464.0 \\
433.0\end{array}$ & $\begin{array}{l}601.0 \\
380.0 \\
341 \bullet 0\end{array}$ & $\begin{array}{l}540 \cdot 0 \\
333 \bullet 0 \\
302 \cdot 0\end{array}$ & $\begin{array}{l}521.0 \\
299.0 \\
270.0\end{array}$ & $\begin{array}{l}471.0 \\
261.0 \\
234.0\end{array}$ & $\begin{array}{l}335.0 \\
187.0 \\
163.0\end{array}$ \\
\hline $\begin{array}{l}1941 \\
1942 \\
1943\end{array}$ & $\begin{array}{l}1380.0 \\
3840.0 \\
4680.0\end{array}$ & $\begin{array}{l}1060 . n \\
2070 . n \\
4090.0\end{array}$ & $\begin{array}{r}680.0 \\
1150.0 \\
2750.0\end{array}$ & $\begin{array}{r}481.0 \\
733.0 \\
1760.0\end{array}$ & $\begin{array}{r}372.0 \\
562.0 \\
1250.0\end{array}$ & $\begin{array}{l}306.0 \\
497.0 \\
945.0\end{array}$ & $\begin{array}{l}247.0 \\
437.0 \\
838.0\end{array}$ & $\begin{array}{l}220 \bullet 0 \\
371 \bullet 0 \\
677 \bullet 0\end{array}$ & $\begin{array}{l}193.0 \\
322.0 \\
603.0\end{array}$ & $\begin{array}{l}181.0 \\
289.0 \\
527.0\end{array}$ & $\begin{array}{l}152.0 \\
233.0 \\
389.0\end{array}$ \\
\hline $\begin{array}{l}1944 \\
1945 \\
1946\end{array}$ & $\begin{array}{l}1400.0 \\
1400.0 \\
5630.0\end{array}$ & $\begin{array}{r}833.0 \\
1150.0 \\
3150.0\end{array}$ & $\begin{array}{r}486.0 \\
946.0 \\
1780.0\end{array}$ & $\begin{array}{r}428.0 \\
724.0 \\
1420.0\end{array}$ & $\begin{array}{l}299.0 \\
565.0 \\
916.0\end{array}$ & $\begin{array}{l}228.0 \\
454.0 \\
740.0\end{array}$ & $\begin{array}{l}236.0 \\
423.0 \\
622.0\end{array}$ & $\begin{array}{l}215 \bullet 0 \\
370 \cdot 0 \\
584 \cdot 0\end{array}$ & $\begin{array}{l}193.0 \\
337.0 \\
528.0\end{array}$ & $\begin{array}{l}185.0 \\
290.0 \\
454.0\end{array}$ & $\begin{array}{l}149.0 \\
208.0 \\
319.0\end{array}$ \\
\hline $\begin{array}{l}1947 \\
1948 \\
1949\end{array}$ & $\begin{array}{l}2150.0 \\
3160.0 \\
3260.0\end{array}$ & $\begin{array}{l}1510.0 \\
2430.0 \\
2430.0\end{array}$ & $\begin{array}{r}976.0 \\
1700.0 \\
1630.0\end{array}$ & $\begin{array}{r}724 \cdot 0 \\
1070.0 \\
1110.0\end{array}$ & $\begin{array}{l}625.0 \\
659.0 \\
719.0\end{array}$ & $\begin{array}{l}390.0 \\
578.0 \\
473.0\end{array}$ & $\begin{array}{l}395.0 \\
520.0 \\
476.0\end{array}$ & $\begin{array}{l}370 \cdot 0 \\
470 \cdot 0 \\
438.0\end{array}$ & $\begin{array}{l}376.0 \\
435.0 \\
399.0\end{array}$ & $\begin{array}{l}328.0 \\
425.0 \\
372.0\end{array}$ & $\begin{array}{l}245.0 \\
342.0 \\
268.0\end{array}$ \\
\hline $\begin{array}{l}1950 \\
1951 \\
1952\end{array}$ & $\begin{array}{l}2550.0 \\
5080.0 \\
1640.0\end{array}$ & $\begin{array}{l}2410.0 \\
3450.0 \\
1540.0\end{array}$ & $\begin{array}{l}1680.0 \\
1890.0 \\
1260.0\end{array}$ & $\begin{array}{r}1070.0 \\
1110.0 \\
910.0\end{array}$ & $\begin{array}{l}872.0 \\
880.0 \\
702.0\end{array}$ & $\begin{array}{l}697.0 \\
648.0 \\
575.0\end{array}$ & $\begin{array}{l}612.0 \\
659.0 \\
566.0\end{array}$ & $\begin{array}{l}531 \bullet 0 \\
625 \bullet 0 \\
512 \bullet 0\end{array}$ & $\begin{array}{l}469.0 \\
574.0 \\
476.0\end{array}$ & $\begin{array}{l}413.0 \\
500.0 \\
423.0\end{array}$ & $\begin{array}{l}293.0 \\
360.0 \\
313.0\end{array}$ \\
\hline $\begin{array}{l}1953 \\
1954 \\
1955\end{array}$ & $\begin{array}{l}4700.0 \\
3990.0 \\
1370.0\end{array}$ & $\begin{array}{l}2860.0 \\
2980.0 \\
1310.0\end{array}$ & $\begin{array}{r}1890.0 \\
1610.0 \\
828.0\end{array}$ & $\begin{array}{r}1320.0 \\
1170.0 \\
619.0\end{array}$ & $\begin{array}{r}1030.0 \\
983.0 \\
575.0\end{array}$ & $\begin{array}{l}709 \cdot 0 \\
724 \cdot 0 \\
472.0\end{array}$ & $\begin{array}{l}610.0 \\
724.0 \\
385.0\end{array}$ & $\begin{array}{l}525 \bullet 0 \\
624 \bullet 0 \\
379 \cdot 0\end{array}$ & $\begin{array}{l}472.0 \\
561.0 \\
347.0\end{array}$ & $\begin{array}{l}434.0 \\
484.0 \\
313.0\end{array}$ & $\begin{array}{l}316.0 \\
345.0 \\
229.0\end{array}$ \\
\hline $\begin{array}{l}1956 \\
1957 \\
1958\end{array}$ & $\begin{array}{l}4340.0 \\
2110.0 \\
3840.0\end{array}$ & $\begin{array}{l}3190 \cdot 0 \\
1700 \cdot 0 \\
2800 \cdot 0\end{array}$ & $\begin{array}{l}2480.0 \\
1260.0 \\
1700.0\end{array}$ & $\begin{array}{r}1690.0 \\
970.0 \\
1270.0\end{array}$ & $\begin{array}{r}1270.0 \\
857.0 \\
807.0\end{array}$ & $\begin{array}{r}1070.0 \\
595.0 \\
726.0\end{array}$ & $\begin{array}{l}853.0 \\
461.0 \\
624.0\end{array}$ & $\begin{array}{l}781 \cdot 0 \\
436 \bullet 0 \\
512 \cdot 0\end{array}$ & $\begin{array}{l}711.0 \\
376.0 \\
455.0\end{array}$ & $\begin{array}{l}621.0 \\
354.0 \\
393.0\end{array}$ & $\begin{array}{l}451.0 \\
264.0 \\
285.0\end{array}$ \\
\hline $\begin{array}{l}1959 \\
1960 \\
1961 \\
1962 \\
1963\end{array}$ & $\begin{array}{l}1810.0 \\
2010.0 \\
5450.0 \\
4550.0 \\
1900.0\end{array}$ & $\begin{array}{l}1390.0 \\
1610.0 \\
3350.0 \\
2740.0 \\
1520.0\end{array}$ & $\begin{array}{r}988.0 \\
1020.0 \\
2010.0 \\
1640.0 \\
1050.0\end{array}$ & $\begin{array}{r}600.0 \\
738.0 \\
1240.0 \\
1050.0 \\
723.0\end{array}$ & $\begin{array}{l}545 \cdot 0 \\
570.0 \\
958.0 \\
834.0 \\
651.0\end{array}$ & $\begin{array}{l}477.0 \\
500.0 \\
737.0 \\
554.0 \\
506.0\end{array}$ & $\begin{array}{l}414.0 \\
436.0 \\
546.0 \\
436.0 \\
399.0\end{array}$ & $\begin{array}{l}356 \cdot 0 \\
417.0 \\
451.0 \\
393.0 \\
369.0\end{array}$ & $\begin{array}{l}334.0 \\
374.0 \\
426.0 \\
400 \cdot 0 \\
311.0\end{array}$ & $\begin{array}{l}295.0 \\
323.0 \\
382.0 \\
364.0 \\
325.0\end{array}$ & $\begin{array}{l}220.0 \\
231.0 \\
272.0 \\
269.0 \\
251.0\end{array}$ \\
\hline
\end{tabular}


COAST FORK WIILAMETTE RTVER BL COTPTAGE GROVE DAM ORBGONATION NUMBER
DURATION TABLE OF DAILY DISCHARGE

CLASS $0 \begin{array}{lllllllllllllllllllllllllllllllllllll} & 0 & 2 & 3 & 4 & 5 & 6 & 7 & 8 & 9 & 10 & 11 & 12 & 13 & 14 & 15 & 16 & 17 & 18 & 19 & 20 & 21 & 22 & 23 & 24 & 25 & 26 & 27 & 28 & 29 & 30 & 31 & 32 & 33 & 34\end{array}$

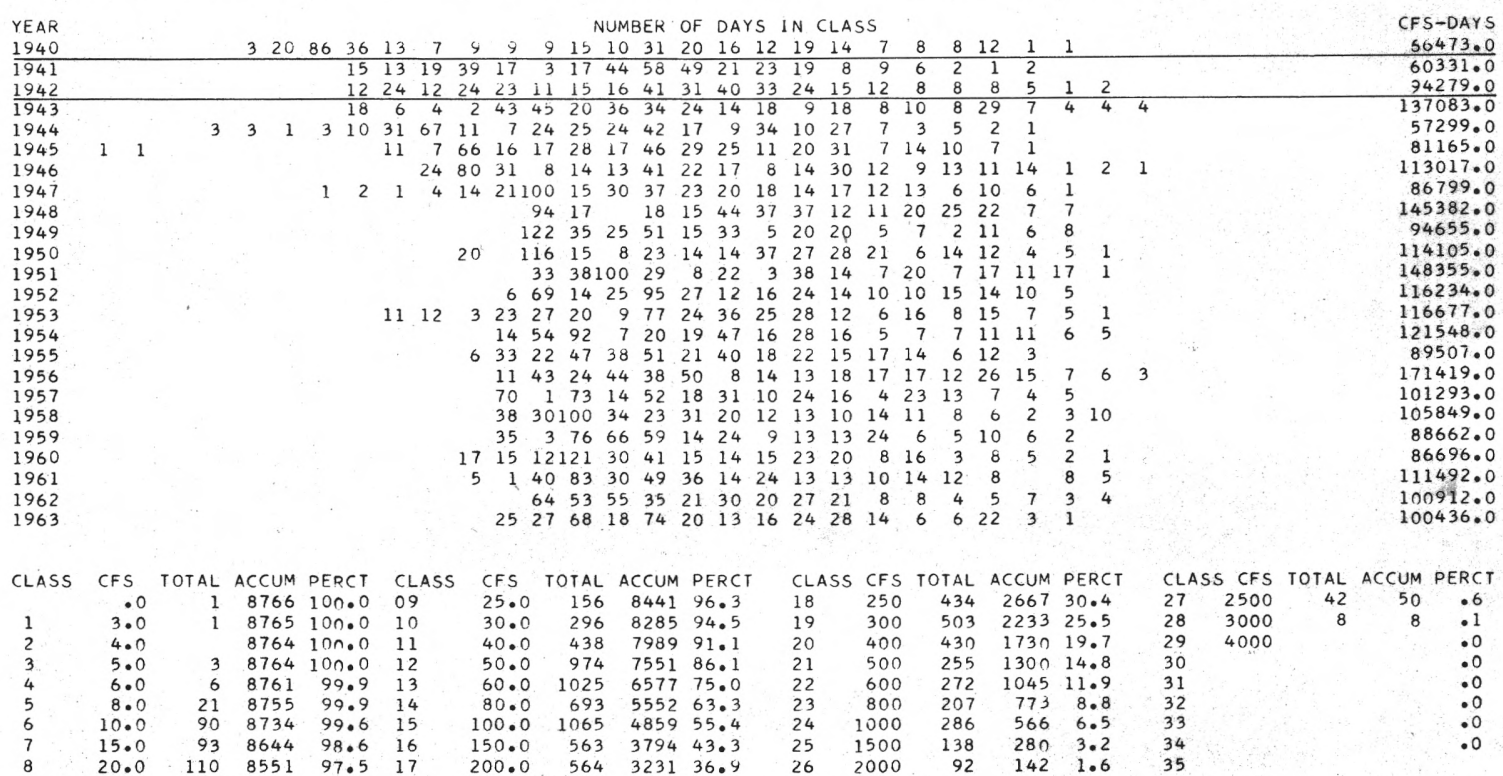

Coast Fork Willamette River below Cottage Grove Dam, Oreg.

Summary for water years 1941-1942

$\begin{array}{cccrrl}\text { CLASS } & \text { CFS } & \text { TOTAL } & \text { ACCUM } & \text { PERCT } & \text { CLASS } \\ & .0 & & 730 & 100.0 & 09 \\ 1 & 3.0 & & 730 & 100.0 & 10 \\ 2 & 4.0 & & 730 & 100.0 & 11 \\ 3 & 5.0 & & 730 & 100.0 & 12 \\ 4 & 6.0 & & 730 & 100.0 & 13 \\ 5 & 8.0 & & 730 & 100.0 & 14 \\ 6 & 10.0 & & 730 & 100.0 & 15 \\ 7 & 15.0 & 27 & 730 & 100.0 & 16 \\ 8 & 20.0 & 37 & 703 & 96.3 & 17\end{array}$

Coast Fork Willamette River below Cottage Grove Dam, Oreg.

Summary for water years 1943-1963

$\begin{array}{rrrrrl} & & & & & \\ \text { CLASS } & \text { CFS } & \text { TOTAL } & \text { ACCUM } & \text { PERCT } & \text { CL.ASS } \\ 1 & .0 & 1 & 7670 & 100.0 & 09 \\ 1 & 3.0 & 1 & 7669 & 100.0 & 10 \\ 2 & 4.0 & & 7668 & 100.0 & 11 \\ 3 & 5.0 & 3 & 7668 & 100.0 & 12 \\ 4 & 6.0 & 3 & 7665 & 99.9 & 13 \\ 5 & 8.0 & 1 & 7662 & 99.9 & 14 \\ 6 & 10.0 & 4 & 7661 & 99.9 & 15 \\ 7 & 15.0 & 30 & 7657 & 99.8 & 16 \\ 8 & 20.0 & 60 & 7627 & 99.4 & 17\end{array}$

\begin{tabular}{rrrrl}
\multicolumn{1}{l}{ CFS } & TOTAL & ACCUM & PERCT & CLASS \\
25.0 & 31 & 666 & 91.2 & 18 \\
30.0 & 63 & 635 & 87.0 & 19 \\
40.0 & 40 & 572 & 78.4 & 20 \\
50.0 & 14 & 532 & 72.9 & 21 \\
60.0 & 32 & 518 & 71.0 & 22 \\
80.0 & 60 & 486 & 66.6 & 23 \\
100.0 & 99 & 426 & 58.4 & 24 \\
150.0 & 80 & 327 & 44.8 & 25 \\
200.0 & 61 & 247 & 33.8 & 26
\end{tabular}

STATION NUMBER

14-1535.00

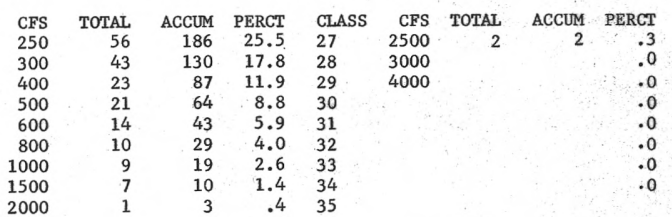

$\begin{array}{rrrrl}\text { CFS } & \text { TOTAL } & \text { ACCUM } & \text { PERCT } & \text { CLASS } \\ 25.0 & 118 & 7567 & 98.7 & 18 \\ 30.0 & 224 & 7449 & 97.1 & 19 \\ 40.0 & 389 & 7225 & 94.2 & 20 \\ 50.0 & 951 & 6836 & 89.1 & 21 \\ 60.0 & 978 & 5885 & 76.7 & 22 \\ 80.0 & 623 & 4907 & 64.0 & 23 \\ 100.0 & 935 & 4284 & 55.9 & 24 \\ 150.0 & 463 & 3349 & 43.7 & 25 \\ 200.0 & 487 & 2886 & 37.6 & 26\end{array}$

$\begin{array}{rr}\text { CFS } & \text { TOTAI } \\ 250 & 366 \\ 300 & 441 \\ 400 & 393 \\ 500 & 227 \\ 600 & 250 \\ 800 & 189 \\ 1000 & 265 \\ 1500 & 130 \\ 2000 & 90\end{array}$

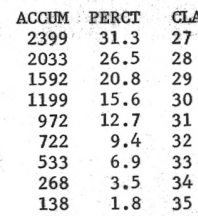

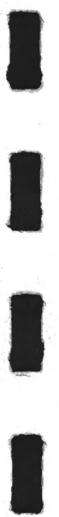

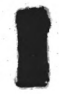

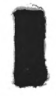
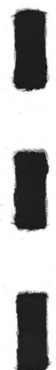

$\begin{array}{rrrrr}\text { CFS } & \text { TOTAL } & \text { ACCUM } & \text { PERCT } \\ 2500 & 40 & 48 & .6 \\ 3000 & 8 & 8 & .1 \\ 4000 & & & .0 \\ & & & .0 \\ & & & .0 \\ & & & .0 \\ & & & .0 \\ & & & .0\end{array}$ 
STATION NUMBER

$14-1535.00$

COAST FORK WILLAMETTE RTVER BL COTTAGE GROVE DAM ORBGG. FOLLOWING NUMBER OF CONSECUTIVE DAYS IN YEAR BEGINNING APRIL 1 LOWEST MEAN DISCHARGE, IN CFS, FOR THE FOLLOWING NUME

\begin{tabular}{|c|c|c|c|c|c|c|c|c|c|c|c|}
\hline YEAR & 1 & 3 & 7 & 14 & 30 & 60 & 90 & 120 & 150 & 183 & 274 \\
\hline $\begin{array}{l}1939 \\
1940\end{array}$ & 5.0 & 5.0 & 5.4 & 6.2 & 7.9 & 10.0 & 11.9 & $13 \cdot 8$ & 13.9 & 18.9 & $\begin{array}{r}54.3 \\
8.0\end{array}$ \\
\hline 1941 & 19.0 & 22.3 & 23.7 & 24.6 & 27.3 & 35.8 & 38.5 & $42 \cdot 3$ & 54.9 & 72.7 & 213.0 \\
\hline 1942 & 16.0 & 16.7 & 17.0 & 17.6 & 18.9 & 20.6 & 24.3 & 32.6 & 56.7 & 101.0 & 258.0 \\
\hline 1943 & 5.0 & 5.0 & 6.1 & 12.1 & 18.3 & 52.6 & 74.5 & 121.0 & $122 \cdot 0$ & $15 b \cdot 0$ & 175.0 \\
\hline 1944 & 17.0 & 17.0 & 17.7 & 19.1 & 22.3 & 24.2 & 31.0 & $44 \cdot 2$ & 74.1 & 109.0 & 114.0 \\
\hline 1945 & .0 & 9.0 & 17.9 & 20.9 & 29.4 & 34.1 & 49.1 & $102 \cdot 0$ & 126.0 & $149 \cdot 0$ & 279.0 \\
\hline 1946 & 28.0 & 28.0 & 28.0 & 29.1 & 31.1 & 32.2 & 35.0 & 42.8 & 41.7 & $79 \cdot 0$ & 201.0 \\
\hline 1947 & 12.0 & 18.7 & 28.1 & 37.2 & 41.3 & 46.0 & 48.4 & 66.2 & 70.7 & 89.4 & 260.0 \\
\hline 1948 & 52.0 & 52.0 & 52.4 & 56.9 & 58.8 & 58.9 & 85.7 & $134 \cdot U$ & 155.0 & 167.0 & 268.0 \\
\hline 1949 & 32.0 & 32.0 & $32 \cdot 6$ & 32.8 & 40.3 & 54.2 & 55.7 & $61 \cdot 3$ & 95.4 & 116.0 & 157.0 \\
\hline 1950 & 52.0 & 52.0 & 54.0 & 54.0 & 54.1 & 54.1 & 65.2 & $80 \cdot 4$ & 101.0 & 136.0 & 333.0 \\
\hline 1951 & 53.0 & 53.7 & 54.0 & 56.5 & 57.3 & 60.0 & 70.1 & $74 \cdot 1$ & 79.8 & 103.0 & 250.0 \\
\hline 1952 & 20.0 & 20.0 & $21 \cdot 3$ & 22.7 & 29.7 & 74.2 & 87.6 & $84 \cdot 8$ & 96.5 & 106.0 & 135.0 \\
\hline 1953 & 41.0 & 44.0 & 45.1 & 45.5 & 51.2 & 88.3 & 111.0 & 146.0 & 147.0 & 187.0 & 320.0 \\
\hline 1954 & 32.0 & $32 \cdot 3$ & $34 \cdot 3$ & 45.4 & 49.6 & 49.8 & 55.7 & $58 \cdot 6$ & 70.6 & 101.0 & 131.0 \\
\hline 1955 & 48.0 & 48.0 & 48.0 & 48.0 & 49.0 & 63.1 & 82.4 & 106.0 & 133.0 & $135 \cdot 0$ & 394.0 \\
\hline 1956 & 53.0 & 53.0 & 53.0 & 53.0 & 53.3 & 69.5 & 96.0 & $106 \cdot 0$ & 119.0 & 143.0 & 206.0 \\
\hline 1957 & 40.0 & 41.7 & 43.0 & 46.0 & 46.0 & 46.9 & 63.2 & $80 \cdot 3$ & 126.0 & 116.0 & 196.0 \\
\hline 1958 & 45.0 & 51.0 & 51.6 & 51.8 & 64.1 & 73.3 & 83.0 & $90 \cdot 5$ & 119.0 & 128.0 & 173.0 \\
\hline 1959 & 36.0 & 36.0 & 36.0 & 36.1 & 41.9 & 57.8 & 66.2 & $73 \cdot 3$ & 99.5 & 114.0 & 107.0 \\
\hline 1960 & 36.0 & 36.7 & 41.6 & 59.9 & 63.9 & 67.4 & 79.0 & 123.0 & 129.0 & 155.0 & 221.0 \\
\hline 1961 & 50.0 & 50.0 & 50.0 & 50.0 & 50.4 & 58.1 & 76.1 & $82 \cdot 2$ & $110 \cdot 0$ & 113.0 & 253.0 \\
\hline 1962 & 50.0 & 50.0 & 50.0 & 50.0 & 50.8 & 54.0 & 67.9 & $88 \cdot 5$ & $100 \cdot 0$ & $129 \cdot 0$ & 194.0 \\
\hline
\end{tabular}

STATION NUMBER $14-1535.00$

COAST PORK WIILAMETTE RIVER BL COITAGE GROVE DAM OREG.

\begin{tabular}{|c|c|c|c|c|c|c|c|c|c|c|c|}
\hline YEAR & 1 & 3 & 7 & 15 & 30 & 60 & 90 & 120 & 150 & $\begin{array}{l}183 \\
345.0\end{array}$ & 274 \\
\hline 1940 & 2010.0 & $1600 \cdot 0$ & 1270.0 & 1020.0 & 937.0 & 670.0 & 524.0 & $460 \cdot 0$ & $\frac{405 \cdot 0}{270 \cdot 0}$ & $\frac{345.0}{253.0}$ & $\frac{237.0}{209.0}$ \\
\hline 1941 & 1980.0 & 1510.0 & 973.0 & 718.0 & 533.0 & 431.0 & 351.0 & $\begin{array}{l}310 \cdot 0 \\
531.0\end{array}$ & $\begin{array}{l}270.0 \\
459.0\end{array}$ & $\begin{array}{l}253.0 \\
415.0\end{array}$ & $\begin{array}{l}209.0 \\
333.0\end{array}$ \\
\hline$\frac{1942}{1943}$ & 2970.0 & 2670.0 & 1560.0 & 1070.0 & 814.0 & $\begin{array}{r}712.0 \\
1300.0\end{array}$ & $\begin{array}{r}622.0 \\
1100.0\end{array}$ & 865.0 & $730 \cdot 0$ & 623.0 & $\frac{335.0}{467.0}$ \\
\hline $\begin{array}{l}1943 \\
1944\end{array}$ & $\begin{array}{l}3210.0 \\
1760.0\end{array}$ & $\begin{array}{l}3180.0 \\
1400.0\end{array}$ & $\begin{array}{r}3030 \bullet 0 \\
937.0\end{array}$ & $\begin{array}{r}2300 \cdot 0 \\
569.0\end{array}$ & $\begin{array}{r}1660.0 \\
474.0\end{array}$ & $\begin{array}{r}1300.0 \\
324.0\end{array}$ & 254.0 & 258.0 & 244.0 & 204.0 & 174.0 \\
\hline 1945 & 1760.0 & 1510.0 & 1200.0 & 861.0 & 634.0 & $467.0^{\prime}$ & 430.0 & 384.0 & 359.0 & 310.0 & 255.0 \\
\hline $\begin{array}{l}1946 \\
1947\end{array}$ & 3040.0 & 2750.0 & 2250.0 & 1870.0 & 1200.0 & 967.0 & 806.0 & 719.0 & 633.0 & 539.0 & 378.0 \\
\hline $\begin{array}{l}1947 \\
1948\end{array}$ & 2030.0 & 1910.0 & 1380.0 & 962.0 & 845.0 & 527.0 & 506.0 & $426 \cdot 0$ & 426.0 & 378.0 & 301.0 \\
\hline $\begin{array}{l}1948 \\
1949\end{array}$ & 2250.0 & 2190.0 & 2030.0 & 1600.0 & 966.0 & 755.0 & 733.0 & $647 \cdot 0$ & 645.0 & 593.0 & 466.0 \\
\hline $\begin{array}{l}1949 \\
1950\end{array}$ & 2330.0 & 2330.0 & 2060.0 & 1550.0 & 987.0 & 633.0 & 642.0 & 537.0 & 468.0 & 418.0 & 310.0 \\
\hline $\begin{array}{l}1950 \\
1951\end{array}$ & 2550.0 & 2450.0 & 2230.0 & 1550.0 & 1230.0 & 858.0 & 749.0 & 645.0 & 564.0 & 493.0 & 375.0 \\
\hline $\begin{array}{l}1951 \\
1952\end{array}$ & 2580.0 & 2440.0 & $2310 \cdot 0$ & 1780.0 & 1320.0 & 973.0 & 959.0 & 915.0 & 811.0 & 708.0 & 4.99 .0 \\
\hline 1952 & 2210.0 & 2110.0 & 1810.0 & 1270.0 & 981.0 & 798.0 & 792.0 & 674.0 & 590.0 & 528.0 & 385.0 \\
\hline 1953 & 2530.0 & 2490.0 & 2340.0 & 1700.0 & 1370.0 & 884.0 & 706.0 & $617 \cdot 0$ & 531.0 & 499.0 & 381.0 \\
\hline 1954 & 2800.0 & 2790.0 & 2090.0 & 1580.0 & 1290.0 & 959.0 & 932.0 & $761 \cdot 0$ & 655.0 & 564.0 & 409.0 \\
\hline 1955 & 1760.0 & $1700 \cdot 0$ & 1160.0 & 774.0 & 711.0 & 517.0 & 398.0 & 432.0 & 396.0 & 346.0 & 285.0 \\
\hline 1956 & 3060.0 & 3030.0 & 3010.0 & 2460.0 & 1930.0 & 1540.0 & 1230.0 & $1080 \cdot 0$ & 944.0 & 810.0 & 580.0 \\
\hline 1957 & 2390.0 & 2360.0 & 2020.0 & 1510.0 & 1100.0 & 668.0 & 519.0 & 509.0 & 454.0 & 422.0 & 315.0 \\
\hline 1958 & 2880.0 & 2810.0 & 2370.0 & 1810.0 & 1150.0 & 976.0 & 806.0 & 635.0 & 545.0 & 466.0 & 356.0 \\
\hline 1959 & 2400.0 & 2100.0 & 1470.0 & 911.0 & & 653.0 & 500.0 & 463.0 & 411.0 & 360.0 & 270.0 \\
\hline 1960 & 2610.0 & 1980.0 & 1500.0 & 997.0 & 733.0 & 618.0 & 509.0 & $481 \cdot 0$ & 428.0 & 370.0 & 290.0 \\
\hline 1961 & 2990.0 & 2760.0 & 2630.0 & 1820.0 & 1370.0 & 963.0 & 686.0 & 631.0 & 574.0 & 494.0 & 362.0 \\
\hline 1962 & 2950.0 & 2.790 .0 & 2460.0 & 1440.0 & 1160.0 & 747.0 & 570.0 & $481 \cdot 0$ & 475.0 & 429.0 & 326.0 \\
\hline 1963 & 2250.0 & 1770.0 & 1380.0 & 920.0 & 806.0 & 622.0 & 445.0 & 414.0 & 357.0 & 393.0 & 317.0 \\
\hline
\end{tabular}


CLASS $00 \begin{array}{llllllllllllllllllllllllllllllllllll}2 & 2 & 4 & 5 & 6 & 7 & 8 & 9 & 10 & 11 & 12 & 13 & 14 & 15 & 16 & 17 & 18 & 19 & 20 & 21 & 22 & 23 & 24 & 25 & 26 & 27 & 28 & 29 & 30 & 31 & 32 & 33 & 34\end{array}$

YEAR
1936
1937
1938
1939
1940
1941
1942
1943
1944
1945
1946
1947
1948
1949
1950
1951
1952
1953
1954
1955
1956
1957
1958
1959
1960
1961
1962
1963

$238202818131917 \quad 1917 \quad$ NUMBER OF DAYS IN CLASS

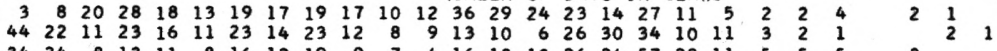

$\begin{array}{lrrrrrrrrrrrrrrrrrrrrrrrrrrrrrr}34 & 24 & 8 & 13 & 11 & 8 & 16 & 12 & 19 & 9 & 7 & 4 & 16 & 10 & 10 & 26 & 24 & 57 & 29 & 11 & 5 & 5 & 5 & 2 & \end{array}$

$\begin{array}{lllllllllllllllllllllll}20 & 23 & 51 & 21 & 26 & 15 & 11 & 12 & 9 & 44 & 23 & 16 & 15 & 21 & 19 & 12 & 23 & 20 & 11 & 11 & 3 & 8 & 3 \\ 14 & 18 & 12 & 18 & 18 & 13 & 19 & 11 & 13 & 13 & 9 & 2 & 1\end{array}$

$\begin{array}{lllllllllllllllllll}10 & 4 & 16 & 17 & 14 & 25 & 14 & 31 & 28 & 32 & 26 & 40 & 32 & 20 & 23 & 12 & 13 & 6 & 1\end{array}$

$\begin{array}{rrrrrrrrrrrrrrrrrrrrr}3 & 8 & 7 & 23 & 9 & 9 & 18 & 13 & 25 & 17 & 25 & 15 & 46 & 28 & 32 & 26 & 12 & 18 & 15 & 5 \\ 3 & 15 & 13 & 26 & 13 & 15 & 16 & 7 & 12 & 12 & 21 & 12 & 29 & 19 & 15 & 18 & 21 & 37 & 23 & 11\end{array}$

$\begin{array}{llllllllllllllllllllllll}8 & 17 & 23 & 25 & 19 & 10 & 15 & 20 & 28 & 23 & 20 & 23 & 38 & 28 & 17 & 10 & 13 & 21 & 4 & 3\end{array}$

$\begin{array}{rrrrrrrrrrrrrrrrrrrr}16 & 32 & 18 & 20 & 14 & 18 & 18 & 11 & 24 & 13 & 17 & 7 & 21 & 17 & 13 & 19 & 15 & 47 & 12 & 6 \\ 13 & 39 & 15 & 24 & 7 & 6 & 14 & 4 & 21 & 12 & 13 & 10 & 18 & 19 & 18 & 32 & 26 & 29 & 18 & 10\end{array}$

$\begin{array}{lllllllllllllllllllll}7 & 4 & 19 & 21 & 15 & 16 & 19 & 32 & 26 & 32 & 13 & 28 & 18 & 13 & 27 & 19 & 22 & 16 & 4\end{array}$

$\begin{array}{lllllllllllllllllllllllllllll}13 & 19 & 19 & 30 & 11 & 10 & 13 & 9 & 15 & 16 & 9 & 13 & 23 & 19 & 15 & 58 & 32 & 39 & 23 & 6 & 5 & 4 & 2 & 1 & 2 & 3\end{array}$

$\begin{array}{llllllllllllllllllllllll}2 & 22 & 14 & 26 & 20 & 13 & 16 & 8 & 13 & 8 & 10 & 10 & 24 & 28 & 19 & 36 & 19 & 39 & 18 & 5\end{array}$

$\begin{array}{rrrrrrrrrrrrrrrrrrr}18 & 11 & 15 & 10 & 4 & 9 & 7 & 11 & 12 & 8 & 13 & 21 & 26 & 11 & 40 & 21 & 28 & 14 & 8 \\ 10 & 10 & 32 & 5 & 10 & 12 & 8 & 16 & 29 & 11 & 7 & 25 & 28 & 26 & 38 & 21 & 37 & 13 & 7\end{array}$

$\begin{array}{llllllllllllllllllll}16 & 37 & 16 & 26 & 14 & 14 & 13 & 6 & 8 & 11 & 11 & 4 & 14 & 19 & 24 & 43 & 19 & 24 & 20 & 11\end{array}$ $\begin{array}{rrrrrrrrrrrrrrrrr}26 & 37 & 19 & 19 & 6 & 26 & 35 & 15 & 13 & 21 & 16 & 15 & 27 & 15 & 30 & 12 & 8 \\ 30 & 24 & 12 & 22 & 10 & 20 & 24 & 16 & 15 & 20 & 19 & 16 & 29 & 22 & 42 & 11 & 6\end{array}$

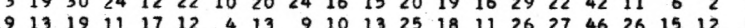

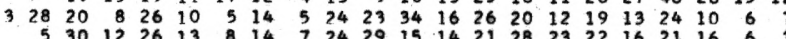

$\begin{array}{rlllllllllllllllllll}5 & 30 & 12 & 26 & 13 & 8 & 14 & 7 & 24 & 29 & 15 & 14 & 21 & 28 & 23 & 22 & 16 & 21 & 16 & 6 \\ 4 & 28 & 25 & 23 & 11 & 5 & 12 & 9 & 12 & 26 & 19 & 10 & 37 & 29 & 17 & 25 & 20 & 15 & 10 & 10\end{array}$

$\begin{array}{lllllllllllllllllllll}11 & 16 & 17 & 22 & 13 & 21 & 27 & 18 & 72 & 13 & 9 & 15 & 15 & 17 & 7 & 27 & 26 & 38 & 15 & 3\end{array}$

$\begin{array}{llllllllllllllllllllll}10 & 25 & 26 & 26 & 14 & 12 & 16 & 10 & 13 & 19 & 22 & 13 & 19 & 24 & 15 & 14 & 12 & 42 & 13 & 10 & 4\end{array}$

$\begin{array}{rrrrrrrrrrrrrrrrrrrrrrrrrrrr}10 & 25 & 26 & 26 & 14 & 12 & 16 & 10 & 13 & 19 & 22 & 13 & 19 & 24 & 15 & 14 & 12 & 42 & 13 & 10 & 4 & 2 & & 1 & 1 & 1 & 1 \\ 3 & 4 & 26 & 34 & 17 & 9 & 16 & 13 & 15 & 12 & 6 & 11 & 28 & 23 & 19 & 39 & 29 & 33 & 11 & 6 & 2 & 2 & 3 & 3 & & & 1 \\ & & 4 & 33 & 18 & 15 & 16 & 23 & 31 & 27 & 13 & 21 & 24 & 16 & 12 & 25 & 17 & 36 & 13 & 8 & 4 & 4 & 1 & 3 & 1 & & \end{array}$

ROW RTVER ABOVE PITCHER CFREGK NEAR DORENA ORRE

Summary of water years $1936-63$
STATTON NUMBRR $\quad 14-1545.00$

CFS TOTAL ACCUM PERCT $80.0 \quad 303$ ACCUM PERCT $\begin{array}{rrrr}80.0 & 303 & 7099 & 69.4 \\ 100.0 & 580 & 6796 & 66.5\end{array}$ $150.0528 \quad 6216 \quad 60$. 200.0 250.0 300.0 489747.9 $590 \quad 421741$. $449 \quad 362735$.

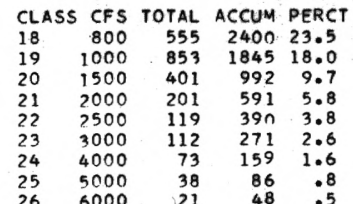

5000

21

$\begin{array}{lrrrr}\text { CLASS CFS } & \text { TOTAL ACCUM PERCT } \\ 27 & 8000 & 14 & 27 & .3 \\ 28 & 10000 & 13 & 13 & .1 \\ 29 & & & & .0 \\ 30 & & & & .0 \\ 31 & & & & .0 \\ 32 & & & & .0 \\ 33 & & & & .0 \\ 34 & & & & .0\end{array}$

CFS-DAYS 199313.0 276514.0 159114.0 130776.0 124919.0 308353.0 119735.0 182855.0 246118.0 205101.0 273155.0 233663.0 279436.0 267140.0 242287.0 256059.0 184576.0 352544.0 214117.0 221179.0 174501.0 203104.0 213550.0 220471.0

\begin{tabular}{ccrrrr} 
CLASS & \multicolumn{1}{c}{ CFS } & TOTAL ACCUM & PERCT & CLASS \\
& .0 & & 10227 & 100.0 & 09 \\
1 & 10.0 & 46 & 10227 & 100.0 & 10 \\
2 & 15.0 & 313 & 10181 & 90.6 & 11 \\
3 & 20.0 & 526 & 9868 & 96.5 & 12 \\
4 & 25.0 & 382 & 9342 & 91.3 & 13 \\
5 & 30.0 & 673 & 8960 & 87.6 & 14 \\
6 & 40.0 & 411 & 8287 & 81.0 & 15 \\
7 & 50.0 & 320 & 7876 & 77.0 & 16 \\
8 & 60.0 & 457 & 7556 & 73.9 & 17
\end{tabular}
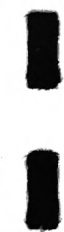

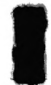

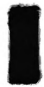

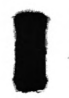
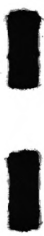

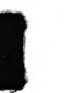


Row River above Pitcher Creek near

Dorena, Oreg.

STATION NUMBER $\quad 14-1545.00$ LOWEST MEAN DISCHARGE, IN CFS, FOR THE FOLLOWING NUMBER OF CONSECUTIVE DAYS IN YEAR BEGINNING APRIL 1

\begin{tabular}{|c|c|c|c|c|c|c|c|c|c|c|c|}
\hline YEAR & 1 & 3 & 7 & 14 & 30 & 60 & 90 & 120 & 150 & 183 & 274 \\
\hline 1936 & 15.0 & 16.0 & $16 \cdot 6$ & 16.9 & 18.7 & 19.0 & 22.5 & $25 \cdot 5$ & 32.9 & 49.8 & 134.0 \\
\hline 1937 & 23.0 & 23.3 & 25.6 & $30 \cdot 1$ & 36.3 & 40.6 & 53.3 & 85.3 & 186.0 & 272.0 & 553.0 \\
\hline 1938 & 17.0 & 17.0 & 17.0 & 17.2 & 18.4 & 21.2 & 22.9 & $27 \cdot 1$ & 43.7 & 111.0 & 254.0 \\
\hline 1939 & 15.0 & 15.0 & $15 \cdot 1$ & 15.9 & 18.8 & 21.6 & 25.2 & $32 \cdot 6$ & 34.6 & 52.1 & 140.0 \\
\hline 1940 & 12.0 & 12.7 & 12.7 & 13.0 & 14.3 & 18.9 & 22.5 & $32 \cdot 5$ & $40 \cdot 5$ & 68.9 & 225.0 \\
\hline 1941 & 34.0 & 34.7 & 37.6 & 37.9 & 43.3 & 65.3 & 78.8 & $87 \cdot 8$ & 135.0 & 197.0 & 474.0 \\
\hline 1942 & 19.0 & 19.0 & 19.6 & 19.9 & 22.7 & 26.7 & 34.8 & 53.7 & 119.0 & 246.0 & 653.0 \\
\hline 1943 & 24.0 & 24.7 & 25.3 & 26.8 & 28.1 & 36.9 & 43.2 & $86 \cdot 6$ & 146.0 & 169.0 & 261.0 \\
\hline 1944 & 16.0 & 17.0 & 17.6 & 19.3 & 22.4 & 28.7 & 29.0 & $33 \cdot 1$ & $48 \cdot 2$ & 67.6 & 162.0 \\
\hline 1945 & 18.0 & 18.0 & 19.0 & 21.1 & 24.1 & 27.5 & 31.6 & $38 \cdot 2$ & 58.4 & 184.0 & 646.0 \\
\hline 1946 & 16.0 & 16.0 & 17.3 & 20.2 & 21.8 & 24.3 & 27.8 & $41 \cdot 3$ & 77.9 & 122.0 & 390.0 \\
\hline 1947 & 34.0 & 34.0 & 34.1 & 35.4 & 44.0 & 49.8 & 73.4 & 103.0 & 132.0 & 163.0 & 474.0 \\
\hline 1948 & 23.0 & 23.3 & 25.4 & 27.6 & 32.2 & 41.8 & 64.6 & $72 \cdot 2$ & 114.0 & 190.0 & 425.0 \\
\hline 1949 & 17.0 & 17.7 & 18.1 & 18.7 & 21.9 & 26.3 & 30.0 & $43 \cdot 8$ & 49.5 & 69.5 & 261.0 \\
\hline 1950 & 18.0 & 18.7 & 20.4 & 20.6 & 23.4 & 31.8 & 46.0 & $91 \cdot 9$ & 156.0 & 255.0 & 640.0 \\
\hline 1951 & 11.0 & 11.7 & 12.4 & 13.3 & 15.4 & 17.0 & 21.0 & $31 \cdot 5$ & 65.2 & 131.0 & 473.0 \\
\hline 1952 & 15.0 & 15.7 & 16.7 & 19.6 & 20.7 & 23.2 & 27.7 & $29 \cdot 3$ & 47.9 & 91.8 & 246.0 \\
\hline 1953 & 26.0 & 26.7 & 27.3 & 27.6 & 33.6 & 41.8 & 49.2 & $63 \cdot 5$ & 91.1 & 247.0 & 658.0 \\
\hline 1954 & 29.0 & 29.0 & 29.6 & 30.6 & 41.5 & 45.3 & 46.3 & $54 \cdot 5$ & 70.6 & 103.0 & 219.0 \\
\hline 1955 & 22.0 & 22.7 & 24.9 & 26.2 & 28.9 & 39.3 & 51.6 & $86 \cdot 3$ & 139.0 & 223.0 & 837. \\
\hline 1956 & 18.0 & 19.7 & 20.4 & 21.4 & 23.3 & 26.5 & 34.2 & $59 \cdot 9$ & 131.0 & 236.0 & 395.0 \\
\hline 1957 & 13.0 & 13.3 & 14.4 & 14.9 & 15.9 & 22.0 & 30.8 & $52 \cdot 6$ & 61.5 & $100 \cdot 0$ & 350.0 \\
\hline 1958 & 11.0 & $12 \cdot 3$ & 16.0 & 17.6 & 21.0 & 22.2 & 26.7 & $38 \cdot 8$ & $82 \cdot 9$ & 124.0 & 330.0 \\
\hline 1959 & 19.0 & 19.0 & 19.4 & 20.7 & 22.8 & 26.2 & 37.3 & $59 \cdot 0$ & 75.6 & 92.3 & 159.0 \\
\hline 1960 & 15.0 & $16 \cdot 3$ & 18.0 & 18.1 & 21.1 & 26.9 & 31.1 & $40 \cdot 4$ & 53.7 & $167 \cdot 0$ & \\
\hline 1961 & 16.0 & 16.7 & $18 \cdot 3$ & 20.9 & 23.7 & 26.5 & 28.2 & $40 \cdot 4$ & 62.4 & 106.0 & $\begin{array}{l}453.0 \\
357\end{array}$ \\
\hline 1962 & 24.0 & 25.0 & 25.6 & 26.9 & 29.8 & 35.3 & 39.4 & $60 \cdot 2$ & 115.0 & 197.0 & \\
\hline
\end{tabular}

Row River above Pitcher lreek near Dorena, Oreg.

STATION NUMBER $\quad 14-1545.00$ HIGHEST MEAN DISCHARGE, IN CFS, FOR THE FOLLOWING NUMBER OF CONSECUTIVE DAYS IN YEAR ENDING SEPTEMBER 30

\begin{tabular}{rrr} 
YEAR & 1 & 3 \\
1936 & 8080.0 & \multicolumn{1}{c}{6670.0} \\
1937 & 10900.0 & 9370.0 \\
1938 & 6840.0 & 4750.0 \\
1939 & 5360.0 & $3670 . n$ \\
1940 & 3550.0 & $2980 . n$ \\
1941 & 3170.0 & 2330.0 \\
1942 & 11600.0 & 6200.0 \\
1943 & 12900.0 & 10800.0 \\
1944 & 3990.0 & 2440.0 \\
1945 & 4570.0 & $3260 . n$ \\
1946 & 14500.0 & 8140.0 \\
1947 & 7380.0 & 5710.0 \\
1948 & 9700.0 & 7410.0 \\
1949 & 10500.0 & 7020.0 \\
1950 & 6380.0 & $5770 . n$ \\
1951 & 12900.0 & $8690 . n$ \\
1952 & 9070.0 & $5840 . n$ \\
1953 & 17000.0 & 7470.0 \\
1954 & 12300.0 & $8730 . n$ \\
1955 & 4480.0 & 4050.0 \\
1956 & 13500.0 & $10100 . n$ \\
1957 & 10400.0 & 6770.0 \\
1958 & 8950.0 & 6620.0 \\
1959 & 5530.0 & 3930.0 \\
1960 & 4990.0 & 4190.0 \\
1961 & 12400.0 & 7800.0 \\
1962 & 10000.0 & 6300.0 \\
1963 & 6420.0 & 4930.0
\end{tabular}

7
4810.0
5340.0
3760.0
2890.0
2450.0
1480.0
3340.0
7480.0
1440.0
2570.0
4600.0
3410.0
5070.0
4420.0
4430.0
4990.0
4040.0
4800.0
4580.0
2390.0
6510.0
4340.0
3950.0
2480.0
3160.0
4920.0
3810.0
3390.0

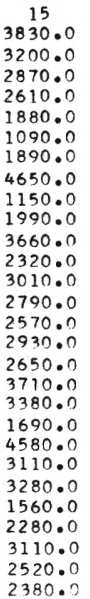

30
2460.0
2170.0
2080.0
1710.0
1730.0
908.0
1690.0
3270.0
860.0
1490.0
2370.0
2050.0
1870.0
1900.0
2090.0
2590.0
1910.0
3030.0
2970.0
1580.0
3420.0
2520.0
2110.0
1460.0
1950.0
2330.0
2120.0
1840.0

1720.0
1930.0

1780.0

1460.0

1320.0

751.0
1490.0

2590.0

781.0
1330.0

330.0

1240.0

1500.0

1340.0

1850.0

1500.

5030.

2000.0

1350.0

2870.0

1800.0

1990.0

1200.0

1600.0

1860.0

1450.0

90
1370.0
1610.0
1650.0
1160.0
1020.0
682.0
1290.0
2270.0
753.0
1290.0
1650.0
1210.0
1360.0
1210.0
1670.0
1740.0
1530.0
1670.0
1980.0
1130.0
2300.0
1330.0
1660.0
1120.0
1380.0
1410.0
1190.0
1200.0

120
1230

1230.0

$1370.0 \quad 1110.0$

$1530.0 \quad 1460.0$

$1000.0 \quad 894.0$

$895.0 \quad 797.0$

$589.0 \quad 511.0$

1
1

1880.0

$656 \cdot 0$
1180.0

1080.0

100.0

1250.0

1120.0

1450 .

$1720 \cdot n$

$1420 \cdot 0$

141000

1680.0

1040.0

1990.0

1310.0

1370.0

996.0

1320.0

$1250 \cdot 0$

$1050 \cdot 0$

1140.0

1420.0

1130.0

1200.0

1080.0

1270.0

1570.0

1380.0

1340.0

1490.0

1020.0

1890.0

1130.0

1250.0

960.0
1190.0

1190.0

1110.0

$\begin{array}{rr}183 & 274 \\ 980.0 & 714.0 \\ 1040.0 & 727.0 \\ 1390.0 & 1000.0 \\ 803.0 & 573.0 \\ 675.0 & 467.0 \\ 489.0 & 434.0 \\ 861.0 & 719.0 \\ 1490.0 & 1110.0 \\ 540.0 & 426.0 \\ 928.0 & 657.0 \\ 1250.0 & 890.0 \\ 985.0 & 726.0 \\ 1160.0 & 977.0 \\ 1040.0 & 747.0 \\ 1150.0 & 838.0 \\ 1400.0 & 1010.0 \\ 1250.0 & 953.0 \\ 1220.0 & 875.0 \\ 1280.0 & 917.0 \\ 911.0 & 659.0 \\ 1720.0 & 1270.0 \\ 1060.0 & 773.0 \\ 1080.0 & 794.0 \\ 845.0 & 624.0 \\ 1020.0 & 730.0 \\ 1080.0 & 770.0 \\ 1060.0 & 792.0 \\ 987.0 & 762.0\end{array}$
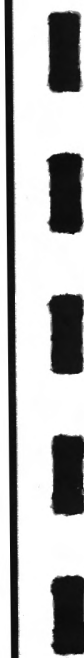
Row R1ver near Cottage Grove, Oreg.

STATION NUMBER $14-1555.00$

DURATION TABLF OF DAILY DISCHARGF

CLASS $0 \begin{array}{llllllllllllllllllllllllllllllllllll} & 0 & 2 & 3 & 4 & 5 & 6 & 7 & 8 & 9 & 10 & 11 & 12 & 13 & 14 & 15 & 16 & 17 & 18 & 19 & 20 & 21 & 22 & 23 & 24 & 25 & 26 & 27 & 28 & 29 & 30 & 31 & 32 & 33 & 34\end{array}$

YEAR

1940
1941

1941

1943

1944

1945

1946

1947

1948
1949

$\frac{1949}{1950}$

1951

1952

1953

1954

1955

1956
1957

$\begin{array}{lll}1957 & 6 & 1 \\ 1959 & 7 & 2\end{array}$

1960

1961

1962
1963

NUMBER OF DAYS IN CLASS

$\begin{array}{lllllllllllllllll}4 & 16 & 38 & 77 & 26 & 19 & 15 & 13 & 28 & 32 & 29 & 23 & 17 & 7 & 16 & 6\end{array}$

$\begin{array}{lllllllllllll}27 & 30 & 29 & 28 & 27.49 & 69 & 44 & 29 & 20 & 7 & 5 & 1\end{array}$

$\begin{array}{rrrrrrrrrrrrrrrrr}6 & 35 & 16 & 16 & 22 & 23 & 33 & 61 & 57 & 36 & 18 & 14 & 17 & 8 & 2 & & 1 \\ 12 & 42 & 25 & 22 & 11 & 8 & 27 & 43 & 33 & 21 & 33 & 32 & 26 & 18 & 8 & 1 & 2\end{array}$

$\begin{array}{lllllllllllll}23 & 55 & 20 & 13 & 35 & 19 & 39 & 63 & 37 & 19 & 23 & 12 & 7\end{array}$

$\begin{array}{lllllllllllllll}28 & 55 & 28 & 24 & 23 & 15 & 25 & 31 & 24 & 23 & 32 & 34 & 16 & 6\end{array}$

$\begin{array}{lllllllllllllllll}22 & 63 & 13 & 20 & 13 & 17 & 20 & 30 & 29 & 37 & 38 & 25 & 19 & 13 & 3 & 2 & \end{array}$

$\begin{array}{llllllllllllllll}1 & 26 & 29 & 28 & 34 & 19 & 46 & 51 & 25 & 31 & 27 & 17 & 17 & 9 & 3 & 2\end{array}$

$\begin{array}{lllllllllllll}34 & 21 & 24 & 12 & 11 & 19 & 37 & 30 & 49 & 65 & 26 & 22 & 10\end{array}$

$\begin{array}{rrrrrrrrrrrrrrrr}32 & 4 & 13 & 62 & 5 & 22 & 68 & 36 & 39 & 31 & 12 & 17 & 16 & 2 \\ 24 & 3.4 & 8 & 53 & 29 & 34 & 19 & 36 & 29 & 30 & 12 & 12 & 40 & 5\end{array}$

$\begin{array}{llllllllll}1 & 20 & 9 & 65101 & 41 & 45 & 24 & 15 & 10 & 34\end{array}$

$\begin{array}{lllllllllllll}2 & 29 & 10 & 54 & 64 & 78 & 28 & 17 & 18 & 19 & 23\end{array}$

$\begin{array}{lllllllllll}25 & 16130 & 55 & 30 & 27 & 28 & 10 & 11 & 32\end{array}$

$\begin{array}{lllllllllllll}4 & 4 & 53 & 13 & 81 & 40 & 34 & 71 & 30 & 17 & 9 & 9\end{array}$

$\begin{array}{lllllllllllll}5 & 74 & 6 & 34 & 40 & 28 & 40 & 38 & 21 & 41 & 26 & 10 & 3\end{array}$

$\begin{array}{llllllllllll}15 & 40 & 59 & 78 & 48 & 23 & 29 & 26 & 11 & 12 & 20 & 4\end{array}$

$\begin{array}{llllllllllllllll}1 & & & 4 & 76 & 15 & 77 & 45 & 35 & 28 & 28 & 19 & 7 & 23 \\ 10 & 2 & 15 & 40 & 14102 & 46 & 35 & 32 & 23 & 12 & 13 & 11\end{array}$

$6 \begin{array}{llllllllllll}57 & 12 & 62 & 75 & 59 & 24 & 30 & 15 & 13 & 13\end{array}$

$\begin{array}{lllllllllllll}6 & 61 & 39 & 94 & 61 & 13 & 14 & 31 & 13 & 11 & 22\end{array}$

$\begin{array}{rrrrrrrrrrrr} & & 46 & 8 & 77 & 44 & 62 & 58 & 33 & 10 & 9 & 18 \\ 2 & 13 & 27 & 15101 & 46 & 46 & 38 & 31 & 14 & 14 & 17\end{array}$
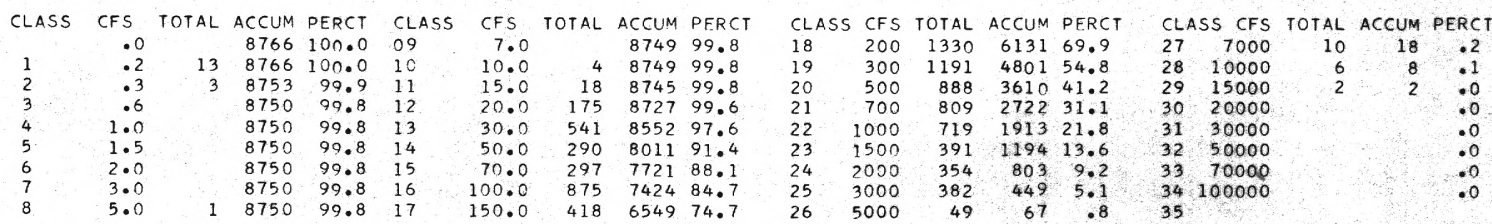

Row River near Cottage Grove, Oreg.

STATION NUMBEK

14-10כנ.vo

Summary for water years 1940-1949

$\begin{array}{lrrrrl}\text { CLASS } & \text { CFS } & \text { TOTAL } & \text { ACCUM } & \text { PERCT } & \text { CLASS } \\ & .0 & 3653 & \mathbf{1 0 0 . 0} & 09 \\ 1 & .2 & & 3653 & \mathbf{1 0 0 . 0} & 10 \\ 2 & .3 & & 3653 & \mathbf{1 0 0 . 0} & 11 \\ 3 & .6 & 3653 & 100.0 & 12 \\ 4 & 1.0 & & 3653 & 100.0 & 13 \\ 5 & 1.5 & & 3653 & 100.0 & 14 \\ 6 & 2.0 & & 3653 & 100.0 & 15 \\ 7 & 3.0 & & 3653 & 100.0 & 16 \\ 8 & 5.0 & & 3653 & 100.0 & 17\end{array}$

Row River near Cottage Grove, Oreg.

\begin{tabular}{r}
\multicolumn{1}{c}{ CFS } \\
7.0 \\
10.0 \\
15.0 \\
20.0 \\
30.0 \\
50.0 \\
70.0 \\
100.0 \\
150.0
\end{tabular}

$\begin{array}{rrr}\text { TOTAL } & \text { ACCUM } & \text { PERCT } \\ & 3653 & 100.0 \\ 4 & 3653 & 100.0 \\ 16 & 3649 & 99.9 \\ 160 & 3633 & 99.5 \\ 460 & 3473 & 95.1 \\ 231 & 3013 & 82.5 \\ 220 & 2782 & 76.2 \\ 212 & 2562 & 70.1 \\ 168 & 2350 & 64.3\end{array}$

\begin{tabular}{l} 
Cl.ASS \\
18 \\
19 \\
20 \\
21 \\
22 \\
23 \\
24 \\
25 \\
26 \\
\hline
\end{tabular}

$\begin{array}{rr}\text { CFS } & \text { TOTAL } \\ 200 & 319 \\ 300 & 439 \\ 500 & 332 \\ 700 & 307 \\ 1000 & 319 \\ 1500 & 192 \\ 2000 & 156 \\ 3000 & 78 \\ 5000 & 25\end{array}$

$\begin{array}{rrr}\text { ACCUM } & \text { PERCT } \\ 2182 & 59.7 & \\ 1863 & 51.0 & \\ 1424 & 39.0 & \\ 1092 & 29.9 & \\ 785 & 21.5 & \\ 466 & 12.8 & \\ 274 & 7.5 & 32 \\ 118 & 3.2 & \\ 40 & 1.1 & \end{array}$

$\begin{array}{lr}\text { CLASS } & \text { CFS } \\ 27 & 7000 \\ 28 & 10000 \\ 29 & 15000 \\ 30 & 20000 \\ 31 & 30000 \\ 32 & 50000 \\ 33 & 70000 \\ 34 & 100000 \\ 35 & \end{array}$

$\begin{array}{rrr}\text { TOTAL } & \text { ACCUM } & \text { PERCT } \\ 7 & 15 & .4 \\ 6 & 8 & .2 \\ 2 & 2 & .1 \\ & & .0 \\ & & .0 \\ & & .0 \\ & & .0 \\ & & .0\end{array}$

Summary for water years 1950-1963

STATION NUMBER

14-1555.00

$\begin{array}{lrrrrl}\text { CLASS } & \text { CFS } & \text { TOTAL } & \text { ACCUM } & \text { PERCT } & \text { CLASS } \\ & .0 & & 5113 & 100.0 & 09 \\ 1 & .2 & 13 & 5113 & 100.0 & 10 \\ 2 & .3 & 3 & 5100 & 99.7 & 11 \\ 3 & .6 & & 5097 & 99.7 & 12 \\ 4 & 1.0 & & 5097 & 99.7 & 13 \\ 5 & 1.5 & & 5097 & 99.7 & 14 \\ 6 & 2.0 & & 5097 & 99.7 & 15 \\ 7 & 3.0 & & 5097 & 99.7 & 16 \\ 8 & 5.0 & 1 & 5097 & 99.7 & 17\end{array}$

$\begin{array}{rrrr}\text { CFS } & \text { TOTAL } & \text { ACCUM } & \text { PERCT } \\ 7.0 & & 5096 & 99.7 \\ 10.0 & & 5096 & 99.7 \\ 15.0 & 2 & 5096 & 99.7 \\ 20.0 & 15 & 5094 & 99.6 \\ 30.0 & 81 & 5079 & 99.3 \\ 50.0 & 59 & 4998 & 97.8 \\ 70.0 & 77 & 4939 & 96.6 \\ 100.0 & 663 & 4862 & 95.1 \\ 150.0 & 250 & 4199 & 82.1\end{array}$

CLASS
18
19
20
21
22
23
24
25
26

$\begin{array}{rr}\text { CFS } & \text { TOTAL } \\ 200 & 1011 \\ 300 & 752 \\ 500 & 556 \\ 700 & 502 \\ 1000 & 400 \\ 1500 & 199 \\ 2000 & 198 \\ 3000 & 304 \\ 5000 & 24\end{array}$

\begin{tabular}{|r|r|}
\hline ACCUM & PERCT \\
3949 & 77.2 \\
2938 & 57.5 \\
2186 & 42.8 \\
1630 & 31.9 \\
1128 & 22.1 \\
728 & 14.2 \\
529 & 10.3 \\
331 & 6.5 \\
27 & .5
\end{tabular}

Cr.ASS
27
28
29
30
31
32
33
34
35

CFS
7000
10000
15000
20000
30000
50000
70000
100000

$\begin{array}{crrr}5 & \text { TOTAL ACCUM PERCT } \\ 0 & 3 & 3 & .1 \\ 0 & & .0 \\ 0 & & .0 \\ 0 & & .0 \\ 0 & & .0 \\ 0 & & .0 \\ 0 & & .0 \\ 0 & & .0\end{array}$


Row River near Cottage Grove, Oreg.

STATION NUMBER $14-1555.00$

LOWEST MEAN DISCHARGF, IN CFS, FOR THE FOLLOWING NUMBER OF CONSECUTIVE DAYS IN YEAR RFGINNING APRIL 1

\begin{tabular}{|c|c|c|c|c|c|c|c|c|c|c|c|}
\hline YEAR & 1 & 3 & 7 & 14 & 30 & 60 & 90 & 120 & 150 & 183 & 274 \\
\hline 1939 & 21.0 & $21 \cdot 0$ & $21 . ?$ & 23.0 & 27.6 & 31.4 & 35.5 & $44 \cdot 5$ & 46.8 & 67.8 & 172.0 \\
\hline 1940 & 14.0 & 14.0 & 14.6 & 15.4 & $18 \cdot 2$ & 25.6 & 30.4 & $42 \cdot 6$ & 52.3 & 87.1 & 285.0 \\
\hline 1941 & 45.0 & 45.0 & 47.1 & 47.9 & 54.7 & 80.3 & 99.3 & 113.0 & $170 \cdot 0$ & 250.0 & 571.0 \\
\hline 1942 & 25.0 & 25.0 & 26.1 & 27.9 & 31.3 & 34.7 & 44.3 & 68.9 & 151.0 & 307.0 & 816.0 \\
\hline $\begin{array}{l}1943 \\
1944\end{array}$ & 25.0 & 26.3 & 28.1 & 30.4 & 32.9 & 45.3 & 53.4 & 110.0 & 185.0 & 215.0 & 334.0 \\
\hline $\begin{array}{l}1944 \\
1945\end{array}$ & 21.0 & 21.7 & 22.9 & 26.9 & 28.8 & 35.7 & 37.4 & 42.0 & 62.2 & 85.1 & 214.0 \\
\hline 1945 & 22.0 & 22.7 & 24.3 & 29.9 & 32.9 & 37.2 & 43.0 & 51.3 & 76.3 & 226.0 & 780.0 \\
\hline $\begin{array}{l}1946 \\
1947\end{array}$ & 23.0 & 23.7 & 25.9 & 28.6 & 30.4 & 33.5 & 37.7 & 53.5 & 97.8 & 150.0 & 487.0 \\
\hline 1947 & 41.0 & 41.7 & 42.9 & 44.1 & 55.0 & 62.6 & 90.9 & $124 \cdot 0$ & 161.0 & 199.0 & 617.0 \\
\hline$\frac{1948}{1949}$ & 31.0 & 31.7 & 33.4 & 36.6 & 43.6 & 55.8 & 78.9 & 89.6 & 139.0 & 229.0 & 533.0 \\
\hline 1949 & 18.0 & 23.0 & 24.6 & 25.9 & 27.2 & 32.3 & 35.5 & 48.6 & 50.5 & 67.8 & 320.0 \\
\hline 1950 & 97.0 & 97.0 & 97.0 & 98.3 & 99.9 & 102.0 & 167.0 & 252.0 & 317.0 & 372.0 & 890.0 \\
\hline 1951 & 41.0 & 42.0 & 42.6 & 44.4 & 49.5 & 57.3 & 71.8 & $85 \cdot 6$ & 116.0 & 154.0 & 662.0 \\
\hline 1952 & 30.0 & 32.3 & 35.0 & 35.1 & 55.3 & 211.0 & 281.0 & 312.0 & 280.0 & 322.0 & 385.0 \\
\hline 1953 & 120.0 & 121.0 & 123.0 & 125.0 & 146.0 & 257.0 & 367.0 & 347.0 & 341.0 & 494.0 & 903.0 \\
\hline 1954 & 54.0 & 54.0 & 66.4 & 114.0 & 132.0 & 169.0 & 182.0 & 234.0 & 280.0 & 281.0 & 339.0 \\
\hline 1955 & 98.0 & 98.0 & 99.4 & 103.0 & 107.0 & 181.0 & 385.0 & $360 \cdot 0$ & 366.0 & 433.0 & 1080.0 \\
\hline 1956 & 100.0 & $100 . ?$ & 101.0 & 105.0 & 106.0 & 223.0 & 388.0 & 332.0 & 371.0 & 436.0 & 597.0 \\
\hline 1957 & 96.0 & 96.0 & 96.3 & 98.5 & 100.0 & 104.0 & 142.0 & 205.0 & 279.0 & 265.0 & 453.0 \\
\hline 1958 & .2 & .2 & .2 & .2 & 11.0 & 66.1 & 204.0 & 345.0 & 327.0 & 315.0 & 469.0 \\
\hline 1959 & 75.0 & 76.0 & 84.1 & 96.2 & 110.0 & 136.0 & 193.0 & 213.0 & 252.0 & 303.0 & 302.0 \\
\hline 1960 & 85.0 & 88.0 & 94.3 & $1 \cap 2 \cdot n$ & 114.0 & 183.0 & 256.0 & 300.0 & 283.0 & 356.0 & 578.0 \\
\hline 1961 & 107.0 & 107.0 & 107.0 & 108.0 & 116.0 & 160.0 & 186.0 & 221.0 & 225.0 & 262.0 & 636.0 \\
\hline 1962 & 62.0 & 69.7 & 90.7 & 94.3 & 139.0 & 220.0 & 333.0 & $364 \cdot 0$ & 369.0 & 403.0 & 541.0 \\
\hline
\end{tabular}

Kow k1ver near lottage lirove, ureg.

SIAIION NUMBEK 14-ISOD.VU

HIGHEST MEAN DISCHARGE, IN CFS, FOR THE FOLLOWING NUMBER OF CONSECUTIVE DAYS IN YEAR ENDING SEPTEMBER 30

\begin{tabular}{|c|c|c|c|c|c|c|c|c|c|c|c|}
\hline YEAR & 1 & 3 & 7 & 15 & 30 & 60 & 90 & 120 & 150 & 183 & 274 \\
\hline 1940 & 4170.0 & $3610 . n$ & 2980.0 & 2390.0 & 2160.0 & 1620.0 & 1250.0 & $1100 \cdot 0$ & 968.0 & 826.0 & 572.0 \\
\hline 1941 & 4040.0 & 2930.0 & $1880 . ?$ & 1370.0 & 1140.0 & 937.0 & 855.0 & 733.0 & 635.0 & 604.0 & 537.0 \\
\hline 1942 & 13300.0 & $732 n \cdot n$ & 40.30 .0 & 2270.0 & 1950.0 & 1750.0 & 1510.0 & $1290 \cdot 0$ & 1130.0 & 1030.0 & 872.0 \\
\hline 1943 & 15400.0 & 12700.0 & $8910 . n$ & 5670.0 & 4030.0 & 3260.0 & $2880 \cdot 0$ & 2390.0 & 2140.0 & $1900 \cdot 0$ & 1410.0 \\
\hline 1944 & 5090.0 & 3160.0 & 1900.0 & 1590.0 & 1160.0 & 1010.0 & 998.0 & 871.0 & 772.0 & 713.0 & 559.0 \\
\hline 1945 & 5310.0 & $3960 . n$ & $309 \cap .0$ & 2480.0 & 1800.0 & 1610.0 & 1590.0 & $1450 \cdot 0$ & 1350.0 & 1150.0 & 817.0 \\
\hline 1946 & 15800.0 & 9630.0 & 5500.0 & 4480.0 & 2920.0 & 2410.0 & 2030.0 & $1920 \cdot 0$ & 1770.0 & 1550.0 & 1100.0 \\
\hline 1947 & 9190.0 & 7180.0 & 4340.0 & 2880.0 & 2570.0 & 1560.0 & 1520.0 & $1380 \cdot 0$ & 1410.0 & 1240.0 & 909.0 \\
\hline 1948 & 11400.0 & $9130 \cdot n$ & 6400.0 & 3840.0 & 2410.0 & 1920.0 & 1780.0 & $1630 \cdot 0$ & 1570.0 & 1520.0 & 1260.0 \\
\hline 1949 & 11000.0 & 8240.0 & 5520.0 & 3530.0 & 2390.0 & 1730.0 & 1540.0 & $1450 \cdot 0$ & 1380.0 & $1320 \cdot 0$ & 944.0 \\
\hline 1950 & 5050.0 & $4990 . n$ & 4640.0 & 3470.0 & 2660.0 & 2170.0 & 1890.0 & 1620.0 & 1440.0 & 1290.0 & 936.0 \\
\hline 1951 & 5080.0 & 5010.0 & 4980.0 & 4270.0 & 3350.0 & 2540.0 & 2450.0 & 2350.0 & 2060.0 & 1820.0 & 1290.0 \\
\hline 1952 & 5020.0 & 4920.0 & 4640.7 & 3240.0 & 2420.0 & $1940 . ?$ & 1970.0 & 1810.0 & 1630.0 & 1550.0 & 1180.0 \\
\hline 1953 & $496 n \cdot n$ & $4920 \cdot n$ & 4670.0 & 3770.0 & $3600 \cdot 0$ & 2370.0 & 1880.0 & $1600 \cdot 0$ & 1440.0 & 1340.0 & 1040.0 \\
\hline 1954 & $5 \cap 00 . n$ & $4980 . n$ & $497 n .0$ & 4220.0 & 3750.0 & 2610.0 & 2490.0 & 2020.0 & 1740.0 & 1490.0 & 1070.0 \\
\hline 1955 & 4230.0 & 3970.0 & 3040.0 & 1900.0 & 1860.0 & 1440.0 & 1160.0 & $1120 \cdot 0$ & 1070.0 & 977.0 & 810.0 \\
\hline 1956 & 7330.0 & $7160 . n$ & $6410 . n$ & 5640.0 & 4250.0 & 3660.0 & 3020.0 & $254 n \cdot 0$ & 2310.0 & 2050.0 & 1510.0 \\
\hline 1957 & 5060.0 & 5010.0 & 4740.0 & 3950.0 & 3070.0 & 1950.0 & 1430.0 & $1460 \cdot 0$ & $1320 \cdot 0$ & 1230.0 & 915.0 \\
\hline 1958 & 4840.0 & $4810 \cdot 0$ & 4690.0 & 4060.0 & 2670.0 & 2270.0 & 1930.0 & 1550.0 & 1360.0 & 1170.0 & 947.0 \\
\hline 1959 & 4700.0 & 3870.0 & 2810.0 & 1920.0 & 1800.0 & 1390.0 & $1210 \cdot 0$ & $1130 \cdot 0$ & 1050.0 & 901.0 & 738.0 \\
\hline 1960 & 4620.0 & $4210 \cdot 0$ & 3490.0 & 2490.0 & 2100.0 & 1680.0 & 1420.0 & $1360 \cdot 0$ & 1250.0 & 1080.0 & 835.0 \\
\hline 1961 & 4940.0 & 4930.0 & 4930.0 & 4150.0 & 2940.0 & 2110.0 & 1530.0 & $1500 \cdot 0$ & 1380.0 & $1200 \cdot 0$ & 867.0 \\
\hline 1962 & 4990.0 & $4820 . n$ & 4490.0 & 3330.0 & 2430.0 & 1930.0 & 1510.0 & $1260 \cdot 0$ & 1250.0 & 1160.0 & 908.0 \\
\hline 1963 & 5830.0 & 4650.0 & 3820.0 & 2620.0 & 2010.0 & 1610.0 & 1220.0 & $1170 \cdot 0$ & 988.0 & $1080 \cdot 0$ & 842.0 \\
\hline
\end{tabular}


Mosby Creek near Cottage Grove, Oreg.

STATION NUMBER 14-156ח.0O

DURATION TABLE OF DAILY DISCHARGE

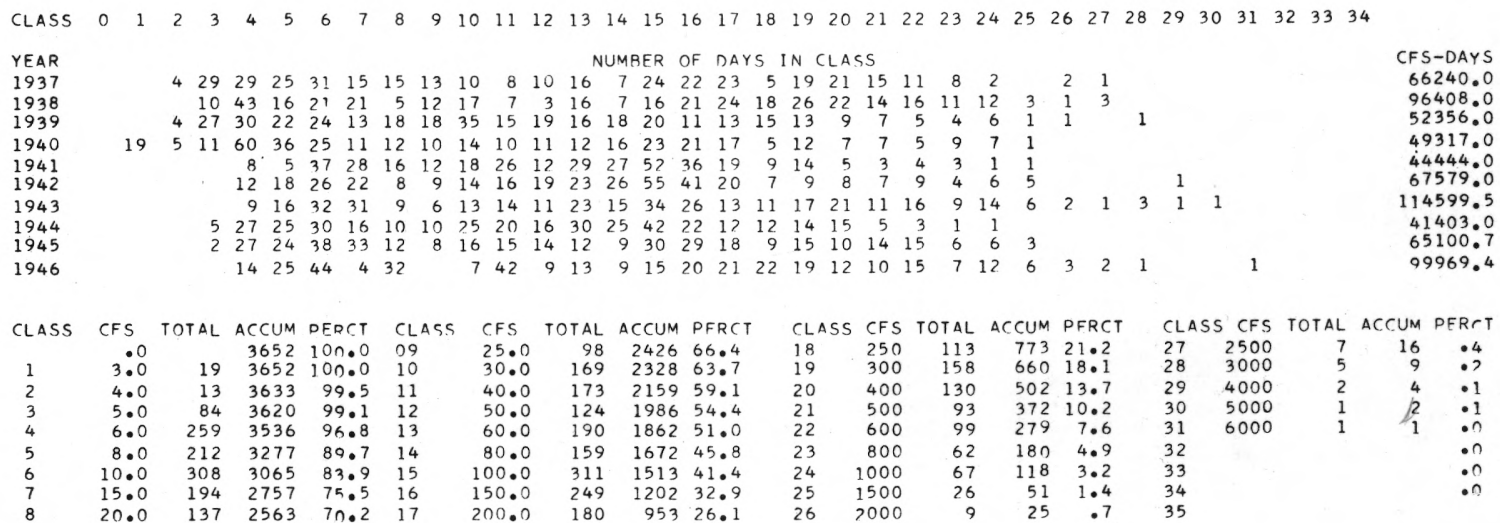

Mosby Creek near Cottage Grove, Oreg. STATION NUMBER 14-1560.00

LOWEST MEAN DISCHARGF, IN CFS, FOR THE FOLLOWING NUMBER OF CONSFCUTIVF DAYS IN YEAR BEGINNING APRIL 1

\begin{tabular}{|c|c|c|c|c|c|c|c|c|c|c|c|}
\hline YEAR & 1 & 3 & 7 & 14 & 30 & 60 & 90 & 120 & 150 & 183 & 274 \\
\hline $\begin{array}{l}1936 \\
1937\end{array}$ & $\begin{array}{l}4.0 \\
7.0\end{array}$ & $\begin{array}{l}4.0 \\
7.0\end{array}$ & $\begin{array}{l}4.4 \\
7.7\end{array}$ & $\begin{array}{l}4.9 \\
8.6\end{array}$ & $\begin{array}{r}5.5 \\
11.0\end{array}$ & $\begin{array}{r}5.8 \\
12.4\end{array}$ & $\begin{array}{r}6.7 \\
17.9\end{array}$ & $\begin{array}{r}7 \cdot 2 \\
29.6\end{array}$ & $\begin{array}{r}9.2 \\
70.1\end{array}$ & $\begin{array}{l}13.5 \\
86.8\end{array}$ & $\begin{array}{r}44.4 \\
185.0\end{array}$ \\
\hline 1938 & 5.0 & 5.0 & 5.0 & 5.8 & 5.9 & 6.7 & 7.5 & 8.6 & 12.3 & 29.9 & 76.9 \\
\hline 1939 & 4.0 & $4 \cdot 3$ & 4.7 & 4.9 & 5.6 & 6.4 & 7.7 & $9 \cdot 7$ & $10 \cdot 2$ & 13.8 & 42.8 \\
\hline 1940 & 3.0 & $3 \cdot n$ & $3 . n$ & 3.0 & 3.7 & $5 \cdot 3$ & 6.5 & $8 \cdot 5$ & 10.7 & 20.7 & 81.2 \\
\hline 1941 & 10.0 & 10.3 & 10.9 & 11.3 & $12 \cdot 6$ & 19.1 & 21.3 & $25 \cdot 7$ & $42 \cdot 1$ & 61.8 & 165.0 \\
\hline 1942 & 6.0 & 6.0 & 6.2 & 6.3 & 7.3 & 8.8 & 10.5 & $15 \cdot 7$ & 32.9 & $70 \cdot 7$ & 227.0 \\
\hline 1943 & 8.5 & 8.5 & 8.7 & 9.4 & 9.9 & 13.2 & 14.8 & $29 \cdot 6$ & 49.7 & 61.6 & 90.7 \\
\hline 1944 & 5.0 & 5.0 & 5.4 & 6.0 & 6.8 & 7.9 & 8.1 & $10 \cdot 0$ & 15.6 & 21.8 & 51.4 \\
\hline 1945 & 6.4 & 6.4 & 6.7 & 7.0 & 8.0 & 10.2 & 10.9 & $12 \cdot 9$ & 18.5 & 54.7 & 245.0 \\
\hline
\end{tabular}

rusuy ureek near cottage Grove, Oreg.

STATION NUMBER $14-1560.00$

HIGHEST MEAN DISCHARGE, IN CFS, FOR THE FOLLOWING NUMRER OF CONSECUTIVF DAYS IN YFAR ENDING SEPTEMBER 30

\begin{tabular}{|c|c|c|c|c|c|c|c|c|c|c|c|}
\hline YEAR & 1 & 3 & 7 & 15 & 30 & 60 & 90 & 120 & 150 & 183 & $\begin{array}{l}274 \\
239\end{array}$ \\
\hline $\begin{array}{l}1937 \\
1938\end{array}$ & $\begin{array}{l}2740.0 \\
2900.0\end{array}$ & $\begin{array}{l}2430.0 \\
2090.0\end{array}$ & $\begin{array}{l}1500.0 \\
1500.0\end{array}$ & $\begin{array}{r}955.0 \\
1140.0\end{array}$ & $\begin{array}{l}648.0 \\
773.0\end{array}$ & $\begin{array}{l}593.0 \\
690.0\end{array}$ & $\begin{array}{l}525.0 \\
617.0\end{array}$ & $\begin{array}{l}439 \cdot 0 \\
565 \cdot 0\end{array}$ & $\begin{array}{l}402.0 \\
549.0\end{array}$ & $\begin{array}{l}344.0 \\
496.0\end{array}$ & $\begin{array}{l}239.0 \\
349.0\end{array}$ \\
\hline $\begin{array}{l}1939 \\
1940\end{array}$ & $\begin{array}{l}3620.0 \\
1710.0\end{array}$ & $\begin{array}{l}2270.0 \\
1310.0\end{array}$ & $\begin{array}{l}1320.0 \\
1040.0\end{array}$ & $\begin{array}{l}838.0 \\
791.0\end{array}$ & $\begin{array}{l}637.0 \\
713.0\end{array}$ & $\begin{array}{l}540.0 \\
513.0\end{array}$ & $\begin{array}{l}425.0 \\
393.0\end{array}$ & $\begin{array}{l}361 \bullet 0 \\
355 \bullet 0\end{array}$ & $\begin{array}{l}315.0 \\
307.0\end{array}$ & $\begin{array}{l}270.0 \\
259.0\end{array}$ & $\begin{array}{l}189.0 \\
177.0\end{array}$ \\
\hline 1941 & 1540.0 & 1200.0 & 773.0 & 531.0 & 405.0 & 314.0 & 264.0 & $226 \cdot 0$ & 196.0 & 178.0 & 156.0 \\
\hline 1942 & 4300.0 & 2310.0 & 1280.0 & 814.0 & 647.0 & 552.0 & 474.0 & $394 \cdot 0$ & 337.0 & 298.0 & 242.0 \\
\hline 1943 & 5370.0 & $4650 . n$ & 3110.0 & 1930.0 & 1320.0 & 1030.0 & 926.0 & $738 \cdot 0$ & 651.0 & 567.0 & 414.0 \\
\hline 1944 & 1460.0 & $886 \cdot n$ & 577.0 & 496.0 & 328.0 & 257.0 & 253.0 & $229 \cdot 0$ & 200.0 & 190.0 & 148.0 \\
\hline 1945 & 1630.0 & 1440.0 & 1210.0 & 872.0 & 653.0 & 521.0 & 498.0 & 433.0 & 390.0 & 332.0 & 234.0 \\
\hline 1946 & 6330.0 & $3650 . n$ & 2160.0 & 1770.0 & 1100.0 & 937.0 & 756.0 & $686 \cdot 0$ & 612.0 & $520 \cdot 0$ & 361.0 \\
\hline
\end{tabular}


Mosby Creek at mouth, near

Cottage Grove, Oreg.
STATION NUMBER $\quad 14-1565.00$

DURATION'TABLE OF DAILY DISCHARGE

CLASS $0 \begin{array}{llllllllllllllllllllllllllllllllllll}1 & 2 & 3 & 4 & 5 & 6 & 7 & 8 & 9 & 10 & 11 & 12 & 13 & 14 & 15 & 16 & 17 & 18 & 19 & 20 & 21 & 22 & 23 & 24 & 25 & 26 & 27 & 28 & 29 & 30 & 31 & 32 & 33 & 34\end{array}$

YEAR
1947
1948
1949
1950
1951
1952
1953
1954
1955
1956
1957
1958
1959
1960
1961
1962
1963

NUMBER OF DAYS IN CLACS

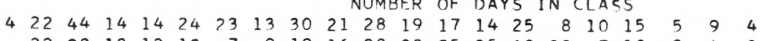

$\begin{array}{rrrrrrrrrrrrrrrrrrrr}22 & 23 & 13 & 12 & 13 & 7 & 9 & 19 & 16 & 28 & 22 & 25 & 25 & 63 & 23 & 7 & 15 & 5 & 9 & 4 \\ 47 & 11 & 15 & 22 & 13 & 9 & 15 & 21 & 19 & 28 & 21 & 18 & 15 & 37 & 11 & 7 & 10 & 4 & 6 & 3\end{array}$

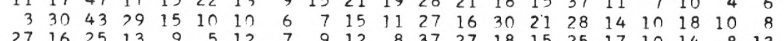

$\begin{array}{rrrrrrrrrrrrrrrrrrrrrrrr}2 & 17 & 27 & 16 & 25 & 13 & 9 & 5 & 12 & 7 & 9 & 12 & 8 & 37 & 27 & 18 & 15 & 25 & 14 & 10 & 18 & 10 & 8 & 1 \\ & & 9 & 31 & 11 & 14 & 12 & 23 & 24 & 11 & 17 & 13 & 27 & 31 & 23 & 24 & 28 & 13 & 7 & 17 & 8 & 13 & 12\end{array}$

$\begin{array}{rrrrrrrrrrrrrrrrrrrrrrr}35 & 52 & 33 & 7 & 8 & 11 & 6 & 6 & 5 & 9 & 32 & 30 & 19 & 17 & 21 & 14 & 13 & 13 & 13 & 12 \\ & 33 & 45 & 19 & 10 & 25 & 22 & 21 & 16 & 12 & 17 & 15 & 27 & 12 & 20 & 17 & 13 & 7 & 1 & 9\end{array}$

$\begin{array}{llllllllllllllllllllllll}21 & 34 & 25 & 20 & 10 & 28 & 16 & 8 & 8 & 25 & 25 & 23 & 33 & 15 & 23 & 16 & 11 & 12 & 4 & 6 & 2\end{array}$

$\begin{array}{rrrrrrrrrrrrrrrrrrrrrrrr}10 & 19 & 26 & 15 & 13 & 6 & 16 & 7 & 5 & 21 & 18 & 28 & 15 & 17 & 15 & 23 & 20 & 19 & 20 & 14 & 22\end{array}$

$\begin{array}{lllllllllllllllllllllllllllll}22 & 20 & 20 & 21 & 15 & 7 & 19 & 21 & 21 & 17 & 13 & 29 & 31 & 15 & 15 & 17 & 14 & 7 & 14 & 10 & 6 & 4 & 1 & 2 & 2 & 1\end{array}$

$\begin{array}{llllllllllllllllllllllllll}4 & 21 & 22 & 29 & 19 & 16 & 12 & 10 & 7 & 17 & 21 & 24 & 40 & 26 & 26 & 16 & 14 & 9 & 2 & 12 & 5 & 5 & 6 & 2\end{array}$

$\begin{array}{rrrrrrrrrrrrrrrrrrrrrrrrrrrrr}13 & 15 & 17 & 25 & 18 & 27 & 11 & 20 & 18 & 10 & 18 & 13 & 27 & 31 & 17 & 14 & 15 & 14 & 15 & 11 & 4 & 8 & 3 & 2\end{array}$

$\begin{array}{rrrrrrrrrrrrrrrrrrrrrrrrr}13 & 22 & 27 & 16 & 14 & 13 & 19 & 9 & 9 & 24 & 24 & 38 & 17 & 9 & 11 & 16 & 12 & 17 & 12 & 10 & 12 & 1 & \\ 13 & 13 & 28 & 24 & 14 & 7 & 16 & 14 & 9 & 10 & 15 & 44 & 29 & 29 & 26 & 26 & 10 & 11 & 11 & 2 & 6 & 4 & 2 & 1 \\ & 6 & 24 & 25 & 15 & 13 & 26 & 19 & 15 & 25 & 18 & 38 & 23 & 16 & 14 & 20 & 13 & 15 & 16 & 9 & 8 & 2 & 3\end{array}$

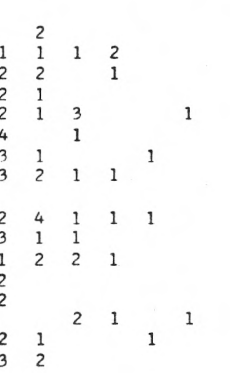

CFS-DAYS 78006.0 80532.0 80532.0
84893.0 84893.0
170917.0 102194.1 98999.9 100423.0 68511.2 141301.8 81860.1 91108.2

69184.1 69184.1
72877.1 $90082: 5$ 83694.0

MOSBY CREHFK AT MOUTH NEAR COTTTAGE GROVE OREC

Summary of water years $1947-63$

STATION NUMBER $\quad 14-1565.00$

\begin{tabular}{|c|c|c|c|c|c|c|c|c|c|c|c|c|c|c|c|c|c|c|c|}
\hline CLASS & CFS & TOTAL & ACCUM & PERCT & CLASS & CFS & TOTAL & ACCUM & PFRCT & CLASS & CFS & TOTAL & ACCUM & PFRCT & CLASS & CFS & TOTAL & ACCUM & PERCT \\
\hline & & & 6209 & 100.0 & 09 & 30.0 & 290 & 4382 & 70.6 & 18 & 300 & 415 & 1505 & 24.2 & 27 & 3000 & 12 & 24 & .4 \\
\hline 1 & 4.0 & 7 & 6209 & 100.0 & 10 & 40.0 & 228 & 4092 & 65.9 & 19 & 400 & 239 & $109 n$ & 17.6 & 28 & 4000 & 7 & 12 & .2 \\
\hline 2 & 5.0 & 51 & 6202 & 90.9 & 11 & 50.0 & 198 & 3864 & 62.2 & 20 & 500 & 184 & 851 & 13.7 & 29 & 5000 & 3 & 5 & .1 \\
\hline 3 & 6.0 & 166 & 6151 & 99.1 & 12 & 60.0 & 310 & 3666 & 59. & 21 & 600 & 230 & 667 & 10.7 & 30 & 6000 & 2 & $?$ & .0 \\
\hline 4 & 8.0 & 270 & 5985 & 96.4 & 13 & 80.0 & 286 & 3356 & 54. & 22 & 800 & 130 & 437 & 7.0 & 31 & & & & .0 \\
\hline 5 & 10.0 & 514 & 5715 & 97.0 & 14 & 100.0 & 524 & 3070 & 49.4 & 23 & 1000 & 154 & 307 & 4.9 & 32 & & & & .0 \\
\hline 6 & 15.0 & 388 & 5201 & 83. & 15 & 150 & 399 & 2546 & 41 & 24 & 15 & 77 & 153 & 2 & 33 & & & & $\bullet 0$ \\
\hline 7 & 20.0 & 249 & 4813 & 77.5 & 16 & 200.0 & 361 & 2147 & 34. & 25 & 2000 & 32 & 76 & 1.2 & 34 & & & & .0 \\
\hline 8 & 25.0 & 182 & 4564 & 72.5 & 17 & 250.0 & 281 & 1786 & 28.8 & 26 & 2500 & 20 & 44 & .7 & 35 & & & & \\
\hline
\end{tabular}

Mosby Creek at mouth, near

STATION NUMBER $14-1565.00$

Cottage Grove mouth.

LOWEST MEAN DISCHARGE, IN CFS, FOR THE FOLLOWING NUMBER OF CONSECUTIVE DAYS IN YEAR REGINNING APRIL 1

\begin{tabular}{|c|c|c|c|c|c|c|c|c|c|c|c|}
\hline YEAR & 1 & 3 & 7 & 14 & 30 & 60 & 90 & 120 & 150 & 183 & 274 \\
\hline 1947 & 12.0 & 12.0 & $12 \cdot 3$ & 12.5 & 15.6 & 17.5 & 23.0 & 29.6 & 40.0 & 48.6 & 172.0 \\
\hline 1948 & 11.0 & 11.7 & 12.1 & 12.9 & 14.9 & 18.7 & 26.5 & 29.6 & 43.8 & 64.0 & 171.0 \\
\hline 1949 & 6.0 & 6.0 & 6.3 & 6.8 & 8.4 & 10.1 & 11.1 & 14.1 & 16.1 & 21.7 & 92.5 \\
\hline 1950 & 7.0 & 7.3 & 7.7 & 7.9 & 8.8 & 10.5 & 13.4 & $26 \cdot 2$ & 39.8 & 64.0 & 244.0 \\
\hline 1951 & 4.0 & $4 \cdot 3$ & 4.7 & 5.2 & 6.3 & 6.6 & 8.5 & $12 \cdot 5$ & 23.2 & 41.7 & 185.0 \\
\hline 1952 & 8.8 & 8.8 & 8.9 & 9.2 & 9.2 & 10.0 & 11.5 & $13 \cdot 2$ & 17.5 & 33.0 & 82.9 \\
\hline 1953 & 11.0 & 12.0 & 12.0 & 12.4 & 14.1 & 16.4 & 19.0 & $24 \cdot 6$ & 34.1 & 96.3 & 240.0 \\
\hline 1954 & 12.0 & 12.0 & $12 \cdot 1$ & 12.9 & 14.2 & 15.5 & 16.2 & 20.9 & 25.6 & 31.6 & 80.6 \\
\hline 1955 & 8.5 & 8.6 & 8.8 & 9.2 & 9.7 & 13.3 & 16.5 & $22 \cdot 6$ & 33.4 & 54.2 & 312.0 \\
\hline 1956 & 6.9 & 7.3 & 7.7 & 8.3 & 8.6 & 9.5 & 12.3 & $19 \cdot 6$ & 38.1 & 72.1 & 140.0 \\
\hline 1957 & 5.0 & 5.2 & 5.7 & 6.3 & 6.7 & 8.1 & 11.1 & $19 \cdot 2$ & 23.5 & 38.0 & 144.0 \\
\hline 1958 & 5.2 & 5.3 & 6.2 & 7.2 & 7.8 & 8.3 & 10.1 & 14.5 & 28.5 & 42.4 & 115.0 \\
\hline 1959 & 5.6 & 6.0 & 6.6 & 7.4 & 8.4 & 10.7 & 14.9 & $20 \cdot 2$ & 23.3 & 28.1 & 32.9 \\
\hline 1960 & 4.7 & 4.9 & 5.0 & 5.4 & 6.9 & 9.1 & 11.2 & 15.3 & 20.7 & 64.4 & 147.0 \\
\hline 1961 & 4.7 & 4.7 & 5.2 & 5.6 & $\begin{array}{r}7.5 \\
.0\end{array}$ & 9.3 & 10.6 & 16.6 & 23.7 & 37.5 & 174.0 \\
\hline 1962 & 6.3 & 6.5 & 7.1 & 7.9 & 10.1 & 13.7 & 15.7 & 23.3 & 48.7 & 72.8 & 147.0 \\
\hline
\end{tabular}

Mosby Creek at mouth, near Cottage Grove, Oreg.

STATION NUMRER $14-1565.00$

HIGHEST MEAN DISCHARGF, IN CFS, FOR THE FOLLOWING NUMBER OF CONSFCUTIVF DAYS IN YFAR ENDING SEPTFMBER 30

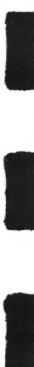

YEAR
1947
1948
1949
1950
1951
1952
1953
1954
1955
1956
1957
1958
1959
1960
1961
1962
1963

1
2990.0
4300.0
4030.0
2500.0
6220.0
3010.0
5120.0
4850.0
1680.0
5340.0
3150.0
4080.0
2300.0
2250.0
6560.0
5040.0
2760.0

3
1980.0
3400.0
2920.0
2300.0
4240.0
2050.0
3360.0
3450.0
1540.0
4760.0
2580.0
3210.0
1810.0
1880.0
3970.0
3100.0
2120.0

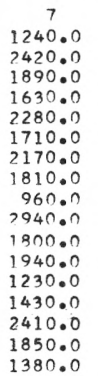

15
978.0
1420.0
1240.0
1020.0
1410.0
1060.0
1610.0
1360.0
691.0
1990.0
1260.0
1510.0
717.0
949.0
1460.0
1150.0
954.0

30
785.0
857.0
806.0
901.0
1140.0
865.0
1260.0
1110.0
635.0
1500.0
1050.0
945.0
672.0
713.0
1130.0
920.0
781.0

60
469.0
701.0
528.0
745.0
787.0
668.0
855.0
823.0
528.0
1250.0
705.0
879.0
567.0
597.0
871.0
609.0
617.0

90
466.0
609.0
527.0
650.0
820.0
679.0
714.0
808.0
426.0
982.0
539.0
737.0
479.0
507.0
633.0
482.0
489.0

120
437.0
541.0
479.0
559.0
779.0
605.0
610.0
685.0
423.0
899.0
506.0
596.0
419.0
473.0
565.0
433.0
464.0

150
447.0
511.0
441.0
493.0
721.0
568.0
546.0
607.0
385.0
819.0
434.0
528.0
392.0
430.0
527.0
439.0
386.0

183
385.0
489.0
408.0
434.0
620.0
500.0
500.0
515.0
342.0
713.0
407.
452.0
341.0
370.
463.0
405.0
418.0

274
277.0
390.0
290.0
306.0
439.0
364.0
357.0
361.0
245.0
511.0
295.0
328.0
248.0
262.0
325.0
301.0
319.0 
Coast Fork Willamette R. at Saginaw, Oreg. STATION NUMBER 14-1570.00

\author{
DURATION TABLE OF DAILY DISCHARGE
}

CLASS $0 \begin{array}{lllllllllllllllllllllllllllllllllll} & 0 & 2 & 3 & 4 & 5 & 6 & 7 & 8 & 9 & 10 & 11 & 12 & 13 & 14 & 15 & 16 & 17 & 18 & 19 & 20 & 21 & 22 & 23 & 24 & 25 & 26 & 27 & 28 & 29 & 30 & 31 & 32 & 33 & 34\end{array}$

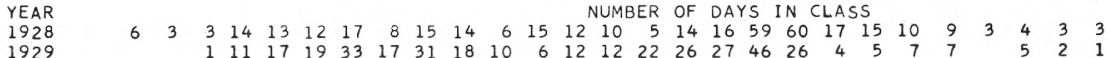

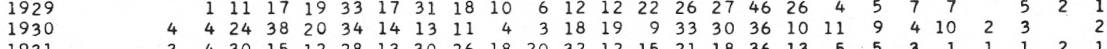

$\begin{array}{llllllllllllrrrrrrrrrrrrrrrrr}1931 & 3 & 4 & 30 & 15 & 12 & 28 & 13 & 30 & 26 & 18 & 20 & 32 & 12 & 15 & 21 & 18 & 36 & 13 & 5 & 5 & 3 & 1 & 1 & 1 & 2 & 1 \\ 1932 & & & 18 & 26 & 11 & 20 & 11 & 19 & 14 & 3 & 9 & 2 & 4 & 2 & 18 & 25 & 60 & 38 & 32 & 8 & 16 & 13 & 5 & 7 & 1 & 3 & 1\end{array}$

$\begin{array}{lrrrrrrrrrrrrrrrrrrrrrrrrrr}1932 & 18 & 26 & 11 & 20 & 11 & 19 & 14 & 3 & 9 & 2 & 4 & 2 & 18 & 25 & 60 & 38 & 32 & 8 & 16 & 13 & 5 & 7 & 1 & 3 & 1 \\ 1933 & 13 & 3 & 8 & 20 & 8 & 40 & 14 & 11 & 9 & 9 & 11 & 11 & 10 & 8 & 47 & 41 & 33 & 28 & 20 & 13 & 2 & 3 & 1 & 1 & 1\end{array}$

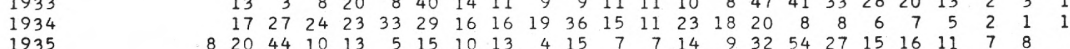

$\begin{array}{llrrrrrrrrrrrrrrrrrrrrrrrr}1935 & 8 & 20 & 44 & 10 & 13 & 5 & 15 & 10 & 13 & 4 & 15 & 7 & 7 & 14 & 9 & 32 & 54 & 27 & 15 & 16 & 11 & 7 & 8 \\ 1936 & 4 & 2 & 16 & 10 & 15 & 28 & 13 & 31 & 23 & 7 & 5 & 9 & 15 & 19 & 36 & 32 & 46 & 18 & 9 & 8 & 12 & 7 & 3 & 6\end{array}$

$\begin{array}{rrrrrrrrrrrrrrrrrrrrrrrrrrrrrrr}1936 & 4 & 2 & 1 & 10 & 15 & 28 & 13 & 31 & 23 & 7 & 5 & 9 & 15 & 19 & 36 & 32 & 46 & 18 & 9 & 8 & 12 & 7 & 3 & 6 & 1 & 4 & 2 \\ 1937 & 1 & 3 & 8 & 45 & 11 & 9 & 22 & 25 & 15 & 15 & 11 & 11 & 8 & 13 & 19 & 10 & 42 & 20 & 23 & 13 & 20 & 11 & 3 & 2 & 2 & 1 & 1 \\ 1938 & & & & 29 & 19 & 13 & 11 & 7 & 23 & 12 & 13 & 10 & 14 & 6 & 2 & 19 & 10 & 28 & 44 & 16 & 24 & 21 & 20 & 9 & 6 & 1 & 7 & 1\end{array}$

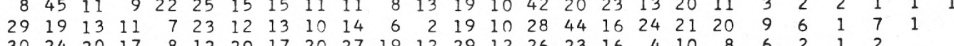

$\begin{array}{lllllllllllllllllllllllllll}4 & 17 & 24 & 40 & 35 & 20 & 19 & 17 & 11 & 3 & 14 & 12 & 11 & 21 & 16 & 29 & 24 & 5 & 7 & 9 & 6 & 9 & 2 & 1 & 2\end{array}$

1940

1941

$\frac{1942}{1943}$

1944

1944

1946

1947

1948

1949

$\begin{array}{lllllllllllllllllllll}3 & 7 & 4 & 21 & 41 & 21 & 8 & 10 & 34 & 28 & 26 & 37 & 35 & 44 & 23 & 5 & 8 & 4 & 3\end{array}$

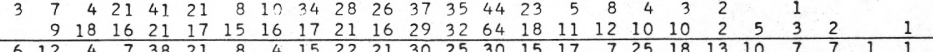

$\begin{array}{rrrrrrrrrrrrrrrrrr}9 & 18 & 16 & 21 & 17 & 15 & 16 & 17 & 21 & 16 & 29 & 32 & 64 & 18 & 11 & 12 & 10 & 10 \\ 12 & 4 & 7 & 38 & 21 & 8 & 4 & 15 & 22 & 21 & 30 & 25 & 30 & 15 & 17 & 7 & 25 & 18 \\ 17 & 35 & 8 & 17 & 10 & 23 & 16 & 44 & 11 & 25 & 53 & 21 & 37 & 20 & 14 & 8 & 4 & 2\end{array}$

$\begin{array}{rrrrrrrrrrrrrrrrrrrrr}35 & 8 & 17 & 10 & 23 & 16 & 44 & 11 & 25 & 53 & 21 & 37 & 20 & 14 & 8 & 4 & 2 & & 1 & \\ 10 & 23 & 38 & 32 & 15 & 13 & 17 & 21 & 25 & 21 & 29 & 27 & 24 & 15 & 14 & 17 & 14 & 4 & 4 & 2\end{array}$

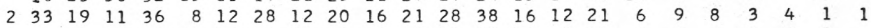

$\begin{array}{rrrrrrrrrrrrrrrrrrrrrr}1 & 43 & 32 & 24 & 17 & 24 & 24 & 28 & 28 & 15 & 38 & 28 & 13 & 8 & 20 & 5 & 5 & 6 & 2 & 3 & 1 & \\ & 45 & 17 & 6 & 12 & 16 & 7 & 2 & 26 & 25 & 52 & 53 & 37 & 17 & 16 & 10 & 9 & 6 & 4 & 3 & 2 & 1\end{array}$

$\begin{array}{lllllllllllllllllllllll}39 & 35 & 29 & 21 & 8 & 20 & 23 & 23 & 16 & 18 & 34 & 34 & 24 & 5 & 11 & 4 & 8 & 5 & & 7 & & 1\end{array}$

$\begin{array}{rrrrrrrrrrrrrrrrrrrrr}6 & 34 & 60 & 3 & 3 & 8 & 18 & 15 & 51 & 16 & 48 & 27 & 16 & 13 & 11 & 11 & 7 & 6 & 7 & 5 \\ 2 & 72 & 8 & 23 & 4 & 52 & 11 & 19 & 19 & 31 & 27 & 19 & 13 & 8 & 7 & 9 & 12 & 20 & 8\end{array}$

CFS-DAYS

475254.0
318039.0

307311.0

232187.0

35051.0

23336.0

226518.0

33725.0

442283.0

618139.0

338790.0

300917.0

284904.0

723262.0

270167.0

422400.0

459310.0

665095.0

480218.0

545585.0

1951

2
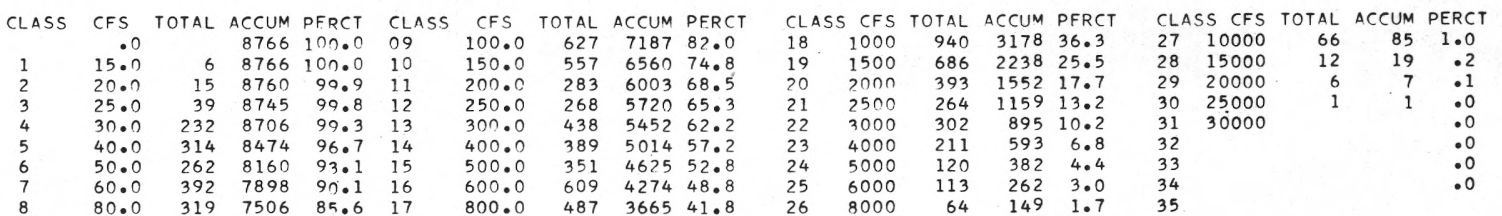

Coast Fork Willamette River

STATION NUMBER

14-1570.00

at Saginaw, Oreg.

Summary for water years 1928-42

\begin{tabular}{lrrrrllr} 
CLASS & CFS & TOTAL & ACCUM & PERCT & CLASS & \multicolumn{1}{c}{ CFS } & TOTAL \\
& .0 & & 5479 & 100.0 & 09 & 100.0 & 364 \\
1 & 15.0 & 6 & 5479 & 100.0 & 10 & 150.0 & 248 \\
2 & 20.0 & 15 & 5473 & 99.9 & 11 & 200.0 & 167 \\
3 & 25.0 & 39 & 5458 & 99.6 & 12 & 250.0 & 160 \\
4 & 30.0 & 232 & 5419 & 98.9 & 13 & 300.0 & 262 \\
5 & 40.0 & 308 & 5187 & 94.7 & 14 & 400.0 & 199 \\
6 & 50.0 & 231 & 4879 & 89.1 & 15 & 500.0 & 181 \\
7 & 60.0 & 310 & 4648 & 84.8 & 16 & 600.0 & 349 \\
8 & 80.0 & 216 & 4338 & 79.2 & 17 & 800.0 & 298
\end{tabular}

$\begin{array}{rrl}\text { ACCUM } & \text { PERCT } & \text { CLASS } \\ 4122 & 75.2 & 18 \\ 3758 & 68.6 & 19 \\ 3510 & 64.1 & 20 \\ 3343 & 61.0 & 21 \\ 3183 & 58.1 & 22 \\ 2921 & 53.3 & 23 \\ 2722 & 49.7 & 24 \\ 2541 & 46.4 & 25 \\ 2192 & 40.0 & 26\end{array}$

$\begin{array}{rrrrlrrrr}\text { CFS } & \text { TOTAL } & \text { ACCUM } & \text { PERCT } & \text { CLASS } & \text { CFS } & \text { TOTAL } & \text { ACCUM } & \text { PERCT } \\ 1000 & 615 & 1894 & 34.6 & 27 & 10000 & 29 & 37 & .7 \\ 1500 & 420 & 1279 & 23.3 & 28 & 15000 & 6 & 8 & .1 \\ 2000 & 222 & 859 & 15.7 & 29 & 20000 & 2 & 2 & .0 \\ 2500 & 167 & 637 & 11.6 & 30 & 25000 & & & .0 \\ 3000 & 169 & 470 & 8.6 & 31 & 30000 & & & .0 \\ 4000 & 134 & 301 & 5.5 & 32 & & & & .0 \\ 5000 & 56 & 167 & 3.0 & 33 & & & & .0 \\ 6000 & 55 & 111 & 2.0 & 34 & & & & .0\end{array}$

Coast Fork Willamette River

STATION NUMBER

$14-1570.00$

Summary for water years 1943-51

$\begin{array}{llrrrlrrrrrr}\text { CLASS } & \text { CFS } & \text { TOTAL } & \text { ACCUM } & \text { PERCT } & \text { CLASS } & \text { CFS } & \text { TOTAL } & \text { ACCUM } & \text { PERCT } & \text { CLASS } & \text { CF } \\ & .0 & & 3287 & 100.0 & 09 & 100.0 & 263 & 3065 & 93.2 & 18 & 1000 \\ 1 & 15.0 & & 3287 & 100.0 & 10 & 150.0 & 309 & 2802 & 85.2 & 19 & 1500 \\ 2 & 20.0 & & 3287 & 100.0 & 11 & 200.0 & 116 & 2493 & 75.8 & 20 & 2000 \\ 3 & 25.0 & & 3287 & 100.0 & 12 & 250.0 & 108 & 2377 & 72.3 & 21 & 2500 \\ 4 & 30.0 & & 3287 & 100.0 & 13 & 300.0 & 176 & 2269 & 69.0 & 22 & 3000 \\ 5 & 40.0 & 6 & 3287 & 100.0 & 14 & 400.0 & 190 & 2093 & 63.7 & 23 & 4000 \\ 6 & 50.0 & 31 & 3281 & 99.8 & 15 & 500.0 & 170 & 1903 & 57.9 & 24 & 5000 \\ 7 & 60.0 & 82 & 3250 & 98.9 & 16 & 600.0 & 260 & 1733 & 52.7 & 25 & 6000 \\ 8 & 80.0 & 103 & 3168 & 96.4 & 17 & 800.0 & 189 & 1473 & 44.8 & 26 & 8000\end{array}$

$\begin{array}{rrrrlrrrr}\text { CFS } & \text { TOTAL } & \text { ACCUM } & \text { PERCT } & \text { CLASS } & \text { CFS } & \text { TOTAL } & \text { ACCUM } & \text { PERCT } \\ 1000 & 325 & 1284 & 39.1 & 27 & 10000 & 37 & 48 & 1.5 \\ 1500 & 266 & 959 & 29.2 & 28 & 15000 & 6 & 11 & .3 \\ 2000 & 171 & 693 & 21.1 & 29 & 20000 & 4 & 5 & .2 \\ 2500 & 97 & 522 & 15.9 & 30 & 25000 & 1 & 1 & .0 \\ 3000 & 133 & 425 & 12.9 & 31 & 30000 & & & .0 \\ 4000 & 77 & 292 & 8.9 & 32 & & & & .0 \\ 5000 & 64 & 215 & 6.5 & 33 & & & & .0 \\ 6000 & 58 & 151 & 4.6 & 34 & & & & .0 \\ 8000 & 45 & 93 & 2.8 & 35 & & & & \end{array}$


Coast Fork Willamette R. at Saginaw, Oreg. STATION NUMBER 14-1570.00 LOWEST MEAN DISCHARGE, IN CFS, FOR THE FOLLOWING NUMBER OF CONSECUTIVF DAYS IN YEAR REGINNING APRIL 1

\begin{tabular}{|c|c|c|c|c|c|c|c|c|c|c|c|}
\hline YEAR & 1 & 3 & 7 & 14 & 30 & 60 & 90 & 120 & 150 & 183 & \\
\hline 1928 & 15.0 & 16.7 & 18.7 & 23.1 & 31.4 & 42.3 & 56.8 & $65 \cdot 1$ & 85.0 & 117.0 & $\begin{array}{l}459.0 \\
576.0\end{array}$ \\
\hline $\begin{array}{l}1929 \\
1930\end{array}$ & 22.0 & 23.3 & 26.7 & 37.8 & 40.2 & 47.4 & 51.1 & 53.8 & 61.9 & 140.0 & \\
\hline $\begin{array}{l}1930 \\
1931\end{array}$ & 32.0 & 33.3 & 38.0 & 39.4 & 43.7 & 52.6 & 64.8 & 76.2 & 105.0 & 230.0 & $\begin{array}{l}410.0 \\
678.0\end{array}$ \\
\hline $\begin{array}{l}1931 \\
1932\end{array}$ & 23.0 & 23.3 & 25.1 & 29.5 & 34.6 & 39.4 & 42.7 & 65.9 & 99.1 & 141.0 & $\begin{array}{l}678.0 \\
829.0\end{array}$ \\
\hline $\begin{array}{l}1932 \\
1933\end{array}$ & 33.0 & 33.0 & 33.4 & 35.1 & 40.0 & 47.8 & 63.2 & $94 \cdot 3$ & 158.0 & 328.0 & $\begin{array}{l}829 . \\
674 .\end{array}$ \\
\hline $\begin{array}{l}1933 \\
1934\end{array}$ & 48.0 & 49.3 & 52.9 & 57.7 & 63.3 & 86.5 & 91.8 & $118 \cdot 0$ & 129.0 & 236.0 & $\begin{array}{l}674^{\circ} \\
837 .\end{array}$ \\
\hline $\begin{array}{l}1934 \\
1935\end{array}$ & 32.0 & 34.0 & 35.7 & 36.6 & 38.1 & 40.5 & 44.9 & $55 \cdot 8$ & $92 \cdot 8$ & 150.0 & $\begin{array}{l}837 . \\
549 .\end{array}$ \\
\hline $\begin{array}{l}1935 \\
1936\end{array}$ & 25.0 & 25.0 & 26.7 & 31.3 & 34.8 & 39.4 & 48.7 & $75 \cdot 5$ & 98.4 & 157.0 & $\begin{array}{l}549 \\
327\end{array}$ \\
\hline $\begin{array}{l}1936 \\
1937\end{array}$ & 20.0 & $22 . n$ & $26 \cdot 1$ & 35.2 & 39.3 & 42.2 & 48.9 & 53.0 & 66.1 & 103.0 & $\begin{array}{r}327 \\
1210\end{array}$ \\
\hline $\begin{array}{l}1937 \\
1938\end{array}$ & 55.0 & 60.3 & 68.7 & 77.9 & 92.8 & 104.0 & 137.0 & $213 \cdot 0$ & 443.0 & 576.0 & $\begin{array}{r}1210 \\
549\end{array}$ \\
\hline $\begin{array}{l}1938 \\
1939\end{array}$ & 30.0 & 30.7 & 32.0 & 32.3 & 38.0 & 44.8 & 54.9 & $64 \cdot 2$ & 99.6 & 239.0 & \\
\hline $\begin{array}{l}1939 \\
1940\end{array}$ & $\begin{array}{l}30.0 \\
25.0\end{array}$ & $\begin{array}{l}32.7 \\
26.0\end{array}$ & $\begin{array}{l}33.7 \\
26.9\end{array}$ & $\begin{array}{l}35.1 \\
27.8\end{array}$ & $\begin{array}{l}39.9 \\
30.4\end{array}$ & $\begin{array}{l}45.5 \\
41.5\end{array}$ & $\begin{array}{l}53.1 \\
51.3\end{array}$ & $\begin{array}{l}69.0 \\
70.4\end{array}$ & $\begin{array}{l}70.3 \\
87.9\end{array}$ & $\begin{array}{l}104.0 \\
149.0\end{array}$ & $\begin{array}{l}288 \\
548\end{array}$ \\
\hline 1941 & 80.0 & 82.3 & 85.0 & 86.8 & 99.9 & 144.0 & 169.0 & 190.0 & 274.0 & 390.0 & 1050 \\
\hline 1942 & 43.0 & 44.7 & 47.7 & 51.7 & 58.4 & 65.4 & 83.3 & $124 \cdot 0$ & 248.0 & 487.0 & $\begin{array}{r}1430 \\
655\end{array}$ \\
\hline 1943 & 96.0 & 97.0 & 101.0 & 112.0 & 127.0 & 142.0 & 214.0 & 297.0 & 423.0 & 497.0 & 655 \\
\hline 1944 & 51.0 & 51.7 & 53.7 & 56.4 & 60.3 & 68.9 & 106.0 & 171.0 & 205.0 & 231.0 & 424 \\
\hline 1945 & 64.0 & 65.0 & 72.3 & 77.4 & 81.7 & 101.0 & 182.0 & 207.0 & 231.0 & 464.0 & 1410 \\
\hline 1946 & 57.0 & 58.0 & 61.7 & 65.5 & 69.2 & 78.8 & 121.0 & $200 \cdot 0$ & $264 \cdot 0$ & 314.0 & 923 \\
\hline 1947 & 99.0 & 102.0 & 115.0 & 121.0 & 136.0 & 138.0 & 174.0 & 245.0 & 291.0 & 372.0 & 1090 \\
\hline 1948 & 113.0 & 115.0 & 116.0 & 129.0 & 134.0 & 171.0 & 309.0 & 298.0 & 355.0 & 473.0 & 1020 \\
\hline 1949 & 83.0 & 83.0 & $84 \cdot 1$ & 85.4 & 87.0 & 96.7 & 117.0 & $167 \cdot 0$ & 200.0 & 217.0 & $\begin{array}{r}624 \\
1670\end{array}$ \\
\hline 1950 & 144.0 & 147.0 & 150.0 & 152.0 & 158.0 & 162.0 & 248.0 & 371.0 & 472.0 & $575 \cdot 0$ & 1670 \\
\hline
\end{tabular}

Coast Fork W1llamette R. at Saginaw, Oreg. STATION NUMBER 14-1570.00 HIGHEST MEAN DISCHARGE, IN CFS, FOR THE FOLLOWING NUMRER OF CONSECUTIVE DAYS IN YEAR ENDING SEPTEMBER 30

\begin{tabular}{|c|c|c|c|c|c|c|c|c|c|c|c|}
\hline YEAR & 1 & 3 & 7 & 15 & 30 & 60 & 90 & 120 & 150 & 183 & 274 \\
\hline 1928 & 12500.0 & $1060 n \cdot n$ & $781 \cap . ?$ & 5040.0 & 3700.0 & 2840.0 & 2440.0 & 2350.0 & 2440.0 & 2290.0 & 1710.0 \\
\hline 1929 & 10800.0 & 7460.0 & 5160.0 & 3780.0 & 2870.0 & 2220.0 & 2000.0 & 1950.0 & 1760.0 & 1590.0 & 1140.0 \\
\hline 1930 & 12700.0 & 9540.0 & $683 n .0$ & 4290.0 & 3550.0 & 2410.0 & 2380.0 & $1940 \cdot 0$ & 1730.0 & 1580.0 & 1100.0 \\
\hline 1931 & 14700.0 & $11000 . ?$ & 6700.0 & 4180.0 & 3160.0 & 2150.0 & 1640.0 & $1410 \cdot 0$ & 1300.0 & 1150.0 & 829.0 \\
\hline 1932 & $18200 \cdot 0$ & 14000.0 & 9460.0 & 6660.0 & 4300.0 & 3290.0 & 3210.0 & $3020 \cdot 0$ & 2810.0 & 2590.0 & 1930.0 \\
\hline 1933 & 17700.0 & 11400.0 & $741 \cap .0$ & 5900.0 & 4330.0 & 3490.0 & 3270.0 & $2930 \cdot 0$ & 2710.0 & 2500.0 & 1880.0 \\
\hline 1934 & 9750.0 & 7220.0 & 5190.0 & 3760.0 & 2560.0 & 2150.0 & 1670.0 & $1500 \cdot 0$ & $1300 \cdot 0$ & $1130 \cdot 0$ & 810.0 \\
\hline 1935 & 11000.0 & 8810.0 & 7700.0 & 5530.0 & 4100.0 & 3270.0 & 2960.0 & $2650 \cdot 0$ & 2590.0 & 2430.0 & 1760.0 \\
\hline 1936 & 18400.0 & 15300.0 & 11300.0 & 8680.0 & 5620.0 & 3940.0 & 3090.0 & $2700 \cdot 0$ & 2420.0 & 2130.0 & 1560.0 \\
\hline 1937 & 21500.0 & 16800.0 & 10600.0 & 6520.0 & 4400.0 & 3930.0 & 3410.0 & $2900 \cdot 0$ & 2630.0 & 2260.0 & $1600 \cdot 0$ \\
\hline 1938 & 19500.0 & $12400 . ?$ & 9350.0 & 7290.0 & 5040.0 & 4340.0 & 3850.0 & $3570 \cdot 0$ & 3430.0 & 3140.0 & 2240.0 \\
\hline 1939 & 10700.0 & $8050 . ?$ & $5930 . ?$ & 4680.0 & 3390.0 & 3160.0 & 2540.0 & $2190 \cdot 0$ & 1950.0 & 1710.0 & 1220.0 \\
\hline 1940 & 10400.0 & $7870 . n$ & $602 n \cdot n$ & 4590.0 & 4230.0 & $3050 . ?$ & 2370.0 & $2080 \cdot 0$ & 1840.0 & 1560.0 & 1080.0 \\
\hline 1941 & 9050.0 & 6330.0 & $414 \pi \cdot n$ & 3060.0 & 2370.0 & 1890.0 & 1670.0 & $1440 \cdot 0$ & 1250.0 & 1160.0 & 992.0 \\
\hline 1942 & 22600.0 & $139 n n \cdot n$ & 7740.0 & 4880.0 & 3960.0 & 3460.0 & 2980.0 & $2530 \cdot 0$ & 2190.0 & 1970.0 & 1590.0 \\
\hline 1943 & 26800.0 & $22700 . n$ & 16600.0 & 11400.0 & 8040.0 & 6350.0 & 5600.0 & $4550 \cdot 0$ & 4020.0 & 3520.0 & 2590. \\
\hline 1944 & 7910.0 & $5100 \cdot 0$ & 2980.0 & 2460.0 & 1740.0 & 1420.0 & 1460.0 & $1330 \cdot 0$ & 1190.0 & 1150.0 & 932.0 \\
\hline 1945 & 8800.0 & $7250 \cdot 0$ & 5610.0 & 4500.0 & 3470.0 & 2910.0 & 2870.0 & $2570 \cdot 0$ & 2380.0 & 2040.0 & 1470.0 \\
\hline 1946 & 24700.0 & 16600.0 & 10400.0 & 8500.0 & 5520.0 & 4590.0 & 3860.0 & 3610.0 & 3260.0 & 2810.0 & 1990.0 \\
\hline 1947 & $15100 \cdot 0$ & 11600.0 & 7690.0 & 5350.0 & 4660.0 & 2880.0 & 2790.0 & $2520 \cdot 0$ & 2540.0 & 2220.0 & 1640.0 \\
\hline 1948 & 21900.0 & 17400.0 & 12900.0 & 8180.0 & 4820.0 & 3960.0 & 3470.0 & $3100 \cdot 0$ & 3000.0 & 2870.0 & 2320.0 \\
\hline 949 & 20200.0 & $14900 . ?$ & 9847.0 & 6740.0 & 4450.0 & 3160.0 & 2960.0 & 2750.0 & 2530.0 & 2370.0 & $\begin{array}{l}1700.0 \\
1890.0\end{array}$ \\
\hline 950 & 11100.0 & 10600.0 & 10400.0 & 7430.0 & 5940.0 & 4620.0 & $4000 \cdot 0$ & $3400 \cdot ?$ & 2990.0 & 2630.0 & 1890.0 \\
\hline 51 & 16000.0 & 12700.0 & $11000 . ?$ & 8370.0 & 6750.0 & 5160.0 & 4910.0 & $4670 \cdot 0$ & 4170.0 & 3650.0 & 2590 . \\
\hline
\end{tabular}


Coast Fork Willamette River near Goshen, Oreg.
STATION NUMBER 14-1575.00

DURATION TABLF OF DAILY DISCHARGE

CLASS $010 \begin{array}{llllllllllllllllllllllllllllllllllll} & 1 & 2 & 4 & 4 & 5 & 6 & 7 & 8 & 9 & 10 & 11 & 12 & 13 & 14 & 15 & 16 & 17 & 18 & 19 & 20 & 21 & 22 & 23 & 24 & 25 & 26 & 27 & 28 & 29 & 30 & 31 & 32 & 33 & 34\end{array}$

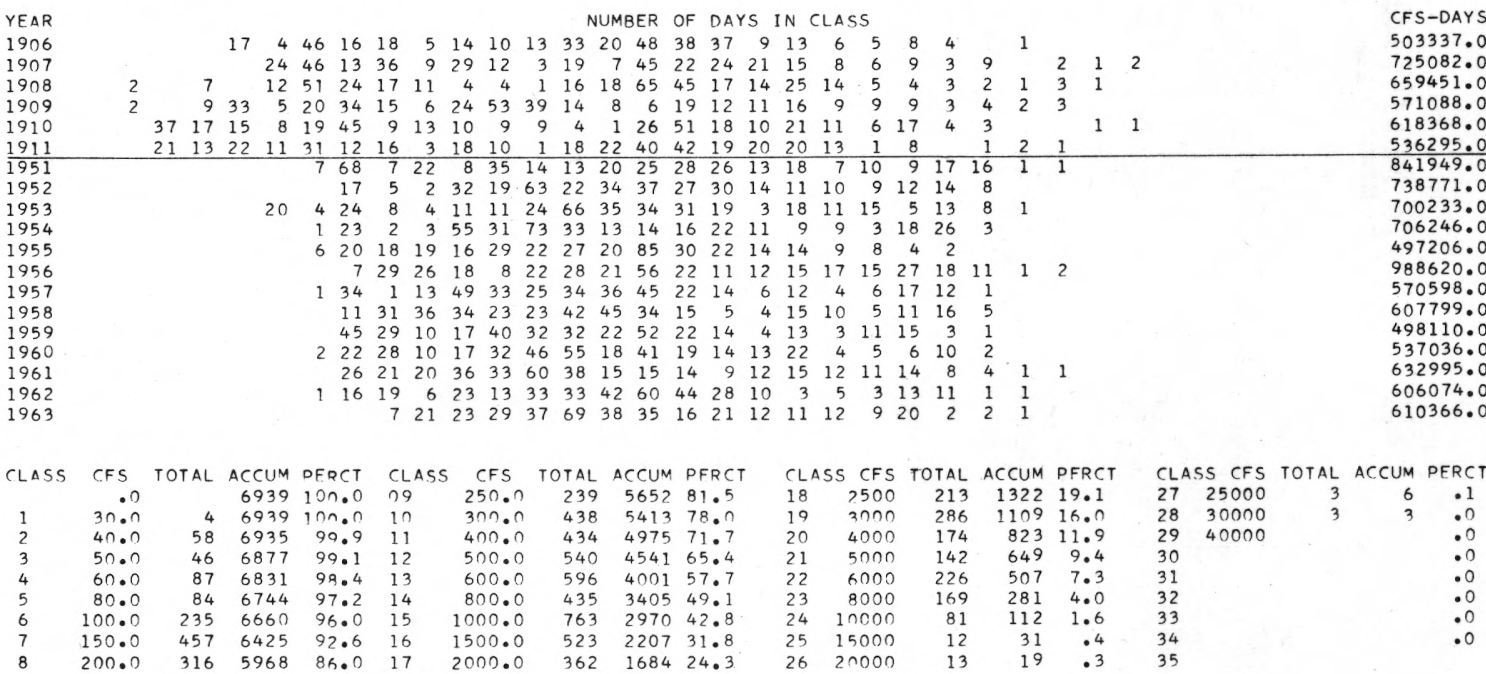

Coast Fork Willamette River near Goshen, Oreg.

Summary for water years 1906-1911
STATION NUMBER

$14-1575.00$
Coast Fork Willamette River near Goshen, Oreg.

Summary for water years 1951-1963

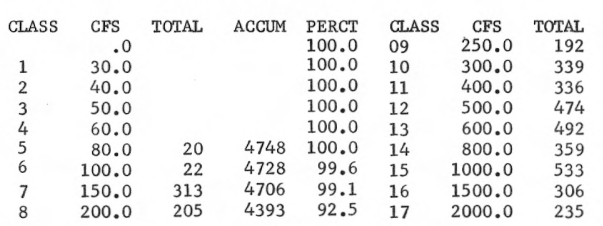

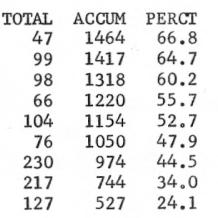

STATION NUMBER
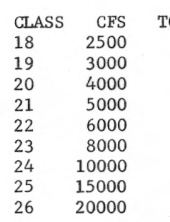

$\begin{array}{rrrlrrrr}\text { TOTAL } & \text { ACCUM } & \text { PERCT } & \text { CLASS } & \text { CFS } & \text { TOTAL } & \text { ACCUM } & \text { PERCT } \\ 85 & 400 & 18.3 & 27 & 25000 & 3 & 6 & .3 \\ 110 & 315 & 14.4 & 28 & 30000 & 3 & 3 & .1 \\ 61 & 205 & 9.4 & 29 & 40000 & & & .0 \\ 32 & 144 & 6.6 & 30 & & & & .0 \\ 55 & 112 & 5.1 & 31 & & & & .0 \\ 17 & 57 & 2.6 & 32 & & & & .0 \\ 19 & 40 & 1.8 & 33 & & & & .0 \\ 6 & 21 & 1.0 & 34 & & & & \end{array}$

$14-1575.00$ \begin{tabular}{lrrrrl} 
CLASS & \multicolumn{1}{c}{ CFS } & TOTAL & ACCUM & PERCT & CLASS \\
1 & 30.0 & 4 & 2191 & 100.0 & 09 \\
2 & 40.0 & 58 & 2187 & 99.8 & 10 \\
3 & 50.0 & 46 & 2129 & 97.2 & 12 \\
4 & 60.0 & 87 & 2083 & 95.1 & 13 \\
5 & 80.0 & 64 & 1996 & 91.1 & 14 \\
6 & 100.0 & 213 & 1932 & 88.2 & 15 \\
7 & 150.0 & 144 & 1719 & 78.5 & 16 \\
8 & 200.0 & 111 & 1575 & 71.9 & 17
\end{tabular}

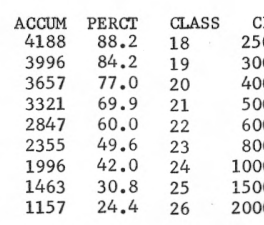

$\begin{array}{rrrrlrrr}\text { CFS } & \text { TOTAL } & \text { ACCUM } & \text { PERCT } & \text { CLASS } & \text { CFS } & \text { TOTAL ACCUM } & \text { PERCT } \\ 2500 & 128 & 922 & 19.4 & 27 & 25000 & & .0 \\ 3000 & 176 & 794 & 16.7 & 28 & 30000 & & .0 \\ 4000 & 113 & 618 & 13.0 & 29 & 40000 & & .0 \\ 5000 & 110 & 505 & 10.6 & 30 & & & .0 \\ 6000 & 171 & 395 & 8.3 & 31 & & & .0 \\ 8000 & 152 & 224 & 4.7 & 32 & & & .0 \\ 10000 & 62 & 72 & 1.5 & 33 & & & .0 \\ 15000 & 6 & 10 & .2 & 34 & & & .0 \\ 20000 & 4 & 4 & .1 & 35 & & & \end{array}$


Coast Fork W1llamette River near

STATION NUMBER 14-1575.00

Goshep OWESTPB MEAN DISCHARGE, IN CFS, FOR THE FOLLOWING NUMBER OF CONSECUTIVE DAYS IN YEAR BFGINNING APRIL 1

\begin{tabular}{|c|c|c|c|c|c|c|c|c|c|c|c|}
\hline YEAR & 1 & 3 & 7 & 14 & 30 & 60 & 90 & 120 & 150 & 183 & 274 \\
\hline 1906 & 68.0 & 68.0 & 68.0 & 68.0 & 84.9 & 126.0 & 149.0 & $222 \cdot 0$ & 370.0 & 682.0 & 1110.0 \\
\hline 1907 & 80.0 & 80.0 & 82.1 & 87.5 & 108.0 & 121.0 & 133.0 & 139.0 & 172.0 & 213.0 & 1370.0 \\
\hline 1908 & 36.0 & $42 . ?$ & 48.9 & 60.7 & 79.0 & 101.0 & 124.0 & 274.0 & 390.0 & 495.0 & 903.0 \\
\hline 1909 & 54.0 & 54.0 & 58.6 & 64.3 & 70.5 & 86.3 & 110.0 & 143.0 & 187.0 & 258.0 & 1190.0 \\
\hline 1910 & 46.0 & 46.0 & 46.0 & 46.9 & 48.5 & 52.7 & 64.2 & 84.3 & 99.0 & 182.0 & 883.0 \\
\hline 1951 & 134.0 & 142.0 & 149.0 & 152.0 & 161.0 & 164.0 & 179.0 & 223.0 & 288.0 & 356.0 & 1370.0 \\
\hline 1952 & 86.0 & 86.0 & 89.7 & 94.1 & 118.0 & 357.0 & 422.0 & 449.0 & 409.0 & 499.0 & 729.0 \\
\hline 1953 & 159.0 & $164 . ?$ & 168.0 & $172 . n$ & 226.0 & 396.0 & 521.0 & 549.0 & 560.0 & 911.0 & 1810.0 \\
\hline 1954 & 121.0 & $126 . ?$ & 137.0 & 158.0 & 188.0 & 307.0 & 317.0 & 362.0 & 402.0 & 415.0 & 633.0 \\
\hline 1955 & 162.0 & 166.0 & 170.0 & 173.0 & 235.0 & 437.0 & 635.0 & 562.0 & 586.0 & 708.0 & 2320.0 \\
\hline 1956 & 201.0 & 205.0 & 209.0 & 217.0 & 257.0 & 479.0 & 552.0 & $504 \cdot 0$ & 571.0 & 697.0 & 1130.0 \\
\hline 1957 & 149.0 & 154.0 & 162.0 & 165.0 & 170.0 & 192.0 & 288.0 & 389.0 & 434.0 & 444.0 & 989.0 \\
\hline 1958 & 156.0 & 157.0 & 178.0 & 183.0 & 197.0 & 224.0 & 396.0 & 483.0 & 479.0 & 489.0 & 869.0 \\
\hline 1959 & 146.0 & 149.0 & 165.0 & 179.0 & 198.0 & 265.0 & 313.0 & 344.0 & 447.0 & 450.0 & 505.0 \\
\hline 1960 & 170.0 & 192.0 & 222.0 & 228.0 & 299.0 & 417.0 & 477.0 & 472.0 & 461.0 & 693.0 & 1210.0 \\
\hline 1961 & 160.0 & 160.0 & 164.0 & 172.0 & 184.0 & 245.0 & 316.0 & 363.0 & 370.0 & 484.0 & 1330.0 \\
\hline 1962 & 148.0 & 157.0 & 164.0 & 167.0 & 208.0 & 319.0 & 452.0 & 519.0 & 595.0 & 684.0 & 1090.0 \\
\hline
\end{tabular}

Coast Fork W1llamette R1ver near

STATION NUMBER $14-1575.00$

Goshen, Oreg HIGHEST DISCHARGE, IN CFS, FOR THE FOLLOWING NUMBER OF CONSFCUTIVE DAYS IN YEAR ENDING SEPTEMBER 30

\begin{tabular}{|c|c|c|c|c|c|c|c|c|c|c|c|}
\hline YEAR & 1 & 3 & 7 & 15 & 30 & 60 & 90 & 120 & 150 & 183 & 274 \\
\hline 1906 & 15000.0 & $8900 . n$ & 7760.0 & 5610.0 & 3740.0 & 3420.0 & 2900.0 & $2580 \cdot 0$ & 2370.0 & $2300 \cdot 0$ & 1780.0 \\
\hline $19 \cap 7$ & 36500.0 & 25300.0 & 18300.0 & 12300.0 & 7220.0 & 6160.0 & 4850.0 & $4780 \cdot 0$ & 4250.0 & 3710.0 & 2600.0 \\
\hline $19 \cap 8$ & 28400.0 & 23000.0 & 19600.0 & 12200.0 & 8600.0 & 5670.0 & 4470.0 & $3860 \cdot 0$ & 3390.0 & $3100 \cdot 0$ & 2360.0 \\
\hline 1909 & 22600.0 & 18700.0 & $16800 \cdot ?$ & 11400.0 & 8240.0 & 6230.0 & 4990.0 & $3990 \cdot 0$ & 3340.0 & 2860.0 & 2050.0 \\
\hline 1910 & 30500.0 & $2320 n \cdot n$ & $144 n n .0$ & 9300.0 & 6960.0 & $4410 . n$ & 3840.0 & $4090 \cdot 0$ & 3660.0 & 3170.0 & 2230.0 \\
\hline 1911 & 23600.0 & $16600 \cdot n$ & $1000 n \cdot 0$ & 6750.0 & 4690.0 & 3910.0 & 3790.0 & 3240.0 & 2870.0 & 2640.0 & 1910.0 \\
\hline 1951 & 21300.0 & $15000 \cdot n$ & $1210 n .0$ & 9560.0 & 7670.0 & 5800.0 & 5690.0 & $5410 \cdot 0$ & 4860.0 & 4250.0 & $3000 \cdot 0$ \\
\hline 1952 & 13000.0 & 11800.0 & 10900.0 & 7490.0 & 5810.0 & 4660.0 & 4740.0 & $4120 \cdot 0$ & 3800.0 & 3440.0 & 2540.0 \\
\hline 1953 & 15100.0 & 11700.0 & 10800.0 & 9400.0 & 8390.0 & 5720.0 & 4650.0 & $4000 \cdot 0$ & 3500.0 & 3240.0 & 2400.0 \\
\hline 1954 & 13900.0 & 10600.0 & 9770.0 & 8310.0 & 7320.0 & 5640.0 & 5560.0 & $4640 \cdot 0$ & 4030.0 & 3430.0 & 2440.0 \\
\hline 1955 & 8280.0 & 7820.0 & 5810.0 & 4220.0 & 3980.0 & 3080.0 & 2540.0 & $2570 \cdot 0$ & 2380.0 & $2160 \cdot 0$ & 1670.0 \\
\hline 1956 & 22800.0 & $18000 \cdot 0$ & 17000.0 & 13200.0 & 10300.0 & 8660.0 & 6960.0 & $6140 \cdot 0$ & 5470.0 & 4750.0 & 3430.0 \\
\hline 1957 & 10800.0 & $88 \cap 0 \cdot n$ & 8260.0 & 8070.0 & 6650.0 & 4360.0 & 3310.0 & $3220 \cdot 0$ & 2830.0 & 2640.0 & 1920.0 \\
\hline 1958 & 14000.0 & 10800.0 & $9800 . n$ & 9240.0 & 6230.0 & 5410.0 & 4790.0 & 3810.0 & 3320.0 & 2830.0 & 2100.0 \\
\hline 1959 & 11200.0 & 8510.0 & 7630.0 & 5180.0 & 4820.0 & 3900.0 & 3120.0 & 2820.0 & 2620.0 & 2240.0 & 1690.0 \\
\hline 1960 & 10700.0 & $10000 \cdot n$ & 9230.0 & 6380.0 & 4920.0 & 4060.0 & $339 n \cdot 0$ & 3170.0 & $290 n .0$ & 2490.0 & 1820.0 \\
\hline 1961 & ? & 15400.0 & $135 n n \cdot 0$ & 10300.0 & 7700.0 & $567 n . n$ & 4130.0 & $3860 \cdot 0$ & 3530.0 & 3070.0 & 2180.0 \\
\hline 1962 & $16>00 \cdot 0$ & 11000.0 & $992 n \cdot 0$ & 7660.0 & 5670.0 & 4280.0 & $3310 \cdot 0$ & $2890 \cdot 0$ & $294 n \cdot 0$ & $2650 \cdot 0$ & $2020 \cdot 0$ \\
\hline 1963 & $15000 \cdot 0$ & 12200.0 & 8770.0 & 5720.0 & $4860 \cdot 0$ & 4010.0 & 3060.0 & $2920 \cdot 0$ & 2440.0 & $2640 \cdot n$ & $2020 \cdot 0$ \\
\hline
\end{tabular}




\begin{tabular}{|c|c|c|c|c|c|c|c|c|c|c|c|c|c|c|c|c|c|c|c|c|c|c|c|c|c|c|c|}
\hline YEAR. & & & & & & & & & & & & & IUMBER & OF $D$ & DAYS & IN & CLAS & & & & & & & & & & DA \\
\hline 1912 & & & & 5 & 5 & 532 & 23 & 1014 & 141 & 1710 & 8 & 132 & 2940 & 322 & 2325 & 518 & 8 & 10 & 6 & 4 & 3 & 32 & 2 & 1 & & & $\begin{array}{l}2389530 . \\
2368600^{\circ}\end{array}$ \\
\hline 913 & & & & & 31 & 311 & 13 & 812 & 121 & $18 \quad 13$ & 14 & 102 & 2026 & 372 & 2441 & 124 & 11 & 19 & 4 & 1 & 2 & & & 1 & & & 2368 \\
\hline 926 & & 11 & 21 & 33 & 17 & 14 & 8 & 203 & 332 & 2922 & 13 & 141 & $15 \quad 31$ & 21 & 821 & 112 & 10 & 4 & 4 & 1 & 2 & 1 & & & & & 1642873 \\
\hline 921 & & & 16 & 12 & 21 & 131 & 11 & 916 & 16 & 718 & 5 & 41 & $14 \quad 273$ & 351 & 1952 & 233 & 10 & 9 & 6 & 8 & 8 & 2 & 2 & & 1 & & 2645270 \\
\hline 92 & & 2 & 20 & 32 & 28 & 261 & 18 & 713 & 131 & 1219 & 8 & 121 & $15 \quad 14$ & 162 & 2651 & 118 & 12 & 7 & 1 & & 2 & & & 1 & & & 2001420 \\
\hline 1923 & & 3 & 4 & 33 & 19 & 303 & 32 & 12 & 91 & $10 \quad 14$ & 23 & 292 & 2928 & 221 & 1713 & 312 & 6 & 8 & 7 & 1 & 1 & & & 1 & 1 & & 1811040 \\
\hline 92 & 7 & 57 & 114 & 17 & 11 & 273 & 32 & 1114 & 14 & 2728 & 28 & 11 & 1721 & 11 & 5 & 8 & & 4 & 2 & & & 1 & & & & & \\
\hline 192 & 8 & 8 & 5 & 46 & 28 & 13 & 7 & 5 & 61 & 16 & 14 & 272 & $26 \quad 27$ & 241 & 1015 & 59 & 18 & 12 & 8 & $\begin{array}{ll}310 \\
10\end{array}$ & & 2 & 2 & 22 & & & \\
\hline 92 & 5 & 31 & 38 & 47 & 26 & 151 & 11 & 2628 & 283 & $31 \quad 17$ & 21 & 101 & 114 & 6 & 511 & 3 & 5 & 4 & 1 & 2 & 1 & 2 & & 1 & & & 125 \\
\hline 192 & & & 12 & 25 & 23 & 193 & 30 & 14 & 3 & 75 & 6 & 152 & 2459 & 241 & 1336 & 9 & 13 & 7 & 3 & 4 & 4 & & 3 & & & 1 & \\
\hline 192 & & 2 & 26 & 25 & 22 & 92 & 27 & 231 & 111 & 159 & 6 & 71 & 1336 & 282 & 2930 & $0 \quad 15$ & 5 & 7 & 5 & 2 & 2 & & & & & & 21 \\
\hline 92 & 2 & 16 & 32 & 39 & 40 & 171 & 15 & $\begin{array}{ll}8 & 1\end{array}$ & 191 & 9 & 16 & 121 & 1635 & 281 & 1214 & 48 & 2 & 5 & 1 & 1 & I & & & & & & 13 \\
\hline 33 & 10 & 74 & 47 & 1 & 8 & 7 & 6 & $\begin{array}{ll}72 \\
2\end{array}$ & 212 & $9 \quad 27$ & 25 & 181 & 14 & 6 & 511 & 3 & 6 & 7 & 5 & 5 & & & & 1 & & & \\
\hline . & 35 & 21 & 33 & 31 & 23 & 82 & 27 & 253 & 343 & $33 \quad 23$ & 18 & 51 & 11 & 5 & 111 & 1 & 2 & 5 & 1 & 1 & 1 & & 1 & & & & 10 \\
\hline 1932 & 18 & 5 & 23 & 24 & 19 & 12 & 5 & 111 & 121 & $14 \quad 19$ & 15 & 12 & 719 & 293 & $39 \quad 29$ & 921 & 8 & 9 & 5 & 2 & & 1 & & 1 & 1 & & 21 \\
\hline 9 & & 13 & 1 & 29 & 23 & 181 & 14 & 131 & 131 & 117 & 8. & 9 & 822 & 253 & 3152 & $2 \quad 39$ & 11 & 9 & 5 & & & & & & & & \\
\hline 19 & 23 & 35 & 21 & 42 & 30 & 92 & 20 & 164 & 452 & $\begin{array}{lll}21 & 14\end{array}$ & 9 & 10 & 712 & 91 & 10 & 6 & 7 & 6 & 1 & 1 & 1 & & & & & & \\
\hline 193 & 1 & 49 & 21 & 14 & 12 & 7 & 9 & 81 & 11 & 810 & 9 & 211 & 1030 & 323 & $\begin{array}{ll}35 & 27\end{array}$ & $\begin{array}{ll}7 & 17\end{array}$ & 10 & 13 & 2 & 3 & 3 & 1 & & & & & \\
\hline 19 & & 13 & 23 & 48 & 29 & 102 & 21 & 121 & 121 & $11 \quad 17$ & 22 & $\begin{array}{ll}9 & 1\end{array}$ & 1136 & 241 & $16 \quad 14$ & 8 & 11 & 4 & 5 & 2 & 3 & 2 & 2 & & & & \\
\hline 193 & & 66 & & 43 & 24 & 182 & 20 & 111 & 11 & 106 & 3 & 5 & 820 & 152 & $26 \quad 27$ & $\begin{array}{ll}7 & 17\end{array}$ & 15 & 11 & 4 & 1 & & 1 & & 2 & & & 178 \\
\hline 93 & & 1 & 41 & 35 & 17 & 121 & 16 & 8 & 61 & 7 & 9 & 10 & 714 & 221 & 1554 & 418 & 22 & 17 & 7 & 4 & 2 & 1 & & 1 & & & 241 \\
\hline & & 12 & 48 & 35 & 8 & 11 & 6 & 1628 & 282 & 2729 & 21 & 111 & 1024 & 251 & 158 & 86 & 6 & 11 & 4 & 2 & & & & & & & \\
\hline 194 & 34 & 23 & 62 & 40 & 13 & 71 & 11 & 131 & 122 & 2016 & 12 & 151 & 1120 & 12 & 5 & 97 & 6 & 11 & 3 & & & & & & & & \\
\hline 19 & & 12 & 8 & 37 & 24 & 201 & 12 & 235 & 113 & $\begin{array}{ll}38 & 35\end{array}$ & 21 & 201 & 1218 & 11 & 7 & 74 & 1 & 3 & 1 & & & & & & & & 11 \\
\hline 1 & & 11 & 20 & 21 & 17 & 181 & 16 & 151 & In 1 & $18 \quad 35$ & 26 & 272 & $24 \quad 28$ & 181 & $11 \quad 12$ & 6 & 11 & 7 & 2 & 2 & 3 & 2 & & 2 & & & 175 \\
\hline & & 17 & 4 & 6 & 33 & 261 & 15 & 71 & 11 & 613 & 29 & 141 & 1118 & 211 & 1627 & $7 \quad 19$ & 19 & 16 & 810 & 4 & 6 & 2 & 2 & 2 & & 2 & 28 \\
\hline 19 & & 12 & 24 & 34 & 26 & 72 & 20 & 172 & 263 & $30 \quad 46$ & 26 & 161 & $16 \quad 22$ & 14 & 711 & 16 & 3 & 1 & 1 & & & & & & & & \\
\hline & & 2 & 15 & 58 & 42 & 192 & 212 & 22 & 181 & 138 & 9 & 61 & 1012 & 171 & 1920 & 023 & 13 & 11 & ? & 1 & & & 1 & & & & \\
\hline & & & & 53 & 22 & 231 & 16 & 61 & 12 & 412 & 13 & 132 & 22.32 & 351 & 1726 & $\begin{array}{ll}6 & 14\end{array}$ & 11 & 7 & 7 & 5 & 5 & & & 1 & 1 & & \\
\hline 1947 & & & & 6 & 42 & 242 & 251 & 1336 & $\begin{array}{ll}36 & 1\end{array}$ & 1925 & 23 & 171 & 1323 & 161 & $16 \quad 19$ & $9 \quad 15$ & 11 & 6 & 6 & 1 & 1 & 2 & & 3 & & & 15 \\
\hline 194 & & & & 2 & 11 & 242 & 24 & 142 & 201 & $18 \quad 8$ & 9 & 81 & $15 \quad 27$ & 285 & 5346 & $6 \quad 15$ & 12 & 13 & 7 & 3 & & & 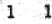 & 1 & & 1 & \\
\hline 194 & & & & 38 & 20 & 351 & 15 & 231 & 191 & 8 & 12 & 121 & 1122 & 142 & 2747 & $7 \quad 17$ & 6 & 6 & 4 & 1 & 1 & 2 & & & 1 & & \\
\hline 1 & & & & 14 & 38 & 233 & 31 & 151 & 10 & 712 & 5 & 91 & 1233 & 383 & 3128 & 817 & 12 & 11 & 5 & 4 & 4 & 2 & & & & & \\
\hline 1 & & & & 9 & 48 & 231 & 17 & 111 & 16 & 9 & 10 & 162 & 20223 & 322 & $28 \quad 23$ & $\begin{array}{ll}318 \\
\end{array}$ & 9 & 11 & 912 & 8 & 6 & 1 & 1 & 1 & & 1 & \\
\hline & & & & & & 56 & 601 & 131 & 121 & 1010 & 16 & 92 & $\begin{array}{lll}25 & 27\end{array}$ & 422 & 2840 & 022 & 8 & 9 & 411 & & 8 & & & & & & \\
\hline 19 & & & & 23 & 11 & 174 & 413 & 321 & 191 & $14 \quad 15$ & 11 & 141 & 13292 & 212 & $20 \quad 21$ & 19 & 8 & 19 & 75 & 6 & 6 & & & 1 & 1 & & \\
\hline & & & & & & & & 459 & 931 & $17 \quad 15$ & 14 & 8 & $\begin{array}{l}781 \\
18\end{array}$ & 141 & 1312 & $2 \quad 13$ & 16 & 11 & 1110 & 15 & 3 & 1 & 1 & & & & \\
\hline 19 & & & & 1 & 2 & & 72 & 2210 & 04 & $\begin{array}{lll}48 & 15\end{array}$ & 22 & 211 & 1130 & 181 & 1322 & 210 & 15 & 4 & 3 & & & & & & & & \\
\hline 195 & & & & & & & 11 & 1542 & +22 & 2430 & 8 & 161 & 1238 & 272 & 2122 & 217 & 15 & 10 & 1522 & 2. 14 & 413 & 3 & 1 & & & & 34 \\
\hline 1957 & & & & & 4 & 1 & 63 & 3050 & 503 & 3447 & 25 & 221 & 1025 & 171 & 1116 & 615 & 10 & 11 & 88 & 315 & & & & & & & 23257 \\
\hline WIII & & $\mathrm{RI}$ & & & Spri & ingf & & $1 d, 0$ & Oreg & & & STAT & TION & NUM & IBER & & $14-1$ & 10 & 0.00 & & & & & & & & \\
\hline CLASS & CFS & & TAL & $\mathrm{ACC}$ & CUM & PERC & $\mathrm{RC}$ & CLA & $A S O$ & & & OTAL & ACCUM & & $R C$ & $\mathrm{CLA}$ & ASS & CFS & $\mathrm{s}$ & & M. & & & & & & CCUM PER \\
\hline & & & & & & 100. & 0.0 & 09 & & 2000.0 & & 928 & 9588 & & 6 & 18 & & 8000 & 96 & 61 & 2952 & 20. & & $z$ & & 3 & 71 \\
\hline 1 & 500.0 & & 143 & & 611 & $10 n$. & n. $n$ & 10 & & 250 & & 72 & $866 n$ & 59. & & 1 & in & noon & 5 & 5 & & & & 2 & & 1 & \\
\hline 2 & $0 . n$ & & 496 & & & & 9.0 & 1 & & 30 & & 68 & 7935 & 554. & . & 2 & & 2000 & 38 & 8 & & & & 2 & & 12 & \\
\hline 3 & $n . n$ & & 579 & & 972 & & 5.6 & 12 & & 3500.0 & & 59 & 7251 & 149. & & 2 & & 4000 & 0 & & & & & & & 6 & 13 \\
\hline 4 & & & 975 & & & & 1. & 1 & & & & 557 & 66 & 45. & & 2 & & & 17 & 7 & & & & 3 & & 1 & 7 \\
\hline 5 & & & 817 & & & & 5.0 & 14 & & & & 57 & 61 & & & 2 & & nc & 19 & 9 & & & & & & & \\
\hline 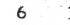 & & & 651 & 116 & 601 & 79. & 9.4 & 15 & & 50 & & 97 & 5525 & $5 \quad 37$ & & 24 & & 5000 & 13 & 36 & & & & & & 1 & 1 \\
\hline 7 & 0.0 & & 746 & 109 & & & 4.9 & 16 & & $6000 \cdot 0$ & & 871 & 4550 & 31. & . & 2 & & oc & & 7 & 189 & 1.3 & .3 & 34 & & & \\
\hline 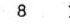 & $1700 \cdot 0$ & & 616 & 102 & 204 & & 9.8 & 17 & & $7000 \cdot 0$ & & 727 & 3679 & 925. & .2 & 26 & & 5000 & & 42 & 113 & & .8 & 35 & & & \\
\hline
\end{tabular}

Willamette River at Springfield, Oreg.

Summary for water years 1912-13, 1920-42

\begin{tabular}{crrrrrr} 
CLASS & \multicolumn{1}{c}{ CFS } & TOTAL & ACCUM & PERCT & CLASS & CFS \\
& .0 & & 9132 & 100.0 & 09 & 2000.0 \\
1 & 500.0 & 143 & 9132 & 100.0 & 10 & 2500.0 \\
2 & 600.0 & 465 & 8989 & 98.4 & 11 & 3000.0 \\
3 & 700.0 & 536 & 8524 & 93.3 & 12 & 3500.0 \\
4 & 800.0 & 731 & 7988 & 87.5 & 13 & 4000.0 \\
5 & 1000.0 & 518 & 7257 & 79.5 & 14 & 4500.0 \\
6 & 1200.0 & 424 & 6739 & 73.8 & 15 & 5000.0 \\
7 & 1400.0 & 410 & 6315 & 69.2 & 16 & 6000.0 \\
8 & 1700.0 & 331 & 5905 & 64.7 & 17 & 7000.0
\end{tabular}

Willamette River at Springfield, Oreg.

Summary for water years 1943-57

\begin{tabular}{|c|c|c|c|c|c|c|c|}
\hline CLAASS & (3FS & TOTAL & ACCUM & PERCT & CLASS & CFS & TOTAL \\
\hline & .0 & & 5479 & 100.0 & 09 & 2000.0 & 484 \\
\hline 1 & 500.0 & & 5479 & 100.0 & 10 & 2500.0 & 268 \\
\hline 2 & 600.0 & 31 & 5479 & 100.0 & 11 & 3000.0 & 267 \\
\hline 3 & 700.0 & 43 & 5448 & 99.4 & 12 & 3500.0 & 232 \\
\hline 4 & 800.0 & 244 & 5405 & 98.7 & 13 & 4000.0 & 201 \\
\hline 5 & 1000.0 & 299 & 5161 & 94.2 & 14 & 4500.0 & 208 \\
\hline 6 & 1200.0 & 227 & 4862 & 88.7 & 15 & 5000.0 & 368 \\
\hline 7 & 1400.0 & 336 & 4635 & 84.6 & 16 & 6000.0 & 354 \\
\hline 8 & 1700.0 & 285 & 4299 & 78.5 & 17 & 7000.0 & 320 \\
\hline
\end{tabular}

STATION NUMBER

$\begin{array}{rrrlr}\text { TOTAL } & \text { ACCUM } & \text { PERCT } & \text { CLASS } & \text { CFS } \\ 444 & 5574 & 61.0 & 18 & 8000 \\ 457 & 5130 & 56.2 & 19 & 10000 \\ 417 & 4673 & 51.2 & 20 & 12000 \\ 360 & 4256 & 46.6 & 21 & 14000 \\ 356 & 3896 & 42.7 & 22 & 17000 \\ 369 & 3540 & 38.8 & 23 & 20000 \\ 607 & 3171 & 34.7 & 24 & 25000 \\ 517 & 2564 & 28.1 & 25 & 30000 \\ 407 & 2047 & 22.4 & 26 & 35000\end{array}$

$14-1580.00$

STATION NUMBER

14-1580.00

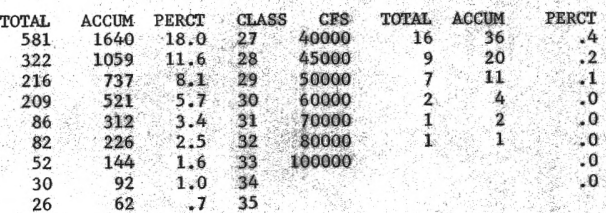

$\begin{array}{rrlrrr}\text { ACCUM } & \text { PERCT } & \text { CLASS } & \text { CFS } & \text { TOTAL } & \text { ACC } \\ 4014 & 73.3 & 18 & 8000 & 380 & 1 \\ 3530 & 64.4 & 19 & 10000 & 230 & \\ 3262 & 59.5 & 20 & 12000 & 168 & \\ 2995 & 54.7 & 21 & 14000 & 146 & \\ 2763 & 50.4 & 22 & 17000 & 92 & \\ 2562 & 46.8 & 23 & 20000 & 115 & \\ 2354 & 43.0 & 24 & 25000 & 84 & \\ 1986 & 36.2 & 25 & 30000 & 46 & \\ 1632 & 29.8 & 26 & 35000 & 16 & \end{array}$

$\begin{array}{rrrrrrr}\text { ACCUM } & \text { PERCT } & \text { CLASS } & \text { CFS } & \text { TOTAL } & \text { ACGUM } & \text { PERCT } \\ 1312 & 23.9 & 27 & 40000 & 15 & 35 & .6 \\ 932 & 17.0 & 28 & 45000 & 3 & 20 & .4 \\ 702 & 12.8 & 29 & 50000 & 8 & 17 & .3 \\ 534 & 9.7 & 30 & 60000 & 4 & 9 & .2 \\ 388 & 7.1 & 31 & 70000 & & 5 & .1 \\ 296 & 5.4 & 32 & 80000 & 4 & 5 & .1 \\ 181 & 3.3 & 33 & 100000 & 1 & 1 & .0 \\ 97 & 1.8 & 34 & & & & .0\end{array}$ 
Willamette River at Springfield, Oreg. STATION NUMBER 14-1580.00 LOWEST MEAN DISCHARGF, IN CFS, FOR THE FOLLOWING NUMBER OF CONSECUTIVE DAYS IN YEAR BEGINNING APRIL 1

\begin{tabular}{|c|c|c|c|c|c|c|c|c|c|c|c|}
\hline YEAR & 1 & 3 & 7 & 14 & 30 & 60 & 90 & 120 & 150 & 183 & 274 \\
\hline 1912 & 1100.0 & 1100.0 & 1110.0 & 1170.0 & 1190.0 & 1500.0 & 1530.0 & 1850.0 & 2780.0 & 3410.0 & 4830.0 \\
\hline 1920 & 693.0 & 693.0 & 700.0 & 725.0 & 777.0 & 905.0 & 1190.0 & 1660.0 & 2260.0 & $2620 \cdot 0$ & 5420.0 \\
\hline 1921 & 780.0 & 780.0 & $780 \cdot 0$ & 780.0 & 856.0 & 952.0 & 1030.0 & $1140 \cdot 0$ & 1410.0 & $2200 \cdot 0$ & 4180.0 \\
\hline 1922 & 680.0 & $680 . n$ & 726.0 & 754.0 & 815.0 & 866.0 & 945.0 & 1070.0 & 1240.0 & $1770 \cdot 0$ & 4120.0 \\
\hline 1923 & 900.0 & 900.0 & $9 \cap n \cdot 0$ & 934.0 & 994.0 & 1120.0 & 1300.0 & $1410 \cdot 0$ & 1660.0 & 2140.0 & 3380.0 \\
\hline 1924 & 550.0 & $550 . n$ & 550.0 & 580.0 & 613.0 & 640.0 & 685.0 & $710 \cdot 0$ & 820.0 & $1090 \cdot 0$ & 3660.0 \\
\hline 1925 & 620.0 & $653 . n$ & 695.0 & 723.0 & 771.0 & $862 \cdot 0$ & 883.0 & $929 \cdot 0$ & $1080 \cdot 0$ & 1370.0 & 2590.0 \\
\hline $19 ? 6$ & $500 \cdot 0$ & $532 \cdot n$ & $551 \cdot 0$ & 585.0 & 644.0 & 750.0 & 805.0 & $878 \cdot 0$ & $981 \cdot 0$ & $1100 \cdot 0$ & 2990.0 \\
\hline $19 ? 7$ & 750.0 & 750.0 & $750 \cdot 0$ & 761.0 & 831.0 & 1020.0 & 1330.0 & $1730 \cdot 0$ & 2120.0 & $2800 \cdot 0$ & 4700.0 \\
\hline 1928 & $6 \cap 0.0$ & $690 \cdot n$ & 701.0 & 742.0 & 785.0 & 823.0 & 847.0 & $910 \cdot 0$ & $994 \cdot 0$ & 1100.0 & 2060.0 \\
\hline 1929 & 535.0 & $540 . ?$ & 567.0 & 596.0 & 615.0 & 669.0 & 671.0 & 695.0 & 790.0 & $1230 \cdot 0$ & 2780.0 \\
\hline 1930 & 618.0 & $618 \cdot n$ & 621.0 & 631.0 & 650.0 & 680.0 & 734.0 & $768 \cdot 0$ & 884.0 & 1210.0 & 1830.0 \\
\hline 1931 & 515.0 & 522.0 & 526.0 & 538.0 & 565.0 & 599.0 & 616.0 & $747 \cdot 0$ & 878.0 & 1100.0 & 2440.0 \\
\hline 1932 & 650.0 & $658 \cdot 0$ & 660.0 & 666.0 & 700.0 & 768.0 & 884.0 & 1050.0 & 1640.0 & 2050.0 & 4320.0 \\
\hline 1933 & 832.0 & 835.0 & 845.0 & 877.0 & 952.0 & 1040.0 & 1070.0 & $1150 \cdot 0$ & 1310.0 & 2010.0 & 3510.0 \\
\hline 1934 & 582.0 & $585 . n$ & 587.0 & 591.0 & 597.0 & 616.0 & 635.0 & $705 \cdot 0$ & 883.0 & 1110.0 & 3540.0 \\
\hline 1935 & 638.0 & $643 . n$ & $652 . n$ & 659.0 & 680.0 & 692.0 & 781.0 & $854 \cdot 0$ & 1060.0 & 1280.0 & 2800.0 \\
\hline 1936 & 622.0 & $622 \cdot 0$ & 622.0 & $622 \cdot 0$ & 627.0 & 639.0 & 688.0 & $728 \cdot 0$ & $808 \cdot 0$ & 959.0 & 1930.0 \\
\hline 1937 & 760.0 & $78 \cap \cdot n$ & $8 \cap 6 . ?$ & 869.0 & $924 \cdot 0$ & 962.0 & 1070.0 & $1320 \cdot 0$ & 2330.0 & 3170.0 & $5340 . ?$ \\
\hline 1938 & 696.0 & $7 \cap 1 \cdot n$ & 706.0 & 715.0 & 746.0 & $770 . n$ & 792.0 & $888 \cdot 0$ & $12 n 0 \cdot 0$ & 1680.0 & 2900.0 \\
\hline 1939 & 645.0 & $650 . n$ & $656 . n$ & 672.0 & 716.0 & $752 \cdot 0$ & $8 \cap 1 \cdot 0$ & $849 \cdot 0$ & 880.0 & $1100 \cdot 0$ & 1960.0 \\
\hline 1940 & 509.0 & $516 \cdot n$ & 517.0 & 519.0 & 535.0 & 599.0 & 650.0 & $704 \cdot 0$ & $805 \cdot 0$ & 1080.0 & 2290.0 \\
\hline 1941 & 792.0 & $801 \cdot 0$ & 832.0 & 840.0 & 883.0 & 1100.0 & 1140.0 & $1230 \cdot 0$ & 1540.0 & 1880.0 & 4030.0 \\
\hline 1942 & 651.0 & $653 . n$ & 662.0 & 670.0 & 695.0 & 732.0 & 811.0 & $1020 \cdot 0$ & 1520.0 & 2450.0 & 5660.0 \\
\hline 1943 & 1000.0 & 1000.0 & 1010.0 & 1040.0 & 1130.0 & 1150.0 & 1240.0 & $1730 \cdot 0$ & 2290.0 & 2380.0 & 3170.0 \\
\hline 1944 & 674.0 & 682.0 & 689.0 & 707.0 & 712.0 & 783.0 & 877.0 & $910 \cdot 0$ & $1010 \cdot 0$ & 1130.0 & 2090,0 \\
\hline 1945 & 793.0 & 793.0 & 810.0 & 819.0 & 859.0 & 966.0 & 966.0 & $1010 \cdot 0$ & $1200 \cdot 0$ & 2210.0 & 5510.0 \\
\hline 1946 & 838.0 & $849 \cdot n$ & $881 \cdot 0$ & 940.0 & 956.0 & 1070.0 & 1160.0 & $1430 \cdot 0$ & 1760.0 & 2220.0 & 4560.0 \\
\hline 1947 & 980.0 & $985 . ?$ & 987.0 & 1010.0 & 1090.0 & 1130.0 & 1310.0 & $1600 \cdot 0$ & 1920.0 & 2390.0 & 4540.0 \\
\hline 1948 & $1 \cap 80.0$ & 1090.7 & 1120.0 & 1240.0 & 1340.0 & 1490.0 & 1590.0 & $1680 \cdot 0$ & 2050.0 & 2970.0 & 4700.0 \\
\hline 1949 & 860.0 & $871 . n$ & $873 . ?$ & 884.0 & 927.0 & 1010.0 & 1120.0 & $1210 \cdot 0$ & 1270.0 & 1660.0 & 3500.0 \\
\hline 1950 & $1060 \cdot 0$ & $107 n \cdot n$ & $108 n \cdot n$ & 1160.0 & 1170.0 & 1350.0 & 1590.0 & 2130.0 & 3030.0 & 3710.0 & 6990.0 \\
\hline 1951 & 950.0 & $950 \cdot n$ & $95 \cap \cdot 0$ & 968.0 & 1020.0 & 1060.0 & 1160.0 & $1400 \cdot 0$ & 1810.0 & 2440.0 & 5150.0 \\
\hline 1952 & 800.0 & $8 \cap 0 . n$ & 800.0 & 900.0 & 942.0 & 1170.0 & 1290.0 & $1370 \cdot 0$ & 1470.0 & 2040.0 & 3450.0 \\
\hline 1953 & 1390.0 & $1400 \cdot 0$ & 1427.0 & 1520.0 & 1620.0 & 1740.0 & 1760.0 & $1810 \bullet 0$ & 2100.0 & 3360.0 & 6530.0 \\
\hline 1954 & 900.0 & 1170.0 & 1490.0 & 1670.0 & 1810.0 & 2160.0 & 2170.0 & $2200 \cdot 0$ & $2200 \cdot 0$ & 2290.0 & 2830.0 \\
\hline 1955 & 1600.0 & $1650 . n$ & $186 n .0$ & 1990.0 & 2160.0 & 2330.0 & 2340.0 & $2820 \bullet 0$ & 3510.0 & 3860.0 & 7880.0 \\
\hline 1956 & 1100.0 & 1100.0 & 1237.0 & 1470.0 & 1940.0 & 2150.0 & 2280.0 & $2380 \cdot 0$ & 3010.0 & 3760.0 & 5250.0 \\
\hline
\end{tabular}

$14-1580.00$ HIGHEST MEAN DISCHARGE, IN CFS, FOR THE FOLLOWING NUMBER OF CONSECUTIVE DAYS IN YEAR ENDING SEPTEMBER 30

\begin{tabular}{|c|c|c|c|c|c|c|c|c|c|c|c|}
\hline YEAR & 1 & 3 & 7 & 15 & 30 & 60 & 90 & 10 & 150 & 183 & 274 \\
\hline 1912 & 59600.0 & 46200.0 & $3170 h .0$ & 25100.0 & 18100.0 & 15600.0 & 12600.0 & 11300.0 & 10300.0 & 9710.0 & 8230.0 \\
\hline 1913 & 48900.0 & 39100.0 & $2680 \cap .0$ & 19800.0 & 14300.0 & 10900.0 & 9710.0 & $10200 \bullet 0$ & 9620.0 & 9190.0 & 8180.0 \\
\hline 1920 & 36500.0 & $25300 . ?$ & 16000.0 & 13000.0 & 11100.0 & 9050.0 & 7920.0 & $6720 \cdot 0$ & 6820.0 & 6840.0 & 5510.0 \\
\hline 1921 & 62300.0 & 48200.0 & 38700.0 & 28700.0 & 20500.0 & 16700.0 & $15000 \cdot 0$ & $13500 \cdot 0$ & 12300.0 & 11400.0 & 9220.0 \\
\hline 1922 & $46100 \cdot 0$ & $39900 . ?$ & $27907 . ?$ & 25000.0 & 15300.0 & 10000.0 & 9320.0 & $8670 \cdot 0$ & 8530.0 & 8660.0 & 6980.0 \\
\hline 1923 & $746 \cap 0.0$ & 54000.0 & 34500.0 & 25900.0 & 19100.0 & 12600.0 & 10900.0 & $9690 \cdot 0$ & 8760.0 & 8060.0 & 6250.0 \\
\hline $19 ? 4$ & 37400.0 & $2>80 n \cdot n$ & $140 \cap n \cdot n$ & 10500.0 & 8100.0 & $71 \cap 0.0$ & $68 \cap 0.0$ & $5960 \cdot 0$ & 5640.0 & 5090.0 & 3910.0 \\
\hline 1925 & $55 n n n \cdot n$ & $454 n n \cdot 0$ & $3660 n \cdot n$ & 31700.0 & $2210 n .0$ & 18300.0 & 14700.0 & $14300 \cdot 0$ & 12300.0 & 11400.0 & 8810.0 \\
\hline 1926 & 52800.0 & $457 \cap 0.0$ & 32500.0 & 21100.0 & 17700.0 & 11400.0 & 9200.0 & 7640.0 & 6660.0 & $5890 \cdot 0$ & 4310.0 \\
\hline 1927 & 85700.0 & $557 \cap 0.0$ & $3640 n \cdot 0$ & 22900.0 & 16900.0 & 14400.0 & $132 n n \cdot 0$ & $12400 \cdot 0$ & .11200 .0 & 10300.0 & 8030.0 \\
\hline 1928 & 40300.0 & 32000.0 & 26100.0 & 17900.0 & 14200.0 & 12100.0 & 10600.0 & $9890 \cdot 0$ & 10200.0 & 9670.0 & 7360.0 \\
\hline 1979 & 26000.0 & 21900.0 & 14600.0 & 11100.0 & 9340.0 & 8320.0 & 7310.0 & $6860 \cdot 0$ & 6480.0 & 6180.0 & 4620.0 \\
\hline 1930 & 45200.0 & $26900 \cdot 0$ & 19000.0 & 16900.0 & 13300.0 & 8670.0 & 8290.0 & 7020.0 & 6340.0 & 5890.0 & 4280.0 \\
\hline 1931 & 43700.0 & 35700.0 & 23500.0 & 16300.0 & 12300.0 & 8280.0 & 6430.0 & $5590 \cdot 0$ & 5020.0 & 4600.0 & 3480.0 \\
\hline 1932 & 60700.0 & $48400 \cdot 0$ & 33500.0 & 26000.0 & 17900.0 & 13700.0 & 11900.0 & $10700 \cdot 0$ & 10200.0 & 9580.0 & 7450.0 \\
\hline 1933 & 37700.0 & $30800 \cdot n$ & 21600.0 & 18300.0 & 13900.0 & 10900.0 & 10500.0 & $9880 \cdot 0$ & $956 \cap .0$ & 9800.0 & 7770.0 \\
\hline 1934 & 31000.0 & $26800 \cdot n$ & 20200.0 & 14700.0 & 10600.0 & 8930.0 & 7140.0 & $6650 \cdot 0$ & 5930.0 & 5210.0 & 3900.0 \\
\hline 1935 & 39800.0 & $34200 \cdot n$ & 28900.0 & 20000.0 & 14800.0 & 11900.0 & 10800.0 & 9770.0 & 9530.0 & 9030.0 & 7100.0 \\
\hline 1936 & 42400.0 & 37900.0 & 32500.0 & 26000.0 & 17500.0 & 12800.0 & 10600.0 & $9790 \cdot 0$ & 9170.0 & 8190.0 & 6120.0 \\
\hline 1937 & 54000.0 & $47500 . n$ & $3200 n \cdot 0$ & 21100.0 & 15400.0 & 13300.0 & 11700.0 & 10700.0 & 10000.0 & 8680.0 & 6270.0 \\
\hline 1938 & 47500.0 & 33200.0 & 25400.0 & 20500.0 & 15000.0 & $134 n \cap .0$ & 12800.0 & $12200 \cdot 0$ & 11800.0 & 11200.0 & 8520.0 \\
\hline 1939 & 27800.0 & 22700.0 & $172 n n \cdot n$ & 16400.0 & 11800.0 & $108 \cap 0.0$ & 8960.0 & 7980.0 & 7390.0 & 6750.0 & 5250.0 \\
\hline 1940 & 23400.0 & 20700.0 & $1780 \cap .0$ & 14700.0 & 13600.0 & 10700.0 & 8630.0 & $7530 \cdot 0$ & 6800.0 & 5940.0 & 4280.0 \\
\hline 1941 & 21200.0 & 15400.0 & $106 n n .0$ & 8820.0 & 7230.0 & & & 5040 & 4470.0 & 4270.0 & 3730.0 \\
\hline 1942 & 50200.0 & 37700.0 & 24200.0 & 16900.0 & 14200.0 & 12000.0 & 10600.0 & $9110 \cdot 0$ & 8040.0 & 7340.0 & 6240 . \\
\hline 1943 & 92100.0 & $79200 . n$ & 56400.0 & 37600.0 & 26600.0 & 22400.0 & 19600.0 & 16400.0 & 14700.0 & 13200.0 & 10200.0 \\
\hline 1944 & 24400.0 & 18400.0 & 11500.0 & 8690.0 & 7060.0 & 6050.0 & 5940.0 & $5450 \cdot 0$ & 5070.0 & 4830.0 & 4110.0 \\
\hline 1945 & 40600.0 & 30100.0 & 21800.0 & 16800.0 & 11200.0 & 10000.0 & 10100.0 & 9630.0 & $9080 \cdot 0$ & 7920.0 & 5760.0 \\
\hline 1946 & 106000.0 & $73700 . n$ & 44800.0 & 34400.0 & 22900.0 & 16500.0 & 14400.0 & $13600 \cdot 0$ & 12300.0 & 11100.0 & 8400.0 \\
\hline 1947 & 64900.0 & $5 \cap 300 . n$ & $3>10 n .0$ & 18600 & 17900.0 & 11400.0 & 10800.0 & 9750.0 & 9770.0 & 8760.0 & 6770.0 \\
\hline 1948 & 82400.0 & 58100.0 & 39300.0 & $266 \mathrm{nn} \cdot n$ & 16300.0 & 14100.0 & 1230 & $11300 \cdot 0$ & 10600.0 & 10300.0 & 9010.0 \\
\hline 1949 & $6320 n \cdot 0$ & 49nกn.n & $331 \mathrm{nn} \cdot \mathrm{n}$ & $227 n 0 . n$ & 15700.0 & 11300.0 & 11000.0 & $989 n \cdot n$ & 9730.0 & 9600.0 & 7370.0 \\
\hline 1950 & $38 \cap 00 \cdot 0$ & $36 \cap n n \cdot n$ & $287 n 0 . n$ & $2 n \cap n n \cdot n$ & 16300.0 & 14700.0 & 13300.0 & $11700 \cdot 0$ & $1 \cap 80 n \cdot 0$ & 9920.0 & 7670.0 \\
\hline 1951 & $85 n \cap 0 . n$ & 58300.0 & $394 n n \cdot n$ & 25300.0 & $2 n n n n \cdot 0$ & 16600.0 & 15800.0 & $15300 \cdot 0$ & 13900.0 & 12500.0 & 9550.0 \\
\hline 1952 & 33300.0 & $32400 . n$ & $2770 n .0$ & 19000.0 & 16000.0 & 12900.0 & 13000.0 & 11900.0 & 11600.0 & 10800.0 & 9040. \\
\hline 1953 & 65700.0 & $56100 \cdot n$ & $4090 \cap .0$ & 30700.0 & 27900.0 & 18900.0 & 15400.0 & $12900 \cdot 0$ & $12200 \cdot 0$ & 11200.0 & 8420 , \\
\hline 1954 & 52700.0 & $43100 \cdot n$ & 34100.0 & 26900.0 & 23900.0 & 17700.0 & 17200.0 & $14800 \cdot 0$ & $13200 \cdot 0$ & $11300 \cdot 0$ & 8340.0 \\
\hline 1955 & 22400.0 & $18700 . ?$ & 14300.0 & 13700.0 & 12200.0 & 9170.0 & 8270.0 & $7560 \cdot 0$ & 7140.0 & 6900.0 & 5460.0 \\
\hline 1956 & 44300.0 & $38700 \cdot ?$ & 37200.0 & 32600.0 & 29000.0 & 25800.0 & 21200.0 & 17900.0 & 16300.0 & 14700.0 & 11700.0 \\
\hline 57 & 29000.0 & 26900.0 & 26800.0 & 23000.0 & 19600.0 & 14300.0 & 11100.0 & $11500 \cdot 0$ & 10400.0 & 9990.0 & 7690 \\
\hline
\end{tabular}


McKenzie River at outlet of Clear Lake,

STATION NUMBER $14-1585 \cdot 00$

DURATION TABLE OF DAILY DISCHARGE CLASS $0 \begin{array}{llllllllllllllllllllllllllllllllllllllll} & 0 & 2 & 3 & 4 & 5 & 6 & 7 & 8 & 9 & 10 & 11 & 12 & 13 & 14 & 15 & 16 & 17 & 18 & 19 & 20 & 21 & 22 & 23 & 24 & 25 & 26 & 27 & 28 & 29 & 30 & 31 & 32 & 33 & 34\end{array}$

YEAR
1913
1914
1915
1948
1949
1950
1951
1952
1953
1954
1955
1956
1957
1958
1959
1960
1961
1962
1963
NUMBER OF DAYS 1 N CLASS
6

$\begin{array}{rrrrrrrrrrrrrrrrrrrrrr} & & & 10 & 11 & 10 & 32 & 42 & 55 & 34 & 34 & 18 & 6 & 9 & 18 & 19 & 10 & 8 & 20 & 12 & 14 & 3 \\ 23 & 31 & 34 & 34 & 15 & 19 & 24 & 33 & 56 & 42 & 35 & 29 & 11 & 21 & 15 & 12 & 13 & 3 & 7 & 1 & & \\ 21 & 34 & 26 & 41 & 10 & 26 & 25 & 8 & 9 & 5 & 8 & & & & & & & \\ \end{array}$

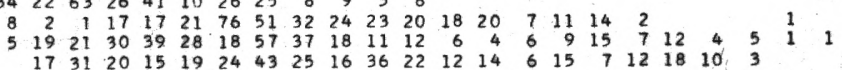
$\begin{array}{lllllllllllllllllllll}11 & 12 & 21 & 15 & 30 & 18 & 25 & 29 & 21 & 22 & 20 & 25 & 21 & 35 & 35 & 14 & 6 & 3 & 1 & 1\end{array}$

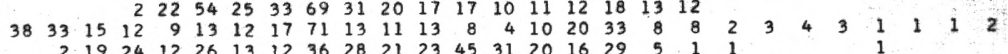
$\begin{array}{llllllllllllllllllllll}2 & 19 & 24 & 12 & 26 & 13 & 12 & 36 & 28 & 21 & 23 & 45 & 31 & 20 & 16 & 29 & 5 & 1 & 1\end{array}$ $\begin{array}{rrrrrrrrrrrrrrrrrrrrrrrrrrrrrr}16 & 16 & 14 & 18 & 26 & 43 & 27 & 39 & 45 & 20 & 14 & 11 & 12 & 12 & 17 & 19 & 14 & 6 & 5 & 2 & 2 & 2 & 1\end{array}$ $\begin{array}{lllllllllllllllllll}5 & 66 & 21 & 9 & 14 & 10 & 8 & 44 & 46 & 21 & 19 & 21 & 19 & 22 & 26 & 6 & 4 & 3 & 1\end{array}$ $\begin{array}{rrrrrrrrrrrrrrrrrrrrrrrrr} & 22 & 28 & 29 & 41 & 16 & 30 & 45 & 37 & 15 & 12 & 9 & 19 & 5 & 10 & 4 & 18 & 16 & 5 & 4 & 1 & & 1 & 1\end{array}$ $\begin{array}{rrrrrrrrrrrrrrrrrrr}15 & 22 & 26 & 21 & 30 & 41 & 36 & 40 & 26 & 19 & 22 & 13 & 5 & 11 & 4 & 23 & 9 & 2 \\ 14 & 34 & 16 & 11 & 15 & 26 & 33 & 31 & 48 & 28 & 16 & 15 & 26 & 21 & 8 & 15 & 5 & 3\end{array}$

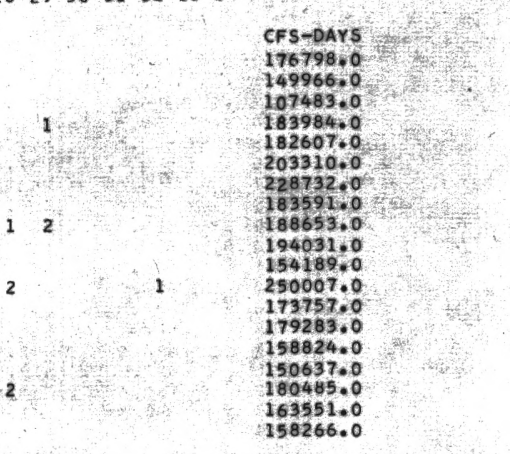

$\begin{array}{ccrrrlllrl}\text { CLASS } & \text { CFS TOTAL ACCUM } & \text { PFRCT } & \text { CLASS } & \text { CFS } & \text { TOTAL } & \text { ACCUM PERCT } \\ & .0 & & 6939 & 100.0 & 09 & 330.0 & 490 & 4824 & 69.5 \\ 1 & 160.0 & 23 & 6939 & 100.0 & 10 & 360.0 & 631 & 4334 & 62.5 \\ 2 & 180.0 & 37 & 6916 & 90.7 & 11 & 400.0 & 789 & 3703 & 53.4 \\ 3 & 200.7 & 163 & 6879 & 99.1 & 12 & 450.0 & 453 & 2914 & 42.0 \\ 4 & 220.0 & 322 & 6716 & 96.8 & 13 & 500.0 & 316 & 2461 & 35.5 \\ 5 & 240.0 & 264 & 6394 & 92.1 & 14 & 550.0 & 319 & 2145 & 30.9 \\ 6 & 260.0 & 389 & 6130 & 88.3 & 15 & 600.0 & 336 & 1826 & 26.3 \\ 7 & 280.0 & 374 & 5741 & 87.7 & 16 & 650.0 & 274 & 1490 & 21.5 \\ 8 & 300.0 & 543 & 5367 & 77.3 & 17 & 700.0 & 230 & 1216 & 17.5\end{array}$

$\begin{array}{lrrrr}\text { CLASS } & \text { CFS } & \text { TOTAL } & \text { ACCUM PERCT } \\ 18 & 750 & 200 & 986 & 14.2 \\ 19 & 800 & 312 & 786 & 11.3 \\ 20 & 900 & 187 & 474 & 6.8 \\ 21 & 1000 & 118 & 287 & 4.1 \\ 22 & 1100 & 74 & 169 & 2.4 \\ 23 & 1200 & 44 & 95 & 1.4 \\ 24 & 1300 & 24 & 51 & .7 \\ 25 & 1400 & 11 & 27 & .4 \\ 26 & 1500 & 4 & 16 & .2\end{array}$

$\begin{array}{crrrr}\text { CLASS CFS } & \text { TOTAL ACCUM PERCT } \\ 27 & 1600 & 3 & 12 & 02 \\ 28 & 1800 & 5 & 9 & : 1 \\ 29 & 2000 & 3 & 4 & 11 \\ 30 & 2200 & & 1 & 0 \\ 31 & 2400 & & 1 & 0 \\ 32 & 2600 & 1 & 1 & * 0 \\ 33 & 2800 & & & .0 \\ 34 & & & & .0\end{array}$

McKenzie River at outlet of Clear Lake,

STATION NUMBER

$14-1585.00$

Oreg. LOWEST MEAN DISCHARGF, IN CFS, FOR THF FOLLOWING NUMRER OF CONSECUTIVE DAYS IN YEAR BEgINNING APRIL 1

\begin{tabular}{|c|c|c|c|c|c|c|c|c|c|c|c|}
\hline YEAR & 1 & 3 & 7 & 14 & 30 & 60 & 90 & 120 & 150 & 183 & 274 \\
\hline 1913 & 269.0 & $270 . n$ & 272.0 & 278.0 & 293.0 & 317.0 & 326.0 & 327.0 & 333.0 & 3.49 .0 & 408,0 \\
\hline 1914 & 209.0 & 209.0 & 209.0 & 209.0 & 218.0 & 230.0 & 244.0 & 255.0 & 261.0 & 258.0 & 273,0 \\
\hline 1948 & 238.0 & 239.0 & 240.0 & 242.0 & 251.0 & 300.0 & 307.0 & $327 \cdot 0$ & 353.0 & 349.0 & 381.0 \\
\hline 1949 & 247.0 & 248.0 & 250.0 & 254.0 & 262.0 & 272.0 & 282.0 & $305 \cdot 0$ & 323,0 & 335.0 & 405.0 \\
\hline 1950 & 322.0 & $323 . n$ & 326.0 & 330.0 & 345.0 & 371.0 & 403.0 & $484 \cdot 0$ & 548.0 & 594.0 & 644.0 \\
\hline 1951 & 270.0 & 270.0 & 272.0 & 276.0 & 285.0 & 302.0 & 324.0 & $356 \cdot 0$ & 377.0 & 387.0 & 394.0 \\
\hline 1952 & 204.0 & 205.0 & 205.0 & 207.0 & 211.0 & 217.0 & 226.0 & $242 \cdot 0$ & 264.0 & 298.0 & 462.0 \\
\hline 1953 & 238.0 & 239.0 & 241.0 & 243.0 & 253.0 & 266.0 & $290 \cdot 0$ & $320 \cdot 0$ & 363.0 & 441.0 & 495.0 \\
\hline 1954 & 230.0 & 232.0 & 233.0 & 236.0 & 251.0 & 283.0 & 301.0 & 303.0 & 305.0 & 303.0 & 329.0 \\
\hline 1955 & 283.0 & $288 . n$ & 292.0 & 294.0 & 304.0 & 335.0 & 353.0 & 384.0 & 438.0 & 517.0 & 575.0 \\
\hline 1956 & 27.3 .0 & 273.0 & 274.0 & 279.0 & 295.0 & $342 . n$ & 367.0 & $388 \cdot 0$ & 419.0 & 425.0 & 499.0 \\
\hline 1957 & 204.0 & 204.0 & 206.0 & 210.0 & 219.0 & 226.0 & 238.0 & $251 * 0$ & 267.0 & 293.0 & 402.0 \\
\hline 1958 & 214.0 & 215.0 & 218.0 & 221.0 & 224.0 & 238.0 & 258.0 & $282 \cdot 0$ & 311.0 & 351.0 & 415.0 \\
\hline 1959 & 206.0 & $206 . n$ & 207.0 & 210.0 & 221.0 & 243.0 & 256.0 & $276 \cdot 0$ & 283.0 & 276.0 & 301.0 \\
\hline 1960 & 194.0 & 196.0 & 197.0 & 200.0 & 204.0 & 214.0 & 227.0 & $245 \cdot 0$ & $274 \cdot 0$ & 340.0 & $424 \times 0$ \\
\hline 1961 & 226.0 & 228.0 & 229.0 & 233.0 & 240.0 & 252.0 & 264.0 & $282 \cdot 0$ & $298 \cdot 0$ & 322.0 & 368.0 \\
\hline 1962 & 234.0 & 235.0 & 236.0 & 238.0 & 246.0 & 265.0 & 292.0 & $307 \cdot 0$ & 327.0 & 363.0 & 426.0 \\
\hline
\end{tabular}

McKenzie River at outlet of Clear Lake, STATION NUMBER 14-1585.00

Oreg. HIGHEST MEAN DISCHARGF, IN CFS, FOR THF FOLLOWING NUMBER OF CONSECUTIVE DAYS. IN YEAR ENDING SEPTEMBER 30

YEAR
1913
1914
1915
1948
1949
1950
1951
1952
1953
1954
1955
1956
1957
1958
1959
1960
1961
1962
1963

$\begin{array}{rr}1 & 3 \\ 1130.0 & 1110.0 \\ 902.0 & 876.0 \\ 645.0 & 628.0 \\ 2050.0 & 1540.0 \\ 1510.0 & 1380.0 \\ 1370.0 & 1340.0 \\ 1720.0 & 1450.0 \\ 1080.0 & 1060.0 \\ 2180.0 & 2050.0 \\ 1540.0 & 1220.0 \\ 1340.0 & 1320.0 \\ 2740.0 & 2130.0 \\ 1420.0 & 1290.0 \\ 1340.0 & 1250.0 \\ 1000.0 & 933.0 \\ 1120.0 & 1080.0 \\ 1990.0 & 1760.0 \\ 1020.0 & 993.0 \\ 946.0 & 897.0\end{array}$

7
1070.0
851.0
620.0
1180.0
1310.0
1290.0
1210.0
1040.0
1750.0
1030.0
1250.0
1700.0
1140.0
1140.0
823.0
1020.0
1440.0
935.0
834.0

15
1060.0
794.0
597.0
937.0
1250.0
1260.0
1040.0
1020.0
1390.0
915.0
1130.0
1330.0
988.0
1090.0
753.0
956.0
1230.0
922.0
792.0

30
1010.0
699.0
543.0
856.0
1180.0
1190.0
928.0
967.0
1280.0
881.0
1000.0
1229.0
826.0
902.0
708.0
824.0
990.0
886.0
701.0

60
898.0
670.0
471.0
755.0
1030.0
1070.0
866.0
874.0
943.0
729.0
865.0
1100.0
757.0
774.0
631.0
762.0
808.0
771.0
582.0

90
811.0
636.0
443.0
678.0
879.0
925.0
828.0
794.0
773.0
687.0
741.0
989.0
735.0
732.0
604.0
720.0
751.0
678.0
567.0

120
742.0
585.0
405.0
611.0
771.0
848.0
832.0
716.0
732.0
667.0
653.0
878.0
669.0
710.0
561.0
638.0
733.0
607.0
574.0

150
672.0
548.0
379.0
587.0
709.0
819.0
772.0
652.0
751.0
650.0
594.0
795.0
615.0
700.0
558.0
592.0
686.0
567.0
547.0

574.0
547.0

183

612.0
521.0

521.0
354.0

354.0
592.0

653.0

758.0
771.0

771.0
606.0

606.0
735.0

735.0
664.0

664.0
542.0

542.0
819.0

611.0

611.0
672.0

672.0
567.0

567.0
546.0

667.0

564.0
274

543.0
460.0

460.0
327.0

327.0
558.0

558.0
566.0

566.0
640.0

640.0
723.0

552.0
612.0

612.0
614.0

614.0
463.0

783.0

540.0
572.0

572.0
502.0

502.0
458.0

580.0

508.0
495.0 
MC KENZIE RIVER NR BELKNIAP SPRINGS OREG
STATION NUMBER $14-1587.00$

DURATION TABLE OF DAILY DISCHARGE

CLASS $0101120 \begin{array}{lllllllllllllllllllllllllllllllll} & 4 & 5 & 6 & 7 & 8 & 9 & 10 & 11 & 12 & 13 & 14 & 15 & 16 & 17 & 18 & 19 & 20 & 21 & 22 & 23 & 24 & 25 & 26 & 27 & 28 & 29 & 30 & 31 & 32 & 33 & 34\end{array}$

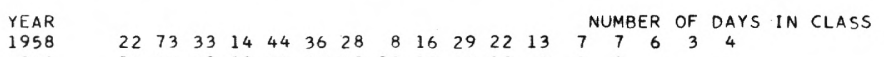

$\begin{array}{rrrrrrrrrrrrrrr}1958 & 22 & 73 & 33 & 14 & 44 & 36 & 28 & 8 & 16 & 29 & 22 & 13 & 7 & 7 \\ 1959 & 59 & 43 & 20 & 30 & 44 & 39 & 28 & 22 & 20 & 46 & 10 & 2 & 1 & 1\end{array}$

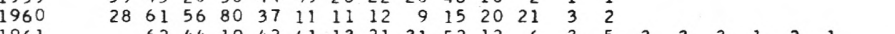

CLASS CFS TOTAL ACCUM PERCT CLASS CFS TOTAL ACCUM PERCT

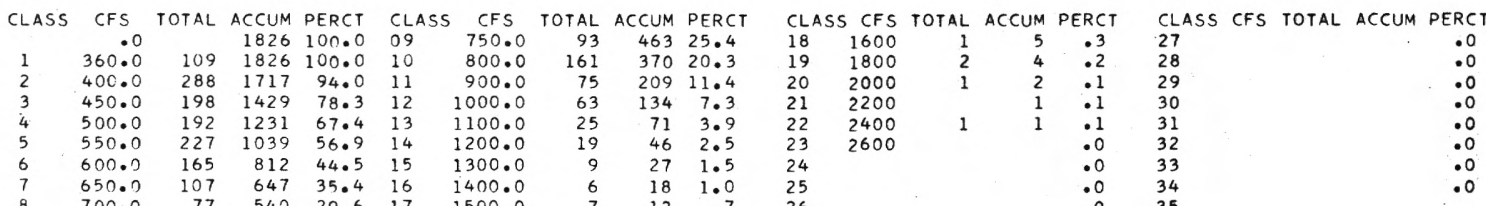

MC KENZIE RIVER NR BELLKNAP SPRINGS OREG

STATION NUMBER $14-1587.00$

LOWEST MEAN DISCHARGF, IN CFS, FOR THE FOLLOWING NUMBER OF CONSECUTIVE DAYS IN YEAR BEGINNING APRIL I

\begin{tabular}{|c|c|c|c|c|c|c|c|c|c|c|c|}
\hline YEAR & 1 & 3 & 7 & 14 & 30 & 60 & 90 & 120 & 150 & 183 & 274 \\
\hline 1958 & 362.0 & $362 . n$ & 364.0 & 368.0 & 370.0 & 387.0 & 406.0 & 433.0 & 464.0 & 505.0 & 576.0 \\
\hline 1959 & 367.0 & 367.0 & 367.0 & 370.0 & 381.0 & 409.0 & 426.0 & 445.0 & 450.0 & 442.0 & 468.0 \\
\hline $\begin{array}{l}1960 \\
1961\end{array}$ & $\begin{array}{l}405.0 \\
411.0\end{array}$ & $\begin{array}{l}406 \cdot 0 \\
414 \cdot 0\end{array}$ & $\begin{array}{l}407.0 \\
414.0\end{array}$ & $\begin{array}{l}408.0 \\
418.0\end{array}$ & $\begin{array}{l}410.0 \\
425.0\end{array}$ & $\begin{array}{l}417.0 \\
439.0\end{array}$ & $\begin{array}{l}431.0 \\
452.0\end{array}$ & $\begin{array}{l}451 \cdot 0 \\
472 \cdot 0\end{array}$ & $\begin{array}{l}477.0 \\
492.0\end{array}$ & $\begin{array}{l}541.0 \\
519.0\end{array}$ & $\begin{array}{l}617.0 \\
562.0\end{array}$ \\
\hline
\end{tabular}

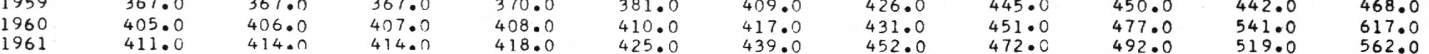

MC KEINZIE RIVER NR BELLENAP SPRTNGS OREG STATION NUMBER 14-1587.00

HIGHEST MEAN DISCHARGF, IN CFS, FOR THE FOLLOWING NUMBER OF CONSECUTIVE DAYS IN YEAR ENDING SEPTEMBER 30

\begin{tabular}{|c|c|c|c|c|c|c|c|c|c|c|c|}
\hline YEAR & 1 & 3 & 7 & 15 & 30 & 60 & 90 & 120 & 150 & 183 & 274 \\
\hline 1958 & 1520.0 & $1490 . n$ & 1380.0 & 1340.0 & 1110.0 & 963.0 & 914.0 & $889 \cdot 0$ & 876.0 & 845.0 & 741.0 \\
\hline 1959 & 1240.0 & 1080.0 & 964.0 & 894.0 & 852.0 & 791.0 & 772.0 & 719.0 & 714.0 & 725.0 & 666.0 \\
\hline 1960 & 1280.0 & 1220.0 & 1150.0 & 1080.0 & 986.0 & 942.0 & 898.0 & 819.0 & 764.0 & 723.0 & 631.0 \\
\hline 1961 & 2480.0 & $2100 \cdot 0$ & 1770.0 & 1520.0 & 1220.0 & 1010.0 & 943.0 & $918 \cdot i$ & 867.0 & 848.0 & 762.0 \\
\hline 1962 & 1260.0 & 1200.0 & 1150.0 & 1130.0 & 1100.0 & 975.0 & 868.0 & 791.0 & 762.0 & 755.0 & 699.0 \\
\hline
\end{tabular}



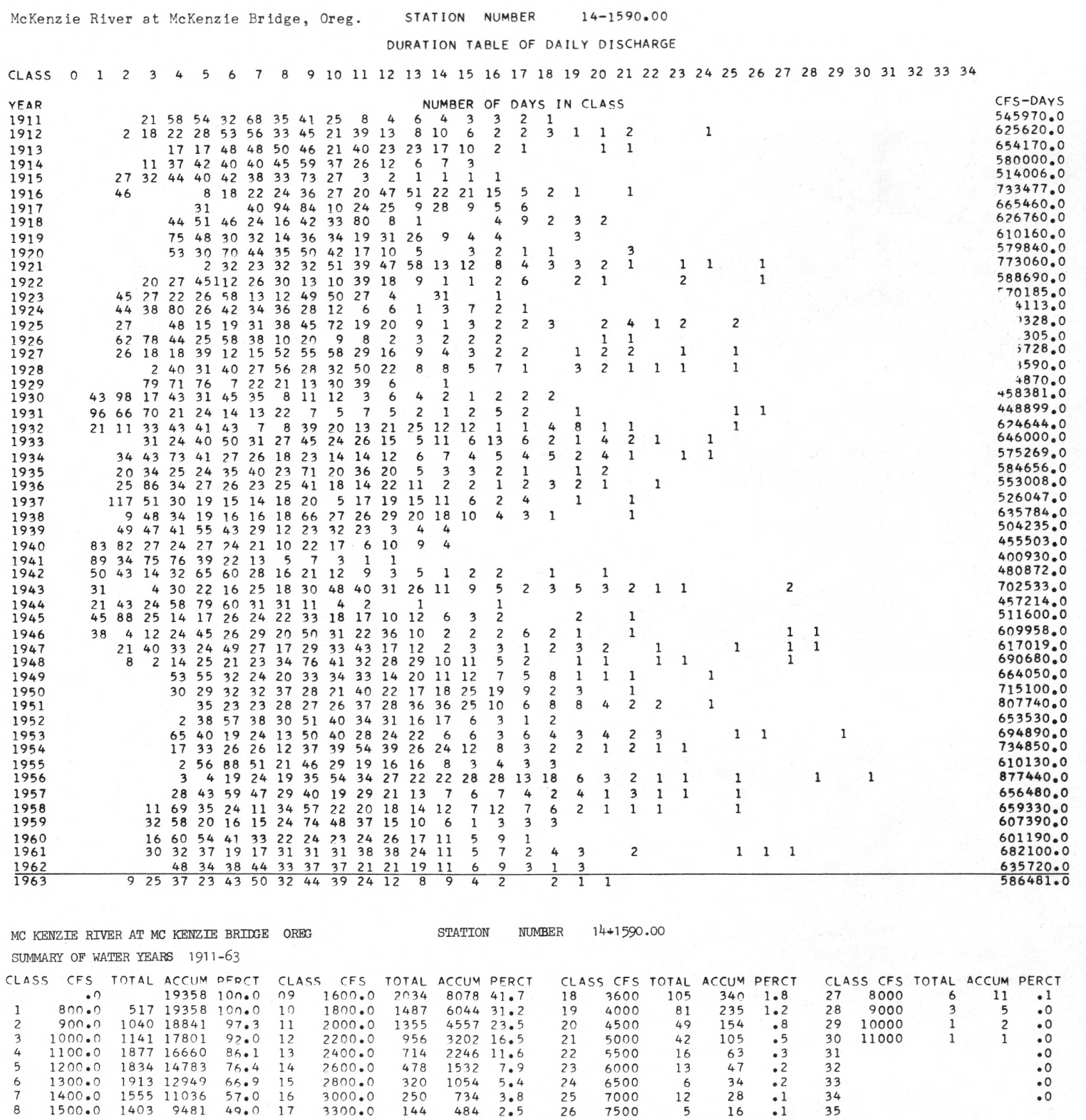

McKenzie River at McKenzie Bridge, Oreg.

STATION NUMBER

14-1590.00

Summary for water years 1911-1962
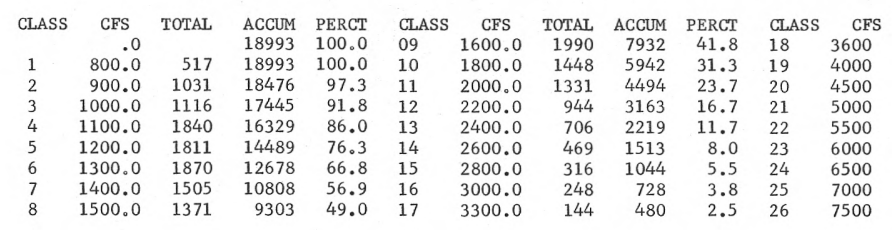

TOTAL AC
103
80
48
42
16
13
6
12
5

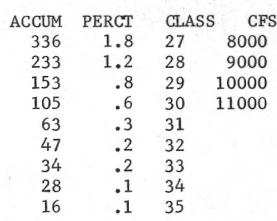

McKenzie River at McKenzie Bridge, Oreg.

STATION NUMBER

$14-1590.00$

Summary for vater year 1963
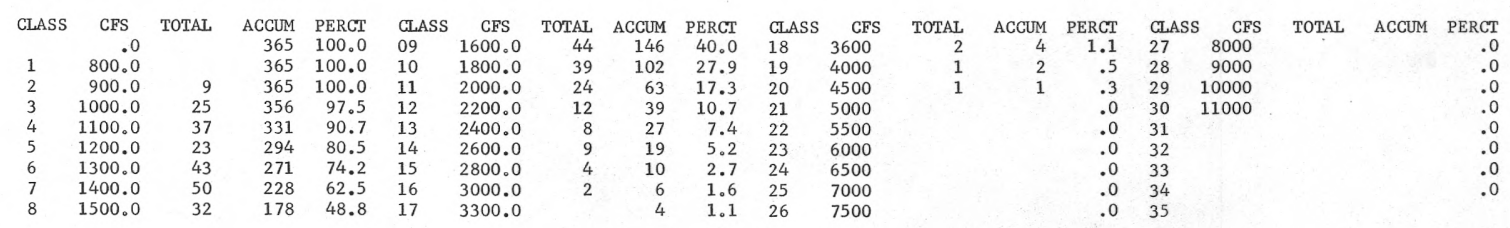
LOWEST MEAN DISCHARGF, IN CFS, FOR THE FOLLOWING NUMBER OF CONSECUTIVE DAYS IN YEAR BEGINNING APRIL 1

\begin{tabular}{|c|c|c|c|c|c|c|c|c|c|c|c|}
\hline YEAR & 1 & 3 & 7 & 14 & 30 & 60 & 90 & 120 & 150 & 183 & 274 \\
\hline $\begin{array}{l}1911 \\
1912\end{array}$ & $\begin{array}{r}950.0 \\
1150.0\end{array}$ & $\begin{array}{r}967.0 \\
1150.0\end{array}$ & $\begin{array}{r}986.0 \\
1150.0\end{array}$ & $\begin{array}{l}1010.0 \\
1170.0\end{array}$ & $\begin{array}{l}1050.0 \\
1180.0\end{array}$ & $\begin{array}{l}1110.0 \\
1280.0\end{array}$ & 1120.0 & $1150 \cdot 0$ & 1220.0 & 1280.0 & 1420.0 \\
\hline 1913 & 1210.0 & 1210.0 & 1230.0 & 1240.0 & 1280.0 & 1350.0 & 1370.0 & 1410.0 & 1420.0 & 1470.0 & 1640.0 \\
\hline 1914 & 1080.0 & $1080 \cdot n$ & 1080.0 & 1000.0 & 1110.0 & 1150.0 & 1190.0 & $1>30.0$ & 1250.0 & 1250.0 & 1380.0 \\
\hline 1915 & 924.0 & 930.0 & 938.0 & 944.0 & 947.0 & 954.0 & 972.0 & 1010.0 & 1050.0 & 1150.0 & 1400.0 \\
\hline $\begin{array}{l}1916 \\
1917\end{array}$ & $\begin{array}{l}1250.0 \\
1190.0\end{array}$ & $\begin{array}{l}1250 . n \\
1190.0\end{array}$ & $\begin{array}{l}1250 . ? \\
1190.0\end{array}$ & $\begin{array}{l}1250.0 \\
1220.0\end{array}$ & 1250.0 & 1330.0 & 1370.0 & $\begin{array}{l}1410.0 \\
1440.0\end{array}$ & 1450.0 & 1480.0 & 1600.0 \\
\hline 1918 & 1110.0 & 1110.0 & 1110.0 & 1120.0 & 1140.0 & 1140.0 & $\begin{array}{l}1360.0 \\
1170.0\end{array}$ & $\begin{array}{l}1440.0 \\
1190.0\end{array}$ & $\begin{array}{l}1550.0 \\
1210.0\end{array}$ & $\begin{array}{l}1770.0 \\
1230.0\end{array}$ & $\begin{array}{l}1890.0 \\
1370.0\end{array}$ \\
\hline 1919 & 1110.0 & 1110.0 & 1130.0 & 1140.0 & 1180.0 & 1200.0 & 1240.0 & $1300 \cdot 0$ & 1420.0 & 1510.0 & 1580.0 \\
\hline 1920 & 1110.0 & 1110.0 & 1130.0 & 1160.0 & 1170.0 & 1210.0 & 1260.0 & 1330.0 & 1410.0 & 1440.0 & 1670.0 \\
\hline 1921 & 1180.0 & 1200.0 & 1230.0 & 1260.0 & 1290.0 & 1310.0 & 1330.0 & $1390 \cdot 0$ & 1480.0 & 1640.0 & 1600.0 \\
\hline 1972 & 962.0 & 967.0 & 975.0 & 977.0 & 984.0 & 993.0 & 1010.0 & 1040.0 & 1090.0 & $1200 \cdot 0$ & 1440.0 \\
\hline 1973 & 1100.0 & 1100.0 & 1100.0 & 1100.0 & 1120.0 & 1150.0 & 1160.0 & $1200 \cdot 0$ & 1250.0 & 1330.0 & $1500 \cdot 0$ \\
\hline $19>4$ & 920.0 & $920 . n$ & $9>0.0$ & 923.0 & 936.0 & 959.0 & 977.0 & $1000 \cdot 0$ & 1030.0 & 1080.0 & 1290.0 \\
\hline 1975 & 1030.0 & 1030.0 & 1040.0 & 1050.0 & 1050.0 & 1080.0 & 1100.0 & 1230.0 & 1160.0 & 1220.0 & 1380.0 \\
\hline 1976 & 934.0 & $934 \cdot n$ & 926.0 & 948.0 & 962.0 & 970.0 & 980.0 & 985.0 & $100 n \cdot 0$ & 1040.0 & 1270.0 \\
\hline 1927 & 1130.0 & $1130 . n$ & 1120.0 & 1120.0 & 1180.0 & $12>0.0$ & 1310.0 & 1320.0 & 1410.0 & 1550.0 & 1750.0 \\
\hline $19>8$ & 1030.0 & 1040.0 & 1050.0 & $1050 . n$ & 1060.0 & 1090.0 & 1110.0 & 1120.0 & 1140.0 & 1160.0 & 1200.0 \\
\hline $19>9$ & 920.0 & 920.0 & $9>0.0$ & $9>0.0$ & 940.0 & 965.0 & 990.0 & 1020.0 & 1070.0 & 1160.0 & 1350.0 \\
\hline 1930 & 832.0 & $832 \cdot n$ & 835.0 & 843.0 & 848.0 & 861.0 & 868.0 & $884 \cdot 0$ & 900.0 & 927.0 & 1000.0 \\
\hline 1931 & 810.0 & 812.0 & 822.0 & 843.0 & 854.0 & 861.0 & 877.0 & 919.0 & 953.0 & 984.0 & 1130.0 \\
\hline 1932 & 1030.0 & 1030.0 & 1050.0 & 1050.0 & 1060.0 & 1080.0 & 1120.0 & 1190.0 & 1300.0 & 1340.0 & 1360.0 \\
\hline 1933 & 1120.0 & 1120.0 & 1130.0 & 1130.0 & 1180.0 & 1200.0 & 1220.0 & $1250 \cdot 0$ & 1340.0 & 1580.0 & 1820.0 \\
\hline 1934 & 932.0 & $932 \cdot 0$ & 933.0 & 937.0 & 949.0 & 966.0 & 989.0 & $1020 \cdot 0$ & 1060.0 & 1110.0 & 1390.0 \\
\hline 1935 & 970.0 & 970.0 & 971.0 & 981.0 & 998.0 & 1010.0 & 1020.0 & 1040.0 & 1060.0 & 1110.0 & 1380.0 \\
\hline 1936 & 905.0 & 907.0 & 910.0 & 914.0 & 923.0 & 943.0 & 955.0 & 955.0 & 966.0 & 985.0 & 1090.0 \\
\hline 1927 & 985.0 & 985.0 & 989.0 & 1000.0 & 1020.0 & 1040.0 & 1070.0 & 1140.0 & 1290.0 & 1450.0 & 1630.0 \\
\hline 1938 & 979.0 & 979.0 & 979.0 & 983.0 & 997.0 & 1030.0 & 1070.0 & $1100 \cdot 0$ & 1150.0 & 1230.0 & 1280.0 \\
\hline 1939 & 872.0 & 872.0 & $872 \cdot 0$ & 874.0 & 878.0 & 898.0 & 914.0 & 936.0 & 967.0 & 1030.0 & 1170.0 \\
\hline 1940 & 835.0 & 835.0 & 837.0 & 838.0 & $848 \cdot 0$ & $859 . ?$ & 872.0 & 891.0 & 914.0 & 945.0 & 1070.0 \\
\hline 1941 & $847 \cdot 0$ & $849 \cdot 0$ & 855.0 & 859.0 & 865.0 & 870.0 & 878.0 & $892 \cdot 0$ & $922 \cdot 0$ & 959.0 & 1170.0 \\
\hline 1942 & 810.0 & 812.0 & 813.0 & 818.0 & 832.0 & 853.0 & 890.0 & 950.0 & 1010.0 & 1080.0 & 1440.0 \\
\hline 1943 & 1090.0 & 1100.0 & 1100.0 & 1110.0 & 1120.0 & 1160.0 & 1220.0 & 1290.0 & 1300.0 & 1300.0 & 1330.0 \\
\hline 1944 & 836.0 & 838.0 & $841 \cdot 0$ & $845 \cdot 0$ & $857 \cdot 0$ & 873.0 & 891.0 & $907 \cdot 0$ & 914.0 & 934.0 & 1070.0 \\
\hline 1945 & 858.0 & $858 \cdot 0$ & $861 \cdot 0$ & $865 \cdot 0$ & 867.0 & 883.0 & 912.0 & $958 \cdot 0$ & 1020.0 & 1120.0 & 1450.0 \\
\hline 1946 & 1000.0 & 1000.0 & $1000 . ?$ & 1020.0 & 1050.0 & 1110.0 & 1170.0 & 1210.0 & 1300.0 & 1460.0 & 1760.0 \\
\hline 1947 & 949.0 & $949 \cdot 0$ & 952.0 & 957.0 & 972.0 & 1010.0 & 1070.0 & 1140.0 & 1190.0 & 1300.0 & 1520.0 \\
\hline 1948 & 1130.0 & 1140.0 & 1140.0 & 1140.0 & 1190.0 & 1210.0 & 1240.0 & $1290 \cdot 0$ & 1380.0 & 1470.0 & 1580.0 \\
\hline 1949 & 1100.0 & 1100.0 & 1110.0 & 1110.0 & 1140.0 & 1160.0 & 1180.0 & $1220 \cdot 0$ & 1300.0 & 1340.0 & 1560.0 \\
\hline 1950 & 1290.0 & $1300 \cdot 0$ & 1310.0 & $13>0.0$ & 1350.0 & 1420.0 & 1480.0 & 1640.0 & 1930.0 & 2030.0 & 2290.0 \\
\hline 1951 & 1180.0 & 1190.0 & 1220.0 & 1230.0 & 1250.0 & 1290.0 & 1340.0 & $1400 \cdot 0$ & 1470.0 & 1540.0 & 1590.0 \\
\hline $195 ?$ & 1100.0 & 1100.0 & $1100 \cdot 0$ & 1110.0 & 1120.0 & 1140.0 & 1170.0 & $1190 \cdot 0$ & 1230.0 & 1200.0 & 1620.0 \\
\hline 1953 & 1170.0 & 1170.0 & 1180.0 & 1190.0 & 1210.0 & 1230.0 & 1270.0 & 1230.0 & 1420.0 & 1580.0 & 1900.0 \\
\hline 1954 & 1250.0 & 1250.0 & 1260.0 & 1270.0 & 1290.0 & 1310.0 & 1340.0 & $1260 \cdot 0$ & 1380.0 & 1410.0 & 1460.0 \\
\hline 1955 & 1170.0 & 1180.0 & 1190.0 & 1200.0 & 1230.0 & 1300.0 & 1370.0 & $1470 \cdot 0$ & 1600.0 & 1820.0 & 2130.0 \\
\hline 1956 & 1220.0 & 1220.0 & 1230.0 & 1240.0 & 1290.0 & 1410.0 & 1480.0 & $1510 \cdot 0$ & 1570.0 & 1650.0 & 1840.0 \\
\hline 1957 & 1080.0 & 1080.0 & 1080.0 & 1090.0 & 1120.0 & 1150.0 & 1170.0 & $1200 \cdot 0$ & 1230.0 & 1280.0 & 1590.0 \\
\hline 1958 & 1040.0 & 1040.0 & $1040 \cdot 0$ & 1060.0 & 1070.0 & 1100.0 & 1130.0 & 1190.0 & 1270.0 & $1350 \cdot 0$ & 1610.0 \\
\hline 1959 & 1090.0 & 1100.0 & 1100.0 & 1110.0 & 1120.0 & 1140.0 & 1190.0 & $1250 \cdot 0$ & 1270.0 & $1270 \cdot 0$ & 1350.0 \\
\hline 960 & $1000 \cdot 0$ & $1000 \cdot 0$ & 1000.0 & 1010.0 & 1030.0 & 1060.0 & 1090.0 & $1140 \cdot 0$ & 1220.0 & 1390.0 & $1630: 0$ \\
\hline 1961 & 1130.0 & 1130.0 & 1130.0 & 1140.0 & 1160.0 & 1180.0 & 1210.0 & $1260 \cdot 0$ & $1300 \cdot 0$ & 1390.0 & 1590.0 \\
\hline 1962 & 1120.0 & 1120.0 & 1130.0 & 1140.0 & 1150.0 & 1190. & 1240. & 1280.0 & 1320 & 1400.0 & 1570.0 \\
\hline
\end{tabular}


McKenzie River at McKenzie Bridge, Oreg.

STATION NUMBER

$14-1590.00$

HIGHEST MEAN DISCHARGE, IN CFS, FOR THE FOLLOWING NUMBER OF CONSECUTIVE DAYS IN YEAR ENDING SEPTEMBER 30

\begin{tabular}{|c|c|c|c|c|c|c|c|c|c|c|c|}
\hline YEAR & 1 & 3 & 7 & 15 & 30 & 60 & 90 & 120 & 150 & 183 & 274 \\
\hline 1911 & 3710.0 & 3370.0 & 3140.0 & 2820.0 & 2480.0 & 2000.0 & 1820.0 & 1690.0 & 1650.0 & 1680.0 & 1620.0 \\
\hline 1912 & 6810.0 & 5700.0 & 4300.0 & 3360.0 & 2890.0 & 2710.0 & 2320.0 & $2130 \cdot 0$ & 2120.0 & 2060.0 & 1870.0 \\
\hline 1913 & 5030.0 & 4380.0 & 3400.0 & 2840.0 & 2690.0 & 2480.0 & 2430.0 & $2^{2} 10 \cdot 0$ & 2160.0 & 2040.0 & 1940.0 \\
\hline 1914 & 2920.0 & 2810.0 & 2550.0 & 2450.0 & 2260.0 & 2130.0 & 2020.0 & 1980.0 & 1930.0 & 1850.0 & 1720.0 \\
\hline 1915 & 3000.0 & 2570.0 & 2140.0 & 1940.0 & 1850.0 & 1740.0 & 1750.0 & 1710.0 & 1690.0 & 1630.0 & 1520.0 \\
\hline 1916 & 5960.0 & $397 n$.n & $3 \times 30.0$ & $33 n 0.0$ & 2700.0 & 2560.0 & 2540.0 & 2510.0 & 2500.0 & 2440.0 & 2270.0 \\
\hline 1917 & 3450.0 & 3330.0 & 3310.0 & $3 \cap 80.0$ & $29 ? 0.0$ & 2710.0 & 2590.0 & 2450.0 & $2 ? 90.0$ & 2160.0 & 1970.0 \\
\hline 1918 & 4500.0 & 4170.0 & 2960.0 & 3670.0 & 3030.0 & 2550.0 & 2360.0 & $\gg 20000$ & $2 ? 00.0$ & 2120.0 & 1870.0 \\
\hline $\begin{array}{l}1919 \\
1990\end{array}$ & $\begin{array}{l}4400.0 \\
5370.0\end{array}$ & $3890 \cdot n$ & 2330.0 & 2820.0 & 2460.0 & 2440.0 & 2290.0 & 2140.0 & 2170.0 & 2080.0 & 1830.0 \\
\hline 1930 & & 3930.0 & 3130.0 & 2520.0 & 2110.0 & 2050.0 & 1990.0 & $1900 \bullet 0$ & 1800.0 & 1790.0 & 1700.0 \\
\hline 1921 & $\begin{array}{l}7750.0 \\
7500.0\end{array}$ & 6030.0 & 5350.0 & 4300.0 & 3310.0 & 2870.0 & 2700.0 & $2570 \cdot 0$ & 2560.0 & 2520.0 & 2320.0 \\
\hline 1922 & $\begin{array}{l}7500.0 \\
3240.0\end{array}$ & 6070.0 & 4750.0 & 3840.0 & 2970.0 & 2320.0 & 1990.0 & $1820 \cdot 0$ & 1740.0 & 1770.0 & 1750.0 \\
\hline 1923 & 3240.0 & 2680.0 & 2680.0 & $2680 \cdot 0$ & 2680.0 & 2230.0 & 1970.0 & 1910.0 & 1930.0 & 1920.0 & 1740.0 \\
\hline $19>4$ & 3540.0 & $3340 \cdot 0$ & $308 n \cdot 0$ & 2680.0 & 2220.0 & 2020.0 & 1920.0 & $1820 \cdot 0$ & 1730.0 & 1660.0 & 1500.0 \\
\hline 1925 & 7420.0 & 7070.0 & 6300.0 & 5210.0 & 3760.0 & 2880.0 & 2570.0 & 2370.0 & 2260.0 & $2200 \cdot 0$ & $2000 \cdot 0$ \\
\hline 1976 & $5000 \cdot 0$ & 4250.0 & 3460.0 & 2770.0 & 2390.0 & 1950.0 & 1790.0 & $1700 \cdot 0$ & 1630.0 & 1560.0 & 1410.0 \\
\hline 1977 & 7470.0 & 6300.0 & 4970.0 & 3480.0 & 2650.0 & 2240.0 & 2230.0 & $2160 \cdot 0$ & 2040.0 & 2020.0 & 1910.0 \\
\hline 1978 & $74>0.0$ & $570 n \cdot n$ & 5280.0 & 3000.0 & $3050 \cdot 0$ & 2600.0 & 2360.0 & 2230.0 & 2230.0 & $P 0 n .0$ & 1970.0 \\
\hline 1979 & $2670 \cdot 0$ & 2280.0 & 2170.0 & 2110.0 & 2070.0 & 2010.0 & 1910.0 & $183 n \cdot 0$ & 1720.0 & 1620.0 & 1480.0 \\
\hline $\begin{array}{l}1920 \\
1931\end{array}$ & $\begin{array}{l}3850.0 \\
7660.0\end{array}$ & $\begin{array}{l}3640.0 \\
6540.0\end{array}$ & $\begin{array}{l}3150.0 \\
4700.0\end{array}$ & $\begin{array}{l}2740.0 \\
3730.0\end{array}$ & $\begin{array}{l}2340.0 \\
2930.0\end{array}$ & $\begin{array}{l}1880.0 \\
2260.0\end{array}$ & $\begin{array}{l}1780.0 \\
1940.0\end{array}$ & $\begin{array}{l}1680.0 \\
1750.0\end{array}$ & $\begin{array}{l}160 n \cdot 0 \\
1640.0\end{array}$ & $\begin{array}{l}1540.0 \\
1540.0\end{array}$ & $\begin{array}{l}1370.0 \\
1350.0\end{array}$ \\
\hline 1932 & 7260.0 & 5540.0 & 4630.0 & 4340.0 & 3440.0 & 2980.0 & 2840.0 & $2620 \cdot 0$ & 2400.0 & 2260.0 & 1930.0 \\
\hline 1933 & 6750.0 & 5690.0 & 5230.0 & 4420.0 & 3830.0 & 3160.0 & 2810.0 & $2540 \cdot 0$ & 2340.0 & 2160.0 & 1950.0 \\
\hline 1934 & 6550.0 & 5400.0 & 4740.0 & 3920.0 & 3270.0 & 2980.0 & 2550.0 & 2360.0 & 2200.0 & 2040.0 & 1760.0 \\
\hline 1935 & 4740.0 & 4520.0 & 3710.0 & 2870.0 & 2370.0 & 2180.0 & 1960.0 & 1870.0 & 1780.0 & 1830.0 & 1780.0 \\
\hline 1936 & 5500.0 & 4800.0 & 4070.0 & 3510.0 & 2740.0 & 2110.0 & 1930.0 & 1940.0 & 1980.0 & 1910.0 & 1680.0 \\
\hline 1937 & 5090.0 & 4320.0 & 3500.0 & 2790.0 & 2610.0 & 2540.0 & 2430.0 & 2200 & 2050.0 & 1890.0 & 1600.0 \\
\hline 1938 & 5280.0 & 4090.0 & 3280.0 & 2810.0 & 2590.0 & 2410.0 & 2240.0 & $2100 \cdot 0$ & 2120.0 & 2130.0 & 1960.0 \\
\hline 1939 & 2760.0 & 2600.0 & 2450.0 & 2720.0 & 2070.0 & 2020.0 & 1860.0 & 1740.0 & 1660.0 & 1640.0 & 1510.0 \\
\hline $\begin{array}{l}1940 \\
1941\end{array}$ & $\begin{array}{l}2730.0 \\
2330.0\end{array}$ & $\begin{array}{l}2650.0 \\
1950.0\end{array}$ & $\begin{array}{l}2590.0 \\
1710.0\end{array}$ & $\begin{array}{l}2410.0 \\
1690.0\end{array}$ & $\begin{array}{l}2160.0 \\
1520.0\end{array}$ & $\begin{array}{l}2060.0 \\
1410.0\end{array}$ & $\begin{array}{l}1880.0 \\
1370.0\end{array}$ & $\begin{array}{l}1730.0 \\
1310.0\end{array}$ & $\begin{array}{l}1660.0 \\
1260.0\end{array}$ & $\begin{array}{l}1570.0 \\
1250.0\end{array}$ & $\begin{array}{l}1360.0 \\
1180.0\end{array}$ \\
\hline 1942 & 4520.0 & 3760.0 & $31 \geqslant 0.0$ & 2500.0 & $2 ? 60.0$ & 1910.0 & 1790.0 & 1690.0 & 1600.0 & 1560.0 & 1460.0 \\
\hline 1943 & 8540.0 & $7540 . n$ & 5700.0 & 4330.0 & 3400.0 & 3220.0 & 2820.0 & $2550 \cdot 0$ & 2510.0 & 2450.0 & 2210.0 \\
\hline & 3180.0 & 2610.0 & 2090.0 & 1760.0 & 1570.0 & 1480.0 & 1410.0 & $1390 \cdot 0$ & 1370.0 & 1380.0 & 1340.0 \\
\hline $\begin{array}{l}1945 \\
1946\end{array}$ & 5040.0 & $4100 \cdot 0$ & 3520.0 & 2950.0 & 2400.0 & 2120.0 & 1960.0 & $2010 \cdot 0$ & $1930 \cdot 0$ & 1830.0 & 1570.0 \\
\hline $\begin{array}{l}1946 \\
1947\end{array}$ & 9560.0 & $\begin{array}{l}7700.0 \\
8520.0\end{array}$ & $\begin{array}{l}5350.0 \\
6230.0\end{array}$ & $\begin{array}{l}4140.0 \\
4320.0\end{array}$ & $\begin{array}{l}3150.0 \\
3530.0\end{array}$ & 2410.0 & 2210.0 & 2060.0 & 2080.0 & 2090.0 & 1890.0 \\
\hline 1948 & 8840.0 & 6940.0 & 4950.0 & 3730.0 & $\begin{array}{l}3530.0 \\
2790.0\end{array}$ & 2670.0 & 2490.0 & $2330 \cdot 0$ & 2270.0 & $2180 \cdot 0$ & 1900.0 \\
\hline 1949 & 6590.0 & 5300.0 & 4240.0 & 3940.0 & $\begin{array}{l}2790.0 \\
3450.0\end{array}$ & $\begin{array}{l}2380.0 \\
2980.0\end{array}$ & $\begin{array}{l}2240.0 \\
2620.0\end{array}$ & $2170 \cdot 0$ & 2170.0 & $2170 \cdot 0$ & 2090.0 \\
\hline 1950 & 5110.0 & $4480 . n$ & 3620.0 & 3280.0 & 3090.0 & $\begin{array}{l}2980.0 \\
2880.0\end{array}$ & $\begin{array}{l}2620.0 \\
2650.0\end{array}$ & 2490.0 & 2340.0 & 2180.0 & 2020.0 \\
\hline 1951. & 6720.0 & $5460 . n$ & $48>0.0$ & 3790.0 & 3400.0 & $\begin{array}{l}2880.0 \\
3130.0\end{array}$ & $\begin{array}{l}2.650 .0 \\
3020.0\end{array}$ & $\begin{array}{l}2680 \cdot 0 \\
3040.0\end{array}$ & 2620.0 & $2490 \cdot 0$ & 2190.0 \\
\hline 1952 & $3800 \cdot 0$ & 3360.0 & 3040.0 & 2640.0 & 2510.0 & $24>0.0$ & $\gg 97.0$ & $\begin{array}{l}3040.0 \\
2150.0\end{array}$ & $\begin{array}{r}2830.0 \\
2040.0\end{array}$ & 2750.0 & $2500 \cdot 0$ \\
\hline 1953 & 10800.0 & 8530.0 & 6510.0 & $49>0.0$ & 4450.0 & 3360.0 & 2850.0 & 2640.0 & 2580.0 & $\begin{array}{l}1980.0 \\
2490.0\end{array}$ & \\
\hline 1954 & 6230.0 & 5330.0 & 4110.0 & 3620.0 & 3360.0 & 2810.0 & 2740.0 & 2620.0 & 2540.0 & 2480.0 & $\begin{array}{l}2150.0 \\
2250.0\end{array}$ \\
\hline 1955 & 3830.0 & 3770.0 & 3550.0 & 3120.0 & 2850.0 & 2560.0 & 2330.0 & $2000 \cdot 0$ & 2060.0 & 1960.0 & 1800.0 \\
\hline 1956 & 11400.0 & 9290.0 & 6510.0 & 4980.0 & 4070.0 & 3710.0 & 3280.0 & 2940.0 & 2810.0 & 2880.0 & 2700.0 \\
\hline 1957 & 7090.0 & 6100.0 & 4660.0 & 3980.0 & 3290.0 & 2840.0 & 2590.0 & 225000 & 2280.0 & 2200.0 & 1980.0 \\
\hline 1958 & 7180.0 & 5480.0 & 4170.0 & 3570.0 & 3110.0 & 2830.0 & 2720.0 & $2470 \cdot 0$ & 2440.0 & 2320.0 & 2020.0 \\
\hline 1959 & 3690.0 & $3480 \cdot 0$ & 2960.0 & 2560.0 & 2450.0 & 2240.0 & 2190.0 & $2070 \cdot 0$ & 2050.0 & 2030.0 & 1840.0 \\
\hline $\begin{array}{l}1960 \\
1961\end{array}$ & $\begin{array}{l}3370.0 \\
8480.0\end{array}$ & 3150.0 & 3130.0 & 2970.0 & 2670.0 & 2510.0 & 2390.0 & 2270.0 & 2160.0 & 2020.0 & 1790.0 \\
\hline $\begin{array}{l}1961 \\
1962\end{array}$ & $\begin{array}{l}8480 \cdot 0 \\
4220 \cdot 0\end{array}$ & 6990.0 & $5340 \cdot 0$ & 4260.0 & 3420.0 & 2940.0 & 2670.0 & $2550 \cdot 0$ & 2420.0 & 2370.0 & 2110.0 \\
\hline$\frac{1962}{1963}$ & $\frac{4220.0}{4960.0}$ & $\frac{3910.0}{4310.0}$ & $\frac{3450 \cdot 0}{3430 \cdot 0}$ & 2940.0 & 2690.0 & 2480.0 & 2240.0 & 2080.0 & 2150.0 & 2120.0 & 1920.0 \\
\hline & & 4310.0 & $3430 . n$ & 2690.0 & 2410.0 & 1970.0 & 1990.0 & 1950.0 & 1890.0 & 1910.0 & 1760 \\
\hline
\end{tabular}


South Fork McKenz1e R1ver above Cougar

STATION NUMBER $14-1592.00$

Reservolr near Ralnbow, Oreg.

DURATION TABLE OF DAILY DISCHARGE

CLASS $0 \begin{array}{llllllllllllllllllllllllllllllllllll} & 0 & 2 & 3 & 4 & 5 & 6 & 7 & 8 & 9 & 10 & 11 & 12 & 13 & 14 & 15 & 16 & 17 & 18 & 19 & 20 & 21 & 22 & 23 & 24 & 25 & 26 & 27 & 28 & 29 & 30 & 31 & 32 & 33 & 34\end{array}$

YEAR

1958

1959

$\begin{array}{lllllllllllllll}77 & 45 & 21 & 11 & 22 & 17 & 20 & 23 & 15 & 47 & 21 & 16 & 14 & 4 & 6\end{array}$

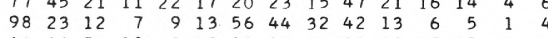

$\begin{array}{lllllllllllllllllll}62 & 66 & 54 & 20 & 9 & 12 & 10 & 17 & 14 & 32 & 20 & 27 & 15 & 7 & 1\end{array}$

1961

$\begin{array}{lllllllllllllllllll}1103 & 28 & 10 & 7 & 10 & 14 & 23 & 20 & 29 & 60 & 33 & 9 & 5 & 5 & 3 & 1\end{array}$

$\begin{array}{rrrrrrrrrrrrrrrrrrr}6 & 48 & 54 & 17 & 11 & 16 & 18 & 33 & 23 & 27 & 35 & 31 & 17 & 17 & 4 & 3 & 1 & 4 \\ 55 & 38 & 31 & 31 & 20 & 22 & 35 & 25 & 26 & 37 & 18 & 9 & 8 & 5 & 3 & 1 & 1\end{array}$

12

1963
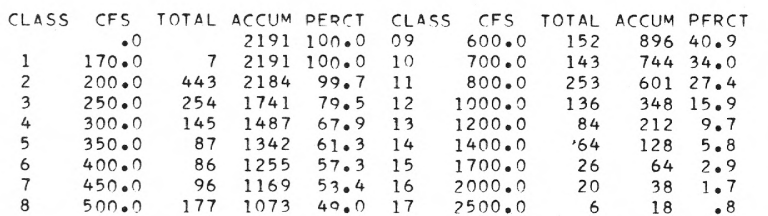

\begin{tabular}{lrrrr}
\multicolumn{6}{c}{ CLASS CFS } & TOTAL & ACCUM & PFRCT \\
18 & 3000 & 7 & 12 & .5 \\
19 & 3500 & & 5 & .2 \\
20 & 4000 & 1 & 5 & .2 \\
21 & 4500 & & 4 & .2 \\
22 & 5000 & 2 & 4 & .2 \\
23 & 6000 & & 2 & .1 \\
24 & 7000 & 2 & 2 & .1 \\
25 & 8000 & & & .0 \\
26 & & & & .0
\end{tabular}

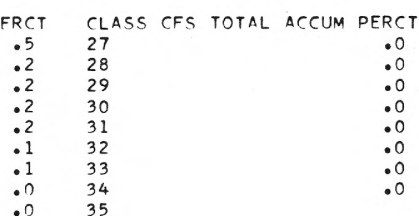

South Fork McKenzie River above Cougar

STATION NUMBER $\quad 14-1592.00$

Reservol near Ra1nbow Oreg

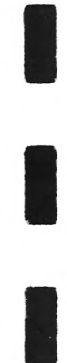

YEAR
1958
1959
1960
1961
1962

$\begin{array}{cc}1 & 3 \\ 212.0 & 213.0 \\ 210.0 & 211.0 \\ 203.0 & 204.0 \\ 196.0 & 196.0 \\ 220.0 & 223.0\end{array}$

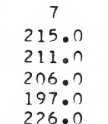

14
217.0
213.0
$209 \cdot 0$
$200 \cdot 0$
230.0

30
222.0
216.0
215.0

206.0
235.0

60
230.0
222.0
226.0
212.0
244.0

90
236.0
235.0
233.0

120
249.0
265.0

243.0

$\begin{array}{lll}150 & 183 & 274 \\ 295.0 & 395.0 & 525.0 \\ 281.0 & 302.0 & 357.0 \\ 266.0 & 398.0 & 567.0 \\ 254.0 & 317.0 & 499.0 \\ 330.0 & 420.0 & 537.0\end{array}$

South Fork McKenzie River abave Cougar

STATION NUMBER

$14-1592.00$

Reservo1r near Rainbow, Oreg, HIGHEST MEAN DISCHARG, IN CFS, FOR THE FOLLOWING NUMBFR OF CONSFCUTIVF DAYS IN YEAR ENDING SEPTFMBER 30

YEAR
1958
1959
1960
1961
1962
1963

5640.0

2410.0

2030.0

7160.0

3400.0

$3830^{3} .0$

7
2660.0

1920.0

3830.0
2090.0

$2090 . ?$

4960.0

1520.0

1140.0

1540.0

2250.0

1640.0

30
1540.0
983.0
1340.0
1640.0
1440.0
1220.0

60

1360.

873.0
1190.0

1320.0

1270.0

1270.0
919.0
90
1240.0
878.0
1180.0
1150.0
1130.0
865.0

120
1060.0
$822 \cdot 0$
$1090 \cdot 0$
$1090 \cdot 0$
971.0
897.0

150
1080.0
$814 \cdot 0$
1000.0
1040.0
980.0

$\begin{array}{rl}183 & 274 \\ 1020.0 & 811.0 \\ 800.0 & 671.0 \\ 876.0 & 708.0 \\ 1000.0 & 813.0 \\ 963.0 & 815.0 \\ 823.0 & 706.0\end{array}$

CFS-DAYS

243737.0

205225.0

242768.0

245428.0

216686.0 
CLASS $0 \begin{array}{lllllllllllllllllllllllllllllllllll} & 1 & 2 & 3 & 4 & 5 & 6 & 7 & 8 & 9 & 10 & 11 & 12 & 13 & 14 & 15 & 16 & 17 & 18 & 19 & 20 & 21 & 22 & 23 & 24 & 25 & 26 & 27 & 28 & 29 & 30 & 31 & 32 & 33 & 34\end{array}$

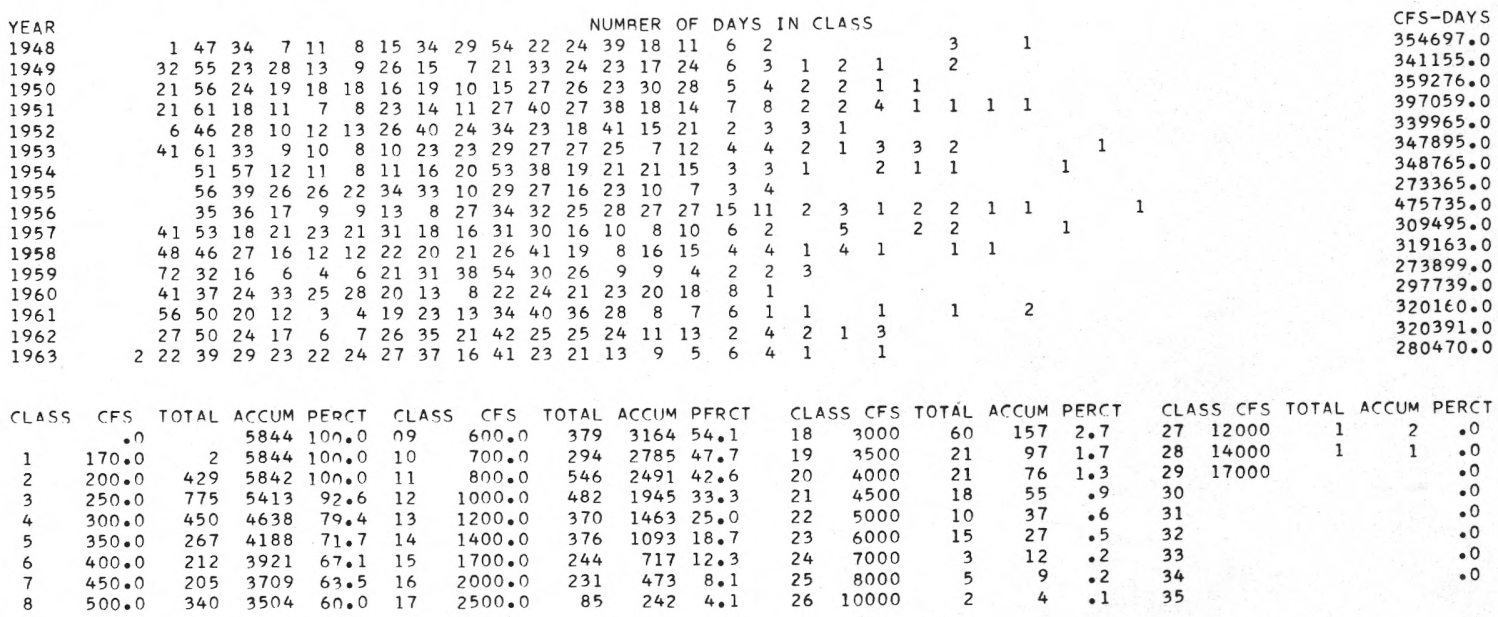

South Fork McKenzie River near Rainbow,

STATION NUMBER $14-1595.00$

Oreg.

\begin{tabular}{|c|c|c|c|c|c|c|c|c|c|c|c|}
\hline YEAR & 1 & 3 & 7 & 14 & 30 & 60 & 90 & 120 & 150 & 183 & 274 \\
\hline $\begin{array}{l}1948 \\
1949\end{array}$ & 256.0 & 257.0 & 261.0 & 264.0 & 273.0 & 287.0 & 311.0 & $327 \cdot 0$ & 384.0 & 528.0 & 701.0 \\
\hline 1949 & 214.0 & 215.0 & 217.0 & 222.0 & 234.0 & 246.0 & 265.0 & $268 \cdot 0$ & 285.0 & 362.0 & 558.0 \\
\hline $\begin{array}{l}1950 \\
1951\end{array}$ & 238.0 & 238.0 & 242.0 & 246.0 & 258.0 & 284.0 & 335.0 & $408 \cdot 0$ & 692.0 & $861 \cdot 0$ & 1150.0 \\
\hline 1951 & 222.0 & 222.0 & 223.0 & 227.0 & 238.0 & 253.0 & 265.0 & $296 \cdot 0$ & 373.0 & 483.0 & 679.0 \\
\hline 1952 & 237.0 & 238.0 & 240.0 & 241.0 & 241.0 & 247.0 & 258.0 & 267.0 & 281.0 & 332.0 & 646.0 \\
\hline 1953 & 264.0 & 265.0 & 267.0 & 268.0 & 277.0 & 291.0 & 297.0 & 303.0 & 358.0 & 535.0 & 934.0 \\
\hline 1954 & 277.0 & $278 . n$ & $282 . ?$ & 287.0 & 296.0 & 307.0 & 315.0 & $328 \cdot 0$ & 350.0 & 373.0 & 493.0 \\
\hline 1955 & 262.0 & 263.0 & 263.0 & 265.0 & 274.0 & 283.0 & 321.0 & 383.0 & 511.0 & $746 \cdot 0$ & 1190.0 \\
\hline 1956 & 251.0 & 263.0 & 266.0 & 269.0 & 274.0 & 285.0 & 302.0 & 349.0 & 431.0 & 507.0 & 723.0 \\
\hline 1957 & 211.0 & 211.0 & 211.0 & 212.0 & 219.0 & 238.0 & 250.0 & $258 \cdot 0$ & 283.0 & $322 \cdot 0$ & 610.0 \\
\hline 1958 & 227.0 & 227.0 & 229.0 & 233.0 & 237.0 & 244.0 & 256.0 & $272 \cdot 0$ & 331.0 & 454.0 & 686.0 \\
\hline 1959 & 215.0 & 218.0 & 221.0 & 224.0 & 226.0 & 233.0 & 254.0 & $308 \cdot 0$ & 348.0 & 388.0 & 468.0 \\
\hline 1960 & 200.0 & $200 \cdot 0$ & 200.0 & 205.0 & 221.0 & 243.0 & 251.0 & $266 \cdot 0$ & 306.0 & 502.0 & 740.0 \\
\hline 1961 & 213.0 & 217.0 & 227.0 & 229.0 & 234.0 & 243.0 & 248.0 & $261 \cdot 0$ & 318.0 & 393.0 & 664.0 \\
\hline 1962 & 242.0 & 243.0 & 246.0 & 250.0 & 256.0 & 271.0 & 283.0 & 350.0 & 392.0 & 496.0 & 666.0 \\
\hline
\end{tabular}

South Fork McKenzle River near Rainbow,

STATION NUMBER

$14-1595.00$

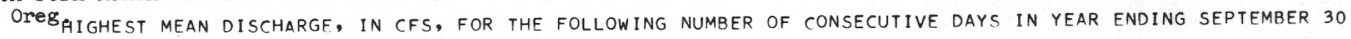

\begin{tabular}{|c|c|c|c|c|c|c|c|c|c|c|c|}
\hline YEAR & 1 & 3 & 7 & 15 & 30 & 60 & 90 & 120 & 150 & 183 & 274 \\
\hline 1948 & 8440.0 & 7110.0 & 4360.0 & 2870.0 & 1870.0 & 1530.0 & 1380.0 & $1340 \cdot 0$ & 1330.0 & 1290.0 & 1190.0 \\
\hline 1949 & 6960.0 & 5060.0 & 3370.0 & 2950.0 & 2500.0 & 1990.0 & 1850.0 & $1690 \cdot 0$ & 1450.0 & $1400 \cdot 0$ & 1160.0 \\
\hline 1950 & 5050.0 & 4390.0 & 3030.0 & 2570.0 & 2210.0 & 1890.0 & 1770.0 & $1830 \cdot 0$ & 1720.0 & 1570.0 & 1220.0 \\
\hline 1951 & 9160.0 & 5960.0 & 5110.0 & 3110.0 & 2630.0 & 2010.0 & 1950.0 & $1980 \cdot 0$ & 1750.0 & 1670.0 & 1360.0 \\
\hline 1952 & 4040.0 & 3570.0 & 2970.0 & 2090.0 & 1750.0 & 1670.0 & 1470.0 & 1420.0 & 1310.0 & 1270.0 & 1140.0 \\
\hline 1953 & 13600.0 & 8720.0 & 5870.0 & 4290.0 & 3660.0 & 2490.0 & 1950.0 & 1720.0 & 1660.0 & 1560.0 & 1180.0 \\
\hline 1954 & 10600.0 & 7350.0 & 4320.0 & 2950.0 & 2840.0 & $1990 . n$ & 1910.0 & $1700 \cdot 0$ & 1570.0 & 1450.0 & 1170.0 \\
\hline 1955 & 3280.0 & 3100.0 & 2790.0 & 2150.0 & 1920.0 & 1600.0 & 1480.0 & $1300 \cdot 0$ & 1180.0 & 1110.0 & 896.0 \\
\hline 1956 & 14300.0 & 10100.0 & 6190.0 & $4660 \cdot 0$ & 3790.0 & $3040 . n$ & 2500.0 & 2070.0 & 1930.0 & 1920.0 & 1630.0 \\
\hline 1957 & 11400.0 & 8030.0 & 5130.0 & 3410.0 & 2520.0 & 1930.0 & 1620.0 & $1490 \cdot 0$ & 1400.0 & $1300 \cdot 0$ & 1050.0 \\
\hline 1958 & 7520.0 & $5500 \cdot 0$ & 3870.0 & 2830.0 & 2200.0 & 1930.0 & 1770.0 & $1500 \cdot 0$ & 1480.0 & 1390.0 & 1080.0 \\
\hline 1959 & 3930.0 & 3360.0 & 2280.0 & 1750.0 & 1440.0 & 1210.0 & 1250.0 & $1150 \cdot 0$ & 1150.0 & 1110.0 & 914.0 \\
\hline 1960 & 3030.0 & 2690.0 & 2540.0 & 2220.0 & 1900.0 & 1710.0 & 1700.0 & $1560 \cdot 0$ & 1420.0 & $1240 \cdot 0$ & 998.0 \\
\hline 1961 & 8750.0 & 6390.0 & 4310.0 & 3020.0 & 2260.0 & 1830.0 & 1570.0 & $1470 \cdot 0$ & 1410.0 & 1350.0 & 1090.0 \\
\hline 1962 & 4990.0 & 4510.0 & 3270.0 & 2280.0 & 1970.0 & 1690.0 & 1450.0 & $1270 \cdot 0$ & 1320.0 & $1300 \cdot 0$ & 1080.0 \\
\hline 1963 & 4610.0 & $3880 . n$ & 3030.0 & 1930.0 & 1650.0 & 1240.0 & 1150.0 & 1190.0 & 1060.0 & 1100.0 & 926.0 \\
\hline
\end{tabular}


Blue Rlver above Quentin Creek, Oreg.

STATION NUMBER $14-1610.00$

DURATION TABLE OF DAILY DISCHARGE

CLASS $001120 \begin{array}{llllllllllllllllllllllllllllllllll} & 0 & 4 & 5 & 6 & 7 & 8 & 9 & 10 & 11 & 12 & 13 & 14 & 15 & 16 & 17 & 18 & 19 & 20 & 21 & 22 & 23 & 24 & 25 & 26 & 27 & 28 & 29 & 30 & 31 & 32 & 33 & 34\end{array}$

YEAR NUMBER OF DAYS IN CLASS

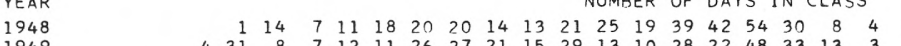

1949

$\begin{array}{lrrrrrrrrrrrrrrrrrrrrrr}4 & 31 & 8 & 7 & 12 & 11 & 20 & 20 & 14 & 13 & 21 & 25 & 19 & 39 & 42 & 54 & 30 & 8 & 4 & 1 & 3 & & 2\end{array}$

$\begin{array}{rrrrrrrrrrrrrrrrrrrrrrrrrrr}13 & 12 & 15 & 16 & 15 & 22 & 21 & 11 & 17 & 21 & 14 & 21 & 44 & 19 & 45 & 22 & 15 & 8 & 9 & 1 & 1 & 2 & 1 \\ 26 & 16 & 7 & 10 & 8 & 14 & 12 & 7 & 15 & 23 & 12 & 5 & 34 & 37 & 69 & 20 & 10 & 6 & 7 & 4 & 2 & 2 & 1\end{array}$

$\begin{array}{llllllllllllllllllll}1951 & 14 & 4 & 26 & 16 & 7 & 10 & 8 & 14 & 12 & 7 & 15 & 23 & 12 & 5 & 34 & 37 & 69 & 20 & 10 \\ 1952 & & & 14 & 11 & 12 & 8 & 10 & 18 & 9 & 18 & 9 & 35 & 44 & 18 & 36 & 27 & 48 & 30 & 11\end{array}$

1952

1953

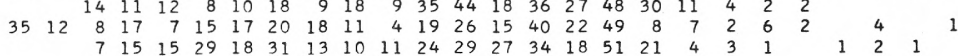

$\begin{array}{lllllllllllllllllllll}11 & 12 & 17 & 9 & 14 & 27 & 12 & 24 & 28 & 46 & 23 & 19 & 36 & 16 & 43 & 19 & 6 & 3\end{array}$

$\begin{array}{lll}1 & 4 & \\ & 2 & 1\end{array}$

CFS-DAYS

28706.3

25299.9

30037.2

31805.4

26812.4

25017.6

24208.1
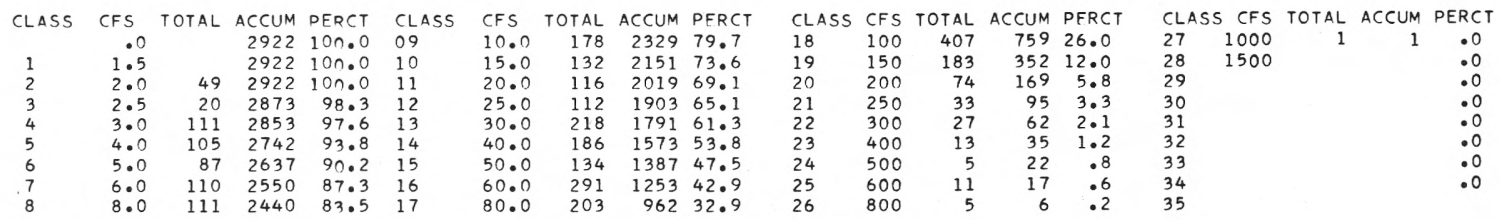

Blue R1ver above Quentin Creek, Oreg.

STATION NUMBER

$14-1610.00$

LOWEST MEAN DISCHARGE, IN CFS, FOR THE FOLLOWING NUMRER OF CONSECUTIVE DAYS IN YEAR BEGINNING APRIL 1

YEAR
1948
1949
1950
1951
1952
1953
1954
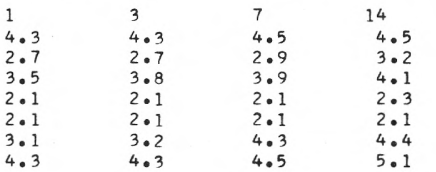

$\begin{array}{rr}30 & 60 \\ 5.7 & 7 . \\ 3.7 & 4 . \\ 5.0 & 7 . \\ 2.8 & 3.4 \\ 2.2 & 2 . \\ 5.5 & 6.9 \\ 6.3 & 7 .\end{array}$

60
7.9
4.2
7.5
3.4
2.8
6.9
7.9

90
11.1
6.1
13.1
5.0
3.5
7.8
8.6
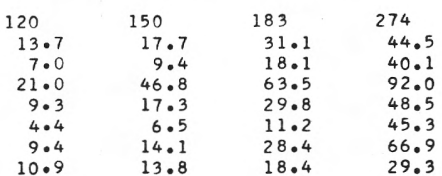

Blue River above Quentin Creek, Oreg.

STATION NUMBER $\quad 14-1610.00$

HIGHEST MEAN DISCHARGE, IN CFS, FOR THE FOLLOWING NUMBER OF CONSECUTIVE DAYS IN YEAR ENDING SEPTEMBER 30

\begin{tabular}{|c|c|c|c|c|c|c|c|c|c|c|c|}
\hline YEAR & 1 & 3 & 7 & 15 & 30 & 60 & 90 & 120 & 150 & 183 & 274 \\
\hline 1948 & 962.0 & 742.0 & 466.0 & 287.0 & 193.0 & 146.0 & 144.0 & $120 \cdot 0$ & 114.0 & 105.0 & 101.0 \\
\hline 1949 & 691.0 & 516.0 & 326.0 & 257.0 & 220.0 & 170.0 & 159.0 & $140 \cdot 0$ & 118.0 & 114.0 & 90.1 \\
\hline 1950 & 884.0 & 683.0 & 399.0 & 299.0 & 231.0 & 190.0 & 160.0 & 162.0 & 156.0 & 139.0 & 107.0 \\
\hline 1951 & 832.0 & 607.0 & 509.0 & 302.0 & 215.0 & 196.0 & 173.0 & 174.0 & 149.0 & 144.0 & 114.0 \\
\hline 1952 & 452.0 & 341.0 & 260.0 & 177.0 & 159.0 & 154.0 & 131.0 & 122.0 & 110.0 & 106.0 & 93.9 \\
\hline 1953 & 1250.0 & 887.0 & 594.0 & 445.0 & 335.0 & 212.0 & 163.0 & $145 \cdot 0$ & 137.0 & 125.0 & 90.0 \\
\hline 1954 & 965.0 & 654.0 & 366.0 & 248.0 & 247.0 & 163.0 & 154.0 & 139.0 & 127.0 & 114.0 & 85.6 \\
\hline 1955 & 258.0 & 255.0 & 231.0 & 187.0 & 170.0 & 140.0 & 127.0 & $108 \cdot 0$ & 97.7 & 89.7 & 71.4 \\
\hline
\end{tabular}


CLASS $00 \begin{array}{lllllllllllllllllllllllllllllllllll} & 2 & 3 & 4 & 5 & 6 & 7 & 8 & 9 & 10 & 11 & 12 & 13 & 14 & 15 & 16 & 17 & 18 & 19 & 20 & 21 & 22 & 23 & 24 & 25 & 26 & 27 & 28 & 29 & 30 & 31 & 32 & 33 & 34\end{array}$

\begin{tabular}{|c|c|c|c|c|c|c|c|c|c|c|c|c|}
\hline YEAR & & & & & & & & & & IUM & ABER & OF DAYS \\
\hline 1950 & & & $27 \quad 38$ & 2414 & 16 & 8 & 9 & 251 & $14 \quad 42$ & 40 & 30 & $28 \quad 24$ \\
\hline 1951 & & 16 & 3523 & 1411 & 16 & 11 & 8 & 231 & $15 \quad 59$ & 43 & 31 & 1216 \\
\hline 1952 & & & 1143 & 227 & 9 & 11 & 22 & 323 & 3560 & 35 & 202 & $21 \quad 13$ \\
\hline 1953 & & $15 \quad 37$ & 2538 & 1610 & 6 & 12 & 12 & 131 & 1361 & 36 & 26 & 811 \\
\hline 195 & & & 2564 & 2313 & 9 & 10 & 14 & 362 & $22 \quad 39$ & 29 & 21 & 1627 \\
\hline 1955 & & & 551 & 3013 & 19 & 19 & 17 & 423 & 3138 & 39 & 23 & $16 \quad 14$ \\
\hline CLASS & CFS & TOTAL & ACCUM & PERCT & & LASS & & FS & TOTAL & & CCUM & 1 PERCT \\
\hline & 0 & & 2191 & 100.0 & & & & 0.0 & 7 & & 1466 & 66. \\
\hline 1 & 5.0 & & 2191 & 100.0 & 1 & 0 & & 50 & 8 & & 1395 & 63. \\
\hline 2 & 6 & 15 & 2191 & 100 & & & & & 17 & & 1313 & 59. \\
\hline 3 & 8. & 53 & 2176 & 90 & 1 & 2 & & 30 & 13 & & 1142 & 52. \\
\hline 4 & 10 & 128 & 2123 & & & & & 0 & 295 & & 1012 & 46 \\
\hline 5 & 15 & 2 & 1995 & 91 & $i$ & 4 & & 0.0 & 222 & & 713 & 32.5 \\
\hline 6 & & 129 & 17 & & & 5 & & & 15 & & 491 & 22 \\
\hline 7 & & 68 & 16 & 73 & 1 & 6 & & 0.0 & 101 & & 340 & 1 \\
\hline 8 & 30. & 75 & 1541 & $7 n$. & & 7 & & $u_{\bullet}$ & 10 & & 239 & 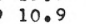 \\
\hline
\end{tabular}

Lookout Creek near Blue R1ver, Oreg.

STATION NUMBER $14-1615.00$

LOWEST MEAN DISCHARGF, IN CFS, FOR THE FOLLOWING NUMBER OF CONSECUTIVE DAYS IN YEAR REGINNING APRIL 1

\begin{tabular}{|c|c|c|c|c|c|c|c|c|c|c|c|}
\hline YEAR & 1 & 3 & 7 & 14 & 30 & 60 & 90 & 120 & 150 & 183 & 274 \\
\hline 1950 & 12.0 & $12 \cdot 0$ & 12.1 & 12.4 & 13.7 & 16.6 & 22.7 & 37.4 & 73.4 & $\begin{array}{r}105.0 \\
53.7\end{array}$ & $\begin{array}{r}169.0 \\
95.3\end{array}$ \\
\hline $\begin{array}{l}1951 \\
1952\end{array}$ & $\begin{array}{l}8.7 \\
6.4\end{array}$ & $\begin{array}{l}8.7 \\
6.4\end{array}$ & $\begin{array}{l}9.0 \\
6.5\end{array}$ & $\begin{array}{l}9.2 \\
7.4\end{array}$ & $\begin{array}{r}10.1 \\
8.4\end{array}$ & $\begin{array}{r}11.8 \\
9.4\end{array}$ & $\begin{array}{l}14.6 \\
11.4\end{array}$ & $\begin{array}{l}20 \cdot 3 \\
13 \cdot 0\end{array}$ & $\begin{array}{l}31.7 \\
15.6\end{array}$ & $\begin{array}{l}53.7 \\
23.5\end{array}$ & $\begin{array}{l}95.3 \\
70.4\end{array}$ \\
\hline 1953 & 12.0 & 12.0 & 12.1 & 12.6 & 13.8 & 15.9 & 16.7 & 18.5 & 24.4 & 46.6 & 123.0 \\
\hline 1954 & 13.0 & 13.0 & 13.4 & 13.9 & 14.9 & 16.5 & 17.7 & 22.1 & 26.4 & 33.5 & 58.3 \\
\hline
\end{tabular}

Lookout Creek near Blue River, Oreg.

STATION NUMBER $14-1615.00$ HIGHEST MEAN DISCHARGE, IN CFS, FOR THE FOLLOWING NUMBER OF CONSECUTIVE DAYS IN YEAR ENDING SEPTEMBER 30

\begin{tabular}{|c|c|c|c|c|c|c|c|c|c|c|c|}
\hline YEAR & 1 & 3 & 7 & 15 & 30 & 60 & 90 & 120 & 150 & 183 & 274 \\
\hline $\begin{array}{l}1950 \\
1951\end{array}$ & $\begin{array}{l}1500.0 \\
1530.0\end{array}$ & $\begin{array}{l}1170.0 \\
1060.0\end{array}$ & $\begin{array}{l}720.0 \\
842.0\end{array}$ & $\begin{array}{l}560.0 \\
492.0\end{array}$ & $\begin{array}{l}461.0 \\
460.0\end{array}$ & $\begin{array}{l}368.0 \\
335.0\end{array}$ & $\begin{array}{l}327.0 \\
336.0\end{array}$ & $\begin{array}{l}312.0 \\
331.0\end{array}$ & $\begin{array}{l}297.0 \\
287.0\end{array}$ & $\begin{array}{l}264.0 \\
269.0\end{array}$ & $\begin{array}{l}205.0 \\
209.0\end{array}$ \\
\hline $\begin{array}{l}1952 \\
1953\end{array}$ & $\begin{array}{r}862.0 \\
2830.0\end{array}$ & $\begin{array}{r}753.0 \\
2020.0\end{array}$ & $\begin{array}{r}630.0 \\
1330.0\end{array}$ & $\begin{array}{l}410.0 \\
932.0\end{array}$ & $\begin{array}{l}317.0 \\
737.0\end{array}$ & $\begin{array}{l}253.0 \\
477.0\end{array}$ & $\begin{array}{l}238.0 \\
371.0\end{array}$ & $\begin{array}{l}219.0 \\
319.0\end{array}$ & $\begin{array}{l}210.0 \\
292.0\end{array}$ & $\begin{array}{l}204.0 \\
259.0\end{array}$ & $\begin{array}{l}175.0 \\
187.0\end{array}$ \\
\hline 1954 & 1840.0 & 1280.0 & 708.0 & 513.0 & 494.0 & 338.0 & 328.0 & 294.0 & 266.0 & 234.0 & 173.0 \\
\hline 1955 & 897.0 & 707.0 & 446.0 & 281.0 & 264.0 & 236.0 & 226.0 & $194 \cdot 0$ & 184.0 & 180.0 & 139.0 \\
\hline
\end{tabular}

Blue River near Blue River, Oreg.

$$
\text { STATION NUMBER, } \quad 14-1620.00
$$

DURATION TABLE OF DAILY DISCHARGE

CLASS $00 \begin{array}{lllllllllllllllllllllllllllllllllllll}1 & 2 & 3 & 4 & 5 & 6 & 7 & 8 & 9 & 10 & 11 & 12 & 13 & 14 & 15 & 16 & 17 & 18 & 19 & 20 & 21 & 22 & 23 & 24 & 25 & 26 & 27 & 28 & 29 & 30 & 31 & 32 & 33 & 34\end{array}$

YEAR NUMBER OF DAYS IN CLACS

$\begin{array}{llllllllllllllllllllll}1936 & 5 & 12 & 34 & 30 & 24 & 7 & 11 & 18 & 21 & 20 & 28 & 25 & 35 & 24 & 20 & 23 & 11 & 8 & 3 & 1\end{array}$

$\begin{array}{llllllllllllllllllllllllllll}1937 & 6 & 47 & 12 & 15 & 26 & 18 & 16 & 29 & 14 & 20 & 10 & 4 & 12 & 30 & 14 & 13 & 34 & 19 & 19 & 3 & 1 & & 2 & 1 \\ 1938 & 1 & 15 & 18 & 24 & 21 & 17 & 7 & 19 & 9 & 15 & 8 & 10 & 12 & 30 & 29 & 28 & 46 & 21 & 18 & 8 & 3 & 5 & \end{array}$

$\begin{array}{rrrrrrrrrrrrrrrrrrrrrrr}1938 & 1 & 15 & 18 & 24 & 21 & 17 & 7 & 19 & 9 & 15 & 8 & 10 & 12 & 30 & 29 & 28 & 46 & 21 & 18 & 8 & 3 \\ 1939 & 2 & 23 & 34 & 18 & 17 & 9 & 5 & 7 & 7 & 37 & 30 & 30 & 30 & 34 & 19 & 12 & 25 & 9 & 6 & 6 & 5\end{array}$

1940

1941

1942

1944

1945

1947

1948

1949

1951

1952

1953

1955

1956

1958

1959

1961

1962

$\begin{array}{rrrrrrrrrrrrrrrrrrrr}32 & 57 & 35 & 33 & 12 & 4 & 11 & 8 & 18 & 19 & 16 & 15 & 32 & 16 & 11 & 17 & 6 & 13 & 10 & 10\end{array}$ $\begin{array}{lllllllllllllllllllll}19 & 15 & 18 & 22 & 9 & 16 & 14 & 68 & 49 & 32 & 23 & 34 & 14 & 11 & 8 & 3 & 7 & 2 & 1\end{array}$

$\begin{array}{lllllllllllllllllllllll}4 & 12 & 26 & 19 & 8 & 5 & 18 & 15 & 23 & 39 & 66 & 29 & 30 & 14 & 10 & 14 & 10 & 10 & 6 & 4 & 1 & 2 \\ 8 & 12 & 19 & 20 & 20 & 11 & 11 & 9 & 9 & 15 & 26 & 17 & 31 & 24 & 23 & 26 & 30 & 27 & 7 & 8 & 4 & 3\end{array}$

$\begin{array}{rrrrrrrrrrrrrrrrrrrr}4 & 28 & 17 & 22 & 33 & 13 & 19 & 9 & 32 & 44 & 27 & 27 & 40 & 22 & 10 & 7 & 4 & 5 & 2\end{array}$

$\begin{array}{rrrrrrrrrrrrrrrrrrr}1 & 35 & 34 & 22 & 17 & 10 & 21 & 19 & 22 & 18 & 23 & 8 & 19 & 17 & 24 & 31 & 16 & 20 & 4 \\ 18 & 15 & 23 & 28 & 10 & 8 & 15 & 8 & 15 & 10 & 10 & 21 & 45 & 34 & 26 & 37 & 11 & 14 & 7\end{array}$

$\begin{array}{lllllllllllllllll}21 & 40 & 14 & 10 & 20 & 16 & 36 & 29 & 19 & 24 & 28 & 14 & 23 & 39 & 6 & 10\end{array}$

$\begin{array}{rlllllllllllllllll}15 & 11 & 18 & 13 & 9 & 17 & 5 & 23 & 19 & 17 & 23 & 44 & 36 & 25 & 29 & 28 & 17 \\ 22 & 16 & 20 & 27 & 12 & 11 & 22 & 35 & 17 & 15 & 20 & 17 & 16 & 21 & 35 & 24 & 21\end{array}$

$\begin{array}{lllllllllllllllllllll}7 & 12 & 30 & 21 & 14 & 20 & 11 & 17 & 19 & 19 & 14 & 31 & 17 & 31 & 37 & 27 & 22 & 6\end{array}$

$\begin{array}{rrrrrrrrrrrrrrrrrrrr}9 & 7 & 33 & 18 & 14 & 8 & 14 & 10 & 14 & 17 & 16 & 10 & 33 & 36 & 32 & 34 & 15 & 20 & 10 \\ 11 & 7 & 11 & 32 & 8 & 5 & 10 & 4 & 20 & 23 & 24 & 32 & 44 & 33 & 27 & 29 & 17 & 15 & 7\end{array}$

$\begin{array}{rrrrrrrrrrrrrrrrr}36 & 10 & 15 & 17 & 13 & 26 & 11 & 11 & 11 & 40 & 31 & 20 & 30 & 10 & 8 \\ 10 & 32 & 34 & 28 & 19 & 17 & 14 & 33 & 24 & 31 & 16 & 18 & 23 & 17 & 35 \\ 30 & 22 & 7 & 25 & 17 & 22 & 27 & 37 & 28 & 42 & 28 & 19 & 21 & 19 & 17\end{array}$

$\begin{array}{lllllllllllllllllll}30 & 22 & 7 & 25 & 17 & 22 & 27 & 37 & 28 & 42 & 28 & 19 & 21 & 19 & 17 & 2\end{array}$

$\begin{array}{rrrrrrrrrrrrrrrrr}26 & 26 & 7 & 16 & 8 & 11 & 17 & 6 & 28 & 22 & 28 & 21 & 25 & 31 & 34 & 35 & 11\end{array}$

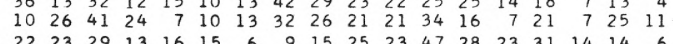

$\begin{array}{rrrrrrrrrrrrrrrrrrrrr}1 & 22 & 23 & 29 & 13 & 16 & 15 & 6 & 9 & 15 & 25 & 23 & 47 & 28 & 23 & 31 & 14 & 14 & 6 \\ 2 & 13 & 12 & 33 & 13 & 8 & 23 & 14 & 41 & 36 & 19 & 12 & 13 & 17 & 18 & 38 & 17 & 23 & 10\end{array}$

$\begin{array}{rrrrrrrrrrrrrrrrrrrrr}2 & 13 & 12 & 33 & 13 & 8 & 23 & 14 & 41 & 36 & 19 & 12 & 13 & 17 & 18 & 38 & 17 & 23 & 10 & 4 \\ 27 & 32 & 11 & 23 & 9 & 10 & 15 & 9 & 9 & 14 & 15 & 16 & 32 & 26 & 31 & 34 & 13 & 20 & 11 & 3\end{array}$

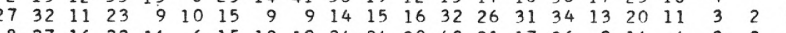

$\begin{array}{rrrrrrrrrrrrrrrrrrr}23 & 14 & 6 & 15 & 10 & 19 & 24 & 24 & 30 & 40 & 21 & 17 & 36 & 9 & 14 & 4 & 3 & 2 & 3 \\ 19 & 15 & 26 & 22 & 25 & 52 & 28 & 20 & 17 & 38 & 33 & 17 & 16 & 13 & 10 & 8 & 3 & 2 & \end{array}$

Blue River near Blue River, Oreg.

STATION NUMBER 14-1620.00

Summary for water years 1936-63

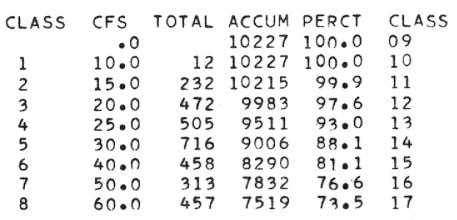

CFS TOTAL ACCUM PERCT $80.0 \quad 348 \quad 706269.1$ $100.0 \quad 711 \quad 671465.6$ 150.0 $200.0 \quad 638 \quad 538752.7$ $250.0 \quad 568 \quad 474946.4$ $300.0 \quad 927 \quad 418140.9$ $400.0 \quad 641 \quad 3254 \quad 31.8$ $\begin{array}{llll}500.0 & 556 & 2613 & 25.6\end{array}$

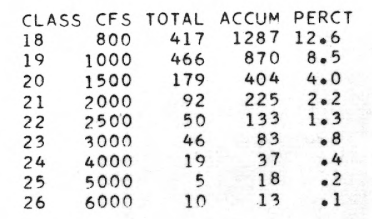

$\begin{array}{lrrr}\text { CLASS CFS TOTAL ACCUM PERCT } \\ 27 \\ 28 & 8000 & 3 & : 0 \\ 29 & & : 0 \\ 30 & & : 0 \\ 31 & & : 0 \\ 32 & & : 0 \\ 33 & & : 0 \\ 34 & & : 0\end{array}$ 
Blue River near Blue River, Oreg.

STATION NUMBFR $14-1620.00$

LOWEST MEAN DISCHARGF, IN CFS, FOR THF FOLLOWING NUMBER OF CONSFCUTIVF DAYS IN YFAR BEGINNING APRIL 1

\begin{tabular}{|c|c|c|c|c|c|c|c|c|c|c|c|}
\hline YEAR & 1 & 3 & 7 & 14 & 30 & 60 & 90 & 120 & 150 & 183 & 274 \\
\hline $\begin{array}{l}1936 \\
1937\end{array}$ & $\begin{array}{l}14.0 \\
26.0\end{array}$ & $\begin{array}{l}14.0 \\
26.0\end{array}$ & $\begin{array}{l}14.3 \\
26.9\end{array}$ & $\begin{array}{l}15.4 \\
28.6\end{array}$ & $\begin{array}{l}17 \cdot 3 \\
31 \cdot 2\end{array}$ & $\begin{array}{l}18.2 \\
36.2\end{array}$ & $\begin{array}{l}22.0 \\
43.4\end{array}$ & $\begin{array}{l}23 \cdot 9 \\
60.9\end{array}$ & $\begin{array}{r}30.4 \\
140.0\end{array}$ & $\begin{array}{r}48.9 \\
214.0\end{array}$ & $\begin{array}{r}98.1 \\
409.0\end{array}$ \\
\hline $\begin{array}{l}1938 \\
1939\end{array}$ & $\begin{array}{l}14.0 \\
16.0\end{array}$ & $\begin{array}{l}14.7 \\
16.0\end{array}$ & $\begin{array}{l}15.7 \\
16.7\end{array}$ & $\begin{array}{l}17.3 \\
16.9\end{array}$ & $\begin{array}{l}18.9 \\
18.7\end{array}$ & $\begin{array}{l}22.1 \\
21.9\end{array}$ & $\begin{array}{l}23.7 \\
25.0\end{array}$ & $\begin{array}{l}29 \cdot 7 \\
31 \cdot 2\end{array}$ & $\begin{array}{l}47.2 \\
33.3\end{array}$ & $\begin{array}{l}92.7 \\
52.1\end{array}$ & $\begin{array}{l}208.0 \\
147.0\end{array}$ \\
\hline 1940 & $\begin{array}{l}16.0 \\
15.0\end{array}$ & $\begin{array}{l}16 \cdot 0 \\
15 . n\end{array}$ & 16.1 & 16.6 & 17.5 & 20.0 & 22.1 & $24 \cdot 3$ & 31.0 & 53.9 & 158.0 \\
\hline 1941 & 22.0 & 22.0 & 23.4 & 25.6 & 30.4 & 42.2 & 56.6 & $70 \cdot 5$ & 82.9 & 105.0 & 262.0 \\
\hline 1942 & 17.0 & 17.0 & 17.6 & $18 \cdot 1$ & 20.4 & 23.8 & 26.8 & $38 \cdot 6$ & 64.8 & 119.0 & 420.0 \\
\hline 1943 & 24.0 & $24 . n$ & 24.0 & $25 \cdot 3$ & 26.4 & 32.6 & 38.8 & $67 \cdot 5$ & 121.0 & $158 \cdot 0$ & 201.0 \\
\hline 1944 & 18.0 & $18 . ?$ & $19 . ?$ & 21.8 & 23.3 & 30.5 & 32.3 & $35 \cdot 2$ & $46 \cdot 1$ & 62.7 & 129.0 \\
\hline 1945 & $17 \cdot 0$ & 17.7 & 17.9 & $17 \cdot 9$ & 20.1 & 23.1 & 24.1 & $30 \cdot 0$ & 43.4 & 103.0 & 384.0 \\
\hline 1946 & 23.0 & $23 . n$ & 23.9 & 26.4 & 29.3 & 30.9 & 33.8 & $50 \cdot 1$ & 91.9 & 134.0 & 370.0 \\
\hline 1947 & 29.0 & 29.0 & 29.3 & 30.4 & 34.6 & 37.6 & $46 \cdot 6$ & $72 \cdot 8$ & 93.3 & 136.0 & 311.0 \\
\hline 1948 & 21.0 & 21.0 & $21 \cdot 3$ & $22 \cdot 5$ & 26.6 & 34.4 & 50.4 & $66 \cdot 1$ & $90 \cdot 3$ & 153.0 & 267.0 \\
\hline 1949 & 20.0 & 20.0 & 20.5 & 21.6 & 24.7 & 27.9 & 35.2 & $46 \cdot 5$ & $57 \cdot 2$ & 87.9 & 213.0 \\
\hline 1950 & 23.0 & 23.7 & 25.0 & $26 \cdot 1$ & 28.8 & 38.7 & 55.6 & 107.0 & 202.0 & $302 \cdot 0$ & 513.0 \\
\hline 1951 & 19.0 & 19.0 & $19 \cdot 3$ & $20 \cdot 0$ & 24.1 & 26.3 & 32.3 & $48 \cdot 4$ & 92.8 & $160 \cdot 0$ & 298.0 \\
\hline 1952 & 14.0 & $14 . n$ & 15.1 & 16.0 & 16.8 & 19.4 & 24.0 & $27 \cdot 4$ & $34 \cdot 7$ & $58 \cdot 5$ & 184.0 \\
\hline 1953 & $28 \cdot n$ & $28 . n$ & 28.6 & 29.6 & 34.7 & 44.1 & 47.6 & $55 \cdot 6$ & 75.0 & 146.0 & 410.0 \\
\hline 1954 & 41.0 & 41.7 & 42.9 & 45.6 & 51.1 & 53.9 & 61.5 & $75 \cdot 7$ & 91.0 & 119.0 & 198.0 \\
\hline 1955 & 30.0 & $3 n \cdot n$ & 30.9 & 33.1 & 36.8 & 44.3 & 63.8 & $122 \cdot 0$ & 190.0 & 284.0 & 552.0 \\
\hline 1956 & 22.0 & 23.3 & 24.9 & 25.6 & 26.6 & 28.7 & 34.7 & $49 \cdot 0$ & 104.0 & 139.0 & 251.0 \\
\hline 1957 & 21.0 & 21.0 & 21.3 & 21.6 & $22 \cdot 9$ & $29 \cdot 3$ & 35.1 & $42 \cdot 2$ & & $76 \cdot ?$ & 272.0 \\
\hline 1958 & 19.0 & 20.7 & $21 \cdot 1$ & 23.6 & 26.1 & 28.1 & 30.3 & $37 \cdot 0$ & 57.9 & 81.5 & 274.0 \\
\hline 1959 & 20.0 & 20.3 & $21 \cdot 3$ & 23.9 & 26.0 & 34.3 & 43.7 & $72 \cdot 3$ & 110.0 & $130 \cdot 0$ & 184.0 \\
\hline 1960 & 19.0 & $19 \cdot 0$ & 19.0 & 19.8 & 23.8 & 31.6 & 34.5 & 39.7 & 59.3 & 148.0 & 318.0 \\
\hline 1961 & 16.0 & 16.0 & $16 \cdot 6$ & $17 \cdot 1$ & 18.9 & 25.0 & 26.2 & 38.4 & 62.5 & 97.8 & 293.0 \\
\hline 1962 & 18.0 & 18.0 & $18 \cdot 3$ & 20.5 & 24.5 & 30.7 & 35.6 & $51 \cdot 6$ & 86.2 & 122.0 & 239.0 \\
\hline
\end{tabular}

Blue River near Blue River, Oreg.

STATION NUMBER

$14-1620.00$

HIGHEST MEAN DISCHORGF, IN CFS, FOR THF FOLLOWING NUMRFP OF CONSFCUTIVF DAYS IN YEAR FNDING SEPTEMRER 30

\begin{tabular}{|c|c|c|c|c|c|c|c|c|c|c|c|}
\hline YEAR & 1 & 3 & 7 & 15 & 30 & 60 & 90 & 120 & 150 & 183 & 274 \\
\hline 1936 & 4620.0 & 3350.0 & 2230.0 & 2140.0 & 1380.0 & 901.0 & 780.0 & $7 ? 2 \cdot 0$ & 661.0 & 583.0 & $430 \cdot 0$ \\
\hline 1937 & 4320.0 & 3670.0 & 2260.0 & 1480.0 & 1150.0 & $968 \cdot 0$ & 898.0 & $811 \cdot 0$ & 711.0 & $599 \cdot 0$ & 434.0 \\
\hline 1938 & 4170.0 & 2880.0 & 1720.0 & 1340.0 & 1020.0 & 890.0 & $861 \cdot 0$ & $783 \cdot 0$ & 771.0 & 783.0 & 586.0 \\
\hline 1939 & 2460.0 & 2210.0 & 1780.0 & 1320.0 & 994.0 & 736.0 & 620.0 & $567 \cdot 0$ & 564.0 & 519.0 & 392.0 \\
\hline 1940 & 2080.0 & 1960.0 & 1710.0 & 1240.0 & 1110.0 & 850.0 & 684.0 & $645 \cdot 0$ & $568 \cdot 0$ & 488.0 & 339.0 \\
\hline 1941 & 2040.0 & 1670.0 & 1150.0 & 970.0 & 675.0 & 566.0 & 503.0 & $437 \cdot 0$ & $38 ? \cdot 0$ & 350.0 & 282.0 \\
\hline 1942 & 3560.0 & 2750.0 & 1810.0 & $1150 \cdot 0$ & 1000.0 & 846.0 & 773.0 & $659 \cdot 0$ & 579.0 & 520.0 & 418.0 \\
\hline 1943 & 6160.0 & $4470 . ?$ & 3510.0 & 2480.0 & 1760.0 & 1650.0 & 1330.0 & $1160 \cdot 0$ & 1070.0 & 982.0 & 733.0 \\
\hline 1944 & 2600.0 & 1650.0 & 947.0 & 711.0 & 470.0 & 412.0 & 384.0 & $356 \cdot 0$ & 337.0 & 336.0 & 281.0 \\
\hline 1945 & 3580.0 & 2600.0 & 2130.0 & 1590.0 & 1000.0 & 815.0 & 761.0 & 749.0 & 712.0 & 609.0 & 446.0 \\
\hline 1946 & 10900.0 & 611n.n & $348 \cap \cdot n$ & 2350.0 & 1630.0 & 1220.0 & 990.0 & $922 \cdot n$ & $84 n \cdot ?$ & 783.0 & 582.0 \\
\hline 1947 & 6170.0 & $5770 . n$ & 3580.7 & 1990.0 & 1830.0 & 1090.0 & 964.0 & 852.0 & 793.0 & $713 . n$ & 533.0 \\
\hline 1948 & 6360.0 & 4990.0 & $3190 . ?$ & 1930.0 & 1240.0 & 918.0 & 878.0 & $748 \cdot 0$ & 745.0 & 695.0 & $625 . n$ \\
\hline 1949 & 4350.0 & $2990 \cdot 0$ & 2120.0 & 1470.0 & 1100.0 & 910.0 & 948.0 & $798 \cdot 0$ & 707.0 & 719.0 & 543.0 \\
\hline 1950 & 4080.0 & 3440.0 & 2090.0 & 1670.0 & 1400.0 & 1120.0 & 1000.0 & $944 \cdot 0$ & 886.0 & 782.0 & 602.0 \\
\hline 1951 & 4690.0 & 3430.0 & 2530.0 & 1530.0 & 1420.0 & 1050.0 & 1050.0 & 1030.0 & 893.0 & 840.0 & 648.0 \\
\hline 1952 & 3460.0 & 2530.0 & 2120.0 & 1340.0 & 877.0 & 716.0 & 705.0 & $652 \cdot 0$ & 635.0 & 622.0 & 538.0 \\
\hline 1953 & 8690.0 & 6070.0 & $4080 . ?$ & 2980.0 & 2320.0 & 1450.0 & 1130.0 & $988 \cdot 0$ & 897.0 & 794.0 & 565.0 \\
\hline 1954 & 7380.0 & 4930.0 & 2650.0 & 1790.0 & 1770.0 & 1170.0 & 1130.0 & 1020.0 & 925.0 & $808 \cdot 0$ & 591.0 \\
\hline 1955 & 3130.0 & 2250.0 & 1400.0 & 897.0 & 795.0 & 729.0 & 698.0 & $592 \cdot 0$ & 568.0 & 557.0 & 443.0 \\
\hline 1956 & 8090.0 & $5860 . ?$ & 3340.0 & 2500.0 & 1960.0 & 1550.0 & 1300.0 & $1060 \cdot 0$ & 976.0 & 962.0 & 772.0 \\
\hline 1957 & 6100.0 & 4080.0 & 2700.0 & 2200.0 & 1520.0 & 1100.0 & $836 \cdot 0$ & 790.0 & 704.0 & 637.0 & 480.0 \\
\hline 1958 & 7470.0 & 4470.0 & 2740.0 & 2000.0 & 1500.0 & 1340.0 & 1170.0 & 949.0 & 886.0 & 778.0 & 558.0 \\
\hline 1959 & 3450.0 & 2630.0 & 1610.0 & $1200 \cdot 0$ & 940.0 & 755.0 & 779.0 & 707.0 & 692.0 & 639.0 & 478.0 \\
\hline 1960 & 2470.0 & 2200.0 & 1680.0 & 1330.0 & 1120.0 & 939.0 & 882.0 & $829 \cdot 0$ & 729.0 & 627.0 & 499.0 \\
\hline 1961 & 6500.0 & 4440.0 & 2880.0 & 1960.0 & 1430.0 & 1140.0 & 938.0 & $890 \cdot 0$ & 853.0 & 788.0 & 579.0 \\
\hline 1962 & 3540.0 & 3130.0 & 2070.0 & 1370.0 & 1060.0 & 860.0 & 696.0 & 676.0 & 683.0 & 649.0 & 490.0 \\
\hline 1963 & 4280.0 & 3110.0 & 2130.0 & 1220.0 & 889.0 & 652.0 & 613.0 & $608 \cdot 0$ & 561.0 & 574.0 & 451.0 \\
\hline
\end{tabular}




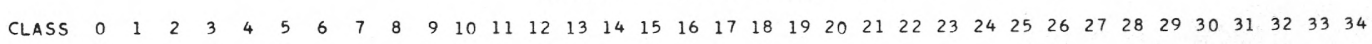

\begin{tabular}{|c|c|c|c|c|c|c|c|c|c|c|c|c|c|c|c|c|c|c|c|c|c|c|c|c|c|c|c|c|c|c|c|c|}
\hline YEAR & & & & & & & & & & & & & & IUMBE & & OF & DAYS & 5 in & $\mathrm{N} C L$ & ASS & & & & & & & & & & & & CFS-DAYS \\
\hline 1925 & & & 26 & 21 & 36 & 12 & 14 & 16 & 19 & 11 & 17 & 55 & 32 & 371 & 18 & 11 & 9 & 5 & 7 & 3 & 2 & 4 & 4 & 1 & 4 & & 1 & & & & & 1675160.0 \\
\hline 1926 & & & 80 & 59 & 13 & 16 & 17 & 41 & 27 & 392 & 26 & 8 & 6 & 12 & 4 & 4 & 2 & 1 & 5 & 1 & 1 & 1 & & & 1 & & 1 & & & & & 1064870.0 \\
\hline 1927 & & & 9 & 26 & 38 & 26 & 12 & 14 & 7 & 16 & 46 & 32 & 29 & 512 & 21 & 16 & 5 & 4 & & 2 & 2 & 2 & & 2 & & & & 1 & 1 & & & 1577530.0 \\
\hline 1928 & & & & 31 & 40 & 13 & 25 & 30 & 29 & 24 & 24 & 18 & 21 & 442 & 24 & 9 & 8 & 4 & 7 & 4 & 1 & 5 & 1 & 1 & 1 & & 1 & 1 & & & & 1595750.0 \\
\hline 1929 & & & 37 & 53 & 38 & 33 & 20 & 29 & 16 & 31 & 16 & 21 & 17 & 321 & 13 & 6 & 1 & & 1 & & & & 1 & & & & & & & & & 1114380.0 \\
\hline 1930 & 1 & 74 & 70 & 17 & 11 & 19 & 5 & 30 & 39 & 37 & 17 & 7 & 8 & 6 & 8 & 3 & 4 & 3 & 3 & & & 2 & 1 & & & & & & & & & 987560.0 \\
\hline 1931 & 24 & 56 & 37 & 21 & 39 & 37 & 23 & 31 & 18 & 31 & 11 & 5 & 10 & 5 & 6 & 2 & 3 & 1 & 2 & & & 1 & & & & & 1 & 1 & & & & 945080.0 \\
\hline 1932 & 18 & 3 & 13 & 33 & 27 & 13 & 28 & 28 & 20 & 21 & 17 & 17 & 16 & 414 & 40 & 11 & 6 & 2 & 4 & 2 & 1 & 2 & & 1 & & & 1 & 1 & & & & 1477710.0 \\
\hline 1933 & & & 16 & 10 & 19 & 17 & 22 & 23 & 25 & 30 & 24 & 30 & 27 & $42 \cdot 4$ & 45 & 121 & 13 & 1 & 3 & 4 & & & 1 & & & 1 & & & & & & $1584180 \bullet 0$ \\
\hline 1934 & & & 53 & 44 & 46 & 19 & 27 & 13 & 41 & 25 & 15 & 14 & 11 & 171 & 12 & 5 & 4 & 5 & 6 & 2 & 1 & 2 & 2 & & 1 & & & & & & & $1214150 \cdot 0$ \\
\hline 1935 & & & 30 & 32 & 19 & 16 & 11 & 10 & 21 & 38 & 27 & 33 & 37 & 522 & 21 & 5 & 3 & 3 & 4 & & & & 2 & 1 & & & & & & & & 1418020.0 \\
\hline 1936 & & & 26 & 58 & 30 & 31 & 15 & 16 & 14 & 36 & 26 & 16 & 25 & 342 & 20 & 5 & 3 & 2 & 2 & 1 & & 4 & & 2 & & & & & & & & 1306630.0 \\
\hline 1937 & & 20 & 61 & 51 & 31 & 22 & 13 & 7 & 12 & 21 & 12 & 8 & 15 & 353 & 31 & 10 & 6 & 3 & 3 & & 1 & 1 & & 1 & & 1 & & & & & & 1259570.0 \\
\hline 1938 & & & 6 & 43 & 41 & 18 & 16 & 7 & 9 & 19 & 15 & 29 & 33 & 473 & 34 & 20 & 9 & 6 & 7 & 3 & 2 & & & & & & 1 & & & & & $1606160 \cdot 0$ \\
\hline 1939 & & & 59 & 38 & 11 & 14 & 14 & 25 & 33 & 48 & 33 & 22 & 20 & 22 & 8 & 4 & 4 & 3 & 7 & & & & & & & & & & & & & $1198840 \cdot C$ \\
\hline 1940 & & 59 & 91 & 29 & 7 & 15 & 15 & 15 & 19 & 20 & 33 & 14 & 8 & 101 & 13 & 5 & 7 & 4 & 2 & & & & & & & & & & & & & 1016950.0 \\
\hline 1941 & 10 & 30 & 48 & 24 & 16 & 36 & 35 & 54 & 33 & 37 & 15 & 10 & 3 & 6 & 2 & 3 & 3 & & & & & & & & & & & & & & & $903560 \cdot \mathrm{C}$ \\
\hline 1942 & & 13 & 45 & 27 & 16 & 14 & 12 & 22 & 46 & 772 & 20 & 19 & 9 & 111 & 10 & 8 & 7 & 3 & 2 & & 1 & & 2 & & 1 & & & & & & & 1208410.0 \\
\hline 1943 & & 22 & 5 & 10 & 22 & 17 & 19 & 15 & 10 & 15 & 34 & 34 & 17 & 353 & 35 & 201 & 18 & 9 & 9 & 3 & & 3 & 3 & 1 & 3 & 1 & 2 & 1 & & 2 & & 1935820.0 \\
\hline 1944 & & 28 & 39 & 32 & 13 & 15 & 32 & 52 & $4 n$ & 46 & 27 & 16 & 11 & 8 & 4 & 1 & & & 1 & & & 1 & & & & & & & & & & 0.0 \\
\hline 1945 & 6 & 26 & 51 & 52 & 26 & 16 & 11 & 16 & 12 & 15 & 15 & 24 & 14 & 262 & 25 & 91 & 10 & 6 & 2 & & & & 2 & & & 1 & & & & & & 0.0 \\
\hline 1946 & & 17 & 13 & 30 & 30 & 12 & 11 & 8 & 9 & 24 & 36 & 35 & 39 & 531 & 14 & 7 & 4 & 5 & 9 & 1 & 2 & 2 & 1 & & 1 & & & & 1 & & 1 & 158 \\
\hline 1947 & & & 9 & 39 & 26 & 20 & 12 & 27 & 33 & 39 & 23 & 26 & 16 & 382 & 29 & 5 & 5 & 4 & 5 & & & 2 & 2 & 1 & 1 & & & 2 & & 1 & & 1517970.0 \\
\hline 1948 & & & 1 & 6 & 19 & 26 & 12 & 27 & 16 & 19 & 38 & 51 & 23 & 454 & 41 & 151 & 10 & 2 & 5 & 4 & 1 & 1 & & 1 & & & 2 & & 1 & & & 1735810.0 \\
\hline 1949 & & & & 15 & 28 & 36 & 36 & 27 & 19 & 26 & 17 & 14 & 16 & 473 & 33 & 211 & 10 & 5 & 6 & 1 & 1 & 3 & 2 & & & & 1 & 1 & & & & $1612430 \cdot 0$ \\
\hline 1950 & & & & 18 & 19 & 31 & 20 & 25 & 28 & 23 & 23 & 14 & 17 & 393 & 30 & 401 & 14 & 6 & 8 & 3 & 2 & 1 & & 1 & 1 & & & & & & & 1740230.0 \\
\hline 1951 & & & & & 29 & 28 & 19 & 28 & 15 & 21 & 21 & 12 & 25 & 553 & 38 & 221 & 10 & 9 & 8 & 5 & 21 & 10 & 4 & 1 & 1 & & 1 & & 1 & & & $80 \cdot 0$ \\
\hline 1952 & & & & & 26 & 25 & 14 & 19 & 18 & 39 & 45 & 27 & 27 & 404 & 45 & 15 & 5 & 6 & 8 & & 3 & 3 & 1 & & & & & & & & & 1673720.0 \\
\hline 1953 & & & 3 & 55 & 5 & 31 & 26 & 24 & 14 & 13 & 27 & 21 & 29 & 392 & 29 & 121 & 10 & 3 & 8 & 2 & 2 & 1 & 3 & 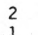 & 1 & 1 & 2 & 1 & & & 1 & 1716870.0 \\
\hline $\begin{array}{l}1954 \\
1955\end{array}$ & & & & & $\begin{array}{r}13 \\
6\end{array}$ & $\begin{array}{l}42 \\
57\end{array}$ & $\begin{array}{l}45 \\
24\end{array}$ & $\begin{array}{l}20 \\
29\end{array}$ & $\begin{array}{l}13 \\
24\end{array}$ & $\begin{array}{l}12 \\
61\end{array}$ & $\begin{array}{l}26 \\
32\end{array}$ & $\begin{array}{l}35 \\
17\end{array}$ & $\begin{array}{l}35 \\
23\end{array}$ & $\begin{array}{ll}32 & 2 \\
39 & 2\end{array}$ & $\begin{array}{l}25 \\
26\end{array}$ & $\begin{array}{ll}20 & 1 \\
12 & \end{array}$ & 12. & $\begin{array}{r}13 \\
5\end{array}$ & $\begin{array}{r}11 \\
3\end{array}$ & 1 & 1 & 3 & $\begin{array}{l}2 \\
1\end{array}$ & 1 & & 1 & & 1 & 1 & & & $\begin{array}{l}1789410 \bullet 0 \\
1438450.0\end{array}$ \\
\hline 1956 & & & & & 3 & 7 & 31 & $3 n$ & 16 & 17 & 17 & 22 & 19 & 424 & 48 & 303 & 301 & 16 & 10 & 7 & 4 & 4 & 5 & 2 & 1 & & 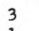 & & & & 1 & $2273210 \cdot 0$ \\
\hline 1957 & & & & 18 & 28 & 50 & 14 & 25 & 26 & 47 & 30 & 26 & 21 & 281 & 12 & 8 & 5 & 7 & 7 & & 3 & 1 & 4 & 1 & 1 & 1 & 1 & & 1 & & & $1548200 \cdot 0$ \\
\hline 1958 & & & & 27 & 51 & 32 & 22 & 12 & 13 & 37 & 33 & 25 & 24 & 201 & 14 & 91 & 13 & 8 & 9 & 4 & 3 & 4 & 2 & & 1 & & 1 & & 1 & & & 1601980.0 \\
\hline 1959 & & & 10 & 72 & 22 & 10 & 9 & 13 & 9 & 26 & 48 & 41 & 32 & 331 & 15 & 7 & 7 & 2 & 5 & & & 1 & 3 & & & & & & & & & 1392380.0 \\
\hline 1960 & & & & 21 & 40 & 35 & 39 & 36 & 28 & 21 & 11 & 13 & 18 & 253 & 34 & 19 & 9 & 6 & 7 & 4 & & & & & & & & & & & & 1451880.0 \\
\hline 1961 & & & & 55 & 32 & 22 & 11 & 18 & 12 & 26 & 16 & 29 & 22 & 452 & 29 & 21 & 6 & 5 & 6 & 3 & 2 & & 1 & 1 & & & & 1 & 1 & 1 & & 1624480.0 \\
\hline 1962 & & & & 41 & 22 & 26 & 19 & 19 & 13 & 41 & 32 & 33 & 20 & 282 & 27 & 20 & 5 & 6 & 4 & 1 & 1 & 3 & 3 & 1 & & & & & & & & 1539080.0 \\
\hline 1963 & & & 12 & 29 & 28 & 16 & 25 & 32 & 19 & 49 & 22 & 21 & 26 & 391 & 16 & 8 & 7 & 4 & 6 & 2 & 1 & 2 & 1 & & & & & & & & & 1416710.0 \\
\hline
\end{tabular}

MC KENZIE RTVER NEAR VIDA OREG

Summary of water years 1925-63

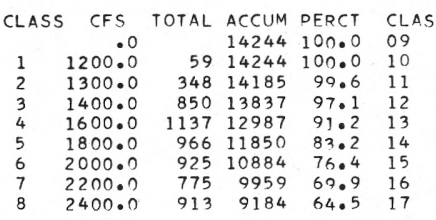

McKenzie River near Vida, Oreg.

Summary for water years 1925-1962

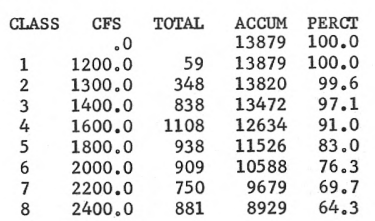

McKenzie River near Vida, Oreg.

Summary for water year 1963

$\begin{array}{ccrrrrr}\text { CLASS } & \text { CFS } & \text { TOTAL } & \text { ACCUM } & \text { PERCT } & \text { CLASS } & \text { CFS } \\ & .0 & & 365 & 100.0 & 09 & 2700.0 \\ 1 & 1200.0 & & 365 & 100.0 & 10 & 3000.0 \\ 2 & 1300.0 & & 365 & 100.0 & 11 & 3500.0 \\ 3 & 1400.0 & 12 & 365 & 100.0 & 12 & 4000.0 \\ 4 & 1600.0 & 29 & 353 & 96.7 & 13 & 4500.0 \\ 5 & 1800.0 & 28 & 324 & 88.8 & 14 & 5000.0 \\ 6 & 2000.0 & 16 & 296 & 81.1 & 15 & 6000.0 \\ 7 & 2200.0 & 25 & 280 & 76.7 & 16 & 7000.0 \\ 8 & 2400.0 & 32 & 255 & 69.9 & 17 & 8000.0\end{array}$

STATION NUMBER

$14-1625.00$

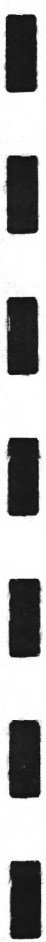

CFS TOTAL ACCUM PERCT $\begin{array}{rrrr}2700.0 & 815 & 8271 & 58.1 \\ 3000.0 & 1178 & 7456 & 52.3\end{array}$ $\begin{array}{rrrr}3000.0 & 1178 & 7456 & 52.3 \\ 3500.0 & 967 & 6278 & 44.1\end{array}$ $4000.0 \quad 894 \quad 5311 \quad 37.3$ $\begin{array}{rrrr}4500.0 & 801 & 4417 & 31.0\end{array}$ $6000.0 \quad 899 \quad 2356 \quad 16.5$ $\begin{array}{rrrr}7000.0 & 460 & 1457 & 10.2 \\ 8000.0 & 293 & 997 & 7.0\end{array}$
STATION NUMBER
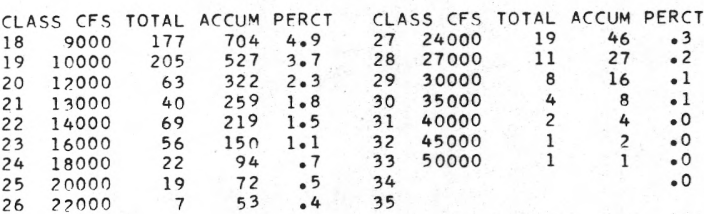

$14-1625.00$

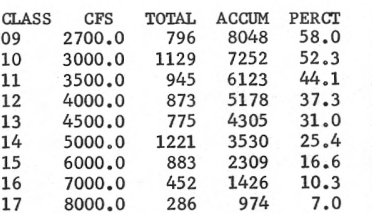

STATION NUMBER
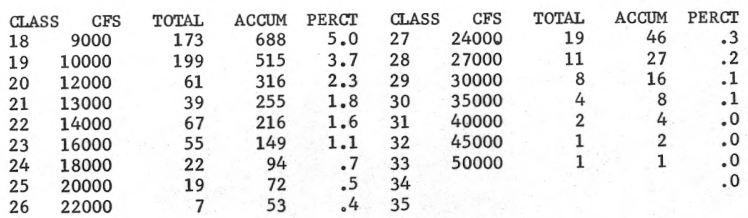
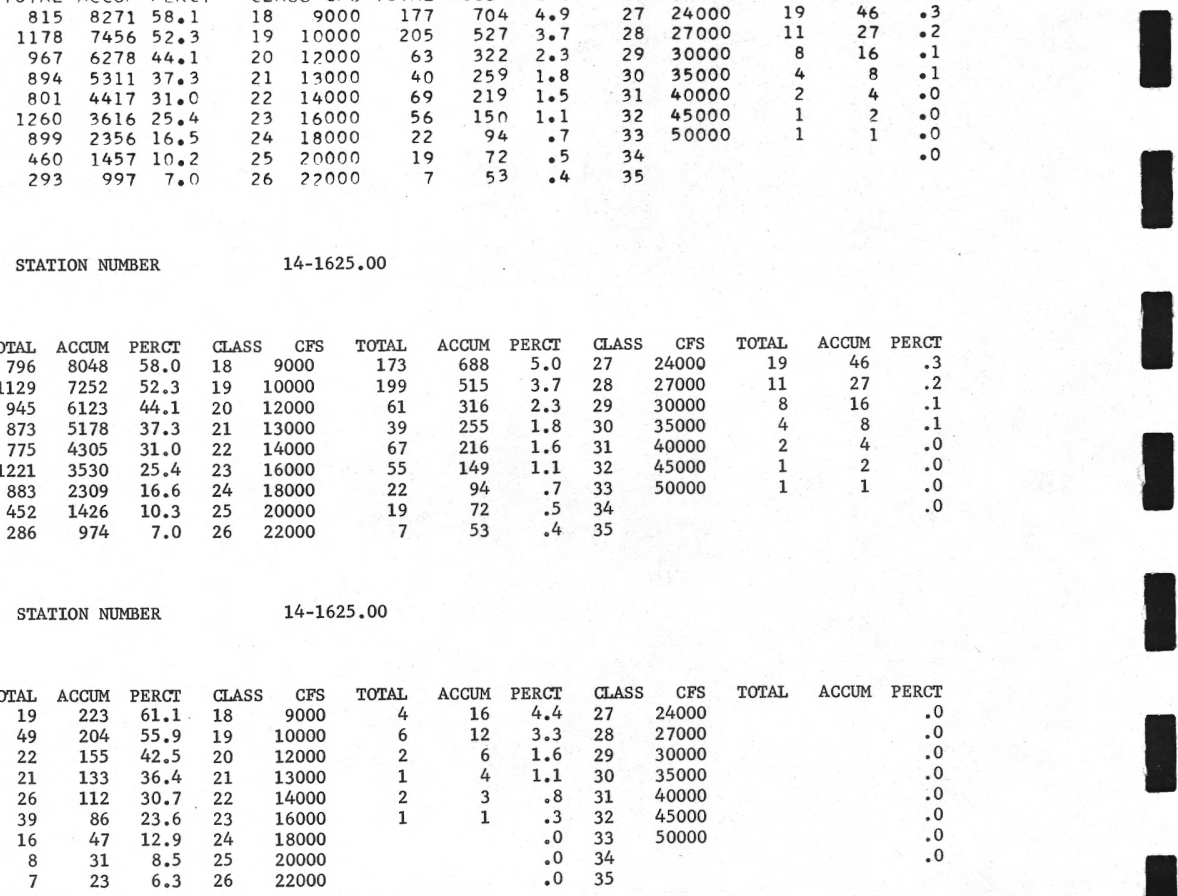

TOTAL ACCUM PERCT

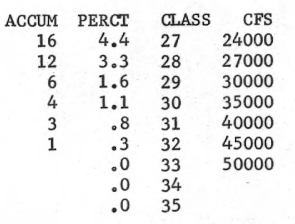



LOWEST MEAN DISCHARGE, IN CFS, FOR THE FOLLOWING NUMBER OF CONSECUTIVE DAYS IN YEAR BEGINNING APRIL 1

\begin{tabular}{|c|c|c|c|c|c|c|c|c|c|c|c|}
\hline YFAR & 1 & 3 & 7 & 14 & 30 & 60 & 90 & 120 & 150 & 183 & 274 \\
\hline 1925 & 1570.0 & 1610.0 & 1630.0 & $1660 \cdot 0$ & 1680.0 & 1700.0 & 1740.0 & $1810 \cdot 0$ & 1980.0 & $\begin{array}{l}2150 \cdot 0 \\
1720.0\end{array}$ & \\
\hline $\begin{array}{l}1926 \\
1927\end{array}$ & 1430.0 & 1450.0 & 1460.0 & $1470 \cdot 0$ & $1500 \cdot 0$ & 1520.0 & $1550 \cdot 0$ & $1590 \cdot 0$ & $\begin{array}{l}1620.0 \\
2740.0\end{array}$ & $\begin{array}{l}1720.0 \\
3260.0\end{array}$ & $\begin{array}{l}2580.0 \\
4210.0\end{array}$ \\
\hline $\begin{array}{l}1927 \\
1928\end{array}$ & 1840.0 & $1840 . n$ & $1860 \cdot 0$ & $1900 \cdot 0$ & 1930.0 & 2010.0 & 2190.0 & $2520 \cdot 0$ & $\begin{array}{l}2740.0 \\
1830.0\end{array}$ & 1900.0 & 2200.0 \\
\hline $\begin{array}{l}1928 \\
1929\end{array}$ & 1570.0 & $1570 \cdot 0$ & $1590 \cdot 0$ & 1630.0 & $\begin{array}{l}1660.0 \\
1360.0\end{array}$ & $\begin{array}{l}1700.0 \\
1390.0\end{array}$ & $\begin{array}{l}1720.0 \\
1420.0\end{array}$ & $\begin{array}{l}1770 \cdot 0 \\
1470.0\end{array}$ & 1580.0 & 1910.0 & 2740.0 \\
\hline $\begin{array}{l}1930 \\
1931\end{array}$ & $\begin{array}{l}1320.0 \\
1260.0\end{array}$ & $\begin{array}{l}1330 . n \\
1270 . n\end{array}$ & $\begin{array}{l}1330 \cdot 0 \\
1280.0\end{array}$ & $\begin{array}{l}1340 \cdot 0 \\
1320 \cdot ?\end{array}$ & 1330.0 & $1350 . ?$ & 1360.0 & $1390 \cdot 0$ & 1470.0 & 1510.0 & 1910.0 \\
\hline $\begin{array}{l}1931 \\
1932\end{array}$ & 1260.0 & $1260 \cdot 0$ & 1270.0 & 1280.0 & 1280.0 & 1310.0 & 1340.0 & $1450 \cdot 0$ & 1570.0 & $1700 \cdot 0$ & 2280.0 \\
\hline $\begin{array}{l}1932 \\
1933\end{array}$ & 1480.0 & $1480 \cdot n$ & 1490.0 & 1510.0 & 1550.0 & 1630.0 & 1710.0 & $1880 \cdot 0$ & $2280 \cdot 0$ & 2560.0 & 3170.0 \\
\hline $\begin{array}{l}1933 \\
1934\end{array}$ & 1770.0 & $1780 . n$ & 1790.0 & $18 \cap 0.0$ & 1880.0 & 1970.0 & 2070.0 & $2090 \cdot 0$ & $22 \cdot 0 \cdot 0$ & 2840.0 & 3830.0 \\
\hline $\begin{array}{l}1934 \\
1935\end{array}$ & $1400 \cdot 0$ & $1410 \cdot 0$ & 1420.0 & $1420 \cdot 0$ & 1450.0 & 1460.0 & 1510.0 & $1580 \cdot 0$ & $1700 \cdot 0$ & $1880 \cdot 0$ & 3000.0 \\
\hline 1935 & 1530.0 & 1540.0 & 1550.0 & $1560 \cdot 0$ & 1590.0 & 1630.0 & 1670.0 & $1760 \bullet 0$ & 1830.0 & 1950.0 & 2840.0 \\
\hline $\begin{array}{l}1936 \\
1937\end{array}$ & 1350.0 & 1350.0 & 1360.0 & 1370.0 & $1390 \cdot 0$ & 1440.0 & 1510.0 & $1580 \cdot 0$ & 1660.0 & $1680 \cdot 0$ & 2060.0 \\
\hline 1937 & 1570.0 & 1570.0 & 1580.0 & 1630.0 & 1660.0 & 1740.0 & 1860.0 & $1980 \cdot 0$ & 2480.0 & $3120 \cdot 0$ & 4090.0 \\
\hline $\begin{array}{l}1938 \\
1939\end{array}$ & 1530.0 & 1530.0 & $1540 \cdot ?$ & 1560.0 & 1600.0 & 1670.0 & 1730.0 & $1840 \cdot 0$ & 2070.0 & 2270.0 & 2850.0 \\
\hline $\begin{array}{l}1939 \\
1940\end{array}$ & $1380 \cdot 0$ & $1380 \cdot 0$ & 1380.0 & 1390.0 & 1410.0 & 1490.0 & 1500.0 & $1520 \cdot 0$ & 1580.0 & 1760.0 & 2270.0 \\
\hline 1940 & 1280.0 & 1280.0 & 1290.0 & 1290.0 & 1340.0 & 1350.0 & 1360.0 & $1400 \cdot 0$ & 1470.0 & 1630.0 & 2190.0 \\
\hline $\begin{array}{l}1941 \\
1942\end{array}$ & 1340.0 & 1350.0 & 1360.0 & 1370.0 & 1390.0 & 1490.0 & 1530.0 & $1600 \cdot 0$ & 1740.0 & 1900.0 & 2810.0 \\
\hline 1942 & 1320.0 & $1320 \cdot n$ & $1330 . n$ & 1350.0 & 1360.0 & 1390.0 & 1460.0 & $1600 \cdot 0$ & 1870.0 & 2230.0 & 3900.0 \\
\hline 1943 & 1640.0 & $1650 . n$ & 1660.0 & 1690.0 & 1740.0 & 1860.0 & 2010.0 & $2300 \cdot 0$ & $2560 \cdot 0$ & 2600.0 & 2800.0 \\
\hline $\begin{array}{l}1944 \\
1945\end{array}$ & 1280.0 & $1280 \bullet 0$ & 1290.0 & 1300.0 & 1330.0 & 1350.0 & 1380.0 & $1450 \cdot 0$ & $1520 \cdot 0$ & $1580 \cdot 0$ & 2100.0 \\
\hline 1945 & 1370.0 & 1370.0 & 1370.0 & $1380 \cdot 0$ & 1400.0 & $1460 \cdot n$ & 1510.0 & $1580 \cdot 0$ & 1750.0 & 2190.0 & 3770.0 \\
\hline 1946 & 1560.0 & 1570.0 & $1590 . ?$ & 1630.0 & 1650.0 & 1730.0 & 1850.0 & $2110 \cdot 0$ & $2300 \cdot 0$ & 2730.0 & 4050.0 \\
\hline 1947 & 1590.0 & 1590.0 & 1590.0 & 1620.0 & 1690.0 & 1760.0 & 1890.0 & $2130 \cdot 0$ & $2320 \cdot 0$ & 2680.0 & 3610.0 \\
\hline 1948 & 1830.0 & 1850.0 & 1870.0 & 1880.0 & 1980.0 & $2100 \cdot 0$ & $2200 \cdot 0$ & $2330 \cdot 0$ & $2540 \cdot 0$ & 3050.0 & 3620.0 \\
\hline 1949 & 1680.0 & 1690.0 & 1710.0 & $1770 \bullet 0$ & 1810.0 & 1910.0 & 1930.0 & $1980 \cdot 0$ & 2120.0 & 2460.0 & 3230.0 \\
\hline 1950 & 1990.0 & 1990.0 & 2020.0 & 2040.0 & 2090.0 & 2230.0 & 2490.0 & 2830.0 & 3690.0 & 4180.0 & 5520.0 \\
\hline 1951 & 1820.0 & $1820 . n$ & 1840.0 & $1860 \cdot 0$ & 1910.0 & 2020.0 & 2150.0 & $2320 \cdot 0$ & 2630.0 & 3050.0 & 3860.0 \\
\hline 1952 & 1590.0 & $1600 \cdot 0$ & 1600.0 & 1630.0 & 1690.0 & 1710.0 & 1780.0 & $1870 \cdot 0$ & 2010.0 & 2190.0 & 3240.0 \\
\hline 1953 & 1920.0 & 1930.0 & $1950 . ?$ & 1980.0 & $208 \cap \cdot 0$ & 2090.0 & 2170.0 & $2270 \cdot 0$ & 2540.0 & $3150 \cdot 0$ & 4690.0 \\
\hline 1954 & 1970.0 & $2000 \cdot 0$ & $20 n 0.0$ & 2020.0 & 2140.0 & 2210.0 & 2260.0 & $2310 \cdot 0$ & 2420.0 & 2550.0 & 3090.0 \\
\hline 1955 & 1920.0 & 1940.0 & $1980 \cdot 0$ & 1990.0 & 2060.0 & 2140.0 & 2390.0 & 2670.0 & 3100.0 & $3810 \cdot 0$ & 5620.0 \\
\hline 1956 & 2000.0 & $2060 . n$ & 2080.0 & 2110.0 & $2] 40.0$ & 2230.0 & 2370.0 & $2660 \cdot 0$ & 2890.0 & 3220.0 & 3920.0 \\
\hline 1957 & 1610.0 & 1610.0 & 1620.0 & 1640.0 & 1770.0 & 1820.0 & 1840.0 & $1900 \cdot 0$ & 2040.0 & $2200 \cdot 0$ & 3390.0 \\
\hline 1958 & 1630.0 & 1650.0 & $1680 \cdot 0$ & 1730.0 & 1780.0 & 1830.0 & 1860.0 & $1960 \cdot 0$ & $2210 \cdot 0$ & 2590.0 & 3550.0 \\
\hline 1959 & 1560.0 & 1570.0 & 1570.0 & 1590.0 & 1630.0 & 1690.0 & $1800 \cdot 0$ & $2010 \cdot 0$ & $2160 \cdot 0$ & 2330.0 & 2630.0 \\
\hline 1960 & 1630.0 & 1640.0 & 1650.0 & 1670.0 & 1720.0 & 1780.0 & 1850.0 & 1940.0 & 2120.0 & 2790.0 & 3710.0 \\
\hline 1961 & 1640.0 & 1640.0 & $164 ? .0$ & 1660.0 & 1710.0 & 1770.0 & 1840.0 & $1960 \cdot 0$ & 2160.0 & 2450.0 & 3540.0 \\
\hline & 40.0 & $1650 . n$ & 1660.0 & 1690.0 & 1740.0 & 1830.0 & 1950.0 & $2170 \cdot 0$ & 2330.0 & 2670.0 & 3390.0 \\
\hline
\end{tabular}
HIGHEST MEAN DISCHARGE, IN CFS, FOR THE FOLLOWING NUMRER OF CONSECUTIVE DAYC IN YEAR ENDING SEPTFMBER 30

\begin{tabular}{|c|c|c|c|c|c|c|c|c|c|c|c|}
\hline YEAR & 1 & 3 & 7 & 15 & 30 & 60 & 90 & 120 & $15 n$ & $\begin{array}{r}183 \\
6550\end{array}$ & $\begin{array}{c}274 \\
5540.0\end{array}$ \\
\hline $\begin{array}{l}1925 \\
1926\end{array}$ & $\begin{array}{l}26700 \cdot 0 \\
24400.0\end{array}$ & $23000 \cdot n$ & 19500.0 & $15800 \cdot 0$ & $11600 \cdot 0$ & $937 n \cdot n$ & 8230.0 & $7530 \cdot 0$ & $\begin{array}{l}6890.0 \\
4410.0\end{array}$ & $\begin{array}{l}6550 \cdot 0 \\
4110.0\end{array}$ & $\begin{array}{l}5540.0 \\
3370.0\end{array}$ \\
\hline 1927 & $\begin{array}{l}2440000 \\
31700.0\end{array}$ & $\begin{array}{l}19600 \cdot 0 \\
26000 \cdot 0\end{array}$ & $\begin{array}{l}14700 \cdot 0 \\
16600 \cdot 0\end{array}$ & $\begin{array}{l}10100.0 \\
11200.0\end{array}$ & $\begin{array}{l}8290.0 \\
8210.0\end{array}$ & $\begin{array}{l}5990.0 \\
6930.0\end{array}$ & $\begin{array}{l}5390.0 \\
6480.0\end{array}$ & $\begin{array}{l}4820 \cdot 0 \\
6320 \cdot 0\end{array}$ & $\begin{array}{l}4410 \cdot 0 \\
5900 \cdot 0\end{array}$ & & $\begin{array}{l}3370.0 \\
5100.0\end{array}$ \\
\hline 1928 & $27800 \cdot 0$ & $20200 . n$ & $17700 \cdot 0$ & 12700.0 & 9500.0 & 8080.0 & $705 n \cdot 0$ & $6400 \cdot 0$ & 6400.0 & 6240.0 & 5200.0 \\
\hline 1929 & $17000 \cdot 0$ & 11400.0 & 7890.0 & $6220 \cdot 0$ & 5780.0 & 5470.0 & 5270.0 & $4870 \cdot 0$ & $4420 . n$ & $4210 \cdot 0$ & 3530.0 \\
\hline 1930 & $17600 \bullet 0$ & $13900 \cdot 0$ & $10000 \cdot 0$ & $9020 \cdot 0$ & 7210.0 & 5210.0 & $4850 \cdot 0$ & $4420 \cdot 0$ & 4090.0 & 3900.0 & 3140.0 \\
\hline 1931 & 29200.0 & $23600 \cdot 0$ & 15300.0 & $10300 \cdot 0$ & 8180.0 & 5840.0 & 4790.0 & $4250 \cdot 0$ & $3860 \cdot 0$ & $3580 \cdot 0$ & $3000 \cdot 0$ \\
\hline 1932 & 29500.0 & 24500.0 & $16800 \cdot n$ & 14000.0 & 10300.0 & 8390.0 & $7600 \cdot 0$ & 6970.0 & $6170 \cdot 0$ & $5850 \cdot 0$ & 4830.0 \\
\hline 1933 & 23400.0 & 17900.0 & 14700.0 & $11600 \cdot 0$ & 9630.0 & 7820.0 & 7040.0 & $6550 \cdot 0$ & $6050 \cdot 0$ & $5740 . n$ & 5120.0 \\
\hline 1934 & $21500 \cdot 0$ & $16800 \cdot n$ & $13700 \cdot 0$ & 10600.0 & 8460.0 & 7740.0 & 6320.0 & $5850 \cdot 0$ & 5330.0 & 4820.0 & 3910.0 \\
\hline 1935 & 19900.0 & $17900 . n$ & 13400.0 & $924 n \cdot ?$ & 6890.0 & $609 n .0$ & $565 n \cdot 0$ & $5340 \cdot 0$ & 5090.0 & 5120.0 & 4610.0 \\
\hline 1936 & 19800.0 & $16600 \cdot 0$ & $1340 n \cdot 0$ & $12200 \cdot n$ & 8720.0 & $6390 . n$ & 5770.0 & $5670 \cdot 0$ & $558 \cap .0$ & 5190.0 & 4200.0 \\
\hline 1937 & 23400.0 & $19400 \cdot ?$ & $1380 n \cdot 0$ & 9670.0 & 8040.0 & 7250.0 & $68 n n \cdot 0$ & $6420 \cdot 0$ & $5820 \cdot 0$ & 5190.0 & 4090.0 \\
\hline 1938 & 24200.0 & $16700 \cdot 0$ & $1160 n \cdot 0$ & 8600.0 & 7220.0 & 6790.0 & 6360.0 & $6300 \cdot 0$ & 6270.0 & 6260.0 & 5250.0 \\
\hline 1939 & 11900.0 & $10800 \cdot 0$ & 10100.0 & 8200.0 & 6760.0 & 5650.0 & 5240.0 & $4770 \cdot 0$ & 4670.0 & 4490.0 & 3850.0 \\
\hline 1940 & $10300 \cdot 0$ & 9830.0 & 9150.0 & 7670.0 & 7030.0 & 6120.0 & 5270.0 & $4780 \cdot 0$ & 4470.0 & 4080.0 & 3240.0 \\
\hline 1941 & 8780.0 & 7620.0 & 6070.0 & 5440.0 & 4380.0 & 3970.0 & 3720.0 & $3460 \cdot 0$ & $3200 \cdot 0$ & $3110 \cdot 0$ & 2810.0 \\
\hline 1942 & $20200 \cdot 0$ & $14900 \cdot 0$ & 11000.0 & 8020.0 & 7090.0 & $6120 \cdot 0$ & $5640 \cdot 0$ & $5100 \cdot 0$ & 4670.0 & $4390 \cdot 0$ & 3890.0 \\
\hline 1943 & 39700.0 & 32900.0 & 24700.0 & $17300 \cdot 0$ & 12800.0 & 11800.0 & $100 n 0.0$ & 8770.0 & 8250.0 & 7760.0 & 6460.0 \\
\hline 1944 & 14700.0 & $10700 \cdot 0$ & $700 n \cdot 0$ & 5060.0 & 4300.0 & 3910.0 & 3650.0 & $3520 \cdot 0$ & $3370 \cdot 0$ & 3350.0 & 3120.0 \\
\hline 1945 & 23800.0 & 17400.0 & $1400 n .0$ & 10600.0 & 7270.0 & 6180.0 & 5970.0 & $5980 \cdot 0$ & 5730.0 & $5190 \cdot 0$ & $4080 . n$ \\
\hline 1946 & 47900.0 & 34400.0 & $213 n n \cdot n$ & $155 \mathrm{nn} \cdot 0$ & 11000.0 & $83 n n \cdot n$ & $718 \cap .0$ & $6790 \cdot 0$ & 6320.0 & 6160.0 & $5240 . n$ \\
\hline 1947 & 35200.0 & $3 n 000 \cdot 0$ & $204 n \cap .0$ & $127 \cap n \cdot 0$ & $108 \cap n \cdot n$ & $754 n .0$ & 7160.0 & $6550 \cdot 0$ & $6340 . n$ & $5950 \cdot n$ & $4940 . n$ \\
\hline 1948 & 33800.0 & $26600 \cdot 0$ & $17500 \cdot 0$ & $124 n 0.0$ & 8520.0 & 7110.0 & $656 n \cdot 0$ & $6200 \cdot 0$ & 6070.0 & 5920.0 & 5590.0 \\
\hline 1949 & 28200.0 & $20300 \cdot 0$ & $1400 n \cdot 0$ & 10700.0 & 8900.0 & 7410.0 & 7350.0 & $6850 \cdot 0$ & 6130.0 & 6010.0 & 5190.0 \\
\hline 1950 & 21200.0 & $18000 \cdot n$ & $1290 ? \cdot 0$ & 11000.0 & 9770.0 & 8330.0 & $765 n .0$ & $7620 \cdot 0$ & $733 n \cdot n$ & $6850 \cdot 0$ & 5670.0 \\
\hline 1951 & $30000 \cdot 0$ & $20800 \cdot 0$ & 18300.0 & $12200 \cdot 0$ & $10900 \cdot 0$ & 9110.0 & 8790.0 & $8800 \cdot 0$ & 7980.0 & $7580 \cdot n$ & 6400.0 \\
\hline 1952 & 16400.0 & $14200 . n$ & 12300.0 & 9120.0 & 7050.0 & 6530.0 & 6010.0 & $602 n \cdot 0$ & 5720.0 & $5800 \cdot 0$ & $5340 \cdot 0$ \\
\hline 1953 & $44200 \cdot 0$ & $32800 \bullet 0$ & 23000.0 & 16900.0 & 15000.0 & 10600.0 & 8780.0 & $7900 \cdot 0$ & 7550.0 & 7100.0 & 5660.0 \\
\hline 1954 & 31600.0 & $25600 \cdot 0$ & $16100 \cdot 0$ & 12200.0 & 12100.0 & 8970.0 & $872 n \cdot 0$ & $7990 \cdot 0$ & 7500.0 & 6990.0 & $5810 \cdot 0$ \\
\hline 1955 & $16000 \cdot 0$ & $12000 \cdot 0$ & 9530.0 & 7840.0 & 7270.0 & 6490.0 & $6260 \cdot 0$ & $5730 \cdot 0$ & 5390.0 & $5250 \cdot 0$ & 4520.0 \\
\hline 1956 & $50000 \cdot 0$ & $38300 \cdot 0$ & 24600.0 & 18300.0 & 14700.0 & 12400.0 & $10500 \cdot 0$ & $9310 \cdot 0$ & 8800.0 & 8610.0 & 7430.0 \\
\hline 1957 & $33800 \cdot 0$ & 26300.0 & 17900.0 & $13800 \cdot 0$ & 10800.0 & 8440.0 & 7140.0 & 6710.0 & 6310.0 & 5890.0 & $5000 \cdot 0$ \\
\hline 1958 & 31600.0 & 21800.0 & 15100.0 & $12400 \cdot 0$ & 9630.0 & 9100.0 & 8320.0 & $7170 \cdot 0$ & 6940.0 & 6450.0 & 5230.0 \\
\hline 1959 & 16800.0 & 14700.0 & $1050 n \cdot ?$ & 7810.0 & 6940.0 & 5990.0 & 5950.0 & $5610 \cdot 0$ & 5520.0 & 5320.0 & 4480.0 \\
\hline 1960 & $12500 \cdot 0$ & $11600 \cdot 0$ & $11000 \cdot 0$ & 9270.0 & 7940.0 & 7210.0 & 7110.0 & $6700 \cdot 0$ & $6180 \cdot 0$ & 5520.0 & 4640.0 \\
\hline 1961 & 35800.0 & 26400.0 & 18500.0 & $13600 \cdot 0$ & 10500.0 & 8750.0 & 7520.0 & 6970.0 & 6730.0 & 6450.0 & 5330.0 \\
\hline 1962 & 18700.0 & 17500.0 & 13300.0 & 9890.0 & 8140.0 & 7080.0 & 6160.0 & $5770 \cdot 0$ & 5910.0 & 5890.0 & $\frac{4990.0}{4530.0}$ \\
\hline 1963 & 17900.0 & 15200.0 & $12200 \cdot 0$ & 8280.0 & 7210.0 & 5790.0 & 5500.0 & $5590 \cdot 0$ & 5100.0 & 5260.0 & 453 \\
\hline
\end{tabular}


Gate Creek at V1da, Oreg.

STATION NUMBER

$14-1630.00$

DURATION TABLE OF DAILY DISCHARGF

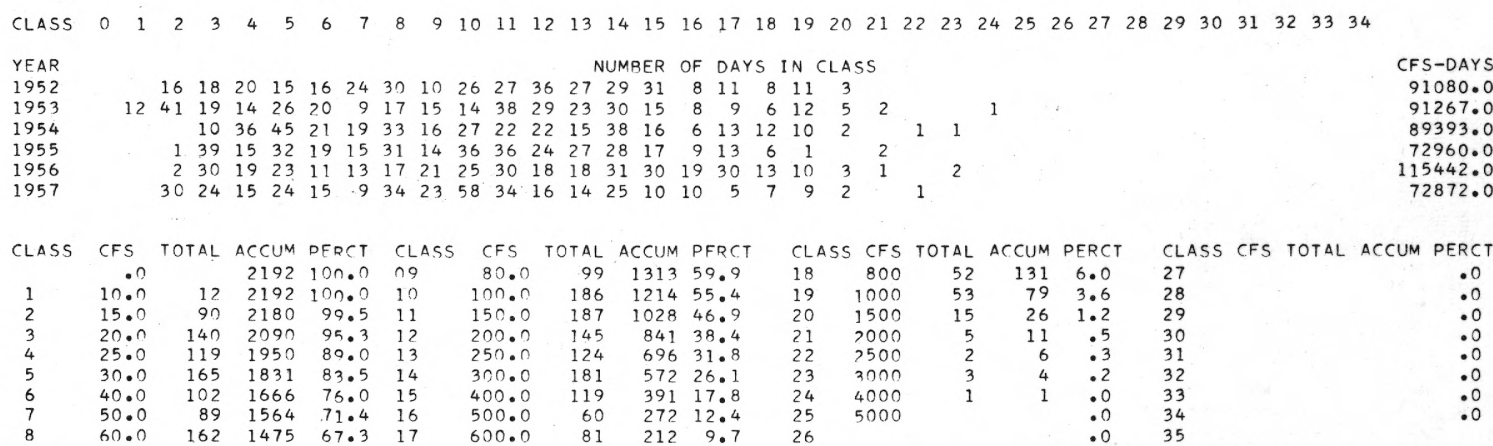

Gate Creek at Vida, Oreg.

STATION NUMBER

$14-1630.00$

LOWEST MEAN DISCHARGE, IN CFS, FOR THE FOLLOWING NUMBER OF CONSECUTIVE DAYS IN YEAR BEGINNING APRIL 1

$\begin{array}{llllllllrrrr}\text { YEAR } & 1 & 3 & 7 & 14 & 30 & 60 & 90 & 120 & 150 & 183 & 274 \\ 1952 & 13.0 & 13.0 & 13.0 & 13.6 & 16.6 & 17.4 & 18.9 & 20.7 & 25.9 & 36.7 & 75.9 \\ 1953 & 21.0 & 21.3 & 21.9 & 22.1 & 25.5 & 31.5 & 33.9 & 37.3 & 46.9 & 85.2 & 222.0 \\ 1954 & 23.0 & 23.0 & 23.0 & 23.9 & 28.6 & 30.8 & 32.6 & 39.4 & 45.6 & 55.0 & 102.0 \\ 1955 & 19.0 & 19.7 & 19.9 & 20.6 & 22.5 & 26.2 & 32.5 & 42.2 & 65.7 & 96.3 & 278.0 \\ 1956 & 18.0 & 18.0 & 18.4 & 18.9 & 20.7 & 22.6 & 26.1 & 33.4 & 45.9 & 70.4 & 134.0\end{array}$

Gate Creek at Vida, Oreg.

STATION NUMBER $\quad 14-1630.00$

HIGHEST MEAN DISCHARGF, IN CFS, FOR THE. FOLLOWING NUMBER OF CONSECUTIVE DAYS IN YEAR ENDING SEPTEMBER 30

\begin{tabular}{|c|c|c|c|c|c|c|c|c|c|c|c|}
\hline YEAR & 1 & 3 & 7 & 15 & 30 & 60 & 90 & 120 & 150 & 183 & 274 \\
\hline 1952 & 1820.0 & 1490.0 & 1280.0 & 880.0 & 613.0 & 506.0 & 504.0 & 477.0 & 461.0 & 425.0 & 321.0 \\
\hline 1953 & 4620.0 & 3030.0 & $2050 . ?$ & 1320.0 & 1200.0 & 818.0 & 680.0 & $568 \cdot 0$ & 509.0 & 451.0 & 326.0 \\
\hline 1954 & 3540.0 & 2520.0 & 1390.0 & 1060.0 & 986.0 & 687.0 & 650.0 & 564.0 & 506.0 & 435.0 & 315.0 \\
\hline 1955 & 2180.0 & 1890.0 & 1120.0 & 641.0 & 596.0 & 477.0 & 413.0 & 410.0 & 385.0 & 343.0 & 256.0 \\
\hline 1956 & 3100.0 & 2570.0 & $1650 . ?$ & 1160.0 & 970.0 & 834.0 & 701.0 & $618 \cdot 0$ & 608.0 & 548.0 & 411.0 \\
\hline 57 & 2600.0 & $2000 \cdot ?$ & 1350 & 898.0 & 740.0 & 545.0 & 418.0 & $432 \cdot 0$ & 375.0 & 343.0 & \\
\hline
\end{tabular}


McKenzle River near Springfield, Oreg. STATION NUMBER 14-1640.00

DURATION TABLE OF DAILY DISCHARGE

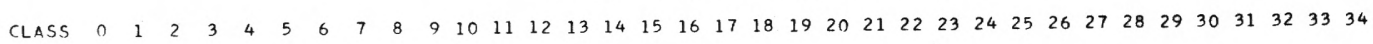
YEAR
1906
1907
1908
1909
1910
1911
1912
1913
1914 NUMBER OF DAYS IN CLASS

$\begin{array}{llllllllllll}59 & 55 & 22 & 31 & 32 & 26 & 34 & 59 & 24 & 11 & 9^{\text {NUMB }} & 3\end{array}$

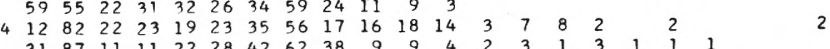

$\begin{array}{lllllllllllllllllll}31 & 87 & 11 & 11 & 22 & 28 & 42 & 62 & 38 & 9 & 9 & 4 & 2 & 3 & 1 & 3 & 1 & 1 & 1\end{array}$

$\begin{array}{llllllllllllllllllll}15 & 75 & 55 & 31 & 23 & 45 & 31 & 32 & 20 & 10 & 14 & 4 & 4 & & 4 & 2 & \\ 60 & 58 & 24 & 38 & 15 & 28 & 46 & 14 & 33 & 16 & 7 & 9 & 7 & 2 & 1 & 4 & 1 & 1\end{array}$

$\begin{array}{lllllllllllll}31 & 49 & 42 & 17 & 41 & 62 & 51 & 25 & 17 & 10 & 4 & 8 & 4\end{array}$

$\begin{array}{llllllllllllllllllllllll}17 & 20 & 59 & 21 & 42 & 45 & 37 & 16 & 37 & 24 & 14 & 13 & 8 & 5 & 3 & 2 & 1 & 1 & 1\end{array}$

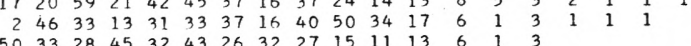

\begin{tabular}{|c|c|c|c|c|c|c|c|c|c|c|c|c|c|c|}
\hline CLASS & CFS & TOTAL & ACCUM & DFPCT & CLASS & CFS & TOTAL & ACCUM & PERCT & $C L A$ & CFS & TOTAL & ACCUM & DERC \\
\hline & .0 & & 3287 & $10 n \cdot 0$ & 09 & 4500.0 & 277 & 1234 & 37.5 & 18 & 20000 & 11 & 26 & \\
\hline 1 & $1200 . ?$ & & 3287 & $10 n \cdot 0$ & 10 & 5000.0 & 344 & 957 & 29.1 & 19 & 25000 & 6 & 15 & \\
\hline 2 & 1400.0 & 104 & 3287 & 100.0 & 11 & 6000.0 & 231 & 613 & 18 & 20 & $3 n$ & 4 & 9 & \\
\hline 3 & $1700 \cdot 0$ & 32 & 3183 & & 12 & 7000.0 & 125 & 382 & 11 & 21 & 350 & 1 & 5 & \\
\hline 4 & 2000.0 & 519 & 2858 & 86 & 13 & 8000.0 & 108 & 257 & & 22 & 40000 & & 4 & \\
\hline 5 & 2500.0 & 23 & 2339 & 71 & 14 & 10000.0 & 58 & 149 & 4. & 23 & 45000 & 1 & 4 & \\
\hline 6 & 3000.0 & 280 & 2109 & 64 & 15 & $12000 \cdot 0$ & 23 & 91 & & 24 & 50 & 3 & 3 & \\
\hline 7 & 3500.0 & 294 & 18 & & 16 & 14000.0 & 24 & 68 & 2. & 25 & 60000 & & & \\
\hline 8 & 4000.0 & 301 & 1535 & 46.7 & 17 & 17000.0 & 18 & 44 & 1.3 & 26 & & & & \\
\hline
\end{tabular}

CFS-DAYS 1448050.0 2009330.0 1745230.0 1586680.0 1749270.0 1352760.0 1679250.0 1415000.0

McKenzle River near Springfield, Oreg.

STATION NUMBER $14-1640.00$

LOWEST MEAN DISCHARGE, IN CFS, FOR THE FOLLOWING NUMBER OF CONSECUTIVF DAYS IN YFAR BEGINNING APRIL 1

$\begin{array}{cccccccccccc}\text { YEAR } & 1 & 3 & 7 & 14 & 30 & 60 & 90 & 120 & 150 & 183 & 274 \\ 1906 & 1630.0 & 1630.0 & 1690.0 & 1740.0 & 1910.0 & 2000.0 & 2060.0 & 2230.0 & 2600.0 & 3070.0 & 4260.0 \\ 1907 & 1830.0 & 1880.0 & 1930.0 & 1960.0 & 1990.0 & 2020.0 & 2070.0 & 2140.0 & 2280.0 & 2620.0 & 4280.0 \\ 1908 & 1820.0 & 1840.0 & 1860.0 & 1870.0 & 1920.0 & 2040.0 & 2160.0 & 2400.0 & 2590.0 & 2740.0 & 3630.0 \\ 1909 & 1990.0 & 1990.0 & 1990.0 & 2040.0 & 2060.0 & 2110.0 & 2210.0 & 2360.0 & 2610.0 & 2990.0 & 4440.0 \\ 1910 & 1540.0 & 1600.0 & 1640.0 & 1730.0 & 1800.0 & 1850.0 & 1890.0 & 1970.0 & 2110.0 & 2340.0 & 3400.0 \\ 1911 & 1490.0 & 1520.0 & 1540.0 & 1580.0 & 1650.0 & 1820.0 & 1850.0 & 1890.0 & 2140.0 & 2670.0 & 3310.0 \\ 1912 & 1700.0 & 1790.0 & 1870.0 & 1920.0 & 1940.0 & 2210.0 & 2230.0 & 2420.0 & 2810.0 & 3320.0 & 3910.0 \\ 1913 & 1600.0 & 1650.0 & 1720.0 & 1780.0 & 1930.0 & 2010.0 & 2280.0 & 2490.0 & 2790.0 & 2970.0 & 4000.0 \\ 1914 & 1420.0 & 1420.0 & 1460.0 & 1480.0 & 1500.0 & 1590.0 & 1790.0 & 1920.0 & 2170.0 & 2220.0 & 2700.0\end{array}$

McKenz1e R1ver near Springfield, Oreg.

STATION NUMBER $14-1640.00$

HIGHEST MEAN DISCHARGF, IN CFS, FOR THF FOLLOWING NUMRFR OF CONSFCUTIVF DAYS IN YEAR ENDING SFPTEMBER 30

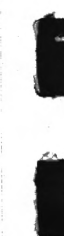

$11800.0 \quad 10500.0$

$46000.036 n .0$

$\begin{array}{cc}7 & 15 \\ 936 n .0 & 7880.0\end{array}$

36500.028300 .0

$28000.0-24200.0$

$51400.0 \quad 42100.0$

$18900.0 \cdot 17200.0$

$30000.0 \quad 24700.0$

$25300.0-16400.0$

$19300 . n$

28800.013400 .0

13400.010800 .0

$24700.0 \quad 17400.0 \quad 13200.0$

15600.0 14500.n 12400.0 9320.0

30
6510.0
14000.0
11900.0
10400.0
13900.0
8400.0
10300.0
9190.0
7390.0

630

6330.0
11000.0

11000.0
9160.0

8680.0

9070.0

6180.0

9160.0

7850.0

90
5780.0
9520.0
7750.0
7590.0
7830.0
5560.0
7460.0
7190.0
6460.0

120
5600.0
8830.0
7270.0
6750.0
8260.0
5070.0
7030.0
6680.0
6180.0

150
5500.0

8370.0

6940.0

6260.0

7650 .

4890 .

6420.

6390.

$572 n .0$
183

5400.0

$8100.0 \quad 4570.0$

6620.0

5680.0

$5960.0 \quad 5050.0$

$7110.0 \quad 5730.0$

$4890.0 \quad 4300.0$

$\begin{array}{ll}6290.0 & 5840.0 \\ 5360.0 & 4580.0\end{array}$ 
CLASS $0 \begin{array}{lllllllllllllllllllllllllllllllllll} & 0 & 2 & 3 & 4 & 5 & 6 & 7 & 8 & 9 & 10 & 11 & 12 & 13 & 14 & 15 & 16 & 17 & 18 & 19 & 20 & 21 & 22 & 23 & 24 & 25 & 26 & 27 & 28 & 29 & 30 & 31 & 32 & 33 & 34\end{array}$

YEAR
1936
1937
1938
1939
1940
1941
1942
1943
1944
1945
1946
1947
1948
1949
1950
1951
1952

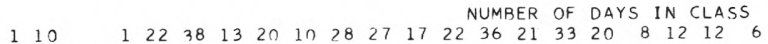

$\begin{array}{llllllllllllllllllllllllllllll}10 & 30 & 22 & 16 & 20 & 15 & 18 & 20 & 24 & 18 & 13 & 24 & 23 & 16 & 17 & 12 & 36 & 15 & 5 & 6 & 3 & 2 & 1\end{array}$

$\begin{array}{rrrrrrrrrrrrrrrrrrrrrrrrrr}11 & 31 & 18 & 9 & 7 & 9 & 7 & 17 & 16 & 26 & 15 & 6 & 7 & 9 & 16 & 14 & 18 & 25 & 50 & 24 & 11 & 8 & 10 & & 1 \\ & 2 & 20 & 31 & 25 & 13 & 7 & 8 & 8 & 54 & 18 & 22 & 21 & 28 & 13 & 7 & 19 & 24 & 26 & 8 & 6 & 3 & 1 & 1\end{array}$

$\begin{array}{lllllllllllllllllllllllll}1 & 26 & 15 & 21 & 36 & 26 & 20 & 21 & 8 & 17 & 12 & 6 & 14 & 34 & 27 & 18 & 16 & 8 & 17 & 8 & 10 & 5\end{array}$

$\begin{array}{rrrrrrrrrrrrrrrrrrrrrrrr}2 & 8 & 19 & 15 & 13 & 24 & 19 & 19 & 36 & 38 & 38 & 42 & 19 & 17 & 27 & 11 & 10 & 5 & 3 & & \\ 12 & 29 & 8 & 5 & 8 & 15 & 32 & 22 & 27 & 19 & 41 & 28 & 27 & 33 & 11 & 16 & 14 & 6 & 5 & 2 & 5\end{array}$

$\begin{array}{rrrrrrrrrrrrrrrrrrrr}5 & 16 & 21 & 31 & 14 & 14 & 19 & 12 & 14 & 13 & 16 & 29 & 28 & 19 & 19 & 21 & 14 & 28 & 24 \\ 16 & 17 & 7 & 26 & 36 & 18 & 23 & 58 & 23 & 17 & 28 & 7 & 12 & 4\end{array}$

$\begin{array}{lllllllllllllllllllll}28 & 42 & 18 & 13 & 18 & 14 & 36 & 18 & 8 & 10 & 16 & 22 & 12 & 38 & 16 & 30 & 15 & 4 & 4 & 3\end{array}$

$\begin{array}{lllllllllllllllllllllllll}19 & 28 & 42 & 11 & 13 & 12 & 19 & 26 & 14 & 7 & 7 & 9 & 19 & 12 & 29 & 24 & 29 & 22 & 9 & 3 & 7 & 2\end{array}$ $\begin{array}{rrrrrrrrrrrrrrrrrrrrrr}5 & 13 & 29 & 14 & 25 & 13 & 31 & 31 & 18 & 10 & 34 & 22 & 13 & 33 & 14 & 31 & 11 & 8 & 2 & 5 \\ 1 & 17 & 16 & 19 & 24 & 7 & 23 & 14 & 10 & 9 & 18 & 28 & 20 & 61 & 34 & 27 & 15 & 11 & 6 & 2\end{array}$

$\begin{array}{lllllllllllllllllllllllllll}15 & 27 & 20 & 15 & 6 & 21 & 20 & 26 & 13 & 17 & 16 & 28 & 18 & 16 & 33 & 21 & 21 & 10 & 7 & 2 & 4 & 5 & 2\end{array}$

$\begin{array}{rrrrrrrrrrrrrrrrrrrrrrrrrrr}2 & 15 & 25 & 25 & 23 & 20 & 18 & 8 & 16 & 13 & 11 & 9 & 18 & 15 & 19 & 34 & 20 & 28 & 24 & 6 & 8 & 4 & 2 & 1 & 1 \\ 21 & 21 & 13 & 16 & 6 & 8 & 15 & 9 & 15 & 15 & 15 & 8 & 17 & 14 & 17 & 18 & 29 & 41 & 15 & 8 & 18 & 10 & 5 & & 1\end{array}$

$\begin{array}{rrrrrrrrrrrrrrrrrrrrrrrr}1 & 6 & 9 & 25 & 19 & 9 & 9 & 8 & 24 & 28 & 18 & 17 & 6 & 17 & 11 & 18 & 36 & 30 & 36 & 17 & 7 & 5 & 8 & 2\end{array}$
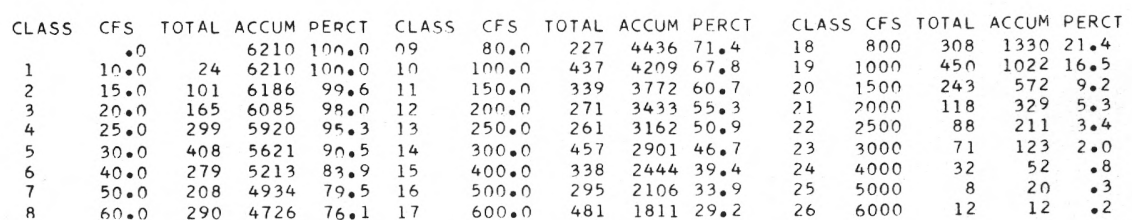

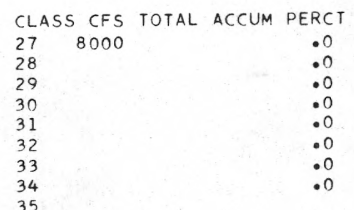

Mohawk River near Springfield, Oreg.

STATION NUMBER $\quad 14-1650.00$

LOWEST MEAN DISCHARGE, IN CFS, FOR THE FOLLOWING NUMBER OF CONSECUTIVE DAYS IN YEAR BEGINNING APRIL 1

\begin{tabular}{|c|c|c|c|c|c|c|c|c|c|c|c|}
\hline YEAR & 1 & 3 & 7 & 14 & 30 & 60 & 90 & 120 & 150 & 183 & 274 \\
\hline 1936 & 21.0 & 21.3 & 21.9 & 23.6 & 25.9 & 28.0 & 35.4 & $36 \cdot 7$ & 45.7 & 61.9 & 153.0 \\
\hline 1937 & 36.0 & 37.7 & $42 \cdot 3$ & 50.4 & 55.0 & 63.9 & 75.2 & 98.7 & $200 \cdot 0$ & 233.0 & 550.0 \\
\hline 1938 & 11.0 & 13.0 & 14.4 & 14.7 & 15.5 & 19.8 & 23.7 & $28 \cdot 5$ & $42 \cdot 6$ & 78.6 & 240.0 \\
\hline 1939 & 22.0 & 23.3 & 24.0 & 24.7 & 27.0 & 32.7 & 35.4 & 47.0 & 49.2 & $66 \cdot 3$ & 141.0 \\
\hline 1940 & 14.0 & $15 . n$ & $15 \cdot 3$ & 16.0 & 17.3 & 22.0 & 26.4 & $30 \cdot 2$ & $44 \cdot 3$ & 76.9 & 247.0 \\
\hline 1941 & 33.0 & 33.7 & 34.9 & 37.1 & 44.3 & 60.2 & 75.5 & 89.0 & 109.0 & 146.0 & 424.0 \\
\hline 1942 & 22.0 & 24.0 & $24 \cdot 1$ & 25.3 & 27.5 & $31 \cdot 2$ & 39.1 & $60 \cdot 7$ & 103.0 & 169.0 & 543.0 \\
\hline 1943 & 29.0 & $31 \cdot n$ & 32.6 & 34.5 & 35.3 & 46.2 & 55.4 & $82 \cdot 1$ & $132 \cdot 0$ & 178.0 & 251.0 \\
\hline 1944 & 18.0 & $19 \cdot 3$ & 20.4 & 22.8 & 24.7 & 28.2 & 30.1 & $36 \cdot 2$ & 49.2 & 65.0 & 164.0 \\
\hline 1945 & 26.0 & $26 . n$ & 28.1 & 31.5 & 33.1 & 39.5 & $40 \cdot 1$ & $48 \cdot 2$ & 67.4 & 155.0 & 490.0 \\
\hline 1946 & 20.0 & 20.0 & 20.7 & $22 \cdot 8$ & 24.9 & 27.7 & $30 \cdot 1$ & 38.9 & 54.9 & $82 \cdot 6$ & 378.0 \\
\hline 1947 & 36.0 & 37.0 & $38 \cdot 6$ & $40 \cdot 1$ & 46.9 & 52.1 & 65.0 & $87 \cdot 3$ & 113.0 & 153.0 & 462.0 \\
\hline 1948 & 28.0 & 30.0 & 32.1 & 34.2 & 39.8 & $49 \cdot 1$ & 66.2 & $75 \bullet 8$ & 94.4 & 133.0 & 399.0 \\
\hline 1949 & 18.0 & 19.3 & 21.0 & 22.0 & 23.6 & 28.0 & 33.0 & $39 \cdot 0$ & 47.0 & 64.5 & 247.0 \\
\hline 1950 & 17.0 & 18.7 & 20.7 & 22.6 & $25 \cdot 3$ & 30.4 & 38.8 & $59 \cdot 7$ & 102.0 & 161.0 & 566.0 \\
\hline 1951 & 11.0 & 11.7 & 12.9 & 14.9 & $18 \cdot 3$ & 19.4 & 26.1 & $42 \cdot 5$ & 67.0 & 112.0 & 409.0 \\
\hline
\end{tabular}

Mohawk River near Springfield, Oreg.

STATION NUMBER $\quad 14-1630.00$

HIGHEST MEAN DISCHARGE, IN CFS, FOR THE FOLLOWING NUMBER OF CONSECUTIVE DAYS IN YEAR ENDING SEPTEMBER 30

YEAR
1936
1937
1938
1939
1940
1941
1942
1943
1944
1945
1946
1947
1948
1949
1950
1951
1952

1
5180.0
4980.0
5230.0
4310.0
2720.0
2270.0
4580.0
7680.0
1800.0
3210.0
6820.0
6220.0
7720.0
7690.0
6380.0
6100.0
4270.0

$$
\begin{gathered}
3 \\
4870.0 \\
4460.0 \\
4050.0 \\
3430.0 \\
2470.0 \\
1780 . n \\
4170 . n \\
6780.0 \\
1520.0 \\
2780.0 \\
5930 . n \\
5480.0 \\
5930.0 \\
5830.0 \\
5340.0
\end{gathered}
$$

\begin{tabular}{|c|c|}
\hline 7 & 15 \\
\hline 850.0 & 2990.0 \\
\hline & 2190.0 \\
\hline 10.0 & 2570.0 \\
\hline & 2070.0 \\
\hline 290.0 & 2150.0 \\
\hline $38 \cap . n$ & 1160.0 \\
\hline 3350.0 & 2300.0 \\
\hline $210 . n$ & 3690.0 \\
\hline 10.0 & 1010.0 \\
\hline 0.0 & 2030.0 \\
\hline 0.0 & $3100 \cdot 0$ \\
\hline 0.0 & $2360 \cdot 0$ \\
\hline $40 \cdot 0$ & 2810.0 \\
\hline 0.0 & 3190.0 \\
\hline 820.0 & 2530.0 \\
\hline 0.0 & 2980.0 \\
\hline & \\
\hline
\end{tabular}

$5930.0 \quad 3830.0$

$5480.0 \quad 3930.0$

$5930.0 \quad 4140.0$

3820.0

3610.0

3210.0

30
2080.0
$1780 \cdot 0$
$1750 \cdot 0$
$1500 \cdot 0$
$1910 \cdot 0$
$987 \cdot 0$
$1880 \cdot 0$
$2890 \cdot 0$
$766 \cdot 0$
$1570 \cdot 0$
$2170 \cdot 0$
$2050 \cdot 0$
1760.0
2090.0
2210.0
2420.0
1760.0

60
1480.0
1540.0
1570.0
1330.0
1350.0
860.0
1530.0
2420.0
606.0
1290.0
1650.0
1330.0
1450.0
1390.0
1990.0
1820.0
1420.0

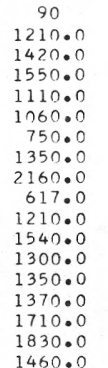

120
$1030 \bullet 0$
$1200 \bullet 0$
$1480 \bullet 0$
$984 \cdot 0$
$933 \cdot 0$
$657 \cdot 0$
$1170 \cdot 0$
$1750 \bullet 0$
$575 \bullet 0$
$1100 \bullet 0$
$1460 \bullet 0$
$1160 \bullet 0$
$1220 \bullet 0$
$1240 \bullet 0$
$1470 \bullet 0$
$1760 \bullet 0$
$1330 \cdot 0$

150
910.0
1060.0
1460.0
873.0
827.0
576.0
1020.0
1590.0
532.0
1000.0
1320.0
1110.0
1200.0
1110.0
1290.0
1620.0
1250.0

183
807.0
939.0
1300.0
760.0
712.0
523.0
885.0
1400.0
504.0
864.0
1140.0
972.0
1140.0
1040.0
1130.0
1420.0
1110.0

274

591.0
678.0

678.0
917.0

547.0

547.0
497.0

416.0
691.0

1020.0

1020.0
397.0

616.0
795.0

795.0
712.0

887.0

742.0

801.0

1010.0

805.0

S-DAYS 89128.0 253703.0 . 19905.0 194867.0 84290 72234.0 . 01780 .

.

9067.0
4849.0 


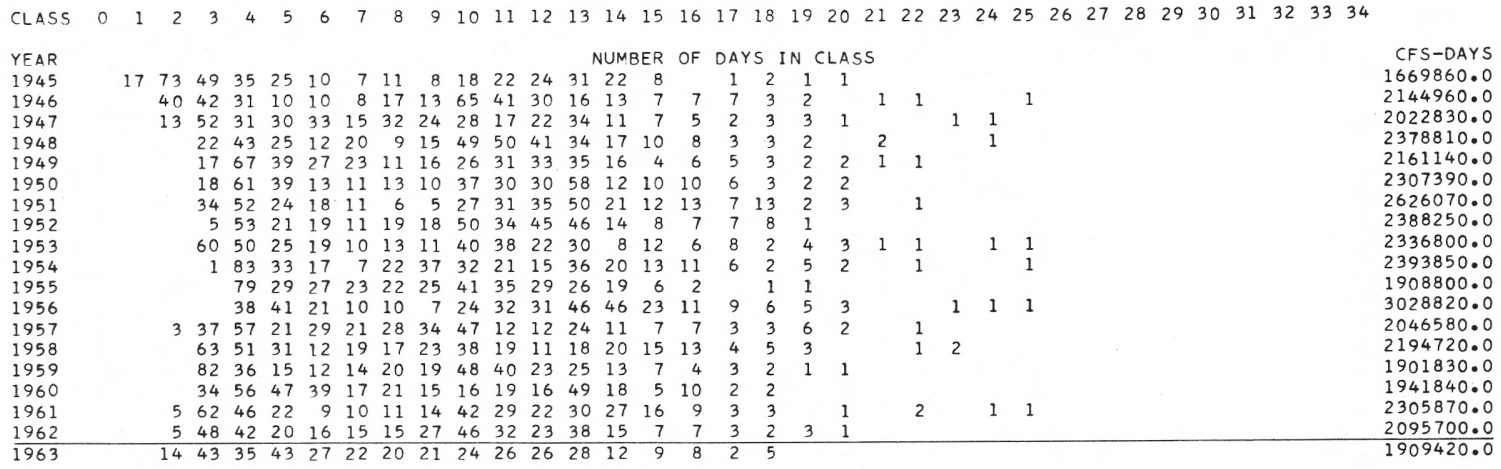

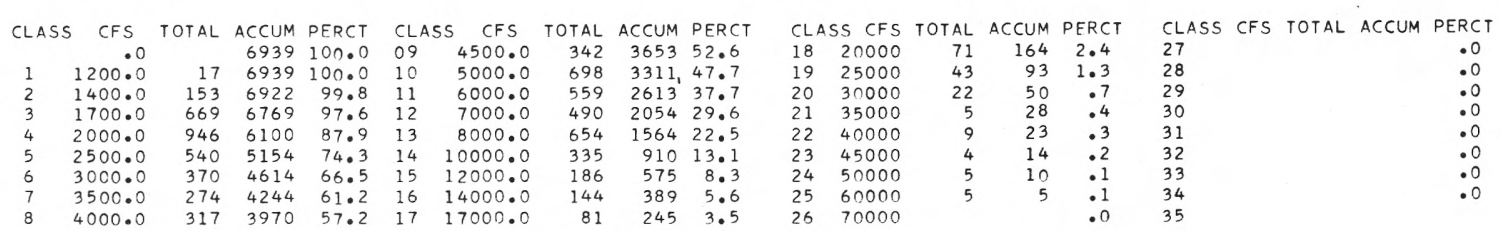

McKenzie River near Coburg, Oreg. Summary for water years 1945-1962

\begin{tabular}{lrrrr} 
CLASS & \multicolumn{1}{c}{ CFS } & TOTAL & ACCUM & PERCT \\
1 & 1200.0 & 17 & 6574 & 100.0 \\
2 & 1400.0 & 139 & 6557 & 100.0 \\
3 & 1700.0 & 626 & 6418 & 99.7 \\
4 & 2000.0 & 911 & 5792 & 88.1 \\
5 & 2500.0 & 497 & 4881 & 74.2 \\
6 & 3000.0 & 343 & 4384 & 66.7 \\
7 & 3500.0 & 252 & 4041 & 61.5 \\
8 & 4000.0 & 297 & 3789 & 57.6
\end{tabular}

STATION NUMBER

$14-1655.00$
McKenzie River near Coburg, Oreg.

Summary for water year 1963

\begin{tabular}{lrrrrrrr} 
CLASS & CFS & TOTAL & ACCUM & PERCT & \multicolumn{1}{l}{ CLASS } & \multicolumn{1}{c}{ CFS } & TOI \\
& .0 & & 365 & 100.0 & 09 & 4500.0 & \\
1 & 1200.0 & & 365 & 100.0 & 10 & 5000.0 & \\
2 & 1400.0 & 14 & 365 & 100.0 & 11 & 6000.0 & \\
3 & 1700.0 & 43 & 351 & 96.2 & 12 & 7000.0 & \\
4 & 2000.0 & 35 & 308 & 84.4 & 13 & 8000.0 & \\
5 & 2500.0 & 43 & 273 & 74.8 & 14 & 10000.0 & \\
6 & 3000.0 & 27 & 230 & 63.0 & 15 & 12000.0 & \\
7 & 3500.0 & 22 & 203 & 55.6 & 16 & 14000.0 & \\
8 & 4000.0 & 20 & 181 & 49.6 & 17 & 17000.0 &
\end{tabular}

STATION NUMBER

$\begin{array}{rrlr}\text { ACCUM } & \text { PERCT } & \text { CLASS } & \text { CF } \\ 3492 & 53.1 & 18 & 2000 \\ 3171 & 48.2 & 19 & 25000 \\ 2497 & 38.0 & 20 & 3000 \\ 1964 & 29.9 & 21 & 35000 \\ 1500 & 22.8 & 22 & 40000 \\ 874 & 13.3 & 23 & 45000 \\ 551 & 8.4 & 24 & 5000 \\ 374 & 5.7 & 25 & 6000 \\ 238 & 3.6 & 26 & 7000\end{array}$
20000 35000

CFS TOTAL ACCUM PERCT CLASS CFS TOTAL ACCUM PERCT 30000 40000
45000 50000 60000 70000

$\begin{array}{rrrllllr}\text { OTAL } & \text { ACCUM } & \text { PERCT } & \text { CLASS } & \text { CFS } & \text { TOTAL } & \text { ACCUM } & \text { PERCT } \\ 66 & 159 & 2.4 & 27 & & & & .0 \\ 43 & 93 & 1.4 & 28 & & & & .0 \\ 22 & 50 & .8 & 29 & & & & .0 \\ 5 & 28 & .4 & 30 & & & & .0 \\ 9 & 23 & .3 & 31 & & & & .0 \\ 4 & 14 & .2 & 32 & & & & .0 \\ 5 & 10 & .2 & 33 & & & & .0 \\ 5 & 5 & .1 & 34 & & & \end{array}$

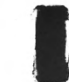

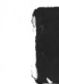

\begin{tabular}{|c|c|c|c|c|c|c|c|c|c|c|c|}
\hline ACCUM & PERCT & CL.ASS & CFS & TOTAL & ACCUM & PERCT & CLASS & CFS & TOTAL & ACCUM & PERCT \\
\hline 21 & 44.1 & 18 & 20000 & 5 & 5 & 1.4 & 27 & & & & .0 \\
\hline 140 & 38.4 & 19 & 25000 & & & .0 & 28 & & & & 0 \\
\hline 116 & 31.8 & 20 & 30000 & & & .0 & 29 & & & & \\
\hline 90 & 24.7 & 21 & 35000 & & & .0 & 30 & & & & \\
\hline 64 & 17.5 & 22 & 40000 & & & .0 & 31 & & & & \\
\hline 36 & 9.9 & 23 & 45000 & & & .0 & 32 & & & & \\
\hline 24 & 6.6 & 24 & 50000 & & & .0 & 33 & & & & \\
\hline 15 & 4.1 & 25 & 60000 & & & .0 & 34 & & & & \\
\hline 7 & 1.9 & 26 & 70000 & & & .0 & 35 & & & & \\
\hline
\end{tabular}


McKenzie River near Coburg, Oreg.

STATION NUMBER $14-1655.00$ LOWEST MEAN DISCHARGE, IN CFS, FOR THE FOLLOWING NUMBER OF CONSECUTIVE DAYS IN YEAR BEGINNING APRIL 1

\begin{tabular}{|c|c|c|c|c|c|c|c|c|c|c|c|}
\hline EAR & 1 & 3 & 7 & 14 & 30 & 60 & 90 & 120 & 150 & 183 & 274 \\
\hline 45 & 1410.0 & 1410.0 & 1420.0 & 1430.0 & 1470.0 & 1540.0 & 1580.0 & 1670.0 & 1860.0 & 2560.0 & 4920.0 \\
\hline & 1540.0 & 1550.0 & 1550.0 & 1630.0 & 1660.0 & 1760.0 & 1880.0 & 2190.0 & 2520.0 & 2990.0 & 5010.0 \\
\hline 47 & 1700.0 & 1710.0 & 1720.0 & 1740.0 & 1830.0 & 1860.0 & 2010.0 & $2320 \cdot 0$ & 2590.0 & 3080.0 & 4750.0 \\
\hline & 1840.0 & 1870.0 & 1870.0 & 1910.0 & 2000.0 & 2180.0 & 2390.0 & $2490 \cdot 0$ & 2780.0 & 3490.0 & 4750.0 \\
\hline & 1840.0 & 1850.0 & 1890.0 & 1970.0 & 2010.0 & 2060.0 & $2100 \cdot 0$ & $2180 \cdot 0$ & 2320.0 & 2790.0 & 4260.0 \\
\hline & 2120.0 & 2130.0 & 2170.0 & 2210.0 & 2280.0 & 2410.0 & 2660.0 & $3030 \cdot 0$ & 3920.0 & 4560.0 & 6840.0 \\
\hline & 1810.0 & 1840.0 & 1860.0 & 1890.0 & 1940.0 & 1980.0 & 2110.0 & $2350 \cdot 0$ & 2740.0 & 3430.0 & 5120.0 \\
\hline & 1790.0 & 1830.0 & 1840.0 & 1860.0 & 1880.0 & 1910.0 & 1970.0 & 2060.0 & 2240.0 & 2590.0 & 3920.0 \\
\hline & 1920.0 & 1960.0 & 1990.0 & $2000 \cdot 0$ & 2090.0 & 2220.0 & 2290.0 & $2400 \cdot 0$ & 2750.0 & 3610.0 & 6010.0 \\
\hline & 2170.0 & 2190.0 & $2200 \cdot 0$ & 2230.0 & 2380.0 & 2400.0 & 2460.0 & $2540 \cdot 0$ & 2740.0 & 2930.0 & 3860.0 \\
\hline & 2100.0 & 2130.0 & 2140.0 & 2170.0 & 2230.0 & 2290.0 & 2570.0 & 2970.0 & 3560.0 & 4360.0 & 7520.0 \\
\hline & 2250.0 & 2260.0 & 2270.0 & 2280.0 & 2310.0 & 2370.0 & 2520.0 & $2820 \cdot 0$ & 3310.0 & 3660.0 & 4830.0 \\
\hline & 1650.0 & 1740.0 & 1760.0 & 1780.0 & 1820.0 & 1940.0 & 1990.0 & $2060 \cdot 0$ & 2240.0 & 2440.0 & 4350.0 \\
\hline & 1730.0 & 1800.0 & 1830.0 & 1850.0 & 1880.0 & 1910.0 & 1960.0 & 2080.0 & 2410.0 & 2870.0 & 4450.0 \\
\hline & 1700.0 & 1720.0 & 1730.0 & 1740.0 & 1760.0 & 1840.0 & 2000.0 & $2250 \cdot 0$ & 2480.0 & 2690.0 & 3270.0 \\
\hline & 1680.0 & 1680.0 & $1690 . ?$ & 1720.0 & 1780.0 & 1880.0 & 1960.0 & $2080 \cdot 0$ & 2300.0 & 3240.0 & 4980.0 \\
\hline & 1620.0 & 1700.0 & 1730.0 & 1760.0 & 1830.0 & 1890.0 & 1970.0 & $2120 \cdot 0$ & 2470.0 & 2840.0 & 4820.0 \\
\hline & 1650.0 & 1660.0 & 1680.0 & 1710.0 & 1820.0 & 1920.0 & 2060.0 & 2450.0 & 2650.0 & 3190.0 & 4320.0 \\
\hline
\end{tabular}

McKenz1e R1ver near Coburg, Oreg.

STATION NUMBER $14-1655.00$

HIGHEST MEAN DISCHARGE, IN CFS, FOR THE FOLLOWING NUMBER OF CONSECUTIVE DAYS IN YEAR ENDING SEPTEMRFR 30

\begin{tabular}{|c|c|c|c|c|c|c|c|c|c|c|c|}
\hline YEAR & 1 & 3 & 7 & 15 & 30 & 60 & 90 & 120 & 150 & 183 & 274 \\
\hline 1945 & 30800.0 & $24800 \cdot 0$ & 19700.0 & 15400.0 & 10700.0 & 9100.0 & 8970.0 & $8630 \cdot 0$ & 8260.0 & $\begin{array}{l}7330.0 \\
8950.0\end{array}$ & $\begin{array}{l}5580.0 \\
7250.0\end{array}$ \\
\hline 46 & $65900 \cdot 0$ & $47900 \cdot 0$ & $30700 \cdot 0$ & 23100.0 & 16400.0 & 12400.0 & $\begin{array}{l}11100.0 \\
10400.0\end{array}$ & $\begin{array}{r}10400 \cdot 0 \\
9360 \cdot 0\end{array}$ & $\begin{array}{l}9570.0 \\
9110.0\end{array}$ & 8370.0 & 6750.0 \\
\hline $\begin{array}{l}1947 \\
1948\end{array}$ & $\begin{array}{l}51400.0 \\
56500.0\end{array}$ & $\begin{array}{l}44200 \cdot 0 \\
41700 \cdot 0\end{array}$ & $30400 \cdot 0$ & $18800 \cdot 0$ & $15700 \cdot 0$ & $\begin{array}{l}10700.0 \\
11100.0\end{array}$ & 9870.0 & $\begin{array}{l}9360.0 \\
9370.0\end{array}$ & 9070.0 & 8670.0 & 7910.0 \\
\hline $\begin{array}{l}1948 \\
1949\end{array}$ & $\begin{array}{l}56500.0 \\
43100.0\end{array}$ & $\begin{array}{l}41700.0 \\
33700.0\end{array}$ & $\begin{array}{l}28400.0 \\
24300.0\end{array}$ & $\begin{array}{l}20100 \cdot 0 \\
17100.0\end{array}$ & $\begin{array}{l}13200 \cdot 0 \\
12800 \cdot 0\end{array}$ & & 10100.0 & 9170.0 & 8510.0 & 8650.0 & 7140.0 \\
\hline 950 & 34700.0 & 26600.0 & 19900.0 & 16700.0 & 14800.0 & 13000.0 & 11900.0 & $10800 \cdot 0$ & 10200.0 & $9390 \cdot 0$ & 7660.0 \\
\hline 51 & 44300.0 & 31100.0 & 25700.0 & 17700.0 & 16400.0 & 13000.0 & 13000.0 & $12900 \cdot 0$ & 11800.0 & 11000.0 & 8890.0 \\
\hline 1952 & 27800.0 & 24500.0 & 21300.0. & 15600.0 & 12000.0 & 9870.0 & 10000.0 & 9320.0 & 9290.0 & 8950.0 & 7870.0 \\
\hline 1953 & 62100.0 & $54600 . n$ & 38500.0 & 26900.0 & 23700.0 & 16600.0 & 13600.0 & $11800 \cdot 0$ & $11000 \cdot 0$ & 10200.0 & 7880.0 \\
\hline 1954 & 64300.0 & 41700.0 & 25100.0 & 18500.0 & 18200.0 & 13300.0 & 13300.0 & $12000 \cdot 0$ & $11100 \cdot 0$ & $10000 \cdot 0$ & 7940.0 \\
\hline 1955 & 27600.0 & 22300.0 & 15202.0 & 10600.0 & 9940.0 & 8990.0 & 8590.0 & $7870 \cdot 0$ & 7460.0 & 7440.0 & 6160.0 \\
\hline 1956 & 68900.0 & 55900.0 & 37100.0 & 27100.0 & 21500.0 & 18400.0 & 15400.0 & $13700 \cdot 0$ & $12900 \cdot 0$ & 12300.0 & 10100.0 \\
\hline 1957 & $40100 \cdot 0$ & $33400 . ?$ & $23600 \cdot 0$ & 20200.0 & 16200.0 & 12300.0 & $10000 \cdot 0$ & $9700 \cdot 0$ & 8960.0 & $8200 \cdot 0$ & 6780.0 \\
\hline 958 & 49100.0 & 40800.0 & 26600.0 & 20700.0 & 15500.0 & 14500.0 & 12800.0 & $11000 \cdot 0$ & 10400.0 & $9440 \cdot 0$ & 7340.0 \\
\hline 359 & $33000 \cdot 0$ & 24100.0 & 17300.0 & 12300.0 & 11500.0 & 9450.0 & 8820.0 & $8430 \cdot 0$ & $8220 \cdot 0$ & $7770 \cdot 0$ & 6290.0 \\
\hline 60 & 21600.0 & 19700.0 & 15300.0 & $12400 \cdot 0$ & 11600.0 & 10300.0 & 9940.0 & $9600 \cdot 0$ & 8740.0 & 7830.0 & 6380.0 \\
\hline 361 & 61600.0 & 47900.0 & 31800.0 & 22200.0 & 17000.0 & 14000.0 & 11500.0 & $11000 \cdot 0$ & $10500 \cdot 0$ & 9850.0 & 7780.0 \\
\hline 62 & 33100.0 & 27800.0 & 21300.0 & 15700.0 & 12500.0 & 10700.0 & 8960.0 & $8570 \cdot 0$ & 8680.0 & 8480.0 & 6980.0 \\
\hline & 2000.0 & 21500.0 & 16800.0 & 12600.0 & 10200.0 & 8810.0 & 8060.0 & $8090 \cdot 0$ & 7310.0 & 7580.0 & $6300 \cdot 0$ \\
\hline
\end{tabular}




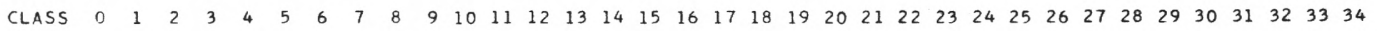

\begin{tabular}{|c|c|c|c|c|c|c|c|c|c|c|c|c|c|c|c|c|c|c|c|c|c|c|c|c|c|c|c|c|}
\hline YEAR & & & & & & & & & & & & & UMBER & OF $D$ & DAY & $S \mathrm{I}$ & $\mathrm{NCl}$ & LASS & & & & & & & & & \multirow{2}{*}{\multicolumn{2}{|c|}{$\begin{array}{r}\text { CFS-DAYS } \\
3459210.0\end{array}$}} \\
\hline 1945 & & 4757 & 34 & 20 & 16 & 11 & 17 & 11 & 7 & 19 & 11 & 222 & $28 \quad 16$ & 231 & 16 & 5 & 2 & & & 2 & & 1 & & \multirow{2}{*}{\multicolumn{3}{|c|}{1}} & & \\
\hline 1946 & & 2547 & 22 & 17 & 5 & 4 & 10 & 6 & 12 & 29 & 55 & 333 & 3316 & 121 & 10 & 4 & 11 & 4 & 3 & 3 & 2 & & 1 & & & & \multicolumn{2}{|c|}{4564690.0} \\
\hline 1947 & & 49 & 29 & 16 & 9 & 7 & 35 & 18 & 29 & 44 & 25 & 162 & 2118 & 22 & 8 & 5 & 4 & 2 & 2 & 1 & 1 & 1 & 2 & & & & \multicolumn{2}{|c|}{4125360.0} \\
\hline 1948 & & 7 & 26 & 30 & 15 & 11 & 10 & 13 & 14 & 16 & 26 & 356 & $66 \quad 34$ & 211 & 13 & 9 & 5 & 4 & 5 & & 2 & 1 & 1 & 1 & & & \multicolumn{2}{|c|}{5321890.0} \\
\hline 1949 & & 26 & 49 & 25 & 11 & 14 & 29 & 16 & 7 & 19 & 21 & 233 & $36 \quad 30$ & 31 & 5 & 4 & 5 & 3 & 1 & 6 & 1 & 1 & 2 & & & & \multirow{2}{*}{\multicolumn{2}{|c|}{4508280.0}} \\
\hline 1950 & & 11 & 25 & 54 & 26 & 9 & 11 & 6 & 8 & 23 & 22 & 274 & $48 \quad 31$ & 211 & 13 & 13 & 6 & 3 & 2 & 4 & 2 & & & & & & 4773040.0 & \\
\hline 951 & & 34 & 31 & 18 & 14 & 10 & 17 & 9 & 10 & 24 & 21 & 304 & $45 \quad 17$ & 29 & 9 & 10 & 81 & 10 & 31 & 10 & 4 & & 1 & & & & \multirow{2}{*}{\multicolumn{2}{|c|}{5524470.0}} \\
\hline 952 & & & 24 & 39 & 13 & 7 & 15 & 10 & 16 & 40 & 29 & 335 & 5128 & 221 & 10 & 9 & 4 & 4 & 5 & 4 & 3 & & & & & & 4992360.0 & \\
\hline 953 & & 333 & 33 & 26 & 33 & 10 & 16 & 13 & 9 & 30 & 32 & 262 & 2316 & 16 & 13 & 10 & 5 & 1 & 1 & 6 & 3 & 2 & 2 & 1 & & & \multicolumn{2}{|c|}{4803050.0} \\
\hline 1954 & & & 10 & 23 & 65 & 20 & 28 & 35 & 22 & 30 & 13 & 13 & 1119 & 241 & 11 & 9 & 6 & 8 & 5 & 7 & 4 & 1 & 1 & & & & \multirow{2}{*}{\multicolumn{2}{|c|}{4880490.0}} \\
\hline 955 & & & 1 & 7 & 36 & 60 & 35 & 27 & 13 & 38 & 29 & 372 & 2518 & 25 & 7 & 5 & & 1 & 1 & & & & & & & & $3760550 \cdot 0$ & \\
\hline 1956 & & & & & 14 & 51 & 19 & 18 & 11 & 14 & 23 & 343 & $\begin{array}{ll}37 & 27\end{array}$ & 331 & 10 & 23 & 20 & 8 & 61 & 10 & 5 & 1 & 1 & & & & \multicolumn{2}{|c|}{6765400.0} \\
\hline 1957 & & & 8 & 34 & 59 & 15 & 19 & 13 & 11 & 56 & 42 & 18 & 167 & 18 & 15 & 6 & 7 & 4 & 2 & 6 & 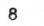 & 1 & & & & & \multirow{2}{*}{\multicolumn{2}{|c|}{4764860.0}} \\
\hline 1958 & & & & 4 & 51 & 46 & 33 & 17 & 27 & 51 & 29 & 12 & 16 & 17 & 12 & 10 & 10 & 9 & 4 & 4 & 2 & 1 & 1 & & & & \multirow{2}{*}{\multicolumn{2}{|c|}{4757800.0}} \\
\hline 1959 & & & 3 & 32 & 58 & 18 & 25 & 18 & 14 & 46 & 31 & 322 & 2221 & 20 & 11 & 6 & 4 & 2 & & 2 & & & & & & & 3991260.0 & \\
\hline 1960 & & & 2 & 6 & 56 & 39 & 36 & 22 & 24 & 42 & 25 & 202 & 2121 & 24 & 8 & 11 & 4 & 5 & & & & & & & & & \multirow{2}{*}{\multicolumn{2}{|c|}{$\begin{array}{l}4052110.0 \\
4697490.0\end{array}$}} \\
\hline 1961 & & & & & 42 & 66 & 22 & 19 & 16 & 59 & 17 & 18 & 1716 & 291 & 14 & 10 & 9 & 2 & 4 & 1 & 2 & 1 & 1 & & & & & \\
\hline 1962 & & & & 6 & 71 & 30 & 22 & 16 & 25 & 49 & 27 & 312 & 2911 & 19 & 7 & 8 & 5 & 3 & 1 & 3 & 2 & & & & & & \multicolumn{2}{|c|}{4168880.0} \\
\hline 1963 & & & & 9 & 40 & 30 & 41 & 35 & 41 & 29 & 30 & 172 & 2120 & 20 & 9 & 10 & 7 & 3 & 1 & 2 & & & & & & & \multicolumn{2}{|c|}{4161720.0} \\
\hline LLASS & CFS & TOTAL & $A C C$ & & PER & & & ASS & & CF & & OTAL & ACCUM & & $R C T$ & & CLA & & CFS & & TAL & ACCUM & PERCT & & & $A L$ & ICCUM & ERCT \\
\hline & . & & & 39 & 100 & & 09 & & & 00.0 & & 322 & 4405 & & 3.5 & & 18 & & 000 & & 167 & & & 7 & 100 & 8 & 11 & .2 \\
\hline 1 & 1700.0 & & & 39 & 100 & & 10 & & & 00.0 & & 316 & 4083 & & .8 & & 19 & & 000 & & 122 & 389 & 5. & 28 & 120 & 1 & 3 & .0 \\
\hline 2 & & 75 & & 39 & ion & & 11 & & 800 & 00.0 & & 658 & 3767 & & .3 & & 20 & 400 & 000 & & 76 & & & 29 & & 1 & 2 & \\
\hline 3 & 2500 & 264 & 68 & 364 & & 3.9 & 12 & & 1000 & 00.0 & & 508 & 3109 & & 4.8 & & 21 & 450 & 000 & & 46 & 191 & 2 . & 30 & 160000 & 1 & 1 & .0 \\
\hline 4 & & 297 & & 60 & & .1 & 13 & & 200 & 00.0 & & 477 & 2601 & & .5 & & 22 & 500 & 0 & & 7 & 14 & 2 . & 31 & 180000 & & & .0 \\
\hline 5 & & 366 & 63 & 303 & in & .8 & 14 & & 400 & 0.0 & & 566 & 2124 & & .6 & & 23 & 600 & 000 & & 41 & 14 & 1. & 32 & & & & .0 \\
\hline 6 & & 634 & & 37 & & & 15 & & & 00.0 & & 375 & 1558 & & & & 24 & & & & 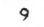 & 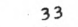 & & 3 & & & & .0 \\
\hline 7 & & 458 & & . & & .4 & 6 & & & ho. & & 426 & 1183 & & & & 25 & $8 \cap 0$ & 000 & & 3 & & - & 34 & & & & .0 \\
\hline 8 & 5000.0 & 440 & & 45 & & .8 & 17 & & 500 & 00.0 & & 201 & 757 & 10. & .9 & & 26 & & $n 00$ & & 8 & 19 & 3 & 35 & & & & \\
\hline
\end{tabular}

W1llamette River at Harrisburg, Oreg. STATION NUMBER 14-1660.00

LOWEST MEAN DISCHARGE, IN CFS, FOR THE FOLLOWING NUMBER OF CONSECUTIVE DAYS IN YEAR BEGINNING APRIL 1

$\begin{array}{cc}1 & 3 \\ 2230.0 & 2240.0 \\ 2470.0 & 2500.0 \\ 2530.0 & 2530.0 \\ 2970.0 & 3000.0 \\ 2820.0 & 2820.0 \\ 3590.0 & 3600.0 \\ 2650.0 & 2650.0 \\ 2420.0 & 2450.0 \\ 3250.0 & 3280.0 \\ 3420.0 & 3650.0 \\ 3800.0 & 3930.0 \\ 3130.0 & 3170.0 \\ 3330.0 & 3470.0 \\ 4070.0 & 4240.0 \\ 3050.0 & 3230.0 \\ 3790.0 & 3880.0 \\ 3780.0 & 3930.0 \\ 4010.0 & 4160.0 \\ & \end{array}$

7
2240.0
2560.0
2530.0
$3050 \cdot 0$
2830.0
3640.0
2670.0
2490.0
3370.0
$4010 \cdot 0$
4120.0
3300.0
3730.0
4290.0
3540.0
4120.0
3990.0
4200.0

14
2260.0
2680.0
2580.0
3380.0
2860.0
3730.0
2720.0
2580.0
3460.0
4060.0
4330.0
3530.0
3830.0
4340.0
3710.0
4230.0
4120.0
4250.0

30
2350.0
2730.0
2750.0
3480.0
2920.0
3790.0
2830.0
2660.0
3640.0
4260.0
4390.0
4210.0
4010.0
4440.0
3850.0
4300.0
4340.0
4310.0
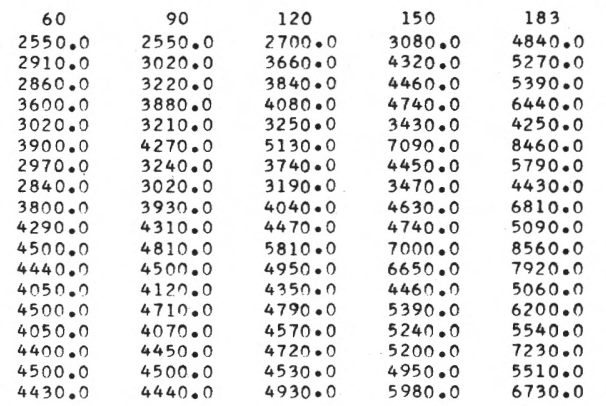

274

10500.0 9900.0 $-10000.0$ 9870.0
8050.0 8050.0
14200.0 14200.0
10200.0 14200.0
7160.0 7160.0
12600.0 12600.0
6500.0 16000.0
10700.0 9240.0 9140.0 6570.0 10300.0 10100.0

9430.0
0

W1llamette River at Harrisburg, Oreg.

STATION NUMBER

$14-1660.00$

HIGHEST MEAN DISCHARGF, IN CFS, FOR THF FOLLOWING NUMBER OF CONSECUTIVE DAYG IN YFAR ENDING SEPTFMRFR 30

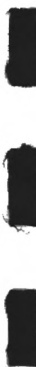

\begin{tabular}{|c|c|c|}
\hline YEAR & 1 & 3 \\
\hline 1945 & 75100.0 & 54400.0 \\
\hline 1946 & 173000.0 & 111000.0 \\
\hline 1947 & 116000.0 & 102000.0 \\
\hline 1948 & 146000.0 & 108000.0 \\
\hline 1949 & 97300.0 & 84200.7 \\
\hline 1950 & 66300.0 & 61000.0 \\
\hline 1951 & 108000.0 & 84900.0 \\
\hline 1952 & 67500.0 & $63500 . n$ \\
\hline 1953 & $134 n n 0.0$ & ח. ח0707י \\
\hline 1954 & 104000.0 & $85500 . n$ \\
\hline 1955 & .47000 .0 & 41800.0 \\
\hline 1956 & 104000.0 & 90300.0 \\
\hline 1957 & 77800.0 & 66600.0 \\
\hline 1958 & 91700.0 & 73700.0 \\
\hline 1959 & 57500.0 & 44100.0 \\
\hline 1960 & 44900.0 & 41900.0 \\
\hline 1961 & 111000.0 & 78600.0 \\
\hline 1962 & 65400.0 & $54500 . n$ \\
\hline 1963 & 58700.0 & 52300.0 \\
\hline
\end{tabular}

43600.0
70600.0
67100.0
74700.0
$60800 . ?$
48300.0
64900.0
$53000 . ?$
87700.0
67500,0
29800.0
74700.0
61200.0
57300.0
37200.0
36400.0
61500.0
45800.0
40800.0

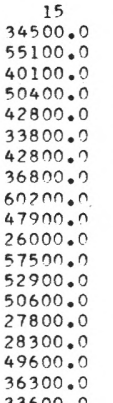

30
23100.0
37800.0
35700.0
32100.0
30700.0
31300.0
38200.0
27400.0
53500.0
44100.0
23700.0
49400.0
43000.0
37300.0
26100.0
26200.0
38600.0
28900.0
27000.0
60
20100.0
28600.0
23500.0
26400.0
22600.0
29600.0
30500.7
23000.0
37000.0
32600.0
19600.0
45300.0
31300.0
33200.0
21400.0
22800.0
30600.0
25400.0
20100.0 90
20000.0
25200.0
22400.0
24100.0
22400.0
26800.0
30000.0
23300.0
$27 n n n .0$
21800.0
18100.0
37900.0
24300.0
30100.0
19700.0
20800.0
24100.0
20100.0
16700.0 120
$19100 \cdot 0$
$24000 \cdot 0$
$20100 \cdot 0$
$22300 \cdot 0$
$20100 \cdot 0$
$23700 \cdot 0$
29500.0
21500.0
25400.0
27600.0
$16200 \cdot 0$
33300.0
24200.0
24800.0
18700.0
$20200 \cdot 0$
$22800 \cdot 0$
17700.0
16400.0 150
18100.0
21900.0
19800.0
21600.0
19600.0
22200.0
26900.0
21100.0
23700.0
24900.0
15500.0
30800.0
21700.0
22600.0
17700.0
18200.0
21700.0
18000.0
15000.0 183
15800.0
20100.0
17900.0
20600.0
19600.0
20500.0
24500.0
19900.0
21700.0
21700.0
15200.0
28500.0
20600.0
20200.0
16300.0
16400.0
19900.0
17100.0
16200.0 274 11800.0 15700.0 14000.0 18200.0
15400.0 16200.0 19100.0 16900.0 16500.0 16400.0 23100.0 23100.0 16000.0 15800.0 13200.0 13300.0 13700.0 13600.0 


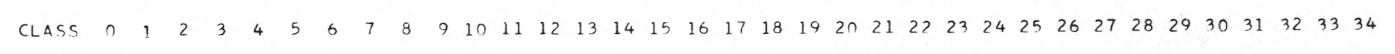

YEAR
1936
1937
1938
1939
1940
1941
1942
1943
1944
1945
1946
1947
1948
1949
1950
1951
1952
1953
1954
1955
1956
1957
1958
1959
1960
1961
1962
1963

NUMBER OF DAYS IN CLACS

$\begin{array}{llllllllllllllllllllll}7 & 37 & 32 & 19 & 22 & 23 & 11 & 31 & 21 & 37 & 25 & 24 & 17 & 16 & 10 & 6 & 5 & 10 & 8 & 1 & 2 & 2\end{array}$

$\begin{array}{rrrrrrrrrrrrrrrrrrrrrrrrrr}10 & 54 & 19 & 23 & 29 & 19 & 11 & 25 & 14 & 29 & 17 & 10 & 13 & 25 & 18 & 13 & 15 & 4 & 10 & 4 & 3 & & \\ 10 & 46 & 17 & 15 & 34 & 13 & 16 & 16 & 9 & 17 & 8 & 10 & 6 & 33 & 27 & 16 & 26 & 12 & 18 & 10 & 2 & 3 & 1\end{array}$

$6 \begin{array}{llllllllllllllllllllll}53 & 21 & 23 & 18 & 15 & 33 & 11 & 33 & 21 & 28 & 23 & 14 & 13 & 15 & 6 & 11 & 10 & 6 & 5\end{array}$

$\begin{array}{rrrrrrrrrrrrrrrrrrrrrrr}1 & 25 & 57 & 41 & 27 & 1 & 14 & 5 & 13 & 19 & 32 & 26 & 16 & 16 & 14 & 11 & 7 & 14 & 6 & 8 & 3 \\ 14 & 44 & 31 & 12 & 14 & 20 & 16 & 32 & 24 & 61 & 23 & 17 & 11 & 22 & 10 & 3 & 6 & 2 & 2 & 1\end{array}$

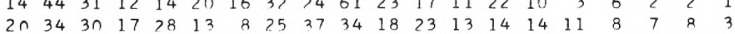

$\begin{array}{lllllllllllllllllllllll}10 & 31 & 27 & 8 & 20 & 13 & 13 & 21 & 15 & 25 & 31 & 23 & 11 & 19 & 12 & 9 & 20 & 11 & 23 & 7 & 4 & 4\end{array}$

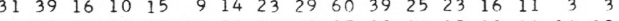

$\begin{array}{lllllllllllllllllllll}10 & 54 & 22 & 12 & >4 & 16 & 13 & 22 & >1 & 37 & 29 & 24 & 15 & 18 & 14 & 10 & 12 & 5 & 5\end{array}$

$\begin{array}{rrrrrrrrrrrrrrrrrrrrrrr}8 & 69 & 27 & 3 & 22 & 15 & 18 & 13 & 13 & 19 & 18 & 13 & 21 & 26 & 18 & 16 & 18 & 10 & 12 & 3 \\ 29 & 31 & 20 & 26 & 25 & 12 & 30 & 13 & 24 & 22 & 31 & 15 & 28 & 23 & 11 & 6 & 10 & 4 & 4\end{array}$

$\begin{array}{rrrrrrrrrrrrrrrrrrrrrrr}10 & 22 & 16 & 22 & 15 & 11 & 15 & 12 & 18 & 27 & 30 & 32 & 61 & 29 & 10 & 14 & 5 & 9 & 5\end{array}$

$\begin{array}{rrrrrrrrrrrrrrrrrrrrr}4 & 40 & 19 & 28 & 20 & 23 & 14 & 11 & 10 & 44 & 24 & 18 & 19 & 36 & 12 & 9 & 9 & 9 & 6 & 5 \\ 31 & 29 & 43 & 19 & 16 & 6 & 15 & 18 & 9 & 22 & 21 & 9 & 13 & 19 & 18 & 13 & 24 & 14 & 19\end{array}$

$\begin{array}{rrrrrrrrrrrrrrrrrrrrrrrr}31 & 29 & 43 & 19 & 16 & 6 & 15 & 18 & 9 & 22 & 21 & 9 & 13 & 19 & 18 & 13 & 24 & 14 & 19 & 5 \\ 15 & 34 & 8 & 15 & 16 & 14 & 6 & 14 & 11 & 41 & 14 & 7 & 14 & 22 & 31 & 21 & 22 & 16 & 13 & 4\end{array}$

$\begin{array}{llllllllllllllllllll}15 & 24 & 28 & 15 & 10 & 8 & 20 & 30 & 11 & 25 & 34 & 8 & 14 & 31 & 24 & 15 & 27 & 10 & 13\end{array}$

$\begin{array}{llllllllllllllllllllll}18 & 18 & 37 & 25 & 27 & 12 & 7 & 9 & 14 & 25 & 30 & 34 & 19 & 20 & 14 & 14 & 12 & 13 & 12 & 12 \\ & & 53 & 30 & 24 & 25 & 7 & 27 & 16 & 19 & 10 & 15 & 12 & 29 & 21 & 11 & 17 & 15 & 19 & 10\end{array}$

$\begin{array}{rrrlllllllllllllllllll}6 & 28 & 25 & 10 & 36 & 28 & 19 & 19 & 12 & 18 & 28 & 24 & 29 & 37 & 20 & 10 & 9 & 4 & 3\end{array}$

$\begin{array}{lllllllllll}2 & 2 & 5 & 1 & 1 & 1\end{array}$

$\begin{array}{llllllllllllllllllllll}18 & 30 & 25 & 15 & 23 & 24 & 13 & 28 & 14 & 40 & 32 & 26 & 15 & 27 & 9 & 5 & 4 & 7 & 4 & 4 & 2\end{array}$

$\begin{array}{rrrrrrrrrrrrrrrrrrrrrrr}28 & 35 & 29 & 12 & 21 & 14 & 17 & 18 & 74 & 21 & 13 & 24 & 13 & 18 & 19 & 15 & 15 & 11 & 9 & 5 & 3 & 1 & \\ 44 & 33 & 18 & 8 & 15 & 16 & 8 & 21 & 17 & 53 & 24 & 23 & 9 & 22 & 17 & 7 & 9 & 7 & 7 & 3 & 3 & & 1\end{array}$

$\begin{array}{rrrrrrrrrrrrrrrrrrrrrrrrrr}16 & 30 & 43 & 25 & 28 & 11 & 12 & 21 & 13 & 28 & 36 & 27 & 16 & 18 & 19 & 7 & 2 & 8 & 4 & & 1 & 1 & \\ 37 & 41 & 25 & 14 & 11 & 12 & 7 & 9 & 12 & 31 & 44 & 23 & 13 & 16 & 10 & 7 & 29 & 6 & 9 & 4 & 2 & & 3\end{array}$

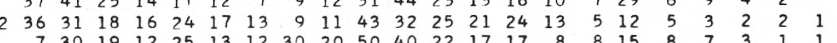

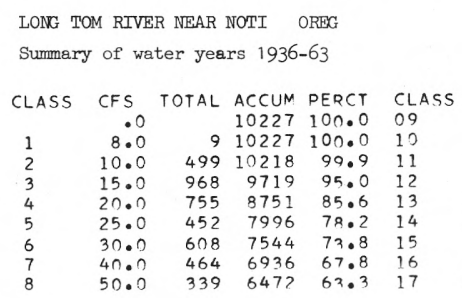

STATION NUMBER

$14-1665.00$

CFS-DAYS

73871.0

R1397.0

54622.0

54622.0

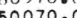

(5)

(1)

$125053 \cdot 0$
47388.0

66776.0

66776.

96423.0

(1)

9558.0

120703.0

2กา

01212.0

930

25343.0

48344.0

त8

ล819

79013.0

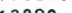

63890.0

$71856 \cdot 1$
CFS TOTAL ACCUM PERCT $60.0 \quad 598 \quad 613360.0$ 80.0 00.0 50.0 00.0 $\begin{array}{ll}452 \\ 00.0 & 282\end{array}$
596513560.0

$907 \quad 507949.7$

$\begin{array}{lll}702 & 4172 & 40.8\end{array}$

$64 \quad 2472 \quad 24.2$

$\begin{array}{lll}52408 & 17.7\end{array}$

135613.3 $\begin{array}{lrrrr}\text { CLASS } & \text { CFS } & \text { TOTAL } & \text { ACCUM } & \text { PERCT } \\ 18 & 600 & 384 & 1074 & 10.5 \\ 19 & 800 & 236 & 690 & 6.7 \\ 20 & 1000 & 267 & 454 & 4.4 \\ 21 & 1500 & 103 & 187 & 1.8 \\ 22 & 2000 & 37 & 84 & .8 \\ 23 & 2500 & 28 & 47 & .5 \\ 24 & 3000 & 14 & 19 & .2 \\ 25 & 4000 & 4 & 5 & .0\end{array}$

$\begin{array}{lc}\text { CLASS CFS TOTAL ACCUM PERCT } \\ 27 \\ 28 & .0 \\ 29 & .0 \\ 30 & .0 \\ 31 & .0 \\ 32 & .0 \\ 33 & \\ 34 & .0 \\ 35 & .0 \\ & \end{array}$ 
$\begin{array}{lllllllllllllllllllllllllllllllllllll}\text { CLASS } & 0 & 1 & 2 & 3 & 4 & 5 & 6 & 7 & 8 & 9 & 10 & 11 & 12 & 13 & 14 & 15 & 16 & 17 & 18 & 19 & 20 & 21 & 22 & 23 & 24 & 25 & 26 & 27 & 28 & 29 & 30 & 31 & 32 & 33 & 34\end{array}$

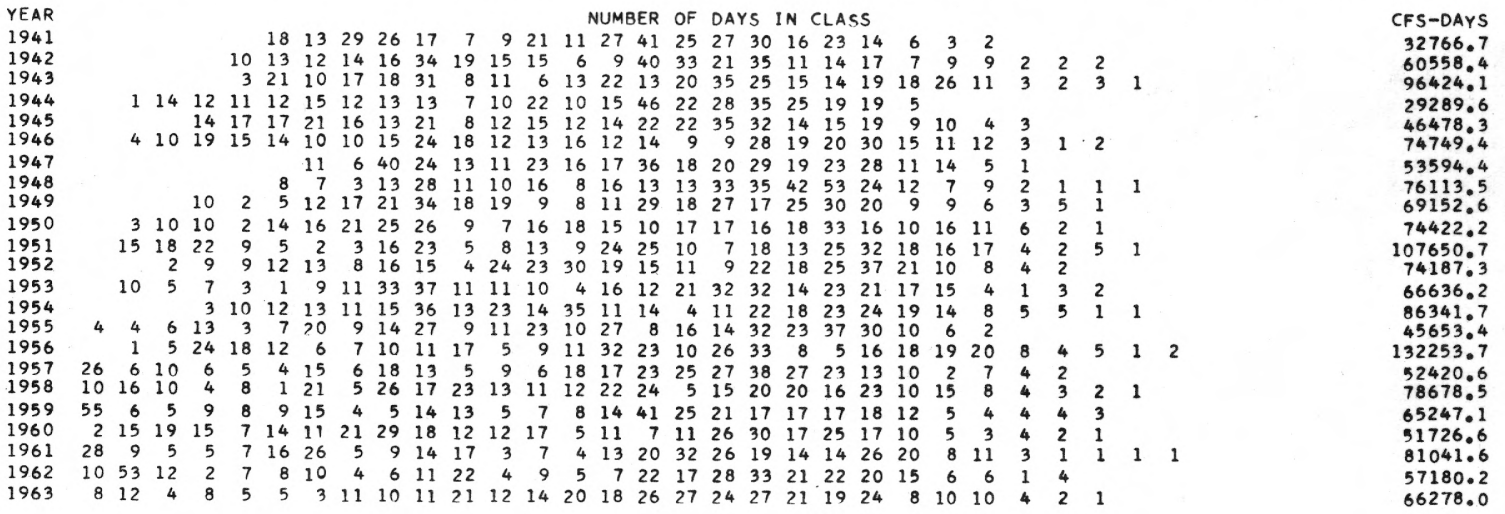

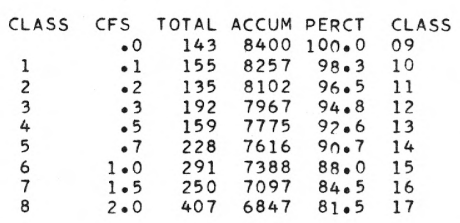

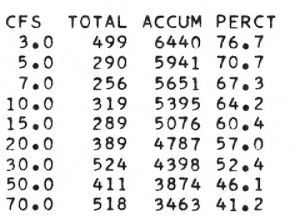
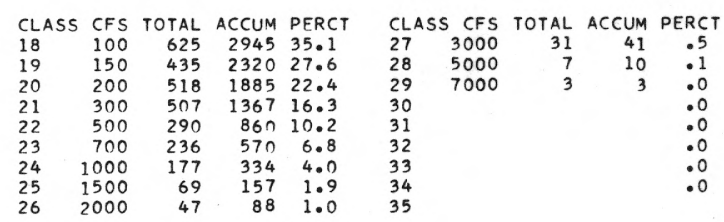

Coyote Creek near Crow, Oreg.

STATION NUMBER

$14-1670.00$

LOWEST MEAN DISCHARGE, IN CFS, FOR THE FOLLOWING NUMBER OF CONSECUTIVE DAYS IN YEAR RFGINNING APRIL 1
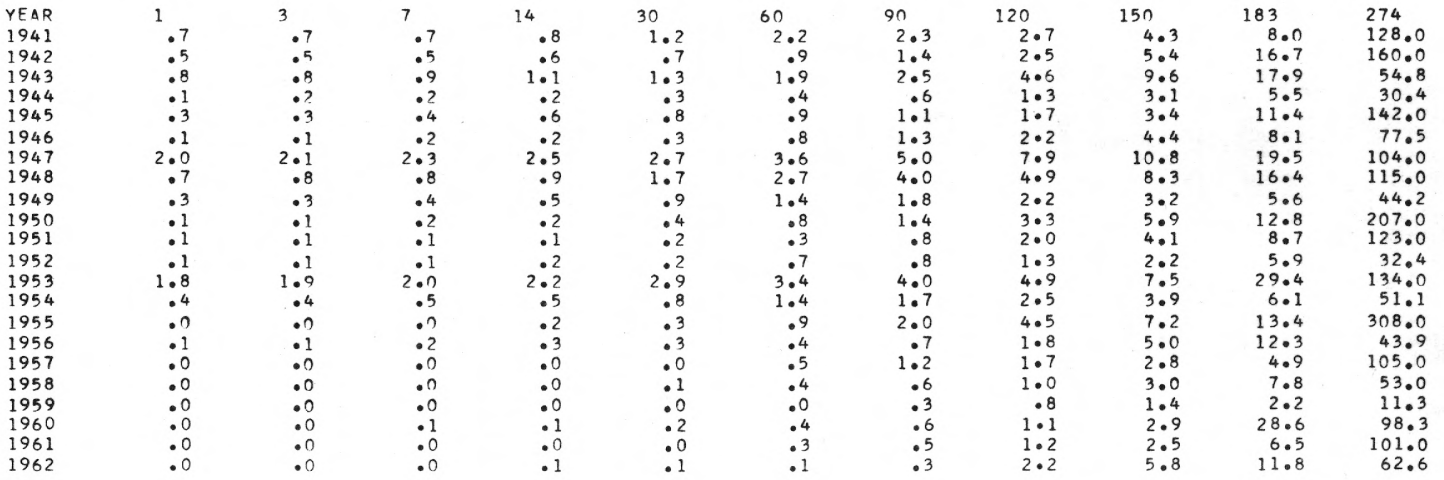

Coyote Creek near Crow, Oreg.

STATION NUMRER

$14-1670.00$

HIGHEST MEAN DISCHARFF, IN CFS, FOR THF FOLLOWING NUMRFR OF CONSFCUTIVF DAYS IN YEAR ENDING SEPTFMBFR 30

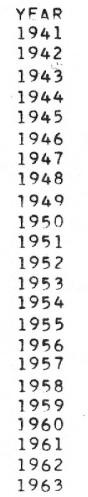

1
1280.0
3700.0
5070.0
599.0
1930.0
4530.0
1620.0
6500.0
4940.0
3570.0
6100.0
2940.0
3270.0
5540.0
1170.0
8650.0
2720.0
5200.0
4930.0
3240.0
7200.0
2170.0
3650.0

\begin{tabular}{|c|}
\hline 914.0 \\
\hline $\begin{array}{l}2840.0 \\
4640.0\end{array}$ \\
\hline \\
\hline 1450.0 \\
\hline 3290.0 \\
\hline $1290 . n$ \\
\hline 3320.7 \\
\hline 3010.7 \\
\hline $2610 . n$ \\
\hline $4620 . n$ \\
\hline $2410 . ?$ \\
\hline 2910.0 \\
\hline 969.0 \\
\hline $6020 . n$ \\
\hline 2210.0 \\
\hline $3940 . ?$ \\
\hline $\begin{array}{l}3200 \cdot n \\
2330 . n\end{array}$ \\
\hline 5220.0 \\
\hline 1850.0 \\
\hline \\
\hline
\end{tabular}

7
727.0
1780.0
2950.0
$468 . n$
1070.0
1860.0
887.0
$2150 . n$
2080.0
2010.0
2620.0
1850.0
2030.0
2360.0
678.0
4840.0
1430.0
2270.0
2260.0
1570.0
2950.0
1250.0
1510.0

15

559.0
1050.0

1800.0

369.0
805.0

1420.0

594.0

$16>0.0$

$1310 . ?$

1430.0

1170.0
1470.0

1470.0
1810.0

546.0
$3090 . ?$

3090.2

1500.0

1520.0

969.
1740.0

1740.0
739.0

934.0

30
428.0
857.0
1330.0
285.0
517.0
881.0
510.0
776.0
997.0
1060.0
1200.0
774.0
1050.0
1120.0
432.0
2140.0
921.0
1020.0
986.0
658.0
1190.0
604.0
785.0

60
343.0
619.0
975.0
232.0
486.0
710.0
364.0
628.0
608.0
837.0
847.0
736.0
714.0
875.0
368.0
1500.0
568.0
904.0
815.0
563.0
922.0
447.0
566.0

90
264.0
567.0
868.0
197.0
412.0
619.0
344.0
513.0
586.0
693.0
838.0
650.0
569.0
752.0
292.0
1180.0
436.0
776.0
593.0
451.0
673.0
350.0
462.0

120
$223 \cdot 0$
$456 \bullet 0$
$679 \cdot 0$
$172 \cdot 0$
$344 \cdot 0$
$563 \cdot 0$
$331 \cdot 0$
$433 \bullet 0$
$498 \cdot 0$
$576 \bullet 0$
$763 \cdot 0$
$547 \bullet 0$
$476 \bullet 0$
$644 \bullet 0$
$301 \cdot 0$
$1040 \bullet 0$
$374 \cdot 0$
$612 \cdot 0$
$492 \bullet 0$
$391 \bullet 0$
$588 \cdot 0$
$366 \bullet 0$
$424 \cdot 0$

$\begin{array}{ll}183 & 274 \\ 169.0 & 119.0 \\ 316.0 & 220.0 \\ 515.0 & 351.0 \\ 148.0 & 107.0 \\ 247.0 & 169.0 \\ 404.0 & 272.0 \\ 281.0 & 194.0 \\ 374.0 & 276.0 \\ 369.0 & 252.0 \\ 401.0 & 271.0 \\ 580.0 & 393.0 \\ 394.0 & 270.0 \\ 354.0 & 243.0 \\ 465.0 & 314.0 \\ 242.0 & 166.0 \\ 710.0 & 482.0 \\ 273.0 & 191.0 \\ 425.0 & 287.0 \\ 351.0 & 238.0 \\ 280.0 & 189.0 \\ 436.0 & 296.0 \\ 306.0 & 209.0 \\ 349.0 & 241.0\end{array}$


Amazon Creek near Eugene, Oreg.

STATION NUMBER 14-1695.00

DURATION TARLE OF DATLY NISCHARGE

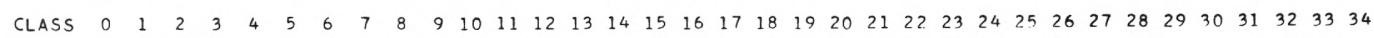

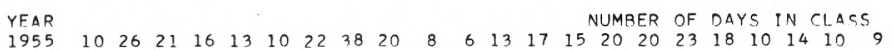

$\begin{array}{rrrrrrrrrrrrrrrrrrrrrrrrr}1955 & 10 & 26 & 21 & 16 & 13 & 10 & 22 & 38 & 20 & 8 & 6 & 13 & 17 & 15 & 20 & 20 & 23 & 18 & 10 & 14 & 10 & 9 & 4 & 2\end{array}$

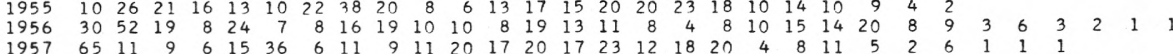

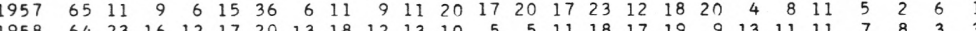

$\begin{array}{rrrrrrrrrrrrrrrrrrrrrrrrrrrrrrrr}1958 & 64 & 23 & 16 & 12 & 17 & 20 & 13 & 18 & 12 & 13 & 10 & 5 & 5 & 11 & 18 & 17 & 19 & 9 & 13 & 11 & 11 & 7 & 8 & 3 & 3 \\ 1959 & 25 & 37 & 25 & 26 & 17 & 19 & 6 & 10 & 5 & 14 & 14 & 9 & 25 & 28 & 11 & 13 & 19 & 14 & 8 & 12 & 9 & 7 & 1 & 3 & 3\end{array}$

$\begin{array}{rrrrrrrrrrrrrrrrrrrrrrrrrrrrrrrrr}1958 & 64 & 23 & 16 & 12 & 17 & 20 & 13 & 18 & 12 & 13 & 10 & 5 & 5 & 11 & 18 & 17 & 19 & 9 & 13 & 11 & 11 & 7 & 8 & 3 & 3 & & 4 & 1 & 1 & 1 \\ 1959 & 25 & 37 & 25 & 26 & 17 & 19 & 6 & 10 & 5 & 14 & 14 & 9 & 25 & 28 & 11 & 13 & 19 & 14 & 8 & 12 & 9 & 7 & 1 & 3 & 3 & 2 & & 2 & 1 & 1 \\ 1960 & 21 & 9 & 25 & 15 & 44 & 39 & 20 & 15 & 8 & 8 & 3 & 5 & 13 & 14 & 8 & 21 & 23 & 17 & 8 & 16 & 11 & 12 & 1 & 4 & 2 & 2 & 2 & & & 1\end{array}$

$\begin{array}{rrrrrrrrrrrrrrrrrrrrrrrrrrrrrrr}1962 & 26 & 16 & 12 & 8 & 14 & 14 & 19 & 32 & 10 & 14 & 7 & 11 & 15 & 22 & 29 & 25 & 32 & 9 & 10 & 8 & 14 & 3 & 6 & 4 & 4 & 1 & \\ 1963 & 5 & 6 & 8 & 3 & 9 & 28 & 15 & 40 & 19 & 22 & 10 & 3 & 13 & 29 & 26 & 17 & 33 & 14 & 16 & 10 & 14 & 5 & 10 & 2 & 2 & 3 & 3\end{array}$

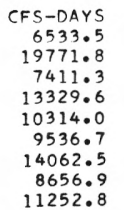

Amazon Creek near Eugene, Oreg.

STATION MIYUMBR $14-1695.00$

Summary for water years 1955-63
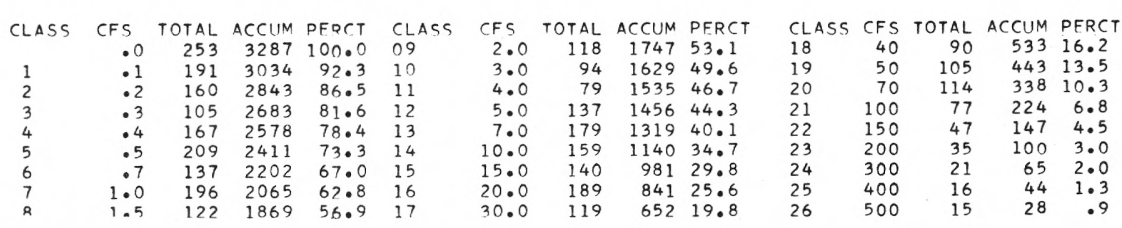

$\begin{array}{lrrrr}\text { CLASS } & \text { CFS } & \text { TOTAL } & \text { ACCUM } & \text { PERCT } \\ 27 & 700 & 6 & 13 & .4 \\ 28 & 1000 & 4 & 7 & .2 \\ 29 & 1500 & 3 & 3 & : 1 \\ 30 & & & & .0 \\ 31 & & & & .0 \\ 32 & & & & .0 \\ 33 & & & & .0 \\ 34 & & & & .0\end{array}$

Amazon Creek near Eugene, Oreg.

STATION NUMBER

$14-10 y 3.00$

LOWEST MEAN DISCHARGE, IN CFS, FOR THE FOLLOWING NUMBER OF CONSECUTIVE DAYS IN YEAR BEGINNING APRIL 1

\begin{tabular}{|c|c|c|c|c|c|c|c|c|c|c|c|}
\hline YEAR & 1 & 3 & 7 & 14 & 30 & 60 & 90 & 120 & 150 & 183 & 274 \\
\hline 1955 & . 1 & $\cdot 1$ & $\cdot 1$ & .1 & .2 & .2 & .5 & .7 & 1.2 & 2.0 & 49.7 \\
\hline 1956 & .0 & . $n$ & .0 & .0 & .1 & .1 & .1 & .2 & .4 & 1.4 & 6.3 \\
\hline 1957 & .0 & - $n$ & . n & .0 & .0 & .0 & .1 & $\cdot 3$ & .3 & .5 & 19.4 \\
\hline 1958 & .0 & . $n$ & .0 & .0 & .0 & .0 & .1 & $\cdot 2$ & .4 & 1.0 & 7.6 \\
\hline 1959 & .0 & $\cdot 0$ & .0 & .0 & .1 & .1 & .1 & $\cdot 2$ & .3 & .4 & 1.4 \\
\hline 1960 & .0 & .0 & .0 & .1 & .1 & .4 & .4 & .5 & .6 & 5.9 & $20 \cdot 3$ \\
\hline 1961 & .0 & .1 & .1 & .1 & . 3 & .4 & .5 & .5 & .8 & 1.7 & 17.2 \\
\hline 196$\rangle$ & .0 & .0 & .1 & .2 & .2 & .6 & .6 & $\cdot 9$ & 2.4 & $4 \cdot 2$ & 16.0 \\
\hline
\end{tabular}

Amazon Creek near Eugene, Oreg.

STATION NIMRFR 14-10Y2.110

HIGHEST MEAN DISCHARGE, IN CFS, FOR THE. FOLLOWING NUMBER OF CONSFCUTIVE DAYS IN YEAR ENDING SEPTFMBER 30

YEAR
1955
1956
1957
1958
1959
1960
1961
1962
1963

$\begin{array}{rr}1 & 3 \\ 260.0 & 206.0 \\ 1990.0 & 1160.0 \\ 520.0 & 403.0 \\ 1780.0 & 954.0 \\ 1280.0 & 597.0 \\ 689.0 & 405.0 \\ 2380.0 & 1220.0 \\ 412.0 & 340.0 \\ 580.0 & 422.0\end{array}$

7
136.0
789.0
233.0
507.0
375.0
306.0
610.0
220.0

15
97.5
504.0
176.0
310.0
210.0
178.0
349.0
134.0

30
72.4
344.0
137.0
185.0
158.0
114.0
213.0
111.0

60
56.2
238.0
83.7
171.0
131.0
91.9
158.0
68.4
83.3

90
43.6
178.0
61.8
137.0
94.6
75.6
113.0
56.6
64.2

120
$43 \cdot 5$
$157 \cdot 0$
$52 \cdot 7$
$107 \cdot 0$
$77 \cdot 6$
70.4
105.0
52.6
60.5

150
40.1
128.0
44.7
87.5
66.6
62.3
88.3
51.1
57.2

$183 \quad 274$

$\begin{array}{rr}34.6 & 23.7 \\ 106.0 & 72.1\end{array}$

$39.1 \quad 27.0$

$72.3 \quad 48.6$

$\begin{array}{ll}55.3 & 37.6 \\ 51.5 & 34.7\end{array}$

$75.7 \quad 51.2$

$45.3 \quad 31.3$ 


\begin{tabular}{|c|c|c|c|c|c|c|c|c|c|c|c|c|c|c|c|c|c|c|c|c|c|c|c|c|c|c|c|c|c|c|c|c|c|}
\hline YEAR & & & & & & & & & & & & & & & UMBE & & OF & DAY & (S I & IN $\mathrm{C}$ & CLASS & & & & & & & & & & & & CFS-DAYS \\
\hline 1922 & & & & 11 & 101 & 18 & 41 & 14 & 24 & 14 & 20 & 10 & 21 & 14 & 3 & 2 & 11 & 9 & 8 & 30 & 182 & 28 & 25 & 20 & 3 & 7 & 6 & 8 & & & & & 287156.0 \\
\hline 1923 & & & & 1 & 52 & 22 & 21 & 16 & 27 & 12 & 28 & 36 & 30 & 10 & 5 & 11 & 7 & 11 & 15 & 15 & 142 & 27 & 10 & 5 & 71 & 12 & 10 & 3 & 2 & 1 & 1 & 1 & 288664.0 \\
\hline 1924 & & & 192 & 281 & 171 & 12 & 19 & 28 & 24 & 24 & 18 & 11 & 24 & 15 & 7 & 14 & 14 & 20 & 20 & 17 & 101 & 11 & 3 & 4 & 4 & 3 & & & & & & & 112581.0 \\
\hline 1975 & & & 21 & 112 & 271 & 19 & 15 & 16 & 12 & 12 & 19 & 8 & 17 & 11 & 15 & 13 & 10 & 11 & 11 & 12 & 82 & 22 & 16 & 15 & 161 & 15 & 2 & 8 & 14 & 7 & 1 & & 448050.0 \\
\hline 1928 & & & & 41 & 131 & 18 & 13 & 12 & 8 & 4 & 20 & 18 & 19 & 43 & 7 & 6 & 10 & 11 & 7 & 28 & 253 & 30 & 25 & 19 & 9 & 7 & 8 & 2 & & & & & 290265.0 \\
\hline 1929 & & & & 74 & 491 & 16 & 16 & 13 & 8 & 9 & 14 & 11 & 41 & 23 & 9 & 9 & 31 & 24 & 19 & 23 & 6 & 9 & 4 & 7 & 6 & 6 & 4 & 1 & & & & & 168275.0 \\
\hline 1930 & & & & & 594 & 45 & 24 & 8 & 15 & 7 & 13 & 7 & 12 & 16 & 16 & 19 & 30 & 19 & 6 & 6 & 111 & 12 & 12 & 7 & 6 & 8 & 4 & 1 & 1 & 1 & & & 189071.0 \\
\hline 1931 & & & & 234 & 422 & 22 & 12 & 26 & 5 & 9 & 30 & 13 & 31 & 15 & 15 & 8 & 17 & 10 & 10 & 16 & 162 & 27 & 12 & 1 & 1 & 2 & 2 & & & & & & 133866.0 \\
\hline 1932 & & & & 44 & 401 & 12 & 18 & 15 & 8 & 19 & 12 & 6 & 17 & 10 & 12 & 5. & 11 & 12 & 21 & 25 & 183 & 30 & 25 & 12 & 91 & 12 & 11 & 2 & & & & & 301611.0 \\
\hline 1933 & & & & 31 & 12 & 1 & 15 & 37 & 19 & 18 & 9 & 12 & 30 & 16 & 20 & 16 & 22 & 7 & 10 & 10 & 63 & 36 & 17 & 13 & 91 & 12 & 10 & 2 & 1 & & 2 & & 303896.0 \\
\hline 1934 & & & & 331 & 191 & 19 & 12 & 39 & 32 & 8 & 14 & 16 & 17 & 22 & 10 & 7 & 18 & 10 & 9 & 19 & 111 & 13 & 16 & 4 & 5 & 5 & 2 & 1 & 4 & & & & 181035.0 \\
\hline 1935 & & & & 262 & 202 & 23 & 6 & 21 & 6 & 12 & 21 & 8 & 16 & 13 & 7 & 5 & 12 & 14 & 11 & 16 & 213 & 31 & 19 & 25 & 91 & 18 & 1 & 2 & 2 & & & & 304563.0 \\
\hline 1936 & & & & 12 & 233 & 37 & 13 & 31 & 7 & 5 & 19 & 11 & 24 & 23 & 19 & 19 & 25 & 13 & 14 & 15 & 14 & 15 & 7 & 2 & 4 & 9 & 9 & 2 & 1 & 1 & 1 & 2 & 253283.0 \\
\hline 1937 & & & & 73 & 332 & 26 & 13 & 35 & 15 & 10 & 17 & 9 & 21 & 16 & 15 & 10 & 16 & 11 & 4 & 18 & 18 & 19 & 15 & 11 & 6 & 7 & 4 & 3 & 1 & 3 & 2 & & 265789.0 \\
\hline 1938 & & & & 73 & 351 & 14 & 12 & 13 & 18 & 16 & 21 & 17 & 18 & 8 & 6 & 7 & 8 & 4 & 6 & 19 & 112 & 27 & 24 & 14 & 82 & 20 & 11 & 7 & 6 & 5 & 3 & & 443739.0 \\
\hline 1939 & & 1 & 45 & 501 & 151 & 19 & 14 & 9 & 16 & 8 & 24 & 14 & 24 & 17 & 29 & 8 & 11 & 19 & 5 & 16 & 12 & 10 & 10 & 12 & 9 & 8 & 1 & & & & & & 171295.0 \\
\hline 1940 & & 1 & & 344 & $48 \quad 3$ & 33 & 16 & 18 & 11 & 4 & 11 & 4 & 14 & 10 & 4 & 17 & 26 & 14 & 10 & 14 & 151 & 14 & 11 & 5 & 81 & 15 & 5 & 4 & & & & & 223977.0 \\
\hline 1941 & & & 1 & 63 & $30 \quad 2$ & 24 & 18 & 16 & 9 & 7 & 16 & 14 & 24 & 16 & 241 & 18 & 24 & 22 & 16 & 20 & 151 & 18 & 6 & 11 & 4 & 6 & & & & & & & 163098.0 \\
\hline 1942 & & & 1 & 2 & 52 & 29 & 16 & 20 & 15 & 21 & 45 & 21 & 18 & 9 & 11 & 3 & 4 & 15 & 38 & 3 & 3 & 7 & 14 & 313 & 30 & 3 & 1 & & & & & & 249061.0 \\
\hline 1943 & & & 72 & 28 & 7 & 7 & 10 & 9 & 6 & 4 & 14 & 12 & 29 & 29 & 8 & 3 & 23 & 18 & 6 & 34 & 31 & 16 & 5 & 122 & 282 & 27 & 11 & 2 & 4 & 1 & 1 & 1 & 406224.0 \\
\hline 1944 & & & & 2 & 77 & 79 & 24 & 14 & 15 & 10 & 22 & 8 & 25 & 14 & 11 & 9 & 7 & 12 & 8 & 20 & 153 & 37 & 12 & 13 & 2 & & & & & & & & 154635.0 \\
\hline 1945 & 3 & & & 21 & 191 & 16 & 27 & 46 & 8 & 9 & 12 & 10 & 16 & 24 & 201 & 14 & 26 & 17 & 15 & 7 & 16 & 9 & 13 & 17 & 91 & 10 & & & & & & & 195708.0 \\
\hline 1946 & & & & & & & & 63 & 31 & 15 & 23 & 15 & 11 & 10 & 10 & 6 & 15 & 5 & 12 & 20 & 132 & 29 & 25 & 10 & 43 & 37 & 10 & 1 & & & & & 344182.0 \\
\hline 1947 & & & & & & & & 52 & 44 & 17 & 28 & 17 & 19 & 12 & 12 & 7 & 23 & 15 & 12 & 20 & 141 & 15 & 23 & 12 & 81 & 11 & 3 & 1 & & & & & 225327.0 \\
\hline 1948 & & & & & & & & 11 & 18 & 33 & 37 & 4 & 10 & 6 & 7 & 9 & 19 & 18 & 24 & 21 & 282 & 23 & 342 & 23 & 102 & 23 & 6 & 1 & 1 & & & & 361631.0 \\
\hline 1949 & & & & 2 & 5 & 3 & 3 & 33 & 18 & 16 & 29 & 21 & 27 & 15 & 5 & 9 & 16 & 21 & 7 & 17 & 102 & 25 & 32 & 4 & 92 & 23 & 8 & 5 & 2 & & & & 329352.0 \\
\hline 1950 & & & & & & & & 8 & 35 & 71 & 36 & 11 & 21 & 8 & 8 & 6 & 6 & 6 & 9 & 28 & 132 & 29 & 11 & 7 & 81 & 15 & 19 & 9 & 1 & & & & 97.0 \\
\hline 1951 & & & & & & & & 17 & 19 & 9 & 25 & 42 & 15 & 5 & 6 & 4 & 20 & 2 & 2 & 56 & 122 & 24 & 20 & 16 & 51 & 18 & 41 & 6 & 1 & & & & 470645.0 \\
\hline 1952 & & & & & & & & 7 & 23 & 16 & 47 & 26 & 31 & 23 & 10 & 5 & 30 & 15 & 10 & 23 & 4 & 18 & 21 & 13 & 71 & 19 & 16 & 2 & & & & & 315796.0 \\
\hline 1953 & & & & & & 2 & 3 & 7 & 16 & 6 & 7 & 14 & 35 & 58 & 302 & 22 & 29 & 9 & 22 & 23 & 121 & 15 & 10 & 4 & 11 & 112 & 26 & 3 & & & & & 296323.0 \\
\hline 1954 & & & & & & & & 1 & 6 & 7 & 18 & 40 & 25 & 12 & 213 & 38 & 28 & 11 & 10 & 33 & 16 & 10 & 13 & 8 & 73 & 332 & 26 & 2 & & & & & 398243.0 \\
\hline 1955 & & & & & & & & & 7 & 19 & 27 & 7 & 15 & 20 & 20 & 35 & 22 & 14 & 38 & 37 & 21 & 46 & 19 & 7 & 5 & 5 & 1 & & & & & & 241564.0 \\
\hline 1956 & & & & & & & & & 5 & 22 & 17 & 60 & 43 & 13. & 161 & 17 & 26 & 15 & 9 & 12 & 4 & 12 & 1 & 7 & 22 & 22 & 19 & 12 & 27 & 3 & 2 & & 555206.0 \\
\hline 1957 & & & & & & & & 3 & 30 & 17 & 11 & 5 & 40 & 27 & 152 & 20 & 35 & 22 & 38 & 45 & 13 & 12 & 5 & 2 & 4 & 9 & 11 & 1 & & & & & 233970.0 \\
\hline 1958 & & & & & & & & & 15 & 12 & 27 & 28 & 38 & 21 & 25 & 8 & 27 & 43 & 31 & 8 & 5 & 6 & 2 & 6 & 132 & 212 & 22 & 7 & & & & & 346657.0 \\
\hline 1959 & & & & & & & & 47 & 3 & 8 & 25 & 12 & 29 & 22 & 142 & 20 & 14 & 17 & 4 & 77 & 8 & 12 & 8 & 5 & 61 & 15 & 9 & 6 & 4 & & & & 301351.0 \\
\hline 1960 & & & & & & & & 1 & 86 & 16 & 17 & 7 & 24 & 17 & 133 & 35 & 43 & 11 & 10 & 23 & 20 & 14 & 6 & 5 & 2 & 6 & 9 & 1 & & & & & 193502.0 \\
\hline 1961 & & & & & & & & 62 & 31 & 1 & 12 & 18 & 10 & 27 & 91 & 10 & 8 & 10 & 16 & 39 & 132 & 20 & 25 & 12 & 61 & 11 & 14 & 7 & 4 & & & & 349548.0 \\
\hline 1962 & & & & & & 8 & 14 & 58 & 10 & 7 & 16 & 8 & 18 & 12 & 15 & 19 & 22 & 20 & 16 & 35 & 23 & 26 & 11 & 7 & 2 & 7 & 9 & 2 & & & & & 236524.0 \\
\hline 63 & & & & & & & & & & & & & & & 18 & & & & & & 27 & & & & & & & & & & & & 271865 \\
\hline
\end{tabular}

Long Tom River at Monroe, Oreg.

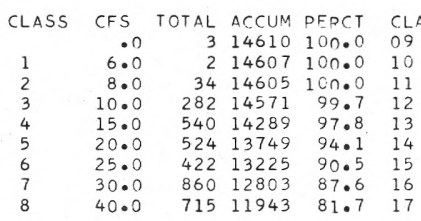

Long Tom River at Monroe, Oreg.

Summary for water years 1922-25, 1928-41

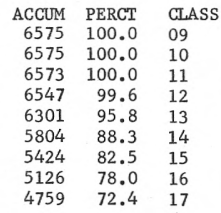

STATION NUMBER

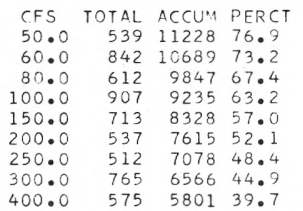

STATION NUMBER
14-1700.00

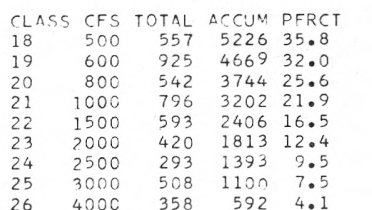

$14-1700.00$

$\begin{array}{llllllllllllllllllllllll}1 & 15 & 37 & 19 & 18 & 9 & 12 & 30 & 16 & 20 & 16 & 22 & 7 & 10 & 10 & 6 & 36 & 17 & 13 & 9 & 12 & 10 & 2\end{array}$

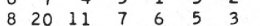

265789.0

443739.0

223977.0

$\frac{163098.0}{249061.0}$

154635.0

195708.0
344182.0

225327.0

361631.0

341997.0

470645.0

296323.0

98243.0

555206.0

33970.0

.

271865.0

Long Tom River at Monroe, Oreg.

$\begin{array}{rrrrl}\text { SS CFS } & \text { TOTAL } & \text { ACCUM } & \text { PERCT } & \text { CLASS } \\ 50.0 & 198 & 4495 & 68.4 & 18 \\ 60.0 & 326 & 4297 & 65.4 & 19 \\ 80.0 & 225 & 3971 & 60.4 & 20 \\ 100.0 & 400 & 3746 & 57.0 & 21 \\ 150.0 & 298 & 3346 & 50.9 & 22 \\ 200.0 & 223 & 3048 & 46.4 & 23 \\ 250.0 & 194 & 2825 & 43.0 & 24 \\ 300.0 & 303 & 2631 & 40.0 & 25 \\ 400.0 & 241 & 2328 & 35.4 & 26\end{array}$
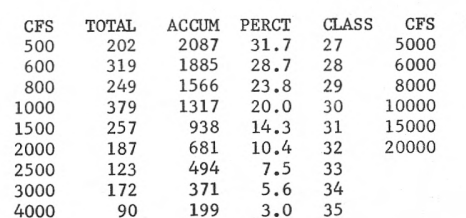

$\begin{array}{rrr}\text { TOTAL } & \text { ACCUM } & \text { PERCT } \\ 46 & 109 & 1.7 \\ 32 & 63 & 1.0 \\ 18 & 31 & .5 \\ 10 & 13 & .2 \\ 3 & 3 & .0 \\ & & .0 \\ & & .0 \\ & & .0\end{array}$

STATION NUMBER

$14-1700.00$

Summary for water years 1942-63

CLASS
1
2
3
4
5
6
7
8

$\begin{array}{rrrr}\text { CFS } & \text { TOTAL } & \text { ACCUM } & \text { PERCT } \\ .0 & 3 & 8035 & 100.0 \\ 6.0 & & 8032 & 100.0 \\ 8.0 & 8 & 8032 & 100.0 \\ 10.0 & 36 & 8024 & 99.9 \\ 15.0 & 43 & 7988 & 99.4 \\ 20.0 & 144 & 7945 & 98.9 \\ 25.0 & 124 & 7801 & 97.1 \\ 30.0 & 493 & 7677 & 95.5 \\ 40.0 & 451 & 7184 & 89.4\end{array}$

CLASS
09
10
11
12
13
14
15
16
17

$\begin{array}{rrrrl}\text { CFS } & \text { TOTAL } & \text { ACCUM } & \text { PERCT } & \text { CLASS } \\ 50.0 & 341 & 6733 & 83.8 & 18 \\ 60.0 & 516 & 6392 & 79.6 & 19 \\ 80.0 & 387 & 5876 & 73.1 & 20 \\ 100.0 & 507 & 5489 & 68.3 & 21 \\ 150.0 & 415 & 4982 & 62.0 & 22 \\ 200.0 & 314 & 4567 & 56.8 & 23 \\ 250.0 & 318 & 4253 & 52.9 & 24 \\ 300.0 & 462 & 3935 & 49.0 & 25 \\ 400.0 & 334 & 3473 & 43.2 & 26\end{array}$

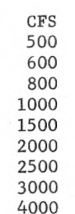

TOTAL
355
606
293
417
336
233
170
336
268

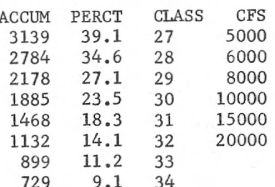

TOTAL ACCUM PERCT

$\begin{array}{rrr}72 & 125 & 1.6\end{array}$

45

4
3

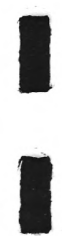

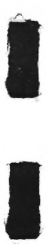

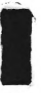

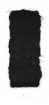

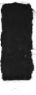

,

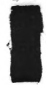


Long Tom Rlver at Monroe, Oreg.

StATION NUMBER $14-1700.00$

LOWEST MEAN DISCHARGE, IN CFS, FOR THE FOLLOWING NUMBER OF CONSECUTIVE DAYS IN YEAR BEGINNING APRIL 1

\begin{tabular}{|c|c|c|c|c|c|c|c|c|c|c|c|}
\hline YEAR & 1 & 3 & 7 & 14 & 30 & 60 & 90 & 120 & 150 & 183 & 274 \\
\hline 1922 & $12 \cdot 0$ & 15.7 & 19.9 & 21.0 & 22.9 & 23.9 & 27.4 & 31.8 & 40.2 & 53.5 & 355.0 \\
\hline 1923 & 23.0 & 23.7 & 23.7 & 23.9 & 24.2 & 26.6 & 29.1 & $32 \cdot 2$ & 38.6 & 49.4 & 173.0 \\
\hline 1924 & 8.0 & 8.0 & 8.1 & 8.5 & 10.0 & 13.2 & 16.2 & $20 \cdot 1$ & 27.2 & 38.7 & 650.0 \\
\hline 1925 & 9.0 & 10.0 & 13.0 & 15.9 & 19.3 & 20.0 & 20.5 & 23.7 & 31.6 & 43.3 & 156.0 \\
\hline 1928 & 13.0 & 15.0 & 17.1 & 18.1 & 20.3 & 20.4 & 21.9 & $28 \cdot 5$ & 41.3 & 60.1 & 323.0 \\
\hline 1929 & 14.0 & 14.0 & 14.1 & 14.6 & 14.8 & 16.0 & 16.6 & $18 \cdot 2$ & 23.2 & 37.0 & 328.0 \\
\hline 1930 & 17.0 & 17.3 & 17.7 & 18.1 & 20.4 & 22.8 & 24.2 & 27.0 & 35.1 & 55.7 & 123.0 \\
\hline 1931 & 11.0 & 11.0 & 11.6 & 12.1 & 13.6 & 14.5 & 15.4 & 21.9 & 33.1 & 46.1 & 468.0 \\
\hline 1932 & 14.0 & $14 \cdot 3$ & 14.6 & 15.2 & 16.2 & 19.3 & 22.4 & $28 \cdot 1$ & 40.9 & 70.2 & 356.0 \\
\hline 1933 & 25.0 & 27.7 & 29.0 & 29.8 & 33.1 & 35.6 & 37.3 & 42.9 & 47.7 & 77.0 & 416.0 \\
\hline 1934 & 12.0 & 12.0 & 12.4 & 12.8 & 13.3 & 13.9 & 15.9 & 20.0 & 29.2 & 48.9 & 467.0 \\
\hline 1935 & 13.0 & 13.0 & 13.1 & 14.2 & 16.5 & 19.2 & 22.8 & 26.9 & 33.7 & 49.9 & 170.0 \\
\hline 1936 & 14.0 & 14.0 & 14.3 & 14.6 & 15.6 & 17.2 & 18.3 & 19.3 & 22.8 & 30.9 & 88.7 \\
\hline 1937 & 25.0 & 25.7 & 27.9 & 29.4 & 33.2 & 38.2 & 44.7 & $54 \cdot 1$ & 134.0 & 161.0 & 827.0 \\
\hline 1938 & 10.0 & 14.0 & 14.9 & 15.4 & 16.5 & 17.9 & 19.8 & $24 \cdot 6$ & 34.7 & 57.7 & 214.0 \\
\hline 1939 & 7.0 & $7 \cdot 3$ & 8.4 & 9.6 & 11.3 & 12.0 & 13.0 & 15.0 & 17.3 & 23.7 & 90.0 \\
\hline 1940 & 12.0 & $12 \cdot 3$ & 12.6 & 12.9 & 13.8 & 17.4 & 19.4 & $24 \cdot 2$ & 37.5 & 67.6 & 305.0 \\
\hline 1941 & 8.0 & 12.3 & 13.9 & 14.6 & 17.0 & 24.7 & 24.7 & $28 \cdot 1$ & 37.5 & 56.6 & 423.0 \\
\hline 1942 & 18.0 & 19.3 & 19.7 & 20.6 & 21.9 & 30.7 & 44.4 & 51.8 & 89.1 & 168.0 & 611.0 \\
\hline 1943 & 8.0 & 8.0 & 8.9 & 10.8 & $12 \cdot 3$ & 14.7 & 42.0 & 59.1 & 75.2 & 123.0 & 442.0 \\
\hline 1944 & .0 & . 3 & 8.4 & 13.2 & 16.5 & $18 \cdot 6$ & 19.2 & 21.0 & 25.3 & 55.6 & 194.0 \\
\hline 1945 & 19.0 & 20.3 & $22 \cdot 1$ & 23.3 & 25.8 & 26.4 & 28.5 & 51.0 & 98.8 & 191.0 & 630.0 \\
\hline 1946 & 30.0 & 30.0 & 30.0 & 30.7 & 32.6 & 35.9 & 38.8 & 43.0 & 49.6 & 140.0 & 504.0 \\
\hline 1947 & 32.0 & 34.0 & 34.6 & 35.3 & 36.8 & 38.6 & 39.2 & 43.9 & 49.8 & 162.0 & 582.0 \\
\hline 1948 & 38.0 & 38.0 & 38.0 & 38.9 & 41.8 & 48.6 & 53.4 & 74.5 & 165.0 & 251.0 & 676.0 \\
\hline 1949 & 14.0 & 15.3 & 15.7 & 21.2 & 30.5 & 34.8 & 42.3 & $51 \cdot 9$ & 73.6 & 191.0 & 315.0 \\
\hline 1950 & 44.0 & 44.0 & 47.0 & 47.7 & 50.2 & 52.0 & 53.6 & $58 \cdot 0$ & 72.5 & 146.0 & 895.0 \\
\hline 1951 & 33.0 & 33.0 & 33.0 & 34.5 & 39.4 & 49.3 & 61.9 & $70 \cdot 1$ & 152.0 & 227.0 & 619.0 \\
\hline 1952 & 23.0 & 23.7 & $26 \cdot 6$ & 40.1 & 44.8 & 58.1 & 62.5 & 73.8 & 110.0 & 186.0 & 241.0 \\
\hline 1953 & 38.0 & 39.0 & 40.6 & 41.4 & 50.8 & 90.7 & 129.0 & $227 \cdot 0$ & 245.0 & 293.0 & 683.0 \\
\hline 1954 & 30.0 & 37.3 & 43.0 & 51.1 & 60.1 & 76.5 & 83.8 & 119.0 & 154.0 & 226.0 & 400.0 \\
\hline 1955 & 46.0 & 46.0 & $48 \cdot 6$ & 50.3 & 54.5 & $64 \cdot 3$ & 109.0 & $168 \cdot 0$ & 318.0 & 333.0 & 1140.0 \\
\hline 1956 & 47.0 & 47.0 & 49.0 & 50.5 & 54.7 & 65.7 & 73.9 & 83.1 & 96.4 & 196.0 & 333.0 \\
\hline 1957 & 36.0 & 36.0 & $44 \cdot 3$ & $45 \cdot 1$ & 46.9 & 56.0 & 87.5 & $121 \cdot 0$ & 170.0 & 234.0 & 463.0 \\
\hline 1958 & 43.0 & 44.0 & 45.1 & 46.6 & 50.7 & 61.8 & 89.2 & $107 \cdot 0$ & 163.0 & 214.0 & 413.0 \\
\hline 1959 & 31.0 & 31.7 & 32.1 & 32.8 & 33.6 & 39.5 & 51.4 & 66.8 & 99.3 & 206.0 & 208.0 \\
\hline 1960 & 39.0 & 39.7 & $40 \cdot 0$ & $40 \cdot 3$ & 40.3 & 41.5 & 41.9 & 53.4 & 194.0 & 284.0 & 601.0 \\
\hline 1961 & 31.0 & 33.0 & 33.6 & 34.6 & 35.1 & 36.8 & 37.4 & $47 \cdot 4$ & 94.6 & 113.0 & 523.0 \\
\hline 1962 & 21.0 & 21.0 & 21.9 & 25.7 & 29.8 & 30.8 & 33.1 & $44 \cdot 3$ & 96.6 & 139.0 & 426.0 \\
\hline
\end{tabular}

Long Tom River at Monroe, Oreg.

STATION NUMBER $\quad 14-1700 \cdot 00$

HIGHEST MEAN DISCHARGE, IN CFS, FOR THE FOLLOWING NUMBER OF CONSECUTIVE DAYS IN YEAR ENDING SEPTEMBER 30

\begin{tabular}{|c|c|c|c|c|c|c|c|c|c|c|c|}
\hline YEAR & 1 & 3 & 7 & 15 & 30 & 60 & 90 & 120 & 150 & 183 & 274 \\
\hline 1922 & 5880.0 & 5760.0 & 5290.0 & 4870.0 & 3080.0 & 1980.0 & 1780.0 & $1830 \cdot 0$ & 1750.0 & 1510.0 & 1040.0 \\
\hline 1923 & 15800.0 & 12100.0 & 8640.0 & 6480.0 & 4940.0 & 3420.0 & 2630.0 & $2160 \cdot 0$ & 1820.0 & 1520.0 & \\
\hline 1924 & 3840.0 & 3610.0 & 3040.0 & 2320.0 & 1460.0 & 1140.0 & 975.0 & $817 \cdot 0$ & 691.0 & 585.0 & 405.0 \\
\hline 1925 & 11900.0 & 9990.0 & 7910.0 & 7060.0 & 4870.0 & 3890.0 & 3120.0 & $3300 \cdot 0$ & 2770.0 & 2380.0 & 1630.0 \\
\hline 1928 & 5650.0 & 5240.0 & 4650.0 & 3800.0 & 2740.0 & 2050.0 & 1700.0 & $1780 \cdot 0$ & 1650.0 & 1500.0 & \\
\hline 1929 & 5450.0 & 5080.0 & 4460.0 & 3220.0 & 1900.0 & 1520.0 & $1170 \cdot 0$ & $1170 \cdot 0$ & 1010.0 & 861.0 & 608.0 \\
\hline 1930 & 8890.0 & 6830.0 & 5490.0 & 3520.0 & 2380.0 & 1790.0 & 1730.0 & $1390 \cdot 0$ & 1180.0 & $1000 \cdot 0$ & 683.0 \\
\hline 1931 & 4770.0 & $4360 \cdot 0$ & 3330.0 & 2200.0 & 1750.0 & 1300.0 & 1080.0 & $956 \cdot 0$ & 811.0 & 693.0 & 483.0 \\
\hline 1932 & 5560.0 & $5230 . n$ & 4890.0 & 4110.0 & 3180.0 & 2580.0 & $2110 \cdot 0$ & $1940 \cdot 0$ & 1780.0 & 1570.0 & 1090.0 \\
\hline 1933 & 10600.0 & 8710.0 & 5730.0 & $4940 \cdot 0$ & 3350.0 & 2930.0 & 2650.0 & $2190 \cdot 0$ & 1850.0 & 1580.0 & 1100.0 \\
\hline 1934 & 7390.0 & 6730.0 & 5840.0 & 3950.0 & 2670.0 & 2170.0 & $1620 \cdot 0$ & $1360 \cdot 0$ & 1140.0 & 955.0 & 654.0 \\
\hline 1935 & 7860.0 & 6840.0 & 5270.0 & 4180.0 & 3480.0 & 2560.0 & $2150 \cdot 0$ & $1960 \cdot 0$ & 1840.0 & 1600.0 & 1100.0 \\
\hline 1936 & 15300.0 & $14500 \cdot 0$ & 9810.0 & 6890.0 & 4430.0 & 2970.0 & $2270 \cdot 0$ & $1880 \cdot 0$ & 1560.0 & 1320.0 & 916.0 \\
\hline 1937 & 11800.0 & 10700.0 & 7440.0 & 4940.0 & 3820.0 & 2520.0 & 2450.0 & 1970.0 & 1640.0 & 1410.0 & 963.0 \\
\hline 1938 & $11500 \cdot 0$ & 10500.0 & 7840.0 & 5670.0 & 3500.0 & 3330.0 & 3240.0 & $2930 \cdot 0$ & 2790.0 & 2370.0 & 1610.0 \\
\hline 1939 & 4280.0 & 3910.0 & 3260.0 & 3050.0 & 2350.0 & 1740.0 & 1460.0 & $1260 \cdot 0$ & 1060.0 & 898.0 & 620.0 \\
\hline 1940 & 5790.0 & 5510.0 & 4740.0 & $3810 \cdot 0$ & 3590.0 & 2480.0 & $2000 \cdot 0$ & $1670 \cdot 0$ & 1420.0 & 1200.0 & 812.0 \\
\hline 1941 & 3590.0 & 3350.0 & 2800.0 & 2320.0 & 1890.0 & 1640.0 & 1270.0 & 1070.0 & 935.0 & 8.24 .0 & 587.0 \\
\hline 1942 & 4010.0 & 3520.0 & 2820.0 & 2680.0 & 2610.0 & 2470.0 & 2190.0 & $1780 \cdot 0$ & 1450.0 & 1200.0 & 822.0 \\
\hline 1943 & 16400.0 & $13100 . ?$ & 8820.0 & $6130 . ?$ & 5070.0 & 4080.0 & $3560 \cdot 0$ & 2870.0 & 2460.0 & 2110.0 & 1460.0 \\
\hline 1944 & 2610.0 & 2450.0 & 2210.0 & $2120 \cdot 0$ & 1830.0 & $1220 \cdot 0$ & $1100 \cdot 0$ & $1080 \cdot 0$ & 903.0 & 787.0 & 558.0 \\
\hline 1945 & 3910.0 & 3790.0 & 3540.0 & 2720.0 & $1750 \cdot 0$ & 1590.0 & $1410 \cdot 0$ & $1200 \cdot 0$ & 1040.0 & 967.0 & 697.0 \\
\hline 1946 & $5560: 0$ & 4840.0 & 4270.0 & 4230.0 & 3630.0 & 2940.0 & 2590.0 & $2230 \cdot 0$ & 1910.0 & 1740.0 & 1190.0 \\
\hline 1947 & 5060.0 & 4510.0 & 3790.0 & 2900.0 & 2480.0 & 1570.0 & 1540.0 & 1330.0 & 1260.0 & 1080.0 & 807.0 \\
\hline 1948 & 7180.0 & 5770.0 & 4780.0 & 4180.0 & 3410.0 & 2530.0 & 2070.0 & $1910 \cdot 0$ & 1870.0 & 1720.0 & 1300.0 \\
\hline 1949 & 6340.0 & 5660.0 & 4650.0 & 4430.0 & 3710.0 & 2140.0 & 2260.0 & 1970.0 & 1790.0 & 1610.0 & 1120.0 \\
\hline 1950 & 6070.0 & 5810.0 & 5380.0 & 5050.0 & 4400.0 & 3560.0 & 2920.0 & $2420 \cdot 0$ & 2020.0 & 1730.0 & 1190.0 \\
\hline 1951 & 6500.0 & 5490.0 & 4790.0 & 4640.0 & $4190 \cdot 0$ & 3200.0 & $3220 \cdot 0$ & $3020 \cdot 0$ & 2720.0 & 2340.0 & 1590.0 \\
\hline 1952 & 5180.0 & 4690.0 & 4100.0 & 3860.0 & 3110.0 & 2990.0 & 2650.0 & $2220 \cdot 0$ & 1840.0 & 1540.0 & 1060.0 \\
\hline 1953 & 5210.0 & 4870.0 & 4630.0 & 4480.0 & 4340.0 & 3060.0 & 2340.0 & $1920 \cdot 0$ & 1580.0 & 1400.0 & 1000.0 \\
\hline 1954 & 5810.0 & 5380.0 & 5060.0 & $4680 \cdot 0$ & 4360.0 & 3440.0 & 3240.0 & $2680 \cdot 0$ & 2270.0 & 1940.0 & 1360.0 \\
\hline 1955 & 4510.0 & 3770.0 & 2810.0 & 2130.0 & 1890.0 & 1380.0 & 1110.0 & $1090 \cdot 0$ & 1060.0 & 965.0 & 739.0 \\
\hline 1956 & $10700 \cdot 0$ & 10200.0 & 8960.0 & $8080 \cdot 0$ & 7220.0 & 5870.0 & 4620.0 & $4140 \cdot 0$ & 3380.0 & 2840.0 & 1940.0 \\
\hline 1957 & 5060.0 & 4560.0 & 4270.0 & 4230.0 & 3340.0 & 1900.0 & 1510.0 & $1360 \cdot 0$ & 1150.0 & 1050.0 & 771.0 \\
\hline 1958 & 5680.0 & 5470.0 & $5240 \cdot 0$ & 4670.0 & 3880.0 & 3380.0 & 3070.0 & $2360 \cdot 0$ & 1970.0 & 1680.0 & 1200.0 \\
\hline 1959 & 6610.0 & 6340.0 & 6050.0 & 4930.0 & 3980.0 & 3110.0 & 2260.0 & 1960.0 & 1690.0 & 1440.0 & 1000.0 \\
\hline 1960 & 5010.0 & 4930.0 & 4510.0 & 2980.0 & 1930.0 & 1770.0 & 1420.0 & $1260 \cdot 0$ & 1100.0 & 934.0 & 692.0 \\
\hline 1961 & 6640.0 & 6430.0 & 5850.0 & 5370.0 & 4140.0 & 3120.0 & 2390.0 & $2240 \cdot 0$ & 1980.0 & 1800.0 & 1260.0 \\
\hline 1962 & 5340.0 & $5140 . ?$ & 4380.0 & 3120.0 & 1980.0 & 1580.0 & 1360.0 & $1210 \cdot 0$ & 1220.0 & 1170.0 & 852.0 \\
\hline 1963 & 6040.0 & $5550 . ?$ & 4000.0 & 3050.0 & 2610.0 & 1930.0 & 1380.0 & 1330.0 & 1130.0 & 1160.0 & 966.0 \\
\hline
\end{tabular}


Rock Creek near Phllomath, Oreg.

STATION NUMBER 14-1705.00

DURATION TABLE OF DAILY DISCHARGE

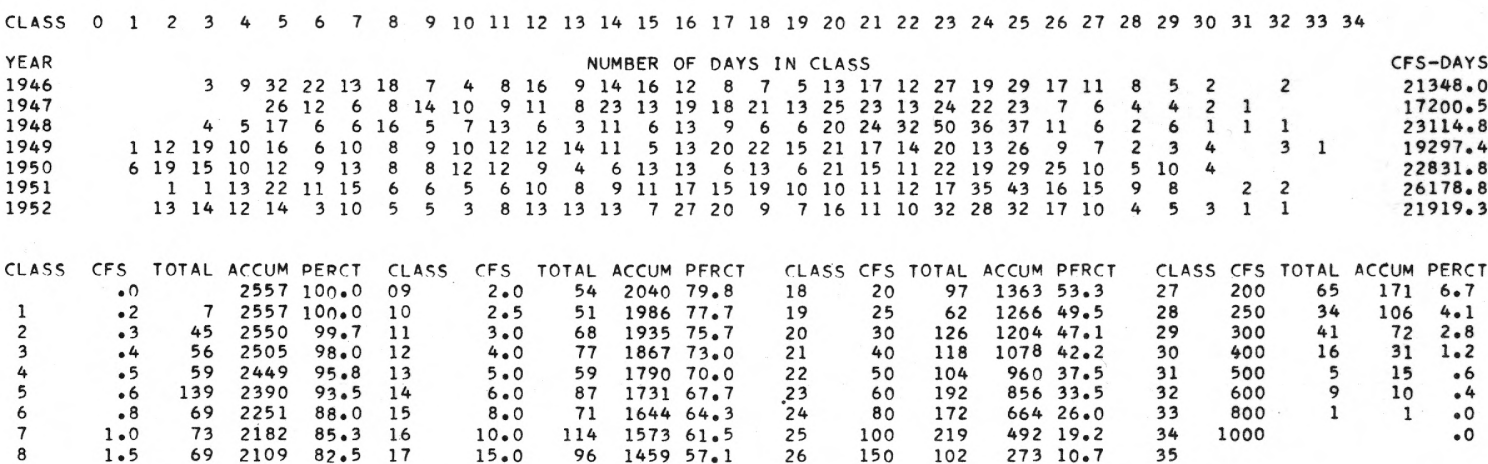

Rock Creek near Philomath, Oreg.

STATION NUMBER $\quad 14-1705.00$

LOWEST MEAN DISCHARGE, IN CFS, FOR THE FOLLOWING NUMBER OF CONSECUTIVE DAYS IN YEAR BEGINNING APRIL I

\begin{tabular}{|c|c|c|c|c|c|c|c|c|c|c|c|}
\hline YEAR & 1 & 3 & 7 & 14 & 30 & 60 & 90 & 120 & 150 & 183 & 274 \\
\hline 1946 & .4 & .4 & .5 & .6 & .6 & 1.0 & 1.3 & $2 \cdot 2$ & 3.2 & 5.8 & 30.6 \\
\hline 1947 & .6 & .6 & .6 & .7 & 1.2 & 1.4 & 2.3 & 4.2 & 6.5 & 9.8 & 36.2 \\
\hline 1948 & .4 & .4 & .5 & .5 & .7 & 1.3 & 1.9 & $2 \cdot 6$ & 4.7 & 9.1 & 33.9 \\
\hline 1949 & .2 & $\cdot 3$ & $\cdot 3$ & .4 & .5 & .7 & 1.1 & $1 \cdot 4$ & 2.0 & $4 \cdot 1$ & 19.1 \\
\hline 1950 & .2 & $\cdot 2$ & $\cdot 3$ & $\cdot 3$ & .3 & .5 & 1.1 & 2.9 & 5.3 & 10.0 & 48.7 \\
\hline 1951 & .5 & .5 & .5 & .5 & .7 & .8 & 1.5 & 3.1 & 5.7 & 10.0 & 42.6 \\
\hline
\end{tabular}

Rock Creek near Philomath, Oreg.

STATION NUMBER $\quad 14-1705.00$

HIGHEST MEAN DISCHARGF, IN CFS, FOR THE FOLLOWING NUMBER OF CONSECUTIVE DAYS IN YEAR FNDING SEPTEMBER 30

\begin{tabular}{|c|c|c|c|c|c|c|c|c|c|c|c|}
\hline YEAR & 1 & 3 & 7 & 15 & 30 & 60 & 90 & 120 & 150 & 183 & 274 \\
\hline 1946 & 687.0 & $496 . ?$ & 311.0 & 262.0 & 184.0 & 172.0 & 165.0 & 149.0 & 132.0 & 113.0 & 77.6 \\
\hline 1947 & 519.0 & 420.0 & 290.0 & 188.0 & 179.0 & 111.0 & 116.0 & 109.0 & 99.9 & 86.1 & 62.1 \\
\hline 1948 & 756.0 & 561.0 & 386.0 & 278.0 & 176.0 & 141.0 & 121.0 & 111.0 & 109.0 & 104.0 & 83.6 \\
\hline 1949 & 925.0 & 720.0 & 572.0 & 407.0 & 259.0 & 166.0 & 145.0 & 132.0 & 115.0 & 101.0 & 70.0 \\
\hline 1950 & 444.0 & $400 \cdot 0$ & 295.0 & 265.0 & 230.0 & 204.0 & 184.0 & 160.0 & 139.0 & 120.0 & 83.0 \\
\hline 1951 & 714.0 & 547.0 & 400.0 & 313.0 & 239.0 & 183.0 & 189.0 & 166.0 & 152.0 & 134.0 & 95.1 \\
\hline 952 & 620.0 & 507.0 & 371.0 & 266.0 & 193.0 & 163.0 & 163.0 & 144.0 & $128 \cdot 0$ & 113.0 & 79.5 \\
\hline
\end{tabular}




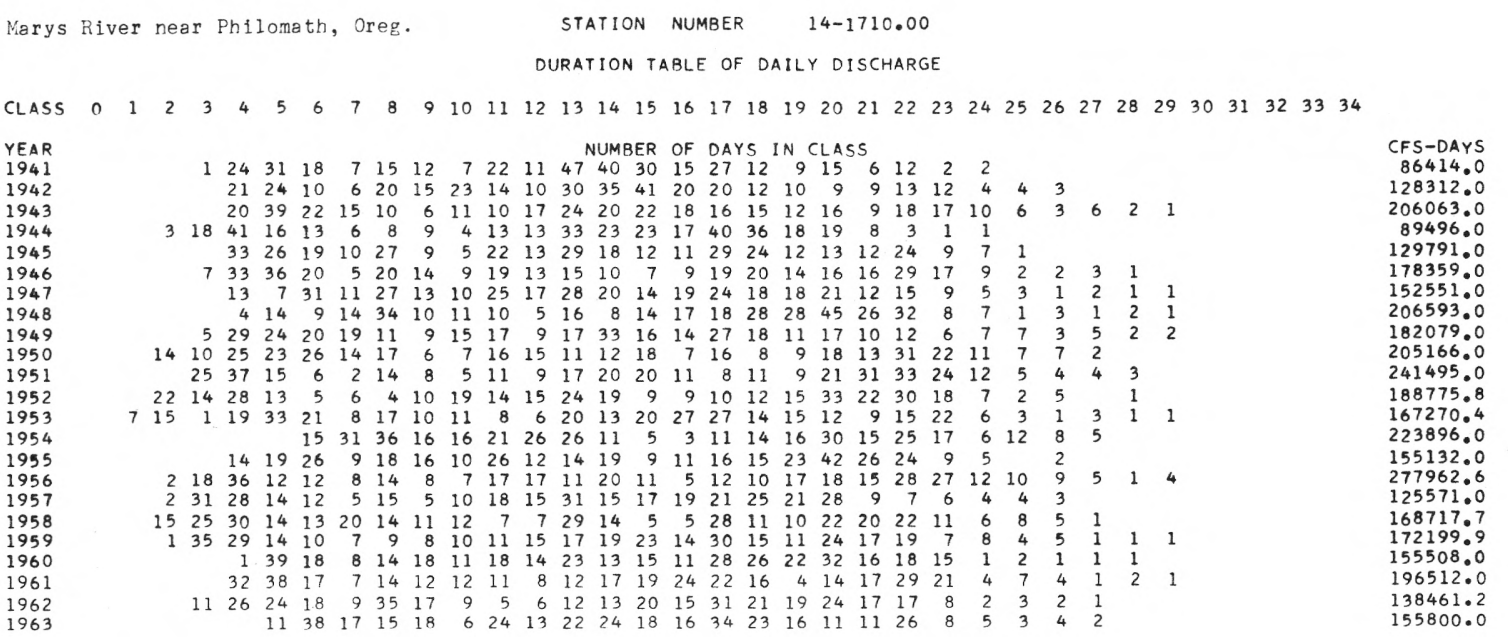

Marys River near Philomath, Oreg.

STATION NUMBER 14-1710.00

Summary for water years 1941-63
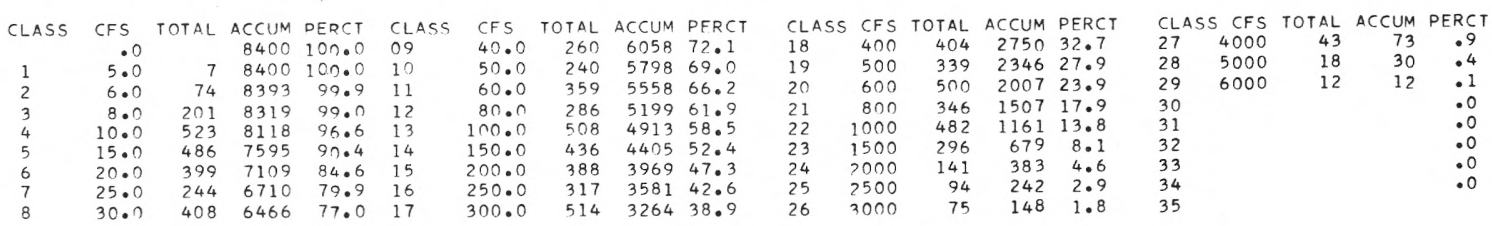

Marys River near Philomath, Oreg.

STATION NUMBER $14-1710.00$

LOWEST MEAN DISCHARGE, IN CFS, FOR THE FOLLOWING NUMBER OF CONSECUTIVE DAYS IN YEAR BEGINNING APRIL 1

\begin{tabular}{|c|c|c|c|c|c|c|c|c|c|c|c|}
\hline YEAR & 1 & 3 & 7 & 14 & 30 & 60 & 90 & 120 & 150 & 183 & 274 \\
\hline 1941 & 9.0 & $10 \cdot 3$ & 10.9 & 11.4 & 13.0 & 19.2 & 29.9 & $33 \cdot 4$ & 39.2 & 54.6 & 258.0 \\
\hline 1942 & 11.0 & 11.0 & 11.3 & $12 \cdot 3$ & 13.2 & 14.7 & $17 \cdot 3$ & $24 \cdot 9$ & 38.8 & 61.2 & 336.0 \\
\hline 1943 & 11.0 & 11.0 & 11.9 & 12.2 & 13.4 & 17.0 & 20.0 & 29.4 & 45.9 & $75 \cdot 9$ & 177.0 \\
\hline 1944 & 6.0 & 6.3 & 7.3 & $8 \cdot 1$ & 9.1 & 11.1 & 12.1 & $15 \cdot 5$ & 25.9 & 37.2 & 108.0 \\
\hline 1945 & 10.0 & 11.7 & 12.9 & 13.9 & 16.7 & 18.8 & 19.9 & $23 \cdot 8$ & 34.4 & $80 \cdot 6$ & 356.0 \\
\hline 1946 & 8.0 & $8 \cdot 3$ & 9.7 & 11.3 & 12.6 & 14.0 & 15.5 & $21 \cdot 7$ & 30.9 & $48 \cdot 8$ & 290.0 \\
\hline 1947 & 18.0 & 19.7 & 20.4 & 21.0 & 24.0 & 25.3 & 30.3 & 40.2 & 54.0 & 79.5 & 296.0 \\
\hline 1948 & 11.0 & 11.3 & 14.3 & $15 \cdot 6$ & 19.2 & 25.0 & 29.4 & 33.8 & 48.4 & 82.0 & 356.0 \\
\hline 1949 & 9.0 & 9.3 & 9.4 & 9.8 & 11.8 & 14.1 & 17.1 & $19 \cdot 4$ & 26.1 & 38.5 & 172.0 \\
\hline 1950 & 6.0 & 6.0 & 6.7 & 7.3 & 8.9 & 11.9 & 16.1 & $27 \cdot 6$ & 41.0 & 67.0 & 417.0 \\
\hline 1951 & $8 \cdot 0$ & 8.0 & $8 \cdot 3$ & $8 \cdot 6$ & 9.6 & 10.5 & $14 \cdot 5$ & $24 \cdot 7$ & $42 \cdot 9$ & 77.1 & 353.0 \\
\hline 1952 & 5.4 & 5.7 & $5 \cdot 8$ & 6.2 & 6.4 & 7.4 & 8.6 & $10 \cdot 5$ & 14.2 & 22.8 & 83.7 \\
\hline 1953 & 14.0 & 14.7 & 15.0 & $15 \cdot 2$ & 18.9 & 24.3 & 30.3 & $36 \cdot 9$ & $48 \cdot 6$ & 89.4 & 368.0 \\
\hline 1954 & 20.0 & 20.7 & 22.0 & $22 \cdot 5$ & 26.7 & 27.7 & 29.9 & $38 \cdot 0$ & 49.0 & 58.4 & 217.0 \\
\hline 1955 & 12.0 & 12.0 & 12.9 & 13.9 & 14.8 & 19.7 & 25.4 & $38 \cdot 1$ & 57.2 & 96.6 & 551.0 \\
\hline 1956 & 7.6 & 7.7 & 8.2 & 9.1 & 9.8 & 11.4 & 13.0 & $18 \cdot 3$ & 29.3 & $44 \cdot 3$ & 159.0 \\
\hline 1957 & 7.6 & 7.7 & 7.9 & $8 \cdot 5$ & 8.9 & $11 \cdot 3$ & 15.4 & $18 \cdot 6$ & 27.0 & 43.5 & 230.0 \\
\hline 1958 & 7.0 & 7.5 & $7 \cdot 7$ & 7.9 & 8.1 & 10.0 & 10.7 & $15 \cdot 5$ & $24 \cdot 0$ & 44.3 & 218.0 \\
\hline 1959 & 7.6 & 7.9 & $8 \cdot 4$ & 8.6 & 9.3 & $12 \cdot 3$ & $21 \cdot 6$ & $33 \cdot 9$ & 44.9 & 54.6 & 128.0 \\
\hline 1960 & 14.0 & 14.0 & 14.4 & 15.1 & 16.0 & $18 \cdot 3$ & $21 \cdot 2$ & $27 \cdot 4$ & 43.2 & 114.0 & 309.0 \\
\hline 1961 & 11.0 & 11.0 & 11.4 & 12.4 & 13.6 & 15.3 & $17 \cdot 6$ & $22 \cdot 9$ & 28.0 & $41 \cdot 1$ & 214.0 \\
\hline 1962 & 8.2 & 8.5 & 8.9 & 9.5 & 11.4 & 15.4 & 18.9 & 29.8 & 44.1 & 68.6 & 205.0 \\
\hline
\end{tabular}

Marys River near Philomath, Oreg.

STATION NUMBER

14-1110.UU

HIGHEST MEAN DISCHARGE, IN CFS, FOR THE FOLLOWING NUMBER OF CONSECUTIVE DAYS IN YEAR ENDING SEPTEMBER 30

YEAR
1941
1942
1943
1944
1945
1946
1947
1948
1949
1950
1951
1952
1953
1954
1955
1956
1957
1958
1959
1960
1961
1962
196

$\begin{array}{cc}1 & 3 \\ 2120.0 & 1930 \cdot 0 \\ 3610.0 & 3370 \cdot 0 \\ 7330.0 & 5800 \cdot 0 \\ 2400.0 & 1830 \cdot 0 \\ 2550.0 & 2360.0 \\ 5280.0 & 4120.0 \\ 7380.0 & 5940.0 \\ 7520.0 & 5410.0 \\ 6960.0 & 6300.0 \\ 4650.0 & 4130.0 \\ 5420.0 & 4950.0 \\ 5760.0 & 4220.0 \\ 6010.0 & 5600.0 \\ 4880.0 & 4160.0 \\ 3520.0 & 2970.0 \\ 7180.0 & 6120.0 \\ 3180.0 & 2920.0 \\ 4730.0 & 3620.0 \\ 6600.0 & 5310.0 \\ 5850.0 & 4560.0 \\ 6800.0 & 5360.0 \\ 4100.0 & 3300.0 \\ 4950.0 & 4030.0\end{array}$

7
1440.0
2540.0
4130.0
1230.0
1990.0
2600.0
3780.0
3680.0
4990.0
3200.0
4100.0
3090.0
4230.0
3330.0
2150.0
4330.0
2240.0
2660.0
4370.0
3280.0
4040.0
2470.0
2740.0

1160.0

$1790 \cdot 0$
$2730 \cdot 0$

2730.0
774.0

1600.0
2250.0

2250.0

2170.0

3840.0

2340.0

2110.0

3140.0

2600.0

1400.0
3220.0

3220.0
1840.0
2160.0

2160.0

2360.0
2760.0

2760.0

1610.0
1920.0

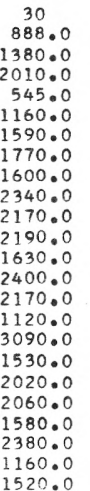

1520.0

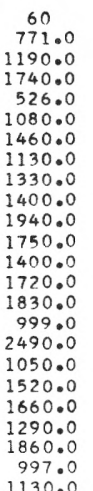

90
612.0
1040.0
1630.0
$510 \cdot 0$
1000.0
1390.0
1110.0
1170.0
1480.0
1710.0
1730.0
1410.0
1430.0
1720.0
881.0
2070.0
844.0
1420.0
1310.0
1120.0
1490.0
823.0
974.0

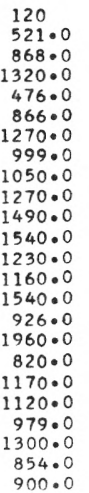

120
$521 \cdot 0$
$868 \cdot 0$
$1320 \cdot 0$
$476 \cdot 0$
$866 \cdot 0$
$1270 \cdot 0$
$999 \cdot 0$
$1050 \cdot 0$
$1270 \cdot 0$
$1490 \cdot 0$
$1540 \cdot 0$
$1230 \cdot 0$
$1160 \cdot 0$
$1540 \cdot 0$
$926 \cdot 0$
$1960 \cdot 0$
$820 \cdot 0$
$1170 \cdot 0$
$1120 \cdot 0$
$979 \cdot 0$
$1300 \cdot 0$
$854 \cdot 0$
$900 \cdot 0$

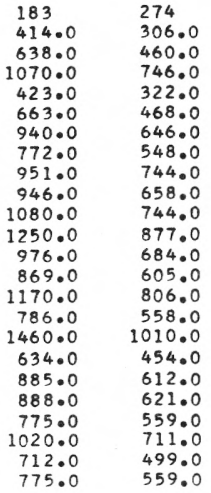




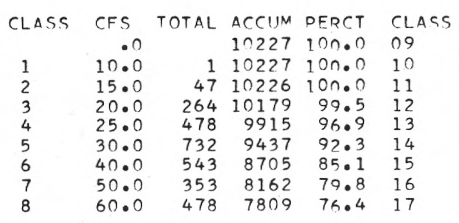

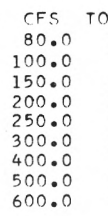

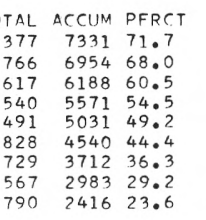

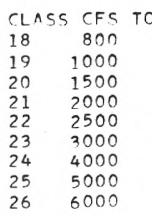

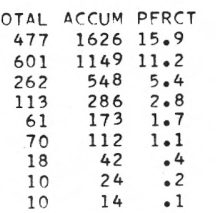

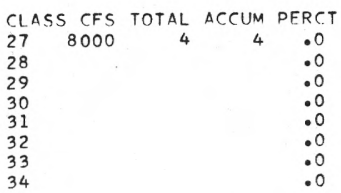

LOWEST MEAN DISCHARGE, IN CFS, FOR THE FOLLOWING NUMBER OF CONSECUTIVE DAYS IN YEAR BEGINNING APRIL 1

$\begin{array}{ll}\text { YEAR } & 1 \\ 1936 & 17.0 \\ 1937 & 27.0 \\ 1938 & 21.0 \\ 1939 & 19.0 \\ 1940 & 13.0 \\ 1941 & 34.0 \\ 1942 & 24.0 \\ 1943 & 25.0 \\ 1944 & 20.0 \\ 1945 & 23.0 \\ 1946 & 25.0 \\ 1947 & 34.0 \\ 1948 & 32.0 \\ 1949 & 23.0 \\ 1950 & 26.0 \\ 1951 & 20.0 \\ 1952 & 20.0 \\ 1953 & 28.0 \\ 1954 & 40.0 \\ 1955 & 35.0 \\ 1956 & 24.0 \\ 1957 & 26.0 \\ 1958 & 25.0 \\ 1959 & 30.0 \\ 1960 & 23.0 \\ 1961 & 23.0 \\ 1962 & 25.0\end{array}$
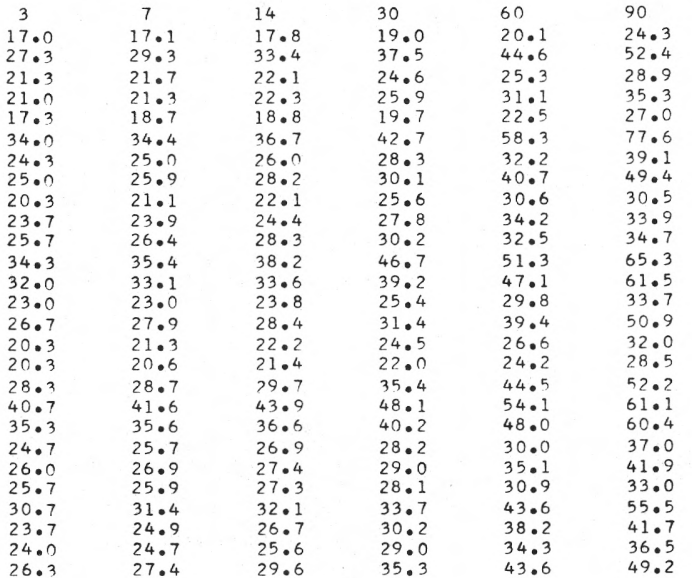

$$
\begin{aligned}
& 120 \\
& 27 \cdot 4 \\
& 74 \cdot 0 \\
& 32 \cdot 9 \\
& 44 \cdot 3 \\
& 30 \cdot 4 \\
& 94 \cdot 8 \\
& 56 \cdot 0 \\
& 77 \cdot 4 \\
& 35 \cdot 7 \\
& 39 \cdot 0 \\
& 45 \cdot 2 \\
& 95 \cdot 7 \\
& 85 \cdot 0 \\
& 45 \cdot 8 \\
& 91 \cdot 3 \\
& 44 \cdot 6 \\
& 32 \cdot 2 \\
& 62 \cdot 5 \\
& 77 \cdot 1 \\
& 90 \cdot 6 \\
& 53 \cdot 1 \\
& 47 \cdot 7 \\
& 39 \cdot 9 \\
& 81 \cdot 3 \\
& 48 \cdot 3 \\
& 46 \cdot 4 \\
& 65 \cdot 7
\end{aligned}
$$

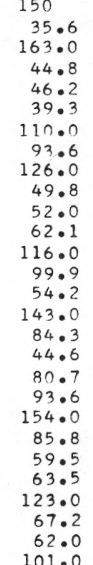

35.6
63.0

183

274
134.0 $\begin{array}{ll}54.6 & 134.0 \\ 219.0 & 465.0\end{array}$ $89.3 \quad 226.0$ $\begin{array}{ll}67.8 & 147.0 \\ 63.7 & 192.0\end{array}$

134.0

139.0

161.0

64.9
128.0

128.0
95.9

151.0

142.0
72.0

208.0

133.0
71.8

166.0

112.0

221.0
145.0

93.7

84.6 .

139.0

160.0

92.7

318.0

442.0
216.0

138.0

443.0
328.0

361.0

336.0

239.0
508.0

357.0

178.0

463.0
232.0

631.0

265.0
316.0

287.0

202.0

336.0

316.0
254.0 
HIGHEST MEAN DISCHARGE, IN CFS, FOR THE FOLLOWING NUMAER OF CONSECUTIVE DAYS IN YEAR ENDING SEPTFMBER 30

YEAR
1936
1937
1938
1939
1940
1941
1942
1943
1944
1945
1946
1947
1948
1949
1950
1951
1952
1953
1954
1955
1956
1957
1958
1959
1960
1961
1962
1963

\begin{tabular}{|c|c|}
\hline 1 & 3 \\
\hline $\begin{array}{l}4370.0 \\
4370.0\end{array}$ & $\begin{array}{l}4120 \cdot 0 \\
3880 \cdot 0\end{array}$ \\
\hline $\begin{array}{l}3950.0 \\
3550.0\end{array}$ & $\begin{array}{l}3070 \cdot 0 \\
2500 \cdot 0\end{array}$ \\
\hline $2160 \cdot 0$ & 1940.0 \\
\hline 1960.0 & 1520.0 \\
\hline 4010.0 & 3280.0 \\
\hline 7380.0 & 6170.0 \\
\hline 2150.0 & $1450 \cdot 0$ \\
\hline 3970.0 & $2480 \cdot 0$ \\
\hline 8970.0 & 5490.0 \\
\hline 5650.0 & $5000 \cdot 0$ \\
\hline 6390.0 & 4920.0 \\
\hline 6650.0 & 4490.0 \\
\hline 4580.0 & $3800 \cdot 0$ \\
\hline 5280.0 & 3940.0 \\
\hline 3310.0 & $3000 \cdot 0$ \\
\hline 7940.0 & 6010.0 \\
\hline $7000 \cdot 0$ & $5200 \cdot 0$ \\
\hline 3650.0 & 3250.0 \\
\hline 9310.0 & $6670 . n$ \\
\hline 5200.0 & 3760.0 \\
\hline 9420.0 & 6300.0 \\
\hline $4100 \cdot 0$ & 2860.0 \\
\hline $2800 \cdot 0$ & 2570.0 \\
\hline 9080.0 & 5970.0 \\
\hline 4220.0 & 3690.0 \\
\hline 2990.0 & $2250 . n$ \\
\hline
\end{tabular}

7
$2970 \cdot 0$
$2580 \cdot 0$
$2300 \cdot 0$
$1810 \cdot 0$
$1680 \cdot 0$
$1140 \cdot 0$
$2300 \cdot 0$
$4290 \cdot 0$
$881 \cdot 0$
$2240 \cdot 0$
$3120 \cdot 0$
$3250 \cdot 0$
$3290 \cdot 0$
$3330 \cdot 0$
$2430 \cdot 0$
$3010 \cdot 0$
$2520 \cdot 0$
$4120 \cdot 0$
$2900 \cdot 0$
$2100 \cdot 0$
$4320 \cdot 0$
$2630 \cdot 0$
$3890 \cdot 0$
$1970 \cdot 0$
$1790 \cdot 0$
$3990 \cdot 0$
$2450 \cdot 0$
$1650 \cdot 0$

\begin{tabular}{|c|}
\hline $\begin{array}{c}15 \\
2500.0 \\
1740.0\end{array}$ \\
\hline $1770 \cdot 0$ \\
\hline $\begin{array}{l}1360.0 \\
1410.0\end{array}$ \\
\hline $\begin{array}{r}962.0 \\
1510.0\end{array}$ \\
\hline $\begin{array}{l}1510.0 \\
2840.0\end{array}$ \\
\hline $688 \cdot 0$ \\
\hline $1650 \cdot 0$ \\
\hline $2310 \cdot 0$ \\
\hline 1870.0 \\
\hline 2080.0 \\
\hline $\begin{array}{l}2240.0 \\
1770.0\end{array}$ \\
\hline $\begin{array}{l}1770.0 \\
2050.0\end{array}$ \\
\hline $\begin{array}{l}2050.0 \\
1730.0\end{array}$ \\
\hline 2880.0 \\
\hline 2240.0 \\
\hline 1270.0 \\
\hline $2940 \cdot 0$ \\
\hline $2230 \cdot n$ \\
\hline 1340.0 \\
\hline 1360.0 \\
\hline $2640 \cdot 0$ \\
\hline $\begin{array}{l}1670.0 \\
1300.0\end{array}$ \\
\hline \\
\hline
\end{tabular}

\begin{tabular}{|c|}
\hline \\
\hline $\begin{array}{c}30 \\
1660.0 \\
1290.0\end{array}$ \\
\hline 946.0 \\
\hline 1300.0 \\
\hline $718 \cdot 0$ \\
\hline $\begin{array}{l}1250.0 \\
2120.0\end{array}$ \\
\hline 524.0 \\
\hline $\begin{array}{l}1100.0 \\
1600.0\end{array}$ \\
\hline 1700.0 \\
\hline 1330.0 \\
\hline 1480.0 \\
\hline 1500.0 \\
\hline 1680.0 \\
\hline 1240.0 \\
\hline 2370.0 \\
\hline 2110.0 \\
\hline 1180.0 \\
\hline 2240.0 \\
\hline $\begin{array}{l}1710.0 \\
1820.0\end{array}$ \\
\hline 1220.0 \\
\hline $1170 \cdot 0$ \\
\hline $\begin{array}{l}1920.0 \\
1290.0\end{array}$ \\
\hline 1020.0 \\
\hline
\end{tabular}

60
1130.0
1150.0
1130.0
918.0
951.0
643.0
1030.0
1840.0
451.0
999.0
1360.0
1050.0
1070.0
1080.0
1380.0
1310.0
987.0
1650.0
1460.0
961.0
1910.0
1210.0
1580.0
999.0
1040.0
1550.0
1010.0
919.0

\begin{tabular}{|c|}
\hline \\
\hline $\begin{array}{r}944.0 \\
1030.0\end{array}$ \\
\hline $\begin{array}{l}788.0 \\
747.0\end{array}$ \\
\hline $\begin{array}{l}583.0 \\
914.0\end{array}$ \\
\hline $\begin{array}{r}914.0 \\
1580.0\end{array}$ \\
\hline 449.0 \\
\hline $\begin{array}{r}962.0 \\
1190.0\end{array}$ \\
\hline 999.0 \\
\hline 991.0 \\
\hline 965.0 \\
\hline 1210.0 \\
\hline 1330.0 \\
\hline 1020.0 \\
\hline 1350.0 \\
\hline 1440.0 \\
\hline $\begin{array}{r}805.0 \\
1580.0\end{array}$ \\
\hline 925.0 \\
\hline 1350.0 \\
\hline 890.0 \\
\hline $\begin{array}{r}911.0 \\
1200.0\end{array}$ \\
\hline 801.0 \\
\hline 785.0 \\
\hline
\end{tabular}
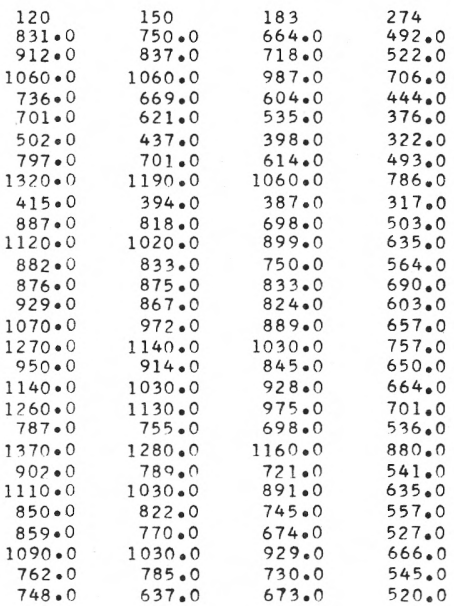

Calapooya River at Albany, Oreg.

STATION NUMBER

$14-1735.00$

DURATION TABLE OF DAILY DISCHARGE

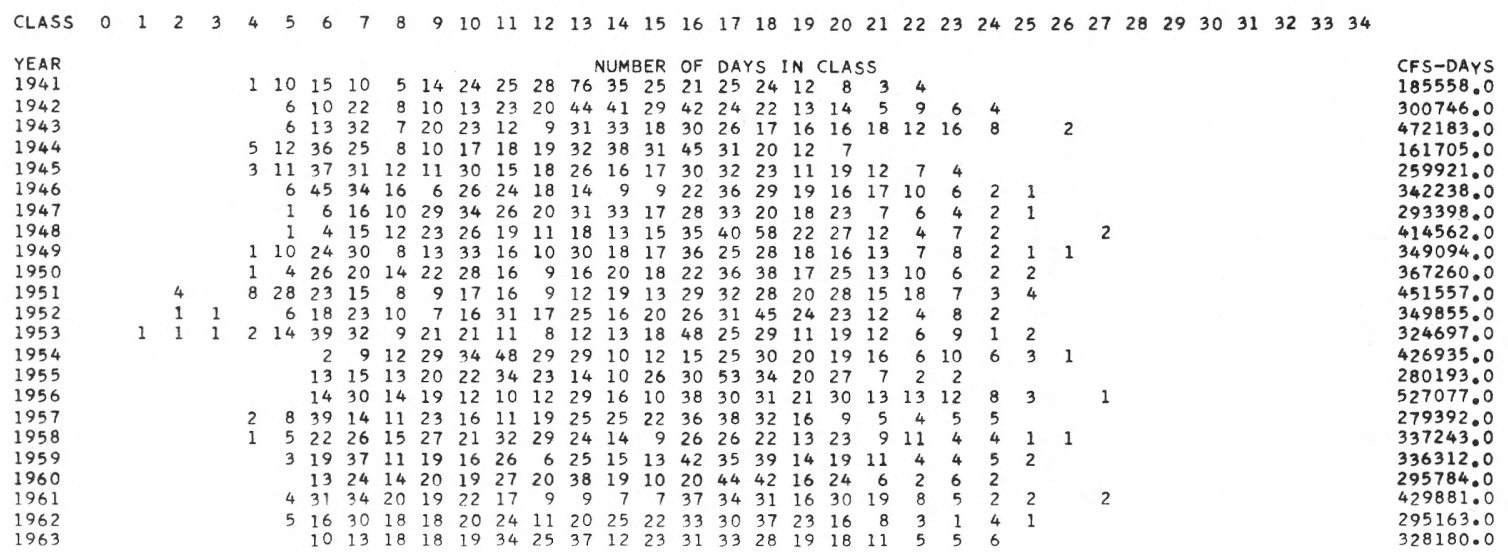

\footnotetext{
Calapooya River at Albany, Oreg.
}

Summary for water years 1941-63

STATION NUMBER $\quad 14-1735.00$

\begin{tabular}{|c|c|c|c|c|c|c|c|c|c|c|c|c|c|c|c|c|c|c|}
\hline CLASS & CFS & TOTAL & ACCUM & PFRCT & CLASS & CFS & TOTAL & ACCUM & PERCT & CLASS & 5 CFS & TOTAL & ACCUM & PERCT & $\begin{array}{l}\text { CLASS CFS } \\
27 \quad 20000\end{array}$ & TOTAL & ACCUM & $\begin{array}{c}\text { PERCT } \\
\cdot 1\end{array}$ \\
\hline 1 & 4.0 & 1 & $\begin{array}{l}8400 \\
8400\end{array}$ & $\begin{array}{l}100.0 \\
100.0\end{array}$ & $\begin{array}{l}09 \\
10\end{array}$ & $\begin{array}{l}50.0 \\
70.0\end{array}$ & $\begin{array}{l}407 \\
509\end{array}$ & $\begin{array}{l}6942 \\
6535\end{array}$ & $\begin{array}{l}82.6 \\
77.8\end{array}$ & $\begin{array}{l}18 \\
19\end{array}$ & $\begin{array}{l}1000 \\
1500\end{array}$ & $\begin{array}{l}707 \\
391\end{array}$ & $\begin{array}{l}2191 \\
1484\end{array}$ & $\begin{array}{l}26.1 \\
17.7\end{array}$ & $\begin{array}{l}27 \\
28\end{array}$ & & & $\begin{array}{l}.1 \\
.0\end{array}$ \\
\hline 2 & 5.0 & 6 & 8399 & 100.0 & 11 & 100.0 & 515 & 6026 & 71.7 & 20 & 2000 & 456 & 1093 & 13.0 & 29 & & & .0 \\
\hline 3 & 7.0 & 2 & 8393 & 99.9 & 12 & 150.0 & 379 & 5511 & 65.6 & 21 & 3000 & 244 & 637 & 7.6 & 30 & & & .0 \\
\hline 4 & 10.0 & 24 & 8391 & 99.9 & 13 & $200 \cdot 0$ & 617 & 5132 & & 22 & 4000 & 155 & 393 & 4.7 & 31 & & & .0 \\
\hline 5 & 15.0 & 140 & 8367 & 99.6 & 14 & 300.0 & 457 & 4515 & 53. & 23 & 5000 & 135 & 238 & 2.8 & 32 & & & .0 \\
\hline 6 & 20.0 & 475 & 8227 & 97.9 & 15 & 400.0 & 401 & 4058 & 48 & 24 & 7000 & 70 & 103 & 1.2 & 33 & & & .0 \\
\hline 7 & 30.0 & 537 & 7752 & $9 ? .3$ & 16 & 500.0 & 722 & 3657 & 43 & 25 & 10000 & 23 & 33 & .4 & 34 & & & .0 \\
\hline 8 & 40.0 & 273 & 7215 & 85.9 & 17 & 700.0 & 744 & 2935 & 34.9 & 26 & 15000 & 5 & 10 & .1 & 35 & & & \\
\hline
\end{tabular}


Calapooya River at Albany, Oreg.

STATION NUMBER $\quad 14-1735.00$

LOWEST MEAN DISCHARGE, IN CFS, FOR THE FOLLOWING NUMBER OF CONSECUTIVE DAYS IN YEAR BEGINNING APRIL 1

\begin{tabular}{|c|c|c|c|c|c|c|c|c|c|c|c|}
\hline YEAR & 1 & 3 & 7 & 14 & 30 & 60 & 90 & 120 & 150 & 183 & 274 \\
\hline 1941 & 12.0 & $16 \cdot 0$ & 17.4 & 23.6 & 37.9 & 63.1 & 91.6 & 116.0 & 137.0 & 176.0 & 656.0 \\
\hline 1942 & 16.0 & 20.3 & 23.7 & 24.4 & 25.8 & 31.3 & 38.6 & 59.5 & 109.0 & 197.0 & 856.0 \\
\hline 1943 & 16.0 & $24 \cdot 3$ & 28.4 & 29.3 & 30.2 & 41.3 & 50.7 & 88.7 & 149.0 & 205.0 & 357.0 \\
\hline 1944 & 12.0 & 14.3 & 16.3 & 18.1 & 19.7 & 25.9 & 26.7 & 32.9 & 52.1 & 74.2 & 211.0 \\
\hline 1945 & 14.0 & 19.3 & 22.0 & $22 \cdot 3$ & 25.3 & 30.0 & 31.1 & $38 \cdot 1$ & 56.1 & 154.0 & 699.0 \\
\hline 1946 & 19.0 & 23.3 & 25.3 & $25 \cdot 6$ & 26.9 & 29.1 & 32.2 & 45.5 & 65.0 & 104.0 & 547.0 \\
\hline 1947 & 19.0 & 32.7 & 38.9 & 41.4 & 51.4 & 58.5 & 71.9 & $104 \cdot 0$ & 130.0 & 176.0 & 683.0 \\
\hline 1948 & 18.0 & 25.0 & 29.7 & 30.6 & 41.0 & 52.0 & 71.4 & $97 \cdot 1$ & 119.0 & 184.0 & 674.0 \\
\hline 1949 & 10.0 & $18 \cdot 0$ & 21.3 & 23.3 & 24.2 & 27.5 & 32.4 & $47 \cdot 6$ & 57.1 & 80.4 & 326.0 \\
\hline 1950 & 14.0 & 20.3 & 22.6 & 23.5 & 27.5 & 33.5 & 46.2 & 89.6 & 150.0 & 237.0 & 971.0 \\
\hline 1951 & 6.0 & 11.3 & 14.3 & 15.3 & 17.7 & 18.5 & 25.9 & $42 \cdot 4$ & 86.2 & 147.0 & 645.0 \\
\hline 1952 & 4.0 & 12.0 & 16.4 & 16.7 & 17.9 & 21.2 & 25.1 & 28.9 & 40.0 & $71 \cdot 2$ & 207.0 \\
\hline 1953 & 18.0 & 25.7 & 28.6 & 30.3 & 39.3 & 46.2 & 56.7 & $71 \cdot 8$ & 96.6 & 261.0 & 860.0 \\
\hline 1954 & 26.0 & 34.0 & $41 \cdot 3$ & 44.1 & 50.8 & 63.2 & 69.9 & $86 \cdot 2$ & 109.0 & 128.0 & 401.0 \\
\hline 1955 & 22.0 & $24 \cdot 0$ & 26.6 & 29.2 & 34.1 & 47.8 & 65.1 & 99.7 & 184.0 & 282.0 & 1290.0 \\
\hline 1956 & 19.0 & 22.7 & 26.7 & 26.9 & 28.4 & 30.6 & 37.4 & $55 \cdot 2$ & 93.9 & 165.0 & 415.0 \\
\hline 1957 & 13.0 & 18.0 & 19.1 & 19.9 & 21.9 & 31.3 & 41.3 & $49 \cdot 2$ & 63.6 & 101.0 & 487.0 \\
\hline 1958 & 14.0 & 18.3 & $21 \cdot 3$ & 23.9 & 25.4 & 32.2 & 35.7 & $44 \cdot 7$ & 70.0 & 96.2 & 467.0 \\
\hline 1959 & 19.0 & 23.0 & $26 \cdot 4$ & 27.4 & 29.5 & 42.7 & 61.8 & $92 \cdot 7$ & 137.0 & 159.0 & 266.0 \\
\hline 1960 & 21.0 & 24.3 & 29.0 & 29.4 & 34.8 & 41.0 & 44.8 & 52.1 & 75.0 & 243.0 & 707.0 \\
\hline 1961 & 16.0 & 21.7 & 24.0 & 24.9 & 27.6 & 32.1 & 34.5 & 47.0 & 68.1 & 108.0 & 586.0 \\
\hline 1962 & 15.0 & 21.0 & & $27 \cdot 1$ & 330 & (1) & & & & & \\
\hline
\end{tabular}

Calapooya River at Albany, Oreg.

STATION NUMBER

$14-1735.00$

HIGHEST MEAN DISCHARGF, IN CFS, FOR THE FOLLOWING NUMBER OF CONSECUTIVE DAYS IN YEAR ENDING SEPTEMBER 30

\begin{tabular}{|c|c|c|c|c|c|c|c|c|c|c|c|}
\hline YEAR & 1 & 3 & 7 & 15 & 30 & 60 & 90 & 120 & 150 & 183 & 274 \\
\hline 1941 & 4920.0 & 4110.0 & 2960.0 & 2370.0 & 1870.0 & 1570.0 & 1360.0 & $1150 \cdot 0$ & 975.0 & 860.0 & 652.0 \\
\hline 1942 & 9820.0 & 7770.0 & 5810.0 & 3850.0 & 3270.0 & 2610.0 & 2360.0 & 1960.0 & 1670.0 & 1420.0 & 1080.0 \\
\hline 1943 & 16500.0 & 14200.0 & 10400.0 & 6950.0 & 4990.0 & 4280.0 & 3900.0 & $3100 \cdot 0$ & 2790.0 & 2420.0 & 1710.0 \\
\hline 1944 & 2820.0 & 2540.0 & 2080.0 & 1530.0 & 1190.0 & 963.0 & 866.0 & $842 \cdot 0$ & 801.0 & 755.0 & 580.0 \\
\hline 1945 & 5340.0 & 4810.0 & 4240.0 & 3550.0 & 2430.0 & 2230.0 & 2000.0 & $1750 \cdot 0$ & 1560.0 & 1330.0 & 938.0 \\
\hline 1946 & 12400.0 & $9290 . n$ & 5950.0 & 4900.0 & 3310.0 & 2730.0 & 2480.0 & 2380.0 & 2100.0 & 1800.0 & 1240.0 \\
\hline 1947 & 11000.0 & 8990.0 & 6050.0 & 3430.0 & 3290.0 & 1970.0 & 1980.0 & 1760.0 & 1690.0 & 1460.0 & 1050.0 \\
\hline 1948 & 23000.0 & 17400.0 & 10800.0 & 6450.0 & 3840.0 & 2730.0 & 2490.0 & $2120 \cdot 0$ & 2060.0 & 1940.0 & 1490.0 \\
\hline 1949 & 18600.0 & 12600.0 & 8670.0 & 5970.0 & 3870.0 & 2540.0 & 2500.0 & 2240.0 & 2000.0 & 1790.0 & 1260.0 \\
\hline 1950 & 11000.0 & 9810.0 & 6890.0 & 4550.0 & 3750.0 & 3380.0 & 2930.0 & $2490 \cdot 0$ & 2160.0 & 1880.0 & 1330.0 \\
\hline 1951 & 14500.0 & $12100 \cdot 0$ & 7830.0 & 4860.0 & 4180.0 & 3160.0 & 3150.0 & $2970 \cdot 0$ & 2700.0 & 2330.0 & 1640.0 \\
\hline 1952 & 9120.0 & 7850.0 & 6410.0 & 4290.0 & 3030.0 & 2500.0 & 2470.0 & $2160 \cdot 0$ & 1980.0 & 1740.0 & 1260.0 \\
\hline 1953 & $13100 \cdot 0$ & $10500 \cdot 0$ & 7540.0 & 5730.0 & 4610.0 & 3200.0 & 2610.0 & $2120 \cdot 0$ & 1910.0 & 1680.0 & 1180.0 \\
\hline 1954 & $16000 \cdot 0$ & 13100.0 & 8600.0 & $6090 \cdot 0$ & 4510.0 & 3790.0 & 3490.0 & $2990 \cdot 0$ & 2610.0 & 2210.0 & 1530.0 \\
\hline 1955 & 5900.0 & $5070 . n$ & 3550.0 & 2730.0 & 2340.0 & 1820.0 & 1500.0 & $1540 \cdot 0$ & 1500.0 & 1370.0 & $1000 \cdot 0$ \\
\hline 1956 & 26400.0 & 17800.0 & 12800.0 & 8200.0 & 6340.0 & 4980.0 & 3920.0 & $3500 \cdot 0$ & 3060.0 & 2670.0 & 1910.0 \\
\hline 1957 & 9020.0 & 7980.0 & 6200.0 & $5010 \cdot 0$ & 3780.0 & 2470.0 & 1930.0 & $1830 \cdot 0$ & 1570.0 & 1390.0 & 1010.0 \\
\hline 1958 & $16800 \cdot 0$ & $12100 \cdot 0$ & $7360 \cdot 0$ & 4990.0 & 3650.0 & 3380.0 & 2870.0 & $2340 \cdot 0$ & 2070.0 & 1750.0 & 1220.0 \\
\hline 1959 & 10900.0 & $9830 . n$ & 7310.0 & $4550 \cdot 0$ & 3740.0 & 2910.0 & 2320.0 & $2060 \cdot 0$ & 1930.0 & 1690.0 & 1210.0 \\
\hline 1960 & 7980.0 & 6950.0 & 5480.0 & 3670.0 & 2750.0 & 2430.0 & 2070.0 & $1880 \cdot 0$ & 1700.0 & 1460.0 & 1060.0 \\
\hline 1961 & 26800.0 & 16400.0 & $1070 n \cdot 0$ & 6730.0 & 5050.0 & 3870.0 & 3010.0 & $2810 \cdot 0$ & 2530.0 & 2240.0 & 1560.0 \\
\hline 1962 & $1050 n \cdot 0$ & $9 n 9 n \cdot n$ & $5810 . n$ & 3660.0 & 2760.0 & 2020.0 & 1680.0 & $1650 \cdot 0$ & 1630.0 & 1490.0 & 1060.0 \\
\hline 1963 & 9980.0 & 7840.0 & $506 n \cdot n$ & 3660.0 & 2800.0 & 2310.0 & 1890.0 & $1830 \cdot 0$ & 1600.0 & 1620.0 & 1170.0 \\
\hline
\end{tabular}

68.1

168.0

469.0

7840.0

3870.0
0

1890.0

620.0

170.0 
Willamette River at Albany, Oreg.

STATION NUMRFR $14-1740.00$

DURATION TARLE OF DAILY DISCHARGE

CLASS $0 \begin{array}{llllllllllllllllllllllllllllllllllll} & 1 & 2 & 3 & 4 & 5 & 6 & 7 & 8 & 9 & 10 & 11 & 12 & 13 & 14 & 15 & 16 & 17 & 18 & 19 & 20 & 21 & 22 & 23 & 24 & 25 & 26 & 27 & 28 & 29 & 30 & 31 & 32 & 33 & 34\end{array}$

YEAR

NUMBER OF DAYS IN CLASS

CFS-DAYS

1896
1897
1898
1899
1900
1901
1902
1903
1904
1905
1906
1907
1908
1909
1910
1911
1912
1913
1914
1915
1916
1917
1918
1919
1920
1921
1922
1923
1924
1925
1926
1927
1928
1929
1930
1931
1932
1933
1934
1935
1936
1937
1938
1

$\begin{array}{lllllllllllllllllllllllllll}20 & 27 & 38 & 16 & 7 & 7 & 19 & 5 & 5 & 10 & 15 & 23 & 29 & 19 & 50 & 26 & 15 & 6 & 4 & 5 & 6 & 5 & 2 & 5 & 1 & 1\end{array}$

$\begin{array}{llllllllllllllllllll}12 & 36 & 37 & 15 & 3 & 6 & 8 & 15 & 23 & 16 & 16 & 48 & 22 & 22 & 19 & 23 & 7 & 10\end{array}$

$\begin{array}{rrrrrrrrrrrrrrrrrrrr}56 & 9 & 6 & 4 & 21 & 5 & 35 & 13 & 42 & 31 & 32 & 15 & 24 & 10 & 7 & 1 & 3 \\ 24 & 8 & 12 & 9 & 34 & 19 & 11 & 7 & 16 & 17 & 31 & 34 & 65 & 28 & 21 & 4 & 5\end{array}$

$\begin{array}{rrrrrrrrrrrrrrrrrrr}27 & 21 & 15 & 23 & 11 & 15 & 22 & 19 & 22 & 30 & 9 & 40 & 33 & 22 & 21 & 8 & 3\end{array}$

$\begin{array}{lllllllllllllllllllll}35 & 31 & 11 & 9 & 5 & 24 & 14 & 18 & 26 & 29 & 33 & 24 & 17 & 38 & 14 & 7 & 5\end{array}$

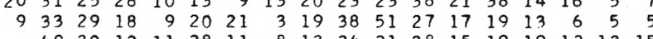

$\begin{array}{llllllllllllllllll}30 & 12 & 11 & 28 & 11 & 8 & 13 & 24 & 21 & 38 & 15 & 19 & 19 & 12 & 13 & 15\end{array}$

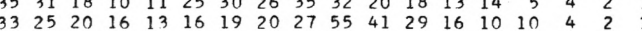

$\begin{array}{lllllllllllllllllll}42 & 25 & 23 & 16 & 7 & 17 & 10 & 22 & 29 & 23 & 22 & 40 & 19 & 21 & 11 & 10 & 4 \\ 38 & 44 & 20 & 14 & 6 & 12 & 4 & 6 & 41 & 52 & 34 & 31 & 16 & 18 & 6 & 8 & 3\end{array}$

$\begin{array}{rrrlllllllllllllll}38 & 44 & 20 & 14 & 6 & 12 & 4 & 6 & 41 & 52 & 34 & 31 & 16 & 18 & 6 & 8 & 3 \\ 39 & 26 & 15 & 11 & 9 & 38 & 23 & 32 & 35 & 27 & 18 & 21 & 12 & 17 & 9 & 5 & 8\end{array}$

$\begin{array}{llllllllllllllllll}59 & 26 & 25 & 9 & 8 & 26 & 5 & 11 & 22 & 48 & 25 & 20 & 16 & 13 & 13 & 11 & 4\end{array}$

$\begin{array}{llllllllllllllllll}4 & 30 & 40 & 22 & 11 & 9 & 13 & 15 & 6 & 29 & 65 & 41 & 26 & 18 & 16 & 5\end{array}$

$\begin{array}{lllllllllllllllll}9 & 13 & 25 & 25 & 28 & 12 & 9 & 24 & 59 & 32 & 42 & 24 & 25 & 12\end{array}$

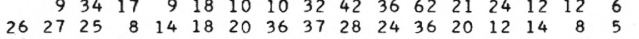

$\begin{array}{lllllllllllllllll}10 & 40 & 5 & 19 & 12 & 6 & 47 & 32 & 21 & 44 & 52 & 36 & 16 & 12 & 7 & 3\end{array}$

$\begin{array}{lllllllllllllllllll}6 & 25 & 8 & 14 & 14 & 10 & 26 & 6 & 5 & 13 & 29 & 33 & 47 & 29 & 21 & 19 & 18 & 14 & 11\end{array}$

$\begin{array}{llllllllllllllllll}29 & 34 & 15 & 14 & 18 & 5 & 6 & 21 & 16 & 23 & 54 & 44 & 46 & 20 & 8 & 6 & 4\end{array}$

$\begin{array}{llllllrllllllllllllll}9 & 19 & 85 & 28 & 11 & 9 & 3 & 11 & 17 & 17 & 18 & 20 & 7 & 19 & 29 & 14 & 12 & 8 & 5 & 2\end{array}$

$\begin{array}{rlllllllllllllllllllllllll}19 & 23 & 45 & 19 & 8 & 9 & 19 & 21 & 19 & 26 & 9 & 26 & 16 & 18 & 27 & 17 & 12 & 8 & 3 & 4 & 8 & 2 & 5 & 2\end{array}$

$\begin{array}{rrrrrrrrrrrrrrrrrrr}12 & 35 & 25 & 17 & 14 & 5 & 15 & 20 & 31 & 37 & 34 & 31 & 23 & 12 & 19 & 13 & 7 & 4 \\ & & 40 & 10 & 17 & 7 & 11 & 11 & 14 & 25 & 25 & 28 & 32 & 19 & 39 & 25 & 14 & 13\end{array}$

$\begin{array}{llllllllllllllllll}12 & 56 & 37 & 9 & 14 & 8 & 4 & 8 & 18 & 25 & 26 & 39 & 37 & 40 & 9 & 6\end{array}$

$\begin{array}{rrrrrrrrrrrrrrrrrr}7 & 56 & 72 & 21 & 12 & 11 & 6 & 14 & 15 & 31 & 45 & 36 & 25 & 20 & 14 & 6 & 5 & 4 \\ 9 & 46 & 42 & 18 & 8 & 11 & 7 & 24 & 27 & 40 & 27 & 25 & 11 & 4 & 2 & 3 & \end{array}$

$\begin{array}{rrrrrrrrrrrrrrrrrr}9 & 46 & 42 & 4 & 12 & 3 & 12 & 8 & 6 & 8 & 54 & 18 & 29 & 21 & 12 & 16 & 19 & 4 \\ 81 & 50 & 20 & 11 & 8 & 6 & 32 & 24 & 29 & 25 & 22 & 9 & 6 & 5 & 10 & 6 & 4 & 4\end{array}$

$\begin{array}{rrrrrrrrrrrrrrrrrr}81 & 50 & 20 & 11 & 8 & 6 & 32 & 24 & 29 & 25 & 22 & 9 & 6 & 5 & 10 & 6 & 4 & 4 \\ 7 & 17 & 39 & 30 & 17 & 12 & 4 & 4 & 9 & 7 & 21 & 33 & 35 & 25 & 35 & 24 & 13 & 7\end{array}$

$\begin{array}{rrrrrrrrrrrrrrrrrrr}23 & 43 & 23 & 5 & 7 & 10 & 27 & 8 & 7 & 15 & 28 & 20 & 19 & 24 & 43 & 23 & 8 & 7\end{array}$

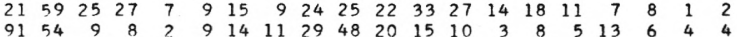

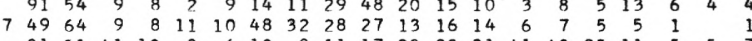

$\begin{array}{rrrrrrrrrrrrrrrrrrrr}31 & 11 & 41 & 10 & 8 & 6 & 10 & 8 & 11 & 17 & 23 & 23 & 21 & 41 & 43 & 25 & 11 & 5 & 5 & 7 \\ 15 & 1 & 28 & 24 & 23 & 7 & 10 & 12 & 7 & 18 & 12 & 13 & 35 & 37 & 50 & 31 & 18 & 12 & 5 & 3\end{array}$

$\begin{array}{lllllllllllllllllll}44 & 35 & 23 & 46 & 14 & 70 & 15 & 30 & 19 & 20 & 11 & 11 & 17 & 11 & 15 & 10 & 9 & 2 & 3\end{array}$

$\begin{array}{lllllllllllllllllll}26 & 47 & 15 & 10 & 6 & 4 & 15 & 11 & 5 & 13 & 27 & 14 & 25 & 51 & 48 & 14 & 15 & 4\end{array}$

$\begin{array}{rrrrrrrrrrrrrrrrrr}14 & 47 & 36 & 16 & 11 & 14 & 22 & 12 & 7 & 19 & 27 & 23 & 44 & 21 & 17 & 6 & 7 & 6 \\ 67 & 11 & 34 & 26 & 14 & 12 & 10 & 13 & 5 & 13 & 7 & 18 & 24 & 23 & 29 & 21 & 19 & 9 \\ & 55 & 29 & 18 & 13 & 10 & 9 & 12 & 3 & 13 & 6 & 14 & 25 & 15 & 50 & 27 & 15 & 14\end{array}$

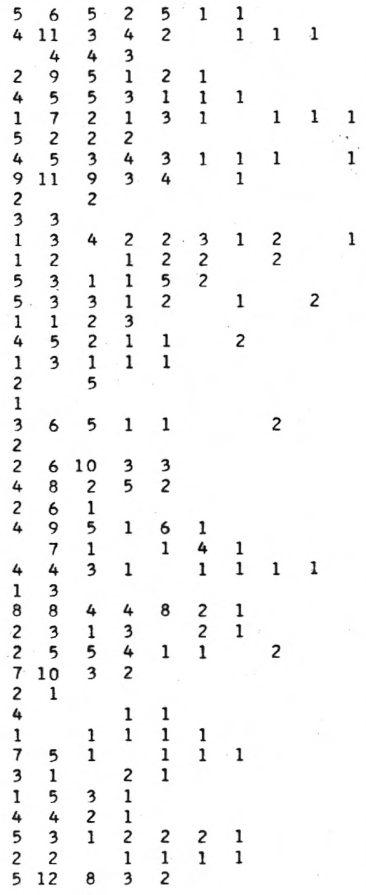

6287810.0

6653850.0

4390390.0

6709490.0

5547180.0
5780270.0

5195680.0

5805410.0

6907880.0
3332760.0

3332760.0
4010730.0

4010730.0

5714600.0
4674990.0

4743760.0

5107940.0

4069400.0

5287330.0

5149620.0

4241860.0

3191350.0

6389340.0

5149900.0

5253300.0

5197980.0

4260460.0

6824120.0

5159900.0

4874910.0

3233420.0

6441400.0

3396700.0

6119540.0

5516740.0

3764250.0

$33614 \mathrm{CO} .0$

2913860.0

5665960.0

5831160.0

3596390.0

5282930.0

4677720.0

4740010.0

6526630.0

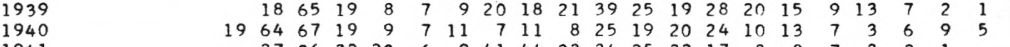

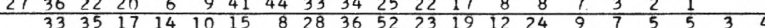

$\begin{array}{lllllllllllllllllllllllllll}13 & 14 & 28 & 20 & 9 & 15 & 10 & 8 & 7 & 27 & 34 & 15 & 24 & 20 & 27 & 18 & 16 & 9 & 12 & 5 & 13 & 7 & 5 & 5 & 1 & 2\end{array}$

$\frac{2981360.0}{4616200.0}$

$\begin{array}{llllllllllllllllllllllll}61 & 25 & 32 & 16 & 13 & 23 & 21 & 8 & 7 & 16 & 10 & 21 & 25 & 29 & 11 & 16 & 10 & 4 & 3 & 3 & 1 & 1\end{array}$

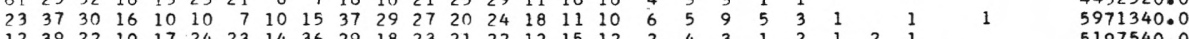
$\begin{array}{llllllllllllllllllllllllllll}12 & 39 & 22 & 10 & 17 & 24 & 23 & 14 & 36 & 29 & 18 & 23 & 21 & 22 & 12 & 15 & 12 & 2 & 4 & 3 & 1 & 2 & 1 & 2 & 1 & 5197540.0\end{array}$ $\begin{array}{lllllllllllllllllllllllllll}9 & 27 & 21 & 16 & 15 & 5 & 10 & 18 & 14 & 8 & 44 & 55 & 52 & 20 & 17 & 10 & 6 & 6 & 2 & 4 & 2 & 2 & 1 & 2 & 6846390.0\end{array}$

$\begin{array}{lllllllllllllllllllllllllll}12 & 25 & 16 & 16 & 22 & 24 & 27 & 14 & 22 & 16 & 16 & 29 & 35 & 31 & 24 & 6 & 6 & 4 & 3 & 5 & 4 & 3 & 2 & 2 & 1 & & 5765940.0\end{array}$ $\begin{array}{llllllllllllllllllllllll}24 & 36 & 28 & 21 & 14 & 8 & 10 & 11 & 12 & 20 & 38 & 40 & 29 & 21 & 12 & 10 & 9 & 5 & 8 & 4 & 2 & 3 & & \end{array}$ $\begin{array}{lllllllllllllllllllllllllll}10 & 64 & 12 & 10 & 14 & 13 & 7 & 12 & 14 & 23 & 31 & 26 & 24 & 23 & 17 & 12 & 8 & 4 & 10 & 11 & 12 & 7 & 1 & 7694030.0\end{array}$ $\begin{array}{llllllllllllllllllllll}1 & 60 & 14 & 11 & 15 & 13 & 25 & 22 & 29 & 40 & 30 & 42 & 16 & 14 & 7 & 6 & 6 & 3 & 6 & 5 & 1 & \end{array}$ $\begin{array}{lllllllllllllllllllllllllll}4 & 33 & 20 & 47 & 24 & 14 & 15 & 13 & 10 & 24 & 23 & 32 & 22 & 22 & 13 & 9 & 6 & 6 & 9 & 4 & 5 & 6 & 2 & 1 & 1 & 5910460.0\end{array}$ $\begin{array}{lllllllllllllllllllllll}9 & 49 & 67 & 12 & 29 & 37 & 23 & 11 & 11 & 15 & 13 & 17 & 19 & 9 & 8 & 7 & 12 & 6 & 7 & 3 & 1 & 6410800.0\end{array}$

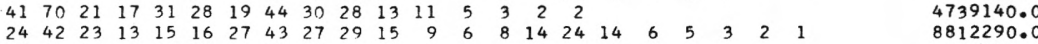
$\begin{array}{llllllllllllllllllllllll}38 & 57 & 24 & 14 & 14 & 19 & 52 & 35 & 28 & 13 & 17 & 11 & 8 & 6 & 5 & 5 & 5 & 6 & 7 & 1 & 5504030.0\end{array}$ $\begin{array}{lllllllllllllllllllllll}11 & 53 & 37 & 31 & 17 & 28 & 38 & 35 & 18 & 10 & 9 & 14 & 10 & 13 & 11 & 5 & 16 & 3 & 3 & 2 & 1 & 6046190.0\end{array}$ $\begin{array}{lllllllllllllllllllllll}56 & 71 & 32 & 28 & 14 & 33 & 32 & 23 & 30 & 19 & 24 & 14 & 9 & 6 & 5 & 6 & 6 & 3 & 2 & 5160130.0\end{array}$ $\begin{array}{rllllllllllllllllllllll}56 & 30 & 26 & 28 & 13 & 42 & 23 & 30 & 24 & 20 & 25 & 18 & 9 & 4 & 5 & 6 & 5 & 2 & & 4934720.0 \\ 3 & 44 & 63 & 17 & 20 & 28 & 4 & 14 & 22 & 18 & 18 & 17 & 1 & 12 & 7 & 9 & 5 & 3 & 3 & 1 & 2 & 1 & 5994300.0\end{array}$

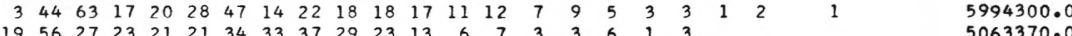
$\begin{array}{rrrrrrrrrrrrrrrrrrrrr}19 & 56 & 27 & 23 & 21 & 21 & 34 & 33 & 37 & 29 & 23 & 13 & 6 & 7 & 3 & 3 & 6 & 1 & 3 & 5063370.0 \\ 8 & 24 & 55 & 40 & 17 & 46 & 21 & 32 & 26 & 13 & 21 & 21 & 14 & 8 & 6 & 3 & 8 & & 2 & 5297780.0\end{array}$ 
Willamette River at Albany, Oreg.

Summary for water years 1896-1963

$\begin{array}{lrrrrl}\text { CLASS } & \text { CFS } & \text { TOTAL } & \text { ACCUM } & \text { PERCT } & \text { CLASS } \\ & 0 & & 24837 & 100.0 & 09 \\ 1 & 1000 & & 24837 & 100.0 & 10 \\ 2 & 1200 & & 24837 & 100.0 & 11 \\ 3 & 1400 & & 24837 & 100.0 & 12 \\ 4 & 1700 & 26 & 24837 & 100.0 & 13 \\ 5 & 2000 & 765 & 24811 & 99.9 & 14 \\ 6 & 2500 & 1583 & 24046 & 96.8 & 15 \\ 7 & 3000 & 1720 & 22463 & 90.4 & 16 \\ 8 & 3500 & 1203 & 20743 & 83.5 & 17\end{array}$

Willamette River at Albany, Oreg.

Summary for water years 1896-1941

$\begin{array}{lrrrrl}\text { CLASS } & \text { CFS } & \text { TOTAL } & \text { ACCUM } & \text { PERCT } & \text { CLAS } \\ & 0 & & 16802 & 100.0 & 09 \\ 1 & 1000 & & 16802 & 100.0 & 10 \\ 2 & 1200 & & 16802 & 100.0 & 11 \\ 3 & 1400 & & 16802 & 100.0 & 12 \\ 4 & 1700 & 26 & 16802 & 100.0 & 13 \\ 5 & 2000 & 705 & 16776 & 98.8 & 14 \\ 6 & 2500 & 1398 & 16071 & 95.6 & 15 \\ 7 & 3000 & 1424 & 14673 & 87.3 & 16 \\ 8 & 3500 & 904 & 13249 & 78.9 & 17\end{array}$

Willamette River at Albany, Oreg.

Summary for water years 1942-1963

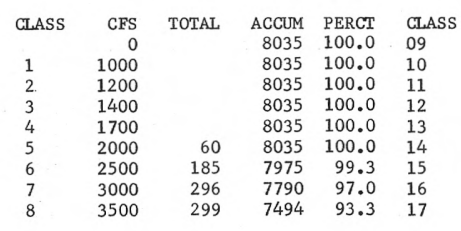

STATION NUMBER

$14-1740.00$

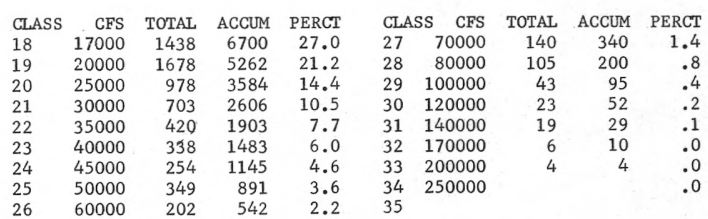

$14-1740.00$

TOTAL ACCUM PERCT

$\begin{array}{llr}570 & 12345 & 73.5 \\ 434 & 11775 & 70.1\end{array}$

$\begin{array}{lll}434 & 11775 & 70.1 \\ 866 & 11341 & 67.5\end{array}$

$\begin{array}{lll}660 & 10475 & 62.3\end{array}$

$\begin{array}{lll}716 & 9815 & 58.4\end{array}$

$\begin{array}{lll}1089 & 9099 & 54.2\end{array}$

$\begin{array}{lll}1319 & 8010 & 47.7\end{array}$

$\begin{array}{lll}1124 & 6691 & 39.8 \\ 1316 & 5567 & 33.1\end{array}$

STATION NUMBER

14-1740.00

\begin{tabular}{lrrrrrrrrr} 
CLASS & CFS & TOTAL & ACCUM & PERCT & \multicolumn{2}{l}{ CLASS CFS } & TOTAL & ACCUM & PERCT \\
18 & 17000 & 924 & 4251 & 25.3 & 27 & 70000 & 69 & 197 & 1.2 \\
19 & 20000 & 1134 & 3327 & 19.8 & 28 & 80000 & 64 & 128 & .8 \\
20 & 25000 & 636 & 2193 & 13.1 & 29 & 100000 & 28 & 64 & .4 \\
21 & 30000 & 454 & 1557 & 9.3 & 30 & 120000 & 16 & 36 & .2 \\
22 & 35000 & 247 & 1103 & 6.6 & 31 & 140000 & 12 & 20 & .1 \\
23 & 40000 & 208 & 856 & 5.1 & 32 & 170000 & 5 & 8 & .0 \\
24 & 45000 & 141 & 648 & 3.9 & 33 & 200000 & 3 & 3 & .0 \\
25 & 50000 & 196 & 507 & 3.0 & 34 & 250000 & & & .0 \\
26 & 60000 & 114 & 311 & 1.9 & 35 & & & &
\end{tabular}

TOTAL ACCUM PERCT

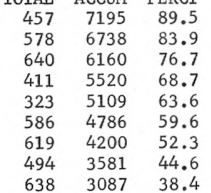

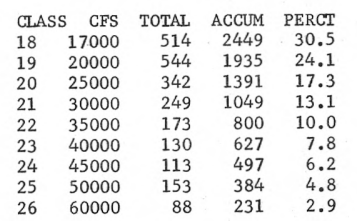

$\begin{array}{lrrrr}\text { CLASS } & \text { CFS } & \text { TOTAL } & \text { ACCUM } & \text { PERCT } \\ 27 & 70000 & 71 & 143 & 1.8 \\ 28 & 80000 & 41 & 72 & .9 \\ 29 & 100000 & 15 & 31 & .4 \\ 30 & 120000 & 7 & 16 & .2 \\ 31 & 140000 & 7 & 9 & .1 \\ 32 & 170000 & 1 & 2 & .0 \\ 33 & 200000 & 1 & 1 & .0 \\ 34 & 250000 & & & .0\end{array}$ 
W1llamette R1ver at Albany, Oreg.

STATION NUMBER $14-1740.00$ LOWEST MEAN DISCHARGF, IN CFS, FOR THF FOLLOWING NUMBER OF CONSECUTIVF DAYS IN YFAR RFGINNING APRIL 1

\begin{tabular}{|c|c|c|c|c|c|c|c|c|c|c|c|}
\hline YEAR & 1 & 3 & 7 & 14 & 30 & 60 & 90 & 120 & 150 & 183 & 274 \\
\hline 1895 & 2220.0 & 2220.0 & $2220 \cdot 0$ & 2250.0 & 2420.0 & 2780.0 & 2860.0 & $3000 \cdot 0$ & 3290.0 & $4060 \cdot 0$ & 8810.0 \\
\hline 1896 & 2950.0 & 2950.0 & 2950.0 & 2980.0 & 3150.0 & 3360.0 & 3520.0 & $4320 \cdot 0$ & 5950.0 & 8970.0 & 16500.0 \\
\hline 1897 & 3330.0 & 3330.0 & 3330.0 & 3330.0 & 3340.0 & 3550.0 & 3660.0 & $3970 \cdot 0$ & $4840 . \mathrm{C}$ & 6040.0 & 12200.0 \\
\hline 1898 & 2760.0 & 2760.0 & 2760.0 & 2760.0 & 2760.0 & 2940.0 & 3160.0 & 3340.0 & 3820.0 & 4670.0 & 8810.0 \\
\hline 1899 & 3730.0 & 3730.0 & 3880.0 & 4280.0 & 4350.0 & 5280.0 & 5520.0 & 6950.0 & 8070.0 & 10100.0 & 15300.0 \\
\hline $19 \cap 0$ & 2580.0 & $2640 . n$ & 2760.0 & 2760.0 & 2930.0 & 3010.0 & 3310.0 & $3810 \cdot 0$ & 4560.0 & 5530.0 & 9400.0 \\
\hline 1901 & 2950.0 & 2950.0 & $2950 . ?$ & 2950.0 & 2950.0 & 3210.0 & 3560.0 & $3760 \cdot 0$ & 4010.0 & 4860.0 & 9170.0 \\
\hline 1902 & 2950.0 & 2950.0 & 2950.0 & 2950.0 & 3060.0 & 3130.0 & 3330.0 & $3890 \cdot 0$ & 4980.0 & 7200.0 & 13700.0 \\
\hline $19 \cap 3$ & 3330.0 & $3330 . n$ & 3330.0 & 3470.0 & 3760.0 & 4110.0 & 4240.0 & 4350.0 & 4940.0 & 6030.0 & 9560.0 \\
\hline $19 \cap 4$ & 3140.0 & $314 n \cdot n$ & $314 n \cdot n$ & 3190.0 & 3270.0 & 3410.0 & 3540.0 & $3780 \cdot 0$ & 4250.0 & $4800 \cdot 0$ & 8560.0 \\
\hline 1905 & 2400.0 & 2400.0 & $24 n \cap .0$ & 2400.0 & 2400.0 & $2 \in \cap n \cdot n$ & 2910.0 & 3490.0 & $371 n .0$ & $4380 \cdot 0$ & 6510.0 \\
\hline $19 \cap 6$ & 2580.0 & $27 n n \cdot n$ & 2730.0 & 2870.0 & 2960.0 & 3070.0 & 3350.0 & $3760 \cdot 0$ & 5030.0 & 6410.0 & 9770.0 \\
\hline $19 n 7$ & 2580.0 & 2580.0 & $258 \cap .0$ & 2670.0 & 2860.0 & 2900.0 & 2980.0 & $3150 \cdot 0$ & 3520.0 & 4280.0 & 11100.0 \\
\hline 1908 & 2760.0 & $2760 . n$ & 2810.0 & 2880.0 & 2960.0 & 3190.0 & 3600.0 & $4330 \cdot 0$ & 4880.0 & 5630.0 & 7960.0 \\
\hline 1909 & 2950.0 & 2950.0 & 2950.0 & 2950.0 & 2980.0 & 3180.0 & 3340.0 & 3750.0 & 4440.0 & 5360.0 & 11400.0 \\
\hline 1910 & 2580.0 & 2580.0 & 2580.0 & 2640.0 & 2720.0 & 2820.0 & 3030.0 & 3110.0 & 3440.0 & 4150.0 & $8300 \cdot 0$ \\
\hline 1911 & 2400.0 & 2400.0 & 2500.0 & 2610.0 & 2830.0 & 3330.0 & 3690.0 & 3770.0 & 4390.0 & $5900 \cdot 0$ & $8380 \cdot 0$ \\
\hline 1912 & 3730.0 & $3730 . n$ & 3790.0 & 3880.0 & 3990.0 & 4770.0 & 4810.0 & $5480 \cdot 0$ & 6820.0 & 8170.0 & 10700.0 \\
\hline 1913 & 3330.0 & 3330.0 & 3330.0 & 3430.0 & 3740.0 & 4080.0 & 4810.0 & $5290 \cdot 0$ & 6670.0 & 7500.0 & $10200 . ?$ \\
\hline 1914 & 2760.0 & 2760.0 & 2810.0 & 2880.0 & 2940.0 & 3150.0 & 3660.0 & $4120 \cdot 0$ & 4850.0 & 5410.0 & 7290.0 \\
\hline 1915 & 2400.0 & $2400 . n$ & 2400.0 & 2450.0 & 2550.0 & 2600.0 & 2730.0 & 2980.0 & 3420.0 & 4970.0 & 10100.0 \\
\hline 1916 & 3220.0 & $3220 . n$ & 3330.0 & 3380.0 & 3510.0 & 3920.0 & 4290.0 & 4910.0 & $616 n .0$ & 7090.0 & 10300.0 \\
\hline 1917 & 3030.0 & $3030 . n$ & $3 \cap 3 \cap \cdot n$ & $3 \cap 30 . n$ & 3070.0 & $3230 . n$ & $333 n .0$ & $3610 \cdot 0$ & $476 n \cdot 0$ & $6840 . n$ & $142 n n \cdot n$ \\
\hline 1918 & 2140.0 & 2140.0 & 2230.0 & 2310.0 & 2480.0 & 2750.0 & 2920.0 & 3120.0 & 3400.0 & 4100.0 & 5940.0 \\
\hline 1919 & 2140.0 & 2140.0 & 2140.0 & 2270.0 & 2550.0 & 2990.0 & 3080.0 & 3430.0 & 4340.0 & 5970.0 & 10200.0 \\
\hline 1920 & 2360.0 & $2360 . n$ & 2360.0 & 2390.0 & 2580.0 & 2950.0 & 3640.0 & $4680 \cdot 0$ & 6110.0 & 7050.0 & 13500.0 \\
\hline 1971 & 3030.0 & $3030 . n$ & 3030.0 & 3030.0 & 3070.0 & 3330.0 & 3480.0 & $3780 \cdot 0$ & 4310.0 & 5710.0 & 11900.0 \\
\hline 1922 & 2780.0 & 2780.0 & $2870 . ?$ & 2930.0 & 3050.0 & 3140.0 & 3280.0 & $3430 \cdot 0$ & 3740.0 & 4800.0 & 9460.0 \\
\hline 1923 & 3090.0 & 3090.0 & 3120.0 & 3120.0 & 3140.0 & 3280.0 & 3700.0 & 3710.0 & 4380.0 & 5400.0 & 8370.0 \\
\hline 1924 & 2460.0 & $2460 . n$ & 2460.0 & 2540.0 & 2690.0 & 2760.0 & 2870.0 & 2920.0 & 3100.0 & 3710.0 & 9970.0 \\
\hline 1925 & 2400.0 & 2400.0 & 2400.0 & 2410.0 & 2550.0 & 2720.0 & 2730.0 & 2890.0 & 3360.0 & $3970 . n$ & 6750.0 \\
\hline 1926 & 2020.0 & 2020.0 & 2020.0 & 2140.0 & 2280.0 & 2300.0 & 2420.0 & 2550.0 & 2780.0 & 3080.0 & 7790.0 \\
\hline 1927 & 2400.0 & 2400.0 & $246^{n} \cdot n$ & 2570.0 & 2840.0 & 3340.0 & 4000.0 & $4980 \cdot 0$ & 5730.0 & $7330 . n$ & 11800.0 \\
\hline 1928 & 2400.0 & $24 n 0 . n$ & 2400.0 & 2430.0 & 2490.0 & $2540 . ?$ & $264 n .0$ & $2700 \cdot n$ & 2980.0 & 3350.0 & 6700.0 \\
\hline 1929 & 2340.0 & $234 n \cdot 0$ & 2340.0 & $234 n \cdot n$ & 2340.0 & 2380.0 & 2390.0 & $2460 \cdot n$ & 2670.0 & 3720.0 & 7750.0 \\
\hline 1930 & 2160.0 & $2160 \cdot n$ & 2160.0 & 2200.0 & 2280.0 & 2340.0 & 2450.0 & $2540 \cdot 0$ & 2830.0 & 3490.0 & 5150.0 \\
\hline 1931 & 1890.0 & 1940.0 & 1950.0 & 2050.0 & 2100.0 & $2160 . n$ & 2240.0 & $2540 \cdot 0$ & 3130.0 & 3700.0 & 7860.0 \\
\hline 1932 & 2400.0 & $2400 \cdot n$ & 2400.0 & 2400.0 & 2440.0 & $2720 . n$ & 2970.0 & 3370.0 & 4580.0 & 5780.0 & 10800.0 \\
\hline 1933 & 3070.0 & 3070.0 & 3100.0 & 3260.0 & 3470.0 & 3610.0 & 3770.0 & $4010 \cdot 0$ & 4410.0 & 6280.0 & 11400.0 \\
\hline 1934 & 2210.0 & $2210 \cdot 0$ & 2210.0 & 2210.0 & 2210.0 & 2230.0 & 2350.0 & $2570 \cdot 0$ & 2970.0 & 3570.0 & 9590.0 \\
\hline 1935 & 2340.0 & 2350.0 & 2380.0 & 2410.0 & 2590.0 & 2620.0 & 2840.0 & $2990 \cdot 0$ & 3460.0 & 3940.0 & 7250.0 \\
\hline 1936 & 2170.0 & 2180.0 & 2.190 .0 & 2210.0 & 2230.0 & 2260.0 & 2390.0 & $2520 \cdot 0$ & 2770.0 & 3210.0 & 5620.0 \\
\hline 1937 & 2840.0 & 2850.0 & 2920.0 & 3020.0 & 3110.0 & 3240.0 & 3560.0 & $4020 \cdot 0$ & 6390.0 & 8270.0 & 14900.0 \\
\hline 1938 & 2600.0 & 2600.0 & 2600.0 & 2610.0 & 2730.0 & $2780 . ?$ & 2830.0 & 3060.0 & 3730.0 & 4830.0 & 8230.0 \\
\hline 1939 & 2230.0 & 2240.0 & 2260.0 & 2300.0 & 2420.0 & 2540.0 & 2610.0 & $2700 \cdot 0$ & 2810.0 & 3320.0 & 5570.0 \\
\hline 1940 & 1860.0 & $1870 . n$ & $19 n n \cdot n$ & 1970.0 & 1970.0 & $2120 \cdot n$ & 2260.0 & 2350.0 & 2640.0 & 3290.0 & 6490. \\
\hline 1941 & 2340.0 & 2370.0 & $2410 . n$ & 2450.0 & 2570.0 & $3040 . n$ & 3190.0 & 3450.0 & 4000.0 & 4660.0 & 9960 \\
\hline 1942 & 2250.0 & 2290.0 & 2310.0 & 2430.0 & 2530.0 & $2690 . n$ & 2850.0 & $327 n \cdot 0$ & 4300.0 & $6000 \cdot n$ & 13300.0 \\
\hline
\end{tabular}

\begin{tabular}{|c|c|c|c|c|c|c|c|c|c|c|c|}
\hline 1943 & 3140.0 & 3150.0 & 3170.0 & 3250.0 & 3280.0 & 3490.0 & 3800.0 & 4930.0 & 6290.0 & 7000.0 & 8790.0 \\
\hline 1944 & 2130.0 & 2150.0 & 2180.0 & 2260.0 & 2320.0 & 2530.0 & 2590.0 & 2770.0 & 3090.0 & 3420.0 & \\
\hline 1945 & 2550.0 & 2560.0 & 2590.0 & 2670.0 & 2760.0 & 2950.0 & 3180.0 & $3330 \cdot 0$ & 3780.0 & 5880.0 & $13000 \cdot 0$ \\
\hline 1946 & 2690.0 & 2710.0 & 2770.0 & 2870.0 & 2910.0 & 3200.0 & 3530.0 & $4140 \cdot 0$ & 4920.0 & 5860.0 & 11700.0 \\
\hline 1947 & 2790.0 & 2820.0 & 2840.0 & 2900.0 & 3090.0 & 3280.0 & 3700.0 & $4500 \cdot 0$ & 5170.0 & 6390.0 & 1270 \\
\hline 1948 & 3120.0 & 3140.0 & 3220.0 & 3560.0 & 3730.0 & $3940 . ?$ & 4480.0 & $4950 \cdot 0$ & 5690.0 & 7590.0 & 12500 \\
\hline 1949 & 2870.0 & 2880.0 & 2907.0 & 2940.0 & 3050.0 & 3420.0 & 3820.0 & $3880 \cdot 0$ & 4090.0 & 5010.0 & 9400 \\
\hline 1950 & $34>0.0$ & 3440.0 & $3490 . n$ & 3600.0 & $3750 . n$ & $407 n . n$ & 4670.0 & 5910.0 & 7990.0 & 9530.0 & 18400 \\
\hline 1951 & $338 n .0$ & 3470.0 & 3430.0 & $3470 . ?$ & $3570 . n$ & 3620.0 & $376 n .0$ & $430 n \cdot n$ & $517 n \cdot 0$ & $6750 \cdot 0$ & $\begin{array}{r}12800 \\
798\end{array}$ \\
\hline 1952 & 2970.0 & 2990.7 & $x \cap \cap n \cdot ?$ & 3090.0 & $3190 . n$ & 3420.1 & 3680.0 & 3820.0 & 4100.0 & $5 n 9 n \cdot n$ & 798 \\
\hline 1953 & 4160.0 & 4200.0 & 4250.0 & 4330.0 & $4450 . n$ & $4520 . ?$ & 4640.0 & $4740 \cdot 0$ & 5370.0 & 7940.0 & 1530 \\
\hline 1954 & 4480.0 & $4640 . n$ & 4720.0 & 4810.0 & 4930.0 & 5000.0 & 5070.0 & $5240 \cdot 0$ & 5650.0 & 6140.0 & 820 \\
\hline 1955 & 4760.0 & 4790.0 & 4830.0 & 4890.0 & 4950.0 & 5140.0 & 5400.0 & 6540.0 & 7910.0 & 9460.0 & 2040 \\
\hline 1956 & 4160.0 & 4190.0 & 4290.0 & 4490.0 & 4940.0 & 5160.0 & $5210 \cdot 0$ & $5730 \cdot 0$ & 7390.0 & 8510.0 & 12100 \\
\hline 1957 & 4210.0 & $4350 . ?$ & 4390.0 & 4420.0 & 4490.0 & 4540.0 & 4690.0 & $5040 \cdot 0$ & 5180.0 & 5810.0 & 1060 \\
\hline 1958 & 4320.0 & 4330.0 & 4400.0 & 4440.0 & 4520.0 & 4710.0 & 5190.0 & $5440 \cdot 0$ & 6140.0 & 7080.0 & 11000 \\
\hline 1959 & 3910.0 & 3960.0 & 4030.0 & 4120.0 & 4200.0 & 4320.0 & 4510.0 & $5110 \cdot 0$ & 5990.0 & 6340.0 & 7450 \\
\hline 1960 & 4020.0 & $4050 . n$ & 4220.0 & 4290.0 & 4350.0 & 4480.0 & 4500.0 & $4970 \cdot 0$ & 5510.0 & 7850.0 & 12200 \\
\hline 1961 & 4520.0 & 4650.0 & 4707.0 & $4870 . ?$ & 4940.0 & 5020.0 & 5020.0 & $5260 \cdot 0$ & 5720.0 & 6300.0 & 11600 \\
\hline 1962 & 4260.0 & 4270.7 & $4320 . ?$ & 4390.0 & 4490.0 & 4670.0 & 4750.0 & 5510.0 & 6800.0 & 7890.0 & 11200 \\
\hline
\end{tabular}


W1llamette River at Albany, Oreg.

STATION NUMBER

$14-1740.00$

HIGHEST MEAN DISCHARGE, IN CFS, FOR THE FOLLOWING NUMRER OF CONSECUTIVE DAYS IN YEAR FNDING SEPTFMBER 30

\begin{tabular}{|c|c|c|c|c|c|c|c|c|c|c|c|}
\hline EAR & 1 & 3 & 7 & 15 & 30 & 60 & 90 & 120 & 150 & 183 & 274 \\
\hline 396 & 00.0 & $\ln 8 r$ & $n$ & $644 n n \cdot n$ & $0 n .0$ & $0, n$ & $0 n, n$ & $n n \cdot n$ & 00.0 & 0.0 & 21900,0 \\
\hline 1897 & 178000.0 & 158000.0 & $10300 n$. & $70100 . n$ & 49700.0 & 39100.0 & 22400.0 & 32000.0 & 31500.0 & 30200.0 & 23100.0 \\
\hline 1898 & 73900.0 & 71200.0 & $6500 n .0$ & 53200.0 & 40100.0 & 29000.0 & 25700.0 & 23600.0 & 21000.0 & 19200.0 & 15000.0 \\
\hline 1899 & 112000.0 & 99500.0 & 70000.0 & 47800.0 & 41600.0 & 36600.0 & 33100.0 & 30600.0 & 29400.0 & 27600.0 & 22800.0 \\
\hline 1900 & 120000.0 & 102000.0 & 79800.0 & 53400.0 & 37700.0 & 36900.0 & 31800.0 & 29200.0 & 27200.0 & 24400.0 & 19000.0 \\
\hline 1901 & 214000.0 & 168000.0 & 108000.0 & 64800.0 & 47900.0 & 39300.0 & 35200.0 & $31800 \cdot 0$ & 28400.0 & 25500.0 & 19900.0 \\
\hline 1902 & 79700.0 & 64100.0 & 50100.0 & 40600.0 & 36200.0 & 28900.0 & 26000.0 & 24000.0 & 23500.0 & 22400.0 & 17800.0 \\
\hline 1903 & 233000.0 & 163000.0 & 115000.0 & 74600.0 & 48900.0 & 42900.0 & 37800.0 & $32600 \cdot 0$ & $29200: 0$ & 26000.0 & 19900.0 \\
\hline 1904 & 174000.0 & 104000.0 & 72100.0 & 66600.0 & 63700.0 & 50000.0 & 42100.0 & 37600.0 & 33100.0 & 31300.0 & 23900.0 \\
\hline 1905 & 69400.0 & 60100.0 & 42100.0 & 29400.0 & 23700.0 & 19800.0 & 16400.0 & 17000.0 & 15400.0 & 14300.0 & 11200.0 \\
\hline 1906 & 56300.0 & 51900.0 & 47100.0 & 36500.0 & 25400.0 & 24200.0 & 20900.0 & 18900.0 & 17200.0 & 16600.0 & 13400.0 \\
\hline $19 \cap 7$ & 212000.0 & 176000.0 & 125000.0 & 83000.0 & 50100.0 & $403 n 0.0$ & $32.6 \cap 0.0$ & 31500.0 & 28600.0 & 26300.0 & 19800.0 \\
\hline 1908 & 147000.0 & 135000.0 & 110000.0 & 70600.0 & 48100.0 & $3280 n \cdot 0$ & 26600.0 & 24100.0 & 22000.0 & 20400.0 & 16000.0 \\
\hline 1909 & 115000.0 & 109000.0 & 97600.0 & $65900 . n$ & 48200.0 & 39700.0 & 222000.0 & 26900.0 & 23300.0 & 20700.0 & 16200.0 \\
\hline 1910 & 193000.0 & 165000.0 & 107000.0 & 68700.0 & 50600.0 & $31400 . n$ & 28100.0 & 29700.0 & 26400.0 & 23500.0 & $17600 . n$ \\
\hline 1911 & 78000.0 & 72600.0 & 50700.0 & 37800.0 & 28300.0 & 24600.0 & 22600.0 & 19700.0 & 18000.0 & 17000.0 & 13700.0 \\
\hline 1912 & 130000.0 & 113000.0 & 77600.0 & 56800.0 & 41400.0 & 34900.0 & 28800.0 & $25200 \cdot 0$ & 23000.0 & 21300.0 & 17700.0 \\
\hline 1913 & 91500.0 & 79200.0 & 56700.0 & 41800.0 & 30200.0 & 23600.0 & 22000.0 & $22000 \cdot 0$ & 20600.0 & 20000.0 & 17300.0 \\
\hline 1914 & 67100.0 & 65400.0 & 58800.0 & 41100.0 & 31600.0 & 26500.0 & 23700.0 & $21600 \cdot 0$ & 19600.0 & 17900.0 & 14300.0 \\
\hline 1915 & 48000.0 & 41400.0 & 30100.0 & 23400.0 & 19600.0 & 16000.0 & 14200.0 & 13100.0 & 12800.0 & 12200.0 & 10600.0 \\
\hline 1916 & 149000.0 & 130000.0 & 90400.0 & 62800.0 & 44600.0 & 38100.0 & 22600.0 & 31400.0 & 30100.0 & 27800.0 & 22000.0 \\
\hline 1917 & 45600.0 & 41600.0 & 38800.0 & 34700.0 & 32000.0 & 27700.0 & 24400.0 & $22400 \cdot 0$ & 21400.0 & 20500.0 & 17500.0 \\
\hline 1918 & 89000.0 & 77800.0 & 67800.0 & 58700.0 & 51100.0 & 43200.0 & 36700.0 & $32300 \cdot 0$ & 28500.0 & 25000.0 & 18200.0 \\
\hline 1919 & 86800.0 & 81800.0 & 77000.0 & 54400.0 & 43100.0 & 39600.0 & 34700.0 & 30700.0 & 27000.0 & 23900.0 & 18000.0 \\
\hline 1920 & 69000.0 & 59000.0 & 46900.0 & 35200.0 & 27500.0 & $22300 . n$ & 20500.0 & 17700.0 & 17800.0 & 17600.0 & 0.0 \\
\hline 1921 & 115000.0 & 101000.0 & $91500 . ?$ & 71200.0 & 57400.0 & 43400.0 & 29400.0 & 35600.0 & 32300.0 & 29600.0 & 23600.0 \\
\hline 1922 & 120000.0 & 107000.0 & 76500.0 & 74600.0 & 46900.0 & 29100.0 & 25000.0 & 23500.0 & 23600.0 & 22500.0 & 00.0 \\
\hline 1923 & 199000.0 & 156000.0 & 111000.0 & 79700.0 & 58600.0 & 37900.0 & 30900.0 & $26800 \cdot 0$ & 24000.0 & 21700.0 & 16700.0 \\
\hline 1924 & 52000.0 & $46200 . n$ & 43400.0 & 34300.0 & 23700.0 & 20100.0 & 19100.0 & 16900.0 & 158 & 20.0 & 10800.0 \\
\hline 1925 & 126000.0 & 113000.0 & 93400.0 & 79800.0 & 56300.0 & 46100.0 & 38300.0 & 37100.0 & 32200.0 & 29400.0 & 22500.0 \\
\hline 1926 & 131000.0 & 117000.0 & 87200.0 & 56500.0 & 47300.0 & 30600.0 & 24500.0 & 20400.0 & 17700.0 & 15700.0 & 00.0 \\
\hline 1927 & 158000.0 & 135000.0 & 91500.0 & 59200.0 & 46200.0 & 40000.0 & 36100.0 & $33800 \cdot 0$ & 30200.0 & 27400.0 & 21200.0 \\
\hline 1928 & 74000.0 & 65000.0 & 56800.0 & 43300.0 & 37200.0 & 30000.0 & 26900.0 & 25500.0 & 26100.0 & 25000.0 & 19200.0 \\
\hline 1929 & 50800.0 & 43200.0 & 37600.0 & 31300.0 & 23500.0 & 21100.0 & 18400.0 & 18100.0 & 17800.0 & 16800.0 & 12800.0 \\
\hline 1930 & 84200.0 & 70000.0 & 50400.0 & 38800.0 & 33700.0 & 22500.0 & 22200.0 & 19000.0 & 17000.0 & 15600.0 & 11500.0 \\
\hline 1931 & 108000.0 & 91000.0 & 64100.0 & 42800.0 & 32900.0 & 22400.0 & 17800.0 & 15700.0 & 14000.0 & 12700.0 & 9850.0 \\
\hline 1932 & 126000.0 & 106000.0 & 76200.0 & 61100.0 & 43700.0 & 32500.0 & 29200.0 & 23500.0 & 27000.0 & $? 4800.0$ & 19700.0 \\
\hline 1933 & 90000.0 & 77400.0 & 57900.0 & 48300.0 & 37400.0 & 30200.0 & 29300.0 & 00.0 & & 00.0 & 20100.0 \\
\hline 1934 & 70200.0 & 63200.0 & 55200.0 & 41400.0 & 32100.0 & 29500.0 & 232000.0 & $21000 \cdot 0$ & 18600.0 & 16300.0 & 00.0 \\
\hline 1935 & 72600.0 & 68400.0 & 59400.0 & 49100.0 & 38300.0 & 30700.0 & 27900.0 & 10.0 & 0.0 & 0.0 & 00.0 \\
\hline 1936 & 120000.0 & 113000.0 & 89700.0 & 69600.0 & 47500.0 & 34300.0 & 28700.0 & & .0 & & $n 0.0$ \\
\hline 1937 & 124000.0 & 108000.0 & 77800.0 & 52100.0 & 38000.0 & 31900.0 & 30200.0 & $27500 \cdot 0$ & 0.0 & 22400.0 & 16500.0 \\
\hline 1938 & 92600.0 & 85400.0 & 70200.0 & 55500.0 & 39500.0 & 35100.0 & 25000.0 & 33700.0 & 326 & 0.0 & 00.0 \\
\hline 1939 & 58900.0 & 51700.0 & 42400.0 & 4100.0 & 26400.0 & 25800.0 & 22300.0 & .0 & .0 & 0.0 & 00.0 \\
\hline 1940 & 58300.0 & 55200.0 & 49400.0 & 0700.0 & 38400.0 & 0.0 & 24000.0 & & & & 0.0 \\
\hline 1941 & 40100.0 & 35900.0 & 28700.0 & .0 & 20300.0 & 18000.0 & 15800.0 & 0.0 & 0.0 & 0.0 & 9910.0 \\
\hline$\overline{1942}$ & 400.0 & 749 & 0.0 & .0 & .0 & 0.0 & 28100.0 & & & 0.0 & 15800.0 \\
\hline 1943 & 210000.0 & 174000.0 & 128000.0 & 88000.7 & 64300.0 & 54100.0 & 48800.0 & 40900.0 & 37100.0 & 33500.0 & 25700.0 \\
\hline & & & & & & & .0 & 00 & $127 n n .0$ & 12600.0 & 1 \\
\hline & 0.0 & & 0.0 & & & & & & & & \\
\hline & 185000.0 & 134 & & 72 & .0 & 39 & .0 & & 0 & 27 & $0 . n$ \\
\hline & 133000.0 & 00.0 & 0.0 & & 0 & 2 & & & & & 00.0 \\
\hline 194 & 167000.0 & $133000 \cdot 0$ & 97900.0 & 67900.0 & 44100.0 & 35100.0 & 32000.0 & 296 & 28700.0 & $27300 \cdot 0$ & 500.0 \\
\hline & 122000.0 & 104000.0 & 0.0 & & & 31100.0 & 39100.0 & 278 & 25900.0 & 25500.0 & 19800.0 \\
\hline 1950 & 95900.0 & 89900.0 & 72800.0 & 51400.0 & 46100.0 & 41600.0 & 37400.0 & 326 & & 0.0 & 0.0 \\
\hline 1951 & 138000.0 & 109000.0 & 87100.0 & 65600.0 & 58000.0 & 44500.0 & 44200.0 & $43300 \cdot 0$ & 39500.0 & 35300.0 & 26800.0 \\
\hline & 800.0 & 76500.0 & $0 . ?$ & & 39100.0 & & & & & & 21 \\
\hline 195 & 164000.0 & 134000.0 & 102000.0 & 75900.0 & 67000.0 & 47000.0 & 38300.0 & $3 ? 200.0$ & 29800.0 & 27100.0 & 00.0 \\
\hline 1954 & 106000.0 & 91200.0 & 70500.0 & 56600.0 & 55000.0 & $43100 . ?$ & 42900.0 & 3740 & 33500.0 & 29100.0 & 21700.0 \\
\hline & 400.0 & & & & & & & & & 300.0 & \\
\hline & 150000.0 & 13 & 110 & & & . $n$ & & & & & 30300.0 \\
\hline 19 & 80200.0 & 77700.0 & 0.0 & 61000.0 & 50800.0 & 36100.0 & 28400.0 & $28500 \cdot 0$ & 25500.0 & 23700.0 & 18500.0 \\
\hline 1958 & 101000.0 & 87400.0 & 0.0 & 60 & 47600.0 & $436 n n \cdot n$ & $n .0$ & $33000 \cdot 0$ & 29900.0 & & 2 \\
\hline & 75000.0 & 68000.0 & 56800.0 & 42300.0 & 40600.0 & 32900.0 & 27300.0 & & & & 17300.0 \\
\hline 1960 & 62400.0 & 58200.0 & 50100.0 & 39300.0 & 33500.0 & 29900.0 & 26500.0 & 257 & 23200.0 & 20600.0 & 16500.0 \\
\hline 19 & 153000.0 & 120000.0 & 91400.0 & & 55100.0 & 42700.0 & 33400.0 & 31300.0 & 29100.0 & 26200.0 & 20200.0 \\
\hline 196 & 73000.0 & 67700.0 & 10.0 & 44100.0 & 33200.0 & 28900.0 & 23600.0 & $21500 \cdot 0$ & 22200.0 & $21100 \cdot 0$ & 16900.0 \\
\hline 1963 & 70800.0 & 65500.0 & 52200.0 & 39400.0 & 31700.0 & 27800.0 & 23000.0 & $22600 \cdot 0$ & 20100.0 & 21300.0 & 17500 \\
\hline
\end{tabular}


North Santiam River below

Boulder Creek near Detrait, Oreg.
CTATION NUMRER $14-1780.00$

DURATION TABLF OF DAILY DISCHARGF

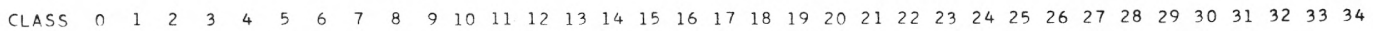

YEAR
1908
1909
1929
1930
1931
1932
1933
1934
1935
1936
1937
1938
1939
1940
1941
1942
1943
1944
1945
1946
1947
1948
1949
1950
1951
1952
1953
1954
1955
1956
1957
1958
1959
1960
1961
1962
1963 NUMBER OF DAYS IN CLASS

$\begin{array}{lllllllllllllllllllll}18 & 9 & 30 & 57 & 47 & 30 & 22 & 37 & 63 & 52 & 25 & 9 & 6 & 8 & 1 & & 3 & 1 & 1 & 4 \\ & 9 & 58 & 16 & 28 & 84 & 51 & 14 & 15 & 4 & 6 & 1 & 2 & & 1 & 1 & 1\end{array}$

$\begin{array}{lllllllllllll}23 & 50 & 36 & 69 & 32 & 25 & 28 & 12 & 26 & 36 & 17 & 10\end{array}$

$\begin{array}{llllllllllllll}37 & 94 & 22 & 16 & 25 & 19 & 19 & 74 & 20 & 2 & 13 & 7 & 8 \\ 83 & 29 & 40 & 37 & 44 & 25 & 20 & 31 & 30 & 4 & 5 & 8 & 4\end{array}$

$91214 \quad 4042 \quad 45231424 \quad 27 \quad 1930 \quad 26 \quad 28$

$\begin{array}{lllllllllllll}14 & 10 & 16 & 48 & 42 & 24 & 39 & 51 & 29 & 35 & 20 & 19\end{array}$

$\begin{array}{rrrrrrrrrrrrr}3 & 43 & 26 & 50 & 43 & 29 & 30 & 44 & 16 & 17 & 20 & 12 & 12 \\ 10 & 32 & 31 & 14 & 13 & 21 & 20 & 64 & 55 & 24 & 53 & 20 & 4\end{array}$

$\begin{array}{llllllllllllll}39 & 55 & 33 & 31 & 29 & 26 & 25 & 39 & 21 & 27 & 11 & 20 \\ 56 & 39 & 22 & 30 & 25 & 12 & 14 & 75 & 14 & 24 & 18 & 22\end{array}$

$\begin{array}{rrrrrrrrrrrrr}50 & 56 & 39 & 22 & 30 & 25 & 12 & 14 & 25 & 14 & 24 & 18 & 22 \\ & 7 & 53 & 28 & 28 & 10 & 14 & 27 & 60 & 32 & 35 & 22 & 34\end{array}$

$\begin{array}{rrrrrrrrrrrrrrr}3 & 61 & 25 & 23 & 31 & 40 & 37 & 50 & 23 & 19 & 35 & 9 & 7 \\ 64 & 91 & 22 & 10 & 26 & 15 & 14 & 42 & 24 & 20 & 12 & 11 & 8 \\ 30 & 60 & 24 & 15 & 83 & 48 & 31 & 35 & 24 & 6 & 4 & 4 & 1\end{array}$

$\begin{array}{llllllllllllll}15 & 40 & 31 & 13 & 27 & 34 & 49 & 67 & 39 & 16 & 14 & 6 & 6\end{array}$

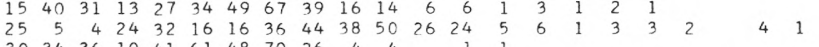

$25 \quad 1502313 \quad 11$

$27 \quad 9 \begin{array}{lllllllllllllll}22 & 33 & 26 & 17 & 23 & 46 & 47 & 26 & 46 & 23 & 8 \\ & 55 & 23 & 33 & 31 & 47 & 31 & 51 & 42 & 21 & 12 & 6\end{array}$

$\begin{array}{lllllllllll}18 & 41 & 41 & 20 & 62 & 48 & 28 & 32 & 29 & 28\end{array}$

$\begin{array}{llllllllllll}31 & 74 & 45 & 23 & 28 & 35 & 22 & 29 & 25 & 32 & 10 \\ 19 & 57 & 42 & 33 & 36 & 15 & 32 & 32 & 31 & 42 & 17\end{array}$

$\begin{array}{llllllllll}30 & 39 & 28 & 12 & 31 & 50 & 21 & 47 & 50 & 29 \\ 21 & 42 & 51 & 33 & 54 & 37 & 26 & 37 & 25 & 34\end{array}$

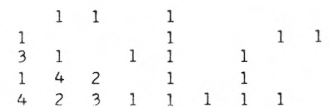

$\begin{array}{lllllllllll}32 & 43 & 27 & 12 & 46 & 38 & 24 & 36 & 17 & 9\end{array}$

$\begin{array}{lllllllllllll}8 & 20 & 50 & 40 & 11 & 31 & 32 & 43 & 75 & 26 & 18 \\ 3 & 28 & 67 & 69 & 44 & 47 & 34 & 17 & 27 & 15 & 9\end{array}$

$\begin{array}{llllllllllllll}3 & 28 & 67 & 69 & 44 & 47 & 24 & 17 & 27 & 15 & 9 & 5 \\ 3 & 6 & 38 & 38 & 26 & 52 & 25 & 33 & 37 & 36 & 35 & 21\end{array}$

$\begin{array}{rrrrrrrrrrrr}4 & 38 & 28 & 57 & 51 & 28 & 35 & 33 & 21 & 31 & 16 & 7 \\ 12 & 30 & 47 & 42 & 16 & 32 & 51 & 23 & 27 & 31 & 12 & 24\end{array}$

$\begin{array}{llllllllllll}19 & 45 & 60 & 37 & 31 & 33 & 31 & 25 & 29 & 22 & 17\end{array}$

$\begin{array}{lllllllllllll}11 & 19 & 45 & 60 & 37 & 31 & 33 & 31 & 25 & 29 & 22 & 17 \\ 16 & 29 & 32 & 45 & 15 & 21 & 26 & 42 & 48 & 42 & 25 & 11\end{array}$

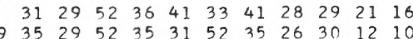

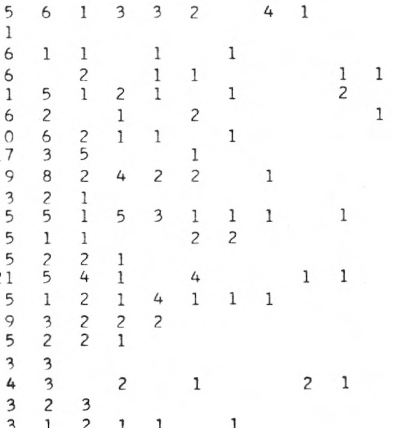

CFS-DAYS

338982.0

311317.0
307801.0

275126.0

373792.0

410141.0

353715.0
361805.0

342718.0

331824.0

300502.0

269640.0

309887.0

480724.0
248370.0

305499.0

393877.0

377897.0

420925.0

462351.0

409460.0

410403.0

440472.0

347977.0

369282.0

383333.0

350248.0

413880.0

$363991 \cdot 0$

North Santiam River below Boulder Creek

near Detroit, Oreg.
Summary for water years 1908-09, 1929-63

STATION NUMBER $\quad 14-1780.00$

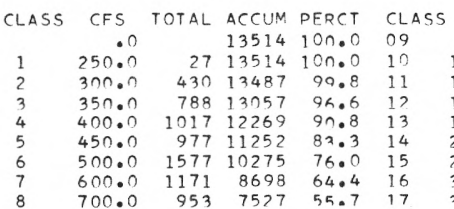

CFS TOTAL ACCUM PERCT $800.0 \quad 1613 \quad 6574 \quad 48.6$ $\begin{array}{llll}800.0 & 1329 & 4961 & 36.7\end{array}$ $\begin{array}{rrrr}1200.0 & 868 & 3637 & 26.9\end{array}$ $1400.0 \quad 1081 \quad ? 764 \quad 20 \cdot 5$ $2000.0 \quad 579 \quad 1036 \quad 7.7$ $2500.0 \quad 188 \quad 457 \quad 3.4 \quad 24 \quad 10000$ $\begin{array}{lllllllll}600.0 & 1171 & 8698 & 64.4 & 16 & 3000.0 & 85 & 269 & 2.0\end{array}$

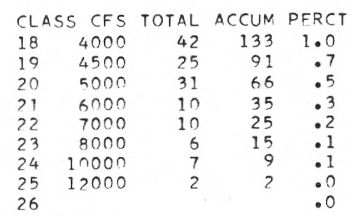
$\begin{array}{lc}\text { CLASS CFS TOTAL ACCUM PERCT } \\ 27 & .0 \\ 28 & .0 \\ 29 & .0 \\ 30 & .0 \\ 31 & .0 \\ 32 & .0 \\ 33 & .0 \\ 34 & .0\end{array}$

North Santiam River below

STATION NUMBER $\quad 14-1780.00$

troit, Oreg.

YFAR

1907

1908

193

1932

1933

1935

1936
1937

1938

192

1941

1942

1944

1946

1947

1948

1949

1951

1952

1953
1954

1955

1956

1958

1958
1959
1960

1961

\begin{tabular}{|c|c|c|}
\hline 1 & 3 & 7 \\
\hline 400.0 & $400 \cdot n$ & $400 . ?$ \\
\hline 375.0 & 375.0 & 377.0 \\
\hline 342.0 & 342.0 & 342.0 \\
\hline 330.0 & 333.0 & 339.0 \\
\hline 295.0 & $295 \cdot 0$ & 296.0 \\
\hline 367.0 & 369.0 & 371.0 \\
\hline 450.0 & 454.0 & 456.0 \\
\hline 343.0 & 346.0 & 349.0 \\
\hline 361.0 & 364.0 & 367.0 \\
\hline 321.0 & $322 . n$ & 323.0 \\
\hline 385.0 & 386.0 & 391.0 \\
\hline 377.0 & 377.0 & 378.0 \\
\hline 338.0 & 340.0 & 344.0 \\
\hline 305.0 & $305 . n$ & $306 . ?$ \\
\hline 346.0 & 346.0 & 350.0 \\
\hline 322.0 & 325.0 & 325.0 \\
\hline 420.0 & 422.0 & 424.0 \\
\hline 315.0 & 315.0 & 317.0 \\
\hline 326.0 & 327.0 & 327.0 \\
\hline 404.0 & 405.0 & 408.0 \\
\hline 408.0 & 410.0 & 413.0 \\
\hline 479.0 & 481.0 & 486.0 \\
\hline 461.0 & 464.0 & 469.0 \\
\hline 512.0 & 512.0 & 516.0 \\
\hline 461.0 & 462.0 & 464.0 \\
\hline 386.0 & 387.0 & 390.0 \\
\hline 446.0 & $446 \cdot n$ & $449 . ?$ \\
\hline 502.0 & 502.0 & 505.0 \\
\hline 431.0 & 436.0 & 451.0 \\
\hline 465.0 & 465.0 & 472.0 \\
\hline 375.0 & 375.0 & 380.0 \\
\hline $400 \cdot 0$ & $400 \cdot 0$ & 409.0 \\
\hline 425.0 & 427.0 & 430.0 \\
\hline 366.0 & 366.0 & 368.0 \\
\hline 415.0 & 418.0 & 423.0 \\
\hline 425.0 & 428.0 & 432.0 \\
\hline
\end{tabular}

14
$414 \cdot 0$
$389 \cdot 0$
$345 \cdot 0$
$344 \cdot 0$
$297 \cdot 0$
$377 \cdot 0$
$468 \cdot 0$
$353 \cdot 0$
$373 \cdot 0$
$326 \cdot 0$
$404 \cdot 0$
$385 \cdot 0$
$350 \cdot 0$
$310 \cdot 0$
$355 \cdot 0$
$330 \cdot 0$
$430 \cdot 0$
$318 \cdot 0$
$329 \cdot 0$
$416 \cdot 0$
$416 \cdot 0$
$487 \cdot 0$
$473 \cdot 0$
$522 \cdot 0$
$468 \cdot 0$
$402 \cdot 0$
$460 \cdot 0$
$511 \cdot 0$
$453 \cdot 0$
$484 \cdot 0$
$393 \cdot 0$
$418 \cdot 0$
$434 \cdot 0$
$376 \cdot 0$
$427 \cdot 0$
$440 \cdot 0$

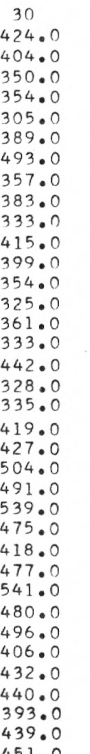

\begin{tabular}{|c|}
\hline $\begin{array}{c}60 \\
432.0\end{array}$ \\
\hline \\
\hline 362.0 \\
\hline 357.0 \\
\hline 313.0 \\
\hline $\begin{array}{l}412.0 \\
512.0\end{array}$ \\
\hline $\begin{array}{l}512.0 \\
366.0\end{array}$ \\
\hline $\begin{array}{l}366.0 \\
395.0\end{array}$ \\
\hline 342.0 \\
\hline 433.0 \\
\hline 413.0 \\
\hline $36 ? \cdot 0$ \\
\hline 331.0 \\
\hline $\begin{array}{l}379.0 \\
344.0\end{array}$ \\
\hline $\begin{array}{l}344.0 \\
475.0\end{array}$ \\
\hline 340.0 \\
\hline 360.0 \\
\hline $442 \cdot 0$ \\
\hline $\begin{array}{l}439.0 \\
533.0\end{array}$ \\
\hline 504.0 \\
\hline $\begin{array}{l}582.0 \\
506.0\end{array}$ \\
\hline 423.0 \\
\hline 484.0 \\
\hline $568 \cdot 0$ \\
\hline $501 \cdot 0$ \\
\hline $\begin{array}{l}526.0 \\
416.0\end{array}$ \\
\hline 446.0 \\
\hline $458 \cdot 0$ \\
\hline $\begin{array}{l}408.0 \\
468.0\end{array}$ \\
\hline 471.0 \\
\hline
\end{tabular}

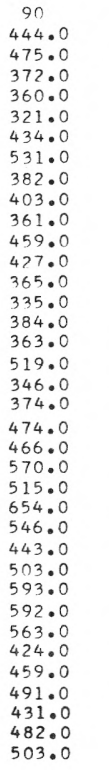

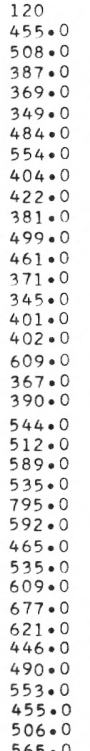

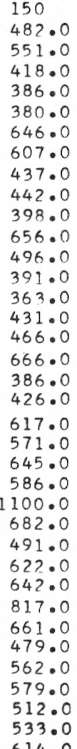

83

37.0

$15 \cdot 0$

15.0

(1)

4.0

$0.0 \quad 675.0$

$\begin{array}{ll}623.0 \\ 4.0 & 539.0\end{array}$

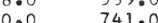

541.0
$573.0 \quad 956.0$

$668.0 \quad 706.0$

$\begin{array}{ll}58.0 & 706.0 \\ 03.0 & 540.0\end{array}$

1070.0

$\quad 1070.0$

889.0
775.0

852.0
65.0

893.0
$0.0 \quad 1410.0$

878.0
2.0

907.0
98.0

1110.0
717.0

1270.0

937.0
$81.0 \quad 937.0$

812.0
70.0

$18.0 \quad 687.0$

687.0
$611.0 \quad 943.0$

$\begin{array}{ll}630.0 & 810.0 \\ 05.0 & 898.0\end{array}$ 
North Santiam River below

Boulder Creek near Detroit, Oreg.

STATION NUMBER $14-1780.00$

HIGHEST MEAN DISCHARGE, IN CFS, FOR THE FOLLOWING NUMBER OF CONSECUTIVE DAYS IN YEAR ENDING SEPTEMBER 30

\begin{tabular}{|c|c|c|c|c|c|c|c|c|c|c|c|}
\hline YEAR & 1 & 3 & 7 & 15 & 30 & 60 & 90 & 120 & 150 & 183 & 274 \\
\hline 1908 & 5820.0 & 5000.0 & 4230.0 & 2690.0 & 1930.0 & 1510.0 & 1340.0 & $1260 \cdot 0$ & 1280.0 & 1250.0 & 1090.0 \\
\hline 1909 & 5150.0 & 4710.0 & $3660 \cdot 0$ & 2580.0 & 1840.0 & 1500.0 & 1300.0 & $1240 \cdot 0$ & $1220 \cdot 0$ & 1180.0 & 1010.0 \\
\hline 1929 & 2520.0 & $2320 \cdot 0$ & $2260 \cdot 0$ & 2010.0 & 1880.0 & 1700.0 & 1540.0 & $1410 \cdot 0$ & 1250.0 & $1150 \cdot 0$ & 982.0 \\
\hline 1930 & 5480.0 & 4050.0 & $2640 \cdot 0$ & 2420.0 & 1950.0 & 1430.0 & 1330.0 & $1240 \cdot 0$ & 1160.0 & $1100 \cdot 0$ & 884.0 \\
\hline 1931 & 10100.0 & 8240.0 & 4870.0 & 3100.0 & 2330.0 & 1670.0 & 1370.0 & $1240 \cdot 0$ & 1110.0 & $1010 \cdot 0$ & 819.0 \\
\hline 1932 & 7620.0 & 5970.0 & 4170.0 & 3420.0 & 2530.0 & 2220.0 & 2090.0 & $1880 \cdot 0$ & 1670.0 & 1520.0 & 1230.0 \\
\hline 1933 & 7000.0 & 5470.0 & 4580.0 & 3590.0 & 2930.0 & 2300.0 & $2000 \cdot 0$ & $1790 \cdot 0$ & 1610.0 & 1450.0 & 1330.0 \\
\hline 1934 & 9800.0 & 7400.0 & $5480 . n$ & 3860.0 & 2820.0 & 2450.0 & 1950.0 & $1790 \bullet 0$ & $16=0.0$ & 1460.0 & 1160.0 \\
\hline 1935 & 4870.0 & $4300 \cdot 0$ & 3120.0 & 2150.0 & 1680.0 & 1580.0 & 1410.0 & $1290 \cdot 0$ & 1250.0 & $1280 \cdot 0$ & 1180.0 \\
\hline 1936 & $5>10.0$ & 4310.0 & $3>40.0$ & 3000.0 & 2060.0 & 1730.0 & 1490.0 & $1420 \cdot 0$ & 1480.0 & 1400.0 & 1110.0 \\
\hline 1937 & 6690.0 & 5410.0 & 3740.0 & 2580.0 & 2260.0 & 2180.0 & 2010.0 & $1790 \cdot 0$ & $1590 \cdot 0$ & $1410 \cdot 0$ & 1090.0 \\
\hline 1938 & 5860.0 & $3960 \cdot 0$ & 2940.0 & 2550.0 & 2120.0 & 1860.0 & 1720.0 & $1550 \cdot 0$ & 1560.0 & 1590.0 & 1390.0 \\
\hline 1939 & $2810 \cdot 0$ & $2500 \cdot 0$ & 2180.0 & 1840.0 & 1640.0 & 1550.0 & 1360.0 & $1240 \cdot 0$ & 1140.0 & $1130 \cdot 0$ & 967.0 \\
\hline 1940 & 4380.0 & $3210 \cdot 0$ & 2550.0 & 2060.0 & 1920.0 & 1660.0 & 1440.0 & $1310 \cdot 0$ & 1230.0 & 1110.0 & 867.0 \\
\hline 1941 & 2450.0 & 1840.0 & $1400 \cdot 0$ & $1280 \cdot 0$ & 1050.0 & 948.0 & 897.0 & $832 \cdot 0$ & 785.0 & 805.0 & 715.0 \\
\hline 1942 & 4900.0 & 3970.0 & 3010.0 & 2020.0 & 1930.0 & 1530.0 & 1390.0 & $1260 \cdot 0$ & 1170.0 & $1120 \cdot 0$ & 999.0 \\
\hline 1943 & 8350.0 & 6690.0 & 5520.0 & 4140.0 & 2800.0 & 2600.0 & 2150.0 & $1900 \cdot 0$ & 1910.0 & 1840.0 & 1600.0 \\
\hline $\begin{array}{l}1944 \\
1945\end{array}$ & $\begin{array}{l}2850.0 \\
6050.0\end{array}$ & $\begin{array}{l}2290 \cdot 0 \\
4110 \cdot 0\end{array}$ & $\begin{array}{l}1600 \cdot 0 \\
3650.0\end{array}$ & 1220.0 & 1010.0 & 944.0 & 897.0 & $851 \cdot 0$ & $819 \cdot 0$ & 810.0 & 780.0 \\
\hline $\begin{array}{l}1945 \\
1946\end{array}$ & $\begin{array}{r}6050.0 \\
13000.0\end{array}$ & $\begin{array}{l}4110.0 \\
9730.0\end{array}$ & $\begin{array}{l}3650.0 \\
5800.0\end{array}$ & $\begin{array}{l}2610.0 \\
3810.0\end{array}$ & $\begin{array}{l}1870.0 \\
2580.0\end{array}$ & $\begin{array}{l}1540.0 \\
1920.0\end{array}$ & $\begin{array}{l}1400.0 \\
1610.0\end{array}$ & $\begin{array}{l}1450 \cdot 0 \\
1510.0\end{array}$ & $\begin{array}{l}1380.0 \\
1520.0\end{array}$ & $\begin{array}{l}1250.0 \\
1510.0\end{array}$ & $\begin{array}{r}991.0 \\
1300.0\end{array}$ \\
\hline 1947 & 11500.0 & 9850.0 & 6270.0 & 3740.0 & 3020.0 & 2020.0 & 1800.0 & 1640.0 & 1560.0 & 1480.0 & 1230.0 \\
\hline 1948 & 12100.0 & 7680.0 & 4660.0 & 3130.0 & 2110.0 & 1750.0 & 1640.0 & 1480.0 & 1460.0 & 1470.0 & 1400.0 \\
\hline 1949 & 6600.0 & 5030.0 & 3700.0 & 3420.0 & 2900.0 & 2420.0 & 2080.0 & $1930 \bullet 0$ & 1730.0 & 1540.0 & 1350.0 \\
\hline 1950 & 5940.0 & 4550.0 & 3270.0 & 2670.0 & 2500.0 & 2210.0 & 1980.0 & $20>0 \cdot 0$ & 1930.0 & $1800 \cdot 0$ & $1500 \cdot 0$ \\
\hline 1951 & 7070.0 & 5190.0 & 4300.0 & 3040.0 & 2460.0 & 2300.0 & $2120 \cdot 0$ & 2140.0 & 1920.0 & 1870.0 & 1650.0 \\
\hline 1952 & 3560.0 & 2930.0 & 2560.0 & 2140.0 & 1960.0 & 1900.0 & 1750.0 & $1560 \cdot 0$ & 1500.0 & 1390.0 & 1300.0 \\
\hline 1953 & 11200.0 & 8300.0 & 5900.0 & 4610.0 & 3740.0 & 2510.0 & 2000.0 & 1830.0 & 1780.0 & $1700 \cdot 0$ & 1350.0 \\
\hline 1954 & 6600.0 & 5240.0 & 3440.0 & 2700.0 & 2460.0 & 1900.0 & 1840.0 & $1740 \cdot 0$ & 1670.0 & 1630.0 & 1430.0 \\
\hline 1955 & 4070.0 & 3930.0 & 3450.0 & 2700.0 & 2340.0 & 1960.0 & 1680.0 & 1510.0 & 1340.0 & 1240.0 & 1090.0 \\
\hline 1956 & 11600.0 & $8810 \cdot 0$ & 5520.0 & 4000.0 & 3130.0 & 2640.0 & 2270.0 & $1930 \cdot 0$ & 1760.0 & 1850.0 & 1690.0 \\
\hline 1957 & 7540.0 & 5940.0 & 4040.0 & 3300.0 & 2410.0 & 1970.0 & 1780.0 & $1560 \cdot 0$ & 1470.0 & 1420.0 & 1190.0 \\
\hline 1958 & 4560.0 & 4120.0 & 3150.0 & 2690.0 & 2300.0 & 1900.0 & 1810.0 & $1580 \cdot 0$ & 1590.0 & 1530.0 & 1250.0 \\
\hline 1959 & 4430.0 & $3800 \cdot 0$ & 2730.0 & 1980.0 & 1840.0 & 1610.0 & 1580.0 & $1430 \cdot 0$ & 1380.0 & 1340.0 & 1160.0 \\
\hline 1960 & 3450.0 & 2940.0 & 2600.0 & 2430.0 & 2010.0 & 1780.0 & 1690.0 & $1570 \cdot 0$ & 1470.0 & $1310 \cdot 0$ & 1120.0 \\
\hline 1961 & 11100.0 & 8010.0 & 5360.0 & 3680.0 & 2740.0 & 2140.0 & 1860.0 & $1760 \cdot 0$ & 1690.0 & 1630.0 & 1360.0 \\
\hline 1962 & 3980.0 & 3430.0 & 2690.0 & $2230 \cdot 0$ & 1970.0 & 1750.0 & 1540.0 & $1350 \cdot 0$ & 1400.0 & $1350 \cdot 0$ & 1170.0 \\
\hline 1963 & 6360.0 & 4890.0 & $344 n \cdot 0$ & 2310.0 & 1900.0 & 1400.0 & 1390.0 & $1330 \cdot 0$ & 1290.0 & $1300 \cdot 0$ & 1120.0 \\
\hline
\end{tabular}

Breitenbush River above Canyon Creek near Detroit, Oreg.
STATION NUMRER $\quad 14-1790.00$

DURATION TABLF OF DAILY NISCHARGF

CLASS $010 \begin{array}{lllllllllllllllllllllllllllllllllll}2 & 3 & 4 & 5 & 6 & 7 & 8 & 9 & 10 & 11 & 12 & 13 & 14 & 15 & 16 & 17 & 18 & 19 & 20 & 21 & 22 & 23 & 24 & 25 & 26 & 27 & 28 & 29 & 30 & 31 & 32 & 33 & 34\end{array}$

YEAR
1933
1934
1935
1936
1937
1938
1939
1940
1941
1942
1943
1944
1945
1946
1947
1948
1949
1950
1951
1952
1953
1954
1955
1956
1957
1958
1959
1960
1961
1962
1963

NUMRER OF DAYS IN CLACS

$\begin{array}{llllllllllllllllllllll}10 & 2 & 8 & 24 & 31 & 32 & 23 & 24 & 13 & 12 & 24 & 30 & 25 & 39 & 23 & 14 & 13 & 7 & 7 & 2 \\ 36 & 2 & 28 & 28 & 3 & 23 & 17 & 24 & 18 & 11 & 16 & 7 & 11 & 15 & 20 & 7 & 7 & 4 & 6 & 5\end{array}$

$\begin{array}{rrrrrrrrrrrrrrrrrrrrrr}4 & 36 & 22 & 28 & 28 & 38 & 23 & 17 & 24 & 18 & 11 & 16 & 7 & 11 & 15 & 20 & 7 & 7 & 4 & 6 \\ 5 & 36 & 20 & 17 & 10 & 7 & 17 & 21 & 14 & 18 & 26 & 34 & 30 & 39 & 41 & 13 & 3 & 9 & 1 & 1\end{array}$

$\begin{array}{lllllllllllllllllllll}15 & 34 & 44 & 17 & 77 & 21 & 26 & 18 & 10 & 15 & 27 & 22 & 23 & 23 & 16 & 11 & 7 & 2 & 2\end{array}$

$\begin{array}{rrrrrrrrrrrrrrrrrrrr}73 & 38 & 43 & 19 & 14 & 14 & 10 & 14 & 12 & 4 & 10 & 12 & 21 & 30 & 22 & 13 & 4 & 6 & 2 \\ 12 & 33 & 35 & 17 & 14 & 13 & 9 & 13 & 18 & 14 & 25 & 40 & 28 & 28 & 23 & 16 & 9 & 4 & 5\end{array}$

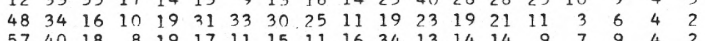

$\begin{array}{llllllllllllllllllll}29 & 26 & 27 & 15 & 31 & 70 & 40 & 24 & 16 & 16 & 26 & 16 & 5 & 7 & 3 & 3 & 1 & 1\end{array}$

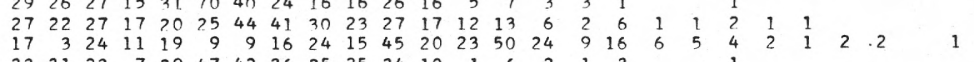

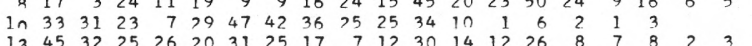

$\begin{array}{rrrrrrrrrrrrrrrrrrrrrrr}12 & 45 & 32 & 25 & 26 & 20 & 31 & 25 & 17 & 7 & 12 & 30 & 14 & 12 & 26 & 8 & 7 & 8 & 2 & 3 & \\ 18 & 10 & 7 & 41 & 18 & 13 & 10 & 16 & 5 & 35 & 13 & 31 & 35 & 35 & 32 & 16 & 10 & 10 & 2 & 3 & 1 \\ 1\end{array}$

$\begin{array}{rrrrrrrrrrrrrrrrrrr}23 & 42 & 17 & 22 & 26 & 24 & 29 & 19 & 12 & 29 & 42 & 26 & 17 & 12 & 7 & 2 & 5 & ? & 2 \\ 16 & 19 & 22 & 23 & 23 & 16 & 20 & 15 & 19 & 48 & 27 & 21 & 25 & 30 & 15 & 13 & 3 & 4 & 3 \\ & 20 & 50 & 43 & 26 & 20 & 15 & 17 & 4 & 25 & 20 & 16 & 26 & 23 & 19 & 24 & 7 & 7 & 2\end{array}$

$\begin{array}{lllllllllllllllll}15 & 32 & 35 & 17 & 24 & 24 & 16 & 14 & 21 & 27 & 22 & 30 & 34 & 23 & 16\end{array}$

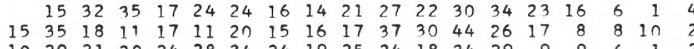

$\begin{array}{rrrrrrrrrrrrrrrrrrrr}10 & 29 & 21 & 20 & 24 & 28 & 34 & 24 & 19 & 25 & 24 & 18 & 34 & 29 & 9 & 9 & 6 & 1 \\ 5 & 60 & 25 & 22 & 21 & 19 & 7 & 17 & 13 & 18 & 36 & 28 & 23 & 27 & 10 & 7 & 4 & 3 & 7\end{array}$

$\begin{array}{llllllllllllllllllllll}10 & 24 & 24 & 36 & 17 & 8 & 11 & 7 & 14 & 40 & 31 & 26 & 44 & 35 & 12 & 11 & 5 & 6 \\ & 36 & 19 & 25 & 36 & 48 & 32 & 23 & 13 & 31 & 17 & 15 & 26 & 21 & 4 & 8 & 5 & 4\end{array}$

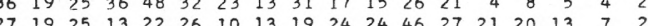

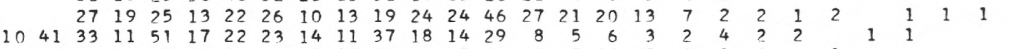

$\begin{array}{rrrrrrrrrrrrrrrrrrrrr}55 & 42 & 16 & 15 & 16 & 16 & 14 & 29 & 18 & 25 & 20 & 15 & 26 & 17 & 5 & 17 & 5 & 9 & 1 \\ 238 & 38 & 12 & 10 & 17 & 6 & 13 & 20 & 34 & 48 & 36 & 24 & 21 & 16 & 9 & 7 & 4 & 5 & 4\end{array}$

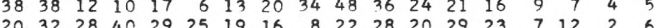

$\begin{array}{rrrrrrrrrrrrrrrrrrrrr}20 & 32 & 28 & 40 & 29 & 25 & 19 & 16 & 8 & 22 & 28 & 20 & 29 & 23 & 7 & 12 & 2 & 6 & & \\ 23 & 46 & 22 & 14 & 11 & 12 & 16 & 19 & 11 & 23 & 34 & 28 & 35 & 22 & 16 & 11 & 9 & 3 & 3 & 2\end{array}$

$\begin{array}{rrrrrrrrrrrrrrrrrrrrrrr}15 & 34 & 15 & 23 & 24 & 49 & 19 & 24 & 23 & 30 & 23 & 15 & 27 & 16 & 11 & 9 & 1 & 2 & 3 & 2 \\ 16 & 23 & 26 & 17 & 23 & 34 & 30 & 27 & 15 & 20 & 27 & 27 & 19 & 25 & 9 & 9 & 5 & -7 & 1 & 2 & 1\end{array}$

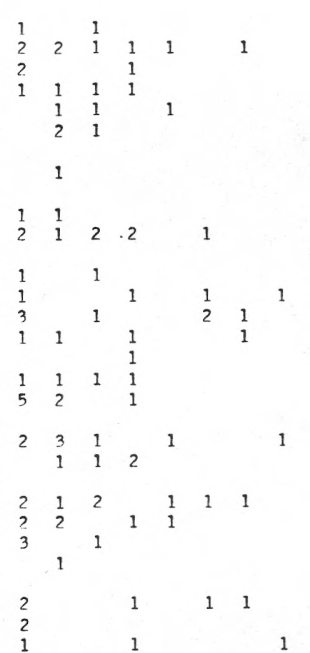

CFS-DAYS 239746.0 199454.0 102101.0 234688.0 159164.0 122672.0 158909.0 271279.0 124122.0 221164.0 211837.0 242614.0 235136.0 254230.0 210264.0 223919.0 249574.0 299357.0 193706.0 212267.0 210081.0 246494.0

198534.0
197170.0

TACTUM PERCT

Summary for water years 1933-63

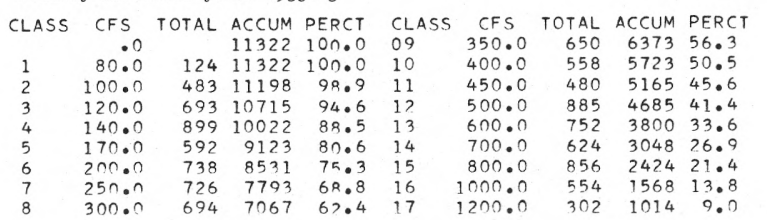

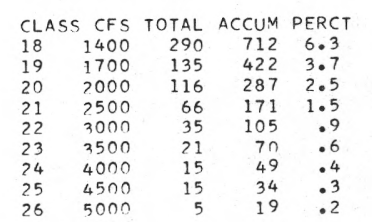

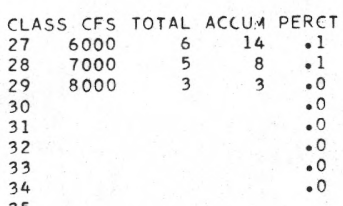

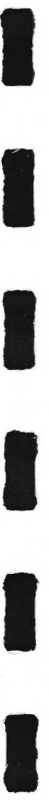

I

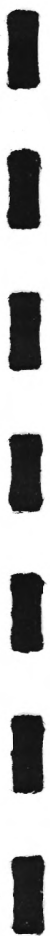

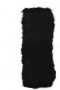


Breitenbush River above Canyon Creek CTATION NUMAFR

$14-1790.0 n$ LOWEST MEAN DISCHARGF, IN CFS, FOR THF FOLLOWING NUMBFR OF CONSECUTIVE DAYC IN YFAR RFGINNING APRIL 1

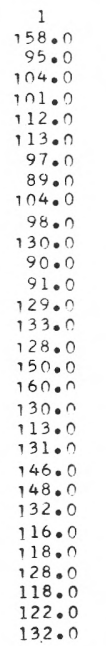

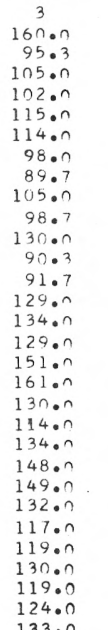

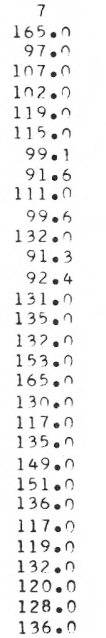

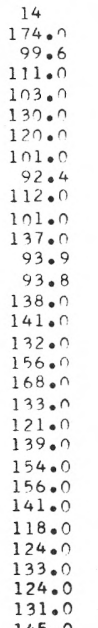
30
188.0
101.0
117.0
106.0
135.0
122.0
108.0
94.7
117.0
103.0
144.0
103.0
99.9
139.0
151.0
144.0
184.0
181.0
139.0
128.0
149.0
186.0
170.0
147.0
122.0
130.0
138.0
130.0
139.0
15500 60
225.0
104.0
122.0
107.0
141.0
129.0
115.0
101.0
133.0
109.0
165.0
108.0
110.0
147.0
157.0
166.0
191.0
208.0
153.0
129.0
15700
198.0
184.0
163.0
132.0
133.0
159.0
142.0
158.0
168.0 90
234.0
110.0
135.0
114.0
165.0
136.0
119.0
109.0
149.0
120.0
201.0
110.0
115.0
167.0
178.0
202.0
220.0
272.0
184.0
135.0
168.0
224.0
256.0
185.0
141.0
142.0
192.0
150.0
172.0
198.0

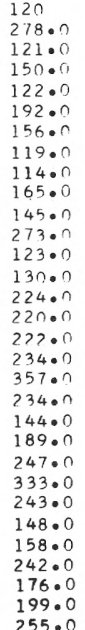

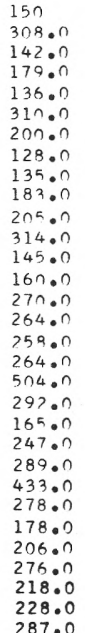
183
438.0 274 PEAR
933
934
935
935
936
937
938
939
940
940
941
1942
943
944
944
1945
946
1947
1948
1949
1950
1951
1952
1953
1954
1955
1956
1957
1958
1959
1960
1961
1962 $133 . n$

Breitenbush River above Canyon Creek near Detroit, Oreg.

CTATION NUMBER

$14-1790.00$

HIGHEST MEAN DISCHARGE, IN CFS, FOR THE FOLLOWING NUMBER OF CONSFCUTIVE DAYS IN YFAR ENDING SEPTFMBER 30

\begin{tabular}{|c|c|c|}
\hline YFAR & 1 & 3 \\
\hline 1933 & 4450.0 & $3480 . n$ \\
\hline 1934 & 7500.0 & 5370.0 \\
\hline 1935 & $4 \times 60.0$ & 3380.0 \\
\hline 1936 & 4080.0 & $3490 . n$ \\
\hline 1937 & $514 n .0$ & $432 n \cdot n$ \\
\hline 1938 & $446 n \cdot 0$ & $3 n 3 n \cdot n$ \\
\hline 1939 & 2230.0 & $2 n 3 n \cdot n$ \\
\hline $\begin{array}{l}1940 \\
1941\end{array}$ & $\begin{array}{l}3690.0 \\
2010.0\end{array}$ & $\begin{array}{l}2460.0 \\
1750: n\end{array}$ \\
\hline 1942 & 3030.0 & $270 n \cdot n$ \\
\hline $\begin{array}{l}1943 \\
1944\end{array}$ & 6560.0 & $4030 \cdot n$ \\
\hline $\begin{array}{l}1944 \\
1945\end{array}$ & $\begin{array}{l}2740.0 \\
4480.0\end{array}$ & $\begin{array}{l}1720 . n \\
2740 . n\end{array}$ \\
\hline 1946 & 9410.0 & $6350 . ?$ \\
\hline 1947 & 7590.0 & 6780.7 \\
\hline 1948 & $7 n 6 n \cdot n$ & $495 n \cdot n$ \\
\hline 1949 & $47 n n . n$ & $337 n \cdot n$ \\
\hline 1950 & 4760.0 & $384 n \cdot n$ \\
\hline 1951 & 4690.0 & $3500 . n$ \\
\hline 1952 & 22030.0 & 2130.0 \\
\hline 1953 & $8 n 2 n \cdot n$ & $5920 . n$ \\
\hline 1954 & $4980 . ?$ & $3890 . n$ \\
\hline 1955 & 2590.0 & 2440.0 \\
\hline 1956 & $7>20 \cdot 0$ & $5790 . n$ \\
\hline 1957 & $5>40.0$ & $4160 . n$ \\
\hline 1958 & $4 n 90.0$ & $312 n \cdot n$ \\
\hline 1959 & $3 \times 60.0$ & 2990.0 \\
\hline 1960 & 2420.0 & $2190 \cdot 0$ \\
\hline 1961 & 7370.0 & $5160 . ?$ \\
\hline 1962 & 3230.0 & 2780.0 \\
\hline 1963 & 8250.0 & 4450.0 \\
\hline
\end{tabular}

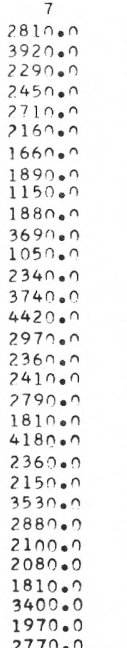

2130.0 30 60

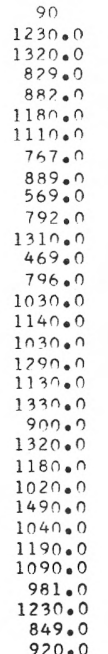

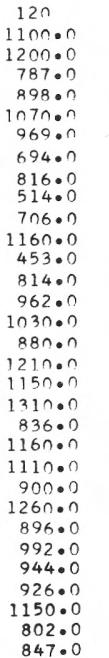

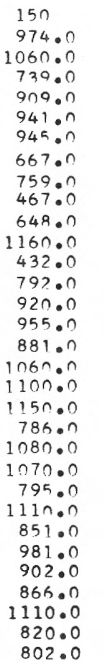

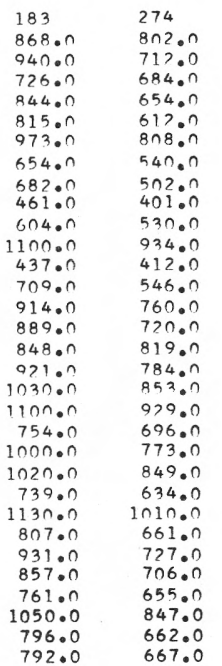




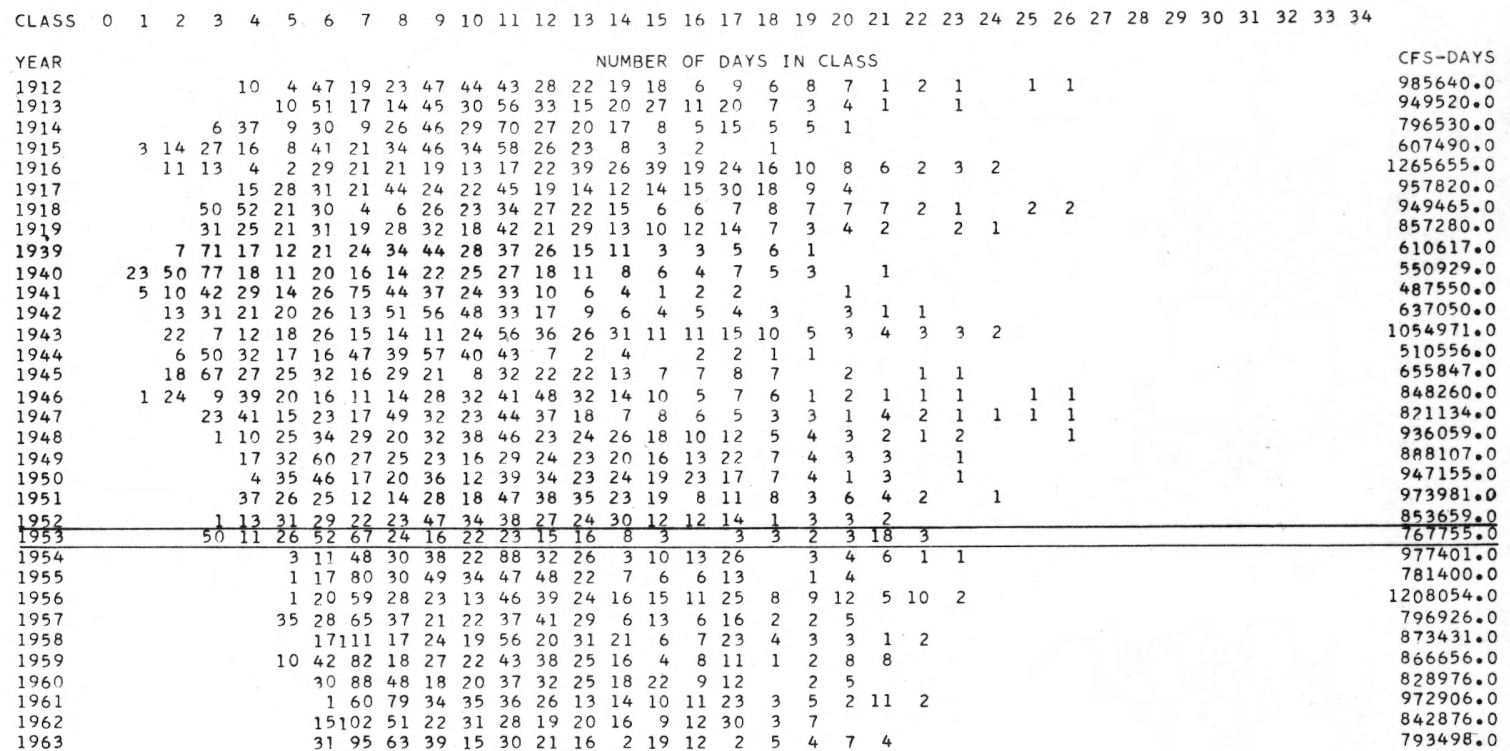

North Santiam River at Niagara, Oreg.

STATION NUMBER

$14-1815.00$

Summary for water years 1912-19, 1939-63.

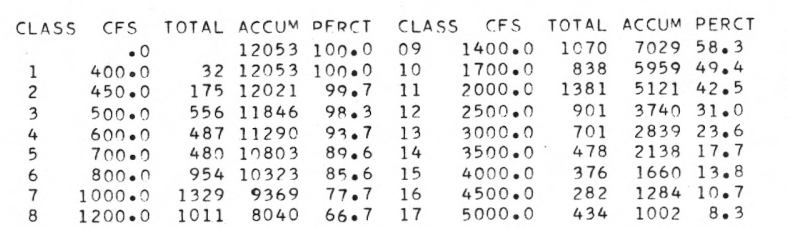

\begin{tabular}{|c|c|c|c|c|c|c|c|}
\hline$C L A S$ & S CFS & TOTAL & ACCUM & PERCT & CLASS CFS & TOTAL ACCUM & PERCT \\
\hline 18 & 6000 & 166 & 568 & 4.7 & 27 & & $\cdot 0$ \\
\hline 19 & 7000 & 117 & 402 & $3 \cdot 3$ & 28 & & .0 \\
\hline 20 & 8000 & 116 & 285 & 2.4 & 29 & & $\cdot 0$ \\
\hline 21 & 10000 & 95 & 169 & 1.4 & 30 & & $\cdot 0$ \\
\hline $\begin{array}{l}22 \\
23\end{array}$ & 12000 & 35 & 74 & .6 & 31 & & .0 \\
\hline $\begin{array}{l}23 \\
24\end{array}$ & 14000 & 21 & 39 & $\cdot 3$ & 32 & & $\cdot 0$ \\
\hline 24 & 17000 & 7 & 18 & .1 & 33 & & $\cdot 0$ \\
\hline 25 & 20000 & 5 & 11 & .1 & 34 & & - 0 \\
\hline 26 & 25000 & 6 & 6 & .0 & 35 & & \\
\hline
\end{tabular}

North Santiam River at Niagara, Oreg.

STATION NUMBER

$14-1815.00$

Sumnary for water years 1912-19, 1939-52

\begin{tabular}{lrrrrlll} 
CLASS & \multicolumn{1}{c}{ CFS } & TOTAL & ACCUM & PERCT & CLASS & \multicolumn{1}{c}{ CFS } & TOTA \\
& .0 & & 8036 & 100.0 & 09 & 1400.0 & 75 \\
1 & 400.0 & 32 & 8036 & 100.0 & 10 & 1700.0 & 583 \\
2 & 450.0 & 175 & 8004 & 99.6 & 11 & 2000.0 & 91 \\
3 & 500.0 & 506 & 7829 & 97.4 & 12 & 2500.0 & 57 \\
4 & 600.0 & 476 & 7323 & 91.1 & 13 & 3000.0 & 45 \\
5 & 700.0 & 404 & 6847 & 85.2 & 14 & 3500.0 & 35 \\
6 & 800.0 & 690 & 6443 & 80.2 & 15 & 4000.0 & 25 \\
7 & 1000.0 & 472 & 5753 & 71.6 & 16 & 4500.0 & 187 \\
8 & 1200.0 & 586 & 5281 & 65.7 & 17 & 5000.0 & 25
\end{tabular}

North Santiam River at Niagara, Oreg.

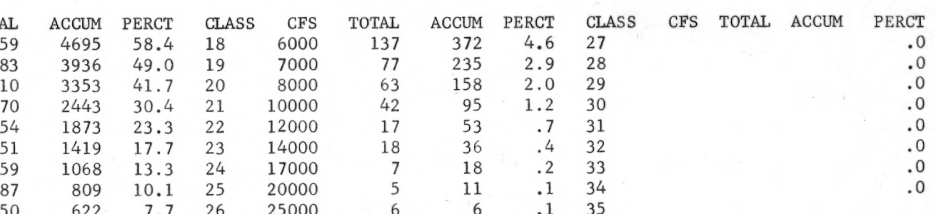

Summary for water years 1954-1963

STATION NUMBER

$14-1815.00$

\begin{tabular}{|c|c|c|c|c|c|c|c|c|c|c|c|c|c|c|c|c|c|c|}
\hline CLASS & CFS & TOTAL & ACCUM & PERCT & CL.ASS & CFS & TOTAL & ACCUM & PERCT & CLASS & CFS & TOTAL & ACCUM & PERCT & CLASS CFS & TOTAL & ACCUM & PERCT \\
\hline & .0 & & 3652 & 100.0 & 09 & 1400.0 & 295 & 2199 & 60.2 & 18 & 6000 & 26 & 167 & 4.6 & 27 & & & .0 \\
\hline 1 & 400.0 & & 3652 & 100.0 & 10 & 1700.0 & 233 & 1904 & 52.1 & 19 & 7000 & 38 & 141 & 3.9 & 28 & & & .0 \\
\hline 2 & 450.0 & & 3652 & 100.0 & 11 & 2000.0 & 448 & 1671 & 45.8 & 20 & 8000 & 50 & 103 & 2.8 & 29 & & & .0 \\
\hline 3 & 500.0 & & 3652 & 100.0 & 12 & 2500.0 & 316 & 1223 & 33.5 & 21 & 10000 & 35 & 53 & 1.5 & 30 & & & .0 \\
\hline 4 & 600.0 & & 3652 & 100.0 & 13 & 3000.0 & 231 & 907 & 24.8 & 22 & 12000 & 15 & 18 & .5 & 31 & & & .0 \\
\hline 5 & 700.0 & 50 & 3652 & 100.0 & 14 & 3500.0 & 119 & 676 & 18.5 & 23 & 14000 & 3 & 3 & .1 & 32 & & & .0 \\
\hline 6 & 800.0 & 212 & 3602 & 98.6 & 15 & 4000.0 & 114 & 557 & 15.3 & 24 & 17000 & & & .0 & 33 & & & .0 \\
\hline 7 & 1000.0 & 790 & 3390 & 92.8 & 16 & 4500.0 & 95 & 443 & 12.1 & 25 & 20000 & & & .0 & 34 & & & .0 \\
\hline 8 & 1200.0 & 401 & 2600 & 71.2 & 17 & 5000.0 & 181 & 348 & 9.5 & 26 & 25000 & & & .0 & 35 & & & \\
\hline
\end{tabular}


North Santiam River at N1agara, Oreg.

STATION NUMBER $14-1815.00$ LOWEST MEAN DISCHARGF, IN CFS, FOR THF FOLLOWING NUMBER OF CONSECUTIVE DAYS IN YEAR REGINNING APRIL 1

\begin{tabular}{|c|c|c|c|c|c|c|c|c|c|c|c|}
\hline YEAR & 1 & 3 & 7 & 14 & 30 & 60 & 90 & 120 & 150 & 183 & 274 \\
\hline 1912 & 720.0 & 720.0 & 743.0 & 783.0 & 819.0 & 1130.0 & 1110.0 & $1230 \cdot 0$ & 1480.0 & 1840.0 & $\begin{array}{l}2070.0 \\
2190.0\end{array}$ \\
\hline $\begin{array}{l}1913 \\
1914\end{array}$ & 720.0 & $720 \cdot 0$ & 766.0 & 766.0 & 850.0 & 905.0 & 1070.0 & $1310 \cdot 0$ & 1510.0 & 1630.0 & \\
\hline $\begin{array}{l}1914 \\
1915\end{array}$ & 580.0 & 580.0 & 586.0 & 603.0 & 625.0 & 685.0 & 873.0 & $1010 \cdot 0$ & 1160.0 & $1290 \cdot 0$ & \\
\hline $\begin{array}{l}1915 \\
1916\end{array}$ & 430.0 & $430 \cdot 0$ & 447.0 & 471.0 & 491.0 & 515.0 & 554.0 & $620 \cdot 0$ & 703.0 & 998.0 & \\
\hline $\begin{array}{l}1916 \\
1917\end{array}$ & 650.0 & 650.0 & 665.0 & 680.0 & 711.0 & 880.0 & 994.0 & $1160 \cdot 0$ & $1500 \cdot 0$ & 1590.0 & $\begin{array}{l}1870.0 \\
3040.0\end{array}$ \\
\hline $\begin{array}{l}1917 \\
1918\end{array}$ & 550.0 & 570.0 & 576.0 & 588.0 & 593.0 & 628.0 & 689.0 & $774 \cdot 0$ & 1160.0 & $\begin{array}{r}1890.0 \\
969.0\end{array}$ & \\
\hline $\begin{array}{l}1918 \\
1939\end{array}$ & $520 \cdot 0$ & $520 \cdot 0$ & 520.0 & 546.0 & $564 \cdot 0$ & $612 . n$ & 640.0 & $677 \cdot 0$ & $765 \cdot 0$ & $\begin{array}{l}969.0 \\
690.0\end{array}$ & 1350.0 \\
\hline $\begin{array}{l}1939 \\
1940\end{array}$ & 470.0 & 470.0 & 475.0 & 487.0 & 508.0 & $528 \cdot 0$ & 542.0 & $545 \cdot 0$ & $572 \cdot 0$ & $\begin{array}{l}690.0 \\
676.0\end{array}$ & 1080.0 \\
\hline $\begin{array}{l}1940 \\
1941\end{array}$ & 432.0 & $436 \cdot n$ & $441 \cdot 0$ & $\begin{array}{l}443.0 \\
525.0\end{array}$ & $\begin{array}{l}448 \cdot 0 \\
545 \cdot 0\end{array}$ & $\begin{array}{l}470 \cdot 0 \\
606.0\end{array}$ & $\begin{array}{l}490.0 \\
665.0\end{array}$ & $\begin{array}{l}506 \cdot 0 \\
721 \cdot 0\end{array}$ & $\begin{array}{l}557.0 \\
793.0\end{array}$ & 921.0 & 1530.0 \\
\hline $\begin{array}{l}1941 \\
1942\end{array}$ & $\begin{array}{l}504.0 \\
456.0\end{array}$ & $\begin{array}{l}509 . n \\
459.0\end{array}$ & $\begin{array}{l}518.0 \\
463.0\end{array}$ & $\begin{array}{l}525 \cdot 0 \\
476.0\end{array}$ & $\begin{array}{l}545.0 \\
479.0\end{array}$ & $\begin{array}{l}606 \cdot 0 \\
501 \cdot 0\end{array}$ & $\begin{array}{l}665.0 \\
542.0\end{array}$ & 629.0 & 827.0 & $1020 \cdot 0$ & 2040.0 \\
\hline 1943 & 635.0 & 637.0 & $640 \cdot 0$ & 654.0 & 671.0 & 731.0 & 821.0 & $1070 \cdot 0$ & 1240.0 & 1320.0 & 1430.0 \\
\hline 1944 & 450.0 & $452 . n$ & 456.0 & 466.0 & 506.0 & 527.0 & 535.0 & $575 \cdot 0$ & 650.0 & 716.0 & $1020 \cdot 0$ \\
\hline 1945 & 448.0 & 452.0 & 456.0 & 461.0 & $482 \cdot 0$ & 516.0 & 529.0 & $572 \cdot 0$ & 658.0 & 888.0 & $1890 \cdot 0$ \\
\hline 1946 & 552.0 & $552 \cdot n$ & $562 \cdot 0$ & 597.0 & 603.0 & 640.0 & 699.0 & $908 \cdot 0$ & 1090.0 & $1400 \cdot 0$ & 2340.0 \\
\hline 1947 & 570.0 & 574.0 & 578.0 & 585.0 & 631.0 & 646.0 & 710.0 & $854 \cdot 0$ & 991.0 & 1220.0 & 1790.0 \\
\hline 1948 & 682.0 & 688.0 & 697.0 & 697.0 & 733.0 & 806.0 & 911.0 & 993.0 & 1130.0 & 1520.0 & 1780.0 \\
\hline 1949 & 610.0 & 619.0 & 628.0 & 640.0 & 703.0 & 738.0 & 849.0 & 885.0 & 982.0 & 1280.0 & 1760.0 \\
\hline 1950 & 700.0 & 700.0 & 700.0 & 718.0 & 755.0 & 839.0 & 1020.0 & 1310.0 & 1900.0 & 2240.0 & 2840.0 \\
\hline 1951 & 605.0 & $605 . n$ & $612 \cdot n$ & $624 . n$ & 642.0 & $68 ? .0$ & 762.0 & 913.0 & 1110.0 & 1330.0 & 1770.0 \\
\hline 952 & 515.0 & 524.0 & 535.0 & 556.0 & 570.0 & 583.0 & 616.0 & 657.0 & 738.0 & 902.0 & 1670.0 \\
\hline 1953 & 620.0 & 736.0 & 751.0 & $757 . ?$ & 821.0 & 872.0 & 1030.0 & 1260.0 & 1420.0 & 1510.0 & 2380.0 \\
\hline 1954 & 728.0 & 803.0 & $867 . n$ & 956.0 & 1010.0 & 1090.0 & 1500.0 & 1640.0 & 1900.0 & 1980.0 & 1910.0 \\
\hline 1955 & 775.0 & 872.0 & 925.0 & 970.0 & 1000.0 & 1180.0 & 1470.0 & $1910 \cdot 0$ & 2060.0 & $2130 \cdot 0$ & 3130.0 \\
\hline 1956 & 782.0 & 939.0 & $960 . n$ & 982.0 & 1030.0 & 1210.0 & 1470.0 & $1650 \cdot 0$ & 1890.0 & 2190.0 & 2230.0 \\
\hline 1957 & 727.0 & 754.0 & 767.0 & 775.0 & 777.0 & 868.0 & $1000 \cdot 0$ & $1180 \cdot 0$ & 1420.0 & 1590.0 & 2010.0 \\
\hline 1958 & 760.0 & 762.0 & 767.0 & 832.0 & 954.0 & 1180.0 & $1200 \cdot 0$ & $1300 \cdot 0$ & 1460.0 & 1620.0 & 2410.0 \\
\hline 1959 & 814.0 & 897.0 & 923.0 & 934.0 & 948.0 & 985.0 & 1120.0 & $1320 \cdot 0$ & 1510.0 & 1700.0 & 1930.0 \\
\hline 1960 & 1040.0 & 1110.0 & 1140.0 & 1160.0 & 1170.0 & 1180.0 & 1290.0 & 1470.0 & 1630.0 & 1940.0 & 2530.0 \\
\hline 1961 & 980.0 & 983.0 & 994.0 & 1000.0 & 1030.0 & 1070.0 & 1280.0 & $1350 \cdot 0$ & 1540.0 & 1650.0 & $2220 \cdot 0$ \\
\hline 1962 & 926.0 & $948 . ?$ & 972.0 & 983.0 & 1090.0 & 1160.0 & 1250.0 & $1360 \cdot 0$ & 1560.0 & 1840.0 & 2190.0 \\
\hline
\end{tabular}

North Santiam River at Niagara, Oreg.

STATION NUMBER $14-1815.00$ HIGHEST MEAN DISCHARGE, IN CFS, FOR THE FOLLOWING NUMBER OF CONSECUTIVE DAYS IN YEAR ENDING SEPTEMBER 30

\begin{tabular}{|c|c|c|c|c|c|c|c|c|c|c|c|}
\hline YEAR & 1 & 3 & 7 & 15 & 30 & 60 & 90 & 120 & 150 & 183 & 274 \\
\hline 1912 & 30000.0 & $23300 . n$ & 14000.0 & 9180.0 & 7240.0 & 5880.0 & 4570.0 & $4530 \cdot 0$ & 3960.0 & 3650.0 & 3230.0 \\
\hline 1913 & 16200.0 & 11000.0 & 8680.0 & 6620.0 & 4940.0 & 4600.0 & 4210.0 & $3780 \cdot 0$ & 3460.0 & 3320.0 & 3140.0 \\
\hline 1914 & 8300.0 & 7670.0 & $604 n .0$ & 5320.0 & 4340.0 & 3730.0 & 3540.0 & $3440 \cdot 0$ & 3190.0 & 3010.0 & 2610.0 \\
\hline 1915 & 6240.0 & 4670.0 & 3430.0 & $3040 . n$ & 2910.0 & 2490.0 & 2440.0 & $2390 \cdot 0$ & 2340.0 & 2180.0 & 2000.0 \\
\hline 1916 & 19500.0 & 15400.0 & 11200.0 & 8690.0 & 6470.0 & 5900.0 & 5370.0 & 5010.0 & 4930.0 & 4880.0 & 4320.0 \\
\hline 1917 & 8060.0 & 8060.0 & 7490.0 & 6830.0 & 6170.0 & 5860.0 & 5340.0 & 4730.0 & 4110.0 & 3740.0 & 3220.0 \\
\hline 1918 & 34200.0 & 28200.0 & 19200.0 & 14400.0 & 10500.0 & 7820.0 & 6300.0 & 5340.0 & 4850.0 & 4370.0 & 3260.0 \\
\hline 1919 & 19000.0 & $14000 . n$ & 12200.0 & 8320.0 & 5590.0 & 4600.0 & 3940.0 & 4070.0 & 3950.0 & 3570.0 & 2890.0 \\
\hline 1939 & 7000.0 & 6640.0 & $5980 . n$ & 4760.0 & 3920.0 & 3340.0 & $2900 \cdot 0$ & 261000 & 2530.0 & 2460.0 & 2040.0 \\
\hline $\begin{array}{l}1940 \\
1941\end{array}$ & 11350.0 & 8270.0 & $654 n . ?$ & 4980.0 & 4710.0 & 3830.0 & 3210.0 & $3010 \cdot 0$ & 2760.0 & 2470.0 & 1840.0 \\
\hline 1941 & 8180.0 & 5410.0 & 3780.0 & 3310.0 & 2570.0 & 2340.0 & 2170.0 & $1990 \cdot 0$ & 1820.0 & $1790 \cdot 0$ & 1570.0 \\
\hline $\begin{array}{l}1942 \\
1943\end{array}$ & 12700.0 & $9300 \cdot n$ & 6820.0 & 4610.0 & 4480.0 & 3450.0 & 3180.0 & $2850 \cdot 0$ & 2610.0 & 2440.0 & \\
\hline 1943 & 18800.0 & $15200 \cdot 0$ & $12400 \cdot 0$ & 9500.0 & 6680.0 & 6250.0 & $5220 \cdot 0$ & $4600 \cdot 0$ & $4570 \cdot 0$ & $\begin{array}{l}4340.0 \\
1780.0\end{array}$ & $\begin{array}{l}3610.0 \\
1670.0\end{array}$ \\
\hline 1944 & 7650.0 & $5860 \cdot 0$ & 3830.0 & 3160.0 & 2360.0 & 2060.0 & 1900.0 & & & 2870.0 & \\
\hline 1945 & 15400.0 & $10400 \cdot 0$ & $903 n .0$ & 6420.0 & 4310.0 & 3530.0 & $\begin{array}{l}3370.0 \\
3980.0\end{array}$ & $\begin{array}{l}3390 \cdot 0 \\
3710.0\end{array}$ & $\begin{array}{l}3240.0 \\
3510.0\end{array}$ & 3480.0 & $\begin{array}{l}2220.0 \\
2890.0\end{array}$ \\
\hline 1946 & 29800.0 & $22300 \cdot n$ & 13500.0 & 9250.0 & 6290.0 & 4750.0 & $\begin{array}{l}3980.0 \\
4460.0\end{array}$ & $\begin{array}{l}3710 \cdot 0 \\
3990 \cdot 0\end{array}$ & $\begin{array}{l}3510 \bullet 0 \\
3710 \cdot 0\end{array}$ & 3420.0 & $\begin{array}{l}2890.0 \\
2780.0\end{array}$ \\
\hline 1947 & 25500.0 & $23000 \bullet n$ & $1560 n \cdot n$ & 9090.0 & 7740.0 & 4890.0 & $\begin{array}{l}4460 \cdot 0 \\
3780 \cdot 0\end{array}$ & 3400.0 & 3370.0 & 3310.0 & $\begin{array}{l}2780.0 \\
3120.0\end{array}$ \\
\hline 1948 & 26700.0 & 18600.0 & 11500.0 & 7630.0 & 4950.0 & 3930.0 & $\begin{array}{l}3780.0 \\
4620.0\end{array}$ & 4320.0 & 3780.0 & 3430.0 & $\begin{array}{l}3120.0 \\
2950.0\end{array}$ \\
\hline 1949 & 15400.0 & $12000 \cdot 0$ & 8240.0 & 7430.0 & 6310.0 & 5110.0 & 4220.0 & 4310.0 & 4100.0 & 3800.0 & 3160.0 \\
\hline 1950 & 16800.0 & $12700 \cdot 0$ & 8550.0 & 6760.0 & 5510.0 & $\begin{array}{l}4540.0 \\
5050.0\end{array}$ & 4610.0 & 4630.0 & 4100.0 & 3910.0 & 3300.0 \\
\hline 1951 & $19300 \cdot 0$ & $13000 \cdot n$ & $1050 n \cdot n$ & 7090.0 & 5580.0 & $\begin{array}{l}5050.0 \\
3980.0\end{array}$ & 3570.0 & 3410.0 & 3180.0 & 3090.0 & 2810.0 \\
\hline 1952 & 11300.0 & 9170.0 & $7570 . n$ & 5260.0 & 4150.0 & $\frac{3980.0}{6330.0}$ & 4710.0 & $3800 \cdot 0$ & 3390.0 & $\frac{3090.0}{3230.0}$ & $\frac{2810.0}{2570.0}$ \\
\hline 1953 & 12500.0 & 12200.0 & 11700.0 & 9760.0 & $\begin{array}{l}9250.0 \\
7470.0\end{array}$ & $\frac{6330.0}{5670.0}$ & 4850.0 & $4190 \cdot 0$ & 3770.0 & 3450.0 & 3070.0 \\
\hline 1954 & 15800.0 & 13400.0 & 10000.0 & 8890.0 & & 3140.0 & 2870.0 & 2670.0 & 2360.0 & 2210.0 & 2360.0 \\
\hline 1955 & 9660.0 & 9360.0 & 8000.0 & 5570.0 & $\begin{array}{l}4290.0 \\
8600.0\end{array}$ & 7510.0 & 6660.0 & & 5030.0 & 4350.0 & 3910.0 \\
\hline 1956 & 14800.0 & 14200.0 & 11900.0 & $10900 \cdot 0$ & $\begin{array}{l}8600.0 \\
4350.0\end{array}$ & 4140.0 & 3620.0 & 3110.0 & 2910.0 & 2850.0 & 2510,0 \\
\hline 1957 & $\begin{array}{r}9990.0 \\
13000.0\end{array}$ & $\begin{array}{r}9820.0 \\
12400.0\end{array}$ & $\begin{array}{l}8740.0 \\
9050.0\end{array}$ & $\begin{array}{l}6790.0 \\
6850.0\end{array}$ & 5620.0 & 4750.0 & 4170.0 & 3760.0 & 3540.0 & 3120.0 & 2750.0 \\
\hline 1958 & $\begin{array}{l}13000.0 \\
11800.0\end{array}$ & $\begin{array}{l}12400.0 \\
11500.0\end{array}$ & 10600.0 & 8060.0 & 6410.0 & 4870.0 & 4510.0 & $3990 \cdot 0$ & 3500.0 & 3070.0 & 2700.0 \\
\hline $\begin{array}{l}1959 \\
1960\end{array}$ & & 8910.0 & 8280.0 & 5890.0 & 4070.0 & 3830.0 & 3300.0 & 2890.0 & 2620.0 & 2460.0 & 2570.0 \\
\hline 1961 & 12100.0 & $12000 \cdot 0$ & 10100.0 & $8230 . ?$ & 6120.0 & 4700.0 & 4230.0 & $4150 \cdot 0$ & 3990.0 & 3660.0 & 3130.0 \\
\hline 1962 & 7460.0 & 7430.0 & $7390 . ?$ & 6370.0 & 5140.0 & 4510.0 & 4100.0 & 3540.0 & 3040.0 & 2840.0 & 2650.0 \\
\hline 1963 & 10200.0 & 10100.0 & $992 n . ?$ & 8100.0 & 6510.0 & $4750 . ?$ & 4050.0 & 3360.0 & 2990.0 & 2650.0 & 2460.0 \\
\hline
\end{tabular}




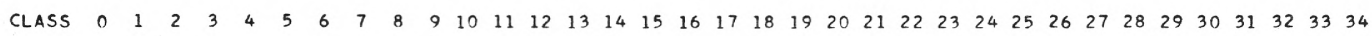

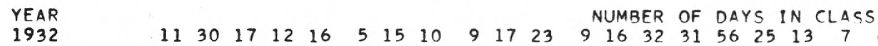

1933

1934
1935

1936

1937
1938

1939

1940
1941

1940
1942

1943

1944
1945

1946

1946
1947
1948

1948
1949

1950
1951

1952

1952
1954

1955

1956
1957

1958
1959

1960

1961
1962

1963

$\begin{array}{rrrrrrrrrrrrrrrrrrrrr}11 & 11 & 30 & 17 & 12 & 16 & 5 & 15 & 10 & 9 & 17 & 23 & 9 & 16 & 32 & 31 & 56 & 25 & 13 & 7 & 6 \\ 10 & 1 & 10 & 13 & 8 & 9 & 11 & 10 & 4 & 13 & 16 & 21 & 167 & 37 & 37 & 81 & 29 & 17 & 6 & 6 & 3\end{array}$

$\begin{array}{llllllllllllllllllllllllllll}10 & 21 & 26 & 13 & 11 & 9 & 11 & 23 & 51 & 25 & 17 & 22 & 19 & 10 & 13 & 9 & 27 & 15 & 9 & 8 & 3 & 4 & 3 & 5 & & 1\end{array}$

$\begin{array}{rrrrrrrrrrrrrrrrrrrrrrrrr}2 & 20 & 38 & 12 & 8 & 12 & 5 & 12 & 10 & 5 & 8 & 15 & 24 & 29 & 56 & 26 & 39 & 21 & 12 & 3 & 4 & 1 & 3 & \\ 5 & 28 & 20 & 10 & 9 & 11 & 25 & 20 & 9 & 9 & 31 & 19 & 24 & 48 & 28 & 33 & 21 & 6 & 2 & 3 & 2 & 2 & 1\end{array}$

$\begin{array}{llllllllllllllllllllll}20 & 23 & 26 & 21 & 21 & 14 & 10 & 13 & 24 & 3 & 9 & 16 & 10 & 20 & 24 & 27 & 46 & 18 & 10 & 4 & 2\end{array}$

$\begin{array}{rrrrrrrrrrrrrrrrrrrrrr}14 & 15 & 27 & 16 & 7 & 13 & 12 & 12 & 14 & 7 & 7 & 14 & 7 & 29 & 41 & 35 & 47 & 11 & 12 & 6 & 7 \\ 4 & 15 & 30 & 23 & 6 & 10 & 7 & 15 & 11 & 12 & 21 & 29 & 33 & 27 & 41 & 26 & 26 & 10 & 4 & 7 & 5\end{array}$

$\begin{array}{lllllllllllllllllllllllll}19 & 22 & 28 & 18 & 11 & 15 & 20 & 39 & 18 & 11 & 8 & 21 & 13 & 22 & 22 & 16 & 27 & 13 & 7 & 7 & 5 & 2 & 1\end{array}$

$\begin{array}{rrrrrrrrrrrrrrrrrrrrr}2 & 13 & 13 & 17 & 11 & 10 & 17 & 11 & 32 & 37 & 29 & 43 & 35 & 18 & 35 & 22 & 23 & 4 & 3 & 1 & 2 \\ & 24 & 5 & 5 & 8 & 3 & 9 & 10 & 15 & 11 & 11 & 44 & 59 & 29 & 50 & 17 & 18 & 14 & 8 & 5 & 3\end{array}$

$\begin{array}{llllllllllllllllllllllll}24 & 21 & 14 & 13 & 11 & 13 & 13 & 5 & 4 & 5 & 15 & 29 & 17 & 32 & 29 & 44 & 23 & 11 & 10 & 13 & 6\end{array}$

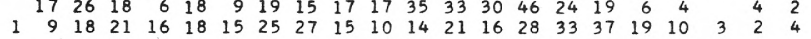

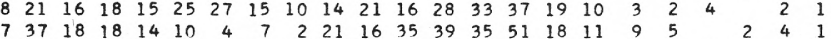

$\begin{array}{rrrrrrrrrrrrrrrrrrrrrrrrrr}14 & 20 & 26 & 14 & 29 & 15 & 15 & 19 & 31 & 21 & 24 & 25 & 27 & 45 & 15 & 6 & 3 & 6 & 3 & 1 & 2 & 1 & 3 \\ 2 & 12 & 9 & 23 & 17 & 15 & 14 & 18 & 14 & 13 & 19 & 23 & 48 & 35 & 43 & 27 & 12 & 3 & 7 & 3 & 5 & 2 & 1 & 1\end{array}$

$\begin{array}{rrrrrrrrrrrrrrrrrrrrrrrrrrrrr} & 1 & 15 & 10 & 4 & 17 & 10 & 24 & 32 & 29 & 10 & 19 & 18 & 17 & 34 & 23 & 49 & 32 & 6 & 4 & 2 & 4 & 1 & 1 & \\ 3 & 34 & 17 & 10 & 6 & 7 & 7 & 18 & 19 & 14 & 7 & 11 & 13 & 18 & 38 & 47 & 69 & 22 & 11 & 7 & 7 & 1 & 3 & 2 & 1\end{array}$

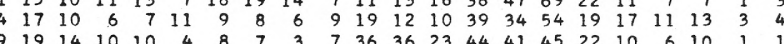

$\begin{array}{rrrrrrrrrrrrrrrrrrrrrr}10 & 95 & 19 & 14 & 10 & 10 & 4 & 8 & 7 & 3 & 7 & 36 & 36 & 23 & 44 & 41 & 45 & 22 & 10 & 6 & 10 & 1\end{array}$

$\begin{array}{rrrrrrrrrrrrrrrrrrr}11 & 7 & 16 & 19 & 12 & 16 & 12 & 8 & 36 & 36 & 27 & 58 & 30 & 39 & 20 & 12 & 2 & 1\end{array}$

$\begin{array}{rrrrrrrrrrrrrrrrrr}11 & 7 & 16 & 19 & 12 & 16 & 12 & 8 & 36 & 36 & 27 & 58 & 30 & 39 & 20 & 12 & 2 \\ 23 & 11 & 6 & 14 & 12 & 10 & 8 & 6 & 6 & 13 & 17 & 32 & 27 & 37 & 60 & 34 & 15 & 12\end{array}$

$\begin{array}{rrrrrrrrrrrrrrrrrrrrrrr}4 & 9 & 31 & 17 & 15 & 14 & 12 & 10 & 8 & 6 & 6 & 13 & 17 & 32 & 27 & 37 & 60 & 34 & 15 & 12 & 6 & 2 & 2 \\ & 17 & 16 & 9 & 7 & 14 & 12 & 11 & 9 & 17 & 13 & 24 & 29 & 33 & 33 & 28 & 33 & 11 & 7 & 4 & 5 & 3 & 3\end{array}$

$\begin{array}{rrrrrrrrrrrrrrrrrrrrrrrrr}17 & 16 & 9 & 7 & 14 & 17 & 26 & 17 & 17 & 18 & 28 & 23 & 23 & 38 & 15 & 29 & 13 & 12 & 9 & 9 & 4 & & 2 & 2 \\ 11 & 22 & 6 & 10 & 11 & 22 & 17 & 9 & 5 & 14 & 25 & 25 & 48 & 40 & 43 & 27 & 8 & 6 & 7 & 3 & 4 & 2 & \end{array}$

$\begin{array}{rrrrrrrrrrrrrrrrrrrrrrrrrrrr}19 & 7 & 7 & 12 & 112 & 14 & 15 & 12 & 13 & 12 & 9 & 15 & 12 & 49 & 25 & 15 & 32 & 30 & 52 & 20 & 11 & 6 & 5 & 3 & & & \\ & & 4 & 13 & 16 & 9 & 15 & 14 & 16 & 9 & 5 & 4 & 19 & 18 & 20 & 34 & 27 & 56 & 19 & 12 & 8 & 5 & 4 & 3 & 2 & 2\end{array}$

$\begin{array}{rrrrrrrrrrrrrrrrrrrrrrrrrrrrrr}4 & 13 & 16 & 8 & 18 & 14 & 20 & 13 & 12 & 14 & 21 & 23 & 33 & 42 & 29 & 41 & 20 & 11 & 3 & 3 & 4 & & 3 & \\ 9 & 8 & 8 & 7 & 10 & 9 & 27 & 30 & 31 & 16 & 39 & 15 & 12 & 32 & 28 & 39 & 18 & 12 & 4 & 5 & 4 & 1 & & 1\end{array}$

\footnotetext{
Little North Santiam River near Mehama, Oreg.

STATION NUMBER

$14-1825.00$
}

Summary for water years 1932-63

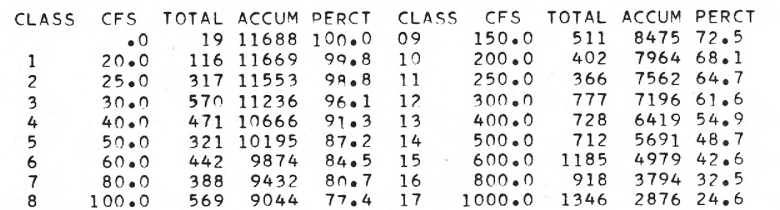

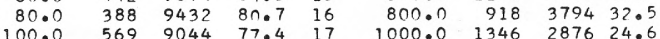
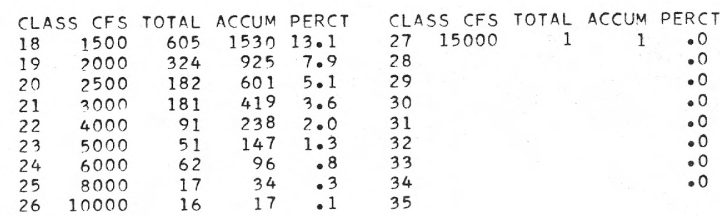

$16-17-110$

CFS-DAYS

299874.0
354378.0

260449.0

261465.0

241953.0

226857.0

311097.0

202720.0

173834.0

234783.0

405158.0

182082.0

326062.0

291672.0

265126.0

330690.0

329934.0

290535.0

309576.0

343961 .0

260682.0

391353.0

251344.0

277833.0

315512.0

318546.0

270166.0

264786.0 
Mehama, Oreg.

STATION NUMBER $14-1825.00$

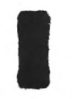

$\begin{array}{llll}1 & 3 & 7 & 14 \\ 22.0 & 22.7 & 23.1 & 24.4 \\ 39.0 & 41.7 & 46.7 & 49.8 \\ 21.0 & 22.0 & 23.1 & 23.9 \\ 24.0 & 24.3 & 26.3 & 27.3 \\ 22.0 & 22.0 & 22.1 & 23.0 \\ 34.0 & 34.7 & 38.1 & 46.6 \\ 21.0 & 21.7 & 22.1 & 23.0 \\ 23.0 & 23.3 & 24.0 & 24.9 \\ 21.0 & 21.7 & 22.0 & 22.7 \\ 35.0 & 36.0 & 36.1 & 37.6 \\ 22.0 & 24.0 & 24.7 & 25.6 \\ 25.0 & 25.7 & 26.0 & 28.7 \\ 26.0 & 26.3 & 27.3 & 29.5 \\ 24.0 & 25.0 & 25.6 & 27.8 \\ 38.0 & 38.7 & 40.1 & 43.1 \\ 46.0 & 47.3 & 51.9 & 52.9 \\ 38.0 & 39.3 & 42.7 & 43.9 \\ 30.0 & 30.3 & 31.1 & 32.6 \\ 28.0 & 29.3 & 30.9 & 32.2 \\ 23.0 & 23.3 & 23.7 & 24.8 \\ 24.0 & 24.0 & 24.0 & 24.6 \\ 36.0 & 37.0 & 37.7 & 40.5 \\ 71.0 & 75.0 & 76.1 & 79.4 \\ 41.0 & 41.7 & 43.3 & 46.7 \\ 24.0 & 24.3 & 25.9 & 28.2 \\ 24.0 & 24.3 & 29.0 & 31.4 \\ 25.0 & 25.7 & 26.4 & 28.9 \\ 34.0 & 35.0 & 37.4 & 39.6 \\ 29.0 & 30.0 & 32.0 & 35.4 \\ 13.0 & 14.0 & 14.6 & 15.6 \\ 26.0 & 27.0 & 29.0 & 32.7 \\ & & & \end{array}$

$\begin{array}{ll}30 & 60 \\ 28.3 & 36.0 \\ 56.3 & 88 \cdot 6 \\ 25.9 & 31.2 \\ 31.6 & 34.7 \\ 25.1 & 27.1 \\ 53 \cdot 3 & 54.5 \\ 28.1 & 30.9 \\ 28.7 & 36.4 \\ 24.3 & 30.6 \\ 42.4 & 70.1 \\ 27.1 & 31.0 \\ 29.7 & 42.1 \\ 31.3 & 41.3 \\ 33.3 & 44.6 \\ 45.5 & 50.3 \\ 60.1 & 86.5 \\ 53.7 & 67.2 \\ 37.3 & 59.6 \\ 45.3 & 63.4 \\ 27.0 & 27.7 \\ 25.6 & 28.2 \\ 55.3 & 67.6 \\ 96.2 & 118.0 \\ 58.0 & 85.7 \\ 31.6 & 38.3 \\ 32.4 & 43.2 \\ 30.3 & 44.9 \\ 43.2 & 72.7 \\ 44.9 & 81.5 \\ 18.8 & 35.8 \\ 45.6 & 61.5\end{array}$

90
51.1
219.0
34.0
$43 \cdot 5$
34.7
69.3
35.1
53.2
35.4
143.0
39.9
54.7
60.0
63.9
60.2
103.0
93.1
86.7
100.0
37.0
33.1
86.2
141.0
145.0
51.7
53.5
58.1
146.0
77.6
65.0
84.5

120
$97 \cdot 8$
$255 \cdot 0$
$42 \cdot 2$
$80 \cdot 9$
$37 \cdot 0$
$133 \cdot 0$
$49 \cdot 9$
$78 \cdot 0$
$48 \cdot 1$
$199 \cdot 0$
$71 \cdot 2$
$101 \cdot 0$
$71 \cdot 2$
$66 \cdot 0$
$129 \cdot 0$
$189 \cdot 0$
$166 \cdot 0$
$137 \cdot 0$
$239 \cdot 0$
$68 \cdot 0$
$38 \cdot 6$
$122 \cdot 0$
$216 \cdot 0$
$281 \cdot 0$
$97 \cdot 2$
$88 \cdot 6$
$94 \cdot 2$
$247 \cdot 0$
$90 \cdot 2$
$83 \cdot 1$
$123 \cdot 0$

\begin{tabular}{|c|}
\hline $\begin{array}{l}50 \\
90.0 \\
54.0\end{array}$ \\
\hline $\begin{array}{r}62.3 \\
106.0 \\
52.4\end{array}$ \\
\hline $\begin{array}{r}32.4 \\
279.0 \\
77.2\end{array}$ \\
\hline 102.0 \\
\hline $\begin{array}{r}60.8 \\
242.0\end{array}$ \\
\hline 163.0 \\
\hline 221.0 \\
\hline $\begin{array}{r}107.0 \\
91.5\end{array}$ \\
\hline 207.0 \\
\hline $\begin{array}{l}226.0 \\
219.0\end{array}$ \\
\hline 206.0 \\
\hline 406.0 \\
\hline $\begin{array}{r}186.0 \\
60.8\end{array}$ \\
\hline 164.0 \\
\hline 280.0 \\
\hline 485.0 \\
\hline $210 \cdot 0$ \\
\hline $\begin{array}{l}117.0 \\
137.0\end{array}$ \\
\hline 340.0 \\
\hline 165.0 \\
\hline 222.0 \\
\hline
\end{tabular}

183
$329 \cdot 0$
$511 \cdot 0$
$131 \cdot 0$
$170 \cdot 0$
$109 \cdot 0$
$408 \cdot 0$
$184 \cdot 0$
$150 \cdot 0$
$139 \cdot 0$
$287 \cdot 0$
$271 \cdot 0$
$324 \cdot 0$
$173 \cdot 0$
$239 \cdot 0$
$326 \cdot 0$
$319 \cdot 0$
$316 \cdot 0$
$271 \cdot 0$
$531 \cdot 0$
$295 \cdot 0$
$150 \cdot 0$
$317 \cdot 0$
$372 \cdot 0$
$613 \cdot 0$
$317 \cdot 0$
$172 \cdot 0$
$177 \cdot 0$
439.0
$356 \cdot 0$
$245 \cdot 0$
307.0

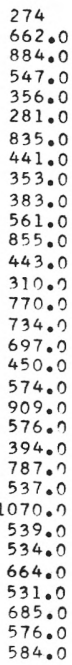

Little North Santiam River near

STATION NUMBER

$14-1825.00$

HIGHEST MEAN DISCHARGE, IN CFS, FOR THE FOLLOWING NUMBER OF CONSECUTIVE DAYS IN YEAR ENDING SEPTFMBER 30

\begin{tabular}{|c|c|c|c|}
\hline YEAR & 1 & 3 & 7 \\
\hline 1937 & 11600.0 & $754 n . n$ & $46>0.0$ \\
\hline 1923 & 8640.0 & $6>10 . n$ & $2900 . ?$ \\
\hline $\begin{array}{l}1934 \\
1925\end{array}$ & $\begin{array}{r}7,700.0 \\
7790.0\end{array}$ & $\begin{array}{l}8410.0 \\
5960.0\end{array}$ & $\begin{array}{l}6280.0 \\
3590.0\end{array}$ \\
\hline $\begin{array}{l}1926 \\
1937\end{array}$ & 9960.0 & 6490.0 & $4 ? 90.0$ \\
\hline $\begin{array}{l}1937 \\
1938\end{array}$ & $\begin{array}{r}7380.0 \\
14000.0\end{array}$ & $\begin{array}{l}6400.0 \\
9060.0\end{array}$ & $\begin{array}{l}3790.0 \\
5170.0\end{array}$ \\
\hline 1939 & 6980.0 & 4300.0 & 3060.0 \\
\hline 1940 & 7080.0 & 4810.0 & 3480.0 \\
\hline 1941 & 6860.0 & 3710.0 & 2270.0 \\
\hline 1942 & 6580.0 & 4520.0 & 3220.0 \\
\hline 1943 & 13800.0 & 8640.0 & 7440.0 \\
\hline 1944 & 5170.0 & 3230.0 & 1990.0 \\
\hline 1945 & $84>0.0$ & 5090.0 & $4080 . ?$ \\
\hline 1946 & 18000.0 & $11600 . n$ & 6450.0 \\
\hline 1947 & 13000.0 & 11800.0 & 7510.0 \\
\hline 1948 & 10300.0 & 7660.0 & 4810.0 \\
\hline $\begin{array}{l}1949 \\
1950\end{array}$ & $\begin{array}{l}6070.0 \\
8000.0\end{array}$ & $\begin{array}{l}4590.0 \\
6500.0\end{array}$ & $\begin{array}{l}3670.0 \\
3930.0\end{array}$ \\
\hline 1951 & 5940.0 & 4360.0 & 3930.0 \\
\hline 1952 & 5520.0 & 4140.0 & 3530.0 \\
\hline 1953 & $1 ? 000.0$ & 9330.0 & 6610.0 \\
\hline 1954 & 11100.0 & $6910 . n$ & 4100.0 \\
\hline 1955 & 9310.0 & 6720.0 & $3700 . ?$ \\
\hline 1956 & 9570.0 & 6980.0 & $4340 . ?$ \\
\hline 1957 & 10100.0 & $7240 . n$ & $4870 . ?$ \\
\hline 1958 & 8820.0 & $6>80.0$ & 4060.0 \\
\hline 1959 & 7300.0 & 5790.0 & 3680.0 \\
\hline 1960 & 4590.0 & 3470.0 & 2510.0 \\
\hline 1961 & 11600.0 & 7460.0 & 4770.0 \\
\hline 1962 & 7560.0 & 6140.0 & 3860.0 \\
\hline 1963 & 12100.0 & 6170.0 & 3730.0 \\
\hline
\end{tabular}

\begin{tabular}{|c|c|}
\hline 15 & \\
\hline & 2820.0 \\
\hline $28 ? 0.0$ & 2060.0 \\
\hline $4 ? 10.0$ & 3190.0 \\
\hline 2260.0 & 1690.0 \\
\hline 3790.0 & 2530.0 \\
\hline $\begin{array}{l}2390.0 \\
2950.0\end{array}$ & 1800.0 \\
\hline $\begin{array}{l}2950.0 \\
1950.0\end{array}$ & $\begin{array}{l}2330.0 \\
1440.0\end{array}$ \\
\hline 2490.0 & 2190.0 \\
\hline 1590.0 & 1280.0 \\
\hline $2030 . ?$ & 1930.0 \\
\hline $\begin{array}{l}5080 . ? \\
1690 . ?\end{array}$ & 3660.0 \\
\hline 1690.0 & 1110.0 \\
\hline $\begin{array}{l}2670.0 \\
4350.0\end{array}$ & 1670.0 \\
\hline 4350.0 & 3110.0 \\
\hline $\begin{array}{r}4110.0 \\
2930.0\end{array}$ & 3340.0 \\
\hline 2680.0 & 1910.0 \\
\hline $\begin{array}{l}3020.0 \\
2730.0\end{array}$ & $\begin{array}{l}2310.0 \\
2380.0\end{array}$ \\
\hline $2320 \cdot 0$ & 1590.0 \\
\hline 5310.0 & 4350.0 \\
\hline 3440.0 & 3320.0 \\
\hline 2120.0 & $1400 . ?$ \\
\hline $\begin{array}{l}3620 . ? \\
3040.0\end{array}$ & $3050 . n$ \\
\hline $\begin{array}{l}3040.0 \\
2010.0\end{array}$ & $2140 . n$ \\
\hline $\begin{array}{r}2010.0 \\
3390.0\end{array}$ & 2290.0 \\
\hline $\begin{array}{l}3390.0 \\
1910.0\end{array}$ & 2360.0 \\
\hline $\begin{array}{l}1910.0 \\
3610.0\end{array}$ & 2580.0 \\
\hline 630.0 & 1990.0 \\
\hline & 2220.0 \\
\hline
\end{tabular}

60
2160.0
1660.0
2530.0
1620.0
1660.0
1580.0
2070.0
1290.0
1590.0
1030.0
1570.0
3110.0
901.0
1540.0
2350.0
2160.0
1650.0
1550.0
1990.0
1870.0
1300.0
2850.0
2270.0
1290.0
2590.0
1660.0
2070.0
1830.0
1410.0
2040.0
1580.0
1460.0

90
1790.0
1500.0
1910.0
1450.0
1430.0
1460.0
1820.0
1110.0
1420.0
1010.0
1390.0
2650.0
817.0
1370.0
2020.0
1830.0
1750.0
1630.0
1770.0
1900.0
1270.0
2210.0
2120.0
1240.0
2320.0
1380.0
1830.0
1820.0
1340.0
1740.0
1270.0
1260.0

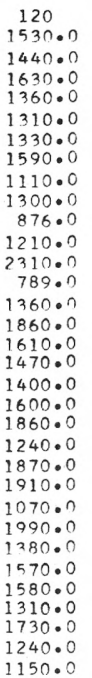

150

1470.0

1290.0

$1400 \cdot 0$
1250.0

1250.0
1220.0

1530.0

1090.0

1190.0
767.0

1070.0

2120.0

767.0

1340.0
1680.0

1440.0

1170.0

1510.0

1160.0

1700.0

1750.0

1050.0
1750.0

1240 . ?

1490.0
1500.0

1500.0
1180.0

1650.0

1240.0
1110.0

\begin{tabular}{|c|c|}
\hline 3 & \\
\hline $1350 . n$ & 1080.0 \\
\hline $1390 . n$ & 1230.0 \\
\hline $\begin{array}{l}1280.0 \\
1180.0\end{array}$ & $\begin{array}{l}938.0 \\
941.0\end{array}$ \\
\hline 1120.0 & 863.0 \\
\hline $\begin{array}{l}1080.0 \\
1510.0\end{array}$ & 817.0 \\
\hline $\begin{array}{l}1510.0 \\
1020.0\end{array}$ & 1120.0 \\
\hline $\begin{array}{l}1020.0 \\
1020.0\end{array}$ & $\begin{array}{l}783.0 \\
726.0\end{array}$ \\
\hline 674.0 & 578.0 \\
\hline 974.0 & 830.0 \\
\hline 1960.0 & 1460.0 \\
\hline 771.0 & 647.0 \\
\hline 1170.0 & $873 . n$ \\
\hline 1550.0 & 1170.0 \\
\hline 1360.0 & 1040.0 \\
\hline 1370.0 & 1200.0 \\
\hline 1170.0 & 937.0 \\
\hline $\begin{array}{l}1430.0 \\
1540.0\end{array}$ & 1170.0 \\
\hline $\begin{array}{l}1540.0 \\
1150.0\end{array}$ & 1190.0 \\
\hline $\begin{array}{l}1150.0 \\
1560.0\end{array}$ & $\begin{array}{l}1020.0 \\
1120.0\end{array}$ \\
\hline 1550.0 & 1210.0 \\
\hline 1050.0 & 910.0 \\
\hline 1680.0 & 1400.0 \\
\hline 1160.0 & $903 . n$ \\
\hline 1360.0 & 993.0 \\
\hline 1390.0 & 1080.0 \\
\hline 1060.0 & 938.0 \\
\hline $1520 \cdot 0$ & 1140.0 \\
\hline 1200.0 & $9 \div 9.0$ \\
\hline & \\
\hline
\end{tabular}

274
080.0

230.0

938.0

863.0

120.0

783.0

460.0
647.0

873.0

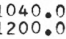

937.0

190.0

1020.0

210.0

400.0

(

938.0

$9 \div 9.0$

927.0 
CLASS $0 \begin{array}{lllllllllllllllllllllllllllllllllll} & 0 & 2 & 3 & 4 & 5 & 6 & 7 & 8 & 9 & 10 & 11 & 12 & 13 & 14 & 15 & 16 & 17 & 18 & 19 & 20 & 21 & 22 & 23 & 24 & 25 & 26 & 27 & 28 & 29 & 30 & 31 & 32 & 33 & 34\end{array}$

\begin{tabular}{|c|c|c|c|c|c|c|c|c|c|c|c|c|c|c|c|c|c|c|c|c|c|c|c|c|c|c|c|c|c|c|}
\hline YEAR & & & & & & & & & & & & & NUMB & BER & OF & DAY & IS I & IN C & CLAS & & & & & & & & & & & CFS-DAYS \\
\hline 1906 & & & & 24 & 33 & 19 & 17 & 10 & 18 & 26 & 53 & 48 & 21 & 27 & 20 & 24 & 7 & 6 & 7 & 1 & 1 & 2 & 1 & & & & & & & 1143080.0 \\
\hline $\begin{array}{l}1911 \\
1912\end{array}$ & & & $\begin{array}{r}12 \\
3\end{array}$ & $\begin{array}{r}23 \\
8\end{array}$ & $\begin{array}{l}52 \\
10\end{array}$ & $\begin{array}{r}8 \\
34\end{array}$ & $\begin{array}{l}10 \\
27\end{array}$ & $\begin{array}{l}33 \\
29\end{array}$ & $\begin{array}{r}14 \\
7\end{array}$ & $\begin{array}{l}40 \\
34\end{array}$ & $\begin{array}{l}40 \\
46\end{array}$ & $\begin{array}{l}31 \\
31\end{array}$ & $\begin{array}{l}21 \\
16\end{array}$ & $\begin{array}{r}9 \\
15\end{array}$ & $\begin{array}{l}11 \\
16\end{array}$ & $\begin{array}{l}24 \\
20\end{array}$ & $\begin{array}{l}10 \\
16\end{array}$ & $\begin{array}{r}8 \\
13\end{array}$ & $\begin{array}{l}8 \\
8\end{array}$ & $\begin{array}{r}4 \\
12\end{array}$ & $\begin{array}{l}3 \\
6\end{array}$ & $\frac{2}{7}$ & $\begin{array}{l}1 \\
5\end{array}$ & $\begin{array}{l}1 \\
1\end{array}$ & & 1 & 1 & & & $\begin{array}{l}1115490.0 \\
1522280.0\end{array}$ \\
\hline 1913 & & & 1 & 11 & 32 & 27 & 8 & 12 & 10 & 10 & 34 & 27 & 31 & 22 & 21 & 29 & 40 & 17 & 12 & 7 & 3 & 8 & 1 & & 1 & 1 & & & & 1576700.0 \\
\hline 1914 & & & 16 & 23 & 22 & 15 & 16 & 22 & 24 & 34 & 45 & 28 & 26 & 16 & 11 & 21 & 14 & 6 & 15 & 7 & 2 & 1 & 1 & & & & & & & 1173605.0 \\
\hline 1922 & & & 11 & 24 & 45 & 20 & 14 & 18 & 19 & 54 & 21 & 17 & 17 & 14 & 17 & 23 & 12 & 10 & 10 & 4 & 6 & 2 & 1 & & 2 & 1 & 1 & 1 & 1 & 1354683.0 \\
\hline $\begin{array}{l}1923 \\
1924\end{array}$ & 134 & 25 & $\begin{array}{l}10 \\
12\end{array}$ & $\begin{array}{l}36 \\
15\end{array}$ & $\begin{array}{l}43 \\
34\end{array}$ & $\begin{array}{l}23 \\
19\end{array}$ & $\begin{array}{l}10 \\
18\end{array}$ & $\begin{array}{l}19 \\
15\end{array}$ & $\begin{array}{l}20 \\
19\end{array}$ & $\begin{array}{l}21 \\
43\end{array}$ & $\begin{array}{l}28 \\
36\end{array}$ & $\begin{array}{l}23 \\
22\end{array}$ & $\begin{array}{l}40 \\
29\end{array}$ & $\begin{array}{r}20 \\
9\end{array}$ & $\begin{array}{r}17 \\
6\end{array}$ & $\begin{array}{r}20 \\
8\end{array}$ & $\begin{array}{r}10 \\
3\end{array}$ & $\begin{array}{l}4 \\
2\end{array}$ & $\begin{array}{l}3 \\
5\end{array}$ & $\begin{array}{l}2 \\
4\end{array}$ & $\begin{array}{l}5 \\
2\end{array}$ & $\begin{array}{l}5 \\
3\end{array}$ & 2 & 1 & 3 & & & 1 & 1 & $\begin{array}{r}1282756.0 \\
894426.0\end{array}$ \\
\hline 1925 & 134 & 4 & 33 & 30 & 28 & 11 & $\begin{array}{r}18 \\
9\end{array}$ & $\begin{array}{r}15 \\
6\end{array}$ & $\begin{array}{r}19 \\
7\end{array}$ & $\begin{array}{l}43 \\
19\end{array}$ & $\begin{array}{l}30 \\
33\end{array}$ & 20 & 38 & 17 & 21 & 28 & 13 & 10 & 16 & 6 & 1 & $\begin{array}{l}3 \\
6\end{array}$ & 2 & 4 & 3 & & & & & 1465530.0 \\
\hline 1926 & 8 & 62 & 23 & 17 & 22 & 16 & 14 & 35 & 33 & 37 & 26 & 14 & 13 & 7 & 7 & 12 & 4 & 1 & 6 & 4 & & 2 & & 1 & & 1 & & & & 833087.0 \\
\hline 1927 & & & & 13 & 33 & 20 & 19 & 16 & 13 & 26 & 36 & 27 & 40 & 22 & 15 & 28 & 18 & 11 & 12 & 7 & 1 & 4 & 1 & & & 3 & & & & 035.0 \\
\hline 1928 & & & 45 & 17 & 15 & 13 & 17 & 14 & 15 & 33 & 30 & 26 & 14 & 25 & 8 & 36 & 19 & 4 & 16 & 6 & 6 & 1 & 2 & 3 & & 1 & & & & 1371515.0 \\
\hline 1929 & & 33 & 18 & 21 & 26 & 14 & 24 & 25 & 18 & 43 & 37 & 21 & 8 & 15 & 6 & 30 & 12 & 5 & 8 & & 1 & & & & & & & & & 945425.0 \\
\hline 1930 & 34 & 86 & 15 & 12 & 12 & 18 & 11 & 10 & 15 & 61 & 26 & 9 & 5 & 9 & 6 & 7 & 6 & 4 & 12 & 3 & 2 & 1 & 1 & & & & & & & 5.0 \\
\hline 1931 & 29. & 37 & 27 & 13 & 26 & 9 & 26 & 37 & 21 & 53 & 30 & 11 & 11 & 7 & 6 & 5 & 5 & 4 & 3 & 2 & & 1 & & & & & 1 & 1 & & 25.0 \\
\hline 1932 & 17 & 7 & 23 & 20 & 22 & 8 & 11 & 17 & 24 & 33 & 18 & 17 & 9 & 22 & 20 & 34 & 27 & 15 & 13 & 3 & 2 & 2 & & & 1 & 1 & & & & 1294190.0 \\
\hline 1933 & & 12 & & 3 & 31 & 21 & 12 & 20 & 10 & 33 & 22 & 26 & 13 & 27 & 24 & 36 & 24 & 24 & 12 & 10 & 2 & 2 & & 1 & & & & & & 40.0 \\
\hline 1934 & & 45 & 25 & 19 & 28 & 19 & 37 & 28 & 23 & 30 & 14 & 10 & 9 & 8 & 10 & 16 & 13 & 3 & 11 & 3 & 2 & 6 & 1 & 3 & 1 & & & 1 & & 1078641.0 \\
\hline 1935 & & 44 & 28 & 11 & 12 & 8 & 8 & 8 & 13 & 27 & 26 & 35 & 35 & 21 & 20 & 34 & 13 & 10 & 6 & 2 & 1 & 2 & & & 1 & & & & & 1164366.0 \\
\hline 1936 & & 27 & 41 & 20 & 23 & 12 & 20 & 14 & 18 & 40 & 25 & 16 & 17 & 19 & 12 & 32 & 8 & 10 & 2 & 3 & 1 & 2 & 2 & & $i$ & 1 & & & & 1066033.0 \\
\hline 1937 & 26 & 39 & 17 & 28 & 42 & 19 & 17 & 9 & 9 & 7 & 14 & 16 & 19 & 14 & 14 & 28 & 21 & 9 & 10 & 1 & 3 & & & 2 & & 1 & & & & 1046972.0 \\
\hline 1938 & & 10 & 42 & 18 & 28 & 17 & 10 & 8 & 11 & 12 & 19 & 26 & 18 & 22 & 26 & 34 & 19 & 12 & 11 & 10 & 6 & 3 & 1 & 1 & 1 & & & & & 1373239.0 \\
\hline 1939 & & 26 & 51 & 15 & 12 & 11 & 6 & 21 & 26 & 45 & 34 & 33 & 15 & 17 & 12 & 17 & 4 & 5 & 8 & 4 & 2 & 1 & & & & & & & & 958271.0 \\
\hline 1940 & 17 & 62 & 51 & 24 & 20 & 13 & 10 & 13 & 12 & 22 & 24 & 18 & 16 & 8 & 8 & 13 & 8 & 8 & 8 & 7 & 3 & & 1 & & & & & & & 7.0 \\
\hline 1941 & & 25 & 27 & 13 & 21 & 15 & 26 & 71 & 33 & 43 & 26 & 22 & 14 & 8 & 3 & 8 & 3 & 2 & 4 & & & 1 & & & & & & & & 752301.0 \\
\hline 1942 & & 23 & 27 & 9 & 16 & 15 & 17 & 18 & 39 & 72 & 48 & 18 & 11 & 7 & 6 & 11 & 7 & 6 & 7 & 2 & 2 & 1 & 3 & & & & & & & 970453.0 \\
\hline 1943 & & 24 & 3 & 24 & 27 & 12 & 9 & 11 & 4 & 23 & 40 & 24 & 23 & 18 & 12 & 31 & 25 & 11 & 17 & 8 & 4 & 6 & 3 & 1 & 3 & 2 & & & & 1578778.0 \\
\hline 1944 & & 31 & 23 & 26 & 25 & 12 & 15 & 38 & 27 & 60 & 37 & 32 & 14 & 9 & 5 & & 5 & 1. & 4 & 2 & & & & & & & & & & 766653.0 \\
\hline 1945 & & 24 & 49 & 20 & 28 & 30 & 13 & 20 & 17 & 31 & 10 & 13 & 17 & 15 & 15 & 23 & 10 & 10 & 11 & 4 & 2 & 1 & & 1 & 1 & & & & & 10266 \\
\hline 1946 & & 12 & 25 & 29 & 25 & 11 & 8 & 6 & 9 & 20 & 45 & 23 & 34 & 33 & 23 & 19 & 12 & 8 & 11 & 4 & $i$ & 2 & 1 & 1 & $i$ & 1 & & & 1 & 37.0 \\
\hline 1947 & & & 17 & 33 & 28 & 16 & 12 & 35 & 22 & 37 & 36 & 18 & 17 & 29 & 16 & 12 & 7 & 7 & 8 & 1 & 2 & 6 & 1 & 1 & 1 & 1 & 1 & 1 & & 1241633.0 \\
\hline 1948 & & & & 18 & 35 & 20 & 12 & 20 & 15 & 28 & 35 & 31 & 19 & 31 & 23 & 23 & 17 & 12 & 8 & 7 & 5 & 2 & 2 & 1 & 1 & & & 1 & & 1419931.0 \\
\hline 1949 & & & 7 & 15 & 36 & 34 & 28 & 22 & 18 & 26 & 17 & 14 & 19 & 14 & 17 & 28 & 18 & 14 & 19 & 8 & 3 & 5 & $i$ & 1 & $i$ & & & & & 31.0 \\
\hline 1950 & & & 4 & 11. & 33 & 30 & 15 & 20 & 12 & 25 & 23 & 17 & 30 & 19 & 17 & 30 & 32 & 18 & 12 & 6 & 3 & 4 & 1 & 1 & $i$ & 1 & & & & 1473019.0 \\
\hline 1951 & & & 15 & 32 & 28 & 13 & 11 & 12 & 15 & 22 & 13 & 19 & 32 & 22 & 24 & 32 & 18 & 9 & 17 & 11 & 7 & 6 & 5 & 1 & & 1 & & & & 3.0 \\
\hline 1952 & & & 10 & 16 & 34 & 11 & 9 & 16 & 16 & 46 & 38 & 33 & 20 & 8 & 25 & 35 & 18 & 8 & 6 & 9 & 4 & 2 & 2 & & & & & & & 1294555.0 \\
\hline 1953 & & & 56 & 4 & 29 & 33 & 16 & 24 & 27 & 41 & 27 & 19 & 11 & 13 & 11 & 6 & 5 & & 6 & 5 & 15 & 16 & & 1 & & & & & & 1215616.0 \\
\hline 1954 & & & & & 3 & 7 & 28 & 26 & 38 & 51 & 41 & 25 & 25 & 25 & 13 & 13 & 20 & 11 & 16 & 8 & 9 & 5 & & 1 & & & & & & 1453733.0 \\
\hline 1955 & & & & & & 26 & 12 & 31 & 31 & 77 & 44 & 40 & 16 & 16 & 19 & 27 & 8 & 8 & 4 & 5 & 1 & & & & & & & & & 090.0 \\
\hline 1956 & & & & & & & 34 & 29 & 22 & 45 & 18 & 24 & 17 & 18 & 24 & 26 & 22 & 17 & 23 & 16 & 17 & 13 & 1 & & & & & & & 1845290.0 \\
\hline 1957 & & & & & 38 & 15 & 28 & 27 & 37 & 51 & 33 & 20 & 21 & 23 & 13 & 10 & 21 & 9 & 11 & 3 & 4 & 1 & & & & & & & & 1158857.0 \\
\hline 1958 & & & & & 5 & 51 & 15 & 32 & 32 & 58 & 34 & 13 & 25 & 13 & 8 & 13 & 14 & 25 & 16 & 7 & 2 & 2 & & & & & & & & 15.0 \\
\hline 1959 & & & & & 16 & 44 & 11 & 10 & 26 & 68 & 25 & 36 & 18 & 22. & 10 & 20 & 23 & 8 & 7 & 10 & 11 & & & & & & & & & 1319500.0 \\
\hline 1960 & & & & & & 24 & 44 & 32 & 34 & 43 & 25 & 39 & 27 & 17 & 11 & 26 & 17 & 11 & 8 & 3 & 4 & 1 & & & & & & & & 1208650.0 \\
\hline 1961 & & & & & & $i$ & 66 & 22 & 34 & 51 & 23 & 34 & 17 & 16 & 8 & 19 & 27 & 11 & 13 & 3 & 11 & 7 & 1 & 1 & & & & & & 1436020.0 \\
\hline 1962 & & & & & & 1 & 65 & 30 & 41 & 54 & 30 & 22 & 13 & 18 & 14 & 25 & 23 & 12 & 14 & 3 & & & & & & & & & & 1198770.0 \\
\hline 1963 & & & & & & 9 & 62 & 72 & 28 & 39 & 33 & 11 & 15 & 19 & 11 & 23 & 13 & 3 & 11 & 10 & 4 & 1 & 1 & & & & & & & 1179630.0 \\
\hline
\end{tabular}

North Santiam River at Mehama, Ureg.

STATION NUMBER 14-1830.00

Summary for water years 1906, 1911-14, 1922-63

\begin{tabular}{|c|c|c|c|c|c|c|c|c|c|c|c|c|c|c|c|c|c|c|c|}
\hline CLASS & CFS & TOTAL & ACCUM & DFRCT & CLASS & CFS & TOTAL & ACCUM & PERCT & CLAS & CFS & TOTAL & ACCUM & PFRCT & CLA & SS CFS & TOTAL & ACCUM & PERCT \\
\hline & & & 17166 & $10 n .0$ & 09 & 1400.0 & 1063 & 12051 & 70.2 & 18 & 6000 & 691 & 2279 & 13.3 & 27 & 30000 & 17 & 30 & $\cdot 2$ \\
\hline 1 & 400.0 & 1 & 17166 & 100.0 & 10 & 1700.0 & 976 & 10988 & 64.0 & 19 & 7000 & 422 & 1588 & 9.3 & 28 & 35000 & 4 & 13 & . \\
\hline 2 & 450.0 & 165 & 17165 & $10 n .0$ & 11 & 2000.0 & 1794 & 10012 & 58 & 20 & 8000 & 475 & 1166 & 6. & 29 & 40000 & 4 & 9 & - 1 \\
\hline 3 & 500.0 & 658 & 17000 & 99.0 & 12 & 2500.0 & 1418 & 8218 & & 21 & 10000 & 247 & 691 & 4. & 30 & 45000 & 2 & 5 & .0 \\
\hline 4 & 600.0 & 767 & 16342 & 95.2 & 13 & 3000.0 & 1099 & 6800 & 39 & 22 & 12000 & 172 & 444 & 2. & 31 & 50000 & 3 & 3 & .0 \\
\hline 5 & 700.0 & 695 & 15575 & & 14 & 350 & 936 & 57 & & 23 & 140 & 145 & 272 & 1. & 32 & & & & .0 \\
\hline 6 & $800 \cdot 0$ & 1078 & 14880 & 86.7 & 15 & 4000.0 & 805 & 4765 & & 24 & 17000 & 45 & 127 & .7 & 33 & & & & .0 \\
\hline 7 & 1000.0 & 824 & 13802 & 80.4 & 16 & 4500.0 & 662 & 3960 & 23 & 25 & 20000 & 29 & 82 & .5 & 34 & & & & \\
\hline 8 & 1200.0 & 927 & 12978 & 75.6 & 17 & 5000.0 & 1019 & 3298 & & 26 & 25000 & 23 & 53 & .3 & 35 & & & & \\
\hline
\end{tabular}

North Santiam River at Mehama, Oreg.

STATION NUMBER

$14-1830.00$

Summary for water years 1906, 1911-14, 1922-52

\begin{tabular}{lrrrrrr} 
CLASS & \multicolumn{1}{c}{ CFS } & TOTAL & ACCUM & PERCT & \multicolumn{2}{l}{ CLASS CFS } \\
& .0 & & 13149 & 100.0 & 09 & 1400.0 \\
1 & 400.0 & 1 & 13149 & 100.0 & 10 & 1700.0 \\
2 & 450.0 & 165 & 13148 & 100.0 & 11 & 2000.0 \\
3 & 500.0 & 658 & 12983 & 98.7 & 12 & 2500.0 \\
4 & 600.0 & 711 & 12325 & 93.7 & 13 & 3000.0 \\
5 & 700.0 & 691 & 11614 & 88.3 & 14 & 3500.0 \\
6 & 800.0 & 987 & 10923 & 83.1 & 15 & 4000.0 \\
7 & 1000.0 & 613 & 9936 & 75.6 & 16 & 4500.0 \\
8 & 1200.0 & 546 & 9323 & 70.9 & 17 & 5000.0
\end{tabular}

North Santiam River at Mehama, Oreg.

Summary for water years 1954-63

\begin{tabular}{lrrrrr} 
CLASS & \multicolumn{1}{c}{ CFS } & TOTAL & ACCUM & PERCT & CL \\
& .0 & & 3652 & $\mathbf{1 0 0 . 0}$ & 09 \\
$\mathbf{1}$ & 400.0 & & 3652 & $\mathbf{1 0 0 . 0}$ & 10 \\
2 & 450.0 & & 3652 & $\mathbf{1 0 0 . 0}$ & 11 \\
3 & 500.0 & & 3652 & 100.0 & 12 \\
4 & 600.0 & & 3652 & $\mathbf{1 0 0 . 0}$ & 13 \\
5 & 700.0 & & 3652 & 100.0 & 14 \\
6 & 800.0 & 62 & 3652 & 100.0 & 15 \\
7 & 1000.0 & 178 & 3590 & 98.3 & 16 \\
8 & 1200.0 & 365 & 3412 & 93.4 & 17
\end{tabular}

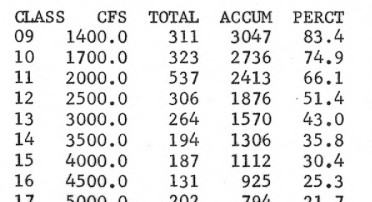

5000.0

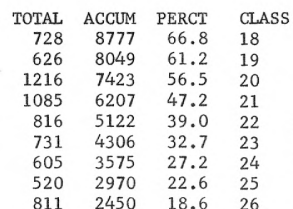

STATION NUMBER

14- 1830.00 
North Santiam River at Mehama, Oreg.

STATION NUMBER

$14-1830.00$ LOWEST MEAN DISCHARGE, IN CFS, FOR THE FOLLOWING NUMBER OF CONSECUTIVE DAYS IN YEAR BEGINNING APRIL 1

\begin{tabular}{|c|c|c|c|c|c|c|c|c|c|c|c|}
\hline YEAR & 1 & 3 & 7 & 14 & 30 & 60 & 90 & 120 & 150 & 183 & 274 \\
\hline $\begin{array}{l}1906 \\
1911\end{array}$ & $\begin{array}{l}700.0 \\
660.0\end{array}$ & $\begin{array}{l}700.0 \\
660.0\end{array}$ & $\begin{array}{l}700.0 \\
660.0\end{array}$ & $\begin{array}{l}720.0 \\
671.0\end{array}$ & $\begin{array}{l}755.0 \\
726.0\end{array}$ & $\begin{array}{l}859.0 \\
828.0\end{array}$ & $\begin{array}{l}965.0 \\
915.0\end{array}$ & $\begin{array}{r}1220 \cdot 0 \\
932 \cdot 0\end{array}$ & $\begin{array}{l}1540.0 \\
1170.0\end{array}$ & $\begin{array}{l}1860.0 \\
1840.0\end{array}$ & $\begin{array}{l}3530.0 \\
2620.0\end{array}$ \\
\hline 1912 & 660.0 & 713.0 & 729.0 & 747.0 & 916.0 & 1430.0 & 1420.0 & $1580 \cdot 0$ & 2050.0 & & \\
\hline $\begin{array}{l}1913 \\
1922\end{array}$ & $\begin{array}{l}830.0 \\
621.0\end{array}$ & $\begin{array}{l}830.0 \\
627.0\end{array}$ & $\begin{array}{l}856.0 \\
635.0\end{array}$ & 862.0 & 931.0 & $\begin{array}{l}992.0 \\
740.0\end{array}$ & $\begin{array}{r}1210.0 \\
776.0\end{array}$ & $\begin{array}{r}1750.0 \\
844.0\end{array}$ & $\begin{array}{r}2070.0 \\
983.0\end{array}$ & $\begin{array}{l}2250.0 \\
1160.0\end{array}$ & $\begin{array}{l}3210.0 \\
2960.0\end{array}$ \\
\hline $\begin{array}{l}1922 \\
1923\end{array}$ & $\begin{array}{l}621.0 \\
720.0\end{array}$ & $\begin{array}{l}627.0 \\
732.0\end{array}$ & $\begin{array}{l}635.0 \\
745.0\end{array}$ & $\begin{array}{l}658.0 \\
766.0\end{array}$ & $\begin{array}{l}700.0 \\
776.0\end{array}$ & 849.0 & 1010.0 & $1040 \cdot 0$ & 1290.0 & 1700.0 & 2620.0 \\
\hline 1924 & 420.0 & 440.0 & 446.0 & 453.0 & 458.0 & 490.0 & 584.0 & 633.0 & 755.0 & 1040.0 & 2440.0 \\
\hline $\begin{array}{l}1925 \\
1926\end{array}$ & $\begin{array}{l}580.0 \\
480.0\end{array}$ & $\begin{array}{l}580.0 \\
480.0\end{array}$ & 580.0 & 580.0 & $\begin{array}{l}580.0 \\
530.0\end{array}$ & $\begin{array}{l}621.0 \\
643.0\end{array}$ & 660.0 & $\begin{array}{l}716.0 \\
843.0\end{array}$ & $\begin{array}{r}909.0 \\
1000.0\end{array}$ & $\begin{array}{l}1200.0 \\
1140.0\end{array}$ & $\begin{array}{l}2090.0 \\
2140.0\end{array}$ \\
\hline $\begin{array}{l}1926 \\
1927\end{array}$ & $\begin{array}{l}480.0 \\
740.0\end{array}$ & $\begin{array}{l}480.0 \\
740.0\end{array}$ & 480.0 & $\begin{array}{l}493.0 \\
756.0\end{array}$ & $\begin{array}{l}530.0 \\
807.0\end{array}$ & $\begin{array}{l}643.0 \\
977.0\end{array}$ & $\begin{array}{r}712.0 \\
1190.0\end{array}$ & $\begin{array}{r}843.0 \\
1830.0\end{array}$ & 2100.0 & 2630.0 & $\begin{array}{l}2140.0 \\
3630.0\end{array}$ \\
\hline 1928 & 626.0 & 626.0 & $\begin{array}{l}740.0 \\
634.0\end{array}$ & $\begin{array}{l}756.0 \\
641.0\end{array}$ & 657.0 & $\begin{array}{l}977.0 \\
677.0\end{array}$ & 762.0 & 806.0 & $900 \cdot 0$ & 1200.0 & $\begin{array}{l}3630.0 \\
1670.0\end{array}$ \\
\hline 1929 & 464.0 & 464.0 & 468.0 & 474.0 & 486.0 & 509.0 & 520.0 & $547 \cdot 0$ & 627.0 & 979.0 & 2020.0 \\
\hline 1930 & 495.0 & 498.0 & 508.0 & 518.0 & 538.0 & 545.0 & 587.0 & $647 \cdot 0$ & 731.0 & 956.0 & 1420.0 \\
\hline $\begin{array}{l}1931 \\
1932\end{array}$ & 454.0 & 454.0 & 454.0 & 466.0 & 485.0 & 497.0 & 523.0 & $655 \cdot 0$ & 795.0 & $1000 \cdot 0$ & 1790.0 \\
\hline $\begin{array}{l}1932 \\
1933\end{array}$ & 540.0 & 542.0 & 554.0 & 565.0 & 600.0 & 659.0 & 759.0 & 929.0 & 1410.0 & 2060.0 & $\begin{array}{l}2770.0 \\
3480.0\end{array}$ \\
\hline $\begin{array}{l}1933 \\
1934\end{array}$ & 788.0 & 796.0 & 824.0 & 843.0 & 867.0 & 1060.0 & 1170.0 & $1460 \cdot 0$ & 1570.0 & 2260.0 & \\
\hline $\begin{array}{l}1934 \\
1935\end{array}$ & 518.0 & 525.0 & 529.0 & 537.0 & 544.0 & 555.0 & 581.0 & $\begin{array}{l}634.0 \\
729.0\end{array}$ & $\begin{array}{l}743.0 \\
901.0\end{array}$ & $\begin{array}{l}1000.0 \\
1080.0\end{array}$ & $\begin{array}{l}2380.0 \\
1960.0\end{array}$ \\
\hline $\begin{array}{l}1935 \\
1936\end{array}$ & 524.0 & 526.0 & 533.0 & 546.0 & 576.0 & 592.0 & $667 \cdot 0$ & $\begin{array}{l}129.0 \\
568.0\end{array}$ & 642.0 & 843.0 & $\begin{array}{l}1960.0 \\
1310.0\end{array}$ \\
\hline $\begin{array}{l}1936 \\
1937\end{array}$ & 468.0 & 473.0 & $\begin{array}{l}474.0 \\
642.0\end{array}$ & 479.0 & 490.0 & 505.0 & 540.0 & $\begin{array}{r}568 \cdot 0 \\
1020.0\end{array}$ & 1680.0 & 2350.0 & 3610.0 \\
\hline $\begin{array}{l}1937 \\
1938\end{array}$ & $\begin{array}{l}628.0 \\
582.0\end{array}$ & $\begin{array}{l}631.0 \\
584.0\end{array}$ & $\begin{array}{l}642.0 \\
587.0\end{array}$ & $\begin{array}{l}694.0 \\
595.0\end{array}$ & $\begin{array}{l}716.0 \\
609.0\end{array}$ & $\begin{array}{l}758.0 \\
643.0\end{array}$ & $\begin{array}{l}818.0 \\
673.0\end{array}$ & 745.0 & 951.0 & 1310.0 & 2120.0 \\
\hline 1939 & 519.0 & $\begin{array}{l}584.0 \\
538.0\end{array}$ & 541.0 & 552.0 & 577.0 & 611.0 & 645.0 & $702 \cdot 0$ & 729.0 & 916.0 & 1660.0 \\
\hline 1940 & 477.0 & 479.0 & 483.0 & 488.0 & 499.0 & 534.0 & 565.0 & $602 \cdot 0$ & 669.0 & $\begin{array}{r}911.0 \\
1320.0\end{array}$ & \\
\hline 1941 & 556.0 & 563.0 & 566.0 & 579.0 & 613.0 & 710.0 & 894.0 & $1030 \bullet 0$ & 1130.0 & 1320.0 & $\begin{array}{l}2320.0 \\
3110.0\end{array}$ \\
\hline 1942 & 500.0 & 505.0 & 509.0 & $522 \cdot 0$ & 531.0 & 552.0 & 605.0 & 742.0 & $\begin{array}{l}1070.0 \\
1620.0\end{array}$ & $\begin{array}{l}1420.0 \\
1810.0\end{array}$ & $\begin{array}{l}3110.0 \\
2070.0\end{array}$ \\
\hline $\begin{array}{l}1943 \\
1944\end{array}$ & $\begin{array}{l}676.0 \\
517.0\end{array}$ & $\begin{array}{l}679.0 \\
517.0\end{array}$ & $\begin{array}{l}685 \cdot 0 \\
529.0\end{array}$ & $\begin{array}{l}701.0 \\
547.0\end{array}$ & $\begin{array}{l}720.0 \\
558.0\end{array}$ & $\begin{array}{l}800.0 \\
632.0\end{array}$ & $\begin{array}{l}905.0 \\
644.0\end{array}$ & $\begin{array}{r}1240.0 \\
694.0\end{array}$ & $\begin{array}{r}1620.0 \\
845.0\end{array}$ & 996.0 & 1490.0 \\
\hline $\begin{array}{l}1945 \\
1946\end{array}$ & $\begin{array}{l}538.0 \\
634.0\end{array}$ & $\begin{array}{l}548.0 \\
637.0\end{array}$ & $\begin{array}{l}557.0 \\
643.0\end{array}$ & $\begin{array}{l}573.0 \\
667.0\end{array}$ & $\begin{array}{l}618.0 \\
688.0\end{array}$ & $\begin{array}{l}659.0 \\
726.0\end{array}$ & $\begin{array}{l}663.0 \\
794.0\end{array}$ & $\begin{array}{r}709.0 \\
1100 \cdot 0\end{array}$ & $\begin{array}{r}862.0 \\
1500.0\end{array}$ & $\begin{array}{l}1350.0 \\
1870.0\end{array}$ & $\begin{array}{l}3100.0 \\
3410.0 \\
2850.0\end{array}$ \\
\hline $\begin{array}{l}1947 \\
1948\end{array}$ & 680.0 & 692.0 & 698.0 & 716.0 & 792.0 & 819.0 & 925.0 & $\begin{array}{l}1170.0 \\
1230.0\end{array}$ & $\begin{array}{l}1370.0 \\
1430.0\end{array}$ & $\begin{array}{l}1700.0 \\
1960.0\end{array}$ & $\begin{array}{l}2850.0 \\
2590.0\end{array}$ \\
\hline $\begin{array}{l}1948 \\
1949\end{array}$ & $\begin{array}{l}731.0 \\
632.0\end{array}$ & $\begin{array}{l}731.0 \\
645.0\end{array}$ & $\begin{array}{l}746.0 \\
656.0\end{array}$ & $\begin{array}{l}748.0 \\
678.0\end{array}$ & $\begin{array}{l}803.0 \\
812.0\end{array}$ & $\begin{array}{l}894.0 \\
841.0\end{array}$ & $\begin{array}{r}1040.0 \\
959.0\end{array}$ & 1120.0 & 1250.0 & 1640.0 & 2650.0 \\
\hline 1950 & 749.0 & 749.0 & 757.0 & 775.0 & 829.0 & 951.0 & 1190.0 & 1720.0 & 2490.0 & $3030 \cdot 0$ & $\begin{array}{l}4460.0 \\
2690.0\end{array}$ \\
\hline$\frac{1951}{1952}$ & 650.0 & 653.0 & 663.0 & 676.0 & 704.0 & 748.0 & 841.0 & 1060.0 & 1420.0 & 1870.0 & $\frac{2690.0}{2240.0}$ \\
\hline$\frac{1952}{1953}$ & 626.0 & 631.0 & 636.0 & 643.0 & 648.0 & 672.0 & 697.0 & 737.0 & 853.0 & $1180 \cdot 0$ & \\
\hline $\begin{array}{l}1953 \\
1954\end{array}$ & 714.0 & 867.0 & 886.0 & 892.0 & 994.0 & 1030.0 & 1200.0 & $1470 \cdot 0$ & $\begin{array}{l}1670.0 \\
2250.0\end{array}$ & $\begin{array}{l}2060 \cdot 0 \\
2460.0\end{array}$ & $\begin{array}{l}3480.0 \\
2650.0\end{array}$ \\
\hline $\begin{array}{l}1954 \\
1955\end{array}$ & 921.0 & $986 \cdot 0$ & 1090.0 & 1120.0 & 1200.0 & 1400.0 & $1720 \cdot 0$ & $\begin{array}{l}1970.0 \\
2360.0\end{array}$ & 2780.0 & $3020 \cdot 0$ & 4760.0 \\
\hline $\begin{array}{l}1955 \\
1956\end{array}$ & $\begin{array}{l}1070.0 \\
1230.0\end{array}$ & $\begin{array}{l}1090 \cdot 0 \\
1250.0\end{array}$ & $\begin{array}{l}1120.0 \\
1260.0\end{array}$ & $\begin{array}{l}1130.0 \\
1270.0\end{array}$ & $\begin{array}{l}1150.0 \\
1300.0\end{array}$ & $\begin{array}{l}1420.0 \\
1390.0\end{array}$ & $\begin{array}{l}1660.0 \\
1630.0\end{array}$ & $1820 \cdot 0$ & $2240 \cdot 0$ & 2670.0 & 3140.0 \\
\hline 1957 & 808.0 & 808.0 & 817.0 & 838.0 & 867.0 & 976.0 & 1200.0 & $1360 \cdot 0$ & 1660.0 & 1850.0 & 2730.0 \\
\hline 1958 & 952.0 & 955.0 & 979.0 & 1020.0 & 1030.0 & 1230.0 & 1320.0 & 1510.0 & 1650.0 & $1840 \cdot 0$ & 3320.0 \\
\hline 1959 & 970.0 & 970.0 & 979.0 & 983.0 & 992.0 & 1070.0 & 1320.0 & 1670.0 & 2080.0 & $2320 \cdot 0$ & 2710.0 \\
\hline 1960 & 1140.0 & 1160.0 & 1170.0 & 1170.0 & 1180.0 & 1230.0 & 1360.0 & 1520.0 & 1760.0 & 2390.0 & 3560.0 \\
\hline 1961 & 1120.0 & 1220.0 & 1240.0 & 1260.0 & 1280.0 & 1310.0 & 1410.0 & 1510.0 & 1780.0 & $2110 \cdot 0$ & $\begin{array}{l}3010.0 \\
3140.0\end{array}$ \\
\hline 1962 & 1190.0 & 1250.0 & 1260.0 & 1270.0 & 1290.0 & 1300.0 & 1410.0 & 1550.0 & 1910.0 & 2280.0 & 3140.0 \\
\hline
\end{tabular}

North Santiam River at Mehama, Oreg.

STATION NUMBEK

$14-1030 \cdot 00$

HIGHEST MEAN DISCHARGE. IN CFS, FOR THE FOLLOWING NUMBER OF CONSECUTIVE DAYS IN YEAR ENDING SEPTEMBER 30

\begin{tabular}{|c|c|c|c|c|c|c|c|c|c|c|c|}
\hline $\begin{array}{l}\text { YEAR } \\
1906 \\
1911\end{array}$ & $\begin{array}{c}1 \\
17100.0 \\
21700.0\end{array}$ & $\begin{array}{c}14100^{3} \cdot 0 \\
16400.0\end{array}$ & $\begin{array}{c}{ }^{7} \\
11300.0 \\
11500.0\end{array}$ & $\begin{array}{c}15 \\
8690.0 \\
10700.0\end{array}$ & $\begin{array}{c}30 \\
6440.0 \\
7830.0\end{array}$ & $\begin{array}{l}60 \\
5900.0 \\
5690.0\end{array}$ & $\begin{array}{c}90 \\
5170.0 \\
5080.0\end{array}$ & $\begin{array}{l}120 \\
4750.0 \\
4330.0\end{array}$ & $\begin{array}{l}150 \\
4570.0 \\
4380.0\end{array}$ & $\begin{array}{l}183 \\
4320.0 \\
4390.0\end{array}$ & $\begin{array}{c}274 \\
3830.0 \\
3760.0\end{array}$ \\
\hline 1912 & 37200.0 & 31000.0 & 21000.0 & 15100.0 & 12300.0 & 10500.0 & 8050.0 & 7470.0 & 6480.0 & 5940.0 & 5080.0 \\
\hline $\begin{array}{r}1913 \\
1914\end{array}$ & 30200.0 & $20600 \cdot n$ & 16500.0 & 11900.0 & 9010.0 & 7670.0 & 6840.0 & $6100 \cdot 0$ & 5790.0 & 5760.0 & 5410.0 \\
\hline $\begin{array}{l}1914 \\
1922\end{array}$ & 18400.0 & $12400 \cdot n$ & 10500.0 & $915 n .0$ & 7080.0 & 6160.0 & 5730.0 & $5470 \cdot 0$ & 5010.0 & 4660.0 & 3920.0 \\
\hline $\begin{array}{l}1922 \\
1973\end{array}$ & 50500.0 & $41300 \cdot n$ & 26400.0 & 22600.0 & 13800.0 & 8080.0 & 6250.0 & $530 n \cdot 0$ & 5180.0 & 5390.0 & 4670.0 \\
\hline $\begin{array}{l}1973 \\
1924\end{array}$ & $\begin{array}{l}58000.0 \\
19600.0\end{array}$ & $\begin{array}{l}44500.0 \\
14500.0\end{array}$ & $\begin{array}{l}27100 \cdot 0 \\
12700.0\end{array}$ & $\begin{array}{r}20800.0 \\
9710.0\end{array}$ & 15100.0 & $\begin{array}{l}8830.0 \\
5360.0\end{array}$ & $\begin{array}{l}7090.0 \\
5230.0\end{array}$ & $\begin{array}{l}6460.0 \\
4630.0\end{array}$ & $\begin{array}{l}6070.0 \\
4300.0\end{array}$ & $\begin{array}{l}5650.0 \\
3980.0\end{array}$ & $\begin{array}{l}4390.0 \\
3070.0\end{array}$ \\
\hline 1925 & 27500.0 & 23400.0 & 20400.0 & $\begin{array}{r}9710.0 \\
16000.0\end{array}$ & 11500.0 & 8840.0 & 7960.0 & 7440.0 & 6670.0 & 6270.0 & 5100.0 \\
\hline $\begin{array}{l}1926 \\
1927\end{array}$ & 30000.0 & $23200 \cdot 0$ & $15200 \cdot 0$ & $9800 \cdot 0$ & 8240.0 & 5830.0 & 5240.0 & $4620 \cdot 0$ & $4090 \cdot 0$ & 3680.0 & 2780.0 \\
\hline $\begin{array}{l}1927 \\
1928\end{array}$ & $33000 \cdot 0$ & 25900.0 & 15900.0 & 10200.0 & 7690.0 & 6610.0 & 6290.0 & $6180 \cdot 0$ & 5640.0 & 5420.0 & 4670.0 \\
\hline $\begin{array}{l}1928 \\
1929\end{array}$ & 33000.0 & $22800 \cdot 0$ & 18900.0 & 13700.0 & 9790.0 & 7570.0 & 6500.0 & $5860 \cdot 0$ & 5930.0 & 5810.0 & 4740.0 \\
\hline $\begin{array}{l}1929 \\
1930\end{array}$ & 13800.0 & 9880.0 & 7440.0 & 6400.0 & 6040.0 & 5320.0 & 4840.0 & $4390 \cdot 0$ & 3950.0 & 3840.0 & 3200.0 \\
\hline $\begin{array}{l}1930 \\
1931\end{array}$ & 19200.0 & 13800.0 & 11000.0 & 9360.0 & 7520.0 & 5160.0 & 4840.0 & $4320 \cdot 0$ & 3930.0 & 3700.0 & 2740.0 \\
\hline $\begin{array}{l}1931 \\
1932\end{array}$ & 43800.0 & 31500.0 & 18100.0 & 11700.0 & 8650.0 & 5770.0 & 4720.0 & 4170.0 & 3740.0 & 3430.0 & \\
\hline $\begin{array}{l}1932 \\
1933\end{array}$ & 30700.0 & $250 n 0 \cdot n$ & $16500 \cdot 0$ & $13200 \cdot 0$ & $\begin{array}{l}9840.0 \\
8960.0\end{array}$ & $\begin{array}{l}7960.0 \\
7450.0\end{array}$ & $\begin{array}{l}7240.0 \\
6640.0\end{array}$ & $\begin{array}{l}6460 \cdot 0 \\
6140 \cdot 0\end{array}$ & $\begin{array}{l}5750.0 \\
5570.0\end{array}$ & $\begin{array}{l}5560.0 \\
5460.0\end{array}$ & $\begin{array}{l}4490.0 \\
4910.0\end{array}$ \\
\hline $\begin{array}{l}1933 \\
1934\end{array}$ & $\begin{array}{l}24800.0 \\
41700.0\end{array}$ & $\begin{array}{l}17700.0 \\
27700.0\end{array}$ & 21000.0 & 14400.0 & 10900.0 & 8940.0 & 6790.0 & 6160.0 & 5450.0 & 4880.0 & 3730.0 \\
\hline $\begin{array}{l}1935 \\
1936\end{array}$ & 26300.0 & 18400.0 & 13200.0 & 8890.0 & 6550.0 & 6020.0 & 5230.0 & $5000 \cdot 0$ & 4700.0 & 4620.0 & $4040 . n$ \\
\hline 1936 & 30100.0 & 19500.0 & 14300.0 & 13600.0 & 9050.0 & 6090.0 & 5440.0 & $5260 \cdot 0$ & 5190.0 & 4730.0 & 3660.0 \\
\hline $\begin{array}{l}1937 \\
1938\end{array}$ & 30400.0 & 23900.0 & 15400.0 & 10300.0 & 8260.0 & 7080.0 & 6450.0 & $6130 \cdot 0$ & 5520.0 & 4750.0 & 3630.0 \\
\hline $\begin{array}{l}1938 \\
1939\end{array}$ & 27500.0 & $17800 \cdot 0$ & 12100.0 & 9410.0 & 8010.0 & 7020.0 & 6740.0 & 6030.0 & 5900.0 & 5990.0 & 4770.0 \\
\hline $\begin{array}{l}1939 \\
1940\end{array}$ & 14800.0 & $11700 \cdot 0$ & 9630.0 & 7430.0 & 6090.0 & 5010.0 & 4550.0 & $4210 \cdot 0$ & 4210.0 & $4000 \cdot 0$ & 3280.0 \\
\hline $\begin{array}{l}1940 \\
1941\end{array}$ & 17300.0 & $13800 \cdot n$ & 11300.0 & 8690.0 & 8110.0 & 6390.0 & 5300.0 & $5030 \cdot 0$ & 4620.0 & 4060.0 & 2970.0 \\
\hline $\begin{array}{l}1941 \\
1942\end{array}$ & 15200.0 & 10100.0 & 6930.0 & 5460.0 & 4170.0 & 3950.0 & 3730.0 & 3340.0 & 3010.0 & $2800 \cdot 0$ & 2460.0 \\
\hline $\begin{array}{l}1942 \\
1943\end{array}$ & 19000.0 & 14600.0 & 10900.0 & 7440.0 & 7120.0 & 5590.0 & 5140.0 & $4560 \cdot 0$ & 4130.0 & 3810.0 & 3280.0 \\
\hline $\begin{array}{l}1943 \\
1944\end{array}$ & 34100.0 & 25500.0 & 20700.0 & 15100.0 & 10900.0 & 10100.0 & 8550.0 & $\begin{array}{l}7530 \cdot 0 \\
2910 \cdot 0\end{array}$ & 7190.0 & $6800 \cdot 0$ & $\begin{array}{l}5500.0 \\
2570.0\end{array}$ \\
\hline $\begin{array}{l}1944 \\
1945\end{array}$ & $\begin{array}{l}11900 . ? \\
25900.0\end{array}$ & $\begin{array}{r}9380 \cdot n \\
17000 . \hat{n}\end{array}$ & $\begin{array}{r}6050.0 \\
14900.0\end{array}$ & $\begin{array}{r}5150 \cdot 0 \\
10300 . ?\end{array}$ & $\begin{array}{l}3760 . n \\
6690 . n\end{array}$ & $\begin{array}{l}3300.0 \\
5630.0\end{array}$ & $\begin{array}{l}3030.0 \\
5440.0\end{array}$ & & $\begin{array}{l}2780.0 \\
5260.0\end{array}$ & $\begin{array}{l}283 n \bullet 0 \\
4610.0\end{array}$ & 3520.0 \\
\hline 1946 & $58800 \cdot 0$ & 37300.0 & 2 200n.ก & 14900.0 & 10100.0 & 8110.0 & 6900.0 & 6330.0 & 5810.0 & 5590.0 & $4590 . n$ \\
\hline 1947 & $44000 \cdot 0$ & 39200.0 & 25800.0 & 14700.0 & 12700.0 & 8050.0 & 7060.0 & 6220.0 & 5760.0 & 5330.0 & 4260.0 \\
\hline 1948 & 45000.0 & 30500.0 & 19300.0 & 12500.0 & 8290.0 & 6320.0 & 6550.0 & $5570 \bullet 0$ & 5580.0 & 5190.0 & 4840.0 \\
\hline 1949 & 28500.0 & $20200 \cdot 0$ & 13700.0 & 11000.0 & 9200.0 & 7270.0 & 7330.0 & 6580.0 & 5690.0 & 5580.0 & 4610.0 \\
\hline 1950 & 31100.0 & 23800.0 & 14900.0 & 11500.0 & 9250.0 & 7760.0 & 7090.0 & 6710.0 & 6550.0 & 6000.0 & 4990.0 \\
\hline 1951 & 32200.0 & 21400.0 & 18300.0 & 11700.0 & 10200.0 & 8320.0 & 8060.0 & 8050.0 & 7170.0 & 6740.0 & 5480.0 \\
\hline 1952 & 18400.0 & 15300.0 & 13100.0 & 8870.0 & 6380.0 & 5680.0 & 5150.0 & 5070.0 & 4750.0 & 4800.0 & 4350.0 \\
\hline 1953 & 20700.0 & 17100.0 & 15500.0 & 15000.0 & 13900.0 & 10400.0 & 7790.0 & 6490.0 & 5830.0 & 5410.0 & 4170.0 \\
\hline 1954 & 21600.0 & 14700.0 & 13300.0 & 12400.0 & 11200.0 & 8900.0 & 7930.0 & $6960 \cdot 0$ & 6200.0 & 5630.0 & 4740.0 \\
\hline 1955 & 13300.0 & 11500.0 & 9970.0 & 7200.0 & 5560.0 & 4480.0 & 4600.0 & 4240.0 & $3800 \cdot 0$ & 3780.0 & 3680.0 \\
\hline 1956 & 17700.0 & $16000 \cdot 0$ & 14500.0 & 13600.0 & 12200.0 & 11600.0 & 10400.0 & $9160 \cdot 0$ & $7800 \cdot 0$ & 7030.0 & 6190.0 \\
\hline 1957 & 16200.0 & $12700 . n$ & $116 \cap 0 . n$ & 10300.0 & 6810.0 & 5980.0 & 5060.0 & 4590.0 & $464 n \cdot 0$ & 4520.0 & $3810 . n$ \\
\hline 1958 & 14500.0 & 14100.0 & 11400.0 & 9770.0 & 8630.0 & 7590.0 & 6860.0 & $5960 \cdot 0$ & 5400.0 & 5050.0 & 4140.0 \\
\hline 1959 & 13400.0 & 13100.0 & 12700.0 & 11700.0 & 9650.0 & 7390.0 & 7190.0 & 6150.0 & 5450.0 & 5070.0 & 4270.0 \\
\hline 1960 & 14400.0 & 12600.0 & 12200.0 & 8890.0 & 6390.0 & $60 n 0.0$ & 5300.0 & 4830.0 & 4370.0 & 3940.0 & 3940.0 \\
\hline 1961 & 23900.0 & 16100.0 & $1>400.0$ & 11700.0 & 9460.0 & 7320.0 & 6390.0 & $6710 \cdot 0$ & 6410.0 & 5830.0 & 4770.0 \\
\hline 1962 & 11800.0 & $10 \cap n n \cdot n$ & $94 n n . n$ & 8600.0 & 7460.0 & 6390.0 & 5580.0 & $4780 \cdot 0$ & 4330.0 & 4410.0 & 3900.0 \\
\hline 1963 & 17500.0 & 13300.0 & 12400.7 & 10900.0 & 9530.0 & $659 n$. & $568 \cap .0$ & 4850.0 & $449 n .0$ & $43 n \cap .0$ & 3810.0 \\
\hline
\end{tabular}


NORTH SANTIAM RIVER NEAR JEFFERSON OREGON

STATION NUMBER

$14-1841.00$

LOWEST

$\begin{array}{rrrrrrrrrrr}\text { YEAR } & 1 & 3 & 7 & 14 & 30 & 60 & 90 & 120 & 150 & 183 \\ 1955 & 739.0 & 745.0 & 782.0 & 795.0 & 828.0 & 1100.0 & 1360.0 & 2000.0 & - & - \\ 1956 & 842.0 & 842.0 & 857.0 & 866.0 & 896.0 & 980.0 & 1230.0 & 1430.0 & - & - \\ 1957 & 465.0 & 472.0 & 483.0 & 501.0 & 545.0 & 629.0 & 853.0 & 1020.0 & - & - \\ 1958 & 610.0 & 616.0 & 631.0 & 697.0 & 709.0 & 872.0 & 989.0 & 1190.0 & - & - \\ 1959 & 603.0 & 606.0 & 611.0 & 616.0 & 629.0 & 694.0 & 969.0 & 1360.0 & - & - \\ 1960 & 725.0 & 751.0 & 756.0 & 760.0 & 777.0 & 869.0 & 1020.0 & 1220.0 & - & - \\ 1961 & 805.0 & 842.0 & 854.0 & 860.0 & 866.0 & 897.0 & 1030.0 & 1140.0 & - & - \\ 1962 & 780.0 & 787.0 & 793.0 & 805.0 & 862.0 & 881.0 & 1010.0 & 1170.0 & - & - \\ & & & & & & & & & - & -\end{array}$

South Santiam River below Cascadia, Oreg.

STATION NUMRER 14-18b1.00

DURATION TABLF OF DAILY DISCHARGF

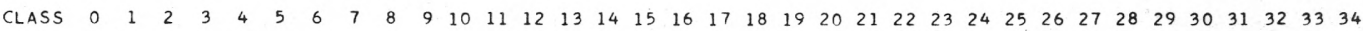

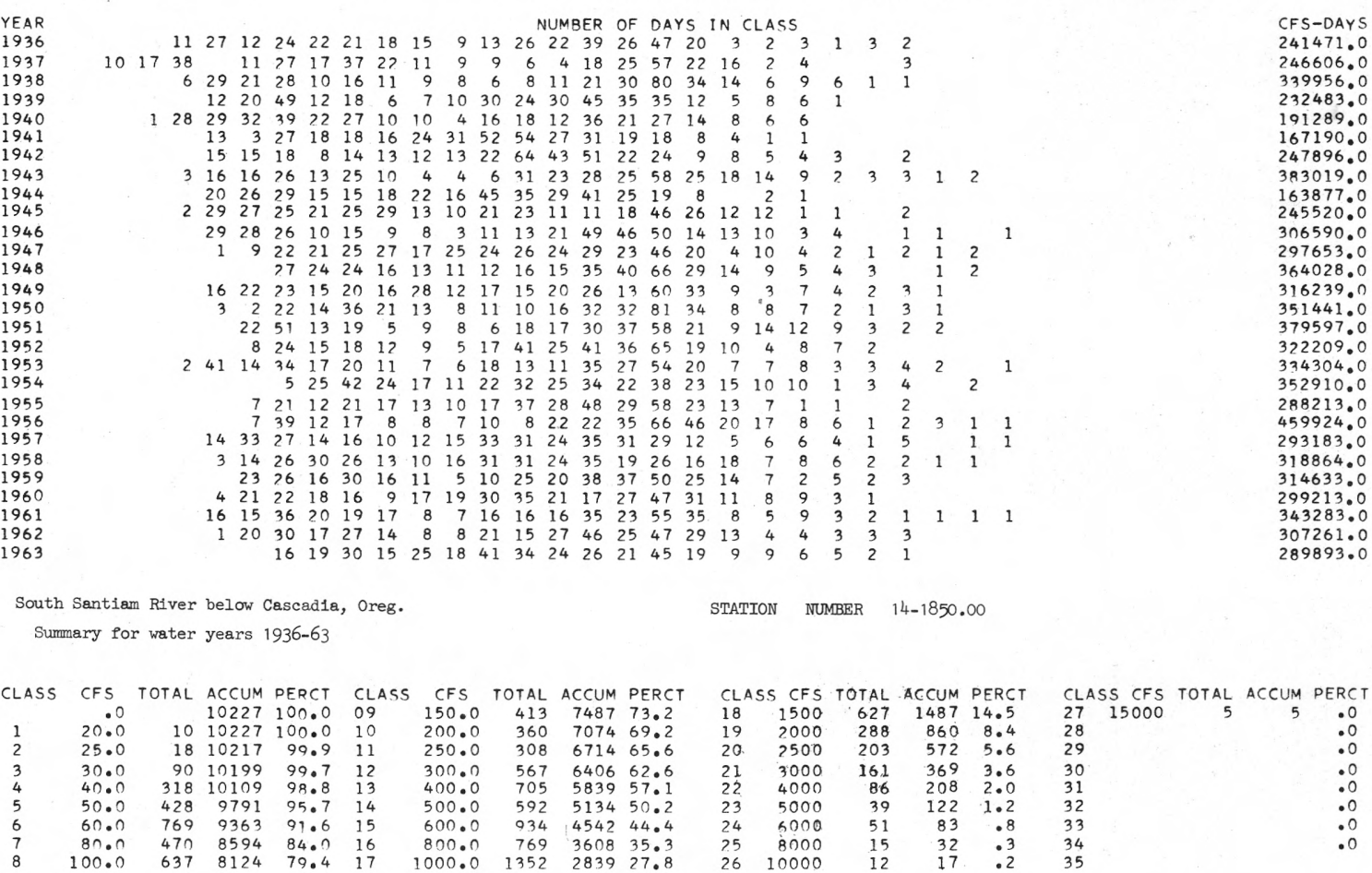


South Santiam River below Cascadia, Oreg. STATION NUMRER 14-1850.00

LOWEST MEAN DISCHARGE, IN CFS, FOR THE FOLLOWING NUMBFR OF CONSFCUTIVE DAYS IN YEAR BEGINNING APRIL 1

\begin{tabular}{|c|c|c|c|c|c|c|c|c|c|c|c|}
\hline YEAR & 1 & 3 & 7 & 14 & 30 & 60 & 90 & 120 & 150 & 183 & 274 \\
\hline 1936 & 23.0 & 23.7 & 23.9 & $24 \cdot 6$ & 26.7 & 29.9 & 37.4 & $42 \cdot 2$ & 55.6 & $90 \cdot 9$ & 219.0 \\
\hline 1937 & 51.0 & 51.7 & $54 \cdot 3$ & $60 \cdot 1$ & 65.8 & 74.9 & 86.7 & $152 \cdot 0$ & 329.0 & $504 \cdot 0$ & 875.0 \\
\hline 1938 & 38.0 & 39.0 & 39.4 & 39.9 & 44.7 & 47.4 & 52.5 & $62 \cdot 3$ & 103.0 & 209.0 & 446.0 \\
\hline 1939 & $51 \cdot 0$ & $51 \cdot ?$ & 51.7 & 53.1 & 58.7 & 64.7 & 71.7 & $89 \cdot 7$ & 92.7 & $147 \cdot 0$ & 327.0 \\
\hline 1940 & 29.0 & 30.2 & 31.4 & 32.7 & 36.5 & 41.7 & 45.7 & $51 \cdot 2$ & 66.0 & 116.0 & 339.0 \\
\hline 1941 & $61 \cdot 0$ & 62.3 & $64 \cdot 1$ & 67.9 & 74.7 & 106.0 & 156.0 & $201 \cdot 0$ & 240.0 & 288.0 & 580.0 \\
\hline 1942 & 39.0 & 39.0 & 39.9 & 40.9 & 44.0 & 49.8 & 61.8 & 93.1 & 176.0 & 289.0 & 794.0 \\
\hline 1943 & 48.0 & $48 . ?$ & 49.0 & 52.1 & 54.0 & 68.3 & 83.0 & $156 \cdot 0$ & 262.0 & 300.0 & 392.0 \\
\hline 1944 & 38.0 & $39 \cdot 0$ & $41 \cdot 6$ & $44 \cdot 6$ & $49 \cdot 6$ & 59.9 & 63.7 & 71.4 & 103.0 & 137.0 & 281.0 \\
\hline 1945 & 41.0 & $41 \cdot ?$ & 41.4 & 42.5 & $52 \cdot 6$ & 60.7 & 61.1 & $70 \cdot 0$ & 99.1 & 254.0 & 742.0 \\
\hline 1946 & 45.0 & $45 \cdot 7$ & 47.7 & 51.9 & 53.6 & 60.1 & 66.3 & $113 \cdot 0$ & 209.0 & 299.0 & 712.0 \\
\hline 1947 & $66 \cdot 0$ & $67 \cdot 0$ & $68 \cdot 3$ & $75 \cdot 8$ & $94 \cdot 1$ & 99.3 & 126.0 & $197 \bullet 0$ & 238.0 & 325.0 & 687.0 \\
\hline 1948 & 60.0 & $61 . n$ & 63.7 & $64 \cdot 1$ & 73.8 & 85.3 & $123 \cdot 0$ & $178 \cdot 0$ & 234.0 & 382.0 & 630.0 \\
\hline 1949 & 47.0 & $47 \cdot 0$ & 47.4 & 48.5 & 51.7 & 59.6 & 70.5 & $111 \cdot 0$ & 131.0 & 191.0 & 520.0 \\
\hline 1950 & 58.0 & 59.3 & 62.9 & 63.9 & 72.7 & 96.6 & 146.0 & $271 \cdot 0$ & 459.0 & 605.0 & 1060.0 \\
\hline 1951 & 54.0 & 54.0 & 54.1 & 56.6 & 60.1 & 61.7 & 70.0 & 99.2 & 183.0 & 303.0 & 610.0 \\
\hline 1952 & 37.0 & 39.0 & 40.3 & $41 \cdot 8$ & 42.8 & 49.7 & 57.5 & $65 \cdot 7$ & 93.4 & $182 \cdot 0$ & 436.0 \\
\hline 1953 & 57.0 & $58 \cdot 2$ & $59 \cdot 6$ & $61 . ?$ & 73.0 & 88.0 & $104 \cdot 0$ & $131 \cdot 0$ & $18 ? \cdot 0$ & $365 \cdot 0$ & 907.0 \\
\hline 1954 & 76.0 & 76.7 & 79.9 & 89.1 & 99.5 & 112.0 & 150.0 & $190 \cdot 0$ & 236.0 & 307.0 & 480.0 \\
\hline 1955 & 55.0 & $55 . ?$ & 55.0 & 58.9 & 69.5 & 93.9 & 157.0 & 287.0 & 454.0 & 634.0 & 1230.0 \\
\hline 1956 & 49.0 & $50 \cdot ?$ & $51 \cdot 3$ & 53.7 & $58 \cdot 7$ & 64.9 & 79.3 & 133.0 & 291.0 & 371.0 & 622.0 \\
\hline 1957 & 42.0 & 43.3 & $46 \cdot 1$ & 47.2 & 50.2 & 61.4 & 77.3 & $99 \cdot 7$ & $128 \cdot 0$ & 198.0 & 594.0 \\
\hline 1958 & 47.0 & 48.3 & 49.3 & 54.2 & 58.1 & 68.8 & 77.5 & 99.6 & 158.0 & 214.0 & 635.0 \\
\hline 1959 & 52.0 & 53.3 & 56.0 & 56.6 & 60.5 & 86.7 & 118.0 & $196 \cdot 0$ & 304.0 & 362.0 & 483.0 \\
\hline 1960 & 42.0 & 43.3 & 44.6 & 47.9 & 56.5 & 69.5 & 78.4 & 94.0 & 150.0 & 354.0 & 697.0 \\
\hline 1961 & 41.0 & 43.3 & 44.4 & 46.3 & 54.0 & $75 \cdot 8$ & 78.0 & $107 \cdot 0$ & $170 \cdot 0$ & 261.0 & 658.0 \\
\hline 1962 & 48.0 & 50.3 & 52.7 & 56.3 & 66.6 & $82 . ?$ & 90.8 & $131 \cdot 0$ & 218.0 & 312.0 & 546.0 \\
\hline
\end{tabular}

South Santiam River below Cascadia, Oreg. STATION NUMBER 14-1850.00

HIGHEST MEAN DISCHARGF, IN CFS, FOR THE FOLLOWING NUMBFR OF CONSECUTIVE DAYS IN YEAR ENDING SEPTFMBER 30

\begin{tabular}{|c|c|c|c|c|c|c|c|c|c|c|c|}
\hline YEAR & 1 & 3 & 7 & 15 & 30 & 60 & 90 & 120 & 150 & 183 & 274 \\
\hline 1936 & 7360.0 & 5730.0 & 4020.0 & 3680.0 & 2440.0 & 1680.0 & 1450.0 & $1360 \cdot 0$ & 1290.0 & 1150.0 & 859.0 \\
\hline 1937 & 7940.0 & 6860.0 & 4420.0 & 2840.0 & 2090.0 & 1830.0 & 1720.0 & 1610.0 & 1450.0 & 1230.0 & 887.0 \\
\hline 1938 & 7360.0 & $5330 . n$ & 3220.0 & 2410.0 & 1950.0 & 1830.0 & 1740.0 & 1610.0 & 1600.0 & 1600.0 & 1220.0 \\
\hline 1939 & 4980.0 & 3540.0 & 2920.0 & 2340.0 & 1750.0 & 1490.0 & 1270.0 & 1190.0 & 1140.0 & 1060.0 & 827.0 \\
\hline 1940 & 3880.0 & 3460.0 & 3010.0 & 2260.0 & 2160.0 & 1650.0 & 1320.0 & 1240.0 & 1120.0 & 968.0 & 681.0 \\
\hline 1941 & 3850.0 & 2580.0 & 1740.0 & 1410.0 & 1050.0 & 1010.0 & 934.0 & $808 \cdot 0$ & 709.0 & 663.0 & 566.0 \\
\hline 1942 & 7200.0 & 4940.0 & 3390.0 & 2240.0 & 1990.0 & 1660.0 & 1470.0 & 1280.0 & 1140.0 & 1040.0 & 871.0 \\
\hline 1943 & 10900.0 & $8820 . n$ & 6370.0 & 4200.0 & 3140.0 & 2960.0 & 2470.0 & 2120.0 & $194 n .0$ & 1780.0 & $1370 . n$ \\
\hline 1944 & 3880.0 & 2820.0 & 1680.0 & 1270.0 & 950.0 & 890.0 & 820.0 & 749.0 & 690.0 & 662.0 & 576.0 \\
\hline 1945 & 7210.0 & 4650.0 & $4110 . ?$ & 2950.0 & 1820.0 & 1540 ? & 1510.0 & $1470 . n$ & 1410.0 & $1210 . n$ & 875.0 \\
\hline 1946 & 16700.0 & $10200 . n$ & 5730.0 & 4020.0 & 2710.0 & 2070.0 & 1810.0 & $1700 \cdot 0$ & 1550.0 & 1440.0 & 1100.0 \\
\hline 1947 & 11600.0 & $10400 . n$ & 6790.0 & 3770.0 & 3320.0 & 2040.0 & $18 ? 0.0$ & 1580.0 & 1500.0 & 1370.0 & 1050.0 \\
\hline 1948 & 12600.0 & 9230.0 & 5850.0 & 3580.0 & 2320.0 & 1750.0 & 1780.0 & 1510.0 & 1500.0 & 1420.0 & 1290.0 \\
\hline 1949 & 8600.0 & 6370.0 & 4810.0 & 3240.0 & 2410.0 & 1800.0 & 1850.0 & 1560.0 & 1460.0 & 1480.0 & 1130.0 \\
\hline 1950 & 8180.0 & $6440 \cdot 0$ & $3930 . n$ & 3080.0 & 2540.0 & 2230.0 & 1990.0 & $1800 \cdot 0$ & 1710.0 & 1560.0 & 1240.0 \\
\hline 1951 & 9710.0 & $7000 \cdot 0$ & 6090.0 & 3610.0 & 2950.0 & 2300.0 & 2260.0 & $2200 \cdot 0$ & 1950.0 & 1780.0 & 1360.0 \\
\hline 1952 & 5800.0 & 4870.0 & $\begin{array}{l}4020.0 \\
6990.0\end{array}$ & $\begin{array}{l}2620.0 \\
5010.0\end{array}$ & $\begin{array}{l}1910.0 \\
4420.0\end{array}$ & $\begin{array}{l}1430.0 \\
2850.0\end{array}$ & $\begin{array}{l}1450.0 \\
2280.0\end{array}$ & $\begin{array}{l}1350 \cdot 0 \\
1970.0\end{array}$ & $\begin{array}{l}1330.0 \\
1840.0\end{array}$ & $\begin{array}{l}1280.0 \\
1660.0\end{array}$ & 1120.0 \\
\hline $\begin{array}{l}1953 \\
1954\end{array}$ & $\begin{array}{l}15600.0 \\
17800.0\end{array}$ & $\begin{array}{r}10300.0 \\
9560.0\end{array}$ & 5160.0 & 3870.0 & $\begin{array}{l}4420.0 \\
3760.0\end{array}$ & 2470.0 & $\begin{array}{l}2280.0 \\
2390.0\end{array}$ & $\begin{array}{l}1970 \cdot 0 \\
2080.0\end{array}$ & $\begin{array}{l}1840.0 \\
1890.0\end{array}$ & $\begin{array}{l}1660.0 \\
1660.0\end{array}$ & $\begin{array}{l}1200.0 \\
1240.0\end{array}$ \\
\hline 1955 & 7490.0 & 6100.0 & 3620.0 & 2080.0 & 1690.0 & 1560.0 & 1500.0 & 1300.0 & 1250.0 & 1240.0 & 1010.0 \\
\hline 1956 & 18400.0 & 13200.0 & 7730.0 & 5380.0 & 4000.0 & 3230.0 & 2750.0 & 2270.0 & $209 n .0$ & $2000 \cdot 0$ & 1640.0 \\
\hline 1957 & 16200.0 & $10300 \cdot n$ & 6460.0 & 4080.0 & 2850.0 & 2100.0 & 1660.0 & $1660 \cdot n$ & 1470.0 & 1360.0 & $105 n \cdot n$ \\
\hline 1958 & 14800.0 & 9510.0 & 5680.0 & 4080.0 & 2860.0 & 2560.0 & 27.50 .0 & $1840 \cdot 0$ & 1740.0 & 1540.0 & 1130.0 \\
\hline 1959 & 7640.0 & 5250.0 & 3540.0 & 2730.0 & 2190.0 & 1780.0 & 1790.0 & 1610.0 & 1530.0 & 1430.0 & 1100.0 \\
\hline 1960 & 5490.0 & 4130.0 & 3210.0 & 2380.0 & 2140.0 & 1830.0 & 1710.0 & 1640.0 & 1450.0 & 1260.0 & 1060.0 \\
\hline 1961 & 16600.0 & 10600.0 & 6640.0 & 4560.0 & 3240.0 & 2460.0 & 1950.0 & $1890 \cdot 0$ & 1790.0 & 1650.0 & 1230.0 \\
\hline 1962 & 6520.0 & 5700.0 & 3970.0 & 2740.0 & 2250.0 & 1860.0 & 1480.0 & $1370 \cdot 0$ & 1440.0 & 1390.0 & 1090.0 \\
\hline 1963 & 6330.0 & 4960.0 & 3600.0 & 2480.0 & 1850.0 & 1570.0 & 1380.0 & 1370.0 & 1210.0 & 1260.0 & 1010.0 \\
\hline
\end{tabular}


CLASS $0 \begin{array}{llllllllllllllllllllllllllllllllllll} & 0 & 2 & 3 & 4 & 5 & 6 & 7 & 8 & 9 & 10 & 11 & 12 & 13 & 14 & 15 & 16 & 17 & 18 & 19 & 20 & 21 & 22 & 23 & 24 & 25 & 26 & 27 & 28 & 29 & 30 & 31 & 32 & 33 & 34\end{array}$

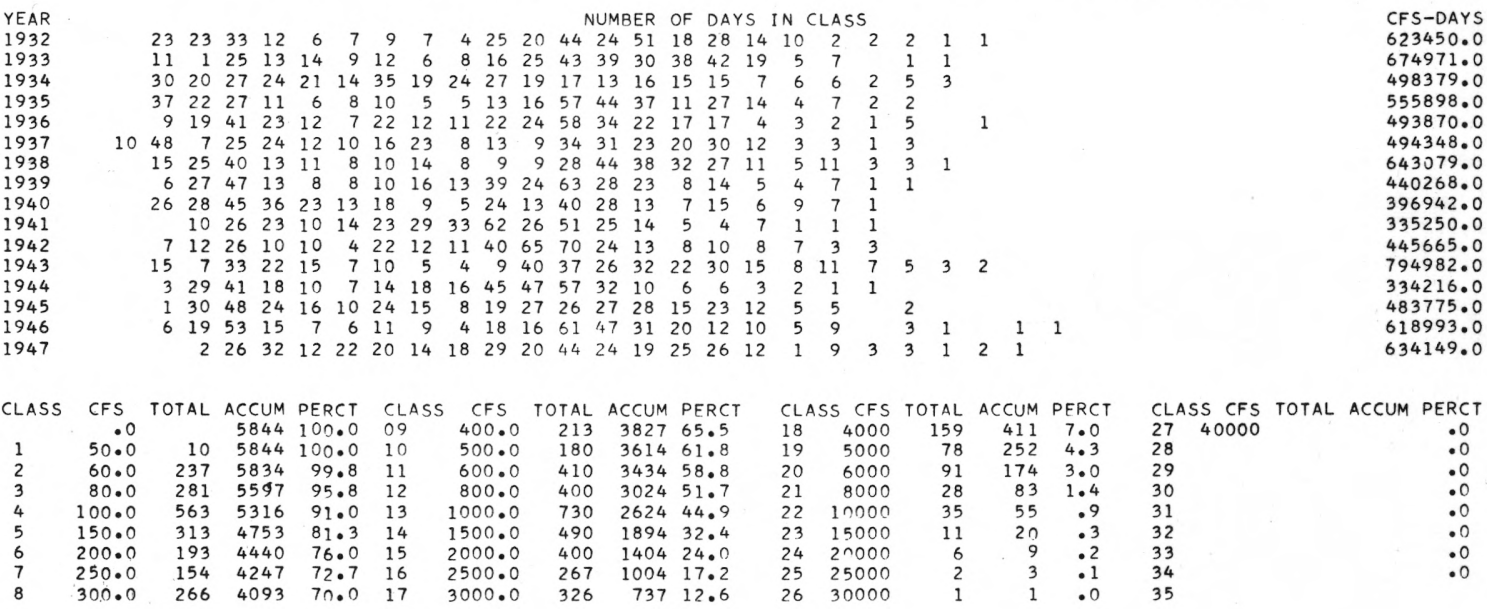

Middle Santiam River near Foster, Oreg.

STATION NUMBER $\quad 14-1860.00$

LOWEST MEAN DISCHARGE, IN CFS, FOR THE FOLLOWING NUMRER OF CONSECUTIVE DAYS IN YEAR BEGINNING APRIL 1

\begin{tabular}{|c|c|c|c|c|c|c|c|c|c|c|c|}
\hline YEAR & 1 & 3 & 7 & 14 & 30 & 60 & on & 120 & 150 & 183 & 274 \\
\hline 1932 & 62.0 & 62.7 & 63.3 & 67.3 & 75.9 & 91.3 & 119.0 & 188.0 & 336.0 & 618.0 & 1170.0 \\
\hline 1933 & 111.0 & $113 . n$ & $125 . n$ & $128 . ?$ & 137.0 & 192.0 & 266.0 & $365 . ?$ & 476.0 & 830.0 & 1640.0 \\
\hline 1934 & 64.0 & 65.0 & 68.4 & 69.8 & 72.5 & $79 . \cap$ & 87.0 & 107.0 & 149.0 & 241.0 & 1090.0 \\
\hline 1935 & 64.0 & 65.7 & 67.4 & 70.4 & 82.1 & 86.1 & $1 \cap 4.0$ & 149.0 & 194.0 & 295.0 & 716.0 \\
\hline 1936 & 57.0 & 57.3 & 57.7 & 59.1 & 63.3 & 67.6 & 82.9 & 91.4 & 118.0 & 202.0 & 486.0 \\
\hline 1937 & 107.0 & $108 . ?$ & $113 \cdot 0$ & 128.0 & 136.0 & $159 . ?$ & 188.0 & 316.0 & 628.0 & 931.0 & 1670.0 \\
\hline 1938 & 73.0 & 75.3 & 76.4 & 78.2 & 85.8 & 93.6 & 105.0 & 122.0 & 189.0 & 402.0 & 862.0 \\
\hline 1939 & 77.0 & 77.0 & 77.4 & 79.9 & 91.4 & 107.0 & 134.0 & 176.0 & 199.0 & 274.0 & 665.0 \\
\hline 1940 & 66.0 & $67 . n$ & 68.6 & 69.5 & 75.1 & 86.7 & $\ln \cdot n^{\circ}$ & 113.0 & 141.0 & 245.0 & 687.0 \\
\hline 1941 & 113.0 & 115.0 & $117 . ?$ & $124 . ?$ & 143.0 & 199.0 & 325.0 & 39000 & 434.0 & 520.0 & 1020.0 \\
\hline 1942 & 72.0 & 72.3 & 73.9 & 75.4 & 81.5 & $92 . \circ$ & 119.0 & 183.0 & 331.0 & 494.0 & 1590.0 \\
\hline 1943 & 91.0 & 91.7 & 92.6 & 07.7 & 103.0 & 130.0 & 159.0 & 277.0 & 490.0 & 667.0 & 829.0 \\
\hline 1944 & 76.0 & 77.7 & 87.1 & 89.5 & 93.7 & 116.0 & 125.0 & 141.0 & 210.0 & 300.0 & 562.0 \\
\hline 1945 & 77.0 & 77.7 & 79.1 & $81 \cdot 1$ & 99.0 & $12^{2} \cdot \cap$ & 121.0 & 136.0 & $19 n .0$ & 443.0 & 1520.0 \\
\hline 1946 & $95 . ?$ & 95.7 & 98.4 & $106 . ?$ & $111 \cdot 0$ & 122.1 & 136.0 & $221 \cdot 0$ & 368.0 & 574.0 & 1440.0 \\
\hline
\end{tabular}

Middle Santiam River near Foster, Oreg.

STATION NUMBER

$14-1860.00$

HIGHEST MEAN DISCHARGE, IN CFS, FOR THF. FOLLOWING NUMBFR OF CONSECUTIVE DAYS IN YFAR ENDING SEPTEMBER 30

\begin{tabular}{|c|c|c|c|c|c|}
\hline YEAR & 1 & 3 & 7 & 15 & 30 \\
\hline 1932 & 22600.0 & $18400 \cdot n$ & 11300.0 & $8630 . ?$ & 6160.0 \\
\hline 1933 & 16500.0 & $11700 . n$ & 7840.0 & 5250.0 & 4310.0 \\
\hline 1934 & 19700.0 & 15300.0 & 12400.0 & 8240.0 & 6150.0 \\
\hline 1935 & 13000.0 & $9330 . n$ & 7290.0 & 4950.0 & 3650.0 \\
\hline 1936 & 20500.0 & 13700.0 & 9480.0 & 8720.0 & 5600.0 \\
\hline 1937 & 14000.0 & 11700.0 & 7770.0 & 5320.0 & 4080.0 \\
\hline 1938 & $17200 \cdot 0$ & 11500.0 & 6770.0 & 4810.0 & 4190.0 \\
\hline 1939 & 11900.0 & $7560 . n$ & 5820.0 & 4380.0 & 3340.0 \\
\hline 1940 & 9520.0 & $7330 . n$ & 6360.0 & 4900.0 & 4560.0 \\
\hline 1941 & 9970.0 & 5870.0 & 3930.0 & 3260.7 & 2360.0 \\
\hline 1942 & 9560.0 & 7330.0 & 5510.0 & 3770.0 & 3530.0 \\
\hline 1943 & 23400.0 & $17700 \cdot n$ & $13000 \cdot ?$ & $9060 . n$ & 6530.0 \\
\hline 1944 & 8600.0 & 5640.0 & 3330.0 & 2670.0 & 1930.0 \\
\hline 1945 & 14900.0 & 9230.0 & 8340.0 & 5750.0 & 3600.0 \\
\hline 1946 & 35900.0 & 23600.0 & 13100.0 & 8580.0 & 5770.0 \\
\hline 1947 & 25200.0 & 23000.0 & 14900.0 & 8240.0 & 7030.0 \\
\hline
\end{tabular}

$\begin{array}{lllll}25200.0 & 23600.0 & 13100.0 & 8580.0 & 5770.0 \\ & 23000 \cdot 0 & 14900.0 & 8240.0 & 7030.0\end{array}$

60
$4580 \cdot 0$
$3570 \cdot 0$
$5040 \cdot 0$
$3390 \cdot 0$
$3640 \cdot 0$
$3530 \cdot 0$
$3470 \cdot 0$
$2810 \cdot 0$
$3320 \cdot 0$
$2150 \cdot 0$
$2940 \cdot 0$
6180.0
$1640 \cdot 0$
$3050 \cdot 0$
$4500 \cdot 0$
4320.0

3770.0
3220.0

3220.0

2950.0

3110.0

3330.0
3400.0

$3400 \cdot 0$
2370.0

2830.0

1980.0

2660.0
5190.0

1560.0

1560.0
2870.0

3840.0
3900.0

120
$3270 \cdot 0$
$3040 \cdot 0$
$3270 \cdot 0$
$2810 \cdot 0$
$2850 \cdot 0$
$3060 \cdot 0$
$3120 \cdot 0$
$2310 \cdot 0$
$2600 \cdot 0$
$1710 \cdot 0$
$2350 \cdot 0$
$4500 \cdot 0$
$1440 \cdot 0$
$2820 \cdot 0$
$3560 \cdot 0$
$3440 \cdot 0$

150
$3110 \cdot 0$
$2690 \cdot 0$
$2810 \cdot 0$
$2630 \cdot 0$
$2650 \cdot 0$
$2800 \cdot 0$
$3090 \cdot 0$
$2210 \cdot 0$
$2310 \cdot 0$
$1510 \cdot 0$
$2090 \cdot 0$
$4130 \cdot 0$
$1360 \cdot 0$
$2730 \cdot 0$
$3200 \cdot 0$
$3250 \cdot 0$

\begin{tabular}{|c|c|}
\hline 183 & 274 \\
\hline $2840 \cdot 0$ & 2240.0 \\
\hline 2760.0 & 2380.0 \\
\hline 2480.0 & 1780.0 \\
\hline 2530.0 & 2000.0 \\
\hline 2350.0 & 1760.0 \\
\hline 2400.0 & 1780.0 \\
\hline 3070.0 & 2310.0 \\
\hline 2070.0 & 1570.0 \\
\hline 1990.0 & 1410.0 \\
\hline 1350.0 & 1130.0 \\
\hline 890.0 & 1560.0 \\
\hline 3800.0 & 2860.0 \\
\hline 1380.0 & 1180.0 \\
\hline 340.0 & 1720.0 \\
\hline 2970.0 & 2220.0 \\
\hline
\end{tabular}


Middle Santiam River at mouth, near Foster, Oreg.
STATION NUMBER $14-1865.00$

DURATION TABLF OF DAILY DISCHARGE

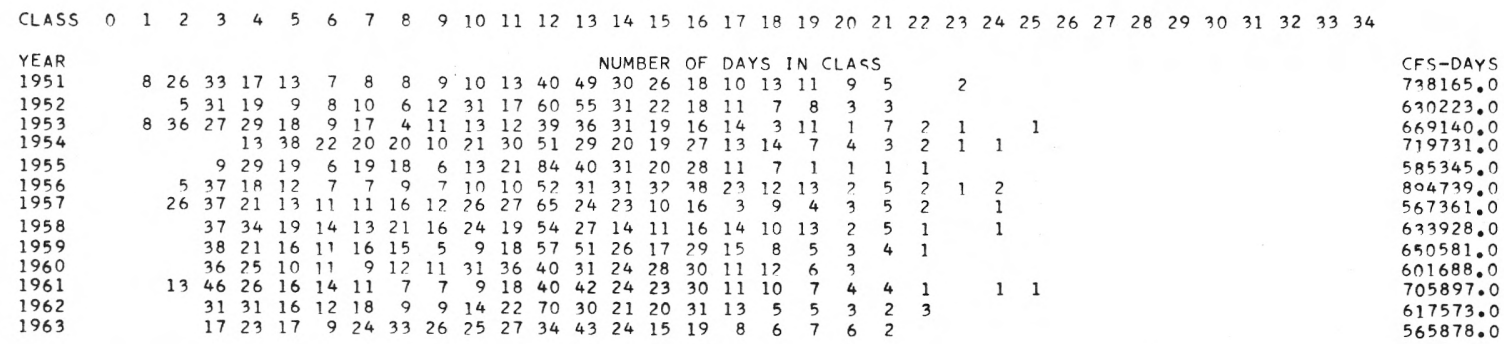

Middle Santiam River at mouth near

STATION NUMBER $\quad 14-1865.00$

Summary for water years 1951-63

CLASS CFS TOTAL ACCUM DFRCT CLACS

ACCUM PERCT $\begin{array}{rrrrr}.0 & & 4748 & 100.0 & 09 \\ 60.0 & 16 & 4748 & 10 n .0 & 10 \\ 80.0 & 111 & 4732 & 99.7 & 11 \\ 100.0 & 379 & 4621 & 97.3 & 12 \\ 150.0 & 306 & 4242 & 89.3 & 13 \\ 200.0 & 216 & 3936 & 87.9 & 14 \\ 250.0 & 141 & 3720 & 78.3 & 15 \\ 300.0 & 183 & 3579 & 75.4 & 16\end{array}$
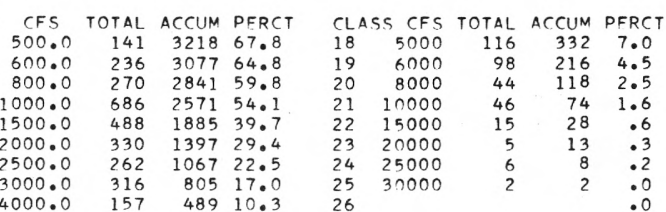

$\begin{array}{lc}\text { CLASS CFS TOTAL ACCUM PERCT } \\ 27 & .0 \\ 28 & .0 \\ 29 & .0 \\ 30 & .0 \\ 31 & .0 \\ 32 & .0 \\ 33 & .0 \\ 34 & .0\end{array}$

Middle Santiam River at mouth, near Foster, Oreg.

STATION NUMBER $\quad 14-1865.00$

LOWEST MEAN DISCHARGF, IN CFS, FOR THF FOLLOWING NUMRER OF CONSECUTIVF DAYS IN YFAR REGINNING APRIL 1

\begin{tabular}{|c|c|c|c|c|c|c|c|c|c|c|c|}
\hline YEAR & 1 & 3 & 7 & 14 & 30 & 60 & 90 & 120 & 150 & 183 & 274 \\
\hline 1951 & 72.0 & 72.3 & 73.7 & 78.6 & 90.7 & 100.0 & 126.0 & 185.0 & 379.0 & 622.0 & 1230.0 \\
\hline 1952 & 77.0 & 77.0 & 77.6 & 80.1 & 84.7 & 93.8 & 112.0 & $124 \cdot 0$ & 166.0 & 303.0 & 814.0 \\
\hline 1953 & 115.0 & 116.0 & 120.0 & 125.0 & 151.0 & 193.0 & 231.0 & 285.0 & 360.0 & 678.0 & 1750.0 \\
\hline 1954 & 176.0 & 178.0 & 182.0 & 197.0 & 214.0 & 250.0 & 293.0 & 381.0 & 478.0 & 603.0 & 1010.0 \\
\hline 1955 & 132.0 & 134.0 & 136.0 & 145.0 & 163.0 & 213.0 & 321.0 & 603.0 & 964.0 & 1300.0 & 2440.0 \\
\hline 1956 & 87.0 & 87.7 & 90.3 & $94 \cdot 3$ & 104.0 & 117.0 & 145.0 & 224.0 & 450.0 & 641.0 & 1160.0 \\
\hline 1957 & 86.0 & 86.7 & 89.0 & 91.1 & 98.8 & 129.0 & 156.0 & 206.0 & 254.0 & 358.0 & 1170.0 \\
\hline 1958 & 101.0 & 102.0 & 103.0 & 111.0 & 118.0 & 140.0 & 155.0 & 196.0 & 285.0 & 381.0 & 1340.0 \\
\hline 1959 & 111.0 & 116.0 & 121.0 & 122.0 & 134.0 & 194.0 & 285.0 & $440 \cdot 0$ & 622.0 & 774.0 & 1030.0 \\
\hline 1960 & 96.0 & 97.0 & 99.4 & 106.0 & 125.0 & 161.0 & 174.0 & 208.0 & 348.0 & 735.0 & 1480.0 \\
\hline 1961 & 94.0 & 95.7 & 96.9 & 99.6 & 110.0 & 145.0 & 157.0 & 197.0 & 303.0 & 464.0 & 1310.0 \\
\hline 1962 & 105.0 & $108 \cdot 0$ & 111.0 & 118.0 & 141.0 & 183.0 & 207.0 & 281.0 & 485.0 & 641.0 & 1180.0 \\
\hline
\end{tabular}

Middle Santiam River at mouth,

near Foster, Oreg.

STATION NUMBER $14-1865.00$

HIGHEST MEAN DISCHARGE, IN CFS, FOR THE FOLLOWING NUMBER OF CONSECUTIVE DAYS IN YEAR ENDING SEPTEMBER 30

\begin{tabular}{|c|c|c|c|c|c|c|c|c|c|c|c|}
\hline YEAR & 1 & 3 & 7 & 15 & 30 & 60 & 90 & 120 & 150 & 183 & 274 \\
\hline & 20000.0 & 14300.0 & 12100.0 & 7160.0 & 5790.0 & 4470.0 & 4410.0 & $4300 \cdot 0$ & $378 n .0$ & 3470.0 & 2650.0 \\
\hline 1952 & 12300.0 & $102 n 0 \cdot n$ & $8600 . n$ & $56 ? 0.0$ & 3690.0 & 2900.0 & 2910.0 & 2760.0 & 2650.0 & $2590 . n$ & 2210.0 \\
\hline 1953 & 31800.0 & 23900.0 & 16200.0 & 11100.0 & 9330.0 & 6010.0 & $47 \cap 0.0$ & $40 \cap 0 \cdot 0$ & 3690.0 & $334 n \cdot n$ & 2400.0 \\
\hline 1954 & 29100.0 & $19300 . n$ & $1 \cap 6 \cap 0 \cdot n$ & 7590.0 & 7500.0 & 5020.0 & 4740.0 & $4>40 \cdot 0$ & 3850.0 & 3380.0 & 2530.0 \\
\hline 1955 & 16400.0 & $12900 . n$ & 7570.0 & 4380.0 & 3330.0 & 3120.0 & 2950.0 & 2540.0 & 2530.0 & 2490.0 & 2040.0 \\
\hline 1956 & 26600.0 & 21300.0 & 12400.0 & 9620.0 & 7730.0 & 6350.0 & 5470.0 & 4560.0 & $408 \cap .0$ & 3920.0 & 3200.0 \\
\hline 1957 & $28600 \cdot 0$ & $18700 \cdot 0$ & $11800 \cdot 0$ & 7950.0 & 5620.0 & 4140.0 & 3300.0 & 3270.0 & 2910.0 & 2680.0 & 2030.0 \\
\hline $\begin{array}{l}1958 \\
1959\end{array}$ & $\begin{array}{r}25500.0 \\
15900.0\end{array}$ & 17400.0 & 10800.0 & 7820.0 & 5630.0 & 4950.0 & 4400.0 & $3670 \cdot 0$ & $3480 \cdot 0$ & 3110.0 & 2250.0 \\
\hline $\begin{array}{l}59 \\
590\end{array}$ & $\begin{array}{r}15900.0 \\
8960.0\end{array}$ & $\begin{array}{r}12300.0 \\
8140.0\end{array}$ & $\begin{array}{l}7490.0 \\
5980.0\end{array}$ & $6010 \cdot 0$ & 4290.0 & 3560.0 & 3740.0 & $3300 \cdot 0$ & 3170.0 & 2960.0 & 2260.0 \\
\hline 61 & $\begin{array}{r}8960.0 \\
30600.0\end{array}$ & $\begin{array}{r}8140.0 \\
20500.0\end{array}$ & $\begin{array}{r}5980.0 \\
12900.0\end{array}$ & 4630.0 & 4030.0 & 3560.0 & 3280.0 & $3200 \cdot 0$ & 2840.0 & 2530.0 & 2130.0 \\
\hline 62 & 16500.0 & 14800.0 & $\begin{array}{r}12900 . n \\
9330.0\end{array}$ & 9050.0 & 6340.0 & 4980.0 & $4080 \cdot 0$ & $3950 \cdot 0$ & $3760 \cdot 0$ & 3410.0 & 2520.0 \\
\hline 163 & 13300.0 & 10400.0 & 7070.0 & 5950.0 & $\begin{array}{l}47800 \\
4280.0\end{array}$ & $\begin{array}{l}3890.0 \\
2840.0\end{array}$ & $\begin{array}{l}3090.0 \\
2570.0\end{array}$ & $2860 \cdot 0$ & $\begin{array}{l}2970.0 \\
2430 \cdot 0\end{array}$ & $\begin{array}{l}2840.0 \\
2470.0\end{array}$ & 2190.0 \\
\hline
\end{tabular}


CLASS $0 \begin{array}{lllllllllllllllllllllllllllllllllllll} & 1 & 2 & 3 & 4 & 5 & 6 & 7 & 8 & 9 & 10 & 11 & 12 & 13 & 14 & 15 & 16 & 17 & 18 & 19 & 20 & 21 & 22 & 23 & 24 & 25 & 26 & 27 & 28 & 29 & 30 & 31 & 32 & 33 & 34\end{array}$

YEAR
1948
1949
1950
1951
1952
1953
1954
1955
1956
1957
1958
1959
1960
1961
1962
1963

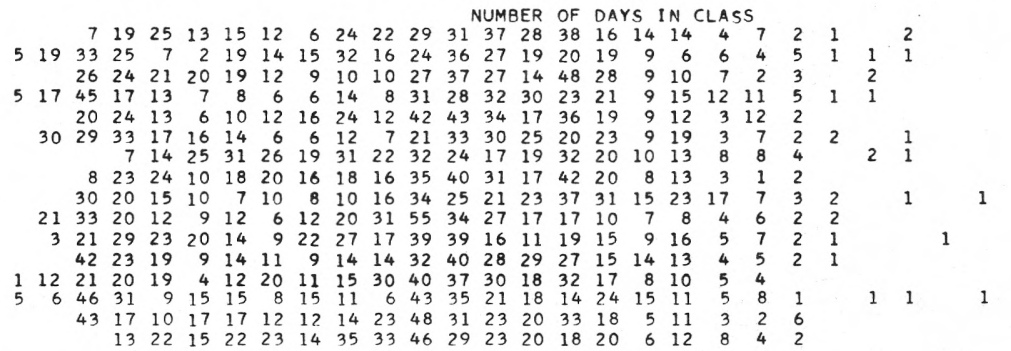

LOWEST MEAN DISCHARGE, IN CFS, FOR THE FOLLOWING NUMBER OF CONSECUTIVE DAYS IN YEAR BEGINNING APRIL 1

\begin{tabular}{|c|c|c|c|c|c|c|c|c|c|c|c|}
\hline YEAR & 1 & 3 & 7 & 14 & 30 & 60 & 90 & 120 & 150 & 183 & 274 \\
\hline 1948 & 13.0 & 13.7 & 14.6 & 14.7 & 18.8 & 21.7 & 28.0 & $41 \cdot 0$ & 50.8 & 69.8 & 156.0 \\
\hline 1949 & 6.9 & 7.3 & 7.6 & 8.2 & 9.1 & 11.4 & 13.6 & $18 \cdot 4$ & 24.0 & 31.9 & 112.0 \\
\hline 1950 & 11.0 & 11.0 & 11.7 & $12 \cdot 3$ & 14.2 & 17.4 & 21.5 & 39.0 & 60.0 & 97.6 & 236.0 \\
\hline 1951 & 7.4 & 7.7 & $8 \cdot 0$ & 8.7 & 10.5 & 10.8 & 12.8 & $18 \cdot 4$ & 37.4 & 60.8 & 164.0 \\
\hline 1952 & 8.4 & 8.7 & 8.9 & 9.1 & 9.6 & 10.4 & 12.9 & $14 \cdot 6$ & 21.1 & 37.8 & 86.4 \\
\hline 1953 & 14.0 & 14.0 & 14.3 & 14.7 & 17.8 & 21.0 & 25.4 & 33.5 & 42.3 & 86.3 & 217.0 \\
\hline 1954 & 17.0 & 17.0 & 17.6 & 19.3 & 24.0 & 29.2 & 33.9 & 43.0 & 55.6 & 63.8 & 119.0 \\
\hline 1955 & 14.0 & 14.0 & $14 \cdot 0$ & 14.9 & 16.5 & 20.9 & 27.6 & $38 \cdot 4$ & 68.2 & 102.0 & 295.0 \\
\hline 1956 & 9.5 & 9.8 & 10.2 & 11.0 & 11.8 & 13.2 & 16.4 & $26 \cdot 5$ & 45.9 & 78.4 & 124.0 \\
\hline 1957 & 8.2 & 8.2 & 8.9 & 9.0 & 9.7 & 13.2 & 18.1 & $22 \cdot 0$ & $29 \cdot 3$ & $45 . y$ & 142.0 \\
\hline 1958 & 9.0 & 9.3 & 10.1 & 11.4 & 12.9 & 14.8 & 16.9 & 21.5 & 35.9 & 44.8 & 140.0 \\
\hline 1959 & 11.0 & 11.3 & 12.0 & 12.2 & 12.8 & 16.9 & 24.2 & $37 \cdot 7$ & 57.7 & 65.7 & 97.9 \\
\hline 1960 & 6.7 & 6.7 & 7.1 & 7.7 & 10.5 & 15.0 & $17 \cdot 2$ & $20 \cdot 4$ & 30.0 & 71.6 & 152.0 \\
\hline 1961 & 9.0 & 9.3 & 9.5 & 10.0 & 11.2 & 14.2 & 14.8 & $19 \cdot 2$ & $26 \cdot 1$ & 41.6 & 144.0 \\
\hline 1962 & 10.0 & 10.3 & 10.9 & 11.7 & 14.2 & 18.0 & 20.7 & $29 \cdot 3$ & 46.9 & 68.9 & 126.0 \\
\hline
\end{tabular}

\begin{tabular}{|c|c|c|c|c|c|c|c|c|c|c|c|}
\hline YEAR & 1 & 3 & 7 & 15 & 30 & 60 & 90 & 120 & 150 & 183 & 274 \\
\hline 1948 & 3620.0 & 2730.0 & 1790.0 & 1090.0 & 684.0 & 543.0 & $481 \cdot 0$ & $428 \cdot 0$ & 422.0 & 398.0 & 327.0 \\
\hline 1949 & 3310.0 & 2300.0 & 1740.0 & 1130.0 & 730.0 & 524.0 & 455.0 & $437 \cdot 0$ & 407.0 & 387.0 & 284.0 \\
\hline 1950 & 2750.0 & 2280.0 & 1390.0 & 806.0 & 660.0 & 640.0 & 557.0 & $491 \cdot 0$ & 449.0 & 413.0 & 302.0 \\
\hline 1951 & 2950.0 & 2180.0 & 1580.0 & 942.0 & 821.0 & 627.0 & 615.0 & $584 \cdot 0$ & 524.0 & 476.0 & 351.0 \\
\hline 1952 & 1570.0 & 1420.0 & 1220.0 & 826.0 & 588.0 & $448 \cdot 0$ & $468 \cdot 0$ & $433 \cdot 0$ & 416.0 & 385.0 & 299.0 \\
\hline 1953 & 3390.0 & 2500.0 & 1670.0 & 1250.0 & 1020.0 & 697.0 & 579.0 & $496 \cdot 0$ & 459.0 & 416.0 & 299.0 \\
\hline 1954 & 3100.0 & 2330.0 & 1360.0 & 994.0 & 923.0 & 663.0 & 651.0 & $570 \cdot 0$ & 515.0 & $445 \cdot 0$ & 325.0 \\
\hline 1955 & 1540.0 & 1410.0 & $9 n 0 . n$ & 586.0 & 566.0 & 469.0 & 391.0 & $361 \cdot 0$ & 354.0 & 330.0 & 256.0 \\
\hline 1956 & 5500.0 & 3550.0 & 2210.0 & 1440.0 & 1060.0 & 897.0 & 741.0 & 639.0 & $601 \cdot 0$ & 546.0 & 418.0 \\
\hline 1957 & 2420.0 & 1900.0 & 1240.0 & 987.0 & 735.0 & 532.0 & 403.0 & $389 \cdot 0$ & $342 \cdot 0$ & $321 \cdot 0$ & 245.0 \\
\hline 1958 & 4080.0 & 2750.0 & 1660.0 & 1160.0 & 770.0 & 686.0 & 581.0 & $478 \cdot 0$ & $438 \cdot 0$ & 382.0 & 277.0 \\
\hline 1959 & 2280.0 & 1560.0 & 1080.0 & 702.0 & 616.0 & 501.0 & 456.0 & $429 \cdot 0$ & 413.0 & 374.0 & 279.0 \\
\hline 1960 & 1380.0 & $1230 \cdot 0$ & 824.0 & 617.0 & 553.0 & 482.0 & 425.0 & 401.0 & $360 \cdot 0$ & $315 \cdot 0$ & 247.0 \\
\hline 1961 & 5270.0 & 3240.0 & $2 n 7 n . n$ & $1350 . ?$ & 939.0 & 734.0 & 557.0 & $512 \cdot 0$ & 477.0 & 426.0 & 305.0 \\
\hline 1962 & 1970.0 & $1640 . n$ & $1090 . n$ & 732.0 & 615.0 & 462.0 & 371.0 & $349 \cdot 0$ & 366.0 & $346 \cdot 0$ & 263.0 \\
\hline 1963 & 1840.0 & 1230.0 & 851.0 & 675.0 & 511.0 & 457.0 & 390.0 & $364 \cdot 0$ & 310.0 & 326.0 & 252.0 \\
\hline
\end{tabular}


$\begin{array}{lllllllllllllllllllll} & 19 & 5 & 1 & 20 & 12 & 17 & 16 & 12 & 27 & 31 & 46 & 30 & 46 & 31 & 20 & 19 & 4 & 9\end{array}$

$\begin{array}{rrrrrrrrrrrrrrrrrrrrr}23 & 40 & 18 & 8 & 19 & 29 & 21 & 15 & 6 & 37 & 27 & 19 & 34 & 36 & 8 & 4 & 6 & 7 & 6 & 1 & 2 \\ 16 & 33 & 19 & 12 & 15 & 15 & 6 & 10 & 6 & 5 & 17 & 44 & 33 & 46 & 15 & 9 & 17 & 14 & 20 & 9 & 2\end{array}$

$\begin{array}{lllllllllllllllllllllllll}51 & 31 & 15 & 8 & 9 & 14 & 12 & 16 & 40 & 59 & 23 & 17 & 10 & 17 & 12 & 5 & 12 & 2 & 7 & 4 & & 1\end{array}$

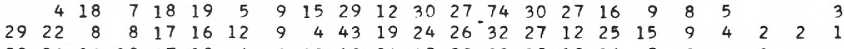

$\begin{array}{llllllllllllllllllll}22 & 20 & 10 & 18 & 27 & 18 & 4 & 6 & 10 & 43 & 24 & 45 & 22 & 33 & 19 & 19 & 16 & 5 & 3\end{array}$

$\begin{array}{lllllllllllllllllllllll}75 & 35 & 22 & 6 & 11 & 12 & 5 & 6 & 12 & 34 & 49 & 19 & 17 & 14 & 16 & 6 & 12 & 5 & 6 & 2 & 1\end{array}$

$\begin{array}{rlllllllllllllllllllll}36 & 29 & 22 & 14 & 26 & 12 & 11 & 14 & 31 & 41 & 41 & 27 & 13 & 22 & 6 & 7 & 4 & 4 & 2 & 1\end{array}$

$\begin{array}{rrrrrrrrrrrrrrrrrrrrr}25 & 26 & 16 & 17 & 11 & 4 & 3 & 17 & 6 & 15 & 27 & 26 & 23 & 34 & 39 & 17 & 35 & 10 & 10 & 3\end{array}$

$\begin{array}{rrrrrrrrrrrrrrrrrrrr}11 & 2 & 17 & 13 & 7 & 15 & 13 & 8 & 5 & 25 & 15 & 25 & 23 & 37 & 28 & 52 & 38 & 19 & 10 & 1 \\ 34 & 22 & 17 & 12 & 23 & 10 & 25 & 33 & 9 & 59 & 16 & 15 & 6 & 24 & 11 & 13 & 10 & 9 & 9 & 6\end{array}$

$\begin{array}{rrrrrrrrrrrrrrrrrrrr}46 & 23 & 11 & 9 & 10 & 4 & 9 & 9 & 6 & 17 & 21 & 29 & 24 & 52 & 30 & 18 & 31 & 4 & 10 & 1 \\ 11 & 19 & 28 & 12 & 25 & 11 & 8 & 22 & 11 & 30 & 31 & 31 & 24 & 38 & 18 & 16 & 15 & 5 & 5 & 2\end{array}$

$\begin{array}{llllllllllllllllllllll}47 & 26 & 13 & 19 & 14 & 13 & 10 & 21 & 17 & 21 & 10 & 11 & 24 & 25 & 31 & 21 & 25 & 8 & 6\end{array}$

$\begin{array}{rrrrrrrrrrrrrrrrrrr}24 & 20 & 23 & 13 & 16 & 10 & 8 & 13 & 12 & 15 & 8 & 11 & 12 & 43 & 47 & 24 & 34 & 8 & 18 \\ 12 & 41 & 25 & 9 & 9 & 10 & 7 & 11 & 12 & 52 & 25 & 30 & 30 & 37 & 16 & 10 & 12 & 8 & 8\end{array}$

$\begin{array}{llllllllllllllllllllllll}1 & 43 & 28 & 21 & 21 & 33 & 17 & 14 & 16 & 7 & 25 & 22 & 17 & 20 & 25 & 13 & 11 & 12 & 8 & 12\end{array}$

$\begin{array}{rrrrrrrrrrrrrrrrrrrrrr}4 & 8 & 15 & 13 & 24 & 11 & 13 & 21 & 36 & 79 & 45 & 24 & 18 & 26 & 9 & 7 & 7 & 2 & 3 \\ & 18 & 12 & 18 & 12 & 7 & 5 & 18 & 15 & 41 & 81 & 42 & 25 & 21 & 11 & 7 & 12 & 7 & 8\end{array}$

$\begin{array}{lllllllllllllllllll}4 & 24 & 14 & 14 & 22 & 17 & 7 & 10 & 5 & 12 & 41 & 20 & 19 & 25 & 32 & 19 & 28 & 16 & 20\end{array}$

$\begin{array}{rrrrrrrrrrrrrrrrrrr}4 & 34 & 26 & 10 & 17 & 12 & 9 & 15 & 22 & 50 & 54 & 33 & 28 & 23 & 11 & 7 & 6 & 2 & 3 \\ 3 & 36 & 29 & 12 & 22 & 18 & 14 & 24 & 15 & 23 & 29 & 12 & 15 & 27 & 26 & 21 & 17 & 13 & 6\end{array}$

$\begin{array}{llllllllllllllllllll}1 & 29 & 41 & 9 & 17 & 5 & 8 & 11 & 6 & 24 & 19 & 26 & 30 & 50 & 31 & 13 & 16 & 9 & 13\end{array}$ $\begin{array}{rrrrrrrrrrrrrrrrrrr}8 & 11 & 19 & 21 & 21 & 18 & 18 & 14 & 44 & 37 & 24 & 20 & 30 & 23 & 19 & 14 & 5 & 10 \\ 10 & 14 & 13 & 18 & 19 & 6 & 8 & 9 & 33 & 21 & 23 & 27 & 59 & 34 & 17 & 25 & 9 & 13\end{array}$

$\begin{array}{llllllllllllllllllll}28 & 19 & 7 & 17 & 6 & 12 & 19 & 29 & 26 & 25 & 20 & 16 & 36 & 33 & 25 & 14 & 7 & 10\end{array}$

$\begin{array}{rrrrrrrrrrrrrrrrrrr}17 & 17 & 12 & 16 & 18 & 16 & 13 & 16 & 10 & 12 & 14 & 22 & 17 & 61 & 43 & 32 & 20 & 6 & 13 \\ 13 & 37 & 11 & 16 & 13 & 13 & 5 & 11 & 7 & 9 & 18 & 27 & 17 & 54 & 31 & 22 & 14 & 13 & 23\end{array}$

$\begin{array}{rrrrrrrrrrrrrrrrrrr}18 & 16 & 13 & 13 & 9 & 7 & 7 & 8 & 32 & 29 & 33 & 29 & 63 & 31 & 11 & 20 & 7 & 12\end{array}$

$\begin{array}{lllllllrrrrrrrrrrrr}32 & 26 & 18 & 19 & 73 & 11 & 9 & 9 & 8 & 19 & 15 & 17 & 26 & 38 & 30 & 16 & 14 & 11 & 12\end{array}$ $\begin{array}{rrrrrrrrrrrrrrrrrrr} & 4 & 44 & 26 & 16 & 18 & 17 & 34 & 33 & 23 & 12 & 31 & 27 & 15 & 23 & 15 & 17 & 3 \\ 1 & 15 & 26 & 12 & 5 & 22 & 13 & 24 & 38 & 47 & 24 & 36 & 33 & 20 & 22 & 8 & 6 & 2\end{array}$

$\begin{array}{rrrrrrrrrrrrrrrrrrr}2 & 9 & 26 & 13 & 19 & 11 & 3 & 6 & 12 & 14 & 14 & 31 & 21 & 32 & 36 & 27 & 39 & 18 & 21 \\ & 16 & 21 & 16 & 18 & 16 & 7 & 10 & 20 & 26 & 44 & 45 & 17 & 23 & 18 & 10 & 12 & 8 & 10\end{array}$ $\begin{array}{rrrrrrrrrrrrrrrrrr}19 & 17 & 24 & 25 & 18 & 10 & 16 & 17 & 39 & 28 & 29 & 26 & 15 & 15 & 12 & 13 & 14 & 16 \\ 14 & 33 & 10 & 23 & 12 & 8 & 16 & 10 & 15 & 23 & 36 & 20 & 46 & 28 & 19 & 20 & 14 & 9\end{array}$ $\begin{array}{rrrrrrrrrrrrrrrrrrrrr}16 & 24 & 17 & 13 & 12 & 4 & 13 & 14 & 39 & 42 & 24 & 13 & 34 & 22 & 31 & 20 & 12 & 12 \\ 28 & 23 & 20 & 16 & 16 & 9 & 11 & 7 & 19 & 21 & 17 & 27 & 36 & 23 & 20 & 35 & 6 & 13\end{array}$

$\begin{array}{rrrrrrrrrrrrrrrrrrr}8 & 28 & 23 & 20 & 16 & 16 & 9 & 11 & 7 & 19 & 21 & 17 & 27 & 36 & 23 & 20 & 35 & 6 & 13 \\ 13 & 23 & 16 & 19 & 17 & 11 & 15 & 13 & 23 & 26 & 43 & 25 & 28 & 25 & 28 & 16 & 11 & 6 \\ & 14 & 15 & 19 & 14 & 10 & 28 & 30 & 49 & 29 & 22 & 16 & 42 & 17 & 14 & 21 & 7 & 12\end{array}$

$$
1
$$

STATION NUMBER

$14-1875.00$

South Santiam River at Waterloo, Oreg.

Summary for water years 1906, 1924-63

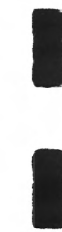

$\begin{array}{ccrrrrl}\text { CLASS } & \text { CFS } & \text { TOTAL ACCUM } & \text { PERCT } & \text { CLASS } \\ & .0 & & 14975 & 100.0 & 09 \\ 1 & 80.0 & 1 & 14975 & 100.0 & 10 \\ 2 & 100.0 & 583 & 14974 & 100.0 & 11 \\ 3 & 150.0 & 864 & 14391 & 96.1 & 12 \\ 4 & 200.0 & 738 & 13527 & 90.3 & 13 \\ 5 & 250.0 & 536 & 12789 & 85.4 & 14 \\ 6 & 300.0 & 769 & 12253 & 81.8 & 15 \\ 7 & 400.0 & 560 & 11484 & 76.7 & 16 \\ 8 & 500.0 & 399 & 10924 & 72.9 & 17\end{array}$

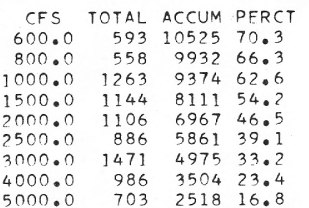

$\begin{array}{lrrrr}\text { CLASS CFS } & \text { TOTAL } & \text { ACCUM } & \text { PERCT } \\ 18 & 6000 & 767 & 1815 & 12.1 \\ 19 & 8000 & 364 & 1048 & 7.0 \\ 20 & 10000 & 426 & 684 & 4.6 \\ 21 & 15000 & 139 & 258 & 1.7 \\ 22 & 20000 & 51 & 119 & .8 \\ 23 & 25000 & 31 & 68 & .5 \\ 24 & 30000 & 23 & 37 & .2 \\ 25 & 40000 & 9 & 14 & .1\end{array}$
$\begin{array}{lr}\text { CLASS CFS TOTAL ACCUM PERCT } \\ 27 \\ 28 & .0 \\ 29 & .0 \\ 30 & .0 \\ 31 & .0 \\ 32 & .0 \\ 33 & .0 \\ 34 & .0 \\ 35 & .0\end{array}$ 
South Santiam River at Waterloo, Oreg.

STATION NUMBER $14-1875.00$ LOWEST MEAN DISCHARGE, IN CFS, FOR THE FOLLOWING NUMBER OF CONSECUTIVE DAYS IN YEAR BEGINNING APRIL 1

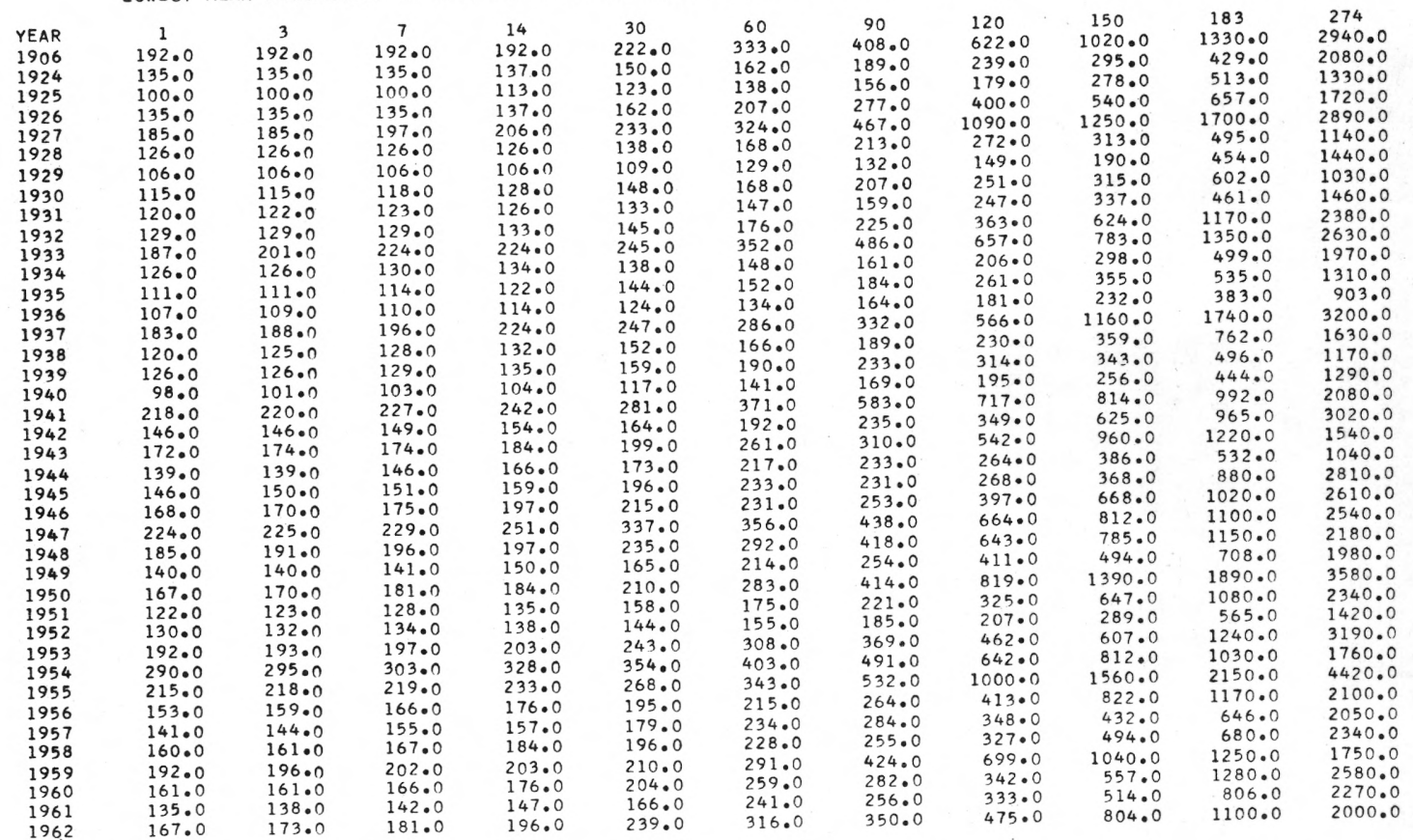

South Santiam River at Waterloo, Oreg. STATION NUMBER 14-1875.00 HIGHEST MEAN DISCHARGE, IN CFS, FOR THE FOLLOWING NUMBER OF CONSECUTIVE DAYS IN YEAR ENDING SEPTEMBER 30

\begin{tabular}{|c|c|c|c|c|c|c|c|c|c|c|c|}
\hline YEAR & 1 & 3 & 7 & 15 & 30 & 60 & 70 & 120 & 150 & $\begin{array}{c}183 \\
4060.0\end{array}$ & $\begin{array}{c}274 \\
3430.0\end{array}$ \\
\hline $\begin{array}{l}1906 \\
1924\end{array}$ & $14000 \cdot 0$ & $\begin{array}{l}12400.0 \\
13700.0\end{array}$ & & $\begin{array}{l}8590.0 \\
9630.0\end{array}$ & $\begin{array}{l}6300.0 \\
6330.0\end{array}$ & $\begin{array}{l}6070.0 \\
5140.0\end{array}$ & $\begin{array}{l}5310.0 \\
4960.0\end{array}$ & $\begin{array}{l}4830 \cdot 0 \\
4250 \cdot 0\end{array}$ & $\begin{array}{l}4430.0 \\
3930.0\end{array}$ & $\begin{array}{l}4060.0 \\
3450.0\end{array}$ & $\begin{array}{l}3430.0 \\
2500.0\end{array}$ \\
\hline $\begin{array}{l}1924 \\
1925\end{array}$ & $\begin{array}{l}23400.0 \\
26200.0\end{array}$ & $\begin{array}{l}13700 \cdot 0 \\
20700 \cdot 0\end{array}$ & $\begin{array}{l}12200 \cdot 0 \\
18200.0\end{array}$ & $\begin{array}{r}9630.0 \\
14800.0\end{array}$ & $\begin{array}{r}6330.0 \\
11200.0\end{array}$ & $\begin{array}{l}5140.0 \\
8920.0\end{array}$ & $\begin{array}{l}4960.0 \\
8110.0\end{array}$ & $7670 \bullet 0$ & 6630.0 & 6140.0 & 4590.0 \\
\hline 1926 & 27400.0 & 20800.0 & 14700.0 & 9870.0 & 8630.0 & 5970.0 & 5300.0 & $4420 \cdot 0$ & 3770.0 & 3320.0 & 2370.0 \\
\hline $\begin{array}{l}1927 \\
1928\end{array}$ & $38500 \cdot 0$ & $30000 \cdot 0$ & 17700.0 & 10900.0 & 8780.0 & 7190.0 & 6610.0 & $6380 \cdot 0$ & 5760.0 & $5360 \cdot 0$ & 4380.0 \\
\hline $\begin{array}{l}1928 \\
1929\end{array}$ & $33000 \cdot 0$ & 25000.0 & 19900.0 & 13700.0 & 9800.0 & 7240.0 & 6130.0 & 5530.0 & 5750.0 & 5440.0 & 4170.0 \\
\hline $\begin{array}{l}1929 \\
1930\end{array}$ & $21000 \cdot 0$ & 13700.0 & $9510 . n$ & 7610.0 & 6310.0 & 5570.0 & 4760.0 & $4180 \cdot 0$ & 4000.0 & 3710.0 & 2870.0 \\
\hline $\begin{array}{l}1930 \\
1931\end{array}$ & $21100 \cdot 0$ & $14800 \bullet n$ & 11000.0 & 9610.0 & 7170.0 & 4860.0 & 4590.0 & $4050 \cdot 0$ & 3590.0 & 3350.0 & 2360.0 \\
\hline $\begin{array}{l}1931 \\
1932\end{array}$ & 46400.0 & 33900.0 & 19400.0 & 11700.0 & 8240.0 & 5370.0 & 4280.0 & $3720 \cdot 0$ & 3320.0 & $3010 \cdot 0$ & 2210.0 \\
\hline $\begin{array}{l}1932 \\
1933\end{array}$ & $35600 \cdot 0$ & $28300 \cdot 0$ & $18800 \cdot 0$ & $14800 \cdot 0$ & 10800.0 & 8170.0 & 6820.0 & $6170 \cdot 0$ & $5880 \cdot 0$ & 5390.0 & 4210.0 \\
\hline $\begin{array}{l}1933 \\
1934\end{array}$ & $22000 \cdot 0$ & 17200.0 & $12600 \cdot 0$ & 9120.0 & 7500.0 & 6360.0 & 5750.0 & $5620 \cdot 0$ & 5120.0 & $5420 \cdot 0$ & 4610.0 \\
\hline $\begin{array}{l}1934 \\
1935\end{array}$ & $22300 \cdot 0$ & $18400 \cdot 0$ & 17000.0 & $12200 \cdot 0$ & 9250.0 & 7700.0 & 5810.0 & $5270 \cdot 0$ & 4580.0 & 4010.0 & 2940.0 \\
\hline $\begin{array}{l}1935 \\
1936\end{array}$ & $21300 \cdot 0$ & $16200 \cdot n$ & $13000 \cdot 0$ & $9000 \cdot 0$ & 6490.0 & 5900.0 & 5360.0 & $5090 \cdot 0$ & 4810.0 & 4690.0 & $\begin{array}{l}3690.0 \\
3260.0\end{array}$ \\
\hline $\begin{array}{l}1936 \\
1937\end{array}$ & 28200.0 & $22400 \bullet 0$ & $15900 \cdot 0$ & $14900 \cdot 0$ & 9840.0 & 6680.0 & 5720.0 & $5260 \cdot 0$ & 4900.0 & 4370.0 & $\begin{array}{l}3260.0 \\
3360.0\end{array}$ \\
\hline $\begin{array}{l}1937 \\
1938\end{array}$ & 29100.0 & $25400 \cdot 0$ & $16500 \cdot 0$ & 10800.0 & 8000.0 & 6930.0 & 6390.0 & $5800 \cdot 0$ & $5360 \cdot 0$ & 4580.0 & $\begin{array}{l}3360.0 \\
4550.0\end{array}$ \\
\hline $\begin{array}{l}1938 \\
1939\end{array}$ & $29100 \cdot 0$ & $18800 \cdot 0$ & $12200 \cdot 0$ & 9570.0 & 7540.0 & 7000.0 & $\begin{array}{l}6650.0 \\
4600.0\end{array}$ & $\begin{array}{l}6200 \cdot 0 \\
4410.0\end{array}$ & $\begin{array}{l}6190.0 \\
4150.0\end{array}$ & $\begin{array}{l}6090.0 \\
3880.0\end{array}$ & $\begin{array}{l}4550.0 \\
2940.0\end{array}$ \\
\hline $\begin{array}{l}1939 \\
1940\end{array}$ & $\begin{array}{l}19900.0 \\
12900.0\end{array}$ & $\begin{array}{l}13600.0 \\
12100.0\end{array}$ & $\begin{array}{r}9740.0 \\
11100 \cdot 0\end{array}$ & $\begin{array}{l}8030.0 \\
9120.0\end{array}$ & $\begin{array}{l}6010.0 \\
8460.0\end{array}$ & $\begin{array}{l}5350.0 \\
6240.0\end{array}$ & 5100.0 & $\begin{array}{l}4410 \cdot 0 \\
4750 \cdot ?\end{array}$ & 4240.0 & 3650.0 & 2580.0 \\
\hline 1941 & 14800.0 & 9940.0 & 6890.0 & 5740.0 & 4240.0 & 4020.0 & 3690.0 & 3180.0 & 2800.0 & 2540.0 & 2120.0 \\
\hline 1942 & $24400 \cdot 0$ & 17900.0 & $12800 \cdot 0$ & 8420.0 & 7470.0 & 6230.0 & 5540.0 & $4850 \cdot 0$ & 4300.0 & 3840.0 & 3150.0 \\
\hline 1943 & 44500.0 & $34200 \cdot 0$ & 24700.0 & $17000 \cdot 0$ & 12700.0 & $12000 \cdot 0$ & $10200 \cdot 0$ & $8710 \cdot 0$ & 7940.0 & 7240.0 & 5440.0 \\
\hline 1944 & $13200 \cdot 0$ & $10500 \cdot 0$ & 6360.0 & 4920.0 & 3530.0 & 3210.0 & 3060.0 & $2780 \cdot 0$ & 2590.0 & 2610.0 & 2210.0 \\
\hline 1945 & $26500 \cdot 0$ & $17000 \cdot 0$ & 15500.0 & 10800.0 & 6780.0 & 5640.0 & 5580.0 & 5370.0 & 5160.0 & 4420.0 & 3240.0 \\
\hline 1946 & 56300.0 & $39000 \cdot 0$ & $22000 \cdot 0$ & 15300.0 & 10500.0 & 8180.0 & 7210.0 & 6760.0 & 6140.0 & 5630.0 & 4180.0 \\
\hline 1947 & 44600.0 & $40600 \cdot 0$ & $26300 \cdot 0$ & $14700 \cdot 0$ & 12600.0 & 7760.0 & 6950.0 & $6050 \cdot 0$ & 5670.0 & 5170.0 & 3910.0 \\
\hline 1948 & $47000 \cdot 0$ & $32700 \cdot 0$ & $21200 \cdot 0$ & 13300.0 & 8730.0 & 6800.0 & 6780.0 & $5800 \cdot 0$ & 5820.0 & 5440.0 & 4670.0 \\
\hline 1949 & 35700.0 & $24600 \cdot 0$ & 19100.0 & $13000 \cdot 0$ & 8870.0 & 6560.0 & 6490.0 & $5500 \cdot 0$ & 5300.0 & 5350.0 & 4060.0 \\
\hline 1950 & 32500.0 & $24100 \cdot 0$ & 15000.0 & 11600.0 & 9560.0 & 8390.0 & 7410.0 & $6620 \cdot 0$ & $6100 \cdot 0$ & 5660.0 & 4470.0 \\
\hline 1951 & 33600.0 & $23100 \cdot 0$ & $20200 \cdot 0$ & 12200.0 & 10500.0 & 8050.0 & 8040.0 & 7870.0 & 7040.0 & 6460.0 & $\begin{array}{l}4910.0 \\
4170.0\end{array}$ \\
\hline 1952 & 21600.0 & 18800.0 & 15800.0 & 10400.0 & 7420.0 & 5660.0 & 5850.0 & 5400.0 & 5200.0 & 4990.0 & $\begin{array}{l}4170.0 \\
4290.0\end{array}$ \\
\hline 1953 & $51600 \cdot 0$ & 37200.0 & 25700.0 & $18500 \cdot 0$ & 15700.0 & 10400.0 & 8290.0 & $7090 \cdot 0$ & 6590.0 & $\begin{array}{l}5940.0 \\
6240.0\end{array}$ & $\begin{array}{l}4290.0 \\
4620.0\end{array}$ \\
\hline & $\begin{array}{l}27000 \cdot 0 \\
52400 \cdot 0\end{array}$ & $41900 \cdot 0$ & 25500.0 & $\begin{array}{r}7660.0 \\
18300.0\end{array}$ & $\begin{array}{r}6550.0 \\
14200.0\end{array}$ & $\begin{array}{r}5860.0 \\
12000 \cdot 0\end{array}$ & 10300.0 & 8440.0 & 7890.0 & 7430.0 & 5960.0 \\
\hline 1957 & $\begin{array}{l}52400 \cdot 0 \\
44900.0\end{array}$ & $31400 \cdot 0$ & 20200.0 & 14100.0 & 10400.0 & 7680.0 & $5930 \cdot 0$ & $6030 \cdot 0$ & 5340.0 & 4920.0 & 3720.0 \\
\hline & 47200.0 & $31000 \cdot 0$ & $19800 \cdot 0$ & 14500.0 & 10100.0 & 9150.0 & 8140.0 & $6690 \cdot 0$ & 6390.0 & $5640 \cdot 0$ & 4080.0 \\
\hline 1959 & 25800.0 & $20800 \cdot 0$ & 12900.0 & 10500.0 & 8170.0 & 6620.0 & 6830.0 & $6130 \cdot 0$ & 5860.0 & $\begin{array}{l}5440.0 \\
4690.0\end{array}$ & $\begin{array}{l}4120.0 \\
3880.0\end{array}$ \\
\hline 1960 & 16600.0 & $15600 \cdot 0$ & 11300.0 & 8550.0 & 7790.0 & 6780.0 & 6120.0 & $5980 \cdot 0$ & & 6210.0 & 4570.0 \\
\hline 1961 & 55100.0 & 37200.0 & 24100.0 & 17000.0 & $12000 \cdot 0$ & 9440.0 & 7580.0 & $\begin{array}{l}7180 \cdot 0 \\
5020.0\end{array}$ & $\begin{array}{l}6820.0 \\
5180.0\end{array}$ & 4960.0 & 3830.0 \\
\hline 1962 & 26000.0 & 22900.0 & 15300.0 & 10400.0 & 8030.0 & $6660 \cdot 0$ & $\begin{array}{l}5360.0 \\
4820.0\end{array}$ & 4780.0 & 4400.0 & 4540.0 & 3590.0 \\
\hline 1963 & 21000.0 & $18000 \cdot 0$ & 12600.0 & 9510.0 & 7020.0 & 5450.0 & & & & & \\
\hline
\end{tabular}


CLASS $\begin{array}{llllllllllllllllllllllllllllllllllllllll}1 & 1 & 2 & 3 & 4 & 5 & 6 & 7 & 8 & 9 & 10 & 11 & 12 & 13 & 14 & 15 & 16 & 17 & 18 & 19 & 20 & 21 & 22 & 23 & 24 & 25 & 26 & 27 & 28 & 29 & 30 & 31 & 32 & 33 & 34\end{array}$

YEAR
1908
1909
1910
1911
1912
1913
1914
1915
1916
1940
1941
1942
1943
1944
1945
1946
1947
1948
1949
1950
1951
1952
1953
1954
1955
1956
1957
1958
1959
1960
1961
1962
1963
$\begin{array}{rrrrrrrrrrrrrrrrrrrrrrrrrrrrrr}\text { NUMBER OF } & \text { DAYS IN CLASS } \\ 14 & 17 & 32 & 37 & 13 & 23 & 10 & 4 & 3 & 11 & 29 & 41 & 33 & 47 & 38 & 7 & 6 & 4 & 1 & 5 & 2 & 1 & 2\end{array}$ $\begin{array}{rrrrrrrrrrrrrrrrrrrrrrrrr}14 & 36 & 7 & 10 & 4 & 25 & 20 & 19 & 14 & 21 & 23 & 34 & 50 & 22 & 28 & 13 & 11 & 6 & 3 & 4 & 1 & & \\ 8 & 47 & 12 & 35 & 5 & 23 & 13 & 10 & 4 & 15 & 18 & 24 & 33 & 25 & 32 & 22 & 14 & 8 & 8 & 1 & 3 & 3 & 1 & 1\end{array}$ $\begin{array}{rrrrrrrrrrrrrrrrrrrr}33 & 22 & 18 & 36 & 11 & 3 & 6 & 16 & 27 & 35 & 62 & 48 & 19 & 9 & 7 & 8 & 4 & 1 & & \\ 1 & 8 & 2 & 36 & 24 & 22 & 10 & 6 & 17 & 58 & 63 & 29 & 37 & 21 & 13 & 8 & 7 & 2 & 1 & 1\end{array}$ $\begin{array}{llllllllllllllllllll}1 & 8 & 2 & 36 & 24 & 22 & 10 & 6 & 17 & 58 & 63 & 29 & 37 & 21 & 13 & 8 & 7 \\ \end{array}$ $\begin{array}{llllllllllllllllll}37 & 11 & 23 & 13 & 10 & 14 & 37 & 31 & 30 & 34 & 39 & 46 & 15 & 12 & 7 & 6\end{array}$ $\begin{array}{lllllllllllllllllll}7 & 41 & 6 & 20 & 23 & 9 & 14 & 34 & 40 & 31 & 58 & 38 & 33 & 7 & 2 & 2\end{array}$

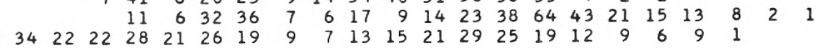
$\begin{array}{lllllllllllllllllll}9 & 12 & 15 & 29 & 8 & 15 & 10 & 40 & 36 & 56 & 32 & 23 & 31 & 19 & 17 & 9 & 1 & 3\end{array}$

$\begin{array}{rrrrrrrrrrrrrrrrrrrrrrrrrrr}9 & 30 & 8 & 8 & 8 & 11 & 17 & 14 & 9 & 31 & 71 & 45 & 34 & 14 & 25 & 14 & 7 & 2 & 3 & 4 & 1 & & \\ 15 & 8 & 4 & 30 & 19 & 22 & 9 & 4 & 3 & 7 & 35 & 26 & 33 & 25 & 46 & 28 & 21 & 8 & 8 & 5 & 4 & 4 & 1\end{array}$

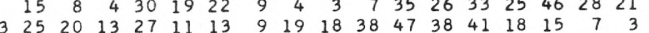

CFS-DAYS

2636745.0

2409750.0

2296600.0

3125950.0

3305070.0

3305070.0
2713270.0

1995230.0

4257820.0

1950064.0

1583859.0

1583859.0

3247239.0

1554139.0

2222576.0

2868611.0

2789501.0

3340561.0

2975837.0

3340216.0

3613640.0

2985970.0

2864662.0

3416650.0

2635041.0

4222790.0

2832697.0

3083466.0

2757463.0

3387801.0

2714343.0

2714343.0
2650485.0

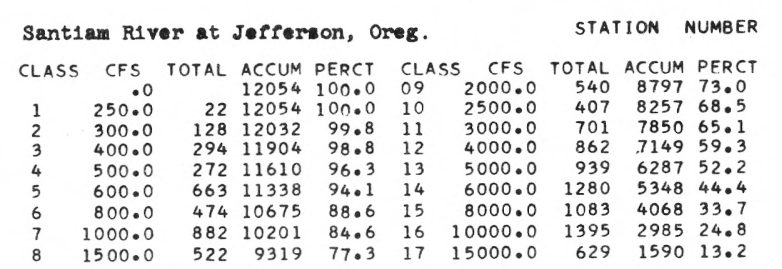

14-1890.00

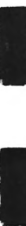

Sant1am River at Jefferson, Oreg.

STATION NUMBER

$14-1890.00$

LOWEST MEAN DISCHARGE, IN CFS, FOR THE FOLLOWING NUMBER OF CONSECUTIVE DAYS IN YEAR BEGINNING APRIL 1

\begin{tabular}{|c|c|c|c|c|c|c|c|c|c|c|c|}
\hline YEAR & 1 & 3 & 7 & 14 & 30 & 60 & 90 & 120 & 150 & 183 & 274 \\
\hline 1908 & 440.0 & $440 \cdot 0$ & 440.0 & 440.0 & 452.0 & 527.0 & 637.0 & $1260 \cdot 0$ & 1670.0 & 2400.0 & 4410.0 \\
\hline 1909 & 350.0 & 350.0 & 363.0 & 379.0 & 417.0 & 530.0 & 653.0 & $997 \cdot 0$ & 1450.0 & $2480 \cdot 0$ & 6600.0 \\
\hline 1910 & 350.0 & 350.0 & 350.0 & 392.0 & $418 \cdot 0$ & 456.0 & 534.0 & $727 \bullet 0$ & 843.0 & $1330 \cdot 0$ & 4500.0 \\
\hline 1911 & 540.0 & 540.0 & 540.0 & 540.0 & 558.0 & 778.0 & 980.0 & $1050 \cdot 0$ & 1500.0 & 2840.0 & 4800.0 \\
\hline 1912 & 1040.0 & 1040.0 & 1100.0 & 1140.0 & 1370.0 & 2100.0 & 2390.0 & $2710 \bullet 0$ & 3600.0 & 4640.0 & 6730.0 \\
\hline 1913 & $900 \cdot 0$ & $900 \cdot 0$ & 920.0 & 960.0 & 1060.0 & 1250.0 & 1750.0 & $2860 \cdot 0$ & $3700 \cdot 0$ & 4550.0 & 6820.0 \\
\hline 1914 & 650.0 & 650.0 & 650.0 & 650.0 & 698.0 & 832.0 & 1250.0 & $1920 \bullet 0$ & 2350.0 & 3000.0 & 4450.0 \\
\hline 1915 & 510.0 & 510.0 & 510.0 & 570.0 & 619.0 & 697.0 & 813.0 & $1070 \cdot 0$ & $1380 \cdot 0$ & 2670.0 & 6690.0 \\
\hline 1940 & 260.0 & 260.0 & $260 \cdot 0$ & 260.0 & 285.0 & 357.0 & 397.0 & $471 \cdot 0$ & 619.0 & 1180.0 & 3390.0 \\
\hline 1941 & 365.0 & 367.0 & 387.0 & $450 \cdot 0$ & 534.0 & 714.0 & 1230.0 & 1570.0 & 1820.0 & $2320 \cdot 0$ & 5070.0 \\
\hline 1942 & 365.0 & 378.0 & $384 \cdot 0$ & 395.0 & $400 \cdot 0$ & 422.0 & 504.0 & 803.0 & 1560.0 & 2470.0 & 7120.0 \\
\hline 1943 & 580.0 & 585.0 & 596.0 & 614.0 & 638.0 & 738.0 & 879.0 & $1530 \cdot 0$ & 2530.0 & 3180.0 & 4030.0 \\
\hline 1944 & 294.0 & 298.0 & 303.0 & 322.0 & 347.0 & $442 \cdot 0$ & 493.0 & $564 \cdot 0$ & 907.0 & $1330 \cdot 0$ & 2590.0 \\
\hline 1945 & 398.0 & $398 \cdot 0$ & $404 \cdot 0$ & 425.0 & 452.0 & 530.0 & 553.0 & $612 \cdot 0$ & 893.0 & $2100 \cdot 0$ & 6610.0 \\
\hline 1946 & 527.0 & 534.0 & 543.0 & 564.0 & 584.0 & 634.0 & 693.0 & $1170 \cdot 0$ & 1940.0 & $2750 \cdot 0$ & 6700.0 \\
\hline 1947 & 702.0 & 707.0 & $714 \cdot 0$ & 772.0 & 910.0 & 976.0 & 1200.0 & $1760 \cdot 0$ & $2150 \cdot 0$ & 2880.0 & 6360.0 \\
\hline 1948 & 670.0 & 672.0 & 686.0 & 700.0 & 781.0 & 898.0 & 1230.0 & 1730.0 & $2130 \cdot 0$ & 3180.0 & 5640.0 \\
\hline 1949 & 620.0 & 622.0 & 627.0 & 638.0 & 654.0 & 775.0 & 911.0 & $1340 \cdot 0$ & 1570.0 & 2210.0 & 5650.0 \\
\hline 1950 & 760.0 & $760 \cdot 0$ & 763.0 & 771.0 & 824.0 & 990.0 & 1370.0 & $2460 \cdot 0$ & 3980.0 & 5220.0 & 9550.0 \\
\hline 1951 & 555.0 & $557 \cdot 0$ & 563.0 & 575.0 & 605.0 & 623.0 & 729.0 & $1080 \cdot 0$ & 1910.0 & 2970.0 & $6000 \cdot 0$ \\
\hline 1952 & 396.0 & $404 \cdot 0$ & 406.0 & 414.0 & $428 \cdot 0$ & 471.0 & 535.0 & $582 \cdot 0$ & 802.0 & 1590.0 & 3750.0 \\
\hline 1953 & 586.0 & 593.0 & 610.0 & 632.0 & 753.0 & $858 \cdot 0$ & 1140.0 & $1620 \cdot 0$ & 2020.0 & $3430 \cdot 0$ & 7860.0 \\
\hline 1954 & 880.0 & $940 \cdot 0$ & 1010.0 & 1050.0 & 1180.0 & 1530.0 & $2000 \cdot 0$ & $2490 \cdot 0$ & 3040.0 & 3620.0 & 5260.0 \\
\hline 1955 & 774.0 & 789.0 & 823.0 & 839.0 & 933.0 & 1340.0 & 1840.0 & $3150 \cdot 0$ & 4700.0 & $5640 \cdot 0$ & 11000.0 \\
\hline 1956 & 970.0 & 973.0 & $1000 \cdot 0$ & 1040.0 & 1100.0 & 1280.0 & 1570.0 & $1940 \cdot 0$ & 2880.0 & 3920.0 & 5810.0 \\
\hline 1957 & 634.0 & 643.0 & 656.0 & 697.0 & 766.0 & 894.0 & 1160.0 & $1590 \cdot 0$ & 1910.0 & 2400.0 & 5610.0 \\
\hline 1958 & 780.0 & 785.0 & 796.0 & 804.0 & 812.0 & 1030.0 & 1320.0 & $1580 \cdot 0$ & 1980.0 & 2320.0 & 6320.0 \\
\hline 1959 & 695.0 & $702 \cdot 0$ & 709.0 & 713.0 & 735.0 & 944.0 & 1500.0 & $2330 \cdot 0$ & 3240.0 & 4020.0 & 5030.0 \\
\hline 1960 & 890.0 & $896 . ?$ & 902.0 & 920.0 & 954.0 & 1170.0 & 1370.0 & $1590 \cdot 0$ & 2180.0 & 3900.0 & 7380.0 \\
\hline 1961 & 757.0 & 829.0 & 835.0 & 840.0 & 865.0 & 999.0 & 1180.0 & $1410 \cdot 0$ & 1980.0 & 2780.0 & 6110.0 \\
\hline 1962 & 944.0 & 973.0 & 985.0 & 998.0 & 1100.0 & 1180.0 & 1370.0 & $1700 \bullet 0$ & $2700 \cdot 0$ & 3370.0 & $5860 \cdot 0$ \\
\hline
\end{tabular}


Santiam River at Jefferson, Oreg.

STATION NUMBER $14-1890.00$

HIGHEST MEAN DISCHARGE, IN CFS, FOR THE FOLLOWING NUMBER OF CONSECUTIVE DAYS IN YEAR ENDING SEPTEMBER 30

\begin{tabular}{|c|c|c|c|c|c|c|c|c|c|c|c|}
\hline EAR & 1 & 3 & 7 & 15 & 30 & 60 & 90 & 120 & 150 & 183 & 274 \\
\hline & 94900.0 & 62900.0 & 54000.0 & 32800.0 & 23100.0 & 18300.0 & 14600.0 & $14100 \cdot 0$ & 13000.0 & 12400.0 & 9400.0 \\
\hline 09 & 58100.0 & 49500.0 & 44500.0 & 29200.0 & 22700.0 & 18500.0 & 15700.0 & $13400 \cdot 0$ & 11900.0 & 10900.0 & 8570.0 \\
\hline & $120000 \cdot 0$ & $91600 . n$ & $65500 . ?$ & $44000 \cdot 0$ & 30900.0 & 20000.0 & 16700.0 & $18600 \cdot 0$ & 17100.0 & 15400.0 & 11000.0 \\
\hline & 41300.0 & $31600 \cdot 0$ & 26400.0 & 23600.0 & 18200.0 & 13200.0 & 12700.0 & 10900.0 & 10400.0 & 9800.0 & 8060.0 \\
\hline 912 & 120000.0 & 75700.0 & 48000.0 & 35100.0 & 27000.0 & 22500.0 & 17500.0 & $16100 \cdot 0$ & 14100.0 & 13000.0 & 10600.0 \\
\hline 1913 & 51800.0 & 40700.0 & $32000 \cdot 0$ & 25500.0 & 19400.0 & $15800 \cdot 0$ & 13900.0 & $13500 \bullet 0$ & 13200.0 & 13000.0 & 11600.0 \\
\hline 1914 & 38700.0 & 37100.0 & 29900.0 & 24800.0 & 18100.0 & 16600.0 & 15000.0 & $14100 \cdot 0$ & 13000.0 & 11900.0 & 9390.0 \\
\hline 5 & 29100.0 & $24900 \cdot 0$ & 17800.0 & 14600.0 & $12000 \cdot 0$ & 9950.0 & 8990.0 & $8380 \cdot 0$ & 8370.0 & 7730.0 & 6930.0 \\
\hline 16 & 73200.0 & 57800.0 & 45100.0 & 34200.0 & 27400.0 & 23400.0 & 20400.0 & $19400 \cdot 0$ & 19500.0 & 18800.0 & 15100.0 \\
\hline 1940 & 41500.0 & $34200 \cdot 0$ & 30500.0 & 24900.0 & 22800.0 & 17000.0 & 13700.0 & $12800 \cdot 0$ & 11500.0 & 9910.0 & 6970.0 \\
\hline 1941 & $29000 \cdot 0$ & 24800.0 & $17000 \cdot 0$ & 13900.0 & 10400.0 & 10100.0 & 9330.0 & $8110 \cdot 0$ & 7170.0 & 6480.0 & 5400.0 \\
\hline 1942 & 54600.0 & $41600 \cdot 0$ & 30100.0 & 20300.0 & 18600.0 & 15100.0 & 13700.0 & $12000 \cdot 0$ & 10600.0 & 9570.0 & 7910.0 \\
\hline 1943 & 103000.0 & $7370 \cap .0$ & 54300.0 & 39700.0 & 29500.0 & 27500.0 & 23900.0 & $20400 \cdot 0$ & 19000.0 & 17400.0 & 13200.0 \\
\hline 1944 & 30600.0 & 22300.0 & 14300.0 & 11600 . & 8370.0 & 7540.0 & 7250.0 & 6770.0 & 6450.0 & 6460.0 & 5500.0 \\
\hline 1945 & 48400.0 & 38700.0 & 35900.0 & 25300.0 & 15900.0 & 13700.0 & 13300.0 & $12900 \cdot 0$ & 12500.0 & 1080 & 7920.0 \\
\hline 1946 & 117000.0 & 81200.0 & 49800.0 & 35300.0 & 24700.0 & 19500.0 & 17200.0 & $16200 \cdot 0$ & 14600.0 & 13500.0 & 10300.0 \\
\hline 1947 & & 0.0 & 634 & 359 & 30 & & & & 14200.0 & 12900.0 & 9850.0 \\
\hline 1948 & $117000 \cdot 0$ & 78100.0 & $51700 \cdot 0$ & $33800 \cdot 0$ & $21800 \cdot 0$ & 16800.0 & 17000.0 & $14300 \cdot 0$ & $14500 \cdot 0$ & 13600.0 & 11800.0 \\
\hline 1949 & 81700.0 & 63600.0 & 48400.0 & 3300 & 22000 & 16400.0 & 16700.0 & 145 & 13300.0 & 13600.0 & 10500.0 \\
\hline 1950 & $82300 \cdot 0$ & $57200 \cdot 0$ & $37500 \cdot 0$ & 29100.0 & 23900.0 & 21400.0 & 18900.0 & $16900 \cdot 0$ & 15700.0 & 1480 & 0.0 \\
\hline 1951 & 74500.0 & $51400 \cdot 0$ & 47400.0 & 30100.0 & 26300.0 & 21100.0 & 21000.0 & $20600 \cdot 0$ & 18400.0 & 16900.0 & 12900.0 \\
\hline 1952 & 51300.0 & 42900.0 & 37200.0 & 24500.0 & 17800.0 & 14000.0 & 14400.0 & 132 & 128 & 1230 & 10500.0 \\
\hline 1953 & 85000.0 & 71400.0 & 54700.0 & 42400.0 & 375 & 26400.0 & 20600.0 & 1710 & 15600.0 & 14100.0 & 10200.0 \\
\hline 1954 & 93900.0 & $61800 \cdot 0$ & 39000.0 & 33400 & 321 & 23400.0 & 22100.0 & & & & \\
\hline 1955 & $58000 \cdot 0$ & $43000 \cdot 0$ & $29600 \cdot 0$ & 18600.0 & 15400 & $12900 \cdot 0$ & $12100 \cdot 0$ & $10700 \cdot 0$ & 10400.0 & 104 & 9040.0 \\
\hline 1956 & 106000.0 & 77400.0 & 51000.0 & 3790 & 331 & 30400.0 & 2610 & & & 1820 & 14900.0 \\
\hline 1957 & 64000.0 & $53400 \cdot 0$ & 37500.0 & 27300.0 & 22200.0 & 16500 & 13000.0 & $13400 \cdot 0$ & $12200 \cdot 0$ & 11600.0 & 9090.0 \\
\hline 1958 & 81100.0 & 62400.0 & 41000.0 & 32400.0 & 24000.0 & 21500.0 & 18900.0 & $15800 \bullet 0$ & 14700.0 & $13200 \cdot 0$ & 9890.0 \\
\hline 1959 & 55300.0 & 43300.0 & 31100.0 & 25400.0 & 20900.0 & 17000.0 & 18000.0 & $15900 \cdot 0$ & 14800.0 & 13600.0 & 10600.0 \\
\hline & $00 \cdot 0$ & $30000 \cdot 0$ & $27200 \cdot 0$ & 20100.0 & 16700.0 & 14800.0 & 14300.0 & $13700 \cdot 0$ & $12200 \cdot 0$ & $00 \cdot 0$ & 9600.0 \\
\hline & $115000 \cdot 0$ & $83000 \cdot 0$ & 53900.0 & 40000.0 & 29700.0 & $23000 \cdot 0$ & 18800.0 & $18700 \cdot 0$ & 17600.0 & 15900.0 & 12000.0 \\
\hline & & & & & & & & 11700 & 122 & 11800.0 & \\
\hline 19 & 43900.0 & $35200 \cdot 0$ & $25200 \cdot 0$ & 23600.0 & 19700.0 & $13200 \cdot 0$ & 11600.0 & $11000 \cdot 0$ & 10900.0 & 11200.0 & 9110.0 \\
\hline
\end{tabular}

1935 NUMBER OF DAYS IN CLASS

$\begin{array}{llllllllllllllllllllllll}1935 & 41 & 43 & 15 & 16 & 9 & 15 & 12 & 2 & 11 & 6 & 22 & 32 & 31 & 25 & 23 & 10 & 13 & 19 & 10 & 10 \\ 1936 & 7 & 53 & 37 & 12 & 7 & 17 & 15 & 12 & 33 & 25 & 37 & 35 & 22 & 8 & 8 & 7 & 4 & 7 & 12 & 4\end{array}$

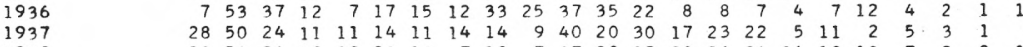

$\begin{array}{rrrrrrrrrrrrrrrrrrrrrrrrrr}1937 & 28 & 50 & 24 & 11 & 11 & 14 & 11 & 14 & 14 & 9 & 40 & 20 & 30 & 17 & 23 & 22 & 5 & 11 & 2 & 5 & 3 & 1 & 1 & 1 & 1 \\ 1938 & 20 & 54 & 24 & 13 & 12 & 20 & 16 & 7 & 10 & 7 & 17 & 20 & 19 & 20 & 26 & 24 & 14 & 18 & 10 & 7 & 2 & 2 & 1 & 1 & 1 \\ 1939 & 26 & 58 & 15 & 27 & 16 & 33 & 12 & 12 & 18 & 22 & 23 & 21 & 18 & 9 & 23 & 11 & 9 & 5 & 2 & 2 & 3 & & & & \end{array}$

1940

1941

1943

1944

1946

1947

1949

1950

1951

1952

1954

1955

1956

1958
1959

1960

1962

1962

$\begin{array}{rrrrrrrrrrrrrrrrrrrrrr}26 & 58 & 15 & 27 & 16 & 33 & 12 & 12 & 18 & 22 & 23 & 21 & 18 & 9 & 23 & 11 & 9 & 5 & 2 & 2 & 3\end{array}$

$\begin{array}{llllllllllllllllllllll}21 & 47 & 28 & 25 & 25 & 17 & 9 & 14 & 13 & 11 & 32 & 22 & 17 & 15 & 21 & 16 & 6 & 12 & 9 & 4 & 1\end{array}$

$\begin{array}{rrrrrrrrrrrrrrrrrrrrrrrr}8 & 41 & 20 & 12 & 8 & 22 & 21 & 22 & 49 & 42 & 49 & 24 & 8 & 14 & 10 & 6 & 2 & 4 & 1 & 1 & 1 & \\ 1 & 30 & 19 & 10 & 7 & 16 & 17 & 21 & 52 & 32 & 63 & 30 & 13 & 7 & 13 & 7 & 6 & 10 & 6 & 3 & 1 & 1\end{array}$

$\begin{array}{llllllllllllllllllllllllllll}2 & 56 & 17 & 19 & 12 & 12 & 12 & 11 & 22 & 23 & 39 & 31 & 13 & 12 & 20 & 13 & 13 & 12 & 9 & 8 & 6 & 1 & 2\end{array}$

$\begin{array}{rrrrrrrrrrrrrrrrrrrrrr}13 & 42 & 23 & 17 & 8 & 11 & 11 & 12 & 28 & 26 & 54 & 39 & 33 & 12 & 21 & 6 & 2 & 3 & 2 & 2 & 1 \\ & 58 & 24 & 18 & 8 & 19 & 11 & 8 & 15 & 25 & 46 & 32 & 25 & 8 & 17 & 13 & 15 & 11 & 6 & 5 & 1\end{array}$

$\begin{array}{lllllllllllllllllllllllll}3 & 48 & 29 & 11 & 15 & 31 & 30 & 12 & 11 & 7 & 16 & 14 & 20 & 18 & 29 & 14 & 15 & 16 & 11 & 11 & 2 & 1 & 1\end{array}$

$\begin{array}{rrrrrrrrrrrrrrrrrrrrrrrr}10 & 29 & 26 & 22 & 24 & 16 & 12 & 19 & 17 & 40 & 40 & 22 & 20 & 20 & 8 & 8 & 8 & 7 & 9 & 5 & & 2 & 1 \\ 25 & 27 & 25 & 14 & 11 & 9 & 6 & 10 & 14 & 27 & 38 & 42 & 25 & 33 & 18 & 14 & 13 & 4 & 8 & 2 & 1 & & \end{array}$

$\begin{array}{rllllrrrrrrrrrrrrrrrrr}51 & 27 & 14 & 15 & 23 & 10 & 11 & 31 & 33 & 27 & 23 & 16 & 12 & 19 & 11 & 11 & 8 & 6 & 6 & 6 & 4 & 1\end{array}$

$\begin{array}{rrrrrrrrrrrrrrrrrrrrrrrrrr}7 & 3 & 44 & 31 & 23 & 12 & 28 & 14 & 10 & 14 & 9 & 19 & 14 & 16 & 18 & 22 & 17 & 19 & 22 & 15 & 11 & 4 & \\ 7 & 26 & 23 & 18 & 12 & 13 & 11 & 7 & 9 & 17 & 15 & 34 & 20 & 11 & 15 & 32 & 21 & 20 & 27 & 11 & 12 & 3 & 1\end{array}$

$\begin{array}{rrrrrrrrrrrrrrrrrrrrrrrrr}18 & 21 & 39 & 8 & 5 & 10 & 26 & 12 & 10 & 12 & 13 & 22 & 19 & 27 & 35 & 38 & 18 & 9 & 8 & 5 & 7 & 4 & & \\ 20 & 8 & 40 & 34 & 16 & 13 & 12 & 8 & 11 & 10 & 10 & 50 & 30 & 14 & 11 & 15 & 12 & 5 & 10 & 17 & 12 & 1 & 1 & 2 & 1\end{array}$

$\begin{array}{rrrrrrrrrrrrrrrrrrrrr} & 10 & 35 & 32 & 29 & 32 & 22 & 23 & 14 & 16 & 15 & 14 & 15 & 33 & 18 & 12 & 17 & 7 & 14 & 6 \\ 31 & 28 & 22 & 15 & 24 & 20 & 10 & 20 & 13 & 22 & 26 & 36 & 21 & 31 & 19 & 8 & 9 & 4 & 4 & 2\end{array}$

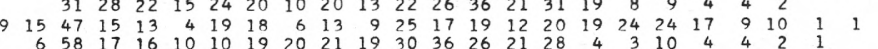

$19 \begin{array}{rrrrrrrrrrrrrrrrrrrrrr}6 & 58 & 17 & 16 & 10 & 10 & 19 & 20 & 21 & 19 & 30 & 36 & 26 & 21 & 28 & 4 & 3 & 10 & 4 & 4 & 2 & 1 \\ 19 & 38 & 23 & 18 & 18 & 12 & 15 & 14 & 10 & 4 & 25 & 26 & 24 & 14 & 23 & 11 & 10 & 14 & 9 & 10 & 2 & 1\end{array}$

$\begin{array}{rrrrrrrrrrrrrrrrrrrrrr}25 & 38 & 23 & 18 & 18 & 12 & 15 & 14 & 10 & 4 & 25 & 26 & 24 & 14 & 23 & 11 & 10 & 14 & 9 & 10 & 2 \\ 13 & 32 & 17 & 14 & 14 & 10 & 16 & 5 & 19 & 18 & 32 & 25 & 28 & 31 & 34 & 17 & 15 & 14 & 4 & 6 & 1 \\ 1 & 37 & 21 & 11 & 7 & 13 & 11 & 9 & 15 & 10 & 44 & 46 & 31 & 25 & 30 & 19 & 15 & 13 & 2 & 5 & 1\end{array}$

$\begin{array}{lllllllllllllllllllll}1 & 37 & 21 & 11 & 7 & 13 & 11 & 9 & 15 & 10 & 44 & 46 & 31 & 25 & 30 & 19 & 15 & 13 & 2 & 5 & 1\end{array}$

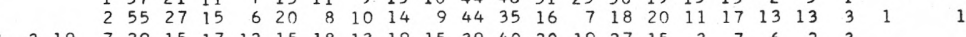

$\begin{array}{rrrrrrrrrrrrrrrrrrrrrrr}7 & 29 & 15 & 17 & 12 & 15 & 18 & 13 & 19 & 15 & 39 & 40 & 20 & 19 & 27 & 15 & 3 & 7 & 6 & 2 & 3 & \\ 1 & 39 & 22 & 22 & 17 & 27 & 12 & 9 & 19 & 11 & 45 & 43 & 23 & 12 & 25 & 12 & 9 & 5 & 6 & 4 & 1 & 1\end{array}$

CFS-DAYS 79715.0
63144.0

67503.0

95311.0

51033.0

63706.0
40200.0

40200.0
58798.0

86686.0

50424.0

66045.0

86608.0

$85859: 0$

81126.0

92693.5

78695.7

84065.0

69446.0

112133.8

74999.1

77355.4

92348.2

63451.9

64233.8

STATION NUMBER $\quad 14-1895.00$

Summary for water years 1935-63
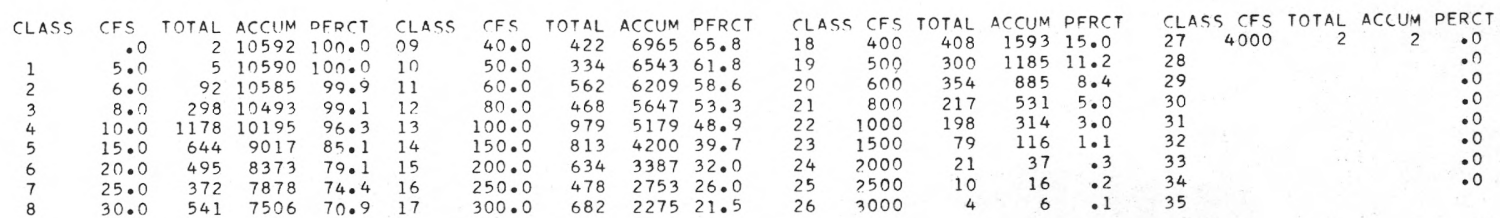
Luckiamute River near Hoskins, Oreg.

STATION NUMBER

$14-1895.00$

LOWEST MEAN DISCHARGF, IN CFS, FOR THF. FOLLOWING NUMRER OF CONSECUTIVE DAYS IN YFAR RFGINNING APRIL 1

\begin{tabular}{|c|c|c|c|c|c|c|c|c|c|c|c|}
\hline $\begin{array}{l}\text { YEAR } \\
1935\end{array}$ & $\begin{array}{l}1 \\
8.0\end{array}$ & $\begin{array}{l}3 \\
8.0\end{array}$ & $\begin{array}{l}7 \\
8.0\end{array}$ & $\begin{array}{l}14 \\
8.4\end{array}$ & $\begin{array}{l}30 \\
9.7\end{array}$ & $\begin{array}{l}60 \\
11.3\end{array}$ & $\begin{array}{l}90 \\
12.2\end{array}$ & $\begin{array}{l}120 \\
13.8\end{array}$ & $\begin{array}{l}150 \\
16.0\end{array}$ & $\begin{array}{c}183 \\
21.3\end{array}$ & $\begin{array}{c}274 \\
60.4\end{array}$ \\
\hline 1936 & 9.0 & 9.0 & 9.0 & 9.2 & 9.4 & 9.8 & 10.4 & $11 \cdot 2$ & 13.7 & 20.7 & 64.6 \\
\hline 1937 & 13.0 & 13.0 & 13.0 & 13.7 & 15.6 & 18.6 & 22.4 & 30.7 & 60.0 & 71.2 & 234.0 \\
\hline 1938 & 8.0 & 8.7 & 8.9 & 9.1 & 10.0 & 10.5 & 11.4 & 13.0 & 16.0 & 24.8 & 82.7 \\
\hline 1939 & 8.0 & $8 \cdot 0$ & 8.0 & 8.1 & 8.9 & 10.0 & 12.0 & $15 \cdot 0$ & 17.9 & 20.7 & 66.3 \\
\hline 1940 & 8.0 & $8 \cdot 3$ & 8.7 & 9.1 & 9.5 & 10.9 & 12.7 & $14 \cdot 2$ & 19.0 & $31 \cdot 2$ & 87.0 \\
\hline 1941 & 8.0 & 8.3 & 8.7 & 9.4 & 11.2 & 15.1 & 22.6 & 33.6 & 39.8 & 47.0 & 131.0 \\
\hline 1942 & 9.0 & 9.7 & 9.7 & 9.9 & 10.3 & 11.6 & 14.2 & 19.9 & 29.1 & $42 \cdot 0$ & 169.0 \\
\hline 1943 & 11.0 & 11.3 & 11.9 & 12.4 & 12.9 & 15.1 & 17.7 & $23 \cdot 6$ & 32.7 & 49.1 & 110.0 \\
\hline 1944 & 8.0 & 8.0 & 8.6 & 9.2 & 10.9 & 11.6 & 12.2 & $14 \cdot 9$ & 21.9 & 31.9 & 68.9 \\
\hline 1945 & 10.0 & 10.0 & 10.4 & 10.9 & 11.8 & 16.7 & 19.4 & $20 \cdot 5$ & 25.4 & 54.1 & 182.0 \\
\hline 1946 & 9.0 & 9.0 & 9.6 & 11.6 & 13.2 & 14.1 & $16 \cdot 3$ & $22 \cdot 9$ & 25.7 & $34 \cdot 2$ & 180.0 \\
\hline 1947 & 16.0 & 16.0 & 16.7 & 17.9 & 20.3 & 22.0 & 25.4 & $39 \cdot 1$ & 43.8 & 62.3 & 156.0 \\
\hline 1948 & 11.0 & 11.0 & 11.1 & 12.1 & 13.1 & 15.7 & 18.2 & $24 \cdot 0$ & 30.6 & $45 \cdot 1$ & 165.0 \\
\hline 1949 & 11.0 & 11.0 & 11.0 & 11.3 & 12.1 & 13.8 & 14.9 & $18 \cdot 7$ & $22 \cdot 1$ & 30.3 & 121.0 \\
\hline 1950 & 8.7 & 9.2 & 9.6 & 10.0 & 11.2 & 13.8 & 15.5 & 20.1 & 29.7 & 45.5 & 195.0 \\
\hline 1951 & $7 \cdot 3$ & 7.3 & 7.5 & 7.7 & 9.0 & 10.5 & 13.9 & $20 \cdot 3$ & 38.7 & $54 \cdot 6$ & 161.0 \\
\hline 1952 & 5.0 & $5 \cdot 3$ & 5.9 & 6.2 & 6.7 & 7.6 & 8.4 & $9 \cdot 5$ & 11.5 & $15 \cdot 0$ & 56.8 \\
\hline 1953 & 13.0 & 13.7 & 14.0 & 14.1 & 16.1 & 17.7 & 21.2 & 26.0 & 31.3 & 51.6 & 176.0 \\
\hline 1954 & 15.0 & 15.3 & 15.9 & 16.4 & 20.4 & 22.1 & 24.4 & $30 \cdot 2$ & $37 \cdot 1$ & $42 \cdot 1$ & 116.0 \\
\hline 1955 & 10.0 & 10.0 & 10.1 & 10.6 & 11.6 & 15.8 & 19.2 & $23 \cdot 5$ & 33.7 & 62.0 & 241.0 \\
\hline 1956 & 7.1 & $7 \cdot 1$ & 7.3 & 7.9 & 8.9 & 10.3 & 11.0 & $13 \cdot 8$ & 18.7 & 29.4 & 99.0 \\
\hline 1957 & 9.8 & 10.0 & 10.4 & 10.8 & 11.1 & 12.5 & 14.4 & $17 \cdot 0$ & 21.1 & 28.9 & 128.0 \\
\hline 1958 & 6.0 & 6.3 & 6.6 & $7 \cdot 1$ & 7.3 & 9.0 & 9.9 & $12 \cdot 5$ & 16.4 & 24.7 & 135.0 \\
\hline 1959 & 11.0 & 11.0 & 11.6 & 11.7 & 12.9 & 18.6 & 29.7 & $41 \cdot 6$ & 57.6 & 78.8 & 130.0 \\
\hline 1960 & 9.9 & 10.3 & 10.7 & 11.2 & 11.4 & 14.4 & 16.3 & 19.9 & $32 \cdot 3$ & 62.1 & 170.0 \\
\hline 1961 & 9.3 & 10.0 & $11 \cdot 1$ & 11.3 & 11.9 & 13.8 & 15.1 & 19.9 & 27.7 & 36.9 & 127.0 \\
\hline 1962 & 4.7 & 5.2 & 5.4 & $6 \cdot 3$ & 8.6 & 11.2 & 14.2 & 19.8 & 27.9 & 38.9 & 127.0 \\
\hline
\end{tabular}

Luckiamute River near Hoskins, Oreg.

STATION NUMBER

$14-1895.00$

HIGHEST MEAN DISCHARGE, IN CFS, FOR THE FOLLOWING NUMBER OF CONSECUTIVE DAYS IN YEAR ENDING SEPTFMBER 30

YEAR
1935
1936
1937
1938
1939
1940
1941
1942
1943
1944
1945
1946
1947
1948
194
1950
195
195
195
195
195
195
195
195
195
196
1961
196
196

1
1450.0
2620.0
2080.0
4580.0
1830.0
2120.0
1520.0
2380.0
2630.0
1550.0
1540.0
2560.0
3280.0
2240.0
4040.0
1980.0
2260.0
1910.0
3320.0
2370.0
1910.0
2850.0
2280.0
2460.0
1500.0
1830.0
3200.0
1890.0
2460.0

3
1270.0
2100.0
1760.0
3320.0
1470.0
1520.0
1180.0
1740.0
1750.0
1330.0
1280.0
1900.0
3020.0
1650.0
2680.0
1620.0
1550.0
1720.0
2850.0
1780.0
1640.0
1910.0
1700.0
1850.0
1290.0
1370.0
2110.0
1680.0
1440.0

7
1090.0
1500.0
1120.0
2200.0
1220.0
1080.0
747.0
1170.0
1400.0
873.0
1010.0
1270.0
1010.0
1080.0
1920.0
1090.0
1300.0
1480.0
2130.0
1260.0
1020.0
1280.0
1150.0
1340.0
918.0
1050.0
1380.0
1190.0
1190.0

15
846.0
1340.0
764.0
1230.0
$855 \cdot 0$
846.0
560.0
745.0
939.0
521.0
743.0
1050.0
1260.0
792.0
1400.0
946.0
1150.0
969.0
1540.0
918.0
620.0
1070.0
745.0
1050.0
622.0
848.0
1120.0
827.0
931.0

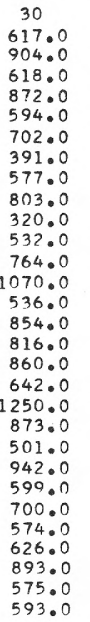

60
562.0
591.0
501.0
729.0
441.0
512.0
318.0
459.0
698.0
280.0
474.0
653.0
638.0
471.0
559.0
712.0
732.0
525.0
860.0
651.0
421.0
889.0
430.0
663.0
488.0
480.0
715.0
445.0
389.0

90
$518 \cdot 0$
$487 \cdot 0$
463.0
$624 \cdot 0$
$386 \cdot 0$
$461 \cdot 0$
$266 \cdot 0$
429.0
$624 \cdot 0$
271.0
449.0
631.0
611.0
426.0
590.0
662.0
705.0
517.0
688.0
649.0
384.0
751.0
387.0
579.0
459.0
442.0
601.0
374.0
339.0

120
$459 \cdot 0$
$415 \cdot 0$
$418 \cdot 0$
$573 \cdot 0$
$346 \cdot 0$
$421 \cdot 0$
$225 \cdot 0$
$363 \cdot 0$
$526 \cdot 0$
$253 \cdot 0$
$410 \cdot 0$
$575 \cdot 0$
$532 \cdot 0$
$386 \cdot 0$
$519 \cdot 0$
$603 \cdot 0$
$632 \cdot 0$
$461 \cdot 0$
$578 \cdot 0$
$608 \cdot 0$
$401 \cdot 0$
$684 \cdot 0$
$384 \cdot 0$
$481 \cdot 0$
$431 \cdot 0$
$388 \cdot 0$
$582 \cdot 0$
$345 \cdot 0$
$309 \cdot 0$

150
438.0
353.0

375.0

561.0

375.0

196.0

316.0

492.0

242.0

515.0

469.0

396.

458.0

550.0

577.0

423.0

493.0

549.0

374.0
633.0

633.0
340.0

444.0
408.0

308.0

367.0
538.0

333.0
319.0

$\begin{array}{ll}183 & 274 \\ 403.0 & 287.0 \\ 314.0 & 226.0 \\ 328.0 & 243.0 \\ 494.0 & 344.0 \\ 260.0 & 182.0 \\ 326.0 & 228.0 \\ 179.0 & 136.0 \\ 275.0 & 207.0 \\ 436.0 & 311.0 \\ 232.0 & 179.0 \\ 324.0 & 235.0 \\ 446.0 & 311.0 \\ 413.0 & 299.0 \\ 380.0 & 307.0 \\ 408.0 & 291.0 \\ 477.0 & 333.0 \\ 505.0 & 365.0 \\ 392.0 & 283.0 \\ 434.0 & 302.0 \\ 471.0 & 332.0 \\ 343.0 & 247.0 \\ 571.0 & 405.0 \\ 299.0 & 220.0 \\ 388.0 & 270.0 \\ 369.0 & 270.0 \\ 343.0 & 275.0 \\ 466.0 & 332.0 \\ 311.0 & 227.0 \\ 311.0 & 229.0\end{array}$


CLASS $0 \begin{array}{llllllllllllllllllllllllllllllllllll} & 0 & 2 & 3 & 4 & 5 & 6 & 7 & 8 & 9 & 10 & 11 & 12 & 13 & 14 & 15 & 16 & 17 & 18 & 19 & 20 & 21 & 22 & 23 & 24 & 25 & 26 & 27 & 28 & 29 & 30 & 31 & 32 & 33 & 34\end{array}$

YEAR
1941
1942
1943
1944
1945
1946
1947
1948
1949
1950
1951
1952
1953
1954
1955
1956
1957
1958
1959
1960
1961
1962
1963

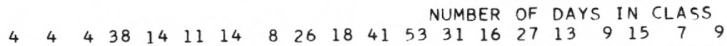

$19 \begin{array}{llllllllllllllllllllllllll} & 10 & 29 & 11 & 10 & 10 & 29 & 14 & 49 & 35 & 31 & 19 & 28 & 20 & 5 & 13 & 11 & 14 & 10 & 2 & 1 & 3 & 1 & \end{array}$

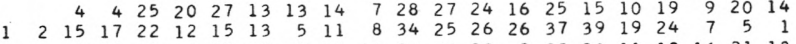

$\begin{array}{lllllllllllllll}36 & 22 & 9 & 33 & 20 & 11 & 12 & 16 & 21 & 13 & 4 & 1 & 2\end{array}$

$\begin{array}{rrrrrrrrrrrrrrrrrrrrrrrrrr}3 & 44 & 19 & 27 & 14 & 13 & 31 & 18 & 19 & 7 & 7 & 7 & 17 & 30 & 13 & 18 & 15 & 26 & 16 & 9 & 7 & 2 & 3 & & \\ & 2 & 13 & 51 & 19 & 16 & 21 & 11 & 24 & 17 & 23 & 23 & 37 & 20 & 16 & 20 & 8 & 16 & 10 & 7 & 2 & 5 & 1 & 1 & 2\end{array}$

$\begin{array}{lllllllllllllllllllllllllll}8 & 13 & 21 & 16 & 8 & 13 & 17 & 9 & 18 & 8 & 25 & 11 & 21 & 45 & 28 & 45 & 14 & 25 & 9 & 2 & 6 & 2 & & 2 & 2 \\ \end{array}$

$\begin{array}{rrrrrrrrrrrrrrrrrrrrrrrrr}19 & 22 & 16 & 18 & 13 & 15 & 28 & 8 & 21 & 34 & 21 & 17 & 22 & 24 & 9 & 16 & 13 & 20 & 7 & 6 & 2 & 6 & 2 & 3 & 2\end{array}$

$\begin{array}{rrrrrrrrrrrrrrrrrrrrrrrrrr}3 & 30 & 19 & 12 & 25 & 24 & 7 & 19 & 21 & 20 & 9 & 11 & 10 & 12 & 11 & 10 & 19 & 20 & 36 & 26 & 6 & 10 & 3 & 2 & \\ 6 & 27 & 24 & 5 & 11 & 13 & 9 & 13 & 9 & 22 & 22 & 23 & 11 & 14 & 10 & 11 & 31 & 20 & 40 & 21 & 10 & 4 & 7 & & 2\end{array}$

$\begin{array}{rrrrrrrrrrrrrrrrrrrrrrrrrrrrrrr} & 25 & 30 & 8 & 13 & 9 & 3 & 10 & 27 & 8 & 19 & 18 & 17 & 11 & 12 & 16 & 23 & 47 & 21 & 29 & 6 & 7 & & 5 & 2 & & \\ 2 & 21 & 15 & 17 & 32 & 18 & 14 & 11 & 15 & 7 & 15 & 8 & 19 & 30 & 36 & 21 & 10 & 15 & 9 & 14 & 19 & 7 & 5 & 1 & 1 & 1 & 2\end{array}$

$\begin{array}{rllllllllllllllllllllll}5 & 44 & 20 & 17 & 25 & 18 & 48 & 15 & 7 & 6 & 12 & 14 & 16 & 32 & 16 & 31 & 12 & 10 & 6 & 9 & 2\end{array}$

$\begin{array}{rrrrrrrrrrrrrrrrrrrrrrrrrrrrrrrrr}11 & 5 & 19 & 25 & 17 & 19 & 9 & 32 & 20 & 13 & 14 & 12 & 12 & 21 & 21 & 27 & 37 & 23 & 24 & 8 & 3 & 2 & 1 & 1 & & \\ 11 & 47 & 11 & 8 & 9 & 15 & 4 & 15 & 18 & 17 & 14 & 8 & 13 & 13 & 16 & 16 & 16 & 16 & 48 & 20 & 10 & 4 & 7 & 7 & 2 & 1\end{array}$

$\begin{array}{llllllllrlllllllllllllll}18 & 29 & 14 & 6 & 19 & 12 & 11 & 8 & 13 & 37 & 25 & 18 & 14 & 34 & 25 & 21 & 31 & 5 & 10 & 8 & 2 & 3 & 1 & 1\end{array}$

$\begin{array}{rrrrrrrrrrrrrrrrrrrrrrrrrrrrr}3 & 33 & 21 & 14 & 26 & 23 & 8 & 11 & 9 & 7 & 30 & 10 & 10 & 12 & 26 & 22 & 11 & 19 & 17 & 23 & 14 & 7 & 4 & 2 & 2 & 1 \\ 6 & 13 & 25 & 13 & 15 & 18 & 7 & 12 & 13 & 23 & 21 & 13 & 11 & 33 & 21 & 27 & 34 & 14 & 23 & 13 & 2 & 5 & 2 & 1 & \end{array}$

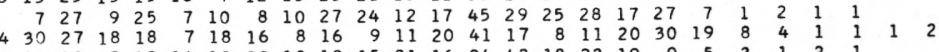

$\begin{array}{rrrrrrrrrrrrrrrrrrrrrrrrr}8 & 24 & 18 & 15 & 18 & 14 & 12 & 20 & 18 & 19 & 15 & 21 & 16 & 24 & 42 & 18 & 23 & 19 & 9 & 5 & 3 & 1 & 2 & 1 \\ & 4 & 19 & 21 & 22 & 17 & 9 & 77 & 15 & 24 & 17 & 19 & 18 & 47 & 28 & 11 & 14 & 17 & 18 & 8 & 2 & 4 & 3 & 1\end{array}$

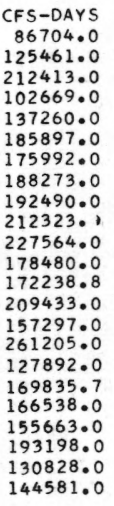

Luckiamute River at Pedee, Oreg.

STATION NUMBER $\quad 14-1900.00$

Summary for water years 1941-63

$\begin{array}{ccrrrl} & & & \\ \text { CLASS } & \text { CFS } & \text { TOTAL ACCUM } & \text { PERCT } & \text { CLASS } \\ & .0 & & 8400 & 100.0 & 09 \\ 1 & 6.0 & 1 & 8400 & 10 n .0 & 10 \\ 2 & 8.0 & 11 & 8399 & 100.0 & 11 \\ 3 & 10.0 & 161 & 8388 & 99.9 & 12 \\ 4 & 15.0 & 366 & 8227 & 97.9 & 13 \\ 5 & 20.0 & 434 & 7861 & 92.6 & 14 \\ 6 & 25.0 & 376 & 7427 & 88.4 & 15 \\ 7 & 30.0 & 478 & 7051 & 83.9 & 16 \\ 8 & 40.0 & 313 & 6573 & 78.3 & 17\end{array}$
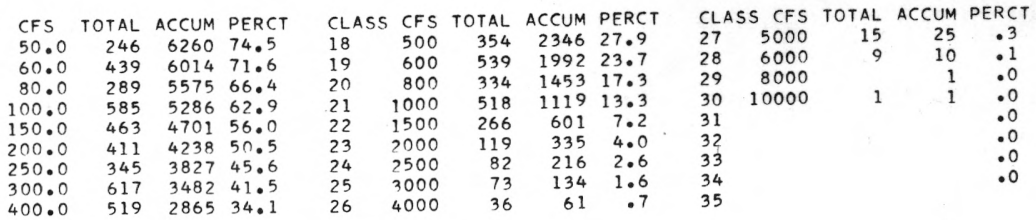

Luckiamute River at Pedee, Oreg.

STATION NUMBER 14-1900.00

LOWEST MEAN DISCHARGE, IN CFS, FOR THE FOLLOWING NUMBER OF CONSECUTIVE DAYS IN YEAR BEGINNING APRIL 1

\begin{tabular}{|c|c|c|c|c|c|c|c|c|c|c|c|}
\hline YEAR & 1 & 3 & 7 & 14 & 30 & 60 & 90 & 120 & 150 & 183 & 274 \\
\hline 1941 & 9.0 & $12 \cdot 3$ & 15.7 & $16 \cdot 6$ & 18.6 & 26.1 & 39.6 & $54 \cdot 6$ & 62.9 & 77.3 & 265.0 \\
\hline 1942 & $12 \cdot 0$ & 14.0 & 17.4 & 18.7 & 19.6 & 22.2 & 25.3 & 33.6 & 50.8 & 76.5 & 388.0 \\
\hline 1943 & 14.0 & 19.0 & 20.7 & 22.9 & 23.8 & 30.0 & 35.2 & $46 \cdot 7$ & $64.2 \%$ & 9.7 .9 & 216,0 \\
\hline 1944 & 7.0 & 9.0 & 9.9 & 13.0 & 15.7 & 17.7 & 18.4 & $24 \cdot 8$ & $39.7^{\circ}$ & 56.0 & $129: 0$ \\
\hline 1945 & 15.0 & 20.7 & 22.7 & 23.0 & 24.0 & 30.2 & 31.9 & $34 \cdot 9$ & 44.6 & 95.6 & 364.0 \\
\hline 1946 & 18.0 & 18.7 & 20.3 & 23.7 & 24.7 & 27.3 & 29.9 & 40.7 & 48.0 & 67.3 & 373.0 \\
\hline 1947 & 25.0 & 25.3 & 27.6 & 29.9 & 32.8 & 36.6 & 41.6 & $63 \cdot 1$ & 77.0 & $108 \cdot 0$ & 299.0 \\
\hline 1948 & 16.0 & 16.0 & 17.4 & 19.1 & 22.0 & 29.6 & 37.3 & $52 \cdot 2$ & 62.8 & 94.6 & 371.0 \\
\hline 1949 & 16.0 & 16.3 & 17.3 & 18.0 & 20.2 & 23.3 & $27 \cdot 6$ & $34 \cdot 5$ & 40.8 & 60.5 & 245.0 \\
\hline 1950 & 14.0 & $14 \cdot 3$ & 14.6 & 15.0 & 17.0 & 21.5 & 25.6 & $37 \cdot 4$ & 53.3 & 81.6 & 413.0 \\
\hline 1951 & 14.0 & 14.0 & 14.6 & 15.9 & 19.1 & 19.7 & 27.1 & $42 \cdot 7$ & 74.5 & 108.0 & $\begin{array}{l}352.0 \\
105.0\end{array}$ \\
\hline 1952 & 9.4 & 9.9 & 11.0 & 11.4 & 11.6 & 13.7 & 15.0 & $17 \cdot 0$ & 21.3 & 29.2 & 105.0 \\
\hline 1953 & 23.0 & 23.0 & 23.6 & 24.1 & 28.3 & 33.1 & 42.2 & $51 \cdot 3$ & 64.5 & $109 \cdot 0$ & $380 \cdot 0$ \\
\hline 1954 & 25.0 & $25 \cdot 7$ & 26.4 & 28.4 & 33.0 & 35.4 & 39.0 & $51 \cdot 4$ & 67.4 & 76.5 & 234.0 \\
\hline 1955 & 17.0 & 17.7 & 18.7 & 19.6 & 21.7 & 30.0 & 36.1 & $47 \cdot 1$ & 73.5 & 126.0 & 562.0 \\
\hline 1956 & 10.0 & 10.7 & 11.6 & 13.5 & 14.9 & 17.1 & 18.2 & 24.1 & 35.3 & 56.3 & 190.0 \\
\hline 1957 & 10.0 & 11.0 & 11.7 & 12.6 & 14.2 & 19.2 & 24.4 & $27 \cdot 7$ & 37.9 & 61.4 & 271.0 \\
\hline 1958 & 9.9 & 9.9 & 10.8 & 11.8 & 12.3 & 15.2 & 17.2 & $22 \cdot 9$ & 32.3 & 54.0 & 258.0 \\
\hline 1959 & 16.0 & 16.7 & 18.1 & 18.6 & 20.5 & 30.5 & 47.0 & 67.5 & 92.1 & 11400 & 219.0 \\
\hline 1960 & 17.0 & 17.0 & 17.3 & 18.6 & 20.7 & 26.2 & 28.7 & $35 \cdot 3$ & 52.7 & 113.0 & 333.0 \\
\hline 1961 & 13.0 & 13.3 & 14.6 & 16.9 & 18.7 & 20.2 & 23.1 & 32.5 & 45.8 & 60.0 & 246.0 \\
\hline 1962 & 11.0 & 11.7 & 12.9 & 14.1 & 17.7 & 22.0 & 27.5 & 38.5 & 54.7 & 80.9 & 259.0 \\
\hline
\end{tabular}

Luckiamute River at Pedee, Oreg.

STATION NUMBER

$14-1900.00$

HIGHEST MEAN DISCHARGE, IN CFS, FOR THE FOLLOWING NUMBER OF CONSECUTIVE DAYS IN YEAR ENDING SEPTEMBER 30

\begin{tabular}{|c|c|c|c|c|c|c|c|c|c|c|c|}
\hline YEAR & 1 & 3 & 7 & 15 & 30 & 60 & 90 & 120 & 150 & 183 & 274 \\
\hline $\begin{array}{l}1941 \\
1942\end{array}$ & 3260.0 & $2540 \cdot 0$ & 1660.0 & $1220 \cdot 0$ & 868.0 & 733.0 & $601 \cdot 0$ & $508 \cdot 0$ & $444 \cdot 0$ & $401 \cdot 0$ & 300.0 \\
\hline $\begin{array}{l}1942 \\
1943\end{array}$ & 4420.0 & $3480 \cdot 0$ & $2470 \bullet 0$ & 1680.0 & 1290.0 & 1070.0 & 964.0 & $816 \cdot 0$ & $702 \cdot 0$ & & $446 \cdot 0$ \\
\hline $\begin{array}{l}1943 \\
1944\end{array}$ & 5410.0 & $4480 . n$ & 3570.0 & 2410.0 & 2050.0 & 1780.0 & 1630.0 & $1340 \cdot 0$ & 1250.0 & 1080.0 & 765.0 \\
\hline $\begin{array}{l}1944 \\
1945\end{array}$ & 3090.0 & $2570 \cdot 0$ & $1660 \cdot 0$ & 1020.0 & 657.0 & 602.0 & 573.0 & $532 \cdot 0$ & $509 \cdot 0$ & $471 \cdot 0$ & $367 \cdot 0$ \\
\hline $\begin{array}{l}1945 \\
1946\end{array}$ & 3310.0 & 2870.0 & $2200 \cdot 0$ & $1730 \cdot 0$ & 1190.0 & 1030.0 & 995.0 & $898 \cdot 0$ & 793.0 & 687.0 & $\begin{array}{l}491.0 \\
669.0\end{array}$ \\
\hline $\begin{array}{l}1946 \\
1947\end{array}$ & 4990.0 & 3930.0 & 2770.0 & 2380.0 & $1750 \cdot 0$ & 1480.0 & 1400.0 & $1270 \cdot 0$ & $1120 \cdot 0$ & & $\begin{array}{l}669.0 \\
630.0\end{array}$ \\
\hline $\begin{array}{l}1947 \\
1948\end{array}$ & $7600 \cdot 0$ & 6700.0 & $4360 \cdot 0$ & $2720 \cdot 0$ & 2260.0 & 1360.0 & $1310 \cdot 0$ & $1150 \cdot 0$ & $1010 \cdot 0$ & $\begin{array}{l}879.0 \\
850.0\end{array}$ & $\begin{array}{l}630.0 \\
674.0\end{array}$ \\
\hline $\begin{array}{l}1948 \\
1949\end{array}$ & 5900.0 & 3890.0 & $2800 \cdot 0$ & $1990 \cdot 0$ & 1290.0 & $1120 \cdot 0$ & $\begin{array}{l}1020.0 \\
1490.0\end{array}$ & $\begin{array}{r}916 \bullet 0 \\
1280 \cdot 0\end{array}$ & $\begin{array}{r}864 \cdot 0 \\
1110.0\end{array}$ & 979.0 & $\begin{array}{l}674.0 \\
693.0\end{array}$ \\
\hline $\begin{array}{l}1949 \\
1950\end{array}$ & 10100.0 & $7630 \cdot 0$ & $5300 \cdot 0$ & $3880 \cdot 0$ & $2340 \cdot 0$ & $\begin{array}{l}141000 \\
1750.0\end{array}$ & 1630.0 & $1440 \cdot 0$ & 1290.0 & 1110.0 & 767.0 \\
\hline $\begin{array}{l}1950 \\
1951\end{array}$ & 4250.0 & $3700 \cdot n$ & 2750.0 & $\begin{array}{l}2190.0 \\
2810.0\end{array}$ & $\begin{array}{l}1880.0 \\
2010.0\end{array}$ & 1650.0 & 1610.0 & $1420 \cdot 0$ & 1330.0 & 1150.0 & 821.0 \\
\hline $\begin{array}{l}1951 \\
1952\end{array}$ & 5650.0 & 4230.0 & $\begin{array}{l}3460.0 \\
3070.0\end{array}$ & $\begin{array}{l}2810.0 \\
2090.0\end{array}$ & $\begin{array}{l}2010.0 \\
1480.0\end{array}$ & 1220.0 & 1230.0 & $1090 \cdot 0$ & 996.0 & 899.0 & 644.0 \\
\hline $\begin{array}{l}1952 \\
1953\end{array}$ & 4310.0 & 3640.0 & 3070.0 & 3270.0 & 2560.0 & 1760.0 & 1420.0 & 1170.0 & 1010.0 & 886.0 & 620.0 \\
\hline $\begin{array}{l}1953 \\
1954\end{array}$ & 7240.0 & 6200.0 & 4630.0 & $3<100$ & & 1530.0 & 1500.0 & $1380 \cdot 0$ & 1240.0 & 1070.0 & 749.0 \\
\hline 1954 & 4410.0 & 3630.0 & 2860.0 & 2120.0 & 1900.0 & 1300 & & 934 & 868.0 & 787.0 & $\begin{array}{l}149.0 \\
563.0\end{array}$ \\
\hline 1955 & 4470.0 & 3510.0 & 2240.0 & $1400 \cdot 0$ & $1170 \cdot 0$ & 1010.0 & $905 \cdot 0$ & $934 \cdot 0$ & 1530.0 & 1350.0 & $\begin{array}{l}563.0 \\
946.0\end{array}$ \\
\hline 1956 & 7450.0 & $5000 \cdot 0$ & 3410.0 & 2760.0 & 2470.0 & 2240.0 & $1820 \cdot 0$ & $\begin{array}{r}1710 \cdot 0 \\
812 \cdot 0\end{array}$ & $\begin{array}{r}1530.0 \\
721.0\end{array}$ & $\begin{array}{r}625.0 \\
\end{array}$ & $\begin{array}{l}946.0 \\
459.0\end{array}$ \\
\hline 1957 & 4590.0 & 3280.0 & 2260.0 & 1590.0 & 1320.0 & 939.0 & $805 \cdot 0$ & $812 \cdot 0$ & 1030.0 & 882.0 & $\begin{array}{l}459.0 \\
614.0\end{array}$ \\
\hline 1958 & 5260.0 & 4470.0 & 3160.0 & 2440.0 & 1690.0 & 1590.0 & $1380 \cdot 0$ & $1140 \cdot 0$ & 920.0 & 821.0 & \\
\hline 1959 & 4200.0 & 3330.0 & 2710.0 & 1740.0 & 1530.0 & 1270.0 & 1040.0 & $977 \cdot 0$ & 781.0 & 724.0 & 557.0 \\
\hline 1960 & 4960.0 & 3660.0 & 2650.0 & $2000 \cdot 0$ & $1430 \cdot 0$ & $1080 \cdot 0$ & $974 \cdot 0$ & $843 \cdot 0$ & 1140.0 & & 697.0 \\
\hline 1961 & 6680.0 & 4710.0 & 3420.0 & 2510.0 & 2030.0 & 1590.0 & 1320.0 & $\begin{array}{r}1240 \cdot 0 \\
738.0\end{array}$ & 696.0 & 652.0 & 469.0 \\
\hline 1962 & 4210.0 & $3700 \cdot 0$ & $\begin{array}{l}2590.0 \\
2340.0\end{array}$ & $\begin{array}{l}1740.0 \\
1940.0\end{array}$ & $\begin{array}{l}1180.0 \\
1240.0\end{array}$ & $\begin{array}{l}868.0 \\
826.0\end{array}$ & 742.0 & $\begin{array}{l}738 \cdot 0 \\
684 \cdot 0\end{array}$ & 721.0 & 705.0 & 527.0 \\
\hline 1963 & 4270.0 & 3060.0 & 2340.0 & 1940.0 & 1240.0 & 826.0 & $741 \cdot 0$ & $684 \cdot 0$ & & & \\
\hline
\end{tabular}


Luekiamute River near Suver, Oreg.

STATION NUMBER $\quad 14-1905.00$

DURATION TABLE OF DAILY DISCHARGF

CLASS $0 \begin{array}{lllllllllllllllllllllllllllllllllll} & 0 & 2 & 3 & 4 & 5 & 6 & 7 & 8 & 9 & 10 & 11 & 12 & 13 & 14 & 15 & 16 & 17 & 18 & 19 & 20 & 21 & 22 & 23 & 24 & 25 & 26 & 27 & 28 & 29 & 30 & 31 & 32 & 33 & 34\end{array}$

$\begin{array}{lrrrrrrrrrrrrrrrrrrrrrrr}\text { NEAR } & & 2 & 12 & 16 & 17 & 34 & 25 & 20 & 19 & 44 & 29 & 26 & 25 & 26 & 21 & 12 & 8 & 8 & 8 & 2 & 7 & 3 \\ 1906 & 2 & 3 & 33 & 19 & 18 & 14 & 21 & 17 & 16 & 15 & 19 & 9 & 6 & 20 & 25 & 47 & 26 & 18 & 4 & 7 & 9 & 4 & 6\end{array}$

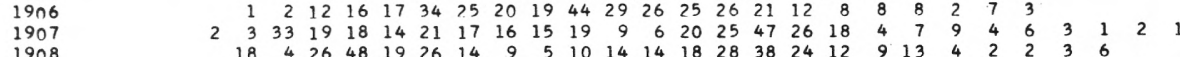

$\begin{array}{lrrrrrrrrrrrrrrrrrrrrrrrrrrrrr}1908 & 18 & 4 & 26 & 48 & 19 & 26 & 14 & 9 & 5 & 10 & 14 & 14 & 18 & 28 & 38 & 24 & 12 & 9 & 13 & 4 & 2 & 2 & 3 & 6 & \\ 1909 & 3 & 2 & 24 & 43 & 12 & 24 & 19 & 25 & 23 & 39 & 16 & 15 & 15 & 12 & 24 & 19 & 15 & 14 & 10 & 2 & 2 & 2 & 1 & 2 & 2\end{array}$

$\begin{array}{lllllllllllllllllllllllllllllll}1910 & 6 & 18 & 24 & 8 & 30 & 14 & 39 & 20 & 9 & 9 & 12 & 4 & 5 & 25 & 23 & 27 & 16 & 16 & 17 & 11 & 11 & 3 & 5 & 4 & 7 & 2 \\ 1911 & & & 1 & 1 & 19 & 45 & 36 & 26 & 13 & 4 & 15 & 29 & 25 & 39 & 21 & 38 & 22 & 9 & 5 & 6 & 3 & 5 & & 1 & 1 & 1\end{array}$

$\begin{array}{llllllllllllllllllllllllll}1911 & 7 & & 1 & 1 & 19 & 45 & 36 & 26 & 13 & 4 & 15 & 29 & 25 & 39 & 21 & 38 & 22 & 9 & 5 & 6 & 3 & 5 & \\ 1941 & & 75 & 23 & 8 & 12 & 11 & 34 & 22 & 23 & 24 & 42 & 28 & 19 & 26 & 8 & 17 & 15 & 4 & 3 & 1 & 1 & 1 & 1\end{array}$

$\begin{array}{rrrrrrrrrrrrrrrrrrrrrrrrrrrr}1942 & 2 & 17 & 25 & 5 & 14 & 13 & 39 & 18 & 19 & 29 & 32 & 25 & 22 & 22 & 17 & 18 & 14 & 9 & 9 & 7 & 4 & 1 & 4 & \\ 1943 & 1 & 22 & 34 & 13 & 24 & 12 & 15 & 13 & 16 & 10 & 27 & 19 & 14 & 26 & 14 & 25 & 17 & 8 & 14 & 11 & 7 & 9 & 9 & 2 & 3\end{array}$

$\begin{array}{llllllllllllllllllllllll}1944 & 1 & 8 & 31 & 32 & 13 & 14 & 6 & 16 & 13 & 15 & 17 & 30 & 22 & 20 & 37 & 30 & 45 & 9 & 3 & & 2 & 1 & 1\end{array}$

1945

1946

1947
1948

1949
1950

1951

1952

1954

1955

1956

1958

1959

1960

1962

$\begin{array}{rrrrrrrrrrrrrrrrrrrrrrr}32 & 26 & 26 & 23 & 10 & 19 & 10 & 14 & 7 & 33 & 18 & 17 & 32 & 19 & 24 & 15 & 13 & 11 & 11 & 3 & 1\end{array}$

$\begin{array}{rrrrrrrrrrrrrrrrrrrrrrrr}39 & 34 & 13 & 16 & 10 & 39 & 20 & 11 & 7 & 8 & 4 & 8 & 14 & 23 & 38 & 19 & 15 & 13 & 12 & 13 & 4 & 3 & 1 & 1\end{array}$

$\begin{array}{rrrrrrrrrrrrrrrrrrrrrrrr}11 & 15 & 35 & 26 & 37 & 16 & 21 & 14 & 12 & 12 & 12 & 18 & 22 & 36 & 16 & 34 & 17 & 9 & 4 & 10 & 7 & 4 & 3 & 2 \\ & 15 & 21 & 24 & 9 & 18 & 14 & 20 & 37 & 79 & 23 & 10 & 10 & 8 & 5 & 3 & 3 & 2\end{array}$

$\begin{array}{lllllllllllllllllllllllllllll}23 & 26 & 18 & 19 & 18 & 30 & 12 & 14 & 10 & 33 & 14 & 15 & 22 & 19 & 32 & 18 & 6 & 6 & 7 & 7 & 4 & 3 & 4 & 4 & 1\end{array}$

$\begin{array}{llllllllllllllllllllllllll}8 & 42 & 17 & 23 & 25 & 9 & 22 & 19 & 11 & 6 & 9 & 9 & 10 & 7 & 16 & 29 & 22 & 18 & 16 & 27 & 11 & 4 & 3 & \text { ? }\end{array}$

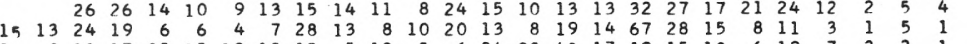

$\begin{array}{llllllllllllllllllllllllllll}21 & 3 & 11 & 17 & 35 & 15 & 19 & 12 & 15 & 5 & 12 & 5 & 6 & 24 & 23 & 40 & 17 & 19 & 15 & 10 & 6 & 19 & 7 & 3 & 2 & 1 & 3\end{array}$ $\begin{array}{llllllllllllllllllllllll}6 & 30 & 26 & 14 & 35 & 29 & 25 & 16 & 13 & 7 & 5 & 15 & 16 & 35 & 22 & 18 & 10 & 15 & 12 & 5 & 10 & 1\end{array}$

$\begin{array}{rrrrrrrrrrrrrrrrrrrrrrrrrrrrr}6 & 22 & 11 & 12 & 21 & 16 & 34 & 26 & 7 & 7 & 16 & 10 & 9 & 26 & 35 & 44 & 32 & 14 & 7 & 5 & 3 & 1 & 1 & \\ 33 & 25 & 7 & 10 & 14 & 5 & 18 & 23 & 9 & 4 & 12 & 9 & 9 & 19 & 21 & 24 & 18 & 18 & 24 & ? 3 & 9 & 7 & 10 & 2 & 4\end{array}$

$\begin{array}{llllllllllllllllllllllllll}1 & 23 & 32 & 14 & 14 & 13 & 9 & 13 & 33 & 16 & 14 & 22 & 14 & 13 & 43 & 27 & 33 & 6 & 6 & 3 & 8 & 5 & 2 & 1\end{array}$

$\begin{array}{rrrrrrrrrrrrrrrrrrrrrrrrrrrrrrr}17 & 21 & 26 & 20 & 19 & 20 & 10 & 16 & 18 & 15 & 8 & 11 & 9 & 12 & 27 & 17 & 25 & 20 & 12 & 8 & 14 & 9 & 6 & 4 & 1 \\ 14 & 33 & 13 & 9 & 19 & 8 & 20 & 9 & 18 & 12 & 16 & 14 & 14 & 34 & 26 & 46 & 16 & 15 & 7 & 12 & 3 & 4 & 2 & 1\end{array}$

$\begin{array}{llllllllllllllllllllll}34 & 16 & 11 & 13 & 8 & 17 & 17 & 13 & 13 & 20 & 18 & 16 & 45 & 30 & 36 & 20 & 13 & 11 & 10 & 2 & 2 & 1\end{array}$

$\begin{array}{lllllllllllllllllllllllllll}16 & 50 & 17 & 13 & 9 & 14 & 19 & 11 & 9 & 6 & 7 & 13 & 24 & 38 & 14 & 14 & 18 & 12 & 15 & 26 & 8 & 5 & 4 & 1 & 2\end{array}$

$\begin{array}{rrrrrrrrrrrrrrrrrrrrrrrrrr}11 & 10 & 34 & 13 & 12 & 15 & 11 & 29 & 19 & 8 & 8 & 18 & 20 & 16 & 29 & 30 & 31 & 26 & 10 & 2 & 7 & 3 & 1 & 1 & 1 \\ & & 22 & 21 & 15 & 17 & 11 & 33 & 14 & 8 & 16 & 18 & 20 & 23 & 34 & 30 & 25 & 21 & 12 & 7 & 7 & 6 & 2 & 3 & \end{array}$

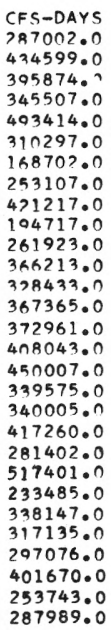

Luckiarnute River near Suver, Oreg.

STATION NUMBER $\quad 14-1905.00$

surmary for water years 1906-11, 1941-63
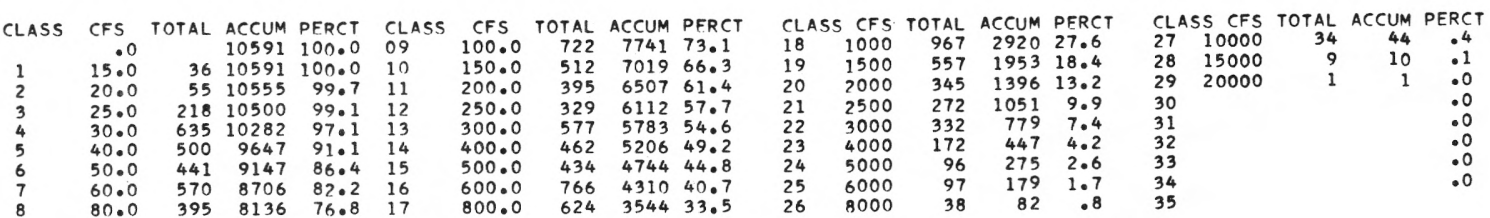

Lucklamute River near Suver, Oreg.

CTATION NUMBER $14-1905 \cdot 00$

LAWEST MEAN DISCHARGE, IN CFS, FOR THF FOLLOWING NUMRER OF CONSFCUTIVF DAYG IN YFAR RFGINNING ADRIL 1

\begin{tabular}{|c|c|c|c|c|c|c|c|c|c|c|c|}
\hline YEAR & 1 & 3 & 7 & 14 & 30 & 60 & 90 & 120 & 150 & 183 & 274 \\
\hline 1906 & 37.0 & 45.7 & 51.1 & 53.1 & 66.1 & 83.5 & 101.0 & $159 \cdot 0$ & $221 \cdot 0$ & 246.0 & 749.0 \\
\hline $\begin{array}{l}19 \cap 7 \\
1908\end{array}$ & 26.0 & 27.3 & 32.7 & 33.9 & 44.6 & 51.4 & 50.6 & $56 \cdot 7$ & 66.6 & 95.0 & 847.0 \\
\hline $\begin{array}{l}1908 \\
1909\end{array}$ & 44.0 & 49.0 & $51 \cdot 3$ & 54.6 & 61.5 & 69.2 & 72.1 & $94 \cdot 5$ & $128 \cdot 0$ & $171 \cdot n$ & 422.0 \\
\hline $\begin{array}{l}1909 \\
1910\end{array}$ & 37.0 & 37.0 & 45.0 & 51.1 & 57.3 & 64.4 & 70.1 & $91 \cdot 6$ & 119.0 & $151 \cdot 0$ & 956.0 \\
\hline $\begin{array}{l}1910 \\
1941\end{array}$ & 26.0 & 26.0 & 27.1 & 30.6 & 34.0 & 44.9 & 54.9 & 70.4 & 96.8 & $117 \cdot 0$ & 577.0 \\
\hline 1941 & 28.0 & 28.3 & 29.3 & 30.3 & 33.8 & 45.3 & 69.4 & $92 \cdot 2$ & 106.0 & 132.0 & 510.0 \\
\hline $\begin{array}{l}1942 \\
1943\end{array}$ & 26.0 & 30.0 & 30.7 & 32.4 & 34.3 & 38.8 & 45.3 & 61.0 & 92.8 & 140.0 & $745 \cdot 0$ \\
\hline 1943 & $33 . n$ & 33.0 & 35.4 & 37.9 & $39 . ?$ & 48.7 & 56.4 & $78 \cdot 0$ & 112.0 & 178.0 & 392.0 \\
\hline $\begin{array}{l}1944 \\
1945\end{array}$ & 24.0 & 25.0 & 26.6 & 28.8 & 33.1 & 36.6 & 38.3 & $45 \cdot 3$ & 60.7 & $105 \cdot n$ & 249.0 \\
\hline $\begin{array}{l}1945 \\
1946\end{array}$ & 33.0 & 35.7 & 37.9 & 38.6 & 41.9 & 53.5 & 56.2 & $59 \cdot 7$ & $75 \cdot 6$ & $171 \cdot 0$ & 705.0 \\
\hline 1946 & $33 . n$ & 33.3 & 34.6 & 36.2 & 37.4 & 45.1 & 49.4 & $68 \cdot 7$ & 85.1 & $126 \cdot n$ & 679.0 \\
\hline $\begin{array}{l}1947 \\
1948\end{array}$ & 46.0 & 47.0 & 49.1 & 52.0 & 59.7 & 64.6 & 72.6 & $106 \cdot 0$ & 132.0 & $197 \cdot 0$ & 553.0 \\
\hline 1948 & 33.0 & 33.7 & 34.9 & 37.3 & 42.6 & 52.9 & 64.2 & $86 \cdot 7$ & $106 \cdot 0$ & $170 \cdot 0$ & 699.0 \\
\hline 19.49 & 32.0 & 32.0 & 33.1 & 34.4 & 37.9 & 43.7 & 50.3 & $61 \cdot 1$ & 71.6 & 109.0 & 441.0 \\
\hline 1950 & 29.0 & 29.0 & 29.4 & 29.6 & 31.4 & 36.4 & 41.6 & 60.7 & $9 n .5$ & 148.0 & $828 . n$ \\
\hline 1951 & 25.0 & 25.0 & 25.7 & $26 \cdot 6$ & 31.1 & 32.4 & 43.4 & $68 \cdot 6$ & 126.0 & $188 \cdot 0$ & 650.0 \\
\hline 1952 & 15.0 & 15.7 & 16.3 & 17.3 & 17.3 & 20.7 & 22.9 & $25 \bullet 9$ & 34.4 & 49.6 & 207.0 \\
\hline 1953 & 36.0 & 36.7 & 37.7 & 40.4 & 49.1 & 53.9 & 69.7 & 88.7 & 112.0 & 195.0 & 713.0 \\
\hline 1954 & 44.0 & 45.7 & 48.4 & 49.9 & 55.1 & 61.9 & 67.7 & $91 \cdot 4$ & 122.0 & 141.0 & 435.0 \\
\hline 1955 & 26.0 & 26.0 & 27.3 & 29.6 & 32.2 & 51.4 & 65.0 & $85 \cdot 8$ & $142 . ?$ & 237.0 & 1050.0 \\
\hline 1956 & $2 n .0$ & 21.0 & 23.1 & 24.8 & 25.8 & $3 n .6$ & 33.0 & $43 \cdot 7$ & 64.5 & $106 \cdot n$ & 328.0 \\
\hline 1957 & 24.0 & 25.3 & 25.9 & 26.9 & 27.9 & 34.3 & 40.7 & $49 \cdot 1$ & 66.0 & $105 \cdot n$ & 491.0 \\
\hline 1958 & 23.0 & 23.0 & 23.1 & 23.7 & 24.5 & 29.4 & 32.6 & $41 \cdot 4$ & 58.0 & 97.6 & $480 . n$ \\
\hline 1959 & 27.0 & 28.3 & 30.4 & 30.6 & 31.8 & 47.4 & 76.0 & $118 \cdot 0$ & 159.0 & 190.0 & 376.0 \\
\hline 1960 & 30.0 & 31.3 & 31.9 & 33.9 & 36.1 & 42.5 & 46.8 & 59.5 & 91.5 & 197.0 & 645.0 \\
\hline 1961 & 25.0 & 25.0 & 26.6 & 28.4 & 29.9 & 33.3 & 36.7 & $51 \cdot 6$ & 77.5 & 103.0 & 467.0 \\
\hline 1962 & 21.0 & 21.7 & 22.4 & 23.1 & 28.1 & 35.5 & 46.5 & $68 \cdot 4$ & 111.0 & 159.0 & 509.0 \\
\hline
\end{tabular}


Luckiamute River near Suver, Oreg.

STATION NUMRER $14-1905.00$

HIGHEST MEAN DISCHARGE, IN CFS, FOR THE FOLLOWING NUMRFR OF CONSFCUTIVF DAYC IN YFAR ENDING SEPTFMRFR 30

\begin{tabular}{|c|c|c|c|c|c|c|c|c|c|c|c|}
\hline YEAR & 1 & 3 & 7 & 15 & 30 & $6 n$ & 90 & 120 & $15 n$ & 183 & 274 \\
\hline $\begin{array}{l}1906 \\
j 907\end{array}$ & 6590.0 & $6250 . n$ & 6030.0 & $4330 . n$ & $2600 \cdot 0$ & 2470.0 & $215 n .0$ & $1800 \cdot 0$ & 1530.0 & 1320.0 & $\ln 1 \cap .0$ \\
\hline $\begin{array}{l}19 n 7 \\
1908\end{array}$ & 2nonnen & $137 n n \cdot n$ & $9 n 7 n \cdot n$ & $5690 \cdot n$ & 3570.0 & $338 n . n$ & $3 n 1 n \cdot 0$ & $27 n n \cdot n$ & $24 n n \cdot n$ & $222 n \cdot n$ & $1570 . n$ \\
\hline $19 \cap 8$ & 14200.0 & $11100 \cdot n$ & 10300.0 & 6940.0 & 5110.0 & $363 n .0$ & 2930.0 & 2690.0 & 2320.0 & $2 n 4 n .0$ & 1420.0 \\
\hline 1909 & $18000 \cdot 0$ & $16100 \cdot 0$ & $11300 \cdot 0$ & 6440.0 & 4410.0 & 3590.0 & 2920.0 & $2400 \cdot 0$ & 2030.0 & 1730.0 & 1240.0 \\
\hline 1910 & 19000.0 & $15200 \cdot 0$ & $10200 \cdot n$ & 7330.0 & 5650.0 & 3670.0 & 3150.0 & 3420.0 & 3000.0 & $2590 . n$ & 1780.0 \\
\hline 1911 & 17500.0 & 12500.0 & 7090.0 & 4200.0 & 2860.0 & 2330.0 & 2260.0 & $1960 \cdot 0$ & $1680 \cdot 0$ & 1480.0 & 1100.0 \\
\hline $\begin{array}{l}1941 \\
1942\end{array}$ & 6290.0 & 5240.0 & 3520.0 & $2580 \cdot 0$ & 1830.0 & 1530.0 & 1220.0 & $1020 \cdot 0$ & 889.0 & 797.0 & 589.0 \\
\hline $\begin{array}{l}1942 \\
1943\end{array}$ & 7740.0 & $6690 . n$ & 5050.0 & 3470.0 & 2650.0 & 2260.0 & 1980.0 & $1680 \cdot 0$ & 1440.0 & $1240 \cdot 0$ & anz. 0 \\
\hline $\begin{array}{l}1943 \\
1944\end{array}$ & $12500 \cdot 0$ & $8520 \cdot n$ & 7290.0 & 4990.0 & 3960.0 & 3530.0 & 3230.0 & $2650 \cdot 0$ & 2500.0 & $216 n \cdot 0$ & $1520 . n$ \\
\hline $\begin{array}{l}1944 \\
1945\end{array}$ & 5420.0 & $4560 . n$ & 3060.0 & 1880.0 & 1300.0 & 1170.0 & 1130.0 & $1050 \cdot 0$ & 1000.0 & 909.0 & 696.0 \\
\hline $\begin{array}{l}1945 \\
1946\end{array}$ & $6900 \cdot 0$ & $5610 \cdot n$ & $433 n \cdot n$ & 3340.0 & 2330.0 & $200 n \cdot n$ & 1900.0 & $1710 \cdot n$ & 1510.0 & 1310.0 & 938.0 \\
\hline 1946 & 10700.0 & $8 \cap 20 \cdot n$ & $579 n \cdot 0$ & 4840.0 & 3420.0 & $296 n . n$ & $277 n .0$ & $252 n \cdot 0$ & 2220.0 & 1910.0 & 1320.0 \\
\hline $\begin{array}{l}1947 \\
1948\end{array}$ & 17600.0 & $124 n 0 \cdot n$ & 7940.0 & $4850 \cdot 0$ & 4090.0 & 2480.0 & 2450.0 & $2170 \cdot 0$ & 1910.0 & $165 n \cdot n$ & 1180.0 \\
\hline 1948 & $8800 \cdot 0$ & $7780 . n$ & $576 n \cdot n$ & 4080.0 & 2620.0 & 2260.0 & 2040.0 & $1810 \bullet n$ & $17 n n \cdot 0$ & $166 n \cdot n$ & $132 n .0$ \\
\hline $\begin{array}{l}1949 \\
1950\end{array}$ & $188 \cap 0 \cdot n$ & 15300.0 & $1 \cap 8 \cap n \cdot n$ & $774 n .0$ & 4690.0 & $286 n . n$ & 2920.0 & 2520.0 & 2190.0 & $191 n_{0} n$ & 1340.0 \\
\hline $\begin{array}{l}1950 \\
1951\end{array}$ & 8650.0 & $7470 . n$ & $5570 \cdot 0$ & 4140.0 & $362 n \cdot 0$ & 3450.0 & 3160.0 & $2790 \cdot 0$ & 2500.0 & $\begin{array}{l}2140 \cdot n \\
2290 \cdot 0\end{array}$ & $\begin{array}{l}1480.0 \\
1630.0\end{array}$ \\
\hline $\begin{array}{l}1951 \\
1952\end{array}$ & 9670.0 & $8010 \cdot n$ & 6510.0 & 5430.0 & 4040.0 & 3300.0 & 3280.0 & $289 n \cdot 0$ & 1910.0 & & $\begin{array}{l}1630.0 \\
1230.0\end{array}$ \\
\hline $\begin{array}{l}1952 \\
1953\end{array}$ & 8760.0 & 7460.0 & 6130.0 & 4110.0 & 2910.0 & 2390.0 & 2380.0 & $2100 \cdot 0$ & $\begin{array}{l}1910 \cdot 0 \\
2010.0\end{array}$ & $\begin{array}{l}170.0 \\
1760 \cdot 0\end{array}$ & $\begin{array}{l}1230.0 \\
1230.0\end{array}$ \\
\hline $\begin{array}{l}1953 \\
1954\end{array}$ & 13800.0 & $11700 \cdot 0$ & $904 n \cdot n$ & $\begin{array}{l}6540.0 \\
4330.0\end{array}$ & $\begin{array}{l}5100.0 \\
3670.0\end{array}$ & $\begin{array}{l}3540.0 \\
3100.0\end{array}$ & 2840.0 & $\begin{array}{l}2350 \cdot 0 \\
280000\end{array}$ & 2500.0 & 2140.0 & $\begin{array}{l}1230.0 \\
1500.0\end{array}$ \\
\hline $\begin{array}{l}1954 \\
1955\end{array}$ & 8120.0 & $652 n \cdot n$ & $\begin{array}{l}521 n \cdot n \\
362 n \cdot 0\end{array}$ & $\begin{array}{l}433 n \cdot n \\
2330.0\end{array}$ & $\begin{array}{l}3670.0 \\
2090.0\end{array}$ & $\begin{array}{l}3100.0 \\
1780.0\end{array}$ & $\begin{array}{l}3050.0 \\
1580.0\end{array}$ & $\begin{array}{l}280 n \bullet 0 \\
1620 \cdot 0\end{array}$ & 1530.0 & 1400.0 & 1010.0 \\
\hline $\begin{array}{l}1955 \\
1956\end{array}$ & 6140.0 & $\begin{array}{r}5270.0 \\
10800.0\end{array}$ & $\begin{array}{l}362 n \cdot 0 \\
787 n .0\end{array}$ & 6310.0 & 5530.0 & $466 n .0$ & 3710.0 & 3440.0 & $307 n .0$ & 2680.0 & 1880.0 \\
\hline $\begin{array}{l}1956 \\
1957\end{array}$ & $\begin{array}{r}14000.0 \\
6250.0\end{array}$ & $5410 . n$ & $4320 . n$ & 3380.0 & 2730.0 & 1880.0 & 151000 & 1500.0 & 1330.0 & 1150.0 & 839.0 \\
\hline 1958 & 9270.0 & 7540 . n & 5860.0 & $4640 . n$ & 3590.0 & $320 n .0$ & 2790.0 & $2300 \cdot 0$ & $206 n \cdot 0$ & 1760.0 & 1220.0 \\
\hline 1959 & 8110.0 & $6780 . n$ & $5650 . n$ & 3610.0 & 3090.0 & $253 n .0$ & 2060.0 & 1890.0 & $178 n .0$ & 1580.0 & $113 n .0$ \\
\hline 1960 & 7750.0 & $59300 n$ & 4810.0 & 3810.0 & 2850.0 & 2180.0 & 1980.0 & $1690 \cdot 0$ & 1550.0 & $1410 . n$ & 1060.0 \\
\hline 1961 & 13700.0 & 9190.0 & 7190.0 & 5300.0 & 4310.0 & 3430.0 & 2850.0 & $2620 \cdot 0$ & 2400.0 & 2080.0 & 1450.0 \\
\hline 1962 & 8200.0 & 6710.0 & 4960.0 & 3420.0 & 2320.0 & 1660.0 & 1470.0 & 1470.0 & 1370.0 & 1280.0 & 912.0 \\
\hline 1963 & 6860.0 & 6000.0 & 4370.0 & 3630.0 & 2390.0 & 1660.0 & 1480.0 & 1370.0 & 1430.0 & 1400.0 & 1030.0 \\
\hline
\end{tabular}

Rickreall Creek near Dallas, Oreg. STATION NUMBER 14-1907.00

DURATION TABLE OF DAILY DISCHARGE

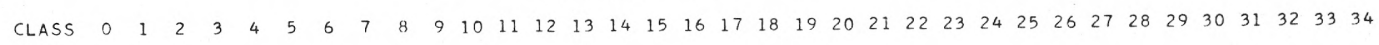

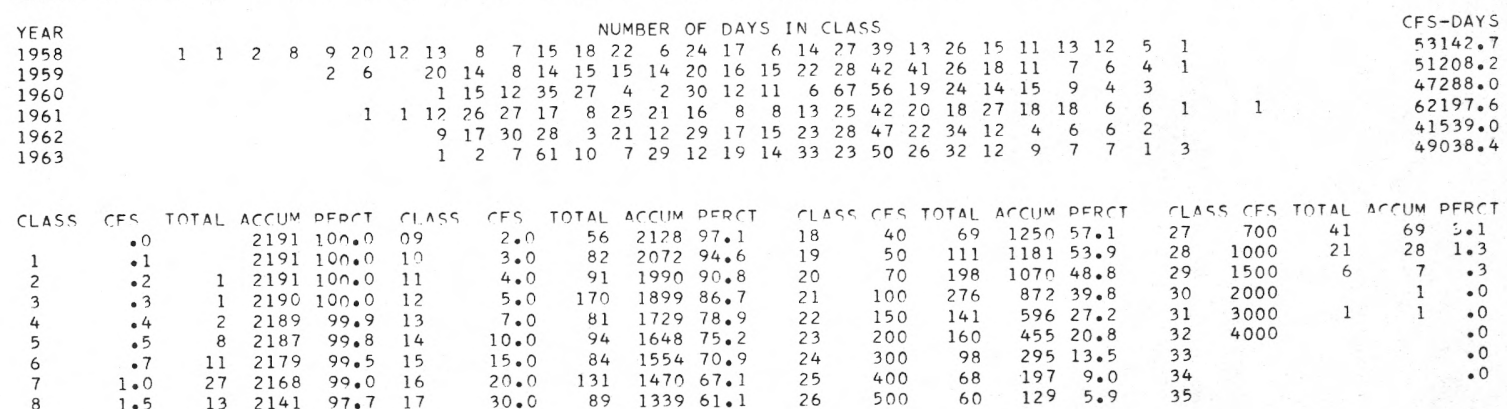

Rickreall Creek near Dallas, Oreg.

STATION NUMBER $\quad 14-1907.00$

LOWEST MEAN DISCHARGF, IN CFS, FOR THE FOLLOWING NUMBER OF CONSECUTIVE DAYS IN YEAR BEGINNING APRIL 1

\begin{tabular}{|c|c|c|c|c|c|c|c|c|c|c|c|}
\hline YEAR & 1 & 3 & 7 & 14 & 30 & 60 & 90 & 120 & 150 & 183 & 274 \\
\hline 1958 & .2 & .5 & .5 & .7 & .8 & 1.4 & 1.7 & 3.5 & 6.4 & 12.1 & 78.7 \\
\hline 1959 & .8 & $2 \cdot 3$ & 2.6 & 2.8 & 2.9 & 6.0 & 9.7 & 16.8 & 23.8 & 27.4 & 57.5 \\
\hline 1960 & 1.4 & 3.2 & 3.6 & 3.8 & 4.3 & 5.3 & 6.0 & 7.6 & 13.8 & 29.0 & 106.0 \\
\hline 1961 & 1.9 & 2.4 & 2.7 & 3.0 & 3.1 & 3.7 & $\begin{array}{l}4.1 \\
5.5\end{array}$ & $\begin{array}{l}6.4 \\
8.5\end{array}$ & $\begin{array}{l}10.2 \\
19.7\end{array}$ & $\begin{array}{l}14.7 \\
32.5\end{array}$ & $\begin{array}{l}76.6 \\
97.7\end{array}$ \\
\hline 1962 & 2.0 & $2 \cdot 3$ & 2.8 & 3.5 & & & & & & & \\
\hline
\end{tabular}

Rickeall Creek near Dallas, Oreg.

STATION NUMBER $\quad 14-1907.00$

HIGHEST MEAN DISCHARGE, IN CFS, FOR THE FOLLOWING NUMBER OF CONSECUTIVE DAYS IN YEAR FNDING SEPTEMBER 30

\begin{tabular}{|c|c|c|c|c|c|c|c|c|c|c|c|}
\hline YEAR & 1 & 3 & 7 & 15 & 30 & 60 & 90 & 120 & 150 & 183 & 274 \\
\hline 1958 & 1740.0 & 1220.0 & 903.0 & 711.0 & $551 \cdot 0$ & 513.0 & 436.0 & $358 \cdot 0$ & 322.0 & 280.0 & 193.0 \\
\hline $\begin{array}{l}1959 \\
1960\end{array}$ & 1900.0 & 1430.0 & 1110.0 & 653.0 & 527.0 & 409.0 & 332.0 & $310 \cdot 0$ & 290.0 & 257.0 & 183.0 \\
\hline $\begin{array}{l}1960 \\
1961\end{array}$ & 1280.0 & 1140.0 & $843 . ?$ & 692.0 & 511.0 & 365.0 & 334.0 & 281.0 & 253.0 & 229.0 & 171.0 \\
\hline $\begin{array}{l}1961 \\
1962\end{array}$ & 3180.0 & 1860.0 & 1140.0 & 751.0 & $567 \cdot 0$ & 496.0 & 419.0 & $408 \cdot 0$ & $373 \cdot 0$ & 323.0 & 225.0 \\
\hline 362 & 1180.0 & 1110.0 & 796.0 & 532.0 & 362.0 & 277.0 & 241.0 & $235 \cdot 0$ & $223 \cdot 0$ & $211 \cdot 0$ & $150 \cdot 0$ \\
\hline 63 & 1780.0 & 1370.0 & 951.0 & 740.0 & 472.0 & 303.0 & 280.0 & $242 \cdot 0$ & 245.0 & 237.0 & 177.0 \\
\hline
\end{tabular}




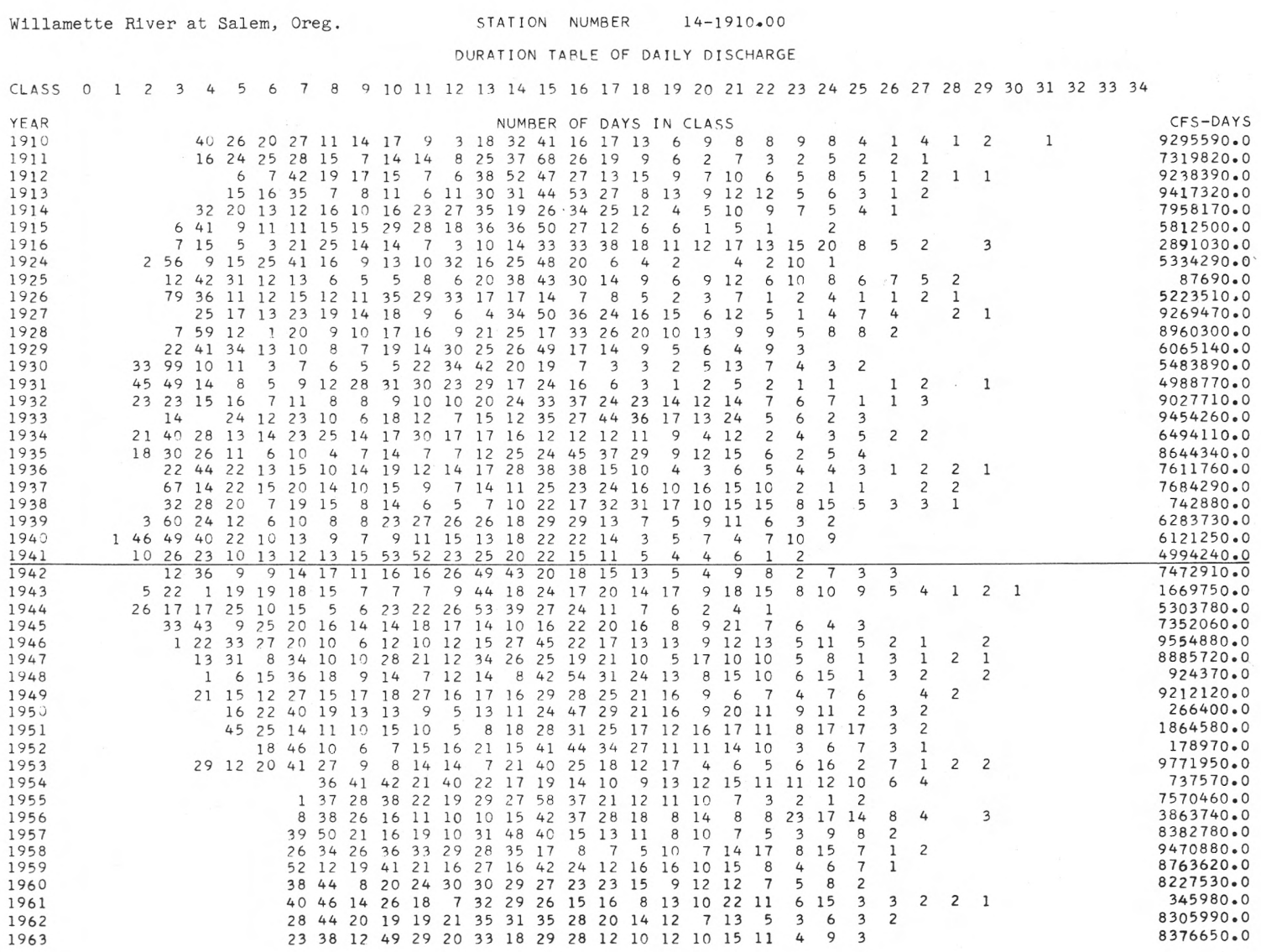

Willamette River at Salem, Oreg.

STATION NUMBER

$14-1910.00$

Summary for water years 1910-16, 1924-63
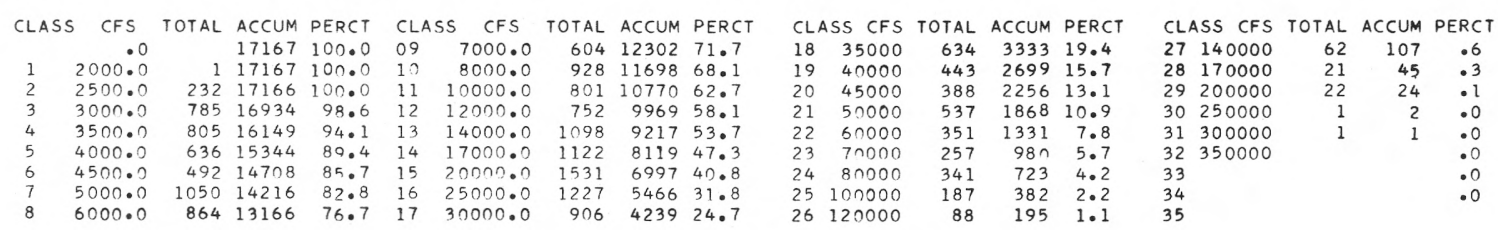

Willamette River at Salem, Oreg.

STATION NUMBER

14-1910.00

Summary for water years 1910-16, 1924-41

\begin{tabular}{|c|c|c|c|c|c|c|c|c|c|}
\hline CLASS & CFS & TOTAL & ACCUM & PERCT & CLAASS & CFS & TOTAL & ACCUM & PERCT \\
\hline & .0 & & 9132 & 100.0 & 09 & 7000.0 & 271 & 6128 & 67.1 \\
\hline 1 & 2000.0 & 1 & 9132 & 100.0 & 10 & 8000.0 & 450 & 5857 & 64.1 \\
\hline 2 & 2500.0 & 201 & 9131 & 100.0 & 11 & 10000.0 & 408 & 5407 & 59.2 \\
\hline 3 & 3000.0 & 700 & 8930 & 97.8 & 12 & 12000.0 & 380 & 4999 & 54.7 \\
\hline 4 & 3500.0 & 622 & 8230 & 90.1 & 13 & 14000.0 & 532 & 4619 & 50.6 \\
\hline 5 & 4000.0 & 416 & 7608 & 83.3 & 14 & 17000.0 & 605 & 4087 & 44.8 \\
\hline 6 & 4500.0 & 282 & 7192 & 78.8 & 15 & 20000.0 & 835 & 3482 & 38.1 \\
\hline 7 & 5000.0 & 470 & 6910 & 75.7 & 16 & 25000.0 & 647 & 2647 & 29.0 \\
\hline 8 & 6000.0 & 312 & 6440 & 70.5 & 17 & 30000.0 & 478 & 2000 & 21.9 \\
\hline
\end{tabular}

$\begin{array}{lrrr}\text { CLASS } & \text { CFS } & \text { TOTAL } & \text { ACC } \\ 18 & 35000 & 321 & 15 \\ 19 & 40000 & 192 & 12 \\ 20 & 45000 & 179 & 1009 \\ 21 & 50000 & 257 & 830 \\ 22 & 60000 & 157 & 573 \\ 23 & 70000 & 126 & 416 \\ 24 & 80000 & 131 & 290 \\ 25 & 100000 & 72 & 159 \\ 26 & 120000 & 33 & \end{array}$

\begin{tabular}{rrrr} 
ACCUM & PERCT & \multicolumn{2}{l}{ CLASS } \\
1522 & 16.7 & 27 & 140000 \\
1201 & 13.2 & 28 & 170000 \\
1009 & 11.0 & 29 & 200000 \\
830 & 9.1 & 30 & 250000 \\
573 & 6.3 & 31 & 300000 \\
416 & 4.6 & 32 & 350000 \\
290 & 3.2 & 33 & \\
159 & 1.7 & 34 & \\
87 & 1.0 & 35 &
\end{tabular}

$14-1910.00$

\section{Summary for water yẹars 1942-63}

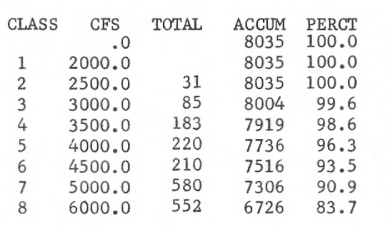

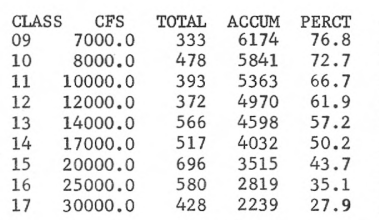

$\begin{array}{lr}\text { CLASS } & \text { CFS } \\ 18 & 35000 \\ 19 & 40000 \\ 20 & 45000 \\ 21 & 50000 \\ 22 & 60000 \\ 23 & 70000 \\ 24 & 80000 \\ 25 & 100000 \\ 26 & 120000\end{array}$

\begin{tabular}{rrrlrrrr} 
TOTAL & ACCUM & PERCT & \multicolumn{1}{l}{ CLASS CFS } & TOTAL & ACCUM & PERCT \\
313 & 1811 & 22.5 & 27 & 140000 & 30 & 53 & .7 \\
251 & 1498 & 18.6 & 28 & 170000 & 9 & 23 & .3 \\
209 & 1247 & 15.5 & 29 & 200000 & 13 & 14 & .2 \\
280 & 1038 & 12.9 & 30 & 250000 & 1 & 1 & .0 \\
194 & 758 & 9.4 & 31 & 300000 & & & .0 \\
131 & 564 & 7.0 & 32 & 350000 & & & .0 \\
210 & 433 & 5.4 & 33 & & & & .0 \\
115 & 223 & 2.8 & 34 & & & & .0
\end{tabular} 
Willamette RIver at Salem, Oreg.

STATION NUMBER 14-1910.00

LOWEST MEAN DISCHARGE, IN CFS, FOR THE FOLLOWING NUMBER OF CONSECUTIVE DAYS IN YEAR BEGINNING APRIL 1

\begin{tabular}{|c|c|c|c|c|c|c|c|c|c|c|c|}
\hline YEAR & 1 & 3 & 7 & 14 & 30 & 60 & 90 & 120 & 150 & 183 & 274 \\
\hline 1910 & 3750.0 & 3750.0 & 3750.0 & 3780.0 & 3850.0 & 3940.0 & 4240.0 & $4560 \cdot 0$ & 5020.0 & 6410.0 & $14800 \cdot 0$ \\
\hline 1911 & 3750.0 & 3750.0 & 3750.0 & 3800.0 & 4050.0 & 4830.0 & 5410.0 & $5480 \cdot 0$ & 6550.0 & 9540.0 & 14500.0 \\
\hline 1912 & 4400.0 & 4400.0 & 4570.0 & 4750.0 & 5020.0 & 7190.0 & 6870.0 & $8020 \cdot 0$ & $10100 \cdot 0$ & 12600.0 & $18200 \cdot 0$ \\
\hline 1913 & 4240.0 & 4240.0 & 4240.0 & 4360.0 & 4840.0 & 5460.0 & 6930.0 & $8290 \cdot 0$ & 10900.0 & $12900 \cdot 0$ & 18300.0 \\
\hline 1914 & 3510.0 & 3510.0 & 3510.0 & 3540.0 & 3640.0 & 4040.0 & 5130.0 & $6270 \cdot 0$ & 7480.0 & 8980.0 & 13000.0 \\
\hline 1915 & 3310.0 & 3380.0 & 3420.0 & 3480.0 & 3500.0 & 3560.0 & 3780.0 & 4240.0 & 4910.0 & $7900 \cdot 0$ & 19100.0 \\
\hline 1923 & 3890.0 & 3890.0 & 3920.0 & 3980.0 & 4210.0 & 4700.0 & 5410.0 & $5630 \cdot 0$ & 6650,0 & 8610.0 & 14100.0 \\
\hline 1924 & 2900.0 & 2940.0 & 2990.0 & 3070.0 & 3160.0 & 3230.0 & 3620.0 & $3810 \cdot 0$ & 4200.0 & 5310.0 & 15800.0 \\
\hline 1925 & 3020.0 & $3020 \cdot 0$ & 3020.0 & 3070.0 & 3220.0 & 3450.0 & 3550.0 & $3800 \cdot 0$ & $4600 \cdot 0$ & $5800 \cdot 0$ & 10600.0 \\
\hline 1926 & 3020.0 & 3110.0 & 3130.0 & 3140.0 & 3190.0 & 3500.0 & 3670.0 & $4070 \cdot 0$ & 4730.0 & $5300 \cdot 0$ & 12500.0 \\
\hline 1927 & 3730.0 & 3730.0 & 3730.0 & 3730.0 & 3860.0 & 4630.0 & 5440.0 & $7880 \cdot 0$ & 9080.0 & $11400 \cdot 0$ & 19100.0 \\
\hline 1928 & 3200.0 & 3270.0 & 3400.0 & 3500.0 & 3560.0 & 3610.0 & 3770.0 & $3890 \cdot 0$ & 4290.0 & $5400 \cdot 0$ & 10900.0 \\
\hline 1929 & 3100.0 & 3170.0 & 3190.0 & 3190.0 & 3240.0 & 3270.0 & 3270.0 & $3370 \cdot 0$ & 3610.0 & 5410.0 & $12100 \cdot 0$ \\
\hline 1930 & 2860.0 & 2860.0 & 2860.0 & 2860.0 & 2920.0 & 3040.0 & 3250.0 & 3410.0 & 3910.0 & $5210 \cdot 0$ & 8270.0 \\
\hline 1931 & $2500 \cdot 0$ & $2500 \cdot 0$ & 2520.0 & 2580.0 & 2640.0 & 2660.0 & 2810.0 & 3430.0 & 4350.0 & 5360.0 & 12700.0 \\
\hline 1932 & 3170.0 & 3170.0 & 3170.0 & 3170.0 & 3170.0 & 3470.0 & 3990.0 & $4790 \cdot 0$ & 6820.0 & 9680.0 & 17500.0 \\
\hline 1933 & 3700.0 & 3700.0 & 3750.0 & 4020.0 & 4360.0 & 5260.0 & 5330.0 & $6650 \cdot 0$ & 7230.0 & 11100.0 & 20800.0 \\
\hline 1934 & 2760.0 & 2790.0 & 2830.0 & $2860 \cdot 0$ & 2900.0 & 2950.0 & 3090.0 & $3390 \cdot 0$ & 4000.0 & $5300 \cdot 0$ & 16000.0 \\
\hline 1935 & 3080.0 & 3080.0 & 3140.0 & 3220.0 & 3350.0 & 3390.0 & 3760.0 & 4040.0 & 4940.0 & 5900.0 & 11500.0 \\
\hline 1936 & 3060.0 & 3070.0 & 3080.0 & 3090.0 & 3130.0 & 3150.0 & 3300.0 & 3420.0 & 3800.0 & $4660 \cdot 0$ & 8850.0 \\
\hline 1937 & 3680.0 & 3700.0 & 3800.0 & 4000.0 & 4110.0 & 4300.0 & 4800.0 & $5820 \cdot 0$ & 9890.0 & 13200.0 & 25300.0 \\
\hline 1938 & 3240.0 & 3250.0 & 3250.0 & 3270.0 & 3410.0 & 3480.0 & 3590.0 & 3940.0 & 4950.0 & 6980.0 & 12800.0 \\
\hline 1939 & 2890.0 & 2900.0 & $2940 . ?$ & 3060.0 & 3170.0 & 3260.0 & 3450.0 & $3760 \cdot 0$ & 3890.0 & $4760 \cdot 0$ & 9270.0 \\
\hline 1940 & 2.480 .0 & 2500.0 & 2520.0 & 2520.0 & 2570.0 & 2800.0 & 2960.0 & $3120 \cdot 0$ & 3580.0 & 4790.0 & 10700.0 \\
\hline 1941 & 3100.0 & 3120.0 & 3140.0 & 3210.0 & 3390.0 & 4010.0 & 4710.0 & $5310 \cdot 0$ & 6120.0 & 7330.0 & 16300.0 \\
\hline 1942 & 2930.0 & $2950 . n$ & 2980.0 & 3160.0 & 3220.0 & 3370.0 & 3620.0 & $4400 \cdot 0$ & 6350.0 & 8970.0 & 21400.0 \\
\hline 1943 & 4150.0 & 4160.0 & 4200.0 & 4300.0 & 4360.0 & 4650.0 & 5090.0 & 6750.0 & 9240.0 & $11200 \cdot 0$ & 13900.0 \\
\hline 1944 & 2730.0 & 2740.0 & 2750.0 & 2830.0 & 2880.0 & 3260.0 & 3350.0 & $3680 \cdot 0$ & 4500.0 & 5350.0 & 8980.0 \\
\hline 1945 & 3090.0 & 3110.0 & 3170.0 & 3300.0 & 3390.0 & 3690.0 & 3990.0 & $4210 \cdot 0$ & 4970.0 & 8480.0 & 20700.0 \\
\hline 1946 & 3460.0 & $3510 \cdot 0$ & 3650.0 & 3770.0 & 3830.0 & 4250.0 & 4540.0 & $5630 \cdot 0$ & 7490.0 & 9010.0 & 20200.0 \\
\hline 1947 & 3730.0 & 3740.0 & 3800.0 & 3940.0 & 4310.0 & 4550.0 & 5220.0 & $6650 \cdot 0$ & 7700.0 & 9790.0 & $21000 \cdot 0$ \\
\hline 1948 & 4280.0 & 4310.0 & 4400.0 & 4670.0 & 4880.0 & 5180.0 & 6010.0 & 6930.0 & 8100.0 & 10900.0 & 19100,0 \\
\hline 1949 & 3590.0 & 3600.0 & 3670.0 & 3730.0 & 3880.0 & 4410.0 & 4990.0 & $5600 \cdot 0$ & 6110.0 & 7720.0 & 15600.0 \\
\hline 1950 & 4000.0 & 4020.0 & 4100.0 & 4260.0 & 4610.0 & 5060.0 & 6150.0 & 8720.0 & $12300 \cdot 0$ & 15200.0 & 29200.0 \\
\hline 1951 & 4060.0 & $4060 . n$ & 4080.0 & 4200.0 & 4360.0 & 4390.0 & 4710.0 & $5720 \cdot 0$ & 7410.0 & $10100 \cdot 0$ & 20300.0 \\
\hline 1952 & 3500.0 & 3530.0 & $3560 . n$ & 3750.0 & 3980.0 & 4160.0 & 4460.0 & 4660.0 & 5220.0 & 7150.0 & 12800.0 \\
\hline 1953 & 5280.0 & 5280.0 & 5320.0 & 5430.0 & 5770.0 & 5970.0 & 6500.0 & 7130.0 & 8220.0 & 12500.0 & 25300.0 \\
\hline 1954 & 6080.0 & 6120.0 & 6160.0 & 6290.0 & 6560.0 & 6970.0 & 7570.0 & $8460 \cdot 0$ & 9170.0 & 10200.0 & 14100.0 \\
\hline 1955 & 5950.0 & 6010.0 & 6160.0 & 6160.0 & 6280.0 & 6920.0 & 7660.0 & $10100 \cdot 0$ & $12800 \cdot 0$ & $15000 \cdot 0$ & 32500.0 \\
\hline 1956 & 5750.0 & 5870.0 & 5950.0 & 6000.0 & 6150.0 & 6630.0 & 6810.0 & $7680 \cdot 0$ & 10300.0 & $12500 \cdot 0$ & 18100.0 \\
\hline 1957 & 5250.0 & 5310.0 & 5380.0 & 5440.0 & 5670.0 & 5850.0 & 6230.0 & $7100 \cdot 0$ & 7450.0 & 8680.0 & 17000.0 \\
\hline 1958 & 5620.0 & 5650.0 & 5680.0 & 5720.0 & 5830.0 & 6150.0 & 6770.0 & $7330 \cdot 0$ & 8310.0 & 9350.0 & 17700.0 \\
\hline 1959 & $5300 \cdot 0$ & 5350.0 & 5410.0 & 5440.0 & 5460.0 & 5650.0 & 6370.0 & 7770.0 & 9450.0 & 10400.0 & 12800.0 \\
\hline 1960 & 5300.0 & $5370 \cdot 0$ & 5510.0 & 5580.0 & 5680.0 & 5980.0 & 6200.0 & 6850.0 & 8010.0 & 12400.0 & 21000.0 \\
\hline 1961 & 5690.0 & 5740.0 & 5780.0 & 5800.0 & 5840.0 & 6090.0 & 6210.0 & 6780.0 & 7930.0 & 9260.0 & 18800.0 \\
\hline 1962 & 5690.0 & 5720.0 & 5740.0 & 5880.0 & 6090.0 & 6250.0 & 6570.0 & 7690.0 & 9890.0 & 11500.0 & 17600.0 \\
\hline
\end{tabular}

\begin{tabular}{|c|c|c|c|c|c|c|c|c|c|c|c|}
\hline YEAR & 1 & 3 & 7 & 15 & 30 & 60 & 90 & 120 & 150 & 183 & 274 \\
\hline 1910 & 15000.0 & $000 \cdot 0$ & 000.0 & 3000.0 & 800.0 & 58000.0 & 51500.0 & $55200 \cdot 0$ & 49200.0 & $44000 \cdot 0$ & 32500.0 \\
\hline 1911 & $55000 \cdot 0$ & $143000 \cdot 0$ & 97100.0 & 71900.0 & $54000 \cdot 0$ & 45400.0 & 41700.0 & $36200 \cdot 0$ & $33300 \cdot 0$ & 31200.0 & $000 \cdot 0$ \\
\hline 1912 & $214000 \cdot 0$ & 194000.0 & $148000 . ?$ & 110000.0 & 81500.0 & 67500.0 & 53900.0 & $47600 \cdot 0$ & 42700.0 & $38900 \cdot 0$ & 31300.0 \\
\hline 1913 & $51000 \cdot 0$ & 144000.0 & 111000.0 & 82200.0 & 58100.0 & 44100.0 & 42500.0 & $41200 \cdot 0$ & 39000.0 & $38000 \cdot 0$ & $32400 \cdot 0$ \\
\hline 1914 & $123000 \cdot 0$ & $120000 \cdot ?$ & $1090 n n \cdot ?$ & $77000 \cdot ?$ & $62100 \cdot 0$ & $52800 \cdot 0$ & 46900.0 & $42500 \cdot 0$ & $38600 \cdot 0$ & $35000 \cdot 0$ & $300 \cdot 0$ \\
\hline 1915 & $0 n \cdot 0$ & $82100 \cdot 0$ & $6 \cap 8 \cap n \cdot ?$ & 47600.0 & 38700.0 & 31100.0 & 27500.0 & $25000 \cdot 0$ & 23900.0 & 22800.0 & 19700.0 \\
\hline 1916 & $248000 \cdot 0$ & $223000 \cdot n$ & $1750 n 0 \cdot n$ & $125000 \cdot ?$ & $89200 \cdot 0$ & 79700.0 & $66000 \cdot 0$ & & $62400 \cdot 0$ & 57900.0 & 45200.0 \\
\hline 1924 & $82900 \cdot 0$ & 79000.0 & $74900 \cdot 0$ & $59100 \cdot ?$ & $41000 \cdot 0$ & $34500 \cdot 0$ & $8 \cap \cap \cdot 0$ & $29000 \cdot 0$ & $27000 \cdot 0$ & $24200 \cdot 0$ & 200.0 \\
\hline 1925 & 177000.0 & $169000 \cdot 0$ & 152000.0 & 130000.0 & 90600.0 & 72300.0 & $61400 \cdot 0$ & $58800 \cdot 0$ & $51300 \cdot 0$ & 46600.0 & 35500.0 \\
\hline 1926 & $71000 \cdot 0$ & $163000 \cdot 0$ & $126000 \cdot 0$ & $80900 \cdot 0$ & 68100.0 & 45000.0 & $37400 \cdot 0$ & $31400 \cdot 0$ & 27300.0 & $24100 \cdot 0$ & 17800.0 \\
\hline 327 & 38000.0 & $02000 \cdot 0$ & 145000.0 & 90900.0 & 71300.0 & 60700.0 & 54700.0 & $50700 \bullet 0$ & 45500.0 & $41200 \cdot 0$ & $32100 \cdot 0$ \\
\hline 928 & $120000 \cdot 0$ & $113000 \cdot ?$ & 108000.0 & 79300.0 & 60000 & 50600.0 & $44700 \cdot 0$ & $40700 \cdot 0$ & 42500.0 & 40900.0 & $31400 \cdot 0$ \\
\hline 1929 & 73600.0 & $72600 . ?$ & 69000.0 & 52300.0 & $36100 \cdot 0$ & 32500.0 & 28600.0 & $28900 \cdot 0$ & $28500 \cdot 0$ & $26800 \cdot 0$ & 0.0 \\
\hline 1930 & $114000 \cdot 0$ & $107000 \cdot 0$ & $8000 n \cdot n$ & $67200 . ?$ & 56700.0 & 37700.0 & 36900.0 & $31800 \cdot 0$ & 28500.0 & $26100 \cdot 0$ & 19000.0 \\
\hline 1931 & $00000 \cdot 0$ & $175000 \cdot 0$ & $12600 n \cdot ?$ & 80000.0 & 5940 & 39900.0 & $0 \cap \cdot 0$ & 282 & 25000.0 & 22600.0 & 17200.0 \\
\hline 932 & 67000.0 & 159000.0 & 124000.0 & 99900.0 & 70200.0 & $53300 . ?$ & 47100.0 & $46200 \cdot 0$ & 43600.0 & $40100 \cdot 0$ & 31700.0 \\
\hline 1933 & 0 & 1080 & $909 n \cap .0$ & 778 & 609 & & 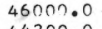 & $42600 \cdot 0$ & $40200 \cdot 0$ & $40200 \cdot 0$ & $32800 \cdot 0$ \\
\hline 1934 & $65000 \cdot 0$ & 147000.0 & $1240 n n \cdot 0$ & $88900 . ?$ & 65900.0 & 57900.0 & $44200 \cdot 0$ & $39500 \cdot 0$ & $34700 \cdot 0$ & 302 & 22600.0 \\
\hline 1935 & 0.0 & $112000 \cdot n$ & n.? & $82700 . n$ & 62900 & 50700.0 & 45700.0 & $42000 \cdot 0$ & $40400 \cdot 0$ & $38500 \cdot 0$ & $30400 \cdot 0$ \\
\hline 1936 & $10 \cdot 0$ & $192000 \cdot 0$ & 0.0 & 121 & 8 & $\cdot 0$ & 47 & & 3950 & $35300 \cdot 0$ & $26400 \cdot 0$ \\
\hline 1937 & 94000.0 & $179000 \cdot 0$ & $136000 \cdot 0$ & 89100.0 & 63400.0 & 52500.0 & 49200.0 & $45000 \cdot 0$ & $41800 \cdot 0$ & 366 & 0.0 \\
\hline 193 & 20.0 & $152000 \cdot 0$ & 120 & 0.0 & & 00.0 & $58300 \cdot 0$ & $55300 \bullet 0$ & 54400.0 & 50900.0 & 37900.0 \\
\hline 1939 & $93900 \cdot 0$ & $87800 \cdot n$ & $72000 \cdot 0$ & $55400 \cdot 0$ & 4220 & $416 n 0.0$ & 00.0 & 334 & 30900.0 & 2830 & 21800.0 \\
\hline 1940 & 0.0 & $100 \cdot 0$ & 0.0 & 74000.0 & 6940 & 52700.0 & 00.0 & $38600 \cdot 0$ & $34400 \cdot 0$ & $29800 \cdot 0$ & $21300 \cdot 0$ \\
\hline 194 & 0.0 & $68800 \cdot 0$ & & & & & & 24900 & $22000 \cdot 0$ & 20300.0 & $16800 \cdot 0$ \\
\hline 1942 & 131000.0 & $124000 . ?$ & 101000.0 & $71500 . ?$ & 62 & no.n & & $40400 \cdot 0$ & 3540 & $31700 \cdot 0$ & \\
\hline 2 & 0 & 244 & $\cdot ?$ & 131 & & 0 & • 0 & 65000 & $5980 n .0$ & $54000 \cdot 0$ & 41100.0 \\
\hline 1944 & 62800.0 & 57300.0 & $43900 . n$ & 33800.0 & 2730 & 23500.0 & 0.0 & 228 & & 21400.0 & 18100.0 \\
\hline 94 & $01000=0$ & . & $9 n 2 n n .0$ & 77300.0 & & $46500 \cdot n$ & & 418 & 39 & 34700.0 & 25600.0 \\
\hline 1946 & $21000 \cdot 0$ & $200000 . ?$ & 146000.0 & 115000.0 & 80 & .0 & & 54400 & 49 & 4430 & 0.0 \\
\hline 947 & 0.0 & 203 & ?.? & 948 & & $0 . n$ & . 0 & $47400 \cdot 0$ & 45100.0 & 40200.0 & 00.0 \\
\hline & & & & $1 \cap \theta$ & & & & 475 & & 44 & $00 \cdot 0$ \\
\hline 1949 & 0.0 & 162 & .0 & 99000.0 & 709 & 51200.0 & 47200.0 & 4510 & .0 & $41400 \cdot 0$ & 0.0 \\
\hline & 0 & 141 & & & & 0.0 & & & 494 & 45700.0 & 35500.0 \\
\hline 1951 & 0.0 & $137000 \cdot 0$ & .0 & 974 & & & & & & & $41700 \cdot 0$ \\
\hline 1952 & 141000.0 & $134000 \cdot 0$ & 115 & 80200.0 & 629 & 00.0 & 53100.0 & $48000 \cdot 0$ & $46100 \cdot 0$ & 42600.0 & 35000.0 \\
\hline 195 & 0.0 & 217 & $n \cdot n$ & $132000 \cdot 0$ & 1160 & & & & & $45800 \cdot 0$ & 34100.0 \\
\hline 195 & 0.0 & 144 & 0.0 & & & 0.0 & 71 & & 56300.0 & $49000 \cdot 0$ & 0.0 \\
\hline $95-2>$ & .0 & 96 & & & & & & 32 & 31400.0 & 30200.0 & 25200.0 \\
\hline 195 & $235 n 00 \cdot 0$ & $2150 n 0 \cdot n$ & $178000 . n$ & $136000 \cdot 0$ & $120 n d$ & 105000.0 & $86400 \cdot 0$ & 7530 & & & 0.0 \\
\hline 195 & 0.0 & $1220 n 0 . n$ & & & & & & 4340 & $3900 n \cdot 0$ & & 28500,0 \\
\hline 195 & 0 & 1400 & $115000 \cdot n$ & $100000 \cdot 0$ & & & & & & & \\
\hline 1959 & 0.0 & 117 & n. 0 & & & & & 4560 & 42300.0 & - 0 & \\
\hline & 103 & & & & & & & & & & \\
\hline & 10.0 & 204000.0 & 159000.0 & $123000 \cdot 0$ & & & 0 & 5610 & & 472 & 0 \\
\hline 196 & $130000 \cdot 0$ & $120000 \cdot 0$ & $100000 \cdot 0$ & 76800.0 & 58000.0 & 49300.0 & $00 \cdot 0$ & $36300 \cdot 0$ & .0 & $35500 \cdot 0$ & \\
\hline 1963 & $110000 \cdot 0$ & $103000 \cdot 0$ & 83200.0 & 63600.0 & 52500.0 & 44700.0 & 37100.0 & $36600 \cdot 0$ & $33100 \cdot 0$ & 34900.0 & 2830 \\
\hline
\end{tabular}




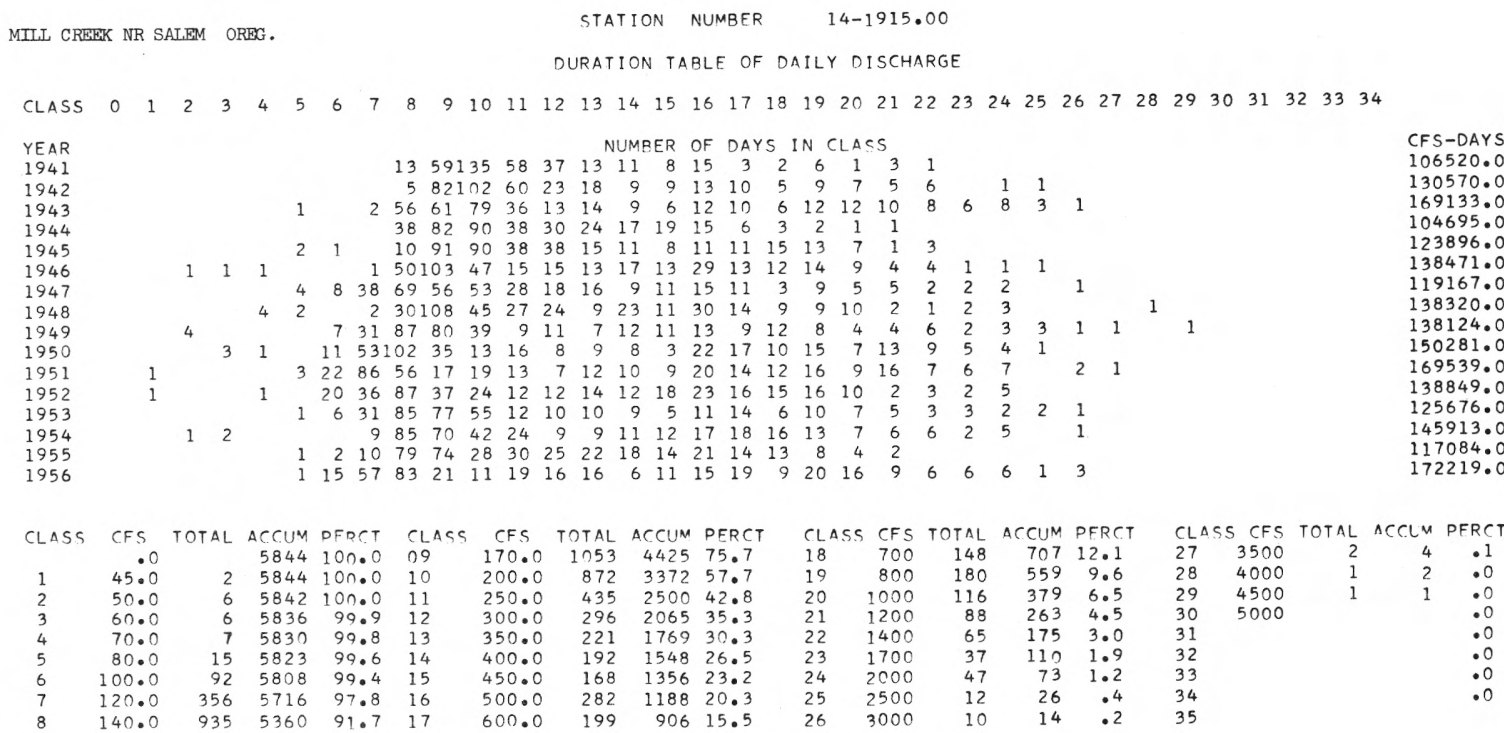

MIIL CREEK NR SALEM OREG. STATION NUMBER $14-1915.00$ LOWEST MEAN DISCHARGE, IN CFS, FOR THE FOLLOWING NUMBER OF CONSECUTIVE DAYS IN YEAR BEGINNING APRIL 1

\begin{tabular}{|c|c|c|c|c|c|c|c|c|c|c|c|}
\hline YEAR & 1 & 3 & 7 & 14 & 30 & 60 & 90 & 120 & 150 & 183 & 274 \\
\hline 1941 & 147.0 & 164.0 & 167.0 & 171.0 & 178.0 & 191.0 & 197.0 & 203.0 & 210.0 & 217.0 & 321.0 \\
\hline 1942 & 158.0 & 158.0 & 171.0 & 175.0 & 180.0 & 184.0 & 185.0 & $187 \cdot 0$ & 195.0 & 226.0 & 389.0 \\
\hline 1943 & 96.0 & 126.0 & 150.0 & 158.0 & 159.0 & 165.0 & 165.0 & 173.0 & 181.0 & 197.0 & 245.0 \\
\hline 1944 & 146.0 & 152.0 & 163.0 & 166.0 & 175.0 & 179.0 & 185.0 & $190 \cdot 0$ & 191.0 & 194.0 & 226.0 \\
\hline 1945 & 88.0 & 94.3 & 136.0 & 153.0 & 172.0 & 176.0 & 182.0 & $186 \cdot 0$ & 187.0 & $206 \cdot 0$ & 321.0 \\
\hline 1946 & 58.0 & 64.0 & 121.0 & 154.0 & 161.0 & 166.0 & 168.0 & $170 \cdot 0$ & 174.0 & 181.0 & 302.0 \\
\hline 1947 & 92.0 & 94.3 & 116.0 & 125.0 & 133.0 & 138.0 & 139.0 & $145 \cdot 0$ & 154.0 & 164.0 & 280.0 \\
\hline 1948 & 73.0 & 76.0 & $88 \cdot 1$ & 124.0 & 151.0 & 167.0 & 167.0 & $170 \cdot 0$ & 172.0 & 189.0 & 323.0 \\
\hline 1949 & 50.0 & 50.7 & 75.1 & 107.0 & 131.0 & 138.0 & 137.0 & $141 \cdot 0$ & 146.0 & 153.0 & 218.0 \\
\hline 1950 & 67.0 & 67.7 & 93.4 & 113.0 & 127.0 & 133.0 & 134.0 & 143.0 & 146.0 & 155.0 & 363.0 \\
\hline 1951 & 48.0 & 76.7 & 111.0 & 117.0 & 122.0 & 125.0 & 128.0 & 129.0 & 137.0 & 142.0 & 273.0 \\
\hline 1952 & 45.0 & 96.3 & 109.0 & 113.0 & 118.0 & 127.0 & 134.0 & $138 \cdot 0$ & 141.0 & 146.0 & 173.0 \\
\hline 1953 & 98.0 & 106.0 & 117.0 & 130.0 & 131.0 & 140.0 & 146.0 & 153.0 & 156.0 & 182.0 & 312.0 \\
\hline 1954 & 50.0 & 60.0 & 112.0 & 127.0 & 141.0 & 157.0 & 160.0 & $160 \cdot 0$ & 168.0 & 175.0 & 255.0 \\
\hline 955 & 90.0 & 118.0 & 124.0 & 130.0 & 144.0 & 155.0 & 162.0 & $166 \cdot 0$ & 171.0 & 193.0 & 424.0 \\
\hline
\end{tabular}

MIIL CREEK NR SALEM OREGON

STATION NUMBER $\quad 14-1915.00$

HIGHEST MEAN DISCHARGE, IN CFS, FOR THE FOLLOWING NUMBER OF CONSECUTIVE DAYS IN YEAR ENDING SEPTFMBER 30

\begin{tabular}{|c|c|c|c|c|c|c|c|c|c|c|c|}
\hline YEAR & 1 & 3 & 7 & 15 & 30 & 60 & 90 & 120 & 150 & 183 & 274 \\
\hline 1941 & 1500.0 & 1210.0 & 915.0 & 760.0 & 607.0 & 545.0 & 483.0 & $430 \cdot 0$ & 393.0 & 371.0 & 323.0 \\
\hline 1942 & 2560.0 & 1830.0 & 1470.0 & 1030.0 & 922.0 & 712.0 & 697.0 & 602.0 & 531.0 & 482.0 & 415.0 \\
\hline 1943 & 3060.0 & 2420.0 & 1980.0 & 1570.0 & 1330.0 & 1130.0 & 1070.0 & $886 \cdot 0$ & 835.0 & 738.0 & 562.0 \\
\hline 1944 & 1220.0 & 927.0 & 682.0 & 575.0 & 535.0 & 479.0 & 450.0 & $415 \cdot 0$ & 398.0 & 373.0 & 321.0 \\
\hline 1945 & 1430.0 & 1380.0 & 1190.0 & 970.0 & 727.0 & 622.0 & 610.0 & $568 \cdot 0$ & 520.0 & 480.0 & 391.0 \\
\hline 1946 & 2600.0 & 2270.0 & 1460.0 & 1050.0 & 791.0 & 759.0 & 732.0 & $714 \cdot 0$ & 654.0 & 579.0 & 449.0 \\
\hline 1947 & 3450.0 & 2470.0 & 1690.0 & 1140.0 & 1070.0 & 698.0 & 646.0 & $574 \cdot 0$ & 542.0 & 493.0 & 389.0 \\
\hline 1948 & 4100.0 & 2930.0 & 1940.0 & 1370.0 & 878.0 & 677.0 & 651.0 & $568 \cdot 0$ & 564.0 & 554.0 & 449.0 \\
\hline 1949 & 4560.0 & 3870.0 & 2740.0 & 2050.0 & 1270.0 & 806.0 & 845.0 & $764 \cdot 0$ & 680.0 & 595.0 & 458.0 \\
\hline 1950 & 2610.0 & 2170.0 & 1980.0 & 1630.0 & 1290.0 & 1180.0 & 1020.0 & 896.0 & 782.0 & 675.0 & 504.0 \\
\hline 1951 & 3870.0 & 3380.0 & 2150.0 & 1600.0 & 1270.0 & 1010.0 & 1030.0 & 962.0 & 906.0 & 786.0 & 576.0 \\
\hline 1952 & 2.380 .0 & 2220.0 & 1760.0 & 1200.0 & 960.0 & 793.0 & 803.0 & $740 \cdot 0$ & 674.0 & 607.0 & 461.0 \\
\hline 1953 & 3470.0 & 3020.0 & 2240.0 & 1760.0 & 1340.0 & 956.0 & 787.0 & 670.0 & 580.0 & 528.0 & 411.0 \\
\hline 1954 & 3140.0 & 2550.0 & 1690.0 & 1260.0 & 1090.0 & 946.0 & 882.0 & $800 \cdot 0$ & 716.0 & 623.0 & 479.0 \\
\hline 1955 & 1370.0 & 1210.0 & 951.0 & 803.0 & 668.0 & 573.0 & 510.0 & $486 \cdot 0$ & 489.0 & 469.0 & 373.0 \\
\hline 1956 & 3350.0 & 2840 . & 2270.0 & 1650.0 & 1560.0 & 1300.0 & 1080.0 & 992.0 & 888.0 & 790.0 & 585.0 \\
\hline
\end{tabular}




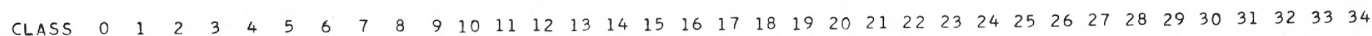

YEAR
1941
1942
1943
1944
1945
1946
1947
1948
1949
1950
1951
1952
1953
1954
1955
1956
1957
1958
1959
1960
1961
1962
1963

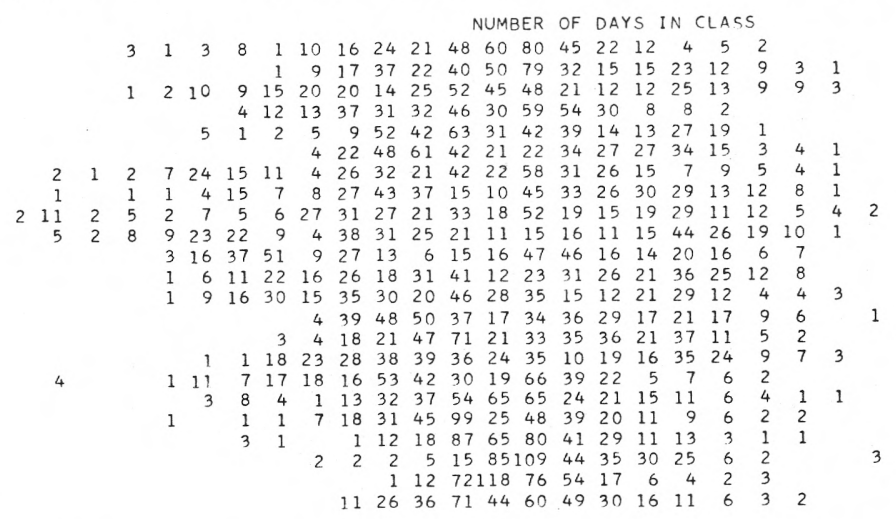

NUMBER OF DAYS IN CLASS

CFS-DAYS 41970.5
54855.0 55212.0 39849.0 49150.0
56517.0 56517.0
44676.0 44676.0 61872.9
57087.9 57087.9
62996.3 62996.3
49970.0 49970.0 63156.0
48803.5 57020.0 55742.0 59814.0 37074.4 44598.0 42236.8 46427.0 60978.0 44031.0
48766.0
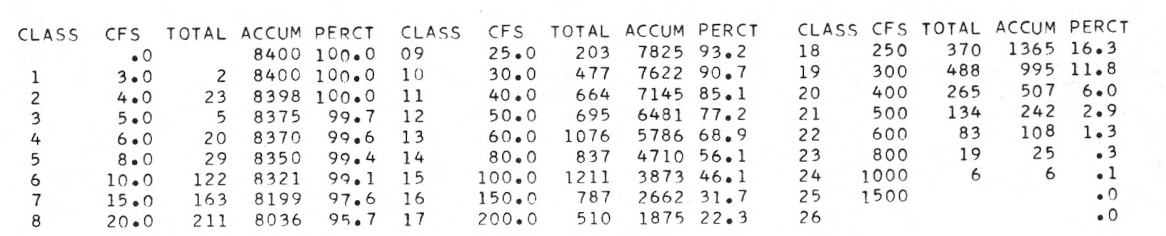

$\begin{array}{lc}\text { CLASS CFS TOTAL ACCUM PERCT } \\ 27 \\ 28 & .0 \\ 29 & .0 \\ 30 & .0 \\ 31 & .0 \\ 32 & .0 \\ 33 & .0 \\ 34 & .0 \\ 35 & .0\end{array}$

M1ll Creek at Salem, Oreg.

STATION NUMBER

$14-1920.00$

LOWEST MEAN DISCHARGE, IN CFS, FOR THE FOLLOWING NUMBER OF CONSECUTIVE DAYS IN YEAR BEGINNING APRIL 1

\begin{tabular}{|c|c|c|c|c|c|c|c|c|c|c|c|}
\hline YEAR & 1 & 3 & 7 & 14 & 30 & 60 & 90 & 120 & 150 & 183 & 274 \\
\hline 1941 & 6.4 & $7 \cdot 2$ & 10.8 & 21.9 & $\begin{array}{l}34.4 \\
39.2\end{array}$ & $\begin{array}{l}47,3 \\
43.5\end{array}$ & $\begin{array}{l}58.6 \\
43.4\end{array}$ & $\begin{array}{l}62 \cdot 8 \\
46 \cdot 6\end{array}$ & $\begin{array}{l}68.7 \\
54.7\end{array}$ & $\begin{array}{l}73.3 \\
76.9\end{array}$ & $\begin{array}{l}127.0 \\
136.0\end{array}$ \\
\hline $\begin{array}{l}1942 \\
1943\end{array}$ & $\begin{array}{r}23.0 \\
6.0\end{array}$ & $\begin{array}{r}26.3 \\
9.0\end{array}$ & $\begin{array}{l}30.3 \\
14.4\end{array}$ & $\begin{array}{l}37.6 \\
16.3\end{array}$ & $\begin{array}{l}39.2 \\
19.9\end{array}$ & 28.3 & 29.3 & 37.8 & 45.2 & 55.2 & $\begin{array}{r}136.0 \\
82.1\end{array}$ \\
\hline 1944 & 10.0 & 15.3 & 23.3 & 24.5 & 33.5 & 39.0 & 45.6 & 49.7 & 51.5 & 54.7 & 74.1 \\
\hline 1945 & 10.0 & 16.3 & 34.4 & 37.6 & 44.4 & 46.6 & 51.6 & 53.9 & 54.0 & 69.2 & 125.0 \\
\hline 1946 & 26.0 & 30.7 & 32.1 & 34.9 & 38.0 & 43.2 & 43.5 & 53.3 & 54.2 & 57.6 & 110.0 \\
\hline 1947 & 4.0 & 5.3 & 6.6 & 9.3 & 19.6 & 21.6 & 23.0 & 32.7 & 38.9 & 55.0 & 128.0 \\
\hline 1948 & 4.0 & 8.2 & 14.2 & 17.0 & 31.7 & 39.8 & 39.5 & $41 \cdot 1$ & 41.9 & 51.2 & 127.0 \\
\hline 1949 & 3.5 & 3.8 & 4.1 & 4.4 & 6.3 & 15.2 & 21.7 & $24 \cdot 5$ & 30.4 & 42.0 & 91.4 \\
\hline 1950 & 7.2 & 7.4 & 9.2 & 11.9 & 13.8 & 21.7 & 23.2 & 31.5 & 33.7 & 43.6 & 91.6 \\
\hline 1951 & 9.0 & 9.0 & 11.1 & 13.4 & 17.5 & 19.7 & 22.9 & $22 \cdot 2$ & 29.9 & 34.7 & 110.0 \\
\hline 1952 & 9.0 & 11.7 & $14 \cdot 3$ & 16.6 & 17.6 & 20.9 & 26.3 & $31 \cdot 3$ & 37.1 & 42.4 & 63.4 \\
\hline 1953 & 9.5 & 11.7 & 14.5 & $18 \cdot 3$ & 19.0 & 24.0 & 25.9 & 33.4 & 36.5 & 57.8 & 106.0 \\
\hline 1954 & 25.0 & 26.7 & 28.4 & 29.6 & 32.6 & 37.2 & 42.1 & $48 \cdot 8$ & 55.5 & 56.3 & 105.0 \\
\hline 1955 & 20.0 & 21.0 & 25.4 & 29.1 & 36.7 & 46.5 & 54.1 & 57.9 & 61.8 & 76.4 & 176.0 \\
\hline 1956 & 13.0 & 13.3 & 14.0 & 15.8 & 23.3 & 29.1 & 30.0 & $31 \cdot 6$ & 35.7 & 37.8 & 57.6 \\
\hline 1957 & 4.0 & 4.2 & 15.6 & 19.6 & 25.4 & 35.2 & 36.9 & 39.4 & 42.6 & 47.7 & 79.9 \\
\hline 1958 & 17.0 & $17 \cdot 3$ & $22 \cdot 4$ & 34.4 & 43.4 & 51.3 & 58.5 & $55 \cdot 6$ & 61.0 & 67.0 & 98.1 \\
\hline 1959 & 9.8 & 25.0 & 26.3 & 31.1 & 38.6 & 48.9 & 53.4 & 55.2 & 58.7 & 61.1 & 73.3 \\
\hline 1960 & 18.0. & $18 \cdot 3$ & 33.0 & 47.5 & 61.6 & 65.2 & 70.2 & 71.1 & 76.5 & 89.4 & 121.0 \\
\hline 1961 & 43.0 & 59.7 & 62.9 & 65.7 & 75.3 & 95.6 & 97.6 & 98.8 & 99.1 & $100 \cdot 0$ & 116.0 \\
\hline 1962 & 30.0 & 34.3 & 36.7 & 37.9 & 67.0 & 72.6 & 79.7 & 79.9 & 79.5 & 83.7 & 111.0 \\
\hline
\end{tabular}

Mill Creek at Salem, Oreg.

STATION NUMBER $14-1920.00$

HIGHEST MEAN DISCHARGE, IN CFS, FOR THE FOLLOWING NUMBER OF CONSECUTIVE DAYS IN YEAR ENDING SEPTEMBER 30

YEAR
1941
1942
1943
1944
1945
1946
1947
1948
1949
1950
1951
1952
1953
1954
1955
1956
1957
1958
1959
1960
1961
1962
1963

$\begin{array}{rc}1 & 3 \\ 532.0 & 478.0 \\ 853.0 & 649.0 \\ 900.0 & 785.0 \\ 463.0 & 387.0 \\ 576.0 & 467.0 \\ 815.0 & 702.0 \\ 928.0 & 709.0 \\ 962.0 & 806.0 \\ 1090.0 & 983.0 \\ 860.0 & 636.0 \\ 708.0 & 643.0 \\ 770.0 & 691.0 \\ 939.0 & 870.0 \\ 1140.0 & 882.0 \\ 626.0 & 550.0 \\ 850.0 & 790.0 \\ 550.0 & 515.0 \\ 896.0 & 683.0 \\ 627.0 & 543.0 \\ 600.0 & 533.0 \\ 1290.0 & 917.0 \\ 569.0 & 501.0 \\ 720.0 & 547.0\end{array}$

$\begin{array}{cc}7 & 15 \\ 375.0 & 301.0 \\ 513.0 & 412.0 \\ 656.0 & 513.0 \\ 287.0 & 253.0 \\ 434.0 & 399.0 \\ 488.0 & 414.0 \\ 553.0 & 493.0 \\ 701.0 & 512.0 \\ 763.0 & 665.0 \\ 596.0 & 538.0 \\ 560.0 & 506.0 \\ 630.0 & 538.0 \\ 697.0 & 578.0 \\ 623.0 & 505.0 \\ 459.0 & 406.0 \\ 619.0 & 522.0 \\ 466.0 & 373.0 \\ 490.0 & 375.0 \\ 457.0 & 328.0 \\ 454.0 & 376.0 \\ 605.0 & 434.0 \\ 365.0 & 274.0 \\ 443.0 & 333.0\end{array}$

30
$254 \bullet 0$
$384 \bullet 0$
442.0
237.0
309.0
336.0
415.0
405.0
472.0
483.0
388.0
435.0
467.0
401.0
359.0
499.0
310.0
314.0
293.0
312.0
362.0
209.0
264.0

60
234.0
326.0
392.0
214.0
266.0
327.0
277.0
301.0
367.0
467.0
368.0
408.0
369.0
349.0
285.0
459.0
230.0
287.0
255.0
246.0
312.0
183.0
221.0

90
211.0
322.0
372.0
198.0
254.0
316.0
248.0
324.0
344.0
440.0
316.0
388.0
315.0
336.0
262.0
406.0
205.0
260.0
228.0
221.0
285.0
166.0
216.0

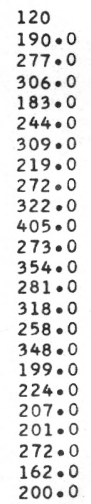

150
172.0
242.0
281.0
173.0
225.0
289.0
207.0
268.0
298.0
363.0
266.0
328.0
243.0
294.0
259.0
316.0
176.0
203.0
193.0
191.0
254.0
160.0
194.0

183
163.0
218.0
251.0
160.0
210.0
254.0
199.0
269.0
261.0
312.0
239.0
295.0
228.0
255.0
244.0
281.0
160.0
183.0
172.0
181.0
234.0
155.0
195.0

274
134.0
185.0
191.0
131.0
162.0
191.0
155.0
212.0
200.0
223.0
175.0
220.0
169.0
193.0
186.0
207.0
124.0
145.0
138.0
146.0
193.0
134.0
157.0 
South Yamhili River near

Willamina, Oreg.
STATION NUMBER $14-1925.00$

DURATION TABLE OF DAILY DISCHARGE

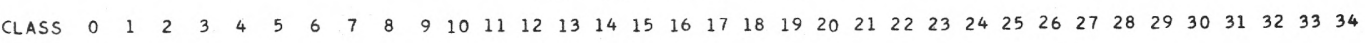

YEAR
1935
1936
1937
1938
1939
1940
1941
1942
1943
1944
1945
1946
1947
1948
1949
1950
1951
1952
1953
1954
1955
1956
1957
1958
1959
1960
1961
1962
1963

NUMBER OF DAYS IN CLASS

$\begin{array}{lllllllllllllllllllllllll}20 & 29 & 20 & 20 & 11 & 13 & 11 & 12 & 8 & 10 & 9 & 5 & 4 & 17 & 20 & 15 & 41 & 16 & 34 & 21 & 10 & 7 & 8 & 3 & 1\end{array}$

$\begin{array}{llllllllllllllllllllllllll}3 & 24 & 36 & 19 & 17 & 10 & 4 & 10 & 11 & 26 & 25 & 25 & 19 & 29 & 23 & 19 & 16 & 7 & 11 & 9 & 9 & 3 & 5 & 1 & 3 \\ 58 & 14 & 8 & 3 & 19 & 5 & 5 & 18 & 12 & 17 & 15 & 6 & 7 & 18 & 3 & 1 & 25 & 20 & 30 & 22 & 17 & 7 & 9 & 1 & 1\end{array}$

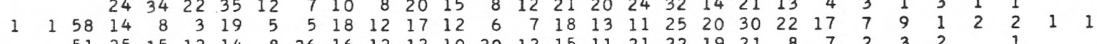

$\begin{array}{rrrrrrrrrrrrrrrrrrrrrrrrrrrrrrrr}51 & 25 & 15 & 12 & 14 & 8 & 26 & 16 & 12 & 12 & 10 & 20 & 12 & 15 & 11 & 21 & 22 & 19 & 21 & 8 & 7 & 2 & 3 & 2 & & 1 & \\ 34 & 38 & 15 & 10 & 19 & 17 & 16 & 15 & 8 & 15 & 11 & 7 & 14 & 16 & 9 & 15 & 21 & 17 & 23 & 19 & 11 & 8 & 3 & 2 & 1 & 1 & 1\end{array}$

$\begin{array}{rrrrrrrrrrrrrrrrrrrrrrrr}34 & 38 & 15 & 10 & 19 & 17 & 16 & 15 & 8 & 15 & 11 & 7 & 14 & 16 & 9 & 15 & 21 & 17 & 23 & 19 & 11 & 8 & 3 & 2 \\ 3 & 21 & 24 & 11 & 15 & 5 & 7 & 21 & 15 & 28 & 29 & 29 & 32 & 27 & 19 & 18 & 21 & 12 & 14 & 9 & 1 & 2 & 1 & 1\end{array}$

$\begin{array}{rrrrrrrrrrrrrrrrrrrrrrrrrrrr}7 & 17 & 12 & 11 & 9 & 8 & 5 & 19 & 19 & 24 & 46 & 20 & 27 & 35 & 39 & 9 & 8 & 8 & 11 & 10 & 9 & 5 & 4 & 2 & & 1\end{array}$

$\begin{array}{llllllllllllllllllllll}18 & 33 & 14 & 22 & 13 & 4 & 10 & 8 & 23 & 29 & 17 & 18 & 20 & 18 & 13 & 15 & 14 & 16 & 17 & 10 & 9 & 10\end{array}$

$\begin{array}{llllllllllllllllllllll}24 & 23 & 23 & 4 & 11 & 10 & 11 & 7 & 3 & 24 & 23 & 19 & 15 & 32 & 29 & 17 & 43 & 21 & 16 & 7 & 1\end{array}$

$\begin{array}{lllllllllllllllllllllllll}26 & 27 & 6 & 28 & 14 & 6 & 13 & 3 & 17 & 11 & 18 & 18 & 20 & 28 & 24 & 28 & 13 & 21 & 18 & 10 & 4 & 3\end{array}$

$\begin{array}{rlllllllllllllllllllllllll}10 & 27 & 21 & 25 & 15 & 4 & 16 & 21 & 34 & 10 & 9 & 9 & 9 & 13 & 17 & 25 & 15 & 27 & 22 & 11 & 5 & 11 & 4 & 1 & 4\end{array}$

$\begin{array}{rrrrrrrrrrrrrrrrrrrrrrrr}11 & 20 & 33 & 10 & 6 & 22 & 14 & 33 & 16 & 24 & 19 & 30 & 22 & 21 & 18 & 11 & 17 & 9 & 7 & 6 & 6 & 4 \\ 4 & 17 & 9 & 21 & 17 & 11 & 11 & 12 & 25 & 6 & 10 & 20 & 19 & 25 & 19 & 33 & 27 & 42 & 19 & 11 & 2 & 5 & 5\end{array}$

$\begin{array}{rrrrrrrrrrrrrrrrrrrrrrrr}11 & 9 & 21 & 17 & 11 & 11 & 12 & 25 & 6 & 10 & 20 & 19 & 25 & 19 & 33 & 27 & 42 & 19 & 11 & 2 & 5 & 5 \\ 31 & 35 & 15 & 14 & 9 & 12 & 10 & 17 & 26 & 33 & 17 & 20 & 20 & 10 & 19 & 12 & 24 & 13 & 7 & 2 & 6 & 6\end{array}$

$\begin{array}{llllllllllllllllllllllllll}17 & 11 & 14 & 20 & 15 & 12 & 12 & 19 & 13 & 24 & 16 & 10 & 9 & 16 & 7 & 12 & 25 & 15 & 34 & 26 & 16 & 7 & 8 & 5\end{array}$

$\begin{array}{rrrrrrrrrrrrrrrrrrrrrrrrrrrrr}7 & 9 & 18 & 20 & 9 & 8 & 12 & 8 & 5 & 13 & 9 & 15 & 13 & 12 & 21 & 21 & 9 & 8 & 20 & 21 & 35 & 29 & 14 & 13 & 9 & 5 & \\ 2 & 13 & 28 & 19 & 5 & 10 & 8 & 4 & 4 & 13 & 10 & 13 & 12 & 17 & 14 & 19 & 12 & 19 & 29 & 31 & 36 & 15 & 12 & 3 & 5 & 6 & 2\end{array}$

$\begin{array}{rrrrrrrrrrrrrrrrrrrrrrrrrrrrrr}2 & 13 & 28 & 19 & 5 & 10 & 8 & 4 & 4 & 18 & 10 & 13 & 12 & 17 & 14 & 19 & 12 & 19 & 29 & 31 & 36 & 15 & 12 & 3 & 5 & 6 & 2 & \\ 4 & 10 & 8 & 23 & 14 & 20 & 19 & 17 & 10 & 14 & 6 & 13 & 11 & 3 & 24 & 37 & 26 & 11 & 20 & 9 & 15 & 16 & 16 & 4 & 7 & 3 & 1 & 2\end{array}$

$\begin{array}{llllllllllllllllllllllll}1 & 8 & 19 & 20 & 14 & 23 & 22 & 48 & 20 & 17 & 9 & 19 & 11 & 7 & 17 & 17 & 32 & 18 & 15 & 7 & 9 & 3\end{array}$

$\begin{array}{rrrrrrrrrrrrrrrrrrrrrrrrrrrrrrrrrr}1 & 8 & 11 & 8 & 19 & 12 & 15 & 19 & 20 & 27 & 19 & 9 & 9 & 22 & 14 & 18 & 38 & 34 & 28 & 19 & 7 & 4 & 1 & 2 & & 1 & \\ 10 & 26 & 26 & 8 & 11 & 10 & 5 & 6 & 19 & 25 & 13 & 9 & 6 & 14 & 17 & 11 & 21 & 18 & 26 & 36 & 11 & 14 & 6 & 7 & 7 & 1 & 2\end{array}$

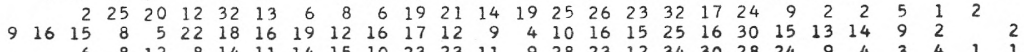

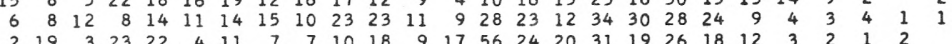

$\begin{array}{rrrrrrrrrrrrrrrrrrrrrrrrrrr}2 & 19 & 3 & 23 & 22 & 4 & 11 & 7 & 7 & 10 & 18 & 9 & 17 & 56 & 24 & 20 & 31 & 19 & 26 & 18 & 12 & 3 & 2 & 1 & 2 \\ 1 & 29 & 0 & 7 & 12 & 15 & 7 & 12 & 6 & 19 & 8 & 8 & 10 & 24 & 26 & 21 & 16 & 10 & 23 & 25 & 17 & 9 & 7 & 4 & 3\end{array}$

$\begin{array}{rrrrrrrrrrrrrrrrrrrrrrrrrrr}1 & 24 & 29 & 20 & 7 & 12 & 15 & 7 & 12 & 6 & 19 & 8 & 8 & 10 & 24 & 26 & 21 & 16 & 10 & 23 & 25 & 17 & 9 & 7 & 4 & 3 \\ 1 & 10 & 18 & 16 & 16 & 14 & 16 & 12 & 12 & 8 & 26 & 17 & 15 & 13 & 24 & 21 & 23 & 28 & 16 & 35 & 10 & 4 & 5 & 2 & 2 & 1\end{array}$

$\begin{array}{llllllllllllllllllllllll}18 & 16 & 16 & 14 & 16 & 12 & 12 & 8 & 26 & 17 & 15 & 13 & 24 & 21 & 23 & 28 & 16 & 35 & 10 & 4 & 5 & 2 & 2 \\ 13 & 15 & 14 & 18 & 12 & 10 & 26 & 9 & 25 & 14 & 12 & 10 & 32 & 21 & 21 & 31 & 25 & 26 & 10 & 8 & 3 & 5 & 1\end{array}$

STATION NUMBER 14-1925.00

South Yamhill River near Willamina, Oreg.

Summary for water years 1935-63
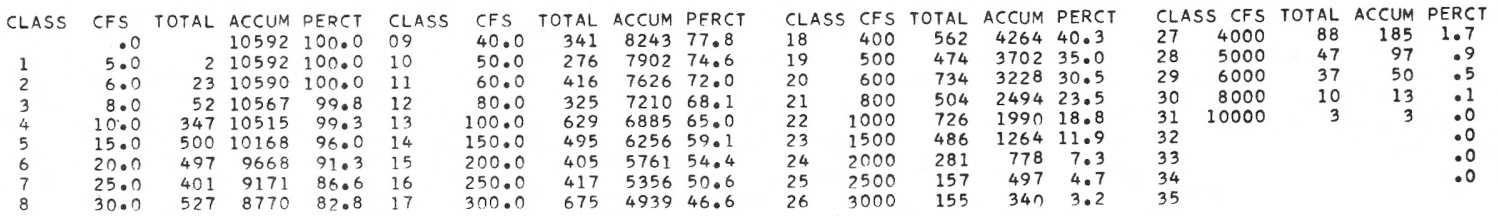

South Yambill River near

STATION NUMBER

$14-1925.00$

Willamina, Oreg. LOWEST MEAN DISCHARGE, IN CFS, FOR THE FOLLOWING NUMBER OF CONSECUTIVE DAYS IN YEAR BEGINNING APRIL 1

YEAR
1935
1936
1937
1938
1939
1940
1941
1942
1943
1944
1945
1946
1947
1948
1949
1950
1951
1952
195
1954
195
195
195
195
195
196
196
196

1
11.0
16.0
25.0
7.0
11.0
13.0
13.0
13.0
17.0
10.0
14.0
16.0
23.0
16.0
20.0
10.0
5.6
5.6
17.0
28.0
12.0
9.4
13.0
6.0
14.0
13.0
9.4
9.5

3
$11 \cdot 0$
$16 \cdot 0$
$25 \cdot 3$
$9 \cdot 7$
$11 \cdot 0$
$13 \cdot 0$
$13 \cdot 7$
$13 \cdot 3$
$17 \cdot 3$
$10 \cdot 3$
$14 \cdot 0$
$17 \cdot 0$
$23 \cdot 3$
$17 \cdot 3$
$20 \cdot 7$
$10 \cdot 7$
$6 \cdot 6$
6.4
$17 \cdot 7$
29.0
$14 \cdot 7$
10.5
$14 \cdot 0$
6.7
16.7
13.0
9.8
9.8

7
11.3
16.9
27.3
9.9
11.0
13.0
14.9
$13 \cdot 7$
$18 \cdot 1$
10.7
$14 \cdot 7$
18.7
$24 \cdot 6$
$18 \cdot 7$
21.7
$11 \cdot 3$
7.3
7.6
18.6
29.7
17.0
11.5
15.1
6.9
18.3
13.6
10.8
10.6

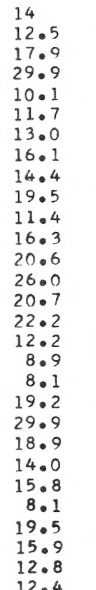

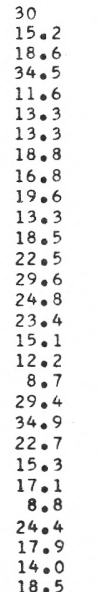

60
60.6
17.0
20.4
34.7
12.1
14.7
15.3
26.0
20.3
24.2
17.4
25.8
23.9
40.0
32.7
26.3
22.1
13.4
11.3
36.4
47.0
36.0
19.8
22.5
14.55
44.7
26.3
18.9
24.9

90
$20 \cdot 5$
$21 \cdot 7$
43.8
$13 \cdot 6$
$17 \cdot 1$
$17 \cdot 3$
$45 \cdot 1$
$25 \cdot 0$
$30 \cdot 3$
$18 \cdot 9$
$43 \cdot 6$
$27 \cdot 1$
$51 \cdot 6$
43.7
28.6
28.4
$21 \cdot 3$
12.5
45.3
50.6
50.0
22.3
28.0
18.1
68.9
30.2
20.2
29.9

120
$23 \cdot 7$
$21 \cdot 9$
$64 \cdot 5$
$18 \cdot 2$
$23 \cdot 7$
$20 \cdot 4$
$59 \cdot 1$
$38 \cdot 1$
$48 \cdot 8$
$23 \cdot 8$
$42 \cdot 8$
$47 \cdot 0$
$81 \cdot 8$
$62 \cdot 1$
$36 \cdot 9$
$42 \cdot 8$
$36 \cdot 1$
$14 \cdot 8$
$68 \cdot 0$
$71 \cdot 6$
$63 \cdot 7$
$29 \cdot 9$
$33 \cdot 9$
$24 \cdot 6$
$103 \cdot 0$
$38 \cdot 8$
$30 \cdot 2$
$46 \cdot 3$

150
29.8
27.4
104.0
26.0
30.6
33.2
73.6
61.2
70.7
45.6
54.2
55.6
97.0
87.4
49.6
70.9
78.2
21.5
85.4
90.4
97.1
44.4
45.9
39.2
158.0
69.7
48.8
88.4

183
42.9
48.7
141.0
54.6
35.8
79.5
107.0
93.8
115.0
74.0
122.0
82.9
128.0
129.0
72.8
107.0
123.0
30.7
136.0
108.0
174.0
74.6
70.1
60.4
204.0
144.0
75.0
135.0

274

165.0 643.0

238.0
194.0 194.0
278.0 394.0 511.0
297.0 198.0 477.0 414.0 480.0 318.0 516.0 476.0
160.0 501.0 338.0 718.0 252.0 391.0 401.0
327.0 327.0
453.0 36.5 .0 450.0 
W1llamina, Oreg.ंN DISCHARGE, IN CFS, FOR THE FOLLOWING NUMBER OF CONSECUTIVE DAYS IN YEAR ENDING SEPTEMBER 30

\begin{tabular}{|c|c|c|c|c|c|c|c|c|c|c|c|}
\hline YEAR & 1 & 3 & 7 & 15 & 30 & 60 & 90 & 120 & 150 & 183 & 274 \\
\hline 1935 & 5350.0 & 4910.0 & 3650.0 & 2670.0 & 1940.0 & 1740.0 & 1620.0 & $1420 \cdot 0$ & 1340.0 & 1240.0 & $875 . C$ \\
\hline 1936 & 9600.0 & $6700 . n$ & $455 n \cdot 0$ & 4400.0 & 2840.0 & 1810.0 & 1470.0 & $1260 \cdot 0$ & 1060.0 & 931.0 & 663.0 \\
\hline 1937 & 6990.0 & $5590 \cdot 0$ & 3510.0 & 2160.0 & 1730.0 & 1330.0 & 1250.0 & $1140 \cdot 0$ & 1020.0 & 873.0 & 623.0 \\
\hline 1938 & 12500.0 & 9760.0 & 6380.0 & 3580.0 & 2550.0 & 2170.0 & 1890.0 & $1690 \cdot 0$ & $1660 \cdot 0$ & 1450.0 & 1000.0 \\
\hline 1939 & 6430.0 & 4840.0 & 3710.0 & 2520.0 & 1750.0 & 1360.0 & $1170 \cdot 0$ & $1050 \cdot 0$ & 942.0 & 806.0 & .556 .0 \\
\hline 1940 & 8030.0 & 5590.0 & 3770.0 & 2840.0 & 2310.0 & 1610.0 & 1630.0 & $1440 \cdot 0$ & 1260.0 & $1090 \cdot 0$ & 750.0 \\
\hline 1941 & 4580.0 & 3700.0 & 2320.0 & 1790.0 & 1320.0 & 1130.0 & 932.0 & $784 \cdot 0$ & 683.0 & $606 \cdot 0$ & 460.0 \\
\hline 1942 & $8400 \cdot 0$ & $6090 \cdot 0$ & 4420.0 & $2880 \cdot 0$ & 2060.0 & 1490.0 & 1340.0 & $1110 \cdot 0$ & $968 \cdot 0$ & 837.0 & 611.0 \\
\hline 1943 & 7600.0 & 6040.0 & $5030 \cdot 0$ & 3230.0 & $2560 \cdot 0$ & $2300 \cdot 0$ & $2100 \cdot 0$ & $1740 \cdot 0$ & $1640 \cdot 0$ & $1430 \cdot 0$ & $1000 \cdot 0$ \\
\hline 1944 & 4000.0 & 3240.0 & 2160.0 & 1310.0 & 923.0 & 829.0 & 804.0 & $748 \cdot 0$ & 711.0 & 677.0 & 516.0 \\
\hline 1945 & 6770.0 & 5150.0 & 3510.0 & 2500.0 & 1750.0 & 1490.0 & 1410.0 & $1260 \cdot 0$ & $1110 \cdot 0$ & 979.0 & 707.0 \\
\hline 1946 & 7790.0 & 5870.0 & $4100 \cdot 0$ & 3640.0 & 2470.0 & 2180.0 & $2080 \cdot 0$ & $1810 \cdot 0$ & 1610.0 & 1390.0 & 961.0 \\
\hline 1947 & 7030.0 & $6550 . n$ & $4800 \cdot n$ & 3370.0 & 3030.0 & $1740 . n$ & 1710.0 & 1470.0 & 1270.0 & $1100 \cdot 0$ & 792.0 \\
\hline 1948 & 7660.0 & 5270.0 & 3950.0 & 2830.0 & 1970.0 & 1530.0 & 1360.0 & $1250 \cdot 0$ & 1210.0 & 1170.0 & 933.0 \\
\hline 1949 & $11300 \cdot 0$ & 6750.0 & 5820.0 & 4720.0 & 2800.0 & 1750.0 & 1790.0 & 1590.0 & 1410.0 & 1240.0 & 881.0 \\
\hline 1950 & 5540.0 & 4850.0 & 3680.0 & $2750 \cdot 0$ & 2390.0 & 2140.0 & 1950.0 & $1740 \cdot 0$ & 1570.0 & 1360.0 & 946.0 \\
\hline 1951 & 7680.0 & 5680.0 & 4390.0 & 3420.0 & 2620.0 & 2110.0 & $2050 \cdot 0$ & $1820 \cdot 0$ & $1680 \cdot 0$ & $1460 \cdot 0$ & 1030.0 \\
\hline 1952 & 5650.0 & 4910.0 & $4440 \cdot 0$ & 2930.0 & 2000.0 & 1660.0 & 1680.0 & $1460 \cdot 0$ & $1350 \cdot 0$ & 1220.0 & 881.0 \\
\hline 1953 & 8420.0 & 7540.0 & 5550.0 & 4450.0 & 3310.0 & 2300.0 & 1850.0 & $1560 \cdot 0$ & 1330.0 & $1170 \cdot 0$ & 811.0 \\
\hline 1954 & 7610.0 & 4950.0 & 4140.0 & 3030.0 & 2610.0 & 2140.0 & $2130 \cdot 0$ & $1950 \cdot 0$ & $1720 \cdot 0$ & 1470.0 & 1030.0 \\
\hline 1955 & 6290.0 & 4860.0 & 3130.0 & 1920.0 & 1460.0 & 1240.0 & 1150.0 & $1160 \cdot 0$ & $1110 \cdot 0$ & 1030.0 & 742.0 \\
\hline 1956 & 8950.0 & 7140.0 & 4730.0 & 3520.0 & 3270.0 & 2830.0 & 2310.0 & $2130 \cdot 0$ & 1950.0 & 1730.0 & 1220.0 \\
\hline 1957 & 5510.0 & 4570.0 & 2990.0 & 2430.0 & 1810.0 & 1320.0 & 1130.0 & $1090 \cdot 0$ & 976.0 & 864.0 & 632.0 \\
\hline 1958 & 6940.0 & $6190 \cdot 0$ & 4440.0 & 3320.0 & 2260.0 & 2120.0 & 1880.0 & $1590 \cdot 0$ & 1460.0 & $1280 \cdot 0$ & 883.0 \\
\hline 1959 & 7280.0 & 5590.0 & 4250.0 & 2590.0 & 2180.0 & 1760.0 & 1510.0 & 1400.0 & 1300.0 & 1150.0 & 829.0 \\
\hline 1960 & 5510.0 & 4490.0 & 3400.0 & 2960.0 & 2180.0 & 1540.0 & 1350.0 & $1150 \cdot 0$ & 1060.0 & 979.0 & 766.0 \\
\hline 1961 & 10000.0 & 6790.0 & 4740.0 & 3270.0 & 2710.0 & 2230.0 & 1850.0 & $1780 \cdot 0$ & 1620.0 & 1410.0 & 990.0 \\
\hline 1962 & 5450.0 & 4320.0 & 3180.0 & 2180.0 & 1590.0 & 1260.0 & 1070.0 & 1020.0 & 982.0 & 917.0 & 658.0 \\
\hline 1963 & 7570.0 & 6020.0 & 4560.0 & 3400.0 & 2240.0 & 1450.0 & 1290.0 & $1130 \cdot 0$ & 1100.0 & 1070.0 & 787.0 \\
\hline
\end{tabular}

Willamina Creek near W1llamina, Oreg.

CLASS $010 \begin{array}{lllllllllllllllllllllllllllllllllllll}2 & 3 & 4 & 5 & 6 & 7 & 8 & 9 & 10 & 11 & 12 & 13 & 14 & 15 & 16 & 17 & 18 & 19 & 20 & 21 & 22 & 23 & 24 & 25 & 26 & 27 & 28 & 29 & 30 & 31 & 32 & 33 & 34\end{array}$

YEAR

1935

1936

1937
1938

1939

1939

1940

1942

1942

1943
1944

1945

1946
1947

1948

1949

1951

1952
1953

1953

1954
1955

1955
1956
1957

1957
1958

1958

1960
1961

1961
1962
1963 $\begin{array}{rrrrrrrrrrrrrrrrrrrr}60 & 25 & 12 & 7 & 17 & 14 & 4 & 12 & 9 & 14 & 11 & 22 & 24 & 36 & 22 & 17 & 26 & 10 & 17 & 6 \\ 27 & 57 & 19 & 17 & 18 & 14 & 10 & 19 & 21 & 38 & 26 & 27 & 14 & 18 & 5 & 6 & 8 & 7 & 9 & 4\end{array}$

$\begin{array}{rrrrrrrrrrrrrrrrrrrrrrrrrrr}41 & 45 & 26 & 11 & 17 & 10 & 8 & 23 & 16 & 28 & 26 & 23 & 22 & 24 & 11 & 9 & 12 & 6 & 4 & 2 & 1 & & & \\ 62 & 20 & 13 & 9 & 26 & 11 & 13 & 13 & 7 & 11 & 14 & 13 & 16 & 31 & 74 & 24 & 21 & 17 & 12 & 3 & 1 & 2 & 1 & 1\end{array}$

$\begin{array}{lllllllllllllllllllllllll}5 & 68 & 19 & 16 & 9 & 19 & 23 & 16 & 20 & 15 & 34 & 12 & 14 & 10 & 34 & 24 & 8 & 7 & 6 & 2 & 2 & 1 & 1 & \end{array}$

$\begin{array}{lrrrrrrrrrrrrrrrrrrrr}57 & 30 & 41 & 11 & 13 & 14 & 7 & 12 & 9 & 20 & 26 & 19 & 19 & 20 & 14 & 13 & 12 & 11 & 11 & 1 \\ 38 & 25 & 12 & 9 & 22 & 20 & 12 & 37 & 43 & 63 & 20 & 24 & 8 & 15 & 8 & 7 & 4 & & 1 & 2\end{array}$

$\begin{array}{rrrrrrrrrrrrrrrrrrrrrr}24 & 24 & 7 & 8 & 24 & 26 & 10 & 20 & 33 & 60 & 54 & 16 & 7 & 12 & 10 & 5 & 11 & 6 & 5 & 2 & & 1 \\ 1 & \end{array}$

$\begin{array}{rrrrrrrrrrrrrrrrrrr}28 & 35 & 15 & 16 & 14 & 9 & 8 & 18 & 16 & 48 & 34 & 18 & 8 & 18 & 11 & 9 & 18 & 8 & 19 \\ 31 & 28 & 19 & 13 & 17 & 7 & 6 & 27 & 24 & 38 & 43 & 39 & 25 & 78 & 12 & 2 & 3 & 1 & 3\end{array}$

$\begin{array}{lllllllllllllllllllll}28 & 40 & 21 & 18 & 14 & 14 & 8 & 14 & 19 & 38 & 31 & 17 & 10 & 33 & 4 & 3 & 41 & 5 & 4 & 2 & 1\end{array}$

$\begin{array}{rrrrrrrrrrrrrrrrrrrrrrrrr}29 & 51 & 17 & 4 & 17 & 25 & 17 & 17 & 5 & 13 & 11 & 16 & 19 & 34 & 15 & 21 & 19 & 14 & 15 & 4 & & 1 & 1\end{array}$

$\begin{array}{llllllllllllllllllllllll}1 & 17 & 9 & 26 & 23 & 10 & 12 & 23 & 7 & 17 & 26 & 22 & 31 & 47 & 35 & 17 & 17 & 13 & 9 & 2 & 1 & 1 & & \\ & 11 & 45 & 18 & 28 & 21 & 11 & 16 & 14 & 53 & 21 & 13 & 18 & 23 & 16 & 11 & 17 & 7 & 7 & 7 & 4 & 1 & 2 & 1\end{array}$

$\begin{array}{rrrrrrrrrrrrrrrrrrr}11 & 45 & 18 & 28 & 21 & 11 & 16 & 14 & 53 & 21 & 13 & 18 & 23 & 16 & 11 & 17 & 7 & 7 & 7 \\ 41 & 20 & 28 & 30 & 10 & 10 & 28 & 15 & 14 & 16 & 9 & 21 & 30 & 18 & 15 & 27 & 12 & 21 & 4\end{array}$

$\begin{array}{lllllllllllllllllllll}31 & 21 & 19 & 11 & 16 & 11 & 9 & 16 & 11 & 42 & 12 & 11 & 11 & 23 & 27 & 15 & 38 & 18 & 18 & 4\end{array}$

$\begin{array}{rrrrrrrrrrrrrrrrrrrrrrrrr}25 & 38 & 18 & 5 & 10 & 21 & 14 & 11 & 9 & 31 & 12 & 15 & 23 & 47 & 33 & 17 & 10 & 10 & 8 & 3 & 5 & 1 & \\ 31 & 35 & 25 & 14 & 71 & 11 & 8 & 15 & 9 & 31 & 44 & 70 & 12 & 21 & 11 & -5 & 12 & 16 & 13 & 4 & 1 & & 2\end{array}$ $\begin{array}{lllllllllllllllllllll}13 & 34 & 39 & 19 & 19 & 43 & 17 & 19 & 14 & 13 & 14 & 27 & 20 & 14 & 20 & 10 & 16 & 11 & 2 & 1\end{array}$

$\begin{array}{rrrrrrrrrrrrrrrrrrrrrrrrr}2 & 23 & 30 & 7 & 27 & 20 & 23 & 22 & 8 & 17 & 17 & 28 & 22 & 45 & 31 & 15 & 14 & 7 & 5 & 1 & 1 & & \\ 13 & 52 & 8 & 12 & 16 & 13 & 12 & 20 & 8 & 18 & 11 & 18 & 12 & 21 & 25 & 17 & 25 & 22 & 23 & 11 & 4 & 3 & 2\end{array}$

$\begin{array}{rrrrrrrrrrrrrrrrrrrrrrr}13 & 40 & 15 & 11 & 24 & 13 & 7 & 30 & 21 & 33 & 33 & 28 & 22 & 26 & 17 & 7 & 9 & 5 & 7 & 2 & 2 & \end{array}$

$\begin{array}{rrrrrrrrrrrrrrrrrrrrr}33 & 42 & 26 & 13 & 13 & 18 & 4 & 21 & 14 & 17 & 13 & 17 & 22 & 27 & 18 & 9 & 17 & 12 & 24 & 3 & 1 \\ 7 & 21 & 34 & 10 & 72 & 6 & 10 & 23 & 14 & 30 & 27 & 29 & 18 & 43 & 14 & 17 & 19 & 12 & 10 & 3 & 1\end{array}$

$\begin{array}{rrrrrrrrrrrrrrrrrrrrrr}1 & 32 & 19 & 11 & 16 & 8 & 10 & 17 & 12 & 40 & 43 & 32 & 24 & 35 & 22 & 9 & 19 & 6 & 6 & 3 & 1 & \\ 25 & 47 & 18 & 12 & 17 & 16 & 6 & 13 & 10 & 15 & 29 & 35 & 13 & 15 & 10 & 13 & 22 & 17 & 21 & 7 & 3 & 1\end{array}$

$\begin{array}{rrrrrrrrrrrrrrrrrrrrrrrr}7 & 31 & 26 & 11 & 26 & 17 & 21 & 17 & 8 & 24 & 28 & 27 & 33 & 37 & 20 & 10 & 8 & 4 & 6 & 3 & 1 & & \\ 111 & 33 & 17 & 21 & 22 & 21 & 14 & 14 & 26 & 37 & 23 & 35 & 33 & 20 & 5 & 14 & 5 & 9 & 2 & & 2 & 1\end{array}$
W11lamine Creak near willowina, Oreg.

Summary for water years 1935-63

CLASS CFS TOTAL ACCUM PERCT CLASS

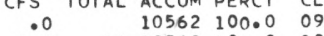

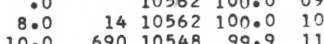

$\begin{array}{rrrrr}10.0 & 690 & 10548 & 99.9 & 11 \\ 15.0 & 892 & 9858 & 93.3 & 12\end{array}$

$\begin{array}{lllll}15.0 & 892 & 9858 & 93.3 & 12 \\ 20.0 & 600 & 8966 & 84.9 & 13\end{array}$

$\begin{array}{lllll}25.0 & 387 & 8366 & 79.2 & 14\end{array}$

$\begin{array}{lllll}30.0 & 579 & 7979 & 75.5 & 15\end{array}$

$\begin{array}{lllll}40.0 & 442 & 7400 & 70.1 & 16 \\ 50.0 & 335 & 6958 & 65.9 & 17\end{array}$
STATION NUMBER $\quad 14-1930.00$

DURATION TABLF OF DAILY DISCHARGE
CFS-DAYS 107231.0 73361.0 66944.0
113492.0 65936.0 77784.0 48610.1 65635.0 113492.0 56942.0

78868.0

103394.0

90261.0

108732.0

107048.0

113907.0

119910.0

105069.0

$92442 . ?$

113523.0

$86974 . n$

150521.0

75700.0
103376.0

103376.0
95685.0

89823.0

117339.0

90814.0
BTATION MUNGERR 14-1930.00

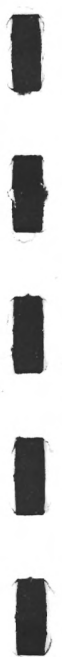

।

।

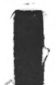

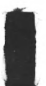

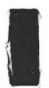

CFS TOTAL ACCUM PERCT $\begin{array}{rrrr}60.0 & 579 & 6623 & 62.7\end{array}$ $80.0 \quad 431 \quad 604457.2$ $150.0724 \quad 475045.0$ $200.0 \quad 614402638.1$ $250.0 \quad 529 \quad 3412 \quad 32.3$ $300.0 \quad 821 \quad 2883 \quad 27.3$ $\begin{array}{llll}300.0 & 821 & 2883 & 27.3 \\ 400.0 & 510 & 2062 & 19.5 \\ 500.0 & 324 & 1552 & 14.7\end{array}$

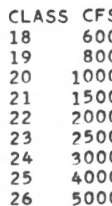

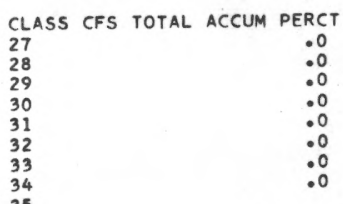


Willamina Creek near Willamina, Oreg.

STATION NUMRER 14-1930.0ก

LAWEST MEAN DISCHARGF, IN CFS, FOR THF FOLLOWING NUMRFR OF CONSFCUTIVF DAYG IN YFAR RFGINNING APRIL 1

\begin{tabular}{|c|c|c|c|c|c|c|c|c|c|c|c|}
\hline YEAR & 1 & 3 & 7 & 14 & 30 & 60 & 90 & 120 & 150 & 183 & 274 \\
\hline 1935 & 10.0 & 10.0 & 10.0 & 10.8 & 12.0 & 13.3 & 14.9 & $16 \cdot 2$ & 19.9 & 24.7 & 71.9 \\
\hline $\begin{array}{l}1936 \\
1937\end{array}$ & 11.0 & 11.7 & $12 \cdot 6$ & 13.0 & 13.5 & 14.0 & 14.5 & 15.1 & 17.5 & 24.9 & 62.0 \\
\hline $\begin{array}{l}1937 \\
1938\end{array}$ & 16.0 & 16.0 & 16.4 & 17.3 & 20.1 & 22.1 & 24.5 & 29.1 & 42.4 & 59.4 & 254.0 \\
\hline $\begin{array}{l}1938 \\
1939\end{array}$ & 10.0 & 10.0 & 10.3 & $10 \cdot 6$ & 12.0 & 12.2 & 13.6 & $15 \cdot 9$ & 21.7 & 35.1 & 99.9 \\
\hline $\begin{array}{l}1939 \\
1940\end{array}$ & 9.0 & 9.0 & 9.3 & 9.8 & 10.9 & 11.8 & 13.4 & 15.9 & 17.3 & 21.5 & 69.5 \\
\hline $\begin{array}{l}1940 \\
1941\end{array}$ & 9.4 & 9.7 & 10.4 & 10.7 & 10.8 & 12.1 & 13.9 & 16.7 & 23.6 & 35.9 & 103.0 \\
\hline $\begin{array}{l}1941 \\
1942\end{array}$ & 10.0 & $10 \cdot 3$ & 10.9 & 11.5 & 12.7 & 17.0 & 25.4 & 30.4 & 35.3 & 46.8 & 144.0 \\
\hline $\begin{array}{l}1942 \\
1943\end{array}$ & 11.0 & $11 \cdot n$ & 11.6 & 12.2 & 13.0 & 14.0 & 16.3 & $\begin{array}{l}22.2 \\
28.5\end{array}$ & $\begin{array}{l}33.0 \\
39.6\end{array}$ & $\begin{array}{l}47.1 \\
57.7\end{array}$ & $\begin{array}{l}208.0 \\
115 \circ \circ\end{array}$ \\
\hline $\begin{array}{l}1943 \\
1944\end{array}$ & 13.0 & $13 \cdot n$ & 13.7 & 14.1 & 14.6 & $17 \cdot 5$ & $\begin{array}{l}20.7 \\
15.7\end{array}$ & 19.1 & $\begin{array}{l}30.6 \\
27.3\end{array}$ & $\begin{array}{l}57.8 \\
37.8\end{array}$ & $\begin{array}{r}174.7 \\
81.7\end{array}$ \\
\hline $\begin{array}{l}1944 \\
1945\end{array}$ & 11.0 & 11.0 & 11.4 & $12 \cdot 3$ & 13.7 & $\begin{array}{l}14.7 \\
20.6\end{array}$ & $\begin{array}{l}15.7 \\
21.0\end{array}$ & 22.7 & 28.7 & 53.0 & 205.0 \\
\hline 1946 & $\begin{array}{l}13.0 \\
12.0\end{array}$ & $\begin{array}{l}13.7 \\
12 . n\end{array}$ & $\begin{array}{l}14.0 \\
12.1\end{array}$ & $\begin{array}{l}15.1 \\
13.6\end{array}$ & $\begin{array}{l}16.0 \\
14.3\end{array}$ & $\begin{array}{l}20.6 \\
14.8\end{array}$ & 16.2 & 22.7 & 28.5 & 41.1 & 188.0 \\
\hline 1947 & 16.0 & 16.0 & 16.9 & 17.7 & 19.3 & 22.5 & 26.3 & 36.5 & 44.9 & 60.0 & 169.0 \\
\hline 1948 & 1400 & 14.7 & 15.0 & 15.6 & 18.9 & 24.0 & 30.6 & $38 \cdot 2$ & 44.8 & 65.8 & 218.0 \\
\hline $\begin{array}{l}1949 \\
1950\end{array}$ & 16.0 & $16 \cdot n$ & 17.0 & 18.1 & 20.7 & 22.3 & 24.2 & 29.5 & 37.2 & 43.4 & 141.0 \\
\hline 1950 & 13.0 & $14 \cdot 3$ & 15.0 & 15.4 & 16.4 & 20.5 & 24.0 & $32 \cdot 1$ & 43.4 & 62.2 & $218 . n$ \\
\hline 1951 & 10.0 & 10.7 & 10.9 & 11.9 & 14.2 & 15.5 & 19.6 & $27 \cdot 6$ & 43.0 & 59.9 & $212 . n$ \\
\hline 1952 & 9.6 & 9.8 & 10.3 & 10.5 & 10.9 & 12.6 & 13.7 & 14.9 & 17.3 & 22.1 & $71 . n$ \\
\hline 1953 & 16.0 & 16.3 & 17.9 & 18.8 & 20.9 & 24.5 & 28.1 & 33.8 & $4 n .1$ & 62.3 & 198.0 \\
\hline 1954 & 20.0 & 20.3 & 21.3 & 22.3 & 27.5 & 29.0 & 31.0 & 37.5 & 45.1 & $51 . n$ & 130.0 \\
\hline 1955 & 14.0 & $14 \cdot 3$ & 15.4 & 16.4 & 18.2 & 27.7 & 28.0 & 35.8 & 52.5 & 84.8 & $321 . n$ \\
\hline 1956 & 11.0 & 11.7 & 12.3 & 14.4 & 15.0 & 16.2 & 17.1 & 21.1 & 28.0 & 41.2 & 114.0 \\
\hline $\begin{array}{l}1957 \\
1958\end{array}$ & 13.0 & $13 . n$ & 13.6 & 14.6 & 15.7 & 18.4 & 20.8 & 23.2 & 28.6 & 40.8 & $164 \cdot n$ \\
\hline 1958 & 10.0 & 10.7 & 11.9 & 12.5 & 12.7 & 14.4 & 15.7 & 19.7 & 25.8 & 38.9 & 161.0 \\
\hline 1959 & 16.0 & 17.0 & 17.7 & 18.5 & 20.2 & 26.0 & 35.0 & 51.1 & 64.9 & 77.8 & 139.0 \\
\hline 1960 & 14.0 & 14.0 & 14.4 & 15.6 & 16.7 & 19.5 & 20.8 & $26 \cdot 1$ & 35.7 & 70.7 & 194.0 \\
\hline 1961 & 12.0 & $12 \cdot 3$ & $13 \cdot 3$ & 13.6 & 14.0 & $15 \cdot 3$ & 17.7 & 22.1 & 31.4 & 40.1 & 151.0 \\
\hline 1962 & 14.0 & $14 \cdot 3$ & 15.0 & 16.3 & 18.6 & 21.9 & 25.9 & 33.7 & 49.7 & 64.4 & 195.0 \\
\hline
\end{tabular}

Willamina Creek near W1llamina, Oreg.

STATION NUMRFR

14-1930.ก0

HITHEST MEAN DISCHARGE, IN CFS, FOR THE FOLLOWING NUMBFR OF CONSECUTIVE DAYS IN YFAR FNDING SEPTFMBER 30

\begin{tabular}{|c|c|c|c|c|c|c|c|c|c|c|c|}
\hline YEAR & 1 & 3 & 7 & 15 & 30 & 60 & 90 & $12 n$ & $15 n$ & 183 & 274 \\
\hline 1935 & 1820.0 & $1680 \cdot n$ & 1470.0 & 1110.0 & 837.0 & 725.0 & 689.0 & 619.0 & 597.0 & 541.0 & 387.0 \\
\hline $\begin{array}{l}1936 \\
1937\end{array}$ & 2900.0 & $2120 \cdot n$ & 1620.0 & 1560.0 & 1070.0 & 704.0 & 588.0 & 496.0 & 424.0 & $370 . n$ & \\
\hline $\begin{array}{l}1937 \\
1938\end{array}$ & 2080.0 & 1780.0 & 1230.0 & 795.0 & 676.0 & 563.0 & 499.0 & 437.0 & 383.0 & 332.0 & 239.0 \\
\hline $\begin{array}{l}1938 \\
1939\end{array}$ & 4900.0 & 3710.0 & 2510.0 & 1450.0 & 1040.0 & 817.0 & 740.0 & $690 \cdot 0$ & 666.0 & 587.0 & 409.0 \\
\hline $\begin{array}{l}1939 \\
1940\end{array}$ & 2680.0 & $2110 . n$ & 1580.0 & 1080.0 & 749.0 & 569.0 & 507.0 & 445.0 & 386.0 & 334.0 & $236 . n$ \\
\hline $\begin{array}{l}1940 \\
1941\end{array}$ & 3100.0 & $2170 \cdot n$ & 1520.0 & 1160.0 & 952.0 & 665.0 & 587.0 & $521 \cdot 0$ & $462 . n$ & $402 \cdot n$ & 279.0 \\
\hline 1941 & 1870.0 & 1580.0 & 1000.0 & 741.0 & 525.0 & 419.0 & 341.0 & 284.0 & 247.0 & 224.0 & 169.0 \\
\hline 1942 & 2690.0 & 2110.0 & 1510.0 & 1010.0 & 688.0 & 537.0 & 472.0 & 406.0 & 356.0 & 311.0 & 232.0 \\
\hline 1943 & 3440.0 & $2470 . n$ & 2060.0 & 1360.0 & 1000.0 & 934.0 & 825.0 & 687.0 & 662.0 & 573.0 & 408.0 \\
\hline 1944 & 1340.0 & $123 n . n$ & $841 . n$ & $505 . n$ & 336.0 & $3 n 4 . n$ & $3 n 5.0$ & $288 \cdot 0$ & 283.0 & 258.0 & $2 n 2.0$ \\
\hline 1945 & 2260.0 & 171non & $130 n \cdot n$ & 935.0 & $666 . n$ & $619 . n$ & 581.0 & 515.0 & $448 . \cap$ & 391.0 & $281 . n$ \\
\hline 1946 & $300 n$. n & 2470.0 & 1660.0 & 1360.0 & 927.0 & $794 . n$ & 745.0 & 671.0 & 608.0 & 533.0 & 372.0 \\
\hline 1947 & 2870.0 & $2670 . n$ & 2040.0 & 1400.0 & 1090.0 & 669.0 & 674.0 & 590.0 & 517.0 & 445.0 & 370.0 \\
\hline 1948 & 2670.0 & 1970.0 & 1360.0 & 1040.0 & 725.0 & 609.0 & 549.0 & 508.0 & 492.0 & 476.0 & 387.0 \\
\hline 1949 & 4590.0 & 2970.0 & $2490 . n$ & 1790.0 & 1150.0 & 745.0 & 755.0 & 688.0 & 610.0 & 536.0 & 383.0 \\
\hline 1950 & 2890.0 & $2430 . n$ & 1670.0 & $1390 . n$ & $115 n .0$ & 909.0 & 824.0 & 737.0 & 667.0 & $578 . n$ & $4 \cap 8.0$ \\
\hline 1951 & 2970.0 & 2130.0 & $1680 . n$ & 1390.0 & 1090.0 & 884.0 & 830.0 & 729.0 & 679.0 & 601.0 & 431.0 \\
\hline 1952 & 2530.0 & $2170 . n$ & 1920.0 & 1230.0 & 821.0 & 664.0 & 700.0 & $617 \cdot 0$ & 578.0 & 521.0 & 377.0 \\
\hline 1953 & 3300.0 & $3000 \cdot n$ & 2280.0 & 1660.0 & 1320.0 & 913.0 & 743.0 & $618 \cdot 0$ & 533.0 & 470.0 & 331.0 \\
\hline 1954 & 2800.0 & $1830 . n$ & 1560.0 & 1140.0 & 940.0 & 840.0 & 821.0 & $750 \cdot 0$ & 666.0 & 571.0 & 404.0 \\
\hline 1955 & 2160.0 & $1810 . n$ & 1200.0 & 743.0 & 603.0 & 516.0 & 491.0 & 504.0 & 467.0 & 426.0 & $3 n 9.0$ \\
\hline 1956 & 3880.0 & $2850 . n$ & 1970.0 & 1470.0 & 1320.0 & 1210.0 & 989.0 & 905.0 & $86 ? .0$ & 764.0 & $542 . n$ \\
\hline 1957 & $2120 . n$ & $185 n . n$ & 1260.0 & 1060.0 & 812 ? & 579.0 & 469.0 & $462 \cdot 0$ & 414.0 & 363.0 & $260^{\circ}$ n \\
\hline 1958 & 3030.0 & $2480 . n$ & $176 n .0$ & 1330.0 & 925.0 & $884 . n$ & 787.0 & 667.0 & 613.0 & 531.0 & $372 . n$ \\
\hline 1939 & 236000 & 1760 on & 145000 & 952 . ก & 870.0 & 722.0 & 600.0 & 554.0 & 519.0 & 462.0 & 337.0 \\
\hline 1960 & 2220.0 & $1810 . n$ & 1390.0 & 1110.0 & 836.0 & 605.0 & 532.0 & 466.0 & $43 n .0$ & 408.0 & $319 . n$ \\
\hline 1961 & 3480.0 & 2400.0 & 1740.0 & 1440.0 & 1210.0 & 979.0 & 809.0 & $750 \cdot 0$ & 686.0 & 601.0 & 423.0 \\
\hline 1962 & 2150.0 & 1730.0 & 1310.0 & 915.0 & 656.0 & 509.0 & 432.0 & 424.0 & 409.0 & 385.0 & 280.0 \\
\hline 1963 & 3280.0 & 2210.0 & 1790.0 & 1410.0 & 903.0 & 594.0 & 507.0 & 449.0 & 451.0 & 433.0 & 322.0 \\
\hline
\end{tabular}


LOWEST. MEAN DISCHARGE, IN CFS, FOR THE FOLLOWING NUMBER OF CONSECUTIVE DAYS IN YEAR BEGINNING APRIL 1

\begin{tabular}{|c|c|c|c|c|c|c|c|c|c|c|c|}
\hline YEAR & 1 & 3 & 7 & 14 & 30 & 60 & 90 & 120 & 150 & 183 & 274 \\
\hline 1959 & 4.6 & 4.7 & 4.9 & 5.0 & 5.3 & 7.5 & 11.6 & 17.4 & 25.2 & 29.0 & 55.9 \\
\hline 1960 & 4.5 & 4.5 & 4.6 & 4.9 & 5.2 & 6.3 & 7.4 & 9.2 & 15.6 & 29.2 & 101.0 \\
\hline 1961 & 3.9 & 4.0 & 4.2 & 4.2 & 4.4 & 4.7 & 5.4 & $7 \cdot 2$ & 10.7 & 16.8 & 77.0 \\
\hline 962 & 3.7 & 3.8 & 3.9 & 4.2 & 5.0 & 5.8 & 7.0 & $10 \cdot 6$ & 19.1 & 31.3 & 92.8 \\
\hline
\end{tabular}

MILI CREFK NR WHLAMINA OREG.

STATION NUMBER $\quad 14-1933.00$

HIGHEST MEAN DISCHARGE, IN CFS, FOR THE FOLLOWING NUMBFR OF CONSECUTIVE DAYS IN YEAR ENDING SEPTEMBER 30

\begin{tabular}{|c|c|c|c|c|c|c|c|c|c|c|c|}
\hline YEAR & 1 & 3 & 7 & 15 & 30 & 60 & 90 & 120 & 150 & 183 & 274 \\
\hline 1959 & 2120.0 & $1530 . ?$ & $1140 . ?$ & 651.0 & 510.0 & 410.0 & 330.0 & $306 \cdot 0$ & 288.0 & 253.0 & 180.0 \\
\hline 1960 & 1500.0 & 1190.0 & $878 . ?$ & 735.0 & 534.0 & 369.0 & 336.0 & $280 \cdot 0$ & $251 \cdot 0$ & 225.0 & 167.0 \\
\hline 1961 & 2980.0 & 1710.0 & 1110.0 & 768.0 & 585.0 & 495.0 & 411.0 & $389 \cdot 0$ & 358.0 & 313.0 & 220.0 \\
\hline 1952 & 1250.0 & 1110.0 & 790.0 & 495.0 & 351.0 & 265.0 & 235.0 & $227 \cdot 0$ & $216 \cdot 0$ & 202.0 & 144.0 \\
\hline 1963 & 2200.0 & 1490.0 & $941 \cdot 0$ & 687.0 & 458.0 & 290.0 & 270.0 & 231.0 & 236.0 & 233.0 & 173.0 \\
\hline
\end{tabular}

South Yambill River near Whiteson, Oreg.

STATION NUNGFR $14-194 \cap \cdot 00$

DURATION TARLF OF MUILY DISCHMRCF

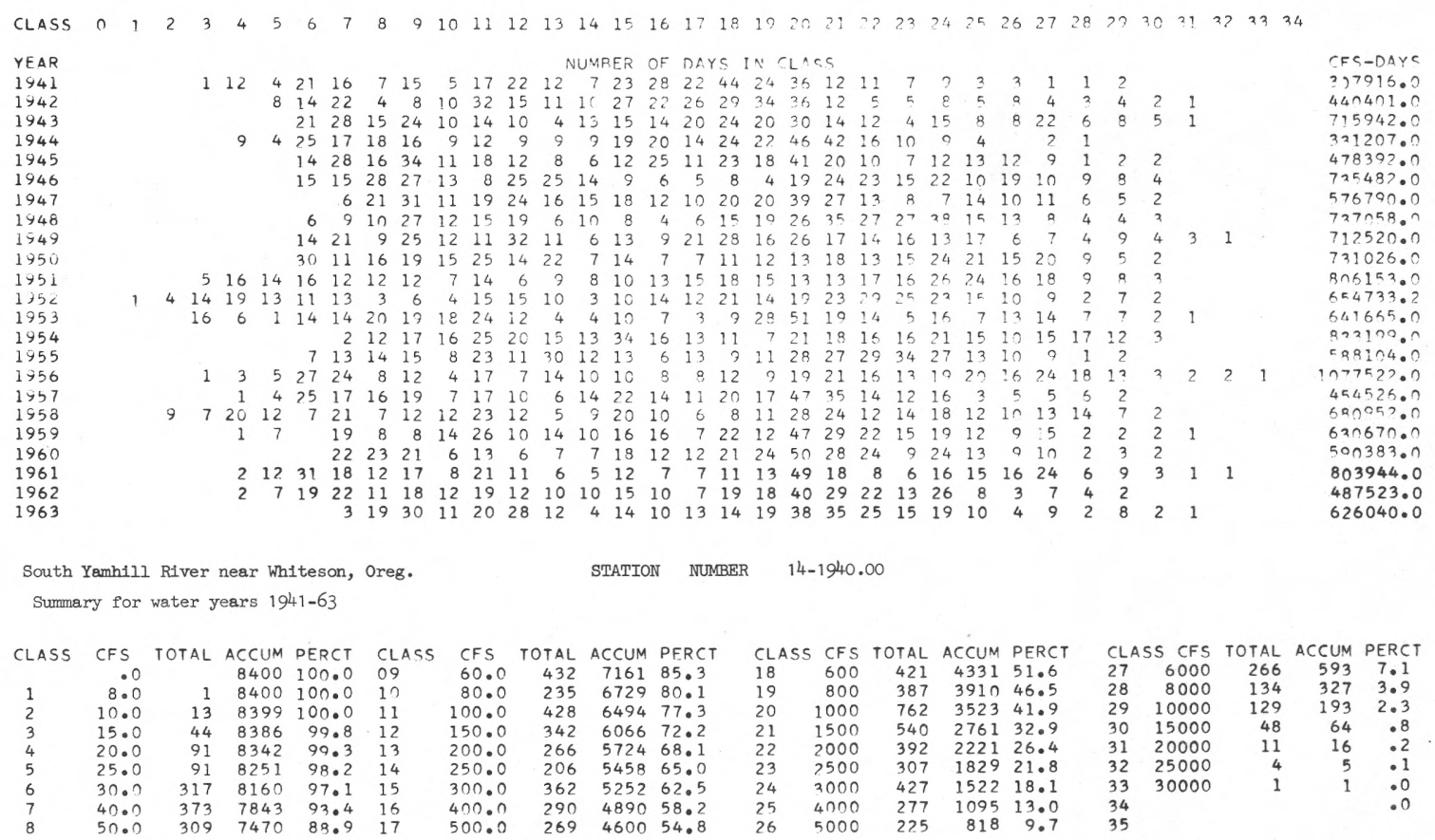


South Yambill River near Whiteson, Oreg.

STATION NUMBFR

$14-1940 \cdot 00$

LAWEST MEAN DISCHARGF, IN CFS, FOR THF FOLLOWING NUMRER OF CONGECUTIVF DAYC IN YFAR RFGINNING APDIL 1

\begin{tabular}{|c|c|c|c|c|c|c|c|c|c|c|c|}
\hline YEAR & 1 & 3 & 7 & 14 & 30 & 60 & $3 n$ & $12 n$ & $15 n$ & 182 & \\
\hline 1941 & 19.0 & $21 . n$ & $21 \cdot 7$ & 22.4 & 27.9 & 44.8 & 85.4 & $109 \cdot 0$ & $13 n .0$ & 195.0 & 950.0 \\
\hline 1942 & $25 \cdot 0$ & 25.7 & $27 \cdot 1$ & 29.4 & 33.0 & 38.2 & 46.4 & $71 \cdot 5$ & $123 \cdot 0$ & $\begin{array}{l}202 \cdot 0 \\
256.0\end{array}$ & $\begin{array}{r}1210.0 \\
65 ? . ?\end{array}$ \\
\hline 1943 & 30.0 & 31.7 & 34.4 & 38.0 & 40.2 & $5 n \cdot 2$ & 62.4 & $97 \cdot 1$ & $147 \cdot 0$ & $\begin{array}{l}256.0 \\
156.0\end{array}$ & $395 . ?$ \\
\hline $\begin{array}{l}1944 \\
1945\end{array}$ & $20 \cdot 0$ & 20.7 & $21 \cdot 4$ & $25 \cdot 1$ & 30.4 & $40 . \hat{9}$ & $\begin{array}{l}43.7 \\
81.7\end{array}$ & $\begin{array}{l}53.1 \\
83.2\end{array}$ & $106 . ?$ & $254 . n$ & I3nn:n \\
\hline $\begin{array}{l}1945 \\
1946\end{array}$ & $\begin{array}{l}30.0 \\
34.0\end{array}$ & $\begin{array}{l}30 \cdot 7 \\
35.3\end{array}$ & $\begin{array}{l}33.4 \\
36.9\end{array}$ & $\begin{array}{l}36.7 \\
40.1\end{array}$ & $\begin{array}{l}41.6 \\
44.9\end{array}$ & $\begin{array}{l}58.9 \\
50.4\end{array}$ & $\begin{array}{l}51.07 \\
57.7\end{array}$ & 94.7 & $12 n \cdot n$ & $20 n \cdot n$ & $1170 . n$ \\
\hline $\begin{array}{l}1946 \\
1947\end{array}$ & $\begin{array}{l}34 \cdot 0 \\
46.0\end{array}$ & $\begin{array}{l}35 \cdot 3 \\
47 \cdot 0\end{array}$ & $\begin{array}{l}36.9 \\
51.7\end{array}$ & $\begin{array}{l}40.1 \\
54 \cdot 2\end{array}$ & 63.1 & 83.3 & $1 \cap 3.0$ & $159 \cdot 0$ & $204 . ?$ & $287 . n$ & $104 n . ?$ \\
\hline 1948 & 35.0 & 35.7 & 36.9 & 43.4 & 50.3 & 62.6 & 88.0 & $132 \cdot n$ & 172.0 & $303 . ?$ & 1360.0 \\
\hline $\begin{array}{l}1949 \\
1950\end{array}$ & 35.0 & 35.7 & 35.9 & $37 \cdot 1$ & 40.9 & 52.9 & 62.2 & 90.3 & $108 \cdot ?$ & $166 . ?$ & $774 . ?$ \\
\hline 1950 & 31.0 & $32 . n$ & 32.3 & 33.4 & 37.7 & 46.8 & 59.3 & $90 . ?$ & $151 . n$ & 242.0 & 1440.0 \\
\hline $\begin{array}{l}1951 \\
1952\end{array}$ & 16.0 & 16.7 & $18 \cdot 3$ & $2.1 \cdot 2$ & 26.8 & 29.6 & 41.8 & 72.9 & $164 . n$ & 255.0 & 1100.0 \\
\hline $\begin{array}{l}1952 \\
1953\end{array}$ & 9.2 & 10.4 & $13 . n$ & $15 \cdot ?$ & 16.6 & $21 \cdot n$ & 22.7 & $27 \cdot 8$ & 44.9 & 65.4 & $378 . ?$ \\
\hline $\begin{array}{l}1953 \\
1954\end{array}$ & 40.0 & $42 . n$ & $45 . ?$ & $48 \cdot 8$ & 69.? & 76.9 & $98 \cdot ?$ & $156 \cdot n$ & $196 . n$ & $35 ? \cdot n$ & $132 n \cdot n$ \\
\hline $\begin{array}{l}1954 \\
1955\end{array}$ & $47 \cdot 0$ & 48.7 & 53.7 & $56 \cdot 0$ & 62.4 & 97.3 & $100 \cdot 0$ & $145 \cdot ?$ & $199 \cdot n$ & $24^{2} \cdot n$ & $90^{2} \cdot 0$ \\
\hline 1955 & 32.0 & 32.3 & 35.1 & 39.7 & 45.1 & 70.1 & $104 \cdot 0$ & $142 \cdot ?$ & 248.0 & $45 ? \cdot n$ & 7160.0 \\
\hline $\begin{array}{l}1956 \\
1957\end{array}$ & 19.0 & 20.7 & $25 \cdot n$ & $31 \cdot 1$ & $32 \cdot 3$ & $41 \cdot 1$ & $45 \cdot 3$ & 61.7 & $101 \cdot n$ & $196 . n$ & $6 \cap 3 \cdot n$ \\
\hline $\begin{array}{l}1957 \\
1958\end{array}$ & $24 \cdot 0$ & $27 \cdot 3$ & $30 \cdot 1$ & 31.7 & 33.8 & $45 \cdot 2$ & $58 \cdot n$ & $74 \cdot 9$ & $103 \cdot n$ & $172 \cdot n$ & $\begin{array}{l}978 \cdot 0 \\
929.0\end{array}$ \\
\hline 1958 & 11.0 & $11 \cdot 3$ & 11.9 & $14 \cdot 6$ & 17.8 & 24.9 & 30.6 & $47 \cdot 4$ & 83.7 & $151 \cdot 0$ & $\begin{array}{l}929.0 \\
725 \%\end{array}$ \\
\hline 1959 & 40.0 & 42.0 & 43.4 & $45 \cdot 6$ & 54.8 & $97 \cdot 1$ & 140.0 & 222.0 & $319 \cdot n$ & $389 \cdot n$ & $\begin{array}{r}725.0 \\
1200.0\end{array}$ \\
\hline 1960 & 40.0 & .40 .7 & $41 \cdot 9$ & $46 \cdot 2$ & 48.4 & $\begin{array}{l}57.4 \\
37.4\end{array}$ & $\begin{array}{l}64.9 \\
42.1\end{array}$ & $\begin{array}{l}83 \cdot 0 \\
64 \cdot 5\end{array}$ & $\begin{array}{l}145.0 \\
109.0\end{array}$ & $\begin{array}{l}345.0 \\
164.0\end{array}$ & 873.0 \\
\hline 1961 & 24.0 & 25.0 & $\begin{array}{l}26.3 \\
25.4\end{array}$ & $\begin{array}{l}29.2 \\
29.2\end{array}$ & $\begin{array}{l}30.7 \\
38.2\end{array}$ & $\begin{array}{l}37.4 \\
50.2\end{array}$ & $\begin{array}{l}42.1 \\
62.8\end{array}$ & 103.0 & 226.0 & 322.0 & 1190.0 \\
\hline & 23.0 & 23.7 & 23.4 & & & & & & & & \\
\hline
\end{tabular}

South Yamb111 River near Whiteson, Oreg.

STATION NUMBER

$14-194 n .00$

HIGHEST MEAN DISCHARGE, IN CFS, FOR THF FOLLOWING NUMBFR OF CONSFCUTIVF DAYC IN YFAR FNMING SFPTFMBFR 30

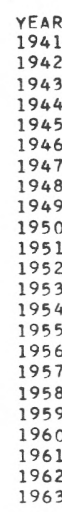

\begin{tabular}{|c|c|}
\hline 1 & 3 \\
\hline 12400.0 & 10500.0 \\
\hline 21100.0 & 18100.0 \\
\hline 21200.0 & $166 n 0 . n$ \\
\hline $8150 \cdot 0$ & 7210.0 \\
\hline $16 \times 00.0$ & $142 n 0 . n$ \\
\hline 18500.0 & $148 \cap 0.0$ \\
\hline $19200 \cdot 0$ & $16200 \cdot n$ \\
\hline $19,00.0$ & $16300 . n$ \\
\hline $26100 \cdot 0$ & $20100 \cdot n$ \\
\hline 17600.0 & $15600 . n$ \\
\hline $18800 \cdot 0$ & $16400 \cdot n$ \\
\hline $17400 \cdot 0$ & $14900 \cdot n$ \\
\hline 20700.0 & $18000 \cdot n$ \\
\hline 17800.0 & 14100.0 \\
\hline 12400.0 & $10800 \cdot n$ \\
\hline 32700.0 & $24400 \cdot n$ \\
\hline $12 k 0 n \cdot 0$ & $11500 \cdot 0$ \\
\hline $18 \cap n 0 \cdot 0$ & $156 n 0 . n$ \\
\hline $22500 \cdot 0$ & $18500 \cdot n$ \\
\hline $15700 \cdot 0$ & $139 n n \cdot n$ \\
\hline $26400 \cdot 0$ & $17600 \cdot 0$ \\
\hline 11900.0 & $11000 \cdot 0$ \\
\hline $24100 \cdot 0$ & $17200 \cdot 0$ \\
\hline
\end{tabular}

\begin{tabular}{|c|c|}
\hline 7 & 15 \\
\hline 6760.0 & $4850 \cdot 0$ \\
\hline $3700 \cdot 0$ & 8580.0 \\
\hline $410 n \cdot n$ & 9140.0 \\
\hline $5050 \cdot 0$ & $3040 \cdot 0$ \\
\hline $978 n . n$ & $6930 . ?$ \\
\hline$n 9 \cap n \cdot n$ & $9850 . n$ \\
\hline $118 n n \cdot n$ & $8310 \cdot n$ \\
\hline $17 n n . n$ & $875 n . n$ \\
\hline $180 n n \cdot 0$ & $142 n 0 . ?$ \\
\hline $11200 \cdot 0$ & $7490 . ?$ \\
\hline $13600 \cdot 0$ & $10500 \cdot 0$ \\
\hline $230 n \cdot n$ & 8310.0 \\
\hline $145 n n \cdot 0$ & $119 n 0.0$ \\
\hline$n 60 n \cdot n$ & $9100 \cdot 0$ \\
\hline $784 n \cdot 0$ & $5180 \cdot ?$ \\
\hline $16800 \cdot n$ & $12400 \cdot 0$ \\
\hline $8410 . n$ & 6920.0 \\
\hline $121 n n \cdot n$ & $9190 \cdot 0$ \\
\hline $43 n n \cdot n$ & $87 n n \cdot n$ \\
\hline$n 5 n n \cdot n$ & $8550 \cdot n$ \\
\hline $0 \bullet$ & $11200 \cdot 0$ \\
\hline $8870 \cdot 0$ & $6100 \cdot 0$ \\
\hline $11500 \cdot 0$ & 9240.0 \\
\hline
\end{tabular}

30
3450.0
5690.0
6390.0
2300.0
4510.0
6660.0
7160.0
5890.0
8740.0
6860.0
7630.0
5810.0
9370.0
7580.0
4270.0
1400.0
5410.0
7000.0
6810.0
6300.0
8980.0
4240.0
6010.0

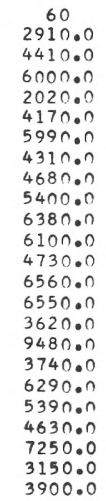

90
2300.0
3660.0
5540.0
1960.0
3720.0
5710.0
4430.0
$412 n \cdot 0$
5470.0
5720.0
6020.0
4790.0
5350.0
6340.0
3260.0
7490.0
3050.0
5520.0
4300.0
4040.0
5870.0
2850.0
3540.0

120
$1920 \cdot 0$
$302 n \cdot 0$
$4530 \cdot 0$
$1810 \cdot 0$
$3260 \cdot 0$
$5090 \cdot 0$
$3910 \cdot 0$
$3760 \cdot 0$
$4800 \cdot 0$
$5050 \cdot 0$
$5240 \cdot 0$
$4180 \cdot 0$
$4470 \cdot 0$
$5690 \cdot 0$
$3360 \cdot 0$
$7110 \cdot 0$
$2910 \cdot 0$
$4620 \cdot 0$
$3890 \cdot 0$
$3430 \cdot 0$
$5380 \cdot 0$
$2820 \cdot 0$
$3110 \cdot 0$
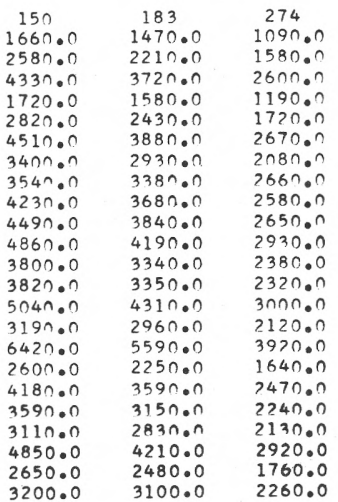

NOELH YAMHILI RTVER NR FAIRDALE OREGON

\author{
STATION NUMBER
}

$14-1943.00$

DURATION TABLE OF DAILY DISCHARGE

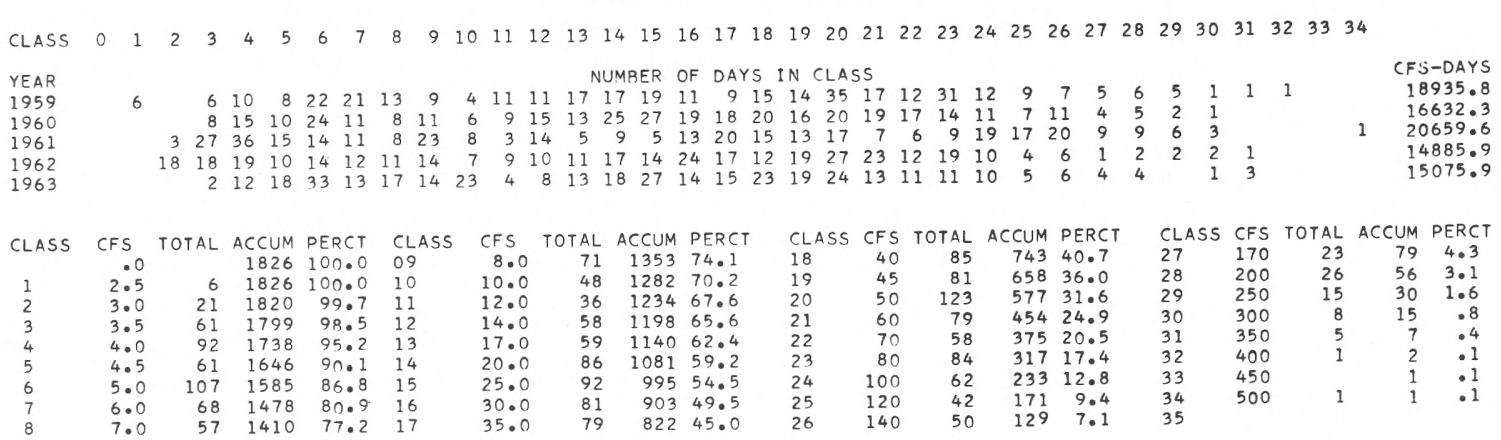

NORTH YAMHIII RIVER NR FAIRDALE ORRG. STATION NUMBER $14-1943.00$
LOWEST MEAN DISCHARGE. IN CFS, FOR THE FOLLOWING NUMBER OF CONSECUTIVE DAYS IN YFAR BEGINNING APRIL I

STATION NUMBER $14-1943.00$

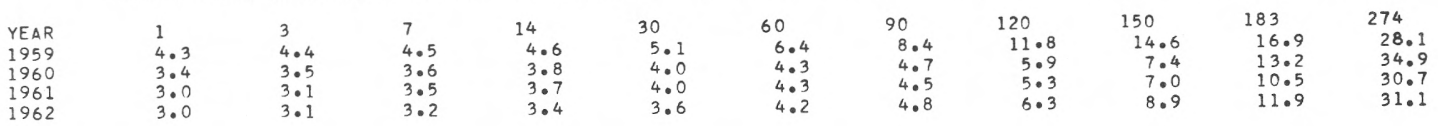

NORTH YAMHCIL RIVER NR FAIRDALE ORBG,

STATION NUMBER $14-1943.00$

HIGHEST MEAN DISCHARGF, IN CFS, FOR THE FOLLOWING NUMBFR OF CONSECUTIVE DAYS IN YEAR ENDING SEPTEMBER 30

\begin{tabular}{|c|c|c|c|c|c|c|c|c|c|c|c|}
\hline YEAR & 1 & 3 & 7 & 15 & 30 & 60 & 90 & 120 & 150 & 183 & 274 \\
\hline 1959 & 441.0 & 356.0 & 260.0 & 164.0 & 155.0 & 124.0 & 113.0 & 104.0 & 99.7 & 89.8 & 66.2 \\
\hline 1960 & 312.0 & 260.0 & 215.0 & 190.0 & 149.0 & 108.0 & 98.4 & 85.6 & 77.9 & 74.2 & $\begin{array}{l}58.7 \\
74.0\end{array}$ \\
\hline 1961 & 539.0 & 358.0 & 277.0 & 222.0 & 189.0 & 163.0 & 139.0 & $126 \cdot 0$ & $\begin{array}{r}118.0 \\
74.6\end{array}$ & $\begin{array}{r}105.0 \\
70.4\end{array}$ & $\begin{array}{l}74.0 \\
52.8\end{array}$ \\
\hline $\begin{array}{l}1962 \\
1963\end{array}$ & $\begin{array}{l}372.0 \\
394.0\end{array}$ & $\begin{array}{l}331.0 \\
334.0\end{array}$ & 238.0 & 173.0 & 125.0 & $\begin{array}{l}94.8 \\
88.5\end{array}$ & $\begin{array}{l}80.2 \\
78.6\end{array}$ & $\begin{array}{l}77 \cdot 3 \\
69 \cdot 8\end{array}$ & $\begin{array}{l}74.6 \\
71.9\end{array}$ & 70.3 & $\begin{array}{l}52.8 \\
53.1\end{array}$ \\
\hline
\end{tabular}


CLASS $0101120 \begin{array}{lllllllllllllllllllllllllllllllll} & 4 & 5 & 6 & 7 & 8 & 9 & 10 & 11 & 12 & 13 & 14 & 15 & 16 & 17 & 18 & 19 & 20 & 21 & 22 & 23 & 24 & 25 & 26 & 27 & 28 & 29 & 30 & 31 & 32 & 33 & 34\end{array}$

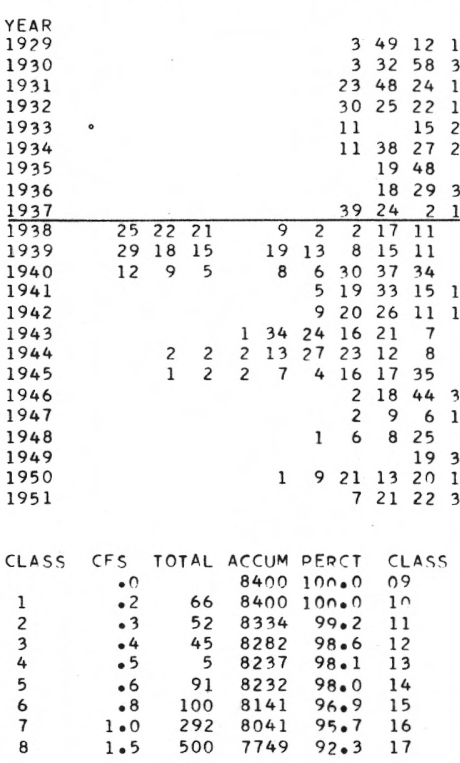

$349 \quad 1215 \quad 51 \quad 3 \quad$ NUMBER OF DAYS IN CLASS

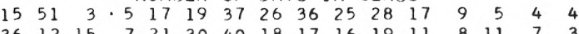

$\begin{array}{llllllllllllllllllllll}48 & 24 & 12 & 21 & 12 & 23 & 27 & 14 & 35 & 17 & 15 & 15 & 19 & 19 & 13 & 13 & 8 & 4\end{array}$

$\begin{array}{lllllllllllllllllll}25 & 22 & 13 & 15 & 17 & 10 & 10 & 12 & 16 & 13 & 16 & 16 & 26 & 25 & 19 & 30 & 22 & 24\end{array}$

$\begin{array}{llllllllllllllllll}38 & 27 & 25 & 29 & 11 & 6 & 21 & 17 & 33 & 25 & 20 & 12 & 16 & 10 & 8 & 14 & 16 & 15\end{array}$

$\begin{array}{llllllllllllllllll}48 & 22 & 9 & 12 & 18 & 7 & 14 & 14 & 7 & 19 & 44 & 29 & 21 & 28 & 21 & 16\end{array}$

$\begin{array}{lllllllllllllllll}2 & 15 & 21 & 17 & 43 & 23 & 75 & 18 & 18 & 15 & 9 & 12 & 3 & 11 & 6 & 3 & 2\end{array}$

$\begin{array}{rrrrrrrrrrrrrrrrrrrrrr}3 & 8 & 15 & 11 & 7 & 17 & 17 & 4 & 18 & 25 & 25 & 13 & 14 & 17 & 29 & 31 & 3 & 5 & 5 & 3 & 3 & 1 \\ 6 & 30 & 37 & 34 & 5 & 13 & 9 & 3 & 16 & 9 & 20 & 22 & 18 & 18 & 24 & 17 & 12 & 10 & 11 & 15 & 2 & 1\end{array}$

$\begin{array}{lllllllllllllllllll} & 3 & 16 & 9 & 20 & 22 & 18 & 18 & 24 & 17 & 12 & 10 & 11 & 15\end{array}$

$\begin{array}{rlllllllllrrrrrrrr}19 & 22 & 16 & 14 & 38 & 32 & 32 & 45 & 15 & 13 & 9 & 8 & 6 & 13 & 8 & 6 & 2 & 1\end{array}$

$\begin{array}{rrrrrrrrrrrrrrrrrrr}12 & 8 & 7 & 10 & 13 & 15 & 16 & 16 & 37 & 39 & 35 & 26 & 42 & 12 & 3 & 3 & 1 & 1 & 1\end{array}$

$\begin{array}{lllllllllllllllllllll}18 & 44 & 31 & 12 & 18 & 13 & 37 & 10 & 13 & 7 & 17 & 16 & 27 & 21 & 21 & 24 & 13 & 16 & 4\end{array}$

$\begin{array}{llllllllllllllllllll}6 & 15 & 50 & 31 & 24 & 39 & 13 & 21 & 21 & 25 & 23 & 13 & 12 & 15 & 19 & 21 & 5 & 1\end{array}$

$\begin{array}{rrrrrrrrrrrrrrrrrrrrrr}9 & 37 & 22 & 34 & 8 & 29 & 8 & 44 & 34 & 18 & 27 & 55 & 32 & 19 & 18 & 11 & 9 & 3 & 2 & & \\ 15 & 19 & 19 & 24 & 14 & 18 & 10 & 7 & 7 & 3 & 3 & 2\end{array}$

$\begin{array}{rrrrrrrrrrrrrrrrrrrr}21 & 22 & 32 & 22 & 15 & 7 & 18 & 8 & 24 & 16 & 12 & 12 & 13 & 23 & 31 & 43 & 16 & 16 & 6 & 16\end{array}$

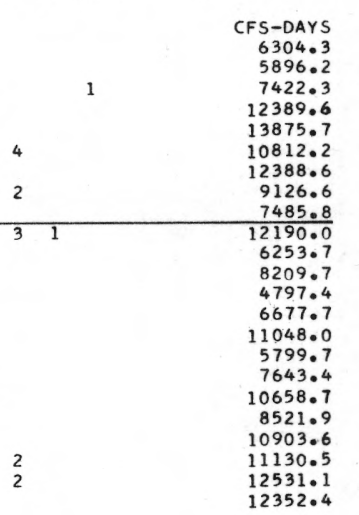
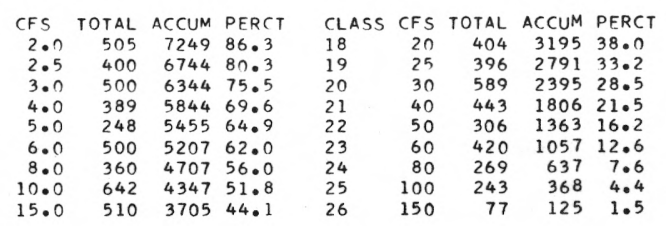

Haskins Creek near McMinnville, Oreg.

STATION NUMBER

$14-1950.00$

Summary for water years 1929-1937
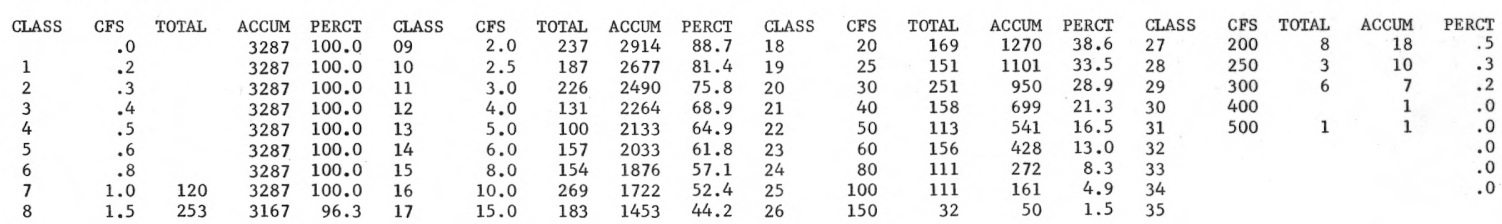

Haskins Creek near McMinnville, Oreg.

STATION NUMBER

$14-1950.00$

Summary for water years 1938-1951

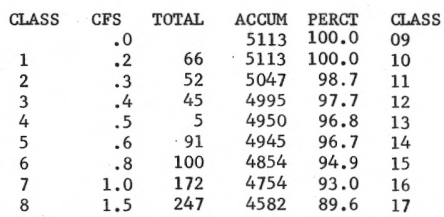

$\begin{array}{rrrrl}\text { CFS } & \text { TOTAL } & \text { ACCUM } & \text { PERCT } & \text { CLASS } \\ 2.0 & 268 & 4335 & 84.8 & 18 \\ 2.5 & 213 & 4067 & 79.5 & 19 \\ 3.0 & 274 & 3854 & 75.4 & 20 \\ 4.0 & 258 & 3580 & 70.0 & 21 \\ 5.0 & 148 & 3322 & 65.0 & 22 \\ 6.0 & 343 & 3174 & 62.1 & 23 \\ 8.0 & 206 & 2831 & 55.4 & 24 \\ 10.0 & 373 & 2625 & 51.3 & 25 \\ 15.0 & 327 & 2252 & 44.0 & 26\end{array}$

$\begin{array}{rrrrl}\text { CFS } & \text { TOTAL } & \text { ACCUM } & \text { PERCT } & \text { CLASS } \\ 20 & 235 & 1925 & 37.6 & 27 \\ 25 & 245 & 1690 & 33.1 & 28 \\ 30 & 338 & 1445 & 28.3 & 29 \\ 40 & 285 & 1107 & 21.7 & 30 \\ 50 & 193 & 822 & 16.1 & 31 \\ 60 & 264 & 629 & 12.3 & 32 \\ 80 & 158 & 365 & 7.1 & 33 \\ 100 & 132 & 207 & 4.0 & 34 \\ 150 & 45 & 75 & 1.5 & 35\end{array}$


Haskins Creek near McMinnville, Oreg.

STATION NUMRER

$14-1950.00$

LOWEST MEAN DISCHARGE, IN CFS, FOR THE FOLLOWING NUMBER OF CONSECUTIVE DAYS IN YEAR BEGINNING APRIL 1

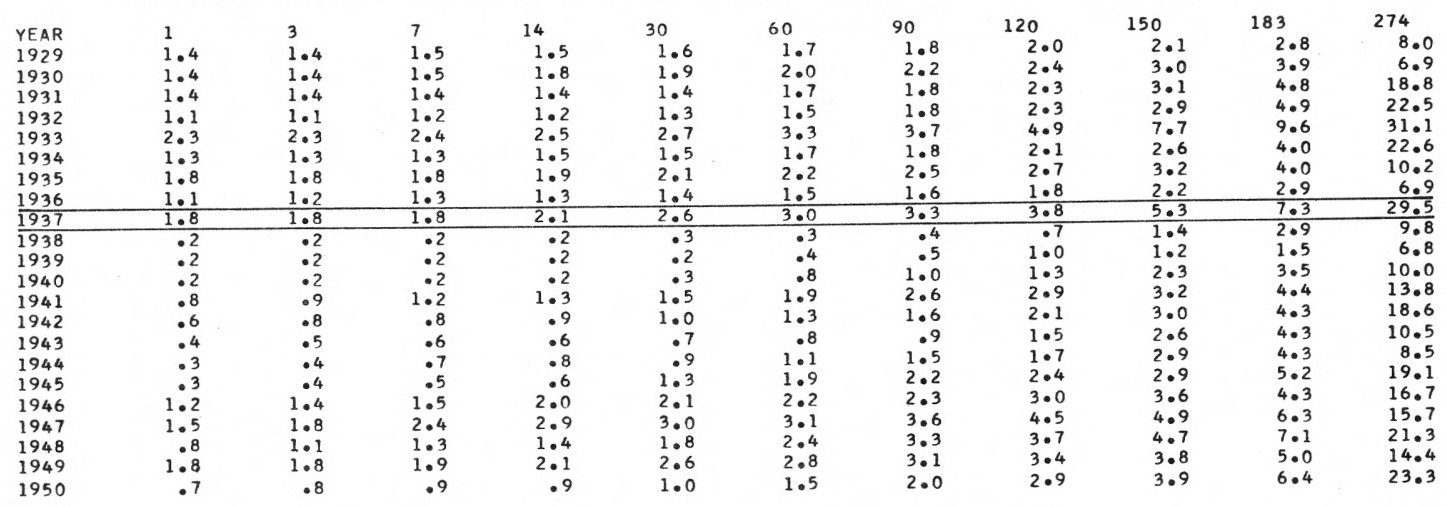

Haskins Creek near McMinnville, Oreg.

STATION NUMBER

$14-1950.00$

HIGHEST MEAN DISCHARGE, IN CFS, FOR THE FOLLOWING NUMBER OF CONSECUTIVE DAYS IN YEAR ENDING SEPTEMBER 30

\begin{tabular}{|c|c|c|c|c|c|c|c|c|c|c|c|}
\hline YEAR & 1 & 3 & 7 & 15 & 30 & 60 & 90 & 120 & 150 & 183 & 274 \\
\hline 1929 & 125.0 & 115.0 & 95.6 & 71.7 & 47.2 & 37.0 & 33.6 & $35 \cdot 7$ & 33.3 & $30 \cdot 2$ & 22.3 \\
\hline 1930 & 210.0 & 138.0 & $112 \bullet 0$ & 87.9 & 77.3 & 53.1 & 42.5 & $38 \cdot 1$ & 32.7 & 29.2 & 20.8 \\
\hline 1931 & 500.0 & 318.0 & 203.0 & 131.0 & 95.8 & 67.1 & 57.9 & $50 \cdot 8$ & 43.4 & 37.2 & 26.5 \\
\hline 1932 & 218.0 & 188.0 & 145.0 & 106.0 & 94.1 & 77.2 & 74.2 & $74 \cdot 8$ & 67.4 & 62.3 & 44.6 \\
\hline 1933 & 200.0 & 159.0 & 151.0 & 127.0 & 114.0 & 82.6 & 78.6 & $74 \cdot 7$ & 71.1 & 65.4 & 49.6 \\
\hline 1934 & 381.0 & 361.0 & 293.0 & $200 \cdot 0$ & 154.0 & 120.0 & 89.7 & $75 \cdot 0$ & 63.3 & 55.1 & 38.8 \\
\hline 1935 & 176.0 & 165.0 & 143.0 & 107.0 & 81.8 & 76.7 & 72.7 & 67.3 & 66.2 & 61.0 & 44.4 \\
\hline 1936 & 338.0 & 247.0 & 192.0 & 184.0 & 126.0 & 84.2 & 72.5 & $61 \cdot 7$ & 52.5 & 45.5 & 32.5 \\
\hline 1937 & 246.0 & 211.0 & 149.0 & 98.0 & 73.0 & 69.1 & 58.6 & $48 \cdot 4$ & 42.3 & 36.9 & 26.7 \\
\hline 1938 & 446.0 & 383.0 & 284.0 & 167.0 & 119.0 & 91.5 & 80.4 & $74 \cdot 3$ & 72.2 & 63.9 & 44.3 \\
\hline 1939 & 237.0 & $189 . n$ & $151 \cdot n$ & 97.3 & 68.5 & 53.8 & 48.3 & $42 \cdot 6$ & 36.9 & 32.4 & 22.7 \\
\hline 1940 & 213.0 & 172.0 & 140.0 & 121.0 & 101.0 & 70.9 & 62.0 & $55 \cdot 2$ & 49.0 & 42.8 & 29.6 \\
\hline 1941 & 146.0 & 124.0 & 84.9 & 67.3 & 50.5 & 41.7 & 32.7 & 27.7 & $24 \cdot 3$ & 22.0 & 16.7 \\
\hline 1942 & 238.0 & 192.0 & 147.0 & 101.0 & 73.3 & 59.4 & 51.5 & $43 \cdot 2$ & 37.5 & 32.4 & 23.7 \\
\hline 1943 & 226.0 & 191.0 & 153.0 & 116.0 & 89.0 & 84.5 & 78.1 & 67.3 & 64.6 & 56.5 & 40.0 \\
\hline 1944 & 156.0 & $128 . n$ & 82.6 & 50.7 & 36.7 & 32.7 & 32.1 & $30 \cdot 8$ & 29.6 & 26.7 & 20.7 \\
\hline 1945 & 179.0 & 142.0 & 110.0 & 87.3 & 66.6 & 59.8 & 55.1 & 49.2 & 43.1 & 37.5 & 27.2 \\
\hline 1946 & 280.0 & 208.0 & 148.0 & 118.0 & 84.5 & 79.1 & 73.6 & 69.3 & 62.7 & 54.3 & 38.2 \\
\hline 1947 & 170.0 & 139.0 & 102.0 & 95.7 & 83.3 & 56.7 & 59.8 & $54 \cdot 7$ & 48.6 & 41.7 & 30.1 \\
\hline 1948 & 238.0 & 183.0 & 136.0 & 105.0 & 71.6 & 62.5 & 58.1 & 52.9 & $50 \cdot 3$ & 46.9 & 38.7 \\
\hline 1949 & 375.0 & 284.0 & 243.0 & 175.0 & 119.0 & 82.6 & 72.6 & $70 \cdot 1$ & 62.7 & 55.3 & 39.5 \\
\hline 1950 & 320.0 & 277.0 & 191.0 & 163.0 & 131.0 & 101.0 & 90.0 & 82.3 & 73.7 & $64 \cdot 3$ & 45.1 \\
\hline 1951 & 238.0 & 189.0 & 160.0 & 147.0 & 119.0 & 95.1 & 86.4 & 76.0 & 69.1 & 62.4 & 44.2 \\
\hline
\end{tabular}


Hask1ns Creek below Reservo1r, near STATION NUMBER 14-1960.00.

LOWEST MEAN DISCHARGE, IN CFS, FOR THE FOLLOWING NUMRER OF CONSECUTIVE DAYS IN YEAR BFGINNING APRIL 1

\begin{tabular}{|c|c|c|c|c|c|c|c|c|c|c|c|}
\hline YEAR & 1 & 3 & 7 & 14 & 30 & 60 & 90 & 120 & 150 & 183 & 274 \\
\hline 1952 & 2. 3 & 2.7 & 2.7 & 2.9 & 3.0 & 3.1 & 3.3 & 3.5 & 3.8 & 4.2 & 10.9 \\
\hline 1953 & 2.3 & 2.5 & 2.7 & 3.1 & 3.6 & 4.1 & 5.1 & $5 \cdot 2$ & 5.7 & 8.2 & 23.9 \\
\hline 1954 & 2.9 & 3.1 & 3.1 & 3.2 & 3.3 & 3.4 & 3.7 & $4 \cdot 3$ & 4.9 & 5.0 & 15.4 \\
\hline 1955 & 2.8 & $2 \cdot 8$ & 2.8 & 2.9 & 3.1 & 3.7 & 4.2 & $5 \cdot 3$ & 7.4 & 11.0 & 37.6 \\
\hline 1956 & 2.5 & 2.7 & 2.7 & 2.7 & 2.8 & 2.9 & 3.3 & $3 \cdot 8$ & 4.4 & 5.5 & 12.2 \\
\hline 1957 & 2.9 & 3.0 & 3.0 & 3.0 & 3.1 & 3.3 & 3.7 & 3.9 & 4.4 & 5.6 & 19.2 \\
\hline 1958 & 1.8 & 1.9 & 2.1 & 2.2 & 2.5 & 2.7 & 3.3 & 3.9 & 4.4 & 5.9 & 18.3 \\
\hline 1959 & 1.6 & 1.6 & 1.7 & 1.8 & 3.8 & 4.8 & 5.5 & $6 \cdot 8$ & 8.2 & 9.1 & 15.3 \\
\hline 1960 & .9 & 2.3 & 2.5 & 3.1 & 3.4 & 3.7 & 3.8 & $4 \cdot 5$ & 5.4 & 9.0 & 23.1 \\
\hline 1961 & 1.8 & $2 \cdot 0$ & $2 \cdot 3$ & 2.4 & 3.4 & 3.8 & 4.4 & $5 \cdot 0$ & 5.4 & 6.6 & 19.1 \\
\hline 1962 & .9 & 2.3 & 3.0 & 3.8 & 3.9 & 4.5 & 5.1 & 5.8 & 7.2 & 8.9 & 23.0 \\
\hline
\end{tabular}

Haskins Creek below Reservoir, near McMinnville, Oreg.

STATION NUMBER 14-1960.00 HIGHEST MEAN DISCHARGE, IN CFS, FOR THF FOLLOWING NUMBER OF CONSECUTIVE DAYS IN YEAR ENDING SEPTEMBER 30

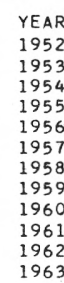

$\begin{array}{cc}1 & 3 \\ 353.0 & 325.0 \\ 359.0 & 306.0 \\ 307.0 & 269.0 \\ 216.0 & 176.0 \\ 378.0 & 302.0 \\ 242.0 & 181.0 \\ 250.0 & 206.0 \\ 260.0 & 240.0 \\ 208.0 & 177.0 \\ 457.0 & 308.0 \\ 207.0 & 182.0 \\ 385.0 & 264.0\end{array}$

7
299.0
248.0
200.0
129.0
215.0
134.0
161.0
200.0
144.0
215.0
140.0
183.0

15
$212 \cdot 0$
$189 \cdot 0$
$186 \cdot 0$
$79 \cdot 3$
$156 \cdot 0$
102.0
$138 \cdot 0$
$127 \cdot 0$
$128 \cdot 0$
182.0
105.0
149.0

30
$134 \cdot 0$
$154 \cdot 0$
$128 \cdot 0$
$71 \cdot 0$
$148 \cdot 0$
$81 \cdot 0$
$112 \cdot 0$
$110 \cdot 0$
$100 \cdot 0$
$152 \cdot 0$
$75 \cdot 0$
$10.7 \cdot 0$

60
103.0
106.0
108.0
57.1
136.0
55.8
99.4
87.7
72.5
127.0
64.6
68.6

90
91.1
86.8
104.0
55.4
112.0
48.7
95.6
73.9
61.2
105.0
54.2
57.9

120
$86 \cdot 1$
$75 \cdot 2$
$96 \cdot 2$
$54 \cdot 9$
93.6
$45 \cdot 4$
$81 \cdot 7$
$67 \cdot 3$
$53 \cdot 6$
$97 \cdot 4$
48.8
50.6

150
79.
62.
84.
53.
91.5
41.
72.
64.
49.
88.
48.
50.9

$\begin{array}{cc}183 & 274 \\ 72.0 & 51.0 \\ 55.3 & 39.0 \\ 71.1 & 49.8 \\ 49.7 & 36.4 \\ 82.8 & 58.6 \\ 37.1 & 27.5 \\ 62.0 & 43.9 \\ 55.3 & 40.2 \\ 46.7 & 36.4 \\ 75.4 & 53.4 \\ 42.8 & 31.6 \\ 46.3 & 35.1\end{array}$


North Yamhill River near Pike, Oreg.

STATION NUMBER $14-1965.00$

DURATION TABLE OF DAILY DISCHARGE

CLASS $0 \begin{array}{lllllllllllllllllllllllllllllllllllll} & 0 & 2 & 3 & 4 & 5 & 6 & 7 & 8 & 9 & 10 & 11 & 12 & 13 & 14 & 15 & 16 & 17 & 18 & 19 & 20 & 21 & 22 & 23 & 24 & 25 & 26 & 27 & 28 & 29 & 30 & 31 & 32 & 33 & 34\end{array}$

YEAR

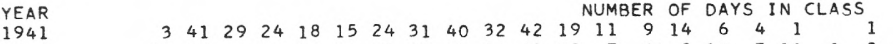

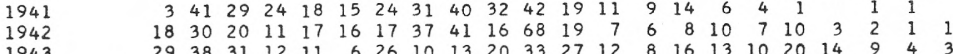

$\begin{array}{rrrrrrrrrrrrrrrrrrrrrrr}1943 & 29 & 38 & 31 & 12 & 11 & 6 & 26 & 10 & 13 & 20 & 33 & 27 & 12 & 8 & 16 & 13 & 10 & 20 & 14 & 9 \\ 1944 & 11 & 28 & 36 & 12 & 13 & 13 & 7 & 23 & 25 & 24 & 20 & 56 & 40 & 23 & 17 & 10 & 2 & 2 & 1 & 2 & 1\end{array}$

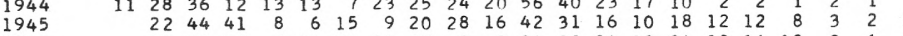

$\begin{array}{rrrrrrrrrrrrrrrrrrrrr}1946 & 12 & 70 & 20 & 7 & 16 & 30 & 21 & 7 & 9 & 2 & 14 & 23 & 24 & 18 & 24 & 22 & 16 & 12 & 9 & 6 \\ 1947 & & 56 & 16 & 18 & 7 & 29 & 22 & 18 & 26 & 10 & 39 & 31 & 14 & 11 & 17 & 13 & 8 & 6 & 9 & 6\end{array}$

$\begin{array}{rrrrrrrrrrrrrrrrrrrrrr}1947 & 56 & 21 & 18 & 7 & 29 & 22 & 18 & 26 & 10 & 39 & 31 & 14 & 11 & 17 & 13 & 8 & 6 & 9 & 6 & 4 \\ 1948 & 20 & 29 & 17 & 16 & 18 & 6 & 8 & 13 & 11 & 30 & 33 & 42 & 42 & 31 & 15 & 12 & 12 & 4 & 5 & 2\end{array}$

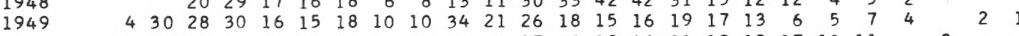

$\begin{array}{rrrrrrrrrrrrrrrrrrrrrrrrr}1950 & 13 & 17 & 32 & 40 & 18 & 10 & 21 & 16 & 12 & 10 & 2 & 17 & 23 & 19 & 16 & 21 & 19 & 19 & 17 & 10 & 11 & & 2 \\ 1951 & 11 & 16 & 42 & 21 & 12 & 8 & 19 & 10 & 11 & 21 & 17 & 12 & 20 & 11 & 16 & 32 & 28 & 17 & 21 & 8 & 10 & 1 & 1\end{array}$

$\begin{array}{crrrrl}\text { CLASS } & \text { CFS } & \text { TOTAL ACCUM } & \text { PERCT } & \text { CLASS } \\ & .0 & & 4017 & 100.0 & 09 \\ 1 & 6.0 & 39 & 4017 & 100.0 & 10 \\ 2 & 8.0 & 175 & 3978 & 99.0 & 11 \\ 3 & 10.0 & 437 & 3803 & 94.7 & 12 \\ 4 & 15.0 & 294 & 3366 & 83.8 & 13 \\ 5 & 20.0 & 156 & 3072 & 76.5 & 14 \\ 6 & 25.0 & 137 & 2916 & 72.6 & 15 \\ 7 & 30.0 & 194 & 2779 & 69.2 & 16 \\ 8 & 40.0 & 184 & 2585 & 64.4 & 17\end{array}$

$\begin{array}{rrrl}\text { CFS } & \text { TOTAL } & \text { ACCUM } & \text { PERCT } \\ 50.0 & 189 & 2401 & 59.8 \\ 60.0 & 259 & 2212 & 55.1 \\ 80.0 & 167 & 1953 & 48.6 \\ 100.0 & 379 & 1786 & 44.5 \\ 150.0 & 284 & 1407 & 35.0 \\ 200.0 & 194 & 1123 & 28.0 \\ 250.0 & 169 & 929 & 23.1 \\ 300.0 & 210 & 760 & 18.9 \\ 400.0 & 157 & 550 & 13.7\end{array}$

$\begin{array}{lrrrr}\text { CLASS } & \text { CFS } & \text { TOTAL } & \text { ACCUM } & \text { PERCT } \\ 18 & 500 & 120 & 393 & 9.8 \\ 19 & 600 & 114 & 273 & 6.8 \\ 20 & 800 & 67 & 159 & 4.0 \\ 21 & 1000 & 60 & 92 & 2.3 \\ 22 & 1500 & 22 & 32 & .8 \\ 23 & 2000 & 7 & 10 & .2 \\ 24 & 2500 & 2 & 3 & .1 \\ 25 & 3000 & 1 & 1 & .0 \\ 26 & & & & .0\end{array}$

$\begin{array}{lc}\text { CLASS CFS TOTAL ACCUM PERCT } \\ 27 & \bullet 0 \\ 28 & \bullet 0 \\ 29 & \bullet 0 \\ 30 & : 0 \\ 31 & \bullet 0 \\ 32 & \bullet 0 \\ 33 & \bullet 0 \\ 34 & \bullet 0\end{array}$

North Yamnill kiver near Pike, Oreg.

STATION NUMBER

$14-1965.00$

LOWEST MEAN DISCHARGE, IN CFS, FOR THE FOLLOWING NUMBER OF CONSECUTIVE DAYS IN YEAR BEGINNING APRIL 1

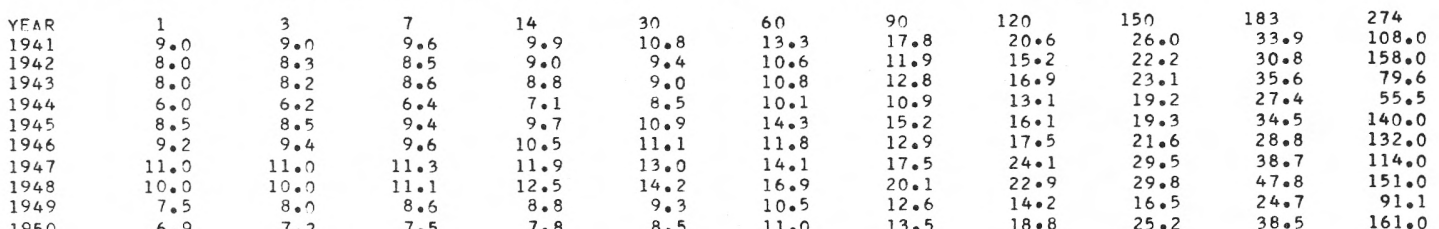

North Yambill River near Pike, Oreg.

STATION NUMBER $\quad 14-1965.00$

HIGHEST MEAN DISCHARGE, IN CFS, FOR THE FOLLOWING NUMBER OF CONSECUTIVE DAYS IN YEAR ENDING SEPTEMBER 30

YEA
194
194
194
194
1945
1946
1947
1948
1949
1950
195

\begin{tabular}{crr}
1 & 3 & \multicolumn{1}{c}{7} \\
1880.0 & 1310.0 & \multicolumn{1}{c}{785.0} \\
2130.0 & 1660.0 & 1180.0 \\
2270.0 & 1630.0 & 1330.0 \\
1110.0 & 961.0 & 608.0 \\
1990.0 & 1330.0 & 929.0 \\
1920.0 & 1340.0 & 961.0 \\
1760.0 & 1590.0 & 1260.0 \\
1650.0 & 1300.0 & 990.0 \\
3290.0 & 2040.0 & 1750.0 \\
2410.0 & 1950.0 & 1330.0
\end{tabular}

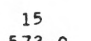

$573 . ?$

910.0

$364 \cdot 0$
$701 \cdot 0$

701.0
867.0

910.0

794.0

1350.0

1080.0

2000.0

1450.0

1250.0

30
$417 \cdot 0$
$558 \cdot 0$
$762 \cdot 0$
$269 \cdot 0$
$504 \cdot 0$
$622 \cdot 0$
$762 \cdot 0$
$558 \cdot 0$
$892 \cdot 0$
$876 \cdot 0$
841.0

60

340.0

446.0
702.0

235.0

468.0
573.0

469.0

466.0

584.0

699.0

$\begin{array}{ll}90 & 120 \\ 269.0 & 227.0 \\ 381.0 & 326.0 \\ 636.0 & 539.0 \\ 230.0 & 213.0 \\ 419.0 & 369.0 \\ 548.0 & 500.0 \\ 489.0 & 435.0 \\ 424.0 & 390 \cdot 0 \\ 533.0 & 498.0 \\ 631.0 & 558.0 \\ 633.0 & 554.0\end{array}$

150

197.0
283.0

512.0

202.0

318.0

452.0

380.0

368.0

447.0

503.0

503.0

$\begin{array}{ll}183 & 274 \\ 175.0 & 132.0 \\ 245.0 & 181.0 \\ 445.0 & 312.0 \\ 184.0 & 142.0 \\ 278.0 & 200.0 \\ 394.0 & 274.0 \\ 325.0 & 233.0 \\ 349.0 & 284.0 \\ 391.0 & 276.0 \\ 435.0 & 302.0\end{array}$

CFS-DAYS

37817.0

86651.0

40001.6

56252.9

655200

79558 .

76776.7

83980.3

87245.2 
North Yamhill River at Pike, Oreg.

STATION NUMBER 14-1970.00

DURATION TABLE OF DAILY DISCHARGE

CLASS $00 \begin{array}{lllllllllllllllllllllllllllllllllllll} & 1 & 2 & 3 & 4 & 5 & 6 & 7 & 8 & 9 & 10 & 11 & 12 & 13 & 14 & 15 & 16 & 17 & 18 & 19 & 20 & 21 & 22 & 23 & 24 & 25 & 26 & 27 & 28 & 29 & 30 & 31 & 32 & 33 & 34\end{array}$

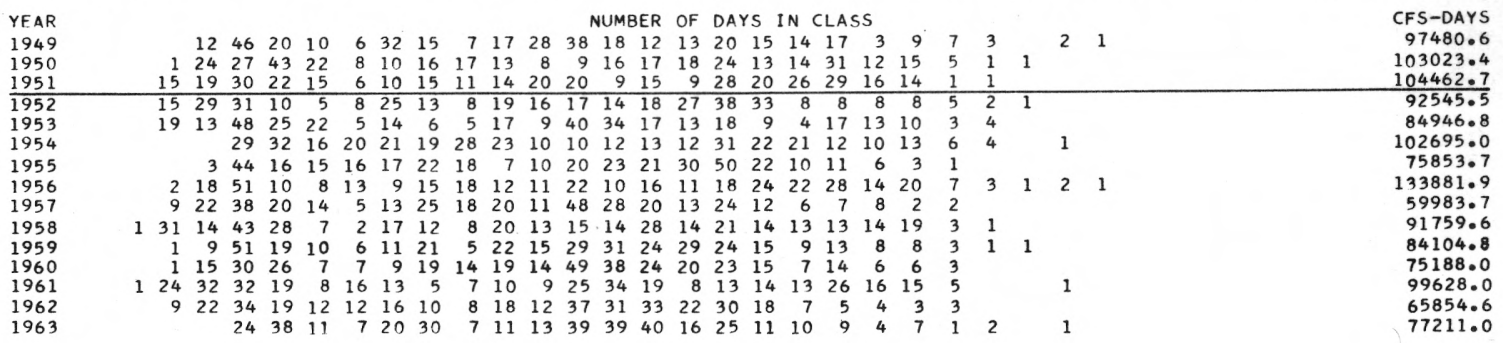

North Yamhill River at Pike, Oreg. $\quad$ STATION NUMBER 14-1970.00

Sumnary for water years 1949-63

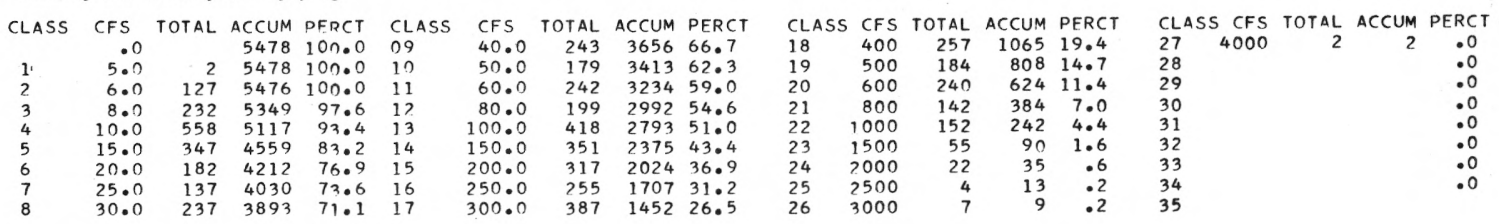

North Yamhi11 River at Pike, Oreg.

STATION NUMBER $\quad 14-1970.00$

Summary for water years $1949-51$

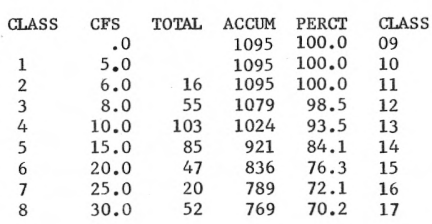

North Yamhil1 River at Pike, Oreg.

Summary for water years 1952-1963

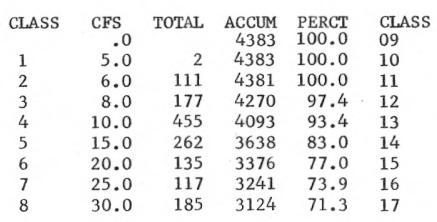

North Yambill River at Pike, Oreg.

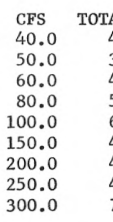

$\begin{array}{rrrl} & & & \\ 46 & \text { ACCUM } & \text { PERCT } & \text { CLASS } \\ 35 & 717 & 65.5 & 18 \\ 44 & 671 & 61.3 & 19 \\ 56 & 636 & 58.1 & 20 \\ 67 & 592 & 54.1 & 21 \\ 43 & 536 & 48.9 & 22 \\ 44 & 469 & 42.8 & 23 \\ 40 & 426 & 38.9 & 24 \\ 72 & 382 & 34.9 & 25 \\ & 342 & 31.2 & 26\end{array}$

STATION NUMBER

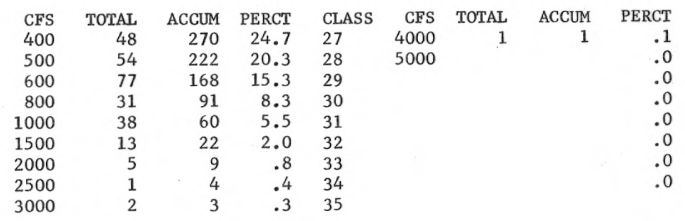

14-1970.00

\begin{tabular}{|c|c|c|c|c|c|c|c|c|c|c|c|}
\hline $\begin{array}{l}\text { YEAR } \\
1949 \\
1950\end{array}$ & 9.0 & $\begin{array}{l}3 \\
9.4\end{array}$ & 79.6 & $\begin{array}{l}14 \\
10.0 \\
8.8\end{array}$ & $\begin{array}{l}30 \\
10.7\end{array}$ & $\begin{array}{l}60 \\
11.6 \\
12.0\end{array}$ & $\begin{array}{l}90 \\
13.7 \\
14.7\end{array}$ & $\begin{array}{l}120 \\
15.5 \\
21.7\end{array}$ & $\begin{array}{c}150 \\
18.6 \\
30.1\end{array}$ & $\begin{array}{c}183 \\
29.0 \\
46.2\end{array}$ & $\begin{array}{l}274 \\
110.0 \\
190.0\end{array}$ \\
\hline & & $\begin{array}{l}8.3 \\
7.4\end{array}$ & $\frac{8.1}{7.6}$ & $\frac{8.8}{8.0}$ & $\begin{array}{l}9.3 \\
8.9\end{array}$ & & $\frac{14.1}{12.2}$ & $\frac{21.1}{17 \cdot 3}$ & 25.4 & $\begin{array}{l}\frac{46.2}{38.8} \\
38.8\end{array}$ & $\frac{190.0}{179.0}$ \\
\hline 1952 & $\begin{array}{l}6.4 \\
8.2\end{array}$ & $\begin{array}{l}6.5 \\
8.5\end{array}$ & $\begin{array}{l}6.7 \\
9.2\end{array}$ & $\begin{array}{r}6.9 \\
10 \cdot 3\end{array}$ & $\begin{array}{r}7.1 \\
14.5\end{array}$ & $\begin{array}{r}8.3 \\
15.7\end{array}$ & $\begin{array}{r}8.8 \\
19.5\end{array}$ & $\begin{array}{r}9.9 \\
24: 1\end{array}$ & $\begin{array}{l}11.8 \\
30.1\end{array}$ & $\begin{array}{l}15.6 \\
49.5\end{array}$ & $\begin{array}{r}57.3 \\
165.0\end{array}$ \\
\hline & 12.0 & 12.0 & 12.3 & 12.7 & 14.0 & 15.5 & 17.1 & 22.4 & 28.5 & & \\
\hline & $\begin{array}{l}8.9 \\
7.6\end{array}$ & $\begin{array}{l}8.9 \\
7.8\end{array}$ & $\begin{array}{l}9.7 \\
8.7\end{array}$ & $\begin{array}{r}10.3 \\
9.7\end{array}$ & $\begin{array}{l}11.2 \\
10.5\end{array}$ & $\begin{array}{l}13.8 \\
11.4\end{array}$ & $\begin{array}{l}19.0 \\
11.6\end{array}$ & $\begin{array}{l}26.4 \\
14.7\end{array}$ & $\begin{array}{l}43.5 \\
21.1\end{array}$ & $\begin{array}{l}69.1 \\
28.9\end{array}$ & 1.0 \\
\hline 957 & $\begin{array}{l}1007 \\
6.7\end{array}$ & $\begin{array}{l}10.9 \\
6.9\end{array}$ & 7.05 & 7.7 & 8.4 & $\begin{array}{l}11.4 \\
10.2\end{array}$ & $\begin{array}{l}11.9 \\
11.9\end{array}$ & 13.9 & 18.3 & 29.5 & 134.0 \\
\hline & 5.7 & 6.1 & 6.3 & 6.8 & 7.0 & 8.1 & 9.6 & 12.5 & 18.2 & 30.5 & 3 \\
\hline & $\begin{array}{l}9: 2 \\
7: 5\end{array}$ & $\begin{array}{l}9.8 \\
7.7\end{array}$ & $\begin{array}{r}10.2 \\
8.2\end{array}$ & 10.9 & $\begin{array}{l}1.3 \\
9.4\end{array}$ & $\begin{array}{l}\begin{array}{l}14.9 \\
11.0\end{array} \\
\end{array}$ & & & & & $\begin{array}{l}99.3 \\
51.0\end{array}$ \\
\hline & 5,6 & 6.1 & 6.3 & 7.0 & 7.9 & .1 & .5 & 13.9 & 21.4 & 33.1 & \\
\hline & 6.5 & 6.7 & 7.0 & 7.9 & 9.6 & 11.8 & 14.2 & 21.1 & & & \\
\hline
\end{tabular}


North Yamhill River at Pike, Oreg.

STATION NUMRER $14-1970.00$

HIGHEST MEAN DISCHARGF, IN CFS, FOR THE FOLLOWING NUMBER OF CONSECUTIVE DAYS IN YEAR ENDING SEPTEMBER 30

\begin{tabular}{|c|c|c|c|c|c|c|c|c|c|c|c|}
\hline YEAR & 1 & 3 & 7 & 15 & 30 & 60 & 90 & 120 & 150 & 183 & 274 \\
\hline 1949 & 4370.0 & 2680.0 & 2300.0 & 1810.0 & 1210.0 & 770.0 & 691.0 & $640 \cdot 0$ & 571.0 & 497.0 & 351.0 \\
\hline 1950 & 2840.0 & 2270.0 & 1580.0 & $1270 \cdot 0$ & 1070.0 & 859.0 & 777.0 & $691 \cdot 0$ & 620.0 & 534.0 & 371.0 \\
\hline 1951 & 2450.0 & 1750.0 & 1500.0 & 1270.0 & 1000.0 & 811.0 & 751.0 & $657 \cdot 0$ & $605 \cdot 0$ & 536.0 & 377.0 \\
\hline 1952 & 2540.0 & $2100 \cdot 0$ & 1720.0 & $1110 \cdot 0$ & 822.0 & $604 \cdot 0$ & 642.0 & $573 \cdot 0$ & 526.0 & 470.0 & 334.0 \\
\hline 1953 & 2350.0 & 2240.0 & $1840 . n$ & 1460.0 & 1220.0 & 846.0 & 700.0 & $580 \cdot 0$ & 499.0 & 438.0 & 306.0 \\
\hline 1954 & 3010.0 & 2290.0 & 1570.0 & 1370.0 & 952.0 & 830.0 & 783.0 & $701 \cdot 0$ & 618.0 & 528.0 & 369.0 \\
\hline 1955 & 1610.0 & 1340.0 & 893.0 & 576.0 & 493.0 & 437.0 & 413.0 & $421 \cdot 0$ & 396.0 & 375.0 & 271.0 \\
\hline 1956 & 4200.0 & 3020.0 & 2080.0 & 1480.0 & 1330.0 & 1150.0 & 914.0 & $834 \cdot 0$ & 788.0 & 689.0 & 484.0 \\
\hline 1957 & 1830.0 & 1560.0 & $104 n \cdot n$ & 843.0 & $687 \cdot 0$ & 486.0 & 399.0 & 373.0 & 339.0 & 296.0 & 215.0 \\
\hline 1958 & 2430.0 & $18 n n \cdot n$ & $138 n \cdot n$ & 1130.0 & 907.0 & 799.0 & 726.0 & $615 \cdot 0$ & 556.0 & $475 \bullet 0$ & 332.0 \\
\hline 1959 & 2900.0 & $2250 . n$ & 1690.0 & $1030 . ?$ & 865.0 & 684.0 & 564.0 & $515 \cdot 0$ & 476.0 & $418 \cdot 0$ & 300.0 \\
\hline 1960 & 1820.0 & $155 n \cdot n$ & $122 n \cdot n$ & 1080.0 & 795.0 & 564.0 & 495.0 & $425 \cdot 0$ & 385.0 & 358.0 & 269.0 \\
\hline 1961 & 3340.0 & 2080.0 & 1440.0 & 1180.0 & 1020.0 & 868.0 & 723.0 & 651.0 & 594.0 & 517.0 & 360.0 \\
\hline 1962 & 1680.0 & 1490.0 & 1150.0 & 813.0 & 566.0 & 441.0 & 375.0 & $355 \cdot 0$ & 346.0 & 323.0 & 236.0 \\
\hline 62 & 2350.0 & 2250.0 & 1460.0 & 1120.0 & 732.0 & 486.0 & 435.0 & 381.0 & 391.0 & 370.0 & 275.0 \\
\hline
\end{tabular}

Willamette River at Wilsonville, Oreg.

$$
\text { STATION NUMBER } \quad 14-1980.00
$$

DURATION TABLE OF DAILY DISCHARGE

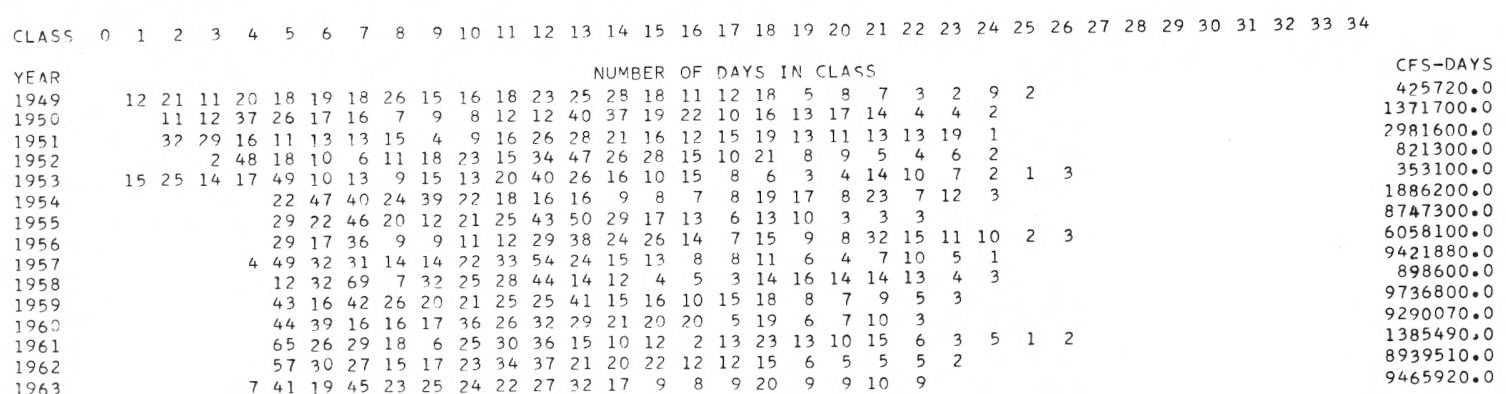
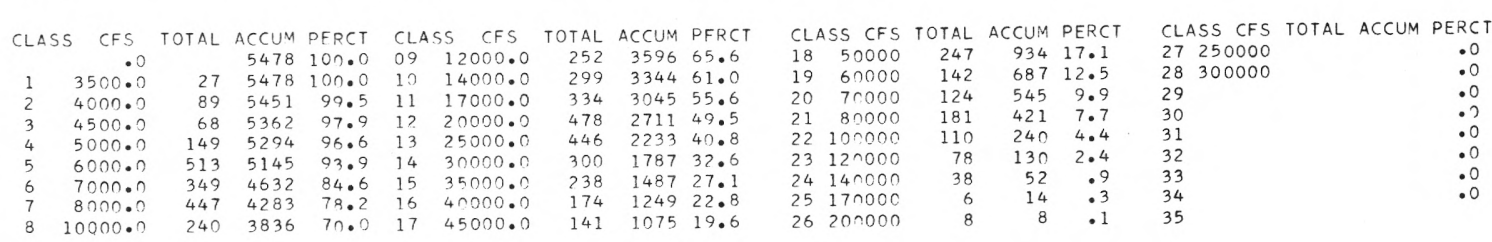

Willamette River at Wilsonville, Oreg.

STATION NUMBER

$14-1980.00$

LOWEST MEAN DISCHARGE, IN CFS, FOR THE FOLLOWING NUMBER OF CONSECUTIVE DAYS IN YEAR BEGINNING APRIL 1

\begin{tabular}{|c|c|c|c|c|c|c|c|c|c|c|c|}
\hline YEAR & 1 & 3 & 7 & 14 & 30 & 60 & 90 & 120 & 150 & 183 & 274 \\
\hline 1949 & 3700.0 & $3700 . n$ & 3760.0 & 3830.0 & 3980.0 & 4530.0 & 5140.0 & $5770 \cdot 0$ & $6400 \cdot 0$ & 7990.0 & 16900.0 \\
\hline 1950 & 4000.0 & $4000 \cdot n$ & 4140.0 & 4310.0 & 4720.0 & 5160.0 & 6290.0 & $8950 \cdot 0$ & $12500 \cdot 0$ & $15600 \cdot 0$ & 31200.0 \\
\hline 1951 & 4200.0 & 4200.0 & 4200.0 & 4340.0 & 4460.0 & 4530.0 & 4920.0 & $5950 \cdot 0$ & 7850.0 & 10500.0 & $21500 \cdot 0$ \\
\hline 1952 & $3600 \cdot 0$ & 3670.0 & 3740.0 & 3910.0 & 4120.0 & 4340.0 & 4630.0 & $4840 \cdot 0$ & 5430.0 & 7330.0 & $12900 \cdot 0$ \\
\hline 1953 & $5500 \cdot 0$ & 5500.0 & 5500.0 & 5640.0 & 5980.0 & 6220.0 & 6770.0 & 7410.0 & 8520.0 & $12800 \cdot 0$ & 26300.0 \\
\hline 1954 & 6200.0 & 6200.0 & 6230.0 & 6390.0 & 6660.0 & 7090.0 & 7740.0 & 8710.0 & 9520.0 & 10500.0 & 15300.0 \\
\hline 1955 & 6200.0 & 6330.0 & 6540.0 & 6570.0 & 6650.0 & 7320.0 & 8120.0 & $11000 \cdot 0$ & 14200.0 & 16900.0 & 37000.0 \\
\hline 1956 & 6200.0 & 6200.0 & 6290.0 & 6340.0 & 6480.0 & 7110.0 & 7550.0 & $8510 \cdot 0$ & 11200.0 & $13600 \cdot 0$ & 19900.0 \\
\hline 1957 & 5500.0 & 5670.0 & 5860.0 & 5930.0 & 6130.0 & 6350.0 & 6760.0 & $7760 \cdot 0$ & 8180.0 & 9630.0 & 18900.0 \\
\hline 1958 & 6500.0 & 6500.0 & 6500.0 & $6570 . ?$ & 6800.0 & 7360.0 & 7920.0 & 8580.0 & 9890.0 & 10900.0 & 19900.0 \\
\hline 1959 & 6500.0 & 6500.0 & $6500 . ?$ & 6500.0 & 6500.0 & 6730.0 & 7420.0 & 8940.0 & 10700.0 & 11800.0 & 14600.0 \\
\hline 1960 & 6000.0 & $6000 \cdot n$ & $6000 . ?$ & 6000.0 & 6250.0 & 6600.0 & 6850.0 & 7500.0 & 8770.0 & 13400.0 & 22900.0 \\
\hline 1961 & $6 \cap 00.0$ & $60 n n \cdot n$ & $60 n \cap . ?$ & 6000.0 & 6040.0 & 6420.0 & 6510.0 & $7070 \cdot 0$ & 8180.0 & 9470.0 & 19500.0 \\
\hline 1962 & 6000.0 & 6000.0 & $600 n .0$ & 6000.0 & 6200.0 & 6390.0 & 6720.0 & $7750 \cdot 0$ & $10100 \cdot 0$ & 11900.0 & 19700.0 \\
\hline
\end{tabular}
HIGHEST MEAN DISCHARGE, IN CFS, FOR THE FOLLOWING NUMBER OF CONSECUTIVE DAYS IN YEAR ENDING SEPTEMBER 30 
Molalla River above Pine Creek near Wilhoit, Oreg.
STATION NUMBER $14-1985.00$

DURATION TARLE OF DAILY DISCHARGE

CLASS $00 \begin{array}{lllllllllllllllllllllllllllllllllll}2 & 2 & 3 & 4 & 5 & 6 & 7 & 8 & 9 & 10 & 11 & 12 & 13 & 14 & 15 & 16 & 17 & 18 & 19 & 20 & 21 & 22 & 23 & 24 & 25 & 26 & 27 & 28 & 29 & 30 & 31 & 32 & 33 & 34\end{array}$

\begin{tabular}{|c|c|c|c|c|c|c|c|c|c|c|c|c|c|c|c|c|c|c|c|c|c|c|c|c|c|c|c|}
\hline YEAR & & & & & & & & & & & & & & UMB & & OF & DAY & S II & $\mathrm{N} \mathrm{Cl}$ & LASS & & & & & & & CFS-DAYS \\
\hline 1936 & & 1 & 6 & 21 & 24 & 21 & 18 & 15 & 26 & 25 & 12 & 22 & 39 & 33 & 19 & 30 & 20 & 21 & 4 & 2 & & 4 & 2 & 1 & & & 163811.0 \\
\hline 1937 & & 26 & 26 & 15 & 21 & 17 & 19 & 10 & 34 & 16 & 13 & 7 & 15 & 15 & 22 & 38 & 20 & 34 & 8 & 5 & 1 & 1 & & 2 & & & 169108.0 \\
\hline 1938 & & 18 & 13 & 29 & 16 & 6 & 16 & 9 & 18 & 13 & 13 & 4 & 16 & 26 & 31 & 47 & 27 & 24 & 15 & 7 & 7 & 6 & 1 & & 2 & & $234415 \cdot 0$ \\
\hline 1939 & & 7 & 15 & 48 & 13 & 5 & 10 & 9 & 11 & 15 & 33 & 33 & 44 & 27 & 26 & 31 & 7 & 14 & 13 & 2 & & 2 & & & & & 145453.0 \\
\hline 1940 & 2 & 31 & 15 & 37 & 22 & 17 & 27 & 18 & 24 & 9 & 9 & 11 & 29 & 19 & 16 & 20 & 11 & 25 & 10 & 9 & & 3 & 2 & & & & 154053.0 \\
\hline 1941 & & & & 27 & 20 & 10 & 14 & 10 & 27 & 47 & 45 & 37 & 40 & 25 & 18 & 23 & 6 & 9 & 5 & 1 & 1 & & & & & & $115900 \cdot 0$ \\
\hline 1942 & & & 12 & 22 & 13 & 7 & 10 & 5 & 22 & 16 & 29 & 58 & 75 & 26 & 10 & 17 & 14 & 12 & 7 & 5 & & 3 & 2 & & & & 157339.0 \\
\hline 1943 & & & 17 & 23 & 19 & 14 & 21 & 10 & 11 & 7 & 11 & 22 & 44 & 19 & 17 & 38 & 20 & 28 & 14 & 13 & 6 & 2 & 5 & 3 & 1 & & 251836.0 \\
\hline 1944 & & 1 & 10 & 38 & 20 & 16 & 7 & 13 & 18 & 30 & 32 & 26 & 63 & 30 & 30 & 16 & 4 & 7 & 4 & & 1 & & & & & & $111144 \cdot 0$ \\
\hline 1945 & & & 10 & 33 & 21 & 19 & 22 & 15 & 40 & 18 & 17 & 9 & 26 & 15 & 10 & 34 & 27 & 25 & 15 & 3 & 3 & 1 & 1 & 1 & & & 172163.0 \\
\hline 1946 & & & & 14 & 44 & 22 & 15 & 4 & 9 & 7 & 11 & 24 & 29 & 29 & 32 & 49 & 28 & 20 & 12 & 6 & 5 & 1 & 2 & & 1 & 1 & 213821.0 \\
\hline 1947 & & & & 10 & 24 & 19 & 22 & 19 & 28 & 26 & 27 & 25 & 38 & 19 & 12 & 35 & 22 & 13 & 9 & 6 & 3 & 2 & 4 & & 2 & & $188679 \cdot 0$ \\
\hline 1948 & & & & 11 & 17 & 21 & 23 & 9 & 17 & 19 & 16 & 12 & 28 & 29 & 36 & 40 & 22 & 36 & 14 & 5 & 1 & 5 & 3 & 1 & & 1 & 231171.0 \\
\hline 1949 & & & & 26 & 19 & 11 & 20 & 11 & 43 & 24 & 12 & 12 & 27 & 27 & 17 & 26 & 28 & 40 & 4 & 8 & 2 & 2 & 6 & & & & 206892.0 \\
\hline 1950 & & & 1 & 19 & 19 & 9 & 14 & 20 & 28 & 15 & 5 & 11 & 22 & 24 & 22 & 52 & 41 & 36 & 12 & 5 & 3 & 3 & 1 & 3 & & & $232066 \cdot 0$ \\
\hline 1951 & & 3 & 20 & 31 & 16 & 13 & 15 & 5 & 13 & 11 & 4 & 17 & 25 & 13 & 20 & 48 & 36 & 29 & 18 & 10 & 8 & 8 & 2 & & & & 249195.0 \\
\hline 1952 & & & & 29 & 12 & 10 & 12 & 5 & 12 & 12 & 32 & 16 & 35 & 37 & 27 & 64 & 18 & 21 & 10 & 6 & 2 & 4 & 2 & & & & 207801.0 \\
\hline 1953 & & & 31 & 20 & 27 & 21 & 22 & 11 & 15 & 14 & 20 & 16 & 27 & 24 & 14 & 42 & 11 & 19 & 8 & 9 & 4 & 5 & 1 & 2 & 1 & 1 & 202348.0 \\
\hline 1954 & & & & & & 6 & 30 & 36 & 21 & 18 & 14 & 19 & 45 & 28 & 17 & 29 & 25 & 32 & 18 & 12 & 5 & 6 & 2 & 1 & 1 & & 248736.0 \\
\hline 1955 & & & & 16 & 7 & 15 & 12 & 10 & 34 & 12 & 15 & 23 & 50 & 38 & 32 & 40 & 24 & 28 & 5 & 1 & 2 & 1 & & & & & $173247 \cdot \mathrm{C}$ \\
\hline 1956 & & & & 17 & 25 & 12 & 19 & 8 & 11 & 7 & 8 & 6 & 30 & 30 & 24 & 43 & 30 & 43 & 33 & 2 & 3 & 6 & 3 & 4 & 1 & 1 & 295722.0 \\
\hline 1957 & & & 17 & 32 & 17 & 14 & 20 & 9 & 17 & 28 & 31 & 18 & 40 & 27 & 27 & 25 & 10 & 16 & 8 & & 3 & 1 & 4 & 1 & & & 157835.0 \\
\hline 1958 & & & 17 & 21 & 19 & 14 & 26 & 12 & 37 & 23 & 19 & 17 & 29 & 28 & 16 & 15 & 14 & 21 & 21 & 4 & 9 & 1 & 2 & & & & 185113.0 \\
\hline 1959 & & & & 18 & 24 & 13 & 20 & 11 & 22 & 11 & 9 & 17 & 40 & 34 & 27 & 40 & 25 & 30 & 12 & 5 & 1 & 5 & & 1 & & & 200324 . \\
\hline 1960 & & & & 6 & 27 & 21 & 18 & 9 & 19 & 26 & 32 & 25 & 31 & 19 & 22 & 39 & 28 & 27 & 9 & 3 & 1 & 4 & & & & & 180700 . \\
\hline 1961 & & & 13 & 30 & 20 & 18 & 16 & 16 & 16 & 11 & 9 & 10 & 13 & 14 & 22 & 30 & 26 & 47 & 24 & 9 & 9 & 6 & 3 & 1 & 1 & 1 & 278402.0 \\
\hline 1962 & & & & 24 & 20 & 21 & 22 & 13 & 19 & 15 & 10 & 24 & 39 & 29 & 19 & 35 & 25 & 30 & 8 & 2 & 5 & 4 & 1 & & & & 183392.0 \\
\hline 1963 & & & & & 16 & 14 & 21 & 9 & 35 & 43 & 26 & 22 & 27 & 17 & 19 & 32 & 24 & 34 & 11 & 3 & 8 & 2 & 1 & & 1 & & 200068 \\
\hline
\end{tabular}

Molalla River above Pine Creek near Wilhoit, Oreg.

STATION NUMBER $\quad 14-1985.00$

Surmary for water years 1936-63

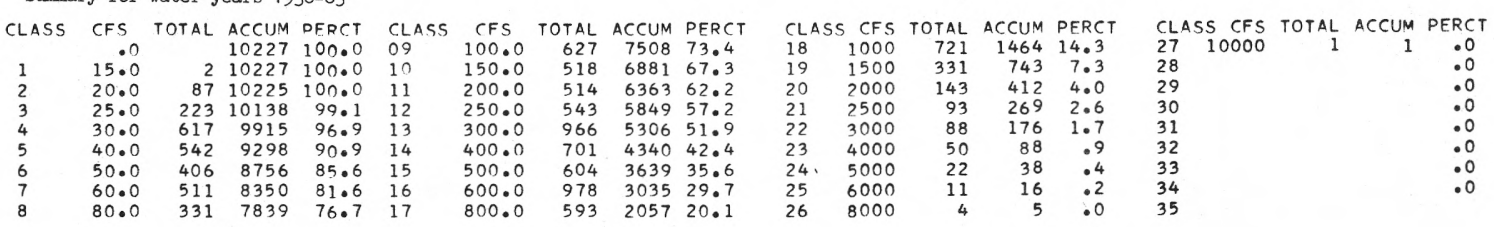

Molalla River above Pine. Creek

STATION NUMBER $14-1985.00$ near Wilhoit, Oreg.

LOWEST MEAN DISCHARGE, IN CFS, FOR THE FOLLOWING NUMBER OF CONSECUTIVE DAYS IN YEAR BEGINNING APRIL 1

\begin{tabular}{|c|c|c|c|c|c|c|c|c|c|c|c|}
\hline YEAR & 1 & 3 & 7 & 14 & 30 & 60 & 90 & 120 & 150 & 183 & 274 \\
\hline 1936 & 21.0 & 21.0 & 21.4 & 21.9 & 23.6 & 25.5 & 32.3 & $35 \cdot 5$ & 47.1 & 84.9 & 181.0 \\
\hline 1937 & 37.0 & 38.0 & 40.7 & 48.3 & 56.9 & 60.1 & 72.8 & 113.0 & 218.0 & 332.0 & 644.0 \\
\hline 1938 & 21.0 & 21.3 & 21.7 & 22.0 & 24.8 & 28.5 & 32.8 & 40.4 & 62.2 & 123.0 & 286.0 \\
\hline 1939 & 24.0 & $24 \cdot n$ & 24.4 & 25.9 & 29.9 & 33.6 & 43.3 & $57 \cdot 8$ & 62.2 & 94.4 & 245.0 \\
\hline 1940 & 19.0 & $19 \cdot 3$ & 20.0 & 20.1 & 21.1 & 26.0 & 29.9 & 37.7 & 47.9 & 95.7 & 251.0 \\
\hline 1941 & $30 \cdot 0$ & 30.7 & 31.3 & 32.7 & 37.6 & 54.3 & 97.3 & $126 \cdot 0$ & 150.0 & 180.0 & 377.0 \\
\hline 1942 & 25.0 & 25.3 & 26.0 & 26.7 & 28.1 & 31.8 & 39.5 & $61 \cdot 5$ & 111.0 & 170.0 & 504.0 \\
\hline 1943 & 30.0 & 30.3 & 31.6 & 34.3 & 35.0 & 45.1 & 52.4 & 84.1 & 139.0 & 206.0 & 272.0 \\
\hline 1944 & 24.0 & 24.7 & 26.0 & 29.4 & 32.2 & 39.7 & 41.0 & $47 \cdot 3$ & 80.4 & 115.0 & 194.0 \\
\hline 1945 & 28.0 & 28.0 & 29.6 & 31.9 & 37.1 & 47.3 & 60.7 & $61 \cdot 1$ & 80.4 & 173.0 & 538.0 \\
\hline 1946 & 34.0 & 34.3 & 35.3 & 37.6 & 40.9 & 43.6 & 49.6 & 93.8 & 140.0 & 200.0 & 479.0 \\
\hline 1947 & 42.0 & 42.7 & 44.7 & 46.9 & 52.8 & 64.0 & 74.7 & $115 \cdot 0$ & 140.0 & 180.0 & 437.0 \\
\hline 1948 & 36.0 & 36.3 & 37.7 & 38.6 & 44.5 & 51.0 & 69.4 & 111.0 & 138.0 & 208.0 & 364.0 \\
\hline 1949 & 32.0 & 32.7 & 33.3 & 34.2 & 36.4 & 47.1 & 57.6 & 82.0 & 99.3 & 147.0 & 369.0 \\
\hline 1950 & 29.0 & 29.7 & $30 \cdot 1$ & 31.2 & 37.7 & 50.3 & 77.8 & 172.0 & 292.0 & 383.0 & 681.0 \\
\hline 1951 & 24.0 & 24.0 & 25.1 & 26.4 & 29.9 & 32.2 & 39.4 & $59 \cdot 6$ & 121.0 & 193.0 & 380.0 \\
\hline 1952 & 26.0 & 26.0 & 26.7 & 27.4 & 28.6 & 30.2 & 34.7 & 39.6 & 57.3 & 118.0 & 277.0 \\
\hline 1953 & 42.0 & 42.7 & 44.0 & 46.1 & 55.4 & 63.9 & 77.1 & $97 \cdot 2$ & 124.0 & 215.0 & 517.0 \\
\hline 1954 & 53.0 & 55.0 & 56.7 & 61.1 & 69.1 & 78.7 & 90.6 & 136.0 & 172.0 & 227.0 & 341.0 \\
\hline 1955 & 32.0 & 33.3 & 33.7 & 35.5 & 41.7 & 71.7 & 99.5 & 176.0 & 315.0 & 404.0 & 784.0 \\
\hline 1956 & 29.0 & 29.0 & 30.3 & 33.5 & 36.8 & 42.4 & 48.4 & 74.2 & 138.0 & 180.0 & 283.0 \\
\hline 1957 & 25.0 & 25.0 & 26.4 & 27.1 & 30.2 & 38.5 & 47.6 & $62 \cdot 0$ & 76.9 & $120 \cdot 0$ & 332.0 \\
\hline 1958 & 26.0 & 26.0 & 27.0 & 28.7 & 29.6 & 35.9 & 42.1 & 54.9 & 76.2 & 101.0 & 372.0 \\
\hline 1959 & 32.0 & 33.3 & 35.6 & 36.9 & 41.3 & 63.0 & 91.9 & 144.0 & 199.0 & 239.0 & 312.0 \\
\hline 1960 & 36.0 & 36.0 & 36.9 & 40.3 & 47.0 & 59.3 & 59.9 & 69.6 & 110.0 & 228.0 & 517.0 \\
\hline 1961 & 25.0 & 25.0 & 27.3 & 29.5 & 30.1 & 41.7 & 56.6 & 66.9 & 95.3 & 135.0 & 346.0 \\
\hline 1962 & 31.0 & 31.7 & 32.3 & 34.0 & 40.0 & 50.1 & 60.4 & $88 \cdot 5$ & 171.0 & 235.0 & 424.0 \\
\hline
\end{tabular}


Molalla River above Pine Creek

STATION NUMBER 14-1985.00

near Wilhoit, Oreg.

\begin{tabular}{|c|c|c|c|c|c|c|c|c|c|c|c|}
\hline YEAR & 1 & 3 & 7 & 15 & 30 & 60 & 90 & 120 & 150 & 183 & 274 \\
\hline 1936 & 5380.0 & 4320.0 & 3180.0 & 2830.0 & $1850 \cdot 0$ & $1200 \cdot 0$ & 1010.0 & $921 \cdot 0$ & 870.0 & 771.0 & 579.0 \\
\hline $\begin{array}{l}1937 \\
1938\end{array}$ & 5560.0 & $5000 \cdot n$ & 3080.0 & $1950 \cdot 0$ & 1460.0 & $1200 \cdot 0$ & 1130.0 & $1040 \cdot 0$ & 942.0 & 811.0 & 605.0 \\
\hline $\begin{array}{l}1938 \\
1939\end{array}$ & 6900.0 & 5520.0 & 3910.0 & 2170.0 & 1840.0 & 1550.0 & 1390.0 & $1210 \cdot 0$ & 1180.0 & 1140.0 & 843.0 \\
\hline $\begin{array}{l}1939 \\
1940\end{array}$ & 3690.0 & 2500.0 & 1780.0 & 1370.0 & 1030.0 & 872.0 & 786.0 & $760 \cdot 0$ & 721.0 & 672.0 & 518.0 \\
\hline $\begin{array}{l}1940 \\
1941\end{array}$ & 4730.0 & 3510.0 & 2350.0 & 1840.0 & 1650.0 & 1260.0 & 1050.0 & $1000 \cdot 0$ & 913.0 & 786.0 & 551.0 \\
\hline 1941 & 2920.0 & $194 n \cdot 0$ & $129 n \cdot n$ & 1080.0 & 766.0 & 694.0 & 655.0 & $575 \cdot 0$ & 512.0 & 462.0 & 390.0 \\
\hline 1942 & 4230.0 & $3430 . n$ & 2250.0 & 1450.0 & 1370.0 & 1050.0 & 978.0 & $849 \cdot 0$ & 746.0 & 666.0 & 552.0 \\
\hline 1943 & 7680.0 & $472 n \cdot n$ & $382 n \cdot n$ & 2650.0 & 2010.0 & 1870.0 & 1600.0 & $1400 \cdot 0$ & 1320.0 & 1210.0 & 904.0 \\
\hline $\begin{array}{l}1944 \\
1945\end{array}$ & 2850.0 & $1840 . n$ & 1170.0 & 906.0 & 629.0 & 522.0 & 463.0 & $465 \cdot 0$ & 443.0 & 447.0 & 391.0 \\
\hline 1945 & 5200.0 & 3960.0 & 2760.0 & 1860.0 & 1210.0 & 1060.0 & 991.0 & $987 \cdot 0$ & 962.0 & 823.0 & 609.0 \\
\hline $\begin{array}{l}1946 \\
1947\end{array}$ & 8880.0 & 5380.0 & 3320.0 & 2490.0 & $1700 \cdot 0$ & 1530.0 & 1320.0 & $1220 \bullet 0$ & $1100 \cdot 0$ & 1010.0 & 764.0 \\
\hline 1947 & 6660.0 & 5980.0 & $4150 . n$ & 2360.0 & 2260.0 & 1390.0 & 1230.0 & $1070 \cdot 0$ & 969.0 & 885.0 & 667.0 \\
\hline 1948 & 8110.0 & 5510.0 & 3500.0 & 2270.0 & 1520.0 & 1130.0 & 1220.0 & $1000 \cdot 0$ & 1020.0 & 949.0 & 819.0 \\
\hline 1949 & 4920.0 & 4390.0 & 3710.0 & 2470.0 & 1640.0 & $1200 \cdot 0$ & 1230.0 & $1050 \cdot 0$ & 933.0 & 957.0 & 735.0 \\
\hline 1950 & 5500.0 & 4590.0 & 2930.0 & $2100 \cdot 0$ & 1650.0 & 1420.0 & 1280.0 & $1160 \cdot 0$ & 1090.0 & $1020 \cdot 0$ & 822.0 \\
\hline 1951 & 4180.0 & 3310.0 & 3050.0 & 2150.0 & 1900.0 & 1470.0 & 1490.0 & $1440 \cdot 0$ & 1270.0 & 1180.0 & 896.0 \\
\hline 1952 & 4530.0 & $4040 . ?$ & 3520.0 & $2200 \cdot 0$ & 1390.0 & 1030.0 & 1010.0 & $911 \cdot 0$ & 899.0 & 849.0 & 726.0 \\
\hline 1953 & 8040.0 & $6480 . n$ & $477 n .0$ & 3970.0 & 2930.0 & $189 n \cdot n$ & 1460.0 & $1220 \cdot 0$ & 1100.0 & 1000.0 & 724.0 \\
\hline 1954 & 6240.0 & $4220 . n$ & 2990.0 & 2310.0 & 2140.0 & 1560.0 & 1570.0 & $1430 \cdot 0$ & 1300.0 & 1140.0 & 875.0 \\
\hline 1955 & 3740.0 & $2980 \cdot n$ & $188 \cap .0$ & $1180 \cdot 0$ & 977.0 & 893.0 & 845.0 & $721 \cdot 0$ & 714.0 & 716.0 & 604.0 \\
\hline 1956 & 8500.0 & $6400 \cdot 0$ & 3930.0 & 2940.0 & 2510.0 & 2140.0 & 1820.0 & $1550 \cdot 0$ & 1370.0 & $1310 \cdot 0$ & 1060.0 \\
\hline 1957 & 5690.0 & 4670.0 & 2990.0 & 2490.0 & 1710.0 & 1240.0 & 967.0 & $861 \cdot 0$ & 788.0 & 712.0 & 562.0 \\
\hline 1958 & 4760.0 & 3750.0 & 2620.0 & 2020.0 & 1540.0 & 1490.0 & 1280.0 & $1060 \cdot 0$ & 1030.0 & 917.0 & 660.0 \\
\hline 1959 & 5010.0 & 3810.0 & 2680.0 & 1690.0 & 1550.0 & 1190.0 & 1140.0 & $1020 \cdot 0$ & 978.0 & 908.0 & 697.0 \\
\hline 1960 & 3450.0 & 3180.0 & 2290.0 & 1600.0 & 1260.0 & 1120.0 & 1020.0 & $974 \cdot 0$ & 862.0 & 774.0 & 637.0 \\
\hline 1961 & 10500.0 & 5960.0 & 4010.0 & 3130.0 & 2400.0 & 1890.0 & 1670.0 & 1650.0 & 1520.0 & 1380.0 & 997.0 \\
\hline 1962 & 4000.0 & 3330.0 & 2230.0 & 1750.0 & 1440.0 & 1150.0 & 937.0 & 883.0 & 882.0 & 843.0 & 649.0 \\
\hline 1963 & 6180.0 & 3510.0 & 2420.0 & 2120.0 & 1570.0 & 1060.0 & 885.0 & 854.0 & 823.0 & 860.0 & 699.0 \\
\hline
\end{tabular}

Molalla River near Molalla, Oreg.

1951
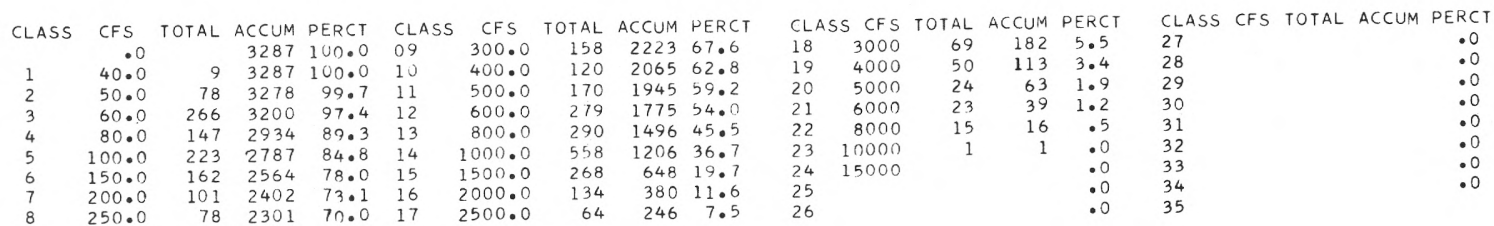

250.0

Molalla River near,Molalla, , Oreg.

STATION NUMBER $14-1990.00$

LOWEST MEAN OISCHARGE, IN CFS, FOR THE FOLLOWING NUMBER OF CONSECUTIVE DAYS IN YEAR BEGINNING APRIL 1

YEAR
1906
1907
1908
1947
1948
1949
1950
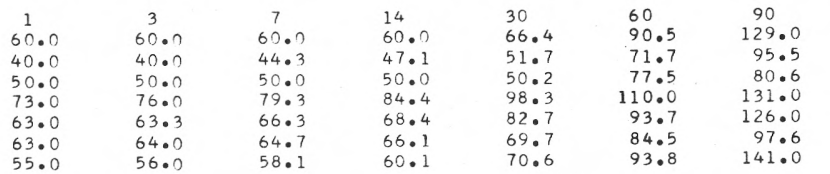

120
$213 \cdot 0$
$108 \cdot 0$
$165 \cdot 0$
$207 \cdot 0$
$197 \bullet 0$
$137 \cdot 0$
$301 \bullet 0$

150
317.0
131.0
247.0
247.0
243.0
160.0
495.0

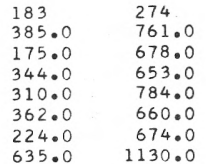

Molalla R1ver near Molalla, Oreg.

STATION NUMBER

$14-1990 \cdot 00$

HIGHEST MEAN DISCHARGE, IN CFS, FOR THE FOLLOWING NUMBER OF CONSECUTIVE DAYS IN YEAR ENDING SEPTEMBER 30

YEAR
1906
1907
1908
1909
1947
1948
1949
1950
1951

$\begin{array}{cc}1 & 3 \\ 3750.0 & 3160.0 \\ 9800.0 & 8390.0 \\ 8550.0 & 5840.0 \\ 7930.0 & 7110.0 \\ 9710.0 & 8530.0 \\ 14200.0 & 9500.0 \\ 9610.0 & 8210.0 \\ 9180.0 & 7920.0 \\ 7250.0 & 5480.0\end{array}$

7
2730.0
6230.0
4800.0
5670.0
6320.0
5990.0
6900.0
5310.0

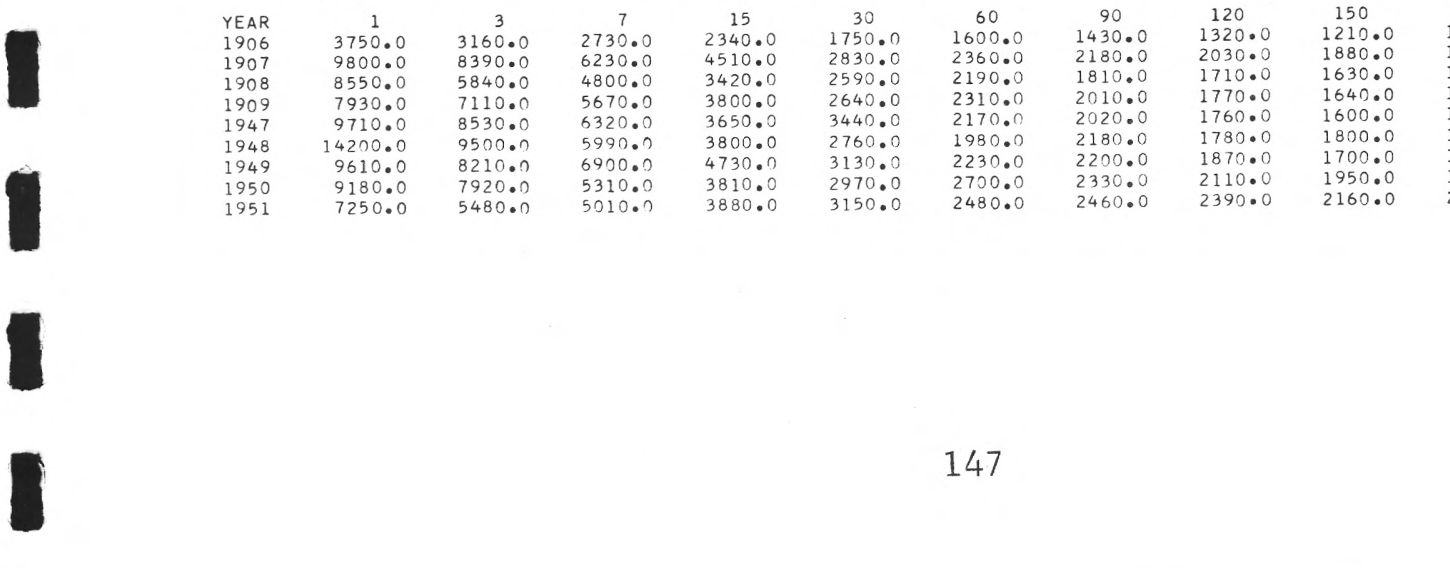

30
$1750 \cdot 0$
$2830 \cdot 0$
$2590 \cdot 0$
$2640 \cdot 0$
$3440 \cdot 0$
$2760 \cdot 0$
$3130 \cdot 0$
$2970 \cdot 0$
$3150 \cdot 0$

60
1600.0
2360.0
$2190 \cdot 0$
2310.0
2170.0
1980.0
2230.0
2700.0
2480.0

90
$1430 \cdot 0$
$2180 \cdot 0$
$1810 \cdot 0$
$2010 \cdot 0$
$2020 \cdot 0$
$2180 \cdot 0$
$2200 \cdot 0$
$2330 \cdot 0$
2460.0

120
$1320 \cdot 0$
$2030 \bullet 0$
$1710 \cdot 0$
$1770 \bullet 0$
$1760 \bullet 0$
$1780 \bullet 0$
$1870 \bullet 0$
$2110 \bullet 0$
$2390 \bullet 0$

150
1210.0
1880.0
1630.0
1640.0
1600.0
1800.0
1700.0
1950.0
2160.0

$1140.0 \quad 1010.0$

1370.0

$\begin{array}{ll}1510.0 & 1240.0 \\ 1210.0\end{array}$

$1460.0 \quad 1110.0$

$1690.0 \quad 1450.0$

$\begin{array}{ll}1320.0 \\ 820.0 & 1440.0\end{array}$

$2000.0 \quad 1510.0$ 
Nolalla River near Canby, Oreg.

STATION NUMBER $14-2000.00$

DURATION TABLF OF DAILY DISCHARGE

CLASS $010 \begin{array}{llllllllllllllllllllllllllllllllll} & 2 & 3 & 4 & 5 & 6 & 7 & 8 & 9 & 10 & 11 & 12 & 13 & 14 & 15 & 16 & 17 & 18 & 19 & 20 & 21 & 22 & 23 & 24 & 25 & 26 & 27 & 28 & 29 & 30 & 31 & 32 & 33 & 34\end{array}$

YEAR
1929
1930
1931
1932
1933
1934
1935
1936
1937
1938
1939
1940
1941
1942
1943
1944
1945
1946
1947
1948
1949
1950
1951
1952
1953
1954
1955
1956
1957
1958
1959

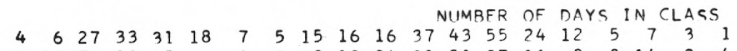
$\begin{array}{rrrrrrrrrrrrrrrrrrrr}2 & 20 & 76 & 28 & 35 & & 6 & 2 & 18 & 12 & 24 & 31 & 31 & 27 & 16 & 8 & 8 & 14 & 2 & 4 \\ 19 & 15 & 30 & 8 & 68 & 8 & 13 & 9 & 26 & 25 & 17 & 24 & 35 & 30 & 14 & 9 & 5 & 6 & 1 & \end{array}$

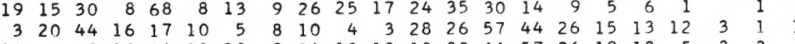
$\begin{array}{lllllllllllllllllllll}1199 & 16 & 24 & 11 & 13 & 9 & 14 & 13 & 10 & 12 & 32 & 66 & 57 & 26 & 19 & 13 & 5 & 3 & 2\end{array}$ $\begin{array}{rrrrrrrrrrrrrrrrrrrrr}3 & 17 & 28 & 22 & 34 & 19 & 12 & 15 & 36 & 23 & 17 & 31 & 11 & 18 & 19 & 18 & 12 & 12 & 4 & 2 \\ 4 & 13 & 40 & 15 & 24 & 13 & 7 & 8 & 8 & 8 & 12 & 15 & 41 & 68 & 35 & 24 & 12 & 10 & 6 & 1\end{array}$

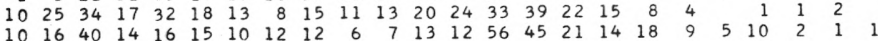
$\begin{array}{llllllllllllllllllllll}10 & 10 & 56 & 17 & 16 & 8 & 6 & 13 & 37 & 26 & 15 & 26 & 40 & 43 & 15 & 10 & 9 & 14 & 1 & 1 & & 1\end{array}$

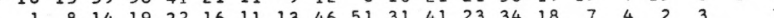

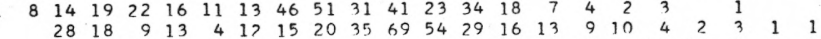
$\begin{array}{rrrrrrrrrrrrrrrrrrrrr}28 & 18 & 9 & 13 & 4 & 17 & 15 & 20 & 35 & 59 & 54 & 29 & 16 & 13 & 9 & 10 & 4 & 2 & 3 & 1 & 1 \\ 16 & 25 & 47 & 12 & 7 & 6 & 5 & 8 & 26 & 28 & 28 & 42 & 28 & 13 & 17 & 20 & 10 & 10 & 6 & 5 & 6\end{array}$

$\begin{array}{lllllllllllllllll}12 & 22 & 20 & 21 & 10 & 5 & 14 & 25 & 22 & 30 & 82 & 32 & 53 & 10 & 1 & 4 & 3\end{array}$ $\begin{array}{lllllllllllllllllllllll}12 & 27 & 49 & 22 & 18 & 19 & 24 & 12 & 14 & 35 & 8 & 31 & 34 & 23 & 16 & 11 & 6 & 2\end{array}$ $\begin{array}{lllllllllllllllllll}21 & 58 & 21 & 5 & 3 & 11 & 12 & 20 & 33 & 21 & 55 & 32 & 22 & 14 & 15 & 10 & 6 & 2\end{array}$

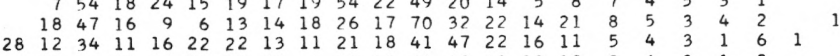

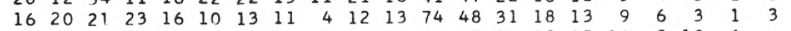
$\begin{array}{lllllllllllllllllllllllll}18 & 37 & 23 & 19 & 9 & 6 & 6 & 8 & 6 & 9 & 13 & 21 & 57 & 37 & 20 & 18 & 15 & 16 & 8 & 13 & 6\end{array}$ $\begin{array}{rrrrrrrrrrrrrrrrrrrrrrrrr}19 & 23 & 26 & 47 & 18 & 10 & 5 & 8 & 10 & 9 & 11 & 23 & 55 & 36 & 17 & 9 & 14 & 12 & 3 & 5 & 1 & 2 & 2\end{array}$

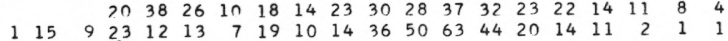
$\begin{array}{rrrrrrrrrrrrrrrrrrrrrrr}5 & 27 & 35 & 11 & 9 & 4 & 5 & 8 & 6 & 15 & 26 & 61 & 43 & 24 & 26 & 25 & 12 & 6 & 7 & 4 & 6 & 1\end{array}$

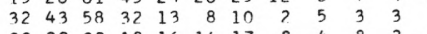
$\begin{array}{rrrrrrrrrrrrrrrrrrrrrrrr}1 & 21 & 25 & 5 & 38 & 15 & 14 & 23 & 26 & 11 & 14 & 23 & 29 & 32 & 18 & 16 & 14 & 17 & 8 & 4 & 8 & 2 & 1 \\ 1 & 2 & 13 & 12 & 23 & 12 & 5 & 13 & 14 & 6 & 8 & 24 & 21 & 66 & 42 & 28 & 17 & 17 & 5 & 1 & 6 & 1 & 1\end{array}$ CFS-DAYS 289413.0 272978.0 441129.0 454533.0 383224.0 369855.0 $338330 \cdot 0$ 517729.0 289519.0 309110.0 244181.0 361902.0 233503.0 35638 . ก 470425.0 397326.0 531813.0 491120.0 531863.0 $587649 \cdot 0$ $46630>.0$ 542121.0 395998.0 666951.0 386632.0 470363,0
Nolalla River near Canby, Oreg.

\begin{tabular}{|c|c|c|c|c|c|}
\hline$\angle L A S$ & CFS & TOTAL & ACCUM & PERCT & $C L$ \\
\hline & on & 5 & 11322 & 100.0 & 09 \\
\hline 1 & $30 . n$ & .15 & 11317 & $10 n$ & 10 \\
\hline 2 & 40 & 99 & 11302 & 99.8 & 11 \\
\hline 3 & $50 . n$ & 257 & 11203 & 98.9 & 12 \\
\hline 4 & 60.0 & 739 & 10946 & & 13 \\
\hline 5 & $80 . n$ & 577 & 10207 & $9 n_{0} 2$ & 14 \\
\hline 6 & $100 \cdot n$ & 991 & 9630 & 85.1 & 15 \\
\hline 7 & $0 . n$ & 456 & 8639 & 76.3 & 16 \\
\hline 8 & on & 330 & 8183 & 7). 3 & 17 \\
\hline
\end{tabular}

STATION NUMBER 14-2000.00

\begin{tabular}{crrlllrrr} 
CFS & TOTAL & ACCUM & PERCT & \multicolumn{2}{l}{ CLASS CFS } & TOTAL & ACCUM & PFRCT \\
250.0 & 307 & 7853 & 69.4 & 18 & 2500 & 382 & 1267 & 11.2 \\
300.0 & 552 & 7546 & 66.6 & 19 & 3000 & 383 & 885 & 7.8 \\
400.0 & 448 & 6994 & 61.8 & 20 & 4000 & 195 & 502 & 4.4 \\
500.0 & 471 & 6546 & 57.8 & 21 & 5000 & 102 & 307 & 2.7 \\
600.0 & 899 & 6075 & 53.7 & 22 & 6000 & 106 & 205 & 1.8 \\
800.0 & 871 & 5176 & 45.7 & 23 & $800 n$ & 49 & 99 & .9 \\
1000.0 & 1537 & 4305 & 38.0 & 24 & 10000 & 42 & 50 & .4 \\
1500.0 & 955 & 2768 & 24.4 & 25 & 15000 & 7 & 8 & .1 \\
2000.0 & 546 & 1813 & 16.0 & 26 & 20000 & 1 & 1 & .0
\end{tabular}

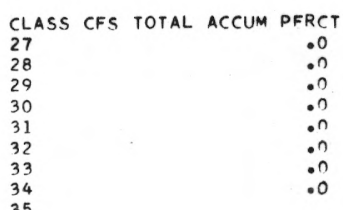

Molalla River near Canby, Oreg.

STATION NUMBER $14-2000.00$

LOWEST MEAN DISCHARGE, IN CFS, FOR THE FOLLOWING NUMRER OF CONSECUTIVE DAYS IN YEAR RFGINNING APRIL 1

$\begin{array}{lrrr}\text { YEAR } & 1 & 3 & 7 \\ 1929 & 41.0 & 51.0 & 54.1 \\ 1930 & 49.0 & 50.0 & 52.1 \\ 1931 & 42.0 & 42.7 & 44.3 \\ 1932 & 40.0 & 41.3 & 42.0 \\ 1933 & 70.0 & 71.3 & 77.3 \\ 1934 & 48.0 & 50.7 & 51.7 \\ 1935 & 38.0 & 44.0 & 46.3 \\ 1936 & 42.0 & 45.3 & 47.0 \\ 1937 & 74.0 & 74.0 & 79.1 \\ 1938 & 42.0 & 42.0 & 44.4 \\ 1939 & 54.0 & 57.0 & 60.0 \\ 1940 & 38.0 & 41.3 & 41.6 \\ 1941 & 49.0 & 53.7 & 54.4 \\ 1942 & 61.0 & 63.0 & 66.6 \\ 1943 & 80.0 & 81.3 & 84.7 \\ 1944 & 52.0 & 53.3 & 54.6 \\ 1945 & 71.0 & 74.7 & 78.4 \\ 1946 & 84.0 & 84.0 & 84.9 \\ 1947 & 92.0 & 93.3 & 99.7 \\ 1948 & 82.0 & 83.3 & 85.3 \\ 1949 & 61.0 & 62.0 & 64.3 \\ 1950 & 69.0 & 70.7 & 72.9 \\ 1951 & 50.0 & 50.0 & 51.1 \\ 1952 & 50.0 & 50.7 & 52.3 \\ 1953 & 78.0 & 80.7 & 82.6 \\ 1954 & 110.0 & 117.0 & 121.0 \\ 1955 & 55.0 & 60.0 & 63.6 \\ 1956 & 78.0 & 78.3 & 83.3 \\ 1957 & 51.0 & 52.3 & 55.6 \\ 1958 & 49.0 & 50.3 & 51.9\end{array}$
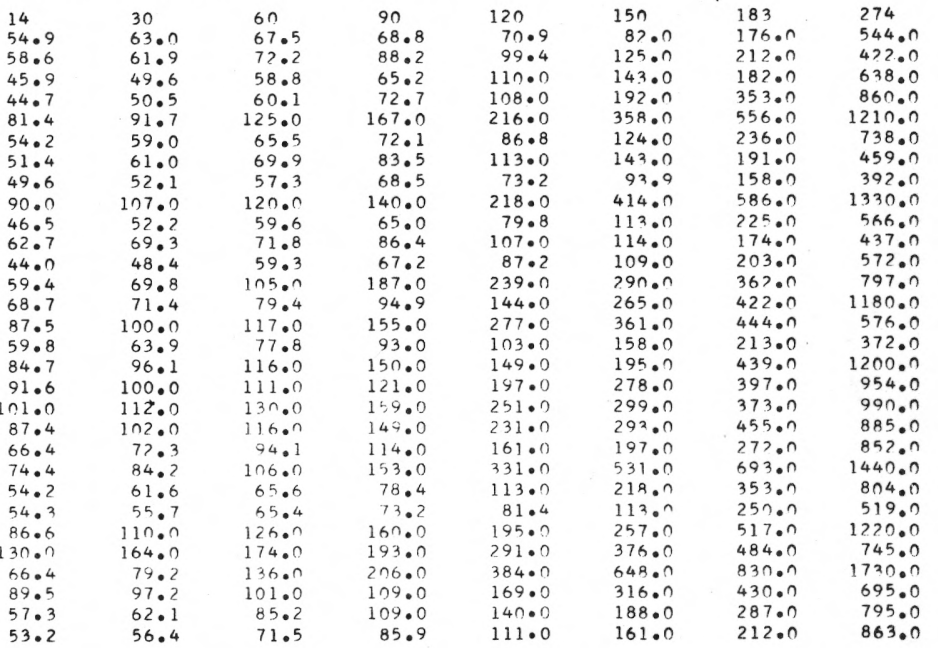
Molla River near Canby, Oreg.

STATION NUMRFR $\quad 14->000.00$

HIG,HEST MEAN DISCHARGF, IN CFS, FOR THE FOLLOWING NUMRFR OF CONSECUTIVF DAYS IN YFAR FNDING SEPTFMRFR 30

\begin{tabular}{|c|c|c|}
\hline 1 & 3 & 7 \\
\hline 5140.0 & $448 \cap \cdot n$ & 3960.0 \\
\hline 7500.0 & $4940 . n$ & $4480 \cdot n$ \\
\hline 15200.0 & $12700 \cdot n$ & $7450 \cdot 0$ \\
\hline 9750.0 & $7550 \cdot 0$ & $523 n \cdot n$ \\
\hline 6710.0 & $5030 \cdot n$ & 4300.0 \\
\hline $13100 \cdot 0$ & $10500 \cdot n$ & 8860.0 \\
\hline 5260.0 & $500 n \cdot 0$ & $432 n \cdot n$ \\
\hline $9220 \cdot 0$ & $862 n \cdot n$ & 6290.0 \\
\hline $10500 \cdot n$ & $9950 \cdot n$ & $664 n \cdot n$ \\
\hline $5 a n n \cdot ?$ & $116 n n \cdot n$ & 8427.0 \\
\hline $80^{80} \cdot 0$ & $5740 . n$ & $4 \cap 9 n \cdot n$ \\
\hline $6>8 \cap \cdot n$ & $554 n \cdot n$ & $44>0 . n$ \\
\hline $6200 \cdot 0$ & $4590 \cdot 0$ & $3130 \cdot 0$ \\
\hline 10700.0 & $6940 \cdot 0$ & $4920 \cdot 0$ \\
\hline 14000.0 & $10500 \cdot n$ & 8810.0 \\
\hline 3600.0 & $2900 \cdot 0$ & 1890.0 \\
\hline 7230.0 & 5470.0 & $441 n \cdot n$ \\
\hline $13500 \cdot 0$ & $1 \cap 400 \cdot n$ & $6570 . ?$ \\
\hline $13200 \cdot 0$ & 10700.0 & $80 n n \cdot n$ \\
\hline $21500 \cdot 0$ & $137 n 0 \cdot n$ & $832 n^{\circ} ?$ \\
\hline $15700 \cdot 0$ & $13700 \cdot 0$ & $\ln 8 \cap n \cdot n$ \\
\hline $1400 \cap .0$ & $1170 n \cdot n$ & $754 n$ •? \\
\hline $996 n \cdot n$ & $8190 . n$ & $681 n \cdot n$ \\
\hline $8140 \cdot 0$ & $7580 \cdot 0$ & $642 n \cdot n$ \\
\hline $16600 \cdot 0$ & $14700 \cdot 0$ & $998 n \cdot n$ \\
\hline $13000 \cdot 0$ & $9180 \cdot n$ & $7100 . ?$ \\
\hline $7990 \cdot 0$ & $5860 \cdot 0$ & 3980.0 \\
\hline 15900.0 & $12100 \cdot 0$ & 8330.0 \\
\hline $9100 \cdot 0$ & $8250 \cdot n$ & $632 n \cdot n$ \\
\hline 11800.0 & $8610 \cdot n$ & $632 n \cdot n$ \\
\hline
\end{tabular}

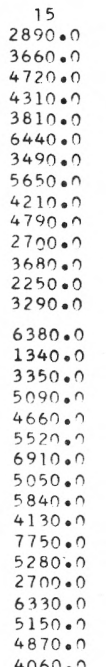

\begin{tabular}{|c|}
\hline \multirow{2}{*}{$\begin{array}{l}30 \\
1940.0\end{array}$} \\
\hline \\
\hline 2950.0 \\
\hline 3410.0 \\
\hline 3390.0 \\
\hline 3020.0 \\
\hline 5010.0 \\
\hline 2640.0 \\
\hline 3770.0 \\
\hline 2960.0 \\
\hline 4010.0 \\
\hline 1950.0 \\
\hline 3330.0 \\
\hline $\begin{array}{l}1720.0 \\
3090.0\end{array}$ \\
\hline 4900.0 \\
\hline 1160.0 \\
\hline 2470.0 \\
\hline 3570.0 \\
\hline $4320 \cdot 0$ \\
\hline $3610 . ?$ \\
\hline $\begin{array}{l}4570 . ? \\
4000 \cdot ? 0\end{array}$ \\
\hline $473 n$. त \\
\hline 2740.0 \\
\hline $5980 \cdot n$ \\
\hline 4950.0 \\
\hline 2630.0 \\
\hline $5540 \cdot 0$ \\
\hline 3460.0 \\
\hline 3680.0 \\
\hline
\end{tabular}

60
1670.0
1950.0
2240.0
2760.0
2090.0
3800.0
2160.0
2530.0
2530.0
3310.0
1890.0
2590.0
1610.0
2380.0
4470.0
1090.0
2230.0
3340.0
2740.0
2670.0
$315 n \cdot n$
3680.0
$352 n \cdot ?$
2170.0
4030.0
$37 n 0.0$
2210.0
5040.0
2910.0
3430.0
2860.0

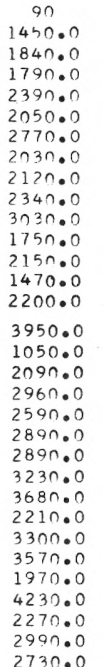

120
$1370 \cdot 0$
$1610 \cdot 0$
$1570 \cdot 0$
$237 n \cdot 0$
$1940 \cdot 0$
$2420 \cdot 0$
$1860 \cdot 0$
$1910 \cdot 0$
$2110 \cdot 0$
$273 n \cdot 0$
$1630 \cdot 0$
$2010 \cdot 0$
$1270 \cdot 0$
$1930 \cdot 0$
$3370 \cdot 0$
$1010 \cdot 0$
$2100 \cdot 0$
$276 n \cdot 0$
$2250 \cdot 0$
$2370 \cdot 0$
$2460 \cdot 0$
$2920 \cdot 0$
$3480 \cdot 0$
$1990 \cdot 0$
$2820 \cdot 0$
$3210 \cdot 0$
$1710 \cdot 0$
$3550 \cdot 0$
$2090 \cdot 0$
$2460 \cdot 0$
$2490 \cdot 0$

150

\begin{tabular}{|c|}
\hline \\
\hline $\begin{array}{l}1300.0 \\
1380.0\end{array}$ \\
\hline $123 n \cdot n$ \\
\hline 2080.0 \\
\hline 1880.0 \\
\hline 1860.0 \\
\hline $1710 . n$ \\
\hline $160 n \cdot 0$ \\
\hline $168 n \cdot n$ \\
\hline $256 n \cdot n$ \\
\hline $137 n .0$ \\
\hline $158 \cap \cdot \cap$ \\
\hline $993 . n$ \\
\hline 2810.0 \\
\hline 940.0 \\
\hline $171 n . n$ \\
\hline $2260 \cdot 0$ \\
\hline $185 n \cdot n$ \\
\hline $2 ? 6 n \cdot n$ \\
\hline $232 n \cdot n$ \\
\hline $247 n \cdot n$ \\
\hline $286 n \cdot n$ \\
\hline 1820.0 \\
\hline $232 n \cdot n$ \\
\hline $252 n \cdot n$ \\
\hline $1640 \cdot 0$ \\
\hline $3020 \cdot 0$ \\
\hline $1730 \cdot n$ \\
\hline $\begin{array}{l}2120 \cdot 0 \\
2180 . n\end{array}$ \\
\hline \\
\hline
\end{tabular}

274

$1400 \cdot 0 \quad 130 n \cdot 0 \quad 1020 \cdot 0$

$\begin{array}{lll}142 n \cdot 0 & 138 n \cdot n & 974 . \\ 137 n .0 & 123 n \cdot n & 896 . n\end{array}$

2230.0 2n80.0 1590.n

$1890.0 \quad 1880.0 \quad 1610.0$

$2080.0 \quad 186 n \cdot n \quad 1370 . n$

1790.0 160n.0 1200.0

$1930 . n$ 168n.n $1240 . n$

$269 n .0 \quad 2560 . n \quad 1870 . n$

$1500.0 \quad 137 n \cdot 0 \quad 1030.0$

$1120.0 \quad 993.0 \quad 830.0$

$1700.0 \quad 1520.0 \quad 1270.0$

$3110.0 \quad 2810.0 \quad 2080.0$

$\begin{array}{rrr}975.0 & 940.0 & 816.0 \\ 1990.0 & 1710.0 & 1250.0\end{array}$

$251 n \cdot n \quad 226 n .0 \quad 168 n . n$

$204 n \cdot 0 \quad 185 n \cdot n \quad 1400 . n$

$24 n n .0 \quad 226 n \cdot 0 \quad 1890.0$

$2710 . n$ 247n.n $189 \pi \cdot n$

$316 n \cdot n \quad 286 n \cdot n \quad 212 n \cdot n$

$1950.0 \quad 1820.0 \quad 1510.0$

$\begin{array}{lll}256 n .0 & 232 n .0 & 1670 . n \\ 288 n .0 & 2520.0 & 1910.0\end{array}$

$1660.0 \quad 1640.0 \quad 1390 . n$

$3240.0 \quad 3020.0 \quad 2390.0$

$\begin{array}{lll}1910.0 & 1730.0 & 1380.0 \\ 2410.0 & 2120.0 & 1520.0\end{array}$

2380.0 2180.n 1660.0

Pudding River near Mount Angel, Oreg.

CTATION NUMRFR 14->011.00

DURATION TARLF OF DATLY NTGCHARGF

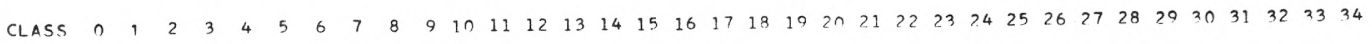

YEAR

1940
1941
1942

NUMRER OF DAYS IN CLASS

.

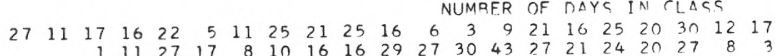

$\begin{array}{lllllllllllllllllllllllll}1 & 25 & 12 & 12 & 3 & 4 & 11 & 4 & 19 & 17 & 23 & 16 & 23 & 53 & 34 & 31 & 15 & 25 & 15 & 11 & 5 & 4 & 2 & & \\ 2\end{array}$

$\begin{array}{rrrrrrrrrrrrrrrrrrrr}10 & 11 & 34 & 12 & 14 & 16 & 7 & 8 & 7 & 13 & 15 & 34 & 27 & 19 & 28 & 17 & 18 & 21 \\ 10 & 16 & 18 & 18\end{array}$

$\begin{array}{rrrrrrrrrrrrrrrrrrrrrrr}1 & 16 & 20 & 20 & 3 & 12 & 14 & 5 & 13 & 7 & 14 & 16 & 15 & 12 & 28 & 27 & 38 & 46 & 34 & 21 & 4 & 19 & 1\end{array}$

$\begin{array}{rrrrrrrrrrrrrrrrrrrrrrrr}12 & 17 & 17 & 24 & 16 & 14 & 16 & 8 & 19 & 13 & 14 & 7 & 28 & 19 & 17 & 19 & 23 & 35 & 21 & 19 & 6 & 1 & \\ 12 & 24 & 13 & 25 & 16 & 5 & 8 & 7 & 36 & 20 & 15 & 5 & 8 & 9 & 10 & 21 & 29 & 35 & 25 & 20 & 16 & 2 & 4\end{array}$

$\begin{array}{llllllllllllllllllllllllll}10 & 24 & 23 & 13 & 7 & 10 & 15 & 20 & 20 & 13 & 10 & 30 & 28 & 19 & 32 & 22 & 28 & 9 & 13 & 10 & 5 & 2 & 1 & 1\end{array}$

$\begin{array}{rrrrrrrrrrrrrrrrrrrrrrrrrrrrrr}1 & 1 & 9 & 29 & 19 & 12 & 5 & 13 & 13 & 15 & 6 & 9 & 14 & 18 & 15 & 27 & 38 & 60 & 29 & 14 & 9 & 8 & 1 & & \\ 12 & 12 & 18 & 20 & 9 & 6 & 11 & 12 & 25 & 12 & 11 & 15 & 20 & 16 & 19 & 38 & 24 & 31 & 13 & 13 & 3 & 6 & 5 & 2 & 4 & 1\end{array}$

$\begin{array}{rrrrrrrrrrrrrrrrrrrrrrrrrrr}18 & 14 & 9 & 16 & 9 & 10 & 13 & 11 & 20 & 14 & 14 & 11 & 17 & 10 & 10 & 23 & 26 & 46 & 28 & 16 & 9 & 14 & 6 & 1 & \\ 2 & 4 & 8 & 7 & 9 & 7 & 8 & 3 & 8 & 7 & 7 & 8 & 16 & 12 & 17 & 28 & 23 & 50 & 25 & 19 & 10 & 15 & 8 & 1 & 1\end{array}$

$\begin{array}{rrrrrrrrrrrrrrrrrrrrrrrrr}23 & 29 & 14 & 8 & 7 & 9 & 7 & 8 & 3 & 8 & 7 & 7 & 8 & 16 & 12 & 17 & 28 & 23 & 5 n & 25 & 19 & 10 & 15 & 8 & 1 \\ 2 & 12 & 7 & 15 & 21 & 5 & 3 & 8 & 13 & 20 & 18 & 4 & 10 & 20 & 19 & 13 & 31 & 43 & 57 & 23 & 10 & 3 & 5 & 3 & 1\end{array}$

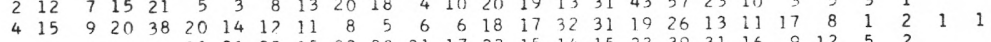

$\begin{array}{rrrrrrrrrrrrrrrrrrrrrrrrrrr}11 & 21 & 25 & 15 & 32 & 20 & 21 & 17 & 2 ? & 15 & 14 & 15 & 23 & 39 & 31 & 16 & 9 & 12 & 5 \\ 7 & 7 & 11 & 6 & 9 & 8 & 16 & 14 & 10 & 18 & 20 & 16 & 6 & 7 & 17 & 28 & 51 & 45 & 36 & 24 & 9 & 1 & 3 & 1\end{array}$

$\begin{array}{llllllllllllllllllllllllllll}7 & 21 & 15 & 10 & 10 & 4 & 8 & 8 & 6 & 11 & 14 & 16 & 12 & 6 & 9 & 20 & 27 & 34 & 24 & 38 & 24 & 13 & 17 & 6 & 5 & 1\end{array}$

$10 \ln \begin{array}{rrrrrrrrrrrrrrrrrrrrrrrrrr}2 & 21 & 22 & 7 & 8 & 16 & 9 & 4 & 15 & 4 & 9 & 13 & 12 & 18 & 39 & 20 & 20 & 55 & 15 & 28 & 10 & 4 & 5 & 5 & 4 & \\ & 20 & 4 & 5 & 14 & 11 & 4 & 16 & 15 & 27 & 29 & 13 & 6 & 8 & 12 & 24 & 25 & 16 & 23 & 23 & 16 & 9 & 11 & 4 & 2\end{array}$

$\begin{array}{rrrrrrrrrrrrrrrrrrrrrrr}13 & 10 & 20 & 12 & 13 & 13 & 11 & 7 & 21 & 15 & 6 & 9 & 16 & 10 & 25 & 27 & 35 & 40 & 33 & 10 & 7 & 7 & 4\end{array}$

$\begin{array}{rrrrrrrrrrrrrrrrrrrrrrrrrrrrrrrrrr}8 & 14 & 10 & 21 & 12 & 9 & 9 & 6 & 4 & 10 & 13 & 12 & 11 & 16 & 24 & 20 & 21 & 48 & 34 & 35 & 23 & 7 & 4 & 3 & & \\ 8 & 8 & 11 & 8 & 17 & 13 & 12 & 13 & 7 & 19 & 8 & 11 & 8 & 10 & 5 & 9 & 12 & 23 & 27 & 29 & 32 & 26 & 23 & 9 & 9 & 3 & 2 & 3\end{array}$

$\begin{array}{rrrrrrrrrrrrrrrrrrrrrrrrrrrrr}5 & 22 & 3 & 16 & 15 & 8 & 8 & 18 & 10 & 23 & 11 & 10 & 8 & 16 & 30 & 19 & 36 & 24 & 60 & 9 & 2 & 3 & 3 & 3 & 3 \\ & 5 & 12 & 13 & 12 & 15 & 9 & 9 & 18 & 30 & 10 & 12 & 15 & 15 & 22 & 19 & 32 & 31 & 35 & 16 & 11 & 12 & 6 & 5 & 1\end{array}$

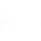

962

Pudding River near Mount Angel, Oreg.

STATION NUMBER $\quad 14-2010.00$

Summary for water years 1940-63

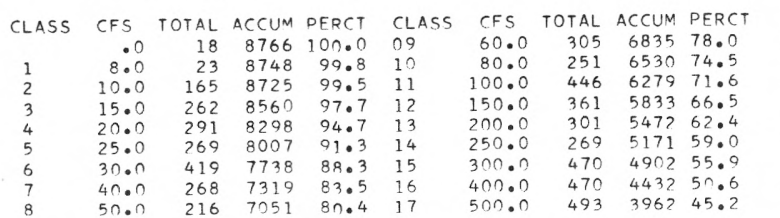

$\begin{array}{llll}405.0 & 470 & 4432 & 50.6\end{array}$

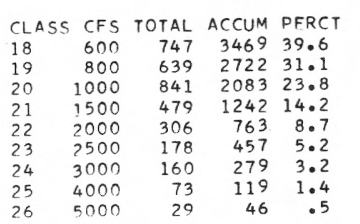

$\begin{array}{lrrrc}\text { CLASS CFS } & \text { TOTAL } & \text { ACCUM } & \text { PERCT } \\ 27 & 6000 & 11 & 17 & .2 \\ 28 & 8000 & 5 & 6 & : 1 \\ 29 & 10000 & 1 & 1 & : 0 \\ 30 & & & & .0 \\ 31 & & & & .0 \\ 32 & & & & .0 \\ 33 & & & & .0 \\ 34 & & & & .0\end{array}$ 
Pudding River near Mount Angel, Oreg.

STATION NUMRFR $14-201 n_{\bullet} \cap 0$ LAWEGT MEAN DISCHARGF, IN CFS, FOR THE FOLLOWING NUMRFR OF CONSFCUTIVF DAYC IN YFAR RFGINNING ADRIL 1

\begin{tabular}{|c|c|c|c|c|c|c|c|c|c|c|}
\hline 1 & 3 & 7 & 14 & 30 & 60 & 90 & $12 n$ & 150 & 183 & 274 \\
\hline $\begin{array}{l}10.0 \\
24.0\end{array}$ & $10 . n$ & 10.4 & 10.9 & $12 \cdot 3$ & 18.9 & 22.9 & 30.7 & 42.9 & $\begin{array}{l}104.0 \\
164.0\end{array}$ & 347.0 \\
\hline $\begin{array}{l}24.0 \\
19.0\end{array}$ & $\begin{array}{l}24.7 \\
19.7\end{array}$ & $\begin{array}{l}25.6 \\
19.9\end{array}$ & $\begin{array}{l}27 \cdot 5 \\
20.3\end{array}$ & $\begin{array}{l}32 \cdot 2 \\
21 \cdot 3\end{array}$ & $\begin{array}{l}48 \cdot 0 \\
24.8\end{array}$ & $\begin{array}{l}81.3 \\
30.8\end{array}$ & $\begin{array}{r}105 \cdot 0 \\
53.6\end{array}$ & $\begin{array}{l}125.0 \\
119.0\end{array}$ & $\begin{array}{l}164.0 \\
208.0\end{array}$ & $\begin{array}{l}441 . n \\
610 . ?\end{array}$ \\
\hline 29.0 & 30.0 & 30.6 & 31.3 & 35.4 & 43.3 & 51.0 & 86.9 & 133.0 & $203 . n$ & 347.0 \\
\hline 9.0 & 9.7 & 10.6 & 12.9 & 14.1 & 19.4 & 22.5 & 27.4 & 49.2 & 81.3 & 198.0 \\
\hline 20.0 & $2 n . n$ & 20.7 & 22.0 & 27.3 & 38.2 & 45.6 & 48.7 & $7 ? \cdot 2$ & $180^{\circ} n$ & $609 \cdot 0$ \\
\hline 16.0 & $16 \cdot n$ & 16.4 & 18.0 & 21.5 & 25.9 & 29.0 & $5 n \cdot 8$ & 71.1 & 115.0 & 505.0 \\
\hline $\begin{array}{l}22.0 \\
24.0\end{array}$ & $22 . n$ & 23.7 & 24.8 & 29.5 & 37.6 & 53.8 & 93.6 & 118.0 & $17^{2} \cdot n$ & $\begin{array}{l}544.0 \\
524.0\end{array}$ \\
\hline $\begin{array}{l}24.0 \\
16.0\end{array}$ & $25 \cdot n$ & 25.9 & 28.9 & 33.8 & 38.5 & 53.5 & $84 \cdot 8$ & $10^{2} \cdot n$ & $\begin{array}{r}178.0 \\
98.9\end{array}$ & $\begin{array}{l}524.0 \\
355.0\end{array}$ \\
\hline $\begin{array}{l}16.0 \\
18.0\end{array}$ & $17 . n$ & 17.7 & 18.1 & 19.6 & 25.7 & 32.1 & $47 \cdot 1$ & 70.4 & $\begin{array}{r}98.9 \\
250 . n\end{array}$ & $\begin{array}{l}355 \cdot 0 \\
765.0\end{array}$ \\
\hline $\begin{array}{r}18.0 \\
9.0\end{array}$ & 18.3 & 18.9 & $19 \cdot 0$ & 21.2 & $28 . ?$ & $4 n \cdot ?$ & $\begin{array}{l}86 \cdot 8 \\
39.9\end{array}$ & $\begin{array}{r}147.0 \\
89.3\end{array}$ & $\begin{array}{l}250 \cdot n \\
156 . n\end{array}$ & 496.0 \\
\hline $\begin{array}{r}9.0 \\
14.0\end{array}$ & 9.3 & $1 \cap .1$ & 11.4 & 13.7 & 15.6 & $\begin{array}{l}20.9 \\
24.8\end{array}$ & $28 \cdot 9$ & 43.1 & 83.3 & 216.0 \\
\hline $\begin{array}{l}14.0 \\
23.0\end{array}$ & $14 \cdot 0$ & 14.6 & 15.6 & 16.4 & 20.7 & 57.4 & $78 \cdot 5$ & 111.0 & $231 \cdot n$ & 629.0 \\
\hline $\begin{array}{l}23.0 \\
41.0\end{array}$ & 23.3 & 24.6 & 27.3 & $35 \cdot 8$ & $\begin{array}{l}43 \cdot 2 \\
69.2\end{array}$ & 76.5 & $116 \cdot 0$ & 166.0 & $195 . ?$ & 417.0 \\
\hline $\begin{array}{l}41.0 \\
14.0\end{array}$ & 42.3 & 46.1 & $49 \cdot 6$ & 55.8 & 40.7 & 59.2 & 96.3 & 211.0 & 330.0 & 927.0 \\
\hline $\begin{array}{l}14.0 \\
12.0\end{array}$ & 14.7 & $16 \cdot 7$ & $18 \cdot 3$ & $22 \cdot 8$ & 21.0 & 25.2 & 49.2 & 84.5 & 154.0 & 323.0 \\
\hline $\begin{array}{r}12.0 \\
9.4\end{array}$ & $\begin{array}{r}12.7 \\
9.7\end{array}$ & $\begin{array}{l}14.6 \\
11.0\end{array}$ & $\begin{array}{l}16.9 \\
11.9\end{array}$ & $\begin{array}{l}18.4 \\
13.2\end{array}$ & 23.2 & 32.2 & $48 \cdot 0$ & 68.0 & 111.0 & 399.0 \\
\hline $\begin{array}{l}9.4 \\
6.7\end{array}$ & $\begin{array}{l}9.7 \\
6.8\end{array}$ & $\begin{array}{r}11.0 \\
7.2\end{array}$ & 8.0 & 9.3 & 15.1 & 21.8 & 32.0 & 54.9 & 86.2 & 455.0 \\
\hline $\begin{array}{r}6.7 \\
12.0\end{array}$ & $\begin{array}{r}6.8 \\
12.7\end{array}$ & $\begin{array}{r}13.2 \\
13.3\end{array}$ & 13.6 & 16.2 & 34.6 & 60.5 & $96 \cdot 3$ & 150.0 & 193.0 & $313 . n$ \\
\hline $\begin{array}{l}12.0 \\
10.0\end{array}$ & 11.0 & 12.0 & 12.9 & 15.4 & 26.9 & 28.4 & 39.8 & 76.4 & 226.0 & 562.0 \\
\hline $\begin{array}{r}10.4 \\
5.4\end{array}$ & 6.0 & 7.4 & 9.4 & 10.2 & 21.8 & 25.4 & $41 \cdot 6$ & 74.4 & 114.0 & $\begin{array}{l}431.0 \\
493.0\end{array}$ \\
\hline 12.0 & 12.7 & 13.9 & 15.4 & 21.0 & 32.4 & 40.7 & 67.7 & 106.0 & 178.0 & 493.0 \\
\hline
\end{tabular}

Pudding River near Mount Angel, Oreg.

STATION NUMBER

$14-7010.00$

HICHEST MEAN DISCHARGF, IN CFS, FOR THE FOLLOWING NUMBER OF CONSECUTIVF DAYS IN YFAR FNDING SFPTFMRFR 30

\begin{tabular}{|c|c|c|c|c|c|c|c|c|c|c|c|}
\hline YEAR & 1 & 3 & 7 & 15 & 30 & 60 & 90 & 120 & 150 & 183 & 274 \\
\hline 1940 & 3490.0 & 3040.0 & 2670.0 & 2410.0 & 2140.0 & 1660.0 & 1400.0 & $1330 \cdot 0$ & 1190.0 & 1030.0 & 716.0 \\
\hline 1941 & 2180.0 & $1900 \cdot n$ & 1570.0 & $1290 . n$ & 1130.0 & 1070.0 & 969.0 & $841 \cdot 0$ & $74 ? \cdot 0$ & 663.0 & $522 . ?$ \\
\hline 1942 & 4450.0 & 4050.0 & $317 n_{0} n$ & 2230.0 & 1910.0 & $150 \cap .0$ & 1440.0 & $1250 \cdot ?$ & $109 n \cdot n$ & $956 \cdot n$ & $768 \cdot ?$ \\
\hline 1943 & 7760.0 & $58 n n \cdot n$ & $447 n \cdot n$ & $347 n \cdot n$ & $283 n \cdot n$ & 2500.0 & 2290.0 & $192 n \cdot 0$ & 1790.0 & $1590 \cdot ?$ & 1150.0 \\
\hline $\begin{array}{l}1944 \\
1945\end{array}$ & 1850.0 & $1690 \cdot n$ & $1370 \cdot n$ & $101 n \cdot 0$ & $951 \cdot n$ & $827 \cdot 0$ & $779 \cdot 0$ & $744 \cdot 0$ & $\begin{array}{r}732 \cdot 0 \\
1220 \cdot 0\end{array}$ & $\begin{array}{r}69 ? \cdot n \\
105 n \cdot 0\end{array}$ & $\begin{array}{l}545 . n \\
7600 ?\end{array}$ \\
\hline 1945 & 3000.0 & $277 n \cdot n$ & $2310 \cdot n$ & $2110 \cdot n$ & 1750.0 & 1470.0 & $138 n \cdot 0$ & $128 n \cdot 0$ & $\begin{array}{l}122 n \cdot 0 \\
158 n \cdot 0\end{array}$ & ח & $973 . n$ \\
\hline 1946 & $4800 \bullet 0$ & $4120 . n$ & 3330.0 & $2920 \cdot 0$ & 2130.0 & 1940.0 & 1820.0 & $1740 \cdot 0$ & 1300.0 & $117 n .0$ & 854.0 \\
\hline $\begin{array}{l}1947 \\
1948\end{array}$ & $\begin{array}{l}8750.0 \\
6850.0\end{array}$ & $\begin{array}{l}6490 \cdot n \\
5890 . n\end{array}$ & $\begin{array}{l}4800 \cdot 0 \\
431 \cap .0\end{array}$ & $\begin{array}{l}308 n \cdot 0 \\
3190.0\end{array}$ & $\begin{array}{l}2620 \bullet 0 \\
2120 \bullet 0\end{array}$ & $\begin{array}{l}1710.0 \\
1610.0\end{array}$ & $\begin{array}{l}1620.0 \\
1630.0\end{array}$ & $\begin{array}{l}142 n \bullet 0 \\
1390 \bullet 0\end{array}$ & 1400.0 & $138 \cap . n$ & $110 n$ \\
\hline 1949 & 10700.0 & $7490 \cdot n$ & 6500.0 & $4600 \cdot 0$ & $304 n \cdot 0$ & 1960.0 & 1850.0 & $1690 \cdot 0$ & 1520.0 & 1380.0 & $99 k \cdot 0$ \\
\hline 1950 & 5300.0 & $4430 \cdot 0$ & 4070.0 & 3060.0 & $272 n .0$ & 2620.0 & 2250.0 & $1980 \cdot 0$ & $178 n \cdot 0$ & $158 \cap \cdot 0$ & $1120 \cdot n$ \\
\hline 1951 & 7030.0 & 5930.0 & 4510.0 & 3580.0 & 2710.0 & $220 n \cdot 0$ & 2280.0 & $212 n \cdot 0$ & 1940.0 & $172 n \cdot n$ & 1250.0 \\
\hline 1952 & 5160.0 & $4830 \cdot n$ & $4160 \cdot 0$ & $2880 \cdot n$ & 2000.0 & $169 n .0$ & 1650.0 & $1500 \cdot 0$ & 1420.0 & $1290 \cdot 0$ & 970.0 \\
\hline 1953 & $85 n 0.0$ & $7160 . n$ & $555 n \cdot n$ & 4220.0 & 3420.0 & 2450.0 & 2040.0 & $169 n \cdot 0$ & 1490.0 & $1330 \cdot 0$ & $945 \cdot 0$ \\
\hline 1954 & $522 n \cdot n$ & $488 \cap \cdot n$ & $398 n \cdot n$ & 3120.0 & $279 n .0$ & $228 \cap . n$ & $216 n \cdot 0$ & $1960 \cdot 0$ & $176 n \cdot ?$ & 1520.0 & 11100 \\
\hline 1955 & 4120.0 & $36 n n \cdot n$ & $265 n . n$ & $206 n \cdot n$ & 1820.0 & $144 n \cdot n$ & $12 n n \cdot 0$ & $118 \cap \cdot 0$ & 1160.0 & 1110.0 & 854.0 \\
\hline 1956 & 6870.0 & $5670 \cdot n$ & $467 n \cdot n$ & $3550 . n$ & 3470.0 & $3 \cap 20.0$ & 2550.0 & $224 n \cdot 0$ & $205 n .0$ & $1860 \cdot 0$ & 1380.0 \\
\hline 1957 & $4580 \cdot 0$ & $437 n \cdot n$ & $391 \cap \cdot n$ & $298 n \cdot n$ & 2430.0 & $168 \cap .0$ & 1350.0 & $127 n \bullet 0$ & 1110.0 & $1 \cap n \cap \cdot n$ & 747.0 \\
\hline 1958 & 5210.0 & $4820 \bullet n$ & 3890.0 & $3120 \cdot n$ & 2340.0 & 2310.0 & 1980.0 & $1650 \cdot 0$ & 1550.0 & 1340.0 & $942 \cdot n$ \\
\hline 1959 & $522^{\prime} 0.0$ & $4800 \cdot 0$ & $400 n \cdot n$ & $2840 \cdot n$ & 2510.0 & 2030.0 & $172 n .0$ & $162 n \cdot 0$ & $152 n \cdot 0$ & 1360.0 & \\
\hline 1960 & 3620.0 & 3320.0 & $279 n \cdot n$ & $217 n \cdot 0$ & 1580.0 & $150 \cap .0$ & 1330.0 & 1250.0 & $115 n \cdot 0$ & 1040.0 & $8 \cap 4 . n$ \\
\hline 1961 & 8640.0 & 7480.0 & 5570.0 & 4010.0 & 3110.0 & 2490.0 & 2060.0 & $1930 \cdot 0$ & $1800 \cdot 0$ & 1620.0 & 1160.0 \\
\hline 1962 & 5500.0 & 4860.0 & 3670.0 & 2590.0 & 1820.0 & 1360.0 & 1160.0 & $1230 \cdot 0$ & 1170.0 & 1110.0 & 818.0 \\
\hline 1963 & 5790.0 & 4870.0 & 3540.0 & 2880.0 & 2080.0 & 1690.0 & 1370.0 & $1280 \cdot 0$ & 1260.0 & 1290.0 & 953.0 \\
\hline
\end{tabular}


Butte Creek at Monitor, Oreg.

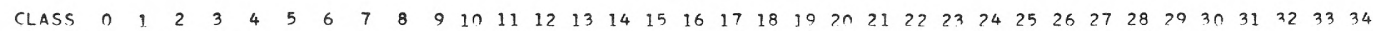

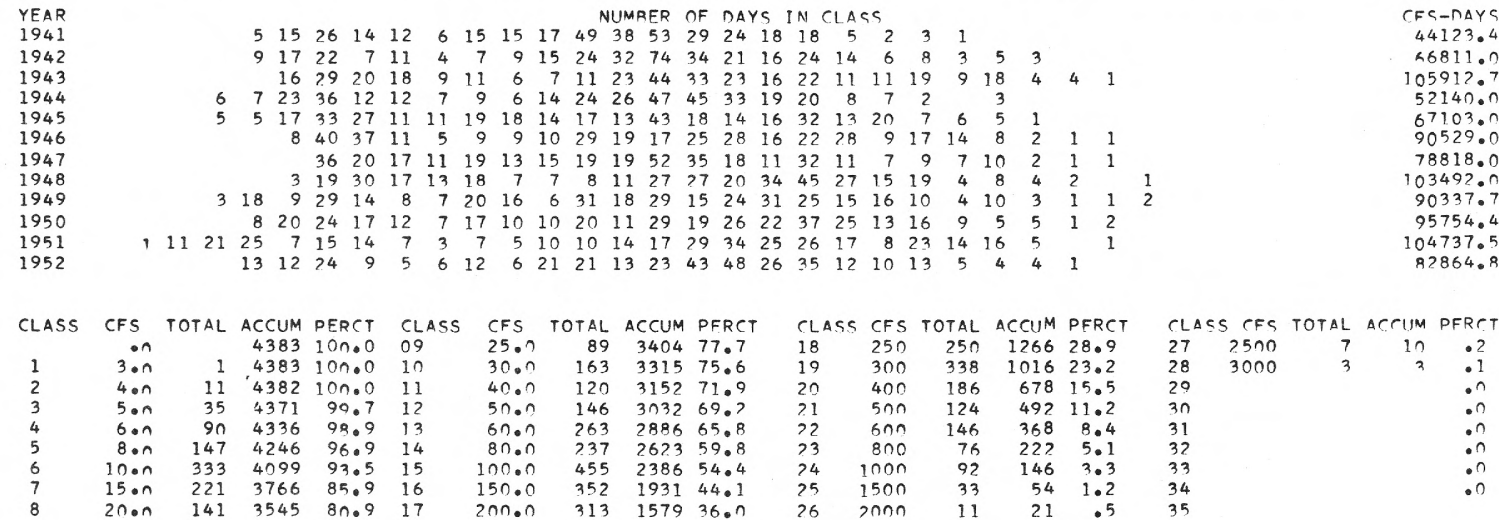

Butte Creek at Monitor. Dreg.

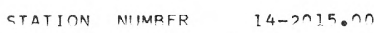

LOWEST MEAN DISCHARGF, IN CFS, FOR THE FOLLOWING NUMRFP OF GONGFCUTIVF DAYS IN YFAR RFGINNING APRTL 1

\begin{tabular}{|c|c|c|c|c|c|c|c|c|c|c|c|}
\hline YEAR & 1 & 3 & 7 & 14 & 30 & $6 \cap$ & $9 n$ & 120 & 150 & 183 & 274 \\
\hline 1941 & 7.3 & 7.4 & 8.2 & 8.7 & 9.5 & $1.5 . ?$ & 26.5 & 34.6 & 42.6 & 56.1 & 147. \\
\hline 1942 & 6.8 & $7 . n$ & 7.3 & 7.7 & 8.4 & 9.8 & 12.4 & $2 n \cdot 6$ & 40.4 & 64.4 & 196. \\
\hline 1943 & 8.2 & 8.7 & 9.7 & 10.4 & 11.2 & 15.4 & 18.7 & 35.6 & 50.3 & 78.7 & 131. \\
\hline 1944 & 5.0 & 5.0 & 5.1 & 6.3 & 7.3 & 9.8 & 11.3 & $13 \cdot 1$ & $21 . ?$ & 32.3 & 68 \\
\hline 1945 & 5.0 & 5.0 & 5.3 & 6.4 & 8.4 & 12.5 & 17.8 & 18.4 & 25.7 & $6^{5} \cdot 1$ & 214 \\
\hline 1946 & $8 . n$ & 8. 0 & 8.3 & 9.4 & 11.9 & 13.8 & $15 . ?$ & $74 . ?$ & 32.7 & 5). 0 & 172 \\
\hline 1947 & $1 n \cdot n$ & 10.7 & 11.0 & $12 . ?$ & 14.5 & 19.0 & 23.2 & 37.5 & 45.9 & 62.6 & 185 \\
\hline 1948 & 9.0 & o. & 10.1 & 10.5 & 13.6 & 16.4 & 20.4 & 20.2 & 37.0 & $6 ? .4$ & 154. \\
\hline 1949 & 5.9 & 5.0 & 6.? & 6.6 & 7.6 & 10.4 & 13.5 & 19.9 & 27.3 & 39.6 & $13 n$. \\
\hline 1950 & 6.8 & 6.0 & 7.3 & 7.8 & 8.8 & 11.7 & 15.8 & 31.4 & 51.7 & 95.9 & 235 \\
\hline 1951 & 3.6 & 3.9 & 3.9 & 4.7 & 6.1 & 6.4 & 8.5 & 15.4 & $3 ! \cdot 1$ & 50.5 & 147 \\
\hline
\end{tabular}

Butte Creek at Nonitor, Oreg.

CTATTON NUMRER $14-2015.00$

HICHECT MEAN DISCHARGE, IN CFS, FOR THF FOLLOWING NUNBER OF CONSECUTIVF DAYG IN YEAR ENDING SEPTFMBFR 30

\begin{tabular}{|c|c|c|c|c|c|c|c|c|c|c|c|}
\hline YEAR & 1 & 2 & 7 & 15 & $3 n$ & 60 & an & 120 & $15 n$ & 182 & 274 \\
\hline 1941 & $978 . \cap$ & $647 . n$ & $461 . n$ & 367.0 & $3 \cap 5.0$ & $296 . n$ & 270.0 & $234 . n$ & $2 n 7.0$ & $185 . n$ & $153 . ?$ \\
\hline 1942 & 1690.0 & $125 n \cdot n$ & $9 \cap 4 . \cap$ & $654 . n$ & 613.0 & $483 . n$ & 454.0 & $388 . n$ & 337.0 & 295.0 & 237. \\
\hline 1943 & 2760.0 & 1890.0 & 1590.0 & 1170.0 & $894 . ?$ & 785.0 & $7>5.0$ & 609.0 & 583.0 & 520.0 & $381 . n$ \\
\hline 1944 & 1270.0 & $974 . n$ & $6 \cap 8.0$ & 440.0 & 306.0 & 271.0 & 242.0 & 250.0 & 237.0 & 234.0 & 186. \\
\hline 1945 & 1580.0 & $1090 . n$ & 861.0 & $668 . n$ & 538.0 & $45 n . n$ & 431.0 & 415.0 & 384.0 & 328.1 & 239. \\
\hline 1946 & 2660.0 & $1850 . n$ & 1250.0 & $1020 . n$ & 718.0 & 646.0 & 597.0 & 573.0 & 517.0 & 457.0 & 326. \\
\hline 1947 & 2820.0 & $2200 \cdot n$ & 1570.0 & 939.0 & 859.0 & 539.0 & 537.0 & 466.0 & 423.0 & 380.0 & 281. \\
\hline 1948 & 3840.0 & 2500.0 & 1597.0 & 1080.0 & 697.0 & 538.0 & 553.0 & $45 \pi \cdot 0$ & 469.0 & 453.0 & 371. \\
\hline 1949 & 3980.0 & $3100 . n$ & 2320.0 & 1570 ? & 1040.0 & 686.0 & 570.0 & $5] 7.0$ & 478.0 & 448.0 & 325 \\
\hline 1950 & 2710.0 & $236 n \cdot n$ & 1600.0 & 1030.0 & 826.0 & $744 . ?$ & 651.0 & 582.0 & 529.0 & 474.0 & 344 \\
\hline 1951 & 2590.0 & 1950.0 & $136 n .0$ & 1120.0 & 883.0 & 548.0 & 680.0 & $640 . n$ & 586.0 & 524.0 & 370 \\
\hline 1952 & 2020.0 & $182 n \cdot n$ & $15 n n .0$ & 938.0 & 607.0 & 489 ? & 483.0 & $4>0.0$ & $4] \cap .0$ & $37 x .0$ & 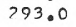 \\
\hline
\end{tabular}


CLASS $0 \begin{array}{lllllllllllllllllllllllllllllllllll} & 0 & 2 & 3 & 4 & 5 & 6 & 7 & 8 & 9 & 10 & 11 & 12 & 13 & 14 & 15 & 16 & 17 & 18 & 19 & 20 & 21 & 22 & 23 & 24 & 25 & 26 & 27 & 28 & 29 & 30 & 31 & 32 & 33 & 34\end{array}$

YEAR
1929
1930
1931
1932
1933
1934
1935
1936
1937
1938
1939
1940
1941
1942
1943
1944
1945
1946
1947
1948
1949
1950
1951
1952
1953
1954
1955
1956
1957
1958
1959
1960
1961
1962
1963 NUMBER OF DAYS IN CLASS

$\begin{array}{lllllllllllllllllll}19 & 38 & 11 & 25 & 38 & 3 & 5 & 22 & 21 & 15 & 31 & 39 & 50 & 25 & 7 & 1 & 7 & 8\end{array}$

$\begin{array}{rrrrrrrrrrrrrrrrrrrrrrrr}1 & 10 & 74 & 45 & 26 & 6 & 8 & 6 & 4 & 5 & 25 & 56 & 17 & 26 & 14 & 5 & 5 & 12 & 19 & 1 & & & \\ 2 & 10 & 34 & 26 & 50 & 17 & 12 & 12 & 20 & 24 & 24 & 32 & 14 & 40 & 21 & 10 & 5 & 3 & 2 & 2 & 2 & 1 & 2\end{array}$

$\begin{array}{lllllrrrrrrrrrrrrrr}12 & 54 & 18 & 25 & 13 & 5 & 8 & 6 & 14 & 11 & 16 & 8 & 39 & 35 & 31 & 16 & 22 & 25 & 8\end{array}$

$\begin{array}{lllllllllllllllllllll}12 & 54 & 18 & 25 & 13 & 5 & 8 & 6 & 14 & 11 & 16 & 8 & 39 & 35 & 31 & 16 & 22 & 25 & 8\end{array}$

$\begin{array}{rrrrrrrrrrrrrrrrrrrrrrr}2 & 10 & 8 & 24 & 27 & 15 & 12 & 7 & 14 & 6 & 12 & 15 & 25 & 52 & 44 & 17 & 23 & 29 & 15 & 3 & 5 & & \\ 2 & 22 & 26 & 28 & 30 & 24 & 6 & 6 & 28 & 13 & 23 & 36 & 17 & 19 & 21 & 7 & 8 & 17 & 11 & 8 & 7 & 4 & 2\end{array}$

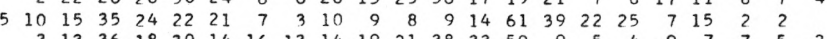

$\begin{array}{lllllllllllllllllrrrrrrr}3 & 13 & 36 & 18 & 30 & 14 & 16 & 13 & 14 & 19 & 21 & 38 & 33 & 50 & 9 & 5 & 4 & 9 & 7 & 7 & 5 & 2\end{array}$

$\begin{array}{llllllllllllllllllllllllll}13 & 30 & 26 & 6 & 29 & 10 & 18 & 7 & 11 & 11 & 6 & 11 & 15 & 26 & 36 & 18 & 20 & 25 & 23 & 14 & 6 & 1 & 2 & 1\end{array}$

$\begin{array}{rrrrrrrrrrrrrrrrrrrrr}21 & 21 & 28 & 18 & 19 & 21 & 28 & 11 & 14 & 10 & 22 & 25 & 20 & 40 & 24 & 16 & 11 & 11 & 4 & 1 \\ 25 & 24 & 36 & 20 & 43 & 16 & 18 & 11 & 9 & 4 & 3 & 25 & 16 & 38 & 17 & 14 & 7 & 11 & 25 & 4\end{array}$

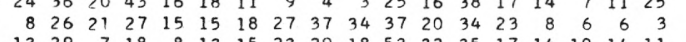

$\begin{array}{rrrrrrrrrrrrrrrrrrrrrrr}13 & 29 & 7 & 18 & 8 & 13 & 15 & 23 & 29 & 18 & 53 & 32 & 35 & 17 & 14 & 10 & 14 & 11 & 5 & 1 & \\ 6 & 20 & 34 & 36 & 10 & 6 & 6 & 8 & 14 & 20 & 40 & 21 & 32 & 19 & 5 & 10 & 17 & 19 & 10 & 24 & 6 & 2\end{array}$

$\begin{array}{llllllllllllllllllll}20 & 21 & 28 & 22 & 16 & 8 & 11 & 12 & 15 & 20 & 18 & 30 & 41 & 62 & 25 & 9 & 5 & 3\end{array}$

$\begin{array}{rrrrrrrrrrrrrrrrrrrrr}5 & 49 & 28 & 28 & 21 & 12 & 11 & 19 & 14 & 16 & 23 & 16 & 43 & 18 & 15 & 12 & 26 & 9 & & \\ 7 & 37 & 29 & 28 & 9 & 22 & 21 & 22 & 8 & 4 & 13 & 9 & 38 & 29 & 14 & 14 & 25 & 19 & 15 & 2\end{array}$

$\begin{array}{rrrrrrrrrrrrrrrrrrrrrr} & 25 & 28 & 34 & 23 & 10 & 11 & 21 & 11 & 14 & 35 & 37 & 37 & 24 & 13 & 7 & 16 & 17 & 2 & 3 & 1 & 1 \\ 15 & 3 & 34 & 43 & 16 & 7 & 5 & 12 & 8 & 7 & 20 & 19 & 38 & 55 & 25 & 26 & 19 & 16 & 7 & 3 & 1 & 2 \\ 15 & 34 & 21 & 15 & 31 & 14 & 7 & 11 & 21 & 14 & 17 & 21 & 44 & 26 & 13 & 14 & 14 & 11 & 8 & 7 & 3 & 3\end{array}$

$\begin{array}{rrrrrrrrrrrrrrrrrrrr}17 & 29 & 16 & 21 & 25 & 11 & 9 & 16 & 18 & 8 & 15 & 15 & 27 & 37 & 19 & 13 & 23 & 21 & 13 & 12\end{array}$

$\begin{array}{lllllllrllllllllllllllll}17 & 31 & 27 & 13 & 17 & 6 & 6 & 5 & 10 & 10 & 11 & 14 & 21 & 32 & 19 & 23 & 14 & 23 & 34 & 15 & 13 & 3\end{array}$

$\begin{array}{lllllrrrrrrrrrrrrrrrrr}18 & 38 & 45 & 28 & 15 & 5 & 5 & 3 & 8 & 12 & 16 & 41 & 41 & 21 & 10 & 10 & 9 & 15 & 14 & 7 & 1 & 3\end{array}$

$\begin{array}{rrrrrrrrrrrrrrrrrrrrrrr}18 & 38 & 45 & 28 & 15 & 5 & 5 & 3 & 8 & 12 & 16 & 41 & 41 & 21 & 10 & 10 & 9 & 15 & 14 & 7 & 1 \\ & & 8 & 46 & 16 & 16 & 19 & 23 & 24 & 25 & 18 & 13 & 24 & 26 & 19 & 15 & 29 & 19 & 10 & 14 & 1\end{array}$

$\begin{array}{llllllrlllllllllllllllll}24 & 29 & 15 & 17 & 10 & 3 & 3 & 24 & 17 & 5 & 14 & 19 & 35 & 27 & 7 & 13 & 30 & 31 & 21 & 14 & 6 & 2\end{array}$

$\begin{array}{rrrrrrrrrrrrrrrrrrrrrrrr}15 & 25 & 15 & 23 & 17 & 11 & 6 & 3 & 4 & 18 & 16 & 27 & 31 & 28 & 61 & 21 & 12 & 6 & 7 & 7 & 7 & 5\end{array}$

$\begin{array}{llllllllrlllllllllllllll}19 & 15 & 18 & 10 & 11 & 23 & 13 & 8 & 13 & 10 & 10 & 11 & 15 & 24 & 41 & 42 & 18 & 13 & 25 & 15 & 6 & 5\end{array}$

$\begin{array}{llllllllllllllllllllllll}2 & 19 & 12 & 2 & 31 & 12 & 9 & 12 & 6 & 9 & 15 & 22 & 17 & 19 & 22 & 54 & 43 & 15 & 15 & 18 & 9 & 3\end{array}$

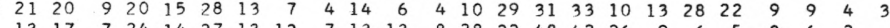

$\begin{array}{rrrrrrrrrrrrrrrrrrrrrrrrr}3 & 17 & 7 & 24 & 14 & 27 & 13 & 12 & 7 & 13 & 13 & 8 & 28 & 22 & 48 & 42 & 26 & 9 & 6 & 5 & 3 & 6 & 2 \\ 16 & 16 & 15 & 11 & 13 & 20 & 25 & 14 & 14 & 18 & 7 & 26 & 18 & 53 & 29 & 14 & 8 & 13 & 14 & 12 & 7 & 2\end{array}$

CFS-DAYS

279557.0

301042.0

497117.0

14501.0

437500.0

346892.0

375130.0

600121.0

295771.0

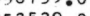

253529.0

370354.0

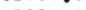

258950.0

450593.0

4750.0

406750.0

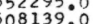

562651.0

62651.0

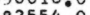

483790.0

568566.0

404707.0

724852.0

461910.0

477251.0

477251.0

402973.0

584703.0

$398034 \cdot 0$
480098.0

Pudding River at Aurora, Oreg.

Summary for water years 1929-63

STATION NUMBER 14-2020.00

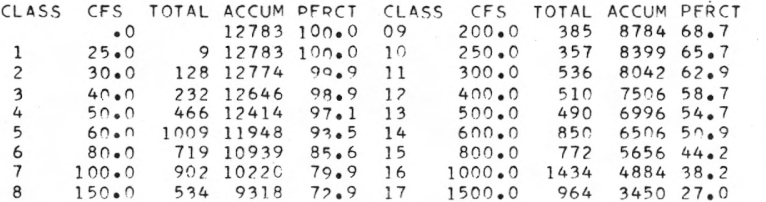

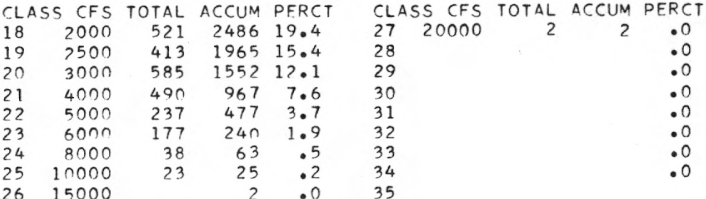


Pudding River at Anrora, Oreg.

STATION NUMBER $\quad 14-2020.00$ LOWEST MEAN DISCHARGE, IN CFS, FOR THE FOLLOWING NUMBER OF CONSECUTIVE DAYS IN YEAR BEGINNING APRIL 1

\begin{tabular}{|c|c|c|c|c|c|c|c|c|c|c|c|}
\hline YEAR & 1 & 3 & 7 & 14 & 30 & 60 & 90 & 120 & 150 & 183 & 274 \\
\hline $\begin{array}{l}1930 \\
1931\end{array}$ & 49.0 & $\begin{array}{l}57.7 \\
50.3\end{array}$ & $\begin{array}{l}58.9 \\
50.7\end{array}$ & $\begin{array}{l}60.4 \\
53.4\end{array}$ & $\begin{array}{l}64.8 \\
62.4\end{array}$ & $\begin{array}{l}74.9 \\
70.9\end{array}$ & $\begin{array}{l}83.9 \\
77.2\end{array}$ & $120 \cdot 0$ & 157.0 & 193.0 & $\begin{array}{l}365.0 \\
827.0\end{array}$ \\
\hline 1932 & 48.0 & $49 \cdot 3$ & 49.4 & 51.6 & 55.7 & 61.7 & 72.2 & $94 \cdot 7$ & 139.0 & 234.0 & 767.0 \\
\hline $\begin{array}{l}1933 \\
1934\end{array}$ & 72.0 & 73.0 & 74.3 & 79.7 & 89.4 & 106.0 & 153.0 & $171 \cdot 0$ & 298.0 & 418.0 & 1140.0 \\
\hline $\begin{array}{l}1934 \\
1935\end{array}$ & 47.0 & 48.0 & 50.4 & 51.7 & 55.6 & 62.6 & 69.6 & $81 \cdot 8$ & 116.0 & 231.0 & 806.0 \\
\hline $\begin{array}{l}1935 \\
1936\end{array}$ & 37.0 & 38.2 & 39.1 & 43.5 & 50.9 & 65.7 & 73.0 & $92 \cdot 2$ & $106 \cdot 0$ & 147.0 & 364.0 \\
\hline $\begin{array}{l}1936 \\
1937\end{array}$ & 53.0 & 54.0 & $57 \cdot 1$ & 59.4 & 64.5 & 68.2 & 70.1 & $72 \cdot 8$ & $87 \cdot 1$ & 144.0 & 343.0 \\
\hline $\begin{array}{l}1938 \\
1939\end{array}$ & $\begin{array}{l}43.0 \\
41.0\end{array}$ & $\begin{array}{l}43.3 \\
42.0\end{array}$ & $45 \cdot 4$ & $48 \cdot 6$ & $\begin{array}{l}52.1 \\
50.6\end{array}$ & 57.6 & $64 \cdot 3$ & $\begin{array}{l}80.0 \\
90.5\end{array}$ & $\begin{array}{l}106 \cdot 0 \\
107.0\end{array}$ & $\begin{array}{l}194.0 \\
138.0\end{array}$ & $\begin{array}{l}547.0 \\
372.0\end{array}$ \\
\hline 1940 & $\begin{array}{l}41.0 \\
43.0\end{array}$ & $\begin{array}{l}42.0 \\
44.7\end{array}$ & $\begin{array}{l}44.6 \\
45.6\end{array}$ & $\begin{array}{l}46 \cdot 4 \\
46.2\end{array}$ & $\begin{array}{l}50.6 \\
47.9\end{array}$ & $\begin{array}{l}58.0 \\
54.9\end{array}$ & $\begin{array}{l}70.1 \\
61.5\end{array}$ & $\begin{array}{l}90.3 \\
73.8\end{array}$ & 95.9 & 182.0 & $\begin{array}{l}372.0 \\
585.0\end{array}$ \\
\hline $\begin{array}{l}1941 \\
1942\end{array}$ & 54.0 & 55.0 & 56.6 & $60 \cdot 5$ & 65.6 & 88.1 & 130.0 & $165 \cdot 0$ & 198.0 & 257.0 & 742.0 \\
\hline 1944 & 40.0 & 40.7 & 41.4 & $\begin{array}{l}80.9 \\
44.4\end{array}$ & 47.3 & 55.0 & 61.1 & 67.9 & $\begin{array}{l}248.0 \\
101.0\end{array}$ & 146.0 & 322.0 \\
\hline 1945 & 54.0 & 55.0 & 57.9 & 64.1 & 70.0 & 85.5 & 104.0 & $108 \cdot 0$ & 140.0 & 330.0 & 994.0 \\
\hline 1946 & 56.0 & 56.3 & 57.3 & 61.3 & 68.5 & 76.2 & 82.5 & 118.0 & 153.0 & 229.0 & 852.0 \\
\hline 1947 & 71.0 & 73.0 & 75.7 & 77.4 & 86.1 & 95.4 & 116.0 & 174.0 & 213.0 & 300.0 & 930.0 \\
\hline 1948 & 78.0 & 79.7 & 80.9 & 83.9 & 93.2 & 102.0 & 119.0 & 167.0 & 199.0 & $315 \cdot 0$ & 938.0 \\
\hline 1949 & 57.0 & 57.3 & 57.4 & 58.1 & 61.4 & 72.2 & 83.8 & $110 \cdot 0$ & $140 \cdot 0$ & 187.0 & 620.0 \\
\hline 1950 & 54.0 & 55.0 & 55.6 & 56.6 & 63.1 & 72.2 & 93.1 & $175 \cdot 0$ & $264 \cdot 0$ & 423.0 & 1370.0 \\
\hline 1951 & 42.0 & $42 \cdot 3$ & 43.7 & 47.4 & 48.9 & 54.9 & 63.9 & 93.7 & 170.0 & 276.0 & $\begin{array}{l}848.0 \\
385.0\end{array}$ \\
\hline 1952 & 53.0 & 53.3 & 54.3 & 55.1 & 56.9 & 62.3 & 65.4 & $75 \cdot 1$ & $94 \cdot 2$ & 163.0 & 385.0 \\
\hline 1953 & 57.0 & 57.7 & 59.0 & 63.6 & 77.5 & 93.4 & 117.0 & 169.0 & 227.0 & 421.0 & 1160.0 \\
\hline 1954 & 84.0 & 86.3 & 93.9 & 98.0 & 110.0 & 133.0 & 146.0 & $212 \cdot 0$ & 287.0 & 333.0 & 716.0 \\
\hline 1955 & 48.0 & 49.3 & 50.0 & 51.7 & 55.7 & 88.0 & 123.0 & $181 \cdot 0$ & 330.0 & 515.0 & 1650.0 \\
\hline 1956 & 54.0 & 54.7 & 55.7 & 56.9 & 62.4 & 71.2 & 74.4 & 107.0 & 165.0 & 270.0 & 546.0 \\
\hline 1957 & 34.0 & 35.0 & 35.1 & 36.9 & 39.0 & 49.6 & 59.2 & $82 \cdot 4$ & 114.0 & 183.0 & 664.0 \\
\hline 1958 & 28.0 & 28.3 & 29.4 & 32.2 & 33.8 & 39.3 & 50.3 & 67.1 & 106.0 & 160.0 & 755.0 \\
\hline 1959 & 32.0 & 33.0 & 35.1 & 36.4 & 38.8 & 61.4 & 105.0 & $162 \cdot 0$ & 244.0 & 304.0 & 508.0 \\
\hline 1960 & 29.0 & 29.3 & 30.6 & 32.6 & 36.9 & 59.5 & 62.8 & 80.8 & 137.0 & 392.0 & 999.0 \\
\hline 1961 & 26.0 & 26.3 & 29.7 & 31.0 & 35.1 & 52.0 & 60.2 & 84.4 & 131.0 & 191.0 & 731.0 \\
\hline 1962 & 30.0 & 30.7 & 33.0 & 41.4 & 50.1 & 64.4 & 78.0 & 122.0 & 192.0 & 312.0 & 863.0 \\
\hline
\end{tabular}

Pudding River at Aurora, Oreg.

STATION NUMBER $\quad 14-2020.00$ HIGHEST MEAN DISCHARGE, IN CFS, FOR THE FOLLOWING NUMBER OF CONSECUTIVE DAYS IN YEAR ENDING SEPTEMBER 30

\begin{tabular}{|c|c|c|c|c|c|c|c|c|c|c|c|}
\hline $\begin{array}{l}\text { YEAR } \\
1929\end{array}$ & 4970.0 & $4870^{3} \cdot 0$ & $4710^{7} .0$ & $\begin{array}{c}15 \\
3720.0\end{array}$ & $\begin{array}{c}30 \\
2330.0\end{array}$ & $\begin{array}{c}60 \\
1750.0\end{array}$ & $\begin{array}{c}90 \\
1490.0\end{array}$ & $\begin{array}{c}120 \\
1560.0\end{array}$ & $\begin{array}{l}150 \\
1460.0\end{array}$ & $\begin{array}{c}183 \\
1330.0\end{array}$ & $\begin{array}{l}274 \\
993.0\end{array}$ \\
\hline 1930 & 5140.0 & 4760.0 & 4520.0 & 4340.0 & 3750.0 & 2400.0 & 2310.0 & $1960 \cdot 0$ & 1710.0 & 1540.0 & $\begin{array}{l}1070.0 \\
1000.0\end{array}$ \\
\hline $\begin{array}{l}1931 \\
1932\end{array}$ & $\begin{array}{r}10600.0 \\
5440.0\end{array}$ & $\begin{array}{r}10100.0 \\
5320.0\end{array}$ & $\begin{array}{l}7970.0 \\
4740.0\end{array}$ & $\begin{array}{l}5410.0 \\
4500.0\end{array}$ & $\begin{array}{l}3790.0 \\
3760.0\end{array}$ & $\begin{array}{l}2490.0 \\
3240.0\end{array}$ & $\begin{array}{l}2040.0 \\
2920.0\end{array}$ & $\begin{array}{l}1800 \cdot 0 \\
2910.0\end{array}$ & $\begin{array}{l}1560.0 \\
2730.0\end{array}$ & $\begin{array}{l}1370.0 \\
2500.0\end{array}$ & $\begin{array}{l}1000.0 \\
1790.0\end{array}$ \\
\hline $\begin{array}{l}1935 \\
1936 \\
1937\end{array}$ & $\begin{array}{r}7140.0 \\
8690.0 \\
7790.0 \\
23900.0\end{array}$ & $\begin{array}{l}6550.0 \\
8350.0 \\
7360.0\end{array}$ & $\begin{array}{r}5600.0 \\
7490.0 \\
6490.0\end{array}$ & $\begin{array}{l}5070.0 \\
6250.0 \\
4650.0\end{array}$ & $\begin{array}{l}4480.0 \\
3700.0\end{array}$ & $\begin{array}{l}3110.0 \\
2830.0\end{array}$ & $\begin{array}{l}2540.0 \\
2920.0\end{array}$ & $\begin{array}{l}2180 \cdot 0 \\
2470 \cdot 0 \\
3510 \cdot 0\end{array}$ & $\begin{array}{l}1920.0 \\
2130.0 \\
3420.0\end{array}$ & $\begin{array}{l}1690.0 \\
1860.0 \\
3070.0\end{array}$ & $\begin{array}{l}1320.0 \\
1240.0 \\
1340.0 \\
2170.0\end{array}$ \\
\hline $\begin{array}{l}1938 \\
1939 \\
1940 \\
1941\end{array}$ & $\begin{array}{r}23900.0 \\
5020.0 \\
5380.0 \\
4180.0\end{array}$ & $\begin{array}{r}16700.0 \\
4800.0 \\
5160.0 \\
3870.0\end{array}$ & $\begin{array}{r}11300.0 \\
4310.0 \\
4780.0 \\
3140.0\end{array}$ & $\begin{array}{l}7220.0 \\
3540.0 \\
4570.0 \\
2440.0\end{array}$ & $\begin{array}{l}5340.0 \\
2690.0 \\
4190.0 \\
2070.0\end{array}$ & $\begin{array}{l}4180.0 \\
2260.0 \\
3180.0 \\
1920.0\end{array}$ & $\begin{array}{l}3860.0 \\
2080.0 \\
2580.0 \\
1680.0\end{array}$ & $\begin{array}{l}3510 \cdot 0 \\
1850 \cdot 0 \\
2450 \cdot 0 \\
1450 \cdot 0\end{array}$ & $\begin{array}{l}3420 \cdot 0 \\
1660 \cdot 0 \\
2170 \cdot 0 \\
1270 \cdot 0\end{array}$ & $\begin{array}{l}1470.0 \\
1850.0 \\
1130.0\end{array}$ & $\begin{array}{r}1060.0 \\
1290.0 \\
883.0\end{array}$ \\
\hline 1942 & .6390 .0 & 5910.0 & 5440.0 & 4090.0 & $\begin{array}{l}3520.0 \\
5430.0\end{array}$ & $\begin{array}{l}2680.0 \\
4990.0\end{array}$ & 2530.0 & $2200 \cdot 0$ & 1910.0 & $\begin{array}{l}1670.0 \\
3170.0\end{array}$ & $\begin{array}{l}1310.0 \\
2280.0\end{array}$ \\
\hline $\begin{array}{l}1943 \\
1944\end{array}$ & $\begin{array}{r}11200.0 \\
3190.0\end{array}$ & $\begin{array}{r}10100.0 \\
3020.0\end{array}$ & $\begin{array}{l}8460.0 \\
2390.0\end{array}$ & $\begin{array}{l}6710.0 \\
1830.0\end{array}$ & $\begin{array}{l}5430.0 \\
1720.0\end{array}$ & $\begin{array}{l}4990.0 \\
1470.0\end{array}$ & $\begin{array}{l}4660.0 \\
1360.0\end{array}$ & $\begin{array}{l}3860 \bullet 0 \\
1280 \bullet 0\end{array}$ & $\begin{array}{l}3620.0 \\
1250.0\end{array}$ & 1180.0 & $\begin{array}{r}2280.0 \\
924.0\end{array}$ \\
\hline 1945 & 4480.0 & $4440 \cdot 0$ & $4090 \cdot 0$ & 3650.0 & 2970.0 & 2520.0 & 2350.0 & $2120 \cdot 0$ & 2010.0 & 1730.0 & 1250.0 \\
\hline $\begin{array}{l}1946 \\
1947\end{array}$ & $\begin{array}{r}6510.0 \\
10000.0\end{array}$ & $\begin{array}{l}6080.0 \\
8820.0\end{array}$ & $\begin{array}{l}5570.0 \\
7150.0\end{array}$ & $\begin{array}{l}5170.0 \\
4970.0\end{array}$ & $\begin{array}{l}3760.0 \\
4220.0\end{array}$ & $\begin{array}{l}3390.0 \\
2800.0\end{array}$ & $\begin{array}{l}3220.0 \\
2730.0\end{array}$ & $\begin{array}{l}3130.0 \\
2420.0\end{array}$ & $\begin{array}{l}2830.0 \\
2230.0\end{array}$ & $\begin{array}{l}2470.0 \\
1980.0\end{array}$ & $\begin{array}{l}1740.0 \\
1450.0\end{array}$ \\
\hline $\begin{array}{l}1948 \\
1949\end{array}$ & $\begin{array}{l}12800.0 \\
21000.0\end{array}$ & $\begin{array}{l}11200.0 \\
16300.0\end{array}$ & $\begin{array}{r}8570.0 \\
12300.0\end{array}$ & $\begin{array}{l}6270.0 \\
8640.0\end{array}$ & $\begin{array}{l}4120.0 \\
5870.0\end{array}$ & $\begin{array}{l}3040.0 \\
3780.0\end{array}$ & $\begin{array}{l}2910.0 \\
3450.0\end{array}$ & $\begin{array}{l}2590 \cdot 0 \\
3140 \cdot 0\end{array}$ & $\begin{array}{l}2530.0 \\
2810.0\end{array}$ & $\begin{array}{l}2480.0 \\
2540.0\end{array}$ & $\begin{array}{l}1980.0 \\
1830.0\end{array}$ \\
\hline 1950 & 7980.0 & $7890 . ?$ & 7190.0 & 5520.0 & 4830.0 & 4760.0 & 4130.0 & $3640 \cdot 0$ & 3230.0 & 2840.0 & 2020.0 \\
\hline 1951 & 10200.0 & 8640.0 & 7440.0 & 6670.0 & 5360.0 & 4100.0 & 4390.0 & $4070 \cdot 0$ & 3790.0 & $\begin{array}{l}3320.0 \\
2290.0\end{array}$ & $\begin{array}{l}2380.0 \\
1720.0\end{array}$ \\
\hline $\begin{array}{l}1952 \\
1953\end{array}$ & $\begin{array}{r}7180.0 \\
14800.0\end{array}$ & $\begin{array}{r}7120 \cdot 0 \\
13500 \cdot 0\end{array}$ & $\begin{array}{r}6580.0 \\
10200.0\end{array}$ & $\begin{array}{l}4950.0 \\
7970.0\end{array}$ & $\begin{array}{l}3540.0 \\
6550.0\end{array}$ & $\begin{array}{l}3020.0 \\
4650.0\end{array}$ & $\begin{array}{l}3010.0 \\
3840.0\end{array}$ & $\begin{array}{l}2730 \cdot 0 \\
3160 \cdot 0\end{array}$ & $\begin{array}{l}2560.0 \\
2760.0\end{array}$ & 2460.0 & $\begin{array}{l}1720.0 \\
1740.0\end{array}$ \\
\hline $\begin{array}{l}1954 \\
1955\end{array}$ & $\begin{array}{l}8000.0 \\
4720.0\end{array}$ & $\begin{array}{l}7170.0 \\
4600.0\end{array}$ & $\begin{array}{l}6840.0 \\
4160.0\end{array}$ & $\begin{array}{l}5660.0 \\
3490.0\end{array}$ & $\begin{array}{l}5100.0 \\
3200.0\end{array}$ & $\begin{array}{l}4330.0 \\
2450.0\end{array}$ & $\begin{array}{l}4040.0 \\
2080.0\end{array}$ & $\begin{array}{l}3610 \cdot 0 \\
2020 \cdot 0\end{array}$ & $\begin{array}{l}3220.0 \\
2000.0\end{array}$ & $\begin{array}{l}2790.0 \\
1890.0\end{array}$ & 2020.0 \\
\hline 1956 & 11500.0 & 10300.0 & 8800.0 & 7170.0 & 6850.0 & 5880.0 & 4890.0 & $4400 \cdot 0$ & 4000.0 & 3580.0 & $\begin{array}{l}2620.0 \\
1310.0\end{array}$ \\
\hline $\begin{array}{l}1957 \\
1958 \\
1959\end{array}$ & $\begin{array}{l}7080.0 \\
7740.0 \\
7700.0\end{array}$ & $\begin{array}{l}6870.0 \\
6990.0 \\
7140.0\end{array}$ & $\begin{array}{l}6430.0 \\
6140.0 \\
6390.0\end{array}$ & $\begin{array}{l}5370.0 \\
5200.0 \\
5010.0\end{array}$ & $\begin{array}{l}4460 . ? \\
4460.0 \\
4440.0\end{array}$ & $\begin{array}{l}3080.0 \\
4030.0 \\
3630.0\end{array}$ & $\begin{array}{l}2460.0 \\
3530.0 \\
3010.0\end{array}$ & $\begin{array}{l}2240.0 \\
2950.0 \\
2770.0\end{array}$ & $\begin{array}{l}1960.0 \\
2760.0 \\
2630.0\end{array}$ & $\begin{array}{l}1750 \cdot 0 \\
2380.0 \\
2340 \cdot 0\end{array}$ & $\begin{array}{l}1310.0 \\
1670.0 \\
1710.0\end{array}$ \\
\hline $\begin{array}{l}1959 \\
1960\end{array}$ & $\begin{array}{l}7700.0 \\
5100.0\end{array}$ & $\begin{array}{l}7140.0 \\
4990.0\end{array}$ & $\begin{array}{l}6390.0 \\
4680.0\end{array}$ & 3880.0 & 2930.0 & 2750.0 & 2470.0 & $2300 \cdot 0$ & 2140.0 & 1920.0 & 1450.0 \\
\hline 1961 & 13600.0 & $12300 \cdot 0$ & 9800.0 & 7510.0 & 5960.0 & 4810.0 & 3920.0 & $3610 \cdot 0$ & 3340.0 & 2990.0 & 2110.0 \\
\hline 1962 & 8790.0 & 8010.0 & 6380.0 & 4660.0 & 3250.0 & 2340.0 & 2100.0 & $2180 \cdot 0$ & 2080.0 & 1940.0 & 1430.0 \\
\hline 1963 & 9060.0 & 8570.0 & $68 \cap 0.0$ & 5270.0 & 3790.0 & 3110.0 & 2490.0 & $2380 \cdot 0$ & 2290.0 & 2330.0 & 1720.0 \\
\hline
\end{tabular}


CLASS $00 \begin{array}{lllllllllllllllllllllllllllllllllll} & 2 & 3 & 4 & 5 & 6 & 7 & 8 & 9 & 10 & 11 & 12 & 13 & 14 & 15 & 16 & 17 & 18 & 19 & 20 & 21 & 22 & 23 & 24 & 25 & 26 & 27 & 28 & 29 & 30 & 31 & 32 & 33 & 34\end{array}$

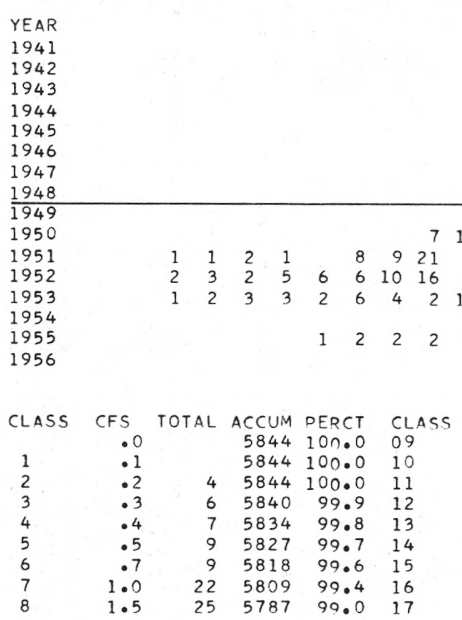

Tualatin River at Gaston, Oreg. Summary for water years 1941-48

$\begin{array}{lcrrrl}\text { CLASS } & \text { CFS } & \text { TOTAL } & \text { ACCUM } & \text { PERCT } & \text { CLASS } \\ & .0 & 2922 & 100.0 & 09 \\ 1 & .1 & 2922 & 100.0 & 10 \\ 2 & .2 & 2922 & 100.0 & 11 \\ 3 & .3 & 2922 & 100.0 & 12 \\ 4 & .4 & 2922 & 100.0 & 13 \\ 5 & .5 & 2922 & 100.0 & 14 \\ 6 & .7 & 2922 & 100.0 & 15 \\ 7 & 1.0 & 2922 & 100.0 & 16 \\ 8 & 1.5 & 2922 & 100.0 & 17\end{array}$

Tualatin River at Gaston, Oreg.

Summary for water years $1949-56$

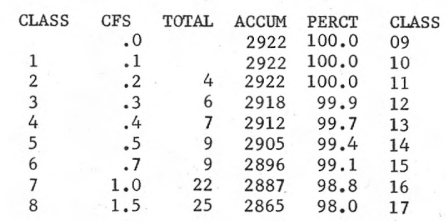

NUMBER OF DAYS IN CLASS

$\begin{array}{llllllllllllllll}16 & 29 & 47 & 31 & 16 & 48 & 51 & 45 & 21 & 24 & 16 & 7 & 11 & 1 & 1 & 1\end{array}$

$\begin{array}{rrrrrrrrrrrrrrrrr}8 & 39 & 24 & 30 & 18 & 39 & 60 & 70 & 19 & 12 & 11 & 6 & 16 & 8 & 4 & & 1 \\ 26 & 44 & 36 & 14 & 12 & 25 & 28 & 39 & 29 & 27 & 25 & 10 & 16 & 17 & 9 & 4 & 4\end{array}$

$\begin{array}{llllllllllllllllll}35 & 41 & 25 & 18 & 17 & 34 & 32 & 56 & 38 & 46 & 16 & 4 & 1 & 1 & 1 & 1\end{array}$

$\begin{array}{rrrrrrrrrrrrrrr}19 & 49 & 42 & 19 & 13 & 24 & 30 & 43 & 37 & 37 & 18 & 13 & 11 & 6 & 2 \\ 36 & 56 & 11 & 33 & 25 & 22 & 12 & 11 & 19 & 47 & 34 & 15 & 19 & 13 & 8\end{array}$

$\begin{array}{lllllllllllllllll}18 & 45 & 36 & 32 & 26 & 25 & 25 & 44 & 25 & 30 & 11 & 10 & 14 & 10 & 9 & 4\end{array}$

$\begin{array}{lllllllllllllllllll}6 & 17 & 29 & 27 & 18 & 9 & 14 & 16 & 38 & 33 & 73 & 35 & 19 & 11 & 14 & 4 & 3 & \\ 34 & 22 & 20 & 33 & 17 & 8 & 23 & 41 & 27 & 15 & 29 & 20 & 17 & 17 & 11 & 8 & 3 & 1\end{array}$

$\begin{array}{rrrrrrrrrrrrrrrrrrrrrrrrrrrrrr}7 & 17 & 4 & 9 & 20 & 28 & 23 & 16 & 22 & 16 & 16 & 14 & 18 & 15 & 43 & 20 & 22 & 26 & 13 & 11 & 2 & 2 & 1\end{array}$

$\begin{array}{lllllllllllllllllll}15 & 8 & 11 & 9 & 16 & 14 & 18 & 24 & 21 & 21 & 21 & 35 & 23 & 31 & 17 & 13 & 1\end{array}$

$\begin{array}{rrrrrrrrrrrrrrrrr}14 & 2 & 5 & 27 & 19 & 10 & 15 & 23 & 18 & 30 & 54 & 39 & 13 & 10 & 9 & 4 & 4 \\ 21 & 25 & 12 & 16 & 10 & 15 & 7 & 28 & 43 & 28 & 21 & 17 & 12 & 17 & 12 & 10 & 1\end{array}$

$\begin{array}{rllllllllllllllllllllll}6 & 57 & 24 & 26 & 33 & 19 & 22 & 14 & 19 & 12 & 17 & 39 & 22 & 24 & 15 & 8 & 6 & 2\end{array}$

$\begin{array}{rlrlrllllllllllllllll}13 & 29 & 27 & 27 & 17 & 26 & 10 & 7 & 12 & 8 & 13 & 24 & 18 & 33 & 26 & 32 & 19 & 16 & 5 & 2 & 2\end{array}$

CFS-DAYS

44352.0

56059.0

87081.0

57328.0

79416.0

68200.0

78884.3

86071 .

88236.3
71912.9

71912.9

65916.2

$894 \epsilon 3.0$
65972.4

12072.3

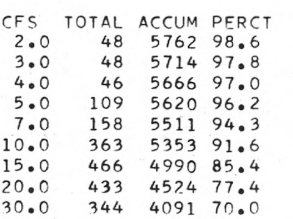

CLASS
18
19
20
21
22
23
24
25
26

$\begin{array}{rrrr}\text { CFS } & \text { TOTAL } & \text { ACCUM } & \text { PERCT } \\ 40 & 231 & 3747 & 64.1 \\ 50 & 355 & 3516 & 60.2 \\ 70 & 423 & 3161 & 54.1 \\ 100 & 528 & 2738 & 46.9 \\ 150 & 393 & 2210 & 37.8 \\ 200 & 565 & 1817 & 31.1 \\ 300 & 402 & 1252 & 21.4 \\ 400 & 239 & 850 & 14.5 \\ 500 & 267 & 611 & 10.5\end{array}$

$\begin{array}{lrrrr}\text { CLASS } & \text { CFS } & \text { TOTAL } & \text { ACCUM } & \text { PERCT } \\ 27 & 700 & 172 & 344 & 5.9 \\ 28 & 1000 & 111 & 172 & 2.9 \\ 29 & 1500 & 42 & 61 & 1.0 \\ 30 & 2000 & 14 & 19 & .3 \\ 31 & 3000 & 5 & 5 & .1 \\ 32 & 4000 & & & .0 \\ 33 & & & & .0 \\ 34 & & & & .0 \\ 35 & & & & \end{array}$

$14-2025.00$

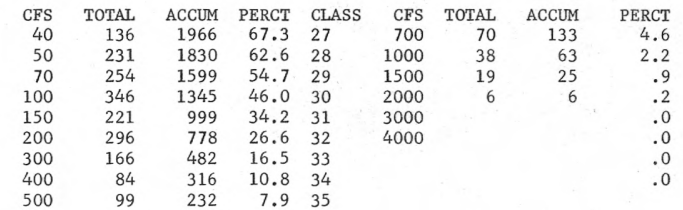

14-2025.00

$\begin{array}{rrrrl}\text { CFS } & \text { TOTAL } & \text { ACCUM } & \text { PERCT } & \text { CLASS } \\ 2.0 & & 2922 & 100.0 & 18 \\ 3.0 & & 2922 & 100.0 & 19 \\ 4.0 & & 2922 & 100.0 & 20 \\ 5.0 & & 2922 & 100.0 & 21 \\ 7.0 & 6 & 2922 & 100.0 & 22 \\ 10.0 & 175 & 2916 & 99.8 & 23 \\ 15.0 & 332 & 2741 & 93.8 & 24 \\ 20.0 & 248 & 2409 & 82.4 & 25 \\ 30.0 & 195 & 2161 & 74.0 & 26\end{array}$

STATION NUMBER

$\begin{array}{rrrrl}\text { CFS } & \text { TOTAL } & \text { ACCUM } & \text { PERCT } & \text { CLASS } \\ 2.0 & 48 & 2840 & 97.2 & 18 \\ 3.0 & 48 & 2792 & 95.6 & 19 \\ 4.0 & 46 & 2744 & 93.9 & 20 \\ 5.0 & 109 & 2698 & 92.3 & 21 \\ 7.0 & 152 & 2589 & 88.6 & 22 \\ 10.0 & 188 & 2437 & 83.4 & 23 \\ 15.0 & 134 & 2249 & 77.0 & 24 \\ 20.0 & 185 & 2115 & 72.4 & 25 \\ 30.0 & 149 & 1930 & 66.1 & 26\end{array}$

$\begin{array}{rr}\text { CFS } & \text { TOTA } \\ 40 & 95 \\ 50 & 124 \\ 70 & 169 \\ 100 & 182 \\ 150 & 172 \\ 200 & 269 \\ 300 & 236 \\ 400 & 155 \\ 500 & 168\end{array}$

$\begin{array}{rrrl}\text { OTAL } & \text { ACCUM } & \text { PERCT } & \text { CLASS } \\ 95 & 1781 & 61.0 & 27 \\ 124 & 1686 & 57.7 & 28 \\ 169 & 1562 & 53.5 & 29 \\ 182 & 1393 & 47.7 & 30 \\ 172 & 1211 & 41.4 & 31 \\ 269 & 1039 & 35.6 & 32 \\ 236 & 770 & 26.4 & 33 \\ 155 & 534 & 18.3 & 34 \\ 168 & 379 & 13.0 & 35\end{array}$

$\begin{array}{rrrr}\text { CFS } & \text { TOTAL } & \text { ACCUM } & \text { PERCT } \\ 700 & 102 & 211 & 7.2 \\ 1000 & 73 & 109 & 3.7 \\ 1500 & 23 & 36 & 1.2 \\ 2000 & 8 & 13 & .4 \\ 3000 & 5 & 5 & .2 \\ 4000 & & & .0 \\ & & & .0 \\ & & & .0\end{array}$


Tualatin River at Gaston, Oreg.

STATION NUMBER $\quad 14-2025.00$

LOWEST MEAN DISCHARGE, IN CFS, FOR THE FOLLOWING NUMBER OF CONSECUTIVE DAYS IN YEAR REGINNING APRIL 1

\begin{tabular}{|c|c|c|c|c|c|c|c|c|c|c|c|}
\hline YEAR & 1 & 3 & 7 & 14 & 30 & 60 & 90 & 120 & 150 & 183 & 274 \\
\hline 1941 & 13.0 & 13.0 & 13.6 & 13.9 & 14.8 & 17.4 & 23.2 & $26 \cdot 8$ & 32.7 & 41.0 & 122.0 \\
\hline 1942 & 14.0 & 14.0 & $14 \cdot 1$ & 14.5 & 14.8 & 16.2 & 17.9 & $22 \cdot 0$ & 29.8 & 39.2 & 161.0 \\
\hline 1943 & 12.0 & $12 \cdot 0$ & $12 \cdot 4$ & 12.9 & 13.3 & 15.6 & 18.0 & $22 \cdot 9$ & 30.4 & 42.3 & 88.9 \\
\hline 1944 & 11.0 & 11.0 & 11.1 & 11.9 & 13.3 & 15.0 & 15.9 & $17 \cdot 9$ & 23.4 & 33.2 & 62.9 \\
\hline 1945 & 14.0 & 14.0 & 14.4 & 14.6 & 15.9 & 19.7 & 21.7 & $22 \cdot 7$ & 26.9 & 43.3 & 151.0 \\
\hline 1946 & 11.0 & 11.3 & 11.7 & 12.6 & 13.5 & 14.0 & 14.9 & 19.7 & 24.4 & 32.4 & 140.0 \\
\hline 1947 & 13.0 & 13.7 & 14.1 & 14.9 & 16.5 & 18.0 & 20.8 & 27.5 & 31.8 & 43.4 & 121.0 \\
\hline 1948 & 7.5 & 7.9 & 8.7 & 10.4 & 12.5 & 16.0 & 19.2 & $22 \cdot 5$ & 28.7 & 47.5 & 157.0 \\
\hline 1949 & 5.4 & 5.6 & 5.7 & 6.2 & 7.5 & 8.3 & 10.0 & 13.5 & 16.6 & 24.8 & 99.5 \\
\hline 1950 & 2.7 & 2.7 & 2.8 & 2.9 & 4.0 & 6.6 & 8.9 & $14 \cdot 8$ & 21.8 & 35.3 & 161.0 \\
\hline 1951 & .2 & $\cdot 3$ & .6 & 1.2 & 2.4 & 2.6 & 5.0 & $10 \cdot 5$ & 19.3 & 32.9 & 138.0 \\
\hline 1952 & .2 & $\cdot 2$ & .4 & .6 & .8 & 2.0 & $2 \cdot 1$ & $3 \cdot 0$ & $4 \cdot 5$ & 8.5 & 48.1 \\
\hline 1953 & 4.9 & 4.9 & 5.3 & 6.3 & 8.6 & 11.1 & 14.4 & $18 \cdot 7$ & 23.0 & 38.0 & 140.0 \\
\hline 1954 & 8.6 & $8 \cdot 8$ & 8.9 & $9 \cdot 3$ & 11.3 & 11.6 & $12 \cdot 5$ & $17 \cdot 7$ & $\begin{array}{l}23.4 \\
32.5\end{array}$ & $\begin{array}{l}27.6 \\
57.4\end{array}$ & $\begin{array}{l}97.2 \\
40.0\end{array}$ \\
\hline
\end{tabular}

Tualatin River at Gaston, Oreg.

STATION NUMBER $\quad 14-2025.00$

HIGHEST MEAN DISCHARGF, IN CFS, FOR THE FOLLOWING NUMBER OF CONSECUTIVE DAYS IN YEAR ENDING SEPTEMBER 30

\begin{tabular}{|c|c|c|c|c|c|c|c|c|c|c|c|}
\hline YEAR & 1 & 3 & 7 & 15 & 30 & 60 & 90 & 120 & 150 & 183 & 274 \\
\hline 1941 & 1760.0 & 1260.0 & 829.0 & 624.0 & 464.0 & 391.0 & 313.0 & 264.0 & 230.0 & 204.0 & 154.0 \\
\hline 1942 & 2570.0 & 1760.0 & 1260.0 & 844.0 & 594.0 & 475.0 & 415.0 & 352.0 & 304.0 & 264.0 & 197.0 \\
\hline 1943 & 2290.0 & 1710.0 & 1490.0 & 940.0 & 739.0 & 685.0 & 613.0 & 527.0 & 500.0 & 440.0 & 312.0 \\
\hline 1944 & 1590.0 & 1070.0 & 654.0 & 398.0 & 280.0 & 253.0 & 245.0 & $230 \cdot 0$ & 218.0 & 198.0 & 153.0 \\
\hline 1945 & 1730.0 & 1310.0 & 895.0 & 683.0 & 492.0 & 450.0 & 406.0 & 358.0 & 312.0 & 276.0 & 202.0 \\
\hline 1946 & 1900.0 & 1570.0 & 1150.0 & 1010.0 & 688.0 & 593.0 & 564.0 & $516 \cdot 0$ & 466.0 & 407.0 & 285.0 \\
\hline 1947 & 2020.0 & 1790.0 & 1380.0 & 966.0 & 809.0 & 493.0 & 515.0 & $456 \cdot 0$ & 396.0 & 339.0 & 243.0 \\
\hline 1948 & 1970.0 & 1330.0 & 1150.0 & 871.0 & 592.0 & 466.0 & 419.0 & 387.0 & 372.0 & 354.0 & 292.0 \\
\hline 1949 & 3500.0 & 2010.0 & 1750.0 & 1300.0 & 891.0 & 594.0 & 536.0 & 512.0 & 461.0 & 402.0 & 284.0 \\
\hline 1950 & 3100.0 & 2530.0 & 1530.0 & 1180.0 & 950.0 & 732.0 & 632.0 & 564.0 & 517.0 & 447.0 & 311.0 \\
\hline 1951 & 1940.0 & 1460.0 & 1280.0 & 1080.0 & 872.0 & 683.0 & 645.0 & 558.0 & 507.0 & 453.0 & 320.0 \\
\hline 1952 & 2120.0 & 1690.0 & 1410.0 & 906.0 & 594.0 & 456.0 & 480.0 & 428.0 & 404.0 & 364.0 & 261.0 \\
\hline 1953 & 1740.0 & 1410.0 & 1300.0 & 1100.0 & 933.0 & 656.0 & 535.0 & 450.0 & 391.0 & 343.0 & 239.0 \\
\hline 1954 & 2380.0 & 1990.0 & 1360.0 & 1180.0 & 823.0 & 722.0 & 687.0 & 617.0 & 542.0 & 462.0 & 322.0 \\
\hline 1955 & 1580.0 & 1140.0 & 756.0 & $512 . n$ & 458.0 & 375.0 & 362.0 & 368.0 & 345.0 & 330.0 & 237.0 \\
\hline 1956 & 3820.0 & 2710.0 & 1760.0 & 1310.0 & 1120.0 & 951.0 & 772.0 & $680 \cdot 0$ & 653.0 & 584.0 & 406.0 \\
\hline
\end{tabular}

Scoggin Creek near Gaston, Oreg.

STATION NUMBER $14-2030.00$

DURATION TABLE OF DAILY DISCHARGE

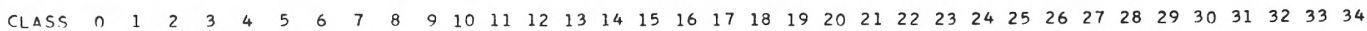

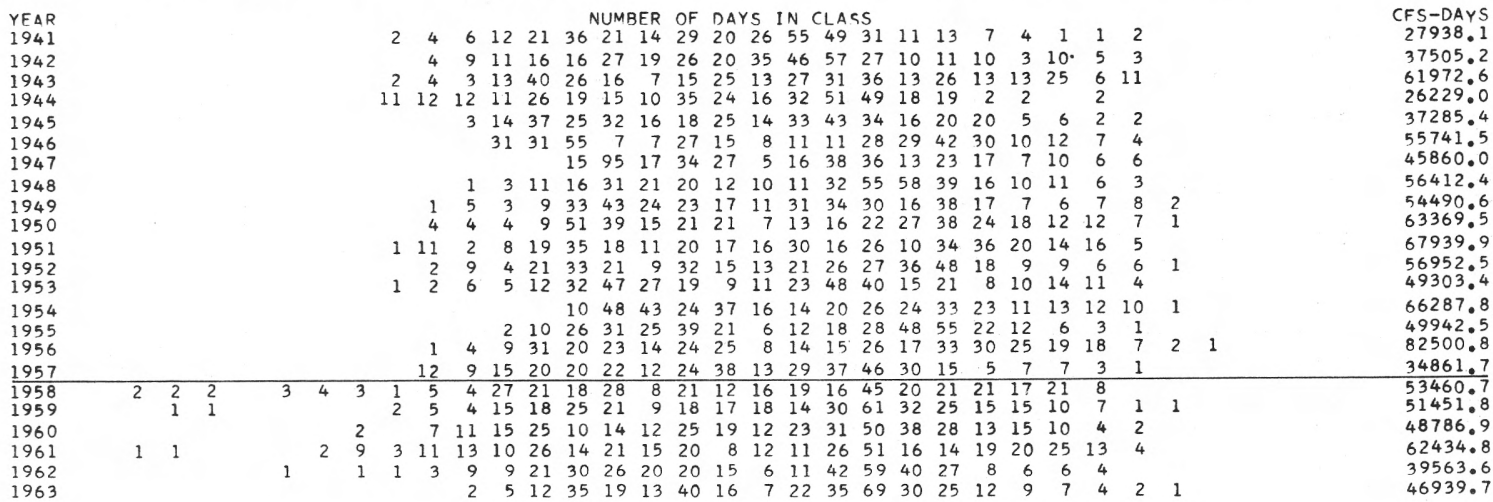


Summary for water years 1941-63

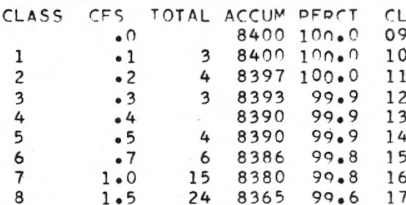

Scoggin Creek near Gaston, Oreg.

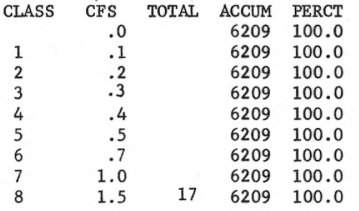

Scoggin Creek near Gaston, Oreg.

Summary for water years 1958-63

CLASS CFS TOTAL ACCUM PERCT

$\begin{array}{lr}\text { CLASS } & \text { CFS } \\ 09 & 2.0 \\ 10 & 3.0 \\ 11 & 4.0 \\ 12 & 5.0 \\ 13 & 7.0 \\ 14 & 10.0 \\ 15 & 15.0 \\ 16 & 20.0 \\ 17 & 30.0\end{array}$

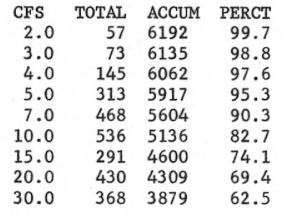

STATION NUMBER

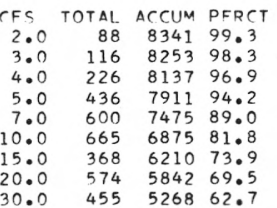

STATION NUMBER
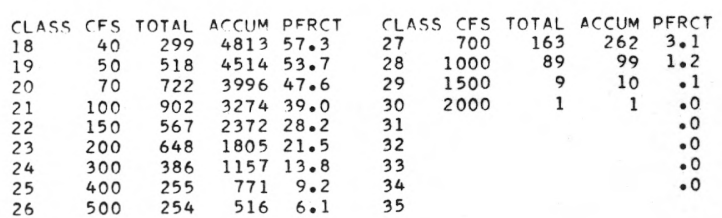

14-2030.00

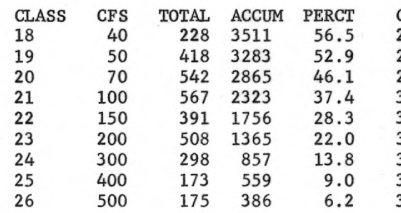

14-2030.00

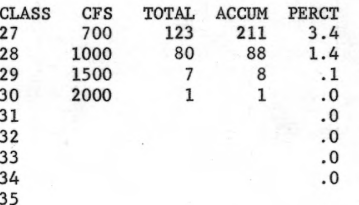$$
35
$$

\begin{tabular}{|c|c|c|c|c|c|c|c|c|c|c|c|c|c|c|c|c|c|c|c|}
\hline & .0 & & 2191 & 100.0 & 09 & 2.0 & 31 & 2149 & 98.1 & 18 & 40 & 71 & 1302 & 59.4 & 27 & 700 & 40 & 51 & 2.3 \\
\hline 1 & .1 & 3 & 2191 & 100.0 & 10 & 3.0 & 43 & 2118 & 96.7 & 19 & 50 & 100 & 1231 & 56.2 & 28 & 1000 & 9 & 11 & .5 \\
\hline 2 & .2 & 4 & 2188 & 99.9 & 11 & 4.0 & 81 & 2075 & 94.7 & 20 & 70 & 180 & 1131 & 51.6 & 29 & 1500 & 2 & 2 & .1 \\
\hline 3 & .3 & 3 & 2184 & 99.7 & 12 & 5.0 & 123 & 1994 & 91.0 & 21 & 100 & 335 & 951 & 43.4 & 30 & 2000 & & & .0 \\
\hline 4 & .4 & & 2181 & 99.5 & 13 & 7.0 & 132 & 1871 & 85.4 & 22 & 150 & 176 & 616 & 28.1 & 31 & & & & .0 \\
\hline 5 & .5 & 4 & 2181 & 99.5 & 14 & 10.0 & 129 & 1739 & 79.4 & 23 & 200 & 140 & 440 & 20.1 & 32 & & & & .0 \\
\hline 6 & .7 & 6 & 2177 & 99.4 & 15 & 15.0 & 77 & 1610 & 73.5 & 24 & 300 & 88 & 300 & 13.7 & 33 & & & & .0 \\
\hline 7 & 1.0 & 15 & 2171 & 99.1 & 16 & 20.0 & 144 & 1533 & 70.0 & 25 & 400 & 82 & 212 & 9.7 & 34 & & & & .0 \\
\hline 8 & 1.5 & 7 & 2156 & 98.4 & 17 & 30.0 & 87 & 1389 & 63.4 & 26 & 500 & 79 & 130 & 5.9 & 35 & & & & \\
\hline
\end{tabular}

Scoggin Creek near Gaston, Oreg.

STATION NUMBER $14-2030.00$

LOWEST MEAN DISCHARGE, IN CFS, FOR THE FOLLOWING NUMBER OF CONSECUTIVE DAYS IN YEAR BEGINNING APRIL 1

\begin{tabular}{|c|c|c|c|c|c|c|c|c|c|c|c|}
\hline YEAR & 1 & 3 & 7 & 14 & 30 & 60 & 90 & 120 & 150 & 183 & 274 \\
\hline $\begin{array}{l}1941 \\
1942 \\
1943\end{array}$ & $\begin{array}{l}1.6 \\
2.0 \\
1.7\end{array}$ & $\begin{array}{l}2.6 \\
2.5 \\
2.1\end{array}$ & $\begin{array}{l}2.8 \\
3.2 \\
3.0\end{array}$ & $\begin{array}{l}3.4 \\
3.8 \\
3.6\end{array}$ & $\begin{array}{l}4.2 \\
4.3 \\
4.1\end{array}$ & $\begin{array}{l}6.5 \\
5.3 \\
5.6\end{array}$ & $\begin{array}{l}8.9 \\
6.4 \\
6.8\end{array}$ & $\begin{array}{r}11 \cdot 5 \\
8 \cdot 7 \\
10.5\end{array}$ & $\begin{array}{l}14 \cdot 8 \\
14.3 \\
15.8\end{array}$ & $\begin{array}{l}21 \cdot 0 \\
20 \cdot 8 \\
22 \cdot 2\end{array}$ & $\begin{array}{r}75.2 \\
109.0 \\
52.0\end{array}$ \\
\hline $\begin{array}{l}1944 \\
1945\end{array}$ & $\begin{array}{l}1.5 \\
4.6\end{array}$ & $\begin{array}{l}1.7 \\
4.9\end{array}$ & $\begin{array}{l}2.0 \\
5.1\end{array}$ & $\begin{array}{l}2.1 \\
5.4\end{array}$ & $\begin{array}{l}3.0 \\
6.0\end{array}$ & $\begin{array}{l}4.3 \\
8.3\end{array}$ & $\begin{array}{l}4.9 \\
9.5\end{array}$ & $\begin{array}{r}6 \cdot 2 \\
10 \cdot 1\end{array}$ & $\begin{array}{r}9.7 \\
12.7\end{array}$ & $\begin{array}{l}14.2 \\
24.0\end{array}$ & $\begin{array}{l}31.8 \\
98.0\end{array}$ \\
\hline $\begin{array}{l}1946 \\
1947\end{array}$ & 4.0 & $\begin{array}{l}4 . n \\
8.0\end{array}$ & $\begin{array}{l}4.0 \\
8.0\end{array}$ & $\begin{array}{l}4.0 \\
8.0\end{array}$ & $\begin{array}{l}4.0 \\
9.6\end{array}$ & $\begin{array}{l}4.5 \\
9.9\end{array}$ & $\begin{array}{r}5.6 \\
10.6\end{array}$ & $\begin{array}{r}7.6 \\
12.3\end{array}$ & $\begin{array}{l}11.8 \\
15.9\end{array}$ & 15.8 & $\begin{array}{l}90.7 \\
72.8\end{array}$ \\
\hline $\begin{array}{l}1947 \\
1948 \\
1949\end{array}$ & $\begin{array}{l}5.9 \\
3.5 \\
2.4\end{array}$ & $\begin{array}{l}8.0 \\
5.1 \\
3.4\end{array}$ & $\begin{array}{l}5.10 \\
5.8\end{array}$ & $\begin{array}{l}6.1 \\
5.8\end{array}$ & $\begin{array}{l}7.7 \\
6.9\end{array}$ & $\begin{array}{r}10.1 \\
7.9\end{array}$ & $\begin{array}{r}13.0 \\
9.5\end{array}$ & $\begin{array}{l}14 \cdot 3 \\
10 \cdot 6\end{array}$ & $\begin{array}{l}19.8 \\
12.5\end{array}$ & $\begin{array}{l}32.7 \\
18.6\end{array}$ & $\begin{array}{r}110.0 \\
66.3\end{array}$ \\
\hline $\begin{array}{l}1950 \\
1951 \\
1952\end{array}$ & $\begin{array}{l}2.2 \\
1.6 \\
1.9\end{array}$ & $\begin{array}{l}2.8 \\
2.4 \\
2.5\end{array}$ & $\begin{array}{l}5.1 \\
2.9 \\
3.9\end{array}$ & $\begin{array}{l}5 \cdot 9 \\
4 \cdot 2 \\
4.1\end{array}$ & $\begin{array}{l}6.3 \\
5.3 \\
4.4\end{array}$ & $\begin{array}{l}7.4 \\
5.6 \\
5.4\end{array}$ & $\begin{array}{l}8.8 \\
7.3 \\
5.9\end{array}$ & $\begin{array}{r}13 \cdot 1 \\
11 \cdot 2 \\
6 \cdot 6\end{array}$ & $\begin{array}{r}17.3 \\
16.1 \\
7.9\end{array}$ & $\begin{array}{l}27 \cdot 6 \\
24 \cdot 7 \\
10 \cdot 6\end{array}$ & $\begin{array}{r}115.0 \\
106.0 \\
35.4\end{array}$ \\
\hline $\begin{array}{l}1953 \\
1954 \\
1955\end{array}$ & $\begin{array}{l}8.3 \\
6.6 \\
4.4\end{array}$ & $\begin{array}{l}9.0 \\
7.6 \\
6.0\end{array}$ & $\begin{array}{l}9 \cdot 3 \\
8 \cdot 7 \\
6 \cdot 5\end{array}$ & $\begin{array}{l}9.6 \\
9.9 \\
6.8\end{array}$ & $\begin{array}{r}10.9 \\
12.0 \\
7.3\end{array}$ & $\begin{array}{r}12.9 \\
12.2 \\
9.8\end{array}$ & $\begin{array}{l}14.8 \\
13.3 \\
13.1\end{array}$ & $\begin{array}{l}17 \cdot 5 \\
16 \cdot 6 \\
17 \cdot 0\end{array}$ & $\begin{array}{l}21.0 \\
20.0 \\
25.7\end{array}$ & $\begin{array}{r}31.3 \\
-23.7 \\
40.7\end{array}$ & $\begin{array}{r}98.8 \\
76.0 \\
177.0\end{array}$ \\
\hline $\begin{array}{l}1956 \\
1957 \\
\end{array}$ & $\begin{array}{l}2.9 \\
2.6 \\
\end{array}$ & $\begin{array}{l}3.6 \\
2.6\end{array}$ & $\begin{array}{l}4.8 \\
2.7 \\
\end{array}$ & $\begin{array}{l}5.6 \\
3.0 \\
\end{array}$ & $\begin{array}{l}5.8 \\
3.5 \\
\end{array}$ & $\begin{array}{l}7.0 \\
4.9 \\
\end{array}$ & $\begin{array}{l}7.2 \\
6.5 \\
\end{array}$ & $\begin{array}{l}9.3 \\
8.6\end{array}$ & $\begin{array}{l}13.3 \\
11.3\end{array}$ & $\begin{array}{l}19.1 \\
17.3\end{array}$ & $\begin{array}{r}47.3 \\
73.1 \\
\end{array}$ \\
\hline 1958 & .1 & .3 & 1.1 & 2.5 & 3.0 & 3.4 & 4.2 & $6 \cdot 0$ & 11.1 & 19.9 & 79.9 \\
\hline 1959 & 1.8 & 1.9 & 4.0 & 4.7 & 4.8 & 8.0 & 11.5 & $17 \cdot 5$ & 20.7 & 26.0 & 60.6 \\
\hline 1960 & 1.1 & 2.5 & 3.2 & 3.7 & 4.3 & 4.7 & 5.7 & $8 \cdot 3$ & 12.5 & 30.0 & 92.6 \\
\hline 1961 & $\cdot 1$ & $\cdot 3$ & 1.8 & 2.4 & 2.6 & 3.6 & 5.0 & 8.0 & 12.1 & 18.1 & 75.8 \\
\hline 1962 & .6 & 1.2 & 3.3 & 4.8 & 6.0 & 6.9 & 8.0 & $12 \cdot 4$ & 19.9 & 30.3 & 93.3 \\
\hline
\end{tabular}

Scoggin Creek near Gaston, Oreg.

STATION NUMBER 14-2030.00

HIGHEST MEAN DISCHARGE, IN CFS, FOR THE FOLLOWING NUMBER OF CONSECUTIVE DAYS IN YEAR ENDING SEPTEMBER 30

\begin{tabular}{|c|c|c|c|c|c|c|c|c|c|c|c|}
\hline YEAR & 1 & 3 & 7 & 15 & 30 & 60 & 90 & 120 & 150 & 183 & 274 \\
\hline $\begin{array}{l}1941 \\
1942\end{array}$ & $\begin{array}{l}1460.0 \\
1470.0\end{array}$ & $\begin{array}{l}1120.0 \\
1180.0\end{array}$ & $\begin{array}{r}679.0 \\
887.0\end{array}$ & $\begin{array}{l}471 \cdot 0 \\
584 \cdot 0\end{array}$ & $\begin{array}{l}320.0 \\
398.0\end{array}$ & $\begin{array}{l}258 \cdot 0 \\
354 \cdot 0\end{array}$ & $\begin{array}{l}205 \cdot 0 \\
291.0\end{array}$ & $\begin{array}{l}173 \cdot 0 \\
247.0\end{array}$ & $\begin{array}{l}151.0 \\
212.0\end{array}$ & $\begin{array}{l}134.0 \\
183.0\end{array}$ & $\begin{array}{r}99.1 \\
134.0\end{array}$ \\
\hline & 1400.0 & 1170.0 & $1000 \cdot 0$ & 677.0 & 534.0 & 499.0 & 451.0 & $385 \cdot 0$ & 367.0 & 318.0 & 224.0 \\
\hline $\begin{array}{l}1944 \\
1945\end{array}$ & $\begin{array}{r}881.0 \\
1360.0\end{array}$ & $\begin{array}{l}680.0 \\
966.0\end{array}$ & $\begin{array}{l}428.0 \\
729.0\end{array}$ & $\begin{array}{l}250.0 \\
541.0\end{array}$ & $\begin{array}{l}203.0 \\
377.0\end{array}$ & $\begin{array}{l}165.0 \\
330.0\end{array}$ & $\begin{array}{l}162.0 \\
287.0\end{array}$ & $\begin{array}{l}151 \cdot 0 \\
248 \cdot 0\end{array}$ & $\begin{array}{l}140.0 \\
215.0\end{array}$ & $\begin{array}{l}124.0 \\
186.0\end{array}$ & $\begin{array}{r}93.9 \\
133.0\end{array}$ \\
\hline 1946 & 1420.0 & 1060.0 & 800.0 & 718.0 & 495.0 & 437.0 & 407.0 & 369.0 & 335.0 & 291.0 & 202.0 \\
\hline 1947 & 1240.0 & 1160.0 & $867 \cdot 0$ & 636.0 & 540.0 & $332 \cdot 0$ & $354 \cdot 0$ & $314 \cdot 0$ & 273.0 & 233.0 & 164.0 \\
\hline 1948 & 1410.0 & 1020.0 & 850.0 & 647.0 & 434.0 & 357.0 & 315.0 & 285.0 & $268 \cdot 0$ & 249.0 & 202.0 \\
\hline 1949 & 1630.0 & 1340.0 & 1170.0 & 848.0 & 604.0 & 402.0 & 378.0 & 355.0 & 318.0 & 277.0 & 196.0 \\
\hline 1950 & 1550.0 & $1400 \cdot 0$ & 978.0 & 820.0 & 695.0 & 557.0 & 476.0 & 422.0 & 381.0 & 328.0 & 228.0 \\
\hline $\begin{array}{l}1951 \\
1952\end{array}$ & $\begin{array}{l}1400.0 \\
1500.0\end{array}$ & $\begin{array}{l}1110.0 \\
1320.0\end{array}$ & $\begin{array}{r}986.0 \\
1130.0\end{array}$ & $\begin{array}{l}866.0 \\
744.0\end{array}$ & $\begin{array}{l}714.0 \\
505.0\end{array}$ & $\begin{array}{l}561.0 \\
377.0\end{array}$ & $\begin{array}{l}505.0 \\
397.0\end{array}$ & $\begin{array}{l}438 \bullet 0 \\
358 \bullet 0\end{array}$ & $\begin{array}{l}399.0 \\
327.0\end{array}$ & $\begin{array}{l}349.0 \\
287.0\end{array}$ & $\begin{array}{l}245.0 \\
205.0\end{array}$ \\
\hline 1953 & 1150.0 & 1040.0 & 998.0 & 851.0 & 733.0 & 503.0 & 406.0 & 333.0 & 287.0 & 251.0 & 177.0 \\
\hline 1954 & 1790.0 & 1440.0 & 1040.0 & 924.0 & 647.0 & 557.0 & 514.0 & $451 \cdot 0$ & 396.0 & 339.0 & 237.0 \\
\hline 1955 & 1020.0 & 797.0 & 562.0 & 367.0 & 325.0 & 287.0 & 273.0 & 277.0 & $264 \cdot 0$ & 248.0 & 178.0 \\
\hline 1956 & 2610.0 & 1910.0 & 1290.0 & 883.0 & 783.0 & 696.0 & 564.0 & 499.0 & 480.0 & 425.0 & 298.0 \\
\hline 1957 & 1200.0 & 879.0 & 629.0 & 515.0 & 420.0 & 293.0 & 237.0 & $215 \cdot 0$ & 196.0 & 170.0 & 125.0 \\
\hline 1958 & 933.0 & 855.0 & 663.0 & 607.0 & 520.0 & 459.0 & 417.0 & 351.0 & 320.0 & 274.0 & 194.0 \\
\hline 1959 & 1700.0 & 1230.0 & 975.0 & 614.0 & 523.0 & 417.0 & 345.0 & $314 \cdot 0$ & 288.0 & 256.0 & 185.0 \\
\hline 1960 & 1300.0 & 970.0 & 766.0 & 696.0 & 519.0 & 372.0 & 332.0 & 286.0 & 258.0 & 236.0 & 176.0 \\
\hline 1961 & 1250.0 & 1000.0 & 890.0 & 733.0 & 644.0 & 553.0 & 465.0 & 409.0 & 371.0 & 324.0 & 226.0 \\
\hline 1962 & 952.0 & 856.0 & 655.0 & 504.0 & 340.0 & 250.0 & 217.0 & 218.0 & 206.0 & 196.0 & 142.0 \\
\hline 1963 & 1670.0 & 1060.0 & 728.0 & 597.0 & 408.0 & 282.0 & 262.0 & 233.0 & 237.0 & 226.0 & 168.0 \\
\hline
\end{tabular}




\begin{tabular}{|c|c|c|c|c|c|c|c|c|c|c|c|c|c|c|c|c|c|c|c|c|c|c|c|c|c|c|c|c|c|}
\hline \multirow{2}{*}{\multicolumn{29}{|c|}{ 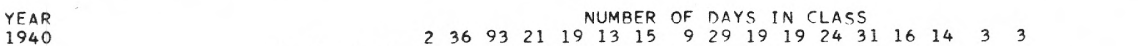 }} & CFS-DAYS \\
\hline & & & & & & & & & & 2 & 36 & 93 & 21 & 19 & 13 & 15 & 9 & 29 & 19 & 19 & 24 & 31 & 16 & 14 & \multicolumn{4}{|c|}{33} & 132149.0 \\
\hline 1941 & & & & & & 1 & 1 & 4 & 22 & 7 & 10 & 29 & 21 & 38 & 22 & 42 & 38 & 47 & 20 & 18 & 18 & 20 & 5 & & 1 & 1 & & & 84547.0 \\
\hline 1942 & & & & & & & & 4 & 12 & 17 & 24 & 14 & 19 & 22 & 22 & 67 & 24 & 55 & 24 & 8 & 12 & 19 & 11 & 5 & 4 & 1 & & & 107486.8 \\
\hline 1943 & & & & & & & & 2 & 20 & 27 & 46 & 10 & 12 & 10 & 26 & 23 & 18 & 31 & 28 & 10 & 23 & 29 & 20 & 9 & 12 & 8 & \multicolumn{2}{|l|}{1} & 168829.0 \\
\hline 1944 & & & & & & & & & 19 & 40 & 30 & 18 & 11 & 6 & 37 & 34 & 23 & 44 & 35 & 29 & 28 & 8 & 2 & 2 & & & & & 79080.0 \\
\hline 1945 & & & & & & & & & 5 & 36 & 49 & 18 & 11 & 18 & 20 & 30 & 24 & 47 & 27 & 17 & 19 & 20 & 14 & 6 & 2 & 1 & \multicolumn{2}{|l|}{1} & 109685.0 \\
\hline 1946 & & & & & & & & 1 & 5 & 43 & 46 & 11 & 16 & 28 & 25 & 14 & 8 & 9 & 19 & 21 & 32 & 35 & 28 & 12 & 8 & 2 & \multicolumn{2}{|l|}{2} & 165353.0 \\
\hline 1947 & & & & & & & & & & 5 & 54 & 33 & 10 & 40 & 26 & 25 & 16 & 34 & 29 & 14 & 17 & 15 & 24 & 11 & 6 & 3 & \multicolumn{2}{|l|}{3} & 147080.0 \\
\hline 1948 & & & & & & & & 1 & 4 & 6 & 24 & 31 & 10 & 22 & 18 & 15 & 16 & 34 & 30 & 37 & 54 & 31 & 12 & 12 & 5 & 4 & & & 162750.0 \\
\hline 1949 & & & & & & & 3 & 3 & 34 & 21 & 17 & 15 & 26 & 19 & 21 & 19 & 41 & 23 & 11 & 13 & 24 & 32 & 14 & 12 & 6 & 5 & 3 & 3 & 172951.0 \\
\hline 1950 & & & & & & 1 & 6 & 21 & 23 & 22 & 26 & 22 & 5 & 16 & 30 & 14 & 10 & 15 & 15 & 20 & 35 & 19 & 42 & 15 & 5 & 2 & \multicolumn{2}{|l|}{1} & 162386.0 \\
\hline 1951 & & 1 & 4 & 8 & 8 & 11 & 10 & 16 & 15 & 10 & 13 & 7 & 4 & 14 & 20 & 20 & 25 & 17 & 17 & 11 & 27 & 33 & 46 & 11 & 14 & 3 & & & 186188.3 \\
\hline 1952 & & & & 7 & 4 & 7 & $? ?$ & 21 & 19 & 6 & 1 & 9 & 13 & 29 & 10 & 22 & 22 & 16 & 12 & 25 & 49 & 40 & 10 & 9 & 7 & 6 & \multicolumn{2}{|l|}{1} & 160500.1 \\
\hline 1953 & & 1 & 2 & 3 & 4 & 4 & 12 & 6 & 32 & 23 & 26 & 16 & 16 & 9 & 15 & 10 & 19 & 43 & 30 & 11 & 24 & 16 & 12 & 11 & 16 & 4 & \multirow{3}{*}{\multicolumn{2}{|c|}{1}} & 146101.3 \\
\hline 1954 & & & & & & & & & 3 & 12 & 49 & 28 & 12 & 19 & 43 & 24 & 12 & 18 & 14 & 13 & 34 & 30 & 23 & 17 & 7 & 6 & & & 172234.0 \\
\hline 1955 & & & & & & 2 & 1 & 6 & 26 & 11 & 27 & 17 & 7 & 41 & 15 & 15 & 11 & 15 & 22 & 39 & 55 & 37 & 13 & 3 & 2 & & & & 125275.6 \\
\hline 1956 & & & & 3 & 2 & 6 & 10 & 11 & 20 & 20 & 20 & 8 & 7 & 15 & 29 & 11 & 10 & 17 & 20 & 15 & 21 & 37 & 35 & 25 & 14 & 5 & \multirow{2}{*}{\multicolumn{2}{|c|}{$\begin{array}{lll}2 & 2 & 1\end{array}$}} & 238075.3 \\
\hline 1957 & & & & & 1 & 4 & 5 & 18 & 26 & 20 & 27 & 9 & 7 & 10 & 38 & 24 & 25 & 37 & 25 & 23 & 31 & 14 & 11 & 7 & 2 & 1 & & & 103081.9 \\
\hline 1958 & & 5 & 6 & 11 & 11 & 10 & 6 & 5 & 18 & 19 & 20 & 15 & 4 & 16 & 22 & 20 & 9 & 15 & 33 & 20 & 19 & 31 & 22 & 21 & 6 & 1 & \multirow{2}{*}{\multicolumn{2}{|c|}{1}} & 149913.9 \\
\hline 1959 & & & & & & & 3 & 15 & 31 & 16 & 19 & 10 & 6 & 13 & 23 & 23 & 16 & 22 & 44 & 31 & 26 & 29 & 22 & 11 & 3 & 1 & & & 146081.4 \\
\hline 1960 & & & & & 1 & 3 & 9 & 15 & 31 & 12 & 10 & 11 & 5 & 7 & 8 & 39 & 18 & 48 & 39 & 24 & 28 & 22 & 22 & 8 & 5 & 1 & \multirow{2}{*}{\multicolumn{2}{|c|}{1}} & 139768.4 \\
\hline 1961 & 6 & 4 & 2 & 4 & 7 & 9 & 10 & 27 & 16 & 12 & 14 & 21 & 10 & 6 & 15 & 12 & 5 & 36 & 36 & 14 & 11 & 18 & 37 & 18 & 14 & & & & 170306.4 \\
\hline 1962 & 3 & 2 & 1 & 3 & 4 & 1 & 6 & 15 & 18 & 15 & 21 & 16 & 16 & 22 & 15 & 14 & 12 & 43 & 37 & 33 & 28 & 20 & 10 & 5 & 3 & 2 & \multirow{2}{*}{\multicolumn{2}{|c|}{1}} & 114714.9 \\
\hline 1963 & & & & & & & & 2 & 6 & 25 & 33 & 11 & 4 & 32 & 25 & 23 & 24 & 27 & 37 & 36 & 29 & 21 & 18 & 6 & 3 & 2 & & & 132023.2 \\
\hline
\end{tabular}

Tualatin River near Dilley, Oreg.

STATION NUMBER $\quad 14-2035.00$

Summary for water years $1940-63$

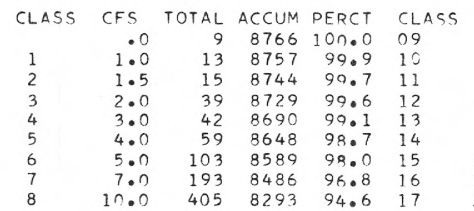

Tualatin River near Dilley, Oreg.

Sunmary for water years 1940-48

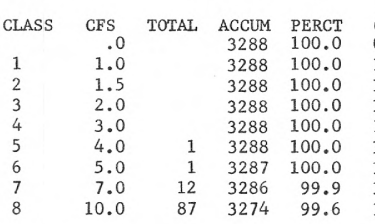

Tualatin River near Dilley, Oreg.

Summary for water years 1949-63

CLASS
1
2
3
4
5
6
7
8

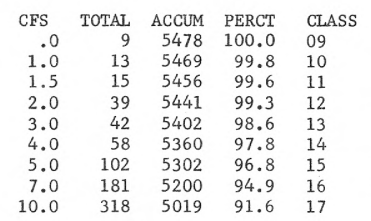

CFS TOTAL ACCUM PERCT

$15.0 \quad 427 \quad 788890.0$

$\begin{array}{llll}15.0 & 427 & 7888 & 90.0 \\ 20.0 & 642 & 7461 & 85.1\end{array}$

$\begin{array}{llll}20.0 & 642 & 7461 & 85.1 \\ 30.0 & 472 & 6819 & 77.8\end{array}$

$40.0 \quad 273 \quad 634772.4$

$\begin{array}{llll}40.0 & 471 & 6074 & 69.3\end{array}$

$70.0 \quad 538 \quad 5603 \quad 63.9$

100.0555506557 .8

$\begin{array}{llll}50.0 & 435 & 4510 & 51.4\end{array}$

$200.0 \quad 722 \quad 4075 \quad 46.5$

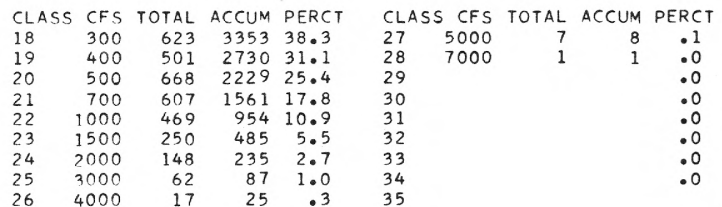

$14-2035.00$

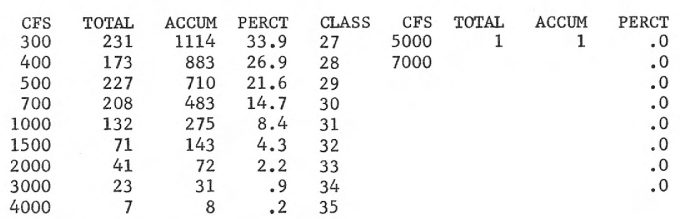

14-2035.00

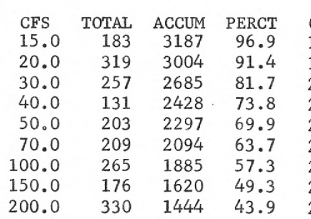

STATION NUMBER

CLASS
18
19
20
21
22
23
24
25
26

14714.9

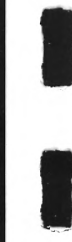

CFS TOTAL ACCUM PERCT CLASS

$\begin{array}{rrrrr}15.0 & 244 & 4701 & 85.8 & 18\end{array}$

$\begin{array}{lllll}20.0 & 323 & 4457 & 81.4 & 19\end{array}$

$\begin{array}{lllll}30.0 & 215 & 4134 & 75.5 & 20\end{array}$

$\begin{array}{lllll}40.0 & 142 & 3919 & 71.5 & 2 \\ 50.0 & 268 & 3777 & 68.9 & 22\end{array}$

70.0

100.0

$\begin{array}{lllll}150.0 & 259 & 2890 & 58.1 & 24 \\ 200.8 & 392 & 2631 & 48.0 & 26\end{array}$

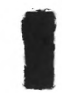

$\begin{array}{rrrrlrrrr}\text { CFS } & \text { TOTAL } & \text { ACCUM } & \text { PERCT } & \text { CLASS } & \text { CFS } & \text { TOTAL } & \text { ACCUM } & \text { PERCT } \\ 300 & 392 & 2239 & 40.9 & 27 & 5000 & 6 & 7 & .1 \\ 400 & 328 & 1847 & 33.7 & 28 & 7000 & 1 & 1 & .0 \\ 500 & 441 & 1519 & 27.7 & 29 & & & & .0 \\ 700 & 399 & 1078 & 19.7 & 30 & & & & .0 \\ 1000 & 337 & 679 & 12.4 & 31 & & & & .0 \\ 1500 & 179 & 342 & 6.2 & 32 & & & & .0 \\ 2000 & 107 & 163 & 3.0 & 33 & & & & .0 \\ 3000 & 39 & 56 & 1.0 & 34 & & & & .0 \\ 4000 & 10 & 17 & .3 & 35 & & & & \end{array}$


Tualatin River near Dilley, Oreg.

STATION NUMBER $14-2035.00$

LOWEST MEAN DISCHARGE, IN CFS, FOR THE FOLLOWING NUMBER OF CONSECUTIVE DAYS IN YEAR BEGINNING APRIL 1

\begin{tabular}{|c|c|c|c|c|c|c|c|c|c|c|c|}
\hline YEAR & 1 & 3 & 7 & 14 & 30 & 60 & 90 & 120 & 150 & 183 & 274 \\
\hline 1940 & 15.0 & $18 \cdot n$ & 19.9 & 22.1 & 25.6 & 29.0 & 32.8 & 35.3 & 44.7 & 66.3 & 182.0 \\
\hline 1941 & 4.0 & 6.7 & 8.7 & 12.6 & 14.5 & 20.4 & 33.6 & 39.4 & 49.8 & 65.2 & 222.0 \\
\hline 1942 & 8.0 & 9.4 & 10.0 & 11.1 & 13.7 & 16.4 & 19.2 & $26 \cdot 0$ & 40.4 & 58.8 & 298.0 \\
\hline 1943 & 11.0 & 12.0 & 13.0 & 14.1 & 15.3 & 18.8 & 21.8 & 29.0 & 41.1 & 62.7 & 148.0 \\
\hline 1944 & 10.0 & 10.3 & 11.0 & 11.8 & 13.9 & 16.6 & 18.7 & 20.9 & 30.0 & 45.2 & 99.4 \\
\hline 1945 & 14.0 & 14.7 & 15.4 & 16.9 & 17.6 & 23.1 & 28.3 & $29 \cdot 6$ & 36.8 & 68.2 & 295.0 \\
\hline 1946 & 9.0 & 12.3 & 13.9 & 14.4 & 15.8 & 17.6 & 20.2 & $27 \bullet 8$ & 36.8 & 49.1 & 276.0 \\
\hline 1947 & 17.0 & 17.0 & 18.3 & 19.9 & 25.3 & 27.6 & 30.9 & 39.3 & 48.2 & 70.3 & 210.0 \\
\hline 1948 & 9.0 & 10.3 & 12.4 & 16.2 & 21.5 & 27.1 & 35.7 & 43.0 & 53.6 & 85.1 & 340.0 \\
\hline 1949 & 6.0 & 7.3 & 9.3 & 10.3 & 12.1 & 14.8 & 16.9 & $21 \cdot 1$ & 26.3 & $41 \cdot 3$ & 167.0 \\
\hline 1950 & 4.0 & 5.3 & 6.9 & 7.8 & 9.3 & 12.3 & 15.7 & $27 \cdot 7$ & 43.4 & 69.2 & 322.0 \\
\hline 1951 & 1.2 & 2.0 & 2.4 & 3.7 & 5.1 & 5.7 & 9.2 & $19 \cdot 1$ & 33.3 & 58.4 & 305.0 \\
\hline 1952 & 1.2 & 1.5 & 3.0 & 3.6 & 4.1 & 6.3 & 6.3 & $7 \cdot 3$ & 10.0 & 17.1 & 88.3 \\
\hline 1953 & 12.0 & 12.7 & 13.4 & 14.8 & 20.8 & 23.5 & 27.7 & 35.8 & $44 \cdot 7$ & 76.5 & 256.0 \\
\hline 1954 & 11.0 & 14.3 & 15.1 & 17.1 & 18.9 & 23.2 & 24.5 & 33.6 & 45.5 & 53.6 & 181.0 \\
\hline 1955 & 4.2 & 5.1 & 7.3 & 9.1 & 10.8 & 15.7 & 22.4 & $32 \cdot 0$ & 55.9 & 102.0 & 497.0 \\
\hline 1956 & 2.3 & 3.1 & 4.5 & 6.0 & 8.3 & 10.6 & 12.5 & $17 \cdot 0$ & 27.0 & 43.5 & 137.0 \\
\hline 1957 & 3.8 & 4.0 & 4.6 & 6.5 & 7.7 & 11.8 & 14.7 & 18.2 & 24.8 & 41.1 & 201.0 \\
\hline 1958 & 1.0 & 1.2 & 1.4 & 1.8 & 2.5 & 4.7 & 7.7 & $12 \cdot 5$ & 23.6 & 46.1 & 222.0 \\
\hline 1959 & 5.5 & 6.7 & 7.3 & 8.8 & 10.3 & 17.8 & 28.5 & $45 \cdot 3$ & 65.5 & 78.5 & 177.0 \\
\hline 1960 & 3.4 & 4.7 & 6.3 & 6.6 & 7.8 & 11.0 & 12.1 & 17.4 & 27.3 & 74.1 & 249.0 \\
\hline 1961 & .4 & .6 & 1.8 & 2.3 & 3.2 & 6.0 & 7.2 & 13.0 & 23.7 & 37.8 & 215.0 \\
\hline 1962 & .3 & .8 & 2.8 & 5.5 & 10.8 & 13.9 & 17.3 & $28 \cdot 3$ & 50.5 & 73.9 & 241.0 \\
\hline
\end{tabular}

Tualatin River near Dilley, Oreg.

STATION NUMRER 14-7USOUUU

HIGHEST MEAN DISCHARGF, IN CFS, FOR THE FOLLOWING NUMBER OF CONSECUTIVE DAYS IN YEAR ENDING SEPTEMBER 30

\begin{tabular}{|c|c|c|c|c|c|c|c|c|c|c|c|}
\hline YEAR & 1 & 3 & 7 & 15 & 30 & 60 & 90 & 120 & 150 & 183 & 274 \\
\hline 1940 & 3780.0 & 3260.0 & 2320.0 & 1820.0 & 1520.0 & 1050.0 & 1010.0 & 891.0 & 785.0 & 682.0 & 472.0 \\
\hline 1941 & 3790.0 & 2630.0 & 1660.0 & 1210.0 & 908.0 & 760.0 & 617.0 & $521 \cdot 0$ & 453.0 & 400.0 & 297.0 \\
\hline 1942 & 5280.0 & 3780.0 & 2730.0 & 1750.0 & 1190.0 & 992.0 & 829.0 & 705.0 & $608 \cdot 0$ & 523.0 & 383.0 \\
\hline 1943 & 4140.0 & 3300.0 & 2850.0 & 1850.0 & 1430.0 & 1350.0 & 1230.0 & $1050 \cdot 0$ & 998.0 & 871.0 & 610.0 \\
\hline 1944 & 1990.0 & $1620 . n$ & 1090.0 & 690.0 & 588.0 & 491.0 & 476.0 & $445 \cdot 0$ & 420.0 & 374.0 & 282.0 \\
\hline 1945 & 4040.0 & 2630.0 & 2010.0 & 1530.0 & 1070.0 & 968.0 & 843.0 & $729 \cdot 0$ & 629.0 & 545.0 & 391.0 \\
\hline 1946 & 4460.0 & 3160.0 & 2240.0 & 2020.0 & 1410.0 & 1270.0 & 1230.0 & $1110 \cdot 0$ & 998.0 & 863.0 & 597.0 \\
\hline 1947 & 4520.0 & 4100.0 & 2960.0 & 2100.0 & 1680.0 & 1120.0 & 1150.0 & $1020 \cdot 0$ & 881.0 & 750.0 & 527.0 \\
\hline 1948 & 3800.0 & $3110 . n$ & $2430 . n$ & 1910.0 & 1300.0 & 1090.0 & 957.0 & $862 \cdot 0$ & 801.0 & 730.0 & 582.0 \\
\hline 1949 & 6570.0 & 4660.0 & 4090.0 & 3030.0 & 2050.0 & 1320.0 & 1280.0 & 1170.0 & 1040.0 & 893.0 & 625.0 \\
\hline 1950 & 4300.0 & 3710.0 & 2470.0 & 2030.0 & 1750.0 & 1390.0 & 1220.0 & 1090.0 & 984.0 & 846.0 & 588.0 \\
\hline 1951 & 3560.0 & 2990.0 & 2750.0 & 2340.0 & 1920.0 & 1480.0 & 1370.0 & $1200 \cdot 0$ & 1090.0 & 961.0 & 676.0 \\
\hline 1952 & 4590.0 & 3640.0 & 2970.0 & 2070.0 & 1500.0 & 1070.0 & 1140.0 & $1020 \cdot 0$ & 937.0 & 824.0 & 582.0 \\
\hline 1953 & 3220.0 & 3130.0 & 2900.0 & 2500.0 & 2220.0 & 1520.0 & 1230.0 & 1010.0 & 874.0 & 762.0 & 528.0 \\
\hline 1954 & 5570.0 & 3810.0 & 2750.0 & 2490.0 & 1740.0 & 1500.0 & 1350.0 & $1190 \cdot 0$ & 1040.0 & 890.0 & 620.0 \\
\hline 1955 & 2260.0 & 1890.0 & 1320.0 & 895.0 & 831.0 & 715.0 & 686.0 & 699.0 & 662.0 & 628.0 & 450.0 \\
\hline 1956 & 8130.0 & 5990.0 & 3870.0 & 2690.0 & 2340.0 & 2070.0 & 1650.0 & $1490 \cdot 0$ & 1410.0 & 1240.0 & 864.0 \\
\hline 1957 & 3280.0 & 2540.0 & 1790.0 & 1470.0 & 1230.0 & 868.0 & 707.0 & 653.0 & 591.0 & 514.0 & 372.0 \\
\hline 1958 & 3140.0 & 2450.0 & 1930.0 & 1650.0 & 1500.0 & 1300.0 & 1180.0 & $991 \cdot 0$ & 908.0 & 781.0 & 544.0 \\
\hline 1959 & 4610.0 & 3610.0 & 2780.0 & $1800 \cdot 0$ & 1510.0 & 1190.0 & 986.0 & $904 \cdot 0$ & 826.0 & 732.0 & 525.0 \\
\hline 1960 & 3310.0 & 2790.0 & 2230.0 & 2020.0 & 1510.0 & 1070.0 & 945.0 & $810 \cdot 0$ & 743.0 & 676.0 & 505.0 \\
\hline 1961 & 4360.0 & 3110.0 & 2450.0 & 2060.0 & 1790.0 & 1540.0 & 1290.0 & $1130 \cdot 0$ & 1020.0 & 894.0 & 619.0 \\
\hline 1962 & 3720.0 & 3160.0 & 2330.0 & 1670.0 & 1110.0 & 779.0 & 666.0 & $669 \cdot 0$ & 623.0 & 580.0 & 414.0 \\
\hline 1963 & 4620.0 & 3410.0 & 2100.0 & 1360.0 & 1000.0 & 734.0 & 716.0 & $640 \cdot 0$ & 671.0 & 641.0 & 473.0 \\
\hline
\end{tabular}


Gales Creek near Gales Creek, Oreg.

STATION NUMBER $\quad 14-2040.00$

DURATION TABLE OF DAILY DISCHARGE

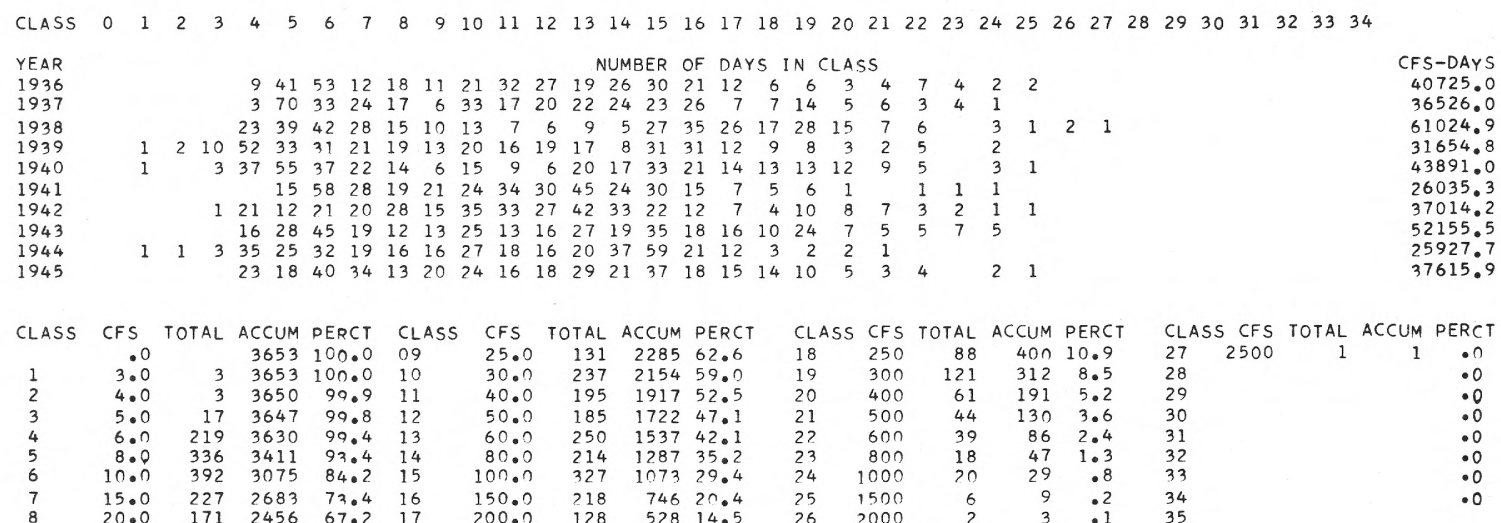

Gales Creek near Gales Creek, Oreg.

STATION NUMBER $\quad 14-204 \cap .00$

LOWEST MEAN DISCHARGE, IN CFS, FOR THE FOLLOWING NUMBER OF CONSECUTIVE DAYS IN YEAR BEGINNING APRIL I

\begin{tabular}{|c|c|c|c|c|c|c|c|c|c|c|c|}
\hline YEAR & 1 & 3 & 7 & 14 & 30 & 60 & 90 & 120 & $15 n$ & 183 & 274 \\
\hline 1936 & 6.0 & 6.7 & 7.4 & 7.8 & 7.9 & 8.0 & 8.3 & 8.8 & 10.6 & 14.6 & 33.3 \\
\hline 1937 & 9.0 & 9.0 & 9.1 & 9.5 & 11.3 & 12.3 & 13.4 & $15 \cdot 4$ & 27.7 & 30.3 & 141.0 \\
\hline 1938 & 6.0 & 6.3 & 6.5 & 6.9 & 7.6 & 8.1 & 8.8 & 10.0 & 12.6 & 18.5 & 50.0 \\
\hline 1939 & 3.4 & 4.9 & 5.4 & 5.7 & 6.1 & 6.4 & 7.1 & 7.9 & 8.4 & 9.8 & 41.4 \\
\hline 1940 & 3.7 & 5.0 & 7.0 & 7.3 & 7.6 & 8.5 & 9.6 & 11.0 & 14.2 & 18.8 & 52.6 \\
\hline 1941 & 8.9 & 8.9 & 9.1 & 9.5 & 10.5 & 12.8 & 14.6 & 17.2 & 20.7 & 27.5 & 81.4 \\
\hline 1942 & 5.9 & 6.7 & 6.9 & 7.4 & 7.8 & 8.5 & 9.7 & $12 \cdot 5$ & 17.1 & 22.9 & 88.5 \\
\hline 1943 & 7.6 & 8.0 & 8.2 & 8.3 & 8.7 & 10.2 & 11.5 & 14.3 & 18.5 & 24.4 & 48.7 \\
\hline 1944 & 3.1 & 5.4 & 5.8 & 6.3 & 6.9 & 7.5 & 7.9 & 9.1 & 12.0 & 15.5 & 33.3 \\
\hline
\end{tabular}

Gales Creek near Gales Creek, Oreg.

STATION NUMBER

$14-2040.00$

HIGHEST MEAN DISCHARGE, IN CFS, FOR THE FOLLOWING NUMBER OF CONSECUTIVE DAYS IN YEAR ENDING SEPTEMBER 30

\begin{tabular}{|c|c|c|c|c|c|c|c|c|c|c|c|}
\hline YEAR & 1 & 3 & 7 & 15 & 30 & 60 & 90 & 120 & 150 & 183 & 274 \\
\hline 1936 & 1900.0 & 1380.0 & 999.0 & 886.0 & 583.0 & 389.0 & 327.0 & 275.0 & 234.0 & 203.0 & 145.0 \\
\hline 1937 & 1020.0 & 980.0 & 669.0 & 512.0 & 444.0 & 351.0 & 289.0 & 237.0 & 210.0 & 181.0 & 130.0 \\
\hline 1938 & 2560.0 & $2390 \cdot n$ & $1600 . ?$ & 882.7 & 615.0 & 469.0 & $4 \cap 8.0$ & 375.0 & 362.0 & 317.0 & 220.0 \\
\hline 1939 & 1170.0 & $888 . n$ & 761.0 & $473 . ?$ & 343.0 & 279.0 & 245.0 & 217.0 & 186.0 & 161.0 & 113.0 \\
\hline 1940 & 1520.0 & $1130 . n$ & $789 . ?$ & $614 . ?$ & $499 . ?$ & 341.0 & 341.0 & 296.0 & $26 ? .0$ & 227.0 & 157.0 \\
\hline 1941 & 1200.0 & $940 . n$ & $574 . ?$ & 409.0 & 282.0 & 224.0 & 176.0 & $150 \cdot 0$ & 133.0 & 120.0 & 90.5 \\
\hline 1942 & 1590.0 & 1240.0 & $89 ? .0$ & 565.0 & 406.0 & 319.0 & 275.0 & 235.0 & 205.0 & 178.0 & 131.0 \\
\hline 1943 & 1410.0 & $1120 \cdot n$ & $846 . ?$ & 609.0 & 405.0 & 375.0 & 360.0 & 307.0 & 300.0 & 262.0 & 187.0 \\
\hline 1944 & 565.0 & 454.0 & 298.0 & 205.0 & 201.0 & 164.0 & 149.0 & 142.0 & 136.0 & 121.0 & 91.7 \\
\hline 45 & 15 & 28 & 0 & 549.0 & 70.0 & 1.0 & 2 & 24 & 21 & 18 & 33 \\
\hline
\end{tabular}


CLASS $00 \begin{array}{lllllllllllllllllllllllllllllllllllll} & 1 & 2 & 3 & 4 & 5 & 6 & 7 & 8 & 9 & 10 & 11 & 12 & 13 & 14 & 15 & 16 & 17 & 18 & 19 & 20 & 21 & 22 & 23 & 24 & 25 & 26 & 27 & 28 & 29 & 30 & 31 & 32 & 33 & 34\end{array}$

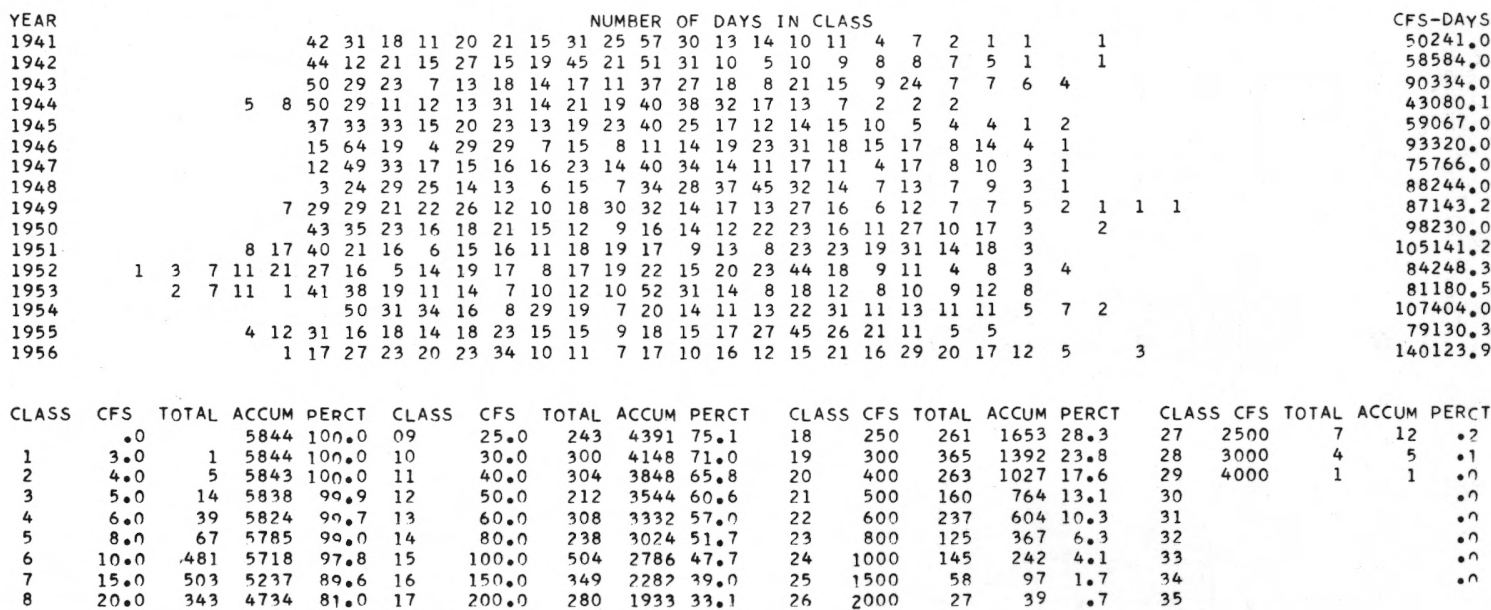

Gales Creek near Forest Grove, Oreg.

STATION NUMBER

$14-7045.00$

LOWEST MEAN DISCHARGE, IN CFS, FOR THE FOLLOWING NUMBER OF CONSECUTIVE DAYS IN YEAR BEGINNING APRIL 1

\begin{tabular}{|c|c|c|c|c|c|c|c|c|c|c|c|}
\hline YEAR & 1 & 3 & 7 & 14 & 30 & 60 & 90 & 120 & $15 n$ & 183 & 274 \\
\hline 1941 & 11.0 & 11.0 & 11.0 & 11.6 & 12.8 & 16.9 & 20.2 & $24 \cdot 2$ & 28.8 & 39.5 & 128.0 \\
\hline 1942 & 10.0 & $10 . n$ & 10.4 & $11 \cdot 1$ & 11.6 & 12.7 & 14.0 & 17.4 & 24.1 & 32.7 & 151.0 \\
\hline 1943 & 11.0 & 11.0 & 11.0 & 11.4 & 11.9 & 14.0 & 16.3 & $21 \cdot 1$ & 28.0 & 36.9 & 79.8 \\
\hline 1944 & 7.0 & 7.3 & 7.5 & 8.6 & 10.3 & 12.0 & 12.8 & $14 \cdot 2$ & 18.5 & 24.2 & 51.5 \\
\hline 1945 & 12.0 & 12.0 & 12.7 & 13.1 & 14.1 & 17.9 & 21.1 & $22 \cdot 1$ & 25.7 & 41.0 & 170.0 \\
\hline 1946 & 13.0 & 13.7 & 13.9 & 14.5 & 15.9 & 16.2 & 17.5 & $22 \cdot 3$ & 27.8 & 37.8 & 156.0 \\
\hline 1947 & 10.0 & 11.3 & 13.9 & 15.1 & 16.6 & 17.5 & 19.2 & $22 \cdot 4$ & 28.5 & 39.9 & 113.0 \\
\hline 1948 & 14.0 & 14.0 & 15.0 & 16.3 & 17.7 & 20.4 & 23.6 & $26 \cdot 2$ & 32.9 & 52.0 & 166.0 \\
\hline 1949 & 8.0 & 9.4 & 9.5 & 10.1 & 11.8 & 13.4 & 15.9 & 18.1 & 21.0 & 29.2 & 103.0 \\
\hline 1950 & 11.0 & 11.3 & 11.7 & 12.1 & 12.5 & 14.7 & 17.6 & 23.4 & 29.0 & 41.0 & 186.0 \\
\hline 1951 & 6.4 & 7.4 & 7.5 & 8.9 & 10.5 & 10.7 & 12.9 & 17.5 & 25.0 & 36.5 & 156.0 \\
\hline 1952 & 3.7 & 4.4 & 5.0 & 6.0 & 6.1 & 7.7 & 8.5 & 9.8 & 11.7 & 15.4 & 52.9 \\
\hline 1953 & 11.0 & 12.3 & $13 . n$ & 14.0 & 16.0 & 18.5 & 21.8 & 25.7 & 30.9 & 46.2 & 154.0 \\
\hline 1954 & 14.0 & 14.3 & 15.3 & 15.7 & 17.5 & 18.9 & 19.5 & $21 \cdot 7$ & 27.3 & 33.0 & 115.0 \\
\hline 1955 & 6.4 & 6.8 & 7.8 & 9.1 & 9.9 & 12.2 & 17.4 & $25 \cdot 5$ & 38.1 & 58.4 & 280.0 \\
\hline
\end{tabular}




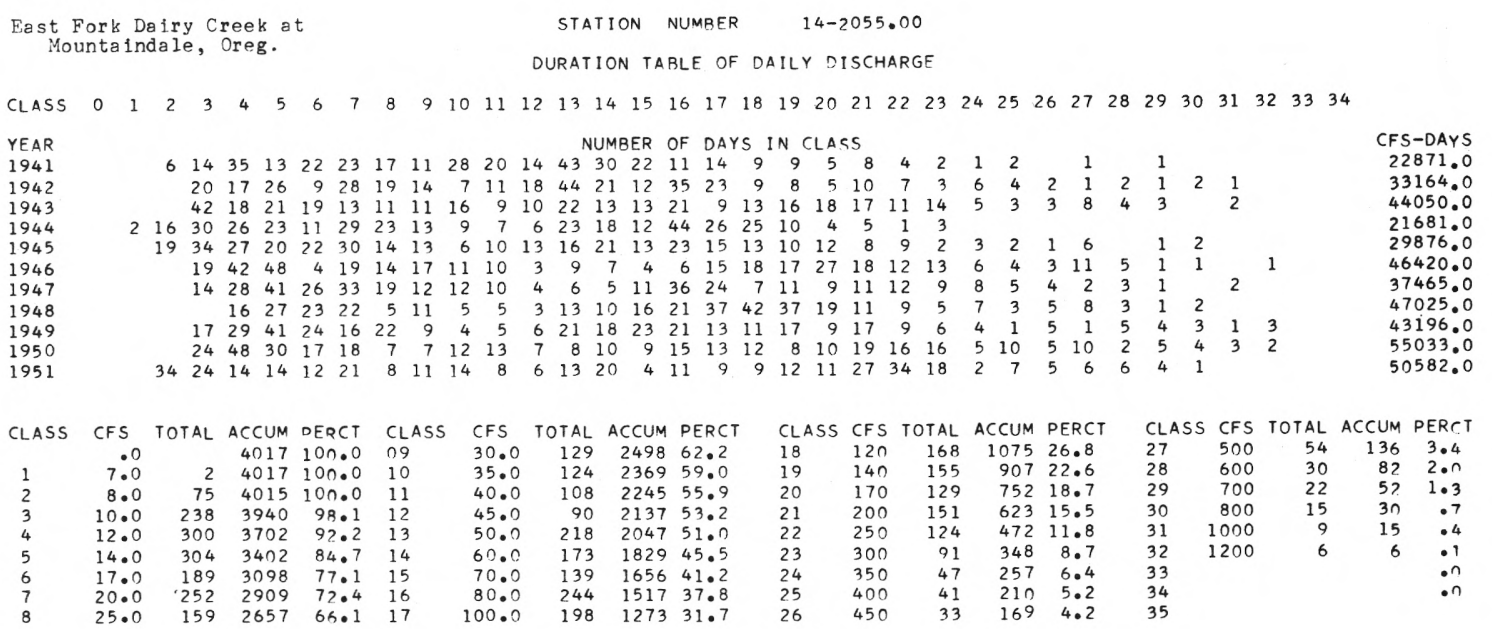

East Fork Dairy Creek at STATION NUMRER 14-2055.00

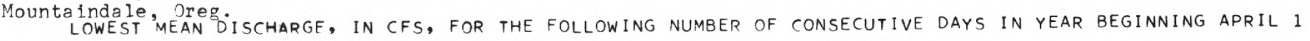

\begin{tabular}{|c|c|c|c|c|c|c|c|c|c|c|c|}
\hline YEAR & 1 & 3 & 7 & 14 & 30 & 60 & 90 & 120 & 150 & 183 & 274 \\
\hline 1941 & 9.0 & $9 . n$ & 9.1 & 9.8 & 11.1 & 13.2 & 14.2 & $16 \cdot 2$ & 18.5 & 23.0 & 62.6 \\
\hline 1942 & 10.0 & 10.0 & 10.0 & 10.4 & 10.7 & 11.3 & 12.6 & $15 \cdot 7$ & 19.9 & 26.0 & 72.4 \\
\hline 1943 & 10.0 & 10.3 & 10.7 & 10.8 & 11.0 & $1 ? \cdot 2$ & 13.7 & $17 \cdot 0$ & $20 \cdot 1$ & 23.4 & 42.8 \\
\hline 1944 & 7.0 & 7.3 & 7.7 & 8.4 & 9.4 & 10.4 & 10.7 & $11 \cdot 8$ & 13.9 & 16.7 & 29.4 \\
\hline 1945 & 10.0 & 10.0 & $10 \cdot 1$ & 10.3 & 11.1 & 12.2 & 12.5 & $13 \cdot 6$ & 16.5 & $24 \cdot 2$ & 74.6 \\
\hline 1946 & 11.0 & 11.7 & 11.9 & 12.5 & 12.7 & $12 \cdot 8$ & 13.5 & $16 \cdot 0$ & 18.0 & 20.6 & 78.5 \\
\hline 1947 & 11.0 & 11.0 & 11.0 & 11.1 & $12 \cdot 1$ & 13.0 & 14.3 & $16 \cdot 3$ & 19.2 & 25.3 & 58.0 \\
\hline 1948 & 12.0 & 12.0 & 12.6 & 13.1 & 14.0 & 15.4 & 16.1 & $18 \cdot 3$ & $22 \cdot 2$ & 32.1 & 82.2 \\
\hline 1949 & 10.0 & $10 . n$ & 10.3 & 10.6 & 11.2 & 11.7 & 12.3 & $13 \cdot 5$ & 15.3 & 18.7 & 47.6 \\
\hline 1950 & 11.0 & 11.0 & 11.4 & 11.7 & 12.2 & 13.1 & 15.1 & $18 \cdot 5$ & 22.5 & 30.3 & 92.3 \\
\hline
\end{tabular}

East Fork Dairy Creek at
Mountaindale, Oreg.

STATION NUMBER $\quad 14-2055.00$

HIGHEST MEAN DISCHARGE, IN CFS, FOR THE FOLLOWING NUMBER OF CONSECUTIVE DAYS IN YEAR ENDING SEPTEMBER 30

YEAR
1941
1942
194
194
194
194
194
194
194
1950
1951

$\begin{array}{rc}1 & 3 \\ 775.0 & 585.0 \\ 1060.0 & 865.0 \\ 1010.0 & 904.0 \\ 323.0 & 298.0 \\ 949.0 & 749.0 \\ 1330.0 & 996 . n \\ 1060.0 & 805 . n \\ 989.0 & 769.0 \\ 1330.0 & 1200.0 \\ 1260.0 & 1210.0 \\ 835.0 & 752.0\end{array}$

7
414.0
652.0
$629 \cdot 0$
$229 \cdot 0$
$623 \cdot 0$
$725 \cdot 0$
648.0
651.0
956.0
927.0

15
291.0
450.0
446.0
175.0
477.0
537.0
484.0
527.0
743.0
851.0

30
217.0
304.0
327.0
156.0
326.0
376.0
404.0
338.0
535.0
695.0

60
185.0
281.0
300.0
136.0
268.0
370.0
265.0
307.0
347.0
511.0
397.0

90
149.0
234.0
290.0
124.0
227.0
329.0
272.0
270.0
298.0
431.0
350.0

120
$128 \cdot 0$
$201 \cdot 0$
$248 \cdot 0$
$115 \cdot 0$
$194 \cdot 0$
$301 \cdot 0$
$245 \cdot 0$
$238 \cdot 0$
$281 \cdot 0$
$379 \cdot 0$
$316 \cdot 0$

150
113.0
176.0
247.0
109.0
168.0
271.0
215.0
224.0
246.0
326.0
292.0

183

$101.0 \quad 78.6$

$\begin{array}{ll}154.0 & 116.0 \\ 217.0 & 157.0\end{array}$

98.9
$145.0 \quad 75.2$

234.0165 .0

$185.0 \quad 132.0$

$206.0 \quad 166.0$

490.0

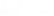

(350.0

(31600

292.0

$\begin{array}{ll}214.0 & 153.0 \\ 281.0 & 197.0 \\ 253.0 & 181.0\end{array}$ 
McKay Creek near North Plains, Oreg.

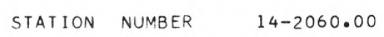

DURATION TABLE OF DAILY DISCHARGE CLASS $0 \begin{array}{lllllllllllllllllllllllllllllllllll} & 0 & 2 & 3 & 4 & 5 & 6 & 7 & 8 & 9 & 10 & 11 & 12 & 13 & 14 & 15 & 16 & 17 & 18 & 19 & 20 & 21 & 22 & 23 & 24 & 25 & 26 & 27 & 28 & 29 & 30 & 31 & 32 & 33 & 34\end{array}$

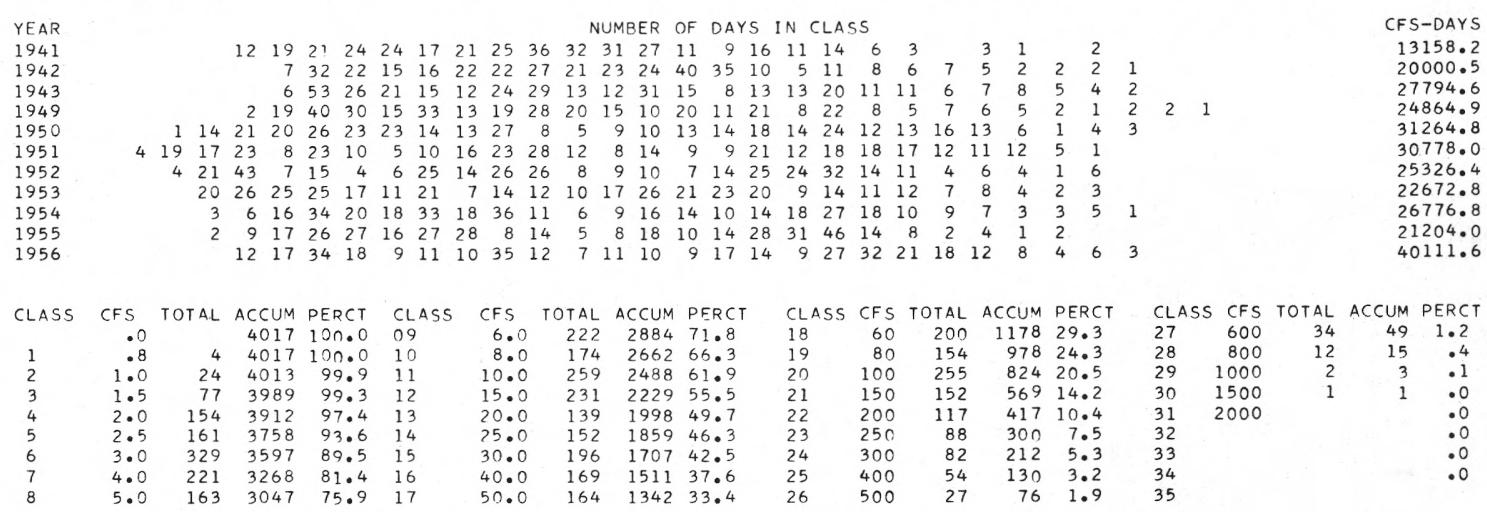

McKay Creek near North Plains, Oreg.

STATION NUMBER $\quad 14-2060.00$

LOWEST MEAN DISCHARGE, IN CFS, FOR THE. FOLLOWING NUMBER OF CONSECUTIVE DAYS IN YEAR BEGINNING APRIL 1

\begin{tabular}{|c|c|c|c|c|c|c|c|c|c|c|c|}
\hline YEAR & 1 & 3 & 7 & 14 & 30 & 60 & 90 & 120 & 150 & 183 & 274 \\
\hline 1941 & 2.0 & $2 \cdot 1$ & $2 \cdot 3$ & 2.4 & 2.6 & 3.5 & 4.1 & 4.9 & 5.8 & 7.6 & 38.9 \\
\hline 1942 & 2.6 & 2.6 & 2.7 & 2.9 & 3.2 & 3.5 & 3.9 & 4.9 & 6.4 & 10.9 & 47.9 \\
\hline 1949 & 2.4 & 2.4 & 2.5 & 2.8 & 3.0 & 3.2 & 3.6 & $4 \cdot 0$ & 4.5 & 5.6 & 21.9 \\
\hline 1950 & 1.4 & 1.6 & 1.7 & 1.8 & 2.0 & 2.4 & 2.9 & $4 \cdot 4$ & 5.7 & 8.9 & 54.7 \\
\hline 1951 & .8 & .9 & 1.0 & 1.2 & 1.5 & 1.7 & 2.1 & $3 \cdot 1$ & 4.6 & 6.8 & 44.2 \\
\hline 1952 & $1 \cdot 1$ & 1.3 & 1.5 & 1.8 & 1.8 & 2.1 & 2.1 & $2 \cdot 3$ & 2.8 & 3.6 & 10.1 \\
\hline 1953 & 1.5 & 1.7 & 1.8 & 2.0 & 2.5 & 3.0 & 3.6 & $4 \cdot 5$ & 5.9 & 10.7 & 41.1 \\
\hline 1954 & 1.5 & 1.7 & 2.1 & 2.4 & 2.9 & 3.5 & 3.7 & $4 \cdot 3$ & 5.0 & 6.1 & 28.5 \\
\hline 1955 & 1.8 & 1.9 & 2.4 & 2.6 & 2.6 & 3.5 & 4.3 & 5.5 & 7.8 & 13.2 & 80.1 \\
\hline
\end{tabular}

McKay Creek near North Plains, Oreg.

STATION NUMBER 14-2060.00

HIGHEST MEAN DISCHARGE, IN CFS, FOR THE FOLLOWING NUMBER OF CONSECUTIVE DAYS IN YEAR ENDING SEPTEMBER 30

\begin{tabular}{|c|c|c|c|c|c|c|c|c|c|c|c|}
\hline YEAR & 1 & 3 & 7 & 15 & 30 & 60 & 90 & 120 & 150 & 183 & 274 \\
\hline 1941 & 723.0 & 581.0 & 367.0 & 230.0 & 173.0 & 135.0 & 106.0 & 86.8 & 74.6 & $\begin{array}{l}64.4 \\
97.7\end{array}$ & $\begin{array}{l}46.6 \\
71.4\end{array}$ \\
\hline 1942 & 960.0 & $770 \cdot 0$ & 557.0 & 352.0 & 235.0 & $189 \cdot 0$ & $157 \cdot 0$ & $132 \cdot 0$ & 113.0 & & $\begin{array}{r}71.4 \\
100.0\end{array}$ \\
\hline 1943 & $\begin{array}{r}965.0 \\
1580.0\end{array}$ & $\begin{array}{l}797.0 \\
933.0\end{array}$ & 549.0 & 340.0 & 253.0 & 218.0 & $\begin{array}{l}208.0 \\
205.0\end{array}$ & $\begin{array}{l}169 \cdot 0 \\
177.0\end{array}$ & $\begin{array}{l}169.0 \\
152.0\end{array}$ & $\begin{array}{l}144.0 \\
129.0\end{array}$ & $\begin{array}{r}100.0 \\
89.5\end{array}$ \\
\hline $\begin{array}{l}1949 \\
1950\end{array}$ & 946.0 & $\begin{array}{l}933.0 \\
847.0\end{array}$ & $\begin{array}{l}767 \cdot 0 \\
649.0\end{array}$ & $\begin{array}{l}561.0 \\
593.0\end{array}$ & $\begin{array}{l}344 \cdot 0 \\
442.0\end{array}$ & 309.0 & 261.0 & $230 \cdot 0$ & 196.0 & 166.0 & 113.0 \\
\hline 1951 & 604.0 & 540.0 & 472.0 & 388.0 & 303.0 & 251.0 & 240.0 & $206 \cdot 0$ & 190.0 & 162.0 & 112.0 \\
\hline 1952 & 786.0 & 662.0 & 576.0 & 384.0 & 256.0 & 187.0 & 196.0 & 174.0 & 154.0 & 132.0 & 91.6 \\
\hline 1953 & 682.0 & 652.0 & 559.0 & 441.0 & 362.0 & 238.0 & 195.0 & $161 \cdot 0$ & 137.0 & 118.0 & 81.8 \\
\hline 1954 & 825.0 & 662.0 & 443.0 & 399.0 & 295.0 & 243.0 & 223.0 & $191 \cdot 0$ & 166.0 & 140.0 & 96.4 \\
\hline 1955 & 536.0 & 412.0 & 275.0 & 180.0 & 159.0 & 144.0 & 129.0 & $124 \cdot 0$ & 119.0 & 109.0 & 76.0 \\
\hline 1956 & 869.0 & 773.0 & 559.0 & 402.0 & 372.0 & 336.0 & 273.0 & 254.0 & 239.0 & 209.0 & 145.0 \\
\hline
\end{tabular}


Tualatin River at Farmington, Oreg.

STATION NUMRFR

$14-9065.00$

DURATION TARLF OF DAILY DISCHARGE CLASS $0 \begin{array}{lllllllllllllllllllllllllllllllllllllllll} & 1 & 2 & 3 & 4 & 5 & 6 & 7 & 8 & 9 & 10 & 11 & 12 & 13 & 14 & 15 & 16 & 17 & 18 & 19 & 20 & 21 & 22 & 23 & 24 & 25 & 26 & 27 & 28 & 29 & 30 & 31 & 32 & 33 & 34\end{array}$ YEAR
1940
1941
1942
1943
1944
1945
1946
1947
1948
1949
1950
1951
1952
1953
1954
1955
1956
1957
1958

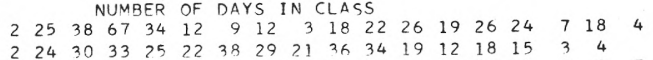

$\begin{array}{llllllllllllllllllllll}2 & 24 & 30 & 33 & 25 & 22 & 38 & 29 & 21 & 26 & 34 & 19 & 12 & 18 & 15 & 3 & 4 & & \\ 12 & 33 & 21 & 20 & 23 & 33 & 29 & 36 & 32 & 29 & 33 & 13 & 17 & 11 & 5 & 8 & 7 & 3\end{array}$ $\begin{array}{rrrrrrrrrrrrrrrrrr}12 & 33 & 21 & 20 & 23 & 33 & 29 & 36 & 32 & 29 & 33 & 13 & 17 & 11 & 5 & 8 & 7 & 3 \\ 8 & 43 & 42 & 17 & 13 & 19 & 20 & 18 & 30 & 26 & 19 & 7 & 26 & 17 & 15 & 26 & 13 & 6\end{array}$ $\begin{array}{llllllllllllllllllll}2 & 13 & 11 & 40 & 25 & 26 & 13 & 43 & 15 & >0 & 28 & 42 & 4 & 25 & 17 & 4\end{array}$ $\begin{array}{rrrrrrrrrrrrrrrrrr}8 & 49 & 44 & 24 & 22 & 29 & 18 & 22 & 25 & 25 & 31 & 13 & 24 & 10 & 8 & 10 & 3 \\ 7 & 10 & 58 & 20 & 23 & 26 & 30 & 10 & 8 & 8 & 11 & 18 & 23 & 39 & 31 & 15 & 17 & 10\end{array}$ $\begin{array}{llllllllllllllllllll}1 & 37 & 51 & 28 & 25 & 40 & 16 & 10 & 7 & 28 & 38 & 9 & 20 & 20 & 15 & 14 & 5 & 1\end{array}$ $\begin{array}{lllllllllllllllllllllll}4 & 30 & 39 & 13 & 44 & 14 & 21 & 11 & 17 & 32 & 21 & 17 & 22 & 27 & 13 & 5 & 17 & 8 & 9 & 1\end{array}$ $\begin{array}{lllllllllllllllllllll}12 & 34 & 10 & 18 & 33 & 21 & 16 & 32 & 9 & 6 & 8 & 22 & 14 & 13 & 28 & 25 & 18 & 29 & 14 & 3\end{array}$ $\begin{array}{lllllllllllllllllllllllll}5 & 16 & 15 & 20 & 17 & 7 & 11 & 15 & 5 & 19 & 17 & 13 & 17 & 19 & 10 & 12 & 22 & 11 & 38 & 48 & 13 & 10 & 4\end{array}$ $\begin{array}{lllllllllllllllllllllllll}12 & 13 & 21 & 19 & 13 & 3 & 3 & 5 & 24 & 17 & 24 & 21 & 15 & 13 & 12 & 13 & 16 & 80 & 19 & 4 & 10 & 5 & 4\end{array}$ $\begin{array}{rrrrrrrrrrrrrrrrrrrrrr}25 & 26 & 15 & 25 & 31 & 21 & 8 & 9 & 14 & 12 & 32 & 40 & 29 & 5 & 22 & 18 & 4 & 16 & 8 & 5 \\ & 1 & 9 & 21 & 23 & 43 & 20 & 45 & 26 & 6 & 12 & 18 & 11 & 17 & 28 & 24 & 17 & 23 & 16 & 5\end{array}$

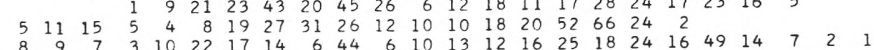
$\begin{array}{rrrrrrrrrrrrrrrrrrrrrrrrrrrr}3 & 4 & 6 & 8 & 9 & 7 & 3 & 10 & 22 & 17 & 14 & 6 & 44 & 6 & 10 & 13 & 12 & 16 & 25 & 18 & 24 & 16 & 49 & 14 \\ 1 & & & & 3 & 8 & 28 & 15 & 7 & 35 & 11 & 13 & 18 & 34 & 15 & 17 & 24 & 33 & 31 & 36 & 14 & 3 & 9 & 13 & 1 \\ 1 & & & 5 & 4 & 5 & 5 & 24 & 28 & 15 & 16 & 32 & 15 & 6 & 16 & 15 & 36 & 18 & 19 & 18 & 18 & 26 & 3\end{array}$

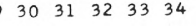

CFS-DAYS 440062.0 268869.0 390662.0 618259.0 238406.0 344317.0 567625.0 442616.0 540573.0 547745.0 637711.0 673246.2 510943.2 467859.0 640464.0 405332.0 851689.6 339140.0
479086.6
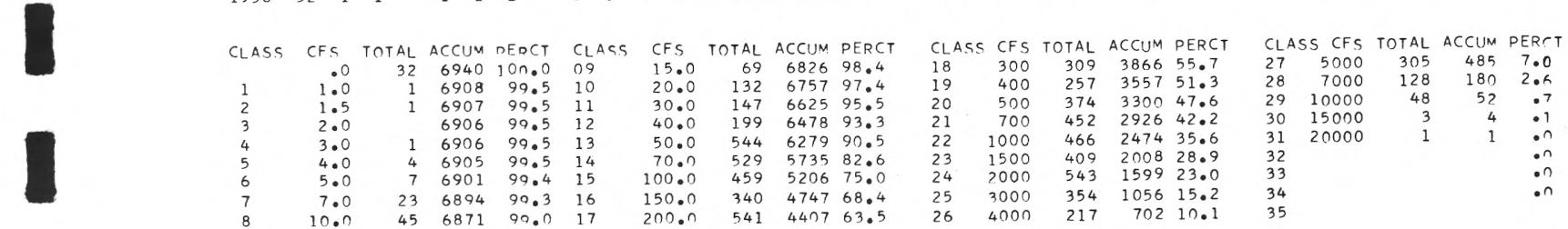

Tualatin River at Farmington, Oreg.

STATION NUMBER

$14-2065.00$

LOWEST MEAN DISCHARGE, IN CFS, FOR THE FOLLOWING NUMBER OF CONSECUTIVE DAYS IN YEAR BEGINNING APRIL 1

\begin{tabular}{|c|c|c|c|c|c|c|c|c|c|c|c|}
\hline YEAR & 1 & 3 & 7 & 14 & 30 & 60 & 90 & 120 & 150 & 183 & 274 \\
\hline 1940 & 37.0 & 39.3 & 43.0 & 43.7 & 49.0 & 57.7 & 65.0 & 73.3 & 99.3 & 140.0 & 521.0 \\
\hline 1941 & 35.0 & $38 \cdot 0$ & 40.4 & $44 \cdot 2$ & 47.4 & 58.9 & $78 \cdot 6$ & $98 \cdot 2$ & 125.0 & $170 \cdot 0$ & 708.0 \\
\hline 1942 & 45.0 & $45 \cdot 3$ & 45.6 & $46 \cdot 5$ & 51.9 & 57.0 & 64.7 & 83.4 & 126.0 & 189.0 & 907.0 \\
\hline 1943 & 45.0 & 45.7 & 46.3 & 49.9 & 53.7 & 65.5 & 74.5 & $98 \cdot 9$ & $14 ? \cdot 0$ & 184.0 & 420.0 \\
\hline 1944 & $28 . n$ & 28.7 & $31 \cdot 0$ & 33.3 & 40.7 & $5 ? .3$ & 55.7 & 65.7 & 89.6 & $120 \cdot 0$ & 268.0 \\
\hline 1945 & 47.0 & 48.7 & 53.4 & $56 \cdot 4$ & $57 \cdot 1$ & 70.4 & 81.4 & $86 \cdot 5$ & 105.0 & $193 . n$ & 857.0 \\
\hline 1946 & $32 . n$ & 35.3 & 37.3 & $4 ? \cdot 6$ & 50.5 & 55.7 & 62.4 & 8 8. 8 & 115.0 & 143.0 & 854.0 \\
\hline 1947 & 47.0 & 52.3 & $56 \cdot 0$ & $58 \cdot 5$ & $6 ? \cdot 4$ & $73 \cdot 1$ & $78 \cdot 5$ & $101 \bullet 0$ & 126.0 & 180.0 & 624.0 \\
\hline 1948 & 53.0 & $54 . ?$ & 57.7 & 63.9 & 74.4 & 89.3 & 110.0 & $125 \cdot 0$ & $16 n \cdot 0$ & 262.0 & 1050.0 \\
\hline 1949 & 38.0 & 39.3 & $39 \cdot 4$ & 40.9 & 43.5 & 50.7 & 56.4 & $67 \cdot 8$ & 79.7 & 116.0 & 437.0 \\
\hline 1950 & 23.0 & $25 \cdot 7$ & 27.9 & $28 \cdot 5$ & 34.1 & 38.6 & $48 \cdot 3$ & $85 \cdot 6$ & 123.0 & 197.0 & 1110.0 \\
\hline 1951 & 6.8 & $7 \cdot 3$ & 8.5 & $11 \cdot 6$ & 12.7 & 20.1 & 27.5 & $51 \cdot 7$ & 89.4 & 157.0 & 849.0 \\
\hline 1952 & $7 \cdot 1$ & 7.6 & $8 \cdot 2$ & 10.0 & 12.6 & 18.4 & 20.6 & $27 \cdot 2$ & $41 \cdot 6$ & 61.7 & 237.0 \\
\hline 1953 & 25.0 & 25.3 & $27 \cdot 3$ & 31.8 & 44.2 & 53.1 & 68.1 & $96 \cdot 9$ & 129.0 & $215 \cdot 0$ & 842.0 \\
\hline 1954 & 38.0 & $42 . ?$ & 46.9 & 48.9 & 53.7 & 76.9 & 83.6 & $109 \cdot 0$ & 138.0 & 165.0 & 578.0 \\
\hline 1955 & 13.0 & 13.7 & $15 . ?$ & $18 \cdot 1$ & 19.0 & 46.3 & 74.9 & $108 \cdot 0$ & 173.0 & 291.0 & 1630.0 \\
\hline 1956 & 4.4 & $4 \cdot 5$ & $5 \cdot 3$ & 7.8 & 17.4 & 28.5 & 45.1 & $58 \cdot 9$ & $9 ? \cdot 3$ & 139.0 & 390.0 \\
\hline 1957 & 16.0 & 16.7 & 18.6 & 20.5 & 21.5 & 32.7 & 39.0 & $51 \cdot 7$ & $7 ? .4$ & 116.0 & 528.0 \\
\hline
\end{tabular}

Tualatin River at Farmington, Oreg.

STATION NUMBER

$14->065.00$

HIGHEST MEAN DISCHARGE, IN CFS, FOR THE FOLLOWING NUMBER OF CONSECUTIVE DAYS IN YEAR ENDING SEPTEMBER 30

\begin{tabular}{|c|c|c|c|}
\hline YEAR & 1 & 3 & 7 \\
\hline 1940 & $86 \cap 0.0$ & $825 n \cdot n$ & $7400 . n$ \\
\hline 1941 & 6810.0 & 6430.0 & 5550.0 \\
\hline 1942 & 13200.0 & $11900 . n$ & $9350 . n$ \\
\hline 1943 & 11900.0 & $\ln 900 . n$ & 9690.0 \\
\hline 1944 & 3440.0 & 3330.0 & $3000 \cdot 0$ \\
\hline 1945 & 7950.0 & $7710 . n$ & 6730.0 \\
\hline 1946 & 11600.0 & $10200 \cdot 0$ & 8170.0 \\
\hline 1947 & 10900.0 & $9760 . n$ & 8020.0 \\
\hline 1948 & 9680.0 & 9320.0 & 8120.0 \\
\hline 1949 & 15100.0 & $13400 \cdot 0$ & 12000.0 \\
\hline 1950 & 13500.0 & 11900.0 & 9710.0 \\
\hline 1951 & $10800 . ?$ & $10300 \cdot n$ & 9920.0 \\
\hline 1952 & 11500.0 & 10400.0 & 8770.0 \\
\hline 1953 & $115 n n .0$ & 11000.0 & $\ln 2 n) \cdot n$ \\
\hline 1954 & 12400.0 & lngno.n & 9080.0 \\
\hline 1955 & 4230.0 & $4090 . n$ & 3750.0 \\
\hline 1956 & 21800.0 & $17900 \cdot n$ & $128 n n \cdot n$ \\
\hline 1957 & 7250.0 & 6760.0 & 5880.0 \\
\hline 1958 & 8220.0 & 7690.0 & 6540.0 \\
\hline
\end{tabular}

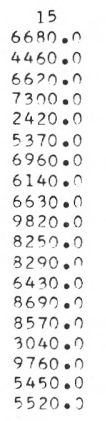

\begin{tabular}{|c|}
\hline \multirow[b]{2}{*}{$585 n \cdot ?$} \\
\hline \\
\hline 3290.0 \\
\hline 4380.0 \\
\hline 5270.0 \\
\hline 2080.0 \\
\hline 3800.0 \\
\hline 4970.0 \\
\hline 5120.0 \\
\hline 4510.0 \\
\hline $7180 \cdot 0$ \\
\hline 7300.0 \\
\hline $6790 . ?$ \\
\hline $\begin{array}{l}4540 . ? \\
7270: ?\end{array}$ \\
\hline 6700.0 \\
\hline 2730.0 \\
\hline 8760.0 \\
\hline 5480.0 \\
\hline
\end{tabular}

60
4060.0
2740.0
3910.0
4840.0
1700.0
3380.0
4680.0
3360.0
3670.0
4460.0
5970.0
5590.0
3610.0
5130.0
5980.0
2500.0
7650.0
3210.0
4400.0

90
3540.0
2110.0
3220.0
4620.0
1490.0
2840.0
4340.0
3500.0
3240.0
4270.0
5310.0
5130.0
3790.0
4070.0
5360.0
2250.0
6110.0
2530.0
4000.0

120
$3110 \cdot 0$
$1770 \cdot 0$
$2650 \cdot 0$
$3840 \cdot 0$
$1390 \cdot 0$
$2400 \cdot 0$
$3940 \cdot 0$
$3110 \cdot 0$
$2910 \cdot 0$
$383 n \cdot 0$
$4600 \cdot 0$
$4540 \cdot 0$
$3440 \cdot 0$
$3330 \cdot 0$
$4570 \cdot 0$
$2290 \cdot 0$
$5620 \cdot 0$
$2230 \cdot 0$
$3300 \cdot 0$

150
$271 n \cdot 0$
$1520 \cdot 0$
$227 n \cdot 0$
$3720 \cdot 0$
$1300 \cdot 0$
$2050 \cdot 0$
$3480 \cdot 0$
$2700 \cdot 0$
$2690 \cdot 0$
$3340 \cdot 0$
$3970 \cdot 0$
$4090 \cdot 0$
$307 n \cdot 0$
$2830 \cdot 0$
$394 n \cdot 0$
$2190 \cdot 0$
$5190 \cdot 0$
$2000 \cdot 0$
2940.0

183

2320.01590 .0

$1320 . n-956 . n$ 14nn.n 850.0 1740.0 $1740.0-1230.0$
2980.0 290.0150 .0 1940.0 $\begin{array}{ll}2450.0 & 1940.0\end{array}$ 33600023100 ? $3530.0 \quad 2450.0$ $2650.0 \quad 1850 \circ$ $2440.0 \quad 16900$ $334 n$. $\quad 23100$ n $2040.0 \quad 1460.0$ 4470.0 3090 $1710.0 \quad 1220.0$ $2500.0 \quad 1740.0$ 
CLASS $0 \begin{array}{lllllllllllllllllllllllllllllllllll}2 & 2 & 3 & 4 & 5 & 6 & 7 & 8 & 9 & 10 & 11 & 12 & 13 & 14 & 15 & 16 & 17 & 18 & 19 & 20 & 21 & 22 & 23 & 24 & 25 & 26 & 27 & 28 & 29 & 30 & 31 & 32 & 33 & 34\end{array}$

\begin{tabular}{|c|c|c|c|c|c|c|c|c|c|c|c|c|c|c|c|c|c|c|c|c|c|c|c|c|c|c|c|c|}
\hline YEAR & & & & & & & & & & & & UMB & & OF & $n \wedge Y$ & Ys II & $N C$ & CLAS & & & & & & & & & & CFC-DAYS \\
\hline 1929 & 1 & 3 & & 8 & 15 & 29 & 7 & 1 & 1 & 16 & 29 & 14 & 25 & 40 & 12 & 164 & 49 & 55 & 16 & 12 & 5 & 4 & 7 & & & & & 277822.0 \\
\hline 1930 & & 6 & 7 & 8 & 13 & 32 & 31 & 17 & 36 & 2 & 7 & 17 & 9 & 21 & 27 & 34 & 29 & 28 & 8 & 4 & 16 & 13 & & & & & & 244920.0 \\
\hline 1931 & & 7 & 16 & 4 & 17 & 21 & 19 & 15 & 23 & 7 & 16 & 22 & 42 & 22 & 8 & 13 & 19 & 17 & 29 & 22 & 13 & 5 & 5 & 3 & & & & 296504.0 \\
\hline 1932 & 6 & 3 & 13 & 11 & 4 & 19 & 20 & 7 & 5 & 3 & 12 & 25 & 15 & 12 & 11 & 13 & 15 & 24 & 23 & 38 & 47 & 19 & 12 & 9 & & & & 585549.0 \\
\hline 1933 & & & 3 & 6 & 2 & 8 & 12 & 24 & 22 & 6 & 12 & 9 & 17 & 12 & 5 & 172 & 44 & 32 & 15 & 36 & 24 & 21 & 26 & 12 & & & & 638465.0 \\
\hline 1934 & & & & 18 & 17 & 13 & 21 & 11 & 7 & 22 & 20 & 6 & 40 & 10 & 8 & 243 & 34 & 27 & 22 & 6 & in & 19 & 11 & in & 4 & 3 & 2 & 566059.0 \\
\hline 1935 & & & 9 & 17 & 4 & 14 & $2 n$ & 23 & 8 & 9 & 14 & 3 & 20 & 12 & 8 & 91 & 10 & 23 & 15 & 44 & 44 & 25 & 20 & 14 & & & & 697693.0 \\
\hline 1936 & & & & 1 & 4 & 21 & 35 & 13 & 25 & 12 & 11 & 24 & 24 & 9 & 28 & 351 & 19 & 35 & 14 & 15 & 7 & 6 & 18 & 6 & 2 & 2 & & 438218.0 \\
\hline 1937 & & & & & 2 & 17 & 33 & 23 & 36 & 22 & 10 & 11 & 23 & 20 & 10 & 15 & 26 & 19 & 22 & 23 & 16 & 19 & 13 & 5 & & & & 430289.0 \\
\hline 1938 & & & & & & 4 & 35 & 30 & 19 & 25 & 24 & 12 & 11 & 11 & 7 & 9 & 9 & 21 & 19 & 22 & 35 & 322 & 23 & 11 & 1 & 3 & 2 & 746858.0 \\
\hline 1939 & 2 & 13 & 19 & 10 & 9 & 17 & 10 & 13 & 19 & 17 & 38 & 11 & 13 & 22 & 16 & 251 & 11 & 25 & 23 & 26 & 15 & 3 & 6 & 2 & & & & 305119.0 \\
\hline 1940 & & 6 & 36 & 13 & 6 & in & 13 & 15 & 31 & 18 & 14 & 14 & 9 & 11 & 4 & 72 & 27 & 28 & 20 & 23 & $2 n$ & 13 & 18 & 10 & & & & 476296.0 \\
\hline 1941 & & & & 19 & 19 & 5 & 14 & 15 & 15 & 21 & 19 & 15 & 32 & 25 & 18 & 412 & 27 & 22 & 14 & 16 & 17 & 4 & 7 & & & & & 287672.0 \\
\hline 1942 & & & 13 & 25 & 6 & 4 & 2 & 12 & 10 & 14 & 26 & 16 & 21 & 29 & 19 & 332 & 21 & 46 & 12 & 15 & 8 & 12 & 10 & 8 & 3 & & & 417330.9 \\
\hline 1943 & & & 1 & 17 & 17 & 17 & $2 n$ & 13 & 15 & 9 & 6 & 6 & 17 & 21 & 13 & 282 & 24 & 27 & 8 & 17 & 19 & 132 & 27 & 26 & 4 & & & 688841.0 \\
\hline 1944 & & 8 & 16 & 27 & 9 & 18 & 11 & 8 & 12 & 6 & 3 & 11 & 35 & 18 & 17 & 294 & 40 & 35 & 37 & 21 & 5 & & & & & & & 253484.0 \\
\hline 1945 & & & & 19 & 22 & 20 & 24 & 13 & 9 & 9 & 12 & 19 & 23 & 18 & 17 & 272 & 25 & 30 & 14 & 29 & 7 & 12 & 14 & 2 & & & & 372251.0 \\
\hline 1946 & & & 112 & 20 & 27 & 16 & 16 & 2 & 6 & 9 & 17 & 12 & 30 & 14 & 5 & 111 & 13 & 14 & 14 & 41 & 30 & 23 & 23 & 11 & & & & 623068.0 \\
\hline 1947 & & 3 & 7 & 13 & 19 & 22 & 13 & 8 & 18 & 12 & 17 & 17 & 36 & 9 & 5 & 112 & 22 & 43 & 9 & 14 & 26 & 18 & 17 & 6 & & & & 473479.0 \\
\hline 1948 & & & & & 1 & 11 & 6 & 8 & 20 & 29 & 8 & 19 & 8 & 16 & 6 & 43 & 30 & 28 & 49 & 69 & 18 & 12 & 14 & 8 & 2 & & & 615433.0 \\
\hline 1949 & & & & 7 & 20 & 36 & 7 & 4 & 8 & 27 & 23 & 14 & 16 & 10 & 11 & 23 & 28 & 20 & 19 & 30 & 18 & 8 & in & 15 & 11 & & & 604635.0 \\
\hline 1950 & & & & 9 & 25 & 29 & 15 & 12 & 9 & 10 & 20 & 18 & 29 & 3 & 7 & 10 & 18 & 15 & 13 & 26 & 29 & 25 & 33 & 8 & 2 & & & 649421.0 \\
\hline 1951 & 4 & 7 & 17 & 41 & 6 & 14 & 8 & 2 & 2 & 5 & 12 & 14 & 24 & 10 & 10 & 181 & 11 & 9 & 16 & 18 & 18 & 67 & 21 & 9 & 2 & & & 702798.8 \\
\hline 1952 & & & & & & 21 & 11 & 23 & 25 & 2 & 6 & 13 & 36 & 21 & 16 & 14 & 19 & 14 & 14 & 65 & 38 & 6 & 16 & 12 & 1 & & & 590135.0 \\
\hline 1953 & & & & & & & 2 & 17 & 44 & 30 & 38 & 14 & 9 & 16 & 7 & $20=$ & 33 & 48 & 12 & 15 & 23 & 6 & 15 & 9 & 7 & & & 524502.0 \\
\hline 1954 & & & & & & 3 & 1 & 2 & 21 & 12 & 33 & 37 & 33 & 41 & 6 & 13 & 16 & 14 & 10 & 30 & 17 & 16 & 35 & 19 & 6 & & & 731609.0 \\
\hline 1955 & & & & & & & 21 & 7 & 6 & 13 & 45 & 24 & 29 & 9 & 12 & 121 & 14 & 21 & 34 & 71 & 37 & 10 & & & & & & 479350.0 \\
\hline 1956 & & & & & & 1 & 7 & 10 & 23 & 32 & 25 & 7 & 19 & 27 & 7 & 15 & 16 & 16 & 13 & 27 & 17 & 20 & 44 & 25 & 11 & 4 & & 967200.0 \\
\hline 1957 & & & & & & & 6 & 32 & 33 & 25 & 13 & 8 & 21 & 20 & 22 & 312 & 27 & 40 & 23 & 31 & 7 & 3 & 23 & & & & & 4043 \\
\hline 1958 & & & & & 31 & 11 & 6 & 3 & 21 & 16 & 37 & 8 & 23 & 13 & 18 & 151 & 16 & 29 & 24 & 14 & 15 & 16 & 45 & 4 & & & & 8.0 \\
\hline 1959 & & & & & & 19 & 11 & 8 & 18 & 24 & 21 & 9 & 25 & 9 & 12 & 183 & 32 & 43 & 18 & 33 & 19 & 19 & 19 & 5 & 3 & & & $567438 \cdot 0$ \\
\hline 1960 & & & & & & & 11 & 13 & 10 & 44 & 15 & 12 & 31 & 11 & 17 & 122 & 24 & 47 & 37 & 28 & 27 & 8 & 12 & 7 & & & & 495914.0 \\
\hline $\begin{array}{l}1961 \\
1962\end{array}$ & & & & & 4 & 19 & 12 & 6 & 27 & 32 & 24 & 16 & 9 & 9 & 8 & 9 & 18 & 32 & 28 & 17 & 15 & 25 & 30 & 25 & & & & 723932.0 \\
\hline 1962 & & & & & & 2 & 7 & 14 & 22 & 38 & 27 & 24 & 11 & 14 & 6 & 13 & 43 & 39 & 36 & 38 & 11 & 7 & 13 & & & & & 416458.0 \\
\hline 1963 & & & & & & & & & 6 & 18 & 33 & 24 & 33 & 24 & 11 & 233 & 38 & 31 & 36 & 31 & 15 & 7 & 26 & 9 & & & & 561270.0 \\
\hline
\end{tabular}

Tualatin River at West Linn, Oreg.

Surmary for water years 1929-63

STATION NUMBER $14-2075.00$

\begin{tabular}{|c|c|c|c|c|c|c|c|c|c|c|c|c|c|c|c|c|c|c|c|}
\hline CLASS & CFS & TOTAL & ACCUM & PERCT & CLASS & CFS & TOTAL & ACCUM & PERCT & CLASS & CFS & TOTAL & ACCUM & PERCT & CLA & SS CFS & TOTAL & ACCUM & DER \\
\hline & .0 & & 12783 & 100.0 & 09 & 30.0 & 487 & 11481 & 89.8 & 18 & 500 & 647 & 6349 & 49.7 & 27 & 10000 & 59 & 75 & \\
\hline 1 & 2.0 & & 12783 & 100.0 & 10 & 40.0 & 424 & 10994 & 86.0 & & 700 & 847 & 5702 & 44.6 & 28 & 15000 & 12 & 16 & 1 \\
\hline 2 & 3.0 & 1 & 12783 & $10 n .0$ & 11 & 50.0 & 612 & 10570 & 82.7 & 20 & 1000 & 997 & 4855 & 38.0 & 29 & 20000 & 4 & 4 & .0 \\
\hline 3 & 4.0 & 12 & 12782 & 100.0 & 12 & 70.0 & 596 & 9958 & 77.9 & 21 & 1500 & 716 & 3858 & 30.2 & 30 & & & & .0 \\
\hline 4 & 5.0 & 56 & 12770 & 99.9 & 13 & 100.0 & 687 & 9362 & 73.2 & 22 & 2000 & 967 & 3142 & 24.6 & 31 & & & & .0 \\
\hline 5 & 7.0 & 168 & 12714 & 99.5 & 14 & 150.0 & 525 & 8675 & 67.9 & 23 & 3000 & 688 & 2175 & 17.0 & 32 & & & & 0 \\
\hline 6 & 10.0 & 293 & 12546 & 98. & 15 & 200.0 & 798 & 8150 & 63 & 24 & 4000 & 516 & 1487 & 11.6 & 33 & & & & 0 \\
\hline 7 & 15.0 & 299 & 12253 & 95.9 & 16 & 300.0 & 589 & 7352 & 57.5 & 25 & 5000 & 606 & 971 & 7. & 34 & & & & .0 \\
\hline 8 & 20.0 & 473 & 11954 & 93. & 17 & 400.0 & 414 & 6763 & 52.9 & 26 & 7000 & 290 & 365 & 2. & 35 & & & & \\
\hline
\end{tabular}

Tualatin River at West Linn, Oreg.

Summary for water years 1952-63

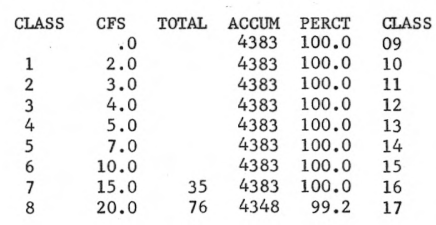

Tualatin River at West Linn, Oreg.

Summary for water years 1929-51

\begin{tabular}{lrrrrl} 
CLASS & \multicolumn{1}{c}{ CFS } & TOTAL & ACCUM & PERCT & CL.ASS \\
1 & .0 & & 8400 & 100.0 & 09 \\
2 & 2.0 & & 8400 & 100.0 & 10 \\
2 & 3.0 & 1 & 8400 & 100.0 & 11 \\
3 & 4.0 & 12 & 8399 & 100.0 & 12 \\
4 & 5.0 & 56 & 8387 & 99.8 & 13 \\
5 & 7.0 & 168 & 8331 & 99.2 & 14 \\
6 & 10.0 & 293 & 8163 & 97.2 & 15 \\
7 & 15.0 & 264 & 7870 & 93.7 & 16 \\
8 & 20.0 & 397 & 7606 & 90.5 & 17
\end{tabular}

STATION NUMBER
14-2075.00

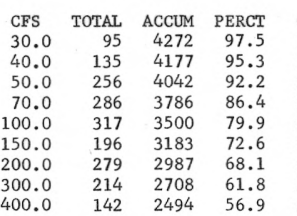

STATION NUMBER

CLASS
18
19
20
21
22
23
24
25
26

14-2075.00

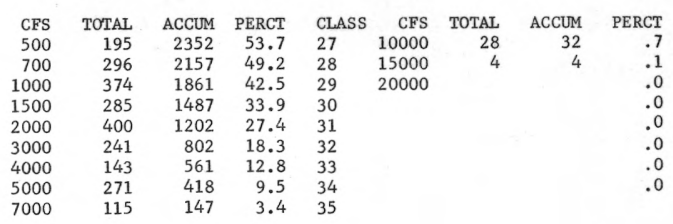


Thalatin River at West Linn, Oreg.

STATION NUMBER $\quad 14-2075.00$ LOWEST MEAN DISCHARGF, IN CFS, FOR THE FOLLOWING NUMRER OF CONSFCUTIVF DAYC IN YFAR RFGTNNING APRIL 1

\begin{tabular}{|c|c|c|c|c|c|c|c|c|c|c|c|}
\hline YEAR & 1 & 3 & 7 & 14 & 30 & 60 & 90 & 120 & 150 & 183 & 274 \\
\hline $\begin{array}{l}1929 \\
1930\end{array}$ & 3.0 & 4.3 & $12 \cdot 6$ & 15.1 & 17.4 & $2 n \cdot 4$ & 25.6 & 33.0 & 47.1 & 85.7 & 298.0 \\
\hline $\begin{array}{l}1930 \\
1931\end{array}$ & $5 . n$ & $5 \cdot n$ & 5.4 & 8.6 & 13.4 & 18.1 & 24.5 & 30.4 & 57.2 & 87.1 & 212.0 \\
\hline $\begin{array}{l}1931 \\
1932\end{array}$ & $5 . n$ & $5 \cdot 3$ & 5.7 & 6.3 & 8.6 & $16 \cdot 1$ & 21.0 & $40 \cdot 7$ & 70.5 & 116.0 & $9 \cap 2 \cdot ?$ \\
\hline $\begin{array}{l}1932 \\
1933\end{array}$ & $4 \cdot n$ & $4 \cdot n$ & $4 \cdot 3$ & 5.7 & 8.4 & 20.4 & 24.0 & $46 \cdot 7$ & 82.1 & $166 \cdot 0$ & 867.0 \\
\hline $\begin{array}{l}1933 \\
1934\end{array}$ & 20.0 & 21.3 & 23.7 & 29.4 & 38.8 & 57.9 & $8 \cap .4$ & $120 \cdot 0$ & 190.0 & $\begin{array}{l}249.0 \\
174.0\end{array}$ & 1320.0 \\
\hline $\begin{array}{l}1934 \\
1935\end{array}$ & 10.0 & 10.7 & 11.4 & 12.9 & 14.9 & 21.7 & 28.3 & 38.2 & 70.4 & $\begin{array}{r}174.0 \\
90.9\end{array}$ & $\begin{array}{r}1210.0 \\
362.0\end{array}$ \\
\hline $\begin{array}{l}1935 \\
1936\end{array}$ & 7.0 & $7 \cdot n$ & 7.7 & 11.2 & 11.6 & 22.9 & 29.4 & $4 n \cdot 5$ & $\begin{array}{l}59.7 \\
43.9\end{array}$ & 81.5 & $\begin{array}{l}362.0 \\
280.0\end{array}$ \\
\hline $\begin{array}{l}1936 \\
1937\end{array}$ & $17 \cdot 0$ & $17 \cdot 3$ & 19.4 & $23 \cdot 4$ & 26.6 & $29 \cdot 3$ & $\begin{array}{l}30.2 \\
69.6\end{array}$ & $\begin{array}{l}33 \cdot 0 \\
82.1\end{array}$ & $14 \% .0$ & 226.0 & 1340 . ? \\
\hline $\begin{array}{l}1937 \\
1938\end{array}$ & 31.0 & $\begin{array}{l}31.3 \\
27.3\end{array}$ & 36.6 & 43.1 & 58.1 & $\begin{array}{l}63.7 \\
38.6\end{array}$ & 44.4 & $5 a .2$ & $76 . ?$ & 132.0 & 477.0 \\
\hline $\begin{array}{l}1938 \\
1939\end{array}$ & $\begin{array}{r}25.0 \\
4.0\end{array}$ & $\begin{array}{r}27 \cdot 3 \\
4.3\end{array}$ & $\begin{array}{r}29.7 \\
5.4\end{array}$ & $\begin{array}{r}31.6 \\
6.2\end{array}$ & $\begin{array}{r}35.3 \\
7.5\end{array}$ & 13.0 & 16.9 & 25.0 & 37.3 & 45.2 & 280.0 \\
\hline 1940 & 6.0 & $6 . n$ & $\begin{array}{l}5.4 \\
6.4\end{array}$ & $\begin{array}{l}6.2 \\
7.0\end{array}$ & 7.9 & 14.5 & 20.8 & 32.9 & 70.0 & 120.0 & 563.0 \\
\hline 1941 & 12.0 & $12 \cdot n$ & $12 . n$ & 12.7 & 13.7 & 25.8 & 47.0 & $64 \cdot 3$ & 85.4 & $13 n .0$ & 724.0 \\
\hline 1942 & 8.4 & 8.4 & 8.5 & 8.7 & 10.0 & 11.9 & 19.2 & 38.9 & 88.4 & $16 n \cdot n$ & $97 n . n$ \\
\hline 1943 & 13.0 & $13 . n$ & 13.9 & 15.4 & 18.8 & 28.2 & 36.7 & 67.1 & 121.0 & 174.0 & 431.0 \\
\hline 1944 & 6.0 & $6 . n$ & 6.1 & 6.6 & 7.8 & 11.0 & 16.9 & 22.6 & 52.8 & 94.9 & $274 . n$ \\
\hline 1945 & $10 \cdot 0$ & $10 \cdot n$ & 10.3 & $11 \cdot 3$ & 13.4 & 24.9 & 38.9 & 43.6 & 67.7 & 179.0 & 927.0 \\
\hline 1946 & $7 \cdot 0$ & $7 . n$ & $7 \cdot 6$ & 8.2 & $12 \cdot 2$ & 14.0 & 18.5 & $47 \cdot 1$ & 82.5 & 122.0 & 912.0 \\
\hline 1947 & 6.0 & $6 \cdot n$ & 7.9 & 9.4 & 15.0 & 19.7 & 30.1 & 53.9 & 82.6 & 156.0 & 656.0 \\
\hline 1948 & 21.0 & 22.3 & 24.3 & 27.2 & 42.8 & 54.6 & 79.0 & 93.1 & 135.0 & 262.0 & 1120.0 \\
\hline 1949 & 11.0 & 11.7 & 12.0 & 14.2 & 17.5 & 21.9 & 27.8 & $40 \cdot 4$ & 57.9 & 104.0 & 490.0 \\
\hline 1950 & 13.0 & 13.7 & 14.3 & 14.9 & 16.5 & 19.7 & 28.5 & $68 \cdot 1$ & 100.0 & 191.0 & 1190.0 \\
\hline 1951 & 4.5 & 4.8 & 5.5 & 7.1 & 8.5 & 9.9 & 14.2 & 37.7 & 79.6 & 149.0 & 951.0 \\
\hline 1952 & $2000_{t}$ & $20 \cdot n$ & 20.6 & 21.2 & 26.3 & 35.6 & 38.6 & $47 \cdot 3$ & 65.0 & 94.6 & 305.0 \\
\hline 1953 & 52.0 & 52.3 & 52.9 & 56.0 & 62.9 & 78.6 & 94.0 & $124 \cdot 0$ & 160.0 & 271.0 & $903 . n$ \\
\hline 1954 & 23.0 & 23.3 & 37.6 & 45.4 & 54.8 & 84.3 & 101.0 & 128.0 & 163.0 & & 666.0 \\
\hline 1955 & 32.0 & $32 \cdot n$ & 32.9 & 35.8 & 38.0 & 69.3 & 35.3 & $13 n \cdot 0$ & $\begin{array}{l}2 n 5.0 \\
131.0\end{array}$ & $\begin{array}{l}347.0 \\
194.0\end{array}$ & $\begin{array}{r}1890.0 \\
501.0\end{array}$ \\
\hline 1956 & $25 \cdot 0$ & $33 . n$ & $35 \cdot n$ & 40.9 & 46.6 & $58 \cdot 4$ & 71.7 & $\begin{array}{l}88 \cdot 1 \\
72.5\end{array}$ & 95.7 & 146.0 & $63 n . n$ \\
\hline $\begin{array}{l}1958 \\
1959\end{array}$ & 15.0 & $\begin{array}{l}15 \cdot 0 \\
20 \cdot 0\end{array}$ & $\begin{array}{l}12.3 \\
20.6\end{array}$ & $\begin{array}{l}13.0 \\
21.6\end{array}$ & $\begin{array}{l}10.3 \\
26.3\end{array}$ & 48.2 & 85.0 & 143.0 & 166.0 & 220.0 & $5 n 9.0$ \\
\hline $\begin{array}{l}1959 \\
1960\end{array}$ & 20.0 & 31.7 & 33.6 & 36.5 & 43.3 & 61.8 & 70.1 & 85.4 & 122.0 & 291.0 & 972.0 \\
\hline $\begin{array}{l}1960 \\
1961\end{array}$ & $\begin{array}{l}31.0 \\
18.0\end{array}$ & $18 \cdot 3$ & 19.3 & 21.6 & 25.9 & 40.4 & 51.9 & 68.7 & 107.0 & 170.0 & 776.0 \\
\hline 1962 & $\begin{array}{l}18.0 \\
27.0\end{array}$ & 28.7 & 32.4 & 43.1 & 55.3 & 62.7 & 76.4 & 112.0 & 172.0 & 273.0 & 975.0 \\
\hline
\end{tabular}

Tualatin River at West Linn, Oreg.

STATION NUMBER $14-2075.00$

HIGHEST MEAN DISCHARGE, IN CFS, FOR THE FOLLOWING NUMBER OF CONSFCUTIVE DAYS IN YFAR FNDING SFPTFMBFR 30

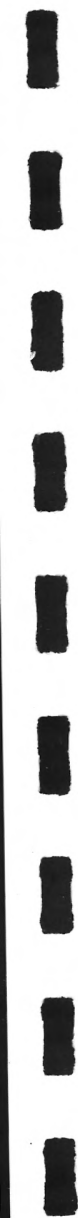

\begin{tabular}{|c|c|c|c|c|c|c|c|c|c|c|c|}
\hline YEAR & 1 & 3 & 7 & 15 & 30 & 60 & $9 n$ & 120 & $15 n$ & 183 & 274 \\
\hline 1929 & $5750 \cdot 0$ & $5660 \cdot n$ & $5430 \cdot 0$ & 4520.0 & 2880.0 & 1940.0 & 1690.0 & 1690.0 & 1550.0 & 1360.0 & 997.0 \\
\hline $\begin{array}{l}1930 \\
1931\end{array}$ & 4760.0 & $476 n \cdot n$ & $4590 \cdot 0$ & $4040 \cdot 0$ & $3800 \cdot 0$ & $2510 . ?$ & 1970.0 & $169 n \cdot ?$ & $1460 \cdot 0$ & 1280.0 & $\begin{array}{r}886.0 \\
1070.0\end{array}$ \\
\hline 1931 & 7190.0 & $7090 \cdot n$ & 6630.0 & $5390 \cdot 0$ & 4070.0 & $2920 \cdot 0$ & 2520.0 & $2180 \cdot 0$ & 1820.0 & 1540.0 & \\
\hline $\begin{array}{l}1932 \\
1933\end{array}$ & $8200 \cdot 0$ & $8100 \cdot n$ & $7380 \cdot 0$ & $6010 \cdot 0$ & $5610 \cdot 0$ & 4520.0 & $4050 \cdot 0$ & $3800 \cdot 0$ & $\begin{array}{l}3390 \cdot 0 \\
366000\end{array}$ & $\begin{array}{l}3030 \cdot 0 \\
3190 \cdot 0\end{array}$ & $\begin{array}{l}2130.0 \\
2310.0\end{array}$ \\
\hline 1935 & 9860.0 & $9730 . n$ & 9320.0 & $\begin{array}{r}130 n 0.0 \\
8130.0\end{array}$ & $\begin{array}{l}8990.0 \\
6300.0\end{array}$ & 5020.0 & 4840.0 & $4270 \cdot 0$ & $407 n \cdot n$ & $362 n \cdot n$ & 2540.0 \\
\hline 1936 & 15900.0 & $14800 \cdot n$ & $1190 n \cdot ?$ & 9340.0 & 6630.0 & 4490.0 & $38 \cap n .0$ & $3180 . ?$ & $2660 \cdot 0$ & $227 n \cdot n$ & $150 n$. n \\
\hline 1937 & 7610.0 & $7380 \cdot n$ & $687 n \cdot n$ & $5630 . ?$ & 4800.0 & $38 \cap 0 \cdot n$ & $3600 \cdot ?$ & $2 n 6 n \cdot n$ & $264 n \cdot n$ & $274 n \cdot n$ & $156 n \cdot ?$ \\
\hline $\begin{array}{l}1938 \\
1939\end{array}$ & $22100 \cdot 0$ & $2 n 9 n \cap \cdot n$ & $16900 \cdot n$ & $114 n n \cdot n$ & 7720.0 & $6 \cap 8 \cap \cdot n$ & $539 n .0$ & $5037 . ?$ & $4590 . ?$ & $3960 \cdot n$ & ? \\
\hline $\begin{array}{l}1939 \\
1940\end{array}$ & $7120 \cdot 0$ & $6980 \cdot n$ & $652 n \cdot n$ & $5250 \cdot 0$ & 4030.0 & $3 \cap 20 \cdot n$ & $26 \cap 0 \cdot 0$ & $22 n n \cdot n$ & $\begin{array}{l}1870.0 \\
2970 \cdot 0\end{array}$ & $\begin{array}{l}160 n \cdot 0 \\
2540.0\end{array}$ & $\begin{array}{l}1110.0 \\
1730.0\end{array}$ \\
\hline 1940 & 8090.0 & $7850 \cdot 0$ & 7550.0 & $7 \cap 50 \cdot 0$ & 6270.0 & $445 \cap .0$ & & $3410 \cdot 0$ & $\begin{array}{l}2970.0 \\
1680.0\end{array}$ & $\begin{array}{l}254 \pi \cdot 0 \\
1450.0\end{array}$ & 1030.0 \\
\hline 1941 & 6160.0 & $6060 . n$ & $5680 \cdot n$ & 4870.0 & 3660.0 & 3050.0 & $236 n \cdot 0$ & & $\begin{array}{l}1680.0 \\
2490.0\end{array}$ & 2130.0 & 1510.0 \\
\hline 1942 & $11500 \cdot 0$ & $11000 \cdot n$ & $9550 \cdot 0$ & $7230 \cdot 0$ & 4850.0 & $\begin{array}{l}4210.0 \\
5410.0\end{array}$ & $\begin{array}{l}3510.0 \\
5170.0\end{array}$ & $\begin{array}{l}2910 \cdot 0 \\
4330 \cdot 0\end{array}$ & 4200.0 & 3600.0 & 2500.0 \\
\hline $\begin{array}{l}1943 \\
1944\end{array}$ & 11500.0 & $11100 \cdot n$ & $9950 \cdot 0$ & $8010 \cdot 0$ & $5800 \cdot 0$ & 1840.0 & 1630.0 & $1500 \cdot 0$ & 1410.0 & 1250.0 & $919 . ?$ \\
\hline 1944 & $3230 \cdot 0$ & $3200 \cdot 0$ & 3040.0 & 2500.0 & $\begin{array}{l}2220.0 \\
4160.0\end{array}$ & 3690.0 & $311 n .0$ & 2630.0 & 2260.0 & 1920.0 & $135 n \cdot 0$ \\
\hline $\begin{array}{l}1945 \\
1946\end{array}$ & $7190 \cdot 0$ & $7110 \cdot 0$ & $\begin{array}{l}6560.0 \\
8160.0\end{array}$ & $\begin{array}{l}5600 \cdot 0 \\
6960 \cdot 0\end{array}$ & 5280.0 & $504 n .0$ & 4670.0 & $432 n \cdot 0$ & $385 n .0$ & 3300.0 & 2270.0 \\
\hline 1947 & $\begin{array}{l}9620.0 \\
8870.0\end{array}$ & $\begin{array}{l}9290 \cdot n \\
8600 \cdot n\end{array}$ & $778 n . n$ & 6220.0 & 5350.0 & 3520.0 & 3730.0 & $3360 \cdot 0$ & $293 n \cdot n$ & $2490 . n$ & 1720.0 \\
\hline & 10400.0 & $\begin{array}{l}8000 \bullet n \\
\ln 10 n \cdot n\end{array}$ & $933 n \cdot n$ & $7570 . n$ & $522 n \cdot 0$ & 4150.0 & 3740.0 & $3370 \cdot 0$ & $3140 \cdot ?$ & $283 n .0$ & $2230 . n$ \\
\hline 1949 & $144 n n \cdot n$ & $1330 n \cdot n$ & $12800 \cdot n$ & $108 n 0.0$ & $813 n \cdot 0$ & $511 \cap . ?$ & 4670.0 & 4240.0 & $371 n \cdot n$ & $318 n \cdot n$ & $22 n n \cdot n$ \\
\hline 1950 & 11000.0 & $104 n n \cdot n$ & $892 n \cdot n$ & $7770 . ?$ & 6330.0 & $581 \cap \cdot n$ & $529 n \cdot 0$ & $464 n \cdot n$ & $404^{n} \cdot n$ & $344 n \cdot n$ & $2350 \cdot 0$ \\
\hline 1951 & 10500.0 & $102 n n \cdot n$ & $96 n n . n$ & 8120.0 & $67 n n .0$ & $564 n .0$ & 5270.1 & $472 n \cdot n$ & $429 n \cdot n$ & $\frac{371 n \cdot n}{3 n 2 n \cdot n}$ & $\frac{256 n .0}{2140.0}$ \\
\hline $\begin{array}{l}1952 \\
1953\end{array}$ & $10200 \cdot 0$ & $9780 \cdot n$ & $8580 \cdot n$ & 6750.0 & $511 n \cdot 0$ & $41 n n \cdot n$ & 4310.0 & $\begin{array}{l}389 n \bullet ? \\
3670.0\end{array}$ & $\begin{array}{l}348 n \cdot 0 \\
314 n \cdot 0\end{array}$ & 2710.0 & 1890.0 \\
\hline $\begin{array}{l}1953 \\
1954\end{array}$ & $11700 \cdot 0$ & $11500 \cdot 0$ & 10900.0 & 9450.0 & $7780 \cdot 0$ & 5530.0 & $\begin{array}{l}4470.0 \\
6070.0\end{array}$ & $5210 \cdot n$ & 4500.0 & 3810.0 & 2630.0 \\
\hline $\begin{array}{l}1954 \\
1955\end{array}$ & $\begin{array}{r}11600.0 \\
4490.0\end{array}$ & $\begin{array}{r}1,1200 \cdot n \\
4420.0\end{array}$ & $\begin{array}{r}10200 \cdot 0 \\
4160 \cdot 0\end{array}$ & $\begin{array}{l}9440.0 \\
3520.0\end{array}$ & $\begin{array}{l}7600 \cdot 0 \\
3200 \cdot 0\end{array}$ & $\begin{array}{l}6760.0 \\
2930.0\end{array}$ & 2640.0 & $2730 \cdot 0$ & 2600.0 & $241 n .0$ & 1720.0 \\
\hline 1956 & 19900.0 & 18600.0 & 14500.0 & 11000.0 & 10100.0 & 8730.0 & 7000.0 & $6380 \cdot 0$ & 5850.0 & 5040.0 & $350 n .0$ \\
\hline 1957 & 6640.0 & $6550 \cdot n$ & $643 n \cdot n$ & $6030 . n$ & $543 n .0$ & $37 n n \cdot 0$ & $294 n \cdot n$ & $2600 \cdot 0$ & $233 n \cdot n$ & $2 n 2 n \cdot n$ & $146 r .0$ \\
\hline 1958 & 7560.0 & $7440 \cdot n$ & 6950.0 & 6140.0 & 6110.0 & $5200 \cdot 0$ & 4740.0 & $3920 \cdot 0$ & $353 n .0$ & $3 n \cap n \cdot n$ & $210 n .0$ \\
\hline 1959 & $11300 \cdot 0$ & $10900 \cdot n$ & 9670.0 & 7610.0 & 6490.0 & $5 \ln n \cdot 0$ & 4160.0 & $3730 \cdot 0$ & $330 n \cdot n$ & $288 n \cdot n$ & $2 n 5 C .0$ \\
\hline 1960 & 8680.0 & $8470 \cdot n$ & $787 n . n$ & $687 n \cdot n$ & 5460.0 & $4 \cap 50.0$ & 3530.0 & 3050.0 & $273 n . n$ & $245 n . n$ & $178 n .0$ \\
\hline 1961 & 9340.0 & 9210.0 & 8780.0 & 7980.0 & 7420.0 & 6590.0 & 5520.0 & $4710 \cdot 0$ & 4290.0 & 3780.0 & 2620.0 \\
\hline 1962 & $\begin{array}{l}6620.0 \\
8140.0\end{array}$ & 6490.0 & $6160 \cdot 0$ & $5350 \cdot 0$ & $3800 \cdot 0$ & 2550.0 & 2450.0 & $2490 \cdot 0$ & $2290 \cdot 0$ & $\begin{array}{l}2090.0 \\
2750.0\end{array}$ & $\begin{array}{l}1500.0 \\
2000.0\end{array}$ \\
\hline 1963 & 8140.0 & 7970.0 & 7240.0 & 6030.0 & 4400.0 & 3110.0 & 3050.0 & $2670 \cdot 0$ & $2880 \cdot 0$ & & $2000 \cdot 0$ \\
\hline
\end{tabular}


CLASS $0101120 \begin{array}{llllllllllllllllllllllllllllllllll} & 4 & 5 & 6 & 7 & 8 & 9 & 10 & 11 & 12 & 13 & 14 & 15 & 16 & 17 & 18 & 19 & 20 & 21 & 22 & 23 & 24 & 25 & 26 & 27 & 28 & 29 & 30 & 31 & 32 & 33 & 34\end{array}$

$\begin{array}{rrrrrrrrrr}45 & 42 & 20 & 17 & 22 & 50 & 39 & 36 & 44 & 27 \\ 100 & 94 & 41 & 22 & 14 & 21 & 15 & 11 & 20 & 12\end{array}$

$\begin{array}{llllllllll}100 & 94 & 41 & 22 & 14 & 21 & 15 & 11 & 20 & 12 \\ 137 & 32 & 53 & 12 & 10 & 16 & 25 & 32 & 23 & 11\end{array}$

NUMBER OF DAYS IN CLASS

$\begin{array}{lllllllllll}95 & 83 & 40 & 49 & 43 & 8 & 20 & 7 & 5 & 8 \\ 38 & 79 & 22 & 21 & 25 & 29 & 47 & 45 & 13 & 23\end{array}$

$\begin{array}{rrrrrrrrrrr}38 & 79 & 22 & 21 & 25 & 29 & 47 & 45 & 13 & 23 \\ 152 & 50 & 65 & 24 & 24 & 11 & 22 & 6 & 4 & 2\end{array}$

$\begin{array}{lllllllllll}31 & 82 & 20 & 37 & 53 & 23 & 29 & 26 & 26 & 19\end{array}$

$\begin{array}{rrrrrrrrrrr}18 & 84 & 58 & 39 & 21 & 18 & 33 & 38 & 23 & 15 \\ 103110 & 38 & 12 & 10 & 25 & 21 & 22 & 9 & 12\end{array}$

$\begin{array}{lllllllllll}2178 & 34 & 52 & 38 & ? ? & 6 & 10 & 8 & 9 & 5\end{array}$

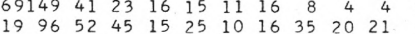

$\begin{array}{lllllllllllll}19 & 96 & 52 & 45 & 15 & 25 & 10 & 16 & 35 & 20 & 21 & 9\end{array}$

$\begin{array}{lllllllllllll}24 & 62 & 70 & 35 & 33 & 22 & 4 & 24 & 18 & 13\end{array}$

$\begin{array}{llllllllll}20135 & 41 & 18 & 38 & 16 & 17 & 22 & 10 & 19 \\ 13 & 83 & 18 & 39 & 37 & 55 & 38 & 26 & 19 & 34\end{array}$

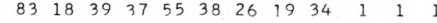

$104 \begin{array}{rrrrrrrrrrr}129 & 47 & 41 & 24 & 22 & 32 & 16 & 10 & 24 & 11 \\ 11 & 94 & 30 & 20 & 12 & 16 & 11 & 11 & 10 & 31\end{array}$

$\begin{array}{rrrrrrrrrrrr}11 & 98 & 18 & 20 & 31 & 21 & 45 & 24 & 22 & 42 & 20 \\ 74 & 70 & 57 & 51 & 22 & 12 & 16 & 36 & 20 & 7\end{array}$

$\begin{array}{rrrrrrrrrrrrr}171 & 47 & 20 & 14 & 18 & 17 & 35 & 9 & 9 & 13 & 6 & 6 & 1\end{array}$

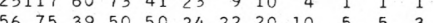

$\begin{array}{rrrrrrrrrrrrrrrr}50 & 55 & 22 & 34 & 20 & 12 & 39 & 21 & 21 & 43 & 20 & 12 & 2 & 1 & 1 & \\ 50 & 3 & 4 & 1\end{array}$

$\begin{array}{lllllllllll}103 & 40 & 89 & 51 & 45 & 19 & 13 & 3 & 1 & 2\end{array}$

$\begin{array}{lllllllllllllll}165 & 38 & 25 & 23 & 29 & 12 & 21 & 16 & 8 & 16 & 6 & 4 & 1 & 1\end{array}$

$\begin{array}{rlllllllll}165 & 38 & 25 & 23 & 29 & 12 & 21 & 16 & 8 & 16 \\ 39 & 65 & 32 & 37 & 22 & 33 & 38 & 30 & 22 & 29\end{array}$

$\begin{array}{rlllllllllll}39 & 65 & 32 & 37 & 22 & 33 & 38 & 30 & 22 & 29 \\ 6 & 97 & 25 & 50 & 25 & 17 & 45 & 53 & 21 & 12\end{array}$

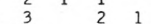

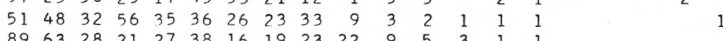

$\begin{array}{llllllllllll}32 & 81 & 42 & 24 & 16 & 25 & 34 & 26 & 28 & 35 & 1\end{array}$

$\begin{array}{llllllllllll}30 & 70 & 18 & 14 & 16 & 47 & 23 & 31 & 70 & 31 & 10\end{array}$

$\begin{array}{llllllllllll}63 & 47 & 55 & 30 & 23 & 38 & 31 & 25 & 34 & 16\end{array}$

$\begin{array}{lllllllllll}6138 & 23 & 16 & 30 & 26 & 26 & 30 & 23 & 19\end{array}$

$\begin{array}{rrrrrrrrrrrr}64 & 54 & 13 & 14 & 15 & 41 & 46 & 38 & 60 & 11 \\ 58 & 90 & 74 & 32 & 22 & 27 & 17 & 13 & 18 & 5\end{array}$

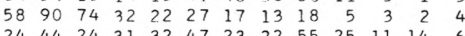

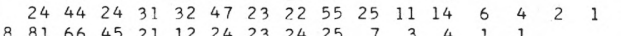

$\begin{array}{lllllllllllll}71 & 65 & 19 & 24 & 37 & 22 & 15 & 26 & 33 & 26 & 15\end{array}$

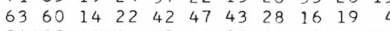

$\begin{array}{lllllllllll}34119 & 41 & 31 & 25 & 13 & 27 & 19 & 28 & 13 & 12\end{array}$

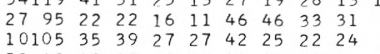

$\begin{array}{rrrrrrrrrrrrrrr}10105 & 35 & 39 & 27 & 27 & 42 & 25 & 22 & 24 & 4 & 4 & & 1 & \\ 59 & 48 & 49 & 33 & 30 & 21 & 49 & 25 & 16 & 11 & 7 & 4 & 9 & 1 & 3\end{array}$

2

$\begin{array}{lllllll}3 & 1 & & & & & \\ 2 & 3 & 1 & 2 & 1 & 1 & 1\end{array}$

CFS-DAYS

241470.0

188969.0

133006.0

$191852 \cdot 0$

180664.0

178004.0

131553.0

114244.0

154927.0

177072.0

183582.0

171123.0

169904.0

162072.0

202300.0

141335.0

137958.0

110411.0

131861.0
214646.0

121659.0

139067.0

185072.0

195725.0

195725.0

196646.0

248261.0

248261.0

195575.0

1950

213279.0

255684.0

171795.0

184153.0

169209.0

159691.0

195805.0

168165.0

174815.0

Clackamas River at Big Bottom, Oregon

CLASS CFS TOTAL ACCUM PERCT CLASS

$\begin{array}{rrrrr}.0 & & 15705 & 100.0 & 09 \\ 170.0 & 171 & 15705 & 100.0 & 10 \\ 200 \cdot 0 & 2138 & 15534 & 98.9 & 11 \\ 250.0 & 3121 & 13396 & 85.3 & 12 \\ 300 \cdot 0 & 1914 & 10275 & 65.4 & 13 \\ 350 \cdot 0 & 1407 & 8361 & 53.2 & 14 \\ 400.0 & 1141 & 6954 & 44.3 & 15 \\ 450 \cdot 0 & 857 & 5813 & 37.0 & 16 \\ 500.0 & 1293 & 4956 & 31.6 & 17\end{array}$

STATION NUMBER

CFS TOTAL ACCUM PERCT $600.0 \quad 1017 \quad 3663 \quad 23.3$ $\begin{array}{llll}700.0 & 781 & 2646 & 16.8\end{array}$ $800.0 \quad 976 \quad 186511.9$ $1200.0 \quad 185 \quad 471 \quad 3.0$ $\begin{array}{llll}1400.0 & 129 & 286 & 1.8\end{array}$ $1700.0 \quad 53 \quad 157 \quad 1.0$ 2500.0
$14-2080.00$

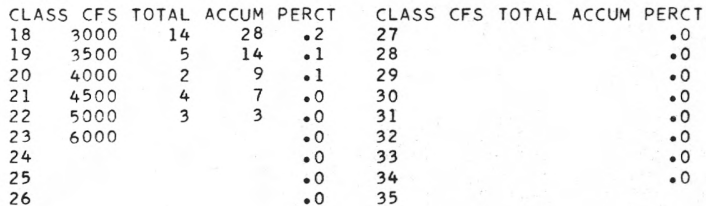


LOWEST MEAN DISCHARGE, IN CFS, FOR THE FOLLOWING NUMBER OF CONSECUTIVE DAYS IN YEAR BEGINNING APRIL 1

\begin{tabular}{|c|c|c|c|c|c|c|c|c|c|c|c|}
\hline YEAR & 1 & 3 & 7 & 14 & 30 & 60 & 90 & 120 & 150 & 183 & 274 \\
\hline $\begin{array}{l}1920 \\
1921\end{array}$ & 280.0 & $\begin{array}{l}280.0 \\
280.0\end{array}$ & 280.0 & 281.0 & 282.0 & 290.0 & 306.0 & $348 \cdot 0$ & 366.0 & 397.0 & 520.0 \\
\hline $\begin{array}{l}1921 \\
1922\end{array}$ & 280.0 & $280 \cdot 0$ & 283.0 & 284.0 & 288.0 & 299.0 & 302.0 & $308 \cdot 0$ & $327 \cdot 0$ & $419 \cdot 0$ & 426.0 \\
\hline $\begin{array}{l}1922 \\
1923\end{array}$ & $\begin{array}{l}256.0 \\
258.0\end{array}$ & $\begin{array}{l}256.0 \\
258.0\end{array}$ & $\begin{array}{l}256.0 \\
258.0\end{array}$ & 257.0 & 262.0 & 265.0 & 268.0 & $\begin{array}{l}270.0 \\
277.0\end{array}$ & $\begin{array}{l}273.0 \\
298.0\end{array}$ & $\begin{array}{l}287.0 \\
331.0\end{array}$ & $\begin{array}{l}438.0 \\
397.0\end{array}$ \\
\hline $\begin{array}{l}1923 \\
1924\end{array}$ & $\begin{array}{l}258.0 \\
212.0\end{array}$ & $\begin{array}{l}258.0 \\
212.0\end{array}$ & $\begin{array}{l}258.0 \\
213.0\end{array}$ & $\begin{array}{l}260 \cdot 0 \\
214.0\end{array}$ & $\begin{array}{l}263.0 \\
216.0\end{array}$ & 269.0 & $\begin{array}{l}274.0 \\
222.0\end{array}$ & 224.0 & 232.0 & 256.0 & $\begin{array}{l}391.0 \\
344.0\end{array}$ \\
\hline 1925 & $\begin{array}{l}212.0 \\
234.0\end{array}$ & 234.0 & $\begin{array}{l}213.0 \\
234.0\end{array}$ & 235.0 & $\begin{array}{l}216.0 \\
236.0\end{array}$ & $\begin{array}{l}219.0 \\
243.0\end{array}$ & 246.0 & $251 \cdot 0$ & 259.0 & 271.0 & 340.0 \\
\hline 1926 & 222.0 & 222.0 & 222.0 & 223.0 & 224.0 & 228 . 0 & 229.0 & $232 \cdot 0$ & 235.0 & 241.0 & 325.0 \\
\hline 1927 & 255.0 & 255.0 & 256.0 & 258.0 & 261.0 & 268.0 & 284.0 & $298 \cdot 0$ & 323.0 & 412.0 & 455.0 \\
\hline 1928 & 235.0 & 235.0 & 240.0 & 244.0 & 248.0 & 253.0 & 255.0 & 259.0 & 263.0 & 268.0 & 277.0 \\
\hline $\begin{array}{l}1929 \\
1930\end{array}$ & 202.0 & $202 \cdot 0$ & 204.0 & 206.0 & 207.0 & 208.0 & 209.0 & $210 \cdot 0$ & $218 \cdot 0$ & 251.0 & 319.0 \\
\hline 1930 & 199.0 & 200.0 & 201.0 & 202.0 & 204.0 & 204.0 & 205.0 & $207 \cdot 0$ & $210 \cdot 0$ & 213.0 & 238.0 \\
\hline 1931 & 190.0 & 190.0 & 190.0 & 190.0 & 192.0 & 194.0 & 195.0 & $201 \cdot 0$ & 212.0 & 223.0 & 263.0 \\
\hline 1932 & 213.0 & 214.0 & 215.0 & 216.0 & 217.0 & 223.0 & 230.0 & $241 \cdot 0$ & $284 \cdot 0$ & 316.0 & 340.0 \\
\hline 1933 & 252.0 & 252.0 & 255.0 & 259.0 & 263.0 & 271.0 & 274.0 & 290.0 & 302.0 & 383.0 & 558.0 \\
\hline 1934 & 246.0 & 246.0 & 247.0 & 249.0 & 250.0 & 252.0 & 255.0 & $261 \cdot 0$ & 270.0 & 290.0 & 369.0 \\
\hline 1935 & 250.0 & 251.0 & 252.0 & 254.0 & 257.0 & 258.0 & 264.0 & 269.0 & 280.0 & 293.0 & 398.0 \\
\hline 1936 & 216.0 & 225.0 & 234.0 & 236.0 & 240.0 & 242.0 & 248.0 & 251.0 & 255.0 & 259.0 & $280 \cdot 0$ \\
\hline 1937 & 241.0 & 241.0 & 242.0 & 246.0 & 251.0 & 253.0 & 258.0 & 268.0 & 323.0 & 403.0 & 496.0 \\
\hline 1938 & 262.0 & 263.0 & 264.0 & 265.0 & 267.0 & 270.0 & 273.0 & $280 \cdot 0$ & 290.0 & 318.0 & 341.0 \\
\hline $\begin{array}{l}1939 \\
1940\end{array}$ & 216.0 & 216.0 & 217.0 & 218.0 & 220.0 & 224.0 & 226.0 & $227 \cdot 0$ & 230.0 & 241.0 & 305.0 \\
\hline $\begin{array}{l}1940 \\
1941\end{array}$ & 201.0 & 203.0 & 205.0 & 207.0 & 217.0 & 220.0 & 220.0 & $223 \cdot 0$ & 229.0 & 239.0 & 291.0 \\
\hline 1941 & 192.0 & 193.0 & 195.0 & 197.0 & 199.0 & 204.0 & 205.0 & 209.0 & 214.0 & 233.0 & 333.0 \\
\hline 1942 & 184.0 & 186.0 & 187.0 & 187.0 & 190.0 & 193.0 & 198.0 & 205.0 & 223.0 & 257.0 & 391.0 \\
\hline 1943 & 239.0 & 239.0 & 241.0 & 242.0 & 244.0 & 252.0 & 261.0 & $288 \cdot 0$ & 311.0 & 324.0 & 338.0 \\
\hline 1944 & 210.0 & 210.0 & 210.0 & 211.0 & 214.0 & 216.0 & 217.0 & $220 \cdot 0$ & 224.0 & 229.0 & 273.0 \\
\hline 1945 & 205.0 & 205.0 & 206.0 & 207.0 & 212.0 & 216.0 & 216.0 & $220 \cdot 0$ & 230.0 & 260.0 & 403.0 \\
\hline 1946 & 277.0 & 278.0 & 280.0 & 284.0 & 285.0 & 288.0 & 292.0 & 314.0 & 335.0 & 413.0 & 529.0 \\
\hline 1947 & 230.0 & 230.0 & 232.0 & 240.0 & 245.0 & 250.0 & 259.0 & $274 \cdot 0$ & 297.0 & 348.0 & 414.0 \\
\hline 1948 & 272.0 & 273.0 & 275.0 & 276.0 & 286.0 & 293.0 & 295.0 & 300.0 & 316.0 & 364.0 & 392.0 \\
\hline 1949 & 279.0 & 279.0 & 280.0 & 282.0 & 287.0 & 291.0 & 294.0 & $300 \cdot 0$ & 320.0 & 350.0 & 447.0 \\
\hline 1950 & 305.0 & 306.0 & 307.0 & 309.0 & 313.0 & 323.0 & 344.0 & 398.0 & 518.0 & 606.0 & 679.0 \\
\hline 1951 & 292.0 & 294.0 & 295.0 & 296.0 & 298.0 & 304.0 & 314.0 & 329.0 & 358.0 & 393.0 & 437.0 \\
\hline 1952 & 244.0 & 247.0 & 249.0 & 252.0 & 258.0 & 259.0 & 263.0 & 267.0 & 274.0 & 284.0 & 435.0 \\
\hline 1953 & 253.0 & 255.0 & 256.0 & 258.0 & 264.0 & 267.0 & 271.0 & 277.0 & 301.0 & 371.0 & 498.0 \\
\hline 1954 & 287.0 & 287.0 & $288 . ?$ & 289.0 & 296.0 & 30.3 .0 & 312.0 & 318.0 & 334.0 & 347.0 & 361.0 \\
\hline 1955 & 262.0 & 263.0 & 272.0 & 273.0 & 278.0 & 283.0 & 312.0 & 351.0 & 408.0 & 528.0 & 640.0 \\
\hline 1956 & 279.0 & 280.0 & 286.0 & 290.0 & 292.0 & 297.0 & 311.0 & 329.0 & 339.0 & 382.0 & 439.0 \\
\hline 1957 & 232.0 & 235.0 & 235.0 & 238.0 & 241.0 & 244.0 & 250.0 & 257.0 & 267.0 & 282.0 & 414.0 \\
\hline 1958 & 229.0 & 229.0 & 231.0 & 235.0 & 239.0 & 242.0 & 246.0 & $254 \cdot 0$ & 277.0 & 332.0 & 437.0 \\
\hline 1959 & 238.0 & 240.0 & 241.0 & 241.0 & 243.0 & 250.0 & 259.0 & 272.0 & 277.0 & 294.0 & 323.0 \\
\hline 1960 & 231.0 & 233.0 & 234.0 & 237.0 & 242.0 & 246.0 & 249.0 & 255.0 & 271.0 & 338.0 & 443.0 \\
\hline 1961 & 247.0 & 248.0 & 250.0 & 250.0 & 253.0 & 258.0 & 260.0 & 269.0 & 276.0 & 307.0 & 384.0 \\
\hline 1962 & 244.0 & 245.0 & 248.0 & 252.0 & 256.0 & 261.0 & 269.0 & 293.0 & 312.0 & 353.0 & 470.0 \\
\hline
\end{tabular}

Clackamas River at Big Bottom, Oregon

STATION NUMBER

$14-2080.00$

HIGHEST MEAN DISCHARGE, IN CFS, FOR THE FOLLOWING NUMBER OF CONSECUTIVE DAYS IN YEAR ENDING SEPTEMBER 30

\begin{tabular}{|c|c|c|c|c|c|c|c|c|c|c|c|}
\hline YEAR & 1 & 3 & 7 & 15 & 30 & 60 & 90 & 120 & 150 & 183 & 274 \\
\hline 1921 & 3280.0 & 2620.0 & 2410.0 & 1740.0 & 1190.0 & 1030.0 & 919.0 & $871 \cdot 0$ & 885.0 & $860 \cdot 0$ & 775.0 \\
\hline $\begin{array}{l}1924 \\
1925\end{array}$ & $\begin{array}{l}1390.0 \\
3050.0\end{array}$ & $\begin{array}{l}1200.0 \\
2950.0\end{array}$ & $\begin{array}{l}1120.0 \\
2500.0\end{array}$ & $\begin{array}{r}956.0 \\
1880.0\end{array}$ & 1320.0 & $\begin{array}{l}603.0 \\
944.0\end{array}$ & $\begin{array}{l}572.0 \\
848.0\end{array}$ & $\begin{array}{l}531 \cdot 0 \\
783 \cdot 0\end{array}$ & 748.0 & 726.0 & 619.0 \\
\hline 1976 & 2680.0 & 2100.0 & 1470.0 & 1010.0 & 790.0 & $\begin{array}{l}944.0 \\
617.0\end{array}$ & 537.0 & 497.0 & 466.0 & 437.0 & 374.0 \\
\hline 1927 & 2900.0 & 2160.0 & $1480 . ?$ & 1040.0 & $869 \cdot 0$ & 788.0 & 666.0 & $688 \cdot 0$ & 651.0 & 625.0 & 571.0 \\
\hline 1928 & 3480.0 & 2290.0 & $1830 . ?$ & 1300.0 & 976.0 & 753.0 & 689.0 & $628 \cdot 0$ & 657.0 & 655.0 & 562.0 \\
\hline 1929 & 1080.0 & $1030 . ?$ & 980.0 & 905.0 & 810.0 & 706.0 & 620.0 & $560 \cdot 0$ & 502.0 & $464 \cdot 0$ & 404.0 \\
\hline 1930 & 1080.0 & 912.0 & 851.0 & 801.0 & 665.0 & 516.0 & 475.0 & $450 \cdot 0$ & 429.0 & $412 \cdot 0$ & 349.0 \\
\hline 1931 & 4130.0 & 3340.0 & 2100.0 & 1390.0 & 1020.0 & 754.0 & 619.0 & $548 \cdot 0$ & 490.0 & $444 \cdot 0$ & 369.0 \\
\hline 1932 & 1920.0 & 1650.0 & 1250.0 & 1150.0 & 914.0 & 833.0 & 793.0 & $708 \cdot 0$ & 629.0 & 590.0 & 491.0 \\
\hline 1933 & 2830.0 & 2220.0 & 1890.0 & 1540.0 & 1260.0 & 988.0 & 844.0 & 749.0 & 670.0 & 616.0 & 559.0 \\
\hline 1934 & 4850.0 & $3700 \cdot 0$ & $275 \mathrm{c} .0$ & $2000 \cdot 0$ & 1470.0 & 1240.0 & 995.0 & & 807.0 & 724.0 & 584.0 \\
\hline 1935 & 1420.0 & 1280.0 & 1030.0 & 872.0 & 841.0 & 767.0 & 675.0 & $616 \cdot 0$ & 586.0 & 582.0 & 538.0 \\
\hline 1936 & 2400.0 & 1870.0 & 1440.0 & 1330.0 & 964.0 & 807.0 & 694.0 & $641 \cdot 0$ & 682.0 & 641.0 & 532.0 \\
\hline 1937 & 2620.0 & 2240.0 & 1640.0 & 1160.0 & 1070.0 & 1030.0 & 917.0 & $796 \cdot 0$ & 706.0 & 630.0 & 510.0 \\
\hline 1938 & 2730.0 & 1960.0 & 1440.0 & 1090.0 & 997.0 & 873.0 & 802.0 & 712.0 & 737.0 & 736.0 & 649.0 \\
\hline 1939 & 970.0 & $859 . ?$ & 786 & 751.0 & 696.0 & 681.0 & 598.0 & $550 \cdot 0$ & 513.0 & 502.0 & 437.0 \\
\hline 1940 & 1870.0 & 1390.0 & $1220 \cdot n$ & $1000 \cdot 0$ & 876.0 & 785.0 & 694.0 & $608 \cdot 0$ & 576.0 & $526 \cdot 0$ & 428.0 \\
\hline 1941 & 1060.0 & 775.0 & 610.0 & 522.0 & 454.0 & 417.0 & 403.0 & $385 \cdot 0$ & 374.0 & 375.0 & 335.0 \\
\hline 1942 & 2070.0 & 1590.0 & 1220.0 & 856.0 & 844.0 & 633.0 & 554.0 & $511 \cdot 0$ & 482.0 & 469.0 & 414.0 \\
\hline 1943 & 2670.0 & 2120.0 & 1750.0 & 1360.0 & 1130.0 & 967.0 & 917.0 & $800 \cdot 0$ & 766.0 & 779.0 & 703.0 \\
\hline 1944 & 876.0 & 772.0 & 598.0 & 501.0 & 462.0 & 434.0 & 408.0 & 395.0 & 387.0 & 391.0 & 369.0 \\
\hline 1945 & 2240.0 & 1530.0 & 1332.0 & 981.0 & 880.0 & $700 \cdot 0$ & 612.0 & $625 \cdot 0$ & 582.0 & 532.0 & 435.0 \\
\hline 1946 & 3490.0 & $2930 \cdot 0$ & 2000.0 & 1510.0 & 1070.0 & 799.0 & 697.0 & 653.0 & 682.0 & 682.0 & 590.0 \\
\hline 1947 & 4760.0 & $4110 \cdot 0$ & 2810.0 & 1740.0 & 1260.0 & 908.0 & 822.0 & $770 \cdot 0$ & 744.0 & 713.0 & 595.0 \\
\hline 1948 & 5060.0 & 3380.0 & 2150.0 & 1450.0 & 959.0 & 780.0 & 686.0 & 667.0 & 666.0 & 669.0 & 616.0 \\
\hline 1949 & 2890.0 & 2220.0 & 1640.0 & 1570.0 & 1370.0 & 1160.0 & 965.0 & 878.0 & 797.0 & 713.0 & 620.0 \\
\hline 1950 & 2390.0 & 1870.0 & 1410.0 & 1240.0 & 1210.0 & 1040.0 & 922.0 & 928.0 & 880.0 & 825.0 & 689.0 \\
\hline 1951 & 2560.0 & 2060.0 & 1790.0 & $1380 . ?$ & 1150.0 & 1050.0 & 1020.0 & $1010 \cdot 0$ & 912.0 & 884.0 & 802.0 \\
\hline 1952 & 1850.0 & 1410.0 & 1200.0 & 1020.0 & 916.0 & 862.0 & 774.0 & $688 \cdot 0$ & 669.0 & 633.0 & 593.0 \\
\hline 1953 & 5320.0 & 4000.0 & $2900 \cdot n$ & 2230.0 & 1750.0 & 1190.0 & 963.0 & 869.0 & 841.0 & 787.0 & 627.0 \\
\hline 1954 & 3270.0 & $2350 . n$ & 1580.0 & 1240.0 & 998.0 & 828.0 & 788.0 & 771.0 & $756 \cdot 0$ & 767.0 & 683.0 \\
\hline 1955 & 1990.0 & 1920.0 & $170 w . ?$ & 1340.0 & 1110.0 & 911.0 & 776.0 & $688 \cdot 0$ & 619.0 & $576 \cdot 0$ & $5 \div 5.0$ \\
\hline 1956 & 3940.0 & 3250.0 & $2250 \cdot 0$ & 1700.0 & 1420.0 & 1310.0 & 1120.0 & 967.0 & 876.0 & 903.0 & 822.0 \\
\hline 1957 & 2170.0 & 1670.0 & 1350.0 & 1250.0 & 980.0 & 852.0 & 818.0 & $716 \cdot 0$ & 647.0 & 635.0 & 539.0 \\
\hline 1958 & 2290.0 & 1960.0 & 1420.0 & 1140.0 & 1020.0 & 868.0 & 821.0 & $751 \cdot 0$ & 751.0 & 722.0 & 590.0 \\
\hline 1959 & 1840.0 & 1570.0 & 1160.0 & 909.0 & 878.0 & 709.0 & 706.0 & 639.0 & $624 \cdot 0$ & 617.0 & 534.0 \\
\hline 1960 & 1610.0 & 1380.0 & 1200.0 & 1120.0 & 935.0 & 812.0 & 750.0 & 694.0 & 644.0 & 580.0 & 497.0 \\
\hline 1961 & 4390.0 & 3260.0 & $2300 . ?$ & 1620.0 & 1210.0 & 980.0 & 864.0 & 813.0 & 778.0 & 752.0 & 630.0 \\
\hline 1962 & 1760.0 & $1380 . ?$ & 1080.2 & 897.0 & 853.0 & 768.0 & 685.0 & $618 \cdot 0$ & 623.0 & 607.0 & 526.0 \\
\hline 963 & 2400.0 & 2030.0 & 1690.0 & 1490.0 & 1150.0 & 812.0 & 730.0 & $680 \cdot 0$ & 644.0 & 637.0 & 556.0 \\
\hline
\end{tabular}


Oak Grove Fork at T1mothy Meadows, Oreg.

STATION NUMBER

$14-2085.00$

DURATION TABLE OF DAILY DISCHARGE

CLASS $00 \begin{array}{lllllllllllllllllllllllllllllllllll}2 & 3 & 4 & 5 & 6 & 7 & 8 & 9 & 10 & 11 & 12 & 13 & 14 & 15 & 16 & 17 & 18 & 19 & 20 & 21 & 22 & 23 & 24 & 25 & 26 & 27 & 28 & 29 & 30 & 31 & 32 & 33 & 34\end{array}$

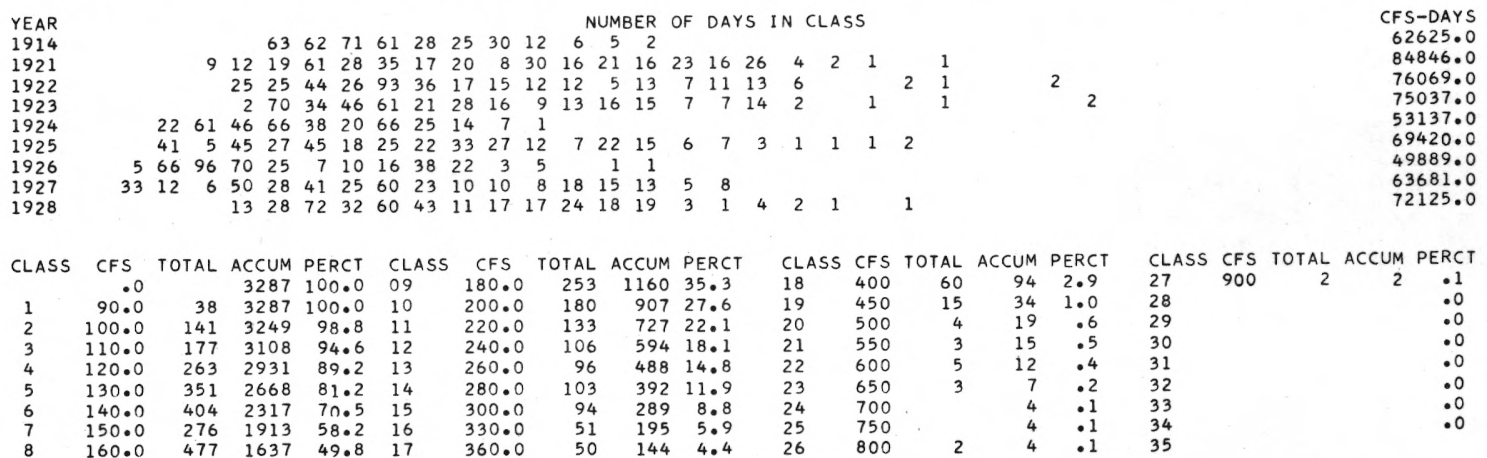

vak urove Fork at Timothy Meadows, Oreg. STATION NUMBER 14-2085.00

LOWEST MEAN DISCHARGE, IN CFS, FOR THE FOLLOWING NUMBER OF CONSECUTIVE DAYS IN YEAR BEGINNING APRIL 1

\begin{tabular}{|c|c|c|c|c|c|c|c|c|c|c|c|}
\hline YEAR & 1 & 3 & 7 & 14 & 30 & 60 & 90 & 120 & 150 & 183 & 274 \\
\hline 1921 & 121.0 & 122.0 & 123.0 & 124.0 & 128.0 & 130.0 & 136.0 & 140.0 & 148.0 & 180.0 & 182.0 \\
\hline 1922 & 127.0 & 128.0 & 129.0 & 130.0 & 130.0 & 134.0 & 135.0 & $139 \cdot 0$ & $144 \cdot 0$ & 151.0 & 174.0 \\
\hline $\begin{array}{l}1923 \\
1924\end{array}$ & 116.0 & 116.0 & 117.0 & 118.0 & $120 \cdot 0$ & $127 \cdot 0$ & $132 \cdot 0$ & $136 \cdot 0$ & 140.0 & $\begin{array}{l}144.0 \\
118.0\end{array}$ & $\begin{array}{l}159.0 \\
132.0\end{array}$ \\
\hline 1924 & 100.0 & $100 \cdot 0$ & 100.0 & 101.0 & 102.0 & 104.0 & 107.0 & $109 \cdot 6$ & $\begin{array}{l}113.0 \\
122.0\end{array}$ & 124.0 & $\begin{array}{l}132.0 \\
141.0\end{array}$ \\
\hline $\begin{array}{l}1925 \\
1926\end{array}$ & $\begin{array}{r}109.0 \\
90.0\end{array}$ & $\begin{array}{r}111.0 \\
90.0\end{array}$ & $\begin{array}{r}113.0 \\
90.0\end{array}$ & $\begin{array}{r}114.0 \\
90.5\end{array}$ & $\begin{array}{r}117.0 \\
91.5\end{array}$ & $\begin{array}{r}118.0 \\
95.8\end{array}$ & $\begin{array}{r}119.0 \\
97.6\end{array}$ & $\begin{array}{r}121.0 \\
99 \cdot 6\end{array}$ & 103.0 & 107.0 & 123.0 \\
\hline $\begin{array}{l}1927 \\
\end{array}$ & 122.0 & 124.0 & 125.0 & 125.0 & 126.0 & 131.0 & 136.0 & 140.0 & 148.0 & 172.0 & 178.0 \\
\hline
\end{tabular}

Oak Grove Fork at Timothy Meadows, Oreg. STATION NUMBER 14-2085.00

HIGHEST MEAN DISCHARGE, IN CFS, FOR THE FOLLOWING NUMBER OF CONSECUTIVE DAYS IN YEAR ENDING SEPTEMBER 30

\begin{tabular}{|c|c|c|c|c|c|c|c|c|c|c|c|}
\hline YEAR & 1 & 3 & 7 & 15 & 30 & 60 & 90 & 120 & 150 & 183 & 274 \\
\hline 1914 & 316.0 & 307.0 & 294.0 & 280.0 & 261.0 & 244.0 & 231.0 & $217 \cdot 0$ & 206.0 & 198.0 & 181.0 \\
\hline 1921 & 670.0 & 588.0 & 505.0 & 412.0 & 400.0 & 375.0 & 353.0 & 332.0 & 319.0 & 312.0 & 264.0 \\
\hline 1922 & 820.0 & 758.0 & 563.0 & 479.0 & 403.0 & 331.0 & 285.0 & 256.0 & 237.0 & 222.0 & 232.0 \\
\hline 1923 & 922.0 & 835.0 & 633.0 & 455.0 & 377.0 & 333.0 & 301.0 & 273.0 & 260.0 & 259.0 & 229.0 \\
\hline 1924 & 252.0 & 233.0 & 222.0 & 216.0 & 205.0 & 187.0 & 182.0 & 177.0 & 173.0 & 168.0 & 155.0 \\
\hline 1925 & 618.0 & 600.0 & 522.0 & 425.0 & 335.0 & 294.0 & 278.0 & $282 \cdot 0$ & 267.0 & 250.0 & 215.0 \\
\hline 1926 & 310.0 & 288.0 & 261.0 & 224.0 & 215.0 & 207.0 & 197.0 & 182.0 & 170.0 & 162.0 & 147.0 \\
\hline 1927 & 399.0 & 394.0 & 372.0 & 346.0 & 327.0 & 295.0 & 259.0 & $242 \cdot 0$ & 230.0 & 216.0 & 195.0 \\
\hline 1928 & 624.0 & 510.0 & 477.0 & 396.0 & 305.0 & 287.0 & 271.0 & $248 \cdot 0$ & 231.0 & 239.0 & 217.0 \\
\hline
\end{tabular}


Oak Grove Fork near Government Camp,

STATION NUMBER $14-2087.00$ Oreg.

DURATION TABLE OF DAILY DISCHARGE

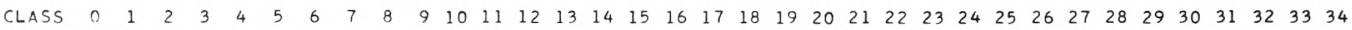

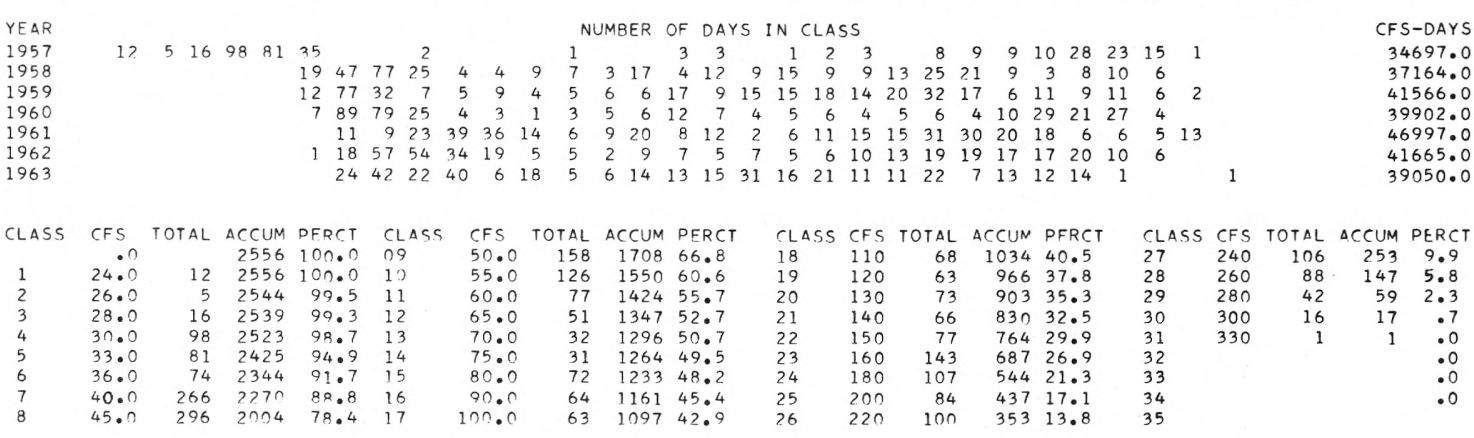

Oak Grove Fork near Government Camp,

StATION NUMBER $\quad 14-2087.00$

Oreg. LOWEST MEAN DISCHARGE, IN CFS, FOR THE FOLLOWING NUMBER OF CONSECUTIVE DAYS IN YEAR REGINNING APRIL 1

\begin{tabular}{|c|c|c|c|c|c|c|c|c|c|c|c|}
\hline YEAR & 1 & 3 & 7 & 14 & 30 & 60 & 90 & 120 & 150 & 183 & 274 \\
\hline 1957 & 29.0 & 29.0 & 29.3 & 29.6 & 30.2 & 31.4 & 32.0 & 32.4 & 32.5 & 33.0 & 71.8 \\
\hline 1958 & 37.0 & 38.0 & 39.0 & 39.5 & 41.1 & 48.4 & 55.8 & 69.1 & 73.1 & 73.9 & 94.2 \\
\hline 1959 & 38.0 & 39.0 & 39.4 & 39.8 & 42.1 & 47.0 & 64.0 & $81 \cdot 3$ & 80.2 & 103.0 & 123.0 \\
\hline 1960 & 38.0 & 38.0 & 38.6 & 43.1 & 45.3 & 50.8 & 63.5 & $61 \cdot 3$ & 58.9 & 86.1 & 117.0 \\
\hline 1961 & 42.0 & 42.7 & 44.4 & 45.4 & 55.3 & 67.0 & 70.4 & 86.0 & 105.0 & 119.0 & 129.0 \\
\hline 1962 & 39.0 & 41.3 & 42.6 & 45.4 & 47.6 & 52.6 & 54.5 & $61 \cdot 1$ & 62.5 & 73.7 & 87 \\
\hline
\end{tabular}

Oak Grove Fork near Government Camp,

STATION NUMBER 14-2U8I.VU

Oreg $_{\text {HIGHEST MEAN DISCHARGE, IN CFS, FOR THE FOLLOWING NUMBER OF CONSECUTIVE DAYS IN YEAR ENDING SEPTEMBER } 30}$

\begin{tabular}{|c|c|c|c|c|c|c|c|c|c|c|c|}
\hline YEAR & 1 & 3 & 7 & 15 & 30 & 60 & 90 & 120 & 150 & 183 & 274 \\
\hline 1957 & 318.0 & 293.0 & 288.0 & 286.0 & 274.0 & 247.0 & 219.0 & $218 \cdot 0$ & 185.0 & 158.0 & 116.0 \\
\hline 1958 & 297.0 & 291.0 & 285.0 & 276.0 & 233.0 & 179.0 & 156.0 & 140.0 & 132.0 & 130.0 & 115.0 \\
\hline 1959 & 301.0 & 300.0 & 286.0 & 272.0 & 221.0 & 150.0 & 136.0 & 123.0 & 123.0 & 125.0 & 116.0 \\
\hline 1960 & 286.0 & 282.0 & 279.0 & 268.0 & 226.0 & 181.0 & 185.0 & 176.0 & 151.0 & 132.0 & 109.0 \\
\hline 1961 & 320.0 & 315.0 & 312.0 & 268.0 & 226.0 & 182.0 & 179.0 & 166.0 & 146.0 & 138.0 & 144.0 \\
\hline 1962 & 296.0 & 284.0 & 277.0 & 272.0 & 213.0 & 191.0 & 177.0 & 161.0 & 161.0 & 152.0 & 134.0 \\
\hline 1963 & 348.0 & 251.0 & 247.0 & 240.0 & 214.0 & 174.0 & 156.0 & 139.0 & 142.0 & 144.0 & 124.0 \\
\hline
\end{tabular}


Oak Grove Fork above powerplant 1ntake, Oreg.STATION NUMBER 14-2090.00

DURATION TABLE OF DAILY DISCHARGE

\begin{tabular}{|c|c|c|c|c|c|c|c|c|c|c|c|c|c|c|c|c|c|c|c|c|c|c|c|c|c|c|c|c|c|c|c|c|}
\hline CLASS & 1 & 2 & 3 & 4 & 5 & 6 & 7 & 8 & 9 & 10 & 11 & 12 & 13 & 14 & 15 & 16 & 1718 & 181 & 192 & 202 & 212 & 222 & 232 & 242 & 252 & 262 & 2728 & 2930 & 31 & 3233 & 34 & \\
\hline YEAR & & & & & & & & & & & & & & NUMB & & OF & DAYS & $S$ IN & $\mathrm{NCL}$ & LASS & & & & & & & & & & & & CFS-DAYS \\
\hline 1910 & & & & & & & 67 & 63 & 29 & 49 & 30 & 15 & 14 & 8 & 27 & 18 & 1910 & 10 & 5 & 4 & 2 & 1 & 1 & & & & 2 & 1 & & & & 218899.0 \\
\hline 1911 & & & & & & 43 & 75 & 65 & 55 & 43 & 20 & 12 & 12 & 13 & 9 & 9 & 7 & & 1 & 1 & & & & & & & & & & & & 178345.0 \\
\hline 1914 & & & & & & 16 & 86 & 74 & 66 & 18 & 18 & 17 & 27 & 20 & 14 & 7 & & & & & & & & & & & & & & & & 181896.0 \\
\hline 1915 & & & & & & 821 & & 33 & 17 & 35 & 14 & 13 & 3 & 8 & 2 & 2 & & & & & & & & & & & & & & & & 155271.0 \\
\hline 1919 & & & & & & & 27 & 54 & 88 & 45 & 38 & 13 & 11 & 9 & 10 & 19 & 3112 & 12 & 2 & 3 & & 2 & & & & 1 & & & & & & 218505.0 \\
\hline 1921 & & & & & 6 & 10 & 47 & 55 & 25 & 34 & 20 & 6 & 13 & 24 & 21 & 24 & 1427 & 272 & 221 & 11 & 1 & 1 & & 3 & & 1 & & & & & & 244649.0 \\
\hline 1922 & & & & & & 221 & 15 & 78 & 28 & 23 & 12 & 8 & 10 & 8 & 5 & 11 & 1011 & 11 & 6 & 9 & 3 & 1 & & 1 & 1 & & 3 & & & & & 201226.0 \\
\hline 1923 & & & & & 6 & 71 & 63 & 36 & 34 & 26 & 9 & 8 & 15 & 11 & 18 & 30 & 146 & 6 & 6 & 4 & 2 & & & 1 & 1 & 1 & & & 1 & 1 & 1 & $212506 \cdot 0$ \\
\hline 1924 & & & & & 78 & 42 & 68 & 53 & 39 & 46 & 14 & 4 & 9 & 4 & 3 & 5 & 1 & & & & & & & & & & & & & & & 156349.0 \\
\hline 1925 & & & & & 31 & 573 & 37 & 29 & 25 & 29 & 27 & 31 & 24 & 24 & 12 & 15 & 45 & 9 & 1 & 2 & 3 & & 1 & 1 & 3 & & & & & & & 202565.0 \\
\hline 1926 & & & & 331 & 12 & 46 & 62 & 22 & 14 & 28 & 19 & 14 & 3 & 2 & 3 & 2 & 21 & 1 & 1 & 1 & & & & & & & & & & & & 147000.0 \\
\hline 1927 & & & & 12 & 28 & 26 & 62 & 46 & 59 & 37 & 14 & 7 & 11 & 8 & 22 & 14 & 67 & 7 & 3 & 2 & & 1 & & & & & & & & & & 186274.0 \\
\hline 1928 & & & & & & 45 & 58 & 50 & 47 & 28 & 20 & 15 & 15 & 15 & 18 & 32 & 103 & 3 & 1 & 1 & 4 & 3 & & & 1 & & & & & & & 204346.0 \\
\hline 1929 & & & & & 611 & 140 & 50 & 19 & 17 & 12 & 4 & 7 & 9 & 9 & 10 & 11 & 96 & 6 & 1 & & & & & & & & & & & & & 160347.0 \\
\hline 1930 & & & 57 & 68 & 62 & 18 & 24 & 39 & 34 & 24 & 23 & 3 & 6 & 3 & 2 & 2 & & & & & & & & & & & & & & & & 139446.0 \\
\hline 1931 & & 361 & 17 & 52 & 47 & 33 & 12 & 5 & 8 & 7 & 14 & 10 & 6 & 5 & 2 & 2 & 22 & 2 & 1 & & 1 & & & 1 & & 1 & 1 & & & & & 133360.0 \\
\hline 1932 & 14 & 34 & 42 & 634 & 46 & $24 z$ & 22 & 15 & 9 & 4 & 6 & 11 & 11 & 10 & 11 & 31 & 9 & 2 & & 1 & & 1 & & & & & & & & & & 158302.0 \\
\hline 1933 & & & 16 & 10 & 42 & 66 & 47 & 42 & 30 & 27 & 11 & 6 & 11 & 7 & 21 & 6 & 5 & 9 & 2 & 4 & 2 & & & 1 & & & & & & & & 176948.0 \\
\hline 1934 & & & 3 & 321 & 11 & 36 & 22 & 15 & 30 & 22 & 15 & 16 & 8 & 8 & 7 & 11 & 5 & 6 & 3 & 3 & 2 & 2 & 1 & 3 & 2 & & 1 & 1 & & & & 181047.0 \\
\hline 1935 & & & 19 & 105 & 56 & 31 & 41 & 66 & 51 & 23 & 7 & 11 & 8 & 5 & 9 & 25 & & 1 & 2 & & & & & & & & & & & & & 166824.0 \\
\hline 1936 & & & & 608 & 80 & 413 & 30 & 32 & 29 & 18 & 11 & 6 & 8 & 5 & 4 & 112 & 21 & 5 & 4 & & & 1 & & & & & & & & & & 167017.0 \\
\hline 1937 & & 3 & 79 & 516 & 60 & 30 & 27 & 15 & 15 & 8 & 3 & 9 & 7 & 12 & 8 & 13 & 11 & 7 & 4 & 1 & & & 2 & & & & & & & & & 158189.0 \\
\hline 1938 & & & & 23 & 21 & 503 & 36 & 30 & 42 & 33 & 26 & 20 & 16 & 10 & 5 & 24 & 18 & 4 & 2 & 1 & 2 & 1 & & & 1 & & & & & & & 194060.0 \\
\hline 1939 & & & & & 96 & 426 & 65 & 57 & 20 & 9 & 8 & 9 & 14 & 24 & 7 & 14 & & & & & & & & & & & & & & & & 161415.0 \\
\hline 1940 & & & & 8410 & & 312 & 23 & 23 & 8 & 12 & 26 & 10 & 6 & 9 & 9 & 10 & 7 & & & & & & & & & & & & & & & 150857.0 \\
\hline 1941 & & 60 & 26 & 526 & 68 & 62 & 66 & 20 & 5 & 3 & & 2 & & & & 1 & & & & & & & & & & & & & & & & 118740.0 \\
\hline 1942 & & 60 & 39 & 203 & 33 & 33 & 44 & 46 & 43 & 21 & 10 & 4 & 3 & 1 & 4 & 1 & 3 & & & & & & & & & & & & & & & 138246.0 \\
\hline 1943 & & & 31 & 5 & 5 & 10 & 49 & 47 & 30 & 24 & 21 & 20 & 9 & 11 & 13 & 222 & 242 & 231 & 15 & 5 & & 1 & & & & & & & & & & 218262.0 \\
\hline 1944 & & & & & 78 & 72 & 85 & 49 & 31 & 38 & 11 & 2 & & & & & & & & & & & & & & & & & & & & 144641.0 \\
\hline 1945 & & 2 & 44 & 766 & 64 & 22 & 26 & 31 & 30 & 14 & 5 & 8 & 6 & 4 & 7 & 9 & 9 & 5 & 3 & & & & & & & & & & & & & 37.0 \\
\hline 1946 & & 15 & 19 & 10 & 7 & 23 & 86 & 57 & 34 & 22 & 10 & 13 & 7 & 13 & 4 & 241 & 13 & 4 & 1 & & & 1 & & 2 & & & & & & & & 179153.0 \\
\hline 1947 & & & & 35 & 58 & 15 & 46 & 41 & 39 & 39 & 27 & 54 & 14 & 8 & 5 & 5 & 2 & 2 & & 1 & & & 1 & & & & 2 & & 1 & 2 & & 191725.0 \\
\hline 1948 & & & & & 15 & 52 & 67 & 34 & 42 & 33 & 21 & 17 & 11 & 28 & 14 & 15 & 92 & 2 & 3 & & 1 & & & 1 & & & 1 & & & & & 193071.0 \\
\hline 1949 & & & & & 10 & 63 & 69 & 37 & 34 & 41 & 18 & 15 & 7 & 5 & 6 & 71 & 131 & 11 & 7 & 7 & 4 & 4 & 6 & & 1 & & & & & & & 205789.0 \\
\hline 1950 & & & & & & 47 & 61 & 73 & 27 & 11 & 14 & 20 & 25 & 15 & 8 & 15 & 812 & 121 & 151 & 12 & 2 & & & & & & & & & & & 210211.0 \\
\hline 1951 & & & & & & 3 & 40 & 51 & 18 & 21 & 26 & 28 & 19 & 26 & 27 & 462 & 261. & $\begin{array}{ll}11 & 1 \\
\end{array}$ & 15 & 4 & 2 & 1 & 1 & & & & & & & & & 245330.0 \\
\hline 1952 & & & & & & 28 & 62 & 79 & 51 & 29 & 16 & 17 & 10 & 21 & 17 & 12 & 912 & 12 & 3 & & & & & & & & & & & & & 195039.0 \\
\hline 1953 & & & & & 59 & 57 & 23 & 38 & 29 & 40 & 16 & 8 & 12 & 20 & 15 & 20 & 56 & 6 & 3 & 1 & 3 & 2 & 4 & 1 & 1 & & 1 & & 1 & & & 200607.0 \\
\hline 1954 & & & & & 6 & 43 & 28 & 44 & 11 & 26 & 44 & 36 & 33 & 23 & 24 & 281 & 115 & 5 & 1 & 1 & & & & 1 & & & & & & & & 213065.0 \\
\hline 1955 & & & & & & 531 & 15 & 72 & 32 & 23 & 8 & 6 & 9 & 7 & 3 & 19 & 8 & 3 & 2 & 3 & 2 & & & & & & & & & & & 176640.0 \\
\hline 1956 & & 32 & 38 & 10 & 9 & 21 & 9 & 9 & 41 & 25 & 32 & 17 & 12 & 11 & 12 & 172 & 2619 & 19 & 7 & 3 & 9 & 3 & 1 & 2 & & 1 & & & & & & 219525.0 \\
\hline 1957 & & 67 & 47 & 13 & 15 & 8 & 8 & 24 & 321 & 13 & 20 & 3 & 4 & 1 & & 6 & 3 & & 1 & & & & & & & & & & & & & 153780.0 \\
\hline 1958 & & 6 & 27 & 47 & 14 & 35 & 13 & 26 & 26 & 97 & 36 & 11 & 8 & 7 & 3 & 5 & 2 & & 1 & & 1 & & & & & & & & & & & 167657.0 \\
\hline 1959 & & 4 & 41 & 7 & 17 & 10 & 12 & 21 & 511 & 54 & 27 & 7 & 5 & 1 & 6 & 2 & & & & & & & & & & & & & & & & 171808.0 \\
\hline 1960 & & & & 27 & 41 & 21 & 32 & 29 & 36 & 86 & 56 & 14 & 5 & 6 & 7 & 5 & 1 & & & & & & & & & & & & & & & 173692.0 \\
\hline 1961 & & 22 & 20 & 134 & 412 & 25 & 9 & 16 & 37 & 87 & 33 & 31 & 21 & 13 & 4 & 3 & 4 & 1 & 1 & & 1 & & & 1 & 2 & & & & & & & 184392.0 \\
\hline 1962 & & & 24 & 362 & 29 & 30 & 13 & 15 & 131 & 35 & 23 & 19 & 14 & 3 & 3 & 3 & 32 & 2 & & & & & & & & & & & & & & 10.0 \\
\hline 1963 & & 13 & 37 & 393 & 37 & 262 & 23 & 53 & 26 & 46 & 30 & 16 & 15 & 5 & 2 & 5 & 1 & & 2 & 1 & & & & & & & & & & & & 160902.0 \\
\hline
\end{tabular}

Oak Grove Fork above powerplant

intake, Oreg.
STATION NUMBER

$\begin{array}{crrr}\text { CFS } & \text { TOTAL } & \text { ACCUM } & \text { PERCT } \\ 450 \cdot 0 & 1537 & 7958 & 45.4 \\ 500 \cdot 0 & 1768 & 6421 & 36 \cdot 6 \\ 550 \cdot 0 & 913 & 4653 & 26.5 \\ 600 \cdot 0 & 649 & 3740 & 21.3 \\ 650 \cdot 0 & 526 & 3091 & 17.6 \\ 700 \cdot 0 & 490 & 2565 & 14.6 \\ 750 \cdot 0 & 443 & 2075 & 11.8 \\ 800 \cdot 0 & 618 & 1632 & 9.3 \\ 900 \cdot 0 & 386 & 1014 & 5.8\end{array}$

$14-2090.00$

\begin{tabular}{|c|c|c|c|c|c|c|c|c|c|}
\hline CLASS & CFS & TOTAL & ACCUM & PERCT & CLASS & 5 CFS & TOTAL & ACCUM & DERCT \\
\hline 18 & 1000 & 247 & 628 & 3.6 & 27 & 2200 & 10 & 20 & .1 \\
\hline 19 & 1100 & 147 & 381 & 2.2 & 28 & 2400 & 1 & 10 & .1 \\
\hline 20 & 1200 & 86 & 234 & 1.3 & 29 & 2600 & 2 & 9 & .1 \\
\hline 21 & 1300 & 47 & 148 & .8 & 30 & 2800 & & 7 & .0 \\
\hline 22 & 1400 & 26 & 101 & .6 & 31 & 3000 & 3 & 7 & .0 \\
\hline 23 & 1500 & 18 & 75 & .4 & 32 & 3300 & 3 & 4 & .0 \\
\hline 24 & 1600 & 19 & 57 & .3 & 33 & 3600 & & 1 & .0 \\
\hline 25 & 1800 & 13 & 38 & .2 & 34 & 4000 & 1 & 1 & .0 \\
\hline 26 & 2000 & 5 & 25 & .1 & 35 & & & & \\
\hline
\end{tabular}

$14-2090.00$

Oak Grove Fork above powerplant

STATION NUMBER

intake, Oreg.

Summary for water years $1910-11,1914-15,1919,1921-55$

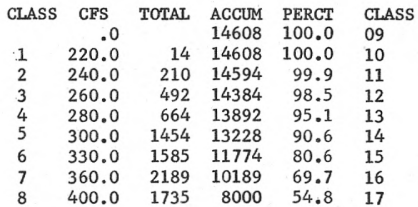

Oak Grove Fork above powerplant intake, Oreg.

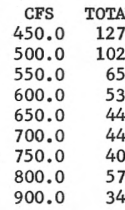

900.0

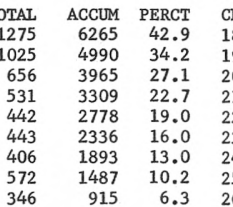

STATION NUMBER

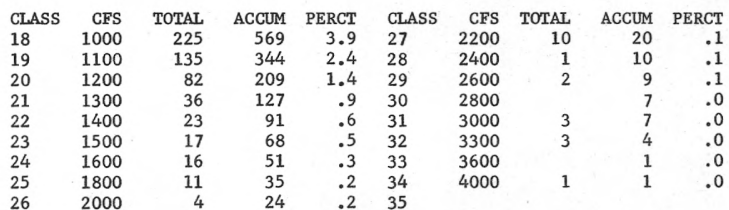

$14-2090.00$
Summary for water years 1957-63

\begin{tabular}{lrrrrlrr} 
CLASS & CFS & TOTAL & ACCUM & PERCT & CLASS & \multicolumn{1}{c}{ CFS } & TOTAL \\
& .0 & & 2556 & 100.0 & 09 & 450.0 & 221 \\
1 & 220.0 & & 2556 & 100.0 & 10 & 500.0 & 718 \\
2 & 240.0 & 80 & 2556 & 100.0 & 11 & 550.0 & 225 \\
3 & 260.0 & 196 & 2476 & 96.9 & 12 & 600.0 & 101 \\
4 & 280.0 & 182 & 2280 & 89.2 & 13 & 650.0 & 72 \\
5 & 300.0 & 194 & 2098 & 82.0 & 14 & 700.0 & 36 \\
6 & 330.0 & 155 & 1904 & 74.5 & 15 & 750.0 & 25 \\
7 & 360.0 & 110 & 1749 & 68.4 & 16 & 800.0 & 29 \\
8 & 400.0 & 184 & 1639 & 64.1 & 17 & 900.0 & 14
\end{tabular}

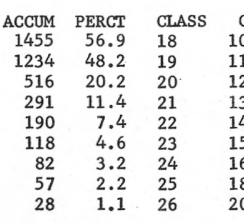

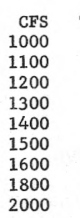

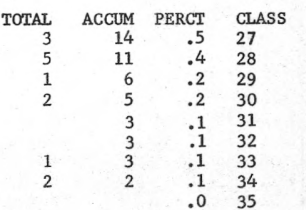

$\begin{array}{rrr}\text { CFS } & \text { TOTAL ACCUM } & \text { PERCT } \\ 2200 & & .0 \\ 2400 & & .0 \\ 2600 & & .0 \\ 2800 & & .0 \\ 3000 & & .0 \\ 3300 & & .0 \\ 3600 & & .0 \\ 4000 & & .0\end{array}$


Oak Grove Fork above powerplant

STATION NUMBER $\quad 14-2090.00$

intake, Oreg.

\begin{tabular}{|c|c|c|c|c|c|c|c|c|c|c|c|}
\hline YEAR & 1 & 3 & 7 & 14 & & & 90 & 120 & 150 & 183 & 274 \\
\hline 1910 & 358.0 & 358.0 & 358.0 & 362.0 & 365.0 & 370.0 & 373.0 & 381.0 & 395.0 & 415.0 & 434.0 \\
\hline 1914 & 350.0 & 350.0 & 350.0 & 353.0 & 357.0 & 362.0 & 367.0 & 370.0 & 371.0 & 370.0 & 383.0 \\
\hline 1915 & 340.0 & 340.0 & 340.0 & 340.0 & 341.0 & 345.0 & 348.0 & $356 \cdot 0$ & 365.0 & 386.0 & 447.0 \\
\hline 1921 & $360 \cdot 0$ & $360 \cdot 0$ & 361.0 & 367.0 & 377.0 & 381.0 & 389.0 & $400 \cdot 0$ & 423.0 & 520.0 & 497.0 \\
\hline 1922 & 324.0 & $324 . n$ & 327.0 & 333.0 & 336.0 & 342.0 & 344.0 & $347 \cdot 0$ & 355.0 & 374.0 & 496.0 \\
\hline 1923 & 340.0 & 340.0 & 340.0 & 346.0 & 352.0 & 359.0 & 362.0 & $370 \cdot 0$ & 382.0 & 398.0 & 445.0 \\
\hline 1924 & 301.0 & 304.0 & 307.0 & 309.0 & 316.0 & 318.0 & 322.0 & 323.0 & 332.0 & 347.0 & 407.0 \\
\hline 1925 & 312.0 & 312.0 & 313.0 & 315.0 & 318.0 & 325.0 & 331.0 & 337.0 & 342.0 & 353.0 & 409.0 \\
\hline 1926 & 286.0 & 286.0 & 289.0 & 291.0 & 296.0 & 299.0 & 302.0 & 305.0 & 308.0 & 317.0 & 375.0 \\
\hline 1927 & 344.0 & 347.0 & 347.0 & 354.0 & 359.0 & 363.0 & 381.0 & 393.0 & 413.0 & 478.0 & 514.0 \\
\hline 1928 & 311.0 & 311.0 & 312.0 & 313.0 & 317.0 & 327.0 & 338.0 & $342 \cdot 0$ & 344.0 & 344.0 & 355.0 \\
\hline 1929 & 271.0 & 271.0 & 274.0 & 277.0 & 278.0 & 288.0 & 298.0 & $308 \cdot 0$ & 318.0 & 331.0 & 382.0 \\
\hline 1930 & 252.0 & 252.0 & 254.0 & 256.0 & 258.0 & 262.0 & 263.0 & $264 \cdot 0$ & 266.0 & 269.0 & 285.0 \\
\hline 1931 & 236.0 & 237.0 & 238.0 & 239.0 & 246.0 & 256.0 & 257.0 & $260 \cdot 0$ & 264.0 & 270.0 & 290.0 \\
\hline 1932 & 266.0 & 267.0 & 269.0 & 271.0 & 277.0 & 283.0 & 291.0 & 303.0 & 334.0 & 353.0 & 367.0 \\
\hline 1933 & 306.0 & 306.0 & 307.0 & 308.0 & 313.0 & 321.0 & 331.0 & 334.0 & 349.0 & $400 \cdot 0$ & 546.0 \\
\hline 1934 & 271.0 & 271.0 & 271.0 & 272.0 & 278.0 & 284.0 & 290.0 & 298.0 & 307.0 & 319.0 & 369.0 \\
\hline 1935 & 282.0 & 282.0 & 283.0 & 288.0 & 293.0 & 295.0 & 296.0 & 299.0 & 304.0 & 314.0 & 379.0 \\
\hline 1936 & 257.0 & 257.0 & 261.0 & 262.0 & 266.0 & 276.0 & 287.0 & $285 \cdot 0$ & 286.0 & 291.0 & 313.0 \\
\hline 1937 & 291.0 & 292.0 & 294.0 & 298.0 & 301.0 & 304.0 & 310.0 & $322 \cdot 0$ & 352.0 & 391.0 & 467.0 \\
\hline 1938 & 315.0 & 315.0 & 315.0 & 317.0 & 324.0 & 329.0 & 335.0 & $342 \cdot 0$ & 350.0 & 369.0 & 385.0 \\
\hline 1939 & 291.0 & 291.0 & 292.0 & 292.0 & 294.0 & 299.0 & 302.0 & $306 \cdot 0$ & 310.0 & 323.0 & 361.0 \\
\hline 1940 & 279.0 & 282.0 & 282.0 & 284.0 & 292.0 & 293.0 & 296.0 & $300 \cdot 0$ & 304.0 & 311.0 & 330.0 \\
\hline 1941 & 243.0 & 243.0 & 244.0 & 246.0 & 252.0 & 255.0 & 256.0 & $258 \cdot 0$ & 263.0 & 279.0 & 336.0 \\
\hline 1942 & 251.0 & 252.0 & 254.0 & 256.0 & 260.0 & 261.0 & 264.0 & $274 \cdot 0$ & 287.0 & 318.0 & 408.0 \\
\hline 1943 & 341.0 & 341.0 & 345.0 & 348.0 & 353.0 & 366.0 & 379.0 & 386.0 & 387.0 & 387.0 & 400.0 \\
\hline 1944 & 281.0 & 285.0 & 286.0 & 286.0 & 293.0 & 298.0 & 298.0 & $302 \cdot 0$ & 304.0 & 308.0 & 346.0 \\
\hline 1945 & 251.0 & 251.0 & 254.0 & 257.0 & 258.0 & 264.0 & 268.0 & 276.0 & 286.0 & 311.0 & 387.0 \\
\hline 1946 & 360.0 & 360.0 & 360.0 & 360.0 & 363.0 & 365.0 & 369.0 & $388 \cdot 0$ & 404.0 & 446.0 & 533.0 \\
\hline 1947 & 296.0 & 296.0 & 301.0 & 302.0 & 305.0 & 311.0 & 321.0 & $338 \cdot 0$ & 360.0 & 381.0 & 432.0 \\
\hline 1948 & 322.0 & 322.0 & 324.0 & 326.0 & 337.0 & 344.0 & 347.0 & 357.0 & 374.0 & 395.0 & 418.0 \\
\hline 1949 & 330.0 & 330.0 & 331.0 & 342.0 & 344.0 & 354.0 & 361.0 & 370.0 & 388.0 & 393.0 & 450.0 \\
\hline 1950 & 352.0 & 352.0 & 355.0 & 359.0 & 367.0 & 384.0 & 396.0 & $439 \cdot 0$ & 500.0 & 558.0 & 623.0 \\
\hline 1951 & 370.0 & 373.0 & 375.0 & 379.0 & 386.0 & 402.0 & 412.0 & $430 \cdot 0$ & 440.0 & 456.0 & 463.0 \\
\hline 1952 & 307.0 & 307.0 & 308.0 & 309.0 & 317.0 & 319.0 & 323.0 & 329.0 & 337.0 & 349.0 & 482.0 \\
\hline 1953 & 325.0 & 327.0 & 329.0 & 332.0 & 335.0 & 340.0 & 351.0 & 365.0 & 387.0 & 435.0 & 513.0 \\
\hline 1954 & 341.0 & 347.0 & 351.0 & 356.0 & 369.0 & 388.0 & 393.0 & 394.0 & 399.0 & 406.0 & 416.0 \\
\hline 1955 & 330.0 & 330.0 & 334.0 & 339.0 & 346.0 & 354.0 & 370.0 & $397 \cdot 0$ & 435.0 & 515.0 & 589.0 \\
\hline 1956 & 247.0 & 247.0 & 247.0 & 249.0 & 252.0 & 256.0 & 260.0 & 274.0 & 314.0 & 348.0 & 414.0 \\
\hline 1957 & 240.0 & 240.0 & 240.0 & 241.0 & 246.0 & 251.0 & 256.0 & $266 \cdot 0$ & 286.0 & 302.0 & 383.0 \\
\hline 1958 & 245.0 & 264.0 & 266.0 & 269.0 & 275.0 & 284.0 & 300.0 & $317 \cdot 0$ & 350.0 & 376.0 & 428.0 \\
\hline 1959 & 261.0 & 266.0 & 268.0 & 268.0 & 271.0 & 284.0 & 318.0 & $364 \cdot 0$ & 379.0 & 404.0 & 423.0 \\
\hline 1960 & 269.0 & 285.0 & 286.0 & 290.0 & 296.0 & 310.0 & 352.0 & $396 \cdot 0$ & 408.0 & 435.0 & 474.0 \\
\hline 1961 & 248.0 & 265.0 & 266.0 & 268.0 & 285.0 & 296.0 & 308.0 & $341 \cdot 0$ & 355.0 & 385.0 & 439.0 \\
\hline 1962 & 257.0 & 261.0 & 264.0 & 278.0 & 281.0 & 288.0 & 296.0 & $309 \cdot 0$ & 316.0 & 350.0 & 416.0 \\
\hline
\end{tabular}

Oak Grove Fork above powerplant

STATION NUMBER $\quad 14-2090.00$

Intake, Oreg.
HIGHEST MEAN DISCHARGE, IN CFS, FOR THE FOLLOWING NUMBER OF CONSECUTIVE DAYS IN YEAR ENDING SEPTEMBER 30

\begin{tabular}{|c|c|c|c|c|c|c|c|c|c|c|c|}
\hline YEAR & 1 & 3 & 7 & 15 & 30 & 60 & 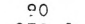 & 120 & 150 & 183 & 274 \\
\hline $\begin{array}{l}1910 \\
1911\end{array}$ & 2670.0 & 2360.0 & 1630.0 & $1230 \cdot 0$ & 1060.0 & 945.0 & $870 \cdot 0$ & $\begin{array}{l}796.0 \\
620.0\end{array}$ & $\begin{array}{l}762.0 \\
581.0\end{array}$ & $\begin{array}{l}771.0 \\
560.0\end{array}$ & $\begin{array}{l}669 \cdot 0 \\
531.0\end{array}$ \\
\hline $\begin{array}{l}1911 \\
1914\end{array}$ & $\begin{array}{l}1270.0 \\
1010.0\end{array}$ & $\begin{array}{r}1130.0 \\
940.0\end{array}$ & $\begin{array}{l}996.0 \\
891.0\end{array}$ & $\begin{array}{l}892.0 \\
835.0\end{array}$ & 847.0 & 742.0 & 671.0 & $\begin{array}{l}620 \cdot 0 \\
649 \cdot 0\end{array}$ & 627.0 & $\begin{array}{l}560.0 \\
599.0\end{array}$ & $\begin{array}{l}531.0 \\
539.0\end{array}$ \\
\hline 1915 & $\begin{array}{r}1010.0 \\
839.0\end{array}$ & $\begin{array}{l}940.0 \\
806.0\end{array}$ & $\begin{array}{l}891.0 \\
771.0\end{array}$ & $\begin{array}{l}835.0 \\
731.0\end{array}$ & $\begin{array}{l}762.0 \\
678.0\end{array}$ & 730.0 & $\begin{array}{l}695.0 \\
574.0\end{array}$ & 536.0 & 507.0 & 484.0 & $\begin{array}{l}539.0 \\
447.0\end{array}$ \\
\hline 1919 & 2000.0 & 1530.0 & 1350.0 & 1100.0 & $\begin{array}{l}678.0 \\
973.0\end{array}$ & $\begin{array}{l}612.0 \\
913.0\end{array}$ & 835.0 & $764 \cdot 0$ & 780.0 & 735.0 & $\begin{array}{l}447.0 \\
654.0\end{array}$ \\
\hline 1921 & 2050.0 & 1790.0 & 1500.0 & 1230.0 & 1140.0 & 1060.0 & 1000.0 & $952 \cdot 0$ & 914.0 & 901.0 & 765.0 \\
\hline $\begin{array}{l}1922 \\
1923\end{array}$ & 2280.0 & 2130.0 & 1550.0 & 1430.0 & 1110.0 & 899.0 & 766.0 & $682 \cdot 0$ & 625.0 & 606.0 & 612.0 \\
\hline 1923 & 4360.0 & 3660.0 & 2570.0 & 1740.0 & 1240.0 & 911.0 & 830.0 & 753.0 & 800.0 & 784.0 & 661.0 \\
\hline 1924 & 905.0 & 851.0 & 813.0 & 755.0 & 674.0 & 580.0 & 554.0 & $537 \cdot 0$ & 526.0 & 509.0 & 462.0 \\
\hline 1925 & 1900.0 & 1880.0 & 1660.0 & 1360.0 & 1030.0 & 820.0 & 796.0 & $789 \cdot 0$ & 754.0 & 713.0 & 627.0 \\
\hline $\begin{array}{l}1926 \\
1927\end{array}$ & 1220.0 & 1150.0 & 974.0 & 780.0 & 720.0 & 639.0 & 584.0 & $540 \cdot 0$ & 516.0 & 488.0 & 436.0 \\
\hline 1927 & 1420.0 & $1210 \cdot 0$ & 1000.0 & 907.0 & 877.0 & 809.0 & 708.0 & $685 \cdot 0$ & 649.0 & 619.0 & 567.0 \\
\hline 1928 & 1860.0 & 1530.0 & 1430.0 & $1210 \cdot 0$ & 929.0 & 815.0 & 766.0 & $690 \cdot 0$ & 679.0 & 700.0 & 624.0 \\
\hline $\begin{array}{l}1929 \\
1930\end{array}$ & 1110.0 & 1080.0 & 1060.0 & 995.0 & 908.0 & 802.0 & 695.0 & $635 \cdot 0$ & 580.0 & 537.0 & 475.0 \\
\hline 1930 & 898.0 & 858.0 & 768.0 & 709.0 & 622.0 & 558.0 & 544.0 & $517 \cdot 0$ & 486.0 & 471.0 & 416.0 \\
\hline 1931 & 2540.0 & 2140.0 & 1580.0 & 1160.0 & 879.0 & 734.0 & 614.0 & $544 \cdot 0$ & 497.0 & 462.0 & 399.0 \\
\hline 1932 & 1420.0 & $1210 \cdot 0$ & 1000.0 & 930.0 & 863.0 & 828.0 & 795.0 & $707 \cdot 0$ & 638.0 & 581.0 & 490.0 \\
\hline 1933 & 1660.0 & 1470.0 & 1320.0 & 1170.0 & 1050.0 & 896.0 & 782.0 & $698 \cdot 0$ & 634.0 & 585.0 & 540.0 \\
\hline 1934 & 2730.0 & $2280 \cdot 0$ & 1940.0 & 1510.0 & 1190.0 & 1030.0 & 874.0 & $800 \cdot 0$ & 739.0 & 674.0 & 562.0 \\
\hline 1935 & 1190.0 & 1120.0 & 936.0 & 834.0 & 827.0 & 732.0 & 642.0 & 589.0 & 562.0 & 561.0 & 506.0 \\
\hline 1936 & 1460.0 & $1140 \cdot 0$ & 1030.0 & 994.0 & 958.0 & 815.0 & 694.0 & 639.0 & 622.0 & 597.0 & 510.0 \\
\hline 1937 & 1530.0 & 1430.0 & 1190.0 & $1000 \cdot 0$ & 940.0 & 887.0 & 786.0 & $695 \cdot 0$ & 631.0 & 576.0 & 482.0 \\
\hline 1938 & $1870 \cdot 0$ & $1490 \cdot 0$ & 1130.0 & $1000 \cdot ?$ & 940.0 & 827.0 & 731.0 & $670 \cdot 0$ & 691.0 & 678.0 & 600.0 \\
\hline 1939 & 859.0 & 838.0 & 826.0 & $820 \cdot 0$ & 778.0 & 737.0 & 659.0 & $600 \cdot 0$ & 560.0 & 537.0 & 484.0 \\
\hline 1940 & 982.0 & 933.0 & 913.0 & 835.0 & 729.0 & $700 \cdot 0$ & 663.0 & $601 \cdot 0$ & 550.0 & $519 \cdot 0$ & 450.0 \\
\hline $\begin{array}{l}1941 \\
1942\end{array}$ & 880.0 & 679.0 & 550.0 & 474.0 & 403.0 & 373.0 & 369.0 & $364 \cdot 0$ & 364.0 & 366.0 & 348.0 \\
\hline $\begin{array}{l}1942 \\
1943\end{array}$ & 967.0 & 878.0 & 771.0 & 641.0 & 617.0 & 494.0 & 471.0 & $448 \cdot 0$ & 453.0 & 458.0 & 418.0 \\
\hline 1943 & 1420.0 & 1320.0 & 1230.0 & 1150.0 & 1070.0 & 1000.0 & 946.0 & $842 \cdot 0$ & 779.0 & 745.0 & 687.0 \\
\hline 1944 & 647.0 & 588.0 & 561.0 & 549.0 & 536.0 & 515.0 & 483.0 & 459.0 & 444.0 & 435.0 & 421.0 \\
\hline 1945 & 1140.0 & $\$ 110 \cdot 0$ & 1040.0 & 975.0 & $908 \cdot 0$ & 736.0 & $640 \cdot 0$ & $614 \cdot 0$ & 570.0 & 536.0 & 459.0 \\
\hline 1946 & 1730.0 & 1590.0 & 1250.0 & 1000.0 & 896.0 & 797.0 & 700.0 & 636.0 & 602.0 & 619.0 & 550.0 \\
\hline 1947 & 3430.0 & 3290.0 & 2460.0 & 1590.0 & 1100.0 & 816.0 & 738.0 & 693.0 & 683.0 & $668 \cdot 0$ & 591.0 \\
\hline 1948 & 2390.0 & 1840.0 & 1340.0 & 983.0 & 855.0 & 781.0 & 700.0 & $678 \cdot 0$ & 652.0 & 651.0 & 584.0 \\
\hline 1949 & 1920.0 & $1640 \cdot 0$ & 1540.0 & 1420.0 & 1340.0 & 1150.0 & 981.0 & $862 \cdot 0$ & 795.0 & 726.0 & 632.0 \\
\hline 1950 & 1350.0 & $1320 \cdot 0$ & 1280.0 & 1250.0 & 1190.0 & 1030.0 & 915.0 & 863.0 & 821.0 & 753.0 & 647.0 \\
\hline 1951 & 1500.0 & 1420.0 & 1280.0 & 1160.0 & 1070.0 & 960.0 & 853.0 & $845 \cdot 0$ & 829.0 & 832.0 & 761.0 \\
\hline 1952 & 1190.0 & 1140.0 & 1080.0 & 1040.0 & 940.0 & 859.0 & 782.0 & $706 \cdot 0$ & 663.0 & 623.0 & 584.0 \\
\hline 1953 & 3040.0 & 2470.0 & 1920.0 & 1410.0 & 1290.0 & 954.0 & 814.0 & $768 \cdot 0$ & 775.0 & 741.0 & 625.0 \\
\hline 1954 & 1610.0 & 1340.0 & 1070.0 & 945.0 & 884.0 & 816.0 & 772.0 & $727 \cdot 0$ & $708 \cdot 0$ & 706.0 & 657.0 \\
\hline 1955 & 1320.0 & 1310.0 & 1230.0 & 1100.0 & 987.0 & 856.0 & .738 .0 & $666 \cdot 0$ & 609.0 & 575.0 & 522.0 \\
\hline 1956 & 2140.0 & 1810.0 & 1510.0 & 1190.0 & 1080.0 & 1010.0 & 890.0 & 798.0 & 777.0 & 819.0 & 710.0 \\
\hline 1957 & 1180.0 & 969.0 & 810.0 & 681.0 & 604.0 & 564.0 & 564.0 & 554.0 & 548.0 & 539.0 & 478.0 \\
\hline 1958 & 1360.0 & 1170.0 & 902.0 & 710.0 & 640.0 & 596.0 & 592.0 & $581 \cdot 0$ & 571.0 & 565.0 & 511.0 \\
\hline 1959 & 848.0 & 752.0 & 682.0 & 606.0 & 567.0 & 551.0 & 544.0 & 543.0 & 538.0 & 538.0 & 509.0 \\
\hline 1960 & 962.0 & 879.0 & 815.0 & 781.0 & 684.0 & 628.0 & 601.0 & 553.0 & 537.0 & 527.0 & 507.0 \\
\hline 1961 & 1990.0 & 1610.0 & 1230.0 & 984.0 & 780.0 & 709.0 & 680.0 & $660 \cdot 0$ & 625.0 & 618.0 & 567.0 \\
\hline 1962 & 1040.0 & 969.0 & 816.0 & 715.0 & 659.0 & 639.0 & 577.0 & 555.0 & 562.0 & 560.0 & 525.0 \\
\hline 1963 & 1200.0 & 1060.0 & 826.0 & 779.0 & 689.0 & 584.0 & 567.0 & 542.0 & 545.0 & $551 \cdot 0$ & 491.0 \\
\hline
\end{tabular}


CLASS $0 \begin{array}{lllllllllllllllllllllllllllllllllll}2 & 2 & 3 & 4 & 5 & 6 & 7 & 8 & 9 & 10 & 11 & 12 & 13 & 14 & 15 & 16 & 17 & 18 & 19 & 20 & 21 & 22 & 23 & 24 & 25 & 26 & 27 & 28 & 29 & 30 & 31 & 32 & 33 & 34\end{array}$

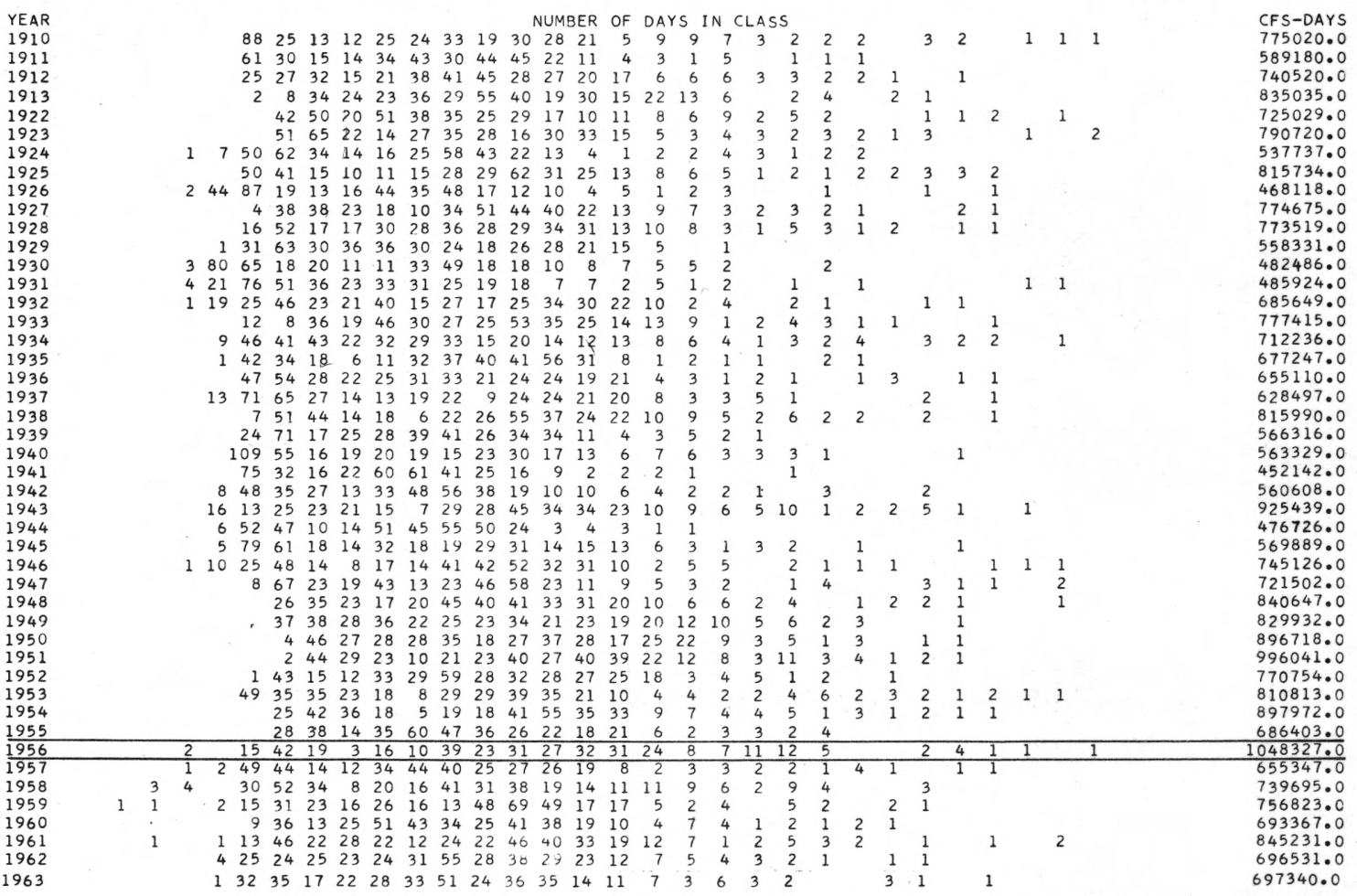

14-2095.00

Summary for water years 1910-13, 1922-55

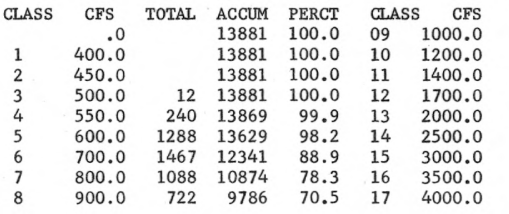

C1ackamas River above Three Lynx, Oreg.

Summary for water years 1957-63

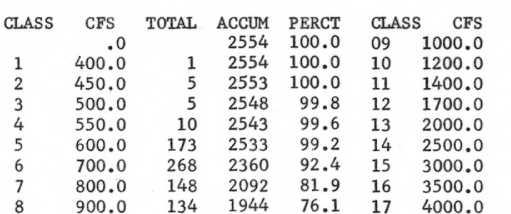

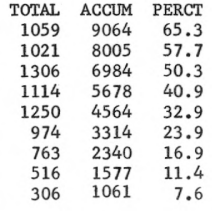

STATION NUMBER

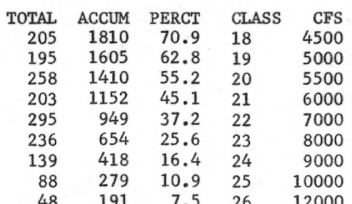

$\begin{array}{lrrrrlrrrr}\text { CLASS } & \text { CFS } & \text { TOTAL } & \text { ACCUM } & \text { PERCT } & \text { CLASS } & \text { CFS } & \text { TOTAL } & \text { ACCUM } & \text { PERCT } \\ 18 & 4500 & 206 & 755 & 5.4 & 27 & 14000 & 19 & 37 & .3 \\ 19 & 5000 & 146 & 549 & 4.0 & 28 & 17000 & 6 & 18 & .1 \\ 20 & 5500 & 71 & 403 & 2.9 & 29 & 20000 & 9 & 12 & .1 \\ 21 & 6000 & 101 & 332 & 2.4 & 30 & 25000 & 3 & 3 & .0 \\ 22 & 7000 & 64 & 231 & 1.7 & 31 & 30000 & & & .0 \\ 23 & 8000 & 43 & 167 & 1.2 & 32 & & & & .0 \\ 24 & 9000 & 23 & 124 & .9 & 33 & & & & .0 \\ 25 & 10000 & 39 & 101 & .7 & 34 & & & & .0 \\ 26 & 12000 & 25 & 62 & .4 & 35 & & & & \end{array}$

$14-2095.00$

\begin{tabular}{rrrlrrrr} 
TOTAL & ACCUM & PERCT & \multicolumn{1}{l}{ CLASS } & CFS & TOTAL & ACCUM & PERCT \\
36 & 143 & 5.6 & 27 & 14000 & 2 & 4 & .2 \\
28 & 107 & 4.2 & 28 & 17000 & & 2 & .1 \\
13 & 79 & 3.1 & 29 & 20000 & 2 & 2 & .1 \\
27 & 66 & 2.6 & 30 & 25000 & & & .0 \\
12 & 39 & 1.5 & 31 & 30000 & & & .0 \\
8 & 27 & 1.1 & 32 & & & & .0 \\
8 & 19 & .7 & 33 & & & & .0 \\
7 & 11 & .4 & 34 & & & & .0
\end{tabular}


Clackamas River above Three Lynx, Oreg. STATION NUMBER 14-2095.00

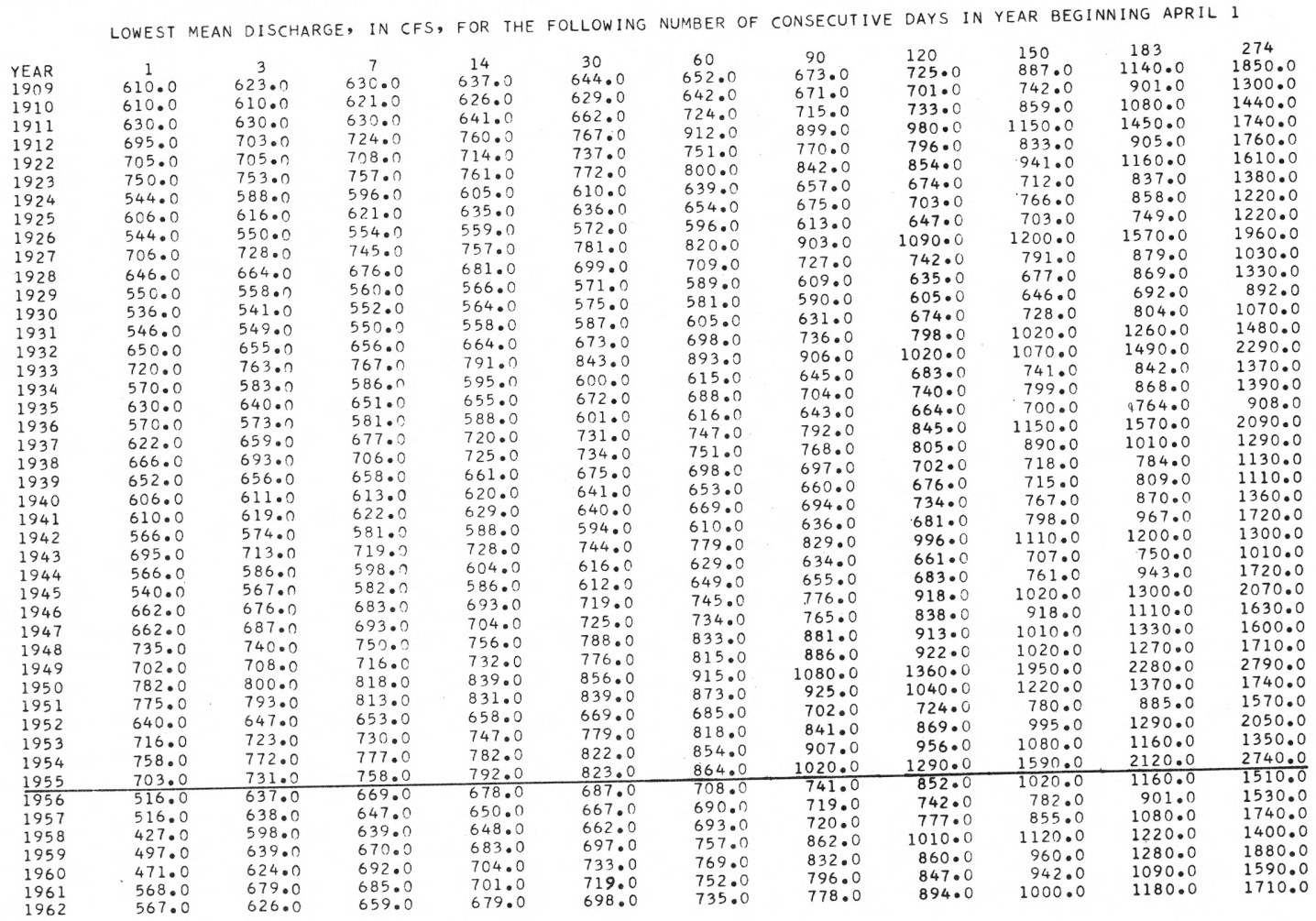

Clackamas River above Three Lynx, Oregon

STATION NUMBER

$14-2095.00$

HIGHEST MEAN DISCHARGE, IN CFS, FOR THE FOLLOWING NUMBER OF CONSECUTIVE DAYS IN YEAR ENDING SEPTEMBER 30

\begin{tabular}{|c|c|c|c|c|c|c|c|c|c|c|c|}
\hline YEAR & 1 & 3 & 7 & 15 & 30 & 00 & 90 & 120 & $\begin{array}{l}150 \\
3480\end{array}$ & $\begin{array}{c}183 \\
3320.0\end{array}$ & 274 \\
\hline $\begin{array}{l}1911 \\
1912\end{array}$ & $\begin{array}{r}8420.0 \\
12900.0\end{array}$ & $\begin{array}{r}7000 \cdot 0 \\
10500 \cdot 0\end{array}$ & & $\begin{array}{l}4170.0 \\
5470.0\end{array}$ & 3310.0 & 2570.0 & 2350.0 & $2100 \cdot 0$ & $\begin{array}{l}1990.0 \\
3090.0\end{array}$ & 2870.0 & $\begin{array}{l}1900.0 \\
2420.0\end{array}$ \\
\hline $\begin{array}{l}1913 \\
1922\end{array}$ & $\begin{array}{l}11600.0 \\
22000.0\end{array}$ & $\begin{array}{r}8680 \cdot 0 \\
14100 \cdot 0\end{array}$ & & & 4390.0 & $\begin{array}{l}4080.0 \\
3730.0\end{array}$ & $\begin{array}{l}3820.0 \\
3070.0\end{array}$ & $2660 \cdot 0$ & 2390.0 & 2560.0 & 2380.0 \\
\hline $\begin{array}{l}1922 \\
1923\end{array}$ & $\begin{array}{l}22000 \cdot 0 \\
28800 \cdot 0\end{array}$ & $\begin{array}{l}14100.0 \\
23900.0\end{array}$ & $\begin{array}{r}9610.0 \\
15500.0\end{array}$ & $\begin{array}{r}9050.0 \\
10600.0\end{array}$ & $\begin{array}{l}6000.0 \\
7920.0\end{array}$ & $\begin{array}{l}3730.0 \\
4790.0\end{array}$ & 3800.0 & $3620 \cdot 0$ & 3590.0 & 3380.0 & 2620.0 \\
\hline $\begin{array}{l}1923 \\
1924\end{array}$ & $\begin{array}{r}28800.0 \\
8590.0\end{array}$ & 7290.0 & $\begin{array}{r}15500.0 \\
6250.0\end{array}$ & & & 2870.0 & 2710.0 & $2480 \cdot 0$ & $2300 \cdot 0$ & 2160.0 & 1740.0 \\
\hline $\begin{array}{l}1924 \\
1925\end{array}$ & $\begin{array}{r}8590.0 \\
15300.0\end{array}$ & 14100.0 & 12000.0 & $\begin{array}{l}4940.0 \\
9020.0\end{array}$ & 6320.0 & 4600.0 & 4070.0 & 3710.0 & 3400.0 & 3280.0 & 2740.0 \\
\hline $\begin{array}{l}1925 \\
1926\end{array}$ & & 11200.0 & 7420.0 & 4870.0 & 3960.0 & 2840.0 & 2460.0 & $2220 \cdot 0$ & 2040.0 & 1870.0 & 1500.0 \\
\hline 1926 & $\begin{array}{l}14500 \cdot 0 \\
14900.0\end{array}$ & 11800.0 & 7520.0 & 5160.0 & 3860.0 & 3200.0 & 3090.0 & $3020 \cdot 0$ & 2840.0 & $2860 \cdot 0$ & 2530.0 \\
\hline 1927 & & 10700.0 & 8810.0 & 6330.0 & 4780.0 & 3650.0 & 3240.0 & $3030 \cdot 0$ & 3130.0 & 3110.0 & 2570.0 \\
\hline 1928 & $\begin{array}{r}16300.0 \\
5260.0\end{array}$ & 4170.0 & 4060.0 & 3820.0 & 3510.0 & 3100.0 & 2810.0 & 2540.0 & 2240.0 & 2130.0 & 1800.0 \\
\hline 1929 & $\begin{array}{l}5260.0 . \\
7410.0\end{array}$ & 5540.0 & 4880.0 & 4090.0 & 3530.0 & 2620.0 & 2390.0 & $2230 \cdot 0$ & 2080.0 & 1990.0 & 1570.0 \\
\hline 1930 & $\begin{array}{r}7410 \cdot 0 \\
22700 \cdot 0\end{array}$ & 17100.0 & 10100.0 & 6480.0 & 4810.0 & 3340.0 & 2720.0 & $2430 \cdot 0$ & 2150.0 & $1940 \cdot 0$ & 1570.0 \\
\hline 1931 & $\begin{array}{l}22700 \cdot 0 \\
13000 \cdot 0\end{array}$ & $10600 \cdot 0$ & 7230.0 & 6060.0 & 4600.0 & 4030.0 & 3680.0 & 3370.0 & 2960.0 & 2810.0 & 2270.0 \\
\hline 1932 & $\begin{array}{l}13000.0 \\
14500.0\end{array}$ & 10900.0 & 8550.0 & 6560.0 & 5340.0 & 4260.0 & 3690.0 & $3320 \cdot 0$ & 2980.0 & 2780.0 & 2540.0 \\
\hline 1933 & & $\begin{array}{l}10900.0 \\
18000.0\end{array}$ & 13500.0 & 9350.0 & 7030.0 & 5630.0 & 4370.0 & 3910.0 & 3440.0 & 3050.0 & 2370.0 \\
\hline 1934 & $\begin{array}{r}24200.0 \\
8350.0\end{array}$ & $\begin{array}{r}18000.0 \\
7850.0\end{array}$ & 5930.0 & 4070.0 & 3090.0 & 2840.0 & 2560.0 & 2410.0 & 2400.0 & 2430.0 & 2240.0 \\
\hline 1935 & $\begin{array}{r}8350.0 \\
14200.0\end{array}$ & $10100 \cdot 0$ & 7420.0 & 7130.0 & 4780.0 & 3210.0 & 2910.0 & $2900 \cdot 0$ & 2970.0 & 2730.0 & 2150.0 \\
\hline 1936 & $\begin{array}{l}14200 \cdot 0 \\
15000 \cdot 0\end{array}$ & $12600 \cdot 0$ & 8270.0 & 5540.0 & 4690.0 & 4270.0 & 3820.0 & $3420 \cdot 0$ & 3030.0 & 2650.0 & 2080.0 \\
\hline 1937 & $\begin{array}{l}15000.0 \\
15600.0\end{array}$ & 11200.0 & 7780.0 & 4970.0 & & 3830.0 & 3660.0 & 3250.0 & 3200.0 & 3240.0 & 2720.0 \\
\hline 1938 & $\begin{array}{r}15600.0 \\
5680.0\end{array}$ & $5010 \cdot 0$ & 4340.0 & $\begin{array}{l}4970.0 \\
3610.0\end{array}$ & 3150.0 & 2870.0 & 2550.0 & $2340 \cdot 0$ & 2220.0 & 2190.0 & 1830.0 \\
\hline 1939 & & 8220.0 & $\begin{array}{l}4340.0 \\
5970.0\end{array}$ & 4480.0 & 4220.0 & 3630.0 & 3160.0 & $2810 \cdot 0$ & 2650.0 & 2370.0 & 1830.0 \\
\hline 1940 & $\begin{array}{r}12300.0 \\
6360.0\end{array}$ & 4350.0 & 3140.0 & 2760.0 & 2190.0 & 1960.0 & 1850.0 & $1730 \cdot 0$ & 1630.0 & 1620.0 & 1420.0 \\
\hline 1941 & $\begin{array}{r}6360.0 \\
11000.0\end{array}$ & $\begin{array}{l}4350 \cdot 0 \\
8040.0\end{array}$ & 5780.0 & 3940.0 & 3840.0 & 2850.0 & 2610.0 & 2390.0 & 2220.0 & 2110.0 & 1810.0 \\
\hline 1942 & $\begin{array}{l}11000.0 \\
17500.0\end{array}$ & $\begin{array}{r}8040.0 \\
11400.0\end{array}$ & 9310.0 & 6980.0 & 4970.0 & 4760.0 & 4150.0 & $3660 \cdot 0$ & 3780.0 & 3680.0 & 3130.0 \\
\hline 1943 & $\begin{array}{r}17500.0 \\
4530.0\end{array}$ & $\begin{array}{r}11400.0 \\
3870.0\end{array}$ & 2750.0 & 2350.0 & 1920.0 & 1830.0 & 1750.0 & 1680.0 & & 1630.0 & 1510.0 \\
\hline 1944 & $\begin{array}{r}4530.0 \\
12700.0\end{array}$ & $\begin{array}{l}3870.0 \\
8590.0\end{array}$ & & $\begin{array}{l}2350.10 \\
4690.0\end{array}$ & 3800.0 & 3030.0 & 2700.0 & 2780.0 & 2620.0 & 2350.0 & 1860.0 \\
\hline 1945 & $\begin{array}{l}12700.0 \\
21800.0\end{array}$ & $\begin{array}{r}8590.0 \\
16200.0\end{array}$ & $\begin{array}{r}6560.0 \\
10000.0\end{array}$ & 7120.0 & 4950.0 & 3890.0 & 3340.0 & 3090.0 & 2920.0 & 2930.0 & 2480.0 \\
\hline 1946 & $\begin{array}{l}21800.0 \\
22000.0\end{array}$ & 19400.0 & & 7900.0 & 6270.0 & 4200.0 & 3820.0 & 3450.0 & 3220.0 & 2970.0 & 2380.0 \\
\hline 1947 & $\begin{array}{l}22000.0 \\
24700.0\end{array}$ & 16100.0 & $\begin{array}{l}13300 \bullet 0 \\
10200.0\end{array}$ & 7010.0 & 4670.0 & 3670.0 & 3460.0 & 3100.0 & 3060.0 & 2960.0 & 2780.0 \\
\hline 1948 & $\begin{array}{l}24700.0 \\
13700.0\end{array}$ & 10200.0 & 7290.0 & 6790.0 & 5800.0 & 4850.0 & 4140.0 & 3980.0 & 3530.0 & 3170.0 & 2740.0 \\
\hline $\begin{array}{l}1949 \\
1950\end{array}$ & $\begin{array}{l}13700.0 \\
13000.0\end{array}$ & $10000 \cdot 0$ & 6960.0 & 5650.0 & 4760.0 & 4120.0 & 3800.0 & 4010.0 & 3790.0 & 3550.0 & 2960.0 \\
\hline 1950 & $\begin{array}{l}13000.0 \\
12700.0\end{array}$ & 9440.0 & 8050.0 & 5980.0 & 5250.0 & 4530.0 & 4490.0 & 4410.0 & 3950.0 & 3830.0 & 3333.0 \\
\hline $\begin{array}{l}1951 \\
1952\end{array}$ & $\begin{array}{r}12700.0 \\
9270.0\end{array}$ & 6900.0 & 5930.0 & 4510.0 & 3580.0 & 3500.0 & 3200.0 & $2980 \cdot 0$ & 2810.0 & 2750.0 & 2510.0 \\
\hline $\begin{array}{l}1952 \\
1953\end{array}$ & $\begin{array}{r}9270.0 \\
24600.0\end{array}$ & 19600.0 & 13800.0 & 11000.0 & 8600.0 & 5790.0 & 4610.0 & 4070.0 & 3810.0 & 3530.0 & 2720.0 \\
\hline $\begin{array}{l}1933 \\
1954\end{array}$ & $\begin{array}{l}24600.0 \\
16100 \cdot 0\end{array}$ & $12000 \cdot 0$ & 7600.0 & 6400.0 & 5470.0 & 4180.0 & 4060.0 & $3880 \cdot 0$ & 3690.0 & 3560.0 & 2990.0 \\
\hline 1955 & 7820.0 & 7380.0 & 6620.0 & 5300.0 & 4660.0 & 3900.0 & 3390.0 & $3000 \cdot 0$ & 2700.0 & 2530.0 & 2200.0 \\
\hline 1956 & 25400.0 & 19300.0 & 12000.0 & 8750.0 & 7060.0 & 6070.0 & 5190.0 & 4430.0 & 3950.0 & 4040.0 & 3560.0 \\
\hline 1957 & 14100.0 & $10100 \cdot 0$ & 7020.0 & 6510.0 & 4700.0 & 3810.0 & 3390.0 & $2880 \cdot 0$ & 2770.0 & $2610 \cdot 0$ & \\
\hline 1958 & 11900.0 & 9680.0 & 6650.0 & 5240.0 & 4760.0 & 4170.0 & 3900.0 & 3350.0 & 3320.0 & 3110.0 & 2460.0 \\
\hline 1959 & 10800.0 & $8600 \cdot 0$ & 6120.0 & 4520.0 & 4220.0 & 3380.0 & 3430.0 & $3090 \cdot 0$ & 3010.0 & 2930.0 & \\
\hline 1960 & .9320 .0 & 7730.0 & 6250.0 & 5320.0 & 4250.0 & 3510.0 & 3240.0 & 3110.0 & $2880 \cdot 0$ & $\begin{array}{l}2590.0 \\
3500.0\end{array}$ & $\begin{array}{l}2250.0 \\
2820.0\end{array}$ \\
\hline 1961 & 24500.0 & 15800.0 & 10700.0 & 7590.0 & 5570.0 & 4550.0 & 3990.0 & $3790 \cdot 0$ & $\begin{array}{l}3690.0 \\
2800.0\end{array}$ & 2720.0 & 2280.0 \\
\hline 1962 & 10300.0 & $\begin{array}{r}8400 \cdot 0 \\
\end{array}$ & $\begin{array}{l}6280.0 \\
6880.0\end{array}$ & 4550.0 & 3890.0 & 3390.0 & $\begin{array}{l}2890.0 \\
2980.0\end{array}$ & 2780.0 & 2720.0 & $2720 \cdot 0$ & 2290.0 \\
\hline 1963 & $15500 \cdot 0$ & & & 5770.0 & 4420.0 & & & & & & \\
\hline
\end{tabular}


CLASS $0 \begin{array}{lllllllllllllllllllllllllllllllllllll} & 0 & 3 & 4 & 5 & 6 & 7 & 8 & 9 & 10 & 11 & 12 & 13 & 14 & 15 & 16 & 17 & 18 & 19 & 20 & 21 & 22 & 23 & 24 & 25 & 26 & 27 & 28 & 29 & 30 & 31 & 32 & 33 & 34\end{array}$

YEAR

1909

1910
1911

1912

1913
1914

1915

1916
1917

1918
1919

1920
1921

1921
1922

1923
1924

1924
1925

1926
1927

1928

1929
1930

1931

1932

1934

1935

1936
1937

1938

1939
1940
1941
1942

1942

1943
1944
1945

9445

1946
1947

1948

1949

1951

1952
1953

1954
1955

$\frac{1955}{1956}$

1956
1957
1958

1958
1959

1960
1961

1962
1963

Clackamas River at Estacada, Oreg.

Summary for water years 1909-63

NUMBER OF DAYS IN CLACS

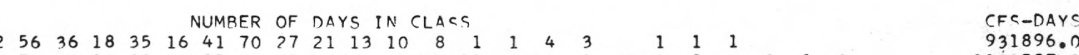

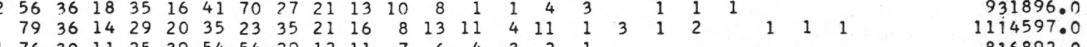

$\begin{array}{llllllllllllllllllll}1 & 76 & 30 & 11 & 25 & 39 & 54 & 54 & 29 & 12 & 11 & 7 & 6 & 4 & 3 & 2 & 1 & & 896897.0\end{array}$

$\begin{array}{rlllllllllllllllllllll}29 & 52 & 17 & 25 & 31 & 69 & 37 & 24 & 14 & 22 & 10 & 15 & 8 & 4 & 4 & 2 & 1 & 1 & 1 & 996990.0 \\ 25 & 48 & 16 & 29 & 34 & 46 & 45 & 21 & 22 & 21 & 23 & 22 & 6 & 1 & 3 & 2 & 1 & & & 1005246.0\end{array}$

$\begin{array}{rrrrrrrrrrrrrrrrrr}25 & 48 & 16 & 29 & 34 & 46 & 45 & 21 & 22 & 21 & 23 & 22 & 6 & 1 & 3 & 2 & 1 & 1005246 \cdot 0 \\ 52 & 30 & 30 & 34 & 26 & 51 & 48 & 22 & 12 & 18 & 8 & 9 & 9 & 2 & 2 & 1 & & 850116.0\end{array}$

$\begin{array}{lllllllllllll}47 & 26 & 37 & 27 & 39 & 39 & 59 & 56 & 17 & 8 & 2 & 5 & 3\end{array}$

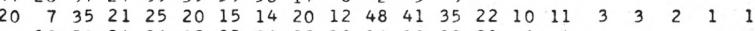

$\begin{array}{rrrrrrrrrrrrrrrrrrrrrrrrr}19 & 50 & 24 & 36 & 32 & 35 & 36 & 23 & 18 & 16 & 13 & 32 & 21 & 6 & 4 & & & & & & \\ 12102 & 36 & 13 & 6 & 10 & 40 & 24 & 25 & 16 & 10 & 9 & 21 & 5 & 6 & 9 & 8 & 2 & 7 & 1 & 1 & 1\end{array}$

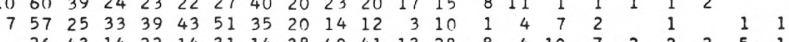

$\begin{array}{rrrrrrrrrrrrrrrrrrrrr}26 & 43 & 14 & 22 & 14 & 31 & 14 & 38 & 40 & 41 & 13 & 28 & 8 & 4 & 10 & 7 & 2 & 2 & 2 & 5 & 1 \\ 60 & 53 & 21 & 42 & 37 & 35 & 26 & 23 & 13 & 14 & 9 & 7 & 10 & 4 & 5 & & & 2 & & 2 & \end{array}$

$\begin{array}{rrrrrrrrrrrrrrrrrrrr}9 & 52 & 75 & 47 & 12 & 20 & 27 & 32 & 25 & 29 & 28 & 15 & 11 & 10 & 6 & 4 & 5 & 4 & 2 \\ 11 & 11 & 20 & 11 & 34 & 50 & 39 & 12 & 8 & 8 & 1 & 3 & 2 & 5 & 5 & 3 & 1\end{array}$

$\begin{array}{llllll}4 & 2 & 5 & 2 & 3 & 2\end{array}$

$\begin{array}{lllllllllllllllllll}29 & 80 & 42 & 17 & 39 & 37 & 40 & 35 & 14 & 8 & 6 & 2 & 2 & 6 & 2 & 2 & 2\end{array}$

$\begin{array}{rrrrrrrrrrrrrrrrrrrrrrr}53 & 37 & 18 & 17 & 10 & 50 & 41 & 40 & 27 & 25 & 9 & 16 & 11 & 3 & 4 & 1 & & 2 & 1 & \\ 7 & 60 & 16 & 26 & 33 & 33 & 39 & 26 & 18 & 23 & 26 & 10 & 17 & 8 & 10 & 6 & 4 & 2 & & 1 & 1\end{array}$

$\begin{array}{lllllllllllllll}23 & 72 & 34 & 36 & 40 & 25 & 26 & 19 & 22 & 17 & 17 & 14 & 17\end{array}$

$\begin{array}{llllllllllllllllllll}1 & 61 & 71 & 26 & 28 & 10 & 15 & 37 & 47 & 17 & 6 & 13 & 2 & 7 & 8 & 6 & 8 & & 1 & 1 \\ 19 & 74 & 47 & 38 & 41 & 31 & 29 & 32 & 16 & 11 & 5 & 4 & 3 & 4 & 4 & 2 & 2 & & 1\end{array}$

$\begin{array}{llllllllllllllll}21 & 29 & 43 & 19 & 29 & 32 & 18 & 21 & 23 & 32 & 28 & 25 & 12 & 15 \\ 10 & 2 & 26 & 32 & 22 & 23 & 30 & 32 & 35 & 39 & 39 & 17 & 16 & 19\end{array}$

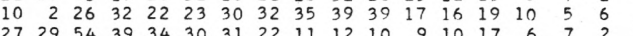

$\begin{array}{lllllllllllllllllllllllll}15 & 84 & 35 & 27 & 22 & 26 & 40 & 18 & 20 & 20 & 17 & 13 & 15 & 3 & 1 & 4 & & 3 & 2 & 1\end{array}$

$\begin{array}{lllllllllllllllllllllll}3 & 75 & 31 & 16 & 11 & 13 & 21 & 33 & 33 & 27 & 26 & 22 & 21 & 8 & 7 & 11 & 3 & 1 & 1 & 1 & 1\end{array}$

$\begin{array}{rrrrrrrrrrrrrrrrrrrr}33 & 66 & 18 & 18 & 34 & 33 & 52 & 31 & 25 & 22 & 12 & 4 & 5 & 4 & 7 & & 1 & \\ 83 & 59 & 18 & 19 & 18 & 15 & 23 & 21 & 19 & 13 & 7 & 7 & 12 & 8 & 4 & 9 & 1 & 1\end{array}$

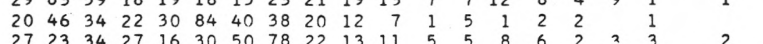

$\begin{array}{rrrrrrrrrrrrrrrrrrrrrrr}27 & 23 & 34 & 27 & 16 & 30 & 50 & 78 & 27 & 13 & 11 & 5 & 5 & 8 & 6 & 7 & 3 & 3 & & 2 & & \\ 20 & 6 & 27 & 30 & 20 & 14 & 10 & 31 & 27 & 35 & 24 & 27 & 17 & 28 & 16 & 7 & 12 & 4 & 3 & 4 & 1 & 1 & 1\end{array}$

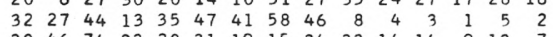

$\begin{array}{rrrrrrrrrrrrrrrrr}32 & 27 & 44 & 13 & 35 & 47 & 41 & 58 & 46 & 8 & 4 & 3 & 1 & 5 & 2 & \\ 20 & 46 & 74 & 23 & 20 & 31 & 19 & 15 & 24 & 23 & 14 & 14 & 9 & 13 & 7 & 6\end{array}$

$\begin{array}{lllllllllllllllllllllll}29 & 61 & 16 & 6 & 13 & 9 & 50 & 34 & 46 & 34 & 22 & 13 & 9 & 4 & 9 & 3 & 1 & 1 & 1 & 1 & 1 & 1\end{array}$

$\begin{array}{lllllllllllllll}42 & 26 & 27 & 33 & 21 & 51 & 29 & 34 & 16 & 10 & 7 & 11\end{array}$

$\begin{array}{llllllllllllllllllllllll}34 & 30 & 25 & 24 & 16 & 47 & 39 & 35 & 33 & 12 & 15 & 17 & 15 & 6 & 7 & 6 & 2 & 2 & 2 & 2 & 2\end{array}$

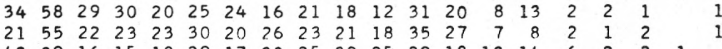

$\begin{array}{lllllllllllllllllllll}42 & 39 & 16 & 15 & 10 & 29 & 17 & 30 & 25 & 32 & 25 & 29 & 18 & 13 & 14 & 6 & 2 & 2 & 1\end{array}$

\begin{tabular}{lllllllllllllllll}
51 & 20 & 15 & 30 & 41 & 52 & 33 & 19 & 25 & 23 & 15 & 21 & 8 & 6 & 4 \\
\hline
\end{tabular}

$\begin{array}{rllllllllllllll}24 & 67 & 21 & 12 & 7 & 23 & 25 & 31 & 34 & 32 & 20 & 28 & 17 & 5 & 11\end{array}$

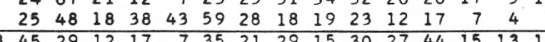

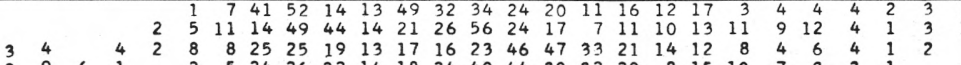

$\begin{array}{rllllllllllllllllllllllll}4 & & 4 & 2 & 8 & 8 & 25 & 25 & 19 & 13 & 17 & 16 & 23 & 46 & 47 & 33 & 21 & 14 & 12 & 8 & 4 & 6 & 4 \\ 9 & 6 & 1 & & 2 & 5 & 24 & 26 & 22 & 14 & 18 & 24 & 40 & 44 & 30 & 32 & 20 & 8 & 15 & 10 & 7 & 3 & 3 \\ 7 & 3 & & & & 2 & 15 & 48 & 42 & 14 & 11 & 8 & 21 & 23 & 49 & 27 & 17 & 20 & 27 & 9 & 5 & 7 & 5\end{array}$

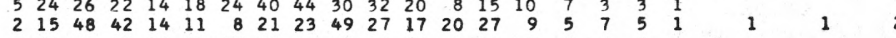

$\begin{array}{llllllllllllllllllllll}6 & 22 & 41 & 28 & 16 & 18 & 27 & 35 & 42 & 28 & 19 & 20 & 6 & 22 & 9 & 5 & 3 & 3 & & 2 & \\ 5 & 20 & 48 & 18 & 27 & 21 & 34 & 31 & 47 & 23 & 21 & 14 & 4 & 19 & 9 & 3 & 7 & & 3 & 1 & 1\end{array}$

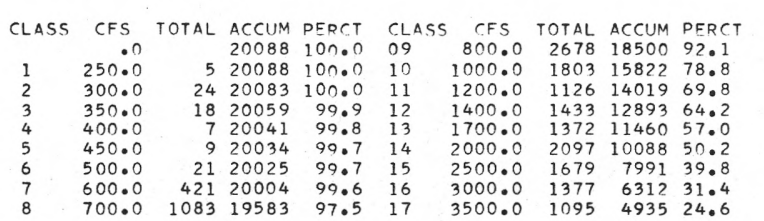

Clackamas River at Estacada, Oreg.

STATION NUMBER
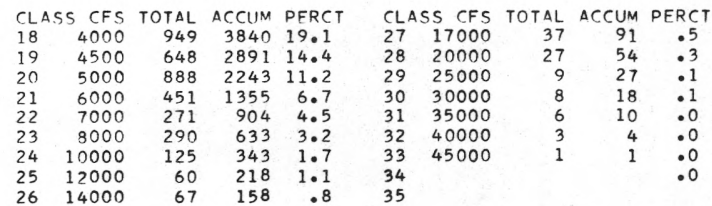

Summary for water years 1909-55

\begin{tabular}{lcrrr} 
CLASS & \multicolumn{1}{c}{ CFS } & TOTAL & ACCUM & PERCT \\
& .0 & & 17166 & 100.0 \\
1 & 250.0 & & 17166 & 100.0 \\
2 & 300.0 & & 17166 & 100.0 \\
3 & 350.0 & & 17166 & 100.0 \\
4 & 400.0 & & 17166 & 100.0 \\
5 & 450.0 & & 17166 & 100.0 \\
6 & 500.0 & 3 & 17166 & 100.0 \\
7 & 600.0 & 377 & 17163 & 100.0 \\
8 & 700.0 & 919 & 16786 & 97.8
\end{tabular}

$\begin{array}{rr}\text { CLASS } & \text { CFS } \\ 09 & 800.0 \\ 10 & 1000.0 \\ 11 & 1200.0 \\ 12 & 1400.0 \\ 13 & 1700.0 \\ 14 & 2000.0 \\ 15 & 2500.0 \\ 16 & 3000.0 \\ 17 & 3500.0\end{array}$

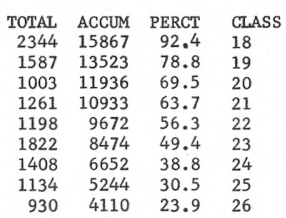

$14-2100.00$

Clackamas River at Estacada, Oreg.

STATION NUMBER
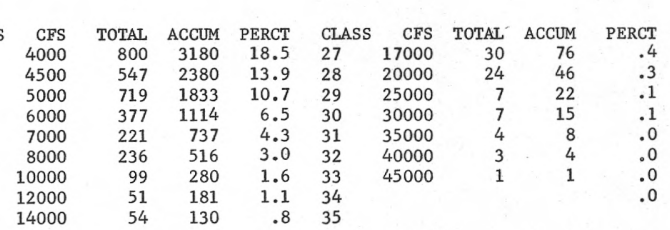

14-2100.00

Summary for water years 1957-63

\begin{tabular}{|c|c|c|c|c|c|c|}
\hline CLASS & $\begin{array}{l}\text { CFS } \\
.0\end{array}$ & TOTAL & $\begin{array}{r}\text { ACCUM } \\
2556\end{array}$ & $\begin{array}{l}\text { PERCT } \\
100.0\end{array}$ & $\begin{array}{l}\text { CLASS } \\
09\end{array}$ & $\begin{array}{l}\text { S CFS } \\
800.0\end{array}$ \\
\hline 1 & 250.0 & 5 & 2556 & 100.0 & 10 & 1000.0 \\
\hline 2 & 300.0 & 24 & 2551 & 99.8 & 11 & 1200.0 \\
\hline 3 & 350.0 & 18 & 2527 & 98.9 & 12 & 1400.0 \\
\hline 4 & 400.0 & 7 & 2509 & 98.2 & 13 & 1700.0 \\
\hline 5 & 450.0 & 9 & 2502 & 97.2 & 14 & 2000.0 \\
\hline 6 & 500.0 & 18 & 2493 & 97.5 & 15 & 2500.0 \\
\hline 7 & 600.0 & 44 & 2475 & 96.8 & 16 & 3000.0 \\
\hline 8 & 700.0 & 161 & 2431 & 95.1 & 17 & 3500.0 \\
\hline
\end{tabular}

$\begin{array}{rrrlr}\text { TOTAL } & \text { ACCUM } & \text { PERCT } & \text { CLASS } & \text { CFS } \\ 289 & 2270 & 88.8 & 18 & 4000 \\ 187 & 1981 & 77.5 & 19 & 4500 \\ 111 & 1794 & 70.2 & 20 & 5000 \\ 155 & 1683 & 65.8 & 21 & 6000 \\ 167 & 1528 & 59.8 & 22 & 7000 \\ 240 & 1361 & 53.2 & 23 & 8000 \\ 250 & 1121 & 43.9 & 24 & 10000 \\ 214 & 871 & 34.1 & 25 & 12000 \\ 150 & 657 & 25.7 & 26 & 14000\end{array}$
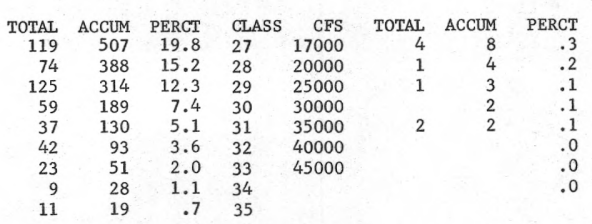
Clackamas River at Estacada, Oreg.

STATION NUMBFR $14->100.00$ LOWEST MEAN DISCHARGE, IN CFS, FOR THE FOLLOWING NUMBFR OF CONSECUTIVF DAYS IN YEAR RFGINNING APRIL 1

\begin{tabular}{|c|c|c|c|c|c|c|c|c|c|c|c|}
\hline YEAR & 1 & 3 & 7 & 14 & 30 & 60 & 90 & 120 & $15 n$ & 183 & 274 \\
\hline $\begin{array}{l}1908 \\
1909\end{array}$ & 640.0 & $640 . n$ & 653.0 & 692.0 & 790.0 & 822.0 & 888.0 & $1080 \cdot 0$ & $123 n \cdot 0$ & 1380.0 & 2150.0 \\
\hline $\begin{array}{l}1909 \\
1910\end{array}$ & $825 \cdot 0$ & $900 . n$ & 912.0 & 923.0 & 944.0 & 972.0 & 1010.0 & $1110 \cdot 0$ & 1330.0 & 1720.0 & $2820 . n$ \\
\hline $\begin{array}{l}1910 \\
1911\end{array}$ & $819 \cdot 0$ & 819.0 & 825.0 & 840.0 & 852.0 & 875.0 & 926.0 & 969.0 & 1040.0 & $125 n \cdot 0$ & 1850.0 \\
\hline $\begin{array}{l}1911 \\
1912\end{array}$ & 798.0 & 806.0 & $816 \cdot 0$ & 833.0 & 867.0 & 953.0 & 961.0 & 988.0 & 1170.0 & $\begin{array}{l}1510.0 \\
1820.0\end{array}$ & $\begin{array}{l}1990.0 \\
2180.0\end{array}$ \\
\hline $\begin{array}{l}1912 \\
1913\end{array}$ & $902 \cdot 0$ & 914.0 & 923.0 & $\begin{array}{l}949 \cdot 0 \\
960.0\end{array}$ & $\begin{array}{r}976.0 \\
1000.0\end{array}$ & $\begin{array}{l}1210.0 \\
1 \cap 60.0\end{array}$ & $\begin{array}{l}1190.0 \\
1180.0\end{array}$ & $\begin{array}{l}13 n n \cdot 0 \\
135 n .0\end{array}$ & $\begin{array}{l}1520.0 \\
1500.0\end{array}$ & 1580.0 & $2.25 n . ?$ \\
\hline 1913 & $\begin{array}{l}930.0 \\
766.0\end{array}$ & $\begin{array}{l}930 . n \\
766 . n\end{array}$ & $\begin{array}{l}950.0 \\
769.0\end{array}$ & $\begin{array}{l}960.0 \\
783 . n\end{array}$ & 809.0 & 863.0 & 987.0 & $112 n \cdot 0$ & 1260.0 & $141 n .0$ & 1630.0 \\
\hline 1915 & $705 \cdot 0$ & $7 n 5 \cdot n$ & $7 n 7 \cdot n$ & 715.0 & 727.0 & 733.0 & $77 n .0$ & $84 n \cdot 0$ & 916.0 & $1170 \cdot n$ & 2150.0 \\
\hline $\begin{array}{l}1916 \\
1917\end{array}$ & 950.0 & $95 n \cdot n$ & $952 \cdot 0$ & $957 . n$ & 978.0 & 1070.0 & $1200 \cdot 0$ & $136 n \cdot n$ & $169 n .0$ & $182 n .0$ & 2130.0 \\
\hline 1917 & 910.0 & $910 \cdot n$ & 924.0 & $940 \cdot 0$ & 957.0 & 975.0 & 1020.0 & $1080 \cdot 0$ & $135 n \cdot 0$ & $2 n 10 \cdot n$ & $3690 . n$ \\
\hline $\begin{array}{l}1918 \\
1919\end{array}$ & $759 \cdot 0$ & $759 . n$ & 763.0 & $772 \cdot 0$ & 799.0 & 843.0 & 889.0 & 936.0 & 1020.0 & $125 n \cdot n$ & $1680 \cdot 0$ \\
\hline $\begin{array}{l}1919 \\
1920\end{array}$ & 786.0 & $794 . n$ & $80 n \cdot 0$ & 827.0 & 878.0 & 912.0 & 934.0 & $1000 \cdot 0$ & 1250.0 & $179 n \cdot 0$ & 2230.0 \\
\hline $\begin{array}{l}1920 \\
1921\end{array}$ & 795.0 & $795 . n$ & $8 \cap 2 \cdot 0$ & 822.0 & 857.0 & 937.0 & 1110.0 & $1330 \cdot 0$ & $1700 \cdot 0$ & $179 n \cdot n$ & $2600 \cdot n$ \\
\hline $\begin{array}{l}1921 \\
1922\end{array}$ & $900 \cdot 0$ & 912.0 & 929.0 & 937.0 & 956.0 & 1020.0 & 1060.0 & $1130 \cdot 0$ & 1220.0 & 1570.0 & 2260.0 \\
\hline $\begin{array}{l}1922 \\
1923\end{array}$ & $760 \cdot 0$ & $767 . n$ & 774.0 & $789 . n$ & 873.0 & 877.0 & 915.0 & 947.0 & 990.0 & 1080.0 & 2100.0 \\
\hline & $880 \cdot 0$ & $880 \cdot 0$ & 889.0 & 903.0 & 931.0 & 982.0 & 1040.0 & $1060 \cdot 0$ & 1210.0 & 1530.0 & 2240.0 \\
\hline $\begin{array}{l}1924 \\
1925\end{array}$ & 635.0 & 676.0 & $69 n \cdot 0$ & 698.0 & $705 . n$ & 730.0 & 760.0 & 785.0 & & $978 . ?$ & 1810.0 \\
\hline $\begin{array}{l}1925 \\
1926\end{array}$ & $708 \cdot 0$ & $715 . n$ & $727 \cdot n$ & $738 \cdot n$ & 747.0 & 777.0 & 805.0 & 836.0 & 923.0 & $105 n \cdot n$ & $1570 . ?$ \\
\hline $\begin{array}{l}1926 \\
1927\end{array}$ & 636.0 & $643 . n$ & $647 . n$ & $654 . n$ & 673.0 & 719.0 & 746.0 & $798 \cdot 0$ & 882.0 & $964 . n$ & $16 n 0.0$ \\
\hline $\begin{array}{l}1927 \\
1928\end{array}$ & 835.0 & $850 . n$ & $865 . n$ & $872 \cdot n$ & $898 . n$ & 984.0 & $\ln 90.0$ & $148 \cap \cdot n$ & 1610.0 & $2 n 8 n \cdot n$ & $\operatorname{sann} 0 n$ \\
\hline $\begin{array}{l}1928 \\
1929\end{array}$ & 760.0 & $768 \cdot n$ & 781.0 & $794 \cdot 0$ & 820.0 & 843.0 & 879.0 & $910 \cdot 0$ & 990.0 & $114 n \cdot n$ & 1380.0 \\
\hline $\begin{array}{l}1929 \\
1930\end{array}$ & $654 \cdot 0$ & $668 \cdot n$ & 674.0 & $690 . n$ & 698.0 & 721.0 & 734.0 & $759 \cdot 0$ & 813.0 & $1080 \cdot n$ & 1840.0 \\
\hline 1930 & 587.0 & 646.0 & 651.0 & 661.0 & 678.0 & 682.0 & 707.0 & 732.0 & 795.0 & 896.0 & 1210.0 \\
\hline $\begin{array}{l}1931 \\
1932\end{array}$ & $590 \cdot 0$ & $602 \cdot 0$ & $605 . n$ & 616.0 & 662.0 & 699.0 & 725.0 & 790.0 & 865.0 & 982.0 & 1460.0 \\
\hline 1932 & 674.0 & 678.0 & 681.0 & $687 \cdot 0$ & 709.0 & 756.0 & 822.0 & 932.0 & 1220.0 & 1670.0 & 2100.0 \\
\hline $\begin{array}{l}1933 \\
1934\end{array}$ & 865.0 & $874 . n$ & $908 \cdot 0$ & 928.0 & 949.0 & 1060.0 & 1080.0 & $1290 \cdot 0$ & $1380 \cdot 0$ & 1960.0 & $3 \cap 60.0$ \\
\hline $\begin{array}{l}1934 \\
1935\end{array}$ & 616.0 & $643 . n$ & 649.0 & 667.0 & 676.0 & 686.0 & $722 \cdot 0$ & 775.0 & 852.0 & 1010.0 & 1850.0 \\
\hline $\begin{array}{l}1935 \\
1936\end{array}$ & 706.0 & $717 . n$ & 725.0 & 746.0 & 761.0 & 784.0 & 828.0 & $875 \cdot 0$ & $964 \cdot 0$ & $1 \cap 7 n \cdot n$ & $\begin{array}{l}1770.0 \\
1210.0\end{array}$ \\
\hline $\begin{array}{l}1936 \\
1937\end{array}$ & $659 \cdot 0$ & $666 . n$ & 672.0 & 679.0 & 696.0 & 716.0 & 754.0 & $784 \cdot 0$ & 837.0 & 950.0 & $\begin{array}{l}1210.0 \\
3000.0\end{array}$ \\
\hline $\begin{array}{l}1937 \\
1938\end{array}$ & $825 \cdot 0$ & $842 \cdot n$ & $850 \cdot 0$ & $9 n 9 \cdot 0$ & 919.0 & 945.0 & $10 \cap n \cdot 0$ & & $\begin{array}{l}1570 \cdot 0 \\
109 n \cdot 0\end{array}$ & $\begin{array}{l}3120.0 \\
127 n .0\end{array}$ & 1780.ก \\
\hline $\begin{array}{l}1938 \\
1939\end{array}$ & $795 \cdot 0$ & $80 n \cdot 0$ & $807 . n$ & $\begin{array}{l}819.0 \\
732.0\end{array}$ & $\begin{array}{l}840 \cdot n \\
756 \cdot n\end{array}$ & $\begin{array}{l}860^{\circ} \cdot 0 \\
787.0\end{array}$ & $\begin{array}{l}887.0 \\
8 \cap 7.0\end{array}$ & $\begin{array}{l}936.0 \\
815.0\end{array}$ & $830^{\circ}$. ? & 967.0 & $1450 . n$ \\
\hline $\begin{array}{l}1939 \\
1940\end{array}$ & $\begin{array}{l}720.0 \\
650.0\end{array}$ & $\begin{array}{l}720 . n \\
654 . n\end{array}$ & $\begin{array}{l}723.0 \\
662.0\end{array}$ & 665.0 & 679.0 & 699.0 & $7 ? 8.0$ & 744.0 & 809.0 & 953.0 & 1490.0 \\
\hline 1941 & 680.0 & 683.0 & 685.0 & 692.0 & 704.0 & 762.0 & 830.0 & $918 \cdot 0$ & 977.0 & $115 n \cdot 0$ & 3890.0 \\
\hline 1942 & 638.0 & $648 \cdot n$ & 656.0 & 657.0 & $660 \cdot 0$ & 678.0 & 722.0 & $811 \cdot 0$ & 1030.0 & $133 n .0$ & 2490.0 \\
\hline 1943 & 865.0 & 872.0 & 876.0 & 911.0 & 937.0 & 987.0 & 1060.0 & $1280 \cdot 0$ & 1480.0 & $1590 \cdot 0$ & 1770.0 \\
\hline 1944 & 632.0 & $649 . n$ & 651.0 & 658.0 & 679.0 & 716.0 & 713.0 & $752 \cdot 0$ & 822.0 & 885.0 & 1270.0 \\
\hline 1945 & 691.0 & $722 \cdot n$ & 733.0 & 739.0 & 769.0 & 807.0 & 803.0 & $838 . ?$ & 965.0 & 1280.0 & 2500.0 \\
\hline 1946 & 767.0 & 777.0 & 791.0 & 800.0 & 826.0 & 853.0 & 902.0 & $1110 \cdot 0$ & 1310.0 & $1680 \cdot 0$ & 2870.0 \\
\hline 1947 & 790.0 & 802.0 & 815.0 & 836.0 & 867.0 & 892.0 & 938.0 & $1070 \cdot 0$ & $1180 \cdot 0$ & 1420.0 & 2380.0 \\
\hline 1948 & 890.0 & 890.0 & 895.0 & 902.0 & 953.0 & 1040.0 & 1130.0 & $1220 \cdot 0$ & 1390.0 & 1850.0 & 2270.0 \\
\hline 1949 & 901.0 & $919 . n$ & 931.0 & 945.0 & 977.0 & 1020.0 & 1120.0 & 1170.0 & 1280.0 & $1660 . n$ & 2320.0 \\
\hline 1950 & 888.0 & $92 n \cdot n$ & $944 . n$ & 965.0 & $100 n \cdot n$ & $108 \cap . n$ & 1290.0 & $1650 \cdot 0$ & $241 n .0$ & $286 n \cdot 0$ & $3810 \cdot 0$ \\
\hline 1951 & $870 \cdot 0$ & $873 . n$ & 877.0 & 894.0 & 919.0 & 964.0 & 1040.0 & $119 n \cdot ?$ & $146 n \cdot 0$ & $1690 \cdot n$ & $2300 \cdot n$ \\
\hline 1952 & 738.0 & $745 \cdot n$ & 760.0 & 780.0 & 799.0 & 809.0 & 832.0 & $862 \cdot 0$ & 930.0 & $1 \cap 8 \cap \cdot 0$ & $1940 . ?$ \\
\hline 1953 & $885 \cdot 0$ & $892 \cdot n$ & 894.0 & 899.0 & 934.0 & 990.0 & 1010.0 & $1050 \cdot 0$ & $1230 \cdot 0$ & 1670.0 & 2850.0 \\
\hline 1954 & 935.0 & 942.0 & $948 \cdot 0$ & 965.0 & 1050.0 & 1070.0 & 1150.0 & $1270 \cdot 0$ & 1470.0 & $1600 \cdot 0$ & 1920.0 \\
\hline 1953 & $900 \cdot 0$ & 923.0 & 948.0 & 974.0 & 1010.0 & 1070.0 & 1280.0 & $1670 \cdot 0$ & 2120.0 & 2770.0 & 3810.0 \\
\hline 1956 & 730.0 & 818.0 & 823.0 & 839.0 & 849.0 & 890.0 & 948.0 & $1110 \cdot 0$ & 1380.0 & 1570.0 & 2020.0 \\
\hline 1997 & 536.0 & 694.0 & 702.0 & 708.0 & 717.0 & 754.0 & 812.0 & 859.0 & 931.0 & 1120.0 & $\begin{array}{l}2160.0 \\
2320.0\end{array}$ \\
\hline 1938 & 285.0 & $381 \cdot 0$ & 507.0 & 644.0 & 761.0 & 785.0 & 811.0 & 887.0 & $993 \cdot 0$ & $1250 \cdot 0$ & $\begin{array}{l}2320.0 \\
1960.0\end{array}$ \\
\hline $\begin{array}{l}1959 \\
1960\end{array}$ & $\begin{array}{l}304.0 \\
292.0\end{array}$ & $\begin{array}{l}571 \cdot 0 \\
480.0\end{array}$ & $757 \cdot 0$ & $786 \cdot 0$ & 813.0 & 897.0 & 1030.0 & $1290 \cdot 0$ & 1520.0 & $\begin{array}{l}1690.0 \\
1680.0\end{array}$ & $\begin{array}{l}1960.0 \\
256000\end{array}$ \\
\hline $\begin{array}{l}1960 \\
1961\end{array}$ & $\begin{array}{l}292.0 \\
340.0\end{array}$ & $\begin{array}{l}480.0 \\
680.0\end{array}$ & $\begin{array}{l}838.0 \\
805.0\end{array}$ & $\begin{array}{l}854.0 \\
838.0\end{array}$ & $\begin{array}{l}883.0 \\
860.0\end{array}$ & $\begin{array}{l}945.0 \\
915.0\end{array}$ & $\begin{array}{r}1010.0 \\
955.0\end{array}$ & $\begin{array}{l}1040 \cdot 0 \\
1020 \cdot 0\end{array}$ & 1160.0 & 1390.0 & 2150.0 \\
\hline $\begin{array}{l}1961 \\
1962\end{array}$ & $\begin{array}{l}340.0 \\
325.0\end{array}$ & $\begin{array}{l}680.0 \\
460.0\end{array}$ & $\begin{array}{l}805.0 \\
636.0\end{array}$ & $\begin{array}{l}830.0 \\
745.0\end{array}$ & $\begin{array}{l}860.0 \\
795.0\end{array}$ & $\begin{array}{l}915.0 \\
844.0\end{array}$ & $\begin{array}{l}955.00 \\
907.0\end{array}$ & 1070.0 & 1250.0 & 1530.0 & 2300.0 \\
\hline
\end{tabular}


Clackamas River at Estacada, Oreg.

STATION NUMBER $\quad 14-2100.00$

HIGHEST MEAN DISCHARGE, IN CFS, FOR THE FOLLOWING NUMBER OF CONSECUTIVE DAYS IN YEAR ENDING SEPTEMBER 30

\begin{tabular}{|c|c|c|c|c|c|c|c|c|c|c|c|}
\hline YEAR & 1 & 3 & 7 & 15 & 30 & 60 & 90 & 120 & 150 & 183 & 274 \\
\hline $19 n 9$ & 22700.0 & $19400 \bullet n$ & 14300.0 & 9260.0 & 6000.0 & 4980.0 & 4320.0 & $408 n \cdot 0$ & 3930.0 & 3690.0 & 3060.0 \\
\hline 1910 & 37600.0 & 32500 .n & 21600.0 & 14300.0 & 9640.0 & 6360.0 & 5230.0 & 5290.0 & $504 n \cdot n$ & 4790.0 & $377 n .0$ \\
\hline 1911 & 10700.0 & $8330 . n$ & 5790.0 & 5400.0 & 4370.0 & 3470.0 & 3250.0 & 2870.0 & 2850.0 & 2940.0 & 2650.0 \\
\hline 1912. & 19300.0 & $15900 \cdot n$ & $105 n n \cdot n$ & 7570.0 & 6580.0 & $567 n \cdot n$ & $454 n \cdot 0$ & 4200.0 & 4050.0 & 3770.0 & 3250.0 \\
\hline 1913 & $13200 \cdot 0$ & $10500 \cdot n$ & 7710.0 & 6140.0 & 5210.0 & $4910 . n$ & 4500.0 & $401 n .0$ & 3700.0 & $368 n \cdot n$ & 3310.0 \\
\hline 1914. & 11400.0 & $8770 . n$ & $6660 \cdot 0$ & $5480 \cdot 0$ & 4570.0 & 4120.0 & 3840.0 & $3800 \cdot 0$ & 3530.0 & 3280.0 & 2760.0 \\
\hline 1915 & 5440.0 & $4780 . n$ & 4170.0 & 3620.0 & 3350.0 & 2860.0 & 2680.0 & 2610.0 & 2550.0 & 2400.0 & 2190.0 \\
\hline 1916 & 22200.0 & 14100.0 & 11800.0 & 9360.0 & 6610.0 & 6320.0 & 5900.0 & 5590.0 & 5470.0 & 5250.0 & 4740.0 \\
\hline 1917 & 8060.0 & 8000.0 & 7390.0 & 6810.0 & 6260.0 & 5910.0 & 5440.0 & $4890 \cdot 0$ & 4310.0 & 3970.0 & 3460.0 \\
\hline 1918 & $40500 \cdot 0$ & $30000 \cdot 0$ & $20600 \cdot 0$ & 16400.0 & 12300.0 & 9710.0 & 7600.0 & 6610.0 & 5970.0 & 5370.0 & 4000.0 \\
\hline 1919 & 18900.0 & 16000.0 & $12700 . n$ & $9080 . n$ & 6360.0 & 4910.0 & 4600.0 & 4640.0 & $445 \cap .0$ & $403 n .0$ & 3290.0 \\
\hline 1920 & 26500.0 & $16700 \cdot 0$ & 10500.0 & $6280 . n$ & 4760.0 & 4370.0 & 4060.0 & $358 \cap \cdot 0$ & $327 n$. $n$ & $32.8 \cap .0$ & 2840.0 \\
\hline 1921 & 29900.0 & $25200 \bullet n$ & 21800.0 & 14300.0 & 9370.0 & 7560.0 & 7140.0 & $6470 . n$ & 6060.0 & 5730.0 & 4720.0 \\
\hline 1922 & 31700.0 & 26100.0 & $160 n 0.0$ & 13800.0 & 8790.0 & 5370.0 & 4180.0 & 3550.0 & 3430.0 & 3570.0 & 3180.0 \\
\hline 1923 & 49700.0 & $380 n$ on & $228 \mathrm{nn} \cdot \mathrm{n}$ & $154 \mathrm{nn} . \mathrm{n}$ & 11200.0 & 6670.0 & 5340.0 & $4990 . n$ & $483 n \cdot n$ & $453 n_{0} n$ & $3490 . n$ \\
\hline 1924 & 1220000 & $105 n n$ on & $936 n \cdot n$ & 7220.0 & 5210.0 & 4220.0 & 4030.0 & 3630.0 & $337 n .0$ & $3 n 9 n . n$ & $242 n . n$ \\
\hline 1925 & $17600 \cdot 0$ & $16 n 0 n$ on & $131 n n \cdot n$ & $10200 \cdot n$ & 7480.0 & 5690.0 & 5130.0 & $4800 \cdot n$ & $440 n \cdot 0$ & $426 n \cdot 0$ & 3540.0 \\
\hline 1926 & $17200 \cdot 0$ & $13300 \cdot n$ & 9140.0 & 6100.0 & 5130.0 & 3690.0 & 3310.0 & 2960.0 & $270 n .0$ & 2470.0 & 1960.0 \\
\hline 1927 & 17500.0 & 13900.0 & 9360.0 & 6680.0 & 5230.0 & 4450.0 & 4310.0 & 4180.0 & 3880.0 & 3890.0 & 3400.0 \\
\hline 1928 & 24700.0 & $16000 \cdot 0$ & $12800 \cdot 0$ & 9140.0 & 6890.0 & 5350.0 & 4620.0 & $4270 \cdot 0$ & 4410.0 & 4350.0 & 3590.0 \\
\hline 1929 & 9250.0 & $6840 . n$ & 5310.0 & $5050 \cdot 0$ & 4860.0 & 4300.0 & 3950.0 & $3580 \cdot 0$ & 3140.0 & 3020.0 & 2320.0 \\
\hline 1930 & 12100.0 & $8510 . n$ & 7770.0 & 6180.0 & 5320.0 & 3850.0 & $3550 \cdot 0$ & 3270.0 & $3020 \cdot 0$ & 2910.0 & 2240.0 \\
\hline $\begin{array}{l}1931 \\
1932\end{array}$ & $37900 \cdot 0$ & $29500 . n$ & 17000.0 & $10400 . n$ & $7430 \cdot 0$ & 4930.0 & 3950.0 & 3490.0 & 3090.0 & 2780.0 & 2210.0 \\
\hline & 19000.0 & $15100 . n$ & $10200 \cdot 0$ & 8510.0 & 6370.0 & 5360.0 & 4980.0 & $4480 \cdot 0$ & 4050.0 & 3870.0 & 3130.0 \\
\hline 1933 & 17000.0 & $12500 \cdot 0$ & 9950.0 & 7750.0 & 6460.0 & 5350.0 & 4730.0 & 4330.0 & 3930.0 & 3840.0 & 3520.0 \\
\hline $\begin{array}{l}1934 \\
1935\end{array}$ & $34800 \cdot 0$ & $24900 \cdot n$ & $\begin{array}{r}18600 \cdot 0 \\
88\end{array}$ & $13000 \cdot n$ & 9880.0 & 7820.0 & 6000.0 & $\begin{array}{l}5320.0 \\
3530.0\end{array}$ & $467 n \cdot 0$ & $\begin{array}{l}4190.0 \\
3430.0\end{array}$ & 3220.0 \\
\hline $\begin{array}{l}1933 \\
1936\end{array}$ & $\begin{array}{l}12100 \cdot 0 \\
20500.0\end{array}$ & $\begin{array}{l}11100 \cdot n \\
145.00 \cdot 0\end{array}$ & $\begin{array}{r}\text { s8in.n } \\
\text { lngnn on }\end{array}$ & $\begin{array}{r}6050 . n \\
103 n 0.0\end{array}$ & $\begin{array}{l}4330.0 \\
6920.0\end{array}$ & $\begin{array}{l}4 n 7 n .0 n \\
466 n \cdot 0 n\end{array}$ & $\begin{array}{l}368 \pi .0 \\
4190.0\end{array}$ & $\begin{array}{l}3530 \cdot n \\
4130 \cdot 0\end{array}$ & $\begin{array}{l}344 n \cdot 0 \\
413 n \cdot n\end{array}$ & $\begin{array}{l}3430.0 \\
3770.0\end{array}$ & $\begin{array}{l}3110.0 \\
2940.0\end{array}$ \\
\hline 1937 & 2010000 & $176 n 0 \cdot n$ & $118 \mathrm{nn} \cdot \mathrm{n}$ & 7870 . & 6540.0 & $5830 . n$ & 5260.0 & $483 n \cdot n$ & 431000 & $375 n \cdot n$ & \\
\hline & 24300.0 & 17900.0 & 12300.0 & 7580.0 & 6970.0 & 5620.0 & 5380.0 & $4820 \cdot 0$ & 4690.0 & 4740.0 & $3880 . n$ \\
\hline 1939 & 10400.0 & 7680.0 & 6820.0 & 5480.0 & 4610.0 & 3960.0 & 3650.0 & $3290 \cdot 0$ & 3220.0 & 3100.0 & $2590 . n$ \\
\hline 1940 & 15800.0 & $11600 \cdot n$ & 8900.0 & $6660 \cdot n$ & 6280.0 & 5290.0 & 4590.0 & 4040.0 & 3790.0 & 3370.0 & $254 n \circ 0$ \\
\hline 1941 & 11400.0 & 7850.0 & $5460 \cdot 0$ & $4130 \cdot 0$ & 3090.0 & 2930.0 & 2730.0 & $2500 \cdot 0$ & 2310.0 & 2270.0 & 1990.0 \\
\hline 1942 & $15200 \cdot 0$ & $11500 \cdot n$ & $856 n .0$ & $5690 \cdot 0$ & 5560.0 & 4140.0 & 3830.0 & $3490 \cdot 0$ & $3230 \cdot n$ & $3 n 60.0$ & $265 n .0$ \\
\hline 1943 & 26500.0 & $17300 \cdot n$ & $13600 \cdot 0$ & 10200.0 & 7540.0 & 7190.0 & 6230.0 & 5490.0 & 5490.0 & $532 n .0$ & 4430.0 \\
\hline 1944 & 6520.0 & 5770.0 & 3960.0 & 3500.0 & 2680.0 & 2580.0 & 2440.0 & 2350.0 & 2280.0 & 224.0 & 2080.0 \\
\hline 1945 & 16100.0 & $10800 \cdot n$ & 9120.0 & 6510.0 & 5930.0 & 4720.0 & 4170.0 & $4170 \cdot 0$ & 3910.0 & $349 n .0$ & $2690 . n$ \\
\hline 1946 & 32500.0 & 2430 & $14900 . n$ & 106 & 745 & $59 n$ & .0 & 4710.0 & 435 & 4280.0 & 3590.0 \\
\hline 194 & & 29 & $2040 n \cdot n$ & $11900 . n$ & & 6130.0 & .0 & $n .0$ & $463 n . n$ & $n . n$ & $3390 . n$ \\
\hline 1948 & 40300.0 & $24900 \cdot n$ & $150 \mathrm{nn} \cdot \mathrm{n}$ & 10200.0 & $6890 . n$ & 5370.0 & 5460.0 & 4730.0 & 4710.0 & 4320.0 & 4020.0 \\
\hline 1949 & 20900.0 & $15200 . n$ & lngon.n & $943 n \cdot n$ & 7870.0 & 6470.0 & 5730.0 & $5440 \cdot 0$ & & & 3850.0 \\
\hline 1950 & 20100.0 & $15800 \cdot n$ & $1 n 7 n n . n$ & 8450.0 & 6930.0 & 5810.0 & 5340.0 & $546 n \cdot n$ & $522 n \cdot 0$ & 4850.0 & 4060.0 \\
\hline 1951 & $18400 \cdot 0$ & $13000 \cdot 0$ & 11100.0 & 8180.0 & 7630.0 & 6330.0 & 6400.0 & 6250.0 & 5630.0 & 5360.0 & 4530.0 \\
\hline 1952 & 11600.0 & 9650.0 & 8440.0 & 6090.0 & 4780.0 & 4490.0 & 4010.0 & 3910.0 & 3650.0 & 3660.0 & 3330.0 \\
\hline 1953 & 3520000 & 2650000 & $18800 . n$ & 15000.0 & 11700.0 & 7840.0 & 6330.0 & $5560 \cdot 0$ & 5240.0 & 4830.0 & 3670.0 \\
\hline 1954 & $23400 \cdot 0$ & $16300 \cdot 0$ & $10400 \cdot 0$ & 9440.0 & 8460.0 & 6350.0 & 6190.0 & $5780 \cdot 0$ & 5400.0 & 5080.0 & 4210.0 \\
\hline & 136 & 100 & 8300.0 & 6670.0 & 596 & 5010.0 & & $4100 \cdot 0$ & & 3570.0 & 3100.0 \\
\hline 1956 & 33000.0 & 2460000 & 15500.0 & 11700.0 & 9850.0 & 141000 & 7310.0 & 6230.0 & 5610.0 & 5580.0 & 480000 \\
\hline 1957 & 22000.0 & 1650000 & 12000.0 & 10800.0 & 7900.0 & 6250.0 & 5430.0 & 455000 & 4220.0 & 3940.0 & 3220.0 \\
\hline 1998 & $18300 \cdot 0$ & $14600 \cdot 0$ & 10000.0 & 8200.0 & 6620.0 & 6140.0 & 5710.0 & 4840.0 & 4770.0 & 4370.0 & 3400.0 \\
\hline 1999 & 2530000 & $12700 \cdot n$ & 8990.0 & 6680.0 & 6250.0 & 5060.0 & 5110.0 & 456000 & 4420.0 & $4220 . n$ & 3470.0 \\
\hline 1960 & 12900.0 & 1110000 & $8810 . n$ & 7460.0 & 3980.0 & 5000.0 & 4680.0 & 4530.0 & $418 n .0$ & 3730.0 & $327 n .0$ \\
\hline 1261 & 36500.0 & $25000 \cdot 0$ & 16400.0 & 11700.0 & 8510.0 & 6840.0 & 5810.0 & $5530 \cdot 0$ & 5340.0 & 5060.0 & 4010.0 \\
\hline 1962 & $16600 \cdot 0$ & 13400.0 & 9870.0 & 7060.0 & 5810.0 & 4920.0 & 4180.0 & 3910.0 & 4030.0 & 3900.0 & 3230.0 \\
\hline 1963 & 19900.0 & 14100.0 & 9260.0 & 8160.0 & 6260.0 & 4370.0 & 4100.0 & $3830 \cdot 0$ & 3810.0 & 3820.0 & 3190.0 \\
\hline
\end{tabular}




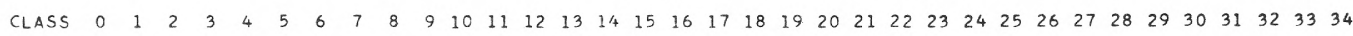

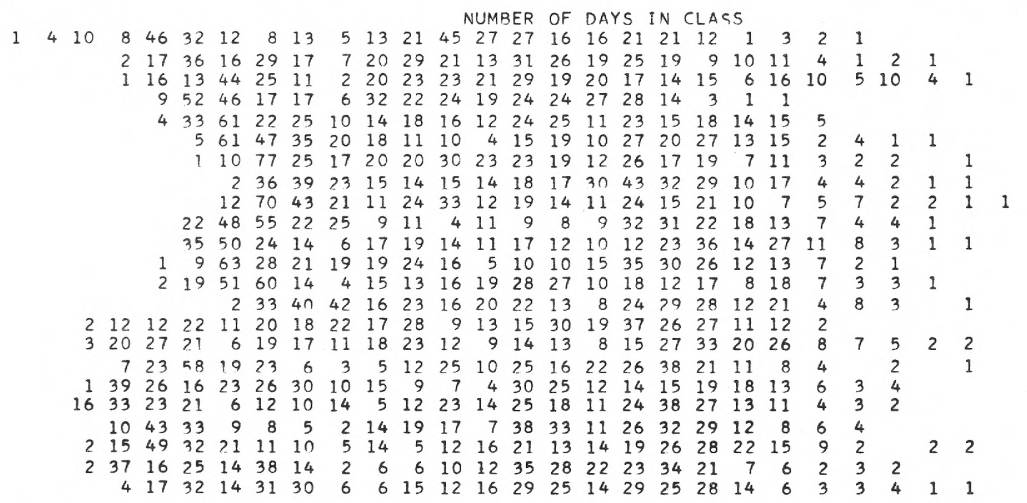

CFS-DAYS

15607.1

28912.2

73637.

15637.3

16177.8

24122.5

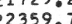

(1)

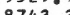

19228.4

(1)

24742.1

31821.0

7653.6

17653.6

9043.5

18738.7

15720.1

$\begin{array}{rrrrrrrrrrrrrrrrrrrrrrrrr}37 & 16 & 25 & 14 & 38 & 14 & 2 & 6 & 6 & 10 & 12 & 35 & 28 & 22 & 23 & 34 & 21 & 7 & 6 & 2 & 3 & 2 & & \\ 4 & 17 & 32 & 14 & 31 & 30 & 6 & 6 & 15 & 12 & 16 & 29 & 25 & 14 & 29 & 25 & 28 & 14 & 6 & 3 & 3 & 4 & 1 & 1\end{array}$

20803.9

Johnson Creek at Sycamore, Oreg.

STATION NUMBER $\quad 14-2115.00$
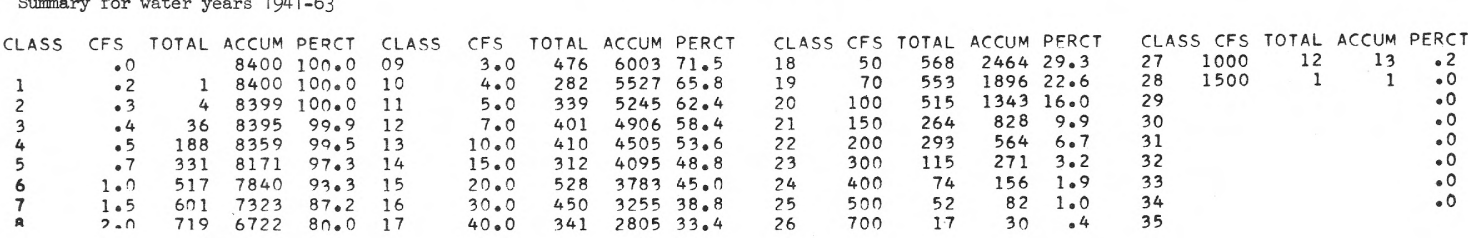

Johnson Creek at Sycamore, Oreg.

STATION NUMBER $\quad 14-2115.00$

LOWEST MEAN DISCHARGE, IN CFS, FOR THE FOLLOWING NUMBER OF CONSECUTIVE DAYS IN YEAR BEGINNING APRIL 1

\begin{tabular}{|c|c|c|c|c|c|c|c|c|c|c|c|}
\hline YEAR & 1 & 3 & 7 & 14 & 30 & 60 & 90 & 120 & 150 & 183 & 274 \\
\hline 1941 & .2 & .3 & .4 & .4 & .6 & .8 & 1.0 & $1 \cdot 3$ & 1.8 & 3.8 & 29.1 \\
\hline 1942 & .6 & .7 & .7 & .8 & .9 & 1.0 & 1.1 & 1.6 & 3.0 & 9.0 & 56.6 \\
\hline 1943 & 1.2 & 1.3 & 1.5 & 1.5 & 1.5 & 1.7 & 1.9 & 3.4 & 5.9 & 8.0 & 13.4 \\
\hline 1944 & .7 & .7 & .8 & .9 & 1.1 & 1.2 & 1.3 & 1.4 & 1.6 & $2 \cdot 3$ & 8.3 \\
\hline 1945 & .7 & .8 & .9 & 1.2 & 1.3 & 1.5 & 1.7 & 1.8 & 2.2 & 9.1 & 43.0 \\
\hline 1946 & 1.3 & 1.5 & 1.5 & 1.6 & 1.7 & 1.9 & 1.9 & $2 \cdot 3$ & 2.8 & $3 \cdot 3$ & 32.2 \\
\hline 1947 & 1.6 & 1.8 & 2.0 & 2.0 & 2.1 & 2.2 & 2.4 & 3.3 & 5.9 & 6.7 & 37.1 \\
\hline 1948 & 1.8 & 2.0 & $2 \cdot 1$ & 2.1 & 2.5 & 3.0 & 3.4 & 3.9 & 4.6 & & \\
\hline $\begin{array}{l}1949 \\
1950\end{array}$ & $\begin{array}{l}1.8 \\
1.0\end{array}$ & $\begin{array}{l}1.8 \\
1.1\end{array}$ & $\begin{array}{l}1.9 \\
1.1\end{array}$ & $\begin{array}{l}2.0 \\
1.1\end{array}$ & $\begin{array}{l}2.3 \\
1.3\end{array}$ & $\begin{array}{l}2.5 \\
1.6\end{array}$ & $\begin{array}{l}2.5 \\
1.8\end{array}$ & $\begin{array}{l}2 \cdot 7 \\
2 \cdot 5\end{array}$ & $\begin{array}{l}2.8 \\
3.2\end{array}$ & $\begin{array}{l}3.1 \\
5.7\end{array}$ & $\begin{array}{l}19.5 \\
56.4\end{array}$ \\
\hline 1951 & 1.2 & 1.2 & 1.3 & 1.4 & 1.5 & 1.6 & 1.6 & 2.0 & 2.6 & 4.5 & 30.9 \\
\hline 1952 & .8 & .9 & 1.2 & 1.3 & 1.5 & 1.6 & 1.6 & 1.7 & 1.7 & $2 \cdot 3$ & 5.5 \\
\hline 1953 & 1.6 & 1.7 & 1.8 & 1.9 & $2 \cdot 1$ & 2.3 & 2.5 & $2 \cdot 9$ & 3.6 & 12.0 & 42.1 \\
\hline 1954 & 1.7 & 1.8 & 1.8 & 2.0 & 3.0 & 3.6 & 3.6 & $5 \cdot 3$ & 8.3 & 10.7 & 29.0 \\
\hline 1955 & .4 & .5 & .5 & .6 & .8 & 1.1 & 1.9 & 2.6 & $4 \cdot 2$ & 9.3 & 68.5 \\
\hline 1956 & .4 & .4 & .5 & .6 & .8 & 1.2 & 1.5 & $2 \cdot 0$ & 2.6 & $4 \cdot 0$ & 21.0 \\
\hline 1957 & .5 & .6 & .6 & .7 & .8 & 1.0 & 1.1 & 1.5 & 1.7 & $2 \cdot 3$ & 22.8 \\
\hline 1958 & .4 & .5 & .5 & .5 & .6 & .7 & .7 & 1.0 & 1.6 & 2.4 & 30.6 \\
\hline $\begin{array}{l}1959 \\
\end{array}$ & .4 & .4 & .4 & .4 & .5 & .6 & 1.1 & $2 \cdot 3$ & 5.3 & 7.1 & 20.0 \\
\hline 1960 & .6 & .8 & .6 & .7 & .8 & .9 & .9 & 1.2 & 1.8 & 10.6 & 39.0 \\
\hline 1961 & .4 & .4 & .5 & .7 & .7 & .8 & .8 & $1 \cdot 1$ & 1.6 & 2.6 & 24.1 \\
\hline 1962 & .4 & .4 & .5 & .6 & .7 & .9 & 1.1 & 2.0 & 3.2 & 9.0 & 36.6 \\
\hline
\end{tabular}

Johnson Creek at Sycamore, Oreg.

STATION NUMBER $14-2115.00$ HIGHEST MEAN DISCHARGE, IN CFS, FOR THE FOLLOWING NUMBER OF CONSECUTIVE DAYS IN YEAR FNDING SEPTEMBER 30

\begin{tabular}{|c|c|c|c|c|c|c|c|c|c|c|c|}
\hline YEAR & 1 & 3 & 7 & 15 & 30 & 60 & 90 & 120 & 150 & 183 & 274 \\
\hline 1941 & 432.0 & 339.0 & 204.0 & 141.0 & 114.0 & 100.0 & 81.7 & 68.6 & 58.4 & 49.8 & 35.8 \\
\hline 1942 & 740.0 & 579.0 & 388.0 & 235.0 & 185.0 & 134.0 & 123.0 & $108 \cdot 0$ & 90.6 & 77.3 & 56.5 \\
\hline 1943 & 1310.0 & 746.0 & 618.0 & 426.0 & 321.0 & 275.0 & 243.0 & 193.0 & 178.0 & 151.0 & 105.0 \\
\hline 1944 & 216.0 & 154.0 & 106.0 & 76.3 & 65.1 & 57.5 & 50.2 & $46 \cdot 3$ & 41.9 & 36.7 & 26.5 \\
\hline 1945 & 398.0 & 328.0 & 248.0 & 202.0 & 160.0 & 126.0 & 122.0 & 102.0 & 96.0 & 81.8 & 56.5 \\
\hline 1946 & 771.0 & 478.0 & 286.0 & 233.0 & 164.0 & 146.0 & 139.0 & $130 \cdot 0$ & 115.0 & $96 \cdot 4$ & 65.7 \\
\hline 1947 & 1110.0 & 686.0 & 486.0 & 265.0 & 227.0 & 132.0 & 126.0 & $107 \cdot 0$ & 96.8 & 82.6 & 58.3 \\
\hline 1948 & 1300.0 & $839 . n$ & 519.0 & 326.0 & $2 n 7.0$ & $153 . ?$ & 141.0 & 125.0 & 121.0 & 115.0 & 86.9 \\
\hline 1949 & 1680.0 & $869 . ?$ & 658.0 & 546.0 & 306.0 & $179 . n$ & 181.0 & $156 \cdot 0$ & 134.0 & 114.0 & 78.5 \\
\hline 1950 & 721.0 & $6 \cap 9 \cdot n$ & $372 \cdot n$ & 272.0 & $239 . n$ & 270.0 & 187.0 & 163.0 & 143.0 & 119.0 & 81.0 \\
\hline 1951 & 1050.0 & $826 . n$ & 509.0 & 363.0 & 278.0 & 216.0 & 229.0 & 202.0 & 185.0 & 156.0 & 107.0 \\
\hline 1952 & 544.0 & 406.0 & 348.0 & 226.0 & 167.0 & 130.0 & 134.0 & $124 \cdot 0$ & 113.0 & 98.6 & 67.7 \\
\hline 1953 & 844.0 & 684.0 & 504.0 & 399.0 & 328.0 & 211.0 & 171.0 & $136 \cdot 0$ & 118.0 & 102.0 & 69.6 \\
\hline 1954 & 1080.0 & 588.0 & 497.0 & 338.0 & 252.0 & 222.0 & 194.0 & 171.0 & 149.0 & 126.0 & 89.1 \\
\hline 1955 & 330.0 & 287.0 & 200.0 & 177.0 & 145.0 & 111.0 & 93.7 & $91 \cdot 4$ & $88 \cdot 3$ & 83.5 & 58.6 \\
\hline 1956 & 1320.0 & 740.0 & 497.0 & 398.0 & 343.0 & 298.0 & 243.0 & $220 \cdot 0$ & 197.0 & 169.0 & 116.0 \\
\hline 1957 & 1110.0 & 749.0 & 490.0 & 325.0 & 230.0 & 154.0 & 126.0 & 119.0 & 103.0 & 92.6 & 64.0 \\
\hline 1958 & 676.0 & 550.0 & 360.0 & 245.0 & 212.0 & 192.0 & 160.0 & $129 \cdot 0$ & $124 \cdot 0$ & $104 \cdot 0$ & 70.6 \\
\hline 1959 & 600.0 & 472.0 & 354.0 & 221.0 & 201.0 & 161.0 & 140.0 & $127 \cdot 0$ & 113.0 & 97.9 & 69.2 \\
\hline 1960 & 485.0 & 436.0 & 290.0 & 214.0 & 167.0 & 140.0 & 125.0 & $109 \cdot 0$ & 101.0 & 93.0 & 68.1 \\
\hline 1961 & 1360.0 & 867.0 & 539.0 & 353.0 & 268.0 & 207.0 & 167.0 & 163.0 & 143.0 & 133.0 & 90.5 \\
\hline 1962 & 540.0 & 437.0 & 305.0 & 199.0 & 132.0 & 110.0 & 94.1 & $95 \cdot 9$ & 89.4 & 80.4 & 57.0 \\
\hline 1963 & 1090.0 & 560.0 & 419.0 & 295.0 & 181.0 & 142.0 & 113.0 & 107.0 & 106.0 & 108.0 & 75.3 \\
\hline
\end{tabular}

